\title{
Gazetteer of Planetary Nomenclature 1994
}

\section{U.S. GEOLOGICAL SURVEY BULLETIN 2129}

Prepared in cooperation with the International Astronomical Union-Working Group for

Planetary System Nomenclature

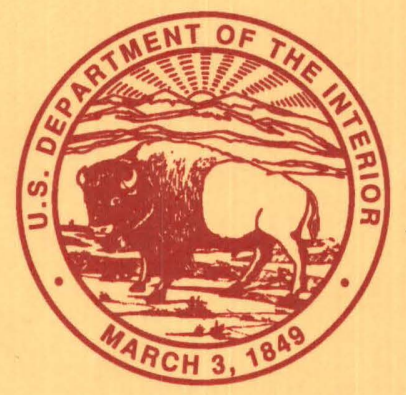





\section{Gazetteer of Planetary Nomenclature 1994}

Edited by Raymond M. Batson and Joel F. Russell

\section{U.S. GEOLOGICAL SURVEY BULLETIN 2129}

Prepared in cooperation with the International Astronomical Union-Working Group for

Planetary System Nomenclature

Information about all names of topographic and albedo features on planets and satellites that the International Astronomical Union has approved from its founding in 1919 through its triennial meeting in 1994 


\title{
U.S. DEPARTMENT OF THE INTERIOR BRUCE BABBITT, Secretary
}

\author{
U.S. GEOLOGICAL SURVEY \\ GORDON P. EATON, Director
}

For sale by U.S. Geological Survey, Information Services

Box 25286, Federal Center, Denver, CO 80225 Any use of trade, product, or firm names in this publication is for descriptive purposes only and
does not imply endorsement by the U.S. Government.

Published in the Eastern Region, Reston, Va.

Manuscript approved for publication February 14, 1995

Library of Congress Cataloging in Publication Data

Gazetteer of planetary nomenclature 1994 / edited by Raymond M. Batson and Joel F.

Russell ; prepared in cooperation with the International Astronomical Union-Working

Group for Planetary System Nomenclature.

p. $\quad \mathrm{cm}$. - (U.S. Geological Survey bulletin ; 2129)

"Information about all names of topographic and albedo features on planets and satellites

that the International Astronomical Union has approved from its founding in 1919

through its triennial meeting in 1994."

Includes bibliographical references.

Supt. of Doc. no.: I 19.3:2129

1. Planets-Nomenclature-Handbooks, manuals, etc. 2. Planets-Names-Handbooks,

manuals, etc. I. Batson, Raymond M. II. Russell, Joel F. III. Series.

QE75.B9 no. 2129

[QB600.5]

$557.3 \mathrm{~s}-\mathrm{dc} 20$

[919.902'03] 


\section{PREFACE}

Planetary nomenclature, like terrestrial nomenclature, is used to uniquely identify a feature on the surface of a planet or satellite so that the feature can be easily located, described, and discussed. This volume contains detailed information about all names of topographic and albedo features on planets and satellites (and some planetary ring and ring-gap systems) that the International Astronomical Union has named and approved from its founding in 1919 through its triennial meeting in 1994.

This edition of the Gazetteer of Planetary Nomenclature supersedes an earlier informal volume distributed by the U.S. Geological Survey in 1986 as Open-File Report 84-692 (Masursky and others, 1986). Named features are depicted on maps of the Moon published first by the U.S. Defense Mapping Agency or the Aeronautical Chart and Information Center and more recently by the U.S. Geological Survey; on maps of Mercury, Venus, Mars, and the satellites of Jupiter, Saturn, and Uranus published by the U.S. Geological Survey; and on maps of the Moon, Venus, and Mars produced by the U.S.S.R.

Although we have attempted to check the accuracy of all data in this volume, we realize that some errors will remain in a work of this size. Readers noting errors or omissions are urged to communicate them to the U.S. Geological Survey, Branch of Astrogeology, Rm. 409, 2255 N. Gemini Drive, Flagstaff, AZ 86001. 



\section{CONTENTS}

\begin{tabular}{|c|c|}
\hline & \\
\hline 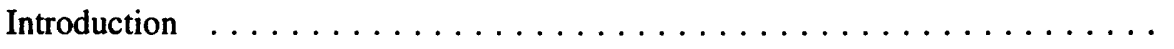 & \\
\hline History of Planetary Nomenclature $\ldots \ldots \ldots \ldots \ldots \ldots$ & \\
\hline How Names Are Approved . . . . & \\
\hline IAU Rules and Conventions. & \\
\hline Naming Conventions . . . . . & \\
\hline Specifics of the Gazetteer . . & \\
\hline Acknowledgments . . . . . & \\
\hline References Cited & \\
\hline Listed by Planet, Satellite, and Feature Type $\ldots \ldots \ldots \ldots$ & \\
\hline$\ldots \ldots \ldots \ldots \ldots \ldots \ldots \ldots \ldots \ldots \ldots \ldots$ & \\
\hline Venus . & \\
\hline$\ldots \ldots \ldots \ldots \ldots \ldots \ldots \ldots \ldots \ldots$ & \\
\hline Mars. & \\
\hline Asteroids & \\
\hline Jovian & \\
\hline Saturnian System & \\
\hline Uraniar & \\
\hline Neptur & \\
\hline Section 2: A & \\
\hline ing Group and Task Groups & \\
\hline Appendix 2. IAU Task & \\
\hline and for Countries or Ethnic Groups & \\
\hline & \\
\hline Appendix 4 & \\
\hline Appendix 5. Descriptor Terms (Feature Types) ...... & \\
\hline Appendix 6. Categories for Naming Features on the Planets and Satellites & \\
\hline Appendix 7. Planet and Satellite Names and Discoverers $\ldots \ldots \ldots \ldots$ & \\
\hline
\end{tabular}

\section{FIGURES}

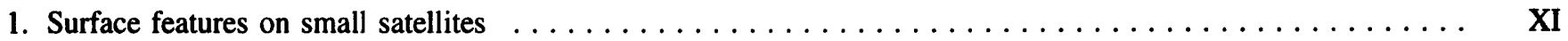

2. Ring-system nomenclature of Saturn, Uranus, and Neptune $\ldots \ldots \ldots \ldots \ldots \ldots \ldots \ldots \ldots \ldots \ldots \ldots$

\section{TABLES}

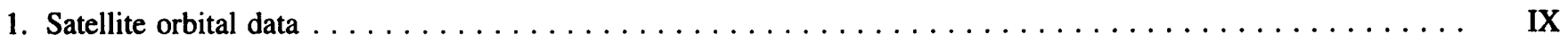

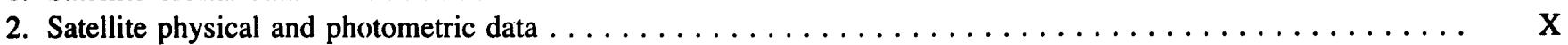





\title{
Gazetteer of Planetary Nomenclature 1994
}

\author{
Edited by Raymond M. Batson and Joel F. Russell
}

\section{INTRODUCTION}

\section{HISTORY OF PLANETARY NOMENCLATURE}

The International Astronomical Union (IAU) has been the arbiter of planetary and satellite nomenclature since its organizational meeting in 1919 in Brussels, Belgium. At that time a committee was appointed to regularize the chaotic lunar and Martian nomenclatures then current. The IAU committee was an outgrowth of an earlier committee established in 1907 by the Council of the International Association of Academies, meeting in General Assembly in Vienna, Austria. This committee had been charged with the task of clarifying the lunar nomenclature but had not published a report, due to a succession of deaths of members. However, a great deal of preliminary work had been done by one member, Mary Blagg.

The IAU appointed Miss Blagg and several other astronomers to the newly commissioned nomenclature committee, chaired by H.H. Turner (IAU, 1922). The report of this committee, "Named Lunar Formations" by Blagg and Müller (1935), was the first systematic listing of lunar nomenclature. Later, "The System of Lunar Craters, quadrants I, II, III, IV" was published by D.W.G. Arthur and others $(1963,1964,1965,1966)$, under the direction of Gerard P. Kuiper. These catalogues listed the names (or other designations) and coordinates of features in the current, greatly expanded lunar nomenclature; the accompanying map (also in four parts) showed their locations. These works were adopted by the IAU and became the recognized sources for lunar nomenclature.

The Martian nomenclature was clarified in 1958, when an ad hoc committee of the IAU chaired by Audouin Dollfus recommended for adoption the names of 128 albedo features (bright, dark, or colored) observed through ground-based telescopes (IAU, 1960). These names were based on a system of nomenclature developed in the late 19 th century by the Italian astronomer G.V. Schiaparelli (1879) and expanded in the early 20th century by E.M. Antoniadi (1930), an Italian-born astronomer working at Meudon, France.

The requirements for extraterrestrial nomenclature were dramatically changed in 1957 when the age of space exploration was inaugurated by the successful flight of Sputnik and by America's consequent determination to land a man on the Moon in the 1960's. As detailed images became available of one newly discriminated extraterrestrial surface after another, the need to name features on these surfaces became evident. Once again the IAU assumed the task of expanding and overseeing planetary nomenclature so that the effort would proceed in an orderly, fair, and evenhanded way.

In 1970, in response to the successful Mariner flyby missions to Mars during the 1960 's and in anticipation of the Mariner 9 Mars Orbiter, a Mars nomenclature working group was formed, chaired by Gerard de Vaucouleurs; this group was asked to designate names for the topographic features shown in the new spacecraft images (de Vaucouleurs and others, 1975). At about the same time, Donald $\mathrm{H}$. Menzel chaired an ad hoc lunar committee that suggested names for features discriminated by the Soviet Zond and American Lunar Orbiter and Apollo cameras (IAU, 1971).

At the 1973 meeting of the IAU in Sydney, Australia, the nomenclature groups were reorganized and expanded. The Working Group for Planetary System Nomenclature (WGPSN) was appointed with Peter Millman of Canada as its first president. Task groups for the Moon, Mercury, Venus, Mars, and the Outer Solar System were formed to conduct the preliminary work of choosing themes and proposing names for features on each newly discriminated planet and satellite. In 1982 at Patras, Greece, Harold Masursky of the United States became president of the WGPSN; he was succeeded in 1991 by Kaare Aksnes of Norway. A new task group was formed in 1984 to name surface features on small primitive bodies (asteroids and comets).

\section{HOW NAMES ARE APPROVED}

When images are first obtained of the surface of a planet or satellite, a theme for naming features is chosen, and a few important 
features are named, usually by members of the appropriate IAU task group. Later, as higher resolution images and maps become available, additional features are named, usually at the request of investigators mapping or describing specific surfaces, features, or geologic formations. However, anyone - either scientist or layman-may suggest a name or ask that a specific feature be named. Names considered appropriate by a task group are submitted to the WGPSN, which meets once a year. The WGPSN transmits its list of recommended names to the yearly meeting of the IAU's Executive Committee, which checks the names for conformity to IAU standards. Successful candidate names are then presented for adoption to the IAU's General Assembly, which meets triennially. A name is not considered to be official-that is, "adopted"-until the General Assembly has given its approval. Names approved by the Executive Committee, but not yet adopted by the General Assembly, are given "provisional" status. Provisional names may be published, so long as their status is noted in the publication.

Suggestions for naming a specific feature or requests that a specific name be used should be sent to the president of the WGPSN or to the chairman of the appropriate task group (see Appendixes 1 and 2 of this volume) or to the U.S. Geological Survey, Branch of Astrogeology in Flagstaff, Ariz.

\section{IAU RULES AND CONVENTIONS}

Names adopted by the IAU must follow various rules and conventions established through the years by the Union. At the first meeting of the WGPSN, the following rules were adopted (paraphrased and condensed from IAU, 1977):

1. Nomenclature is a tool and the first consideration should be to make it simple, clear, and unambiguous. As corollaries to rule 1, the WGPSN decided that names should be easy to pronounce and spell and that single names of no more than three syllables are preferred. Exceptions are allowed for persons or mythical characters known by double names.

2. The number of names chosen for each body should be kept to a minimum and their placement governed by the requirements of the scientific community.

3. Duplication of the same name on two or more bodies is to be avoided. The nearly 2,000 names of asteroids (not included in this volume) are excepted from this rule.

4. Individual names chosen for each body should be expressed in the language of origin. Transliteration and pronunciation for various alphabets should be given, but there will be no translation from one language to another. Most planetary maps are published in the United States; however, maps published in other countries use the language of that country. Maps published in Russian use the Cyrillic typescript; (romanized) Latin and Greek terms are translated, and names are transliterated.

5. Where possible, the practice established by early lunar nomenclature should be used, in which descriptors (feature types) are written (except in Russian usage) in their Latin or Greek form. This rule has been invoked by the IAU when establishing a theme for naming features on newly discriminated satellites or planets. Thus, newly discovered Uranian satellites and features on previously discovered satellites continued the theme established by William Lassell when he named the first four satellites for characters (mostly bright and dark spirits) from Shakespeare and Pope; names for satellites of Neptune continue the "watery" theme established by the names of the planet and first two satellites discovered.

6. Solar system nomenclature should be international in its choice of names. Recommendations submitted to the IAU national committees will be considered, but final approval of any selection is the responsibility of the IAU. The WGPSN strongly supports equitable representation of ethnic groups/countries on each individual map; however, a higher percentage of names from the country planning a landing is allowed on landing site maps.

7. We must look to the future in general discussions of solar system nomenclature and attempt to lay the groundwork for future requirements that will result from the development of the space program.

Additional rules developed since 1976 include the following:

8. No names having political, military or religious significance, or names of modern philosophers, may be used. Names of political figures prior to the 19th century are acceptable.

9. Persons being honored must have been deceased for at least 3 years before his or her name can be assigned to a feature. Exceptions to this rule were made for living astronauts and cosmonauts because their contributions to space exploration were unique.

10. When more than one spelling of a name is extant, the spelling preferred by the person, or referenced in Appendix 4, is used.

11. Diacritical marks are a necessary part of a name and will be used.

12. Ring and ring-gap nomenclature and names for newly discovered small satellites (tables 1 and 2, figs. 1 and 2) are developed by joint deliberation of the Working Group and Commission 20 , which is in charge of naming asteroids and comets. Names will not be assigned to newly discovered satellites until their orbital elements are reasonably well known or definite features have been identified on them. Duplications between the names of asteroids and satellites should be minimized.

In addition to these general rules, each task group develops additional conventions as it formulates an interesting and meaningful nomenclature for individual planetary bodies. Most of these conventions are self evident from study of the appendixes that follow. 
Table 1. Satellite orbital data ${ }^{1}$

\begin{tabular}{|c|c|c|c|c|c|c|c|c|c|}
\hline \multirow{2}{*}{$\begin{array}{l}\text { Planet } \\
\text { Earth }\end{array}$} & \multicolumn{2}{|c|}{ Satellite } & \multirow{2}{*}{$\begin{array}{c}\begin{array}{c}\text { Orbital period }^{2} \\
\mathbf{R}=\text { retrograde }^{\text {(days) }}\end{array} \\
27.321661\end{array}$} & \multicolumn{2}{|c|}{$\begin{array}{c}\text { Max } \\
\text { elongation } \\
\text { at mean } \\
\text { opposition }\end{array}$} & \multirow{2}{*}{$\begin{array}{c}\begin{array}{c}\text { Semi-major } \\
\text { axis } \\
\left(\times 10^{3} \mathrm{~km}\right)\end{array} \\
384.40\end{array}$} & \multirow{2}{*}{$\begin{array}{c}\begin{array}{c}\text { Orbital } \\
\text { eccentricity }\end{array} \\
0.0549004\end{array}$} & \multirow{2}{*}{$\begin{array}{c}\begin{array}{c}\text { Orbital } \\
\text { inclination } \\
\text { to planetary } \\
\text { equator }\left({ }^{\circ}\right)\end{array} \\
18.2-28.6\end{array}$} & \multirow{2}{*}{$\begin{array}{c}\begin{array}{c}\text { Motion of } \\
\text { node on } \\
\text { fixed plane } \\
(\% / y r)\end{array} \\
{ }^{4} 19.34\end{array}$} \\
\hline & & Moon & & 。 & ' & & & & \\
\hline Mars & $\begin{array}{l}\text { l } \\
\text { II }\end{array}$ & $\begin{array}{l}\text { Phobos } \\
\text { Deimos }\end{array}$ & $\begin{array}{l}0.31891023 \\
1.2624407\end{array}$ & & $\begin{array}{r}2 \\
1 \quad 0\end{array}$ & $\begin{array}{r}9.38 \\
23.46\end{array}$ & $\begin{array}{l}0.015 \\
0.0005\end{array}$ & $\begin{array}{l}1.0 \\
0.9-2.7\end{array}$ & $\begin{array}{l}158.8 \\
6.614\end{array}$ \\
\hline Jupiter & $\begin{array}{l}\text { I } \\
\text { II } \\
\text { III } \\
\text { IV } \\
\text { V } \\
\text { VI } \\
\text { VII } \\
\text { VIII } \\
\text { IX } \\
\text { X } \\
\text { XI } \\
\text { XII } \\
\text { XIII } \\
\text { XIV } \\
\text { XV } \\
\text { XVI }\end{array}$ & $\begin{array}{l}\text { Io } \\
\text { Europa } \\
\text { Ganymede } \\
\text { Callisto } \\
\text { Amalthea } \\
\text { Himalia } \\
\text { Elara } \\
\text { Pasiphae } \\
\text { Sinope } \\
\text { Lysithea } \\
\text { Carme } \\
\text { Ananke } \\
\text { Leda } \\
\text { Thebe } \\
\text { Adrastea } \\
\text { Metis }\end{array}$ & $\begin{array}{c}1.76913778 \\
3.55118104 \\
7.15455296 \\
16.6890184 \\
0.49817905 \\
250.5662 \\
259.6528 \\
735 \mathrm{R} \\
758 \mathrm{R} \\
259.22 \\
692 \mathrm{R} \\
631 \mathrm{R} \\
238.72 \\
0.6745 \\
0.29826 \\
0.29478\end{array}$ & $\begin{array}{l}1 \\
1 \\
2 \\
2 \\
2 \\
1 \\
2 \\
1 \\
1\end{array}$ & $\begin{array}{rr}2 & 18 \\
3 & 4 \\
5 & 5 \\
10 & 18 \\
& 5 \\
02 & 4 \\
04 & 1 \\
08 & 26 \\
09 & 3 \\
04 & 0 \\
03 & 3 \\
55 & 5 \\
00 & 3 \\
1 & 1 \\
& 4 \\
& 42\end{array}$ & $\begin{array}{r}422 \\
671 \\
1070 \\
1883 \\
181 \\
11480 \\
11737 \\
23500 \\
23700 \\
11720 \\
22600 \\
21200 \\
11094 \\
222 \\
129 \\
128\end{array}$ & $\begin{array}{l}0.004 \\
0.009 \\
0.002 \\
0.007 \\
0.003 \\
0.1579 \\
0.2072 \\
0.378 \\
0.275 \\
0.107 \\
0.2068 \\
0.1687 \\
0.1476 \\
0.015 \\
\sim 0.0 \\
<0.004\end{array}$ & $\begin{array}{c}0.04 \\
0.47 \\
0.21 \\
0.51 \\
0.40 \\
27.63 \\
24.77 \\
145 \\
153 \\
29.02 \\
164 \\
147 \\
26.07 \\
0.8 \\
-0.0 \\
\sim 0.0\end{array}$ & $\begin{array}{l}48.6 \\
12.0 \\
2.63 \\
0.643 \\
914.6\end{array}$ \\
\hline Saturn & $\begin{array}{l}\text { I } \\
\text { II } \\
\text { III } \\
\text { IV } \\
\text { V } \\
\text { VI } \\
\text { VII } \\
\text { VIII } \\
\text { IX } \\
\text { X } \\
\text { XI } \\
\text { XII } \\
\text { XIII } \\
\text { XIV } \\
\text { XV } \\
\text { XVI } \\
\text { XVII } \\
\text { XVIII }\end{array}$ & $\begin{array}{l}\text { Mimas } \\
\text { Enceladus } \\
\text { Tethys } \\
\text { Dione } \\
\text { Rhea } \\
\text { Titan } \\
\text { Hyperion } \\
\text { lapetus } \\
\text { Phoebe } \\
\text { Janus } \\
\text { Epimetheus } \\
\text { Helene } \\
\text { Telesto } \\
\text { Calypso } \\
\text { Atlas } \\
\text { Prometheus } \\
\text { Pandora } \\
\text { Pan }\end{array}$ & $\begin{array}{c}0.94242181 \\
1.37021786 \\
1.88780216 \\
2.73691474 \\
4.51750044 \\
15.94542068 \\
21.2766088 \\
79.3301825 \\
550.48 \mathrm{R} \\
0.6945 \\
0.6942 \\
2.7369 \\
1.8878 \\
1.8878 \\
0.6019 \\
0.6130 \\
0.6285 \\
0.5750\end{array}$ & & $\begin{array}{rr} & 3 \\
& 38 \\
& 48 \\
1 & 0 \\
1 & 2 \\
3 & 17 \\
3 & 5 \\
9 & 3 \\
34 & 5 \\
& 2 \\
& 2 \\
1 & 0 \\
& 48 \\
& 48 \\
& 22 \\
& 23 \\
& 23\end{array}$ & $\begin{array}{r}185.52 \\
238.02 \\
294.66 \\
377.40 \\
527.04 \\
1221.83 \\
1481.10 \\
3561.30 \\
12952 \\
151.47 \\
151.42 \\
377.40 \\
294.66 \\
294.66 \\
137.67 \\
139.35 \\
141.70 \\
133.60\end{array}$ & $\begin{array}{l}0.0202 \\
0.00452 \\
0.00000 \\
0.00223 \\
0.00100 \\
0.029192 \\
0.104 \\
0.02828 \\
0.16326 \\
0.007 \\
0.0009 \\
0.005 \\
\sim 0.0 \\
\sim 0.0 \\
0.000 \\
0.003 \\
0.004 \\
\sim 0.0\end{array}$ & $\begin{array}{c}1.53 \\
0.00 \\
1.86 \\
0.02 \\
0.35 \\
0.33 \\
0.43 \\
14.72 \\
{ }^{6} 177 . \\
0.14 \\
0.34 \\
0.00 \\
\sim 0.0 \\
\sim 0.0 \\
0.3 \\
0.0 \\
0.0 \\
0.0\end{array}$ & $\begin{array}{c}365.0 \\
{ }^{3} 156.2 \\
72.25 \\
530.85 \\
10.16 \\
{ }^{5} 0.5213\end{array}$ \\
\hline Neptune & $\begin{array}{l}\text { I } \\
\text { II } \\
\text { III } \\
\text { IV } \\
\text { V } \\
\text { VI } \\
\text { VII } \\
\text { VIII }\end{array}$ & $\begin{array}{l}\text { Triton } \\
\text { Nereid } \\
\text { Naiad } \\
\text { Thalassa } \\
\text { Despina } \\
\text { Galatea } \\
\text { Larissa } \\
\text { Proteus }\end{array}$ & $\begin{array}{c}5.8768433 \\
360.2 \\
0.2944 \\
0.3115 \\
0.3347 \\
0.4287 \\
0.5547 \\
1.1223\end{array}$ & & $\begin{array}{ll}17 \\
42 \\
3\end{array}$ & $\begin{array}{c}354.76 \\
5513.4 \\
48.23 \\
50.07 \\
52.53 \\
61.95 \\
73.55 \\
117.64\end{array}$ & $\begin{array}{l}<0.01 \\
0.7512 \\
\sim 0 \\
\sim 0 \\
\sim 0 \\
\sim 0 \\
\sim 0 \\
\sim 0\end{array}$ & $\begin{array}{c}157.345 \\
{ }^{2} 27.6 \\
4.74 \\
0.21 \\
0.07 \\
0.05 \\
0.20 \\
0.55\end{array}$ & $\begin{array}{l}0.5232 \\
0.039 \\
626 \\
551 \\
466 \\
261 \\
143 \\
0.5232\end{array}$ \\
\hline Pluto & 1 & Charon & 6.3871 & & $<$ & 19.70 & $\sim 0$ & ${ }^{7} 99$ & \\
\hline
\end{tabular}

'Taken from Astronomical Almanac, 1993.

${ }^{2}$ Sidereal periods except for satellites of Saturn, for which tropical periods are shown.

${ }^{5}$ Rate of increase in the longitude of the apse.

${ }^{3}$ Rate of decrease (or increase) in the longitude of the ascending node.

${ }^{6}$ Relative to ecliptic plane.

${ }^{4} \mathrm{O}$ en ecliptic plane.

${ }^{7}$ To equator of 1950.0 . 
Table 2. Satellite physical and photometric data'

\begin{tabular}{|c|c|c|c|c|c|c|c|c|c|c|}
\hline Planet & & lite & $\begin{array}{c}\text { Mass } \\
\text { (1/plane) }\end{array}$ & $\begin{array}{c}\text { Radius } \\
\text { (km) }\end{array}$ & $\begin{array}{c}\text { Sidereal period } \\
\text { of rotation } \\
S=\text { synchr. } \\
\text { (days) }\end{array}$ & $\begin{array}{c}\text { Geometric } \\
\text { albedo } \\
(V)^{3}\end{array}$ & $V(1,0)$ & $V_{0}$ & B-V & U-B \\
\hline Earth & & Moon & 0.0123002 & 1738 & $S$ & 0.12 & +0.21 & -12.74 & 0.92 & 0.46 \\
\hline Mars & $\begin{array}{l}\text { I } \\
\text { II }\end{array}$ & $\begin{array}{l}\text { Phobos } \\
\text { Deimos }\end{array}$ & $\begin{array}{l}1.5 \times 10^{-8} \\
3.0 \times 10^{-9}\end{array}$ & $\begin{array}{c}13.5 \times 10.8 \times 9.4 \\
7.5 \times 6.1 \times 5.5\end{array}$ & $\begin{array}{l}S \\
S\end{array}$ & $\begin{array}{l}0.06 \\
0.07\end{array}$ & $\begin{array}{l}+11.8 \\
+12.89\end{array}$ & $\begin{array}{l}11.3 \\
12.4\end{array}$ & $\begin{array}{l}0.6 \\
0.65\end{array}$ & 0.18 \\
\hline Jupiter & $\begin{array}{l}\text { I } \\
\text { II } \\
\text { III } \\
\text { IV } \\
\text { V } \\
\text { VI } \\
\text { VII } \\
\text { VIII } \\
\text { IX } \\
\text { X } \\
\text { XI } \\
\text { XII } \\
\text { XIII } \\
\text { XIV } \\
\text { XV } \\
\text { XVI }\end{array}$ & $\begin{array}{l}\text { Io } \\
\text { Europa } \\
\text { Ganymede } \\
\text { Callisto } \\
\text { Amalthea } \\
\text { Himalia } \\
\text { Elara } \\
\text { Pasiphae } \\
\text { Sinope } \\
\text { Lysithea } \\
\text { Carme } \\
\text { Ananke } \\
\text { Leda } \\
\text { Thebe } \\
\text { Adrastea } \\
\text { Metis }\end{array}$ & $\begin{array}{l}4.7 \times 10^{-5} \\
2.5 \times 10^{-5} \\
7.8 \times 10^{-5} \\
5.7 \times 10^{-5} \\
38 \times 10^{-10} \\
50 \times 10^{-10} \\
4 \times 10^{-10} \\
1 \times 10^{-10} \\
.4 \times 10^{-10} \\
.4 \times 10^{-10} \\
.5 \times 10^{-10} \\
.2 \times 10^{-10} \\
.03 \times 10^{-10} \\
4 \times 10^{-10} \\
.1 \times 10^{-10} \\
.5 \times 10^{-10}\end{array}$ & $\begin{array}{c}1815 \\
1569 \\
2631 \\
2400 \\
135 \times 83 \times 75 \\
93 \\
38 \\
25 \\
18 \\
18 \\
20 \\
15 \\
8 \\
55 \times 45 \\
12.4 \times 10 \times 7.5 \\
20\end{array}$ & $\begin{array}{c}\mathrm{S} \\
\mathrm{S} \\
\mathrm{S} \\
\mathrm{S} \\
\mathrm{S} \\
0.4 \\
0.5\end{array}$ & $\begin{array}{l}0.05 \\
0.05 \\
0.05\end{array}$ & $\begin{array}{l}-1.68 \\
-1.41 \\
-2.09 \\
-1.05 \\
+7.4 \\
+8.14 \\
+10.07 \\
+10.33 \\
+11.6 \\
+11.7 \\
+11.3 \\
+12.2 \\
+13.5 \\
+8.9 \\
+12.4 \\
+10.8\end{array}$ & $\begin{array}{l}5.02 \\
5.29 \\
4.61 \\
5.65 \\
14.1 \\
14.84 \\
16.77 \\
17.03 \\
18.3 \\
18.4 \\
18.0 \\
18.9 \\
20.2 \\
15.6 \\
19.1 \\
17.5\end{array}$ & $\begin{array}{l}1.17 \\
0.87 \\
0.83 \\
0.86 \\
1.50 \\
0.67 \\
0.69 \\
0.63 \\
0.7 \\
0.7 \\
0.7 \\
0.7 \\
0.7 \\
1.3\end{array}$ & $\begin{array}{l}1.30 \\
0.52 \\
0.50 \\
0.55\end{array}$ \\
\hline Saturn & $\begin{array}{l}\text { I } \\
\text { II } \\
\text { III } \\
\text { IV } \\
\text { V } \\
\text { VI } \\
\text { VII } \\
\text { VIII } \\
\text { IX } \\
\text { X } \\
\text { XI } \\
\text { XII } \\
\text { XIII } \\
\text { XIV } \\
\text { XV } \\
\text { XVI } \\
\text { XVII }\end{array}$ & $\begin{array}{l}\text { Mimas } \\
\text { Enceladus } \\
\text { Tethys } \\
\text { Dione } \\
\text { Rhea } \\
\text { Titan } \\
\text { Hyperion } \\
\text { lapetus } \\
\text { Phoebe } \\
\text { Janus } \\
\text { Epimetheus } \\
\text { Helene } \\
\text { Telesto } \\
\text { Calypso } \\
\text { Atlas } \\
\text { Prometheus } \\
\text { Pandora }\end{array}$ & $\begin{array}{l}8.0 \times 10^{-8} \\
1.3 \times 10^{-7} \\
1.3 \times 10^{-6} \\
1.8 \times 10^{-6} \\
4.4 \times 10^{-6} \\
2.4 \times 10^{-4} \\
3 \times 10^{-8} \\
3.3 \times 10^{-6} \\
7 \times 10^{-10}\end{array}$ & $\begin{array}{c}196 \\
250 \\
530 \\
560 \\
765 \\
2575 \\
205 \times 130 \times 110 \\
730 \\
110 \\
110 \times 100 \times 80 \\
70 \times 60 \times 50 \\
18 \times 16 \times 15 \\
17 \times 14 \times 13 \\
17 \times 11 \times 11 \\
20 \times 10 \\
70 \times 50 \times 40 \\
55 \times 45 \times 35\end{array}$ & $\begin{array}{c}S \\
S \\
S \\
S \\
S \\
S \\
\\
S \\
0.4 \\
S \\
S\end{array}$ & $\begin{array}{l}0.5 \\
1.0 \\
0.9 \\
0.7 \\
0.7 \\
0.21 \\
0.3 \\
0.2^{4} \\
0.06 \\
0.8 \\
0.8 \\
0.7 \\
0.5 \\
0.6 \\
0.9 \\
0.6 \\
0.9\end{array}$ & $\begin{array}{l}+3.3 \\
+2.1 \\
+0.6 \\
+0.8 \\
+0.1 \\
-1.28 \\
+4.63 \\
+1.5 \\
+6.89 \\
+4.4 \\
+5.4 \\
+8.4 \\
+8.9 \\
+9.1 \\
+8.4 \\
+6.4 \\
+6.4\end{array}$ & $\begin{array}{l}12.9 \\
11.7 \\
10.2 \\
10.4 \\
9.7 \\
8.28 \\
14.19 \\
11.1 \\
16.45 \\
14 \\
15 \\
18 \\
18.5 \\
18.7 \\
18 \\
16 \\
16\end{array}$ & $\begin{array}{l}0.70 \\
0.73 \\
0.71 \\
0.78 \\
1.28 \\
0.78 \\
0.72 \\
0.70\end{array}$ & $\begin{array}{l}0.28 \\
0.30 \\
0.31 \\
0.38 \\
0.75 \\
0.33 \\
0.30 \\
0.34\end{array}$ \\
\hline Uranus & $\begin{array}{l}\text { I } \\
\text { II } \\
\text { III } \\
\text { IV } \\
\text { V } \\
\text { VI } \\
\text { VII } \\
\text { VIII } \\
\text { IX } \\
\text { X } \\
\text { XI } \\
\text { XII } \\
\text { XIII } \\
\text { XIV } \\
\text { XV }\end{array}$ & $\begin{array}{l}\text { Ariel } \\
\text { Umbriel } \\
\text { Titania } \\
\text { Oberon } \\
\text { Miranda } \\
\text { Cordelia } \\
\text { Ophelia } \\
\text { Bianca } \\
\text { Cressida } \\
\text { Desdemona } \\
\text { Juliet } \\
\text { Portia } \\
\text { Rosalinda } \\
\text { Belinda } \\
\text { Puck }\end{array}$ & $\begin{array}{l}1.8 \times 10^{-5} \\
1.2 \times 10^{-5} \\
6.8 \times 10^{-5} \\
6.9 \times 10^{-5} \\
0.2 \times 10^{-5}\end{array}$ & $\begin{array}{c}579 \\
586 \\
790 \\
762 \\
240 \\
13 \\
15 \\
21 \\
31 \\
27 \\
42 \\
54 \\
27 \\
33 \\
77\end{array}$ & $\begin{array}{c}S \\
S \\
S \\
S \\
S \\
S \\
S \\
.464 \\
.559 \\
S \\
S \\
S \\
S \\
S \\
S\end{array}$ & $\begin{array}{l}0.34 \\
0.18 \\
0.27 \\
0.24 \\
0.27 \\
0.07 \\
0.07 \\
0.07 \\
0.07 \\
0.07 \\
0.07 \\
0.07 \\
0.07 \\
0.07 \\
0.07\end{array}$ & $\begin{array}{l}+1.45 \\
+2.10 \\
+1.02 \\
+1.23 \\
+3.6 \\
+11.4 \\
+11.1 \\
+10.3 \\
+9.5 \\
+9.8 \\
+8.8 \\
+8.3 \\
+9.8 \\
+9.4 \\
+7.5\end{array}$ & $\begin{array}{l}14.16 \\
14.81 \\
13.73 \\
13.94 \\
16.3 \\
24.1 \\
23.8 \\
23.0 \\
22.2 \\
22.5 \\
21.5 \\
21.0 \\
22.5 \\
22.1 \\
20.2\end{array}$ & $\begin{array}{l}0.65 \\
0.68 \\
0.70 \\
0.68\end{array}$ & $\begin{array}{l}0.28 \\
0.20\end{array}$ \\
\hline Neptune & $\begin{array}{l}\text { I } \\
\text { II } \\
\text { III } \\
\text { IV } \\
\text { V } \\
\text { VI } \\
\text { VII } \\
\text { VIII }\end{array}$ & $\begin{array}{l}\text { Triton } \\
\text { Nereid } \\
\text { Naiad } \\
\text { Thalassa } \\
\text { Despina } \\
\text { Galatea } \\
\text { Larissa } \\
\text { Proteus }\end{array}$ & $\begin{array}{r}2.1 \times 10^{-4} \\
2 \times 10^{-7}\end{array}$ & $\begin{array}{c}1350 \\
170 \\
27 \\
40 \\
75 \\
80 \\
104 \times 89 \\
218 \times 208 \times 201\end{array}$ & $\begin{array}{l}\mathrm{S} \\
\mathrm{S} \\
\mathrm{S} \\
\mathrm{S} \\
\mathrm{S} \\
\mathrm{S} \\
\mathrm{S}\end{array}$ & $\begin{array}{l}0.7 \\
0.4 \\
0.06 \\
0.06 \\
0.05 \\
0.06 \\
0.06 \\
0.06\end{array}$ & $\begin{array}{l}-1.24 \\
+4.0 \\
+10.0 \\
+9.1 \\
+7.9 \\
+7.6 \\
+7.3 \\
+5.6\end{array}$ & $\begin{array}{l}13.47 \\
18.7 \\
24.7 \\
23.8 \\
22.6 \\
22.3 \\
22.0 \\
20.3\end{array}$ & $\begin{array}{l}0.72 \\
0.65\end{array}$ & 0.29 \\
\hline Pluto & 1 & Charon & 0.22 & 593 & S & 0.5 & +0.9 & 16.8 & & \\
\hline
\end{tabular}

'Taken from Astronomical Almanac, 1993.

${ }^{2}$ Rotation period same as orbital period.

${ }^{3} V($ Sun $)=-26.8$.

${ }^{4}$ Bright side 0.5 ; faint side 0.05 . 


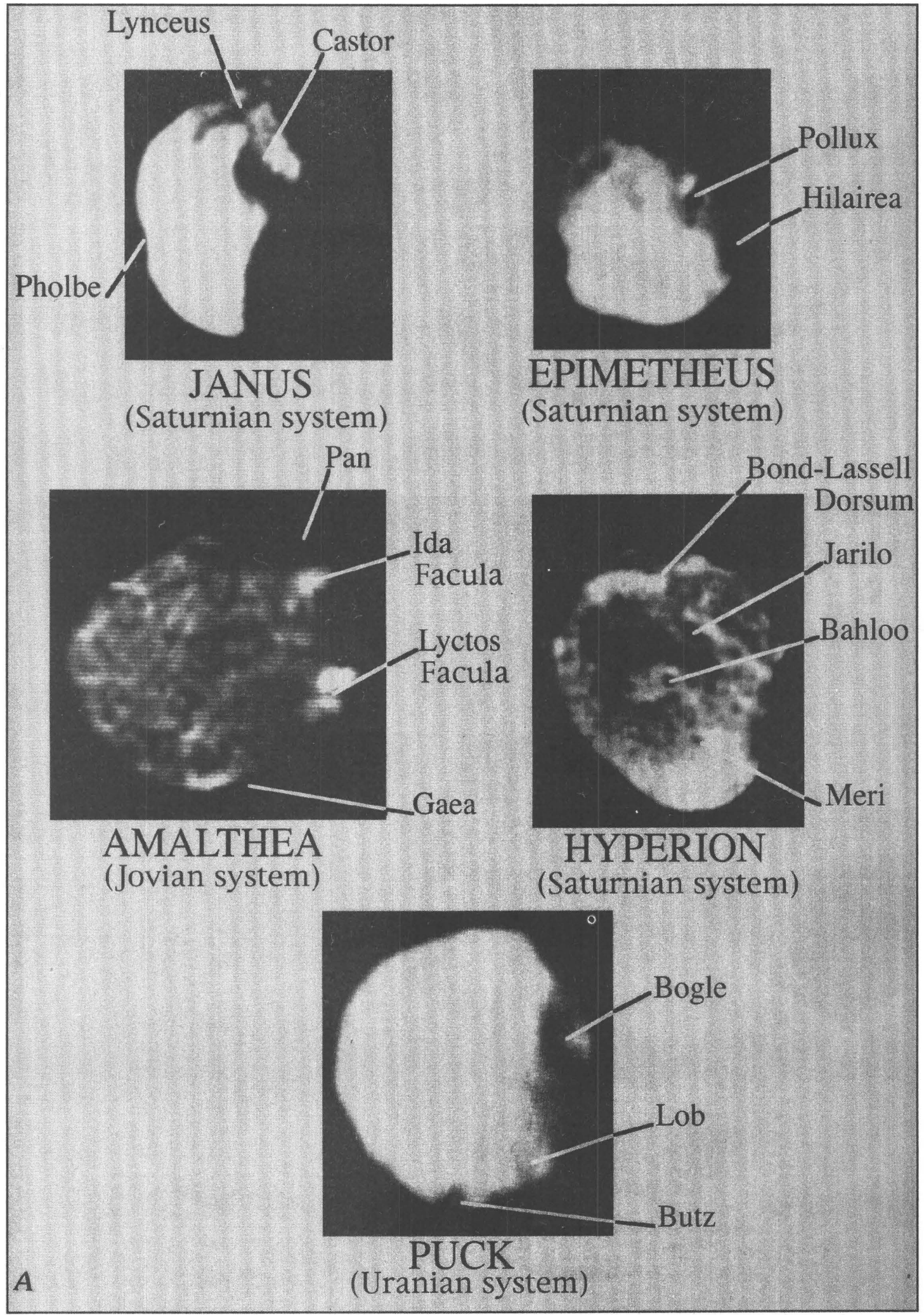

Figure 1A. Surface féatures on small satellites. Note: Images are not at same scale. 


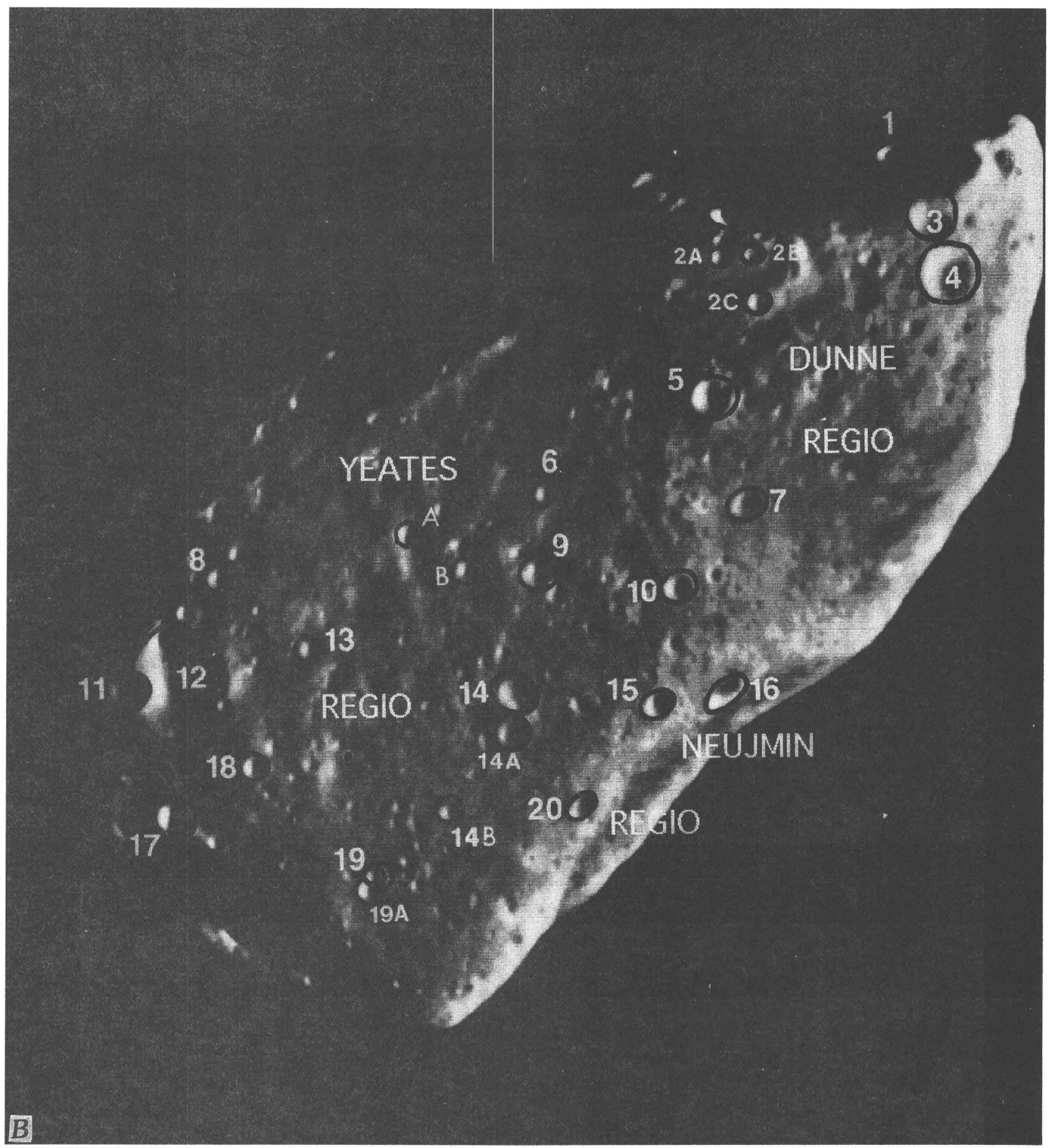

Figure 1B. Surface features on asteroid Gaspra.

Craters:
A. Alupka
4. Bath
11. Aix
B. Katsiveli
5. Rio Hondo
12. Spa
16. Beppu
1. Lisdoonvarna
6. Krynica
13. Bagnoles
2A. Calistoga
7. Rotorua
14. Marienbad
Moree
2B. Badgastein
14A. Carlsbad
18. Loutraki
2C. Yalova
8. Manikaran
14B. Brookton
19. Helwan
9. Baden-Baden
19A. Zohar
20. Ixtapan

3. Ramlosa

15. Miskhor 


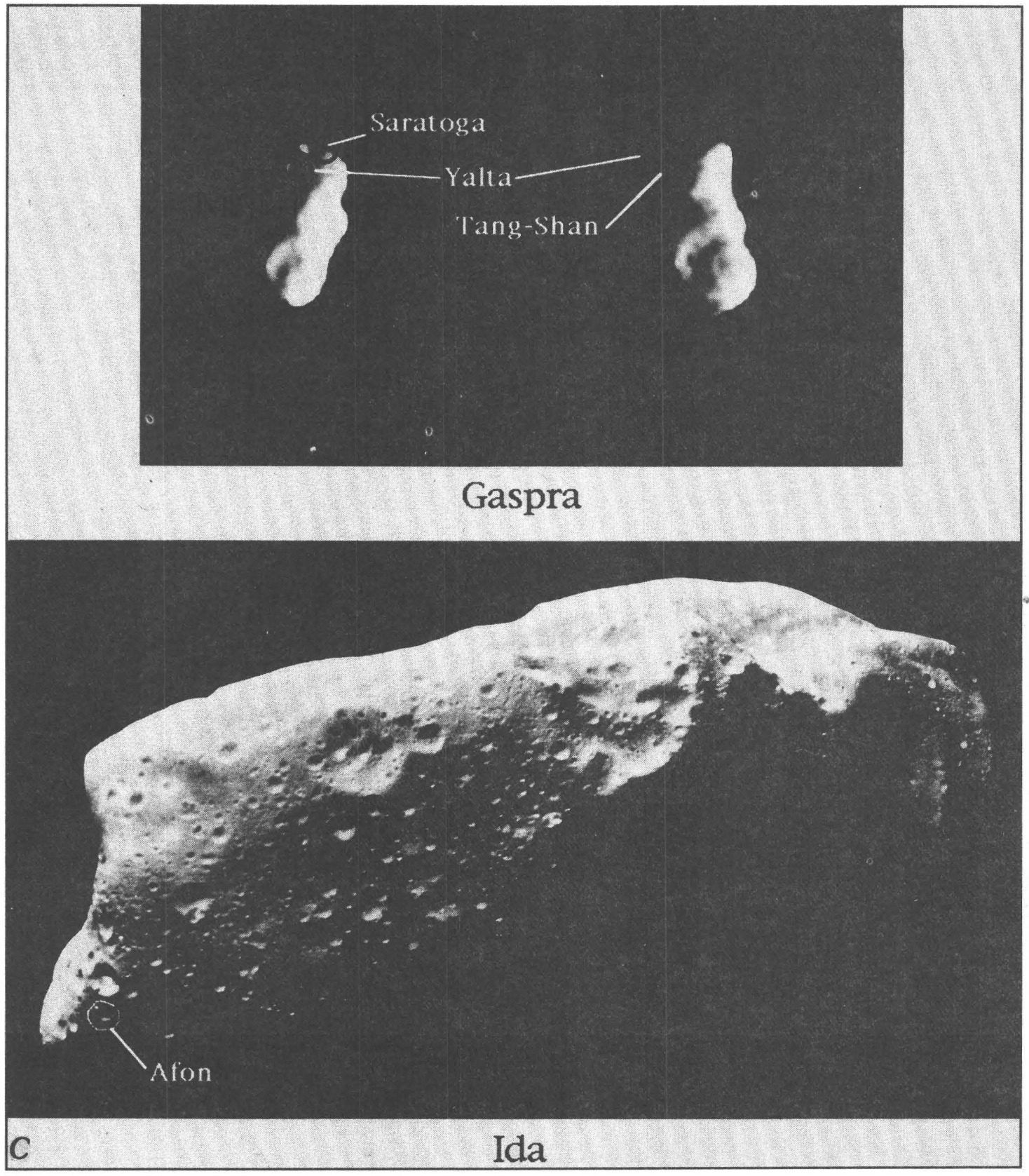

Figure 1C. Surface features on asteroids Gaspra and Ida. 


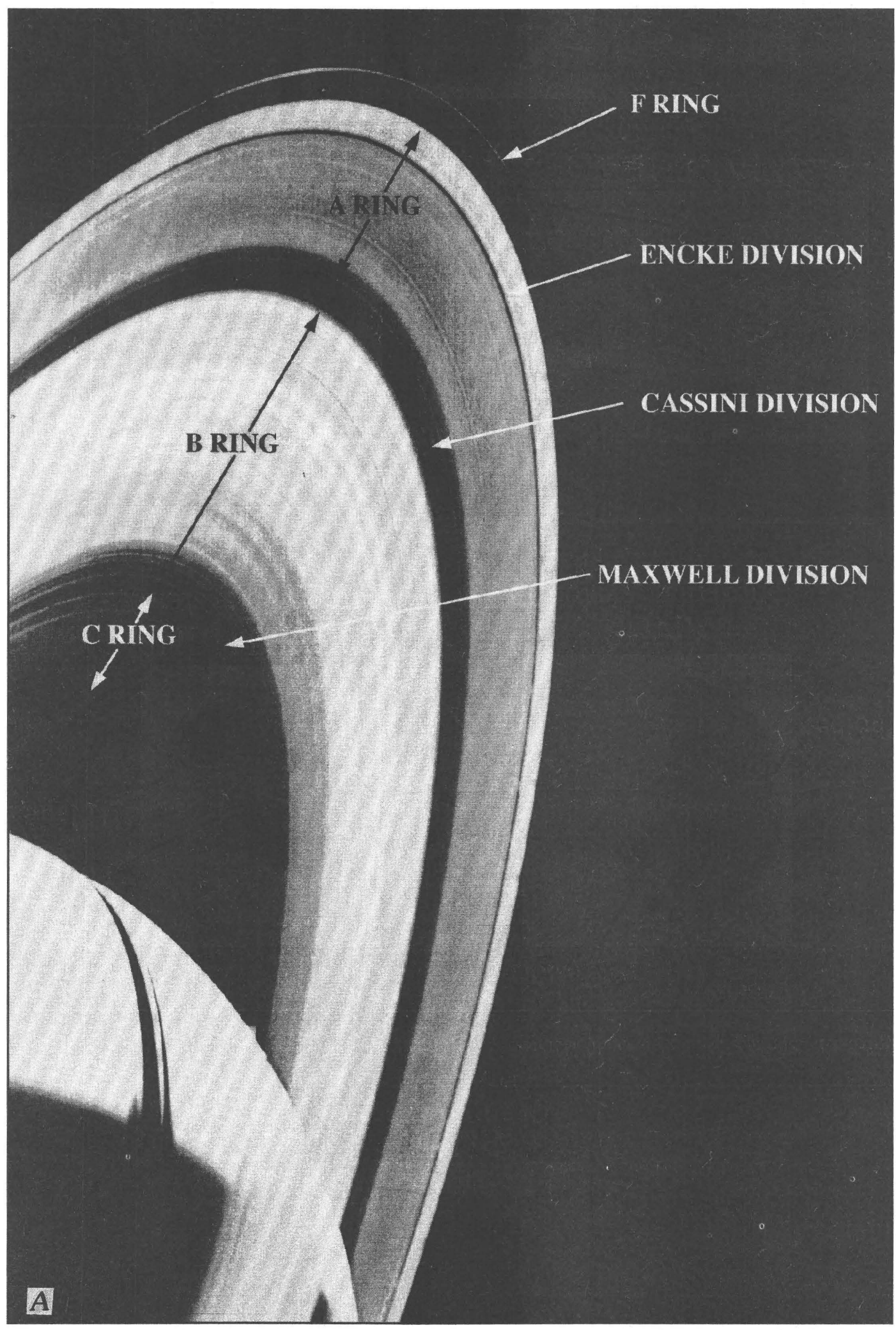

Figure 2A. Ring-system nomenclature of Saturn. 


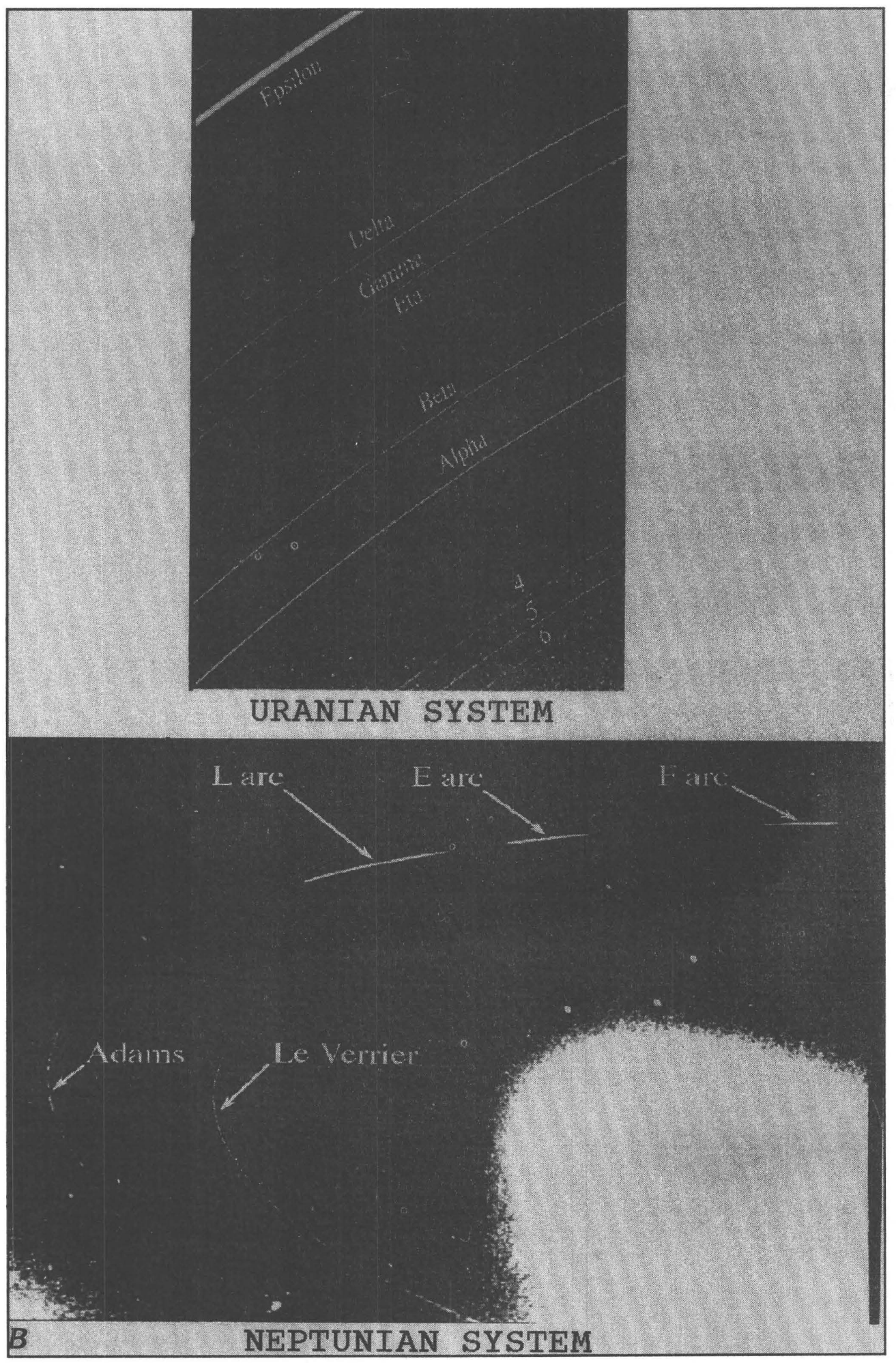

Figure $2 \boldsymbol{B}$. Ring-system nomenclature of Uranus and Neptune. 


\section{NAMING CONVENTIONS}

Names for all planetary features include a descriptor term (Appendix 5), with the exception of two feature types. For craters, the descriptor term is implicit. Some features named on Io and Triton do not carry a descriptor term because they are ephemeral.

In general, the naming convention for a feature type remains the same regardless of its size. Exceptions to this rule are channels (valles) on Mars and craters on the Moon, Mars, and Venus; naming conventions for these features differ according to size. The categories for naming features on each planet or satellite (and the exceptions) are listed in Appendix 6. One feature classification, regio, was originally used on early maps of the Moon and Mercury (drawn from telescopic observations) to describe vague albedo features. It is now used to delineate a broad geographic region.

Named features on bodies so small that coordinates have not yet been determined are identified on drawings of the body that are included in the IAU Transactions volume of the year when the names were adopted (see also fig. $1 A, B$, and $C$ ). Satellite rings and gaps in the rings are named for scientists who have studied these features; drawings that show these names are also included in the pertinent Transactions volume (see also fig. $2 A$ and $B$ ). Names for atmospheric features are informal at present; a formal system will be chosen in the future.

The boundaries of many large features (such as terrae, regiones, planitiae, and plana) are not topographically or geomorphically distinct; the coordinates of these features are identified from an arbitrarily chosen center point. Boundaries (and thus coordinates) may be determined more accurately from geochemical and geophysical data obtained by future missions.

\section{SPECIFICS OF THE GAZETTEER}

The complete file of names is sorted in two ways: first, alphabetically by planetary body, satellite, and feature type; second, alphabetically without respect to planet or feature type. A key to column classification and codes follows at the end of this section.

Coordinates listed in columns labeled "lat" and "long" are taken from the maps identified in columns "quad" and "map." For maps published by the U.S. Geological Survey (USGS), the field "quad" identifies the informal name of the map, and the field "map" lists the USGS map identification ("I") number. In identifying lunar maps published by the Defense Mapping Agency or the Aeronautical Chart and Information Center in the 1960's and early 1970's or Soviet maps of Venus published in 1985 and 1986, we use different systems of identification, as shown in the key below.

We identify continent ("ct") and ethnic group ("et") of a name in order to compute and equalize international representation (see Appendix 3).

\section{COLUMN CLASSIFICATION AND CODES}

\section{LABEL \\ $\mathrm{P}$ (planet)}

Sa (satellite)

\section{DESCRIPTION}

Planet on which name is found (This field does not appear in the sort by planet and feature type.)

Key to Planet codes:

$\begin{array}{lll}\text { A=asteroid belt } & \text { L=Moon (Luna) } & \text { S=Saturn } \\ \text { H=Mercury (Hermes) } & \text { M=Mars } & \text { U=Uranus } \\ \text { J=Jupiter } & \text { N=Neptune } & \text { V=Venus }\end{array}$

Satellite on which name is found (This field does not appear in the sort by planet and feature type.)

Key to satellite codes:

$\begin{array}{lll}\text { am=Amalthea } & \text { gs=Gaspra } & \text { ne=Nereid } \\ \text { ar=Ariel } & \text { hy=Hyperion } & \text { ob=Oberon } \\ \text { ca=Callisto } & \text { ia=Iapetus } & \text { ph=Phobos } \\ \text { de=Deimos } & \text { id=Ida } & \text { pu=Puck } \\ \text { di=Dione } & \text { ss=small satellites of Uranus } & \text { rh=Rhea } \\ \text { en=Enceladus } & \text { io=Io } & \text { um=Umbriel } \\ \text { ep=Epimetheus } & \text { ja=Janus } & \text { te=Tethys } \\ \text { eu=Europa } & \text { mi=Mimas } & \text { ti=Titania } \\ \text { ga=Ganymede } & \text { mr=Miranda } & \text { tr=Triton }\end{array}$




\section{COLUMN CLASSIFICATION AND CODES-Continued}

\section{LABEL}

name (feature name)

lat (latitude)

long (longitude)

diam (diameter)

ct (continent)

et (ethnic group)

quad (map name)*

map (map number)

as (approval status) ad (approval date) ref (reference) $\mathrm{ft}$ (feature type) origin

\section{DESCRIPTION}

As spelled by honoree or by reference shown in Appendix 4; brackets indicate that name has been dropped.

Degrees 0 to 90 followed by $\mathrm{N}$ or $\mathrm{S}$.

Degrees 0 to 360 followed by E or W; 0 to $180 \mathrm{E}$ and 0 to $180 \mathrm{~W}$ on the Moon.

Diameter or longest dimension of feature in kilometers.

Continent or large geographic division that is origin of name (see Appendix 3).

Country or ethnic group that is origin of name (see Appendix 3).

Informal name of map; planet or satellite abbreviation or number, or abbreviation of map name. (This field does not appear in the alphabetical sort.)

USGS, Soviet, or Defense Mapping Agency number of map. (This field does not appear in the alphabetical sort.)

Number indicates IAU level of approval:

$1=$ Suggested

2 = Task Group approval

$3=$ WGPSN approval

$4=$ Executive Committee approval

$5=$ Adopted by IAU General Assembly

$6=$ Dropped, disallowed

Year when name was adopted.

Reference from which spelling and origin information were derived (see Appendix 4).

Latin or Greek descriptor term (see Appendix 5).

Short explanation of name.

*Abbreviations used in this column, by planet or planetary system, are as follows:

Maps of Mercury:

$\mathrm{H}-\mathrm{n}$

Mercury maps $\mathrm{H}-1$ through $\mathrm{H}-15$; scale $1: 5$ million.

\section{Maps of Venus:}

1:50M

B1 to 29

\section{Lunar maps:}

LAC

LOC

LM

LMP

LPC

LTO

Sn

$\mathrm{xxx}$
Preliminary pictorial map of Venus; scale 1:50 million.

Number of Soviet Venera map, scale 1:5 million, on which named feature occurs. I-number denotes USGS Magellan Planning Chart map.

Lunar Charts 1 through 154; published in 1966 by ACIC for U.S. Air Force and NASA; scale 1:1 million.

Lunar Planning Charts 1 through 4; published in 1971 by ACIC for NASA under direction of U.S. Department of Defense (DoD); scale 1:2.75 million.

Lunar Maps 1 through 144; scale 1:1 million; published 1978.

Lunar Earthside, Farside and Polar charts 1 through 3; published in 1970 by ACIC for DoD; scale 1:6 million.

Lunar Chart, published by Defense Mapping Agency (DMA) for NASA; scale 1:10 million.

Lunar Topographic Orthophoto Maps 1 through 104a,b,c,d; published by DMA, 1971-197x; scale 1:250,000.

"Special" map of LTO series, at high resolution (scale usually 1:50,000, may be $1: 25,000$ or $1: 10,000$ ). Full designation would be LTO 41B4 S2.

Adopted name that is not on any published map. 


\section{COLUMN CLASSIFICATION AND CODES-Continued}

\section{Maps of Mars:}

$1: 15 \mathrm{M}$

MC15

$\mathrm{MC} 25 \mathrm{NW}$

Canber

Capri

Chryse

Chry E

Chry W

Cydoni

Erythr

Nereid

Soviet

Triton

Utopia

Yorktn

05072
Synoptic maps; scale 1:15 million.

Mars Charts 1-30; scale 1:5 million; each chart also has a name (MC-9 = Tharsis),

but we refer only to the number.

Northwest section of MC-25; scale 1:2 million.

Canberra; Viking 2 landing site map; scale 1:250,000.

Capri; pre-Viking alternate or safe site; scale 1:1 million.

Chryse (Planitia); proposed Viking-1 prime landing site map; scale 1:1 million.

East Chryse; Viking map, scale 1:1 million.

West Chryse; Viking map, scale 1:1 million.

Cydonia; proposed Viking 2 prime landing site, scale 1:1 million.

Erythraeum, 1973 proposed Soviet landing site map; scale 1:1 million.

Nereidum Montes, 1973 proposed Soviet landing site map; scale 1:1 million.

An unpublished Soviet map compiled from Mars 4 and 5 images.

Tritonis Lacus; proposed pre-Viking 2 backup landing site map, scale 1:1 million. Utopia Planitia; Viking 2 landing site map; scale 1:1 million.

Yorktown; Viking 1 landing site map; scale 1:250,000.

Center coordinates of 1:500,000-scale maps. Latitude given first, then longitude; here, they are $5^{\circ} \mathrm{N}, 72^{\circ} \mathrm{W}$.

\section{Maps of Jovian satellites:}

Prelim

Preliminary shaded relief drawing.

$\mathrm{Ji} 2$

Designation of satellite map and number. $\mathrm{J}=$ Jupiter; $\mathrm{a}=$ Amalthea, $\mathrm{i}=\mathrm{Io}$, $\mathrm{e}=$ Europa, $\mathrm{g}=$ Ganymede, $\mathrm{c}=$ Callisto. Map numbers $1-4$.

\section{Maps of Saturnian satellites:}

Prelim

Janus

Epimetheus

Mimas

Enceladus

Tethys

Dione

Rhea

Hyperian

Iapetus
Preliminary pictorial maps published in 1976.

No map or I-number.

No map or I-number.

Name of mapped satellite; scale 1:5 million.

Name of mapped satellite; scale 1:5 million.

Name of mapped satellite; scale 1:10 million.

Name of mapped satellite; scale 1:10 million.

Name of mapped satellite; scale 1:10 million.

No map or I-number.

Name of mapped satellite, scale 1:10 million.

Maps of Uranian satellites:

Miranda

Ariel

Umbriel

Titania

Oberon

Puck
Name of satellite; scale 1:5 million.

Name of satellite; scale 1:5 million.

Name of satellite; scale 1:5 million.

Name of satellite; scale 1:5 million.

Name of satellite; scale 1:5 million.

Satellite name; no map or coordinates. 


\section{ACKNOWLEDGMENTS}

The Working Group for Planetary System Nomenclature wishes to express its appreciation for the efforts of the following people. The late Dr. Peter Millman, National Research Council of Canada in Ottawa, provided the original list of planetary names and carefully reviewed all planetary nomenclature material. Ewen Whitaker, now retired from the Lunar and Planetary Laboratory, Tucson, Ariz., corrected the lunar list. The late Dr. Harold Masursky aggressively led and managed the planetary nomenclature process. Dr. Jean Duchesne-Guillermin, Liege, Belgium, performed authoritative and careful editing of the 1986 edition of the Gazetteer. We also thank Dr. Audouin Dollfus of the Observatoire de Paris, Dr. A.T. Basilevsky and Dr. G. Burba of the Vernadsky Institute, Moscow, and Mr. William Musielak of the University of Arizona, Tucson, for reviews of maps during the critical prepublication period. Many USGS employees in Flagstaff, Ariz., are responsible for producing the Gazetteer. Mary Strobell, now retired, and Joel F. Russell, no longer with the Survey, coordinated all details of the naming process, from collecting names and submitting them to the appropriate IAU task groups and the WGPSN to applying names to features on maps. They were assisted at various stages by D.L. Applebee, Katherine Beer, Rebecca Birkholst, Jennifer Blue, A.L. Dial, Jr., Elizabeth Dyer, Derrick D. Hirsch, Victoria Lobato, Connie Nordstrom, Jana Ruhlmann, Christine Vargas, and Doris Weir, who made corrections and additions; by Janet Barrett, Robert Gurule, C.E. Isbell, K.D. Knisely, and Bill Woodsmall, who wrote computer programs to sort and print the files; and by R.D. Carroll and Ramon Sabala, who supplied cartographic expertise.

\section{REFERENCES CITED}

Antoniadi, E. M., 1930, La planète Mars, pl. 2-5: Paris, Libraire Scientifique Herman et Cie., 239 p.
Arthur, D.W.G., Agnieray, A.P., Horvath, R.A., Wood, C.A., and Chapman, C.R., 1963, The system of lunar craters, quadrant I: Communications of the Lunar and Planetary Laboratory, v. 2 , no. 30, p. 71-78, 4 unnumbered appendixes, and 12 unnumbered maps.

1964, The system of lunar craters, quadrant II: Communications of the Lunar and Planetary Laboratory, v. 3, no. 40, p. 1-59 and 12 unnumbered maps.

Arthur, D.W.G., Agnieray, A.P., Pellicori, R.H., Wood, C.A., and Weller, T., 1965, The system of lunar craters, quadrant III: Communications of the Lunar and Planetary Laboratory, v. 3 , no. 50, p. 61-62, catalogue p. 1-146, and 12 unnumbered maps.

Arthur, D.W.G., Pellicori, R.H., and Wood, C.A., 1966, The system of lunar craters, quadrant IV: Communications of the Lunar and Planetary Laboratory, v. 5, no. 70, catalogue p. 1-208 and 12 unnumbered maps.

Blagg, Mary, and Müller, Karl, 1935, Named lunar formations: London, Percy Lund, Humphries \& Co. Ltd., 196 p.

International Astronomical Union, 1922, Transactions of the International Astronomical Union, Rome, May 12-20, 1922: London, Imperial College Bookstall, v. 1, p. 52-53.

1960, Transactions of the International Astronomical Union, Moscow, August 12-20, 1958: Cambridge University Press, v. 10 , pl. 1 , p. 262 .

1971, Commission 17: The Moon, in Proceedings of the 14th General Assembly, Brighton, 1970: Transactions of the International Astronomical Union, v. 14B, p. 138-145.

- 1977, Working Group for Planetary System Nomenclature, in Proceedings of the 16th General Assembly, Grenoble, 1976: Transactions of the International Astronomical Union, v. 16B, p. 321-369.

Masursky, Harold, and others, 1986, Annual gazetteer of planetary nomenclature: U.S. Geological Survey Open-File Report 84-692.

Schiaparelli, G.V., 1879, Osservazioni astronomiche e fisiche sull'asse di rotazione e sulla topografia del pianeta Marte, in Atti della R. Accademia del Lincei, Memoria della cl. di scienze fisiche. Memoria 2, ser. 3, v. 10, 1880-81, p. 281-387.

de Vaucouleurs, Gerard, Blunck, Jürgen, Davies, Merton, Dollfus, Audouin, Koval, I.K., Kuiper, G.P., Masursky, Harold, Miyamoto, S., Moroz, V.I., Sagan, Carl, and Smith, Bradford, 1975, The new Martian nomenclature of the International Astronomical Union: Icarus, v. 26, p. 85-98. 
SECTION 1-NAMES LISTED BY PLANET, SATELLITE, AND FEATURE TYPE 


\section{MERCURY}

Name

lat long diam ct et quad

\section{CRATER}

Abu Nuwas

Africanus Horton

Ahmad Baba

Al-Akhtal
Alencar
Al-Hamadhani
Al-Jähiz
Amru Al-Qays
Andal
Aristoxenus

$59.2 \mathrm{~N}$

$51.5 \mathrm{~S}$

$20.4 \mathrm{~W}$

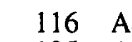

$58.5 \mathrm{~N} \quad 126.8 \mathrm{~W}$

135 A

$\begin{array}{llll}\text { SY } & \mathrm{H}-6 & \mathrm{I1} 822\end{array}$

$5 \quad 1976$

59 AA

Arab poet (c. 1756-1810).

8.5

$26.8 \mathrm{~W}$

127 AF SU H-3 $\quad$ I1822

63.5

102 AS AR 1:10M I1822

$103.5 \mathrm{~W}$

120 SA

BR

$\mathrm{H}-12$

I1 822

$38.8 \mathrm{~N}$

$1.2 \mathrm{~N}$

$89.7 \mathrm{~W}$

$21.5 \mathrm{~W}$

186 AS AR H-2

I1 822

$12.3 \mathrm{~N}$

$47.7 \mathrm{~S}$

$175.6 \mathrm{~W}$

$37.7 \mathrm{~W}$

91 AS AR H-6

I1822

50 AS AR H-8 $\quad$ I1822

Aristoxenus

$82.0 \mathrm{~N}$

Aśvaghosa

Bach

10.4

$21.0 \mathrm{~W}$

69

$\begin{array}{ll}\mathrm{H}-11 & \mathrm{I} 1822 \\ \mathrm{H}-1 & \mathrm{I} 1822\end{array}$

51976

51979

$59 \quad \mathrm{AA}$ author (1835-1883)

Abu-a;-Abbas Aj,Ad Obm Aj,Ad a;-Takruri Al-Massufi; Sudanese writer (1556-1627).

198559 AA Arab poet (c. 640-710). 51979 59 AA Jose de; Brazilian novelist (1829-1877).

$5 \quad 1979$

80 AA

$5 \quad 1976$

1976

$\begin{array}{ll}5 & 1976 \\ 5 & 1979\end{array}$

80 AA

80 AA

80 AA

59 AA

Arab writer (d. 1007).

Arab author (d. 869).

Arab poet (pre-Islamic).

(18th century).

Greek philosopher and musical theorist (fl. 4th century B.C.).

68.5

$103.4 \mathrm{~W}$

90 AS IN

H-6

I1822

$5 \quad 1976$

$59 \quad \mathrm{AA}$

Indian philosopher and poet (fl. A.D. 80-150).

Balagtas

$22.6 \mathrm{~S}$

$13.7 \mathrm{~W}$

214 EU

GE H-15

I1822

51976

59 AA

J. S. German compose (1685-1750).

Balzac

$10.3 \mathrm{~N}$

$144.1 \mathrm{~W}$

$98 \quad$ A

I1822

51976

80 AA

F.; Filipino writer

(1788-1862).

Barma

$41.3 \mathrm{~S}$

$162.8 \mathrm{~W}$

$80 \quad$ EU

FR

H-8

I1822

51976

Bartók

$29.6 \mathrm{~S}$

134.6W

128 UR

$$
\text { RU }
$$

$1: 10 \mathrm{M}$

I1822

$5 \quad 1982$

59 AA

Honore de; French novelist (1799-1850).

Bashō

$32.7 \mathrm{~S} \quad 169.7 \mathrm{~W}$

$112 \mathrm{EU} \mathrm{HU}$

H-12

11822

51979

80 AA

Yakovlev; 16th Century

Russian architect.

Beethoven

$20.8 \mathrm{~S} \quad 123.6 \mathrm{~W}$

80 AS

JA

H-12

11822

51979

59 AA

Bela; Hungarian composer

(1881-1945).

80 AA Matsuo; Japanese poet (1644-1694).

Belinskij

$76.0 \mathrm{~S}$

$103.4 \mathrm{~W}$

643 EU

GE

$\mathrm{H}-7 \quad \mathbf{1 1 8 2 2}$

51976

59 AA

Ludwig van; German composer of Flemish descent (1770-1827).

Vissarion Grigoryevich; Russian literary critic and journalist (1811-1848).

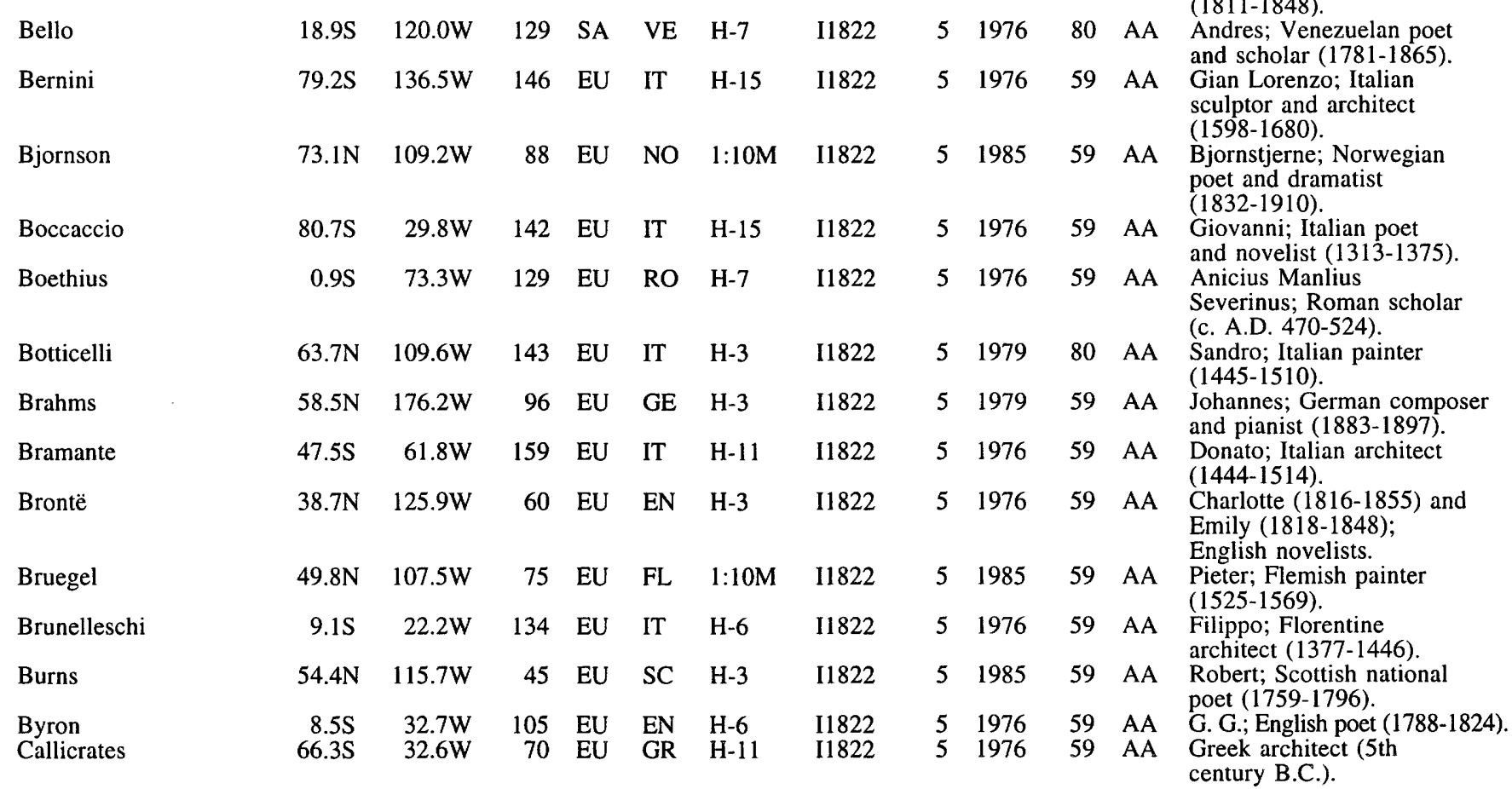

lat: latitude of feature center.

long: longitude of feature center.

diam: diameter or long dimension of feature.

et: ethnicity of name origin (see page $284 \mathrm{ff}$.)

quad: map quadrangle or informal name

(see page xvii ff.).

as: name approval status (see page xvii).

ad: name approval date (year).

map name or USGS map number (see page xvii ff.). ref: reference source for name (see page $287 \mathrm{ff}$.). 


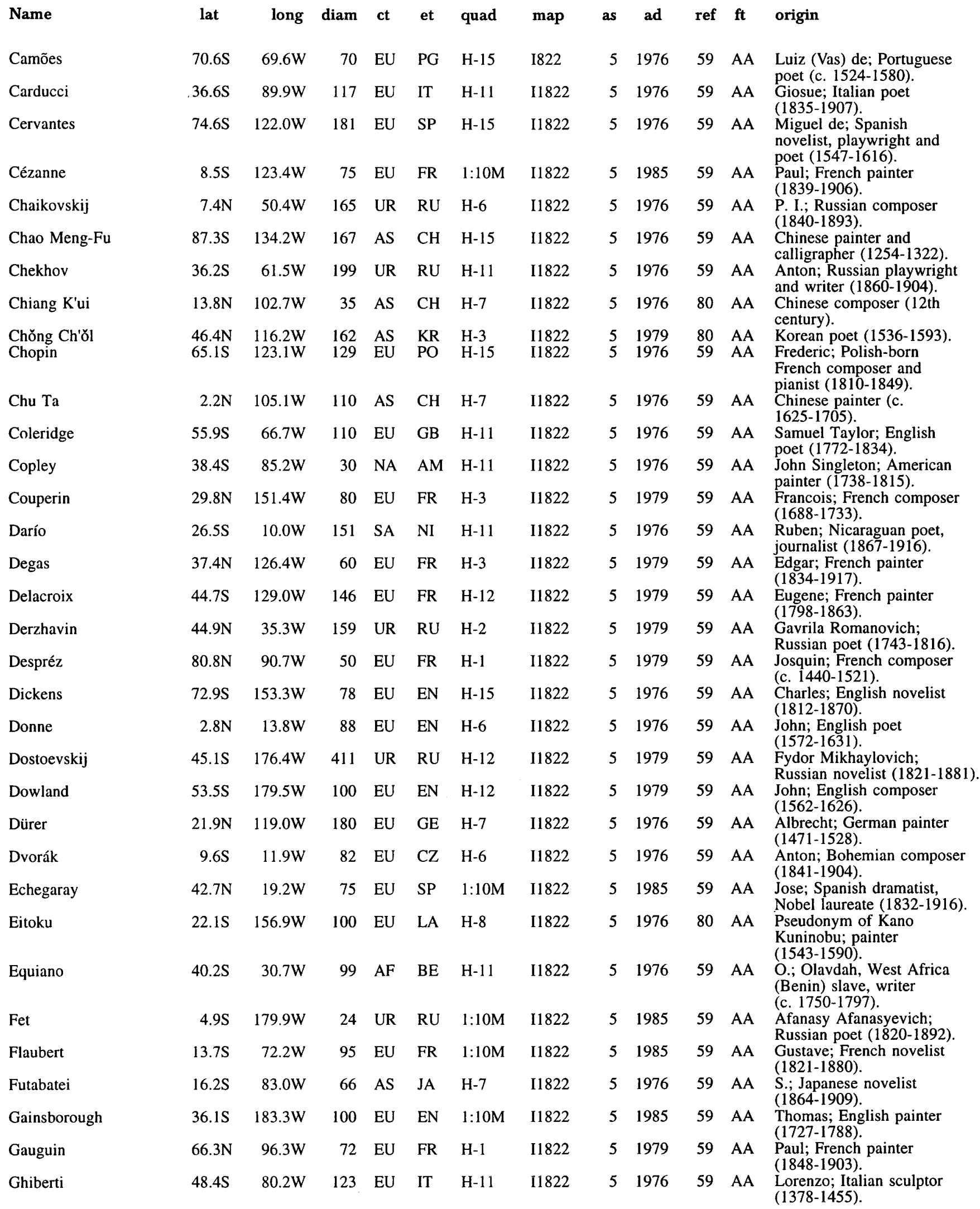

lat: latitude of feature center.

long: longitude of feature center.

diam: diameter or long dimension of feature.

ct: continent of name origin (see page $284 \mathrm{ff}$.) map: map name or USGS map number (see page xvii ff.). ft: feature type (see page 290).

et: ethnicity of name origin (see page $284 \mathrm{ff}$.)

quad: map quadrangle or informal name

(see page xvii ff.). as: name approval status (see page xvii).

ad: name approval date (year).

ref: reference source for name (see page $287 \mathrm{ff}$.). 


\section{MERCURY}

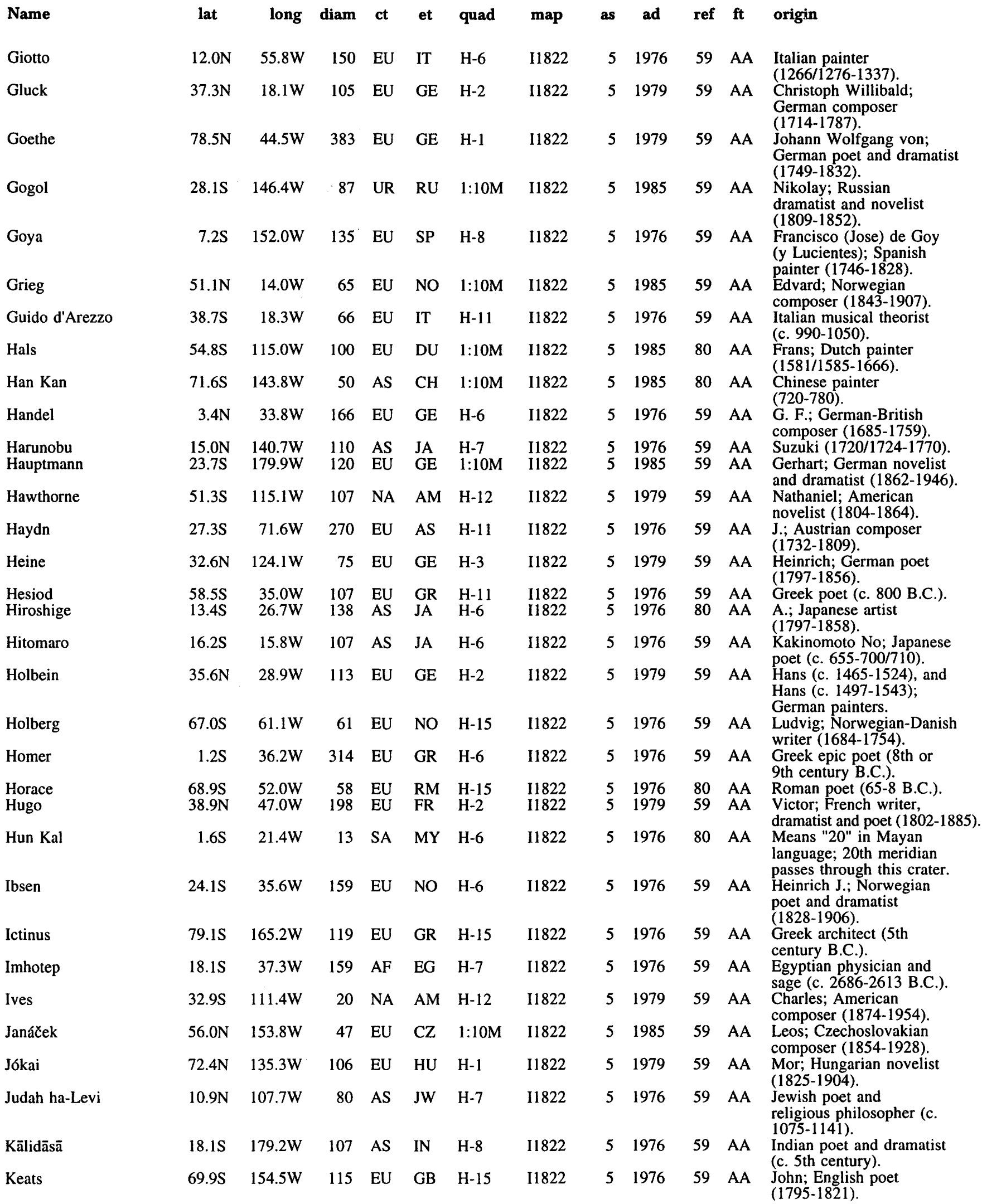

lat: latitude of feature center. long: longitude of feature center.

diam: diameter or long dimension of feature. et: ethnicity of name origin (see page $284 \mathrm{ff}$.)

quad: map quadrangle or informal name (see page xvii ff.). as: name approval status (see page $x v i i$ )

ad: name approval date (year).

ref: reference source for name (see page $287 \mathrm{ff}$.).

ct: continent of name origin (see page $284 \mathrm{ff}$.) map: map name or USGS map number (see page xvii ff.). ft: feature type (see page 290 ). 


\section{MERCURY}

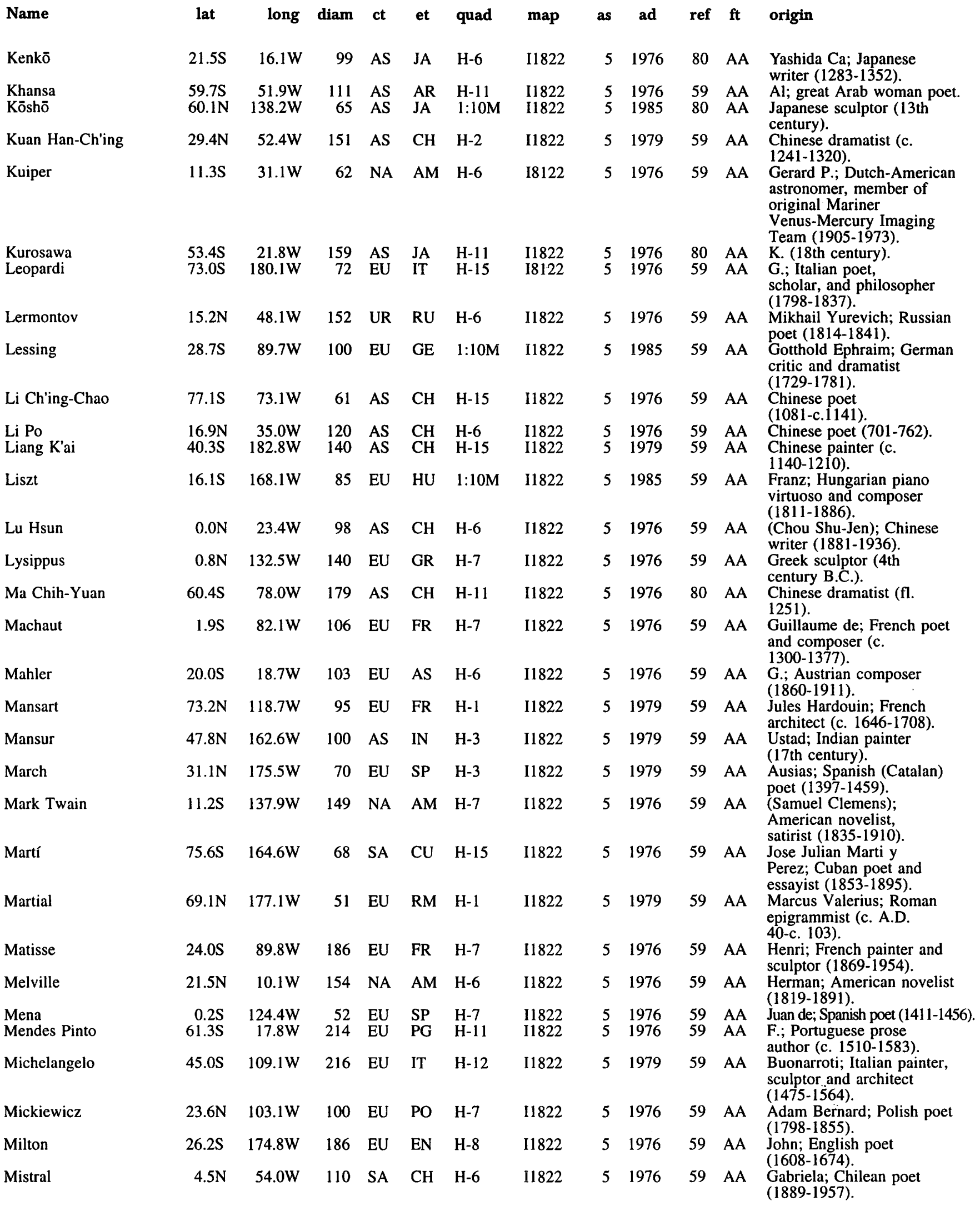

lat: latitude of feature center.

long: longitude of feature center.

diam: diameter or long dimension of feature.

ct: $\quad$ continent of name origin (see page $284 \mathrm{ff}$ ) et: ethnicity of name origin (see page $284 \mathrm{ff}$.)

quad: map quadrangle or informal name

(see page $x$ vii ff.). as: name approval status (see page xvii).

ad: name approval date (year).

ref: reference source for name (see page $287 \mathrm{ff}$.) 


\section{MERCURY}

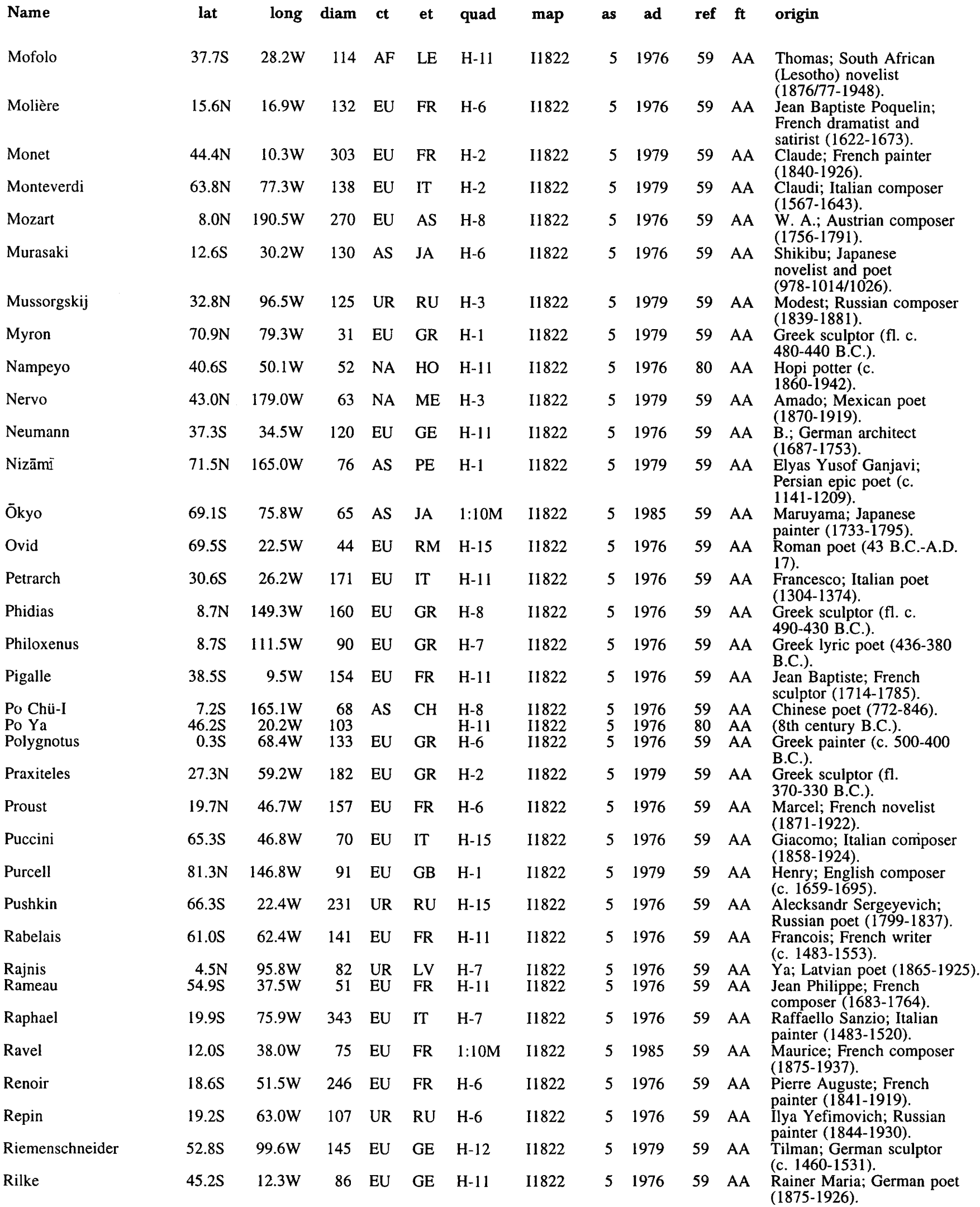

\footnotetext{
lat: latitude of feature center.

long: longitude of feature center.

diam: diameter or long dimension of feature.

et: ethnicity of name origin (see page $284 \mathrm{ff}$.)

as: name approval status (see page xvii).

quad: map quadrangle or informal name

ad: name approval date (year)

(see page xvii ff.)

ref: reference source for name (see page $287 \mathrm{ff}$.)

ct: continent of name origin (see page $284 \mathrm{ff}$.) map: map name or USGS map number (see page xvii ff.). ft: feature type (see page 290 ).
} 


\section{MERCURY}

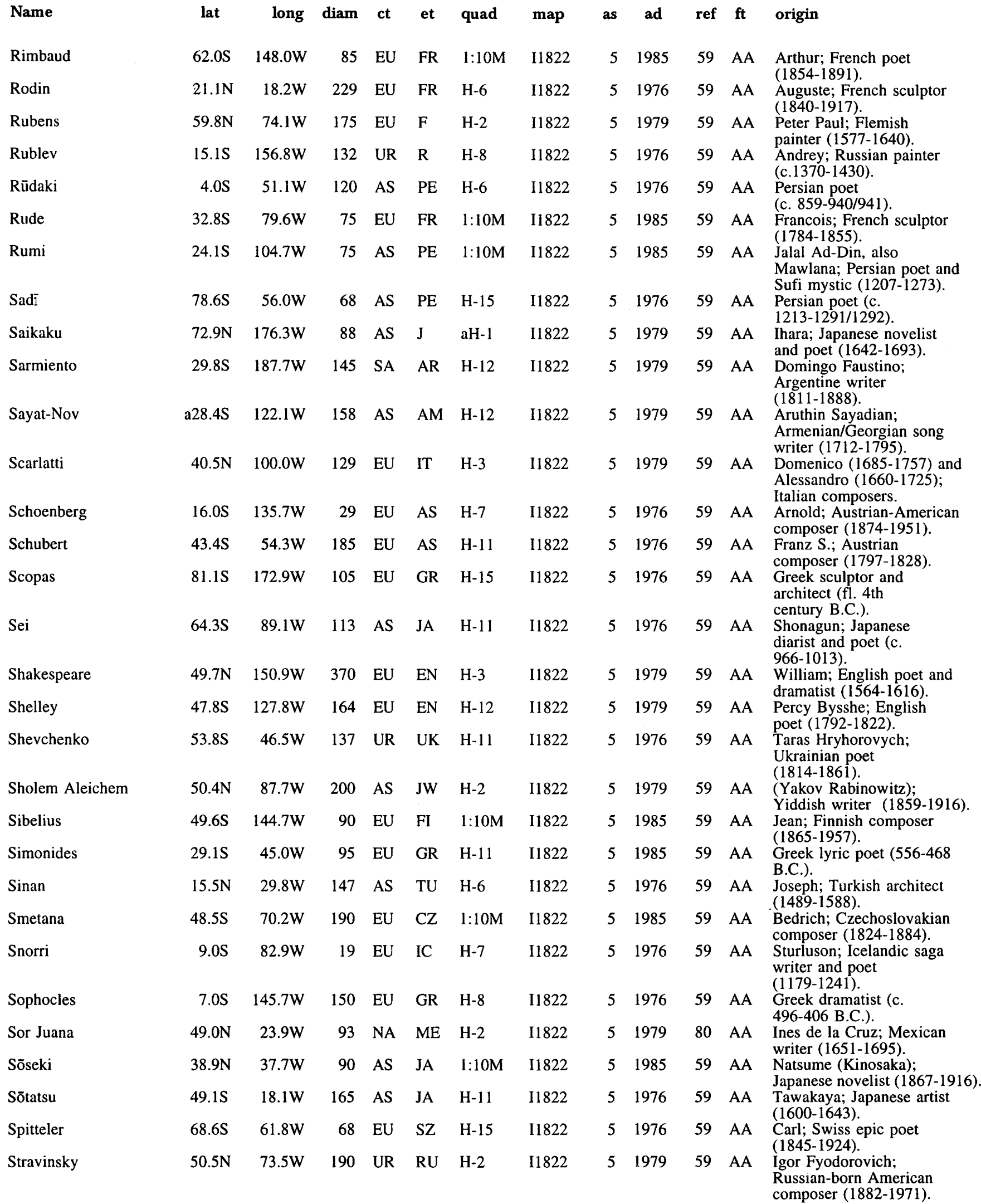

lat: latitude of feature center.

long: longitude of feature center.

diam: diameter or long dimension of feature.

et: ethnicity of name origin (see page $284 \mathrm{ff}$.)

quad: map quadrangle or informal name

(see page xvii ff.).

ct: continent of name origin (see page $284 \mathrm{ff}$.) map: map name or USGS map number (see page xvii ff.). as: name approval status (see page xvii).

ad: name approval date (year).

ref: reference source for name (see page $287 \mathrm{ff}$.) 
MERCURY

\begin{tabular}{|c|c|c|c|c|c|c|c|c|c|c|c|c|}
\hline Name & lat & long & diam & ct & et & quad & map & as & ad & ref & $\mathrm{ft}$ & origin \\
\hline Strindberg & $53.7 \mathrm{~N}$ & $135.3 W$ & 190 & EU & SW & $\mathrm{H}-3$ & I1822 & 5 & 1979 & 59 & $\mathrm{AA}$ & $\begin{array}{l}\text { August; Swedish } \\
\text { playwright, novelist, and } \\
\text { short-story writer } \\
(1849-1912)\end{array}$ \\
\hline Sullivan & $16.9 \mathrm{~S}$ & $86.3 W$ & 145 & NA & $\mathrm{AM}$ & $\mathrm{H}-7$ & 11822 & 5 & 1976 & 59 & AA & $\begin{array}{l}\text { Louis; American architect } \\
\text { (1856-1924). }\end{array}$ \\
\hline $\begin{array}{l}\text { Sūr Dās } \\
\text { Surikov }\end{array}$ & $\begin{array}{l}47.1 \mathrm{~S} \\
37.1 \mathrm{~S}\end{array}$ & $\begin{array}{r}93.3 \mathrm{~W} \\
124.6 \mathrm{~W}\end{array}$ & $\begin{array}{l}132 \\
120\end{array}$ & $\begin{array}{l}\text { AS } \\
\text { UR }\end{array}$ & $\begin{array}{l}\text { IN } \\
\text { RU }\end{array}$ & $\begin{array}{l}\mathrm{H}-12 \\
\mathrm{H}-12\end{array}$ & $\begin{array}{l}11822 \\
11822\end{array}$ & $\begin{array}{l}5 \\
5\end{array}$ & $\begin{array}{l}1979 \\
1979\end{array}$ & $\begin{array}{l}59 \\
80\end{array}$ & $\begin{array}{l}\mathrm{AA} \\
\mathrm{AA}\end{array}$ & $\begin{array}{l}\text { Indian poet }(1483-1563) \\
\text { Vassily; Russian painter } \\
(1848-1916) \text {. }\end{array}$ \\
\hline Takanobu & $30.8 \mathrm{~N}$ & $108.2 \mathrm{~W}$ & 80 & AS & JA & $1: 10 \mathrm{M}$ & 11822 & 5 & 1985 & 59 & $\mathrm{AA}$ & $\begin{array}{l}\text { Fujiwara; Japanese poet } \\
\text { and portrait artist } \\
(1142-1205) \text {. }\end{array}$ \\
\hline Takayoshi & $37.5 S$ & $163.1 \mathrm{~W}$ & 139 & AS & JA & $\mathrm{H}-12$ & 11822 & 5 & 1979 & 80 & $\mathrm{AA}$ & $\begin{array}{l}\text { Japanese painter (12th } \\
\text { century). }\end{array}$ \\
\hline Tansen & $3.9 \mathrm{~N}$ & $70.9 W$ & 34 & AS & IN & H-7 & 11822 & 5 & 1976 & 80 & AA & $\begin{array}{l}\text { Indian (Mogul) composer } \\
\text { from the court of Akbar. }\end{array}$ \\
\hline Thākur & $3.0 \mathrm{~S}$ & $63.5 \mathrm{~W}$ & 118 & AS & IN & H-6 & $\mathrm{I} 1822$ & 5 & 1976 & 80 & $\mathrm{AA}$ & $\begin{array}{l}\text { R.; Bengalese poet and } \\
\text { novelist, Nobel Peace } \\
\text { laureate (1861-1941). }\end{array}$ \\
\hline Theophanes & $4.9 \mathrm{~S}$ & $142.4 \mathrm{~W}$ & 45 & EU & BZ & H-7 & 11822 & 5 & 1976 & 59 & $\mathrm{AA}$ & $\begin{array}{l}\text { Byzantine painter } \\
\text { (c.1330-1405). }\end{array}$ \\
\hline Thoreau & $5.9 \mathrm{~N}$ & $132.3 \mathrm{~W}$ & 80 & NA & $\mathrm{AM}$ & $1: 10 \mathrm{M}$ & I1822 & 5 & 1985 & 59 & $\mathrm{AA}$ & $\begin{array}{l}\text { Henry David; American } \\
\text { poet and philosopher } \\
(1817-1862) \text {. }\end{array}$ \\
\hline Tintoretto & $48.1 \mathrm{~S}$ & $22.9 \mathrm{~W}$ & 92 & EU & IT & $\mathrm{H}-11$ & 11822 & 5 & 1976 & 59 & $\mathrm{AA}$ & $\begin{array}{l}\text { Italian painter } \\
(1518-1594)\end{array}$ \\
\hline Titian & $3.6 \mathrm{~S}$ & $42.1 \mathrm{~W}$ & 121 & EU & IT & H-6 & I1822 & 5 & 1976 & 59 & AA & $\begin{array}{l}\text { (Tiziano) Vecellio; } \\
\text { Italian Renaissance } \\
\text { painter (c.1488/90-1576). }\end{array}$ \\
\hline Tolstoj & $16.3 S$ & $163.5 \mathrm{~W}$ & 390 & UR & RU & H-8 & I1822 & 5 & 1976 & 59 & $\mathrm{AA}$ & $\begin{array}{l}\text { Lev N.; Russian novelist } \\
(1828-1910)\end{array}$ \\
\hline Ts'ai Wen-Chi & $22.8 \mathrm{~N}$ & $22.2 \mathrm{~W}$ & 119 & AS & $\mathrm{CH}$ & H-6 & 11822 & 5 & 1976 & 80 & AA & $\begin{array}{l}\text { Han dynasty composer (A.D } \\
\text { 2nd century). }\end{array}$ \\
\hline Ts'ao Chan & $13.4 \mathrm{~S}$ & $142.0 \mathrm{~W}$ & 110 & AS & $\mathrm{CH}$ & $\mathrm{H}-7$ & 11822 & 5 & 1976 & 59 & AA & $\begin{array}{l}\text { Chinese writer (c. } \\
\text { 1715-1763). }\end{array}$ \\
\hline Tsurayuki & $63.0 \mathrm{~S}$ & $21.3 \mathrm{~W}$ & 87 & AS & JA & $\mathrm{H}-11$ & I1822 & 5 & 1976 & 80 & $\mathrm{AA}$ & $\begin{array}{l}\mathrm{Ki} \text { (Kino); noted Japanese } \\
\text { man of letters (c. 945). }\end{array}$ \\
\hline Tung Yüan & $73.6 \mathrm{~N}$ & $55.0 \mathrm{~W}$ & 64 & AS & $\mathrm{CH}$ & $\mathrm{H}-1$ & 11822 & 5 & 1979 & 80 & AA & $\begin{array}{l}\text { Chinese painter (10th } \\
\text { century). }\end{array}$ \\
\hline Turgenev & $65.7 \mathrm{~N}$ & $135.0 \mathrm{~W}$ & 116 & UR & RU & $\mathrm{H}-1$ & 11822 & 5 & 1979 & 80 & $\mathrm{AA}$ & $\begin{array}{l}\text { Ivan Sergeyevich; Russian } \\
\text { writer (1818-1883). }\end{array}$ \\
\hline Tyagaraja & $3.7 \mathrm{~N}$ & $148.4 W$ & 105 & AS & IN & $\mathrm{H}-8$ & I1822 & 5 & 1976 & 59 & AA & $\begin{array}{l}\text { Indian composer } \\
(1767-1847) \text {. }\end{array}$ \\
\hline Unkei & $31.9 \mathrm{~S}$ & $62.7 \mathrm{~W}$ & 123 & AS & JA & $\mathrm{H}-11$ & I1822 & 5 & 1976 & 59 & AA & $\begin{array}{l}\text { Japanese sculptor (c. } \\
\text { 1 148-1223). }\end{array}$ \\
\hline Ustad Isa & $32.1 \mathrm{~S}$ & $165.3 \mathrm{~W}$ & 136 & AS & TU & $\mathrm{H}-12$ & 11822 & 5 & 1979 & 80 & AA & $\begin{array}{l}\text { Turkish/Persian architect } \\
\text { (17th century). }\end{array}$ \\
\hline Vãlmiki & $23.5 \mathrm{~S}$ & $141.0 \mathrm{~W}$ & 221 & AS & SA & $\mathrm{H}-7$ & 11822 & 5 & 1976 & 80 & AA & $\begin{array}{l}\text { Sanskrit poet, author of } \\
\text { the Ramayala (1st century } \\
\text { B.C.). }\end{array}$ \\
\hline van Dijck & $76.7 \mathrm{~N}$ & $163.8 \mathrm{~W}$ & 105 & EU & FL & $\mathrm{H}-1$ & 11822 & 5 & 1979 & 59 & AA & $\begin{array}{l}\text { Anthony; Flemish } \\
\text { painter (1599-1641). }\end{array}$ \\
\hline van Eyck & $43.2 \mathrm{~N}$ & $158.8 \mathrm{~W}$ & 282 & EU & FL & $\mathrm{H}-3$ & 11822 & 5 & 1979 & 59 & AA & $\begin{array}{l}\text { Jan; Flemish painter } \\
\text { (c. 1395-1441). }\end{array}$ \\
\hline van Gogh & $76.5 \mathrm{~S}$ & $134.9 \mathrm{~W}$ & 104 & EU & DU & $\mathrm{H}-15$ & 11822 & 5 & 1976 & 59 & AA & $\begin{array}{l}\text { Vincent Willem; Dutch } \\
\text { painter }(1853-1890) \text {. }\end{array}$ \\
\hline Velázquez & $37.5 \mathrm{~N}$ & $53.7 \mathrm{~W}$ & 129 & EU & SP & $\mathrm{H}-2$ & 11822 & 5 & 1979 & 59 & $\mathrm{AA}$ & $\begin{array}{l}\text { Diego; Spanish painter } \\
(1599-1660)\end{array}$ \\
\hline Verdı & $64 . \pi N$ & $168.6 \mathrm{~W}$ & 163 & EU & IT & $\mathrm{H}-1$ & 11822 & 5 & 1979 & 59 & $\mathrm{AA}$ & $\begin{array}{l}\text { Giuseppe; Italian } \\
\text { composer (1813-1901). }\end{array}$ \\
\hline Vincente & $56.8 \mathrm{~S}$ & $142.4 \mathrm{~W}$ & 98 & EU & PG & $\mathrm{H}-12$ & 11822 & 5 & 1979 & 80 & AA & $\begin{array}{l}\text { Gil; Portuguese dramatist } \\
\text { (c. 1465-1537). }\end{array}$ \\
\hline Vivaldi & $13.7 \mathrm{~N}$ & $85.0 \mathrm{~W}$ & 213 & EU & IT & $\mathrm{H}-7$ & I1822 & 5 & 1976 & 59 & $\mathrm{AA}$ & $\begin{array}{l}\text { Antonio; Italian composer } \\
(1678-1741) \text {. }\end{array}$ \\
\hline Vlamınck & $28.0 \mathrm{~N}$ & $12.7 \mathrm{~W}$ & 97 & EU & FR & 1:10M & 11822 & 5 & 1985 & 59 & AA & $\begin{array}{l}\text { Maurice de; French } \\
\text { painter (1876-1958) }\end{array}$ \\
\hline $\begin{array}{l}\text { Vyāsa } \\
\text { Wagner }\end{array}$ & $\begin{array}{l}48.3 \mathrm{~N} \\
67.4 \mathrm{~S}\end{array}$ & $\begin{array}{r}81.1 \mathrm{~W} \\
114.0 \mathrm{~W}\end{array}$ & $\begin{array}{l}290 \\
140\end{array}$ & $\begin{array}{l}\text { AS } \\
\text { EU }\end{array}$ & $\begin{array}{l}\text { IN } \\
\text { GE }\end{array}$ & $\begin{array}{l}\mathrm{H}-2 \\
\mathrm{H}-15\end{array}$ & $\begin{array}{l}I 1822 \\
\mathrm{I} 1822\end{array}$ & $\begin{array}{l}5 \\
5\end{array}$ & $\begin{array}{l}1979 \\
1976\end{array}$ & $\begin{array}{l}59 \\
59\end{array}$ & $\begin{array}{l}\mathrm{AA} \\
\mathrm{AA}\end{array}$ & $\begin{array}{l}\text { İndian poet (fl. } 1500 \text { B..C.). } \\
\text { Richard; German composer } \\
(1813-1883) \text {. }\end{array}$ \\
\hline Wang Meng & $8.8 \mathrm{~N}$ & $103.8 \mathrm{~W}$ & 165 & AS & $\mathrm{CH}$ & $\mathrm{H}-7$ & 11822 & 5 & 1976 & 59 & AA & $\begin{array}{l}\text { Chinese painter } \\
(1308-1385)\end{array}$ \\
\hline
\end{tabular}

lat: latitude of feature center.

long: longitude of feature center.

diam: diameter or long dimension of feature.

ct: continent of name origin (see page $284 \mathrm{ff}$.) map: map name or USGS map number (see page xvii ff.). et: ethnicity of name origin (see page $284 \mathrm{ff}$.)

quad: map quadrangle or informal name

(see page xvii ff.). as: name approval status (see page xvii)

ad: name approval date (year).

ref: reference source for name (see page $287 \mathrm{ff}$.) 


\section{MERCURY}

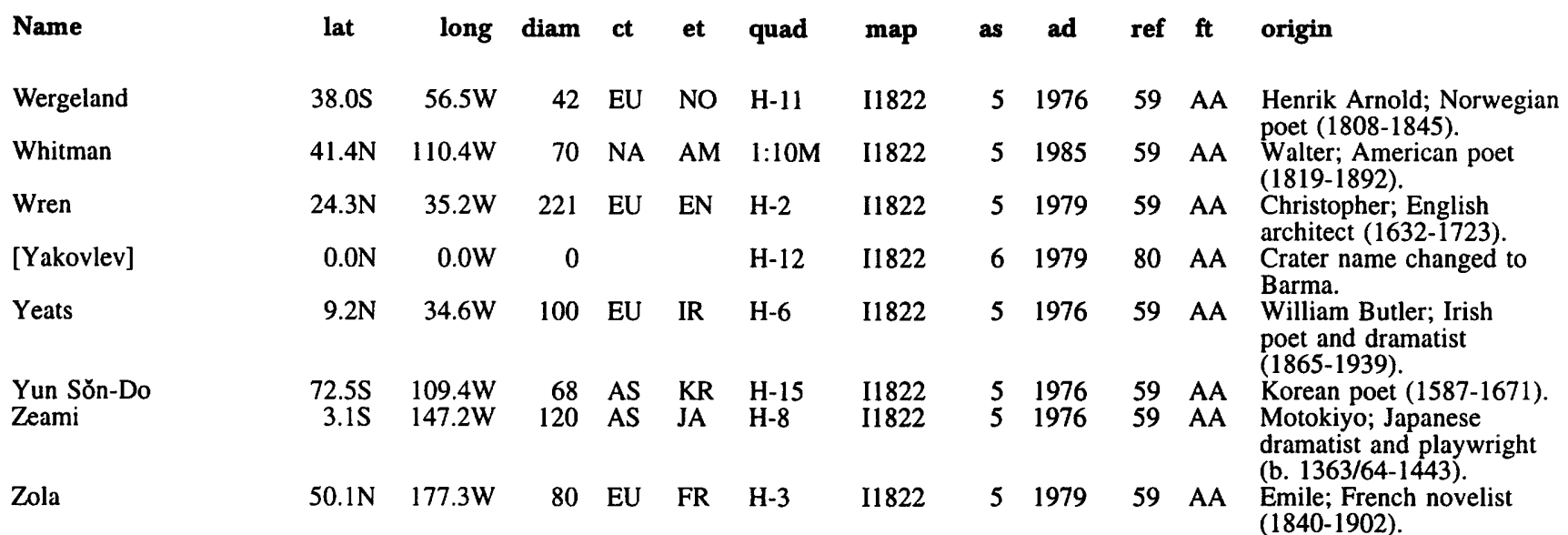

ALBEDO FEATURE

\begin{tabular}{|c|c|c|c|c|c|c|c|}
\hline Apollonia & $45.0 \mathrm{~N}$ & $315.0 \mathrm{~W}$ & 0 & EU & RM & $\mathrm{H}-5$ & I1 822 \\
\hline Aurora & $45.0 \mathrm{~N}$ & $90.0 \mathrm{~W}$ & 0 & EU & $\mathbf{R M}$ & $\mathrm{H}-2$ & I 1822 \\
\hline Australia & $72.5 \mathrm{~S}$ & $0.0 \mathrm{~W}$ & 0 & EU & RM & $\mathrm{H}-15$ & 11822 \\
\hline Borea & $75.0 \mathrm{~N}$ & $0.0 \mathrm{~W}$ & 0 & $\mathrm{EU}$ & RM & $\mathrm{H}-1$ & $\mathrm{I} 1822$ \\
\hline Caduceata & $45.0 \mathrm{~N}$ & $135.0 \mathrm{~W}$ & 0 & $\mathrm{EU}$ & RM & $\mathrm{H}-3$ & I1066 \\
\hline Cyllene & $41.0 \mathrm{~S}$ & $270.0 \mathrm{~W}$ & 0 & EU & RM & $\mathrm{H}-14$ & \\
\hline Heliocaminus & $40.0 \mathrm{~N}$ & $170.0 \mathrm{~W}$ & 0 & EU & RM & & \\
\hline Hesperis & $45.0 \mathrm{~S}$ & $355.0 \mathrm{~W}$ & 0 & $\mathrm{EU}$ & RM & & \\
\hline Liguria & $45.0 \mathrm{~N}$ & $225.0 \mathrm{~W}$ & 0 & EU & RM & $\mathrm{H}-4$ & \\
\hline Pentas & $5.0 \mathrm{~N}$ & $310.0 \mathrm{~W}$ & 0 & EU & RM & & \\
\hline Phaethontias & $0.0 \mathrm{~N}$ & $167.0 \mathrm{~W}$ & 0 & EU & RM & $\mathrm{H}-8$ & 10993 \\
\hline Pieria & $0.0 \mathrm{~N}$ & $270.0 \mathrm{~W}$ & 0 & EU & RM & $\mathrm{H}-10$ & \\
\hline Pleias Gallia & $25.0 \mathrm{~N}$ & $130.0 \mathrm{~W}$ & 0 & EU & RM & & \\
\hline Sinus Argiphontae & $10.0 \mathrm{~S}$ & $335.0 \mathrm{~W}$ & 0 & EU & $\mathrm{RM}$ & & \\
\hline Solitudo Admetei & $55.0 \mathrm{~N}$ & $90.0 \mathrm{~W}$ & 0 & EU & $\mathrm{RM}$ & & \\
\hline Solitudo Alarum & $15.0 \mathrm{~S}$ & $290.0 \mathrm{~W}$ & 0 & EU & RM & & \\
\hline Solitudo Aphrodites & $25.0 \mathrm{~N}$ & $290.0 \mathrm{~W}$ & 0 & EU & $\mathrm{RM}$ & & \\
\hline Solitudo Atlantis & $35.0 \mathrm{~S}$ & $210.0 \mathrm{~W}$ & 0 & EU & RM & & \\
\hline Solitudo Criophori & $0.0 \mathrm{~N}$ & $230.0 \mathrm{~W}$ & 0 & $\mathrm{EU}$ & $\mathrm{RM}$ & $\mathrm{H}-9$ & \\
\hline Solitudo Helii & $10.0 \mathrm{~S}$ & $180.0 \mathrm{~W}$ & 0 & EU & RM & & \\
\hline $\begin{array}{l}\text { Solitudo Hermae } \\
\text { Trismegisti }\end{array}$ & $45.0 \mathrm{~S}$ & $45.0 \mathrm{~W}$ & 0 & EU & RM & $\mathrm{H}-11$ & $\mathrm{I} 1030$ \\
\hline Solitudo Horarum & $25.0 \mathrm{~N}$ & $115.0 \mathrm{~W}$ & 0 & EU & $\mathrm{RM}$ & & \\
\hline Solitudo Iovis & $0.0 \mathrm{~N}$ & $0.0 \mathrm{~W}$ & 0 & EU & RM & & \\
\hline Solitudo Lycaonis & $0.0 \mathrm{~N}$ & $107.0 \mathrm{~W}$ & 0 & EU & $\mathrm{RM}$ & $\mathrm{H}-7$ & 11029 \\
\hline Solitudo Maiae & $15.0 \mathrm{~S}$ & $155.0 \mathrm{~W}$ & 0 & EU & $\mathrm{RM}$ & & \\
\hline Solitudo Martis & $35.0 \mathrm{~S}$ & $100.0 \mathrm{~W}$ & 0 & EU & RM & & \\
\hline
\end{tabular}

$51976 \quad 60 \mathrm{AL}$ Albedo name for unimaged $\mathrm{H}-5$ region.

$5197660 \mathrm{AL}$ Albedo name for $\mathrm{H}-2$, Victoria region.

5197660 AL Albedo name for H-15, Bach region.

$51976 \quad 60$ AL Albedo name for $\mathrm{H}-1$,

$1976 \quad 60$ BL Borealis region

Albedo name for $\mathrm{H}-3$,

51976

Shakespeare region.

$51976 \quad 60 \quad \mathrm{AL} \quad \begin{aligned} & \text { Al-14 region. } \\ & \text { Albedo feature; Antoniadi }\end{aligned}$

$51976 \quad 60$ AL Albedo feature; Antoniadi

1976 60 map.

$60 \mathrm{AL}$ Albedo name for unimaged

51976

H-4 region.

60 AL Albedo feature; Antoniad map.

$5197660 \mathrm{AL}$ Albedo name for H-8, Tolstoj region.

$51976 \quad 60$ AL Albedo name for unimaged

$5-1976$ H-10 region.

$51976 \quad 60$ AL Albedo feature; Antoniadi

$5 \quad 1976$

$60 \mathrm{AL}$ Albedo feature; Antoniad

$51976 \quad 60$ AL Albedo feature; Antoniadi

$51976 \quad 60$ AL $\begin{aligned} & \text { map. } \\ & \text { Albedo feature; Antoniadi }\end{aligned}$

5 map.

$51976 \quad 60$ AL Albedo feature; Antoniadi

$5 \quad 1976$ map.

$1976 \quad 60$ AL Albedo feature; Antoniadi

$5 \quad 1976$ map.

$51976 \quad 60$ AL $\begin{aligned} & \text { H-9 region. } \\ & \text { Albedo feature; Antoniadi }\end{aligned}$

map.

$51976 \quad 60 \mathrm{AL}$ Albedo name for H-11,

51976 Discovery region.

$51976 \quad 60$ AL Albedo feature; Antoniadi

map.

$51976 \quad 60$ AL Albedo feature; Antoniadi

map.

$51976 \quad 60$ AL Albedo name for H-7,

5197660 Beethoven region.

$51976 \quad 60 \mathrm{AL}$ Albedo feature; Antoniadi

$51976 \quad 60$ AL Albedo

map. map.

lat: latitude of feature center.

long: longitude of feature center.

diam: diameter or long dimension of feature.

ct: continent of name origin (see page $284 \mathrm{ff}$ ) et: ethnicity of name origin (see page $284 \mathrm{ff}$.)

quad: map quadrangle or informal name

(see page $x$ vii $f f$.). as: name approval status (see page $\mathrm{xvii}$ ).

ad: name approval date (year).

ref: reference source for name (see page $287 \mathrm{ff}$.).

map: map name or USGS map number (see page xvii ff.). ft: feature type (see page 290). 


\section{MERCURY}

Name

Solitudo Neptuni

Solitudo Persephones

Solitudo Phoenicis

Solitudo Promethei

Tricrena

\section{DORSUM}

Antoniadi Dorsum

Schiaparelli Dorsum

\section{MONS}

Caloris Montes

\section{PLANITIA}

\section{Borealis Planitia}

Budh Planitia

Caloris Planitia

Odin Planitia

Sobkou Planitia

Suisei Planitia

Tir Planitia

\section{RUPES}

Adventure Rupes

Astrolabe Rupes

Discovery Rupes

Endeavour Rupes

Fram Rupes

$56.9 \mathrm{~S} \quad 93.3 \mathrm{~W}$

\section{$65.1 \mathrm{~S} \quad 65.5 \mathrm{~W}$}

lat long

$30.0 \mathrm{~N} \quad 150.0 \mathrm{~W}$

diam ct et quad

$41.0 \mathrm{~S} \quad 225.0 \mathrm{~W}$

0 EU RM

$25.0 \mathrm{~N} \quad 225.0 \mathrm{~W}$

0 EU RM H-13

45.0S $142.5 \mathrm{~W}$

0 EU RM

$0.0 \mathrm{~N} \quad 36.0 \mathrm{~W}$

0 EU RM H-12

0 EU RM H-6

$25.1 \mathrm{~N} \quad 30.5 \mathrm{~W}$

$0 \quad$ EU

IT

H-2

I1057

$23.0 \mathrm{~N} \quad 164.1 \mathrm{~W}$

$\begin{array}{llll}0 & \text { EU } & \text { IT } & \mathrm{H}-8\end{array}$

1993

$39.4 \mathrm{~N} \quad 187.2 \mathrm{~W}$
LA

$\mathrm{H}-8$

1067

map
$\mathrm{I} 1067$
10960

51976

51976

51976

1976

$5 \quad 1976$
80 DO classical nomenclature for albedo features of Mars (1870-1944).

Giovanni V.; Italian astronomer (1835-1910).

"Hot mountains"; surface temperature hottest near this position.

$\begin{array}{lllll}11066 & 5 & 1976 & 80 & \mathrm{MO}\end{array}$

$\begin{array}{rllllllll}73.4 \mathrm{~N} & 79.5 \mathrm{~W} & 0 & \text { EU } & \text { LA } & \text { H-1 } & \text { I1056 } & 5 & 1976 \\ & & & & & & & & \\ 22.0 \mathrm{~N} & 150.9 \mathrm{~W} & 0 & \text { AS } & \text { IN } & \text { H-8 } & \text { I993 } & 5 & 1976 \\ 30.5 \mathrm{~N} & 189.8 \mathrm{~W} & 0 & \text { EU } & \text { LA } & \text { H-4 } & \text { I1066 } & 5 & 1976 \\ & & & & & & & & \\ 23.3 \mathrm{~N} & 171.6 \mathrm{~W} & 0 & \text { EU } & \text { NO } & \text { H-8 } & \text { I } 1066 & 5 & 1976 \\ 39.9 \mathrm{~N} & 129.9 \mathrm{~W} & 0 & & & \text { H-3 } & \text { I1066 } & 5 & 1976 \\ 59.2 \mathrm{~N} & 150.8 \mathrm{~W} & 0 & \text { AS } & \text { JA } & \text { H-3 } & \text { I1056 } & 5 & 1976 \\ 0.8 \mathrm{~N} & 176.1 \mathrm{~W} & 0 & \text { EU } & \text { NS } & \text { H-8 } & \text { I993 } & 5 & 1976\end{array}$

80 PL "Northern plain"; from classical albedo name for quadrangle.

83 PL Hindu word for Mercury.

80 PL "Hot plain"; surface temperature hottest near this position.

80 PL Norse god.

80 PL Messenger god.

$\begin{array}{lll}80 & \text { PL Japanese messenger god. } \\ 80 & \text { PL Norse word for Mercury. }\end{array}$

0 EU EN H-11

11030

51976

$59 \mathrm{RU}$

English; one of Cook's ships on second voyage to the Pacific, 1772-75.

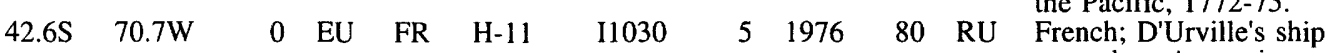
to explore Antarctica, 1838-40.

56.3S 38.3W $\quad 0 \quad$ EU EN H-11 I1030 $51976 \quad 59$ RU English; Cook's ship on last voyage to Pacific, 1776-80.

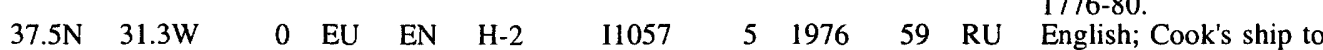
explore Tahiti, New Zealand, Australia, 1768-71.

NO $\mathrm{H}-12$

I1067

51976

80 RU

Norwegian; ship used in Arctic by Nansen, 1892-96, and by Sverdrup and Amundsen in antarctica, 1909.

Gjöa Rupes

$66.7 \mathrm{~S} \quad 159.3 \mathrm{~W}$

0 EU NO $\mathrm{H}-12$

11067

51976

59 RU

Norwegian; Amundsen's ship through Northwest

Heemskerck Rupes

$25.9 \mathrm{~N} \quad 125.3 \mathrm{~W}$

$0 \mathrm{EU}$

I1066

51976

$59 \mathrm{RU}$

passage, 1903-06.

Dutch; one of Tasman's ships to explore Australia, New Zealand, 1642-43.

Hero Rupes

$58.4 \mathrm{~S} \quad 171.4 \mathrm{~W}$

$0 \quad$ NA AM H-12 I1066

51976

$80 \mathrm{RU}$

American; Palmer's ship to explore Antarctic coast, $1820-21$.

Mirni Rupes

$37.3 \mathrm{~S} \quad 39.9 \mathrm{~W}$

$0 \quad$ UR RU H-11 $\quad$ I1030

51976

Russian; Bellingshausen's ship for Antarctic exploration, 1819-21.

\footnotetext{
lat: latitude of feature center.

et: ethnicity of name origin (see page $284 \mathrm{ff}$.)

long: longitude of feature center.

quad: map quadrangle or informal name (see page xvii ff.).

as: name approval status (see page $x$ vii).

ad: name approval date (year).

diam: diameter or long dimension of feature.

ref: reference source for name (see page $287 \mathrm{ff}$.).

ct: continent of name origin (see page $284 \mathrm{ff}$.) map: map name or USGS map number (see page xvii ff.). ft: feature type (see page 290 ).
} 


\section{MERCURY}

\begin{tabular}{|c|c|c|c|c|c|c|c|c|c|c|c|c|}
\hline Name & lat & long & diam & ct & et & quad & map & as & ad & ref & ft & origin \\
\hline Pourquoi-Pas Rupes & $58.1 S$ & $156.0 \mathrm{~W}$ & 0 & EU & FR & H-12 & I1067 & 5 & 1976 & 80 & RU & $\begin{array}{l}\text { French; Charcot's ship to } \\
\text { explore Antarctica, } \\
\text { 1908-10. }\end{array}$ \\
\hline Resolution Rupes & $63.8 \mathrm{~S}$ & $51.7 \mathrm{~W}$ & 0 & EU & EN & $\mathrm{H}-11$ & $\mathrm{I} 1030$ & 5 & 1976 & 59 & RU & $\begin{array}{l}\text { English; one of Cook's } \\
\text { ships, second expedition } \\
\text { to Pacific, } 1772-75 \text {. }\end{array}$ \\
\hline Santa María Rupes & $5.5 \mathrm{~N}$ & $19.7 \mathrm{~W}$ & 0 & $\mathrm{EU}$ & SP & H-6 & $\mathrm{I} 960$ & 5 & 1976 & 59 & RU & $\begin{array}{l}\text { Spanish; Columbus' } \\
\text { flagship, first voyage to } \\
\text { America, } 1492 \text {. }\end{array}$ \\
\hline Victoria Rupes & $50.9 \mathrm{~N}$ & $31.1 \mathrm{~W}$ & 0 & $\mathrm{EU}$ & SP & $\mathrm{H}-2$ & I1057 & 5 & 1976 & 59 & RU & $\begin{array}{l}\text { Spanish; Magellan's and } \\
\text { del Cano's ship, first } \\
\text { voyage around the world. }\end{array}$ \\
\hline Vostok Rupes & $37.7 \mathrm{~S}$ & $19.5 \mathrm{~W}$ & 0 & UR & RU & H-11 & I1030 & 5 & 1976 & 59 & RU & $\begin{array}{l}\text { Russian; Bellingshausen's } \\
\text { ship for Antarctic } \\
\text { exploration, } 1819-21 \text {. }\end{array}$ \\
\hline Zarya Rupes & $42.8 \mathrm{~S}$ & $20.5 \mathrm{~W}$ & 0 & UR & SO & $\mathrm{H}-11$ & I1030 & 5 & 1976 & 59 & RU & $\begin{array}{l}\text { USSR; Motor-sail schooner } \\
\text { to study Earth's magnetic } \\
\text { field, } 1953 \text {. }\end{array}$ \\
\hline Zeehaen Rupes & $51.0 \mathrm{~N}$ & $157.0 \mathrm{~W}$ & 0 & EU & DU & $\mathrm{H}-3$ & I1066 & 5 & 1976 & 59 & RU & $\begin{array}{l}\text { Dutch; one of Tasman's } \\
\text { ships to explore } \\
\text { Australia, New Zealand, } \\
1642-43 \text {. }\end{array}$ \\
\hline \multicolumn{13}{|l|}{ VALLIS } \\
\hline Arecibo Vallis & $27.5 S$ & $28.4 \mathrm{~W}$ & 0 & SA & PR & H-11 & I1030 & 5 & 1976 & 59 & VA & \multirow{4}{*}{$\begin{array}{l}\text { Radio telescope in Puerto } \\
\text { Rico. } \\
\text { Radio telescope facility } \\
\text { in California. } \\
\text { Radio telescope facility } \\
\text { in Massachusetts. } \\
\text { Radio telescope facility, } \\
\text { Crimea, Ukraine. }\end{array}$} \\
\hline Goldstone Vallis & $15.8 \mathrm{~S}$ & $31.7 \mathrm{~W}$ & 0 & NA & AM & H-6 & 1960 & 5 & 1976 & 80 & VA & \\
\hline Haystack Vallis & $4.7 \mathrm{~N}$ & $46.2 W$ & 0 & NA & $\mathrm{AM}$ & H-6 & 1960 & 5 & 1976 & 80 & VA & \\
\hline Simeiz Vallis & $13.2 \mathrm{~S}$ & $64.3 \mathrm{~W}$ & $\mathbf{0}$ & UR & UK & H-6 & 1960 & 5 & 1976 & 80 & VA & \\
\hline
\end{tabular}

lat: latitude of feature center.

long: longitude of feature center.

diam: diameter or long dimension of feature.

ct: continent of name origin (see page $284 \mathrm{ff}$ ) et: ethnicity of name origin (see page $284 \mathrm{ff}$.)

quad: map quadrangle or informal name

(see page xvii ff.). as: name approval status (see page $x$ vii).

ad: name approval date (year).

ref: reference source for name (see page $287 \mathrm{ff}$.). 


\section{VENUS}

Name

CRATER

\begin{tabular}{|c|c|c|c|c|c|c|}
\hline $\begin{array}{l}\text { Abaka } \\
\text { Abigail } \\
\text { Abington }\end{array}$ & $\begin{array}{l}52.6 \mathrm{~S} \\
52.2 \mathrm{~S} \\
47.8 \mathrm{~S}\end{array}$ & $\begin{array}{l}104.4 \mathrm{E} \\
111.3 \mathrm{E} \\
278.0 \mathrm{E}\end{array}$ & $\begin{array}{l}14 \\
18 \\
22\end{array}$ & $\begin{array}{l}\text { UR } \\
\text { AS } \\
\text { EU }\end{array}$ & $\begin{array}{l}\text { RU } \\
\text { HE } \\
\text { EN }\end{array}$ & \\
\hline $\begin{array}{l}\text { Adaiah } \\
\text { Adamson }\end{array}$ & $\begin{array}{l}47.3 \mathrm{~S} \\
14.8 \mathrm{~S}\end{array}$ & $\begin{array}{r}253.3 \mathrm{E} \\
29.7 \mathrm{E}\end{array}$ & $\begin{array}{l}19 \\
20\end{array}$ & $\begin{array}{l}\text { AS } \\
\text { EU }\end{array}$ & $\begin{array}{l}\mathrm{HE} \\
\mathrm{AS}\end{array}$ & \\
\hline Addams & $56.1 \mathrm{~S}$ & $98.0 \mathrm{E}$ & 85 & NA & AM & \\
\hline Adivar & $8.9 \mathrm{~N}$ & $76.1 \mathrm{E}$ & 31 & AS & TU & \\
\hline Aethelflaed & $18.2 \mathrm{~S}$ & $196.4 \mathrm{E}$ & 20 & EU & EN & \\
\hline $\begin{array}{l}\text { Aglaonice } \\
\text { Agnesi }\end{array}$ & $\begin{array}{l}26.5 S \\
39.5 S\end{array}$ & $\begin{array}{r}339.9 \mathrm{E} \\
37.8 \mathrm{E}\end{array}$ & $\begin{array}{l}65 \\
40\end{array}$ & $\begin{array}{l}\mathrm{EU} \\
\mathrm{EU}\end{array}$ & $\begin{array}{l}\text { GR } \\
\text { IT }\end{array}$ & \\
\hline Agrippina & $33.2 \mathrm{~S}$ & $65.6 \mathrm{E}$ & 37 & EU & RM & \\
\hline $\begin{array}{l}\text { Aimee } \\
\text { Aita } \\
\text { Akeley }\end{array}$ & $\begin{array}{r}16.2 \mathrm{~N} \\
8.9 \mathrm{~N} \\
8.0 \mathrm{~N}\end{array}$ & $\begin{array}{l}127.3 \mathrm{E} \\
270.7 \mathrm{E} \\
244.3 \mathrm{E}\end{array}$ & $\begin{array}{l}18 \\
14 \\
25\end{array}$ & $\begin{array}{l}\text { EU } \\
\text { UR } \\
\text { NA }\end{array}$ & $\begin{array}{l}\text { FR } \\
\text { ES } \\
\text { AM }\end{array}$ & \\
\hline Akhmatova & $61.3 \mathrm{~N}$ & $307.9 \mathrm{E}$ & 43 & UR & SO & 4 \\
\hline Akiko & $30.7 \mathrm{~N}$ & 187.1E & 25 & AS & JA & \\
\hline Aksentyeva & $42.0 \mathrm{~S}$ & $271.9 \mathrm{E}$ & 40 & UR & SO & \\
\hline Alcott & $59.5 \mathrm{~S}$ & $354.5 \mathrm{E}$ & 63 & NA & AM & \\
\hline $\begin{array}{l}\text { Alima } \\
\text { Alimat } \\
\text { Alison } \\
\text { Alma } \\
\text { Almeida } \\
\text { Al-Taymuriyya }\end{array}$ & $\begin{array}{r}45.9 \mathrm{~S} \\
29.5 \mathrm{~S} \\
4.0 \mathrm{~S} \\
2.4 \mathrm{~S} \\
46.7 \mathrm{~N} \\
32.9 \mathrm{~N}\end{array}$ & $\begin{array}{l}229.3 \mathrm{E} \\
205.9 \mathrm{E} \\
165.6 \mathrm{E} \\
228.7 \mathrm{E} \\
123.2 \mathrm{E} \\
336.2 \mathrm{E}\end{array}$ & $\begin{array}{l}13 \\
14 \\
18 \\
17 \\
14 \\
22\end{array}$ & $\begin{array}{l}\text { UR } \\
\text { UR } \\
\text { EU } \\
\text { UR } \\
\text { EU } \\
\text { AF }\end{array}$ & $\begin{array}{l}\text { RU } \\
\text { GE } \\
\text { IR } \\
\text { KZ } \\
\text { PG } \\
\text { EG }\end{array}$ & 14 \\
\hline Amalasthuna & $11.5 \mathrm{~S}$ & $342.4 \mathrm{E}$ & 16 & UR & RU & \\
\hline Amaya & $11.3 \mathrm{~N}$ & $89.3 \mathrm{E}$ & 33 & $\mathrm{EU}$ & SP & \\
\hline Amenardes & $15.0 \mathrm{~N}$ & $54.1 \mathrm{E}$ & 25 & $\mathrm{AF}$ & EG & \\
\hline $\begin{array}{l}\text { Anaxandra } \\
\text { Andami } \\
\text { Andreianova }\end{array}$ & $\begin{array}{c}44.2 \mathrm{~N} \\
17.5 \mathrm{~S} \\
3.0 \mathrm{~S}\end{array}$ & $\begin{array}{r}162.2 \mathrm{E} \\
26.5 \mathrm{E} \\
68.8 \mathrm{E}\end{array}$ & $\begin{array}{l}23 \\
30 \\
70\end{array}$ & $\begin{array}{l}\text { EU } \\
\text { AS } \\
\text { UR }\end{array}$ & $\begin{array}{l}\text { GR } \\
\text { IR } \\
\text { RU }\end{array}$ & \\
\hline Anicia & $26.3 \mathrm{~S}$ & $31.3 \mathrm{E}$ & 38 & EU & GR & \\
\hline Annia Faustina & $22.1 \mathrm{~N}$ & 4.7E & 23 & EU & $\mathrm{RM}$ & \\
\hline $\begin{array}{l}\text { Antonina } \\
\text { Anush } \\
\text { Anya } \\
\text { Ariadne }\end{array}$ & $\begin{array}{l}28.2 \mathrm{~N} \\
15.0 \mathrm{~N} \\
39.5 \mathrm{~N} \\
43.9 \mathrm{~N}\end{array}$ & $\begin{array}{r}106.8 \mathrm{E} \\
86.5 \mathrm{E} \\
297.8 \mathrm{E} \\
0.0 \mathrm{E}\end{array}$ & $\begin{array}{l}15 \\
13 \\
20 \\
22\end{array}$ & $\begin{array}{l}\text { UR } \\
\text { UR } \\
\text { UR } \\
\text { EU }\end{array}$ & $\begin{array}{l}\text { RU } \\
\text { AM } \\
\text { RU } \\
\text { GR }\end{array}$ & $\begin{array}{l}25 \\
19 \\
11,12\end{array}$ \\
\hline $\begin{array}{l}\text { Asmik } \\
\text { Astrid } \\
\text { Audrey } \\
\text { Aurelia } \\
\text { Austen }\end{array}$ & $\begin{array}{l}3.9 \mathrm{~N} \\
21.4 \mathrm{~S} \\
23.8 \mathrm{~N} \\
20.3 \mathrm{~N} \\
25.0 \mathrm{~S}\end{array}$ & $\begin{array}{l}166.4 \mathrm{E} \\
335.2 \mathrm{E} \\
348.1 \mathrm{E} \\
331.8 \mathrm{E} \\
168.3 \mathrm{E}\end{array}$ & $\begin{array}{l}17 \\
11 \\
15 \\
31 \\
47\end{array}$ & $\begin{array}{l}\text { UR } \\
\text { EU } \\
\text { EU } \\
\text { EU } \\
\text { EU }\end{array}$ & $\begin{array}{l}\text { AM } \\
\text { SD } \\
\text { EN } \\
\text { RM } \\
\text { EN }\end{array}$ & \\
\hline $\begin{array}{l}\text { Avviyar } \\
\text { Ayana } \\
\text { Badarzewska }\end{array}$ & $\begin{array}{l}18.0 \mathrm{~S} \\
29.1 \mathrm{~S} \\
22.6 \mathrm{~S}\end{array}$ & $\begin{array}{l}353.6 \mathrm{E} \\
175.5 \mathrm{E} \\
137.2 \mathrm{E}\end{array}$ & $\begin{array}{l}21 \\
16 \\
27\end{array}$ & $\begin{array}{l}\text { AS } \\
\text { UR } \\
\text { EU }\end{array}$ & $\begin{array}{l}\text { IN } \\
\text { RU } \\
\text { PO }\end{array}$ & \\
\hline
\end{tabular}

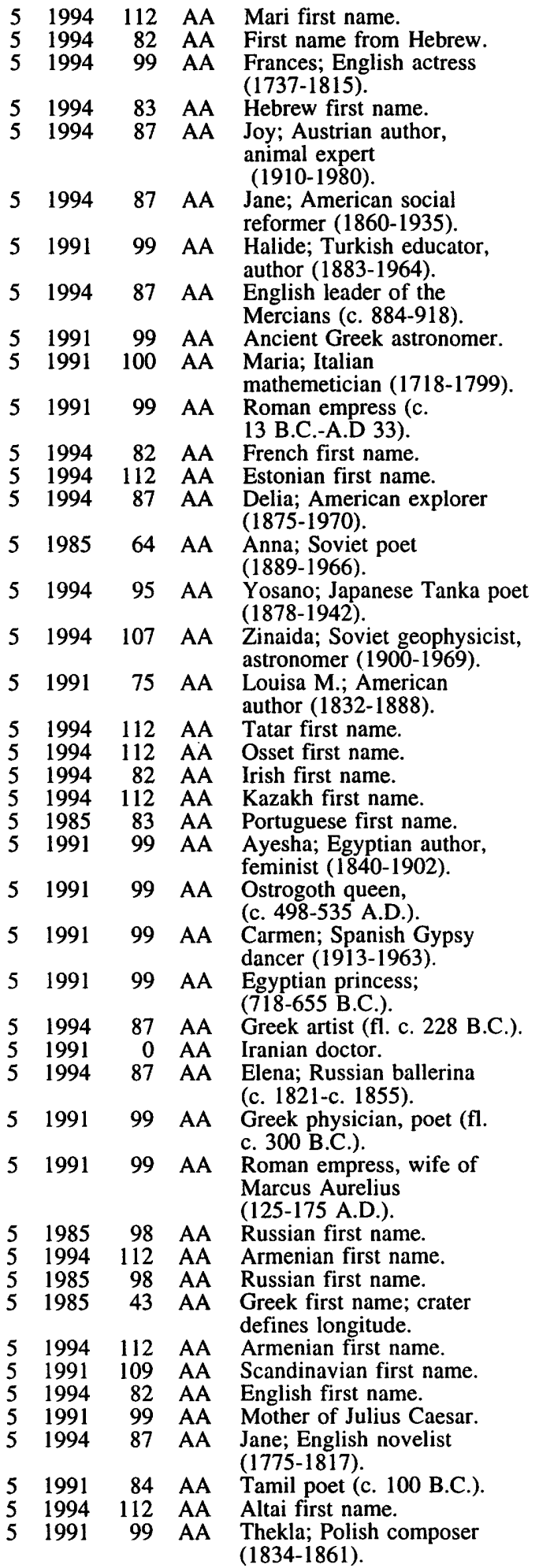

lat: latitude of feature center.

long: longitude of feature center.

diam: diameter or long dimension of feature. et: ethnicity of name origin (see page $284 \mathrm{ff}$.)

quad: map quadrangle or informal name

(see page xvii ff.). as: name approval status (see page xvii)

ad: name approval date (year).

ref: reference source for name (see page $287 \mathrm{ff}$.).

ct. continent of name origin (see page $284 \mathrm{ff}$.) map: map name or USGS map number (see page xvii ff.). ft: feature type (see page 290 ). 
VENUS

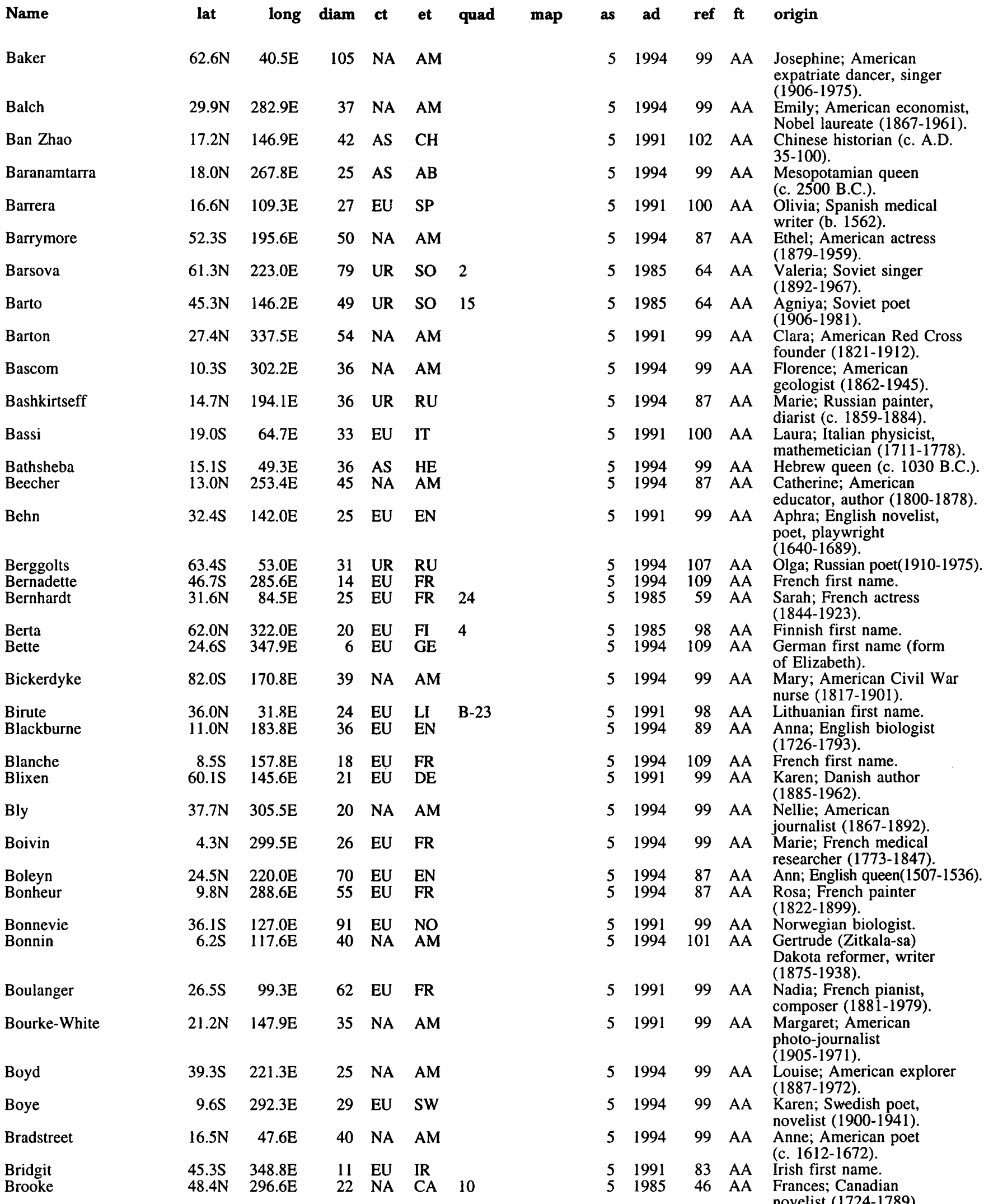
novelist (1724-1789).

lat: latitude of feature center.

long: longitude of feature center.

diam: diameter or long dimension of feature. et: ethnicity of name origin (see page $284 \mathrm{ff}$.)

quad: map quadrangle or informal name

(see page xvii ff.).

map name or USGS map number (see page xvii ff.). as: name approval status (see page xvii)

ad: name approval date (year).

ref: reference source for name (see page $287 \mathrm{ff}$.). 


\section{VENUS}

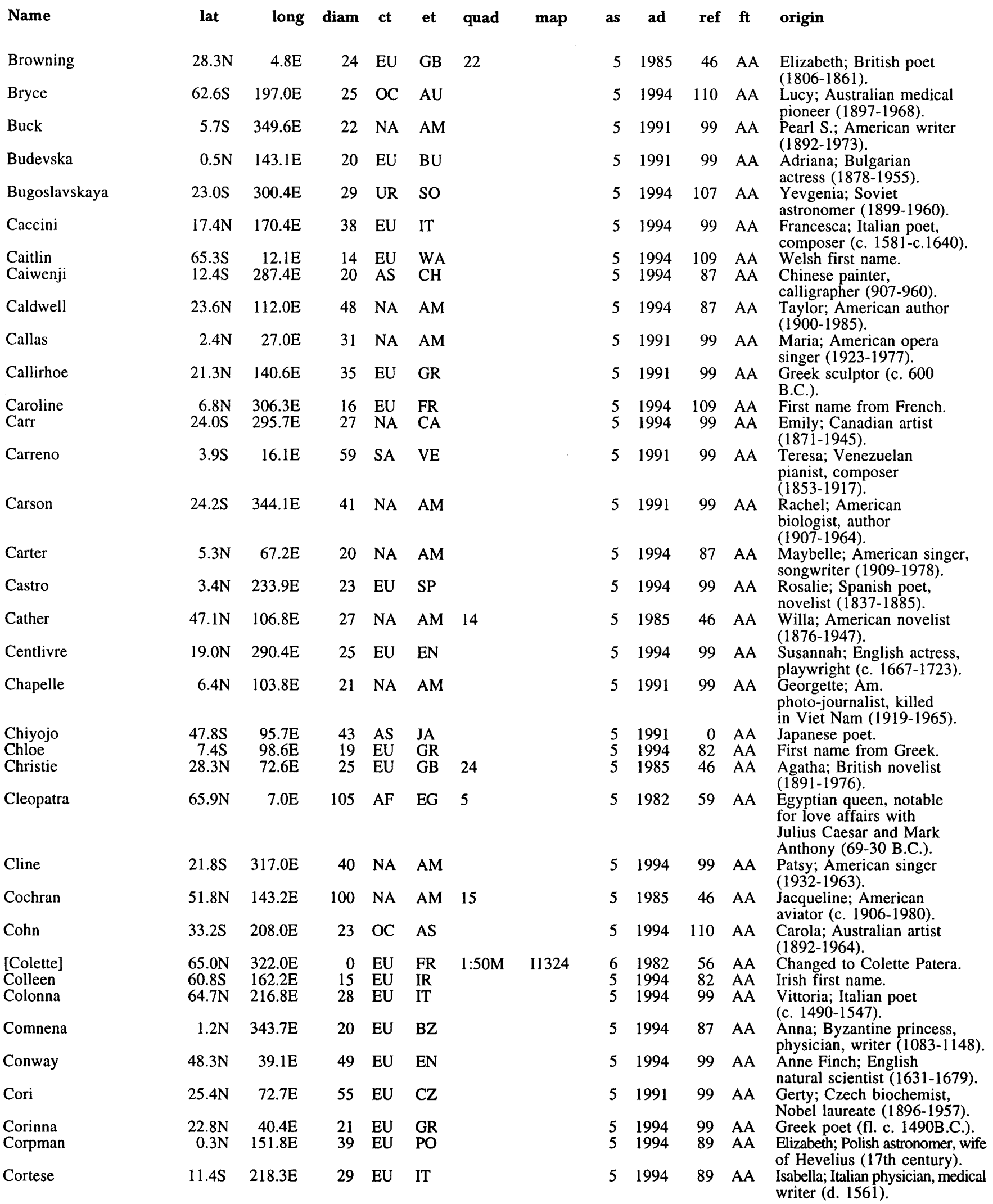

lat: latitude of feature center.

long: longitude of feature center.

diam: diameter or long dimension of feature.

ct: continent of name origin (see page $284 \mathrm{ff}$.) map: et: ethnicity of name origin (see page $284 \mathrm{ff}$.)

quad: map quadrangle or informal name

(see page xvii ff.). as: name approval status (see page xvii).

ad: name approval date (year).

ref: reference source for name (see page $287 \mathrm{ff}$.). 
VENUS

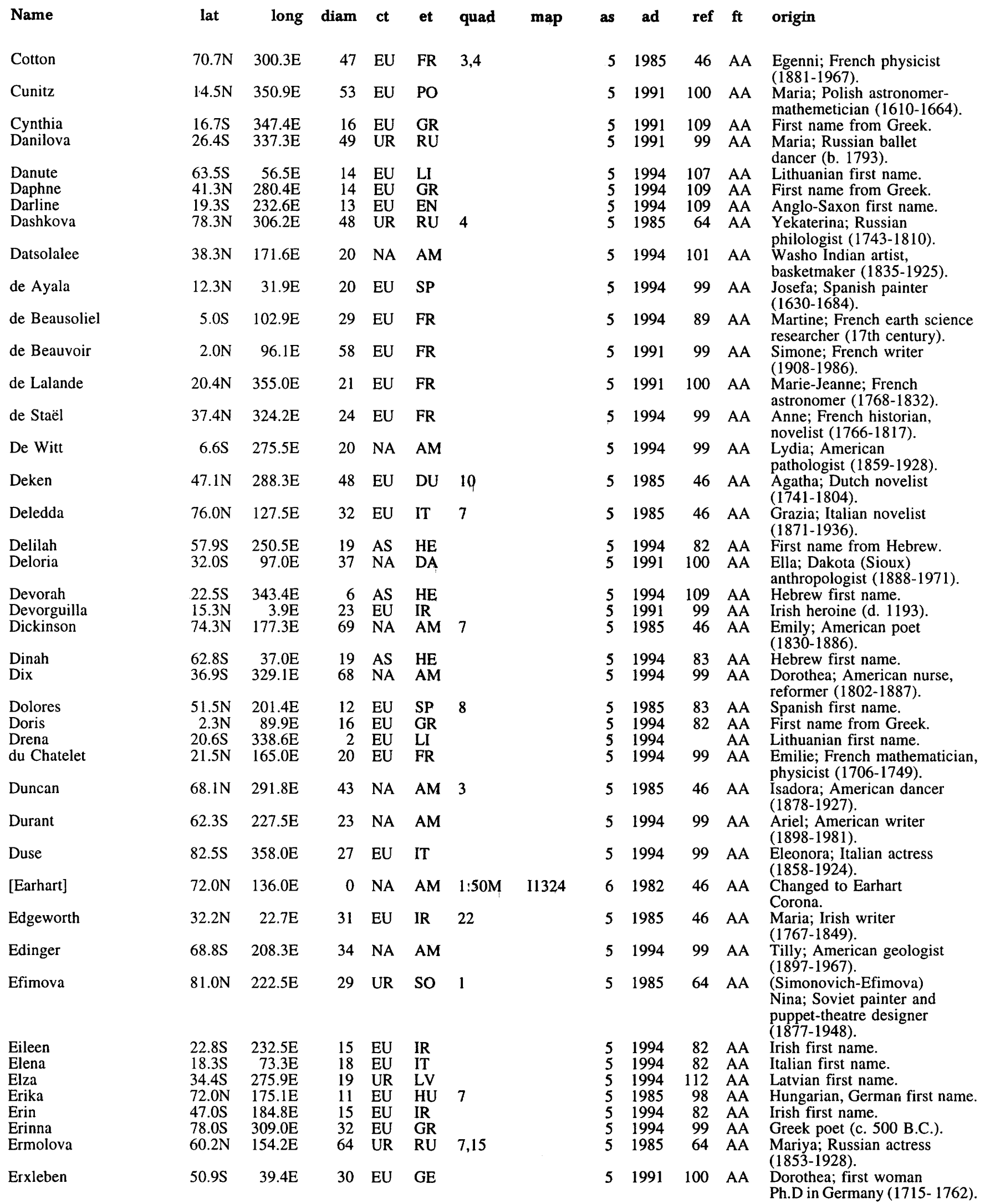

lat: latitude of feature center.

long: longitude of feature center.

diam: diameter or long dimension of feature.

ct: continent of name origin (see page $284 \mathrm{ff}$.) map: et: ethnicity of name origin (see page $284 \mathrm{ff}$.)

quad: map quadrangle or informal name

(see page xvii ff.). as: name approval status (see page xvii).

ad: name approval date (year).

ref: reference source for name (see page $287 \mathrm{ff}$.) 


\section{VENUS}

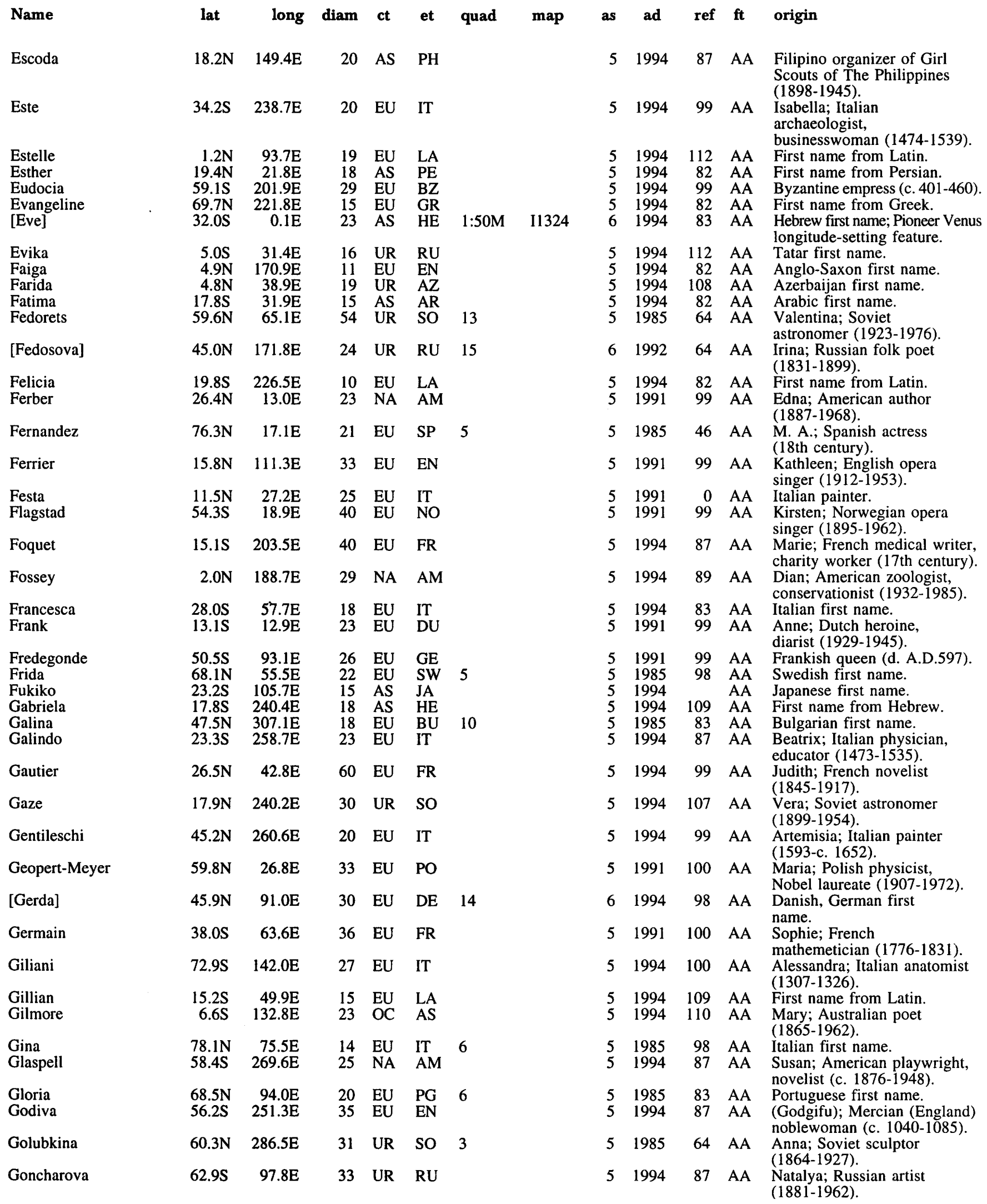

\footnotetext{
lat: latitude of feature center.

long: longitude of feature center.

diam: diameter or long dimension of feature.

et: ethnicity of name origin (see page $284 \mathrm{ff}$.)

quad: map quadrangle or informal name

(see page xvii ff.).

as: name approval status (see page $\mathrm{xvii}$ ).

ad: name approval date (year).

ref: reference source for name (see page $287 \mathrm{ff}$.)

ct: continent of name origin (see page $284 \mathrm{ff}$.) map: map name or USGS map number (see page xvii ff.). ft: feature type (see page 290 ).
} 


\section{VENUS}

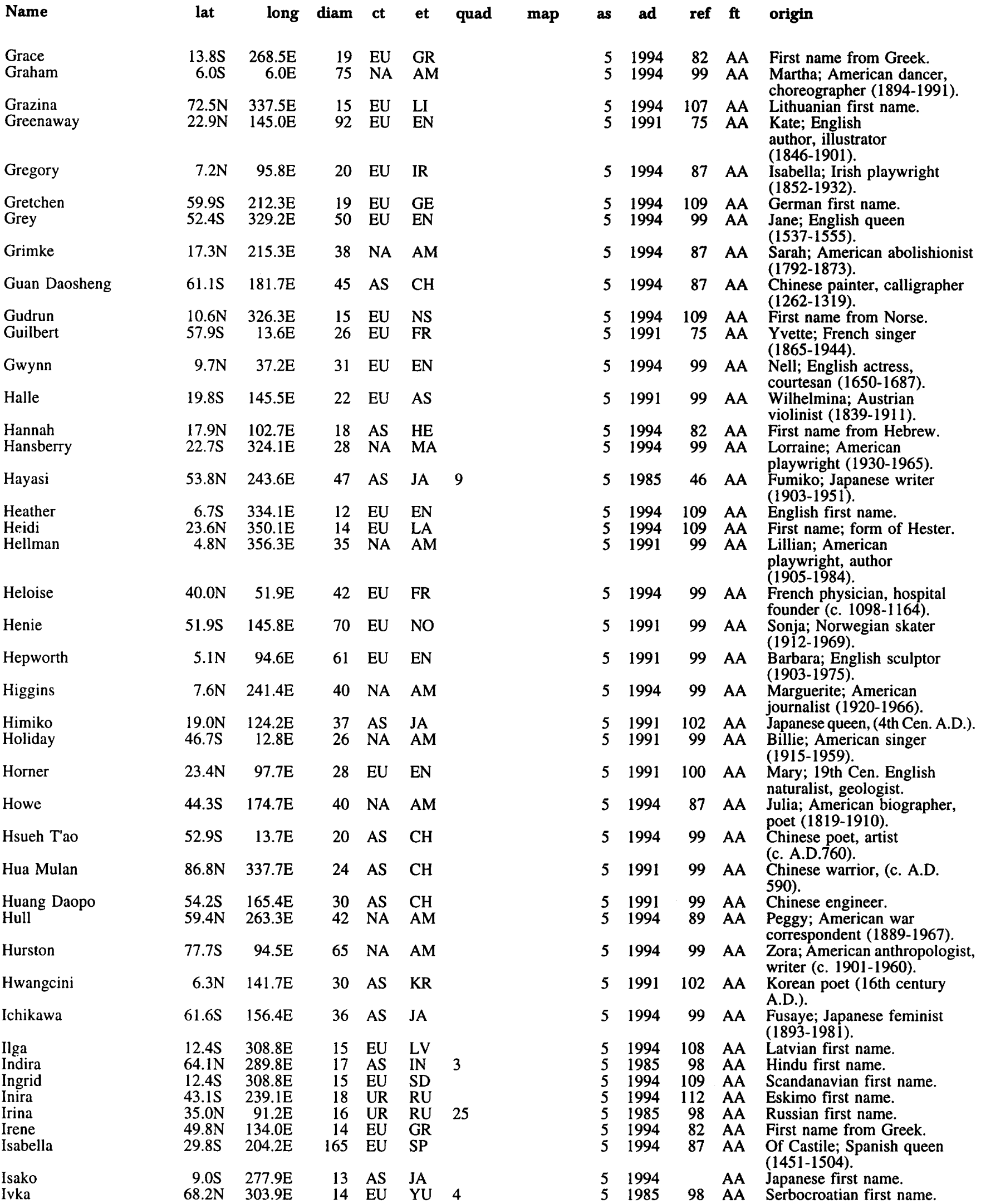

lat: latitude of feature center. long: longitude of feature center.

diam: diameter or long dimension of feature. et: ethnicity of name origin (see page $284 \mathrm{ff}$.)

quad: map quadrangle or informal name (see page xvii ff.). as: name approval status (see page xvii).

ad: name approval date (year).

ref: reference source for name (see page $287 \mathrm{ff}$.).

ct: continent of name origin (see page $284 \mathrm{ff}$.) map: map name or USGS map number (see page xvii ff.). ft: feature type (see page 290). 
VENUS

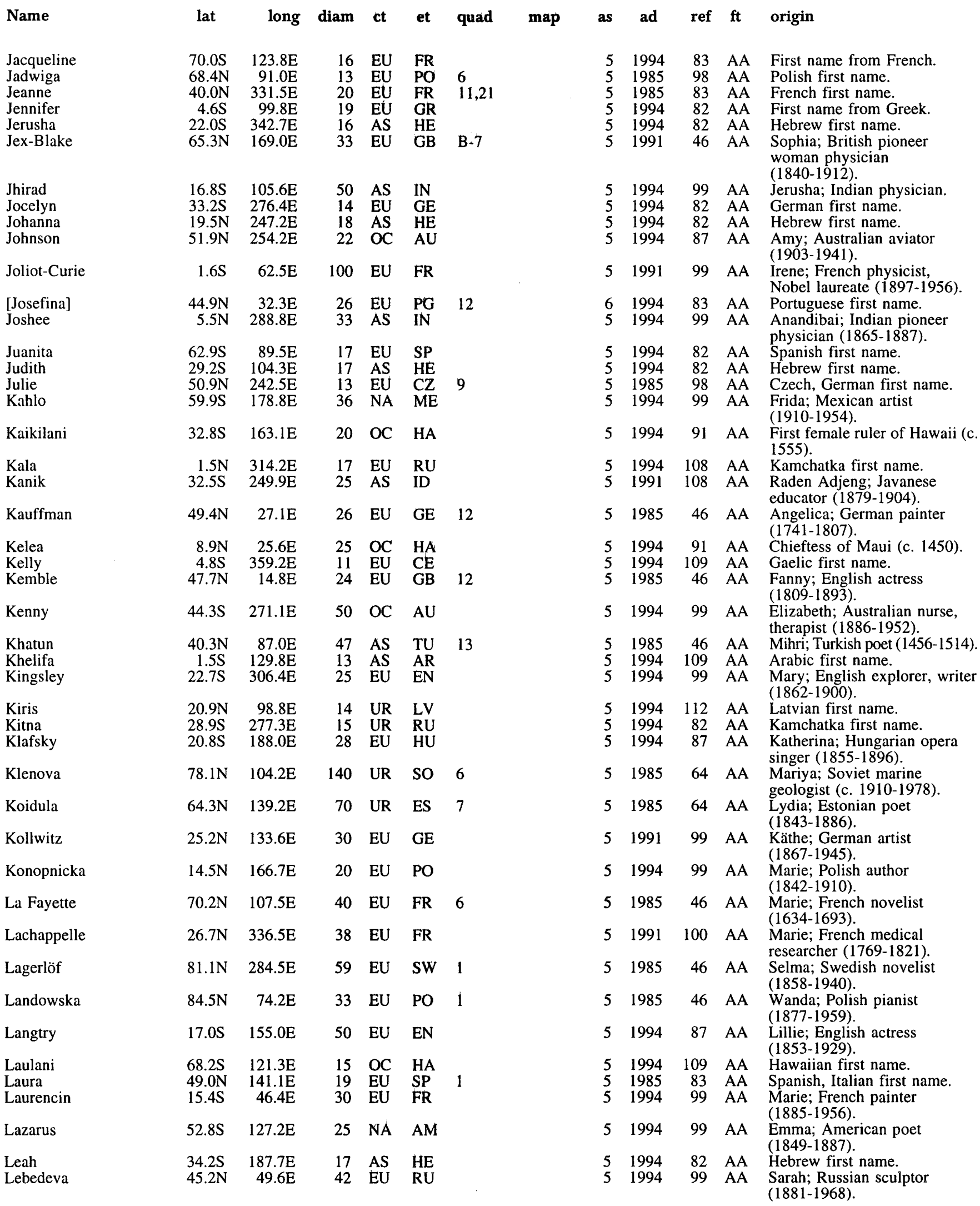

lat: latitude of feature center.

long: longitude of feature center.

diam: diameter or long dimension of feature.

ct: continent of name origin (see page $284 \mathrm{ff}$.) map: et: ethnicity of name otigin (see page $284 \mathrm{ff}$.)

quad: map quadrangle or informal name

(see page $x$ vii $\mathrm{ff}$.)

map name or USGS map number (see page xvii ff.). ft: feature type (see page 290). as: name approval status (see page $x$ vii).

ad: name approval date (year).

ref: reference source for name (see page $287 \mathrm{ff}$.). 


\section{VENUS}

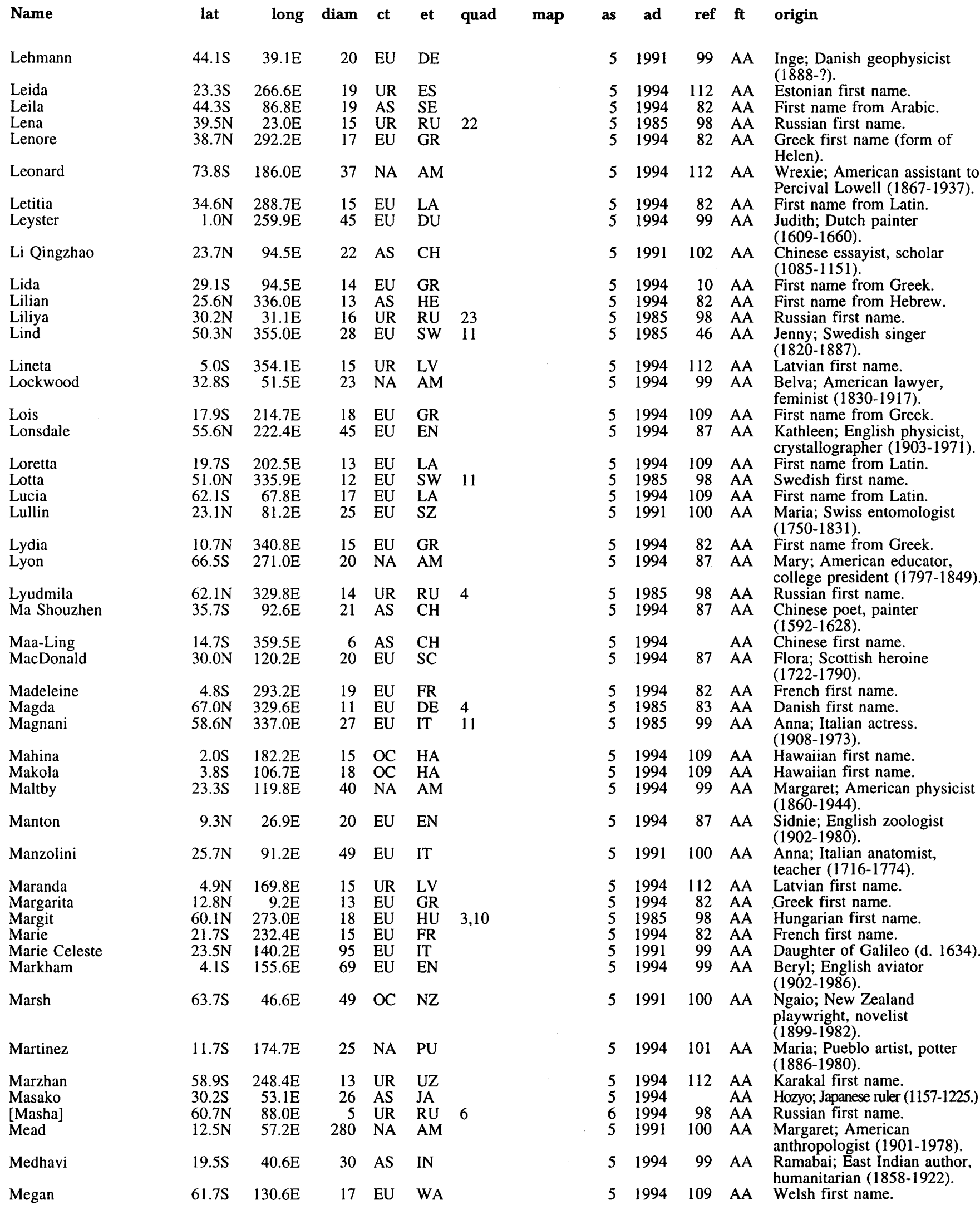

\footnotetext{
lat: latitude of feature center.

long: longitude of feature center.

diam: diameter or long dimension of feature.

et: ethnicity of name origin (see page $284 \mathrm{ff}$.)

quad: map quadrangle or informal name

(see page xvii ff.).

as: name approval status (see page $x v i i$ ).

ad: name approval date (year)

ref: reference source for name (see page $287 \mathrm{ff}$.).
} 


\section{VENUS}

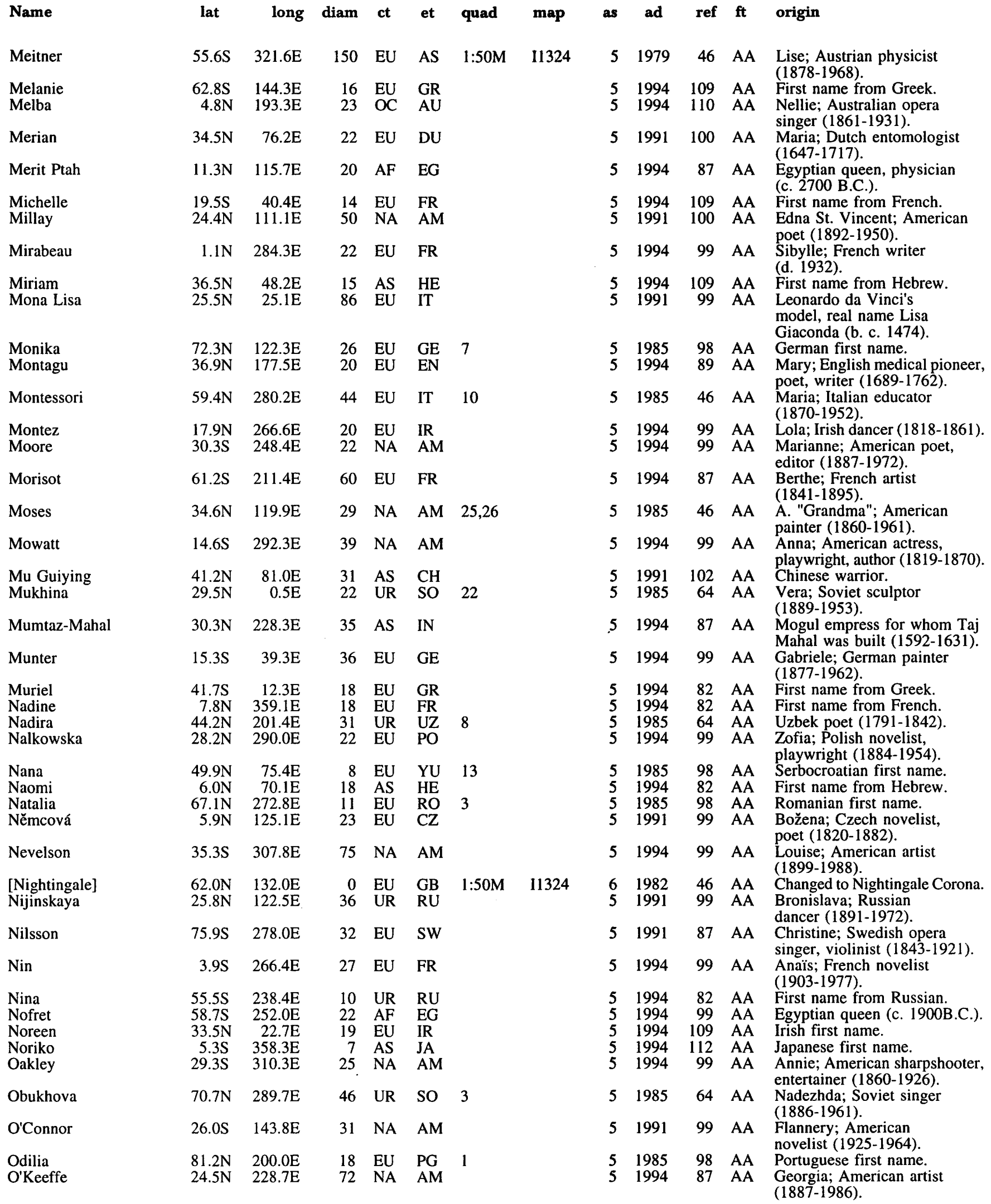

\footnotetext{
lat: latitude of feature center.

et: ethnicity of name origin (see page $284 \mathrm{ff}$.)

as: name approval status (see page $x$ vii).

long: longitude of feature center.

quad: map quadrangle or informal name

ad: name approval date (year).

diam: diameter or long dimension of feature. (see page $x$ vii ff.).

ref: reference source for name (see page $287 \mathrm{ff}$.).

ct: continent of name origin (see page $284 \mathrm{ff}$.) map: map name or USGS map number (see page xvii ff.). ft: feature type (see page 290 ).
} 
VENUS

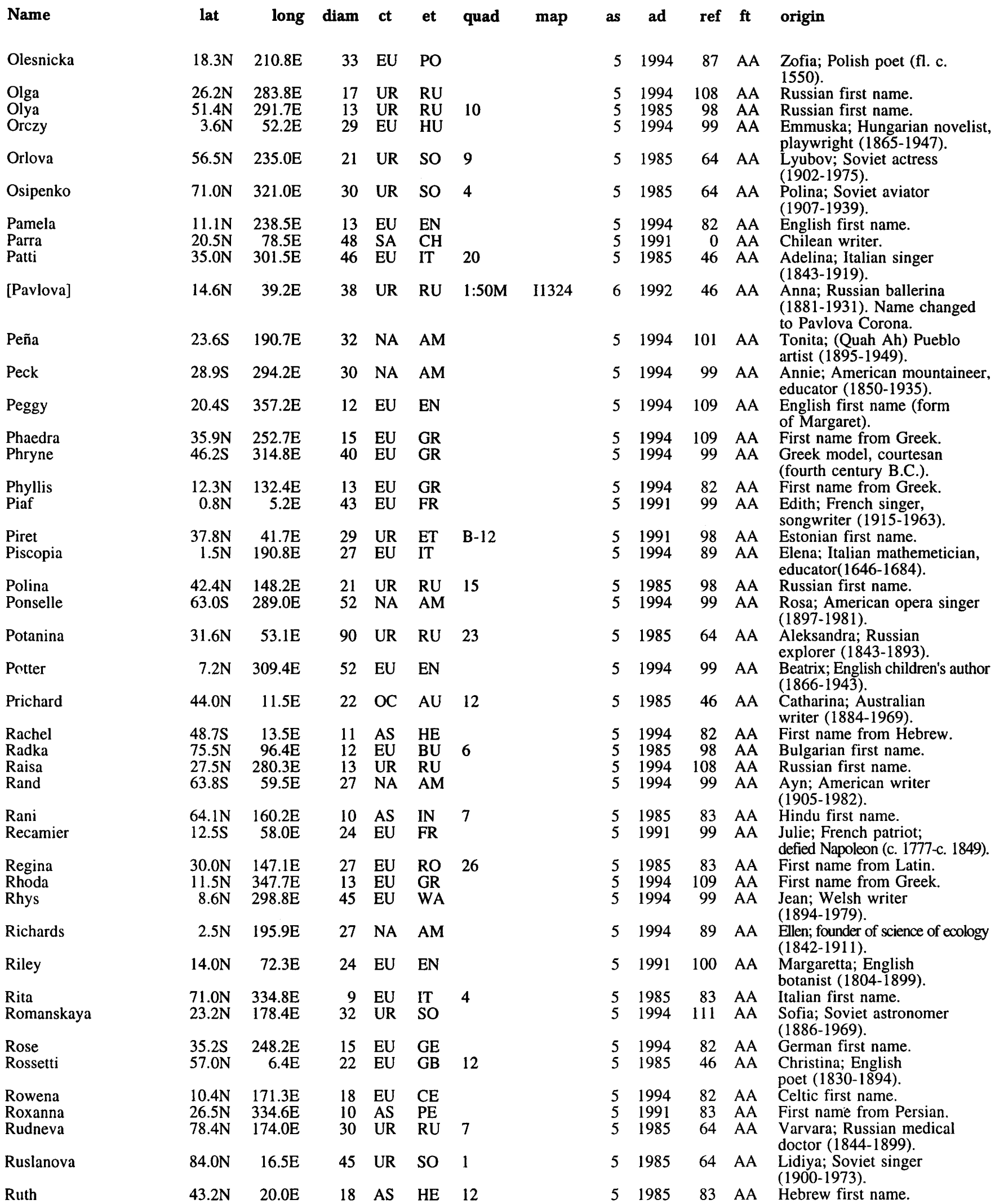

lat: latitude of feature center.

long: longitude of feature center.

diam: diameter or long dimension of feature. et: ethnicity of name origin (see page $284 \mathrm{ff}$.)

quad: map quadrangle or informal name

(see page xvii ff.). as: name approval status (see page xvii)

ad: name approval date (year).

ref: reference source for name (see page $287 \mathrm{ff}$.).

ct: continent of name origin (see page $284 \mathrm{ff}$.) map: map name or USGS map number (see page xvii ff.). ft: feature type (see page 290 ). 


\section{VENUS}

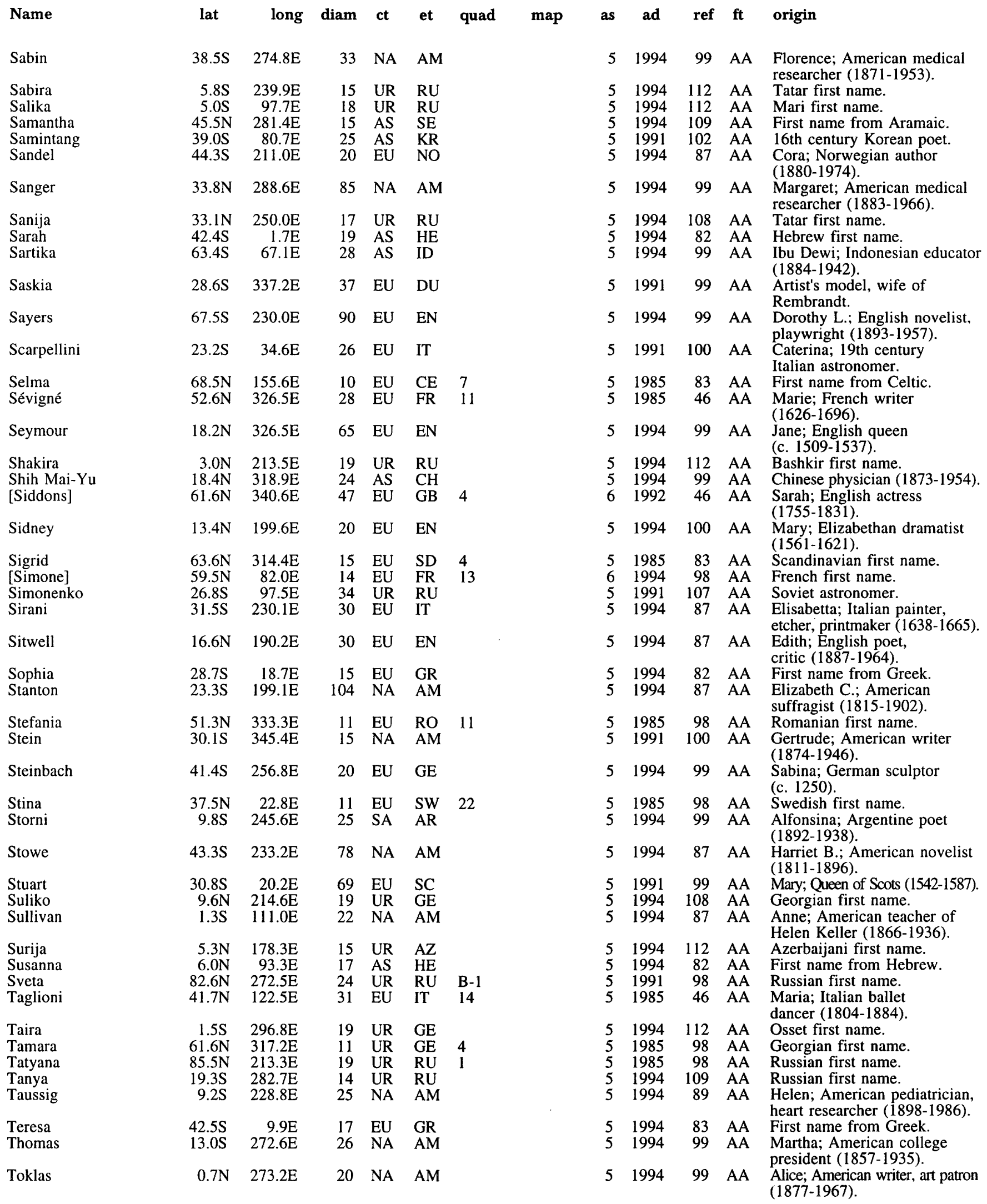

lat: latitude of feature center.

long: longitude of feature center.

diam: diameter or long dimension of feature. et: ethnicity of name origin (see page $284 \mathrm{ff}$.) -

quad: map quadrangle or informal name

(see page xvii ff.) as: name approval status (see page xvii).

ad: name approval date (year).

ref: reference source for name (see page $287 \mathrm{ff}$.)

ct: continent of name origin (see page $284 \mathrm{ff}$.) map: map name or USGS map number (see page xvii ff.). ft: feature type (see page 290 ). 
VENUS

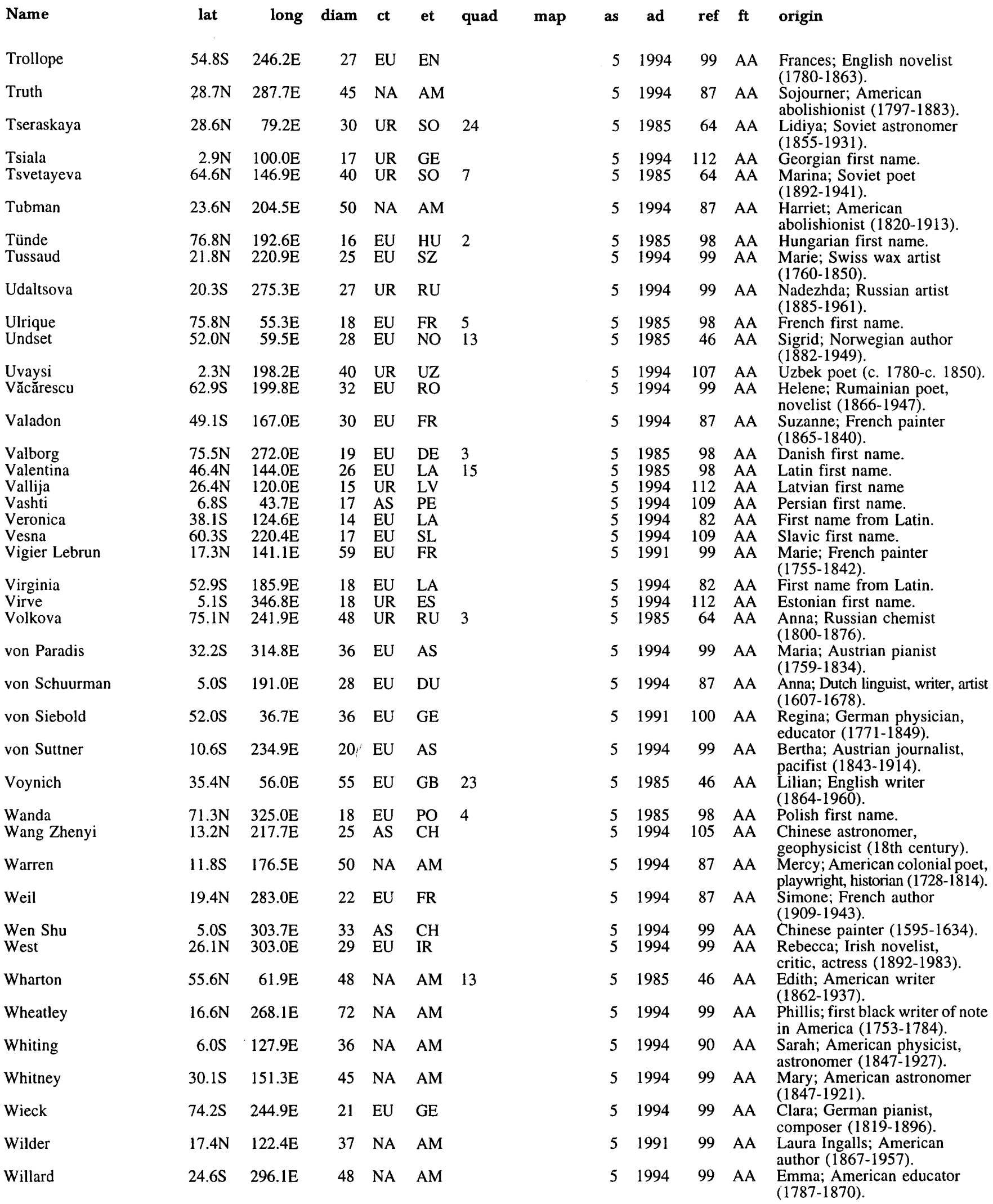

lat: latitude of feature center.

long: longitude of feature center.

diam: diameter or long dimension of feature.

et: ethnicity of name origin (see page $284 \mathrm{ff}$.)

quad: map quadrangle or informal name

(see page xvii ff.).

ct: continent of name origin (see page $284 \mathrm{ff}$ ) map: map name or USGS map number (see page xvii ff.). ft: feature type (see page 290).

as: name approval status (see page xvii)

ad: name approval date (year).

ref: reference source for name (see page $287 \mathrm{ff}$.). 


\section{VENUS}

\begin{tabular}{|c|c|c|c|c|c|}
\hline Name & lat & long & diam & ct & et \\
\hline Winema & $3.1 \mathrm{~N}$ & $168.6 \mathrm{E}$ & 24 & NA & $\mathrm{AM}$ \\
\hline Winnemucca & $15.4 \mathrm{~S}$ & $121.1 \mathrm{E}$ & 30 & NA & AM \\
\hline Wollstonecraft & $39.2 \mathrm{~S}$ & $260.8 \mathrm{E}$ & 45 & EU & EN \\
\hline Woolf & $37.7 \mathrm{~S}$ & $27.1 \mathrm{E}$ & 25 & $\mathrm{EU}$ & GB \\
\hline Workman & $12.9 \mathrm{~S}$ & $299.9 \mathrm{E}$ & 20 & NA & $\mathrm{AM}$ \\
\hline $\begin{array}{l}\text { Wu Hou } \\
\text { Xantippe }\end{array}$ & $\begin{array}{l}25.4 \mathrm{~S} \\
10.8 \mathrm{~S}\end{array}$ & $\begin{array}{r}317.4 \mathrm{E} \\
11.7 \mathrm{E}\end{array}$ & $\begin{array}{l}30 \\
40\end{array}$ & $\begin{array}{l}\text { AS } \\
\text { EU }\end{array}$ & $\begin{array}{l}\mathrm{CH} \\
\text { GR }\end{array}$ \\
\hline Xiao Hong & $43.6 \mathrm{~S}$ & $101.7 \mathrm{E}$ & 38 & AS & $\mathrm{CH}$ \\
\hline Yablochkina & $48.2 \mathrm{~N}$ & $195.1 \mathrm{E}$ & 63 & UR & SO \\
\hline Yale & $13.4 \mathrm{~S}$ & $271.3 \mathrm{E}$ & 20 & NA & AM \\
\hline Yonge & $14.0 \mathrm{~S}$ & $115.1 \mathrm{E}$ & 28 & EU & EN \\
\hline Yoshioka & $32.4 \mathrm{~S}$ & $58.8 \mathrm{E}$ & 20 & AS & JA \\
\hline $\begin{array}{l}\text { Yvonne } \\
\text { Zamudio }\end{array}$ & $\begin{array}{r}56.0 \mathrm{~S} \\
9.6 \mathrm{~N}\end{array}$ & $\begin{array}{l}298.3 \mathrm{E} \\
189.3 \mathrm{E}\end{array}$ & $\begin{array}{l}15 \\
20\end{array}$ & $\begin{array}{l}\text { EU } \\
\text { SA }\end{array}$ & $\begin{array}{l}\text { FR } \\
\text { BO }\end{array}$ \\
\hline $\begin{array}{l}\text { Zdravka } \\
\text { Zenobia }\end{array}$ & $\begin{array}{l}65.0 \mathrm{~N} \\
29.3 \mathrm{~S}\end{array}$ & $\begin{array}{r}299.0 \mathrm{E} \\
28.5 \mathrm{E}\end{array}$ & $\begin{array}{l}12 \\
39\end{array}$ & $\begin{array}{l}\text { EU } \\
\text { AS }\end{array}$ & $\begin{array}{l}\text { BU } \\
\text { SY }\end{array}$ \\
\hline Zhilova & $66.3 \mathrm{~N}$ & $125.5 \mathrm{E}$ & 56 & UR & RU \\
\hline $\begin{array}{l}\text { Zhu Shuzhen } \\
\text { Zija } \\
\text { Zina } \\
\text { Zlata } \\
\text { Zoya } \\
\text { Zvereva }\end{array}$ & $\begin{array}{l}26.5 \mathrm{~S} \\
3.5 \mathrm{~S} \\
41.9 \mathrm{~N} \\
64.6 \mathrm{~N} \\
69.1 \mathrm{~N} \\
45.3 \mathrm{~N}\end{array}$ & $\begin{array}{l}356.5 \mathrm{E} \\
265.0 \mathrm{E} \\
320.0 \mathrm{E} \\
334.0 \mathrm{E} \\
235.9 \mathrm{E} \\
283.0 \mathrm{E}\end{array}$ & $\begin{array}{r}30 \\
18 \\
10 \\
7 \\
21 \\
24\end{array}$ & $\begin{array}{l}\text { AS } \\
\text { UR } \\
\text { EU } \\
\text { EU } \\
\text { UR } \\
\text { UR }\end{array}$ & $\begin{array}{l}\mathrm{CH} \\
\text { AZ } \\
\text { RO } \\
\text { YU } \\
\text { RU } \\
\text { RU }\end{array}$ \\
\hline
\end{tabular}

\section{CHASMA}

Aranyani Chasma

Artemis Chasma

Baba-Jaga Chasma

Britomartis Chasma

Dali Chasma

Daura Chasma

Devana Chasma

Diana Chasma

Ganis Chasma

Hecate Chasma

Heng-O Chasma

Hina Chasma

Ix Chel Chasma

Juno Chasma

Kaygus Chasmata

Kottravey Chasma

Kozhla-Ava Chasma

Kuanja Chasma

Lasdona Chasma

Medeina Chasma

Mežas-Mate Chasma

\begin{tabular}{|c|c|c|c|c|c|c|}
\hline $\begin{array}{l}69.3 \mathrm{~N} \\
41.2 \mathrm{~S}\end{array}$ & $\begin{array}{r}74.4 \mathrm{E} \\
138.5 \mathrm{E}\end{array}$ & $\begin{array}{r}718 \\
3087\end{array}$ & $\begin{array}{l}\text { AS } \\
\text { EU }\end{array}$ & $\begin{array}{l}\text { IN } \\
\text { GR }\end{array}$ & $\begin{array}{l}6 \\
1: 50 M\end{array}$ & I1324 \\
\hline $53.2 \mathrm{~N}$ & $\begin{array}{r}49.5 \mathrm{E}\end{array}$ & 580 & $\begin{array}{l}\text { EU } \\
\text { UR }\end{array}$ & SL & 12,13 & 11524 \\
\hline $33.0 \mathrm{~S}$ & $130.0 \mathrm{E}$ & $\mathrm{EU}$ & GR & & & \\
\hline $17.6 \mathrm{~S}$ & $167.0 \mathrm{E}$ & 2077 & UR & GE & $1: 50 \mathrm{M}$ & I1324 \\
\hline $96 \mathrm{~N}$ & $284.4 \mathrm{E}$ & 1616 & EU & $\mathrm{CZ}$ & $1.50 \mathrm{M}$ & 11324 \\
\hline & $154.8 \mathrm{E}$ & 938 & EU & RM & $1.50 \mathrm{M}$ & 11324 \\
\hline $16.3 \mathrm{~N}$ & $196.4 \mathrm{E}$ & 615 & $\mathrm{EU}$ & LP & $1: 50 \mathrm{M}$ & I1324 \\
\hline $18.2 \mathrm{~N}$ & $254.3 \mathrm{E}$ & 3145 & $\mathrm{EU}$ & GR & $1: 50 \mathrm{M}$ & I1 324 \\
\hline $6.6 \mathrm{~N}$ & $355.5 \mathrm{E}$ & 734 & AS & $\mathrm{CH}$ & $1: 50 \mathrm{M}$ & I1 324 \\
\hline $64.5 \mathrm{~N}$ & $20.0 \mathrm{E}$ & 0 & OC & HA & B-5 & \\
\hline $10.0 \mathrm{~S}$ & $73.4 \mathrm{E}$ & 503 & SA & $\mathrm{AZ}$ & $1: 50 \mathrm{M}$ & I1324 \\
\hline $30.5 \mathrm{~S}$ & $111.1 \mathrm{E}$ & 915 & EU & RM & $1: 50 \mathrm{M}$ & I1324 \\
\hline $49.6 \mathrm{~N}$ & $52.1 \mathrm{E}$ & 503 & UR & SI & 13 & \\
\hline $30.5 \mathrm{~N}$ & $76.8 \mathrm{E}$ & 744 & AS & IN & 24 & \\
\hline $56.2 \mathrm{~N}$ & $50.6 \mathrm{E}$ & 581 & EU & FI & 13 & \\
\hline $12.0 \mathrm{~S}$ & $99.5 \mathrm{E}$ & 890 & AF & MB & $1: 50 \mathrm{M}$ & I1324 \\
\hline $69.3 \mathrm{~N}$ & $34.4 \mathrm{E}$ & 697 & UR & LI & 5 & \\
\hline $46.2 \mathrm{~N}$ & $89.3 \mathrm{E}$ & 606 & UR & LI & 13,14 & \\
\hline $51.0 \mathrm{~N}$ & $50.7 \mathrm{E}$ & 506 & UR & LV & 13 & \\
\hline
\end{tabular}

\begin{tabular}{|c|c|c|c|c|}
\hline is & ad & ref & $\mathrm{ft}$ & origin \\
\hline 5 & 1994 & 101 & AA & Modoc Indian heroine, \\
\hline 5 & 1994 & 101 & AA & $\begin{array}{l}\text { peacemaker (c. 1848-1932). } \\
\text { Sarah; Piute interpreter, } \\
\text { activist (c 1844-1891). }\end{array}$ \\
\hline 5 & 1994 & 87 & AA & $\begin{array}{l}\text { Mary; English author } \\
(1759-1797) \text {. }\end{array}$ \\
\hline 5 & 1991 & 100 & AA & $\begin{array}{l}\text { Virginia; British writer } \\
(1882-1941) \text {. }\end{array}$ \\
\hline 5 & 1994 & 99 & AA & $\begin{array}{l}\text { Fanny; American mountaineer, } \\
\text { author }(1859-1925) \text {. }\end{array}$ \\
\hline 5 & 1994 & 99 & AA & Chinese empress (c. 624-705). \\
\hline 5 & 1991 & 99 & $\mathrm{AA}$ & $\begin{array}{l}\text { Wife of Socrates ( } 5 \text { th } \\
\text { century B.C.). }\end{array}$ \\
\hline 5 & 1991 & 102 & AA & $\begin{array}{l}\text { Chinese novelist } \\
(1911-1942) \text {. }\end{array}$ \\
\hline 5 & 1985 & 64 & AA & $\begin{array}{l}\text { Aleksandra; Soviet } \\
\text { actress (1866-1964). }\end{array}$ \\
\hline 5 & 1994 & 99 & AA & $\begin{array}{l}\text { Caroline; American educator of } \\
\text { the deaf (1848-1933). }\end{array}$ \\
\hline 5 & 1994 & 99 & AA & $\begin{array}{l}\text { Charlotte; English writer } \\
\text { (1823-1901). }\end{array}$ \\
\hline 5 & 1994 & 99 & AA & $\begin{array}{l}\text { Yayoi; Japanese physician, } \\
\text { college founder (c. 1871-1959). }\end{array}$ \\
\hline 5 & 1994 & 109 & AA & French first name. \\
\hline 5 & 1994 & 99 & $\mathrm{AA}$ & $\begin{array}{l}\text { Adela; Bolivian poet } \\
\text { (1854-1928). }\end{array}$ \\
\hline 5 & 1985 & 98 & AA & Bulgarian first name. \\
\hline & 1994 & 99 & AA & $\begin{array}{l}\text { Queen of Palmyra (Syria) } \\
\text { (third century A.D.). }\end{array}$ \\
\hline 5 & 1985 & 64 & AA & $\begin{array}{l}\text { Maria; Russian astronomer } \\
(1870-1934) \text {. }\end{array}$ \\
\hline 5 & 1991 & 99 & AA & Chinese poet (1126-1200). \\
\hline & 1994 & 112 & AA & Azerbaijani first name. \\
\hline 5 & 1985 & 98 & AA & Romanian first name. \\
\hline 5 & 1985 & 98 & AA & Serbocroatian first name. \\
\hline & 1985 & 98 & AA & Russian first name. \\
\hline & & & & $\begin{array}{l}\text { Lidiya; Russian aviator } \\
\text { (1890-1916). }\end{array}$ \\
\hline
\end{tabular}

$5198565 \mathrm{CM}$ Indian forest goddess.

$51982 \quad 1 \quad \mathrm{CM}$ Greek goddess of hunt/moon. $5 \quad 1985 \quad 65$ CM Slavic forest witch.

$51994 \quad 01 \quad$ CM Greek/Cretan goddess of the hunt

$5 \quad 1985 \quad 27$ CM Georgian; goddess of hunt.

5198565 CM Hausa (W. Sudan) great huntress.

$51982 \quad 27$ CM Czechoslovakian goddess of hunt.

$51982 \quad 1 \mathrm{CM}$ Roman goddess of hunt/moon

$5 \quad 1982 \quad 27 \quad$ CM Western Lapp forest maiden.

$5 \quad 1982 \quad 1 \quad \mathrm{CM}$ Greek moon goddess.

$51982 \quad 1 \quad \mathrm{CM}$ Chinese moon goddess.

$5 \quad 1991 \quad 1 \quad \mathrm{CM}$ Hawaiian moon goddess.

519821 CM Aztec wife of the Sun god

probably moon goddess.

$51982 \quad 1 \quad \mathrm{CM}$ Roman sky goddess; sister and consort of Jupiter.

$5198565 \mathrm{CM}$ Ketian (Siberia) ruler of

forest animals.

$5198565 \mathrm{CM}$ Dravidian (India) hunting

$5198565 \mathrm{CM}$ Marian (Volga Finn)

forest goddess.

$51982 \quad 1 \quad \mathrm{CM}$ Mbundu goddess of the

$5198565 \mathrm{CM} \quad \begin{aligned} & \text { spirit of the hunt. } \\ & \text { Lithuanian main forest }\end{aligned}$

goddess.

$51985 \quad 65 \mathrm{CM}$ Lithuanian forest

$5198565 \quad \mathrm{CM}$ Latvian forest goddess. lat: latitude of feature center. long: longitude of feature center.

diam: diameter or long dimension of feature. et: ethnicity of name origin (see page $284 \mathrm{ff}$.)

quad: map quadrangle or informal name (see page xvii ff.). as: name approval status (see page xvii).

ad: name approval date (year).

ref: reference source for name (see page $287 \mathrm{ff}$.).

ct: $\quad$ continent of name origin (see page $284 \mathrm{ff}$.) map: map name or USGS map number (see page xvii ff.). ft: feature type (see page 290 ). 
VENUS

Name

Misne Chasma

Morana Chasma

Mots Chasma

Parga Chasma

Quilla Chasma

Varz Chasma

Vir-Ava Chasma

Vires-Akka Chasma

\section{COLLES}

Akkruva Colles

Jurate Colles

Mena Colles

Molpe Colles

T'ien Hu Colles

\section{CORONA}

Aeracura Corona

Agraulos Corona

Allatu Corona

Ament Corona

Anahit Corona

Anala Corona

Annapurna Corona

Aramaiti Corona

Artemis Corona

Aruru Corona

Ashnan Corona

Atargatis Corona

Atete Corona

Atse Estsan Corona

Audhumla Corona

Bachue Corona

Bau Corona

Beiwe Corona

Belet-Ili Corona

Benten Corona

Beruth Corona

Beyla Corona

Bhumidevi Corona

Bhumiya Corona

Blai Corona

Blathnat Corona

Boann Corona

Bona Corona

Cailleach Corona

Calakomana Corona

Carpo Corona

Cauteovan Corona

Ceres Corona

Chiun Corona

Ciuacoatl Corona

Coatlicue Corona

Colijnsplaat Corona

Copia Corona

Cybele Corona

Demeter Corona

Derceto Corona

Dhisana Corona

Earhart Corona

Eigin Corona

$\begin{array}{crrllll}\text { lat } & \text { long } & \text { diam } & \text { ct } & \text { et } & \text { quad } & \text { map } \\ & & & & & & \\ 77.1 \mathrm{~N} & 316.5 \mathrm{E} & 610 & \mathrm{UR} & \mathrm{SI} & 4 & \\ & & & & & & \\ 68.9 \mathrm{~N} & 24.0 \mathrm{E} & 317 & \mathrm{EU} & \mathrm{CZ} & 5 & \\ 51.9 \mathrm{~N} & 56.1 \mathrm{E} & 464 & \mathrm{UR} & \mathrm{CC} & 13 & \\ & & & & & & \\ 24.5 \mathrm{~S} & 271.5 \mathrm{E} & 1870 & \mathrm{UR} & \mathrm{SM} & & \\ 23.7 \mathrm{~S} & 127.3 \mathrm{E} & 973 & \text { SA } & \text { IN } & 1: 50 \mathrm{M} & \mathrm{I} 1324 \\ 71.3 \mathrm{~N} & 27.0 \mathrm{E} & 346 & \mathrm{UR} & \mathrm{CC} & 5 & \\ & & & & & & \\ 14.7 \mathrm{~S} & 124.1 \mathrm{E} & 416 & \mathrm{UR} & \mathrm{MO} & 1: 50 \mathrm{M} & \mathrm{I} 1324 \\ 75.6 \mathrm{~N} & 341.6 \mathrm{E} & 742 & \mathrm{EU} & \mathrm{FI} & 4 & \\ \end{array}$

51985

51985

51985

$\begin{array}{ll}5 & 1982\end{array}$

$5 \quad 1982$

1985

$5 \quad 1982$

$\begin{array}{ll}5 & 1985\end{array}$

$\begin{array}{llrlll}46.1 \mathrm{~N} & 115.5 \mathrm{E} & 1059 & \text { EU } & \text { LA } & 14 \\ 56.8 \mathrm{~N} & 153.5 \mathrm{E} & 418 & \text { UR } & \text { LI } & 7.15 \\ 52.5 \mathrm{~S} & 160.0 \mathrm{E} & 850 & \text { EU } & \text { RM } & \\ 76.5 \mathrm{~N} & 195.0 \mathrm{E} 0 & \text { EU } & \text { GR } & \text { B-2 } & \\ 30.8 \mathrm{~N} & 16.0 \mathrm{E} 0 & \text { AS } & \text { CH } & \text { B-22 } & \end{array}$

19.0

$27.7 \mathrm{~S}$

$15.5 \mathrm{~N}$

$67.2 \mathrm{~S}$

$77.1 \mathrm{~N}$

$11.0 \mathrm{~N}$

$35.5 \mathrm{~S}$

26.3S

$35.0 \mathrm{~S}$

$50.2 \mathrm{~S}$

$8.0 \mathrm{~S}$

$16.0 \mathrm{~S}$

$8.5 \mathrm{~N}$

$45.5 \mathrm{~N}$

$73.3 \mathrm{~N}$

$53.0 \mathrm{~N}$

$52.6 \mathrm{~N}$
$6.0 \mathrm{~N}$

$16.0 \mathrm{~N} \quad 340.0 \mathrm{E}$

$19.0 \mathrm{~S} \quad 233.5 \mathrm{E}$

$25.0 \mathrm{~N}$

$17.2 \mathrm{~S}$

$15.0 \mathrm{~N}$

$0.4 \mathrm{~S}$

$35.0 \mathrm{~N}$

$27.0 \mathrm{~N}$

$24.0 \mathrm{~S}$

$48.0 \mathrm{~S}$

$6.5 \mathrm{~N}$

$37.5 \mathrm{~S}$

$31.5 \mathrm{~N}$

$16.0 \mathrm{~S}$

$18.3 \mathrm{~N}$

$53.0 \mathrm{~N}$

$63.2 \mathrm{~N}$

$32.0 \mathrm{~S}$

$42.5 \mathrm{~S}$

$7.5 \mathrm{~S}$
$53.9 \mathrm{~N}$

$53.9 \mathrm{~N}$
$46.8 \mathrm{~S}$

$14.5 \mathrm{~N}$

$70.1 \mathrm{~N}$

$5.0 \mathrm{~S}$

$125.0 \mathrm{E}$

238.5E

$65.8 \mathrm{E}$

$114.0 \mathrm{E}$

$277.3 \mathrm{E}$

$14.0 \mathrm{E}$

$152.0 \mathrm{E}$

$82.0 \mathrm{E}$

$135.0 \mathrm{E}$

$262.0 \mathrm{E}$

$357.0 \mathrm{E}$

$243.5 \mathrm{E}$

$92.0 \mathrm{E}$

$12.0 \mathrm{E}$

250 EU

170 EU CE

125 AS AK

115 AF EG

324 AS AM 3

240 AS IN

300 AS IN

350 AS PE

2600 EU GR

450 AS SU

300 AS SU

220 AS TU

600 AF ET

150 NA NV

225 EU NS

463 SA CO

258.0E 0

$306.5 \mathrm{E}$

AS SU

600 EU

B-9

FI

310 AS

$\begin{array}{ll}310 & \text { AS } \\ 350 & \text { AS }\end{array}$

400 EU

$343.6 \mathrm{E}$

$118.0 \mathrm{E}$

$134.5 \mathrm{E}$

$293.8 \mathrm{E}$

$136.5 \mathrm{E}$

$157.5 \mathrm{E}$

88.3E

$43.5 \mathrm{E}$

$3.0 \mathrm{E}$

200 AS

100 AS

125 EU

300 EU

300 EU

275 EU

$125 \mathrm{EU}$

575 NA

215 EU

SA CO

JA

PO

NS

IN

CE

IR

RM

$\mathrm{SC}$

PU

$151.5 \mathrm{E}$

$340.5 \mathrm{E}$

$150.9 \mathrm{E}$

273.0E

$151.0 \mathrm{E}$

$75.5 \mathrm{E}$

20.7E

$294.8 \mathrm{E}$

$20.2 \mathrm{E}$

$136.2 \mathrm{E}$

675 EU

B-26

150 AS HE

100 NA AZ

$199 \mathrm{CA}$ AZ

350 EU TU

500 EU RM

480 AS TU

560 EU GR 10

200 AS SE

100 AS IN

414 NA AM 7

3

200 EU CE
51985

$\begin{array}{ll}5 & 1985\end{array}$

51994

1991

$\begin{array}{ll}5 & 1991\end{array}$

ref $f t$ origin

$65 \mathrm{CM}$ Mansi (Siberia) forest maiden.

$65 \mathrm{CM}$ Czech moon goddess.

65 CM Avarian (Caucasus) moon goddess.

65 CM Samoyed forest spirit.

$48 \mathrm{CM}$ Inca moon goddess.

$65 \mathrm{CM}$ Lezghin (Caucasus) moon goddess.

$27 \mathrm{CM}$ Mordvinian forest mother

65 CM Saami-Lapp forest goddess.

65 CO Saami-Lapp fishing goddess.

65 CO Lithuanian sea goddess.

43 CO Roman goddess of menses.

1 CO Greek; mother of Sirens.

1 CO Chinese sea goddess.

5
5
5
5
5
5
5
5
5
5
5
5
5

$1994 \quad 111$ CR

$\begin{array}{llll}5 & 1994 & 27 & \mathrm{CR}\end{array}$

$\begin{array}{llll}5 & 1994 & 83 & \mathrm{CR}\end{array}$

$\begin{array}{llll}5 & 1985 & 65 & \mathrm{CR}\end{array}$

$\begin{array}{llll}5 & 1994 & 83 & \mathrm{CR}\end{array}$

$199427 \quad C R$

$199401 \quad C R$

1994 01 CR

$1994 \quad 83$ CR

$1994 \quad 83$ CR

$1994 \quad 83 \quad C R$

$\begin{array}{llll}5 & 1994 \quad 107 & \mathrm{CR}\end{array}$

$5 \quad 1994 \quad 83 \quad \mathrm{CR}$

$\begin{array}{llll}5 & 1994 & 83 & \mathrm{CR}\end{array}$

$\begin{array}{llll}5 & 1985 & 65 & \mathrm{CR}\end{array}$

$\begin{array}{rrrr}5 & 1991 & 64 & \text { CR } \\ 5 & 1994 & 111 & \text { CR }\end{array}$

$\begin{array}{rrrr}5 & 1994 & 27 & \text { CR }\end{array}$

$51994 \quad 83 \quad \mathrm{CR}$

$\begin{array}{rrrr}5 & 1994 & 83 & \mathrm{CR} \\ 5 & 1994 & 111 & \mathrm{CR}\end{array}$

$5 \quad 1991 \quad 83$ CR

$\begin{array}{llll}5 & 1994 & 83 & \mathrm{CR}\end{array}$

$\begin{array}{llll}5 & 1994 & 27 & \mathrm{CR}\end{array}$

$5 \quad 1994 \quad 83 \quad C R$

$\begin{array}{llll}5 & 1994 & 27 & \mathrm{CR}\end{array}$

$\begin{array}{llll}5 & 1994 & 27 & \mathrm{CR}\end{array}$

$\begin{array}{llll}5 & 1994 & 83 & \mathrm{CR}\end{array}$

$\begin{array}{llll}5 & 1994 & 83 & \text { CR }\end{array}$

$\begin{array}{llll}5 & 1994 & 83 & \mathrm{CR}\end{array}$

$\begin{array}{llll}5 & 1994 & 83 & \mathrm{CR}\end{array}$

$\begin{array}{llll}5 & 1991 & 64 & \mathrm{CR}\end{array}$

$\begin{array}{llll}5 & 1994 & 01 & \mathrm{CR}\end{array}$

$5 \quad 1994 \quad 83 \quad \mathrm{CR}$

1994

$\begin{array}{ll}5 & 1994 \\ 5 & 1985\end{array}$

5

$5 \quad 1994$

$\begin{array}{ll}5 & 1994 \\ 5 & 1994\end{array}$

51985

$\begin{array}{ll}5 & 1985 \\ 5 & 1994\end{array}$

51994

51985

$5 \quad 1994$

Norse goddess of giving.

Celtic earth goddess.

Greek fertility goddess.

Akkadian earth goddess. 
VENUS

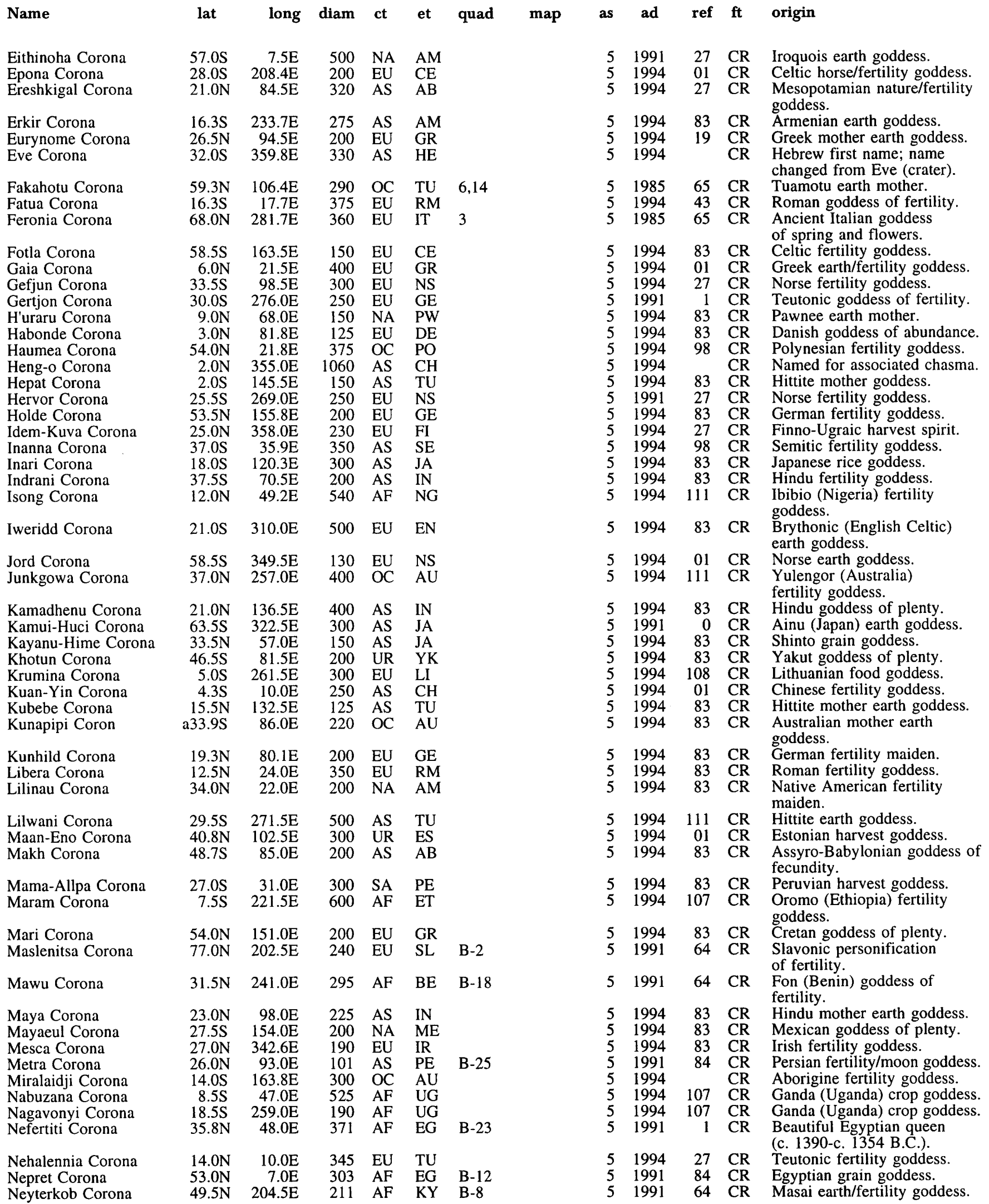

\footnotetext{
lat: latitude of feature center.

et: ethnicity of name origin (see page $284 \mathrm{ff}$.)

as: name approval status (see page $\mathrm{xvii}$ ).

long: longitude of feature center.

quad: map quadrangle or informal name

ad: name approval date (year)

diam: diameter or long dimension of feature.

(see page xvii ff.).

ref: reference source for name (see page $287 \mathrm{ff}$.).

ct: continent of name origin (see page $284 \mathrm{ff}$.) map: map name or USGS map number (see page xvii ff.). ft: feature type (see page 290 ).
} 


\section{VENUS}

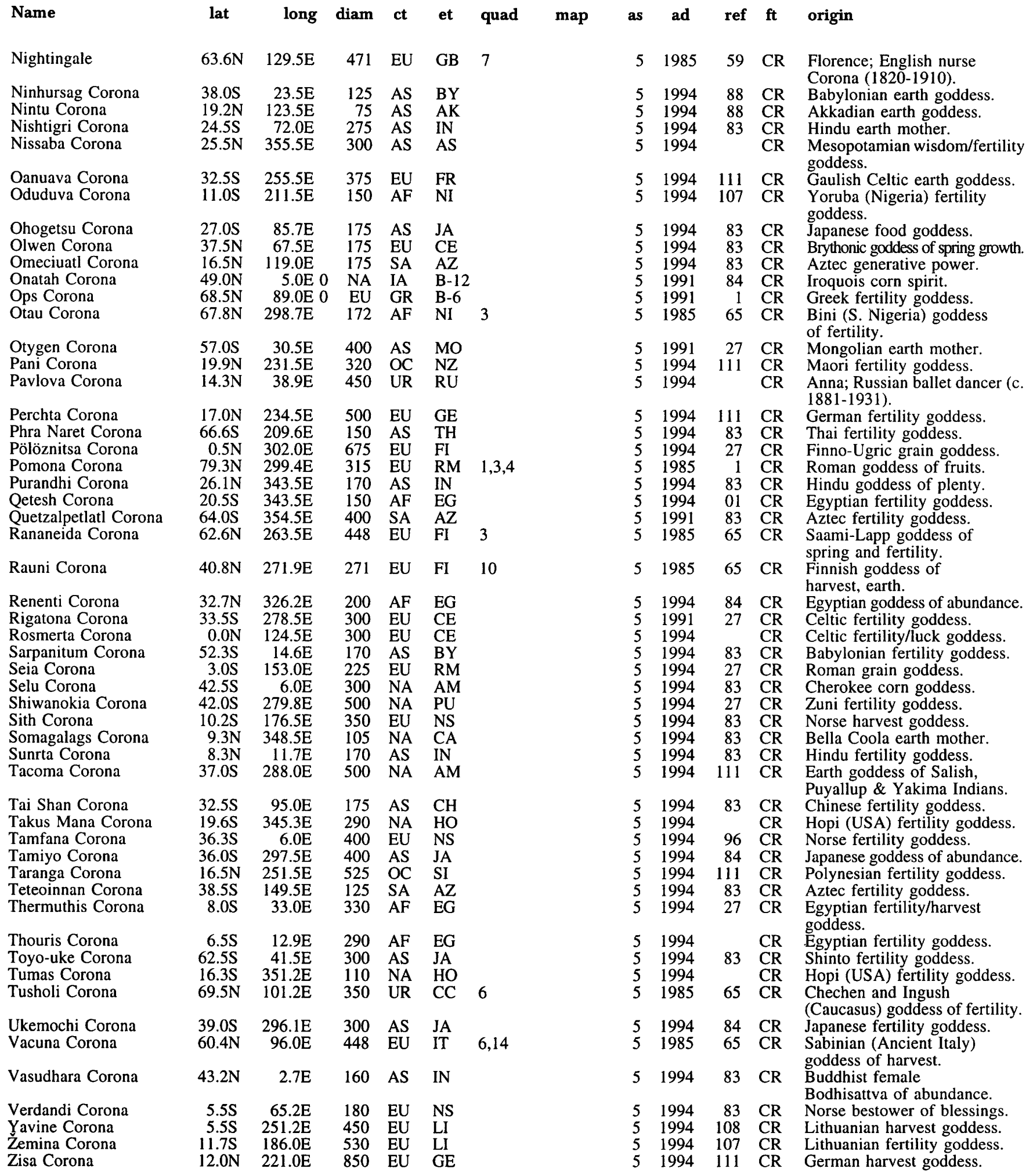

\section{DORSUM}

Ahsonnutli Dorsa

Allat Dorsa

$\begin{array}{llllll}47.9 \mathrm{~N} & 194.8 \mathrm{E} & 1708 & \mathrm{NA} & \mathrm{NV} & 2,8\end{array}$

$63.3 \mathrm{~N}$

302 AS AR 6
51985
11 DO Navajo (N. America) spirit of light and sky.

lat: latitude of feature center.

long: longitude of feature center.

diam: diameter or long dimension of feature.

ct: continent of name origin (see page $284 \mathrm{ff}$.) map: map name or USGS map number (see page xvii ff.).

\footnotetext{
et: $\quad$ ethnicity of name origin (see page $284 \mathrm{ff}$.)

quad: map quadrangle or informal name (see page xvii ff.)
}

Florence; English nurse

Corona (1820-1910).

Akkadian earth goddess.

Mesopotamian wisdom/fertility

Yoruba (Nigeria) fertility oddess.

Brythonic goddess of spring growth

Iroquois corn spirit.

eek fertility goddes

of fertility.

881-1931).

German fertility goddess.

Finno-Ugric grain goddess.

Roman goddess of fruits.

spring and fertility.

harvest, earth.

4 CR Egyptian goddess of abundance

Celtic fertility goddess.

83 CR Babylonian fertility goddess.

CR Roman grain goddess.

C2 CR Cherokee corn goddess.

83 CR Norse harvest goddess.

83 CR Bella Coola earth mother.

CR Hindu fertility goddess.

Earth goddess of Salish,

Chinese fertility goddess.

CR Hopi (USA) fertility goddess.

1 CR Polynesian fertility goddess.

33 CR Aztec fertility goddess.

Egyptian fertility/harvest oddess.

83 CR Shinto fertility goddess.

CR Hopi (USA) fertility goddess.

Chechen and Ingush

Sabinian (Ancient Italy)

dess of harvest.

Bodhisattva of abundance.

Lithuanian fertility goddess.

German harvest goddess. 
VENUS

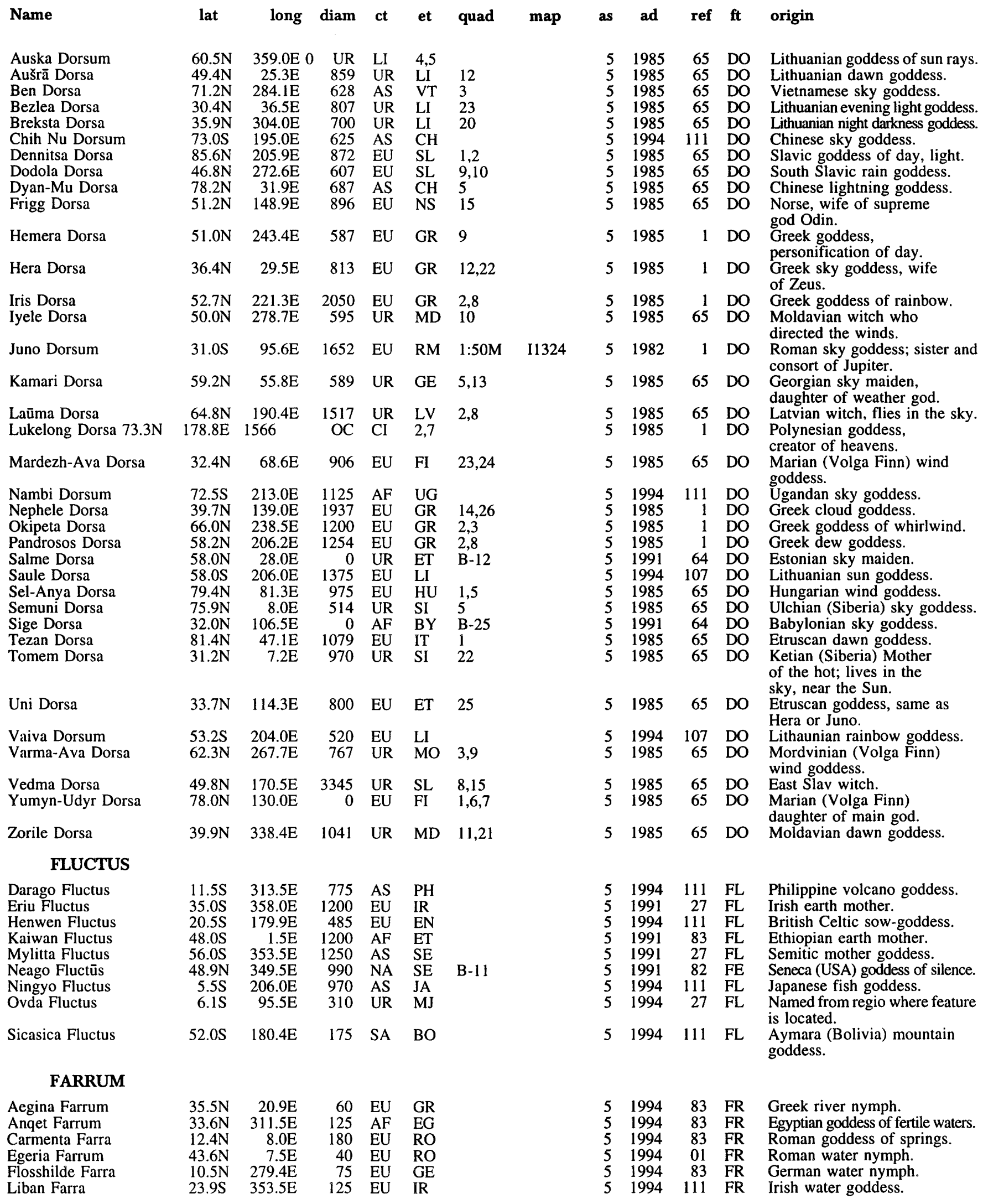

lat: latitude of feature center. long: longitude of feature center.

diam: diameter or long dimension of feature. et: ethnicity of name origin (see page $284 \mathrm{ff}$.)

quad: map quadrangle or informal name (see page xvii ff.). as: name approval status (see page xvii).

ad: name approval date (year).

ref: reference source for name (see page $287 \mathrm{ff}$.). 
VENUS

Name

Oshun Farra

Seoritsu Farra

19.3E

80 AF NI

11.0E $230 \quad$ AS JA

\section{FOSSA}

Arianrod Fossae

Bellona Fossae

Enyo Fossae

Felesta Fossae

Friagabi Fossae

Hildr Fossa

Ilbis Fossae

Manto Fossae

Minerva Fossae

Mist Fossae

Nike Fossae

Rangrid Fossae

Sigrun Fossae

Valkyrie Fossae

\section{LINEA}

Antiope Linea

Guor Linea

Hariasa Linea

Hippolyta Linea

Kalaipahoa Linea

Kara Linea

Lampedo Linea

Molpadia Linea

Morrigan Linea

Penardun Linea

Vihansa Linea

MONS

Akna Montes

Api Mons

Atira Mons

Bécuma Mons

Bunzi Mons

Cotis Mons

Danu Montes

Eostre Mons

Freyja Montes

Furki Mons

Gula Mons

Hallgerda Mons

Hathor Mons

Idunn Mons

Ilithyia Mons

Innini Mons

Jael Mons

Kurukulla Mons

Maat Mons

Maxwell Montes

$\begin{array}{lr}40.0 \mathrm{~S} & 350.0 \mathrm{E} \\ & \\ 17.0 \mathrm{~N} & 2.6 \mathrm{E} \\ & \\ 19.0 \mathrm{~N} & 15.0 \mathrm{E} \\ 42.0 \mathrm{~S} & 345.0 \mathrm{E} \\ 60.5 \mathrm{~S} & 338.0 \mathrm{E} \\ 44.0 \mathrm{~S} & 306.0 \mathrm{E} \\ 57.0 \mathrm{~N} & 295.0 \mathrm{E} \\ 48.0 \mathrm{~S} & 359.0 \mathrm{E} \\ 54.5 \mathrm{~S} & 311.0 \mathrm{E} \\ 54.0 \mathrm{~S} & 344.0 \mathrm{E} \\ 54.0 \mathrm{~N} & 20.0 \mathrm{E}\end{array}$

\begin{tabular}{|c|c|c|c|c|c|}
\hline lat & long & diam & ct & et & quad \\
\hline $4.2 \mathrm{~N}$ & $19.3 \mathrm{E}$ & 80 & $\mathrm{AF}$ & NI & \\
\hline $30.0 \mathrm{~S}$ & $11.0 \mathrm{E}$ & 230 & AS & JA & \\
\hline $\begin{array}{l}37.0 \mathrm{~N} \\
38.0 \mathrm{~N}\end{array}$ & $\begin{array}{l}239.9 \mathrm{E} \\
222.1 \mathrm{E}\end{array}$ & $\begin{array}{l}715 \\
855\end{array}$ & $\begin{array}{l}\text { EU } \\
\text { EU }\end{array}$ & $\begin{array}{l}\text { CE } \\
\text { RO }\end{array}$ & $\begin{array}{l}17,18 \\
8,17\end{array}$ \\
\hline $\begin{array}{l}61.0 \mathrm{~S} \\
35.0 \mathrm{~N}\end{array}$ & $\begin{array}{r}344.0 \mathrm{E} \\
46.5 \mathrm{E}\end{array}$ & $\begin{array}{l}900 \\
240\end{array}$ & $\begin{array}{l}\text { EU } \\
\text { AS }\end{array}$ & $\begin{array}{l}\text { GR } \\
\text { SC }\end{array}$ & B-23 \\
\hline $50.2 \mathrm{~N}$ & $109.5 \mathrm{E}$ & 141 & $\mathrm{EU}$ & GB & 14 \\
\hline $45.4 \mathrm{~N}$ & $159.4 \mathrm{E}$ & 677 & $\mathrm{EU}$ & NS & 15 \\
\hline $71.9 \mathrm{~N}$ & $254.6 \mathrm{E}$ & 512 & UR & YK & 3 \\
\hline $\begin{array}{l}64.5 \mathrm{~N} \\
64.5 \mathrm{~N} \\
39.5 \mathrm{~N} \\
62.0 \mathrm{~S} \\
62.7 \mathrm{~N} \\
52.3 \mathrm{~N} \\
58.8 \mathrm{~N}\end{array}$ & $\begin{array}{r}60.0 \mathrm{E} \\
252.5 \mathrm{E} \\
247.3 \mathrm{E} \\
347.0 \mathrm{E} \\
356.4 \mathrm{E} \\
19.9 \mathrm{E} \\
7.5 \mathrm{E}\end{array}$ & $\begin{array}{r}536 \\
0 \\
244 \\
850 \\
243 \\
971 \\
0\end{array}$ & $\begin{array}{l}\text { EU } \\
\text { EU } \\
\text { EU } \\
\text { EU } \\
\text { EU } \\
\text { EU } \\
\text { EU }\end{array}$ & $\begin{array}{l}\text { GR } \\
\text { RM } \\
\text { NS } \\
\text { GR } \\
\text { NS } \\
\text { NS } \\
\text { NS }\end{array}$ & $\begin{array}{l}\text { B-5,6 } \\
3 \\
18\end{array}$ \\
\hline
\end{tabular}

map

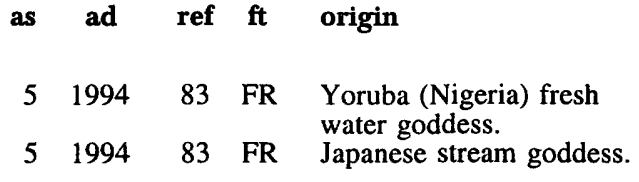

$\begin{array}{ll}5 & 1985 \\ 5 & 1985 \\ 5 & 1991 \\ 5 & 1991 \\ 5 & 1985 \\ 5 & 1985 \\ & \\ 5 & 1985 \\ 5 & 1991 \\ 5 & 1985 \\ 5 & 1985 \\ 5 & 1991 \\ 5 & 1985 \\ 5 & 1985 \\ 5 & 1991\end{array}$

65 FO

Celtic warrior queen.

1 Roman war goddess, wife

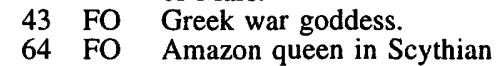

27 FO Opic tales.

17 connected with Mars.

17 FO Norse mythological

warrior.

goddess of bloodshed.

1 FO Greek war goddess.

1 FO Roman goddess of war.

1 FO Norse Valkyrie.

43 FO Greek goddess of victory.

1 FO Norse Valkyrie.

1 FO Norse Valkyrie.

$\begin{array}{rllll}1240 & \text { EU } & \text { GR } & 1: 50 \mathrm{M} & \text { I1324 } \\ 1050 & \text { EU } & \text { IC } & 1: 50 \mathrm{M} & \text { I1324 } \\ & & & & \\ 0 & \text { EU } & \text { GE } & 1: 50 \mathrm{M} & \text { I1324 } \\ 0 & \text { EU } & \text { GR } & 1: 50 \mathrm{M} & \text { I1324 } \\ 2400 & \text { OC } & \text { HA } & & \\ 700 & \text { EU } & \text { IC } & 1: 50 \mathrm{M} & 11324 \\ 0 & \text { UR } & \text { SC } & 1: 50 \mathrm{M} & 11324 \\ 1350 & \text { EU } & \text { GR } & 1: 50 \mathrm{M} & \mathrm{I} 1324 \\ 3200 & \text { EU } & \text { CE } & & \\ 975 & \text { EU } & \text { CE } & & \\ 0 & \text { EU } & \text { GE } & 1: 50 M & 1324\end{array}$

$\begin{array}{rrrr}5 & 1982 & 19 & \text { LI } \\ 5 & 1982 & 35 & \text { LI } \\ 5 & 1982 & 27 & \text { LI } \\ 5 & 1982 & 19 & \text { LI } \\ 5 & 1991 & 27 & \text { LI } \\ 5 & 1982 & 48 & \text { LI } \\ 5 & 1982 & 1 & \text { LI } \\ 5 & 1982 & 19 & \text { LI } \\ 5 & 1991 & 27 & \text { LI } \\ 5 & 1991 & 83 & \text { LI } \\ 5 & 1982 & 27 & \text { LI }\end{array}$

Amazon awarded to

Theseus.

Northern European

Valkyrie.

German war goddess.

Amazon queen.

Hawaiian war goddess.

Icelandic Valkryie.

Scythian Amazon queen.

Amazon.

Celtic war goddess.

Celtic sky goddess.

German war goddess.
$68.9 \mathrm{~N} \quad 318.2 \mathrm{E}$

$38.9 \mathrm{~N} \quad 54.7 \mathrm{E}$

$52.2 \mathrm{~N} \quad 267.6 \mathrm{E}$

830 CA MY 1:50M

190 UR SC 23

152 NA $\mathrm{PW} 9$

$34.0 \mathrm{~N} \quad 22.0 \mathrm{E}$

$46.0 \mathrm{~N} \quad 355.0 \mathrm{E}$

$44.1 \mathrm{~N} \quad 233.1 \mathrm{E}$

130 EU IR B-22

36 AF ZA B-11

$\begin{array}{llllll}58.5 \mathrm{~N} & 334.0 \mathrm{E} & 808 & \mathrm{EU} & \mathrm{CE} & 4,11\end{array}$

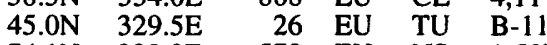

$74.1 \mathrm{~N} \quad 333.8 \mathrm{E}$

$35.9 \mathrm{~N} \quad 236.4 \mathrm{E}$

579 EU

79 UR CC $1: 50 \mathrm{M}$

I1324

$\begin{array}{ll}5 & 1979 \\ 5 & 1985 \\ 5 & 1985\end{array}$

51985

$\begin{array}{ll}5 & 199 \\ 5 & 1991\end{array}$

$\begin{array}{ll}5 & 1991 \\ 5 & 1985\end{array}$

51985

$\begin{array}{ll}5 & 1985 \\ 5 & 1991 \\ 5 & 1979\end{array}$

I1324

$\begin{array}{ll}5 & 1979 \\ 5 & 1985\end{array}$

1 MO Mayan goddess of birth.

27 MO Scythian goddess of earth.

1 MO Pawnee (N. America) wife of Great Spirit Tirawa.

27 MO Irish goddess.

1 MO Woyo (Zaire) rainbow goddess.

1 MO

Thracian goddess, mother of gods, similar to Cybele.

27 MO Celtic mother of gods.

64 MO Teutonic goddess of spring.

27 MO Norse, mother of Odin.

65 MO Chechen and Ingush

(Caucasus) goddess, wife of thunder god Sela.

\begin{tabular}{|c|c|c|c|c|c|c|c|c|c|c|c|}
\hline $21.9 \mathrm{~N}$ & $359.1 \mathrm{E}$ & 276 & AS & BY & $1: 50 \mathrm{M}$ & I1324 & 5 & 1982 & 48 & MO & $\begin{array}{l}\text { Babylonian earth mother, } \\
\text { creative force. }\end{array}$ \\
\hline $55.0 \mathrm{~N}$ & $198.0 \mathrm{E}$ & 57 & EU & IC & B-8 & & & 1991 & 64 & MO & Icelandic goddess of vanity. \\
\hline 875 & 324.7 & 333 & $\mathrm{AF}$ & EG & $1: 50 \mathrm{M}$ & I1324 & & 197 & 1 & MO & $\begin{array}{l}\text { Egyptian sky goddess, usually in } \\
\text { the form of a cow. }\end{array}$ \\
\hline $46.5 \mathrm{~S}$ & $213.5 \mathrm{E}$ & 250 & EU & NS & & & & 1994 & 111 & MO & Norse goddess. \\
\hline $13.5 \mathrm{~S}$ & $315.5 \mathrm{E}$ & 90 & EU & GR & & & & 199 & 01 & MO & Greek goddess of childbirth. \\
\hline $34.6 S$ & $328.5 \mathrm{E}$ & 339 & AS & $\mathrm{BY}$ & $1: 50 \mathrm{M}$ & I1324 & & 1982 & 1 & MO & $\begin{array}{l}\text { Babylonian earth mother } \\
\text { worshipped at Kish. }\end{array}$ \\
\hline $\begin{array}{l}52.0 \mathrm{~N} \\
48.5 \mathrm{~N}\end{array}$ & $121.0 \mathrm{E}$ & $\begin{array}{l}36 \\
59\end{array}$ & AS & HE & B-14 & & & 1991 & 1 & MO & $\begin{array}{l}\text { Hebrew goddess of dawn. } \\
\text { Etan (Tibet) goddess of wea }\end{array}$ \\
\hline $\begin{array}{r}48.5 \mathrm{~N} \\
0.5 \mathrm{~N}\end{array}$ & $\begin{array}{l}103.0 \mathrm{E} \\
194.6 \mathrm{E}\end{array}$ & $\begin{array}{r}59 \\
395\end{array}$ & AF & EG & $\begin{array}{l}\mathrm{B}-14 \\
1: 50 \mathrm{M}\end{array}$ & I1324 & & $\begin{array}{l}1991 \\
1982\end{array}$ & $\begin{array}{l}04 \\
48\end{array}$ & MO & Ancient Egyptian goddess \\
\hline $65.2 \mathrm{~N}$ & $3.3 \mathrm{E}$ & 797 & EU & GB & $1: 50 \mathrm{M}$ & I1324 & 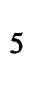 & 1979 & 61 & MO & $\begin{array}{l}\text { of truth and justice. } \\
\text { James C.; British } \\
\text { physicist (1831-1879). }\end{array}$ \\
\hline
\end{tabular}

lat: latitude of feature center.

long: longitude of feature center.

diam: diameter or long dimension of feature.

ct: continent of name origin (see page $284 \mathrm{ff}$.) map: et: ethnicity of name origin (see page $284 \mathrm{ff}$.)

quad: map quadrangle or informal name

(see page $\mathrm{xvii} \mathrm{ff}$.)

map name or USGS map number (see page xvii ff.). as: name approval status (see page xvii).

ad: name approval date (year).

ref: reference source for name (see page $287 \mathrm{ff}$.). 


\section{VENUS}

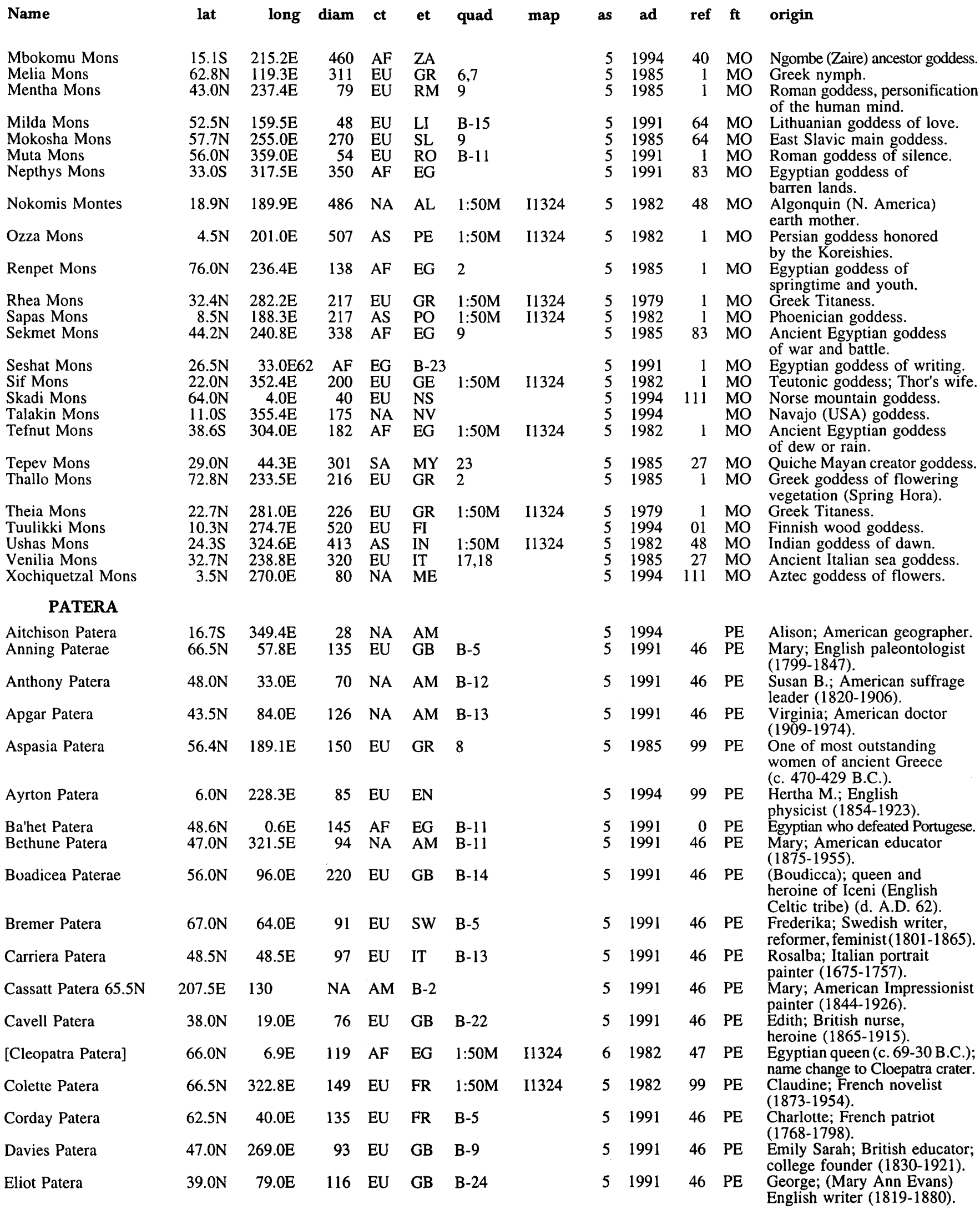

lat: latitude of feature center. long: longitude of feature center. diam: diameter or long dimension of feature. et: ethnicity of name origin (see page $284 \mathrm{ff}$.)

quad: map quadrangle or informal name (see page xvii ff.). as: name approval status (see page xvii).

ad: name approval date (year).

ref: reference source for name (see page $287 \mathrm{ff}$.). 


\section{VENUS}

Name

Hatshepsut Patera

Hiei Chu Patera

Hroswitha Patera

Izumi Patera

Keller Patera

Kottauer Patera

Labé Patera

Ledoux Patera

Malintzin Patera

Nzingha Patera

Pocahontas Patera

Raskova Paterae

Razia Patera

Sacajawea Patera

Sachs Patera

$49.0 \mathrm{~N} \quad 324.0 \mathrm{E}$

65 EU SW B-11

Sand Patera

$42.0 \mathrm{~N}$

15.5E

181 EU

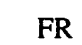

B-12

Sappho Patera

$14.1 \mathrm{~N} \quad 16.5 \mathrm{E}$

Schumann-Heink Patera

Stopes Patera

Tarbell Patera

Teasdale Patera

Tey Patera

[Theodora Patera]

Tipporah Patera

Tituba Patera

Trotula Patera

Woodhull Patera

Yaroslavna Patera
92 AS

$120 \mathrm{EU}$

TU

1:50M

11324

$42.5 \mathrm{~N} \quad 47.0 \mathrm{E}$

$169 \mathrm{E}$

80 NA AM

$58.2 \mathrm{~S} \quad 351.5 \mathrm{E}$

$67.6 \mathrm{~S} \quad 189.1 \mathrm{E}$

17.8S $349.1 \mathrm{E}$

75 NA AM

20 EU SC

$23.0 \mathrm{~N} \quad 280.0 \mathrm{E}$

$38.9 \mathrm{~N} \quad 43.0 \mathrm{E}$

$42.5 \mathrm{~N} \quad 214.0 \mathrm{E}$

$41.3 \mathrm{~N} \quad 18.9 \mathrm{E}$

$37.5 \mathrm{~N} \quad 306.0 \mathrm{E}$

0 EU BZ 1:50M

99 AS HE 23

163 NA AM B-8

146 EU IT 12

83 NA AM B-20

$38.8 \mathrm{~N} \quad 21.2 \mathrm{E}$

112 UR RU 22
$74.0 \mathrm{~N} \quad 215.0 \mathrm{E}$

map

\begin{tabular}{|c|c|c|}
\hline & ad & ref \\
\hline $\begin{array}{l}5 \\
5\end{array}$ & $\begin{array}{l}1985 \\
1985\end{array}$ & $\begin{array}{l}99 \\
80\end{array}$ \\
\hline $\begin{array}{l}5 \\
5 \\
5\end{array}$ & $\begin{array}{l}1985 \\
1985 \\
1991\end{array}$ & $\begin{array}{l}99 \\
80 \\
46\end{array}$ \\
\hline 5 & 1985 & 46 \\
\hline $\begin{array}{l}5 \\
5 \\
5\end{array}$ & $\begin{array}{l}1991 \\
1994 \\
1991\end{array}$ & $\begin{array}{l}46 \\
99 \\
46\end{array}$ \\
\hline 5 & 1991 & 46 \\
\hline 5 & 1991 & 46 \\
\hline 5 & 1994 & 107 \\
\hline 5 & 1985 & 80 \\
\hline & 1982 & 99 \\
\hline
\end{tabular}

$\begin{array}{llll}5 & 1991 \quad 46 & \mathrm{PE}\end{array}$ (1786-1812)

Nelly; German-born

Swedish playwright, poet (1891-1970).

$\begin{array}{llll}5 & 1991 \quad 59 & \mathrm{PE}\end{array}$

George (Aurore Dupin); French novelist (1804-1876).

$5 \quad 1979 \quad 46 \quad \mathrm{PE}$

$51991 \quad 46 \quad \mathrm{PE}$

Lyric poet, Lesbos, Asia Mino (fl. between 160-580 B.C.).

Ernestine; German singer (1861-1936).

5199146 PE Marie; English paleontologist (1880-1959).

5199499 PE Ida; American author, editor (1857-1944).

$51994 \quad 99$ PE $\quad$ Sara; American poet (1884-1933).

$51994 \quad 99 \quad \mathrm{PE} \quad$ Josephine; Scottish author (1897-1952).

$6 \quad 1982 \quad 46$ PE Byzantine empress; not being used

5198580 PE Hebrew medical scholar (1500 B.C.).

5199146 PE Nurse who started Salem witch hunt (c. 1692).

$51985 \quad 80$ PE Italian physician (A.D. 1097)

5199146 PE Victoria; American-English lecturer (1838-1927).

5198564 PE Russian, wife of Prince Igor; patiently waited for his return from captivity (12th century).

\section{PLANITIA}

Atalanta Planitia

Audra Planitia

Bereghinya Planitia

Ganiki Planitia

Guinevere Planitia

Helen Planitia

Kawelu Planitia

Lavinia Planitia

Leda Planitia

Louhii Planitia

\begin{tabular}{|c|c|c|c|c|c|c|}
\hline $40.5 \mathrm{~S}$ & $94.5 \mathrm{E}$ & 4983 & EU & $\mathrm{FI}$ & $1: 50 \mathrm{M}$ & 11324 \\
\hline $45.6 \mathrm{~N}$ & $165.8 \mathrm{E}$ & 2048 & $\mathrm{EU}$ & GR & 1:50M & I1324 \\
\hline $\begin{array}{l}61.5 \mathrm{~N} \\
28.6 \mathrm{~N} \\
25.9 \mathrm{~N}\end{array}$ & $\begin{array}{r}71.5 \mathrm{E} \\
23.6 \mathrm{E} \\
189.7 \mathrm{E}\end{array}$ & $\begin{array}{l}1861 \\
3902 \\
5158\end{array}$ & $\begin{array}{l}\text { AS } \\
\text { EU } \\
\text { AS }\end{array}$ & $\begin{array}{l}\text { LI } \\
\text { SL } \\
\text { SI }\end{array}$ & $\begin{array}{l}\text { B-5,6 } \\
12,22 \\
8,16\end{array}$ & \\
\hline $\begin{array}{l}21.9 \mathrm{~N} \\
51.7 \mathrm{~S}\end{array}$ & $\begin{array}{l}325.0 \mathrm{E} \\
263.9 \mathrm{E}\end{array}$ & $\begin{array}{l}7519 \\
4362\end{array}$ & $\begin{array}{l}\text { EU } \\
\text { EU }\end{array}$ & $\begin{array}{l}\text { GB } \\
\text { GR }\end{array}$ & $\begin{array}{l}10 \mathrm{I1324} \\
1: 50 \mathrm{M}\end{array}$ & I1324 \\
\hline $32.8 \mathrm{~N}$ & $246.5 \mathrm{E}$ & 3910 & OC & HA & 9,17 & \\
\hline $\begin{array}{l}47.3 \mathrm{~S} \\
44.0 \mathrm{~N} \\
80.5 \mathrm{~N}\end{array}$ & $\begin{array}{r}347.5 \mathrm{E} \\
65.1 \mathrm{E} \\
120.5 \mathrm{E}\end{array}$ & $\begin{array}{l}2820 \\
2890 \\
2441\end{array}$ & $\begin{array}{l}\text { EU } \\
\text { EU } \\
\text { EU }\end{array}$ & $\begin{array}{l}\text { RM } \\
\text { GR } \\
\text { FI }\end{array}$ & $\begin{array}{l}1: 50 \mathrm{M} \\
1: 50 \mathrm{M} \\
1,6.7\end{array}$ & $\begin{array}{l}11324 \\
11324\end{array}$ \\
\hline
\end{tabular}

51982

$\begin{array}{ll}5 & 1991\end{array}$

$\begin{array}{ll}5 & 1985 \\ 5 & 1985\end{array}$

51985

$5 \quad 1982$

51982

51985

$5 \quad 1982$

$\begin{array}{ll}5 & 1982\end{array}$

51985 $\begin{array}{llll}5 & 1982 & 48 & \mathrm{PL}\end{array}$
Finnish heroine who became water spirit.

Greek; huntress associated with

64 PL Lolden apples.

64 PL Slavic water spirit.

64 PL Orochian (Siberia) water

spirit, mermaid.

93 PL British, wife of Arthur.

1 PL Greek; "the face that launched 1000 ships."

Hawaiian mythological heroine, died and brought back to life.

1 PL Mother of Helen, Castor.

1 PL Karelo-Finn mother of the North.
1 PL Roman; wife of Aeneas.

lat: latitude of feature center.

long: longitude of feature center.

diam: diameter or long dimension of feature.

et: ethnicity of name origin (see page $284 \mathrm{ff}$.)

quad: map quadrangle or informal name

(see page xvii ff.). as: name approval status (see page xvii).

ad: name approval date (year).

ref: reference source for name (see page $287 \mathrm{ff}$.). 


\section{VENUS}

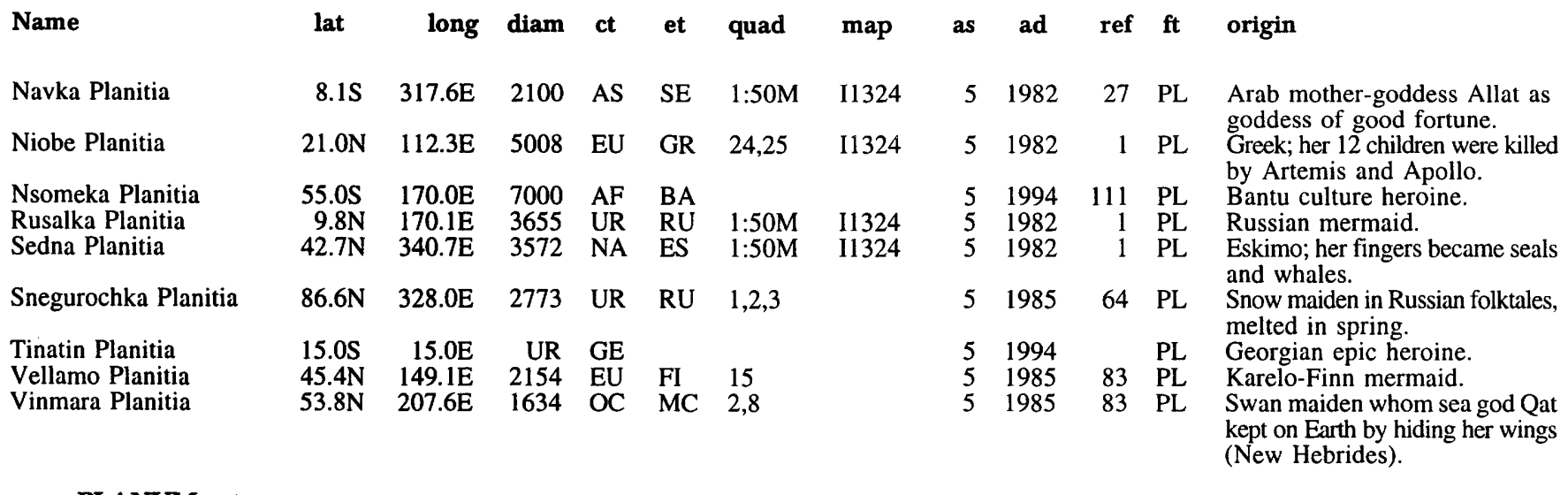

\section{PLANUM}

Lakshmi Planum

$\begin{array}{llllll}68.6 \mathrm{~N} & 339.3 \mathrm{E} & 2343 & \text { AS } & \text { IN } & 4,11\end{array}$

11324

51979

1 PM

Indian goddess of love and war.

\section{REGIO}

Alpha Regio
Asteria Regio
Atla Regio
Bell Regio
Beta Regio
Dione Regio
Eistla Regio
Hyndla Regio
Imdr Regio
Metis Regio
Mnemosyne Regio
Ovda Regio
Phoebe Regio
Tethus Regio
Themis Regio
Thetis Regio
Ulfrun Regio

$\begin{array}{rrrl}25.5 \mathrm{~S} & 1.3 \mathrm{E} & 1897 & \mathrm{EU} \\ 21.6 \mathrm{~N} & 267.5 \mathrm{E} & 1131 & \mathrm{EU} \\ 9.2 \mathrm{~N} & 200.1 \mathrm{E} & 3200 & \mathrm{EU} \\ 32.8 \mathrm{~N} & 51.4 \mathrm{E} & 1778 & \mathrm{EU} \\ 25.3 \mathrm{~N} & 282.8 \mathrm{E} & 2869 & \mathrm{EU} \\ 31.5 \mathrm{~S} & 328.0 \mathrm{E} & 2300 & \mathrm{EU} \\ 10.5 \mathrm{~N} & 21.5 \mathrm{E} & 8015 & \mathrm{EU} \\ 22.5 \mathrm{~N} & 294.5 \mathrm{E} & 2300 & \mathrm{EU} \\ 43.0 \mathrm{~S} & 212.0 \mathrm{E} & 1611 & \mathrm{EU} \\ 72.0 \mathrm{~N} & 256.0 \mathrm{E} & 729 & \mathrm{EU} \\ 65.8 \mathrm{~N} & 277.9 \mathrm{E} & 0 & \mathrm{EU} \\ 2.8 \mathrm{~S} & 85.6 \mathrm{E} & 5280 & \mathrm{UR} \\ 6.0 \mathrm{~S} & 282.8 \mathrm{E} & 2852 & \mathrm{EU} \\ 66.0 \mathrm{~N} & 120.0 \mathrm{E} & 2410 & \mathrm{EU} \\ 37.4 \mathrm{~S} & 284.2 \mathrm{E} & 1811 & \mathrm{EU} \\ 11.4 \mathrm{~S} & 129.9 \mathrm{E} & 2801 & \mathrm{EU} \\ 20.5 \mathrm{~N} & 223.0 \mathrm{E} & 3954 & \mathrm{EU}\end{array}$

$\begin{array}{lll}\text { GR } & 1: 50 \mathrm{M} & \mathrm{I} 1324 \\ \text { GR } & 1: 50 \mathrm{M} & \mathrm{I} 1324 \\ \text { NO } & 1: 50 \mathrm{M} & \mathrm{I} 1324 \\ \text { GB } & 1: 50 \mathrm{M} & \mathrm{I} 1324 \\ \text { GR } & 1: 50 \mathrm{M} & \mathrm{I} 1324 \\ \text { GR } & & \\ \text { NS } & 1: 50 \mathrm{M} & \mathrm{I} 1324 \\ \text { NS } & & \\ \text { NS } & 1: 50 \mathrm{M} & \mathrm{I} 1324 \\ \text { GR } & 1: 50 \mathrm{M} & \mathrm{I} 1324 \\ \text { GR } & 1: 50 \mathrm{M} & \mathrm{I} 1324 \\ \text { MJ } & 1: 50 \mathrm{M} & \mathrm{I} 1324 \\ \text { GR } & 1: 50 \mathrm{M} & \mathrm{I} 1324 \\ \text { GR } & 1: 50 \mathrm{M} & \mathrm{I} 1324 \\ \text { GR } & 1: 50 \mathrm{M} & \mathrm{I} 1324 \\ \text { GR } & 1: 50 \mathrm{M} & \mathrm{I} 1324 \\ \text { NS } & 1: 50 \mathrm{M} & \mathrm{I} 1324\end{array}$

\section{RUPES}

Fornax Rupes

Gabie Rupes

Hestia Rupes

Uorsar Rupes

Ut Rupes

Vesta Rupes

TERRA

Aphrodite Terra

Ishtar Terra

Lada Terra

\section{TESSERA}

Ananke Tessera
Atropos Tessera
Clotho Tessera
Dekla Tessera
Fortuna Tessera
Itzpapalotl Tessera
Kutue Tessera
Lachesis Tessera
Laima Tessera

$\begin{array}{llllll}30.3 \mathrm{~N} & 201.1 \mathrm{E} & 729 & \mathrm{EU} & \mathrm{RM} & 16 \\ 67.5 \mathrm{~N} & 109.9 \mathrm{E} & 350 & \text { UR } & \text { LI } & 6\end{array}$

$\begin{array}{llllll}6.0 \mathrm{~N} & 71.1 \mathrm{E} & 588 & \mathrm{EU} & \mathrm{GR} & 1: 50 \mathrm{M}\end{array}$

$76.8 \mathrm{~N} \quad 341.2 \mathrm{E}$

820 UR

$55.3 \mathrm{~N} \quad 321.9 \mathrm{E}$

676 UR TK 3,11

I1324

$58.3 \mathrm{~N} \quad 323.9 \mathrm{E}$

$788 \quad$ EU

RM 4,11

I1324

5.8S $\quad 104.8 \mathrm{E}$

$70.4 \mathrm{~N} \quad 27.5 \mathrm{E}$

$54.4 \mathrm{~S} \quad 342.5 \mathrm{E}$

9999 EU

5609 AS

GR 1:50M

BY $1: 50 \mathrm{M}$

I1 1324

8614 EU

SL $\quad 1: 50 \mathrm{M}$

$\begin{array}{lrrlll}53.3 \mathrm{~N} & 133.3 \mathrm{E} & 1060 & \mathrm{EU} & \mathrm{GR} & 14,15 \\ 71.5 \mathrm{~N} & 304.0 \mathrm{E} & 469 & \mathrm{EU} & \mathrm{GR} & 3,4 \\ 56.4 \mathrm{~N} & 334.9 \mathrm{E} & 289 & \mathrm{EU} & \mathrm{GR} & 11 \\ 57.4 \mathrm{~N} & 71.8 \mathrm{E} & 1363 & \mathrm{UR} & \mathrm{LV} & 13 \\ 69.9 \mathrm{~N} & 45.1 \mathrm{E} & 2801 & \mathrm{EU} & \mathrm{RM} & 5,6 \\ 75.7 \mathrm{~N} & 317.6 \mathrm{E} & 380 & \mathrm{SA} & \mathrm{AZ} & 4 \\ 39.5 \mathrm{~N} & 108.8 \mathrm{E} & 653 & \mathrm{AS} & \mathrm{SI} & 14,25 \\ & & & & & \\ 44.4 \mathrm{~N} & 300.1 \mathrm{E} & 664 & \mathrm{EU} & \mathrm{GR} & 10 \\ 55.0 \mathrm{~N} & 48.5 \mathrm{E} & 971 & \mathrm{UR} & \mathrm{LV} & 12,13\end{array}$

$\begin{array}{ll}5 & 1979 \\ 5 & 1982 \\ 5 & 1982 \\ 5 & 1982 \\ 5 & 1979 \\ 5 & 1991 \\ 5 & 1982 \\ 5 & 1991 \\ 5 & 1982 \\ 5 & 1982 \\ 5 & 1982 \\ 5 & 1982 \\ 5 & 1982 \\ 5 & 1982 \\ 5 & 1982 \\ 5 & 1982 \\ 5 & 1982\end{array}$

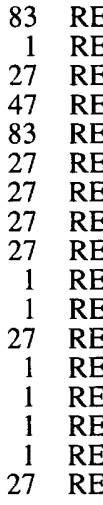

$\begin{array}{rl}83 & \mathrm{RE} \\ 1 & \mathrm{RE} \\ 27 & \mathrm{RE} \\ 47 & \mathrm{RE} \\ 83 & \mathrm{RE} \\ 27 & \mathrm{RE} \\ 27 & \mathrm{RE} \\ 27 & \mathrm{RE} \\ 27 & \mathrm{RE} \\ 1 & \mathrm{RE} \\ 1 & \mathrm{RE} \\ 27 & \mathrm{RE} \\ 1 & \mathrm{RE} \\ 1 & \mathrm{RE} \\ 1 & \mathrm{RE} \\ 1 & \mathrm{RE} \\ 27 & \mathrm{RE}\end{array}$
First letter in Greek alphabet.

Greek Titaness.

Norse giantess, mother of Heimdall.

English giantess

Second letter in Greek alphabet.

Greek Titaness; 1st wife of Zeus.

Norse giantess.

Norse wood giantess.

Norse giantess.

Greek Titaness.

Greek Titaness.

Marijian (Russia) forest giantess.

Greek Titaness.

Greek Titaness.

Greek Titaness.

Greek Titaness.

Norse giantess.
5198583 RU Roman goddess of hearth and baking of bread.

5198564 RU Lithuanian goddess of fire and hearth.

$19821 \mathrm{RU}$ Greek hearth goddess.

5198564 RU Adygan (Caucasus) goddess

of hearth.

$51982 \quad 48$ RU $\begin{aligned} & \text { the hearth fire. } \\ & \text { Roman hearth goddess. }\end{aligned}$

$51979 \quad 1$ TA Greek goddess of love.

$51979 \quad 1$ TA Babylonian goddess of love.

$5 \quad 1982 \quad 30 \quad$ TA Slavic goddess of love.

$\begin{array}{rrrl}5 & 1985 & 27 & \text { TE } \\ 5 & 1985 & 1 & \text { TE } \\ 5 & 1985 & 1 & \text { TE } \\ 5 & 1985 & 64 & \text { TE } \\ 5 & 1985 & 1 & \text { TE } \\ 5 & 1985 & 27 & \text { TE } \\ 5 & 1985 & 64 & \text { TE } \\ 5 & 1985 & 1 & \text { TE } \\ 5 & 1985 & 64 & \text { TE }\end{array}$

Greek goddess of necessity.

Greek; one of three Fates.

Greek; one of three Fates.

Latvian goddess of fate.

Roman goddess of chance.

Aztec goddess of fate.

Ulchian (Siberia) folklore toad that brings happiness.

Greek, one of three Fates.

Latvian and Lithuanian goddess of fate. lat: latitude of feature center. long: longitude of feature center.

diam: diameter or long dimension of feature. et: ethnicity of name origin (see page $284 \mathrm{ff}$.)

quad: map quadrangle or informal name (see page xvii ff.). as: name approval status (see page xvii).

ad: name approval date (year).

ref: reference source for name (see page $287 \mathrm{ff}$.).

ct: continent of name origin (see page $284 \mathrm{ff}$.) map: map name or USGS map number (see page xvii ff.). ft: feature type (see page 290 ). 
VENUS

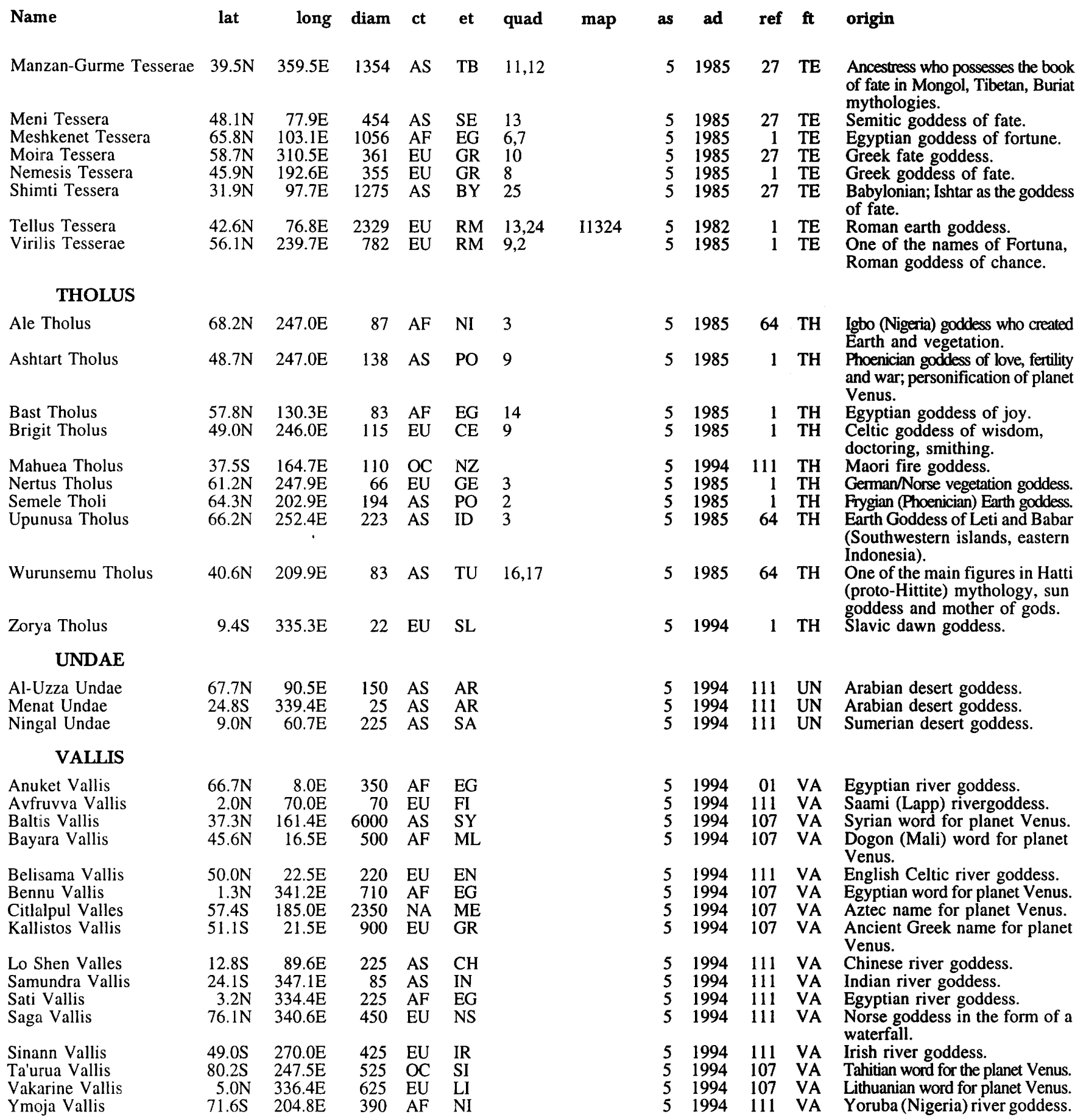

lat: latitude of feature center

long: longitude of feature center.

diam: diameter or long dimension of feature.

ct. continent of name origin (see page $284 \mathrm{ff}$.) map: et: ethnicity of name origin (see page $284 \mathrm{ff}$.)

quad: map quadrangle or informal name

(see page xvii ff.). as: name approval status (see page $\mathrm{xvii}$ ).

ad: name approval date (year).

ref: reference source for name (see page $287 \mathrm{ff}$.). map name or USGS map number (see page xvii ff.). ft: feature type (see page 290). 


\section{MOON}

Name

lat long diam ct et quad map as ad ref $\mathrm{ft}$ origin

\section{CRATER}

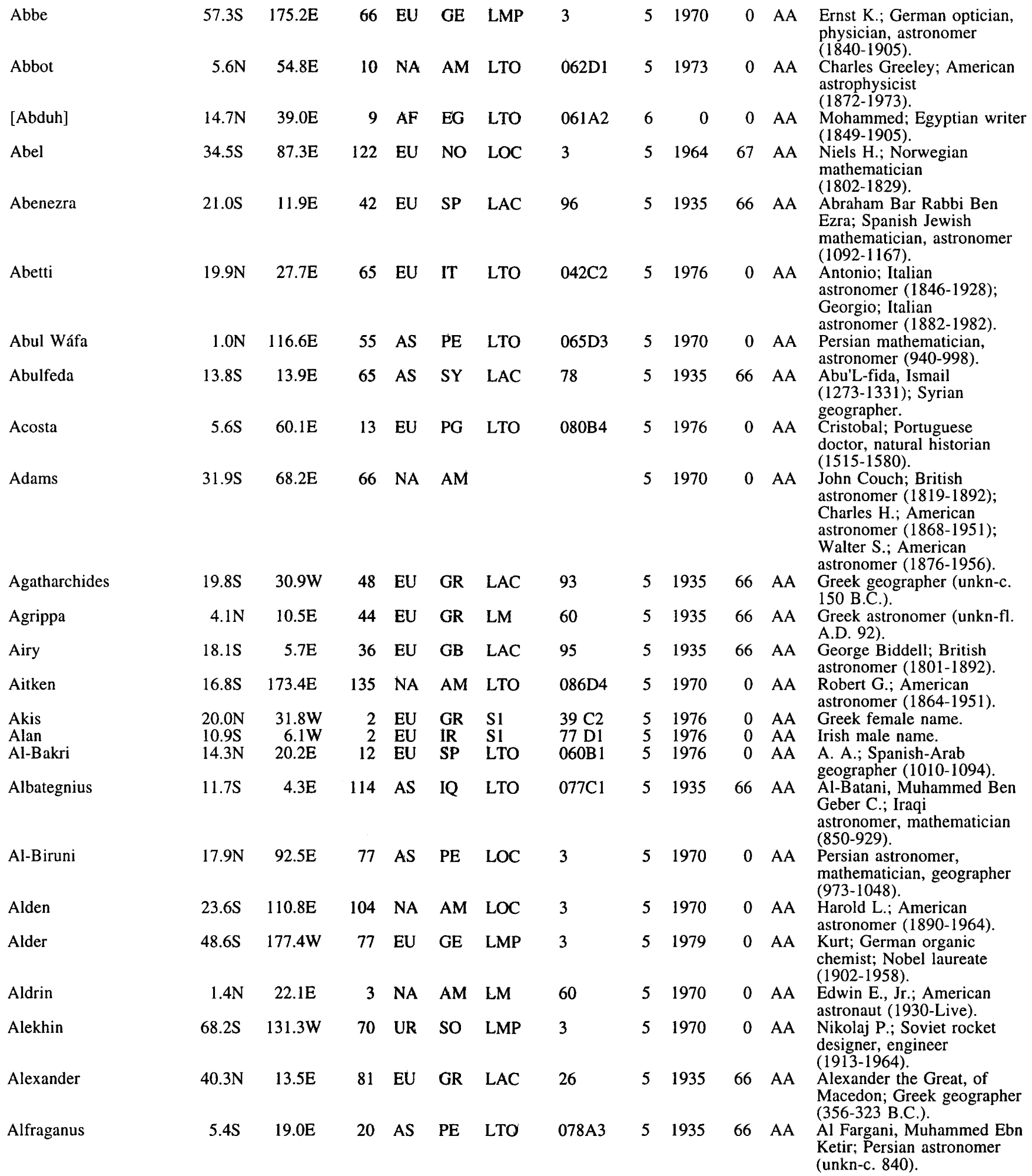

lat: latitude of feature center.

long: longitude of feature center.

diam: diameter or long dimension of feature. et: $\quad$ ethnicity of name origin (see page $284 \mathrm{ff}$.)

quad: map quadrangle or informal name

(see page xvii ff.). as: name approval status (see page $\mathrm{xvii)}$.

ad: name approval date (year).

ref: reference source for name (see page $287 \mathrm{ff}$.). 
MOON

\begin{tabular}{|c|c|c|c|c|c|c|c|c|c|c|c|c|}
\hline Name & lat & long & diam & ct & et & quad & map & as & ad & ref & $\mathbf{f t}$ & origin \\
\hline Alhazen & $15.9 \mathrm{~N}$ & $71.8 \mathrm{E}$ & 32 & AS & IQ & LMP & 1 & 5 & 1935 & 66 & AA & $\begin{array}{l}\text { Abu Ali Al-Hasan Ibn Al } \\
\text { Haitham; Iraqi }\end{array}$ \\
\hline Aliacensis & $30.6 \mathrm{~S}$ & $5.2 \mathrm{E}$ & 79 & EU & FR & LAC & 95 & 5 & 1935 & 66 & AA & $\begin{array}{l}\text { mathematician }(987-1038) \text {. } \\
\text { D'Ailly, Pierre; French } \\
\text { geographer (1350-1420). }\end{array}$ \\
\hline Al-Khwarizmi & $7.1 \mathrm{~N}$ & $106.4 \mathrm{E}$ & 65 & AS & IQ & LOC & 3 & 5 & 1973 & 0 & AA & $\begin{array}{l}\text { Iraqi mathematician } \\
\text { (unkn-c. 825). }\end{array}$ \\
\hline Almanon & $16.8 \mathrm{~S}$ & $15.2 \mathrm{E}$ & 49 & AS & PE & LAC & 96 & 5 & 1935 & 66 & AA & $\begin{array}{l}\text { Abdalla Al Mamun; Persian } \\
\text { astronomer (786-833). }\end{array}$ \\
\hline Al-Marrakushi & $10.4 \mathrm{~S}$ & $55.8 \mathrm{E}$ & 8 & $\mathrm{AF}$ & MR & LTO & 080D2 & 5 & 1976 & 0 & AA & $\begin{array}{l}\text { Moroccan astronomer, } \\
\text { mathematician (fl. c. 1262). }\end{array}$ \\
\hline $\begin{array}{l}\text { Aloha } \\
\text { Alpetragius }\end{array}$ & $\begin{array}{l}29.8 \mathrm{~N} \\
16.0 \mathrm{~S}\end{array}$ & $\begin{array}{r}53.9 \mathrm{~W} \\
4.5 \mathrm{~W}\end{array}$ & $\begin{array}{r}3 \\
39\end{array}$ & $\begin{array}{l}\mathrm{OC} \\
\mathrm{AF}\end{array}$ & $\begin{array}{l}\text { HA } \\
\text { MR }\end{array}$ & $\begin{array}{l}\text { S1 } \\
\text { LAC }\end{array}$ & $\begin{array}{l}38 \mathrm{~B} 2 \\
77\end{array}$ & $\begin{array}{l}5 \\
5\end{array}$ & $\begin{array}{l}1976 \\
1935\end{array}$ & $\begin{array}{r}0 \\
66\end{array}$ & $\begin{array}{l}\text { AA } \\
\text { AA }\end{array}$ & $\begin{array}{l}\text { Hawaiian greeting. } \\
\text { Nur Ed-Din Al Betrugi; }\end{array}$ \\
\hline & & & & & & & & & & & & $\begin{array}{l}\text { Moroccan astronomer } \\
\text { (unkn-c. 1100). }\end{array}$ \\
\hline Alphonsus & $13.7 \mathrm{~S}$ & $3.2 \mathrm{~W}$ & 108 & EU & SP & S1 & 77 D3 & 5 & 1935 & 66 & AA & $\begin{array}{l}\text { Alfonso X; Spanish } \\
\text { astronomer (1223-1284). }\end{array}$ \\
\hline Alter & $18.7 \mathrm{~N}$ & $107.5 \mathrm{~W}$ & 64 & NA & $\mathrm{AM}$ & LOC & 1 & 5 & 1970 & 0 & AA & $\begin{array}{l}\text { Dinsmore; American } \\
\text { astronomer, meteorologist } \\
(1888-1968) \text {. }\end{array}$ \\
\hline Ameghino & $3.3 \mathrm{~N}$ & $57.0 \mathrm{E}$ & 9 & EU & IT & LTO & 062D3 & 5 & 1976 & 0 & AA & $\begin{array}{l}\text { Fiorino (or Florentino); } \\
\text { Italian natural historian } \\
\text { (c. 1854-1911). }\end{array}$ \\
\hline Amici & $9.9 \mathrm{~S}$ & $172.1 \mathrm{~W}$ & 54 & EU & IT & LOC & 4 & 5 & 1970 & 0 & AA & $\begin{array}{l}\text { Giovanni B.; Italian } \\
\text { astronomer, botanist } \\
\text { (1786-1863). }\end{array}$ \\
\hline Ammonius & $8.5 \mathrm{~S}$ & $0.8 \mathrm{~W}$ & 8 & EU & GR & LTO & 077D2 & 5 & 1976 & 0 & AA & $\begin{array}{l}\text { Greek philosopher (unkn-c. } \\
517 \text { ). }\end{array}$ \\
\hline Amontons & $5.3 \mathrm{~S}$ & $46.8 \mathrm{E}$ & 2 & EU & FR & LTO & 079B3 & 5 & 1976 & 0 & AA & $\begin{array}{l}\text { Guillaume; French } \\
\text { physicist (1663-1705). }\end{array}$ \\
\hline Amundsen & $84.3 \mathrm{~S}$ & $85.6 \mathrm{E}$ & 101 & EU & NO & LMP & 3 & 5 & 1964 & 67 & AA & $\begin{array}{l}\text { Roald E.; Norwegian } \\
\text { explorer (1872-1928) }\end{array}$ \\
\hline Anaxagoras & $73.4 \mathrm{~N}$ & $10.1 \mathrm{~W}$ & 50 & EU & GR & LMP & 3 & 5 & 1935 & 66 & AA & $\begin{array}{l}\text { Greek astronomer }(500-428 \\
\text { B.C.). }\end{array}$ \\
\hline Anaximander & $66.9 \mathrm{~N}$ & $51.3 \mathrm{~W}$ & 67 & EU & GR & LMP & 3 & 5 & 1935 & 66 & AA & $\begin{array}{l}\text { Greek astronomer (c. } \\
611-547 \text { B.C.). }\end{array}$ \\
\hline Anaximenes & $72.5 \mathrm{~N}$ & $44.5 \mathrm{~W}$ & 80 & EU & GR & LMP & 3 & 5 & 1935 & 66 & AA & $\begin{array}{l}\text { Greek astronomer }(585-528 \\
\text { B.C.). }\end{array}$ \\
\hline Anděl & $10.4 \mathrm{~S}$ & $12.4 \mathrm{E}$ & 35 & EU & $\mathrm{CZ}$ & LTO & 078D1 & 5 & 1935 & 66 & AA & $\begin{array}{l}\text { Karel; Czechoslovakian } \\
\text { astronomer (1884-1947). }\end{array}$ \\
\hline Anders & $41.3 \mathrm{~S}$ & $142.9 \mathrm{~W}$ & 40 & NA & $\mathrm{AM}$ & LMP & 2 & 5 & 1970 & 0 & AA & $\begin{array}{l}\text { William A.; American } \\
\text { astronaut (1933-Live). }\end{array}$ \\
\hline Anderson & $15.8 \mathrm{~N}$ & 171.1E & 109 & NA & $\mathrm{AM}$ & LOC & 4 & 5 & 1970 & 0 & AA & $\begin{array}{l}\text { John A.; American } \\
\text { astronaut (1876-1959). }\end{array}$ \\
\hline Andersson & $49.7 \mathrm{~S}$ & $95.3 \mathrm{~W}$ & 13 & NA & AM & & & 5 & 1985 & 0 & AA & $\begin{array}{l}\text { Leif Erland; American } \\
\text { astronomer (1943-1979). }\end{array}$ \\
\hline Andronov & $22.7 \mathrm{~S}$ & $146.1 \mathrm{E}$ & 16 & UR & S & LTO & $102 \mathrm{~B} 3$ & 5 & 1976 & $\mathbf{0}$ & AA & $\begin{array}{l}\text { Aleksandr Aleksandrovich; } \\
\text { Soviet physicist } \\
\text { (1901-1952). }\end{array}$ \\
\hline $\begin{array}{l}\text { Ango } \\
\text { Angström }\end{array}$ & $\begin{array}{l}20.5 \mathrm{~N} \\
29.9 \mathrm{~N}\end{array}$ & $\begin{array}{l}32.3 \mathrm{~W} \\
41.6 \mathrm{~W}\end{array}$ & $\begin{array}{l}1 \\
9\end{array}$ & $\begin{array}{l}\mathrm{AF} \\
\mathrm{EU}\end{array}$ & $\begin{array}{l}\text { UN } \\
\text { SW }\end{array}$ & $\begin{array}{l}\text { S1 } \\
\text { LTO }\end{array}$ & $\begin{array}{l}39 \mathrm{C} 2 \\
039 \mathrm{~A} 2\end{array}$ & $\begin{array}{l}5 \\
5\end{array}$ & $\begin{array}{l}1976 \\
1935\end{array}$ & $\begin{array}{r}0 \\
66\end{array}$ & $\begin{array}{l}\text { AA } \\
\text { AA }\end{array}$ & $\begin{array}{l}\text { African male name. } \\
\text { Anders Jonas; Swedish } \\
\text { physicist (1814-1874). }\end{array}$ \\
\hline $\begin{array}{l}\text { Ann } \\
\text { Annegrit }\end{array}$ & $25.1 \mathrm{~N}$ & $0.1 \mathrm{~W}$ & 3 & AS & $\mathrm{HE}$ & S1 & & 5 & 1976 & 0 & AA & Hebrew female name. \\
\hline Ansgarius & $12.7 \mathrm{~S}$ & $79.7 \mathrm{E}$ & 94 & EU & GE & LTO & $081 \mathrm{Cl}$ & 5 & 1935 & 66 & AA & $\begin{array}{l}\text { St. Ansgar; German } \\
\text { theologian (801-864). }\end{array}$ \\
\hline Antoniadi & $69.7 \mathrm{~S}$ & $172.0 \mathrm{~W}$ & 143 & EU & FR & LMP & 3 & 5 & 1970 & 0 & AA & $\begin{array}{l}\text { Eugēne M.; French } \\
\text { astronomer (1870-1944). }\end{array}$ \\
\hline Anuchin & $49.0 \mathrm{~S}$ & $101.3 \mathrm{E}$ & 57 & UR & RU & LMP & 3 & 5 & 1979 & 0 & AA & $\begin{array}{l}\text { Dimitri N.; Russian } \\
\text { geographer (1843-1923). }\end{array}$ \\
\hline Anville & $1.9 \mathrm{~N}$ & $49.5 \mathrm{E}$ & 10 & EU & $\mathrm{F}$ & LTO & $061 \mathrm{C} 3$ & 5 & 1976 & 0 & AA & $\begin{array}{l}\text { Jean-Baptiste Bourguignon; } \\
\text { French cartographer } \\
\text { (1697-1782). }\end{array}$ \\
\hline Apianus & $26.9 \mathrm{~S}$ & $7.9 \mathrm{E}$ & 63 & EU & GE & LAC & 95 & 5 & 1935 & 66 & AA & $\begin{array}{l}\text { Bienewitz, Peter; German } \\
\text { mathematician, astronomer } \\
\text { (1495-1552). }\end{array}$ \\
\hline Apollo & $36.1 \mathrm{~S}$ & $151.8 \mathrm{~W}$ & 537 & EU & GR & LOC & 4 & 5 & 1970 & 0 & AA & $\begin{array}{l}\text { Named to honor Apollo } \\
\text { missions. }\end{array}$ \\
\hline Apollonius & $4.5 \mathrm{~N}$ & $61.1 \mathrm{E}$ & 53 & EU & GR & LTO & $062 \mathrm{Cl}$ & 5 & 1935 & 66 & AA & $\begin{array}{l}\text { Apollonius of Perga 3rd } \\
\text { century B.C.; Greek } \\
\text { mathematician. }\end{array}$ \\
\hline
\end{tabular}

lat: latitude of feature center.

long: longitude of feature center.

diam: diameter or long dimension of feature. et: ethnicity of name origin (see page $284 \mathrm{ff}$.)

quad: map quadrangle or informal name

(see page xvii ff.).

(see page $x v$
Haitham; Iraqi

mathematician (987-1038).

geographer (1350-1420).

(unk

Abdalla Al Mamun; Persian

astronomer (786-833)

mathematician (fl. c. 1262)

Nur Ed-Din Al Betrugi

astronomer (1223-1284)

astronomer, meteorologist

1888-1968)

Italian natural historian

(c. 1854-1911).

Giovanni B.; Italian (1786-1863)

Greek philosopher (unkn-c.

explorer (1872-1928)

B.C.).

B.C.).

William A . American

astronaut (1933-Live)

astronomer (1943-1979).

Aleksandr Aleksandrovich;

African male name.

German female name.

St. Ansgar; German

theologian (801-864).

Eugene M.; French

Dimitri N.; Russian

geographer (1843-1923).

Jean-Baptiste Bourguignon;

(16) cartographer

Bienewitz, Peter; German

mathematician, astronomer (1495-1552). missions.

century B C. Greek

mathematician. 
MOON

\begin{tabular}{|c|c|c|c|c|c|c|c|c|c|c|c|c|}
\hline Name & lat & long & diam & ct & et & quad & map & as & ad & ref & $\mathbf{f t}$ & origin \\
\hline Appleton & $37.2 \mathrm{~N}$ & $158.3 \mathrm{E}$ & 63 & $\mathrm{EU}$ & EN & LOC & 4 & 5 & 1970 & 0 & $\mathrm{AA}$ & $\begin{array}{l}\text { Sir Edward V.; British } \\
\text { physicist; Nobel laureate } \\
\text { (1892-1965). }\end{array}$ \\
\hline Arago & $6.2 \mathrm{~N}$ & $21.4 \mathrm{E}$ & 26 & $\mathrm{EU}$ & FR & LM & 60 & 5 & 1935 & 66 & $\mathrm{AA}$ & $\begin{array}{l}\text { Dominique Francois Jean; } \\
\text { French astronomer } \\
\text { (1786-1853). }\end{array}$ \\
\hline Aratus & $23.6 \mathrm{~N}$ & $4.5 \mathrm{E}$ & 10 & EU & GR & LTO & $041 \mathrm{Cl}$ & 5 & 1935 & 66 & $\mathrm{AA}$ & $\begin{array}{l}\text { Greek astronomer (315-245 } \\
\text { B.C. ?). }\end{array}$ \\
\hline Archimedes & $29.7 \mathrm{~N}$ & $4.0 \mathrm{~W}$ & 82 & EU & GR & LM & 41 & 5 & 1935 & 66 & AA & $\begin{array}{l}\text { Greek physicist, } \\
\text { mathematician (c. } 287-212 \\
\text { B.C.). }\end{array}$ \\
\hline Archytas & $58.7 \mathrm{~N}$ & $5.0 \mathrm{E}$ & 31 & EU & GR & LAC & 12 & 5 & 1935 & 66 & AA & $\begin{array}{l}\text { Greek mathematician } \\
\text { (428-347 B.C. ?). }\end{array}$ \\
\hline Argelander & $16.5 \mathrm{~S}$ & $5.8 \mathrm{E}$ & 34 & EU & GE & LAC & 95 & 5 & 1935 & 66 & $\mathrm{AA}$ & $\begin{array}{l}\text { Friedrich Wilhelm August; } \\
\text { German astronomer } \\
(1799-1875) \text {. }\end{array}$ \\
\hline Ariadaeus & $4.6 \mathrm{~N}$ & $17.3 \mathrm{E}$ & 11 & AS & BY & LM & 60 & 5 & 1935 & 66 & $\mathrm{AA}$ & $\begin{array}{l}\text { Arrhidaeus, Philipus; King } \\
\text { of Babylon chronologer } \\
\text { (unkn.-317 B.C.). }\end{array}$ \\
\hline [Ariosto] & $3.6 \mathrm{~S}$ & $95.6 \mathrm{E}$ & 23 & EU & IT & LTO & $082 \mathrm{~A} 3$ & 6 & 0 & 0 & $\mathrm{AA}$ & $\begin{array}{l}\text { Ludovico; Italian writer } \\
\text { (1474-1533). }\end{array}$ \\
\hline Aristarchus & $23.7 \mathrm{~N}$ & $47.4 \mathrm{~W}$ & 40 & EU & GR & $\mathrm{LAC}$ & 39 & 5 & 1935 & 66 & $\mathrm{AA}$ & $\begin{array}{l}\text { Greek astronomer (310-230 } \\
\text { B.C. ?). }\end{array}$ \\
\hline Aristillus & $33.9 \mathrm{~N}$ & $1.2 \mathrm{E}$ & 55 & EU & GR & LAC & 25 & 5 & 1935 & 66 & AA & $\begin{array}{l}\text { Greek astronomer (fl. c. } \\
280 \text { B.C.). }\end{array}$ \\
\hline Aristoteles & $50.2 \mathrm{~N}$ & $17.4 \mathrm{E}$ & 87 & $\mathrm{EU}$ & GR & LAC & 13 & 5 & 1935 & 66 & $\mathrm{AA}$ & $\begin{array}{l}\text { Greek astronomer, } \\
\text { philosopher (383-322 } \\
\text { B.C.). }\end{array}$ \\
\hline Armínski & $16.4 \mathrm{~S}$ & $154.2 \mathrm{E}$ & 26 & $\mathrm{EU}$ & PO & LTO & $103 \mathrm{Al}$ & 5 & 1976 & 0 & AA & $\begin{array}{l}\text { Franciszek; Polish } \\
\text { astronomer (1789-1848). }\end{array}$ \\
\hline Armstrong & $1.4 \mathrm{~N}$ & $25.0 \mathrm{E}$ & 4 & NA & $\mathrm{AM}$ & LM & 60 & 5 & 1970 & 0 & $\mathrm{AA}$ & $\begin{array}{l}\text { Neil A.; American } \\
\text { astronaut (1930-Live). }\end{array}$ \\
\hline Arnold & $66.8 \mathrm{~N}$ & $35.9 \mathrm{E}$ & 94 & EU & GE & LMP & 3 & 5 & 1935 & 66 & AA & $\begin{array}{l}\text { Christoph; German } \\
\text { astronomer (1650-1695). }\end{array}$ \\
\hline Arrhenius & $55.6 \mathrm{~S}$ & $91.3 \mathrm{~W}$ & 40 & $\mathrm{EU}$ & SW & LMP & 3 & 5 & 1970 & 0 & $\mathrm{AA}$ & $\begin{array}{l}\text { Svante A.; Swedish } \\
\text { chemist; Nobel laureate } \\
\text { (1859-1927). }\end{array}$ \\
\hline Artamonov & $25.5 \mathrm{~N}$ & $103.5 \mathrm{E}$ & 60 & UR & SO & LOC & 3 & 5 & 1970 & 0 & $\mathrm{AA}$ & $\begin{array}{l}\text { Nikolaj N.; Soviet rocket } \\
\text { scientist (1906-1965). }\end{array}$ \\
\hline Artem'ev & $10.8 \mathrm{~N}$ & $144.4 \mathrm{~W}$ & 67 & UR & SO & LOC & 4 & 5 & 1970 & 0 & AA & $\begin{array}{l}\text { Vladimir A.; Soviet rocket } \\
\text { scientist (1885-1962). }\end{array}$ \\
\hline $\begin{array}{l}\text { Artemis } \\
\text { Artsimovich }\end{array}$ & $\begin{array}{l}25.0 \mathrm{~N} \\
27.6 \mathrm{~N}\end{array}$ & $\begin{array}{l}25.4 \mathrm{~W} \\
36.6 \mathrm{~W}\end{array}$ & $\begin{array}{l}2 \\
8\end{array}$ & $\begin{array}{l}\text { EU } \\
\text { UR }\end{array}$ & $\begin{array}{l}\text { GR } \\
\text { SO }\end{array}$ & $\begin{array}{l}\text { SI } \\
\text { LTO }\end{array}$ & $\begin{array}{l}40 \text { A4 } \\
039 \mathrm{~B} 4\end{array}$ & $\begin{array}{l}5 \\
5\end{array}$ & $\begin{array}{l}1976 \\
1973\end{array}$ & $\begin{array}{l}0 \\
0\end{array}$ & $\begin{array}{l}\mathrm{AA} \\
\mathrm{AA}\end{array}$ & $\begin{array}{l}\text { Greek Moon goddess. } \\
\text { Lev Andreevich; Soviet } \\
\text { physicist (1909-1973). }\end{array}$ \\
\hline Aryabhata & $6.2 \mathrm{~N}$ & $35.1 \mathrm{E}$ & 22 & AS & IN & LTO & 061D2 & 5 & 1979 & 0 & $\mathrm{AA}$ & $\begin{array}{l}\text { Indian astronomer, } \\
\text { mathematician } \\
\text { (476-c.550). }\end{array}$ \\
\hline Arzachel & $18.2 \mathrm{~S}$ & $1.9 \mathrm{~W}$ & 96 & $\mathrm{EU}$ & SP & LAC & 95 & 5 & 1935 & 66 & $\mathrm{AA}$ & $\begin{array}{l}\text { Al Zarkala; Spanish-Arabic } \\
\text { astronomer (c. } \\
\text { 1028-1087). }\end{array}$ \\
\hline Asada & $7.3 \mathrm{~N}$ & $49.9 \mathrm{E}$ & 12 & AS & JA & LTO & $061 \mathrm{C} 2$ & 5 & 1976 & 0 & $\mathrm{AA}$ & $\begin{array}{l}\text { Goryu; Japanese astronomer } \\
(1734-1799) \text {. }\end{array}$ \\
\hline Asclepi & $55.1 \mathrm{~S}$ & $25.4 \mathrm{E}$ & 42 & $\mathrm{EU}$ & IT & LAC & 127 & 5 & 1935 & 66 & $\mathrm{AA}$ & $\begin{array}{l}\text { Giuseppe; Italian } \\
\text { astronomer (1706-1776). }\end{array}$ \\
\hline Ashbrook & $81.4 \mathrm{~S}$ & $112.5 \mathrm{~W}$ & 156 & NA & AM & & & 5 & 1994 & 112 & $\mathrm{AA}$ & $\begin{array}{l}\text { Joseph; American } \\
\text { astronomer (1918-1980). }\end{array}$ \\
\hline Aston & $32.9 \mathrm{~N}$ & $87.7 \mathrm{~W}$ & 43 & EU & EN & LOC & 27 & 5 & 1964 & 67 & $\mathrm{AA}$ & $\begin{array}{l}\text { Francis W.; British } \\
\text { chemist, physicist; Nobel } \\
\text { laureate (1877-1945). }\end{array}$ \\
\hline $\begin{array}{l}\text { Atlas } \\
\text { Atwood }\end{array}$ & $\begin{array}{r}46.7 \mathrm{~N} \\
5.8 \mathrm{~S}\end{array}$ & $\begin{array}{l}44.4 \mathrm{E} \\
57.7 \mathrm{E}\end{array}$ & $\begin{array}{l}87 \\
29\end{array}$ & $\begin{array}{l}\mathrm{EU} \\
\mathrm{EU}\end{array}$ & $\begin{array}{l}\text { GR } \\
\text { EN }\end{array}$ & $\begin{array}{l}\text { LAC } \\
\text { LTO }\end{array}$ & $\begin{array}{l}27 \\
080 \mathrm{~A} 3\end{array}$ & $\begin{array}{l}5 \\
5\end{array}$ & $\begin{array}{l}1935 \\
1976\end{array}$ & $\begin{array}{r}66 \\
0\end{array}$ & $\begin{array}{l}\mathrm{AA} \\
\mathrm{AA}\end{array}$ & $\begin{array}{l}\text { Mythological Greek Titan. } \\
\text { G.; British mathematician, } \\
\text { physicist (1745-1807). }\end{array}$ \\
\hline [Austen] & $9.0 \mathrm{~S}$ & $0.0 \mathrm{E}$ & 28 & EU & GB & LTO & 082D1 & 6 & 0 & 0 & $\mathrm{AA}$ & $\begin{array}{l}\text { Jane; British author } \\
(1775-1817) \text {. }\end{array}$ \\
\hline Autolycus & $30.7 \mathrm{~N}$ & $1.5 \mathrm{E}$ & 39 & $\mathrm{EU}$ & GR & LM & 41 & 5 & 1935 & 66 & $\mathrm{AA}$ & $\begin{array}{l}\text { Greek astronomer (unkn-c. } \\
330 \text { B.C.). }\end{array}$ \\
\hline Auwers & $15.1 \mathrm{~N}$ & $17.2 \mathrm{E}$ & 20 & EU & GE & LTO & $60 \mathrm{~A} 2$ & 5 & 1935 & 66 & $\mathrm{AA}$ & $\begin{array}{l}\text { Georg Friedrich Julius } \\
\text { Arthur; German astronomer } \\
\text { (1838-1915). }\end{array}$ \\
\hline Auzout & $10.3 \mathrm{~N}$ & $64.1 \mathrm{E}$ & 32 & EU & FR & LTO & 062B4 & 5 & 1961 & 0 & AA & $\begin{array}{l}\text { Adrien; French astronomer, } \\
\text { physicist (1622-1691). }\end{array}$ \\
\hline
\end{tabular}

\footnotetext{
lat: latitude of feature center.

et: ethnicity of name origin (see page $284 \mathrm{ff}$.)

long: longitude of feature center.

diam: diameter or long dimension of feature.

quad: map quadrangle or informal name

as: name approval status (see page $\mathrm{xvii}$ ).

(see page $x$ vii $\mathrm{ff}$.).

ad: name approval date (year).

ref: reference source for name (see page $287 \mathrm{ff}$.).

ct: continent of name origin (see page $284 \mathrm{ff}$.) map: map name or USGS map number (see page xvii ff.). ft: feature type (see page 290).
} 
MOON

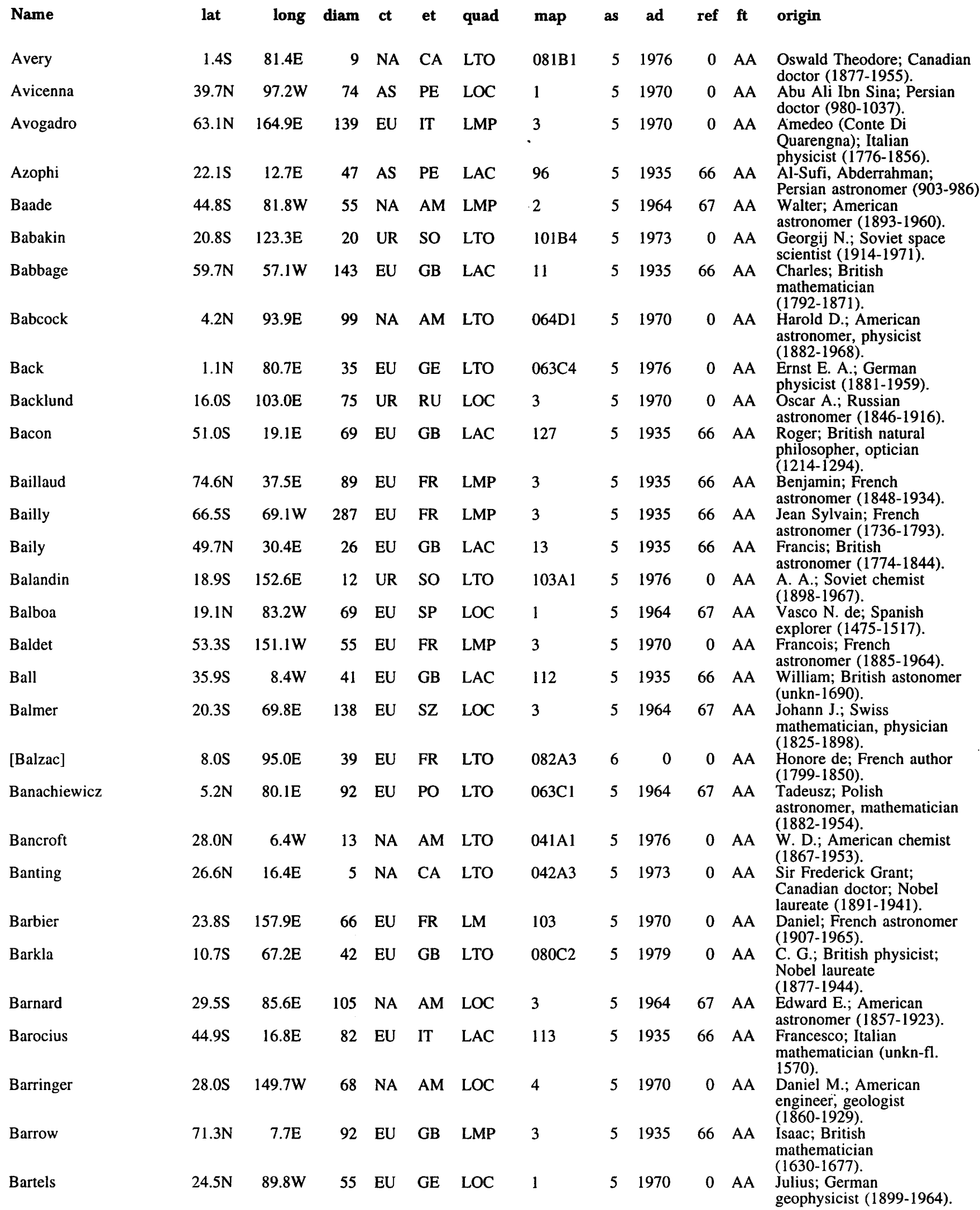

lat: latitude of feature center.

long: longitude of feature center.

diam: diameter or long dimension of feature.

ct: continent of name origin (see page $284 \mathrm{ff}$ ) et: ethnicity of name origin (see page $284 \mathrm{ff}$.)

quad: map quadrangle or informal name

(see page xvii ff.).

as: name approval status (see page $\mathrm{xvii}$.

ad: name approval date (year).

ref: reference source for name (see page $287 \mathrm{ff}$.)

map name or USGS map number (see page xvii ff.). $\mathrm{ft}$ : feature type (see page 290). 


\section{MOON}

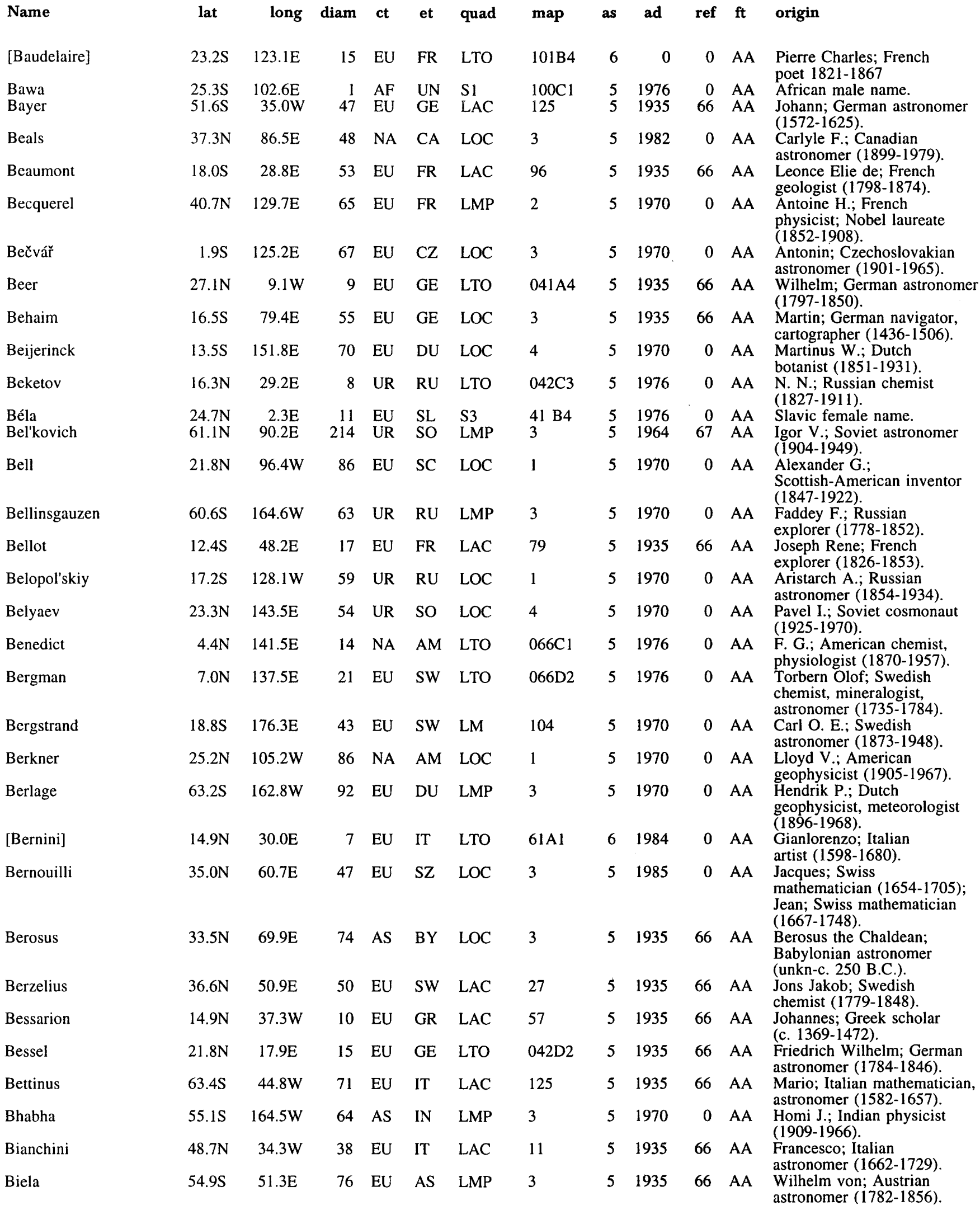

lat: latitude of feature center.

long: longitude of feature center.

diam: diameter or long dimension of feature.

ct: continent of name origin (see page 284 ff. et: ethnicity of name origin (see page $284 \mathrm{ff}$.)

quad: map quadrangle or informal name

(see page xvii ff.). as: name approval status (see page xvii).

ad: name approval date (year).

ref: reference source for name (see page $287 \mathrm{ff}$.). 
MOON

\begin{tabular}{|c|c|c|c|c|c|c|c|c|c|c|c|c|}
\hline Name & lat & long & diam & ct & et & quad & map & as & ad & ref & $\mathbf{f t}$ & origin \\
\hline Bilharz & $5.8 \mathrm{~S}$ & $56.3 \mathrm{E}$ & 43 & EU & GE & LTO & $080 \mathrm{~A} 3$ & 5 & 1976 & 0 & AA & $\begin{array}{l}\text { T.; German doctor } \\
\text { (1825-1862). }\end{array}$ \\
\hline Billy & $13.8 \mathrm{~S}$ & $50.1 \mathrm{~W}$ & 45 & EU & FR & LAC & 74 & 5 & 1935 & 66 & AA & $\begin{array}{l}\text { Jacques de; French } \\
\text { mathematician } \\
(1602-1679) \text {. }\end{array}$ \\
\hline Bingham & $8.1 \mathrm{~N}$ & $115.1 \mathrm{E}$ & 33 & NA & $\mathrm{AM}$ & LTO & $065 \mathrm{~A} 3$ & 5 & 1976 & 0 & AA & $\begin{array}{l}\text { H.; American explorer } \\
(1875-1956) .\end{array}$ \\
\hline Biot & $22.6 \mathrm{~S}$ & $51.1 \mathrm{E}$ & 12 & EU & FR & LAC & 98 & 5 & 1935 & 66 & AA & $\begin{array}{l}\text { Jean-Baptiste; French } \\
\text { astronomer (1774-1862). }\end{array}$ \\
\hline Birkeland & $30.2 \mathrm{~S}$ & $173.9 \mathrm{E}$ & 82 & $\mathrm{EU}$ & NO & LM & 104 & 5 & 1970 & 0 & AA & $\begin{array}{l}\text { Olaf K.; Norwegian } \\
\text { physicist }(1867-1917) .\end{array}$ \\
\hline Birkhoff & $58.7 \mathrm{~N}$ & $146.1 \mathrm{~W}$ & 345 & NA & $\mathrm{AM}$ & LMP & 3 & 5 & 1970 & 0 & AA & $\begin{array}{l}\text { George D.; American } \\
\text { mathematician } \\
\text { (1884-1944). }\end{array}$ \\
\hline Birmingham & $65.1 \mathrm{~N}$ & $10.5 \mathrm{~W}$ & 92 & $\mathrm{EU}$ & IR & LMP & 3 & 5 & 1935 & 66 & AA & $\begin{array}{l}\text { John; Irish astronomer } \\
(1829-1884) \text {. }\end{array}$ \\
\hline Birt & $22.4 \mathrm{~S}$ & $8.5 \mathrm{~W}$ & 16 & EU & GB & $\mathrm{LAC}$ & 95 & 5 & 1935 & 66 & AA & $\begin{array}{l}\text { William R.; British } \\
\text { selenographer } \\
\text { (1804-1881). }\end{array}$ \\
\hline Bjerknes & $38.4 \mathrm{~S}$ & $113.0 \mathrm{E}$ & 48 & EU & NO & LOC & 3 & 5 & 1970 & 0 & AA & $\begin{array}{l}\text { Vilhelm F. K.; Norwegian } \\
\text { physicist (1862-1951). }\end{array}$ \\
\hline Black & $9.2 \mathrm{~S}$ & $80.4 \mathrm{E}$ & 18 & EU & FR & LTO & $081 \mathrm{Cl}$ & 5 & 1976 & 0 & AA & $\begin{array}{l}\text { Joseph; French chemist } \\
(1728-1799) \text {. }\end{array}$ \\
\hline Blackett & $37.5 \mathrm{~S}$ & $116.1 \mathrm{~W}$ & 141 & EU & GB & LMP & 2 & 5 & 1979 & 0 & AA & $\begin{array}{l}\text { Patrick Maynard Stuart; } \\
\text { British physicist; Nobel } \\
\text { laureate (1897-1974). }\end{array}$ \\
\hline Blagg & $1.3 \mathrm{~N}$ & $1.5 \mathrm{E}$ & 5 & EU & GB & LAC & 59 & 5 & 1935 & 66 & AA & $\begin{array}{l}\text { Mary Adela; British } \\
\text { astronomer (1858-1944). }\end{array}$ \\
\hline Blancanus & $63.8 \mathrm{~S}$ & $21.4 \mathrm{~W}$ & 117 & EU & IT & LAC & 125 & 5 & 1935 & 66 & AA & $\begin{array}{l}\text { Biancani, Giuseppe; } \\
\text { Italian mathematician, } \\
\text { astronomer (1566-1624). }\end{array}$ \\
\hline Diantifara & J0.JS & $94.4 \mathrm{~W}$ & 40 & $\mathrm{LU}$ & Th s & & 1985 & 5 & 1991 & 0 & AA & (1753-1809). \\
\hline Blancninus & $25.4 \mathrm{~S}$ & $2.5 \mathrm{E}$ & 61 & EU & IT & LAC & 95 & 5 & 1935 & 66 & AA & $\begin{array}{l}\text { Bianchini, Giovanni; } \\
\text { Italian astronomer } \\
\text { (unkn-fl. 1458). }\end{array}$ \\
\hline BIaznko & $31.01 \mathrm{~V}$ & $148.0 \mathrm{~W}$ & 34 & UK & su & LOC & 4 & 5 & 1970 & 0 & AA & $\begin{array}{l}\text { Sergej N.; Soviet } \\
\text { astronomer (1870-1956). }\end{array}$ \\
\hline Bobillier & $19.6 \mathrm{~N}$ & $15.5 \mathrm{E}$ & 6 & $\mathrm{EU}$ & FR & LTO & 042D3 & 5 & 1976 & 0 & AA & $\begin{array}{l}\text { E.; French geometer } \\
(1798-1840) \text {. }\end{array}$ \\
\hline Bobone & $26.9 \mathrm{~N}$ & $131.8 \mathrm{~W}$ & 3 & SA & AR & LOC & 1 & 5 & 1970 & 0 & AA & $\begin{array}{l}\text { Jorge; Argentinian } \\
\text { astronomer (1901-1958). }\end{array}$ \\
\hline Bode & $6.7 \mathrm{~N}$ & $2.4 \mathrm{~W}$ & 18 & EU & GE & LAC & 59 & 5 & 1935 & 68 & AA & $\begin{array}{l}\text { Johann Elert; German } \\
\text { astronomer (1747-1826). }\end{array}$ \\
\hline Boethius & $5.6 \mathrm{~N}$ & $72.3 \mathrm{E}$ & 10 & EU & GR & LTO & 063D1 & 5 & 1976 & 0 & AA & Greek physicist (c. \\
\hline Boguslawsky & $72.9 \mathrm{~S}$ & $43.2 \mathrm{E}$ & 97 & EU & GE & LMP & 3 & 5 & 1935 & 68 & AA & $\begin{array}{l}\text { Palon Heinrich Ludwig von } \\
\text { German astronomer } \\
(1789-1851) \text {. }\end{array}$ \\
\hline Bonnenberger & $16.2 \mathrm{~S}$ & $40.0 \mathrm{E}$ & 33 & $\mathrm{EU}$ & $\mathrm{GE}$ & LAC & 79 & 5 & 1935 & 68 & AA & $\begin{array}{l}\text { Johann Gottlieb Friedrich } \\
\text { Von; German astronomer } \\
\text { (1765-1831). }\end{array}$ \\
\hline Bohr & $12.4 \mathrm{~N}$ & $86.6 \mathrm{~W}$ & 71 & EU & $\mathrm{DE}$ & LOC & 1 & 5 & 1964 & 0 & AA & $\begin{array}{l}\text { Niels H. D.; Danish } \\
\text { physicist; Nobel laureate } \\
\text { (1885-1962). }\end{array}$ \\
\hline Bok & $20.2 \mathrm{~S}$ & $171.6 \mathrm{~W}$ & 45 & NA & $\mathrm{AM}$ & LM & 104 & 5 & 1979 & 0 & AA & $\begin{array}{l}\text { Priscilla Fairfield; } \\
\text { American astronomer } \\
(1896-1975) \text {. }\end{array}$ \\
\hline Boltzmann & $74.9 \mathrm{~S}$ & $90.7 \mathrm{~W}$ & 76 & EU & AS & LMP & 3 & 5 & 1964 & 67 & AA & $\begin{array}{l}\text { Ludwig E.; Austrian } \\
\text { physicist (1844-1906). }\end{array}$ \\
\hline Bolyai & $33.6 \mathrm{~S}$ & $125.9 \mathrm{E}$ & 135 & $\mathrm{EU}$ & $\mathrm{HU}$ & LOC & 3 & 5 & 1970 & 0 & AA & $\begin{array}{l}\text { Janos; Hungarian } \\
\text { mathematician } \\
(1802-1860)\end{array}$ \\
\hline Bombelli & $5.3 \mathrm{~N}$ & $56.2 \mathrm{E}$ & 10 & EU & IT & LTO & 062D2 & 5 & 1976 & 0 & AA & $\begin{array}{l}\text { R.; Italian mathematician } \\
\text { (1526-1572). }\end{array}$ \\
\hline Bondarenko & $17.8 \mathrm{~S}$ & 136.3E & 30 & UR & SO & SOV & 1985 & 5 & 1991 & 0 & AA & $\begin{array}{l}\text { V. V.; Soviet } \\
\text { student-cosmonaut } \\
(1937-1961) \text {. }\end{array}$ \\
\hline Bonpland & & & 60 & $\mathrm{EU}$ & & TO & & 5 & 1935 & 66 & AA & $\begin{array}{l}\text { Aime; French botanist } \\
(1773-1858) .\end{array}$ \\
\hline
\end{tabular}

lat: latitude of feature center.

long: longitude of feature center.

diam: diameter or long dimension of feature.

ct: continent of name origin (see page 284 ff et: ethnicity of name origin (see page $284 \mathrm{ff}$.)

quad: map quadrangle or informal name

(see page xvii ff.). as: name approval status (see page xvii).

ad: name approval date (year).

ref: reference source for name (see page $287 \mathrm{ff}$.). 
MOON

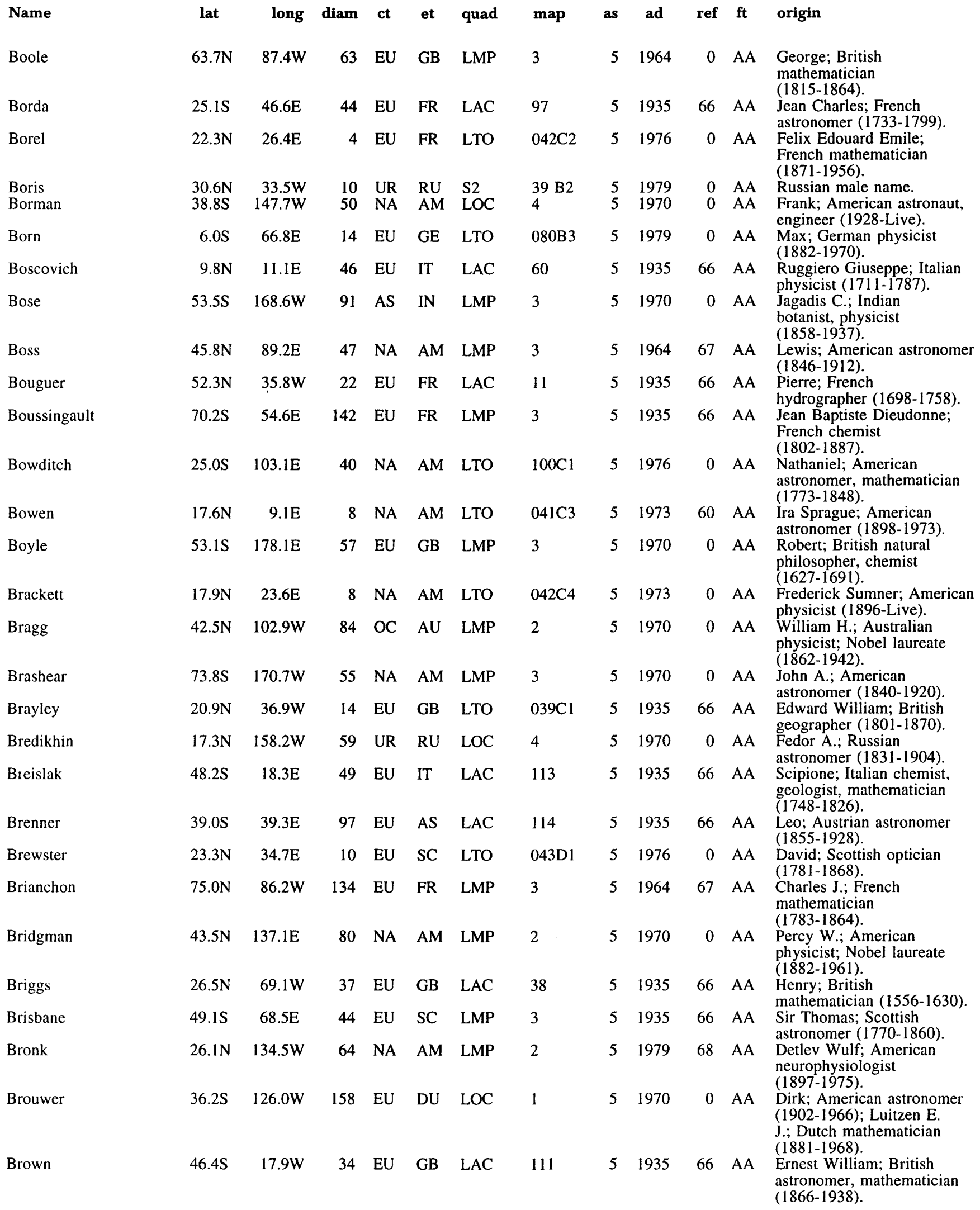

lat: latitude of feature center.

long: longitude of feature center.

diam: diameter or long dimension of feature.

ct: continent of name origin (see page $284 \mathrm{ff}$ ) et: ethnicity of name origin (see page $284 \mathrm{ff}$.)

quad: map quadrangle or informal name (see page xvii ff.). as: name approval status (see page xvii).

ad: name approval date (year).

ref: reference source for name (see page $287 \mathrm{ff}$.)

map: map name or USGS map number (see page xvii ff.). $\mathrm{ft}$ : feature type (see page 290). 
MOON

\begin{tabular}{|c|c|c|c|c|c|c|c|c|c|c|c|c|}
\hline Name & lat & long & diam & ct & et & quad & map & as & ad & ref & ft & origin \\
\hline Bruce & $1.1 \mathrm{~N}$ & $0.4 \mathrm{E}$ & 6 & NA & AM & LAC & 59 & 5 & 1935 & 66 & $\mathrm{AA}$ & $\begin{array}{l}\text { Catherine Wolfe; American } \\
\text { philanthropist, astronomer } \\
(1816-1900) \text {. }\end{array}$ \\
\hline Brunner & $9.9 \mathrm{~S}$ & $90.9 \mathrm{E}$ & 53 & EU & $\mathrm{SZ}$ & LTO & 082D1 & 5 & 1970 & 0 & $\mathrm{AA}$ & $\begin{array}{l}\text { William O.; Swiss } \\
\text { astronomer (1878-1958). }\end{array}$ \\
\hline Buch & $38.8 \mathrm{~S}$ & $17.7 \mathrm{E}$ & 53 & EU & GE & LAC & 113 & 5 & 1935 & 66 & AA & $\begin{array}{l}\text { Christian Leopold von; } \\
\text { German geologist } \\
(1774-1853) \text {. }\end{array}$ \\
\hline Buffon & $40.4 \mathrm{~S}$ & $133.4 \mathrm{~W}$ & 106 & $\mathrm{EU}$ & FR & LMP & 2 & 5 & 1970 & 0 & AA & $\begin{array}{l}\text { Georges L. L.; French } \\
\text { natural historian } \\
(1707-1788) \text {. }\end{array}$ \\
\hline Buisson & $1.4 \mathrm{~S}$ & $112.5 \mathrm{E}$ & 56 & $\mathrm{EU}$ & FR & LMP & 2 & 5 & 1970 & 0 & AA & $\begin{array}{l}\text { Henri; French physicist, } \\
\text { astronomer (1873-1944). }\end{array}$ \\
\hline Bullialdus & $20.7 \mathrm{~S}$ & $22.2 \mathrm{~W}$ & 60 & $\mathrm{EU}$ & FR & $\mathrm{LAC}$ & 94 & 5 & 1935 & 66 & $\mathrm{AA}$ & $\begin{array}{l}\text { Boulliaud, Ismael; French } \\
\text { astronomer (1605-1694). }\end{array}$ \\
\hline Bunsen & $41.4 \mathrm{~N}$ & $85.3 \mathrm{~W}$ & 52 & $\mathrm{EU}$ & GE & LMP & 2 & 5 & 1964 & 67 & $\mathrm{AA}$ & $\begin{array}{l}\text { Robert W.; German chemist } \\
\text { (1811-1899). }\end{array}$ \\
\hline Burckhardt & $31.1 \mathrm{~N}$ & $56.5 \mathrm{E}$ & 56 & EU & GE & LAC & 44 & 5 & 1935 & 68 & AA & $\begin{array}{l}\text { Johann Karl; German } \\
\text { astronomer }(1773-1825) \text {. }\end{array}$ \\
\hline Bürg & $45.0 \mathrm{~N}$ & $28.2 \mathrm{E}$ & 39 & EU & AS & LAC & 26 & 5 & 1935 & 68 & AA & $\begin{array}{l}\text { Johann Tobias; Austrian } \\
\text { astronomer (1766-1834). }\end{array}$ \\
\hline Burnham & $13.9 \mathrm{~S}$ & $7.3 \mathrm{E}$ & 24 & NA & AM & LAC & 77 & 5 & 1935 & 66 & AA & $\begin{array}{l}\text { Sherburne Wesley; American } \\
\text { astronomer (1838-1921). }\end{array}$ \\
\hline Büsching & $38.0 \mathrm{~S}$ & $20.0 \mathrm{E}$ & 52 & EU & GE & LAC & 113 & 5 & 1935 & 66 & AA & $\begin{array}{l}\text { Anton Friedrich; German } \\
\text { geographer (1724-1793). }\end{array}$ \\
\hline Butlerov & $12.5 \mathrm{~N}$ & $108.7 \mathrm{~W}$ & 40 & UR & RU & LOC & 1 & 5 & 1970 & 0 & AA & $\begin{array}{l}\text { Aleksandr M.; Russian } \\
\text { chemist (1828-1886). }\end{array}$ \\
\hline Buys-Ballot & $20.8 \mathrm{~N}$ & 174.5E & 55 & EU & DU & LOC & 4 & 5 & 1970 & 0 & AA & $\begin{array}{l}\text { C. H. D.; Dutch } \\
\text { meteorologist } \\
(1817-1890) .\end{array}$ \\
\hline Byrd & $85.3 \mathrm{~N}$ & $9.8 \mathrm{E}$ & 93 & NA & $\mathrm{AM}$ & LMP & 3 & 5 & 1964 & 67 & AA & $\begin{array}{l}\text { Richard E.; American } \\
\text { explorer, aviator, } \\
\text { navigator (1888-1957). }\end{array}$ \\
\hline Byrgius & $24.7 \mathrm{~S}$ & $65.3 \mathrm{~W}$ & 87 & $\mathrm{EU}$ & $\mathrm{SZ}$ & LAC & 9 & 5 & 1935 & 66 & AA & $\begin{array}{l}\text { Burgi, Joost; Swiss } \\
\text { horologist (1552-1632). }\end{array}$ \\
\hline C. Herschel & $34.5 \mathrm{~N}$ & $31.2 \mathrm{~W}$ & 13 & $\mathrm{EU}$ & GB & LAC & 24 & 5 & 1935 & 66 & AA & $\begin{array}{l}\text { Caroline; British } \\
\text { astronomer (1750-1848). }\end{array}$ \\
\hline C. Mayer & $63.2 \mathrm{~N}$ & $17.3 \mathrm{E}$ & 38 & EU & GE & LAC & 13 & 5 & 1935 & 66 & AA & $\begin{array}{l}\text { Christian; German } \\
\text { astronomer, mathematician, } \\
\text { physicist (1719-1783). }\end{array}$ \\
\hline Cabannes & $60.9 \mathrm{~S}$ & $169.6 \mathrm{~W}$ & 80 & EU & FR & LMP & 3 & 5 & 1970 & 0 & AA & $\begin{array}{l}\text { Jean; French physicist } \\
(1885-1959)\end{array}$ \\
\hline Cabeus & $84.9 \mathrm{~S}$ & $35.5 \mathrm{~W}$ & 98 & $\mathrm{EU}$ & IT & LMP & 3 & 5 & 1935 & 66 & $\mathrm{AA}$ & $\begin{array}{l}\text { Cabeo, Niccolo; Italian } \\
\text { astronomer (1586-1650). }\end{array}$ \\
\hline Cajal & $12.6 \mathrm{~N}$ & $31.1 \mathrm{E}$ & 9 & $\mathrm{EU}$ & SP & LTO & $061 \mathrm{Al}$ & 5 & 1973 & 0 & AA & $\begin{array}{l}\text { Santiago Ramon Y; Spanish } \\
\text { doctor; Nobel laureate } \\
\text { (1852-1934). }\end{array}$ \\
\hline Cajori & $47.4 \mathrm{~S}$ & $168.8 \mathrm{E}$ & 70 & NA & AM & LMP & 3 & 5 & 1970 & 0 & AA & $\begin{array}{l}\text { Florian; American } \\
\text { mathematician } \\
(1859-1930)\end{array}$ \\
\hline Calippus & $38.9 \mathrm{~N}$ & $10.7 \mathrm{E}$ & 32 & EU & GR & LAC & 26 & 5 & 1935 & 66 & AA & $\begin{array}{l}\text { Calippus of Cyzicus; Greek } \\
\text { astronomer (c. } 330 \text { B.C.). }\end{array}$ \\
\hline Cameron & $6.2 \mathrm{~N}$ & $45.9 \mathrm{E}$ & 10 & NA & AM & LTO & $061 \mathrm{C} 2$ & 5 & 1973 & 0 & $\mathrm{AA}$ & $\begin{array}{l}\text { Robert Curry; American } \\
\text { astronomer (1925-1972). }\end{array}$ \\
\hline [Camoens] & $0.8 \mathrm{~N}$ & $84.9 \mathrm{E}$ & 33 & EU & PG & LTO & $063 \mathrm{C} 3$ & 6 & 0 & 0 & AA & $\begin{array}{l}\text { Luis de; Portuguese author } \\
\text { (1524-1530) }\end{array}$ \\
\hline Campanus & $28.0 \mathrm{~S}$ & $27.8 \mathrm{~W}$ & 48 & EU & IT & LAC & 94 & 5 & 1935 & 66 & AA & $\begin{array}{l}\text { Campano, Giovanni; Italian } \\
\text { astronomer (c. } \\
1200 \text {-unkn). }\end{array}$ \\
\hline Campbell & $45.3 \mathrm{~N}$ & $151.4 \mathrm{E}$ & 219 & NA & $\mathrm{AM}$ & LMP & 2 & 5 & 1970 & 0 & AA & $\begin{array}{l}\text { Leon; American astronomer } \\
\text { (1881-1951); William W.; } \\
\text { American astronomer } \\
(1862-1938) \text {. }\end{array}$ \\
\hline Cannizzaro & $55.6 \mathrm{~N}$ & $99.6 \mathrm{~W}$ & 56 & $\mathrm{EU}$ & IT & LMP & 3 & 5 & 1970 & 0 & $\mathrm{AA}$ & $\begin{array}{l}\text { Stanislao; Italian chemist } \\
(1826-1910) \text {. }\end{array}$ \\
\hline Cannon & $19.9 \mathrm{~N}$ & $81.4 \mathrm{E}$ & 56 & NA & $\mathrm{AM}$ & LOC & 3 & 5 & 1964 & 67 & AA & $\begin{array}{l}\text { Annie J.; American } \\
\text { astronomer (1863-1941). }\end{array}$ \\
\hline Cantor & $38.2 \mathrm{~N}$ & $118.6 \mathrm{E}$ & 0 & EU & & LOC & 3 & 5 & 1970 & 0 & AA & $\begin{array}{l}\text { Georg; German } \\
\text { mathematician (1845-1918); } \\
\text { Moritz; German } \\
\text { mathematician (1829-1920). }\end{array}$ \\
\hline
\end{tabular}

lat: latitude of feature center.

long: longitude of feature center.

diam: diameter or long dimension of feature.

ct: continent of name origin (see page $284 \mathrm{ff}$.) et: ethnicity of name origin (see page $284 \mathrm{ff}$.)

quad: map quadrangle or informal name

(see page $x$ vii ff.).

map name or USGS map number (see page xvii ff.). ft: feature type (see page 290). as: name approval status (see page $x$ vii)

ad: name approval date (year).

ref: reference source for name (see page $287 \mathrm{ff}$.). 
MOON

\begin{tabular}{|c|c|c|c|c|c|c|c|c|c|c|c|c|}
\hline Name & lat & long & diam & ct & et & quad & map & as & ad & ref & $\mathbf{f t}$ & origin \\
\hline Capella & $7.5 \mathrm{~S}$ & $35.0 \mathrm{E}$ & 90 & EU & RO & LTO & 079A3 & 5 & 1935 & 66 & AA & $\begin{array}{l}\text { Martianus; Roman } \\
\text { astronomer (c. A.D. } \\
\text { 400-unkn). }\end{array}$ \\
\hline Capuanus & $34.1 \mathrm{~S}$ & $26.7 \mathrm{~W}$ & 59 & $\mathrm{EU}$ & IT & LAC & 111 & 5 & 1935 & 66 & AA & $\begin{array}{l}\text { Francesco Capuano Di } \\
\text { Manfredonia; Italian } \\
\text { astronomer (c. } \\
1400 \text {-unkn). }\end{array}$ \\
\hline Cardanus & $13.2 \mathrm{~S}$ & $72.4 \mathrm{~W}$ & 49 & EU & IT & LOC & 1 & 5 & 1935 & 66 & AA & $\begin{array}{l}\text { Cardano, Girolamo; Italian } \\
\text { mathematician } \\
(1501-1576) \text {. }\end{array}$ \\
\hline Carlini & $33.7 \mathrm{~N}$ & $24.1 \mathrm{~W}$ & 10 & EU & IT & LOC & 2 & 5 & 1935 & 66 & AA & $\begin{array}{l}\text { Francesco; Italian } \\
\text { astronomer (1783-1862). }\end{array}$ \\
\hline Carlos & $24.9 \mathrm{~N}$ & $2.3 \mathrm{E}$ & 4 & EU & SP & S2 & $41 \mathrm{~B} 4$ & 5 & 1976 & 0 & AA & Spanish male name. \\
\hline Carmichael & $19.6 \mathrm{~N}$ & $40.4 \mathrm{E}$ & 20 & NA & $\mathrm{AM}$ & LTO & $043 C 4$ & 5 & 1973 & 0 & AA & $\begin{array}{l}\text { Leonard; American } \\
\text { psychologist (1898-1973). }\end{array}$ \\
\hline Carnot & $52.3 \mathrm{~N}$ & $143.5 \mathrm{~W}$ & 126 & EU & FR & LMP & 3 & 5 & 1970 & 0 & AA & $\begin{array}{l}\text { Nicolas L. S.; French } \\
\text { physicist (1796-1832). }\end{array}$ \\
\hline Carol & $8.5 \mathrm{~N}$ & $122.3 \mathrm{E}$ & 117 & EU & LA & S2 & $65 \mathrm{Cl}$ & 5 & 1979 & 0 & AA & Latin female name. \\
\hline Carpenter & $69.4 \mathrm{~N}$ & $50.9 \mathrm{~W}$ & 59 & $\mathrm{EU}$ & $\mathrm{GB}$ & LMP & & 5 & 1935 & 66 & AA & $\begin{array}{l}\text { James; British astronomer } \\
\text { (1840-1899); Edwin F.; } \\
\text { American Astronomer } \\
(1898-1963) \text {. }\end{array}$ \\
\hline Carrel & $10.7 \mathrm{~N}$ & 26.7E & 15 & EU & FR & LTO & 060B3 & 5 & 1979 & 0 & AA & $\begin{array}{l}\text { Alexis; French doctor, } \\
\text { physiologist; Nobel } \\
\text { laureate (1873-1944). }\end{array}$ \\
\hline Carrillo & $2.2 \mathrm{~S}$ & $80.9 \mathrm{E}$ & 16 & NA & ME & LTO & $081 \mathrm{~B} 1$ & 5 & 1979 & 0 & AA & $\begin{array}{l}\text { Flores Nabor; Mexican soil } \\
\text { engineer (1911-1967). }\end{array}$ \\
\hline Carrington & $44.0 \mathrm{~N}$ & $62.1 \mathrm{E}$ & 30 & EU & GB & LOC & 3 & 5 & 1935 & 66 & AA & $\begin{array}{l}\text { Richard Christopher; } \\
\text { British astronomer } \\
(1826-1875) \text {. }\end{array}$ \\
\hline Cartan & $4.2 \mathrm{~N}$ & $59.3 \mathrm{E}$ & 15 & $\mathrm{EU}$ & FR & LTO & $062 \mathrm{D} 2$ & 5 & 1976 & 0 & AA & $\begin{array}{l}\text { E. J.; French } \\
\text { mathematician } \\
(1869-1951)\end{array}$ \\
\hline Carver & $43.0 \mathrm{~S}$ & $126.9 \mathrm{E}$ & 59 & NA & AM & LMP & 2 & 5 & 1970 & 0 & AA & $\begin{array}{l}\text { George W.; American } \\
\text { botanist (c. 1864-1943). }\end{array}$ \\
\hline Casatus & $72.8 \mathrm{~S}$ & $29.5 \mathrm{~W}$ & 108 & EU & IT & LMP & 3 & 5 & 1935 & 66 & AA & $\begin{array}{l}\text { Casati, Paolo; Italian } \\
\text { mathematician } \\
(1617-1707) .\end{array}$ \\
\hline Cassegrain & $52.4 \mathrm{~S}$ & $113.5 \mathrm{E}$ & 55 & EU & FR & LMP & 3 & 5 & 1970 & 0 & AA & $\begin{array}{l}\text { Giovanni D.; French } \\
\text { astronomer, doctor } \\
(1625-1712) \text {. }\end{array}$ \\
\hline Cassini & $40.2 \mathrm{~N}$ & $4.6 \mathrm{E}$ & 56 & EU & IT & LAC & 25 & 5 & 1935 & 66 & AA & $\begin{array}{l}\text { Giovanni Domenico; } \\
\text { Italian-French astronomer } \\
\text { (1625-1712); Jacques J.; } \\
\text { French astronomer } \\
(1677-1756) \text {. }\end{array}$ \\
\hline Catalán & $45.7 \mathrm{~S}$ & $87.3 \mathrm{~W}$ & 25 & $\mathrm{EU}$ & SP & LMP & 2 & 5 & 1970 & 0 & AA & $\begin{array}{l}\text { Miguel A.; Spanish } \\
\text { spectroscopist } \\
\text { (1894-1957). }\end{array}$ \\
\hline Catharina & $18.1 \mathrm{~S}$ & $23.4 \mathrm{E}$ & 104 & EU & GR & $\mathrm{LOC}$ & 2 & 5 & 1935 & 66 & AA & $\begin{array}{l}\text { St. Catherine of } \\
\text { Alexandria; Greek } \\
\text { theologian, philosopher } \\
\text { (unkn-c. 307). }\end{array}$ \\
\hline Cauchy & $9.6 \mathrm{~N}$ & $38.6 \mathrm{E}$ & 12 & $\mathrm{EU}$ & FR & LOC & 2 & 5 & 1935 & 66 & AA & $\begin{array}{l}\text { Augustin Louis; French } \\
\text { mathematician } \\
(1789-1857) .\end{array}$ \\
\hline Cavalerius & $5.1 \mathrm{~N}$ & $66.8 \mathrm{~W}$ & 57 & $\mathrm{EU}$ & IT & LAC & 56 & 5 & 1935 & 66 & AA & $\begin{array}{l}\text { Cavalieri, Buonaventura; } \\
\text { Italian mathematician } \\
\text { (1598-1647). }\end{array}$ \\
\hline Cavendish & $24.5 \mathrm{~S}$ & $53.7 \mathrm{~W}$ & 56 & EU & GB & LAC & 92 & 5 & 1935 & 66 & AA & $\begin{array}{l}\text { Henry; British chemist, } \\
\text { physicist }(1731-1810) \text {. }\end{array}$ \\
\hline Caventou & $29.8 \mathrm{~N}$ & $29.4 \mathrm{~W}$ & 3 & $\mathrm{EU}$ & FR & LTO & 040A I & 5 & 1976 & 68 & AA & $\begin{array}{l}\text { Joseph Bienaime; French } \\
\text { chemist, pharmacologist } \\
(1795-1877) \text {. }\end{array}$ \\
\hline Cayley & $4.0 \mathrm{~N}$ & $15.1 \mathrm{E}$ & 14 & $\mathrm{EU}$ & GB & LAC & 60 & 5 & 1935 & 66 & AA & $\begin{array}{l}\text { Arthur; British } \\
\text { astronomer, mathematician } \\
(1821-1895) \text {. }\end{array}$ \\
\hline [Cellini] & $7.8 \mathrm{~S}$ & $3.0 \mathrm{E}$ & 34 & EU & IT & LTO & 081B4 & 6 & 0 & 0 & AA & $\begin{array}{l}\text { Benvenuto; Italian artist, } \\
\text { writer }(1500-1571) \text {. }\end{array}$ \\
\hline Celsius & $34.1 \mathrm{~S}$ & $20.1 \mathrm{E}$ & 36 & EU & SW & LAC & 113 & 5 & 1935 & 66 & AA & $\begin{array}{l}\text { Anders; Swedish astronomer } \\
(1701-1744) \text {. }\end{array}$ \\
\hline
\end{tabular}

lat: latitude of feature center.

long: longitude of feature center.

diam: diameter or long dimension of feature.

ct: $\quad$ continent of name origin (see page 284 ff et: ethnicity of name origin (see page $284 \mathrm{ff}$.)

quad: map quadrangle or informal name

(see page xvii ff.). as: name approval status (see page xvii)

ad: name approval date (year).

ref: reference source for name (see page $287 \mathrm{ff}$.) 
MOON

\begin{tabular}{|c|c|c|c|c|c|c|c|c|c|c|c|c|}
\hline Name & lat & long & diam & ct & et & quad & map & as & ad & ref & $\mathbf{f t}$ & origin \\
\hline Censorinus & $0.4 \mathrm{~S}$ & 32.7E & 3 & EU & $\mathrm{RM}$ & LAC & 61 & 5 & 1935 & 66 & AA & $\begin{array}{l}\text { Roman astronomer (fl. } \\
\text { 238-unkn). }\end{array}$ \\
\hline Cepheus & $40.8 \mathrm{~N}$ & $45.8 \mathrm{E}$ & 39 & EU & GR & LAC & 27 & 5 & 1935 & 66 & $\mathrm{AA}$ & $\begin{array}{l}\text { Mythological astronomer, } \\
\text { father of Andromeda. }\end{array}$ \\
\hline [Cervantes] & $3.4 \mathrm{~S}$ & $99.2 \mathrm{E}$ & 25 & EU & SP & LTO & $082 \mathrm{~A} 2$ & 6 & 0 & 0 & AA & $\begin{array}{l}\text { Miguel De; Spanish writer } \\
(1547-1616) \text {. }\end{array}$ \\
\hline Chacornac & $29.8 \mathrm{~N}$ & $31.7 \mathrm{E}$ & 51 & EU & FR & $\mathrm{LAC}$ & 43 & 5 & 1935 & 66 & $\mathrm{AA}$ & $\begin{array}{l}\text { Jean; French astronomer } \\
(1823-1873) \text {. }\end{array}$ \\
\hline Chadwick & $52.7 \mathrm{~S}$ & $101.3 \mathrm{~W}$ & 30 & $\mathrm{EU}$ & GB & & & 5 & 1985 & 0 & $\mathrm{AA}$ & $\begin{array}{l}\text { James; British physicist } \\
(1891-1974) \text {. }\end{array}$ \\
\hline Chaffee & $38.8 \mathrm{~S}$ & $153.9 \mathrm{~W}$ & 49 & NA & AM & $\mathrm{LOC}$ & 4 & 5 & 1970 & 0 & AA & $\begin{array}{l}\text { Roger B.; American } \\
\text { aeronautic engineer, } \\
\text { astronaut (1935-1967). }\end{array}$ \\
\hline Challis & $79.5 \mathrm{~N}$ & $9.2 \mathrm{E}$ & 55 & $\mathrm{EU}$ & GB & LMP & 3 & 5 & 1935 & 66 & AA & $\begin{array}{l}\text { James; British astronomer, } \\
\text { mathematician, physicist } \\
(1803-1862) \text {. }\end{array}$ \\
\hline Chalonge & $21.2 \mathrm{~S}$ & $117.3 \mathrm{~W}$ & 30 & EU & FR & & & 5 & 1985 & 0 & AA & $\begin{array}{l}\text { Daniel; French astronomer } \\
\text { (1895-1977). }\end{array}$ \\
\hline Chamberlin & $58.9 S$ & $95.7 \mathrm{E}$ & 58 & NA & $\mathrm{AM}$ & LMP & 3 & 5 & 1970 & 0 & AA & $\begin{array}{l}\text { Thomas C.; American } \\
\text { geologist (1843-1928). }\end{array}$ \\
\hline Champollion & $37.4 \mathrm{~N}$ & $175.2 \mathrm{E}$ & 58 & EU & FR & LOC & 4 & 5 & 1970 & 0 & AA & $\begin{array}{l}\text { Jean F.; French } \\
\text { egyptologist (1790-1832). }\end{array}$ \\
\hline Chandler & $43.8 \mathrm{~N}$ & $171.5 \mathrm{E}$ & 85 & NA & AM & LMP & 2 & 5 & 1970 & 0 & AA & $\begin{array}{l}\text { Seth C.; American } \\
\text { astronomer (1846-1913). }\end{array}$ \\
\hline Chang Heng & $19.0 \mathrm{~N}$ & $112.2 \mathrm{E}$ & 43 & AS & $\mathrm{CH}$ & LMP & 2 & 5 & 1970 & 0 & AA & $\begin{array}{l}\text { Chinese astronomer } \\
(78-139) .\end{array}$ \\
\hline $\begin{array}{l}\text { Chang-Ngo } \\
\text { Chant }\end{array}$ & $12.7 \mathrm{~S}$ & $2.1 \mathrm{~W}$ & 3 & AS & $\mathrm{CH} \mathrm{S}$ & 1 & 77 D3 & 5 & 1976 & 0 & AA & Chinese female name. \\
\hline & $40.0 s$ & $109.2 \mathrm{~W}$ & 33 & NA & CA & LMP & & 5 & 1970 & 0 & AA & $\begin{array}{l}\text { Clarence A.; Canadian } \\
\text { astronomer, physicist } \\
(1865-1956) \text {. }\end{array}$ \\
\hline Chaplygin & $6.2 \mathrm{~S}$ & $150.3 \mathrm{E}$ & 137 & UR & SO & LTO & 084B3 & 5 & 1970 & 0 & AA & $\begin{array}{l}\text { Sergej A.; Soviet } \\
\text { mathematician } \\
(1869-1942)\end{array}$ \\
\hline Chapman & $50.4 \mathrm{~N}$ & $100.7 \mathrm{~W}$ & 71 & EU & GB & LMP & 3 & 5 & 1970 & 0 & AA & $\begin{array}{l}\text { Sydney; British } \\
\text { geophysicist (1888-1970). }\end{array}$ \\
\hline Chappe & $61.2 \mathrm{~S}$ & $91.5 \mathrm{~W}$ & 59 & EU & FR & & & 5 & 1994 & 68 & AA & $\begin{array}{l}\text { d'Auteroche, } \\
\text { Jean-Baptiste; French } \\
\text { astronomer (1728-1769). }\end{array}$ \\
\hline Chappell & $54.7 \mathrm{~N}$ & $177.0 \mathrm{~W}$ & 80 & NA & $\mathrm{AM}$ & LMP & 3 & 5 & 1970 & 0 & AA & $\begin{array}{l}\text { James F.; American } \\
\text { astronomer (1891-1964). }\end{array}$ \\
\hline $\begin{array}{l}\text { Charles } \\
\text { Charlier }\end{array}$ & $\begin{array}{l}29.9 \mathrm{~N} \\
36.6 \mathrm{~N}\end{array}$ & $\begin{array}{r}26.4 \mathrm{~W} \\
131.5 \mathrm{~W}\end{array}$ & $\begin{array}{r}1 \\
99\end{array}$ & $\begin{array}{l}\text { EU } \\
\text { EU }\end{array}$ & $\begin{array}{l}\text { FR } \\
\text { SW }\end{array}$ & $\begin{array}{l}\text { S1 } \\
\text { LOC }\end{array}$ & $\begin{array}{l}40 \mathrm{Al} \\
4\end{array}$ & $\begin{array}{l}5 \\
5\end{array}$ & $\begin{array}{l}1976 \\
1970\end{array}$ & $\begin{array}{l}0 \\
0\end{array}$ & $\begin{array}{l}\mathrm{AA} \\
\mathrm{AA}\end{array}$ & $\begin{array}{l}\text { French male name. } \\
\text { Carl W. L.; Swedish } \\
\text { astronomer (1862-1934). }\end{array}$ \\
\hline Chaucer & $3.7 \mathrm{~N}$ & $140.0 \mathrm{~W}$ & 45 & EU & GB & LOC & 4 & 5 & 1970 & 0 & AA & $\begin{array}{l}\text { Geoffrey; British writer, } \\
\text { astronomer (c. 1340-1400). }\end{array}$ \\
\hline Chauvenet & $11.5 \mathrm{~S}$ & $137.0 \mathrm{E}$ & 81 & NA & $\mathrm{AM}$ & LOC & 4 & 5 & 1970 & 0 & AA & $\begin{array}{l}\text { William; American } \\
\text { astronomer, mathematician } \\
(1820-1870) \text {. }\end{array}$ \\
\hline Chebyshev & $33.7 \mathrm{~S}$ & $133.1 \mathrm{~W}$ & 178 & UR & RU & LOC & 4 & 5 & 1970 & 0 & AA & $\begin{array}{l}\text { Pafnutif L.; Russian } \\
\text { mathematician (1821-1894). }\end{array}$ \\
\hline [Chekov] & $6.6 \mathrm{~S}$ & $82.0 \mathrm{E}$ & 0 & UR F & $U$ & LTO & 81B4 & 6 & 0 & 0 & AA & $\begin{array}{l}\text { Anton Pavlovich; Russian } \\
\text { author (1860-1904). }\end{array}$ \\
\hline [Chenier] & $17.7 \mathrm{~S}$ & $132.4 \mathrm{E}$ & 33 & EU & FR & LTO & 10281 & 6 & 0 & 0 & AA & Andre Marie; French poet \\
\hline Chernyshev & $47.3 \mathrm{~N}$ & 174.2E & 58 & UR & SO & LMP & 2 & 5 & 1970 & 0 & AA & $\begin{array}{l}\text { Nikolaj G.; Soviet } \\
\text { rocketry engineer } \\
\text { (1906-1963). }\end{array}$ \\
\hline Chevallier & $44.9 \mathrm{~N}$ & $51.2 \mathrm{E}$ & 52 & EU & GB & LAC & 27 & 5 & 1935 & 66 & AA & $\begin{array}{l}\text { Temple; British astronomer } \\
\text { (1794-1873). }\end{array}$ \\
\hline $\begin{array}{l}\text { Ching-Te } \\
\text { Chladni }\end{array}$ & $\begin{array}{r}20.0 \mathrm{~N} \\
4.0 \mathrm{~N}\end{array}$ & $\begin{array}{r}30.0 \mathrm{E} \\
1.1 \mathrm{E}\end{array}$ & $\begin{array}{r}4 \\
13\end{array}$ & $\begin{array}{l}\text { AS } \\
\mathrm{EU}\end{array}$ & $\begin{array}{l}\mathrm{CH} \\
\mathrm{GE}\end{array}$ & $\begin{array}{l}\text { S3 } \\
\text { LAC }\end{array}$ & $\begin{array}{l}42 \mathrm{C} 3 \\
59\end{array}$ & $\begin{array}{l}5 \\
5\end{array}$ & $\begin{array}{l}1976 \\
1935\end{array}$ & $\begin{array}{r}0 \\
66\end{array}$ & $\begin{array}{l}\text { AA } \\
\text { AA }\end{array}$ & $\begin{array}{l}\text { Chinese male name. } \\
\text { Ernst Florens Friedrich; } \\
\text { German physicist } \\
(1756-1827) \text {. }\end{array}$ \\
\hline Chrétien & $45.9 \mathrm{~S}$ & $162.9 \mathrm{E}$ & 88 & EU & FR & LMP & 2 & 5 & 1970 & 0 & AA & $\begin{array}{l}\text { Chrétien, Henri; French } \\
\text { mathematician, astronomer } \\
(1870-1956) \text {. }\end{array}$ \\
\hline $\begin{array}{l}\text { Christel } \\
\text { Cichus }\end{array}$ & $\begin{array}{l}24.5 \mathrm{~N} \\
33.3 \mathrm{~S}\end{array}$ & $\begin{array}{l}11.0 \mathrm{E} \\
21.1 \mathrm{~W}\end{array}$ & $\begin{array}{r}2 \\
40\end{array}$ & $\begin{array}{l}\mathrm{EU} \\
\mathrm{EU}\end{array}$ & $\begin{array}{l}\text { GE } \\
\text { IT }\end{array}$ & $\begin{array}{l}\text { Sl } \\
\text { LAC }\end{array}$ & $\begin{array}{l}42 \mathrm{~A} 4 \\
111\end{array}$ & $\begin{array}{l}5 \\
5\end{array}$ & $\begin{array}{l}1976 \\
1935\end{array}$ & $\begin{array}{r}0 \\
66\end{array}$ & $\begin{array}{l}\text { AA } \\
\text { AA }\end{array}$ & $\begin{array}{l}\text { German female name. } \\
\text { Franceso Degli Stabili } \\
\text { (Cecco D'Ascoli); Italian } \\
\text { astronomer (1257-1327). }\end{array}$ \\
\hline
\end{tabular}

\footnotetext{
lat: latitude of feature center.

long: longitude of feature center.

et: ethnicity of name origin (see page $284 \mathrm{ff}$.)

quad: map quadrangle or informal name

(see page xvii ff.).

as: name approval status (see page $\mathrm{xvii}$.

diam: diameter or long dimension of feature.

ct: continent of name origin (see page $284 \mathrm{ff}$.) map: map name or USGS map number (see page xvii ff.). ft: feature type (see page 290).

ad: name approval date (year).

ref: reference source for name (see page $287 \mathrm{ff}$.)
} 


\section{MOON}

\begin{tabular}{|c|c|c|c|c|c|c|c|c|c|c|c|c|}
\hline Name & lat & long & diam & ct & et & quad & map & as & ad & ref & ft & origin \\
\hline Clairaut & $47.7 \mathrm{~S}$ & $13.9 \mathrm{E}$ & 75 & EU & FR & LAC & 113 & 5 & 1935 & 66 & $\mathrm{AA}$ & $\begin{array}{l}\text { Alexis Claude; French } \\
\text { mathematician } \\
(1713-1765) \text {. }\end{array}$ \\
\hline Clark & $38.4 \mathrm{~S}$ & $118.9 \mathrm{E}$ & 49 & NA & AM & LOC & 3 & 5 & 1970 & 0 & AA & $\begin{array}{l}\text { Alvan; American astronome } \\
\text { (1804-1887); Alvan G.; } \\
\text { American astronomer, } \\
\text { optician (1832-1897). }\end{array}$ \\
\hline Clausius & $36.9 \mathrm{~S}$ & $43.8 \mathrm{~W}$ & 24 & EU & $\mathrm{GE}$ & LAC & 110 & 5 & 1935 & 66 & AA & $\begin{array}{l}\text { Rudolf Julius Emmanuel; } \\
\text { German physicist } \\
(1822-1888) \text {. }\end{array}$ \\
\hline Clavius & $58.8 \mathrm{~S}$ & $14.1 \mathrm{~W}$ & 245 & EU & GE & LAC & 126 & 5 & 1935 & 66 & AA & $\begin{array}{l}\text { Christopher Klau; German } \\
\text { mathematician } \\
(1537-1612) \text {. }\end{array}$ \\
\hline Cleomedes & $27.7 \mathrm{~N}$ & $56.0 \mathrm{E}$ & 125 & EU & GR & LAC & 44 & 5 & 1935 & 66 & AA & $\begin{array}{l}\text { Greek astronomer (unkn-c. } \\
50 \text { B.C.). }\end{array}$ \\
\hline Cleostratus & $60.4 \mathrm{~N}$ & $77.0 \mathrm{~W}$ & 62 & EU & GR & LMP & 3 & 5 & 1935 & 66 & $\mathrm{AA}$ & $\begin{array}{l}\text { Greek astronomer (unkn-c. } \\
500 \text { B.C.). }\end{array}$ \\
\hline Clerke & $21.7 \mathrm{~N}$ & $29.8 \mathrm{E}$ & 6 & EU & GB & LTO & $042 \mathrm{C} 2$ & 5 & 1973 & 0 & AA & $\begin{array}{l}\text { Agnes Mary; British } \\
\text { astronomer (1842-1907). }\end{array}$ \\
\hline Coblentz & $37.9 \mathrm{~S}$ & 126.1E & 33 & NA & $\mathrm{AM}$ & LOC & 3 & 5 & 1970 & 0 & AA & $\begin{array}{l}\text { William W.; American } \\
\text { physicist, astronomer } \\
\text { (1873-1962). }\end{array}$ \\
\hline Cockcroft & $31.3 \mathrm{~N}$ & $162.6 \mathrm{~W}$ & 93 & EU & GB & LOC & 4 & 5 & 1970 & 0 & AA & $\begin{array}{l}\text { Sir John D.; British } \\
\text { nuclear physicist; Nobel } \\
\text { laureate (1897-1967). }\end{array}$ \\
\hline Collins & $1.3 \mathrm{~N}$ & $23.7 \mathrm{E}$ & 2 & NA & AM & LAC & 60 & 5 & 1970 & 0 & AA & $\begin{array}{l}\text { Michael; American } \\
\text { astronaut (1930-Live). }\end{array}$ \\
\hline Colombo & $15.1 \mathrm{~S}$ & $45.8 \mathrm{E}$ & 76 & $\mathrm{EU}$ & SP & LAC & 79 & 5 & 1935 & 66 & AA & $\begin{array}{l}\text { Columbus, Christopher; } \\
\text { Spanish explorer (c. } \\
1446-1506) \text {. }\end{array}$ \\
\hline Compton & $55.3 \mathrm{~N}$ & $103.8 \mathrm{E}$ & 182 & NA & $\mathrm{AM}$ & LMP & 3 & 5 & 1970 & 0 & AA & $\begin{array}{l}\text { Arthur H.; American } \\
\text { physicist, Nobel laureate } \\
\text { (1892-1962); Karl T.; } \\
\text { American physicist } \\
(1887-1954) \text {. }\end{array}$ \\
\hline Comrie & $23.3 \mathrm{~N}$ & $112.7 \mathrm{~W}$ & 59 & EU & GB & LOC & 1 & 5 & 1970 & 0 & AA & $\begin{array}{l}\text { Leslie J.; British } \\
\text { astronomer (1893-1950). }\end{array}$ \\
\hline Comstock & $21.8 \mathrm{~N}$ & $121.5 \mathrm{~W}$ & 72 & NA & AM & LOC & 1 & 5 & 1970 & 0 & AA & $\begin{array}{l}\text { George C.; American } \\
\text { astronomer (1855-1934). }\end{array}$ \\
\hline Condon & $1.9 \mathrm{~N}$ & $60.4 \mathrm{E}$ & 34 & NA & $\mathrm{AM}$ & LTO & $062 C 4$ & 5 & 1976 & 0 & AA & $\begin{array}{l}\text { Edward U.; American } \\
\text { physicist (1902-1974). }\end{array}$ \\
\hline Condorcet & $12.1 \mathrm{~N}$ & $69.6 \mathrm{E}$ & 74 & EU & FR & LTO & 062B2 & 5 & 1935 & 66 & AA & $\begin{array}{l}\text { Jean de; French } \\
\text { mathematician } \\
(1743-1794) \text {. }\end{array}$ \\
\hline Congreve & $0.2 S$ & $167.3 \mathrm{~W}$ & 57 & EU & GB & LOC & 4 & 5 & 1970 & 0 & AA & $\begin{array}{l}\text { Sir William; British } \\
\text { rocket engineer, inventor } \\
\text { (1772-1828). }\end{array}$ \\
\hline Conon & $21.6 \mathrm{~N}$ & $2.0 \mathrm{E}$ & 21 & EU & GR & LTO & $041 \mathrm{Cl}$ & 5 & 1935 & 66 & $\mathrm{AA}$ & $\begin{array}{l}\text { Conon of Samos; Greek } \\
\text { astronomer (c. } 260 \text { B.C.). }\end{array}$ \\
\hline Cook & $17.5 \mathrm{~S}$ & $48.9 \mathrm{E}$ & 46 & EU & GB & LAC & 97 & 5 & 1935 & 66 & AA & $\begin{array}{l}\text { James; British explorer } \\
(1728-1779)\end{array}$ \\
\hline Cooper & $52.9 \mathrm{~N}$ & $175.6 \mathrm{E}$ & 36 & NA & $\mathrm{AM}$ & LMP & 3 & 5 & 1970 & 0 & AA & $\begin{array}{l}\text { John C.; American } \\
\text { humanitarian (1887-1967). }\end{array}$ \\
\hline Copernicus & $9.7 \mathrm{~N}$ & $20.1 \mathrm{~W}$ & 107 & EU & PO & LAC & 58 & 5 & 1935 & 66 & $\mathrm{AA}$ & $\begin{array}{l}\text { Nicholas; Polish } \\
\text { astronomer (1473-1543). }\end{array}$ \\
\hline Cori & $50.6 \mathrm{~S}$ & $151.9 \mathrm{~W}$ & 65 & EU & $\mathrm{CZ}$ & LMP & 3 & 5 & 1979 & 0 & AA & $\begin{array}{l}\text { Gerty Theresa Radnitz; } \\
\text { Czech-American } \\
\text { physiologist; Nobel } \\
\text { laureate (1896-1957). }\end{array}$ \\
\hline Coriolis & $0.1 \mathrm{~N}$ & $171.8 \mathrm{E}$ & 78 & EU & FR & LOC & 4 & 5 & 1970 & 0 & $\mathrm{AA}$ & $\begin{array}{l}\text { Gaspard G. De; French } \\
\text { physicist (1792-1843). }\end{array}$ \\
\hline [Corneille] & $12.3 \mathrm{~N}$ & $134.8 \mathrm{E}$ & 35 & EU & FR & LTO & 084D4 & 6 & 0 & 0 & AA & $\begin{array}{l}\text { Pierre; French lieraure } \\
(1606-1684) \text {. }\end{array}$ \\
\hline Couder & $4.8 \mathrm{~S}$ & $92.4 \mathrm{~W}$ & 21 & $\mathrm{EU}$ & FR & & & 5 & 1985 & 0 & $\mathrm{AA}$ & $\begin{array}{l}\text { Andre; French astronomer } \\
\text { (1897-1978). }\end{array}$ \\
\hline Coulomb & $54.7 \mathrm{~N}$ & $114.6 \mathrm{~W}$ & 89 & EU & FR & LOC & 4 & 5 & 1970 & 0 & AA & $\begin{array}{l}\text { Charles A. De; French } \\
\text { physicist (1736-1806). }\end{array}$ \\
\hline $\begin{array}{l}\text { Courtney } \\
\text { Cremona }\end{array}$ & $\begin{array}{l}25.1 \mathrm{~N} \\
67.5 \mathrm{~N}\end{array}$ & $\begin{array}{l}30.8 \mathrm{~W} \\
90.6 \mathrm{~W}\end{array}$ & $\begin{array}{r}1 \\
85\end{array}$ & $\begin{array}{l}\text { EU } \\
\text { EU }\end{array}$ & $\begin{array}{l}\text { EN } \\
\text { IT }\end{array}$ & $\begin{array}{l}\text { S1 } \\
\text { LMP }\end{array}$ & $\begin{array}{l}39 \mathrm{~B} 3 \\
3\end{array}$ & $\begin{array}{l}5 \\
5\end{array}$ & $\begin{array}{l}1976 \\
1964\end{array}$ & $\begin{array}{r}0 \\
67\end{array}$ & $\begin{array}{l}\text { AA } \\
\text { AA }\end{array}$ & $\begin{array}{l}\text { English male name. } \\
\text { Luigi; Italian } \\
\text { mathematician } \\
(1830-1903) .\end{array}$ \\
\hline
\end{tabular}

\footnotetext{
lat: latitude of feature center.

long: longitude of feature center.

diam: diameter or long dimension of feature.

et: ethnicity of name origin (see page $284 \mathrm{ff}$.)

quad: map quadrangle or informal name

(see page xvii ff.).

as: name approval status (see page $\mathrm{xvii}$ ).

ad: name approval date (year).

ref: reference source for name (see page $287 \mathrm{ff}$.).

ct: continent of name origin (see page $284 \mathrm{ff}$.) map: map name or USGS map number (see page xvii ff.). ft: feature type (see page 290).
} 


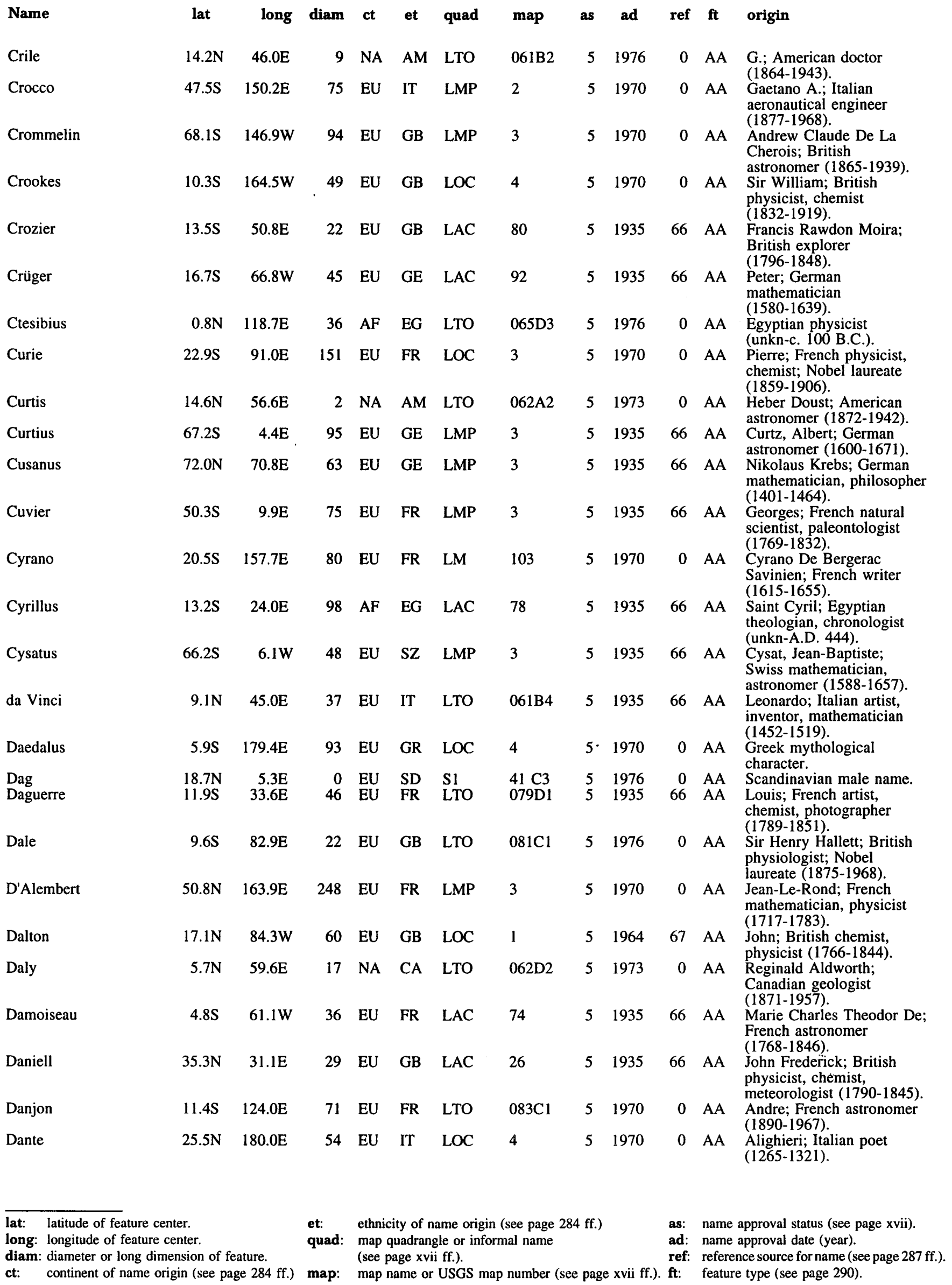




\section{MOON}

\begin{tabular}{|c|c|c|c|c|c|c|c|c|c|c|c|c|}
\hline Name & lat & long & diam & ct & et & quad & map & as & ad & ref & $\mathrm{ft}$ & origin \\
\hline [Dario] & $11.3 \mathrm{~S}$ & $90.7 \mathrm{E}$ & 19 & SA & NI & LTO & $82 \mathrm{D} 1$ & 6 & 0 & 0 & AA & $\begin{array}{l}\text { Ruben; Nicaraguan author } \\
1867-1916\end{array}$ \\
\hline Darney & $14.5 \mathrm{~S}$ & $23.5 \mathrm{~W}$ & 15 & EU & FR & LAC & 76 & 5 & 1935 & 66 & AA & $\begin{array}{l}\text { Maurice; French astronomer } \\
(1882-1958) \text {. }\end{array}$ \\
\hline D'Arrest & $2.3 \mathrm{~N}$ & $14.7 \mathrm{E}$ & 30 & $\mathrm{EU}$ & GE & LAC & 60 & 5 & 1935 & 66 & AA & $\begin{array}{l}\text { Heinrich Ludwig; German } \\
\text { astronomer (1822-1875). }\end{array}$ \\
\hline D'Arsonval & $10.3 \mathrm{~S}$ & $124.6 \mathrm{E}$ & 28 & $\mathrm{EU}$ & FR & LTO & $083 \mathrm{C} 1$ & 5 & 1976 & 68 & AA & $\begin{array}{l}\text { Jacques Arsène; French } \\
\text { physicist (1851-1940). }\end{array}$ \\
\hline Darwin & $20.2 S$ & $69.5 \mathrm{~W}$ & 120 & EU & GB & LAC & 92 & 5 & 1935 & 66 & AA & $\begin{array}{l}\text { Charles; British natural } \\
\text { scientist (1809-1882). }\end{array}$ \\
\hline Das & $26.6 S$ & $136.8 \mathrm{~W}$ & 38 & AS & IN & LOC & 1 & 5 & 1970 & 0 & AA & $\begin{array}{l}\text { Amil K.; Indian astronomer } \\
(1902-1961) .\end{array}$ \\
\hline Daubrée & $15.7 \mathrm{~N}$ & $14.7 \mathrm{E}$ & 14 & $\mathrm{EU}$ & FR & LTO & $060 \mathrm{~A} 1$ & 5 & 1973 & 0 & AA & $\begin{array}{l}\text { Gabriel-Auguste; French } \\
\text { geologist (1814-1896). }\end{array}$ \\
\hline Davisson & $37.5 \mathrm{~S}$ & $174.6 \mathrm{~W}$ & 87 & NA & $\mathrm{AM}$ & LOC & 4 & 5 & 1970 & 0 & AA & $\begin{array}{l}\text { Clinton J.; American } \\
\text { physicist; Nobel laureate } \\
\text { (1881-1958). }\end{array}$ \\
\hline Davy & $11.8 \mathrm{~S}$ & $8.1 \mathrm{~W}$ & 34 & EU & GB & LTO & 077D1 & 5 & 1935 & 55 & AA & $\begin{array}{l}\text { Humphry; British physicist } \\
(1778-1829) \text {. }\end{array}$ \\
\hline Dawes & $17.2 \mathrm{~N}$ & $26.4 \mathrm{E}$ & 18 & $\mathrm{EU}$ & GB & LTO & $042 C 3$ & 5 & 1935 & 66 & AA & $\begin{array}{l}\text { William Rutter; British } \\
\text { astronomer (1799-1868). }\end{array}$ \\
\hline Dawson & $67.4 \mathrm{~S}$ & $134.7 \mathrm{~W}$ & 45 & SA & $\mathrm{AR}$ & LMP & 3 & 5 & 1970 & 0 & AA & $\begin{array}{l}\text { Bernhard H.; Argentinian } \\
\text { astronomer (1890-1960). }\end{array}$ \\
\hline De Forest & $77.3 \mathrm{~S}$ & $162.1 \mathrm{~W}$ & 57 & NA & AM & LMP & 3 & 5 & 1970 & 0 & AA & $\begin{array}{l}\text { Lee; American inventor } \\
(1873-1961) \text {. }\end{array}$ \\
\hline de Gasparis & $25.9 \mathrm{~S}$ & $50.7 \mathrm{~W}$ & 30 & $\mathrm{EU}$ & IT & $\mathrm{LAC}$ & 92 & 5 & 1935 & 66 & AA & $\begin{array}{l}\text { Annibale; Italian } \\
\text { astronomer (1819-1892). }\end{array}$ \\
\hline De La Rue & $59.1 \mathrm{~N}$ & $52.3 \mathrm{E}$ & 134 & EU & GB & LMP & 3 & 5 & 1935 & 66 & AA & $\begin{array}{l}\text { Warren; British astronomer } \\
(1815-1889) \text {. }\end{array}$ \\
\hline De Moraes & $49.5 \mathrm{~N}$ & $143.2 \mathrm{E}$ & 53 & SA & BR & LMP & 3 & 5 & 1979 & 0 & AA & $\begin{array}{l}\text { A.; Brazilian astronomer } \\
(1916-1970)\end{array}$ \\
\hline De Morgan & $3.3 \mathrm{~N}$ & $14.9 \mathrm{E}$ & 10 & EU & GB & $\mathrm{LAC}$ & 60 & 5 & 1935 & 66 & AA & $\begin{array}{l}\text { Augustus; British } \\
\text { mathematician } \\
\text { (1806-1871). }\end{array}$ \\
\hline De Roy & $55.3 \mathrm{~S}$ & $99.1 \mathrm{~W}$ & 43 & EU & $\mathrm{BE}$ & LMP & 3 & 5 & 1970 & 0 & AA & $\begin{array}{l}\text { Felix; Belgian astronomer } \\
\text { (1883-1942). }\end{array}$ \\
\hline De Vico & $80.1 \mathrm{~N}$ & $39.0 \mathrm{E}$ & 64 & $\mathrm{EU}$ & DU & LMP & 3 & 5 & 1964 & 67 & AA & $\begin{array}{l}\text { Willem; Dutch astronomer } \\
\text { (1872-1934). }\end{array}$ \\
\hline De Vries & $19.9 \mathrm{~S}$ & $176.7 \mathrm{~W}$ & 59 & EU & DU & LAC & 92 & 5 & 1970 & 60 & AA & $\begin{array}{l}\text { Francesco; Italian } \\
\text { astronomer ( } 1805-1848) \text {. } \\
\text { Hugo M.: Dutch botanist }\end{array}$ \\
\hline Debes & $29.5 \mathrm{~N}$ & $51.7 \mathrm{E}$ & 30 & $\mathrm{EU}$ & GE & LAC & 44 & 5 & 1961 & 67 & AA & \\
\hline Debye & $49.6 \mathrm{~N}$ & $176.2 \mathrm{~W}$ & 142 & EU & DU & LMP & 3 & 5 & 1970 & 0 & AA & $\begin{array}{l}\text { cartographer (1840-1923). } \\
\text { Peter J. W.; Dutch } \\
\text { physicist, chemist; Nobel } \\
\text { laureate (1884-1966). }\end{array}$ \\
\hline Dechen & $46.1 \mathrm{~N}$ & $68.2 \mathrm{~W}$ & 12 & EU & GE & LMP & 3 & 5 & 1935 & 66 & AA & $\begin{array}{l}\text { Ernst Heinrich Karl von; } \\
\text { German geologist, } \\
\text { mineralogist (1800-1889). }\end{array}$ \\
\hline [Defoe] & $6.0 \mathrm{~S}$ & $80.5 \mathrm{E}$ & 18 & EU & GB & LTO & 081B4 & 6 & 0 & 0 & AA & $\begin{array}{l}\text { Daniel; British author (c. } \\
\text { 1661-1731). }\end{array}$ \\
\hline Delambre & $1.9 \mathrm{~S}$ & $17.5 \mathrm{E}$ & 51 & $\mathrm{EU}$ & FR & LAC & 78 & 5 & 1935 & 66 & AA & $\begin{array}{l}\text { Jean-Baptiste Joseph; } \\
\text { French astronomer } \\
\text { (1749-1822). }\end{array}$ \\
\hline Delaunay & $22.2 \mathrm{~S}$ & $2.5 \mathrm{E}$ & 46 & EU & FR & LAC & 95 & 5 & 1935 & 66 & $\mathrm{AA}$ & $\begin{array}{l}\text { Charles Eugene; French } \\
\text { astronomer (1816-1872). }\end{array}$ \\
\hline $\begin{array}{l}\text { Delia } \\
\text { Delisle }\end{array}$ & $\begin{array}{l}10.9 \mathrm{~S} \\
29.9 \mathrm{~N}\end{array}$ & $\begin{array}{r}6.1 \mathrm{~W} \\
34.6 \mathrm{~W}\end{array}$ & $\begin{array}{r}2 \\
25\end{array}$ & $\begin{array}{l}\mathrm{EU} \\
\mathrm{EU}\end{array}$ & $\begin{array}{l}\text { GR } \\
\text { FR }\end{array}$ & $\begin{array}{l}\text { S1 } \\
\text { LTO }\end{array}$ & $\begin{array}{l}77 \mathrm{D} 1 \\
039 \mathrm{~B} 1\end{array}$ & $\begin{array}{l}5 \\
5\end{array}$ & $\begin{array}{l}1976 \\
1935\end{array}$ & $\begin{array}{r}0 \\
66\end{array}$ & $\begin{array}{l}\text { AA } \\
\text { AA }\end{array}$ & $\begin{array}{l}\text { Greek female name. } \\
\text { Joseph Nicolas; French } \\
\text { astronomer (1688-1768). }\end{array}$ \\
\hline Dellinger & $6.8 \mathrm{~S}$ & $140.6 \mathrm{E}$ & 81 & NA & $\mathrm{AM}$ & LOC & 4 & 5 & 1970 & 0 & $\mathrm{AA}$ & $\begin{array}{l}\text { John H.; American } \\
\text { physicist (1886-1962). }\end{array}$ \\
\hline Delmotte & $27.1 \mathrm{~N}$ & $60.2 \mathrm{E}$ & 32 & EU & FR & LAC & 44 & 5 & 1935 & 66 & AA & $\begin{array}{l}\text { Gabriel; French astronomer } \\
(1876-1950) .\end{array}$ \\
\hline Delporte & $16.0 \mathrm{~S}$ & $121.6 \mathrm{E}$ & 45 & $\mathrm{EU}$ & $\mathrm{BE}$ & LTO & $083 \mathrm{C} 4$ & 5 & 1970 & 0 & AA & $\begin{array}{l}\text { Eugene J.; Belgian } \\
\text { astronomer (1882-1955). }\end{array}$ \\
\hline Deluc & $55.0 \mathrm{~S}$ & $2.8 \mathrm{~W}$ & 46 & EU & SZ & LMP & 3 & 5 & 1935 & 66 & AA & $\begin{array}{l}\text { Jean Andre; Swiss } \\
\text { geologist, physicist } \\
(1727-1817) \text {. }\end{array}$ \\
\hline Dembowski & $2.9 \mathrm{~N}$ & $7.2 \mathrm{E}$ & 26 & $\mathrm{EU}$ & IT & LAC & 60 & 5 & 1935 & 66 & AA & $\begin{array}{l}\text { Baron Ercole; Italian } \\
\text { astronomer (1815-1881). }\end{array}$ \\
\hline
\end{tabular}

lat: latitude of feature center. long: longitude of feature center.

diam: diameter or long dimension of feature. et: ethnicity of name origin (see page $284 \mathrm{ff}$.)

quad: map quadrangle or informal name (see page xvii ff.). as: name approval status (see page xvii).

ad: name approval date (year).

ref: reference source for name (see page $287 \mathrm{ff}$.). 


\section{MOON}

Name

Democritus

lat

$62.3 \mathrm{~N}$

Demonax

Denning

Desargues

$16.4 \mathrm{~S} \quad 142.6 \mathrm{E}$

$70.2 \mathrm{~N} \quad 73.3 \mathrm{~W}$

ong

diam ct et quad

map

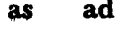

$5 \quad 1935$

66 AA

51935

128 EU GR LMP 3

44 EU GB LOC 3

$5 \quad 1970$

$5 \quad 1964$

0 AA

85 EU FR LMP 3

11.7S $\quad 15.7 \mathrm{E}$

48

EU FR LTO

078D2

51935

$67 \mathrm{AA}$

Descarte

Deseilligny

$21.1 \mathrm{~N}$

20.6E

6 EU FR LTO

042C1

51935

Deslandres

33.1

$4.8 \mathrm{~W}$

256

$24.1 \mathrm{~N} \quad 110.5 \mathrm{E}$

Deutsch

Dewar

Diana

Diderot

Dionysius

2.7S $\quad 165.5 \mathrm{E}$

$14.3 \mathrm{~N} \quad 35.7 \mathrm{E}$

$20.4 \mathrm{~S} \quad 121.5 \mathrm{E}$

$2.8 \mathrm{~N} \quad 17.3 \mathrm{E}$

66 NA AM LOC

50 EU GB LOC

50 EU LA S1

20 EU FR LTO

$61 \mathrm{~A} 2$

101B4

18 EU GR LAC

60

$27.6 \mathrm{~N} \quad 34.3 \mathrm{~W}$

17 EU GR LTO

039B3

$11.1 \mathrm{~N} \quad 151.4 \mathrm{~W}$

47 EU GE LOC

1

$12.8 \mathrm{~S} \quad 129.7 \mathrm{E}$

38 UR

UR

LTO

$083 \mathrm{C} 3$

69.1S 107.9W

68 EU GE

Doerfel

10.4

$14.4 \mathrm{E}$

11 EU GB LTO

078D1

51985

Dollond

Donati

Donna

Donner

Doppelmayer

$20.7 \mathrm{~S}$

$$
5.2 \mathrm{E}
$$$$
36 \text { EU IT }
$$

$7.2 \mathrm{~N} \quad 38.3 \mathrm{E}$

31.4S $98.0 \mathrm{E}$

2 EU IT S1 61 D2

$28.5 \mathrm{~S}$

41.4W

58 EU

63 EU

$12.6 \mathrm{~S} \quad 159.6 \mathrm{~W}$

$110 \mathrm{E}$

Doppler

$12.6 \mathrm{~S}$

.

$35.9 \mathrm{~N} \quad 122.4 \mathrm{~W}$

49 NA AM LOC

1

46.7S $31.5 \mathrm{E}$

30 EU GE LAC

113

$$
2.0 \mathrm{~N}
$$

$84.5 \mathrm{E}$

$17.6 \mathrm{~N}$

$21.7 \mathrm{~W}$

32 EU GB LTO

$063 C 4$

Draper

Drebbel

$40.9 \mathrm{~S}$

$49.0 \mathrm{~W}$

8 NA AM LAC

4

30 EU DU LAC 110

Dreyer

$10.0 \mathrm{~N}$

96.9E

61 EU GB LOC

3

$38.5 \mathrm{~S} \quad 91.8 \mathrm{~W}$

$33.0 \mathrm{~S} \quad 155.2 \mathrm{~W}$

24 EU GE LOC

51 NA AM LOC

$79.3 \mathrm{~S} \quad 84.9 \mathrm{~W}$

149 EU GE LMP 3
51935

$5 \quad 1935$

$5 \quad 1979$

$5 \quad 1970$

51935

$5 \quad 1970$

$5 \quad 1970$

$5 \quad 1935$

0 AA

$66 \mathrm{AA}$

60

0 AA

51935

$66 \mathrm{AA}$

51935

51970

66 AA

0 AA

$5 \quad 1970$

0 AA

51970

51964
0 AA

$67 \mathrm{AA}$
Greek astronomer

philosopher (c. 460-360

B.C.).

Greek philosopher (unkn-c.

100 B.C.).

William F; British

astronomer (1848-1931).

Gerard; French

mathematician, engineer (1593-1662).

mathematician, philosopher (1596-1650).

Jules Alfred Pierrot;

French selenographer (1868-1918).

Henri Alexandre; French astrophysicist (1853-1948)

Armin J.; American astronomer (1918-1969).

Sir James; British chemist (1842-1923).

Latin female name

Denis; French philosopher (1713-1784).

St. Dionysius the Areopagite; Greek astronomer (A.D. 9-120).

Greek mathematician (unkn-c. A.D. 300).

Peter G. L.; German

mathematician (1805-1859).

Georgiy T.; Soviet aeronautical engineer (1928-1971).

Georg Samuel; German astronomer (1643-1688)

John; British optician (1706-1761)

Giovanni Battista; Italian astronomer (1826-1873).

Italian female name.

Anders; Finnish astronomer (1873-1949)

Johann Gabriel; German mathematician, astronomer (1671-1750).

Doppler, J. Christian; Austrian physicist, mathematician, astronomer (1803-1853).

Andrew E.; American astronomer (1867-1962)

Heinrich Wilhelm; German physicist (1803-1879).

Sir Arthur Conan; British novelist (1859-1930).

Henry; American astronomer (1837-1882).

Cornelius; Dutch inventor (1572-1634)

John L. E.; British astronomer (1852-1926).

Paul K. L.; German physicist (1863-1906).

Hugh L.; American physicist, engineer (1898-1965).

Erich D. von; German geographer, geophysicist (1865-1949). lat: latitude of feature center. long: longitude of feature center.

diam: diameter or long dimension of feature. et: ethnicity of name origin (see page $284 \mathrm{ff}$.)

quad: map quadrangle or informal name (see page xvii ff.). as: name approval status (see page xvii).

ad: name approval date (year).

ref: reference source for name (see page $287 \mathrm{ff}$.).

ct: continent of name origin (see page $284 \mathrm{ff}$ ) map: map name or USGS map number (see page xvii ff.). ft: feature type (see page 290). 


\section{MOON}

Name

Dubyago

Dufay

Dugan

[Dumas]

Dunér

Dunthorne

Dyson

Dziewulski

Eckert

Eddington

Edison
Edith
Egede

Ehrlich

Eichstadt

Eijkman

Eimmart

Einstein

Einthoven

[El Greco]

Elger

Ellerman

Ellison

Elmer

Elvey

Emden

Encke

Endymion

28 E

$53.9 \mathrm{~N} \quad 57.0 \mathrm{E}$

123 EU GR LMP 3

43 UR SO LOC 4

Engel'gardt (Engelhardt)

Eötvös

99 EU HU LOC 4

5.3S 81.7E

16 EU FR LTO

$35.3 \mathrm{~S} \quad 29.8 \mathrm{~W}$ map as ad ref $\mathrm{ft}$ origin

062C2 $51964 \quad 0 \quad$ AA Dmitrij I.; Russian

astronomer (1850-1918)

Alexander D.; Soviet

astronomer (1903-1959)

$\begin{array}{llllllllllll}5.5 \mathrm{~N} & 169.5 \mathrm{E} & 39 & \mathrm{EU} & \mathrm{FR} & \text { LOC } & 4 & 5 & 1970 & 0 & \text { AA } & \text { Jean C. B.; French }\end{array}$

$\begin{array}{lllllllll}64.2 \mathrm{~N} & 103.3 \mathrm{E} & 50 & \text { NA } & \text { AM } & \text { LMP } & 3 & 5 & 1970\end{array}$

0 AA

astronomer (1896-1967)

Raymond S.; American

astronomer (1878-1940)

081B4

60

0 AA

(1802-1870)

$5 \quad 1970$

$0 \quad$ AA

Nils C.; Swedish

astronomer (1839-1914)

5193566 AA Richard; British

$5 \quad 1970$

$5 \quad 1970$

0 AA

astronomer (1711-1775)

Sir Frank W.; British astronomer (1868-1939)

0 AA Wladysiaw; Polish astronomer (1878-1962).

$5 \quad 1973$

$0 \quad$ AA

Wallace J.; American

astronomer (1902-1971)

$5 \quad 1964$

67 AA

Sir Arthur S.; British

astrophysicist,

mathematician

(1882-1944).

$51961 \quad 67$ AA Thomas A.; American inventor (1847-1931).

$51976 \quad 0 \quad$ AA English female name.

$5 \quad 1935 \quad 66$ AA

Hans; Danish natural

historian (1686-1758).

$51970 \quad 0 \quad$ AA Paul; German doctor; Nobel

laureate (1854-1915).

5193566 AA Lorentz; German

mathematician, astronomer (1596-1660).

$51970 \quad 0 \quad$ AA Christiaan H.; Dutch

doctor; Nobel laureate (1858-1930).

5193566 AA Georg Christoph; German astronomer (1638-1705).

5196467 AA Albert; German-American physicist; Nobel laureate (1879-1955).

$51970 \quad 0 \quad$ AA Willem; Dutch

physiologist; Nobel laureate (1879-1955)

Spanish artist, born in Crete (c. 1541-1614).

21 EU GB LAC 111

$5 \quad 1935$

0 AA

Thomas Gwyn; British

astronomer (1838-1897)

$5 \quad 1970$

Ferdinand; American

astronomer (1869-1940).

$5 \quad 1970$

51976

0 AA

Mervyn A.; British astronomer (1909-1963)

0 AA Charles W.; American

astronomer (1872-1954).

$5 \quad 1970$

0 AA

Christian T.; American astronomer, geophysicist (1899-1970).

$51970 \quad 0 \quad$ AA J. Robert; Swiss astrophysicist, meteorologist (1862-1940)

Johann Franz; German mathematician, astronomer (1791-1865)

Greek mythological character.

$5 \quad 1970$

Vasilij P.; Soviet astronomer (1828-1915).

$51970 \quad 0$ AA Roland von; Hungarian physicist (1848-1919). lat: latitude of feature center.

long: longitude of feature center.

diam: diameter or long dimension of feature.

et: ethnicity of name origin (see page $284 \mathrm{ff}$.)

quad: map quadrangle or informal name

(see page xvii ff.).

map name or USGS map number (see page xvii ff.). as: name approval status (see page xvii).

ad: name approval date (year).

ref: reference source for name (see page $287 \mathrm{ff}$.). 


\section{MOON}

\begin{tabular}{|c|c|c|c|c|c|c|c|c|c|c|c|c|}
\hline Name & lat & long & diam & ct & et & quad & map & as & ad & ref & $\mathrm{ft}$ & origin \\
\hline Epigenes & $67.5 \mathrm{~N}$ & $4.6 \mathrm{~W}$ & 55 & EU & GR & LMP & 3 & 5 & 1935 & 66 & $\mathbf{A A}$ & $\begin{array}{l}\text { Greek astronomer (unkn-c. } \\
200 \text { B.C.). }\end{array}$ \\
\hline Epimenides & $40.9 S$ & $30.2 \mathrm{~W}$ & 27 & $\mathrm{EU}$ & GR & LAC & 111 & 5 & 1935 & 66 & $\mathbf{A A}$ & $\begin{array}{l}\text { Greek philosopher, writer } \\
\text { (unkn-fl. } 596 \text { B.C.). }\end{array}$ \\
\hline Eppinger & $9.4 \mathrm{~S}$ & $25.7 \mathrm{~W}$ & 6 & EU & $\mathrm{CZ}$ & LTO & 076D1 & 5 & 1976 & 0 & $\mathbf{A A}$ & $\begin{array}{l}\text { H.; Czechoslovakian doctor } \\
(1879-1946) \text {. }\end{array}$ \\
\hline Eratosthenes & $14.5 \mathrm{~N}$ & $11.3 \mathrm{~W}$ & 58 & EU & GR & LAC & 58 & 5 & 1935 & 66 & AA & $\begin{array}{l}\text { Greek astronomer, } \\
\text { geographer (c. } 276-196 \\
\text { B.C.). }\end{array}$ \\
\hline Erro & $5.7 \mathrm{~N}$ & $98.5 \mathrm{E}$ & 61 & NA & ME & LTO & 064D2 & 5 & 1970 & 0 & AA & $\begin{array}{l}\text { Luis E.; Mexican } \\
\text { astronomer (1897-1955). }\end{array}$ \\
\hline Esclangon & $21.5 \mathrm{~N}$ & $42.1 \mathrm{E}$ & 15 & EU & FR & LTO & $043 \mathrm{C} 1$ & 5 & 1976 & $\mathbf{0}$ & AA & $\begin{array}{l}\text { Ernest Benjamin; French } \\
\text { astronomer (1876-1954). }\end{array}$ \\
\hline Esnault-Pelterie & $47.7 \mathrm{~N}$ & $141.4 \mathrm{~W}$ & 79 & EU & FR & LOC & 1 & 5 & 1970 & 0 & AA & $\begin{array}{l}\text { Robert A. C.; French } \\
\text { rocketry engineer } \\
(1881-1957) \text {. }\end{array}$ \\
\hline Espin & $28.1 \mathrm{~N}$ & 109.1E & 75 & EU & GB & LOC & 3 & 5 & 1970 & 0 & AA & $\begin{array}{l}\text { Thomas H. E. C.; British } \\
\text { astronomer (1858-1934). }\end{array}$ \\
\hline Euclides & $7.4 \mathrm{~S}$ & $29.5 \mathrm{~W}$ & 11 & EU & GR & LAC & 76 & 5 & 1935 & 66 & AA & $\begin{array}{l}\text { Euclid; Greek } \\
\text { mathematician (unkn-c. } 300 \\
\text { B.C.). }\end{array}$ \\
\hline Euctemon & $76.4 \mathrm{~N}$ & $31.3 \mathrm{E}$ & 62 & EU & GR & LMP & 3 & 5 & 1935 & 66 & AA & $\begin{array}{l}\text { Greek astronomer (unkn-fl. } \\
432 \text { B.C.). }\end{array}$ \\
\hline Eudoxus & $44.3 \mathrm{~N}$ & $16.3 \mathrm{E}$ & 67 & EU & GR & LAC & 26 & 5 & 1935 & 66 & AA & $\begin{array}{l}\text { Greek astronomer (c. } \\
\text { 408-355 B.C.). }\end{array}$ \\
\hline Euler & $23.3 \mathrm{~N}$ & $29.2 \mathrm{~W}$ & 27 & $\mathrm{EU}$ & SZ & LTO & 040D1 & 5 & 1935 & 66 & AA & $\begin{array}{l}\text { Leonhard; Swiss } \\
\text { mathematician } \\
\text { (1707-1783). }\end{array}$ \\
\hline Evans & $9.5 \mathrm{~S}$ & $133.5 \mathrm{~W}$ & 67 & EU & GB & LOC & 4 & 5 & 1970 & 0 & AA & $\begin{array}{l}\text { Sir Arthur; British } \\
\text { archaeologist } \\
(1851-1941)\end{array}$ \\
\hline Evdokimov & $34.8 \mathrm{~N}$ & $153.0 \mathrm{~W}$ & 50 & UR & SO & LOC & 4 & 5 & 1970 & 0 & AA & $\begin{array}{l}\text { Nikolaj N.; Soviet } \\
\text { astronomer (1868-1940). }\end{array}$ \\
\hline Evershed & $35.7 \mathrm{~N}$ & $159.5 \mathrm{~W}$ & 66 & EU & GB & LOC & 4 & 5 & 1970 & 0 & AA & $\begin{array}{l}\text { John; British astronomer } \\
\text { (1864-1956). }\end{array}$ \\
\hline $\begin{array}{l}\text { Ewen } \\
\text { Fabbroni }\end{array}$ & $\begin{array}{r}7.7 \mathrm{~N} \\
18.7 \mathrm{~N}\end{array}$ & $\begin{array}{r}121.4 \mathrm{E} \\
29.2 \mathrm{E}\end{array}$ & $\begin{array}{r}3 \\
10\end{array}$ & $\begin{array}{l}\mathrm{EU} \\
\mathrm{EU}\end{array}$ & $\begin{array}{l}\text { IR } \\
\text { IT }\end{array}$ & $\begin{array}{l}\text { S2 } \\
\text { LTO }\end{array}$ & $\begin{array}{l}65 \mathrm{C} 1 \\
042 \mathrm{C} 3\end{array}$ & $\begin{array}{l}5 \\
5\end{array}$ & $\begin{array}{l}1979 \\
1976\end{array}$ & $\begin{array}{l}0 \\
0\end{array}$ & $\begin{array}{l}\text { AA } \\
\text { AA }\end{array}$ & $\begin{array}{l}\text { Gaelic male name. } \\
\text { Giovanni Valentino Mattia; } \\
\text { Italian chemist (1752-1822). }\end{array}$ \\
\hline Fabricius & $42.9 \mathrm{~S}$ & $42.0 \mathrm{E}$ & 78 & EU & DU & LAC & 114 & 5 & 1935 & 66 & AA & $\begin{array}{l}\text { Goldschmidt, David; Dutch } \\
\text { astronomer (1564-1617). }\end{array}$ \\
\hline Fabry & $42.9 \mathrm{~N}$ & $100.7 \mathrm{E}$ & 184 & EU & FR & LOC & 3 & 5 & 1970 & 0 & AA & Charles; French physicist \\
\hline Fahrenheit & $13.1 \mathrm{~N}$ & $61.7 \mathrm{E}$ & 6 & EU & DU & LTO & 062B1 & 5 & 1976 & 0 & AA & $\begin{array}{l}\text { Gabriel Daniel; Dutch } \\
\text { physicist (1686-1736). }\end{array}$ \\
\hline $\begin{array}{l}\text { Fairouz } \\
\text { Falcon }\end{array}$ & $\begin{array}{l}26.1 \mathrm{~S} \\
20.4 \mathrm{~N}\end{array}$ & $\begin{array}{r}102.9 \mathrm{E} \\
30.3 \mathrm{E}\end{array}$ & $\begin{array}{l}3 \\
0\end{array}$ & $\begin{array}{l}\text { AS } \\
\text { NA }\end{array}$ & $\begin{array}{l}\text { AR } \\
\text { AM }\end{array}$ & $\begin{array}{l}\text { S1 } \\
\text { S1 }\end{array}$ & $\begin{array}{l}100 \mathrm{Cl} \\
43 \mathrm{D} 1\end{array}$ & $\begin{array}{l}5 \\
5\end{array}$ & $\begin{array}{l}1976 \\
1973\end{array}$ & $\begin{array}{l}0 \\
0\end{array}$ & $\begin{array}{l}\text { AA } \\
\text { AA }\end{array}$ & $\begin{array}{l}\text { Arab female name. } \\
\text { Astronaut-named feature, } \\
\text { Apollo } 17 \text { site. }\end{array}$ \\
\hline Faraday & $42.4 \mathrm{~S}$ & $8.7 \mathrm{E}$ & 69 & EU & GB & LAC & 112 & 5 & 1935 & 66 & AA & $\begin{array}{l}\text { Michael; British chemist, } \\
\text { physicist (1791-1867). }\end{array}$ \\
\hline Faustini & $87.3 \mathrm{~S}$ & $77.0 \mathrm{E}$ & 39 & $\mathrm{EU}$ & IT & & & 5 & 1994 & 0 & AA & $\begin{array}{l}\text { Arnoldo; Italian polar } \\
\text { geographer (1874-1944). }\end{array}$ \\
\hline Fauth & $6.3 \mathrm{~N}$ & $20.1 \mathrm{~W}$ & 12 & EU & GE & LAC & 58 & 5 & 1935 & 68 & AA & $\begin{array}{l}\text { Philipp Johann Heinrich; } \\
\text { German selenographer } \\
\text { (1867-1941). }\end{array}$ \\
\hline Faye & $21.4 \mathrm{~S}$ & $3.9 \mathrm{E}$ & 36 & EU & FR & LAC & 95 & 5 & 1935 & 66 & AA & $\begin{array}{l}\text { Herve; French astronomer } \\
(1814-1902) \text {. }\end{array}$ \\
\hline Fechner & $59.0 S$ & $124.9 \mathrm{E}$ & 63 & EU & GE & LMP & 3 & 5 & 1970 & 0 & AA & $\begin{array}{l}\text { Gustav T.; German } \\
\text { physicist, psychologist } \\
\text { (1801-1887). }\end{array}$ \\
\hline Fedorov & $28.2 \mathrm{~N}$ & $37.0 \mathrm{~W}$ & 6 & UR & RU & LTO & 039B 1 & 5 & 1979 & 0 & AA & $\begin{array}{l}\text { A.P.; Russian rocket } \\
\text { scientist }(1872-1920) \text {. }\end{array}$ \\
\hline $\begin{array}{l}\text { Felix } \\
\text { Fényi }\end{array}$ & $\begin{array}{l}28.2 \mathrm{~N} \\
44.9 \mathrm{~S}\end{array}$ & $\begin{array}{r}37.0 \mathrm{~W} \\
105.1 \mathrm{~W}\end{array}$ & $\begin{array}{r}6 \\
38\end{array}$ & $\begin{array}{l}\text { EU } \\
\text { EU }\end{array}$ & $\begin{array}{l}\text { LA } \\
\text { HU }\end{array}$ & $\begin{array}{l}\text { S1 } \\
\text { LMP }\end{array}$ & $\begin{array}{l}40 \mathrm{~A} 4 \\
2\end{array}$ & $\begin{array}{l}5 \\
5\end{array}$ & $\begin{array}{l}1976 \\
1970\end{array}$ & $\begin{array}{l}0 \\
0\end{array}$ & $\begin{array}{l}\text { AA } \\
\text { AA }\end{array}$ & $\begin{array}{l}\text { Latin male name. } \\
\text { Gyula; Hungarian } \\
\text { astronomer. }(1845-1927) .\end{array}$ \\
\hline Feoktistov & $30.9 \mathrm{~N}$ & $140.7 \mathrm{E}$ & 23 & UR & SO & LOC & 4 & 5 & 1970 & 0 & AA & $\begin{array}{l}\text { Konstantin P.; Soviet } \\
\text { cosmonaut (1926-Live). }\end{array}$ \\
\hline Fermat & $22.6 \mathrm{~S}$ & $19.8 \mathrm{E}$ & 38 & EU & FR & LAC & 96 & 5 & 1935 & 66 & AA & $\begin{array}{l}\text { Pierre De; French } \\
\text { mathematician (1601-1665). }\end{array}$ \\
\hline Fermi & 19.3S & $122.6 \mathrm{E}$ & 183 & EU & IT & LTO & $083 C 4$ & 5 & 1970 & 0 & AA & $\begin{array}{l}\text { Enrico; Italian-American } \\
\text { physicist; Nobel laureate } \\
\text { (1901-1954). }\end{array}$ \\
\hline
\end{tabular}

lat: latitude of feature center.

long: longitude of feature center.

diam: diameter or long dimension of feature. et: ethnicity of name origin (see page $284 \mathrm{ff}$.)

quad: map quadrangle or informal name

(see page xvii ff.).

map name or USGS map number (see page xvii ff.). as: name approval status (see page xvii).

ad: name approval date (year).

ref: reference source for name (see page $287 \mathrm{ff}$.) 
MOON

\begin{tabular}{|c|c|c|c|c|c|c|c|c|c|c|c|c|}
\hline Name & lat & long & diam & ct & et & quad & map & as & ad & ref & $\mathrm{ft}$ & origin \\
\hline Fernelius & $38.1 \mathrm{~S}$ & $4.9 \mathrm{E}$ & 65 & $\mathrm{EU}$ & FR & LAC & 112 & 5 & 1935 & 66 & AA & $\begin{array}{l}\text { Jean; French doctor, } \\
\text { astronomer }(1497-1558)\end{array}$ \\
\hline Fersman & $18.7 \mathrm{~N}$ & $126.0 \mathrm{~W}$ & 151 & UR & SO & LOC & 1 & 5 & 1970 & 0 & $\mathrm{AA}$ & $\begin{array}{l}\text { Aleksandr; Soviet } \\
\text { geochemist (1883-1945). }\end{array}$ \\
\hline Fesenkov & $23.2 \mathrm{~S}$ & $135.1 \mathrm{E}$ & 35 & UR & RU & LTO & $102 \mathrm{~A} 4$ & 5 & 1973 & 0 & $\mathrm{AA}$ & $\begin{array}{l}\text { Vasiliy Grigor'evich; } \\
\text { Soviet astrophysicist } \\
(1889-1972) \text {. }\end{array}$ \\
\hline Feuillée & $27.4 \mathrm{~N}$ & $9.4 \mathrm{~W}$ & 9 & EU & FR & LTO & 041 A4 & 5 & 1935 & 66 & $\mathrm{AA}$ & $\begin{array}{l}\text { Louis; French natural } \\
\text { scientist (1660-1732). }\end{array}$ \\
\hline Finsch & $23.6 \mathrm{~N}$ & $21.3 \mathrm{E}$ & 4 & $\mathrm{EU}$ & GE & LTO & $042 \mathrm{C} 2$ & 5 & 1976 & 0 & $\mathrm{AA}$ & $\begin{array}{l}\text { O. F. H.; German zoologist } \\
(1839-1917) \text {. }\end{array}$ \\
\hline Finsen & $42.0 \mathrm{~S}$ & $177.9 \mathrm{~W}$ & 72 & $\mathrm{EU}$ & $\mathrm{DE}$ & LMP & 2 & 5 & 1979 & 0 & $\mathrm{AA}$ & $\begin{array}{l}\text { Niels Ryberg; Danish } \\
\text { phototherapist; Nobel } \\
\text { laureate }(1860-1904) \text {. }\end{array}$ \\
\hline [Firdausi] & $24.8 \mathrm{~N}$ & $34.0 \mathrm{~W}$ & 6 & AS & PE & LTO & 039B3 & 6 & 0 & 0 & AA & $\begin{array}{l}\text { Hasan; Persian author (c. } \\
940-1020) \text {. }\end{array}$ \\
\hline Firmicus & $7.3 \mathrm{~N}$ & $63.4 \mathrm{E}$ & 56 & $\mathrm{EU}$ & IT & LTO & $062 \mathrm{C} 1$ & 5 & 1935 & 66 & $\mathrm{AA}$ & $\begin{array}{l}\text { Maternus; Italian } \\
\text { astronomer (unkn-c. 330). }\end{array}$ \\
\hline Firsov & $4.5 \mathrm{~N}$ & $112.2 \mathrm{E}$ & 51 & UR & SO & LOC & 3 & 5 & 1970 & 0 & AA & $\begin{array}{l}\text { Georgij F.; Soviet } \\
\text { rocketry engineer } \\
(1917-1960)\end{array}$ \\
\hline Fischer & $8.0 \mathrm{~N}$ & $142.4 \mathrm{E}$ & 30 & EU & GE & LTO & 066B4 & 5 & 1976 & 0 & $\mathrm{AA}$ & $\begin{array}{l}\text { Emil; German chemist } \\
\text { (1852-1919); Hans; German } \\
\text { organic chemist } \\
(1881-1945) \text {. }\end{array}$ \\
\hline Fitzgerald & $27.5 \mathrm{~N}$ & $171.7 \mathrm{~W}$ & 110 & EU & IR & LOC & 4 & 5 & 1970 & 0 & $\mathrm{AA}$ & $\begin{array}{l}\text { George F.; Irish physicist } \\
\text { (1851-1901). }\end{array}$ \\
\hline Fizeau & $58.6 \mathrm{~S}$ & $133.9 \mathrm{~W}$ & 111 & EU & FR & $\mathrm{LOC}$ & 1 & 5 & 1970 & 0 & AA & $\begin{array}{l}\text { Armand H. L.; French } \\
\text { physicist (1819-1896). }\end{array}$ \\
\hline Flammarion & $3.4 \mathrm{~S}$ & $3.7 \mathrm{~W}$ & 74 & EU & FR & LTO & 077A3 & 5 & 1935 & 66 & AA & $\begin{array}{l}\text { Camille; French astronomer } \\
(1842-1925) \text {. }\end{array}$ \\
\hline Flamsteed & $4.5 \mathrm{~S}$ & $44.3 \mathrm{~W}$ & 20 & EU & GB & LAC & 75 & 5 & 1935 & 66 & $\mathrm{AA}$ & $\begin{array}{l}\text { John; British astronomer } \\
(1646-1720) \text {. }\end{array}$ \\
\hline Fleming & $15.0 \mathrm{~N}$ & $109.6 \mathrm{E}$ & 106 & EU & GB & LOC & 3 & 5 & 1970 & 0 & AA & $\begin{array}{l}\text { Alexander; British doctor, } \\
\text { Nobel laureate } \\
\text { (1881-1955); Williamina } \\
\text { P.; American astronomer } \\
\text { (1857-1911). }\end{array}$ \\
\hline Florensky & $25.3 \mathrm{~N}$ & $131.5 \mathrm{E}$ & 0 & UR & SO & & & 5 & 1985 & 0 & $\mathrm{AA}$ & $\begin{array}{l}\text { Kirill P.; Soviet } \\
\text { geologist (1915-1982). }\end{array}$ \\
\hline Focas & $33.7 \mathrm{~S}$ & $93.8 \mathrm{~W}$ & 22 & EU & FR & LOC & 1 & 5 & 1970 & 0 & AA & $\begin{array}{l}\text { Ionnas; French astronomer } \\
\text { (1908-1969). }\end{array}$ \\
\hline Fontana & $16.1 \mathrm{~S}$ & $56.6 \mathrm{~W}$ & 31 & $\mathrm{EU}$ & IT & LAC & 92 & 5 & 1935 & 66 & AA & $\begin{array}{l}\text { Francesco; Italian } \\
\text { astronomer (c. } \\
\text { 1585-1656). }\end{array}$ \\
\hline Fontenelle & $63.4 \mathrm{~N}$ & $18.9 \mathrm{~W}$ & 38 & EU & FR & $\mathrm{LAC}$ & 12 & 5 & 1935 & 66 & AA & $\begin{array}{l}\text { Bernard Le Bovier De; } \\
\text { French astronomer } \\
(1657-1757) \text {. }\end{array}$ \\
\hline Foster & $23.7 \mathrm{~N}$ & $141.5 \mathrm{~W}$ & 33 & NA & CA & LOC & 4 & 5 & 1970 & 0 & $\mathrm{AA}$ & $\begin{array}{l}\text { John S.; Canadian } \\
\text { physicist (1890-1964). }\end{array}$ \\
\hline Foucault & $50.4 \mathrm{~N}$ & $39.7 \mathrm{~W}$ & 23 & EU & FR & LAC & 11 & 5 & 1935 & 66 & AA & $\begin{array}{l}\text { Leon; French physicist } \\
(1819-1868) \text {. }\end{array}$ \\
\hline Fourier & $30.3 \mathrm{~S}$ & $53.0 \mathrm{~W}$ & 51 & EU & FR & LAC & 92 & 5 & 1935 & 66 & AA & $\begin{array}{l}\text { Jean-Baptiste Joseph; } \\
\text { French mathematician } \\
(1768-1830)\end{array}$ \\
\hline Fowler & $42.3 \mathrm{~N}$ & $145.0 \mathrm{~W}$ & 146 & $\mathrm{EU}$ & GB & LOC & 4 & 5 & 1970 & 0 & $\mathrm{AA}$ & $\begin{array}{l}\text { Alfred; British astronomer } \\
\text { (1868-1940); Ralph H.; } \\
\text { British mathematician; } \\
\text { physicist (1889-1944). }\end{array}$ \\
\hline Fox & $0.5 \mathrm{~N}$ & $98.2 \mathrm{E}$ & 24 & NA & AM & LTO & 064D3 & 5 & 1973 & 0 & $\mathrm{AA}$ & $\begin{array}{l}\text { Philip; American } \\
\text { astronomer (1878-1944). }\end{array}$ \\
\hline Fra Mauro & $6.1 \mathrm{~S}$ & $17.0 \mathrm{~W}$ & 101 & $\mathrm{EU}$ & IT & LAC & 76 & 5 & 1935 & 66 & AA & $\begin{array}{l}\text { Italian geographer } \\
\text { (unkn-1459). }\end{array}$ \\
\hline Fracastorius & $21.5 \mathrm{~S}$ & $33.2 \mathrm{E}$ & 112 & EU & IT & LAC & 97 & 5 & 1935 & 66 & AA & $\begin{array}{l}\text { Fracastoro, Girolamo; } \\
\text { Italian doctor, astronomer } \\
(1483-1553) \text {. }\end{array}$ \\
\hline Franck & $22.6 \mathrm{~N}$ & $35.5 \mathrm{E}$ & 12 & EU & GE & LTO & 043D2 & 5 & 1973 & 0 & AA & $\begin{array}{l}\text { James; German physicist; } \\
\text { Nobel laureate } \\
(1882-1964) \text {. }\end{array}$ \\
\hline Franklin & & & 56 & NA & AM & LAC & 27 & 5 & 1935 & 66 & AA & $\begin{array}{l}\text { Benjamin; American } \\
\text { inventor (1706-1790). }\end{array}$ \\
\hline
\end{tabular}

lat: latitude of feature center.

long: longitude of feature center.

diam: diameter or long dimension of feature.

ct: continent of name origin (see page $284 \mathrm{ff}$ ) et: ethnicity of name origin (see page $284 \mathrm{ff}$.)

quad: map quadrangle or informal name

(see page xvii ff.).

map name or USGS map number (see page xvii ff.). as: name approval status (see page xvii).

ad: name approval date (year).

ref: reference source for name (see page $287 \mathrm{ff}$.). 
MOON

\begin{tabular}{|c|c|c|c|c|c|c|c|c|c|c|c|c|}
\hline Name & lat & long & diam & ct & et & quad & map & as & ad & ref & ft & origin \\
\hline Franz & $16.6 \mathrm{~N}$ & $40.2 \mathrm{E}$ & 25 & EU & GE & LTO & $043 \mathrm{C} 4$ & 5 & 1935 & 66 & AA & $\begin{array}{l}\text { Julius Heinrich; German } \\
\text { astronomer (1847-1913). }\end{array}$ \\
\hline Fraunhofer & $39.5 S$ & $59.1 \mathrm{E}$ & 56 & $\mathrm{EU}$ & GE & $\mathrm{LAC}$ & 114 & 5 & 1935 & 66 & $\mathrm{AA}$ & $\begin{array}{l}\text { Joseph von; German } \\
\text { astronomer, optician } \\
(1787-1826) \text {. }\end{array}$ \\
\hline Fredholm & $18.4 \mathrm{~N}$ & $46.5 \mathrm{E}$ & 14 & EU & SW & LTO & $043 C 3$ & 5 & 1976 & 0 & AA & $\begin{array}{l}\text { Erik Ivar; Swedish } \\
\text { mathematician (1866-1927). }\end{array}$ \\
\hline Freud & $25.8 \mathrm{~N}$ & $52.3 \mathrm{~W}$ & 2 & EU & AS & LTO & 038B3 & 5 & 1973 & 0 & AA & $\begin{array}{l}\text { Sigmund; Austrian } \\
\text { psychoanalyst (1856-1939). }\end{array}$ \\
\hline Freundlich & $25.0 \mathrm{~N}$ & $171.0 \mathrm{E}$ & 85 & EU & GE & LOC & 4 & 5 & 1970 & 0 & AA & $\begin{array}{l}\text { Erwin (Finlay-); } \\
\text { German-British astronomer } \\
\text { (1885-1964). }\end{array}$ \\
\hline Fridman & $12.6 \mathrm{~S}$ & $126.0 \mathrm{~W}$ & 102 & UR & SO & LOC & 1 & 5 & 1970 & 0 & AA & $\begin{array}{l}\text { Aleksandr; Soviet } \\
\text { physicist (1888-1925). }\end{array}$ \\
\hline Froelich & $80.3 \mathrm{~N}$ & $109.7 \mathrm{~W}$ & 58 & NA & AM & LMP & 3 & 5 & 1970 & 0 & AA & $\begin{array}{l}\text { Jack E.; American rocket } \\
\text { scientist (1921-1967). }\end{array}$ \\
\hline Frost & $37.7 \mathrm{~N}$ & $118.4 \mathrm{~W}$ & 75 & NA & AM & LOC & 1 & 5 & 1970 & 0 & AA & $\begin{array}{l}\text { Edwin B.; American } \\
\text { astronomer (1866-1935). }\end{array}$ \\
\hline Fryxell & $21.3 \mathrm{~S}$ & $101.4 \mathrm{~W}$ & 18 & NA & AM & & & 5 & 1985 & 0 & AA & $\begin{array}{l}\text { R. H.; American geologist } \\
(1934-1974) \text {. }\end{array}$ \\
\hline Furnerius & $36.0 \mathrm{~S}$ & $60.6 \mathrm{E}$ & 135 & EU & FR & LOC & 3 & 5 & 1935 & 66 & AA & $\begin{array}{l}\text { Furner, Georges; French } \\
\text { mathematician (unkn-fl. } \\
\text { 1643). }\end{array}$ \\
\hline G. Bond & $32.4 \mathrm{~N}$ & $36.2 \mathrm{E}$ & 20 & NA & AM & LAC & 27 & 5 & 1935 & 66 & AA & $\begin{array}{l}\text { George Philip; American } \\
\text { astronomer (1826-1865). }\end{array}$ \\
\hline Gadomski & $36.4 \mathrm{~N}$ & $147.3 \mathrm{~W}$ & 65 & EU & PO & LOC & 4 & 5 & 1970 & 0 & AA & $\begin{array}{l}\text { Jan; Polish astronomer } \\
(1889-1966) .\end{array}$ \\
\hline Gagarin & $20.2 \mathrm{~S}$ & $149.2 \mathrm{E}$ & 265 & UR & SO & LTO & 102B2 & 5 & 1970 & $\mathbf{0}$ & AA & $\begin{array}{l}\text { Yurij A.; Soviet cosmonaut } \\
\text { (1934-1968). }\end{array}$ \\
\hline Galen & $21.9 \mathrm{~N}$ & $5.0 \mathrm{E}$ & 10 & $\mathrm{EU}$ & GR & LTO & $041 \mathrm{Cl}$ & 5 & 1973 & 0 & AA & $\begin{array}{l}\text { Claudius; Greek doctor (c. } \\
129-200 \text { ). }\end{array}$ \\
\hline Galilaei & $10.5 \mathrm{~N}$ & $62.7 \mathrm{~W}$ & 15 & EU & IT & LAC & 56 & 5 & 1935 & 66 & AA & $\begin{array}{l}\text { Galileo; Italian astronomer, } \\
\text { physicist (1564-1642). }\end{array}$ \\
\hline Galle & $55.9 \mathrm{~N}$ & $22.3 \mathrm{E}$ & 21 & $\mathrm{EU}$ & GE & LAC & 11 & 5 & 1935 & 68 & AA & $\begin{array}{l}\text { Johann Gottfried; German } \\
\text { astronomer (1812-1910). }\end{array}$ \\
\hline Galois & $14.2 \mathrm{~S}$ & $151.9 \mathrm{~W}$ & 222 & $\mathrm{EU}$ & FR & LOC & 4 & 5 & 1970 & 0 & AA & $\begin{array}{l}\text { Evariste; French } \\
\text { mathematician (1811-1832). }\end{array}$ \\
\hline Galvani & $49.6 \mathrm{~N}$ & $84.6 \mathrm{~W}$ & 80 & EU & IT & LOC & 1 & 5 & 1961 & 67 & AA & $\begin{array}{l}\text { Luigi; Italian physicist } \\
\text { (1737-1798). }\end{array}$ \\
\hline Gambart & $1.0 \mathrm{~N}$ & $15.2 \mathrm{~W}$ & 25 & $\mathrm{EU}$ & FR & LAC & 58 & 5 & 1935 & 66 & AA & $\begin{array}{l}\text { Jean Felix; French } \\
\text { astronomer }(1800-1836) \text {. }\end{array}$ \\
\hline Ganskiy (Hansky) & $0.3 \mathrm{~N}$ & $145.3 \mathrm{E}$ & 129 & INA & AIM & LIMIP & 3 & 3 & 1970 & 0 & AA & $\begin{array}{l}\text { George; American physicist } \\
\text { (1904-1968). } \\
\text { Aleksey P. Soyiet }\end{array}$ \\
\hline Uanskiy (nansky) & 9.13 & 9 $1.0 \mathrm{E}$ & 43 & UN & S & LiO & $002 \mathrm{DL}$ & J & 1910 & 0 & AA & astronomer (1870-1908). \\
\hline Ganswindt & 19.05 & $110.3 \mathrm{E}$ & 14 & EU & GE & $\mathrm{LMP}$ & 3 & 3 & $19 / 0$ & 0 & AA & $\begin{array}{l}\text { Hermann; German rocketry } \\
\text { engineer (1856-1934). }\end{array}$ \\
\hline Garavito & 4/.JS & $150 . / \mathrm{E}$ & 14 & SA & $\mathrm{Cu}$ & LMP & 3 & 3 & 1970 & 0 & AA & $\begin{array}{l}\text { J.; Colombian astronomer } \\
(1865-1920) \text {. }\end{array}$ \\
\hline Gardner & $17.7 \mathrm{~N}$ & $33.8 \mathrm{E}$ & 18 & NA & AM & LTO & 043D4 & 5 & 1976 & 0 & AA & $\begin{array}{l}\text { Irvine Clifton; American } \\
\text { physicist (1889-1972). }\end{array}$ \\
\hline Gärtner & $59.1 \mathrm{~N}$ & $34.6 \mathrm{E}$ & 115 & $\mathrm{EU}$ & $\mathrm{GE}$ & LAC & 13 & 5 & 1935 & 66 & AA & $\begin{array}{l}\text { Christian; German } \\
\text { mineralogist, geologist } \\
\text { (c. 1750-1813). }\end{array}$ \\
\hline Gassendı & $17.6 \mathrm{~S}$ & $40.1 \mathrm{~W}$ & 101 & $\mathrm{EU}$ & FR & LAC & 93 & 5 & 1935 & 66 & AA & $\begin{array}{l}\text { Pierre; French astronomer, } \\
\text { mathematician (1592-1655). } \\
\text { French male name. }\end{array}$ \\
\hline $\begin{array}{l}\text { Gaston } \\
\text { Gaudibert }\end{array}$ & $\begin{array}{l}30.9 \mathrm{~N} \\
10.9 \mathrm{~S}\end{array}$ & $\begin{array}{l}34.0 \mathrm{~W} \\
37.8 \mathrm{E}\end{array}$ & $\begin{array}{r}2 \\
34\end{array}$ & $\begin{array}{l}\mathrm{EU} \\
\mathrm{EU}\end{array}$ & $\begin{array}{l}\text { FR } \\
\text { FR }\end{array}$ & LTO & $\begin{array}{l}39 \mathrm{~B} 2 \\
079 \mathrm{D} 2\end{array}$ & $\begin{array}{l}5 \\
5\end{array}$ & $\begin{array}{l}1979 \\
1935\end{array}$ & $\begin{array}{r}0 \\
66\end{array}$ & $\begin{array}{l}\mathrm{AA} \\
\mathrm{AA}\end{array}$ & $\begin{array}{l}\text { French male name. } \\
\text { Casimir Marie; French } \\
\text { astronomer (1823-1901). }\end{array}$ \\
\hline Gauricus & $33.8 \mathrm{~S}$ & $12.6 \mathrm{~W}$ & 79 & $\mathrm{EU}$ & IT & LAC & 112 & 5 & 1935 & 66 & AA & $\begin{array}{l}\text { Gaurico, Luca; Italian } \\
\text { astronomer (1476-1558). }\end{array}$ \\
\hline Gauss & $35.7 \mathrm{~N}$ & $79.0 \mathrm{E}$ & 177 & $\mathrm{EU}$ & GE & LOC & 3 & 5 & 1935 & 66 & AA & $\begin{array}{l}\text { Karl Friedrich; German } \\
\text { mathematician (1777-1855). }\end{array}$ \\
\hline Gavrilov & $17.4 \mathrm{~N}$ & $130.9 \mathrm{E}$ & 60 & UR & SO & LOC & 3 & 5 & 1970 & 0 & AA & $\begin{array}{l}\text { Aleksandr I.; Soviet } \\
\text { rocket engineer } \\
\text { (1884-1955), Igor B.; } \\
\text { Soviet astronomer } \\
\text { (1928-1982). } \\
\text { Josenh Louis: French }\end{array}$ \\
\hline & & & & $\mathrm{EU}$ & FR & & & 5 & 1 & & & $\begin{array}{l}\text { Joseph Louis; French } \\
\text { physicist (1778-1850). }\end{array}$ \\
\hline
\end{tabular}

lat: latitude of feature center.

long: longitude of feature center.

diam: diameter or long dimension of feature.

ct: continent of name origin (see page 284 et: ethnicity of name origin (see page $284 \mathrm{ff}$.)

quad: map quadrangle or informal name

(see page xvii ff.) as: name approval status (see page $x$ vii)

ad: name approval date (year).

ref: reference source for name (see page $287 \mathrm{ff}$.).

(st) map: map name or USGS map number (see page xvii ff.). ft: feature type (see page 290). 


\section{MOON}

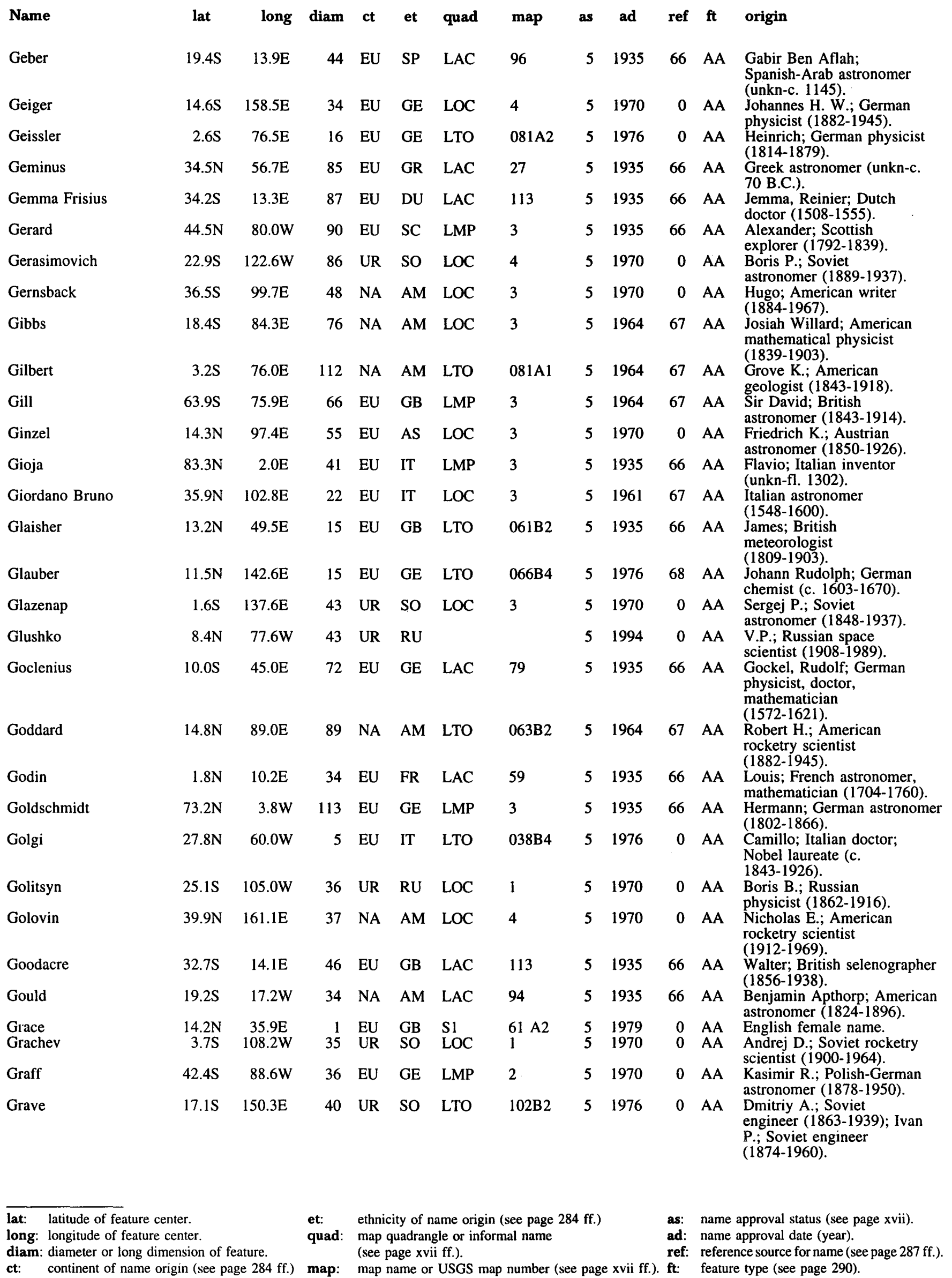


MOON

\begin{tabular}{|c|c|c|c|c|c|c|c|c|c|c|c|c|}
\hline Name & lat & long & diam & ct & et & quad & map & as & ad & ref & $\mathbf{f t}$ & origin \\
\hline Greaves & $13.2 \mathrm{~N}$ & $52.7 \mathrm{E}$ & 13 & EU & GB & LTO & $062 \mathrm{Al}$ & 5 & 1976 & 0 & AA & $\begin{array}{l}\text { William Michael Herbert; } \\
\text { British astronomer } \\
(1897-1955) \text {. }\end{array}$ \\
\hline Green & $4.1 \mathrm{~N}$ & $132.9 \mathrm{E}$ & 65 & $\mathrm{EU}$ & GB & LOC & 3 & 5 & 1970 & 0 & AA & $\begin{array}{l}\text { George; British } \\
\text { mathematician (1793-1841). }\end{array}$ \\
\hline Gregory & $2.2 \mathrm{~N}$ & $127.2 \mathrm{E}$ & 67 & $\mathrm{EU}$ & $\mathrm{SC}$ & LOC & 3 & 5 & 1970 & 0 & AA & $\begin{array}{l}\text { James; Scottish } \\
\text { astronomer, mathematician } \\
(1638-1675) \text {. }\end{array}$ \\
\hline Grigg & $12.9 \mathrm{~N}$ & $129.4 W$ & 36 & $\mathrm{OC}$ & $\mathrm{NE}$ & LOC & 1 & 5 & 1970 & 0 & AA & $\begin{array}{l}\text { J.; New Zealander } \\
\text { astronomer }(1838-1920) \text {. }\end{array}$ \\
\hline Grimaldi & $5.5 \mathrm{~S}$ & $68.3 \mathrm{~W}$ & 172 & EU & IT & LAC & 74 & 5 & 1935 & 66 & AA & $\begin{array}{l}\text { Francesco Maria; Italian } \\
\text { astronomer, physicist } \\
(1618-1663) .\end{array}$ \\
\hline [Grimm] & $15.0 \mathrm{~S}$ & $130.2 \mathrm{E}$ & 15 & EU & GE & LTO & 084D4 & 6 & 0 & 0 & AA & $\begin{array}{l}\text { Wilhelm Karl; German } \\
\text { story-teller (1786-1859). }\end{array}$ \\
\hline Grissom & $47.0 \mathrm{~S}$ & $147.4 W$ & 58 & NA & AM & LMP & 3 & 5 & 1970 & 0 & AA & $\begin{array}{l}\text { Virgil I.; American } \\
\text { astronaut (1926-1967). }\end{array}$ \\
\hline Grotrian & $66.5 \mathrm{~S}$ & $128.3 \mathrm{E}$ & 37 & EU & GE & LMP & 3 & 5 & 1970 & 0 & AA & $\begin{array}{l}\text { W.; German astronomer } \\
(1890-1954) \text {. }\end{array}$ \\
\hline Grove & $40.3 \mathrm{~N}$ & $32.9 \mathrm{E}$ & 28 & $\mathrm{EU}$ & GB & LAC & 26 & 5 & 1935 & 66 & AA & $\begin{array}{l}\text { Sir William Robert; } \\
\text { British physicist } \\
\text { (1811-1896). }\end{array}$ \\
\hline Gruemberger & $66.9 S$ & $10.0 \mathrm{~W}$ & 93 & EU & AS & LMP & 3 & 5 & 1935 & 66 & AA & $\begin{array}{l}\text { Christoph; Austrian } \\
\text { astronomer (1561-1636). }\end{array}$ \\
\hline Gruithuisen & $32.9 \mathrm{~N}$ & $39.7 \mathrm{~W}$ & 15 & $\mathrm{EU}$ & GE & LAC & 23 & 5 & 1935 & 66 & AA & $\begin{array}{l}\text { Franz von; German } \\
\text { astronomer (1774-1852). }\end{array}$ \\
\hline Guericke & $11.5 \mathrm{~S}$ & $14.1 \mathrm{~W}$ & 63 & $\mathrm{EU}$ & GE & LTO & $076 \mathrm{C} 2$ & 5 & 1935 & 66 & AA & $\begin{array}{l}\text { Otto von; German } \\
\text { physicist, engineer, } \\
\text { naturalist (1602-1686). }\end{array}$ \\
\hline Guillaume & $45.4 \mathrm{~N}$ & $173.4 \mathrm{~W}$ & 57 & $\mathrm{EU}$ & SZ & LMP & 2 & 5 & 1979 & 0 & AA & $\begin{array}{l}\text { Charles Edouard; Swiss } \\
\text { metallurgist; Nobel } \\
\text { laureate (1861-1938). }\end{array}$ \\
\hline Gullstrand & $45.2 \mathrm{~N}$ & $129.3 \mathrm{~W}$ & 43 & $\mathrm{EU}$ & SW & $\mathrm{LOC}$ & 1 & 5 & 1970 & 0 & AA & $\begin{array}{l}\text { Allvar; Swedish } \\
\text { ophthalmologist; Nobel } \\
\text { laureate (1862-1930). }\end{array}$ \\
\hline Gum & $40.4 S$ & $88.6 \mathrm{E}$ & 54 & $\mathrm{OC}$ & $\mathrm{AU}$ & LMP & 2 & 5 & 1970 & 0 & AA & $\begin{array}{l}\text { Colin; Australian } \\
\text { astronomer (1924-1960). }\end{array}$ \\
\hline Gutenberg & $8.6 \mathrm{~S}$ & $41.2 \mathrm{E}$ & 74 & $\mathrm{EU}$ & GE & LTO & 079B4 & 5 & 1935 & 66 & AA & $\begin{array}{l}\text { Johann; German inventor } \\
\text { (c. 1398-1468). }\end{array}$ \\
\hline Guthnick & $47.7 \mathrm{~S}$ & $93.9 \mathrm{~W}$ & 36 & $\mathrm{EU}$ & GE & LMP & 3 & 5 & 1970 & 0 & AA & $\begin{array}{l}\text { Paul; German astronomer } \\
(1879-1947) \text {. }\end{array}$ \\
\hline Guyot & $11.4 \mathrm{~N}$ & $117.5 \mathrm{E}$ & 92 & EU & SZ & LTO & $065 A 3$ & 5 & 1970 & 0 & AA & $\begin{array}{l}\text { Arnold H.; Swiss-American } \\
\text { geographer, geologist } \\
(1807-1884) \text {. }\end{array}$ \\
\hline Gyldén & $5.3 \mathrm{~S}$ & $0.3 \mathrm{E}$ & 47 & $\mathrm{EU}$ & SW & LTO & 077A3 & 5 & 1935 & 66 & AA & $\begin{array}{l}\text { Hugo; Swedish astronomer } \\
\text { (1841-1896). }\end{array}$ \\
\hline H. G. Wells & $40.7 \mathrm{~N}$ & $122.8 \mathrm{E}$ & 114 & EU & GB & LMP & 2 & 5 & 1970 & 0 & AA & $\begin{array}{l}\text { Herbert George; British } \\
\text { scientific writer (1866-1946) }\end{array}$ \\
\hline [Hadley] & $25.4 \mathrm{~N}$ & 2.7E & 6 & EU & GB & LTO & 041B4 & 6 & 1985 & 0 & AA & $\begin{array}{l}\text { John; British instrument } \\
\text { maker (1682-1793). }\end{array}$ \\
\hline Hagecius & $59.8 \mathrm{~S}$ & $46.6 \mathrm{E}$ & 76 & EU & $\mathrm{CZ}$ & LMP & 3 & 5 & 1935 & 66 & AA & $\begin{array}{l}\text { Hayek, Thaddaeus; } \\
\text { Czechoslovakian } \\
\text { astronomer, mathematician } \\
(1525-1600) \text {. }\end{array}$ \\
\hline Hagen & $48.3 S$ & $135.1 \mathrm{E}$ & 55 & EU & AS & LMP & 3 & 5 & 1970 & 68 & AA & $\begin{array}{l}\text { Johann G.; Austrian } \\
\text { astronomer (1847-1930). }\end{array}$ \\
\hline Hahn & $31.3 \mathrm{~N}$ & $73.6 \mathrm{E}$ & 84 & $\mathrm{EU}$ & GE & LOC & 3 & 5 & 1935 & 66 & AA & $\begin{array}{l}\text { Friedrich von; German } \\
\text { astronomer }(1741-1805) \text {; } \\
\text { Otto; German chemist } \\
(1879-1968) \text {. }\end{array}$ \\
\hline Haidinger & $39.2 \mathrm{~S}$ & $25.0 \mathrm{~W}$ & 22 & $\mathrm{EU}$ & AS & LAC & 111 & 5 & 1935 & 66 & AA & $\begin{array}{l}\text { Wilhelm Karl von; Austrian } \\
\text { geologist, physicist } \\
(1795-1871) \text {. }\end{array}$ \\
\hline Hainzel & $41.3 \mathrm{~S}$ & $33.5 \mathrm{~W}$ & 70 & $\mathrm{EU}$ & GE & $\mathrm{LAC}$ & 11 & 5 & 1935 & 66 & AA & $\begin{array}{l}\text { Paul; German astronomer } \\
\text { (unkn-fl. 1570). }\end{array}$ \\
\hline Haldane & $1.7 \mathrm{~S}$ & 84.1E & 37 & $\mathrm{EU}$ & GB & LTO & 081B 1 & 5 & 1973 & 0 & AA & $\begin{array}{l}\text { John Burdon Sanderson; } \\
\text { British doctor (1892-1964). }\end{array}$ \\
\hline Hale & $74.2 \mathrm{~S}$ & $90.8 \mathrm{E}$ & 83 & NA & AM & LMP & 3 & 5 & 1964 & 67 & AA & $\begin{array}{l}\text { George Ellery; American } \\
\text { astronomer (1868-1938); } \\
\text { William; British rocket } \\
\text { scientist (1797-1870). }\end{array}$ \\
\hline
\end{tabular}

lat: latitude of feature center. long: longitude of feature center.

diam: diameter or long dimension of feature.

ct: continent of name origin (see page $284 \mathrm{ff}$.) et: ethnicity of name origin (see page $284 \mathrm{ff}$.)

quad: map quadrangle or informal name (see page xvii ff.). as: name approval status (see page xvii) ad: name approval date (year).

ref: reference source for name (see page $287 \mathrm{ff}$.). map name or USGS map number (see page xvii ff.). ft: feature type (see page 290). 


\section{MOON}

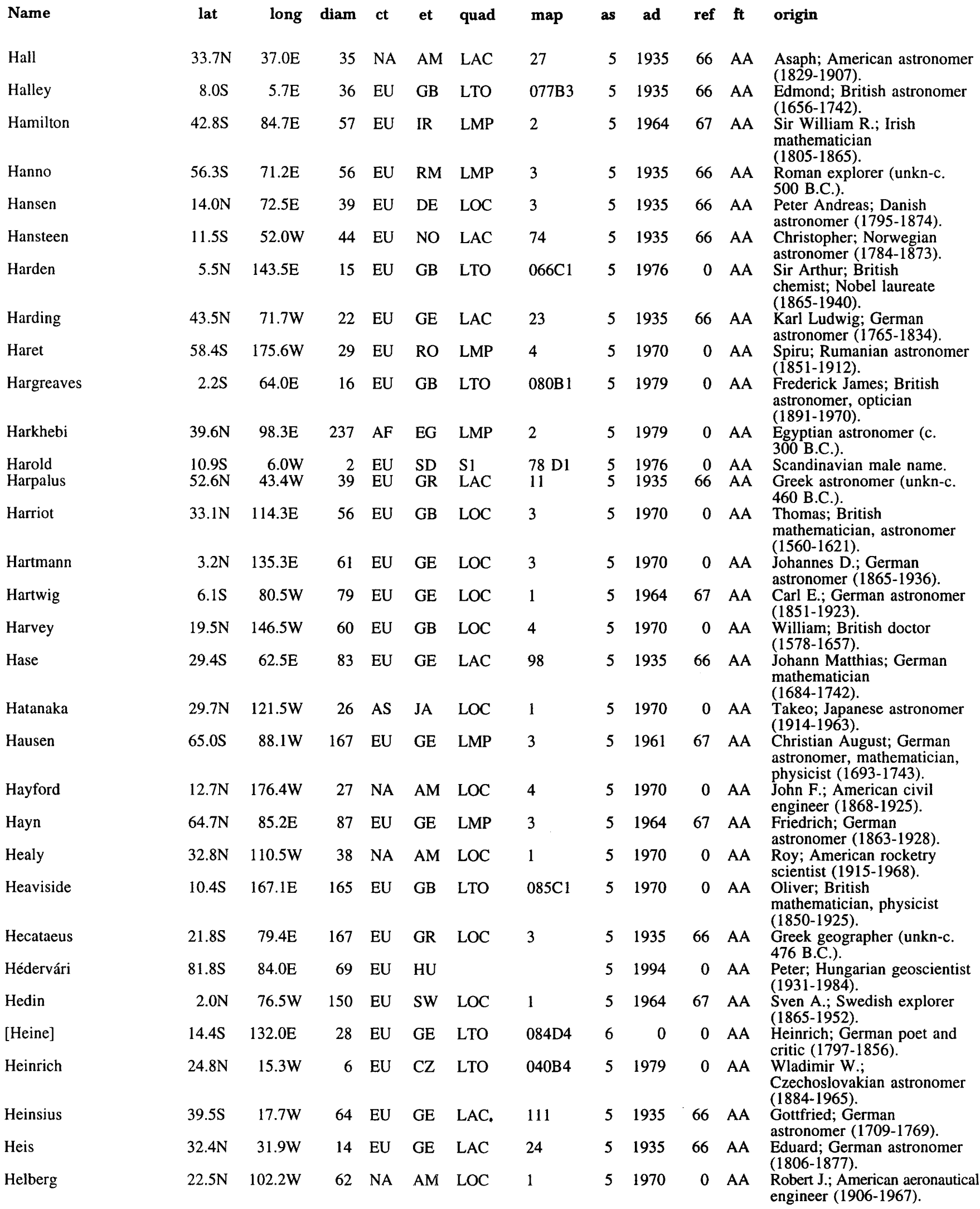

lat: latitude of feature center.

long: longitude of feature center.

diam: diameter or long dimension of feature.

ch: continent of name origin (see page 2 ? et: ethnicity of name origin (see page $284 \mathrm{ff}$.)

quad: map quadrangle or informal name

(see page xvii ff.). as: name approval status (see page xvii).

ad: name approval date (year).

ref: reference source for name (see page $287 \mathrm{ff}$.). 


\section{MOON}

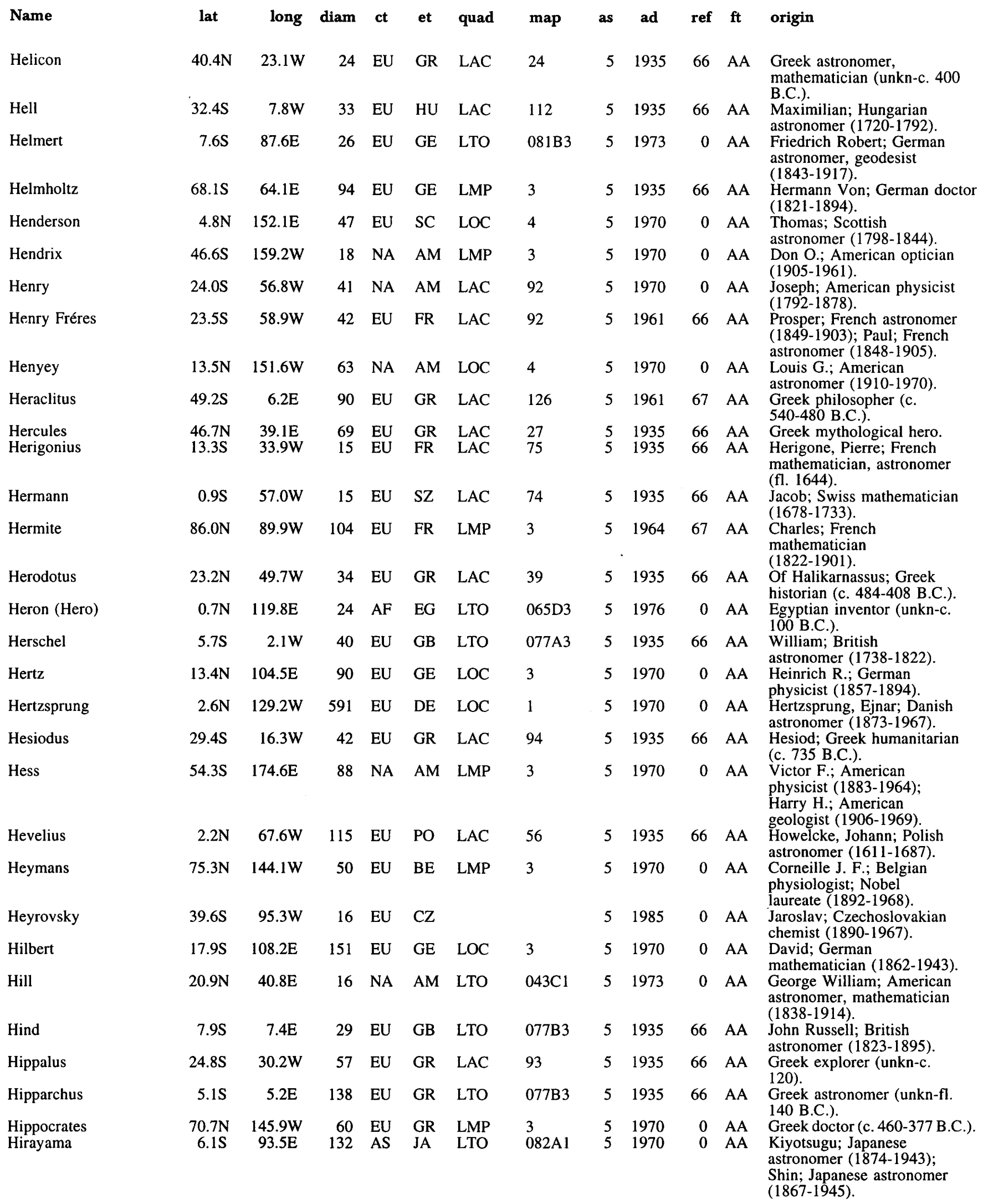

lat: latitude of feature center.

long: longitude of feature center.

diam: diameter or long dimension of feature.

ct: continent of name origin (see page $284 \mathrm{ff}$.) map: map name or USGS map number (see page xvii ff.). ft: feature type (see page 290).

et: $\quad$ ethnicity of name origin (see page $284 \mathrm{ff}$.)

quad: map quadrangle or informal name

(see page xvii ff.). as: name approval status (see page xvii).

ad: name approval date (year).

ref: reference source for name (see page $287 \mathrm{ff}$.). 


\section{MOON}

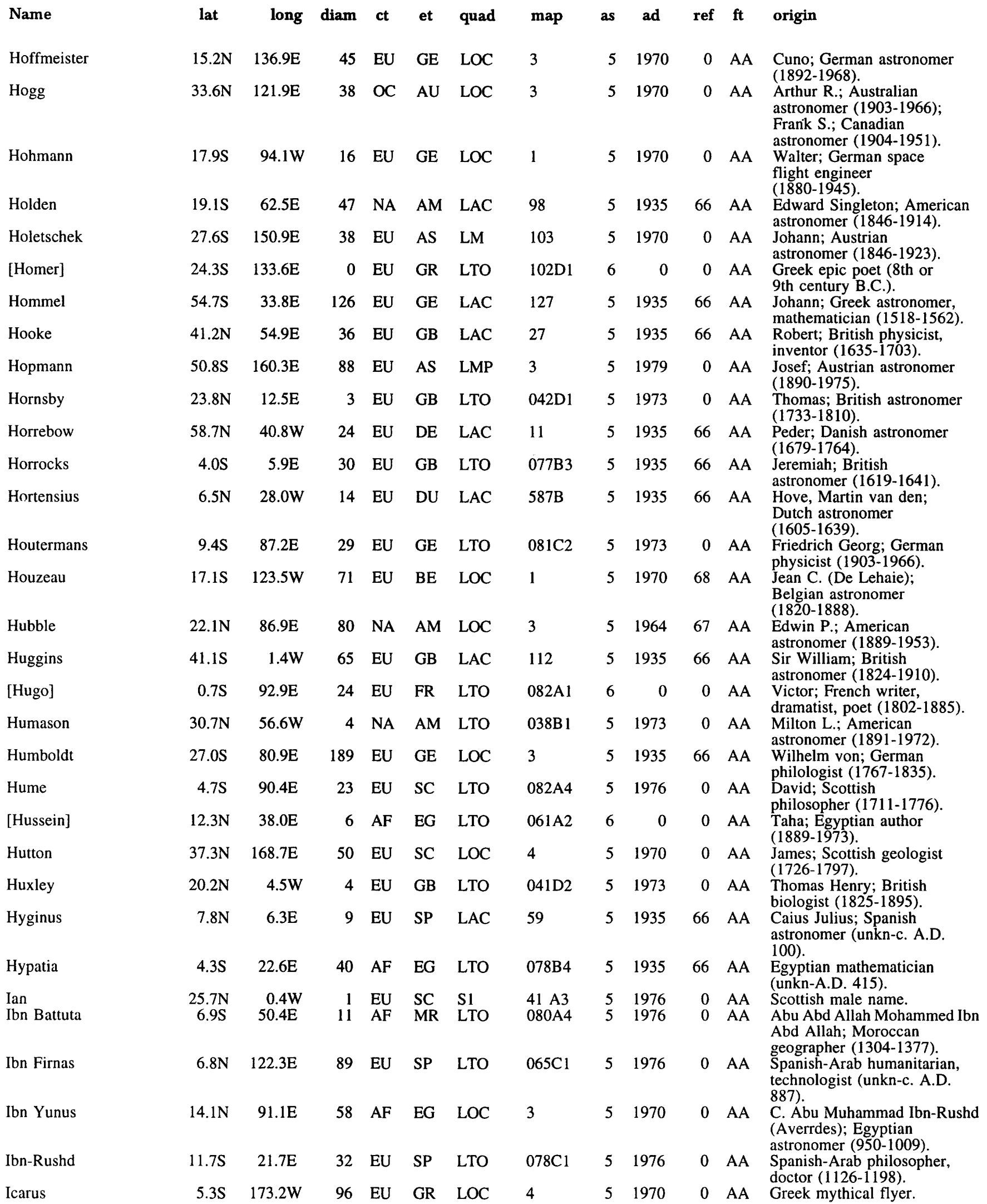

lat: latitude of feature center.

long: longitude of feature center.

diam: diameter or long dimension of feature.

ct: et: ethnicity of name origin (see page $284 \mathrm{ff}$.)

quad: map quadrangle or informal name

(see page xvii ff.). as: name approval status (see page xvii).

ad: name approval date (year).

ref: reference source for name (see page $287 \mathrm{ff}$.). 
MOON

\begin{tabular}{|c|c|c|c|c|c|c|c|c|c|c|c|c|}
\hline Name & lat & long & diam & ct & et & quad & map & as & ad & ref & $\mathbf{f t}$ & origin \\
\hline Ideler & $49.2 S$ & $22.3 \mathrm{E}$ & 38 & $\mathrm{EU}$ & GE & LAC & 127 & 5 & 1935 & 66 & AA & Christian Ludwig; German \\
\hline Idel'son & $81.5 \mathrm{~S}$ & $110.9 \mathrm{E}$ & 60 & UR & SO & LMP & 3 & 5 & 1970 & 0 & AA & $\begin{array}{l}\text { Naum I.; Soviet astronomer } \\
(1885-1951) \text {. }\end{array}$ \\
\hline Il'in & $17.8 \mathrm{~S}$ & $97.5 \mathrm{~W}$ & 13 & UR & SO & & & 5 & 1985 & 0 & AA & $\begin{array}{l}\text { N.Ja.; Soviet rocketry } \\
\text { scientist (1901-1937). }\end{array}$ \\
\hline $\begin{array}{l}\text { Ina } \\
\text { Ingalls }\end{array}$ & $\begin{array}{l}49.2 \mathrm{~S} \\
26.4 \mathrm{~N}\end{array}$ & $\begin{array}{l}22.3 \mathrm{E} \\
153.1 \mathrm{~W}\end{array}$ & $\begin{array}{l}38 \\
37\end{array}$ & $\begin{array}{l}\text { EU } \\
\text { NA }\end{array}$ & $\begin{array}{l}\text { LA } \\
\text { AM }\end{array}$ & $\begin{array}{l}\text { S1 } \\
\text { LOC }\end{array}$ & $\begin{array}{l}41 \mathrm{C} 3 \\
4\end{array}$ & $\begin{array}{l}5 \\
5\end{array}$ & $\begin{array}{l}1976 \\
1970\end{array}$ & $\begin{array}{l}0 \\
0\end{array}$ & $\begin{array}{l}\text { AA } \\
\text { AA }\end{array}$ & $\begin{array}{l}\text { Latin female name. } \\
\text { Albert L.; American } \\
\text { optician (1888-1958). }\end{array}$ \\
\hline Inghirami & $47.5 \mathrm{~S}$ & $68.8 \mathrm{~W}$ & 91 & EU & $\mathrm{IT}$ & LMP & 1 & 5 & 1935 & 66 & AA & $\begin{array}{l}\text { Giovanni; Italian } \\
\text { astronomer (1779-1851). }\end{array}$ \\
\hline Innes & $27.8 \mathrm{~N}$ & $119.2 \mathrm{E}$ & 42 & $\mathrm{EU}$ & SC & LOC & 3 & 5 & 1970 & 0 & AA & $\begin{array}{l}\text { Robert T. A.; Scottish } \\
\text { astronomer (1861-1933). }\end{array}$ \\
\hline Ioffe & $14.4 \mathrm{~S}$ & $129.2 \mathrm{~W}$ & 86 & UR & SO & LOC & 1 & 5 & 1970 & 0 & AA & $\begin{array}{l}\text { Joffe, Abram F.; Soviet } \\
\text { physicist (1880-1960). }\end{array}$ \\
\hline Isabel & $28.2 \mathrm{~N}$ & $34.1 \mathrm{~W}$ & 1 & EU & SP & $S 1$ & 39 B2 & 5 & 1979 & 0 & AA & Spanish female name. \\
\hline Isaev & $17.5 \mathrm{~S}$ & $147.5 \mathrm{E}$ & 90 & UR & SO & LTO & 102B2 & 5 & 1976 & 0 & AA & $\begin{array}{l}\text { Aleksei M.; Soviet space } \\
\text { scientist (1908-1971). }\end{array}$ \\
\hline Isidorus & $8.0 \mathrm{~S}$ & $33.5 \mathrm{E}$ & 42 & EU & RM & LTO & 079A4 & 5 & 1935 & 66 & AA & $\begin{array}{l}\text { St. Isidore of Seville; } \\
\text { Roman astronomer (c. } \\
570-636 \text { ). }\end{array}$ \\
\hline $\begin{array}{l}\text { Isis } \\
\text { Ivan }\end{array}$ & $18.9 \mathrm{~N}$ & $27.5 \mathrm{E}$ & 1 & AF & EG & S1 & $42 \mathrm{C} 3$ & 5 & 1976 & 0 & $\mathrm{AA}$ & Egyptian goddess. \\
\hline $\begin{array}{l}\text { Ivan } \\
\text { Izsak }\end{array}$ & $\begin{array}{l}26.9 \mathrm{~N} \\
23.3 \mathrm{~S}\end{array}$ & $\begin{array}{l}43.3 \mathrm{~W} \\
117.1 \mathrm{E}\end{array}$ & $\begin{array}{r}4 \\
30\end{array}$ & $\begin{array}{l}\text { UR } \\
\text { NA }\end{array}$ & $\begin{array}{l}\text { RU } \\
\text { AM }\end{array}$ & $\begin{array}{l}\text { S1 } \\
\text { LOC }\end{array}$ & $\begin{array}{l}39 \mathrm{~A} 3 \\
3\end{array}$ & $\begin{array}{l}5 \\
5\end{array}$ & $\begin{array}{l}1976 \\
1970\end{array}$ & $\begin{array}{l}0 \\
0\end{array}$ & $\begin{array}{l}\text { AA } \\
\text { AA }\end{array}$ & $\begin{array}{l}\text { Russian male name. } \\
\text { Imre; Hungarian-American } \\
\text { astronomer }(1929-1965) \text {. }\end{array}$ \\
\hline J. Herschel & $62.0 \mathrm{~N}$ & $42.0 \mathrm{~W}$ & 165 & EU & GB & LAC & 11 & 5 & 1935 & 66 & AA & $\begin{array}{l}\text { John; British astronomer } \\
\text { (1792-1871). }\end{array}$ \\
\hline Jackson & $22.4 \mathrm{~N}$ & $163.1 \mathrm{~W}$ & 71 & EU & $\mathrm{SC}$ & LOC & 4 & 5 & 1970 & 0 & AA & $\begin{array}{l}\text { John; Scottish astronomer } \\
(1887-1958) \text {. }\end{array}$ \\
\hline Jacobi & $56.7 \mathrm{~S}$ & $11.4 \mathrm{E}$ & 68 & EU & GE & LMP & 3 & 5 & 1935 & 66 & AA & $\begin{array}{l}\text { Karl Gustav Jacob; German } \\
\text { mathematician } \\
\text { (1804-1851). }\end{array}$ \\
\hline [James] & $10.2 \mathrm{~N}$ & $50.4 \mathrm{E}$ & 28 & NA & $\mathrm{AM}$ & LTO & $062 \mathrm{~A} 4$ & 6 & 0 & 0 & $\mathrm{AA}$ & $\begin{array}{l}\text { Henry; American writer } \\
(1843-1916) \text {. }\end{array}$ \\
\hline Jansen & $13.5 \mathrm{~N}$ & $28.7 \mathrm{E}$ & 23 & $\mathrm{EU}$ & DU & LTO & 060B2 & 5 & 1935 & 66 & AA & $\begin{array}{l}\text { Janszoon, Zacharias; Dutch } \\
\text { optician (1580-c. 1638). }\end{array}$ \\
\hline Jansky & $8.5 \mathrm{~N}$ & $89.5 \mathrm{E}$ & 72 & NA & AM & LTO & 063B3 & 5 & 1964 & 67 & AA & $\begin{array}{l}\text { Karl; American radio } \\
\text { engineer (1905-1950). }\end{array}$ \\
\hline Janssen & $45.4 \mathrm{~S}$ & $40.3 \mathrm{E}$ & 199 & $\mathrm{EU}$ & FR & LAC & 114 & 5 & 1935 & 66 & AA & $\begin{array}{l}\text { Pierre Jules; French } \\
\text { astronomer (1824-1907). }\end{array}$ \\
\hline Jarvis & $34.9 \mathrm{~S}$ & $148.9 \mathrm{~W}$ & 38 & NA & $\mathrm{AM}$ & & & 5 & 1988 & 88 & AA & $\begin{array}{l}\text { Gregory B.; Member of the } \\
\text { Challenger crew } \\
\text { (1944-1986); previous } \\
\text { designation Borman Z. }\end{array}$ \\
\hline Jeans & $55.8 \mathrm{~S}$ & $91.4 \mathrm{E}$ & 79 & EU & GB & LMP & 3 & 5 & 1964 & 67 & AA & $\begin{array}{l}\text { Sir James H.; British } \\
\text { mathematical physicist } \\
(1877-1946) \text {. }\end{array}$ \\
\hline $\begin{array}{l}\text { Jehan } \\
\text { Jenkins }\end{array}$ & $\begin{array}{r}20.7 \mathrm{~N} \\
0.3 \mathrm{~N}\end{array}$ & $\begin{array}{l}31.9 \mathrm{~W} \\
78.1 \mathrm{E}\end{array}$ & $\begin{array}{r}5 \\
38\end{array}$ & $\begin{array}{l}\text { AS } \\
\text { NA }\end{array}$ & $\begin{array}{l}\text { TU } \\
\text { AM }\end{array}$ & $\begin{array}{l}\text { S1 } \\
\text { LTO }\end{array}$ & $\begin{array}{l}39 \mathrm{C} 2 \\
063 \mathrm{D} 3\end{array}$ & $\begin{array}{l}5 \\
5\end{array}$ & $\begin{array}{l}1976 \\
1982\end{array}$ & $\begin{array}{l}\mathbf{0} \\
\mathbf{0}\end{array}$ & $\begin{array}{l}\mathrm{AA} \\
\mathrm{AA}\end{array}$ & $\begin{array}{l}\text { Turkish female name. } \\
\text { Louise F.; American } \\
\text { astronomer (1888-1970). }\end{array}$ \\
\hline Jenner & $42.1 \mathrm{~S}$ & $95.9 \mathrm{E}$ & 71 & $\mathrm{EU}$ & GB & LMP & 1 & 5 & 1970 & 0 & $\mathrm{AA}$ & $\begin{array}{l}\text { Edward; British doctor } \\
(1749-1823) \text {. }\end{array}$ \\
\hline $\begin{array}{l}\text { Jerik } \\
\text { [Johnson] }\end{array}$ & $\begin{array}{r}18.5 \mathrm{~N} \\
8.7 \mathrm{~S}\end{array}$ & $\begin{array}{l}27.6 \mathrm{E} \\
89.0 \mathrm{E}\end{array}$ & $\begin{array}{r}1 \\
22\end{array}$ & $\begin{array}{l}\mathrm{EU} \\
\mathrm{EU}\end{array}$ & $\begin{array}{l}\text { SD } \\
\text { GB }\end{array}$ & $\begin{array}{l}\text { S2 } \\
\text { LTO }\end{array}$ & $\begin{array}{l}42 \mathrm{C} 3 \\
081 \mathrm{C} 2\end{array}$ & $\begin{array}{l}5 \\
6\end{array}$ & $\begin{array}{r}1976 \\
0\end{array}$ & $\begin{array}{l}0 \\
0\end{array}$ & $\begin{array}{l}\text { AA } \\
\text { AA }\end{array}$ & $\begin{array}{l}\text { Scandinavian male name. } \\
\text { Samuel; British writer } \\
(1709-1784) \text {. }\end{array}$ \\
\hline Joliot & $25.8 \mathrm{~N}$ & $93.1 \mathrm{E}$ & 164 & EU & FR & LOC & 3 & 5 & 1970 & 0 & AA & $\begin{array}{l}\text { Frederic Joliot-Curie; } \\
\text { French physicist; Nobel } \\
\text { laureate (1900-1958). }\end{array}$ \\
\hline $\begin{array}{l}\text { Jomo } \\
\text { José }\end{array}$ & $\begin{array}{l}24.4 \mathrm{~N} \\
12.7 \mathrm{~S}\end{array}$ & $\begin{array}{l}1.6 \mathrm{~W} \\
1.6 \mathrm{~W}\end{array}$ & $\begin{array}{l}7 \\
2\end{array}$ & $\begin{array}{l}\mathrm{AF} \\
\mathrm{EU}\end{array}$ & $\begin{array}{l}\text { UN } \\
\text { SP }\end{array}$ & $\begin{array}{l}\text { S3 } \\
\text { S1 }\end{array}$ & $\begin{array}{l}41 \mathrm{~B} 4 \\
77 \mathrm{D} 3\end{array}$ & $\begin{array}{l}5 \\
5\end{array}$ & $\begin{array}{l}1976 \\
1976\end{array}$ & $\begin{array}{l}0 \\
0\end{array}$ & $\begin{array}{l}\text { AA } \\
\text { AA }\end{array}$ & $\begin{array}{l}\text { African male name. } \\
\text { Spanish male name. }\end{array}$ \\
\hline Joule & $27.3 \mathrm{~N}$ & $144.2 \mathrm{~W}$ & 96 & $\mathrm{EU}$ & GB & LOC & 4 & 5 & 1970 & 0 & AA & $\begin{array}{l}\text { James P.; British } \\
\text { physicist (1818-1889). }\end{array}$ \\
\hline Joy & $25.0 \mathrm{~N}$ & $6.6 \mathrm{E}$ & 5 & NA & $\mathrm{AM}$ & LTO & 041B3 & 5 & 1973 & 0 & AA & $\begin{array}{l}\text { Alfred H.; American } \\
\text { astronomer (1882-1973). }\end{array}$ \\
\hline Jules Verne & $35.0 \mathrm{~S}$ & $147.0 \mathrm{E}$ & 143 & EU & FR & LOC & 4 & 5 & 1961 & 67 & AA & French writer \\
\hline $\begin{array}{l}\text { Julienne } \\
\text { Julius Caesar }\end{array}$ & $\begin{array}{r}26.0 \mathrm{~N} \\
9.0 \mathrm{~N}\end{array}$ & $\begin{array}{r}3.2 \mathrm{E} \\
15.4 \mathrm{E}\end{array}$ & $\begin{array}{r}2 \\
90\end{array}$ & $\begin{array}{l}\text { EU } \\
\text { EU }\end{array}$ & $\begin{array}{l}\text { FR } \\
\text { RM }\end{array}$ & $\begin{array}{l}\text { S1 } \\
\text { LAC }\end{array}$ & $\begin{array}{l}41 \mathrm{~B} 4 \\
60\end{array}$ & $\begin{array}{l}5 \\
5\end{array}$ & $\begin{array}{l}1976 \\
1935\end{array}$ & $\begin{array}{r}0 \\
66\end{array}$ & $\begin{array}{l}\text { AA } \\
\text { AA }\end{array}$ & $\begin{array}{l}\text { French female name. } \\
\text { Roman emperor (c. } 102-44 \\
\text { B.C.). }\end{array}$ \\
\hline Kaiser & $36.5 \mathrm{~S}$ & $6.5 \mathrm{E}$ & 52 & $\mathrm{EU}$ & DU & LAC & 112 & 5 & 1935 & 66 & AA & Frederick; Dutch \\
\hline
\end{tabular}

astronomer (1808-1872).

lat: latitude of feature center.

long: longitude of feature center.

diam: diameter or long dimension of feature. et: $\quad$ ethnicity of name origin (see page $284 \mathrm{ff}$.)

quad: map quadrangle or informal name

(see page xvii ff.).

ref: reference source for name (see page $287 \mathrm{ff}$.). 


\section{MOON}

Name

Kane

Kant

Kao

Kapteyn

Karima

Karpinskiy

Karrer

Kasper

Kästner

Katchalsky

$5.9 \mathrm{~N} \quad 116.1 \mathrm{E}$

32 A

IS

LTO

Kathleen

Kearons

Keeler

Kekulé

Keldysh

Kepínski

Kepler

$5.9 \mathrm{~N} \quad 116.1 \mathrm{E}$

32 EU IR S1

$11.4 \mathrm{~S} \quad 112.6 \mathrm{~W}$

23 NA AM LOC

$41 \mathrm{~A} 3$

10.2

$161.9 \mathrm{E}$

160 NA AM LTO

$085 \mathrm{Cl}$

$16.4 \mathrm{~N} \quad 138.1 \mathrm{~W}$

94 EU GE LOC

1

$51.2 \mathrm{~N} \quad 43.6 \mathrm{E}$

33 UR SO

$28.8 \mathrm{~N} \quad 126.6 \mathrm{E}$

31 EU

$8.1 \mathrm{~N} \quad 38.0 \mathrm{~W}$

31 EU GE LAC 57

Khvol'son

13.8S 111.4E

54 UR SO LOC 3

Kibal'chich

$3.0 \mathrm{~N} \quad 146.5 \mathrm{~W}$

92 UR

Kidinnu

$35.9 \mathrm{~N}$

$122.9 \mathrm{E}$

56 AS

RU

LOC

4

Kies

$26.3 S$

$22.5 \mathrm{~W}$

45 EU

$6.4 \mathrm{~S}$

$84.0 \mathrm{E}$

63 NA AM LTO

081B4

$5 \quad 1976$

Kiess

57.1S 118.4E

Kimura

$60.8 \mathrm{~S}$

15.1E

28 A

Kinau

$5.0 \mathrm{~N} \quad 120.5 \mathrm{E}$

41 EU

$\mathrm{JA}$

JA LMP

3

King

$$
5.0
$$

Kira
Kirch

17.6S $132.8 \mathrm{E}$

$\begin{array}{lr}17.6 \mathrm{~S} & 132.8 \mathrm{E} \\ 39.2 \mathrm{~N} & 5.6 \mathrm{~W}\end{array}$

3 UR RU SI

$67.1 \mathrm{~S} \quad 45.3 \mathrm{~W}$

11 EU GE LAC

$102 \mathrm{Al}$

Kircher

Kirchhoff

$30.3 \mathrm{~N}$

$38.8 \mathrm{E}$

72 EU

$G$

Kirkwood

$68.8 \mathrm{~N} \quad 156.1 \mathrm{~W}$

24 EU GE LAC 43

Klaproth

$\begin{array}{rllll}67 & \text { NA } & \text { AM } & \text { LMP } & 3 \\ 119 & \text { EU } & \text { GE } & \text { LMP } & 3\end{array}$

$5 \quad 1973$

51970
$5 \quad 1970$

51970

51982

51979

51935

$5 \quad 1970$

$5 \quad 1970$

$5 \quad 1970$

$5 \quad 1935$

51973

51970

$5 \quad 1935$

$5 \quad 1970$ ref $\mathrm{ft}$ origin

0 AA

Heike Kamerlingh Onnes; Dutch physicist; Nobel

laureate (1853-1926). explorer (1820-1857).

Immanuel; German philosopher (1724-1804).

Ping-Tse; Taiwanese astronomer (1888-1970).

Jacobus C.; Dutch astronomer (1851-1922).

Arabic female name.

Aleksey P.; Soviet geologist (1846-1936).

Paul; Russian/Swiss biochemist; Nobel laureate (1889-1971).

Polish male name.

Abraham Gotthelf; German mathematician, physicist (1719-1800).

Katzir-Katchalsky, Aharon; Polish-Israeli chemist (1914-1972).

AA Irish female name.

0 AA William M.; American astronomer (1878-1948).

0 AA James E.; American astronomer (1857-1900).

0 AA Friedrich A.; German chemist (1829-1896).

Mstislav V.; Soviet mathematician (1911-1978)

F.; Polish astronomer (1885-1966).

$\begin{array}{rll}68 & \text { AA } & \begin{array}{l}\text { Johannes; German } \\ \text { astronomer }(1571-1630) \text {. }\end{array} \\ 0 & \text { AA } & \text { Orest D; Soviet physicis }\end{array}$

$\begin{array}{rll}68 & \text { AA } & \begin{array}{l}\text { Johannes; German } \\ \text { astronomer }(1571-1630) \text {. }\end{array} \\ 0 & \text { AA } & \text { Orest D; Soviet physicis }\end{array}$ (1852-1934).

Nikolaj I.; Russian rocketry scientist (1853-1881).

Or Cidenas; Babylonian astronomer (unkn-c. 343 B.C.).

Johann; German mathematician, astronomer (1713-1781).

Carl Clarence; American astrophysicist (1887-1967).

Hisashi; Japanese astronomer (1870-1943)

C. A.; German botanist, selenographer (unkn-fl. 1850).

Arthur S.; American physicist (1876-1957); Edward S.; American astronomer (1861-1931).

Russian female name.

$5 \quad 1976$

0 AA Gottfried; German astronomer (1639-1710).

51935 $66 \mathrm{AA}$

Athanasius; German

5193566 AA Gustav Robert; German physicist (1824-1887).

51970

0 AA

Daniel; American astronomer (1814-1895).

5193566 AA Martin Heinrich; German chemist, mineralogist (1743-1817). lat: latitude of feature center.

long: longitude of feature center.

diam: diameter or long dimension of feature.

ct: continent of name origin (see page $284 \mathrm{ff}$.) et: ethnicity of name origin (see page $284 \mathrm{ff}$.)

quad: map quadrangle or informal name

(see page xvii ff.) as: name approval status (see page xvii).

ad: name approval date (year).

ref: reference source for name (see page $287 \mathrm{ff}$.). 


\section{MOON}

\begin{tabular}{|c|c|c|c|c|c|c|c|c|c|c|c|c|}
\hline Name & lat & long & diam & ct & et & quad & map & as & ad & ref & $\mathbf{f t}$ & origin \\
\hline Klein & $12.0 \mathrm{~S}$ & $2.6 \mathrm{E}$ & 44 & EU & GE & LTO & $077 \mathrm{Cl}$ & 5 & 1935 & 66 & AA & $\begin{array}{l}\text { Hermann Joseph; German } \\
\text { astronomer (1844-1914). }\end{array}$ \\
\hline Kleymenov & $32.4 S$ & $140.2 \mathrm{~W}$ & 55 & UR & SO & LOC & 4 & 5 & 1970 & 0 & AA & $\begin{array}{l}\text { Ivan T.; Soviet rocketry } \\
\text { scientist (1898-1938). }\end{array}$ \\
\hline Klute & $37.2 \mathrm{~N}$ & $141.3 \mathrm{~W}$ & 75 & NA & $\mathrm{AM}$ & LOC & 4 & 5 & 1970 & 0 & AA & $\begin{array}{l}\text { Daniel O.; American } \\
\text { rocketry scientist } \\
(1921-1964) .\end{array}$ \\
\hline Knox-Shaw & $5.3 \mathrm{~N}$ & $80.2 \mathrm{E}$ & 12 & EU & GB & LTO & $063 \mathrm{Cl}$ & 5 & 1973 & 0 & AA & $\begin{array}{l}\text { Harold; British astronomer } \\
(1885-1970) \text {. }\end{array}$ \\
\hline Koch & $42.8 \mathrm{~S}$ & $150.1 \mathrm{E}$ & 95 & EU & GE & LMP & 2 & 5 & 1970 & 0 & AA & $\begin{array}{l}\text { Robert; German doctor; } \\
\text { Nobel laureate } \\
(1843-1910) \text {. }\end{array}$ \\
\hline Kohlschütter & $14.4 \mathrm{~N}$ & $154.0 \mathrm{E}$ & 53 & EU & GE & LOC & 4 & 5 & 1970 & 0 & AA & $\begin{array}{l}\text { Arnold; German astronomer } \\
(1883-1969) \text {. }\end{array}$ \\
\hline Kolhörster & $11.2 \mathrm{~N}$ & $114.6 \mathrm{~W}$ & 97 & EU & GE & LOC & 1 & 5 & 1970 & 0 & AA & $\begin{array}{l}\text { Werner; German physicist } \\
(1887-1946) \text {. }\end{array}$ \\
\hline Komarov & $24.7 \mathrm{~N}$ & $152.5 \mathrm{E}$ & 78 & UR & SO & LOC & 4 & 5 & 1970 & 0 & AA & $\begin{array}{l}\text { Vladimir M;; Soviet } \\
\text { cosmonaut (1927-1967). }\end{array}$ \\
\hline Kondratyuk & $14.9 \mathrm{~S}$ & $115.5 \mathrm{E}$ & 108 & UR & SO & LOC & 3 & 5 & 1970 & 0 & AA & $\begin{array}{l}\text { Yurij V.; Soviet rocketry } \\
\text { scientist }(1897-1942) \text {. }\end{array}$ \\
\hline König & $24.1 \mathrm{~S}$ & $24.6 \mathrm{~W}$ & 23 & $\mathrm{EU}$ & AS & LAC & 94 & 5 & 1935 & 66 & AA & $\begin{array}{l}\text { Rudolf; Austrian } \\
\text { mathematician, astronomer } \\
(1865-1927) \text {. }\end{array}$ \\
\hline Konoplev & $28.5 \mathrm{~S}$ & $125.5 \mathrm{~W}$ & 25 & AS & SO & SOV & 1985 & 5 & 1991 & 0 & AA & $\begin{array}{l}\text { B.T.; Soviet radio } \\
\text { engineer }(1912-1960) \text {. }\end{array}$ \\
\hline Konstantinov & $19.8 \mathrm{~N}$ & $158.4 \mathrm{E}$ & 66 & UR & RU & LOC & 4 & 5 & 1970 & 0 & AA & $\begin{array}{l}\text { Konstantin I.; Russian } \\
\text { rocketry scientist } \\
\text { (1817-1871). }\end{array}$ \\
\hline Kopff & $17.4 S$ & $89.6 \mathrm{~W}$ & 41 & $\mathrm{EU}$ & GE & LOC & 1 & 5 & 1970 & 0 & AA & $\begin{array}{l}\text { August; German astronomer } \\
(1882-1960) \text {. }\end{array}$ \\
\hline Korolev & $4.0 \mathrm{~S}$ & $157.4 \mathrm{~W}$ & 437 & UR & SO & LOC & 4 & 5 & 1970 & 0 & AA & Sergej P.; Soviet rocketry \\
\hline Kosberg & $20.2 \mathrm{~S}$ & $149.6 \mathrm{E}$ & 15 & UR & SO & LTO & $102 \mathrm{~B} 3$ & 5 & 1976 & 0 & AA & $\begin{array}{l}\text { C. A.; Soviet aeronaut } \\
(1903-1965) \text {. }\end{array}$ \\
\hline Kovalevskaya & $30.8 \mathrm{~N}$ & $118.8 \mathrm{E}$ & 115 & UK & 30 & $\mathrm{LOC}$ & 3 & 5 & 1910 & 0 & AA & $\begin{array}{l}\text { astronomer (1867-1937). } \\
\text { Sofia V.: Russian }\end{array}$ \\
\hline Koval'skij & $21.9 \mathrm{~S}$ & $101.0 \mathrm{E}$ & 49 & UR & RU & LOC & 3 & 5 & 1970 & 0 & AA & $\begin{array}{l}\text { mathematician (1850-1891). } \\
\text { Marian A.; Russsian }\end{array}$ \\
\hline Krafft & $16.6 \mathrm{~N}$ & $72.6 \mathrm{~W}$ & 51 & EU & $\mathrm{GE}$ & LOC & 1 & 5 & 1935 & 66 & AA & $\begin{array}{l}\text { Wolfgang Ludwig; German } \\
\text { astronomer, physicist } \\
(1743-1814) \text {. }\end{array}$ \\
\hline Kramarov & $2.3 \mathrm{~S}$ & $98.8 \mathrm{~W}$ & 20 & UR & RU & SOV & 1985 & 5 & 1991 & 0 & AA & $\begin{array}{l}\text { G.M.; Soviet space } \\
\text { scientist }(1887-1970)\end{array}$ \\
\hline Kramers & $53.6 \mathrm{~N}$ & $127.6 \mathrm{~W}$ & 61 & EU & DU & LMP & 3 & 5 & 1970 & 0 & AA & $\begin{array}{l}\text { Hendrik A.; Dutch } \\
\text { physicist (1894-1952). }\end{array}$ \\
\hline Krasnov & $29.9 \mathrm{~S}$ & $79.6 \mathrm{~W}$ & 40 & UR & RU & LOC & 1 & 5 & 1964 & 67 & AA & $\begin{array}{l}\text { Aleksander V.; Russian } \\
\text { astronomer (1866-1907). }\end{array}$ \\
\hline Krasovskiy & $3.9 \mathrm{~N}$ & $175.5 \mathrm{~W}$ & 59 & UR & SO & LOC & 4 & 5 & 1970 & 0 & AA & $\begin{array}{l}\text { Feodosiy N.; Soviet } \\
\text { geodesist (1878-1948). }\end{array}$ \\
\hline Kreiken & $9.0 \mathrm{~S}$ & $84.6 \mathrm{E}$ & 23 & $\mathrm{EU}$ & DU & LTO & $081 \mathrm{Cl}$ & 5 & 1973 & 0 & AA & $\begin{array}{l}\text { E. A.; Dutch astronomer } \\
(1896-1964) \text {. }\end{array}$ \\
\hline Krieger & $29.0 \mathrm{~N}$ & $45.6 \mathrm{~W}$ & 22 & EU & GE & LTO & 039A1 & 5 & 1935 & 66 & AA & $\begin{array}{l}\text { Johann Nepomuk; German } \\
\text { selenographer (1865-1902). }\end{array}$ \\
\hline $\begin{array}{l}\text { Krishna } \\
\text { Krogh }\end{array}$ & $\begin{array}{r}24.5 \mathrm{~N} \\
9.4 \mathrm{~N}\end{array}$ & $\begin{array}{l}11.3 \mathrm{E} \\
65.7 \mathrm{E}\end{array}$ & $\begin{array}{r}3 \\
19\end{array}$ & $\begin{array}{l}\text { AS } \\
\text { EU }\end{array}$ & $\begin{array}{l}\text { IN } \\
\text { DE }\end{array}$ & $\begin{array}{l}\text { S2 } \\
\text { LTO }\end{array}$ & $\begin{array}{l}42 \mathrm{~A} 4 \\
062 \mathrm{~B} 3\end{array}$ & $\begin{array}{l}5 \\
5\end{array}$ & $\begin{array}{l}1976 \\
1976\end{array}$ & $\begin{array}{l}0 \\
0\end{array}$ & $\begin{array}{l}\text { AA } \\
\text { AA }\end{array}$ & $\begin{array}{l}\text { Indian male name. } \\
\text { Schack August Steenberg; } \\
\text { Danish zoologist, } \\
\text { physiologist; Nobel } \\
\text { laureate (1874-1949). }\end{array}$ \\
\hline Krusenstern & $26.2 \mathrm{~S}$ & $5.9 \mathrm{E}$ & 47 & UR & RU & LAC & 95 & 5 & 1935 & 66 & AA & $\begin{array}{l}\text { Adam Johann, Baron Von; } \\
\text { Russian explorer } \\
\text { (1770-1846). }\end{array}$ \\
\hline Krylov & $35.6 \mathrm{~N}$ & $165.8 \mathrm{~W}$ & 49 & UR & So & LOC & 4 & 5 & 1970 & 0 & AA & $\begin{array}{l}\text { Aleksej } N_{\text {.; }} \text { Soviet } \\
\text { mathematician, mechanical } \\
\text { engineer (1863-1945). }\end{array}$ \\
\hline Kugler & $53.8 \mathrm{~S}$ & 103.7E & 65 & EU & GE & LMP & 3 & 5 & 1970 & 0 & AA & $\begin{array}{l}\text { F. X.; German-Babylonian } \\
\text { chronologist (1862-1929). }\end{array}$ \\
\hline & $9.8 \mathrm{~S}$ & $22.7 \mathrm{~W}$ & 6 & NA & $\mathrm{AM}$ & LTO & 076D2 & 5 & 1976 & 0 & AA & $\begin{array}{l}\text { Gerard Peter; } \\
\text { Dutch-American astronomer } \\
\text { (1905-1973). }\end{array}$ \\
\hline
\end{tabular}

lat: latitude of feature center.

long: longitude of feature center.

diam: diameter or long dimension of feature. et: ethnicity of name origin (see page $284 \mathrm{ff}$.)

quad: map quadrangle or informal name

(see page xvii ff.).

as: name approval status (see page xvii)

ad: name approval date (year).

ref: reference source for name (see page $287 \mathrm{ff}$.). 


\section{MOON}

\begin{tabular}{|c|c|c|c|c|c|c|c|c|c|c|c|c|}
\hline Name & lat & long & diam & ct & et & quad & map & as & ad & ref & $\mathbf{f t}$ & origin \\
\hline Kulik & $42.4 \mathrm{~N}$ & $154.5 \mathrm{~W}$ & 58 & UR & SO & LMP & 2 & 5 & 1970 & 0 & AA & $\begin{array}{l}\text { Leonid A.; Soviet } \\
\text { mineralogist (1883-1942). }\end{array}$ \\
\hline Kundt & $11.5 \mathrm{~S}$ & $11.5 \mathrm{~W}$ & 10 & EU & GE & LTO & $076 \mathrm{C} 2$ & 5 & 1976 & 0 & AA & $\begin{array}{l}\text { August; German physicist } \\
\text { (1839-1894). }\end{array}$ \\
\hline Kunowsky & $3.2 \mathrm{~N}$ & $32.5 \mathrm{~W}$ & 18 & EU & GE & LAC & 57 & 5 & 1935 & 66 & AA & $\begin{array}{l}\text { Georg Karl Friedrich; } \\
\text { German astronomer } \\
(1786-1846) \text {. }\end{array}$ \\
\hline Kuo Shou Ching & $8.4 \mathrm{~N}$ & $133.7 \mathrm{~W}$ & 34 & AS & $\mathrm{CH}$ & LOC & 1 & 5 & 1970 & 0 & AA & $\begin{array}{l}\text { Chinese astronomer } \\
(1231-1316) \text {. }\end{array}$ \\
\hline Kurchatov & $38.3 \mathrm{~N}$ & $142.1 \mathrm{E}$ & 106 & UR & SO & LOC & 4 & 5 & 1970 & 0 & AA & $\begin{array}{l}\text { Igor' V.; Soviet nuclear } \\
\text { physicist (1903-1960). }\end{array}$ \\
\hline La Caille & $23.8 \mathrm{~S}$ & $1.1 \mathrm{E}$ & 67 & $\mathrm{EU}$ & FR & LAC & 95 & 5 & 1961 & 67 & AA & $\begin{array}{l}\text { Nicholas Louis De; French } \\
\text { astronomer (1713-1762). }\end{array}$ \\
\hline La Condamine & $53.4 \mathrm{~N}$ & $28.2 \mathrm{~W}$ & 37 & $\mathrm{EU}$ & FR & LAC & 11 & 5 & 1961 & 67 & AA & $\begin{array}{l}\text { Charles Marie De; French } \\
\text { astronomer, physicist } \\
(1701-1774) \text {. }\end{array}$ \\
\hline La Pérouse & $10.7 \mathrm{~S}$ & $76.3 \mathrm{E}$ & 77 & EU & FR & LTO & 081D1 & 5 & 1935 & 66 & AA & $\begin{array}{l}\text { Jean Francois de Galoup, } \\
\text { Comte De La Pérouse; } \\
\text { French explorer } \\
(1741-1788) \text {. }\end{array}$ \\
\hline Lacchini & $41.7 \mathrm{~N}$ & $107.5 \mathrm{~W}$ & 58 & EU & IT & LOC & 1 & 5 & 1970 & 0 & AA & $\begin{array}{l}\text { Giovanni; Italian } \\
\text { astronomer (1884-1967). }\end{array}$ \\
\hline Lacroix & $37.9 \mathrm{~S}$ & $59.0 \mathrm{~W}$ & 37 & $\mathrm{EU}$ & FR & LAC & 110 & 5 & 1935 & 66 & AA & $\begin{array}{l}\text { Sylvestre Francois De; } \\
\text { French mathematician } \\
\text { (1765-1843). }\end{array}$ \\
\hline Lade & $1.3 \mathrm{~S}$ & $10.1 \mathrm{E}$ & 55 & $\mathrm{EU}$ & GE & LAC & 77 & 5 & 1935 & 66 & AA & $\begin{array}{l}\text { Heinrich Eduard von; } \\
\text { German astronomer } \\
\text { (1817-1904). }\end{array}$ \\
\hline Lagalla & $44.6 \mathrm{~S}$ & $22.5 \mathrm{~W}$ & 85 & EU & IT & $\mathrm{LAC}$ & 111 & 5 & 1935 & 66 & AA & $\begin{array}{l}\text { Giulio Cesare; Italian } \\
\text { philosopher (1571-1624). }\end{array}$ \\
\hline Lagrange & $32.3 \mathrm{~S}$ & $72.8 \mathrm{~W}$ & 225 & EU & IT & LOC & 1 & 5 & 1935 & 68 & AA & $\begin{array}{l}\text { Joseph Louis; Italian } \\
\text { mathematician } \\
(1736-1813)\end{array}$ \\
\hline Lalande & $4.4 \mathrm{~S}$ & $8.6 \mathrm{~W}$ & 24 & $\mathrm{EU}$ & FR & S1 & $77 \mathrm{~A} 4$ & 5 & 1935 & 66 & AA & $\begin{array}{l}\text { Joseph Jerome Le Francois } \\
\text { De; French astronomer } \\
(1732-1807) \text {. }\end{array}$ \\
\hline Lallemand & $14.3 \mathrm{~S}$ & $84.1 \mathrm{~W}$ & 18 & EU & FR & & & 5 & 1985 & 0 & AA & $\begin{array}{l}\text { Andre; French astronomer } \\
(1904-1978) \text {. }\end{array}$ \\
\hline Lamarck & $22.9 \mathrm{~S}$ & $69.8 \mathrm{~W}$ & 100 & $\mathrm{EU}$ & FR & LAC & 92 & 5 & 1964 & 68 & AA & $\begin{array}{l}\text { Jean B. P. A. De M.; } \\
\text { French natural historian } \\
\text { (1744-1829). }\end{array}$ \\
\hline Lamb & $42.9 \mathrm{~S}$ & $100.1 \mathrm{E}$ & 106 & $\mathrm{EU}$ & GB & LOC & 3 & 5 & 1970 & 0 & AA & $\begin{array}{l}\text { Sir Horace; British } \\
\text { mathematician, physicist } \\
\text { (1849-1934). }\end{array}$ \\
\hline Lambert & $25.8 \mathrm{~N}$ & $21.0 \mathrm{~W}$ & 30 & $\mathrm{EU}$ & GE & LTO & 040A3 & 5 & 1935 & 66 & AA & $\begin{array}{l}\text { Johann Heinrich; German } \\
\text { astronomer, mathematician, } \\
\text { physicist (1728-1777). }\end{array}$ \\
\hline Lamé & $14.7 \mathrm{~S}$ & $64.5 \mathrm{E}$ & 84 & $\mathrm{EU}$ & FR & LAC & 80 & 5 & 1964 & 67 & AA & $\begin{array}{l}\text { Gabriel; French } \\
\text { mathematician } \\
(1795-1870)\end{array}$ \\
\hline Lamèch & $42.7 \mathrm{~N}$ & $13.1 \mathrm{E}$ & 13 & $\mathrm{EU}$ & $\mathrm{FR}$ & LAC & 26 & 5 & 1935 & 66 & AA & $\begin{array}{l}\text { Felix Chemla; French } \\
\text { selenographer } \\
\text { (1894-1962). }\end{array}$ \\
\hline Lamont & $4.4 \mathrm{~N}$ & $23.7 \mathrm{E}$ & 106 & EU & SC & LAC & 60 & 5 & 1935 & 68 & AA & $\begin{array}{l}\text { John; Scottish astronomer } \\
(1805-1879) \text {. }\end{array}$ \\
\hline Lampland & $31.0 \mathrm{~S}$ & $131.0 \mathrm{E}$ & 65 & NA & $\mathrm{AM}$ & LOC & 1 & 5 & 1970 & 0 & AA & $\begin{array}{l}\text { Carl O.; American } \\
\text { astronomer (1873-1951). }\end{array}$ \\
\hline Landau & $41.6 \mathrm{~N}$ & $118.1 \mathrm{~W}$ & 214 & UR & SO & LMP & 2 & 5 & 1970 & 0 & AA & $\begin{array}{l}\text { Lev D.; Soviet physicist; } \\
\text { Nobel laureate } \\
\text { (1908-1968). }\end{array}$ \\
\hline Lander & $15.3 \mathrm{~S}$ & $131.8 \mathrm{E}$ & 40 & EU & GB & LTO & 084D4 & 5 & 1976 & 0 & AA & $\begin{array}{l}\text { Richard Lemon; British } \\
\text { explorer (1804-1834). }\end{array}$ \\
\hline Landsteiner & $31.3 \mathrm{~N}$ & $14.8 \mathrm{~W}$ & 6 & EU & AS & LTO & 040B2 & 5 & 1976 & 0 & AA & $\begin{array}{l}\text { Karl; Austrian-American } \\
\text { pathologist; Nobel } \\
\text { laureate (1868-1943). }\end{array}$ \\
\hline Lane & $9.5 \mathrm{~S}$ & $132.0 \mathrm{E}$ & 55 & NA & $\mathrm{AM}$ & LOC & 3 & 5 & 1970 & 0 & AA & $\begin{array}{l}\text { Jonathan } \mathrm{H} . ; \text { American } \\
\text { physicist, astrophysicist } \\
(1819-1880) \text {. }\end{array}$ \\
\hline Langemak & $10.3 \mathrm{~S}$ & $118.7 \mathrm{E}$ & 97 & UR & SO & LTO & $083 \mathrm{C} 1$ & 5 & 1970 & 0 & AA & $\begin{array}{l}\text { Georgij E.; Soviet } \\
\text { rocketry scientist } \\
(1898-1938) .\end{array}$ \\
\hline
\end{tabular}

\footnotetext{
lat: latitude of feature center.

et: ethnicity of name origin (see page $284 \mathrm{ff}$.)

long: longitude of feature center.

quad: map quadrangle or informal name

(see page xvii ff.).

as: name approval status (see page $x$ vii)

diam: diameter or long dimension of feature.

ct: continent of name origin (see page $284 \mathrm{ff}$.) map: map name or USGS map number (see page xvii ff.). ft: feature type (see page 290).

ad: name approval date (year).
ref: reference source for name (see page $287 \mathrm{ff}$.)
} 
Name

Langevin

Langley

Langmuir

Langrenus

Lansberg

Larmor

Lassell

Laue

Lauritsen

Lavoisier

Lawrence

Le Gentil

Le Monnier

Le Verrier

Leakey

Leavitt

Lebedev

Lebedinskiy

Lebesgue

Lee

Leeuwenhoek

Legendre

Lehmann

Leibnitz

Lemaître

Lents (Lenz)

Leonov

Lepaute lat

$44.3 \mathrm{~N}$

$51.1 \mathrm{~N}$

162.7E

35.7S $128.4 \mathrm{~W}$

$91 \mathrm{NA}$

$8.9 S$

$61.1 \mathrm{E}$

127 E

B

$0.3 \mathrm{~S} \quad 26.6 \mathrm{~W}$

38 EU

$32.1 \mathrm{~N} \quad 179.7 \mathrm{~W}$

97 EU

$15.5 S$

7.9W

$28.0 \mathrm{~N} \quad 96.7 \mathrm{~W}$

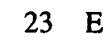

87 EU

$27.6 \mathrm{~S}$

$96.1 \mathrm{E}$

52 EU

$38.2 \mathrm{~N}$

81.2W

$7.4 \mathrm{~N}$

43.2E

$74.6 \mathrm{~S}$

$75.7 \mathrm{~W}$

$26.6 \mathrm{~N}$

$30.6 \mathrm{E}$

$40.3 \mathrm{~N}$

$20.6 \mathrm{~W}$

$3.2 \mathrm{~S}$

$37.4 \mathrm{E}$

$44.8 \mathrm{~S}$

139.3W

$47.3 \mathrm{~S}$

$107.8 \mathrm{E}$

$8.3 \mathrm{~N} \quad 164.3 \mathrm{~W}$

$5.1 \mathrm{~S}$

$89.0 \mathrm{E}$

$30.7 \mathrm{~S}$

$40.7 \mathrm{~W}$

29.3S

$178.7 \mathrm{~W}$

$28.9 S$

70.2E

$40.0 \mathrm{~S}$

$56.0 \mathrm{~W}$

$38.3 S$

179.2E

$61.2 \mathrm{~S} \quad 149.6 \mathrm{~W}$

$2.8 \mathrm{~N} \quad 102.1 \mathrm{~W}$

$19.0 \mathrm{~N} \quad 148.2 \mathrm{E}$

$33.3 \mathrm{~S}$

$33.6 \mathrm{~W}$
128 E

60 E

70 EU

24 NA

FR LOC

AM LTO

$61 \mathrm{Cl}$

$5 \quad 1973$

$5 \quad 1961$

FR LMP 3

FR LTO

043A4

$5 \quad 1935$

20 EU FR LAC 24

$\begin{array}{ll}5 & 1961\end{array}$

12 EU GB LTO

079A2 51976

66 NA AM LMP 2

$5 \quad 1970$

$5 \quad 1970$

102 UR RU LMP 2

62 UR SO LOC 4

$5 \quad 1970$

11 EU FR LTO

$081 \mathrm{~B} 3$

41 EU GB LOC

2

51976

51935

125 EU DU LM 104

$5 \quad 1970$

$\begin{array}{lllll}78 & \text { EU } & \text { FR } & \text { LAC } & 98\end{array}$

53 EU GE LAC 110

$5 \quad 1935$

$5 \quad 1935$

$5 \quad 1970$

245 EU GE LOC 4

4

$5 \quad 1970$

$5 \quad 1970$

$5 \quad 1970$

$5 \quad 1935$
21 UR RU LOC 1

33 UR SO LOC 4

16 EU FR LAC 111

\section{3}

1

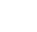

\begin{tabular}{|c|c|c|c|}
\hline 5 & 1970 & 0 & $\mathrm{AA}$ \\
\hline 5 & 1964 & 67 & $\mathrm{AA}$ \\
\hline 5 & 1970 & 0 & $\mathrm{AA}$ \\
\hline 5 & 1935 & 66 & $\mathrm{AA}$ \\
\hline
\end{tabular}

Paul; French physicist (1872-1946)

Samuel P.; American astronomer, physicist (1834-1906).

Irving; American physicist, chemist; Nobel laureate (1881-1957).

Langren, Michel Florent van; Belgian selenographer, engineer (c. 1600-1675).

Philippe van; Belgian astronomer (1561-1632)

Sir Joseph; British mathematician, physicist (1857-1942)

William; British astronomer (1799-1880).

Max T. F. von; German physicist; Nobel laureate (1879-1960).

Charles C.

Danish-American physicist (1892-1968)

68 AA Antoine Laurent; French chemist (1743-1794)

Ernest Orlando; American physicist; Nobel laureate (1901-1958)

Guillaume Hyazinthe; French astronomer (1725-1792).

67 AA

Pierre Charles; French astronomer, physicist (1715-1799).

Urbain Jean; French astronomer, mathematician (1811-1877).

Louis Seymour Bazett; British archaeologist (1903-1972).

Henrietta S.; American astronomer (1868-1921).

Pëtr N.; Russian physicist

68 AA Pëtr N.; Russ

0 AA Aleksandr I.; Soviet astrophysicist (1913-1967).

Henri Leon; French mathematician (1875-1941).

68 AA

John; British astronomer, humanitarian (1783-1866).

0 AA Antony van; Dutch microscopist (1632-1723).

Adrien Marie; French mathematician (1752-1833).

Jacob Heinrich Wilhelm; German astronomer (1800-1863)

Gottfried W.; Germa mathematician, physicist, philosopher (1646-1716).

Georges; Belgian

mathematician (1894-1966).

H. F. Emil; Russian physicist (1804-1865). Aleksej A.; Soviet cosmonaut (1934-Live).

Nicole Reine De La Briere; French astronomer (1723-1788). lat: latitude of feature center.

long: longitude of feature center.

diam: diameter or long dimension of feature.

ct: continent of name origin (see page $284 \mathrm{ff}$.) et: ethnicity of name origin (see page $284 \mathrm{ff}$.)

quad: map quadrangle or informal name

(see page xvii ff.).

map name or USGS map number (see page xvii ff.). as: name approval status (see page xvii).

ad: name approval date (year).

ref: reference source for name (see page $287 \mathrm{ff}$.). 
MOON

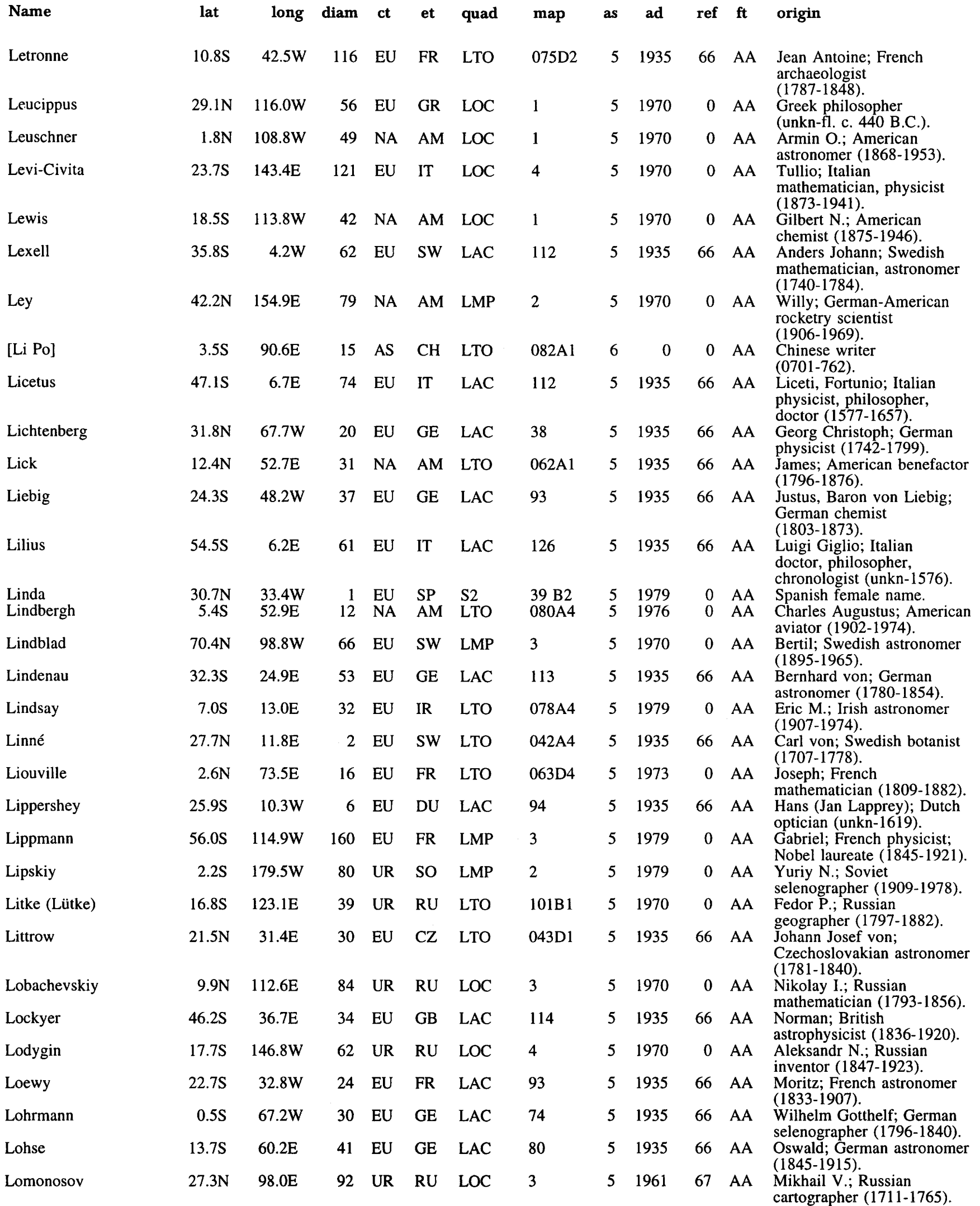

lat: latitude of feature center.

long: longitude of feature center.

diam: diameter or long dimension of feature. et: ethnicity of name origin (see page $284 \mathrm{ff}$.)

quad: map quadrangle or informal name

(see page xvii ff.). as: name approval status (see page xvii).

ad: name approval date (year).

ref: reference source for name (see page $287 \mathrm{ff}$.) 


\section{MOON}

\begin{tabular}{|c|c|c|c|c|c|c|c|c|c|c|c|c|}
\hline Name & lat & long & diam & ct & et & quad & map & as & ad & ref & $\mathbf{f t}$ & origin \\
\hline [Longfellow] & $7.5 \mathrm{~S}$ & $91.3 \mathrm{E}$ & 35 & NA & AM & LTO & $082 \mathrm{~A} 4$ & 6 & 0 & 0 & AA & Henry Wadsworth; American \\
\hline Longomontanus & $49.6 \mathrm{~S}$ & $21.8 \mathrm{~W}$ & 157 & EU & $\mathrm{DE}$ & LAC & 125 & 5 & 1935 & 66 & AA & $\begin{array}{l}\text { Christian Sorensen; Danish } \\
\text { astronomer, mathematician } \\
(1562-1647) \text {. }\end{array}$ \\
\hline [Lorca] & $24.4 \mathrm{~N}$ & $10.9 \mathrm{E}$ & 0 & $\mathrm{EU}$ & SP & LTO & $042 \mathrm{~A} 4$ & 6 & 0 & 0 & AA & $\begin{array}{l}\text { Federico Garcia; Spanish } \\
\text { writer }(1899-1936) \text {. }\end{array}$ \\
\hline Lorentz & $32.6 \mathrm{~N}$ & $95.3 \mathrm{~W}$ & 312 & EU & DU & LOC & 1 & 5 & 1970 & 0 & AA & $\begin{array}{l}\text { Hendrik A.; Dutch } \\
\text { physicist; Nobel laureate } \\
(1853-1928) \text {. }\end{array}$ \\
\hline Louise & $28.5 \mathrm{~N}$ & $34.2 \mathrm{~W}$ & 0 & EU & FR & S1 & $39 \mathrm{~B} 2$ & 5 & 1979 & 0 & AA & French female name. \\
\hline Louville & $44.0 \mathrm{~N}$ & $46.0 \mathrm{~W}$ & 36 & EU & FR & LAC & 23 & 5 & 1935 & 66 & AA & $\begin{array}{l}\text { Jacques D'Allonville, } \\
\text { Chevalier De Louville; } \\
\text { French astronomer, } \\
\text { mathematician (1671-1732). }\end{array}$ \\
\hline Love & $6.3 \mathrm{~S}$ & $129.0 \mathrm{E}$ & 84 & EU & GB & LOC & 3 & 5 & 1970 & 0 & AA & Augustus E. H.; British \\
\hline Lovelace & $82.3 \mathrm{~N}$ & $106.4 \mathrm{~W}$ & 54 & NA & $\mathrm{AM}$ & LMP & 3 & 5 & 1970 & 0 & AA & $\begin{array}{l}\text { William R.; American } \\
\text { doctor, space scientist } \\
\text { (1907-1965). }\end{array}$ \\
\hline Lovell & $36.8 \mathrm{~S}$ & $141.9 \mathrm{~W}$ & 34 & NA & AM & $\mathrm{LOC}$ & 4 & 5 & 1970 & 0 & AA & $\begin{array}{l}\text { James A., Jr.; American } \\
\text { astronaut (1928-Live). }\end{array}$ \\
\hline Lowell & $12.9 \mathrm{~S}$ & $103.1 \mathrm{~W}$ & 66 & NA & $\mathrm{AM}$ & LOC & 1 & 5 & 1970 & 0 & AA & $\begin{array}{l}\text { Percival; American } \\
\text { astronomer (1855-1916). }\end{array}$ \\
\hline Lubbock & $3.9 \mathrm{~S}$ & $41.8 \mathrm{E}$ & 13 & EU & GB & LTO & 079B 1 & 5 & 1935 & 66 & AA & $\begin{array}{l}\text { Sir John William; British } \\
\text { astronomer, mathematician } \\
(1803-1865) \text {. }\end{array}$ \\
\hline Lubiniezky & $17.8 \mathrm{~S}$ & $23.8 \mathrm{~W}$ & 43 & EU & PO & LAC & 94 & 5 & 1935 & 66 & AA & $\begin{array}{l}\text { Stanislaus; Polish } \\
\text { astronomer (1623-1675). }\end{array}$ \\
\hline Lucian & $14.3 \mathrm{~N}$ & $36.7 \mathrm{E}$ & 7 & $\mathrm{EU}$ & GR & LTO & 061A2 & 5 & 1973 & 0 & AA & $\begin{array}{l}\text { Of Samosata; Greek writer } \\
(125-190) \text {. }\end{array}$ \\
\hline Lucretius & $8.2 \mathrm{~S}$ & $120.8 \mathrm{~W}$ & 63 & $\mathrm{EU}$ & RM & LOC & 1 & 5 & 1970 & 0 & AA & $\begin{array}{l}\text { Titus C.; Roman scientific } \\
\text { philosopher (c. } 95-55 \\
\text { B.C.). }\end{array}$ \\
\hline Ludwig & $7.7 \mathrm{~S}$ & $97.4 \mathrm{E}$ & 23 & $\mathrm{EU}$ & GE & LTO & $082 \mathrm{~A} 3$ & 5 & 1973 & 0 & $\mathrm{AA}$ & $\begin{array}{l}\text { Carl Friedrich Wilhelm; } \\
\text { German physiologist } \\
(1816-1895) \text {. }\end{array}$ \\
\hline Lundmark & $39.7 \mathrm{~S}$ & $152.5 \mathrm{E}$ & 106 & $\mathrm{EU}$ & SW & LOC & 4 & 5 & 1970 & 0 & AA & $\begin{array}{l}\text { Knut E.; Swedish } \\
\text { astronomer (1889-1958). }\end{array}$ \\
\hline Luther & $33.2 \mathrm{~N}$ & $24.1 \mathrm{E}$ & 9 & $\mathrm{EU}$ & GM & LAC & 26 & 5 & 1935 & 66 & AA & $\begin{array}{l}\text { Robert; German astronomer } \\
(1822-1900) .\end{array}$ \\
\hline Lyapunov & $26.3 \mathrm{~N}$ & $89.3 \mathrm{E}$ & 66 & UR & RU & LOC & 3 & 5 & 1964 & 67 & AA & $\begin{array}{l}\text { Aleksandr M.; Russian } \\
\text { mathematician, engineer } \\
(1857-1918) \text {. }\end{array}$ \\
\hline Lyell & $13.6 \mathrm{~N}$ & $40.6 \mathrm{E}$ & 32 & $\mathrm{EU}$ & SC & LTO & 061B 1 & 5 & 1935 & 66 & AA & $\begin{array}{l}\text { Sir Charles; Scottish } \\
\text { geologist (1797-1875) }\end{array}$ \\
\hline Lyman & $64.8 \mathrm{~S}$ & $163.6 \mathrm{E}$ & 84 & NA & AM & LMP & 3 & 5 & 1970 & 0 & AA & $\begin{array}{l}\text { Theodore; American } \\
\text { physicist (1874-1954). }\end{array}$ \\
\hline Lyot & $49.8 \mathrm{~S}$ & $84.5 \mathrm{E}$ & 132 & $\mathrm{EU}$ & FR & LMP & 3 & 5 & 1964 & 67 & AA & $\begin{array}{l}\text { Bernard F.; French } \\
\text { astronomer (1897-1952). }\end{array}$ \\
\hline Mach & $18.5 \mathrm{~N}$ & $149.3 \mathrm{~W}$ & 180 & EU & AS & LOC & 4 & 5 & 1970 & 0 & AA & $\begin{array}{l}\text { Ernst; Austrian physicist, } \\
\text { philosopher (1838-1916). }\end{array}$ \\
\hline MacLaurin & $1.9 \mathrm{~S}$ & $68.0 \mathrm{E}$ & 50 & EU & SC & LTO & 080B2 & 5 & 1935 & 66 & AA & $\begin{array}{l}\text { Colin; Scottish } \\
\text { mathematician } \\
(1698-1746)\end{array}$ \\
\hline MacLear & $10.5 \mathrm{~N}$ & $20.1 \mathrm{E}$ & 20 & $\mathrm{EU}$ & IR & LM & 60 & 5 & 1961 & 67 & AA & $\begin{array}{l}\text { Thomas; Irish astronomer } \\
\text { (1794-1879). }\end{array}$ \\
\hline MacMillan & $24.2 \mathrm{~N}$ & $7.8 \mathrm{~W}$ & 7 & NA & $\mathrm{AM}$ & LTO & $041 \mathrm{~A} 4$ & 5 & 1976 & 0 & AA & $\begin{array}{l}\text { William Duncan; American } \\
\text { mathematician, astronomer } \\
\text { (1871-1948). }\end{array}$ \\
\hline Macrobius & $21.3 \mathrm{~N}$ & $46.0 \mathrm{E}$ & 64 & EU & RM & LTO & $043 C 1$ & 5 & 1935 & 66 & AA & $\begin{array}{l}\text { Ambrosius Aurelius } \\
\text { Theodosius; Roman writer } \\
\text { (unkn-fl. c. 410). }\end{array}$ \\
\hline Mädler & $11.0 \mathrm{~S}$ & $29.8 \mathrm{E}$ & 27 & EU & GE & LTO & $078 \mathrm{C} 2$ & 5 & 1935 & 66 & AA & $\begin{array}{l}\text { Johann Heinrich; German } \\
\text { astronomer (1794-1874). }\end{array}$ \\
\hline Maestlin & $4.9 \mathrm{~N}$ & $40.6 \mathrm{~W}$ & 7 & EU & GE & LAC & 57 & 5 & 1961 & 67 & AA & $\begin{array}{l}\text { Michael; German } \\
\text { mathematician (1550-1631). }\end{array}$ \\
\hline Magelhaens & $11.9 \mathrm{~S}$ & $44.1 \mathrm{E}$ & 40 & $\mathrm{EU}$ & PG & LAC & 79 & 5 & 1935 & 66 & AA & $\begin{array}{l}\text { Fernao De (Ferdinand } \\
\text { Magellan); Portuguese } \\
\text { explorer (1480-1521). }\end{array}$ \\
\hline
\end{tabular}

lat: latitude of feature center.

long: longitude of feature center.

diam: diameter or long dimension of feature.

ct: continent of name origin (see page $284 \mathrm{ff}$.) map: map name or USGS map number (see page xvii ff.). ft: feature type (see page 290).

et: ethnicity of name origin (see page $284 \mathrm{ff}$.)

quad: map quadrangle or informal name

(see page xvii ff.). as: name approval status (see page xvii).

ad: name approval date (year).

ref: reference source for name (see page $287 \mathrm{ff}$.). 


\section{MOON}

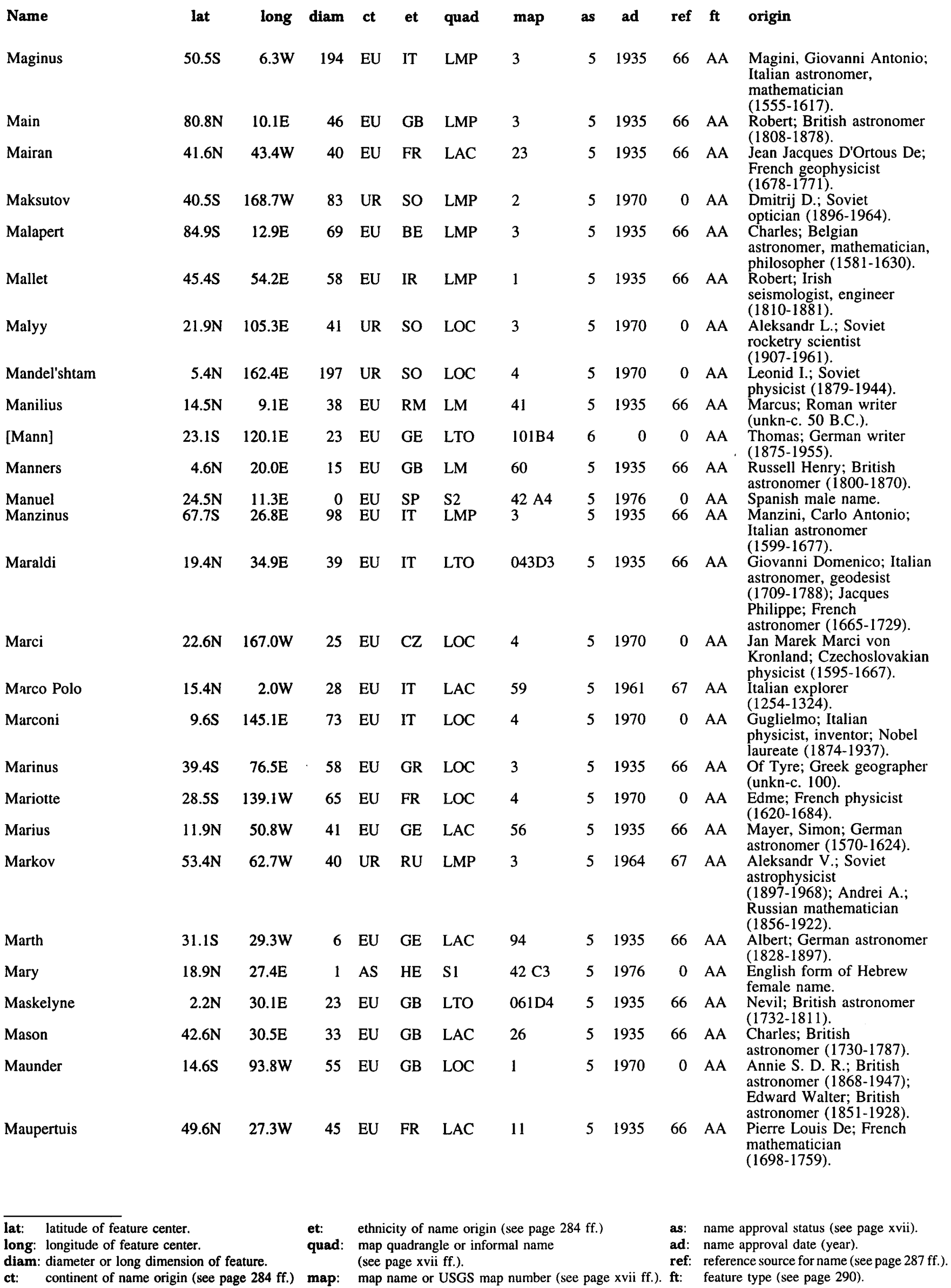




\section{MOON}

Name

Maurolycus

lat

Maury

Mavis

Maxwell

McAdie

McAuliffe

McClure

McDonald

$30.4 \mathrm{~N} \quad 20.9 \mathrm{~W}$

$29.8 \mathrm{~N} \quad 26.4 \mathrm{~W}$

$30.2 \mathrm{~N}$

$98.9 \mathrm{E}$

1 EU SC S1

$2.1 \mathrm{~N}$

92.1E

45 NA

33.0S 148.9W

19 NA AM

AM LTO

$40 \mathrm{Al}$

$5 \quad 1976$

51961

064D4

$5 \quad 1973$

$5 \quad 1988$

$30.4 \mathrm{~N}$

7 NA AM LTO

040A2

$5 \quad 1935 \quad 66 \quad$ AA

$5 \quad 1973$
McKellar
McLaughlin

McMath

McNair

35.7S $147.3 \mathrm{~W} \quad 29 \quad$ NA $\quad$ AM

McNally

Mechnikov

$22.6 \mathrm{~N} \quad 127.2 \mathrm{~W}$

47 NA AM LOC

11.0S $149.0 \mathrm{~W}$

60 UR RU LOC

43.7S $\quad 35.3 \mathrm{~W}$

126

EU SC LAC

$13.6 \mathrm{~N} \quad 96.1 \mathrm{~W}$

50 NA AM LOC

$24.3 \mathrm{~N} \quad 123.0 \mathrm{E}$

52 NA AM LOC 3

Meggers

10.5S 112.7E

87 EU AS LOC

$8.1 \mathrm{~N} \quad 121.8 \mathrm{E}$

$48.8 \mathrm{~S} \quad 109.4 \mathrm{~W}$

18 EU GR S2

138 EU AS LMP

$5.7 \mathrm{~N} \quad 140.9 \mathrm{E}$

313 UR RU LTO

$16.3 \mathrm{~N} \quad 16.0 \mathrm{E}$

$26 \mathrm{EU}$

Menelaus

Menzel

$$
3.4 \mathrm{~N}
$$

$36.9 \mathrm{E}$

3 NA AM LTO

$61 \mathrm{D} 3$

Mercator

$\begin{array}{lrrrrrr}15.7 \mathrm{~S} & 170.8 \mathrm{~W} & 51 & \text { NA } & \text { CA } & \text { LOC } & 4 \\ 47.1 \mathrm{~N} & 92.9 \mathrm{~W} & 79 & \text { NA } & \text { AM } & \text { LOC } & 1 \\ 17.3 \mathrm{~N} & 165.6 \mathrm{~W} & 86 & \text { NA } & \text { AM } & \text { LOC } & 44\end{array}$

$\begin{array}{lllllll}29.3 S & 26.1 \mathrm{~W} & 46 & \mathrm{EU} & \mathrm{BE} & \mathrm{LAC} & 94\end{array}$

$29.3 S \quad 26.1 \mathrm{~W}$

51970

$5 \quad 1970$
$5 \quad 1970$ $\begin{array}{rr}0 & \text { AA } \\ 67 & \text { AA }\end{array}$

0 AA

$88 \quad A A$
Maurolico, Francesco; Italian mathematician (1494-1575).

Matthew Fontaine; American oceanographer (1806-1873); Antonia C.; American astronomer (1866-1952).

Scottish female name.

ames Clerk; British physicist (1831-1879). Alexander George; American meteorologist (1863-1943).

Sharon Christa; civilian school teacher member of the Challenger crew (1948-1986); previous designation Borman Y.

Robert Le Mesurier;

British explorer (1807-1873).

William Johnson; American benefactor (1844-1926); Thomas Logie; Scottish selenographer

(unkn.-1973).

Andrew; Canadian astronomer (1910-1960).

Dean B.; American astronomer (1901-1965).

Francis C.; American engineer, astronomer (1867-1938); Robert R.; American astronomer (1891-1962).

$\begin{array}{llll}5 & 1988 & 88 & \text { AA }\end{array}$ the Challenger crew (1950-1986); previous designation Borman $\mathrm{A}$.

Paul A.; American astronomer (1890-1955). Il'ya I.; Russian-French bacteriologist; Nobel laureate (1845-1916).

Arthur Butler Phillips;

Scottish astronomer (1860-1926).

C. E. Kenneth; Anglo-American photographer (1882-1960).

William F.; American physicist (1888-1968). Lise; Austrian physicist

$51970 \quad 0$ AA $\begin{aligned} & \text { Lise; Austria } \\ & (1878-1968) .\end{aligned}$

$65 \mathrm{Cl} 551979 \quad 0 \quad \mathrm{AA}$ Greek female name.

Gregor J.; Austrian

biologist (1822-1884).

Dmitrij I.; Russian chemist (1834-1907).

Of Alexandria; Greek geometer, astronomer (c. A.D. 98).

Donald Howard; American astrophysicist, Smithsonian researcher (1901-1976).

Gerard De Kremer (Gerhardus Mercator); Belgian cartographer, geographer, mathematician (1512-1594).

\footnotetext{
lat: latitude of feature center. et: ethnicity of name origin (see page $284 \mathrm{ff}$.)

as: name approval status (see page xvii). long: longitude of feature center.

quad: map quadrangle or informal name

ad: name approval date (year).

diam: diameter or long dimension of feature. (see page $x$ vii $f f$.)

ref: reference source for name (see page $287 \mathrm{ff}$.).

ct: continent of name origin (see page $284 \mathrm{ff}$.) map: map name or USGS map number (see page xvii ff.). ft: feature type (see page 290 ).
} 


\section{MOON}

\begin{tabular}{|c|c|c|c|c|c|c|c|c|c|c|c|c|}
\hline Name & lat & long & diam & ct & et & quad & map & as & ad & ref & $\mathbf{f t}$ & origin \\
\hline Mercurius & $46.6 \mathrm{~N}$ & $66.2 \mathrm{E}$ & 67 & EU & RM & LOC & 3 & 5 & 1935 & 66 & AA & $\begin{array}{l}\text { Mercury; Roman mythical } \\
\text { messenger. }\end{array}$ \\
\hline Merrill & $75.2 \mathrm{~N}$ & $116.3 \mathrm{~W}$ & 57 & NA & $\mathrm{AM}$ & LMP & 3 & 5 & 1970 & 0 & $\mathrm{AA}$ & $\begin{array}{l}\text { Paul W.; American } \\
\text { astronomer (1887-1961). }\end{array}$ \\
\hline Mersenius & $21.5 \mathrm{~S}$ & $49.2 W$ & 84 & EU & FR & LAC & 93 & 5 & 1935 & 66 & AA & $\begin{array}{l}\text { Mersenne, Marin; French } \\
\text { mathematician, physicist } \\
(1588-1648) \text {. }\end{array}$ \\
\hline Meshcherskiy & $12.2 \mathrm{~N}$ & $125.5 \mathrm{E}$ & 65 & UR & RU & LOC & 3 & 5 & 1970 & 0 & AA & $\begin{array}{l}\text { Ivan V.; Russian } \\
\text { mathematician } \\
(1859-1935) .\end{array}$ \\
\hline Messala & $39.2 \mathrm{~N}$ & $60.5 \mathrm{E}$ & 125 & AS & JW & LOC & 3 & 5 & 1935 & 66 & AA & $\begin{array}{l}\text { (Ma-Sa-Allah); Jewish } \\
\text { astronomer (unkn- c. } \\
815 \text { ). }\end{array}$ \\
\hline Messier & $1.9 \mathrm{~S}$ & $47.6 \mathrm{E}$ & 11 & EU & FR & LTO & 079B 1 & 5 & 1935 & 66 & AA & $\begin{array}{l}\text { Charles; French astronomer } \\
(1730-1817)\end{array}$ \\
\hline Metius & $40.3 \mathrm{~S}$ & 43.3E & 87 & EU & DU & LAC & 114 & 5 & 1935 & 66 & AA & $\begin{array}{l}\text { Adriaan Adriaanszoon; } \\
\text { Dutch astronomer } \\
\text { (1571-1635). }\end{array}$ \\
\hline Meton & $73.6 \mathrm{~N}$ & $18.8 \mathrm{E}$ & 130 & $\mathrm{EU}$ & GR & LMP & 3 & 5 & 1935 & 66 & AA & $\begin{array}{l}\text { Greek astronomer (unkn-fl. } \\
432 \text { B.C.). }\end{array}$ \\
\hline Mezentsev & $72.1 \mathrm{~N}$ & $128.7 \mathrm{~W}$ & 89 & UR & SO & LMP & 3 & 5 & 1970 & 0 & AA & $\begin{array}{l}\text { Yurij B.; Soviet rocket } \\
\text { scientist }(1929-1965) .\end{array}$ \\
\hline $\begin{array}{l}\text { Michael } \\
\text { Michelson }\end{array}$ & $\begin{array}{r}25.1 \mathrm{~N} \\
7.2 \mathrm{~N}\end{array}$ & $\begin{array}{c}0.2 \mathrm{E} \\
120.7 \mathrm{~W}\end{array}$ & $\begin{array}{r}4 \\
123\end{array}$ & $\begin{array}{l}\text { EU } \\
\text { EU }\end{array}$ & $\begin{array}{l}\text { EN } \\
\text { GE }\end{array}$ & $\begin{array}{l}\text { S1 } \\
\text { LOC }\end{array}$ & $\begin{array}{l}41 \mathrm{~A} 3 \\
1\end{array}$ & $\begin{array}{l}5 \\
5\end{array}$ & $\begin{array}{l}1976 \\
1970\end{array}$ & $\begin{array}{l}0 \\
0\end{array}$ & $\begin{array}{l}\text { AA } \\
\text { AA }\end{array}$ & $\begin{array}{l}\text { English male name. } \\
\text { Albert A.; German-Americar }\end{array}$ \\
\hline & & & & & & & & & & & & $\begin{array}{l}\text { physicist; Nobel laureate } \\
(1852-1931) \text {. }\end{array}$ \\
\hline Milankovič & $77.2 \mathrm{~N}$ & $168.8 \mathrm{E}$ & 101 & EU & YU & LMP & 3 & 5 & 1970 & 0 & AA & $\begin{array}{l}\text { M.; Yugoslavian astronomer } \\
(1879-1958) \text {. }\end{array}$ \\
\hline Milichius & $10.0 \mathrm{~N}$ & $30.2 \mathrm{~W}$ & 12 & EU & GE & LAC & 57 & 5 & 1935 & 66 & AA & $\begin{array}{l}\text { Milich, Jacob; German } \\
\text { doctor, mathematician, } \\
\text { astronomer (1501-1559). }\end{array}$ \\
\hline Miller & $39.3 \mathrm{~S}$ & $0.8 \mathrm{E}$ & 61 & EU & GB & LAC & 112 & 5 & 1935 & 66 & AA & $\begin{array}{l}\text { William Allen; British } \\
\text { chemist (1817-1870). }\end{array}$ \\
\hline Millikan & $46.8 \mathrm{~N}$ & $121.5 \mathrm{E}$ & 98 & NA & $\mathrm{AM}$ & LMP & 3 & 5 & 1970 & 0 & AA & $\begin{array}{l}\text { Robert A.; American } \\
\text { physicist; Nobel laureate } \\
(1868-1953) \text {. }\end{array}$ \\
\hline Mills & $8.6 \mathrm{~N}$ & $156.0 \mathrm{E}$ & 32 & NA & $\mathrm{AM}$ & LOC & 4 & 5 & 1970 & 0 & AA & $\begin{array}{l}\text { Mark M.; American } \\
\text { physicist (1917-1958). }\end{array}$ \\
\hline Milne & $31.4 \mathrm{~S}$ & $112.2 \mathrm{E}$ & 272 & EU & GB & LOC & 3 & 5 & 1970 & 0 & AA & $\begin{array}{l}\text { E. Arthur; British } \\
\text { mathematician, } \\
\text { astrophysicist } \\
(1896-1950) .\end{array}$ \\
\hline [Milton] & $1.6 \mathrm{~S}$ & $90.9 \mathrm{E}$ & 35 & $\mathrm{EU}$ & GB & LTO & 082A1 & 6 & 0 & 0 & AA & $\begin{array}{l}\text { John; British writer } \\
(1608-1674)\end{array}$ \\
\hline Mineur & $25.0 \mathrm{~N}$ & $161.3 \mathrm{~W}$ & 73 & $\mathrm{EU}$ & FR & LOC & 4 & 5 & 1970 & 0 & AA & $\begin{array}{l}\text { Henri; French } \\
\text { mathematician, astronomer } \\
(1899-1954) \text {. }\end{array}$ \\
\hline Minkowskı & $56.5 \mathrm{~S}$ & $146.0 \mathrm{~W}$ & 113 & NA & AM & LMP & 3 & 5 & 1970 & 0 & AA & $\begin{array}{l}\text { Hermann; German } \\
\text { mathematician (1864-1909); } \\
\text { Rudolph L. B.; American } \\
\text { astronomer (1895-1976). }\end{array}$ \\
\hline DVIItacto & $07.0 \mathrm{~s}$ & $177.0 \mathrm{~L}$ & $12 J$ & LU & DU & LIVIT & 3 & J & 1970 & 0 & AA & $\begin{array}{l}\text { astronomer, astrophysicist } \\
(1893-1970) \text {. }\end{array}$ \\
\hline Mitchell & $49.7 \mathrm{~N}$ & $20.2 \mathrm{E}$ & 30 & NA & $\mathrm{AM}$ & LAC & 13 & 5 & 1935 & 66 & AA & $\begin{array}{l}\text { Maria; American astronomer } \\
(1818-1889) \text {. }\end{array}$ \\
\hline Mitra & $18.0 \mathrm{~N}$ & $154.7 \mathrm{~W}$ & 92 & AS & IN & LOC & 4 & 5 & 1970 & 0 & AA & $\begin{array}{l}\text { S. K.; Indian physicist } \\
(1890-1963) .\end{array}$ \\
\hline Möbius & $15.8 \mathrm{~N}$ & 101.2E & 50 & EU & GE & LOC & 3 & 5 & 1970 & 0 & AA & $\begin{array}{l}\text { August F.; German } \\
\text { mathematician, astronomer } \\
(1790-1868) \text {. }\end{array}$ \\
\hline Mohoróvičic & $19.0 \mathrm{~S}$ & $165.0 \mathrm{~W}$ & 51 & EU & YU & LOC & 4 & 5 & 1970 & 0 & AA & $\begin{array}{l}\text { Andrija; Croatian } \\
\text { geophysicist (1857-1936). }\end{array}$ \\
\hline Moigno & $66.4 \mathrm{~N}$ & $28.9 \mathrm{E}$ & 36 & EU & FR & LMP & 3 & 5 & 1935 & 66 & AA & $\begin{array}{l}\text { Francois Napoleon Marie; } \\
\text { French mathematician, } \\
\text { physicist (1804-1884). }\end{array}$ \\
\hline Moiseev & $9.5 \mathrm{~N}$ & $103.3 \mathrm{E}$ & 59 & UR & $\mathrm{SO}$ & LOC & 3 & 5 & $19 / 0$ & 0 & AA & $\begin{array}{l}\text { Nikolaj D.; Soviet } \\
\text { astronomer (1902-1955). }\end{array}$ \\
\hline & & $137.4 \mathrm{E}$ & 21 & EU & FR & LTO & 066D2 & 5 & 1976 & 0 & AA & $\begin{array}{l}\text { Ferdinand Frederic Henri; } \\
\text { French chemist; Nobel } \\
\text { laureate (1852-1907). }\end{array}$ \\
\hline
\end{tabular}

lat: latitude of feature center.

long: longitude of feature center.

diam: diameter or long dimension of feature. et: ethnicity of name origin (see page $284 \mathrm{ff}$.)

quad: map quadrangle or informal name

(see page xvii ff.). as: name approval status (see page xvii).

ad: name approval date (year)

ref: reference source for name (see page $287 \mathrm{ff}$.). 


\section{MOON}

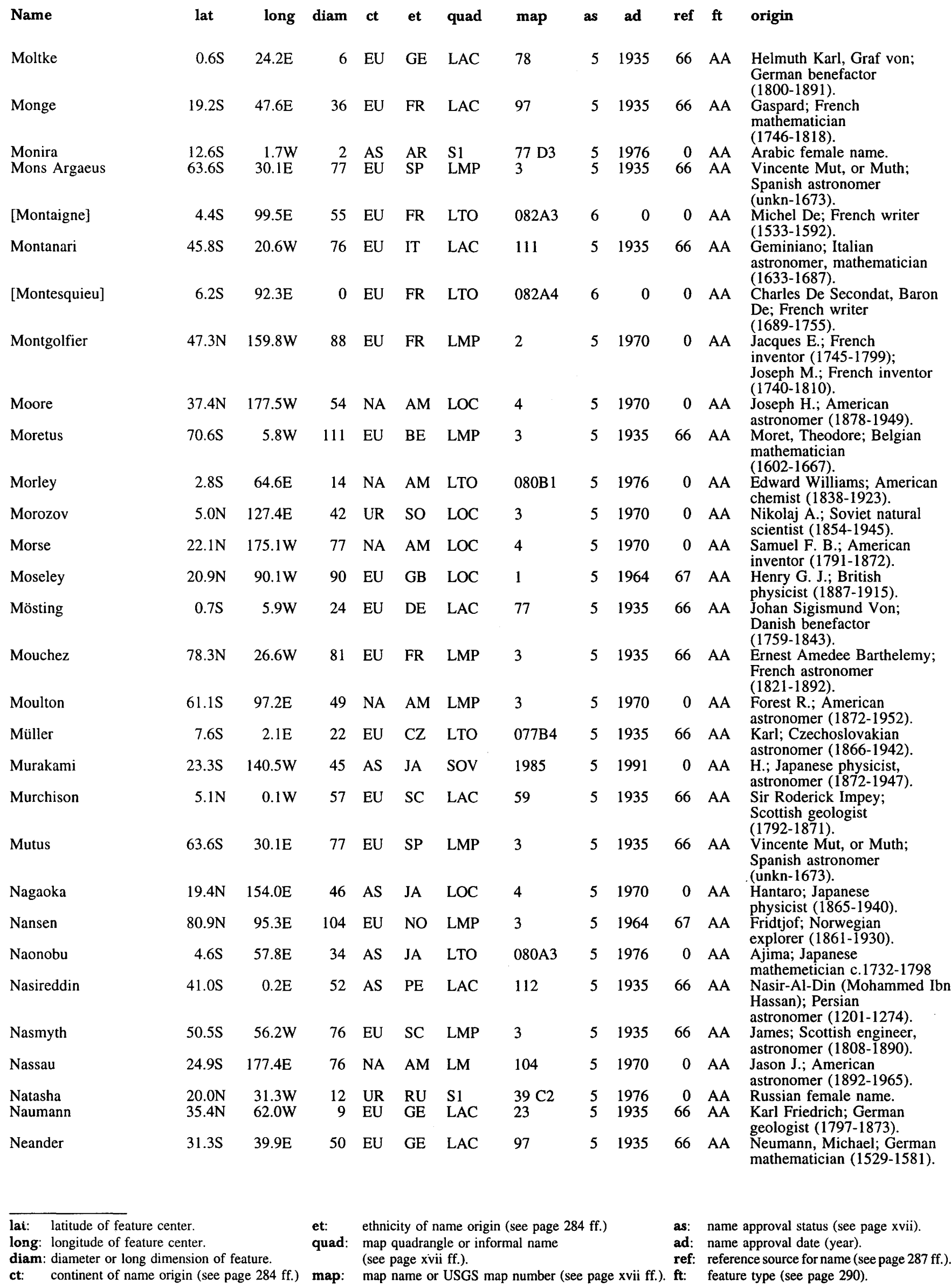


MOON

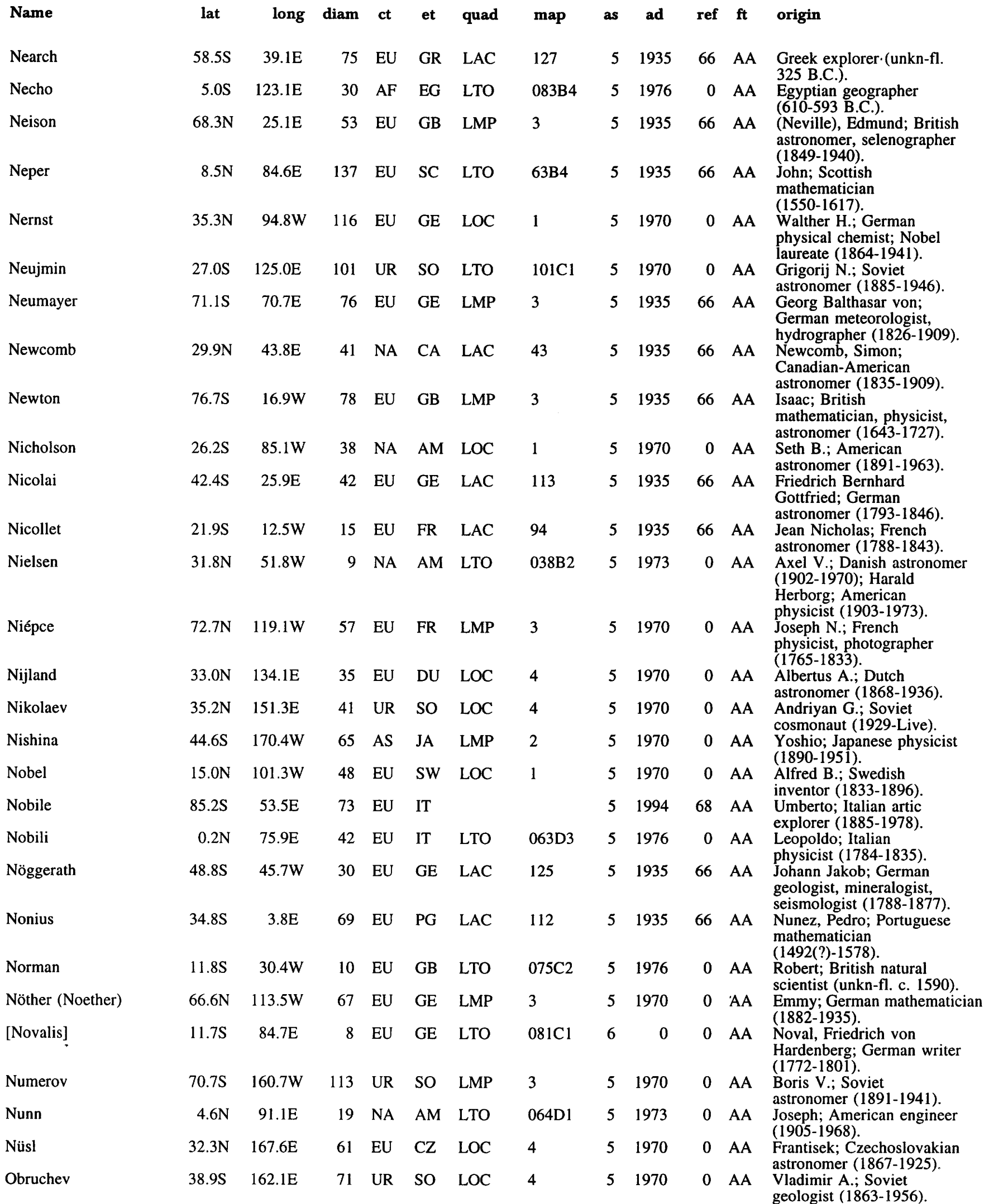

\footnotetext{
lat: latitude of feature center. long: longitude of feature center.

diam: diameter or long dimension of feature.

et: ethnicity of name origin (see page $284 \mathrm{ff}$.)

as: name approval status (see page xvii).

quad: map quadrangle or informal name

ad: name approval date (year).

(see page xvii ff.).

ref: reference source for name (see page $287 \mathrm{ff}$.).

ct: continent of name origin (see page $284 \mathrm{ff}$.) map: map name or USGS map number (see page xvii ff.). ft: feature type (see page 290).
} 
MOON

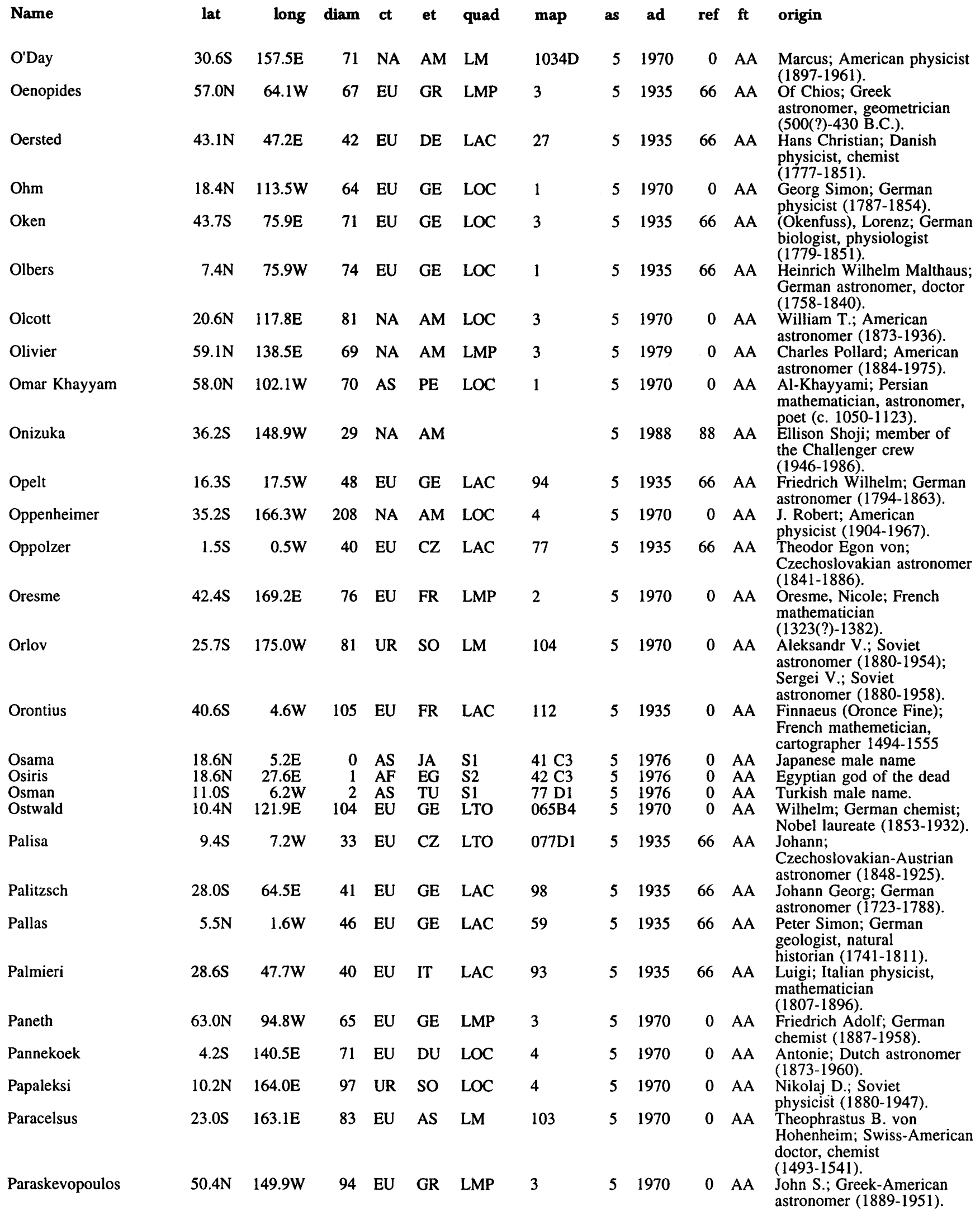

lat: latitude of feature center.

long: longitude of feature center.

diam: diameter or long dimension of feature.

ct: continent of name origin (see page $284 \mathrm{ff}$.) map: map name or USGS map number (see page xvii ff.). et: ethnicity of name origin (see page $284 \mathrm{ff}$.)

quad: map quadrangle or informal name

(see page xvii ff.).

as: name approval status (see page xvii).

ad: name approval date (year).

ref: reference source for name (see page $287 \mathrm{ff}$.) 
MOON

\begin{tabular}{|c|c|c|c|c|c|c|c|c|c|c|c|c|}
\hline Name & lat & long & diam & ct & et & quad & map & as & ad & ref & $\mathrm{ft}$ & origin \\
\hline Parenago & $25.9 \mathrm{~N}$ & $108.5 \mathrm{~W}$ & 93 & UR & SO & LOC & 1 & 5 & 1970 & 0 & $\mathrm{AA}$ & $\begin{array}{l}\text { Pavel P.; Soviet } \\
\text { astronomer (1906-1960). }\end{array}$ \\
\hline Parkhurst & $33.4 S$ & $103.6 \mathrm{E}$ & 96 & NA & $\mathrm{AM}$ & LOC & 3 & 5 & 1970 & 0 & AA & $\begin{array}{l}\text { John A.; American } \\
\text { astronomer (1861-1925). }\end{array}$ \\
\hline Parrot & $14.5 \mathrm{~S}$ & $3.3 \mathrm{E}$ & 70 & UR & RU & LAC & 77 & 5 & 1935 & 66 & AA & $\begin{array}{l}\text { Johann Jacob F. W.; } \\
\text { Russian doctor, physicist } \\
(1792-1840) \text {. }\end{array}$ \\
\hline Parry & $7.9 \mathrm{~S}$ & $15.8 \mathrm{~W}$ & 47 & $\mathrm{EU}$ & GB & LTO & $076 \mathrm{Cl}$ & 5 & 1935 & 66 & AA & $\begin{array}{l}\text { William Edward; British } \\
\text { explorer (1790-1855). }\end{array}$ \\
\hline Parsons & $37.3 \mathrm{~N}$ & $171.2 \mathrm{~W}$ & 40 & NA & $\mathrm{AM}$ & LOC & 4 & 5 & 1970 & 0 & AA & $\begin{array}{l}\text { John W.; American rocketry } \\
\text { scientist }(1913-1952) \text {. }\end{array}$ \\
\hline Pascal & $74.6 \mathrm{~N}$ & $70.3 W$ & 115 & EU & $\mathrm{FR}$ & LMP & 3 & 5 & 1964 & 67 & AA & $\begin{array}{l}\text { Blaise; French } \\
\text { mathematician } \\
(1623-1662)\end{array}$ \\
\hline Paschen & $13.5 \mathrm{~S}$ & $139.8 \mathrm{~W}$ & 124 & EU & GE & LOC & 4 & 5 & 1970 & 0 & AA & $\begin{array}{l}\text { Friedrich; German } \\
\text { physicist }(1865-1940) .\end{array}$ \\
\hline Pasteur & $11.9 \mathrm{~S}$ & $104.6 \mathrm{E}$ & 224 & $\mathrm{EU}$ & FR & LOC & 3 & 5 & 1970 & 0 & AA & $\begin{array}{l}\text { Louis; French chemist, } \\
\text { microbiologist } \\
(1822-1895)\end{array}$ \\
\hline $\begin{array}{l}\text { Patricia } \\
\text { Patsaev }\end{array}$ & $\begin{array}{l}25.0 \mathrm{~N} \\
16.7 \mathrm{~S}\end{array}$ & $\begin{array}{r}0.3 \mathrm{E} \\
133.4 \mathrm{E}\end{array}$ & $\begin{array}{r}5 \\
55\end{array}$ & $\begin{array}{l}\text { EU } \\
\text { UR }\end{array}$ & $\begin{array}{l}\text { GB } \\
\text { SO }\end{array}$ & $\begin{array}{l}\text { S1 } \\
\text { LTO }\end{array}$ & $\begin{array}{l}41 \mathrm{~A} 3 \\
102 \mathrm{~A} 1\end{array}$ & $\begin{array}{l}5 \\
5\end{array}$ & $\begin{array}{l}1976 \\
1973\end{array}$ & $\begin{array}{l}0 \\
0\end{array}$ & $\begin{array}{l}\text { AA } \\
\text { AA }\end{array}$ & $\begin{array}{l}\text { English female name. } \\
\text { Viktor I.; Soviet engineer } \\
(1933-1971) \text {. }\end{array}$ \\
\hline Pauli & $44.5 S$ & $136.4 \mathrm{E}$ & 84 & EU & AS & LMP & 2 & 5 & 1970 & 0 & AA & $\begin{array}{l}\text { Wolfgang; } \\
\text { Austrian-American } \\
\text { physicist; Nobel laureate } \\
(1900-1958) \text {. }\end{array}$ \\
\hline Pavlov & $28.8 \mathrm{~S}$ & 142.5E & 148 & UR & SO & LOC & 4 & 5 & 1970 & 0 & AA & $\begin{array}{l}\text { Ivan P.; Soviet } \\
\text { physiologist; Nobel } \\
\text { laureate (1849-1936). }\end{array}$ \\
\hline Pawsey & $44.5 \mathrm{~N}$ & $145.0 \mathrm{E}$ & 60 & OC & $\mathrm{AU}$ & LMP & 2 & 5 & 1970 & 0 & AA & $\begin{array}{l}\text { Joseph L.; Australian } \\
\text { radio astronomer } \\
(1908-1962) .\end{array}$ \\
\hline Peary & $88.6 \mathrm{~N}$ & $33.0 \mathrm{E}$ & 73 & NA & $\mathrm{AM}$ & LMP & 3 & 5 & 1964 & 67 & AA & $\begin{array}{l}\text { Robert E.; American } \\
\text { explorer (1856-1920). }\end{array}$ \\
\hline Pease & $12.5 \mathrm{~N}$ & $106.1 \mathrm{~W}$ & 38 & NA & AM & LOC & 1 & 5 & 1970 & 0 & AA & $\begin{array}{l}\text { Francis G.; American } \\
\text { astronomer }(1881-1938) \text {. }\end{array}$ \\
\hline Peek & $2.6 \mathrm{~N}$ & $86.9 \mathrm{E}$ & 12 & EU & GB & LTO & $063 \mathrm{C} 3$ & 5 & 1973 & 0 & AA & $\begin{array}{l}\text { Bertrand Meigh; British } \\
\text { astronomer (1891-1965). }\end{array}$ \\
\hline Peirce & $18.3 \mathrm{~N}$ & $53.5 \mathrm{E}$ & 18 & NA & $\mathrm{AM}$ & LTO & 044D4 & 5 & 1935 & 66 & AA & $\begin{array}{l}\text { Benjamin; American } \\
\text { mathematician, astronomer } \\
(1809-1880) \text {. }\end{array}$ \\
\hline Peirescius & $46.5 S$ & $67.6 \mathrm{E}$ & 61 & $\mathrm{EU}$ & FR & LMP & 1 & 5 & 1935 & 66 & AA & $\begin{array}{l}\text { Peiresc, Nicolas Claude } \\
\text { Fabri De; French } \\
\text { astronomer, archaeologist } \\
(1580-1637) \text {. }\end{array}$ \\
\hline Pentland & $64.6 \mathrm{~S}$ & $11.5 \mathrm{E}$ & 56 & $\mathrm{EU}$ & IR & LMP & 3 & 5 & 1935 & 66 & AA & $\begin{array}{l}\text { Joseph Barclay; Irish } \\
\text { geographer (1797-1873). }\end{array}$ \\
\hline Perel'man & $24.0 \mathrm{~S}$ & $106.0 \mathrm{E}$ & 46 & UR & SO & LOC & 3 & 5 & 1970 & 0 & AA & $\begin{array}{l}\text { Yakov I.; Soviet rocketry } \\
\text { scientist }(1882-1942)\end{array}$ \\
\hline Perepelkin & $10.0 \mathrm{~S}$ & $129.0 \mathrm{E}$ & 97 & UR & SO & LOC & 3 & 5 & 1970 & 0 & AA & $\begin{array}{l}\text { Evgenij J.; Soviet } \\
\text { astrophysicist } \\
\text { (1906-1940). }\end{array}$ \\
\hline Perkin & $47.2 \mathrm{~N}$ & $175.9 \mathrm{~W}$ & 62 & NA & $\mathrm{AM}$ & LMP & 2 & 5 & 1970 & 0 & AA & $\begin{array}{l}\text { Richard S.; American } \\
\text { telescope manufacturer } \\
\text { (1906-1969). }\end{array}$ \\
\hline Perrine & $42.5 \mathrm{~N}$ & $127.8 \mathrm{~W}$ & 86 & NA & $\mathrm{AM}$ & LMP & 2 & 5 & 1970 & 0 & AA & $\begin{array}{l}\text { Charles D.; American } \\
\text { astronomer }(1867-1951) \text {. }\end{array}$ \\
\hline Petavius & $25.1 \mathrm{~S}$ & $60.4 \mathrm{E}$ & 188 & $\mathrm{EU}$ & FR & $\mathrm{LAC}$ & 98 & 5 & 1935 & 66 & AA & $\begin{array}{l}\text { Petau, Denis; French } \\
\text { chronologist, astronomer } \\
\text { (1583-1652). }\end{array}$ \\
\hline Petermann & $74.2 \mathrm{~N}$ & $66.3 \mathrm{E}$ & 73 & EU & GE & LMP & 3 & 5 & 1935 & 66 & AA & $\begin{array}{l}\text { August Heinrich; German } \\
\text { geographer (1822-1878). }\end{array}$ \\
\hline Peters & $68.1 \mathrm{~N}$ & $29.5 \mathrm{E}$ & 15 & $\mathrm{EU}$ & GE & LMP & 3 & 5 & 1935 & 66 & AA & $\begin{array}{l}\text { Christian August } \\
\text { Friedrich; German } \\
\text { astronomer (1806-1880). }\end{array}$ \\
\hline Petit & $2.3 \mathrm{~N}$ & $63.5 \mathrm{E}$ & 5 & EU & FR & LTO & $062 \mathrm{C} 4$ & 5 & 1976 & 0 & AA & $\begin{array}{l}\text { Alexis Therese; French } \\
\text { physicist (1771-1820). }\end{array}$ \\
\hline Petrie & $45.3 \mathrm{~N}$ & $108.4 \mathrm{E}$ & 33 & NA & CA & LOC & 3 & 5 & 1970 & 0 & AA & $\begin{array}{l}\text { Robert M.; Canadian } \\
\text { astronomer (1906-1966). }\end{array}$ \\
\hline Petropavlovskiy & $37.2 \mathrm{~N}$ & $114.8 \mathrm{~W}$ & 63 & UR & SO & LOC & 1 & 5 & 1970 & 0 & AA & $\begin{array}{l}\text { Boris S.; Soviet rocketry } \\
\text { engineer (1898-1933). }\end{array}$ \\
\hline
\end{tabular}

\footnotetext{
lat: latitude of feature center.

long: longitude of feature center.

diam: diameter or long dimension of feature.

et: $\quad$ ethnicity of name origin (see page $284 \mathrm{ff}$.)

quad: map quadrangle or informal name

(see page xvii ff.).

as: name approval status (see page xvii).

ad: name approval date (year).

ref: reference source for name (see page $287 \mathrm{ff}$.).

ct: continent of name origin (see page $284 \mathrm{ff}$.) map: map name or USGS map number (see page xvii ff.). ft: feature type (see page 290).
} 
MOON

\begin{tabular}{|c|c|c|c|c|c|c|c|c|c|c|c|c|}
\hline Name & lat & long & diam & ct & et & quad & map & as & ad & ref & $\mathbf{f t}$ & origin \\
\hline Petrov & $61.4 \mathrm{~S}$ & $88.0 \mathrm{E}$ & 49 & UR & SO & LMP & 3 & 5 & 1970 & 0 & AA & $\begin{array}{l}\text { Evgenij S.; Soviet } \\
\text { rocketry scientist } \\
(1900-1942) .\end{array}$ \\
\hline Pettit & $27.5 S$ & $86.6 \mathrm{~W}$ & 35 & NA & $\mathrm{AM}$ & LOC & 1 & 5 & 1970 & 0 & AA & $\begin{array}{l}\text { Edison; American } \\
\text { astronomer (1889-1962). }\end{array}$ \\
\hline Petzval & $62.7 \mathrm{~S}$ & $110.4 \mathrm{~W}$ & 90 & EU & AS & LMP & 3 & 5 & 1970 & 0 & $\mathrm{AA}$ & $\begin{array}{l}\text { Joseph von; Austrian } \\
\text { optician (1807-1891). }\end{array}$ \\
\hline Phillips & $26.6 \mathrm{~S}$ & $75.3 \mathrm{E}$ & 122 & EU & GB & LOC & 3 & 5 & 1935 & 66 & AA & $\begin{array}{l}\text { John; British geologist, } \\
\text { astronomer (1800-1874). }\end{array}$ \\
\hline Philolaus & $72.1 \mathrm{~N}$ & $32.4 \mathrm{~W}$ & 70 & $\mathrm{EU}$ & GR & LMP & 3 & 5 & 1935 & 66 & AA & $\begin{array}{l}\text { Of Croton; Greek } \\
\text { mathematician, astronomer, } \\
\text { philosopher (unkn-fl. } 400 \\
\text { B.C.). }\end{array}$ \\
\hline Phocylides & $52.7 \mathrm{~S}$ & $57.0 \mathrm{~W}$ & 121 & $\mathrm{EU}$ & DU & LMP & 3 & 5 & 1935 & 66 & AA & $\begin{array}{l}\text { Johannes Phocylides } \\
\text { Holwarda (Jan Fokker); } \\
\text { Dutch astronomer } \\
\text { (1618-1651). }\end{array}$ \\
\hline Piazzi & $36.6 \mathrm{~S}$ & $67.9 \mathrm{~W}$ & 134 & EU & IT & LOC & 1 & 5 & 1935 & 66 & AA & $\begin{array}{l}\text { Giuseppe; Italian } \\
\text { astronomer (1746-1826). }\end{array}$ \\
\hline Piazzi Smyth & $41.9 \mathrm{~N}$ & $3.2 \mathrm{~W}$ & 13 & EU & $\mathrm{SC}$ & LMP & 1 & 5 & 1935 & 66 & $\mathrm{AA}$ & $\begin{array}{l}\text { Charles; Scottish } \\
\text { astronomer (1819-1900). }\end{array}$ \\
\hline Picard & $14.6 \mathrm{~N}$ & $54.7 \mathrm{E}$ & 22 & EU & FR & LTO & $062 \mathrm{~A} 2$ & 5 & 1935 & 66 & AA & $\begin{array}{l}\text { Jean; French astronomer } \\
(1620-1682) \text {. }\end{array}$ \\
\hline Piccolomini & $29.7 \mathrm{~S}$ & $32.2 \mathrm{E}$ & 87 & EU & IT & $\mathrm{LAC}$ & 97 & 5 & 1935 & 66 & AA & $\begin{array}{l}\text { Alessandro; Italian } \\
\text { astronomer (1508-1578). }\end{array}$ \\
\hline Pickering & $2.9 \mathrm{~S}$ & $7.0 \mathrm{E}$ & 15 & NA & AM & LAC & 77 & 5 & 1935 & 66 & AA & $\begin{array}{l}\text { Edward Charles; American } \\
\text { astronomer (1846-1919); } \\
\text { Willaim H.; American } \\
\text { astronomer (1858-1938). }\end{array}$ \\
\hline Pictet & $43.6 \mathrm{~S}$ & $7.4 \mathrm{~W}$ & 62 & $\mathrm{EU}$ & $\mathrm{SZ}$ & LAC & 112 & 5 & 1935 & 66 & AA & $\begin{array}{l}\text { Pictet-Turretin, } \\
\text { Marc-Auguste; Swiss } \\
\text { physicist (1752-1825). }\end{array}$ \\
\hline Pikel'ner & $47.9 \mathrm{~S}$ & $123.3 \mathrm{E}$ & 47 & UR & SO & LMP & 3 & 5 & 1979 & 0 & AA & $\begin{array}{l}\text { Solomon Borisovich; Soviet } \\
\text { astronomer, cosmologist } \\
(1921-1975) \text {. }\end{array}$ \\
\hline Pilâtre & $60.2 \mathrm{~S}$ & $86.9 W$ & 50 & EU & FR & SOV & 1985 & 5 & 1991 & 0 & AA & $\begin{array}{l}\text { Rozier, F. De; French } \\
\text { aeronaut (1753-1785). }\end{array}$ \\
\hline Pingré & $58.7 \mathrm{~S}$ & $73.7 \mathrm{~W}$ & 88 & EU & FR & LOC & 1 & 5 & 1961 & 66 & AA & $\begin{array}{l}\text { Alexandre Guy; French } \\
\text { astronomer }(1711-1796) \text {. }\end{array}$ \\
\hline [Pirandello] & $2.8 \mathrm{~N}$ & $88.8 \mathrm{E}$ & 8 & EU & IT & LTO & $063 C 3$ & 6 & 0 & 0 & AA & $\begin{array}{l}\text { Luigi; Italian playwright, } \\
\text { novelist (1867-1936). }\end{array}$ \\
\hline Pirquet & $20.3 \mathrm{~S}$ & $139.6 \mathrm{E}$ & 65 & EU & AS & LOC & 4 & 5 & 1970 & 0 & AA & $\begin{array}{l}\text { Baron Guido von; Austrian } \\
\text { (spacecraft trajectories) } \\
\text { astronaut (1867-1936). }\end{array}$ \\
\hline Pitatus & $29.9 S$ & $13.5 \mathrm{~W}$ & 106 & $\mathrm{EU}$ & IT & LAC & 94 & 5 & 1935 & 66 & AA & $\begin{array}{l}\text { Pitati, Pietro; Italian } \\
\text { astronomer, mathematician } \\
\text { (unkn.-fl. c. 1500). }\end{array}$ \\
\hline Pitiscus & $50.4 \mathrm{~S}$ & $30.9 \mathrm{E}$ & 82 & EU & GE & $\mathrm{LAC}$ & 127 & 5 & 1935 & 66 & AA & $\begin{array}{l}\text { Bartholemaeus; German } \\
\text { mathematician } \\
(1561-1613)\end{array}$ \\
\hline Pizzetti & $34.9 \mathrm{~S}$ & $118.8 \mathrm{E}$ & 44 & EU & IT & LOC & 3 & 5 & 1970 & 0 & AA & $\begin{array}{l}\text { P.; Italian geodesist } \\
\text { (1860-1918). }\end{array}$ \\
\hline Plana & $42.2 \mathrm{~N}$ & $28.2 \mathrm{E}$ & 44 & EU & IT & LAC & 26 & 5 & 1935 & 66 & AA & $\begin{array}{l}\text { Baron Giovanni Antonio } \\
\text { Amedeo; Italian } \\
\text { astronomer, geometrician } \\
(1781-1864) \text {. }\end{array}$ \\
\hline Planck & $57.9 \mathrm{~S}$ & $136.8 \mathrm{E}$ & 314 & $\mathrm{EU}$ & GE & LMP & 3 & 5 & 1970 & 0 & AA & $\begin{array}{l}\text { Max Karl Ernst; German } \\
\text { physicist; Nobel laureate } \\
(1858-1947) \text {. }\end{array}$ \\
\hline Planté & $10.2 S$ & $163.3 \mathrm{E}$ & 37 & $\mathrm{EU}$ & FR & LTO & $085 \mathrm{C} 1$ & 5 & 1979 & 0 & AA & $\begin{array}{l}\text { Gaston; French physicist } \\
\text { (1834-1889). }\end{array}$ \\
\hline Plaskett & $82.1 \mathrm{~N}$ & $174.3 \mathrm{E}$ & 109 & NA & $\mathrm{CA}$ & LMP & 3 & 5 & 1976 & 0 & AA & $\begin{array}{l}\text { John S.; Canadian } \\
\text { astronomer (1865-1941). }\end{array}$ \\
\hline Plato & $51.6 \mathrm{~N}$ & $9.4 \mathrm{~W}$ & 109 & EU & GR & LAC & 12 & 5 & 1935 & 66 & AA & $\begin{array}{l}\text { Greek philosopher } \\
\text { c.428-c. } 347 \text { B.C. }\end{array}$ \\
\hline Playfair & $23.5 \mathrm{~S}$ & $8.4 \mathrm{E}$ & 47 & EU & SC & LAC & 95 & 5 & 1935 & 66 & AA & $\begin{array}{l}\text { John; Scottish } \\
\text { mathematician, geologist } \\
(1748-1819) \text {. }\end{array}$ \\
\hline Plinius & $15.4 \mathrm{~N}$ & $23.7 \mathrm{E}$ & 43 & EU & RM & LTO & 060B1 & 5 & 1935 & 66 & AA & $\begin{array}{l}\text { Gaius Plinius Secundus } \\
\text { (The Elder); Roman natural } \\
\text { scientist (23-79?). }\end{array}$ \\
\hline
\end{tabular}

lat: latitude of feature center.

long: longitude of feature center.

diam: diameter or long dimension of feature

ct: continent of name origin (see page $284 \mathrm{ff}$.) et: ethnicity of name origin (see page $284 \mathrm{ff}$.)

quad: map quadrangle or informal name

(see page $x$ vii $f f$.).

map name or USGS map number (see page xvii ff.) as: name approval status (see page xvii).

ad: name approval date (year).

ref: reference source for name (see page $287 \mathrm{ff}$.). 
MOON

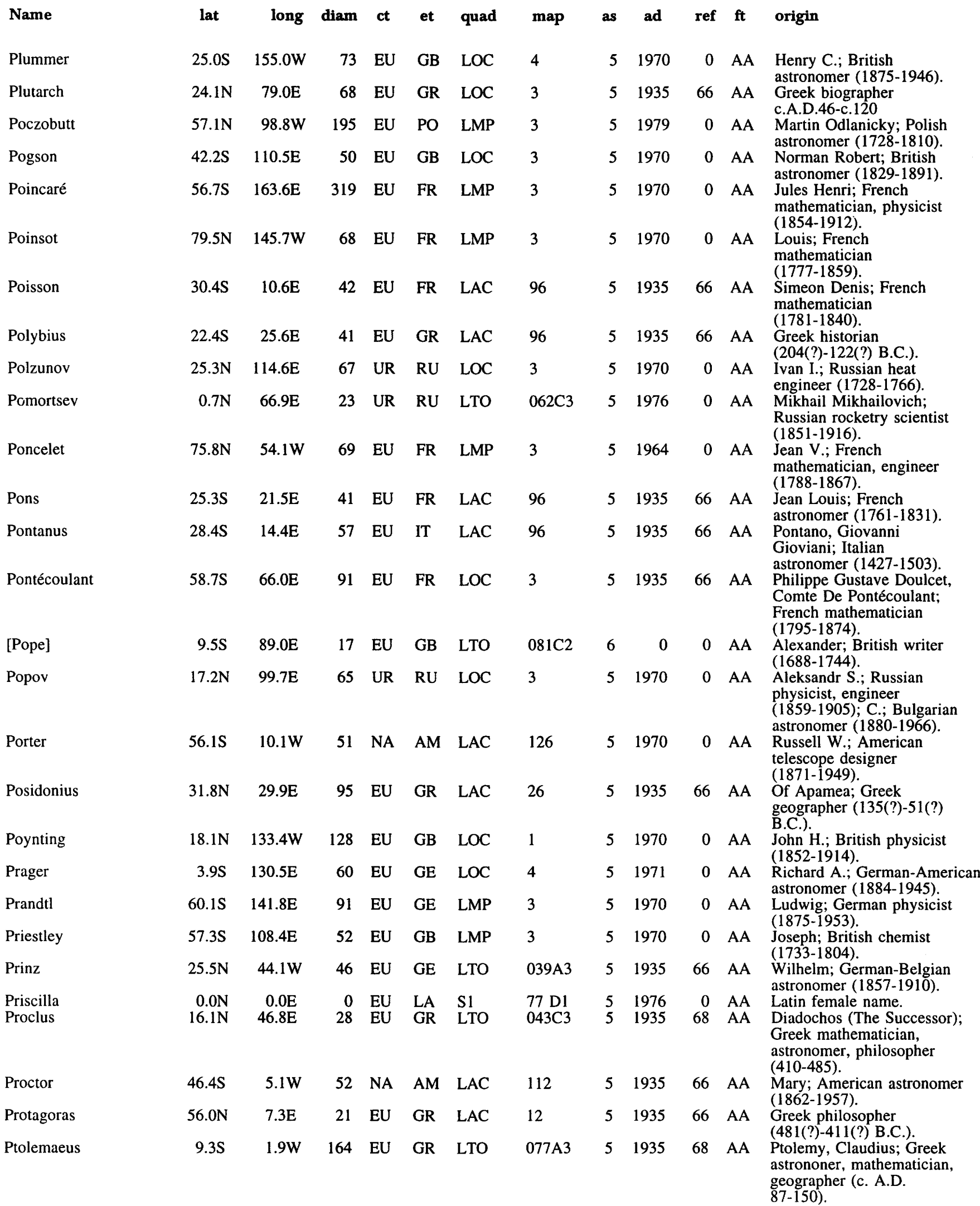

lat: latitude of feature center. long: longitude of feature center. diam: diameter or long dimension of feature. ct: continent of name origin (see page 284 ff.) et: ethnicity of name origin (see page $284 \mathrm{ff}$.)

quad: map quadrangle or informal name (see page xvii ff.).

map name or USGS map number (see page xvii ff.). as: name approval status (see page xvii).

ad: name approval date (year).

ref: reference source for name (see page $287 \mathrm{ff}$.) 
MOON

\begin{tabular}{|c|c|c|c|c|c|c|c|c|c|c|c|c|}
\hline Name & lat & long & diam & ct & et & quad & map & as & ad & ref & $\mathrm{ft}$ & origin \\
\hline Puiseux & $27.8 \mathrm{~S}$ & $39.0 \mathrm{~W}$ & 24 & $\mathrm{EU}$ & FR & LAC & 93 & 5 & 1935 & 66 & AA & $\begin{array}{l}\text { Pierre; French astronomer } \\
(1855-1928) .\end{array}$ \\
\hline Pupin & $23.8 \mathrm{~N}$ & $11.0 \mathrm{~W}$ & 2 & EU & YU & LTO & $040 C 2$ & 5 & 1976 & 0 & AA & $\begin{array}{l}\text { Michael Idvorsky; } \\
\text { Yugoslavian-American } \\
\text { physicist (1858-1935). }\end{array}$ \\
\hline Purbach & $25.5 \mathrm{~S}$ & $2.3 \mathrm{~W}$ & 115 & $\mathrm{EU}$ & AS & LAC & 95 & 5 & 1935 & 66 & AA & $\begin{array}{l}\text { Georg von; Austrian } \\
\text { mathematician, astronomer } \\
\text { (1423-1461). }\end{array}$ \\
\hline Purkyně & $1.6 \mathrm{~S}$ & $94.9 \mathrm{E}$ & 48 & $\mathrm{EU}$ & $\mathrm{CZ}$ & LTO & 082Al & 5 & 1970 & 0 & $\mathrm{AA}$ & $\begin{array}{l}\text { Jan Evangelista; } \\
\text { Czechoslovakian doctor, } \\
\text { physiologist (1787-1869). }\end{array}$ \\
\hline Pythagoras & $63.5 \mathrm{~N}$ & $63.0 \mathrm{~W}$ & 142 & $\mathrm{EU}$ & GR & LMP & 3 & 5 & 1935 & 66 & $\mathrm{AA}$ & $\begin{array}{l}\text { Of Samos; Greek } \\
\text { philosopher, mathematician } \\
\text { (unkn-fl. c. } 532 \text { B.C.). }\end{array}$ \\
\hline Pytheas & $20.5 \mathrm{~N}$ & $20.6 \mathrm{~W}$ & 20 & EU & GR & LTO & 040D2 & 5 & 1935 & 68 & AA & $\begin{array}{l}\text { Of Marseilles; Greek } \\
\text { navigator, geographer (b. } \\
\text { c. } 308 \text { B.C.). }\end{array}$ \\
\hline Quetelet & $43.1 \mathrm{~N}$ & $134.9 \mathrm{~W}$ & 55 & EU & $\mathrm{BE}$ & LMP & 2 & 5 & 1970 & 0 & AA & $\begin{array}{l}\text { Lambert A. J.; Belgian } \\
\text { statistician, astronomer } \\
(1796-1874) \text {. }\end{array}$ \\
\hline Rabbi Levi & $34.7 \mathrm{~S}$ & $23.6 \mathrm{E}$ & 81 & EU & SP & LAC & 113 & 5 & 1935 & 66 & AA & $\begin{array}{l}\text { Gershon, Levi Ben; } \\
\text { Spanish-Jewish } \\
\text { philosopher, } \\
\text { mathematician, astronomer } \\
(1288-1344) \text {. }\end{array}$ \\
\hline Racah & $13.8 \mathrm{~S}$ & $179.8 \mathrm{~W}$ & 63 & AS & IS & LOC & 4 & 5 & 1970 & 0 & AA & $\begin{array}{l}\text { Giulio; Italian-Israeli } \\
\text { physicist (1909-1965). }\end{array}$ \\
\hline [Racine] & $8.3 \mathrm{~S}$ & $99.0 \mathrm{E}$ & 30 & $\mathrm{EU}$ & FR & LTO & 082D2 & 6 & 1984 & 0 & AA & $\begin{array}{l}\text { Jean Baptiste; French } \\
\text { playwright (1639-1699). }\end{array}$ \\
\hline Raimond & $14.6 \mathrm{~N}$ & $159.3 \mathrm{~W}$ & 70 & EU & DU & LOC & 4 & 5 & 1970 & 0 & AA & $\begin{array}{l}\text { J. J., Jr.; Dutch } \\
\text { astronomer (1903-1961). }\end{array}$ \\
\hline Raman & $27.0 \mathrm{~N}$ & $55.1 \mathrm{~W}$ & 10 & AS & IN & LTO & 038B4 & 5 & 1976 & 0 & AA & $\begin{array}{l}\text { Chandrasekhara V.; Indian } \\
\text { physicist; Nobel laureate } \\
(1888-1970) \text {. }\end{array}$ \\
\hline Ramsay & $40.2 S$ & $144.5 \mathrm{E}$ & 81 & EU & GB & LOC & 4 & 5 & 1970 & 0 & AA & $\begin{array}{l}\text { Sir William; British } \\
\text { chemist; Nobel laureate } \\
\text { (1852-1916). }\end{array}$ \\
\hline Ramsden & $0.0 \mathrm{~N}$ & $0.0 \mathrm{E}$ & 24 & EU & GB & LAC & 111 & 5 & 1935 & 66 & $\mathrm{AA}$ & $\begin{array}{l}\text { Jesse; British instrument } \\
\text { maker }(1735-1800) \text {. }\end{array}$ \\
\hline Rankine & $3.9 \mathrm{~S}$ & $71.5 \mathrm{E}$ & 8 & $\mathrm{EU}$ & SC & LTO & 081A1 & 5 & 1976 & 0 & $\mathrm{AA}$ & $\begin{array}{l}\text { William John M.; Scottish } \\
\text { physicist, engineer } \\
(1820-1872) \text {. }\end{array}$ \\
\hline Raspletin & $22.5 \mathrm{~S}$ & $151.8 \mathrm{E}$ & 48 & UR & SO & LTO & $103 \mathrm{~A} 4$ & 5 & 1976 & 0 & AA & $\begin{array}{l}\text { Aleksandr Ándreyevich; } \\
\text { Soviet radio engineer } \\
\text { (1908-1967). }\end{array}$ \\
\hline $\begin{array}{l}\text { Ravi } \\
\text { Rayet }\end{array}$ & $\begin{array}{l}22.5 \mathrm{~S} \\
44.7 \mathrm{~N}\end{array}$ & $\begin{array}{l}151.8 \mathrm{E} \\
114.5 \mathrm{E}\end{array}$ & $\begin{array}{l}48 \\
27\end{array}$ & $\begin{array}{l}\text { AS } \\
\text { EU }\end{array}$ & $\begin{array}{l}\text { IN } \\
\text { FR }\end{array}$ & $\begin{array}{l}\text { S1 } \\
\text { LMP }\end{array}$ & $\begin{array}{l}77 \mathrm{D} 3 \\
2\end{array}$ & $\begin{array}{l}5 \\
5\end{array}$ & $\begin{array}{l}1976 \\
1970\end{array}$ & $\begin{array}{l}0 \\
0\end{array}$ & $\begin{array}{l}\text { AA } \\
\text { AA }\end{array}$ & $\begin{array}{l}\text { Indian male name. } \\
\text { George A. P.; French } \\
\text { astronomer (1839-1906). }\end{array}$ \\
\hline Rayleigh & $29.3 \mathrm{~N}$ & $89.6 \mathrm{E}$ & 114 & EU & GB & LOC & 3 & 5 & 1964 & 67 & AA & $\begin{array}{l}\text { John W. Strutt, Lord } \\
\text { Rayleigh; British } \\
\text { physicist; Nobel laureate } \\
(1842-1919) \text {. }\end{array}$ \\
\hline Razumov & $39.1 \mathrm{~N}$ & $114.3 \mathrm{~W}$ & 70 & UR & SO & LOC & 1 & 5 & 1970 & 0 & AA & $\begin{array}{l}\text { Vladimir V.; Soviet rocket } \\
\text { builder }(1890-1967) \text {. }\end{array}$ \\
\hline Recht & $9.8 \mathrm{~N}$ & $124.0 \mathrm{E}$ & 20 & NA & $\mathrm{AM}$ & LTO & 065B4 & 5 & 1982 & 0 & AA & $\begin{array}{l}\text { Albert W.; American } \\
\text { astronomer, mathematician } \\
(1892-1962) \text {. }\end{array}$ \\
\hline Regiomontanus & $28.3 \mathrm{~S}$ & $1.0 \mathrm{~W}$ & 108 & EU & GE & $\mathrm{LAC}$ & 95 & 5 & 1935 & 66 & AA & $\begin{array}{l}\text { Muller, Johann; German } \\
\text { astronomer, mathematician } \\
(1436-1476) \text {. }\end{array}$ \\
\hline Regnault & $54.1 \mathrm{~N}$ & $88.0 \mathrm{~W}$ & 46 & $\mathrm{EU}$ & FR & LOC & 1 & 5 & 1961 & 67 & AA & $\begin{array}{l}\text { Henri Victor; French } \\
\text { chemist; physicist } \\
(1810-1878) \text {. }\end{array}$ \\
\hline Reichenbach & $30.3 S$ & $48.0 \mathrm{E}$ & 71 & $\mathrm{EU}$ & GE & $\mathrm{LAC}$ & 97 & 5 & 1935 & 66 & AA & $\begin{array}{l}\text { Georg von; German optician } \\
(1772-1826) \text {. }\end{array}$ \\
\hline Reimarus & $47.7 \mathrm{~S}$ & $60.3 \mathrm{E}$ & 48 & $\mathrm{EU}$ & GE & LMP & 1 & 5 & 1935 & 66 & AA & $\begin{array}{l}\text { Baer, Nicolai Reymers; } \\
\text { German mathemetician c. } \\
1550-\text { c. } 1600\end{array}$ \\
\hline Reiner & $7.0 \mathrm{~N}$ & $54.9 \mathrm{~W}$ & 29 & EU & IT & LAC & 56 & 5 & 1935 & 66 & AA & $\begin{array}{l}\text { Reinieri, Vincentio; } \\
\text { Italian astronomer, } \\
\text { mathematician (unkn-1648). }\end{array}$ \\
\hline
\end{tabular}

\footnotetext{
lat: latitude of feature center.

et: ethnicity of name origin (see page $284 \mathrm{ff}$.)

as: name approval status (see page xvii)

long: longitude of feature center.

quad: map quadrangle or informal name

(see page xvii ff.).

ad: name approval date (year).

diam: diameter or long dimension of feature.

ref: reference source for name (see page $287 \mathrm{ff}$.)

ct: continent of name origin (see page $284 \mathrm{ff}$.) map: map name or USGS map number (see page xvii ff.). ft: feature type (see page 290).
} 


\section{MOON}

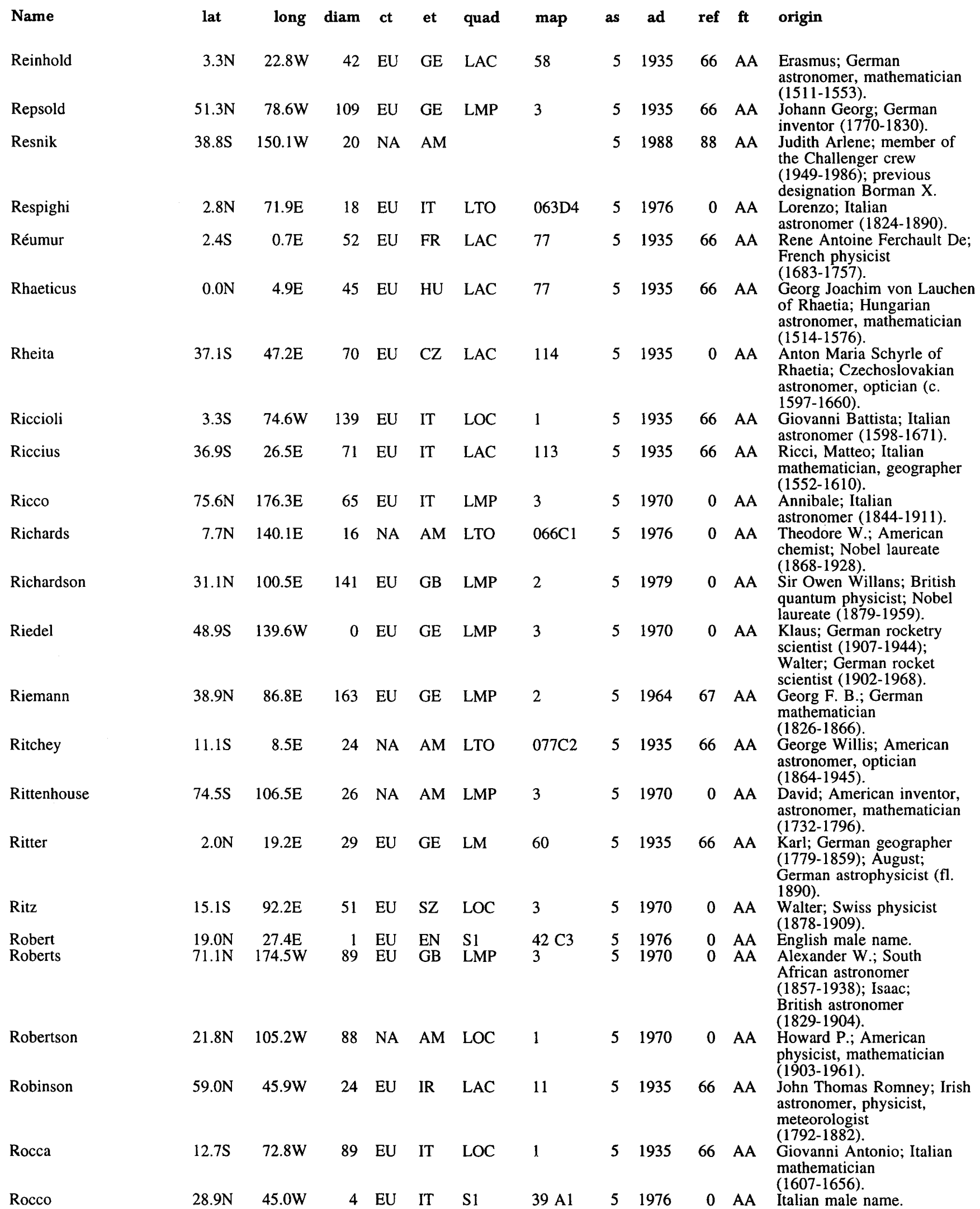

\footnotetext{
lat: latitude of feature center.

et: ethnicity of name origin (see page $284 \mathrm{ff}$.)

as: name approval status (see page xvii).

long: longitude of feature center.

quad: map quadrangle or informal name

ad: name approval date (year).

diam: diameter or long dimension of feature.

(see page xvii ff.)

ref: reference source for name (see page $287 \mathrm{ff}$.)

ct: continent of name origin (see page $284 \mathrm{ff}$.) map: map name or USGS map number (see page xvii ff.). ft: feature type (see page 290 ).
} 


\section{MOON}

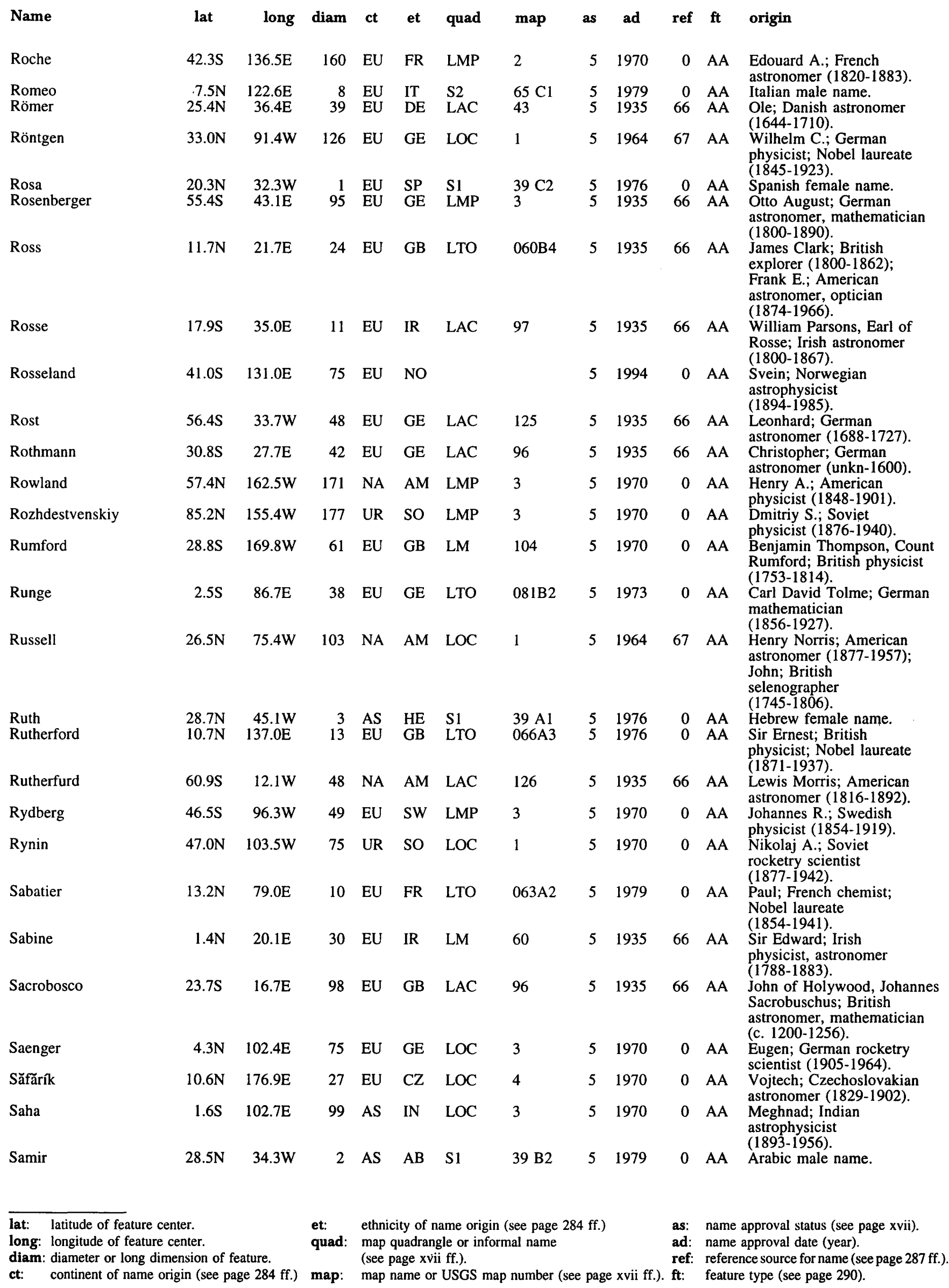


MOON

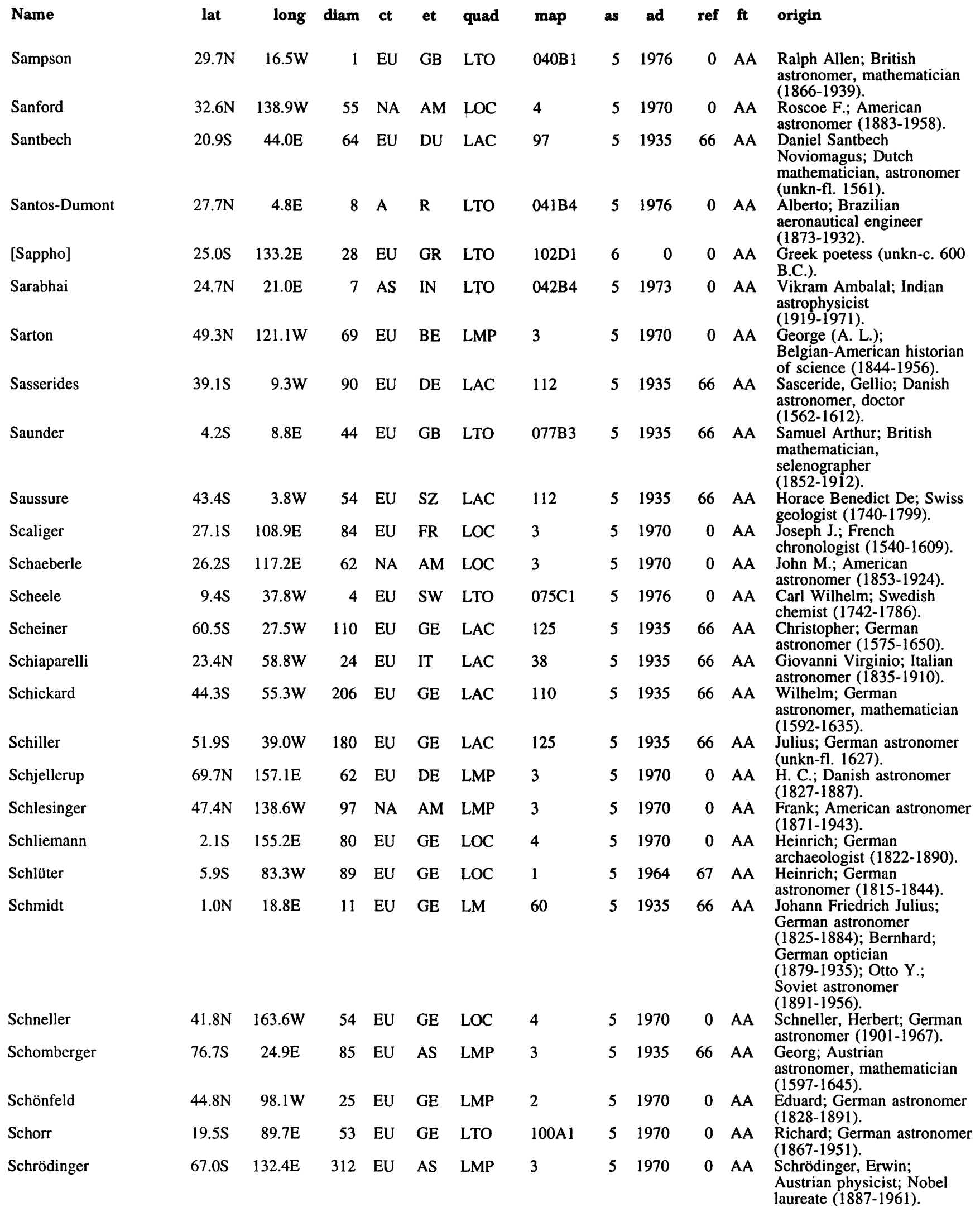

\footnotetext{
lat: latitude of feature center. long: longitude of feature center.

diam: diameter or long dimension of feature.

et: ethnicity of name origin (see page $284 \mathrm{ff}$.)

as: name approval status (see page $\mathrm{xvii}$ ).

quad: map quadrangle or informal name (see page xvii ff.).

ad: name approval date (year).

ref: reference source for name (see page $287 \mathrm{ff}$.).

ct: continent of name origin (see page $284 \mathrm{ff}$.) map: map name or USGS map number (see page xvii ff.). ft: feature type (see page 290).
} 


\section{MOON}

\begin{tabular}{|c|c|c|c|c|c|c|c|c|c|c|c|c|}
\hline Name & lat & long & diam & ct & et & quad & map & as & ad & ref & $\mathrm{ft}$ & origin \\
\hline Schröter & $2.6 \mathrm{~N}$ & $7.0 \mathrm{~W}$ & 35 & EU & GE & LAC & 59 & 5 & 1935 & 66 & AA & $\begin{array}{l}\text { Johann Hieronymus; German } \\
\text { astronomer (1745-1816). }\end{array}$ \\
\hline Schubert & $2.8 \mathrm{~N}$ & $81.0 E$ & 54 & UR & RU & LTO & $063 \mathrm{C} 4$ & 5 & 1935 & 66 & AA & $\begin{array}{l}\text { Theodor Friedrich Von; } \\
\text { Russian cartographer } \\
\text { (1789-1865). }\end{array}$ \\
\hline Schumacher & $42.4 \mathrm{~N}$ & $60.7 E$ & 60 & EU & GE & LMP & 1 & 5 & 1935 & 66 & AA & $\begin{array}{l}\text { Heinrich Christian; German } \\
\text { astronomer (1780-1850). }\end{array}$ \\
\hline Schuster & $4.2 \mathrm{~N}$ & $146.5 \mathrm{E}$ & 108 & $\mathbf{E U}$ & GB & LTO & $066 \mathrm{Cl}$ & 5 & 1970 & 0 & AA & $\begin{array}{l}\text { Sir Arthur; British } \\
\text { mathematician, physicist } \\
(1851-1934) \text {. }\end{array}$ \\
\hline Schwabe & $65.1 \mathrm{~N}$ & $45.6 \mathrm{E}$ & 25 & EU & GE & LMP & 3 & 5 & 1935 & 66 & AA & $\begin{array}{l}\text { Heinrich; German } \\
\text { astronomer (1789-1875). }\end{array}$ \\
\hline Schwarzschild & $70.1 \mathrm{~N}$ & 121.2E & 212 & EU & GE & LMP & 3 & 5 & 1970 & 0 & AA & $\begin{array}{l}\text { Karl; German astronomer } \\
(1873-1916) \text {. }\end{array}$ \\
\hline Scobee & $31.1 \mathrm{~S}$ & $148.9 \mathrm{~W}$ & 40 & NA & $\mathrm{AM}$ & & & 5 & 1988 & 88 & AA & $\begin{array}{l}\text { Francis Richard; member of } \\
\text { the Challenger crew } \\
(1939-1986) \text {; previous } \\
\text { designation Barringer L. }\end{array}$ \\
\hline Scoresby & $77.7 \mathrm{~N}$ & 14.1E & 55 & $\mathbf{E U}$ & GB & LMP & 3 & 5 & 1935 & 66 & $\mathrm{AA}$ & $\begin{array}{l}\text { William; British explorer } \\
(1789-1857) \text {. }\end{array}$ \\
\hline Scott & $82.1 S$ & $48.5 E$ & 103 & EU & GB & LMP & 3 & 5 & 1964 & 67 & AA & $\begin{array}{l}\text { Robert F.; British } \\
\text { explorer (1868-1912). }\end{array}$ \\
\hline Seares & $73.5 \mathrm{~N}$ & $145.8 \mathrm{E}$ & 110 & NA & $\mathrm{AM}$ & LMP & 3 & 5 & 1970 & 0 & AA & $\begin{array}{l}\text { Frederick H.; American } \\
\text { astronomer (1873-1964). }\end{array}$ \\
\hline Secchi & $2.4 \mathrm{~N}$ & $43.5 \mathrm{E}$ & 22 & EU & IT & LTO & $061 C 4$ & 5 & 1935 & 68 & $\mathrm{AA}$ & $\begin{array}{l}\text { Pietro Angelo; Italian } \\
\text { astronomer, astrophysicist } \\
(1818-1878) \text {. }\end{array}$ \\
\hline Sechenov & $7.1 \mathrm{~S}$ & $142.6 \mathrm{~W}$ & 62 & UR & RU & LOC & 4 & 5 & 1970 & 0 & AA & $\begin{array}{l}\text { Ivan M.; Russian } \\
\text { physiologist (1829-1905). }\end{array}$ \\
\hline Seeliger & $2.2 \mathrm{~S}$ & $3.0 \mathrm{E}$ & 8 & EU & GE & LAC & 77 & 5 & 1935 & 66 & AA & $\begin{array}{l}\text { Hugo von; German } \\
\text { astronomer (1849-1924). }\end{array}$ \\
\hline Segers & $47.1 \mathrm{~N}$ & $127.7 \mathrm{E}$ & 17 & SA & AR & LMP & 3 & 5 & 1970 & 0 & AA & $\begin{array}{l}\text { Carlos; Argentinian } \\
\text { astronomer 1900-1967 }\end{array}$ \\
\hline Segner & $58.9 \mathrm{~S}$ & $48.3 W$ & 67 & EU & GE & LMP & 3 & 5 & 1935 & 66 & AA & $\begin{array}{l}\text { Johann Andreas von; Germar } \\
\text { physicist, mathematician } \\
\text { (1704-1777). }\end{array}$ \\
\hline Seidel & $32.8 \mathrm{~S}$ & $152.2 \mathrm{E}$ & 62 & EU & GE & LOC & 4 & 5 & 1970 & 0 & AA & $\begin{array}{l}\text { Ludwig P. Von; German } \\
\text { astronomer, mathematician } \\
(1821-1896) \text {. }\end{array}$ \\
\hline Seleucus & $21.0 \mathrm{~N}$ & $66.6 \mathrm{~W}$ & 43 & AS & BY & LAC & 38 & 5 & 1935 & 66 & AA & $\begin{array}{l}\text { Babylonian astronomer } \\
\text { (unkn-fl. c. } 150 \text { B.C.). }\end{array}$ \\
\hline Seneca & $26.6 \mathrm{~N}$ & $80.2 \mathrm{E}$ & 46 & EU & RM & LOC & 3 & 5 & 1961 & 67 & AA & $\begin{array}{l}\text { Lucius Annaeus; Roman } \\
\text { philosopher, natural } \\
\text { scientist (4 B.C.- A.D. 65). }\end{array}$ \\
\hline Seyfert & $29.1 \mathrm{~N}$ & $114.6 \mathrm{E}$ & 110 & NA & AM & LOC & 3 & 5 & 1970 & 0 & AA & $\begin{array}{l}\text { Carl K.; American } \\
\text { astronomer (1911-1960). }\end{array}$ \\
\hline $\begin{array}{l}\text { Shahinaz } \\
\text { Shakleton }\end{array}$ & $\begin{array}{l}7.5 \mathrm{~N} \\
89.9 \mathrm{~S}\end{array}$ & $\begin{array}{r}122.4 \mathrm{E} \\
0.0 \mathrm{E}\end{array}$ & $\begin{array}{l}15 \\
19\end{array}$ & $\begin{array}{l}\text { AS } \\
\text { EU }\end{array}$ & $\begin{array}{l}\text { TU } \\
\text { EN }\end{array}$ & S2 & $65 \mathrm{Cl}$ & $\begin{array}{l}5 \\
5\end{array}$ & $\begin{array}{l}1979 \\
1994\end{array}$ & $\begin{array}{r}0 \\
68\end{array}$ & $\begin{array}{l}\text { AA } \\
\text { AA }\end{array}$ & $\begin{array}{l}\text { Turkish female name. } \\
\text { Earnest H.; English } \\
\text { antarctic explorer } \\
\text { (1874-1922). }\end{array}$ \\
\hline Shaler & $32.9 \mathrm{~S}$ & $85.2 \mathrm{~W}$ & 48 & NA & AM & LOC & 1 & 5 & 1964 & 67 & AA & $\begin{array}{l}\text { Nathaniel S.; American } \\
\text { geologist, paleontologist } \\
(1841-1906) \text {. }\end{array}$ \\
\hline Shapley & $9.4 \mathrm{~N}$ & $56.9 \mathrm{E}$ & 23 & NA & $\mathrm{AM}$ & LTO & $062 \mathrm{~A} 3$ & 5 & 1973 & 0 & AA & $\begin{array}{l}\text { Harlow; American } \\
\text { astronomer (1885-1972). }\end{array}$ \\
\hline Sharonov & $12.4 \mathrm{~N}$ & $173.3 \mathrm{E}$ & 74 & UR & So & LOC & 4 & 5 & 1970 & 0 & AA & $\begin{array}{l}\text { Vsevolod V.; Soviet } \\
\text { astronomer (1901-1964). }\end{array}$ \\
\hline Sharp & $45.7 \mathrm{~N}$ & $40.2 W$ & 39 & $\mathrm{EU}$ & GB & LAC & 23 & 5 & 1935 & 66 & AA & $\begin{array}{l}\text { Abraham; British } \\
\text { astronomer, mathematician } \\
\text { (1651-1742). }\end{array}$ \\
\hline Shatalov & $24.3 \mathrm{~N}$ & $141.5 \mathrm{E}$ & 21 & UR & So & LOC & 4 & 5 & 1970 & 0 & AA & $\begin{array}{l}\text { Vladimir A.; Soviet } \\
\text { cosmonaut (1927-Live). }\end{array}$ \\
\hline Shayn & $32.6 \mathrm{~N}$ & $172.5 \mathrm{E}$ & 93 & UR & SO & LOC & 4 & 5 & 1970 & 0 & AA & $\begin{array}{l}\text { Grigoriy A.; Soviet } \\
\text { astrophysicist (1892-1956). }\end{array}$ \\
\hline Sheepshanks & $59.2 \mathrm{~N}$ & $16.9 \mathrm{E}$ & 25 & EU & GB & LAC & 13 & 5 & 1935 & 66 & AA & Anne; British benefactor \\
\hline [Shekhov (Chekhov)] & $6.6 \mathrm{~S}$ & 82.0E & 19 & UR & $\mathbf{R U}$ & LTO & 081B4 & 6 & 0 & 0 & AA & $\begin{array}{l}\text { Anton Pavlovich; Russian } \\
\text { writer (1860-1904). }\end{array}$ \\
\hline Sherrington & $11.1 \mathrm{~S}$ & $118.0 \mathrm{E}$ & 18 & EU & GB & LTO & 083D2 & 5 & 1976 & $\mathbf{0}$ & AA & $\begin{array}{l}\text { Sir Charles Scott; British } \\
\text { neurophysiologist; Nobel } \\
\text { laureate (1856-1952). }\end{array}$ \\
\hline
\end{tabular}

lat: latitude of feature center.

long: longitude of feature center.

diam: diameter or long dimension of feature.

ct: continent of name origin (see page $284 \mathrm{ff}$.) et: ethnicity of name origin (see page $284 \mathrm{ff}$.)

quad: map quadrangle or informal name

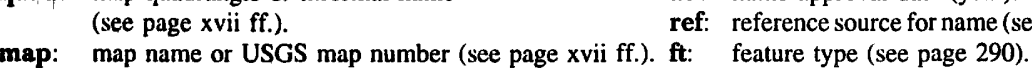

as: name approval status (see page xvii).

ad: name approval date (year).

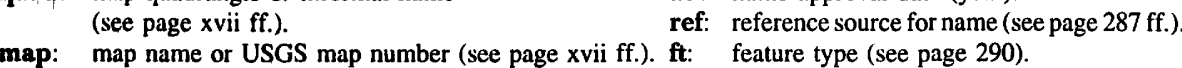




\section{MOON}

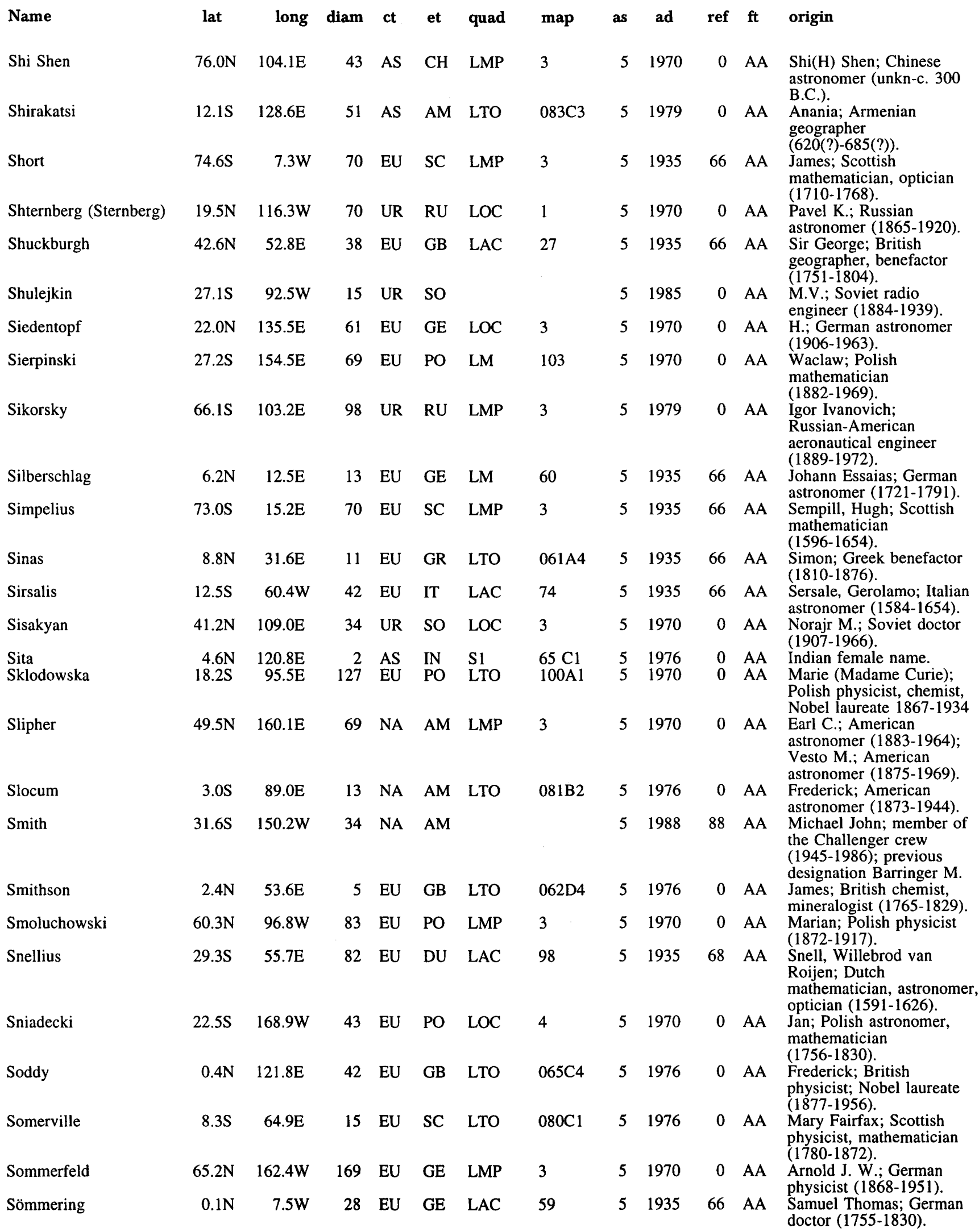

lat: latitude of feature center.

long: longitude of feature center.

diam: diameter or long dimension of feature. et: ethnicity of name origin (see page $284 \mathrm{ff}$.)

quad: map quadrangle or informal name

(see page xvii ff.). as: name approval status (see page xvii).

ad: name approval date (year).

ref: reference source for name (see page $287 \mathrm{ff}$.).

ct: continent of name origin (see page $284 \mathrm{ff}$.) map: map name or USGS map number (see page xvii ff.). ft: feature type (see page 290 ). 
MOON

\begin{tabular}{|c|c|c|c|c|c|c|c|c|c|c|c|c|}
\hline Name & lat & long & diam & ct & et & quad & map & as & ad & ref & $\mathbf{f t}$ & origin \\
\hline [Sophocles] & $21.5 S$ & $119.8 \mathrm{E}$ & 0 & $\mathrm{EU}$ & GR & LTO & $101 \mathrm{~A} 3$ & 6 & 0 & 0 & AA & $\begin{array}{l}\text { Greek philosopher (c. } \\
\text { 495-406 B.C.). }\end{array}$ \\
\hline Soraya & $12.9 \mathrm{~S}$ & $1.6 \mathrm{~W}$ & 2 & AS & PE & S1 & 77 D3 & 5 & 1976 & 0 & AA & Persian female name. \\
\hline Sosigenes & $8.7 \mathrm{~N}$ & $17.6 \mathrm{E}$ & 17 & EU & GR & LM & 60 & 5 & 1935 & 66 & AA & $\begin{array}{l}\text { Greek astronomer, } \\
\text { chronologist (unkn-fl. } 46 \\
\text { B.C.). }\end{array}$ \\
\hline South & $58.0 \mathrm{~N}$ & $50.8 \mathrm{~W}$ & 104 & $\mathrm{EU}$ & GB & LMP & 3 & 5 & 1935 & 66 & AA & $\begin{array}{l}\text { James; British astronomer } \\
(1785-1867) \text {. }\end{array}$ \\
\hline Spallanzani & $46.3 \mathrm{~S}$ & $24.7 \mathrm{E}$ & 32 & $\mathrm{EU}$ & IT & LMP & 2 & 5 & 1935 & 66 & AA & $\begin{array}{l}\text { Lazzaro; Italian natural } \\
\text { scientist, biologist } \\
(1729-1799) \text {. }\end{array}$ \\
\hline Spencer Jones & $13.3 \mathrm{~N}$ & $165.6 \mathrm{E}$ & 85 & EU & GB & LOC & 4 & 5 & 1970 & 0 & AA & $\begin{array}{l}\text { Sir Harold; British } \\
\text { astronomer (1890-1960). }\end{array}$ \\
\hline Spörer & $4.3 \mathrm{~S}$ & $1.8 \mathrm{~W}$ & 27 & EU & GE & LTO & 077A3 & 5 & 1935 & 66 & AA & $\begin{array}{l}\text { Friederich Wilhelm Gustav; } \\
\text { German astronomer } \\
(1822-1895) \text {. }\end{array}$ \\
\hline Spurr & $27.9 \mathrm{~N}$ & $1.2 \mathrm{~W}$ & 11 & NA & AM & LTO & 041A3 & 5 & 1973 & 0 & AA & $\begin{array}{l}\text { Josiah Edward; American } \\
\text { geologist (1870-1950). }\end{array}$ \\
\hline St. John & $10.2 \mathrm{~N}$ & $150.2 \mathrm{E}$ & 68 & NA & AM & LOC & 4 & 5 & 1970 & 0 & AA & $\begin{array}{l}\text { Charles E.; American solar } \\
\text { physicist, astronomer } \\
(1857-1935) \text {. }\end{array}$ \\
\hline Stadius & $10.5 \mathrm{~N}$ & $13.7 \mathrm{~W}$ & 69 & $\mathrm{EU}$ & $\mathrm{BE}$ & LAC & 58 & 5 & 1935 & 66 & AA & $\begin{array}{l}\text { Stade, Jan; Belgian } \\
\text { astronomer, mathematician } \\
(1527-1579) \text {. }\end{array}$ \\
\hline Stark & $25.5 \mathrm{~S}$ & $134.6 \mathrm{E}$ & 49 & EU & GE & LTO & $102 \mathrm{D} 1$ & 5 & 1970 & 0 & AA & $\begin{array}{l}\text { Johannes; German } \\
\text { physicist; Nobel laureate } \\
(1874-1957) \text {. }\end{array}$ \\
\hline Stearns & $34.8 \mathrm{~N}$ & $162.6 \mathrm{E}$ & 36 & NA & AM & LMP & 2 & 5 & 1979 & 0 & AA & $\begin{array}{l}\text { Carl Leo; American } \\
\text { astronomer (1892-1972). }\end{array}$ \\
\hline Stebbins & $64.8 \mathrm{~N}$ & $141.8 \mathrm{~W}$ & 131 & NA & AM & LMP & 3 & 5 & 1970 & 0 & AA & $\begin{array}{l}\text { Joel; American astronomer } \\
(1878-1966) \text {. }\end{array}$ \\
\hline Stefan & $46.0 \mathrm{~N}$ & $108.3 \mathrm{~W}$ & 125 & $\mathrm{EU}$ & AS & LMP & 2 & 5 & 1970 & 0 & AA & $\begin{array}{l}\text { Josef; Austrian physicist } \\
\text { (1835-1893). }\end{array}$ \\
\hline Stein & $7.2 \mathrm{~N}$ & $179.0 \mathrm{E}$ & 33 & EU & DU & LOC & 4 & 5 & 1970 & 0 & AA & $\begin{array}{l}\text { J. W.; Dutch astronomer } \\
\text { (1871-1951). }\end{array}$ \\
\hline Steinheil & $48.6 \mathrm{~S}$ & $46.5 \mathrm{E}$ & 67 & EU & GE & LMP & 3 & 5 & 1935 & 66 & AA & $\begin{array}{l}\text { Karl August von; German } \\
\text { astronomer, physicist } \\
(1801-1870) \text {. }\end{array}$ \\
\hline Steklov & $36.7 \mathrm{~S}$ & $104.9 W$ & 36 & UR & SO & LOC & 1 & 5 & 1970 & 0 & AA & $\begin{array}{l}\text { Vladimir A.; Soviet } \\
\text { mathematician (1864-1926). }\end{array}$ \\
\hline $\begin{array}{l}\text { Stella } \\
\text { Steno }\end{array}$ & $\begin{array}{l}19.9 \mathrm{~N} \\
32.8 \mathrm{~N}\end{array}$ & $\begin{array}{l}29.8 \mathrm{E} \\
161.8 \mathrm{E}\end{array}$ & $\begin{array}{l}36 \\
31\end{array}$ & $\begin{array}{l}\text { EU } \\
\text { EU }\end{array}$ & $\begin{array}{l}\mathrm{LA} \\
\mathrm{DE}\end{array}$ & $\begin{array}{l}\text { S3 } \\
\text { LOC }\end{array}$ & $\begin{array}{l}42 \mathrm{C} 3 \\
4\end{array}$ & $\begin{array}{l}5 \\
5\end{array}$ & $\begin{array}{l}1976 \\
1970\end{array}$ & $\begin{array}{l}0 \\
0\end{array}$ & $\begin{array}{l}\text { AA } \\
\text { AA }\end{array}$ & $\begin{array}{l}\text { Latin female name. } \\
\text { Nicolaus; Danish doctor } \\
(1638-1686)\end{array}$ \\
\hline Sternfeld & $19.6 \mathrm{~S}$ & $141.2 \mathrm{~W}$ & 100 & UR & SO & SOV & 1985 & 5 & 1991 & 0 & AA & $\begin{array}{l}\text { A. A.; Soviet space } \\
\text { scientist }(1905-1980) \text {. }\end{array}$ \\
\hline Stetson & $39.6 \mathrm{~S}$ & $118.3 \mathrm{~W}$ & 64 & NA & AM & LOC & 1 & 5 & 1970 & 0 & AA & $\begin{array}{l}\text { Harlan T.; American } \\
\text { astronomer, geophysicist } \\
(1885-1964) \text {. }\end{array}$ \\
\hline Stevinus & $32.5 \mathrm{~S}$ & $54.2 \mathrm{E}$ & 74 & EU & BE & LAC & 114 & 5 & 1935 & 66 & $\mathrm{AA}$ & $\begin{array}{l}\text { Stevin, Simon; Belgian } \\
\text { mathematician, physicist } \\
(1548-1620) \text {. }\end{array}$ \\
\hline Stewart & $2.2 \mathrm{~N}$ & $67.0 \mathrm{E}$ & 13 & NA & $\mathrm{AM}$ & LTO & $062 \mathrm{C} 3$ & 5 & 1976 & 0 & AA & $\begin{array}{l}\text { John Quincy; American } \\
\text { astrophysicist (1894-1972). }\end{array}$ \\
\hline Stiborius & $34.4 \mathrm{~S}$ & $32.0 \mathrm{E}$ & 43 & EU & GE & LAC & 113 & 5 & 1935 & 66 & AA & $\begin{array}{l}\text { Stoberl, Andreas; German } \\
\text { astronomer, mathematician } \\
(1465-1515) \text {. }\end{array}$ \\
\hline Stöfler & $41.1 \mathrm{~S}$ & $6.0 \mathrm{E}$ & 126 & EU & GE & LAC & 112 & 5 & 1935 & 66 & AA & $\begin{array}{l}\text { Johann; German astronomer, } \\
\text { mathematician (1452-1531). }\end{array}$ \\
\hline Stokes & $52.5 \mathrm{~N}$ & $88.1 \mathrm{~W}$ & 51 & $\mathrm{EU}$ & GB & LMP & 3 & 5 & 1964 & 67 & AA & $\begin{array}{l}\text { Sir George G.; British } \\
\text { mathematician, physicist } \\
(1819-1903) \text {. }\end{array}$ \\
\hline Stoletov & $45.1 \mathrm{~N}$ & $155.2 \mathrm{~W}$ & 42 & UR & RU & LOC & 4 & 5 & 1970 & 0 & AA & $\begin{array}{l}\text { Aleksandr G.; Russian } \\
\text { physicist (1839-1896). }\end{array}$ \\
\hline Stoney & $55.3 \mathrm{~S}$ & $156.1 \mathrm{~W}$ & 45 & EU & IR & LMP & 3 & 5 & 1970 & 0 & AA & $\begin{array}{l}\text { George J.; Irish physicist } \\
(1826-1911)\end{array}$ \\
\hline Störmer & $57.3 \mathrm{~N}$ & $146.3 \mathrm{E}$ & 55 & EU & NO & LMP & 3 & 5 & 1935 & 66 & AA & $\begin{array}{l}\text { F. Carl M.; Norwegian } \\
\text { mathematician and } \\
\text { astronomer, aurora } \\
\text { research (1874-1957). } \\
\text { Greek geographer (54 B.C.- } \\
\text { A.D. 24). }\end{array}$ \\
\hline
\end{tabular}

lat: latitude of feature center.

long: longitude of feature center.

diam: diameter or long dimension of feature.

ct: continent of name origin (see page $284 \mathrm{ff}$.) et: ethnicity of name origin (see page $284 \mathrm{ff}$.)

quad: map quadrangle or informal name

(see page xvii ff.).

as: name approval status (see page xvii).

ad: name approval date (year).

ref: reference source for name (see page $287 \mathrm{ff}$.). 
MOON

\begin{tabular}{|c|c|c|c|c|c|c|c|c|c|c|c|c|}
\hline Name & lat & long & diam & ct & et & quad & map & as & ad & ref & $\mathbf{f t}$ & origin \\
\hline Stratton & $5.8 \mathrm{~S}$ & $164.6 \mathrm{E}$ & 70 & EU & GB & LOC & 4 & 5 & 1970 & 0 & AA & $\begin{array}{l}\text { Frederick J. M.; British } \\
\text { astronomer, astrophysicist } \\
(1881-1960) \text {. }\end{array}$ \\
\hline Street & $46.5 S$ & $10.5 \mathrm{~W}$ & 57 & $\mathrm{EU}$ & GB & $\mathrm{LAC}$ & 112 & 5 & 1935 & 66 & AA & $\begin{array}{l}\text { Thomas; British astronomer } \\
(1621-1689) \text {. }\end{array}$ \\
\hline Strömgren & $21.7 \mathrm{~S}$ & $132.4 \mathrm{~W}$ & 61 & EU & $\mathrm{DE}$ & LOC & 3 & 5 & 1970 & 0 & AA & $\begin{array}{l}\text { Elis; Danish astronomer } \\
(1870-1947) \text {. }\end{array}$ \\
\hline Struve & $22.4 \mathrm{~N}$ & $77.1 \mathrm{~W}$ & 164 & UR & RU & LOC & 1 & 5 & 1964 & 67 & AA & $\begin{array}{l}\text { Otto von; Russian } \\
\text { astronomer (1819-1905); } \\
\text { Otto; American astronomer } \\
\text { (1897-1963); Friedrich G. } \\
\text { W. von; German astronomer } \\
\text { (1973-1864). }\end{array}$ \\
\hline Subbotin & $29.2 \mathrm{~S}$ & 135.3E & 67 & UR & SO & LOC & 4 & 5 & 1970 & 0 & AA & $\begin{array}{l}\text { Mikhail F.; Soviet } \\
\text { astronomer (1893-1966). }\end{array}$ \\
\hline Suess & $4.4 \mathrm{~N}$ & $47.6 \mathrm{~W}$ & 8 & EU & AS & $\mathrm{LAC}$ & 57 & 5 & 1935 & 66 & $\mathrm{AA}$ & $\begin{array}{l}\text { Eduard; Austrian geologist } \\
\text { (1831-1914). }\end{array}$ \\
\hline Sulpicius Gallus & $19.6 \mathrm{~N}$ & $11.6 \mathrm{E}$ & 12 & $\mathrm{EU}$ & RM & LTO & 042D4 & 5 & 1935 & 66 & AA & $\begin{array}{l}\text { Gaius; Roman astronomer } \\
\text { (unkn-fl. c. B.C. 166). }\end{array}$ \\
\hline Sumner & $37.5 \mathrm{~N}$ & 108.7E & 50 & NA & $\mathrm{AM}$ & LOC & 3 & 5 & 1970 & 0 & AA & $\begin{array}{l}\text { Thomas H.; American } \\
\text { geographer (1807-1876). }\end{array}$ \\
\hline Sundman & $10.8 \mathrm{~N}$ & $91.6 \mathrm{~W}$ & 40 & EU & FI & LOC & 1 & 5 & 1970 & 0 & $\mathrm{AA}$ & $\begin{array}{l}\text { K. F.; Finnish astronomer } \\
(1873-1949) \text {. }\end{array}$ \\
\hline $\begin{array}{l}\text { Sung-Mei } \\
\text { Susan }\end{array}$ & $\begin{array}{l}24.6 \mathrm{~N} \\
11.0 \mathrm{~S}\end{array}$ & $\begin{array}{r}11.3 \mathrm{E} \\
6.3 \mathrm{~W}\end{array}$ & $\begin{array}{l}5 \\
1\end{array}$ & $\begin{array}{l}\mathrm{AS} \\
\mathrm{EU}\end{array}$ & $\begin{array}{l}\mathrm{CH} \\
\mathrm{GB}\end{array}$ & $\begin{array}{l}\text { S2 } \\
\text { S1 }\end{array}$ & $\begin{array}{l}42 \mathrm{~A} 4 \\
77 \mathrm{D} 1\end{array}$ & $\begin{array}{l}5 \\
5\end{array}$ & $\begin{array}{l}1976 \\
1976\end{array}$ & $\begin{array}{l}0 \\
0\end{array}$ & $\begin{array}{l}\text { AA } \\
\text { AA }\end{array}$ & $\begin{array}{l}\text { Chinese female name. } \\
\text { English female name. }\end{array}$ \\
\hline Swann & $52.0 \mathrm{~N}$ & 112.7E & 42 & NA & $\mathrm{AM}$ & LMP & 3 & 5 & 1970 & 0 & $\mathrm{AA}$ & $\begin{array}{l}\text { William F. G.; } \\
\text { Anglo-American physicist } \\
\text { (1884-1962). }\end{array}$ \\
\hline Swasey & $5.5 \mathrm{~S}$ & 89.7E & 23 & NA & $\mathrm{AM}$ & LTO & $081 \mathrm{~B} 3$ & 5 & 1976 & 0 & AA & $\begin{array}{l}\text { Ambrose; American inventor } \\
(1846-1937) \text {. }\end{array}$ \\
\hline Swift & $19.3 \mathrm{~N}$ & $53.4 \mathrm{E}$ & 10 & NA & $\mathrm{AM}$ & LTO & 044D4 & 5 & 1976 & 0 & AA & $\begin{array}{l}\text { Lewis; American astronomer } \\
(1820-1913) \text {. }\end{array}$ \\
\hline Sylvester & $82.7 \mathrm{~N}$ & $79.6 \mathrm{~W}$ & 58 & EU & GB & LMP & 3 & 5 & 1964 & 67 & AA & $\begin{array}{l}\text { James J.; British } \\
\text { mathematician } \\
(1814-1897)\end{array}$ \\
\hline Szilard & $34.0 \mathrm{~N}$ & 105.7E & 122 & EU & HU & LOC & 3 & 5 & 1970 & 0 & AA & $\begin{array}{l}\text { Leo; Hungarian-American } \\
\text { physicist }(1898-1964) \text {. }\end{array}$ \\
\hline T. Mayer & $15.6 \mathrm{~N}$ & $29.1 \mathrm{~W}$ & 33 & EU & GE & LAC & 58 & 5 & 1935 & 66 & AA & $\begin{array}{l}\text { Tobias; German astronomer } \\
(1723-1762) \text {. }\end{array}$ \\
\hline Tacchini & $4.9 \mathrm{~N}$ & $85.8 \mathrm{E}$ & 40 & EU & IT & LTO & $063 C 2$ & 5 & 1973 & 0 & AA & $\begin{array}{l}\text { Pietro; Italian astronomer } \\
(1838-1905) \text {. }\end{array}$ \\
\hline Tacitus & $16.2 \mathrm{~S}$ & $19.0 \mathrm{E}$ & 39 & EU & RM & LAC & 96 & 5 & 1935 & 66 & $\mathrm{AA}$ & $\begin{array}{l}\text { Cornelius; Roman historian } \\
\text { (c. 55-120). }\end{array}$ \\
\hline Tacquet & $16.6 \mathrm{~N}$ & $19.2 \mathrm{E}$ & 7 & EU & BE & LTO & 042D3 & 5 & 1935 & 66 & AA & $\begin{array}{l}\text { Andre; Belgian } \\
\text { mathematician (1612-1660). }\end{array}$ \\
\hline $\begin{array}{l}\text { Taizo } \\
\text { Talbot }\end{array}$ & $\begin{array}{r}16.6 \mathrm{~N} \\
2.5 \mathrm{~S}\end{array}$ & $\begin{array}{l}19.2 \mathrm{E} \\
85.3 \mathrm{E}\end{array}$ & $\begin{array}{r}6 \\
11\end{array}$ & $\begin{array}{l}\text { AS } \\
\text { EU }\end{array}$ & $\begin{array}{l}\text { JA } \\
\text { GB }\end{array}$ & $\begin{array}{l}\text { S3 } \\
\text { LTO }\end{array}$ & $\begin{array}{l}\text { 41B4 } \\
081 \mathrm{~B} 2\end{array}$ & $\begin{array}{l}5 \\
5\end{array}$ & $\begin{array}{l}1976 \\
1976\end{array}$ & $\begin{array}{l}0 \\
0\end{array}$ & $\begin{array}{l}\text { AA } \\
\text { AA }\end{array}$ & $\begin{array}{l}\text { Japanese male name. } \\
\text { William Henry Fox; British } \\
\text { photographer, physicist, } \\
\text { archaeologist } \\
(1800-1877) \text {. }\end{array}$ \\
\hline Tamm & $4.4 \mathrm{~S}$ & $146.4 \mathrm{E}$ & 38 & UR & SO & LTO & 084B3 & 5 & 1979 & 0 & AA & $\begin{array}{l}\text { Igor; Soviet physicist; } \\
\text { Nobel laureate } \\
\text { (1895-1971). }\end{array}$ \\
\hline Tannerus & $56.4 \mathrm{~S}$ & $22.0 \mathrm{E}$ & 28 & EU & AS & LMP & 3 & 5 & 1935 & 66 & AA & $\begin{array}{l}\text { Tanner, Adam; Austrian } \\
\text { mathematician } \\
\text { (1572-1632). }\end{array}$ \\
\hline Taruntius & $5.6 \mathrm{~N}$ & $46.5 \mathrm{E}$ & 56 & EU & RM & LTO & $061 C 2$ & 5 & 1935 & 66 & AA & $\begin{array}{l}\text { Firmanus, Lucius; Roman } \\
\text { philosopher (unkn-fl. } 86 \\
\text { B.C.). }\end{array}$ \\
\hline [Tasso] & $0.7 \mathrm{~S}$ & $92.0 \mathrm{E}$ & 52 & EU & IT & LTO & $082 \mathrm{~A} 1$ & 6 & 1984 & 0 & $\mathrm{AA}$ & $\begin{array}{l}\text { Torquato; Italian poet } \\
(1544-1595) \text {. }\end{array}$ \\
\hline Taylor & $5.3 \mathrm{~S}$ & 16.7E & 42 & EU & GB & LTO & 078A3 & 5 & 1935 & 66 & AA & $\begin{array}{l}\text { Brook; British } \\
\text { mathematician (1685-1731). }\end{array}$ \\
\hline Tebbutt & $9.6 \mathrm{~N}$ & $53.6 \mathrm{E}$ & 31 & OC & $\mathrm{AU}$ & LTO & $062 \mathrm{~A} 4$ & 5 & 1973 & 0 & AA & $\begin{array}{l}\text { John; Australian } \\
\text { astronomer (1834-1916). }\end{array}$ \\
\hline Teisserenc & $32.2 \mathrm{~N}$ & $135.9 \mathrm{~W}$ & 62 & EU & FR & LOC & 1 & 5 & 1970 & 0 & AA & $\begin{array}{l}\text { de Bort, Leon-Philippe; } \\
\text { Fernch meteorologist } \\
\text { 1855-1913 }\end{array}$ \\
\hline Tempel & $3.9 \mathrm{~N}$ & $11.9 \mathrm{E}$ & 45 & EU & GE & LM & 60 & 5 & 1935 & 66 & AA & $\begin{array}{l}\text { Ernst Wilhelm Leberecht; } \\
\text { German astronomer } \\
(1821-1889) \text {. }\end{array}$ \\
\hline
\end{tabular}

lat: latitude of feature center.

long: longitude of feature center.

diam: diameter or long dimension of feature.

et: ethnicity of name origin (see page $284 \mathrm{ff}$.)

quad: map quadrangle or informal name

(see page xvii ff.).

ct: continent of name origin (see page $284 \mathrm{ff}$.) map: map name or USGS map number (see page xvii ff.). ft: feature type (see page 290).

as: name approval status (see page xvii).

ad: name approval date (year).

ref: reference source for name (see page $287 \mathrm{ff}$.) 
MOON

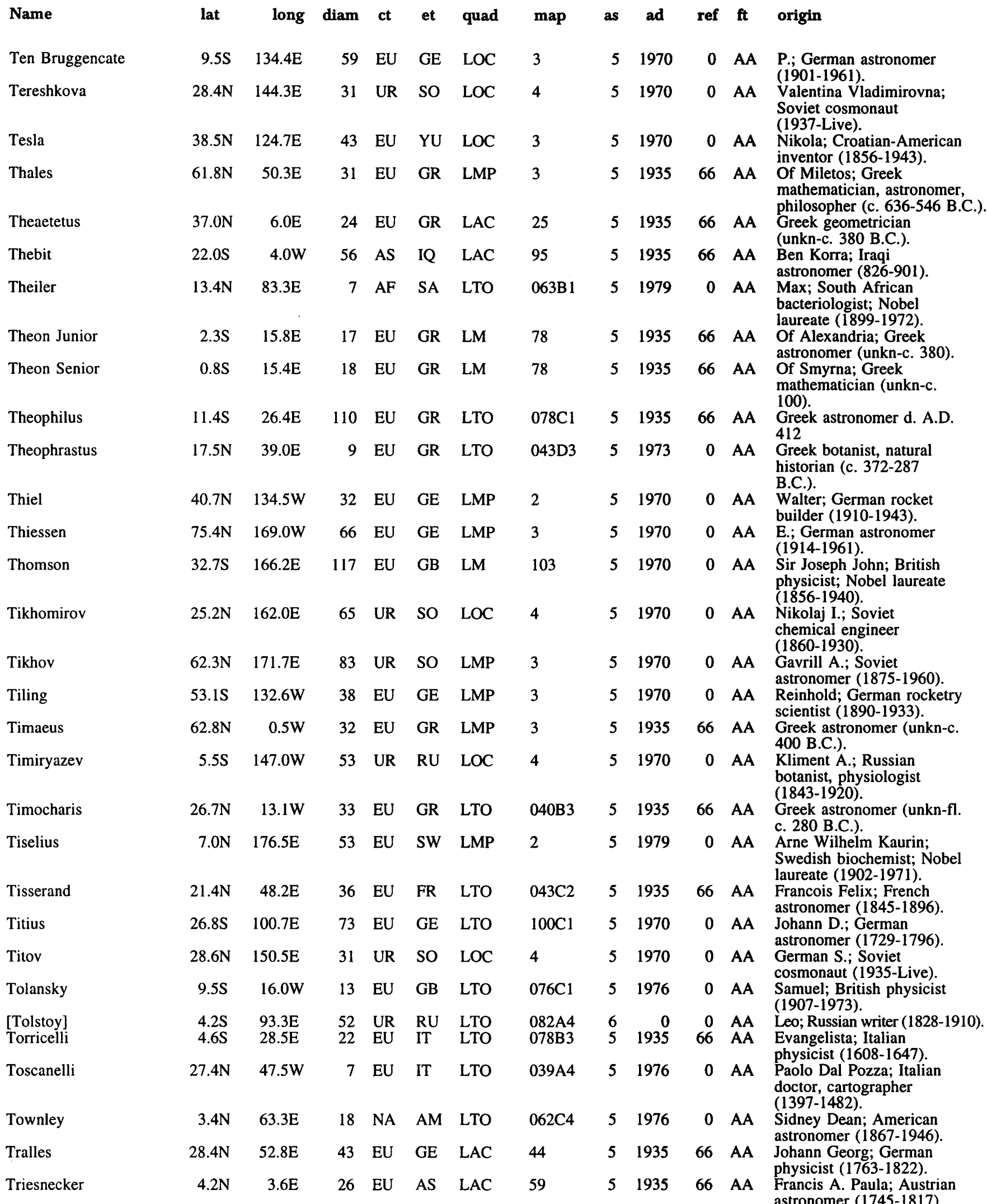

lat: latitude of feature center. long: longitude of feature center. diam: diameter or long dimension of feature.

ct: continent of name origin (see page $284 \mathrm{ff}$.) et: ethnicity of name origin (see page $284 \mathrm{ff}$.)

quad: map quadrangle or informal name

(see page $x$ vii ff.).

map: map name or USGS map number (see page xvii ff.). ft: feature type (see page 290).

as: name approval status (see page $\mathrm{xvii}$ )

ad: name approval date (year).

ref: reference source for name (see page $287 \mathrm{ff}$.) 
MOON

\begin{tabular}{|c|c|c|c|c|c|c|c|c|c|c|c|c|}
\hline Name & lat & long & diam & ct & et & quad & map & as & ad & ref & ft & origin \\
\hline Trouvelot & $49.3 \mathrm{~N}$ & $5.8 \mathrm{E}$ & 9 & $\mathrm{EU}$ & FR & LAC & 12 & 5 & 1935 & 66 & AA & $\begin{array}{l}\text { Etiénne Leopold; French } \\
\text { astronomer }(1827-1895) \text {. }\end{array}$ \\
\hline Trumpler & $29.3 \mathrm{~N}$ & $167.1 \mathrm{E}$ & 77 & NA & AM & LOC & 4 & 5 & 1970 & 0 & AA & $\begin{array}{l}\text { Robert J.; American } \\
\text { astronomer (1866-1956). }\end{array}$ \\
\hline Tsander & $6.2 \mathrm{~N}$ & $149.3 W$ & 181 & UR & SO & LOC & 4 & 5 & 1970 & 0 & AA & $\begin{array}{l}\text { Friedrich A.; Soviet } \\
\text { rocketry scientist } \\
\text { (1887-1933). }\end{array}$ \\
\hline Tseraskiy (Cerasky) & $49.0 \mathrm{~S}$ & 141.6E & 56 & UR & RU & LMP & 3 & 5 & 1970 & 0 & AA & $\begin{array}{l}\text { Vitol'd K.; Polish-Russian } \\
\text { astronomer (1849-1925). }\end{array}$ \\
\hline Tsinger & $56.7 \mathrm{~N}$ & $175.6 \mathrm{E}$ & 44 & UR & RU & LMP & 3 & 5 & 1970 & 0 & AA & $\begin{array}{l}\text { Nikolaj Ya.; Russian } \\
\text { astronomer (1842-1918). }\end{array}$ \\
\hline Tsiolkovskiy & $21.2 \mathrm{~S}$ & $128.9 \mathrm{E}$ & 185 & UR & SO & LTO & 101B2 & 5 & 1961 & 67 & AA & $\begin{array}{l}\text { Konstantin E.; Soviet } \\
\text { physicist (1857-1935). }\end{array}$ \\
\hline Tsu Chung-Chi & $17.3 \mathrm{~N}$ & $145.1 \mathrm{E}$ & 28 & AS & $\mathrm{CH}$ & LOC & 4 & 5 & 1970 & 0 & AA & $\begin{array}{l}\text { Chinese mathematician } \\
(430-501) \text {. }\end{array}$ \\
\hline Tucker & $5.6 \mathrm{~S}$ & $88.2 \mathrm{E}$ & 7 & NA & AM & LTO & 081B3 & 5 & 1979 & 0 & AA & $\begin{array}{l}\text { Richard Hawley; American } \\
\text { astronomer (1859-1952). }\end{array}$ \\
\hline Turner & $1.4 \mathrm{~S}$ & $13.2 \mathrm{~W}$ & 11 & EU & GB & LAC & 76 & 5 & 1935 & 66 & AA & $\begin{array}{l}\text { Herbert Hall; British } \\
\text { astronomer (1861-1930). }\end{array}$ \\
\hline Tycho & $43.4 \mathrm{~S}$ & $11.1 \mathrm{~W}$ & 102 & $\mathrm{EU}$ & DE & LAC & 112 & 5 & 1935 & 66 & AA & $\begin{array}{l}\text { Tycho Brahe; Danish } \\
\text { astronomer (1546-1601). }\end{array}$ \\
\hline Tyndall & $34.9 \mathrm{~S}$ & $117.0 \mathrm{E}$ & 18 & EU & GB & LOC & 3 & 5 & 1970 & 0 & AA & $\begin{array}{l}\text { John; British physicist } \\
(1820-1893)\end{array}$ \\
\hline Ukert & $7.8 \mathrm{~N}$ & $1.4 \mathrm{E}$ & 23 & EU & GE & LAC & 59 & 5 & 1935 & 66 & AA & $\begin{array}{l}\text { Friedrich August; German } \\
\text { historian, humanitarian } \\
(1780-1851) \text {. }\end{array}$ \\
\hline Ulugh Beigh & $32.7 \mathrm{~N}$ & $81.9 \mathrm{~W}$ & 54 & AS & MN & LOC & 1 & 5 & 1961 & 67 & AA & $\begin{array}{l}\text { Ulugh-Beg; Mongolian } \\
\text { astronomer, mathematician } \\
\text { (1394--1449). }\end{array}$ \\
\hline [Undest] & $26.5 \mathrm{~N}$ & $18.5 \mathrm{~W}$ & 7 & EU & NO & LTO & 040B4 & 6 & 1984 & 0 & AA & $\begin{array}{l}\text { Sigrid; Norwegian } \\
\text { novelist; Nobel laureate } \\
\text { (1882-1949). }\end{array}$ \\
\hline Urey & $27.9 \mathrm{~N}$ & $87.4 \mathrm{E}$ & 38 & NA & $\mathrm{AM}$ & & & 5 & 1985 & 0 & AA & $\begin{array}{l}\text { H.; American chemist; } \\
\text { Nobel laureate } \\
(1893-1981) \text {. }\end{array}$ \\
\hline Väisälä & $25.9 \mathrm{~N}$ & $47.8 \mathrm{~W}$ & 8 & EU & FI & LTO & 039A4 & 5 & 1973 & 0 & AA & $\begin{array}{l}\text { Yrjo; Finnish astronomer } \\
\text { (1891-1971). }\end{array}$ \\
\hline Valier & $6.8 \mathrm{~N}$ & $174.5 \mathrm{E}$ & 67 & EU & GE & LOC & 4 & 5 & 1970 & 0 & AA & $\begin{array}{l}\text { Max; German rocketry } \\
\text { engineer (1895-1930). }\end{array}$ \\
\hline van Albada & $9.4 \mathrm{~N}$ & $64.3 \mathrm{E}$ & 21 & EU & DU & LTO & $062 \mathrm{~B} 4$ & 5 & 1976 & 0 & AA & $\begin{array}{l}\text { Gale Bruno; Dutch } \\
\text { astronomer (1912-1972). }\end{array}$ \\
\hline Van Biesbroeck & $28.7 \mathrm{~N}$ & $45.6 \mathrm{~W}$ & 9 & NA & AM & S1 & $39 \mathrm{Al}$ & 5 & 1976 & 0 & AA & $\begin{array}{l}\text { George A.; } \\
\text { Belgian-American } \\
\text { astronomer (1880-1974). }\end{array}$ \\
\hline Van de Graaff & $27.4 S$ & $172.2 \mathrm{E}$ & 233 & NA & AM & $\mathrm{LM}$ & 104 & 5 & 1970 & 0 & AA & $\begin{array}{l}\text { Robert J.; American } \\
\text { physicist (1901-1967). }\end{array}$ \\
\hline van den Bergh & $31.3 \mathrm{~N}$ & $159.1 \mathrm{~W}$ & 42 & EU & DU & LOC & 4 & 5 & 1970 & 0 & AA & $\begin{array}{l}\text { G.; Dutch astronomer } \\
(1890-1966) \text {. }\end{array}$ \\
\hline van den Bos & $5.3 \mathrm{~S}$ & $146.0 \mathrm{E}$ & 22 & $\mathrm{AF}$ & SA & LTO & 084B3 & 5 & 1979 & 0 & AA & $\begin{array}{l}\text { Willem Hendrik; South } \\
\text { African astronomer } \\
(1896-1974) \text {. }\end{array}$ \\
\hline van der Waals & $43.9 \mathrm{~S}$ & $119.9 \mathrm{E}$ & 104 & EU & DU & LMP & 2 & 5 & 1970 & 0 & AA & $\begin{array}{l}\text { Johannes D.; Dutch } \\
\text { physicist; Nobel laureate } \\
\text { (1837-1923). }\end{array}$ \\
\hline van Gent & $15.4 \mathrm{~N}$ & $160.4 \mathrm{E}$ & 43 & EU & DU & LOC & 4 & 5 & 1970 & 0 & AA & $\begin{array}{l}\text { H.; Dutch astronomer } \\
1900-1947\end{array}$ \\
\hline Van Maanen & $35.7 \mathrm{~N}$ & $128.0 \mathrm{E}$ & 60 & NA & AM & LOC & 3 & 5 & 1970 & 0 & AA & $\begin{array}{l}\text { Adriaan; Dutch-American } \\
\text { astronomer (1884-1946). }\end{array}$ \\
\hline van Rhijn & $52.6 \mathrm{~N}$ & $146.4 \mathrm{E}$ & 46 & EU & DU & LMP & 3 & 5 & 1970 & 0 & AA & $\begin{array}{l}\text { Pieter J.; Dutch } \\
\text { astronomer (1886-1960). }\end{array}$ \\
\hline Van Vleck & $1.9 \mathrm{~S}$ & $78.3 \mathrm{E}$ & 31 & NA & AM & LTO & $081 \mathrm{~A} 2$ & 5 & 1976 & 0 & AA & $\begin{array}{l}\text { John Monroe; American } \\
\text { astronomer, mathematician } \\
\text { (1833-1912). }\end{array}$ \\
\hline $\operatorname{Van} W i j k$ & $62.8 \mathrm{~S}$ & $118.8 \mathrm{E}$ & 32 & EU & DU & LMP & 3 & 5 & 1970 & 0 & AA & $\begin{array}{l}\text { Uco; Dutch-American } \\
\text { astronomer (1924-1966). }\end{array}$ \\
\hline van't Hoff & $62.1 \mathrm{~N}$ & $131.8 \mathrm{~W}$ & 92 & EU & DU & LMP & 3 & 5 & 1970 & 0 & AA & $\begin{array}{l}\text { Jacobus H.; Dutch chemist; } \\
\text { Nobel laureate }(1852-1911)\end{array}$ \\
\hline Vasco da Gama & $13.6 \mathrm{~N}$ & $83.9 \mathrm{~W}$ & 83 & EU & PG & LOC & 1 & 5 & 1935 & 66 & AA & $\begin{array}{l}\text { Portuguese navigator, } \\
\text { explorer (1469-1524). }\end{array}$ \\
\hline Vashakidze & $43.6 \mathrm{~N}$ & $93.3 \mathrm{E}$ & 44 & UR & SO & LMP & 2 & 5 & 1970 & 0 & AA & $\begin{array}{l}\text { Mikhail A.; Soviet } \\
\text { astronomer (1909-1956). }\end{array}$ \\
\hline
\end{tabular}

lat: latitude of feature center.

long: longitude of feature center.

diam: diameter or long dimension of feature.

ct: continent of name origin (see page $284 \mathrm{ff}$.) et: $\quad$ ethnicity of name origin (see page $284 \mathrm{ff}$.)

quad: map quadrangle or informal name

(see page $x$ vii $f f$. .).

map name or USGS map number (see page xvii ff.). as: name approval status (see page $x$ vii)

ad: name approval date (year).

ref: reference source for name (see page $287 \mathrm{ff}$.). 


\section{MOON}

Name

Vavilov$$
0.8 \mathrm{~S}
$$

$$
\text { long }
$$

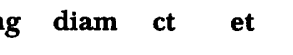

quad
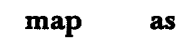

Vega

$45.4 \mathrm{~S}$

Vendelinus

$16.4 \mathrm{~S}$

$63.4 \mathrm{E}$

75 E

U

SO LOC

$61.6 \mathrm{E}$

131

EU B

Vening Meinesz

$0.3 \mathrm{~S} \quad 162.6 \mathrm{E}$

$87 \quad$ EU

Ventris

Vera

[Vergil]

$26.3 \mathrm{~N}$
$26.3 \mathrm{~S}$

Vernadskiy

Verne

Vertregt

Very

Vesalius

Vestine

Vetchinkin

$23.2 \mathrm{~N}$

$24.9 \mathrm{~N}$

$19.8 \mathrm{~S}$

$25.6 \mathrm{~N}$

$3.1 \mathrm{~S}$

$33.9 \mathrm{~N} \quad 93.9 \mathrm{E}$

$10.2 \mathrm{~N} \quad 131.3 \mathrm{E}$

$29.2 \mathrm{~S} \quad 56.3 \mathrm{~W}$

Vieta

Vil'ev

Virchow

6.1S $\quad 144.4 \mathrm{E}$

$9.8 \mathrm{~N}$

83.7E

$15.5 \mathrm{~N} \quad 176.7 \mathrm{E}$

Virtanen

Vitello

Vitruvius

$17.6 \mathrm{~N} \quad 31.3 \mathrm{E}$

42 E
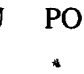

29 EU RM LTO

Viviani

Vlacq

Vogel

Volkov

Volta

[Voltaire]

Volterra

$17.6 \mathrm{~N} \quad 31.3 \mathrm{E}$

$5.2 \mathrm{~N} \quad 117.1 \mathrm{E}$

$53.3 \mathrm{~S} \quad 38.8 \mathrm{E}$

15.1S $5.9 \mathrm{E}$

13.6S 131.7E

$53.9 \mathrm{~N} \quad 84.4 \mathrm{~W}$

11.9S 100.3E

$56.8 \mathrm{~N} \quad 132.2 \mathrm{E}$

\section{EU RM LTO}

26 EU IT LTO

89 EU DU LAC

127

26 EU GE LAC

77

40 UR SO LTO

123 EU IT LMP

0 EU

$52 \mathrm{EU}$
FR LTO $082 \mathrm{Cl}$

3
93

39 A3

102D1

3

$40 \mathrm{~A} 4$

104

042B3

51935

$66 \mathrm{AA}$

51935

66 AA

$5 \quad 1970$

0 AA

$5 \quad 1970$

0 AA

AA
decipherer of Linear B
Cretan script

(1922-1956)

Soviet physiological

optician (1891-1951)

Georg Freiherr von; German

Wendelin, Godefroid;

Belgian astronomer

(1580-1667).

geophysicist, geodesist 1887-1966).

Roman epic poet (70-19

B.C.).

$51970 \quad 0 \quad$ AA Vladimir Ivanovich; Soviet

mineralogist (1863-1945).

$\begin{array}{lllll}5 & 1976 & 0 & \text { AA } & \text { Latin male name. } \\ 5 & 1979 & 0 & \text { AA } & \text { M.; Dutch chemist }\end{array}$

$\begin{array}{lllll}5 & 1976 & 0 & \text { AA } & \text { Latin male name. } \\ 5 & 1979 & 0 & \text { AA } & \text { M.; Dutch chemist }\end{array}$

(1897-1973)

$5 \quad 1973$

0 AA

Frank Washington; American astronomer (1852-1927).

$5 \quad 1970$

0 AA

Andreas; Belgian doctor

(1514-1564).

$5 \quad 1970$

0 AA

Ernest H.; American

physicist (1906-1968).

$5 \quad 1970$

0 AA

Vladimir P.; Soviet

physicist, engineer

(1888-1950).

5193566 AA Francois; French

mathematician

(1540-1603).

Mikhail; Russian chemist

(1893-1919)

Rudolph Ludwig Karl;

German doctor, pathologist

(1821-1902).

519790 AA Artturi Ilmari; Finnish agricultural biochemist; Nobel laureate

(1895-1973).

Witelo, Erazmus Ciokek;

Polish physicist

mathematician

(1210-1285)

043D4 5193566 AA Vitruvius Pollio, Marcus;

Roman engineer, architect

(unkn-fl, c. 25 B.C.)

Vincenzo; Italian

physicist, mathematician

(1622-1703).

Adriaan; Dutch

mathematician (c.

1600-1667).

Hermann Karl; German

astronomer (1841-1907)

Vladislav N.; Soviet

engineer (1935-1971).

Count Allessandro G. A.

A.; Italian physicist

(1745-1827).

Francois; French

philosopher (1694-1778)

Vito; Italian

mathematician, physicist (1860-1940). lat: latitude of feature center.

long: longitude of feature center.

diam: diameter or long dimension of feature.

ct: continent of name origin (see page 284 et: ethnicity of name origin (see page $284 \mathrm{ff}$.)

quad: map quadrangle or informal name

(see page xvii ff.). as: name approval status (see page xvii).

ad: name approval date (year).

ref: reference source for name (see page $287 \mathrm{ff}$.). 
MOON

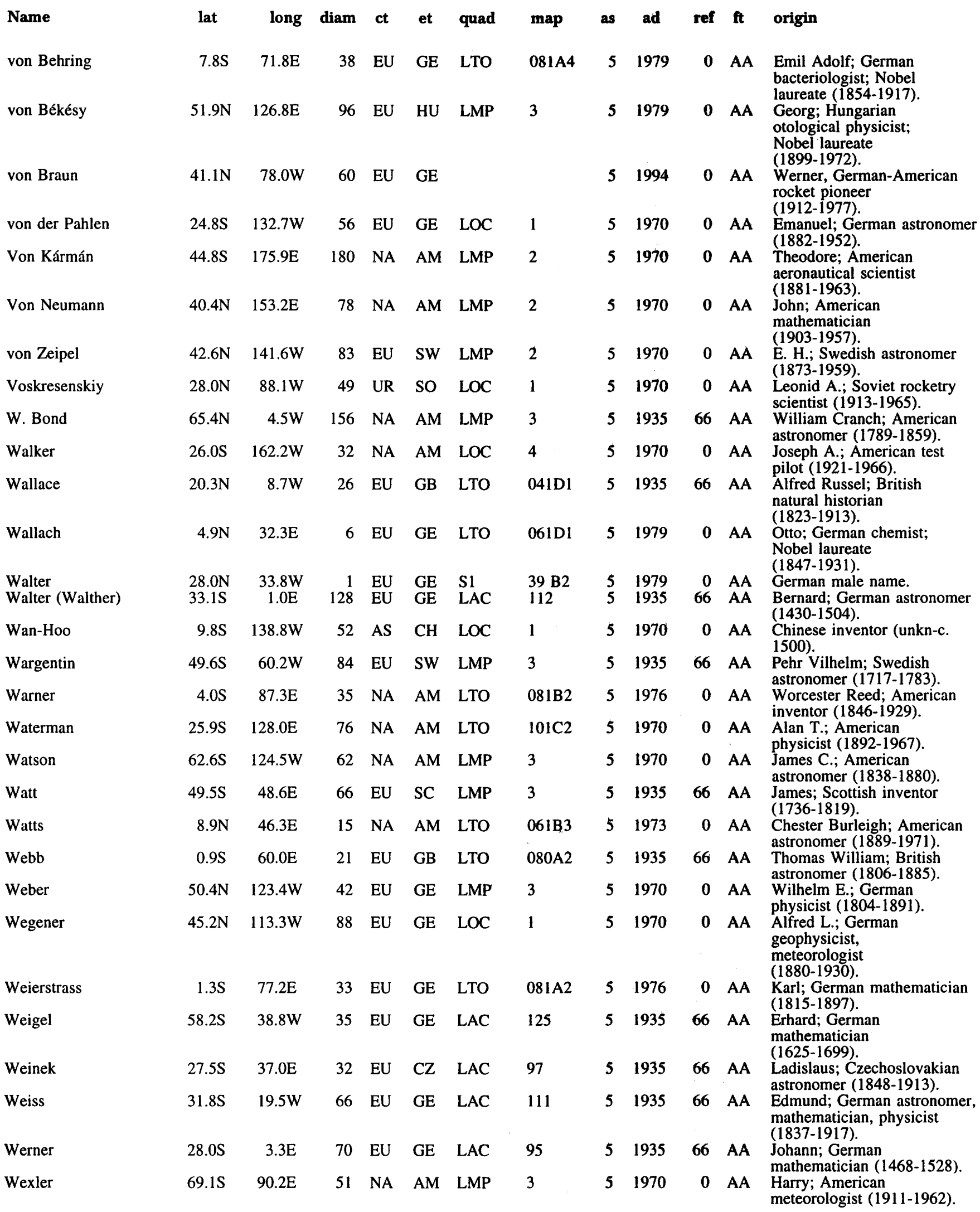

lat: latitude of feature center

long: longitude of feature center.

diam: diameter or long dimension of feature.

ct: continent of name origin (see page 284 ff.) et: ethnicity of name origin (see page $284 \mathrm{ff}$.)

quad: map quadrangle or informal name

(see page $x$ vii $\mathrm{ff}$.).

map name or USGS map number (see page xvii ff.). as: name approval status (see page xvii).

ad: name approval date (year).

ref: reference source for name (see page $287 \mathrm{ff}$.) 
MOON

\begin{tabular}{|c|c|c|c|c|c|c|c|c|c|c|c|c|}
\hline Name & lat & long & diam & ct & et & quad & map & as & ad & ref & $\mathrm{ft}$ & origin \\
\hline Weyl & $17.5 \mathrm{~N}$ & $120.2 \mathrm{~W}$ & 108 & EU & GE & LOC & 1 & 5 & 1970 & 0 & AA & $\begin{array}{l}\text { Hermann; German-American } \\
\text { mathematician (1885-1955). }\end{array}$ \\
\hline Whewell & $4.2 \mathrm{~N}$ & $13.7 \mathrm{E}$ & 13 & EU & GB & $\mathbf{L M}$ & 60 & 5 & 1935 & 66 & AA & $\begin{array}{l}\text { William; British } \\
\text { philosopher (1794-1866). }\end{array}$ \\
\hline White & $44.6 \mathrm{~S}$ & $158.3 \mathrm{~W}$ & 39 & NA & $\mathbf{A M}$ & LMP & 2 & 5 & 1970 & 0 & AA & $\begin{array}{l}\text { Edward H. II; American } \\
\text { astronaut (1930-1967). }\end{array}$ \\
\hline Wichmann & $7.5 \mathrm{~S}$ & $38.1 \mathrm{~W}$ & 10 & EU & GE & LAC & 75 & 5 & 1935 & 66 & AA & $\begin{array}{l}\text { Moritz Ludwig Georg; } \\
\text { German astronomer } \\
(1821-1859) \text {. }\end{array}$ \\
\hline Widmanstätten & $6.1 S$ & $85.5 \mathrm{E}$ & 46 & EU & $\mathrm{GE}$ & LTO & $081 \mathrm{B3}$ & 5 & 1973 & 0 & AA & $\begin{array}{l}\text { Aloys B.; German physicist } \\
\text { (1753-1849). }\end{array}$ \\
\hline Wiechert & $84.5 S$ & $165.0 \mathrm{E}$ & 41 & $\mathrm{EU}$ & $\mathrm{GE}$ & LMP & 3 & 5 & 1970 & 0 & AA & $\begin{array}{l}\text { E.; German geophysicist } \\
(1861-1928) \text {. }\end{array}$ \\
\hline Wiener & $40.8 \mathrm{~N}$ & $146.6 \mathrm{E}$ & 120 & NA & $\mathbf{A M}$ & LMP & 2 & 5 & 1970 & 0 & AA & $\begin{array}{l}\text { Norbert; American } \\
\text { mathematician (1894-1964). }\end{array}$ \\
\hline Wildt & $9.0 \mathrm{~N}$ & $75.8 \mathrm{E}$ & 11 & EU & GE & LTO & $063 \mathrm{~A} 3$ & 5 & 1979 & 0 & AA & $\begin{array}{l}\text { Rupert; German-American } \\
\text { astronomer (1905-1976). }\end{array}$ \\
\hline Wilhelm & $43.4 \mathrm{~S}$ & $20.4 \mathrm{~W}$ & 106 & EU & GE & LAC & 111 & 5 & 1935 & 66 & AA & $\begin{array}{l}\text { Wilhelm IV, Landgrave of } \\
\text { Hesse; German astronomer } \\
(1532-1592) \text {. }\end{array}$ \\
\hline Wilkins & $29.4 \mathrm{~S}$ & $19.6 \mathrm{E}$ & 57 & EU & GB & LAC & 96 & 5 & 1961 & 67 & AA & $\begin{array}{l}\text { Hugh Percy; British } \\
\text { selenographer (1896-1960). }\end{array}$ \\
\hline Williams & $42.0 \mathrm{~N}$ & $37.2 \mathrm{E}$ & 36 & EU & GB & LAC & 27 & 5 & 1935 & 66 & AA & $\begin{array}{l}\text { Arthur Stanley; British } \\
\text { astronomer (1861-1938). }\end{array}$ \\
\hline Wilsing & $21.5 \mathrm{~S}$ & $155.2 \mathrm{~W}$ & 73 & EU & GE & LOC & 4 & 5 & 1970 & 0 & AA & $\begin{array}{l}\text { J.; German astronomer } \\
(1856-1943) \text {. }\end{array}$ \\
\hline Wilson & $69.2 \mathrm{~S}$ & $42.4 \mathrm{~W}$ & 69 & $\mathrm{EU}$ & SC & LMP & 3 & 5 & 1935 & 66 & AA & $\begin{array}{l}\text { Alexander; Scottish } \\
\text { astronomer (1714-1786); } \\
\text { Charles T.R.; Scottish } \\
\text { physicist (1869-1959); } \\
\text { Ralph E.; American } \\
\text { astronomer ((1886-1960). }\end{array}$ \\
\hline Winkler & $42.2 \mathrm{~N}$ & $179.0 \mathrm{~W}$ & 22 & EU & $\mathrm{GE}$ & LMP & 2 & 5 & 1970 & 0 & AA & $\begin{array}{l}\text { Johannes; German rocketry } \\
\text { scientist (1897-1947). }\end{array}$ \\
\hline Winlock & $35.6 \mathrm{~N}$ & $105.6 \mathrm{~W}$ & 64 & NA & AM & LMP & 2 & 5 & 1970 & 0 & AA & $\begin{array}{l}\text { Joseph; American } \\
\text { astronomer (1826-1875). }\end{array}$ \\
\hline Winthrop & $10.7 \mathrm{~S}$ & $44.4 \mathrm{~W}$ & 17 & NA & AM & LTO & 075D2 & 5 & 1976 & 0 & AA & $\begin{array}{l}\text { John; American astronomer } \\
\text { (1714-1779). }\end{array}$ \\
\hline Wöhler & $38.2 S$ & $31.4 \mathrm{E}$ & 27 & EU & $\mathrm{GE}$ & LOC & 3 & 5 & 1935 & 66 & AA & $\begin{array}{l}\text { Friedrich; German chemist } \\
(1800-1882) \text {. }\end{array}$ \\
\hline Wolf & $22.7 \mathrm{~S}$ & $16.6 \mathrm{~W}$ & 25 & $\mathrm{EU}$ & GE & LAC & 94 & 5 & 1935 & 66 & AA & $\begin{array}{l}\text { Maxmilian Franz Joseph } \\
\text { Cornelius; German } \\
\text { astronomer (1863-1932). }\end{array}$ \\
\hline Wollaston & $30.6 \mathrm{~N}$ & $46.9 \mathrm{~W}$ & 10 & EU & GB & LTO & 039A1 & 5 & 1935 & 66 & AA & $\begin{array}{l}\text { William Hyde; British } \\
\text { chemist, physicist } \\
\text { (1766-1828). }\end{array}$ \\
\hline Woltjer & $45.2 \mathrm{~N}$ & $159.6 \mathrm{~W}$ & 46 & EU & $\mathrm{DU}$ & LMP & 2 & 5 & 1970 & 0 & AA & $\begin{array}{l}\text { Jan; Dutch astronomer } \\
(1891-1946)\end{array}$ \\
\hline Wood & $43.0 \mathrm{~N}$ & $120.8 \mathrm{~W}$ & 78 & NA & AM & LMP & 2 & 5 & 1970 & 0 & AA & $\begin{array}{l}\text { Robert W.; American } \\
\text { physicist (1868-1955). }\end{array}$ \\
\hline Wright & $31.6 \mathrm{~S}$ & $86.6 \mathrm{~W}$ & 39 & NA & AM & LOC & 1 & 5 & 1964 & 67 & AA & $\begin{array}{l}\text { Frederick E.; American } \\
\text { astronomer (1878-1953); } \\
\text { Thomas; British } \\
\text { philosopher (1711-1786); } \\
\text { Willian H.; American } \\
\text { astronomer (1871-1959). }\end{array}$ \\
\hline Wróblewski & $24.0 \mathrm{~S}$ & $152.8 \mathrm{E}$ & 21 & EU & $\mathrm{PO}$ & LTO & $103 \mathrm{~A} 4$ & 5 & 1976 & 0 & AA & $\begin{array}{l}\text { Sigmund von; Polish } \\
\text { physicist (1845-1888). }\end{array}$ \\
\hline Wrottesley & $23.9 \mathrm{~S}$ & $56.8 \mathrm{E}$ & 57 & EU & GB & LAC & 98 & 5 & 1935 & 66 & AA & $\begin{array}{l}\text { John, Baron Wrottesley; } \\
\text { British astronomer } \\
\text { (1798-1867). }\end{array}$ \\
\hline Wurzelbauer & $33.9 \mathrm{~S}$ & $15.9 \mathrm{~W}$ & 88 & EU & GE & LAC & 111 & 5 & 1935 & 66 & AA & $\begin{array}{l}\text { Johann Philipp von; German } \\
\text { astronomer (1651-1725). }\end{array}$ \\
\hline Wyld & $1.4 \mathrm{~S}$ & $98.1 \mathrm{E}$ & 93 & NA & AM & LTO & 064D3 & 5 & 1970 & 0 & AA & $\begin{array}{l}\text { James H.; American } \\
\text { rocketry scientist } \\
\text { (1913-1953). }\end{array}$ \\
\hline Xenophanes & $57.5 \mathrm{~N}$ & $82.0 \mathrm{~W}$ & 125 & EU & GR & LMP & 3 & 5 & 1935 & 66 & AA & $\begin{array}{l}\text { Of Colophon; Greek } \\
\text { philosopher (570(?)-478(?) } \\
\text { B.C.). }\end{array}$ \\
\hline Xenophon & $22.8 \mathrm{~S}$ & $122.1 \mathrm{E}$ & 25 & EU & GR & LTO & $101 \mathrm{~B} 4$ & 5 & 1976 & 0 & AA & $\begin{array}{l}\text { Greek natural philosopher, } \\
\text { historian (c. } 430-354 \text { B.C.). }\end{array}$ \\
\hline
\end{tabular}

lat: latitude of feature center.

long: longitude of feature center.

diam: diameter or long dimension of feature.

ct: et: ethnicity of name origin (see page $284 \mathrm{ff}$.)

quad: map quadrangle or informal name

(see page $x$ vii ff.). as: name approval status (see page xvii).

ad: name approval date (year).

ref: reference source for name (see page $287 \mathrm{ff}$.). 


\section{MOON}

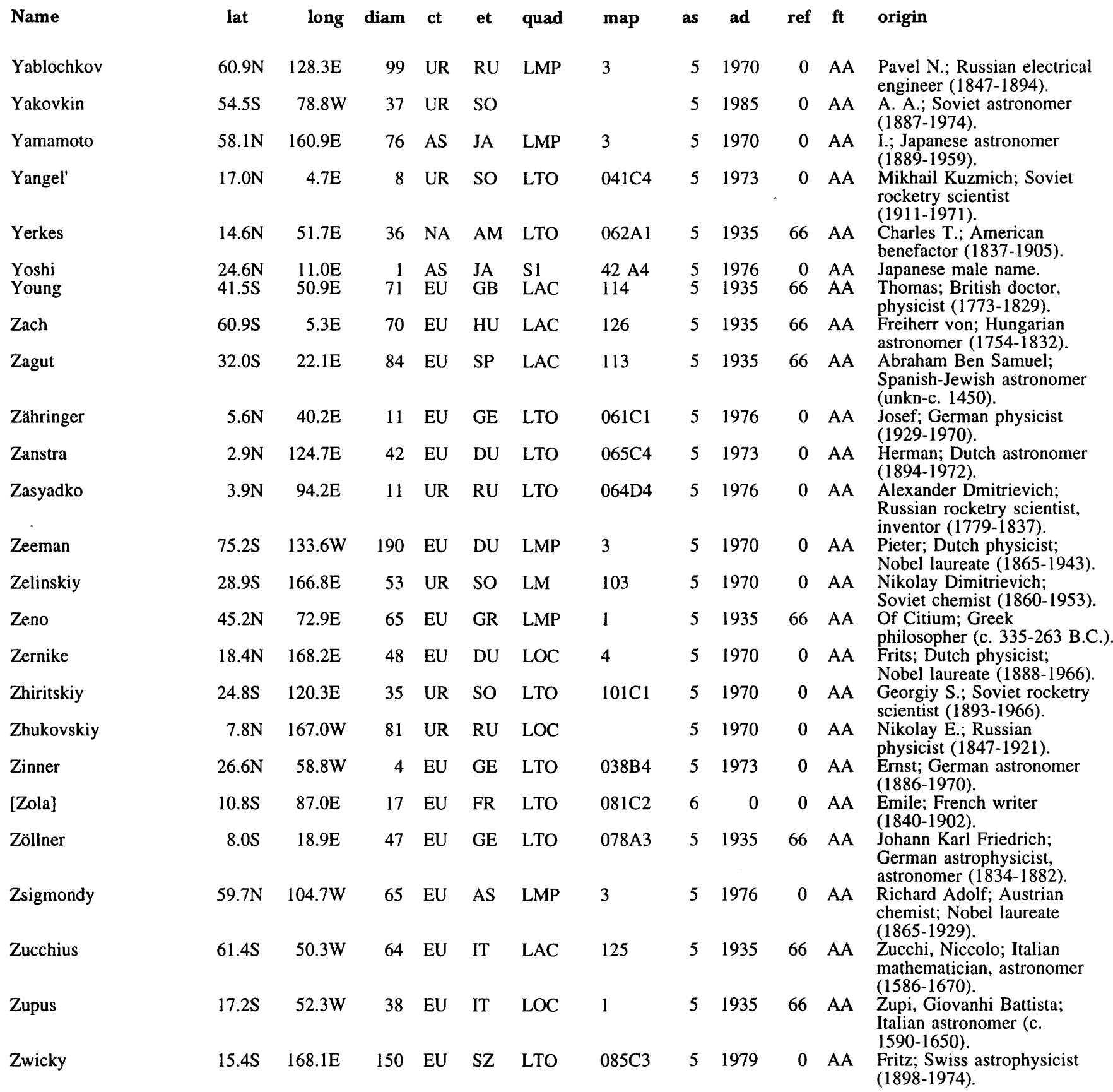

\section{ALBEDO FEATURE}

Reiner Gamma

CATENA

Catena Abulfeda

Catena Artamonov

Catena Brigitte

Catena Davy

Catena Dziewulski

Catena Gregory

Catena Humboldt

Catena Krafft

Catena Kurchatov
$7.5 \mathrm{~N}$

$\begin{array}{rrrllll}16.9 \mathrm{~S} & 17.2 \mathrm{E} & 219 & \text { AS } & \text { SY } & \text { LAC } & 96 \\ 26.0 \mathrm{~N} & 105.9 \mathrm{E} & 134 & \text { UR } & \text { SO } & \text { LOC } & 3 \\ 18.5 \mathrm{~N} & 27.5 \mathrm{E} & 5 & \text { EU } & \text { FR } & \text { S2(10) } & 42 \mathrm{C} 3 \\ 11.0 \mathrm{~S} & 7.0 \mathrm{~W} & 50 & \text { EU } & \text { GB } & \text { S1 } & 77 \mathrm{D} 1 \\ 19.0 \mathrm{~N} & 100.0 \mathrm{E} & 80 & \text { EU } & \text { PO } & \text { LOC } & 3 \\ 0.6 \mathrm{~S} & 129.9 \mathrm{E} & 152 & \text { EU } & \text { SC } & \text { LOC } & 3 \\ 21.5 \mathrm{~S} & 84.6 \mathrm{E} & 165 & \text { EU } & \text { GE } & \text { LOC } & 3 \\ 15.0 \mathrm{~N} & 72.0 \mathrm{~W} & 60 & \text { EU } & \text { GE } & \text { LOC } & 1 \\ 37.2 \mathrm{~N} & 136.3 \mathrm{E} & 226 & \text { UR } & \text { SO } & \text { LOC } & 4\end{array}$

51935

66 AL Bright marking, named from nearby crater.
0 CA Named from nearby crater.

0 CA Named from nearby crater.

0 CA French female name.

0 CA Named from nearby crater.

0 CA Named from nearby crater.

0 CA Named from nearby crater.

0 CA Named from nearby crater.

0 CA Named from nearby crater.

0 CA Named from nearby crater. lat: latitude of feature center.

long: longitude of feature center.

diam: diameter or long dimension of feature.

ct: continent of name origin (see page $284 \mathrm{ff}$ ) et: ethnicity of name origin (see page $284 \mathrm{ff}$.)

quad: map quadrangle or informal name

(see page $x$ vii $\mathrm{ff}$.).

map name or USGS map number (vee page xvii as: name approval status (see page xvii).

ad: name approval date (year).

ref: reference source for name (see page $287 \mathrm{ff}$.) 


\section{MOON}

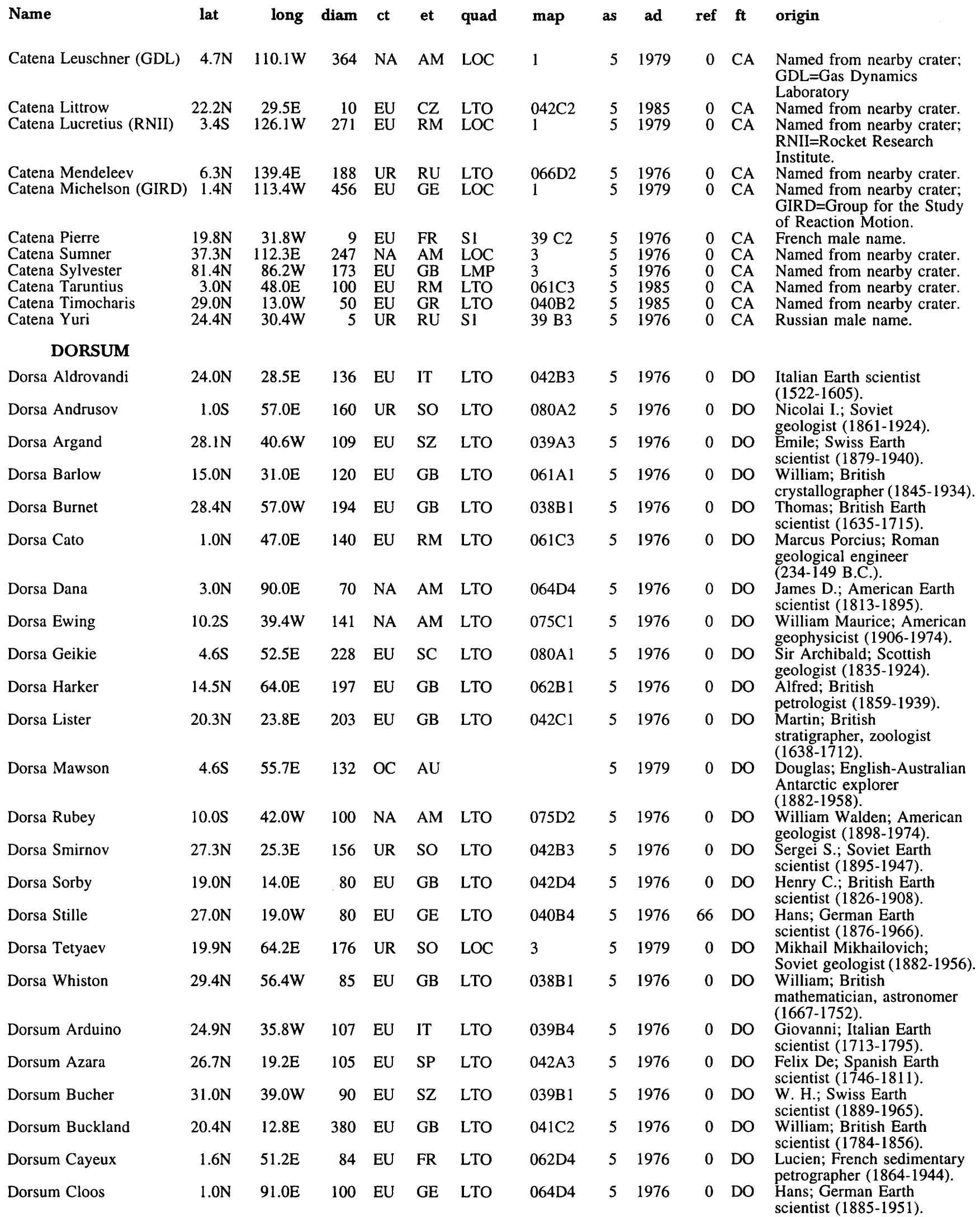

lat: latitude of feature center.

long: longitude of feature center.

diam: diameter or long dimension of feature.

ct: continent of name origin (see page 284 et: ethnicity of name origin (see page $284 \mathrm{ff}$.)

quad: map quadrangle or informal name

(see page xvii ff.). as: name approval status (see page xvii).

ad: name approval date (year).

ref: reference source for name (see page $287 \mathrm{ff}$.). 
MOON

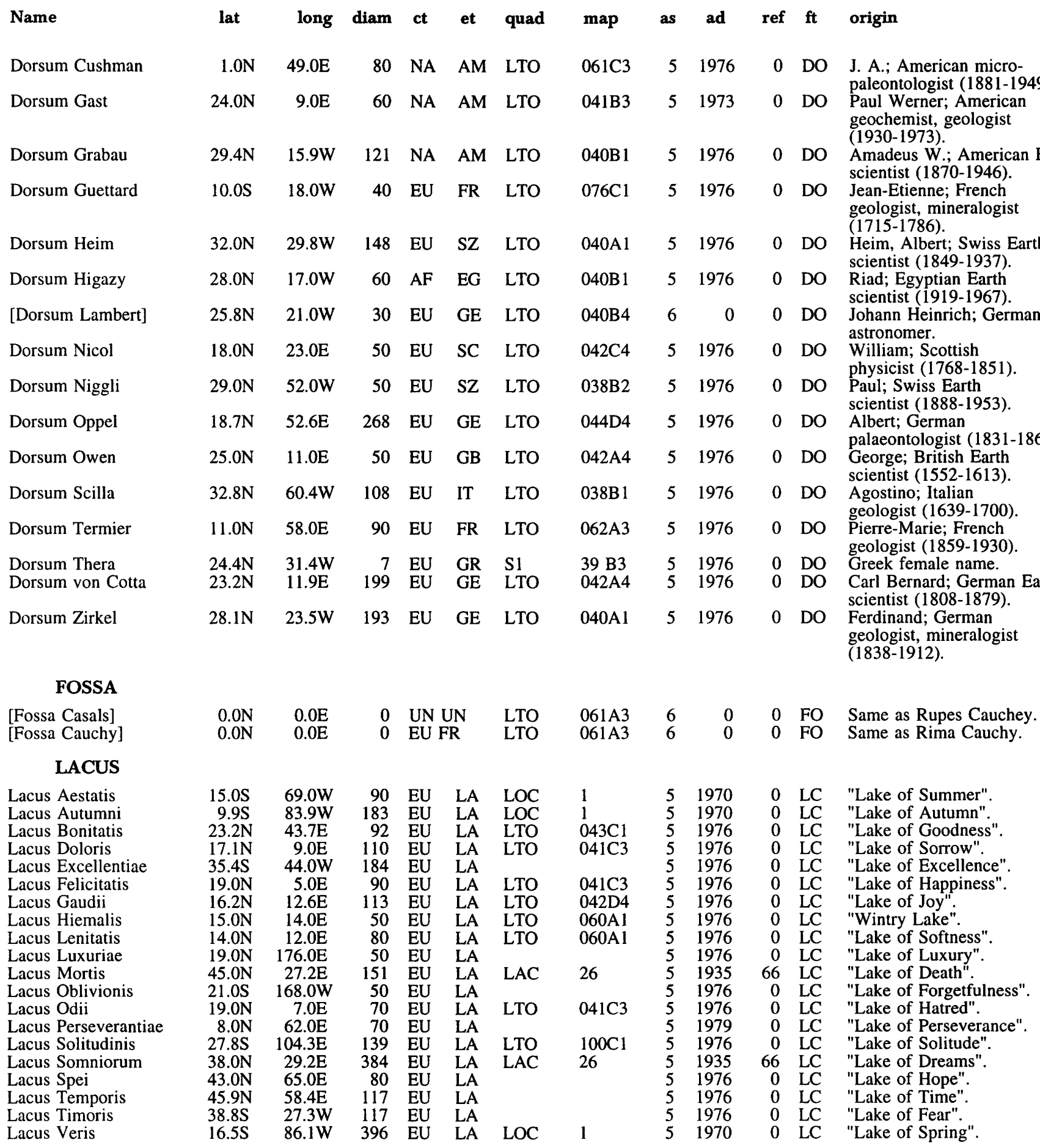

\section{APOLLO LANDING SITE}

\begin{tabular}{|c|c|c|c|c|c|c|c|c|c|c|c|c|}
\hline Apennine Front & $25.9 \mathrm{~N}$ & $3.7 \mathrm{E}$ & 6 & EU & IT & S1 & $41 \mathrm{~B} 4$ & 5 & 1973 & 59 & LF & $\begin{array}{l}\text { Astronaut-named feature, } \\
\text { Apollo } 15 \text { site. }\end{array}$ \\
\hline Baby Ray & $9.1 \mathrm{~S}$ & $15.4 \mathrm{E}$ & 0 & NA & $\mathrm{AM}$ & S2 & $78 \mathrm{D} 2$ & 5 & 1973 & 60 & LF & Astronaut-named feature, \\
\hline Bear Mountain & $20.0 \mathrm{~N}$ & $30.7 \mathrm{E}$ & 0 & NA & $\mathrm{AM}$ & S1 & $43 \mathrm{D} 1$ & 5 & 1973 & 59 & LF & $\begin{array}{l}\text { Astronaut-named feature, } \\
\text { Apollo } 17 \text { site. }\end{array}$ \\
\hline Bench & $3.2 \mathrm{~S}$ & $23.4 \mathrm{~W}$ & 0 & NA & $\mathrm{AM}$ & LAC & 76 & 5 & 1973 & 60 & LF & $\begin{array}{l}\text { Astronaut-named feature, } \\
\text { Apollo } 12 \text { site. }\end{array}$ \\
\hline
\end{tabular}

lat: latitude of feature center.

long: longitude of feature center.

diam: diameter or long dimension of feature.

et: ethnicity of name origin (see page $284 \mathrm{ff}$.)

quad: map quadrangle or informal name

(see page $x$ vii $\mathrm{ff}$.).

ct: continent of name origin (see page $284 \mathrm{ff}$.) map: map name or USGS map number (see page xvii ff.). ft: feature type (see page 290 ).

as: name approval status (see page xvii)

ad: name approval date (year).

ref: reference source for name (see page $287 \mathrm{ff}$.) 
MOON

\begin{tabular}{|c|c|c|c|c|c|c|c|c|c|c|c|c|}
\hline Name & lat & long & diam & ct & et & quad & map & as & ad & ref & $\mathbf{f t}$ & origin \\
\hline Block & $3.2 \mathrm{~S}$ & $23.4 \mathrm{~W}$ & 0 & NA & $\mathrm{AM}$ & LAC & 76 & 5 & 1973 & 60 & LF & $\begin{array}{l}\text { Astronaut-named feature, } \\
\text { Apollo } 12 \text { site. }\end{array}$ \\
\hline Bowen-Apollo & $20.3 N$ & $30.9 \mathrm{E}$ & 0 & NA & $\mathrm{AM}$ & S2 & $43 \mathrm{D} 1$ & 5 & 1973 & 0 & LF & $\begin{array}{l}\text { Astronaut-named feature, } \\
\text { Apollo } 17 \text { site. }\end{array}$ \\
\hline Bridge & $26.0 \mathrm{~N}$ & $3.6 \mathrm{E}$ & 1 & NA & $\mathrm{AM}$ & S4 & $41 \mathrm{~B} 4$ & 5 & 1973 & 60 & LF & $\begin{array}{l}\text { Astronaut-named feature, } \\
\text { Apollo } 15 \text { site. }\end{array}$ \\
\hline Brontë & $20.2 \mathrm{~N}$ & $30.7 \mathrm{E}$ & 0 & NA & AM & S1 & $43 \mathrm{D} 1$ & 5 & 1973 & 60 & LF & $\begin{array}{l}\text { Astronaut-named feature, } \\
\text { Apollo } 17 \text { site. }\end{array}$ \\
\hline Camelot & $20.2 \mathrm{~N}$ & $30.7 \mathrm{E}$ & 1 & $\mathrm{EU}$ & GB & $\mathrm{S} 2$ & $43 \mathrm{DI}$ & 5 & 1973 & 60 & LF & $\begin{array}{l}\text { Astronaut-named feature, } \\
\text { Apollo } 17 \text { site. }\end{array}$ \\
\hline Cinco & $19.1 S$ & $15.5 \mathrm{E}$ & 0 & NA & AM & S2 & $78 \mathrm{D} 2$ & 5 & 1973 & 60 & LF & $\begin{array}{l}\text { Astronaut-named feature, } \\
\text { Apollo } 16 \text { site. }\end{array}$ \\
\hline Cochise & $20.2 S$ & $30.8 \mathrm{E}$ & 1 & NA & $\mathrm{AM}$ & S2 & 43 D1 & 5 & 1973 & 60 & LF & $\begin{array}{l}\text { Astronaut-named feature, } \\
\text { Apollo } 17 \text { site. }\end{array}$ \\
\hline Cone & $3.7 \mathrm{~S}$ & $17.4 \mathrm{~W}$ & 0 & NA & AM & LAC & 76 & 5 & 1973 & 60 & LF & $\begin{array}{l}\text { Astronaut-named feature, } \\
\text { Apollo } 14 \text { site. }\end{array}$ \\
\hline Crescent & $2.9 \mathrm{~S}$ & $23.4 \mathrm{~W}$ & 1 & NA & AM & LAC & 76 & 5 & 1973 & 60 & LF & $\begin{array}{l}\text { Astronaut-named feature, } \\
\text { Apollo } 12 \text { site. }\end{array}$ \\
\hline Doublet & $3.7 \mathrm{~S}$ & $17.5 \mathrm{~W}$ & 0 & NA & AM & LAC & 76 & 5 & 1973 & 60 & LF & $\begin{array}{l}\text { Asrtonaut-named feature, } \\
\text { Apollo } 14 \text { site. }\end{array}$ \\
\hline Dune & $26.0 \mathrm{~N}$ & $3.7 \mathrm{E}$ & 0 & NA & AM & S1 & $41 \mathrm{~B} 4$ & 5 & 1973 & 60 & LF & $\begin{array}{l}\text { Astronaut-named feature, } \\
\text { Apollo } 15 \text { site. }\end{array}$ \\
\hline Earthlight & $26.1 \mathrm{~N}$ & $3.7 \mathrm{E}$ & 0 & NA & $\mathrm{AM}$ & S1 & 41 B4 & 5 & 1973 & 60 & LF & $\begin{array}{l}\text { Astronaut-named feature, } \\
\text { Apollo } 15 \text { site. }\end{array}$ \\
\hline Elbow & $26.0 \mathrm{~N}$ & $3.6 \mathrm{E}$ & 0 & NA & AM & S1 & 41 B4 & 5 & 1973 & 60 & LF & $\begin{array}{l}\text { Astronaut-named feature, } \\
\text { Apollo } 15 \text { site. }\end{array}$ \\
\hline Emory & $20.1 \mathrm{~N}$ & $30.8 \mathrm{E}$ & 1 & NA & AM & S1 & $43 \mathrm{D} 1$ & 5 & 1973 & 60 & LF & $\begin{array}{l}\text { Astronaut-named feature, } \\
\text { Apollo } 17 \text { site. }\end{array}$ \\
\hline End & $8.9 \mathrm{~S}$ & $15.6 \mathrm{E}$ & 0 & NA & AM & S1 & $78 \mathrm{D} 2$ & 5 & 1973 & 60 & LF & $\begin{array}{l}\text { Astronaut-named feature, } \\
\text { Apollo } 16 \text { site. }\end{array}$ \\
\hline Family Mountain & $20.4 \mathrm{~N}$ & $30.3 \mathrm{E}$ & 7 & NA & AM & S1 & $43 \mathrm{D} 1$ & 5 & 1973 & 59 & LF & $\begin{array}{l}\text { Astronaut-named feature, } \\
\text { Apollo } 17 \text { site. }\end{array}$ \\
\hline Flag & $9.0 \mathrm{~S}$ & $15.5 \mathrm{E}$ & 0 & NA & $\mathrm{AM}$ & $\mathrm{S} 2$ & $78 \mathrm{D} 2$ & 5 & 1973 & 60 & LF & $\begin{array}{l}\text { Astronaut-named feature, } \\
\text { Apollo } 16 \text { site. }\end{array}$ \\
\hline Flank & $3.7 \mathrm{~S}$ & $17.4 \mathrm{~W}$ & 0 & NA & AM & LAC & 76 & 5 & 1973 & 60 & LF & $\begin{array}{l}\text { Astronaut-named feature, } \\
\text { Apollo } 14 \text { site. }\end{array}$ \\
\hline Gator & $9.0 \mathrm{~S}$ & $15.6 \mathrm{E}$ & 1 & NA & $\mathrm{AM}$ & S2 & $78 \mathrm{D} 2$ & 5 & 1973 & 60 & LF & $\begin{array}{l}\text { Astronaut-named feature, } \\
\text { Apollo } 16 \text { site. }\end{array}$ \\
\hline Halfway & $9.0 \mathrm{~S}$ & $15.5 \mathrm{E}$ & 0 & NA & $\mathrm{AM}$ & S2 & $78 \mathrm{D} 2$ & 5 & 1973 & 59 & LF & $\begin{array}{l}\text { Astronaut-named feature, } \\
\text { Apollo } 16 \text { site. }\end{array}$ \\
\hline Halo & $3.2 \mathrm{~S}$ & $23.4 \mathrm{~W}$ & 0 & NA & AM & LAC & 76 & 5 & 1973 & 60 & LF & $\begin{array}{l}\text { Astronaut-named feature, } \\
\text { Apollo } 12 \text { site. }\end{array}$ \\
\hline Head & $3.0 \mathrm{~S}$ & $23.4 \mathrm{~W}$ & 0 & NA & $\mathrm{AM}$ & LAC & 76 & 5 & 1973 & 59 & LF & $\begin{array}{l}\text { Astronaut-named feature, } \\
\text { Apollo } 12 \text { site. }\end{array}$ \\
\hline Hess-Apollo & $20.1 \mathrm{~N}$ & $30.7 \mathrm{E}$ & 1 & NA & AM & S1 & $43 \mathrm{D} 1$ & 5 & 1973 & 59 & LF & $\begin{array}{l}\text { Astronaut-named feature, } \\
\text { Apollo } 17 \text { site. }\end{array}$ \\
\hline Horatio & $20.2 \mathrm{~N}$ & $30.7 \mathrm{E}$ & 0 & NA & AM & S1 & $43 \mathrm{D} 1$ & 5 & 1973 & 59 & LF & $\begin{array}{l}\text { Astronaut-named feature, } \\
\text { Apollo } 17 \text { site. }\end{array}$ \\
\hline Index & $26.1 \mathrm{~N}$ & $3.7 \mathrm{E}$ & 0 & NA & $\mathrm{AM}$ & S1 & $41 \mathrm{~B} 4$ & 5 & 1973 & 59 & LF & $\begin{array}{l}\text { Astronaut-named feature, } \\
\text { Apollo } 15 \text { site. }\end{array}$ \\
\hline Kiva & $8.6 \mathrm{~S}$ & $15.5 \mathrm{E}$ & 1 & NA & PU & S1 & $78 \mathrm{D} 2$ & 5 & 1973 & 59 & LF & $\begin{array}{l}\text { Astronaut-named feature, } \\
\text { Apollo } 16 \text { site. }\end{array}$ \\
\hline Lara & $20.4 \mathrm{~N}$ & $30.5 \mathrm{E}$ & 0 & NA & $\mathrm{AM}$ & S2 & $43 \mathrm{D} 1$ & 5 & 1973 & 59 & LF & $\begin{array}{l}\text { Astronaut-named feature, } \\
\text { Apollo } 17 \text { site. }\end{array}$ \\
\hline Last & $26.1 \mathrm{~N}$ & $0.0 \mathrm{E}$ & 0 & NA & $\mathrm{AM}$ & S1 & $41 \mathrm{~B} 4$ & 5 & 1973 & 59 & LF & $\begin{array}{l}\text { Astronaut-named feature, } \\
\text { Apollo } 15 \text { site. }\end{array}$ \\
\hline Light Mantle & $20.2 \mathrm{~N}$ & $30.8 \mathrm{E}$ & 4 & NA & AM & S1 & $43 \mathrm{D} 1$ & 5 & 1973 & 59 & LF & $\begin{array}{l}\text { Astronaut-named feature, } \\
\text { Apollo } 17 \text { site. }\end{array}$ \\
\hline Mackin-Apollo (Mackin) & $20.1 \mathrm{~N}$ & $30.7 \mathrm{E}$ & 0 & NA & $\mathrm{AM}$ & S1 & $43 \mathrm{D} 1$ & 5 & 1973 & 59 & LF & $\begin{array}{l}\text { Astronaut-named feature, } \\
\text { Apollo } 17 \text { site. }\end{array}$ \\
\hline Middle Crescent & $3.2 \mathrm{~S}$ & $23.4 \mathrm{~W}$ & 0 & NA & AM & LAC & 76 & 5 & 1973 & 59 & LF & $\begin{array}{l}\text { Astronaut-named feature, } \\
\text { Apollo } 12 \text { site. }\end{array}$ \\
\hline Nansen-Apollo & $20.1 \mathrm{~N}$ & $30.5 \mathrm{E}$ & 1 & NA & AM & SI & $43 \mathrm{D} 1$ & 5 & 1973 & 59 & LF & $\begin{array}{l}\text { Astronaut-named feature, } \\
\text { Apollo } 17 \text { site. }\end{array}$ \\
\hline North Complex & $26.2 \mathrm{~N}$ & $3.6 \mathrm{E}$ & 2 & NA & AM & S1 & $41 \mathrm{~B} 4$ & 5 & 1973 & 59 & LF & $\begin{array}{l}\text { Astronaut-named feature, } \\
\text { Apollo } 15 \text { site. }\end{array}$ \\
\hline North Massif & $20.4 \mathrm{~N}$ & $30.8 \mathrm{E}$ & 14 & EU & FR & S1 & $43 \mathrm{D} 1$ & 5 & 1973 & 59 & LF & $\begin{array}{l}\text { Astronaut-named feature, } \\
\text { Apollo } 17 \text { site. }\end{array}$ \\
\hline North Ray & $8.8 \mathrm{~S}$ & $15.5 \mathrm{E}$ & 1 & NA & AM & S1 & $78 \mathrm{D} 2$ & 5 & 1973 & 59 & LF & $\begin{array}{l}\text { Astronaut-named feature, } \\
\text { Apollo } 16 \text { site. }\end{array}$ \\
\hline
\end{tabular}

lat: latitude of feature center.

long: longitude of feature center.

diam: diameter or long dimension of feature.

ct: continent of name origin (see page $284 \mathrm{ff}$ ) et: $\quad$ ethnicity of name origin (see page $284 \mathrm{ff}$.)

quad: map quadrangle or informal name

(see page xvii ff.).

map name or USGS map number (see page xvii ff.) as: name approval status (see page $\mathrm{xvii}$.

ad: name approval date (year).

ref: reference source for name (see page $287 \mathrm{ff}$.) 


\section{MOON}

\begin{tabular}{|c|c|c|c|c|c|c|c|c|c|c|c|c|}
\hline Name & lat & long & diam & ct & et & quad & map & as & ad & ref & $\mathbf{f t}$ & origin \\
\hline Old Nameless & $3.7 \mathrm{~S}$ & $17.5 \mathrm{~W}$ & 0 & NA & $\mathrm{AM}$ & LAC & 76 & 5 & 1973 & 59 & LF & $\begin{array}{l}\text { Astronaut-named feature, } \\
\text { Apollo } 14 \text { site. }\end{array}$ \\
\hline Palmetto & $8.9 \mathrm{~S}$ & $15.5 \mathrm{E}$ & 0 & NA & $\mathrm{AM}$ & $\mathrm{S} 1$ & $78 \mathrm{D} 2$ & 5 & 1973 & 59 & LF & $\begin{array}{l}\text { Astronaut-named feature, } \\
\text { Apollo } 16 \text { site. }\end{array}$ \\
\hline Plain & $26.2 \mathrm{~N}$ & $3.6 \mathrm{E}$ & 2 & NA & $\mathrm{AM}$ & $\mathrm{S} 1$ & $41 \mathrm{~B} 4$ & 5 & 1973 & 59 & LF & $\begin{array}{l}\text { Astronaut-named feature, } \\
\text { Apollo } 15 \text { site. }\end{array}$ \\
\hline Plum & $9.0 \mathrm{~S}$ & $15.5 \mathrm{E}$ & 0 & NA & $\mathrm{AM}$ & $\mathrm{S} 1$ & $78 \mathrm{D} 2$ & 5 & 1973 & 59 & LF & $\begin{array}{l}\text { Astronaut-named feature, } \\
\text { Apollo } 16 \text { site. }\end{array}$ \\
\hline Powell & $20.2 \mathrm{~N}$ & $30.8 \mathrm{E}$ & 1 & NA & $\mathrm{AM}$ & $\mathrm{S} 2$ & $43 \mathrm{D} 1$ & 5 & 1973 & 60 & $\mathbf{L F}$ & $\begin{array}{l}\text { Astronaut-named feature, } \\
\text { Apollo } 17 \text { site. }\end{array}$ \\
\hline Ravine & $8.9 \mathrm{~S}$ & $15.6 \mathrm{E}$ & 1 & NA & $\mathrm{AM}$ & S1 & $78 \mathrm{D} 2$ & 5 & 1973 & 59 & LF & $\begin{array}{l}\text { Astronaut-named feature, } \\
\text { Apollo } 16 \text { site. }\end{array}$ \\
\hline Rhysling & $26.1 \mathrm{~N}$ & $3.7 \mathrm{E}$ & 0 & NA & $\mathrm{AM}$ & $\mathrm{S} 1$ & $41 \mathrm{~B} 4$ & 5 & 1973 & 59 & LF & $\begin{array}{l}\text { Astronaut-named feature, } \\
\text { Apollo } 15 \text { site. }\end{array}$ \\
\hline Scarp & $20.3 \mathrm{~N}$ & $30.6 \mathrm{E}$ & 8 & NA & $\mathrm{AM}$ & S1 & $43 \mathrm{Dl}$ & 5 & 1973 & 59 & LF & $\begin{array}{l}\text { Astronaut-named feature, } \\
\text { Apollo } 17 \text { site. }\end{array}$ \\
\hline Sculptured Hills & $20.3 \mathrm{~N}$ & $31.0 \mathrm{E}$ & 8 & NA & $\mathrm{AM}$ & $\mathrm{S} 1$ & $43 \mathrm{D} 1$ & 5 & 1973 & 59 & LF & $\begin{array}{l}\text { Astronaut-named feature, } \\
\text { Apollo } 17 \text { site. }\end{array}$ \\
\hline Shakespeare & $20.2 \mathrm{~N}$ & $30.8 \mathrm{E}$ & 1 & $\mathrm{EU}$ & GB & $\mathrm{S} 1$ & $43 \mathrm{Dl}$ & 5 & 1973 & 59 & LF & $\begin{array}{l}\text { Astronaut-named feature, } \\
\text { Apollo } 17 \text { site. }\end{array}$ \\
\hline Sharp-Apollo & $3.2 \mathrm{~S}$ & $23.4 \mathrm{~W}$ & 0 & NA & $\mathrm{AM}$ & $\mathrm{LAC}$ & 76 & 5 & 1973 & 59 & LF & $\begin{array}{l}\text { Astronaut-named feature, } \\
\text { Apollo } 12 \text { site. }\end{array}$ \\
\hline Sherlock & $20.2 \mathrm{~N}$ & $30.8 \mathrm{E}$ & 0 & $\mathrm{EU}$ & GB & S1 & $43 \mathrm{D} 1$ & 5 & 1973 & 59 & LF & $\begin{array}{l}\text { Astronaut-named feature, } \\
\text { Apollo } 17 \text { site. }\end{array}$ \\
\hline Shorty & $20.2 \mathrm{~N}$ & $30.6 \mathrm{E}$ & 0 & NA & $\mathrm{AM}$ & $\mathrm{S} 1$ & 43 D1 & 5 & 1973 & 59 & LF & $\begin{array}{l}\text { Astronaut-named feature, } \\
\text { Apollo } 17 \text { site. }\end{array}$ \\
\hline Smoky Mountains & $8.8 \mathrm{~S}$ & $15.6 \mathrm{E}$ & 3 & NA & $\mathrm{AM}$ & S1 & $78 \mathrm{D} 2$ & 5 & 1973 & 59 & LF & $\begin{array}{l}\text { Astronaut-named feature, } \\
\text { Apollo } 16 \text { site. }\end{array}$ \\
\hline Snowman & $3.2 \mathrm{~S}$ & $23.4 \mathrm{~W}$ & 1 & NA & $\mathrm{AM}$ & LAC & 76 & 5 & 1973 & 59 & LF & $\begin{array}{l}\text { Astronaut-named feature, } \\
\text { Apollo } 12 \text { site. }\end{array}$ \\
\hline South Cluster & $26.0 \mathrm{~N}$ & $3.7 \mathrm{E}$ & 2 & NA & $\mathrm{AM}$ & $\mathrm{S} 1$ & $41 \mathrm{~B} 4$ & 5 & 1973 & 59 & LF & $\begin{array}{l}\text { Astronaut-named feature, } \\
\text { Apollo } 15 \text { site. }\end{array}$ \\
\hline South Massif & $20.0 \mathrm{~N}$ & $30.4 \mathrm{E}$ & 16 & $\mathrm{EU}$ & FR & S1 & $43 \mathrm{D} 1$ & 5 & 1973 & 59 & LF & $\begin{array}{l}\text { Astronaut-named feature, } \\
\text { Apollo } 17 \text { site. }\end{array}$ \\
\hline Scuth Ray & $9.2 \mathrm{~S}$ & $15.4 \mathrm{E}$ & 1 & NA & $\mathrm{AM}$ & $\mathrm{S} 1$ & $78 \mathrm{D} 2$ & 5 & 1973 & 59 & LF & $\begin{array}{l}\text { Astronaut-named feature, } \\
\text { Apollo } 16 \text { site. }\end{array}$ \\
\hline Spook & $9.0 \mathrm{~S}$ & $15.5 \mathrm{E}$ & 0 & NA & $\mathrm{AM}$ & S1 & $78 \mathrm{D} 2$ & 5 & 1973 & 59 & LF & $\begin{array}{l}\text { Astronaut-named feature, } \\
\text { Apollo } 16 \text { site. }\end{array}$ \\
\hline Spot & $9.0 \mathrm{~S}$ & $15.5 \mathrm{E}$ & 0 & NA & $\mathrm{AM}$ & $\mathrm{S} 2$ & $78 \mathrm{D} 2$ & 5 & 1973 & 59 & LF & $\begin{array}{l}\text { Astronaut-named feature, } \\
\text { Apollo } 16 \text { site. }\end{array}$ \\
\hline Spur & $27.9 \mathrm{~N}$ & $1.2 \mathrm{~W}$ & 13 & NA & $\mathrm{AM}$ & $\mathrm{S} 1$ & $41 \mathrm{~B} 4$ & 5 & 1973 & 59 & LF & $\begin{array}{l}\text { Astronaut-named feature, } \\
\text { Apollo } 15 \text { site. }\end{array}$ \\
\hline St. George & $26.0 \mathrm{~N}$ & $3.5 \mathrm{E}$ & 2 & NA & $\mathrm{AM}$ & S1 & $41 \mathrm{~B} 4$ & 5 & 1973 & 59 & LF & $\begin{array}{l}\text { Astronaut-named feature, } \\
\text { Apollo } 15 \text { site. }\end{array}$ \\
\hline Statio Tranquillitatis & $0.8 \mathrm{~N}$ & $23.5 \mathrm{E}$ & 0 & $\mathrm{EU}$ & LA & LM & 60 & 5 & 1970 & 60 & LF & $\begin{array}{l}\text { "Tranquility Base", Apollo } \\
11 \text { landing site. }\end{array}$ \\
\hline Steno-Apollo & $20.1 \mathrm{~N}$ & $30.8 \mathrm{E}$ & 1 & NA & $\mathrm{AM}$ & $\mathrm{S} 1$ & $43 \mathrm{D} 1$ & 5 & 1973 & 59 & LF & $\begin{array}{l}\text { Astronaut-named feature, } \\
\text { Apollo } 17 \text { site. }\end{array}$ \\
\hline Stone Mountain & $9.1 \mathrm{~S}$ & $15.6 \mathrm{E}$ & 5 & NA & $\mathrm{AM}$ & $\mathrm{S} 1$ & $78 \mathrm{D} 2$ & 5 & 1973 & 59 & LF & $\begin{array}{l}\text { Astronaut-named feature, } \\
\text { Apollo } 16 \text { site. }\end{array}$ \\
\hline Stubby & $9.1 \mathrm{~S}$ & $15.5 \mathrm{E}$ & 1 & NA & $\mathrm{AM}$ & $\mathrm{S} 1$ & $78 \mathrm{D} 2$ & 5 & 1973 & 59 & LF & $\begin{array}{l}\text { Astronaut-named feature, } \\
\text { Apollo } 16 \text { site. }\end{array}$ \\
\hline Surveyor & $3.2 \mathrm{~S}$ & $23.4 \mathrm{~W}$ & 0 & NA & $\mathrm{AM}$ & LAC & 76 & 5 & 1973 & 59 & LF & $\begin{array}{l}\text { Astronaut-named feature, } \\
\text { Apollo } 12 \text { site. }\end{array}$ \\
\hline Taurus-Littrow Valley & $20.0 \mathrm{~N}$ & $31.0 \mathrm{E}$ & 30 & NA & AM & S1 & $43 \mathrm{D} 1$ & 5 & 1973 & 59 & LF & $\begin{array}{l}\text { Astronaut-named feature, } \\
\text { Apollo } 17 \text { site. }\end{array}$ \\
\hline Terrace & $26.1 \mathrm{~N}$ & $3.7 \mathrm{E}$ & 0 & NA & $\mathrm{AM}$ & S1 & $41 \mathrm{~B} 4$ & 5 & 1973 & 59 & LF & $\begin{array}{l}\text { Astronaut-named feature, } \\
\text { Apollo } 15 \text { site. }\end{array}$ \\
\hline Tortilla Flat & $20.2 \mathrm{~N}$ & $30.7 \mathrm{E}$ & 1 & NA & $\mathrm{AM}$ & S1 & $43 \mathrm{D} 1$ & 5 & 1973 & 59 & LF & $\begin{array}{l}\text { Astronaut-named feature, } \\
\text { Apollo } 17 \text { site. }\end{array}$ \\
\hline Trap & $9.1 \mathrm{~S}$ & $15.4 \mathrm{E}$ & 1 & NA & AM & S1 & $78 \mathrm{D} 2$ & 5 & 1973 & 59 & LF & $\begin{array}{l}\text { Astronaut-named feature, } \\
\text { Apollo } 16 \text { site. }\end{array}$ \\
\hline Trident & $20.2 \mathrm{~N}$ & $30.8 \mathrm{E}$ & 0 & NA & $\mathrm{AM}$ & S1 & $43 \mathrm{D} 1$ & 5 & 1973 & 59 & LF & $\begin{array}{l}\text { Astronaut-named feature, } \\
\text { Apollo } 17 \text { site. }\end{array}$ \\
\hline Triplet & $3.7 \mathrm{~S}$ & $17.5 \mathrm{~W}$ & 0 & NA & $\mathrm{AM}$ & LAC & 76 & 5 & 1973 & 59 & LF & $\begin{array}{l}\text { Astronaut-named feature, } \\
\text { Apollo } 14 \text { site. }\end{array}$ \\
\hline Van Serg & $20.2 \mathrm{~N}$ & $30.8 \mathrm{E}$ & 0 & NA & $\mathrm{AM}$ & S2 & $43 \mathrm{D} 1$ & 5 & 1973 & 59 & LF & $\begin{array}{l}\text { Astronaut-named feature, } \\
\text { Apollo } 17 \text { site. }\end{array}$ \\
\hline Victory & $20.2 \mathrm{~N}$ & $30.7 \mathrm{E}$ & 1 & NA & $\mathrm{AM}$ & S2 & 43 D1 & 5 & 1973 & 59 & LF & $\begin{array}{l}\text { Astronaut-named feature, } \\
\text { Apollo } 17 \text { site. }\end{array}$ \\
\hline
\end{tabular}

lat: latitude of feature center.

long: longitude of feature center.

diam: diameter or long dimension of feature.

ct: continent of name origin (see page $284 \mathrm{ff}$.) et: ethnicity of name origin (see page $284 \mathrm{ff}$.)

quad: map quadrangle or informal name

(see page xvii ff.).

map: map name or USGS map number (see page xvii ff.). ft: feature type (see page 290 ).

as: name approval status (see page xvii).

ad: name approval date (year).

ref: reference source for name (see page $287 \mathrm{ff}$.) 
MOON

\begin{tabular}{|c|c|c|c|c|c|c|c|c|c|c|c|c|}
\hline Name & lat & long & diam & ct & et & quad & map & as & ad & ref & $\mathbf{f t}$ & origin \\
\hline Weird & $3.7 \mathrm{~S}$ & $17.5 \mathrm{~W}$ & 0 & NA & $\mathrm{AM}$ & $\mathrm{LAC}$ & 76 & 5 & 1973 & 59 & LF & $\begin{array}{l}\text { Astronaut-named feature, } \\
\text { Apollo } 14 \text { site. }\end{array}$ \\
\hline Wessex Cleft & $.20 .3 \mathrm{~N}$ & $30.9 E$ & 4 & $\mathrm{EU}$ & GB & S1 & $43 \mathrm{D} 1$ & 5 & 1973 & 59 & LF & $\begin{array}{l}\text { Astronaut-named feature, } \\
\text { Apollo } 17 \text { site. }\end{array}$ \\
\hline West & $0.8 \mathrm{~N}$ & $23.5 \mathrm{E}$ & 0 & NA & AM & LM & 60 & 5 & 1973 & 59 & LF & $\begin{array}{l}\text { Astronaut-named feature, } \\
\text { Apollo } 11 \text { site. }\end{array}$ \\
\hline Wreck & $9.1 \mathrm{~S}$ & $15.5 \mathrm{E}$ & 1 & NA & $\mathrm{AM}$ & S1 & $78 \mathrm{D} 2$ & 5 & 1973 & 59 & LF & $\begin{array}{l}\text { Astronaut-named feature, } \\
\text { Apollo } 16 \text { site. }\end{array}$ \\
\hline
\end{tabular}

\section{MARE}

Mare Anguis

Mare Australe

Mare Cognitum

Mare Crisium

Mare Fecunditatis

Mare Frigoris

Mare Humboldtianum

$\begin{array}{clrllll}22.6 \mathrm{~N} & 67.7 \mathrm{E} & 150 & \text { EU } & \text { LA } & \text { LAC } & 44 \\ 38.9 \mathrm{~S} & 93.0 \mathrm{E} & 603 & \text { EU } & \text { LA } & \text { LMP } & 3 \\ 10.0 \mathrm{~S} & 23.1 \mathrm{~W} & 376 & \text { EU } & \text { LA } & \text { LOC } & 2 \\ 17.0 \mathrm{~N} & 59.1 \mathrm{E} & 418 & \text { EU } & \text { LA } & \text { LOC } & 3 \\ 7.8 \mathrm{~S} & 51.3 \mathrm{E} & 909 & \text { EU } & \text { LA } & \text { LOC } & 3 \\ 56.0 \mathrm{~N} & 1.4 \mathrm{E} & 1596 & \text { EU } & \text { LA } & \text { LMP } & 3 \\ 56.8 \mathrm{~N} & 81.5 \mathrm{E} & 273 & \text { EU } & \text { GE } & \text { LMP } & 3\end{array}$

Mare Humorum

Mare Imbrium

Mare Ingenii

Mare Insularum

Mare Marginis

Mare Moscoviense

Mare Nectaris

Mare Nubium

Mare Orientale

Mare Serenitatis

Mare Smythii

$\begin{array}{cc}24.4 \mathrm{~S} & 38.6 \mathrm{~W} \\ 32.8 \mathrm{~N} & 15.6 \mathrm{~W} \\ 33.7 \mathrm{~S} & 163.5 \mathrm{E} \\ 7.5 \mathrm{~N} & 30.9 \mathrm{~W} \\ 13.3 \mathrm{~N} & 86.1 \mathrm{E} \\ 27.3 \mathrm{~N} & 147.9 \mathrm{E} \\ 15.2 \mathrm{~S} & 35.5 \mathrm{E} \\ 21.3 \mathrm{~S} & 16.6 \mathrm{~W} \\ 19.4 \mathrm{~S} & 92.8 \mathrm{~W} \\ 28.0 \mathrm{~N} & 17.5 \mathrm{E} \\ 1.3 \mathrm{~N} & 87.5 \mathrm{E}\end{array}$

389 EU

1123

318

513 EU

420 EU

277

333 EU

333 EU

327 EU LA

707 EU LA

373 EU GB

LAC

LA

LA

LA

LA

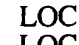

LOC

LOC

LOC

LOC

LOC

LOC

$1.1 \mathrm{~N} \quad 65.1 \mathrm{E}$

$8.5 \mathrm{~N} \quad 31.4 \mathrm{E}$

$6.8 \mathrm{~N} \quad 68.4 \mathrm{E}$

$\begin{array}{lll}139 & \text { EU } & \text { LA } \\ 873 & \text { EU } & \text { LA } \\ 243 & \text { EU } & \text { LA }\end{array}$

LAC

Mare Tranquillitatis

Mare Undarum

$13.3 \mathrm{~N}$

$3.6 \mathrm{E}$

245 EU LA

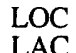

2

62

LAC $\quad 59$

93
2
4
2
3
4
2
2
1
2
3

62
2
62
59

$\begin{array}{llll}5 & 1935 & 66 & \mathrm{ME} \\ 5 & 1935 & 66 & \mathrm{ME} \\ 5 & 1964 & 67 & \mathrm{ME} \\ 5 & 1935 & 66 & \mathrm{ME} \\ 5 & 1935 & 66 & \mathrm{ME} \\ 5 & 1935 & 66 & \mathrm{ME} \\ 5 & 1935 & 66 & \mathrm{ME}\end{array}$

1935

\section{5}

1961

51976

51935

$\begin{array}{ll}5 & 1961\end{array}$

51935

51935

51935

$\begin{array}{ll}5 & 1935 \\ 5 & 1935\end{array}$

51935

$\begin{array}{ll}5 & 1935 \\ 5 & 1935 \\ 5 & 1935 \\ 5 & 1935\end{array}$

"Serpent Sea".

"Southern Sea".

"Sea that has become known".

"Sea of Crises".

"Sea of Fecundity".

"Sea of Cold".

Humboldt, Alexander von; German natural historian (1769-1859)

66 ME "Sea of Moisture".

66 ME "Sea of Showers".

67 ME "Sea of Cleverness".

0 ME "Sea of Islands".

66 ME "Sea of the Edge".

67 ME "Sea of Muscovy".

66 ME "Sea of Nectar".

66 ME "Sea of Clouds".

66 ME "Eastern sea"

66 ME "Sea of Serenity".

66 ME Smyth, William Henry;

British astronomer (1788-1865).

$66 \mathrm{ME}$ "Foaming Sea".

66 ME "Sea of Tranquility".

66 ME "Sea of Waves".

66 ME "Sea of Vapors".
$18.6 \mathrm{~N}$

$19.0 \mathrm{~N}$

$5.2 \mathrm{~N} \quad 120.6 \mathrm{E}$

$5.0 \mathrm{~N} \quad 121.0 \mathrm{E}$

$22.0 \mathrm{~N}$

$1.0 \mathrm{E}$

$29.5 \mathrm{~N} \quad 35.8 \mathrm{~W}$

$5.0 \mathrm{~N} \quad 120.2 \mathrm{E}$

$5.6 \mathrm{~N} \quad 120.8 \mathrm{E}$

$14.6 \mathrm{~N} \quad 35.7 \mathrm{E}$

$23.3 \mathrm{~N} \quad 29.2 \mathrm{~W}$

$5.3 \mathrm{E}$
Mons Delisle

Mons Dieter

Mons Dilip

Mons Esam
[Mons Euler]

$\begin{array}{rlll}1 & \text { EU } & \text { GR } & \text { S }\end{array}$

30 EU FR LM

8 AS PE $\mathrm{S} 1$

30 EU GB LTO

30 EU FR LTO

20 EU GE S1

2 AS IN S1

$\begin{array}{rlll}8 & \text { AS } & \text { AR } & \text { S1 } \\ 27 & \text { EU } & \text { SZ } & \text { LAC }\end{array}$

$41 \mathrm{C} 3$

41

$65 \mathrm{Cl}$

$65 \mathrm{C} 1$

$041 \mathrm{C} 1$

039B 1

$65 \mathrm{Cl}$

$65 \mathrm{C} 1$

$61 \mathrm{~A} 2$

39

$4.8 \mathrm{~N} \quad 120.6 \mathrm{E}$

Mons Ganau

$36.0 \mathrm{~N}$

$39.5 \mathrm{~W}$

$40.5 \mathrm{~W}$

$4.7 \mathrm{E}$

Mons Hadley

$26.5 \mathrm{~N}$

Mons Hadley Delta

Mons Hansteen

Mons Herodotus

Mons Huygens

$25.8 \mathrm{~N}$

$3.8 \mathrm{E}$

$50.0 \mathrm{~W}$

$12.1 \mathrm{~S}$

$27.5 \mathrm{~N}$

$53.0 \mathrm{~W}$

$2.9 \mathrm{~W}$

14 AF

20 EU

20 EU

25 EU GE

15 EU GB S

30 EU SW LAC

5 EU GR LTO

40 EU DU LM

S1

LAC

LAC

S1

$65 \mathrm{Cl}$
23
23
$41 \mathrm{~B} 4$

$41 \mathrm{~B} 4$
74
$038 \mathrm{~B} 3$
41

$5 \quad 1979$

$5 \quad 1935$

$\begin{array}{rr}0 & \mathrm{MO} \\ 66 & \mathrm{MO}\end{array}$

1976

51976

1961

51985

51976

1976

$\begin{array}{ll}5 & 1979\end{array}$

$\begin{array}{ll}6 & 1979\end{array}$

\section{6}

$5 \quad 1976$ 1976
1935

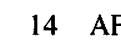

Andre Marie; French

0 MO French male name.

0 MO Persian (Iranian) king's name.

67 MO James; British astronomer (1692-1762).

0 MO Named from nearby crater.

0 MO German male name.

0 MO Indian male name.

0 MO Arabic male name.

0 MO Leonhard; Swiss mathematician (1707-1783); now called Mons Vinogradov.

Mons La Hire

$27.8 \mathrm{~N}$

$25.5 \mathrm{~W}$

25 EU FR LTO

040A4

0 MO African male name

0 MO Named from nearby crater.

0 MO Named from nearby crater.

66 MO Hadley, John; British instrument maker (1682-1743).

193566 MO Named from nearby mountain.

$5 \quad 1976$ 0 MO Named from nearby crater.

$\begin{array}{rrrrr}5 & 1976 & 0 & \text { MO } & \text { Named from nearby crater. } \\ 5 & 1961 & 67 & \text { MO } & \text { Christian; Dutch astronomer, }\end{array}$ mathematician, physicist (1629-1695).

Philippe De; French mathematician, astronomer (1640-1718).

Mons Maraldi

$20.3 \mathrm{~N} \quad 35.3 \mathrm{E}$ $12.0 \mathrm{~S}$
$35.3 \mathrm{E}$
$19.7 \mathrm{~W}$
15 EU IT LTO 10 EU IT LTO $\begin{array}{lll}043 \mathrm{D} 2 & 5 & 1976 \\ 076 \mathrm{C} 1 & 5 & 1976\end{array}$
Named from nearby crater

Antonio Lazzaro; Italian

\footnotetext{
lat: latitude of feature center.

long: longitude of feature center.

et: ethnicity of name origin (see page $284 \mathrm{ff}$.)

as: name approval status (see page xvii).

quad: map quadrangle or informal name

ad: name approval date (year).

diam: diameter or long dimension of feature.

(see page xvii ff.).

ref: reference source for name (see page $287 \mathrm{ff}$.).

ct: continent of name origin (see page $284 \mathrm{ff}$.) map: map name or USGS map number (see page xvii ff.). ft: feature type (see page 290 ).
} 


\section{MOON}

Name

Mons Penck

Mons Pico

Mons Piton

Mons Rümker

Mons Usov

Mons Vinogradov

$12.0 \mathrm{~N}$

63.0E

$22.4 \mathrm{~N}$

$32.4 \mathrm{~W}$

$15 \mathrm{UR}$

SO LTO

25 UR SO LTO

062B 1

039C2

$5 \quad 1979$
$19.4 \mathrm{~N}$

$17.0 \mathrm{~N}$

$45.0 \mathrm{~N}$

Mont Blanc

Montes Agricola

Montes Alpes

Montes Apenninus

Montes Archimedes

Montes Carpatus

Montes Caucasus

Montes Cordillera

Montes Haemus

Montes Harbinger

Montes Jura

Montes Pyrenaeus

Montes Recti

Montes Riphaeus

Montes Rook

Montes Secchi

Montes Spitzbergen

$29.1 \mathrm{~N}$

$46.4 \mathrm{~N}$

$18.9 \mathrm{~N}$

$25.3 \mathrm{~N}$

$14.5 \mathrm{~N}$

$38.4 \mathrm{~N}$

$17.5 S$

$19.9 \mathrm{~N}$

$27.0 \mathrm{~N}$

$47.1 \mathrm{~N}$

$15.6 \mathrm{~S}$

$48.0 \mathrm{~N}$

$7.7 \mathrm{~S}$

$30.8 \mathrm{E}$

$6.8 \mathrm{~W}$

15 EU RM LTO

$1.0 \mathrm{E}$

$54.2 \mathrm{~W}$

$0.8 \mathrm{~W}$

$3.7 \mathrm{~W}$

4.6W

$24.4 \mathrm{~W}$

$10.0 \mathrm{E}$

$81.6 \mathrm{~W}$

9.2E

$41.0 \mathrm{~W}$

$34.0 \mathrm{~W}$

$41.2 \mathrm{E}$

$20.0 \mathrm{~W}$

$28.1 \mathrm{~W}$

$20.6 \mathrm{~S}$

$82.5 \mathrm{~W}$

$3.0 \mathrm{~N}$

$35.0 \mathrm{~N}$

$43.0 \mathrm{E}$

$5.0 \mathrm{~W}$

$28.4 \mathrm{~N}$

41.1E

$47.1 \mathrm{~N}$

043D4

41

25 EU FR LAC

141 EU GE LTO

281 EU SZ LAC

401 EU IT LOC

163 EU GR LTO

361 EU RO LAC

445 UR CC LAC

574 EU SP LOC

560 EU BU LOC

90 EU LA LTO

422 EU GE LMP

164 EU SP LAC

90 EU LA LAC

189 UR RU LTO

791 EU GB LOC

50 EU IT LTO

60 EU GE LAC

25

038B2

25

2

041A3

58

25

1

2

039A3

1

79

12

076D1

1

$061 \mathrm{Cl}$

25

Montes Teneriffe

OCEANUS

Oceanus Procellarum

$18.4 \mathrm{~N}$

$32.0 \mathrm{~S}$
$26.5 \mathrm{~N}$

Palus Putredinis

Palus Somni

\section{PLẢNITIA}

Planitia Descensus

111
$11.8 \mathrm{~W}$

18

$57.4 \mathrm{~W}$

2568

EU

$28.2 \mathrm{~W}$

286 EU

$0.4 \mathrm{E}$

16

$64.4 \mathrm{~W}$

$1 \mathrm{EU}$

43

25
51976

51961

51935

51976

51935

51961

51976

51961

51961

51961

51961

51961

$5 \quad 1961$

$5 \quad 1961$

$\begin{array}{ll}5 & 1961 \\ 5 & 1961\end{array}$

51961

$\begin{array}{ll}5 & 1976 \\ 5 & 1961\end{array}$

1961

51935

51935 ref $\mathbf{f t}$ origin

- MO Albrecht; German

66 MO Spanish for "peak".

66 MO Named from Mt. Piton on Tenerife Islands.

Karl Ludwig Christian; (1788-1862).

MO Mikhail A.; Soviet

MO Aleksandr Pavlovich;

Soviet geochemist and cosmochemist (1895-1975); formerly called Mons Euler.

0 MO Named from nearby crater. philosopher (1679-1754).

Named for terrestrial mountain in Alps.

$66 \mathrm{MO}$

Georgius; German Earth scientist (1494-1555).

66 MO Named from terrestrial Alps.

67 MO Named from terrestrial Apennines.

0 MO Named from nearby crater.

66 MO Named from terrestrial Carpathians.

67 MO Named from terrestrial Caucasus Mountains.

67 MO Spanish for "mountain chain".

67 MO Named for range in the Balkans.

67 MO Harbingers of dawn on crater Aristarchus.

67 MO Named from terrestrial Jura Mountains.

67 MO Named from terrestrial Pyrenees.

67 MO Latin for "straight range".

67 MO Named from range in Asia (now Ural Mountains)

67 MO Lawrence; British astronomer (1622-1666).

0 MO Named from nearby crater.

67 MO German for "sharp peaks", and named for resemblance to the terrestrial island group. to the terrestrial island
Named from terrestrial

66 MO Naurus Mts. island. geographer (1858-1945)

German astronomer geologist (1883-1933).

67 MO Christian, Baron von; German

Named from terrestrial

\section{PROMONTORIUM}

Promontorium Agarum $14.0 \mathrm{~N}$

66.0E 70 UR RU LTO

062B2

51935

$66 \mathrm{OC}$

"Ocean of Storms".

Promontorium Agassi $\quad 42.0 \mathrm{~N}$
20 EU SZ LAC
$043 \mathrm{C} 3$

$\begin{array}{ll}5 & 1935 \\ 5 & 1935 \\ 5 & 1935\end{array}$

66 PA

66 PA
"Marsh of Epidemics".

"Marsh of Decay".

"Marsh of Sleep".
$5 \quad 1970$

$0 \quad P$

Luna 9 landing site ("plain of descent").

5

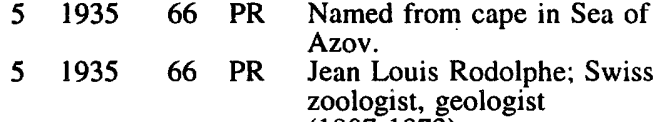
(1807-1873) lat: latitude of feature center.

long: longitude of feature center.

diam: diameter or long dimension of feature.

ct: continent of name origin (see page 284 et: ethnicity of name origin (see page $284 \mathrm{ff}$.)

quad: map quadrangle or informal name

(see page xvii ff.). as: name approval status (see page xvii).

ad: name approval date (year).

ref: reference source for name (see page $287 \mathrm{ff}$.). 


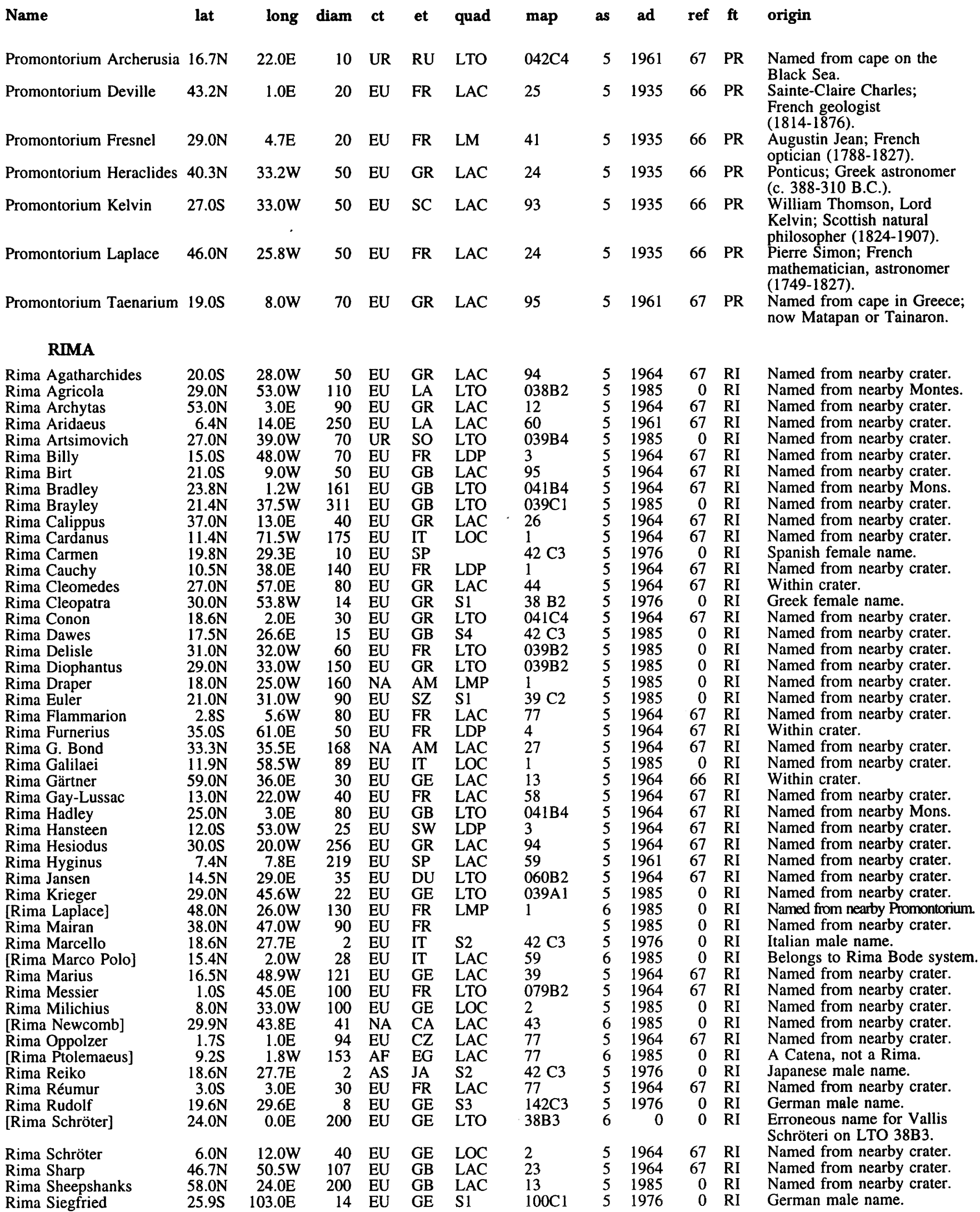

lat: latitude of feature center.

long: longitude of feature center.

diam: diameter or long dimension of feature. et: ethnicity of name origin (see page $284 \mathrm{ff}$.)

quad: map quadrangle or informal name

(see page xvii ff.).

ct: continent of name origin (see page $284 \mathrm{ff}$.) map: map name or USGS map number (see page xvii ff.). ft: feature type (see page 290).

as: name approval status (see page xvii).

ad: name approval date (year).

ref: reference source for name (see page $287 \mathrm{ff}$.). 


\section{MOON}

Name

Rima Sues

Rima Sung-Mei

Rima T. Mayer

Rima Wan-Yu

[Rima Widmannstatten]

Rima Yangel'

Rimae Alphonsus

Rimae Apollonius

Rimae Archimedes

Rimae Aristarchus

Rimae Arzachel

Rimae Atlas

Rimae Bode

Rimae Boscovich

Rimae Bürg

Rimae Chacornac

Rimae Daniell

Rimae Darwin

Rimae de Gasparis

Rimae Doppelmayer

Rimae Focas

Rimae Fresnel

Rimae Gassendi

Rimae Gerard

Rimae Goclenius

[Rimae Golitsyn]

Rimae Grimaldi

Rimae Gutenberg

Rimae Hase

Rimae Herigonius

Rimae Hevelius

Rimae Hippalus

Rimae Hypatia

Rimae Janssen

Rimae Kopff

Rimae Liebig

Rimae Littrow

Rimae MacLear

Rimae Maestlin

Rimae Maupertuis

Rimae Menelaus

Rimae Mersenius

Rimae Opelt

Rimae Palmieri

Rimae Parry

Rimae Petavius

Rimae Pettit

Rimae Pitatus

Rimae Plato

Rimae Plinius

Rimae Posidonius

Rimae Prinz

Rimae Ramsden

Rimae Repsold

Rimae Riccioli

Rimae Ritter

Rimae Römer

Rimae Secchi

Rimae Sirsalis

Rimae Sosigenes

[Rimae Stadius]

Rimae Sulpicius Gallus

Rimae Taruntius

Rimae Theaetetus

Rimae Triesnecker

Rimae Vasco da Gama

Rimae Zupus lat

$6.7 \mathrm{~N}$
$24.6 \mathrm{~N}$

$13.0 \mathrm{~N}$

$20.0 \mathrm{~N}$

$6.1 \mathrm{~S}$

$16.7 \mathrm{~N}$

$25.0 \mathrm{~N}$

$5.0 \mathrm{~N}$

$26.6 \mathrm{~N}$

$26.9 \mathrm{~N}$
$18.0 \mathrm{~S}$

$18.0 \mathrm{~S}$
$47.5 \mathrm{~N}$

$10.0 \mathrm{~N}$

$9.8 \mathrm{~N}$

$44.5 \mathrm{~N}$

$29.0 \mathrm{~N}$

$19.3 \mathrm{~S}$

$24.6 \mathrm{~S}$

$25.9 \mathrm{~S}$

$28.0 \mathrm{~S}$

$28.0 \mathrm{~N}$
$18.0 \mathrm{~S}$

$46.0 \mathrm{~N}$

$8.0 \mathrm{~S}$

$25.1 \mathrm{~N}$
$9.0 \mathrm{~N}$

$9.0 \mathrm{~N}$
$5.0 \mathrm{~S}$

$29.4 \mathrm{~S}$

$13.0 \mathrm{~S}$

$1.0 \mathrm{~N}$
$25.5 \mathrm{~S}$

$25.5 \mathrm{~S}$
$0.4 \mathrm{~S}$

45.6S

$17.4 \mathrm{~S}$

$20.0 \mathrm{~S}$

$22.1 \mathrm{~N}$

$2.0 \mathrm{~N}$

$52.0 \mathrm{~N}$

$17.2 \mathrm{~N}$

21.5S

$13.0 \mathrm{~S}$

$28.0 \mathrm{~S}$

$6.1 \mathrm{~S}$

25.9S

23.0S

$28.5 \mathrm{~S}$

$17.9 \mathrm{~N}$

$32.0 \mathrm{~N}$

$27.0 \mathrm{~N}$

$33.9 \mathrm{~S}$

$50.6 \mathrm{~N}$

$2.0 \mathrm{~N}$

$27.0 \mathrm{~N}$

$1.0 \mathrm{~N}$

$15.7 \mathrm{~S}$

$8.6 \mathrm{~N}$

$10.5 \mathrm{~N}$

$21.0 \mathrm{~N}$

$5.5 \mathrm{~N}$
$33.0 \mathrm{~N}$

$4.3 \mathrm{~N}$

$10.0 \mathrm{~N}$

$15.0 \mathrm{~S}$ long diam ct

48.2W 165 EU AS LOC

$11.3 \mathrm{E}$

40 AS

CH

$31.0 \mathrm{~W}$

$31.5 \mathrm{~W}$

$85.5 \mathrm{E}$

$4.6 \mathrm{E}$

$29.5 \mathrm{~W}$

$2.0 \mathrm{~W}$

$53.0 \mathrm{E}$

$47.5 \mathrm{~W}$

$2.0 \mathrm{~W}$

$43.6 \mathrm{E}$

4.0W

$23.8 \mathrm{E}$

$32.0 \mathrm{E}$

26.0E

$69.5 \mathrm{~W}$

$51.1 \mathrm{~W}$

$45.1 \mathrm{~W}$

$98.0 \mathrm{~W}$

$4.0 \mathrm{E}$

$40.0 \mathrm{~W}$

$84.0 \mathrm{~W}$

$43.0 \mathrm{E}$

$105.0 \mathrm{~W}$

$64.0 \mathrm{~W}$

38.0E

$62.5 \mathrm{E}$
$37.0 \mathrm{~W}$

$68.0 \mathrm{~W}$

29.2W

22.4E

$40.0 \mathrm{E}$
$89.6 \mathrm{~W}$

$89.6 \mathrm{~W}$
$45.0 \mathrm{~W}$

$29.9 \mathrm{E}$

$20.0 \mathrm{E}$

$40.0 \mathrm{~W}$

23.0W

$17.9 \mathrm{E}$

$49.2 \mathrm{~W}$
$18.0 \mathrm{~W}$

$18.0 \mathrm{~W}$

$16.8 \mathrm{~W}$

$58.9 \mathrm{E}$

$92.0 \mathrm{~W}$

$13.8 \mathrm{~W}$

$3.2 \mathrm{~W}$
$23.6 \mathrm{E}$

$23.6 \mathrm{E}$
$28.7 \mathrm{E}$

$43.0 \mathrm{~W}$

$31.4 \mathrm{~W}$

$81.7 \mathrm{~W}$

$74.0 \mathrm{~W}$
$18.0 \mathrm{E}$

$18.0 \mathrm{E}$

$35.0 \mathrm{E}$
$44.0 \mathrm{E}$

$44.0 \mathrm{E}$
$61.7 \mathrm{~W}$

$18.7 \mathrm{E}$

$13.7 \mathrm{~W}$

$10.0 \mathrm{E}$

$46.5 \mathrm{E}$

$6.0 \mathrm{E}$

$4.6 \mathrm{E}$

82.0W
$53.0 \mathrm{~W}$
50 EU GE

12 AS CH SI

46 EU

30 UR

16 AS

80
230
EU

$169 \mathrm{EU}$

121 EU

50 EU

60 EU

70 EU

40 EU

147 EU

200 EU

143 EU

$93 \mathrm{EU}$

162 EU

$100 \mathrm{EU}$

90 EU

70 EU

100 EU

36
UR

230 EU

330 EU

83 EU

$100 \mathrm{EU}$

182 EU

$206 \mathrm{AF}$

114 EU

41 EU

140 EU

115 EU

80 EU

$60 \mathrm{EU}$

131 EU

84 EU

$70 \mathrm{EU}$

150 EU

82 EU

80 EU

450
94
EU

87 EU

124 EU

70 EU

80 EU

108 EU

400 EU

100 EU

110
35
EU

426 EU

190 EU

69 EU

25 EU

50 EU

215 EU

60
$120 \mathrm{EU}$

$\begin{array}{ll}\text { GE } & \text { LT } \\ \text { AR } & \text { St } \\ \text { LA } & \text { L }\end{array}$

GE

GE LR

GE
LA LDP

$\begin{array}{lll}\text { LA } & \text { LAC } & 62 \\ \text { GR } & \text { LAC } & 41\end{array}$

GR LTO

SP LAC

GE LAC

IT LM

AS LAC 26

FR LAC 43

GB LAC 26

GB LAC 92

IT LAC 92

FR LOC

FR LTO

$\begin{array}{lll} & \text { LAC } & 93\end{array}$

GE LAC 79

IT LOC

GE LOC

PO LAC

GR LAC

FR LAC

GE LOC

LOC

CZ LAC

LM

1

1

1
3
2

2

$75 \mathrm{C} 4$

56

94

2

114

93

43

60 
MOON

Name

\section{RUPES}

Rupes Altai

Rupes Boris

Rupes Cauchy

Rupes Kelvin

Rupes Liebig

Rupes Mercator

Rupes Recta

Rupes Toscanelli

\section{SINUS}

Sinus Aestuun

Sinus Amoris

Sinus Asperitatis

Sinus Concordiae

Sinus Fidei

Sinus Honoris

Sinus Iridum

Sinus Lunicus

Sinus Medii

Sinus Roris

Sinus Successus

\section{VALLIS}

Vallis Alpes

Vallis Baade

Vallis Bohr

Vallis Bouvard

Vallis Capella

Vallis Inghirami

Vallis Palitzsch

Vallis Planck

Vallis Rheita

Vallis Schrödinger

Vallis Schröteri

Vallis Snellius lat long diam ct et quad map as ad ref $f t$ origin

\begin{tabular}{|c|c|c|c|c|c|c|c|c|c|c|c|}
\hline $24.3 S$ & $22.6 \mathrm{E}$ & 427 & AS & MN & LAC & 96 & 5 & 1961 & 67 & RU & $\begin{array}{l}\text { Named from terrestrial } \\
\text { Altai Mountains. }\end{array}$ \\
\hline 30.5 & $33.5 \mathrm{~W}$ & 4 & UR & RU & S2 & 39 B2 & 5 & 1985 & 0 & RU & Named from nearby crater. \\
\hline & $37.0 \mathrm{E}$ & 120 & $\mathrm{EU}$ & FR & LTO & $061 \mathrm{A3}$ & 5 & 1964 & 67 & RU & Named from nearby crater. \\
\hline .3 & $33.1 \mathrm{~W}$ & 78 & EU & SC & LOC & & 5 & 196 & 67 & RU & Named from nearby promontorium. \\
\hline $4.4 \mathrm{~S}$ & $48.5 \mathrm{~W}$ & 37 & EU & GE & LOC & 2 & 5 & 1964 & 67 & RU & Named from nearby crater. \\
\hline 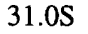 & $22.3 \mathrm{~W}$ & 93 & EU & BE & LO & 2 & 5 & 198 & 0 & RU & Named from nearby crater. \\
\hline 22.1 & $7.8 \mathrm{~W}$ & 134 & $\mathrm{EU}$ & $\overline{L A}$ & LA & 95 & 5 & 1961 & 67 & RU & $\begin{array}{l}\text { Latin for "straight cliff" } \\
\text { (The straight wall). }\end{array}$ \\
\hline $.4 \mathrm{~N}$ & $47.5 \mathrm{~W}$ & 70 & EU & IT & LTO & 039A4 & 5 & 1985 & 0 & RU & Named from nearby crater. \\
\hline
\end{tabular}

$\begin{array}{rrrlllllllll}10.9 \mathrm{~N} & 8.8 \mathrm{~W} & 290 & \text { EU } & \text { LA } & \text { LAC } & 59 & 5 & 1935 & 66 & \text { SI } & \text { "Seething Bay". } \\ 18.1 \mathrm{~N} & 39.1 \mathrm{E} & 130 & \text { EU } & \text { LA } & \text { LTO } & 043 \mathrm{C} 4 & 5 & 1976 & 0 & \text { SI } & \text { "Bay of Love". } \\ 3.8 \mathrm{~S} & 27.4 \mathrm{E} & 206 & \text { EU } & \text { LA } & \text { LTO } & 078 \mathrm{~B} 3 & 5 & 1976 & 0 & \text { SI } & \text { "Bay of Roughness". } \\ 10.8 \mathrm{~N} & 43.2 \mathrm{E} & 142 & \text { EU } & \text { LA } & \text { LTO } & 061 \mathrm{~B} 3 & 5 & 1976 & 0 & \text { SI } & \text { "Bay of Harmony". } \\ 18.0 \mathrm{~N} & 2.0 \mathrm{E} & 70 & \text { EU } & \text { LA } & \text { LTO } & 041 \mathrm{C} 4 & 5 & 1976 & 0 & \text { SI } & \text { "Bay of Trust". } \\ 11.7 \mathrm{~N} & 18.1 \mathrm{E} & 109 & \text { EU } & \text { LA } & \text { LTO } & 060 \mathrm{~A} 2 & 5 & 1976 & 0 & \text { SI } & \text { "Bay of Honor". } \\ 44.1 \mathrm{~N} & 31.5 \mathrm{~W} & 236 & \text { EU } & \text { LA } & \text { LAC } & 24 & 5 & 1935 & 66 & \text { SI } & \text { "Bay of Rainbows". } \\ 31.8 \mathrm{~N} & 1.4 \mathrm{~W} & 126 & \text { EU } & \text { LA } & \text { LOC } & 2 & 5 & 1970 & 0 & \text { SI } & \text { "Lunik Bay"-landing area } \\ & & & & & & & & & & & \\ 2.4 \mathrm{~N} & 1.7 \mathrm{E} & 335 & \text { EU } & \text { LA } & \text { LAC } & 59 & 5 & 1935 & 66 & \text { SI } & \text { "Bay of the center". } \\ 54.0 \mathrm{~N} & 56.6 \mathrm{~W} & 202 & \text { EU } & \text { LA } & \text { LMP } & 3 & 5 & 1935 & 66 & \text { SI } & \text { "Bay of Dew". } \\ 0.9 \mathrm{~N} & 59.0 \mathrm{E} & 132 & \text { EU } & \text { LA } & \text { LTO } & 062 \mathrm{D} 3 & 5 & 1979 & 0 & \text { SI } & \text { "Bay of Success". }\end{array}$

$\begin{array}{lrrllllll}48.5 \mathrm{~N} & 3.2 \mathrm{E} & 166 & \text { EU } & \text { SZ } & \text { LAC } & 12 & 5 & 1961 \\ 45.9 \mathrm{~S} & 76.2 \mathrm{~W} & 203 & \text { EU } & \text { GE } & \text { LMP } & 1 & 5 & 1964 \\ 12.4 \mathrm{~N} & 86.6 \mathrm{~W} & 80 & \text { EU } & \text { DE } & & & 5 & 1976 \\ 38.3 \mathrm{~S} & 83.1 \mathrm{~W} & 284 & \text { EU } & \text { FR } & \text { LMP } & 1 & 5 & 1970 \\ & & & & & & & & \\ 7.6 \mathrm{~S} & 34.9 \mathrm{E} & 49 & \text { EU } & \text { RO } & \text { LTO } & 079 \mathrm{A4} & 5 & 1984 \\ 43.8 \mathrm{~S} & 72.2 \mathrm{~W} & 148 & \text { EU } & \text { IT } & \text { LMP } & 3 & 5 & 1964 \\ 26.4 \mathrm{~S} & 64.3 \mathrm{E} & 132 & \text { EU } & \text { GE } & \text { LAC } & 98 & 5 & 1964 \\ 58.4 \mathrm{~S} & 126.1 \mathrm{E} & 451 & \text { EU } & \text { GE } & \text { LMP } & 3 & 5 & 1970 \\ 42.5 \mathrm{~S} & 51.5 \mathrm{E} & 445 & \text { EU } & \text { CZ } & \text { LAC } & 114 & 5 & 1961 \\ 67.0 \mathrm{~S} & 105.0 \mathrm{E} & 310 & \text { EU } & \text { AS } & \text { LMP } & 3 & 5 & 1970 \\ 26.2 \mathrm{~N} & 50.8 \mathrm{~W} & 168 & \text { EU } & \text { GE } & \text { LTO } & 038 \mathrm{~B} 3 & 5 & 1961 \\ 31.1 \mathrm{~S} & 56.0 \mathrm{E} & 592 & \text { EU } & \text { DU } & \text { LAC } & 98 & 5 & 1964\end{array}$

67 VA "Alpine Valley".

67 VA Named from nearby crater.

0 VA Named from nearby crater.

0 VA Alexis; French astronomer, mathematician (1767-1843).

0 VA Named from nearby crater.

67 VA Named from nearby crater.

67 VA Named from nearby crater.

0 VA Named from nearby crater.

67 VA Named from nearby crater.

0 VA Named from nearby crater.

67 VA Schröter's Valley.

67 VA Named from nearby crater. lat: latitude of feature center.

long: longitude of feature center.

diam: diameter or long dimension of feature.

ct: continent of name origin (see page $284 \mathrm{ff}$.) et: ethnicity of name origin (see page $284 \mathrm{ff}$.)

quad: map quadrangle or informal name

(see page xvii ff.). as: name approval status (see page xvii).

ad: name approval date (year).

ref: reference source for name (see page $287 \mathrm{ff}$.) 
MARS

Name

lat long diam ct et quad map as ad ref ft origin

\section{CRATER}

\begin{tabular}{|c|c|c|c|c|c|c|c|c|c|c|c|c|}
\hline $\begin{array}{l}\text { Aban } \\
\text { Achar } \\
\text { Adams }\end{array}$ & $\begin{array}{l}16.2 \mathrm{~N} \\
45.7 \mathrm{~N} \\
31.3 \mathrm{~N}\end{array}$ & $\begin{array}{l}249.1 \mathrm{~W} \\
236.8 \mathrm{~W} \\
197.1 \mathrm{~W}\end{array}$ & $\begin{array}{r}0 \\
6 \\
100\end{array}$ & $\begin{array}{l}\text { UR } \\
\text { SA } \\
\text { NA }\end{array}$ & $\begin{array}{l}\text { RU } \\
\text { UR } \\
\text { AM }\end{array}$ & $\begin{array}{l}\text { UTOPIA } \\
\text { MC07 }\end{array}$ & $\begin{array}{l}11061 \\
\text { I475 }\end{array}$ & $\begin{array}{l}5 \\
5 \\
5\end{array}$ & $\begin{array}{l}1988 \\
1979 \\
1973\end{array}$ & $\begin{array}{l}36 \\
36 \\
68\end{array}$ & $\begin{array}{l}\text { AA } \\
\text { AA } \\
\text { AA }\end{array}$ & $\begin{array}{l}\text { Town in Russia. } \\
\text { Town in Uruguay. } \\
\text { Walter S.; American } \\
\text { astronomer (1876-1956). }\end{array}$ \\
\hline Agassiz & $70.1 \mathrm{~S}$ & $88.4 \mathrm{~W}$ & 104 & NA & AM & MC30 & I1990 & 5 & 1973 & 68 & $\mathrm{AA}$ & $\begin{array}{l}\text { Jean L.; American } \\
\text { naturalist (1807-1873). }\end{array}$ \\
\hline Airy & $5.2 \mathrm{~S}$ & $0.0 \mathrm{~W}$ & 56 & EU & GB & MC19 & I1293 & 5 & 1973 & 68 & $\mathrm{AA}$ & $\begin{array}{l}\text { George B.; British } \\
\text { astronomer }(1801-1892) \text {. }\end{array}$ \\
\hline $\begin{array}{l}\text { Ajon } \\
\text { Aki } \\
\text { Aktaj } \\
\text { Albany }\end{array}$ & $\begin{array}{l}16.8 \mathrm{~N} \\
36.0 \mathrm{~S} \\
20.7 \mathrm{~N} \\
23.3 \mathrm{~N}\end{array}$ & $\begin{array}{r}257.9 \mathrm{~W} \\
60.5 \mathrm{~W} \\
46.5 \mathrm{~W} \\
49.0 \mathrm{~W}\end{array}$ & $\begin{array}{l}0 \\
8 \\
0 \\
2\end{array}$ & $\begin{array}{l}\text { UR } \\
\text { AS } \\
\text { UR } \\
\text { US }\end{array}$ & $\begin{array}{l}\text { RU } \\
\text { JA } \\
\text { RU } \\
\text { NY }\end{array}$ & $\begin{array}{l}\text { SOVIET } \\
\text { YORKTN }\end{array}$ & I1059 & $\begin{array}{l}5 \\
5 \\
5 \\
5\end{array}$ & $\begin{array}{l}1988 \\
1979 \\
1988 \\
1979\end{array}$ & $\begin{array}{l}36 \\
36 \\
36 \\
36\end{array}$ & $\begin{array}{l}\text { AA } \\
\text { AA } \\
\text { AA } \\
\text { AA }\end{array}$ & $\begin{array}{l}\text { Town in Russia. } \\
\text { Town in Japan. } \\
\text { Town in Russia. } \\
\text { American colonial town } \\
\text { (New York). }\end{array}$ \\
\hline $\begin{array}{l}\text { Albi } \\
\text { Alga } \\
\text { Alitus } \\
\text { Amet } \\
\text { Amsterdam } \\
\text { Angu } \\
\text { Aniak } \\
\text { Annapolis }\end{array}$ & $\begin{array}{l}41.9 \mathrm{~S} \\
24.6 \mathrm{~S} \\
34.5 \mathrm{~S} \\
23.7 \mathrm{~N} \\
23.3 \mathrm{~N} \\
20.3 \mathrm{~N} \\
32.1 \mathrm{~S} \\
23.4 \mathrm{~N}\end{array}$ & $\begin{array}{c}34.7 \mathrm{~W} \\
26.5 \mathrm{~W} \\
49.0 \mathrm{~W} \\
57.4 \mathrm{~W} \\
47.0 \mathrm{~W} \\
254.5 \mathrm{~W} \\
69.6 \mathrm{~W} \\
47.8 \mathrm{~W}\end{array}$ & $\begin{array}{r}8 \\
19 \\
29 \\
0 \\
2 \\
0 \\
58 \\
1\end{array}$ & $\begin{array}{l}\text { EU } \\
\text { UR } \\
\text { UR } \\
\text { AS } \\
\text { EU } \\
\text { AF } \\
\text { US } \\
\text { US }\end{array}$ & $\begin{array}{l}\text { FR } \\
\text { KZ } \\
\text { EU } \\
\text { IN } \\
\text { DU } \\
\text { ZA } \\
\text { AK } \\
\text { MD }\end{array}$ & $\begin{array}{l}\text { NEREID } \\
\text { ERYTHR } \\
\text { SOVIET } \\
25057 \\
\text { YORKTN } \\
\text { SOVIET } \\
\text { YORKTN }\end{array}$ & $\begin{array}{l}\mathrm{I} 1919 \\
\mathrm{I} 1059\end{array}$ & $\begin{array}{l}5 \\
5 \\
5 \\
5 \\
5 \\
5 \\
5 \\
5\end{array}$ & $\begin{array}{l}1976 \\
1976 \\
1979 \\
1988 \\
1979 \\
1988 \\
1979 \\
1979\end{array}$ & $\begin{array}{l}36 \\
36 \\
58 \\
36 \\
36 \\
36 \\
36 \\
36\end{array}$ & $\begin{array}{l}\text { AA } \\
\text { AA } \\
\text { AA } \\
\text { AA } \\
\text { AA } \\
\text { AA } \\
\text { AA } \\
\text { AA }\end{array}$ & $\begin{array}{l}\text { Town in France. } \\
\text { Town in Kazakhstan. } \\
\text { Town in Lithuania. } \\
\text { Town in India. } \\
\text { Dutch port. } \\
\text { Town in Zaire. } \\
\text { Town in Alaska, USA. } \\
\text { American colonial town } \\
\text { (Maryland). }\end{array}$ \\
\hline Antoniadi & $21.7 \mathrm{~N}$ & $299.0 \mathrm{~W}$ & 381 & EU & FR & $\mathrm{MC} 13$ & I967 & 5 & 1973 & 68 & $\mathrm{AA}$ & $\begin{array}{l}\text { Eugene M.; Greco-French } \\
\text { astronomer (1870-1944). }\end{array}$ \\
\hline $\begin{array}{l}\text { Apia } \\
\text { Apt } \\
\text { Arago }\end{array}$ & $\begin{array}{l}37.7 \mathrm{~S} \\
40.1 \mathrm{~N} \\
10.5 \mathrm{~N}\end{array}$ & $\begin{array}{r}271.0 \mathrm{~W} \\
9.5 \mathrm{~W} \\
330.2 \mathrm{~W}\end{array}$ & $\begin{array}{r}0 \\
10 \\
154\end{array}$ & $\begin{array}{l}\text { OC } \\
\text { EU } \\
\text { EU }\end{array}$ & $\begin{array}{l}\text { SA } \\
\text { FR } \\
\text { FR }\end{array}$ & $\begin{array}{l}\text { CYDONI } \\
\text { MC12 }\end{array}$ & $\begin{array}{l}\text { I2044 } \\
\text { I } 946 \\
\text { I1651 }\end{array}$ & $\begin{array}{l}5 \\
5 \\
5\end{array}$ & $\begin{array}{l}1989 \\
1976 \\
1973\end{array}$ & $\begin{array}{l}74 \\
36 \\
68\end{array}$ & $\begin{array}{l}\text { AA } \\
\text { AA } \\
\text { AA }\end{array}$ & $\begin{array}{l}\text { Town in American Samoa. } \\
\text { Town in France. } \\
\text { Dominique F.; French } \\
\text { astronomer (1786-1853). }\end{array}$ \\
\hline $\begin{array}{l}\text { Arandas } \\
\text { Argas } \\
\text { Arica } \\
\text { Arkhangelsky } \\
\text { Arrhenius }\end{array}$ & $\begin{array}{l}42.6 \mathrm{~N} \\
23.5 \mathrm{~N} \\
24.0 \mathrm{~S} \\
41.3 \mathrm{~S} \\
40.2 \mathrm{~S}\end{array}$ & $\begin{array}{r}15.1 \mathrm{~W} \\
50.1 \mathrm{~W} \\
249.7 \mathrm{~W} \\
24.6 \mathrm{~W} \\
237.0 \mathrm{~W}\end{array}$ & $\begin{array}{r}22 \\
0 \\
0 \\
119 \\
132\end{array}$ & $\begin{array}{l}\text { NA } \\
\text { UR } \\
\text { SA } \\
\text { UR } \\
\text { EU }\end{array}$ & $\begin{array}{l}\text { ME } \\
\text { RU } \\
\text { CO } \\
\text { RU } \\
\text { SW }\end{array}$ & $\begin{array}{l}\text { MC26 } \\
\text { MC29 }\end{array}$ & $\begin{array}{l}\text { I946 } \\
\text { I2064 } \\
\text { I1535 } \\
\text { I1 } 170\end{array}$ & $\begin{array}{l}5 \\
5 \\
5 \\
5 \\
5\end{array}$ & $\begin{array}{l}1976 \\
1988 \\
1989 \\
1979 \\
1973\end{array}$ & $\begin{array}{l}36 \\
36 \\
74 \\
68 \\
68\end{array}$ & $\begin{array}{l}\text { AA } \\
\text { AA } \\
\text { AA } \\
\text { AA } \\
\text { AA }\end{array}$ & $\begin{array}{l}\text { Town in Mexico. } \\
\text { Town in Russia. } \\
\text { Town in Colombia. } \\
\text { A.D.; Russian geologist. } \\
\text { Svante; Swedish physical } \\
\text { chemist (1859-1927). }\end{array}$ \\
\hline $\begin{array}{l}\text { Arta } \\
\text { Aspen } \\
\text { Aveiro } \\
\text { Ayacucho } \\
\text { Ayr }\end{array}$ & $\begin{array}{l}21.6 \mathrm{~N} \\
21.6 \mathrm{~S} \\
21.5 \mathrm{~N} \\
38.5 \mathrm{~N} \\
39.5 \mathrm{~S}\end{array}$ & $\begin{array}{r}54.2 \mathrm{~W} \\
22.9 \mathrm{~W} \\
80.0 \mathrm{~W} \\
92.0 \mathrm{~W} \\
268.2 \mathrm{~W}\end{array}$ & $\begin{array}{r}0 \\
16 \\
0 \\
0 \\
0\end{array}$ & $\begin{array}{l}\text { UR } \\
\text { US } \\
\text { EU } \\
\text { SA } \\
\text { OC }\end{array}$ & $\begin{array}{l}\text { RU } \\
\text { CO } \\
\text { PG } \\
\text { BO } \\
\text { AU }\end{array}$ & $\begin{array}{l}\text { ERYTHR } \\
20077 \\
40092\end{array}$ & $\begin{array}{l}\text { I947 } \\
\text { I } 1782 \\
\text { I } 2043\end{array}$ & $\begin{array}{l}5 \\
5 \\
5 \\
5 \\
5\end{array}$ & $\begin{array}{l}1988 \\
1976 \\
1985 \\
1991 \\
1989\end{array}$ & $\begin{array}{l}36 \\
36 \\
36 \\
36 \\
74\end{array}$ & $\begin{array}{l}\text { AA } \\
\text { AA } \\
\text { AA } \\
\text { AA } \\
\text { AA }\end{array}$ & $\begin{array}{l}\text { Town in Russia. } \\
\text { Town in Colorado, USA. } \\
\text { Town in Portugal. } \\
\text { Town in Bolivia. } \\
\text { Town in Queensland, } \\
\text { Australia. }\end{array}$ \\
\hline $\begin{array}{l}\text { Azul } \\
\text { Azusa } \\
\text { Babakin }\end{array}$ & $\begin{array}{r}42.5 S \\
5.5 S \\
36.4 S\end{array}$ & $\begin{array}{l}42.3 \mathrm{~W} \\
40.5 \mathrm{~W} \\
71.4 \mathrm{~W}\end{array}$ & $\begin{array}{l}20 \\
41 \\
78\end{array}$ & $\begin{array}{l}\text { SA } \\
\text { US } \\
\text { UR }\end{array}$ & $\begin{array}{l}\text { AR } \\
\text { CA } \\
\text { RU }\end{array}$ & $\begin{array}{l}\text { NEREID } \\
\text { CAPRI } \\
\text { MC25NE }\end{array}$ & $\begin{array}{l}1957 \\
\mathrm{I} 1026 \\
\mathrm{I} 1602\end{array}$ & $\begin{array}{l}5 \\
5 \\
5\end{array}$ & $\begin{array}{l}1976 \\
1976 \\
1985\end{array}$ & $\begin{array}{l}36 \\
36 \\
72\end{array}$ & $\begin{array}{l}\text { AA } \\
\text { AA } \\
\text { AA }\end{array}$ & $\begin{array}{l}\text { Town in Argentina. } \\
\text { Town in California, USA. } \\
\text { Soviet builder of } \\
\text { unmanned space stations } \\
(1914-1970) \text {. }\end{array}$ \\
\hline $\begin{array}{l}\text { Bacht } \\
\text { Bada } \\
\text { Bahn } \\
\text { Bak } \\
\text { Bakhuysen }\end{array}$ & $\begin{array}{l}18.9 \mathrm{~N} \\
20.5 \mathrm{~N} \\
3.5 \mathrm{~S} \\
18.3 \mathrm{~N} \\
23.1 \mathrm{~S}\end{array}$ & $\begin{array}{r}257.5 \mathrm{~W} \\
50.7 \mathrm{~W} \\
43.5 \mathrm{~W} \\
256.3 \mathrm{~W} \\
344.3 \mathrm{~W}\end{array}$ & $\begin{array}{r}7 \\
0 \\
10 \\
0 \\
162\end{array}$ & $\begin{array}{l}\text { UR } \\
\text { UR } \\
\text { AF } \\
\text { EU } \\
\text { EU }\end{array}$ & $\begin{array}{l}\text { UZ } \\
\text { RU } \\
\text { LI } \\
\text { HU } \\
\text { DU }\end{array}$ & $\begin{array}{l}\text { TRITON } \\
\text { CAPRI } \\
\text { MC20 }\end{array}$ & $\begin{array}{l}\mathrm{I} 1055 \\
\mathrm{I} 1026 \\
\mathrm{I} 1296\end{array}$ & $\begin{array}{l}5 \\
5 \\
5 \\
5 \\
5\end{array}$ & $\begin{array}{l}1976 \\
1988 \\
1976 \\
1988 \\
1973\end{array}$ & $\begin{array}{l}36 \\
36 \\
36 \\
36 \\
68\end{array}$ & $\begin{array}{l}\text { AA } \\
\text { AA } \\
\text { AA } \\
\text { AA } \\
\text { AA }\end{array}$ & $\begin{array}{l}\text { Town in Uzbekistan. } \\
\text { Town in Russia. } \\
\text { Town in Liberia. } \\
\text { Town in Hungary. } \\
\text { Henricus G.; Dutch } \\
\text { astronomer (1838-1923). }\end{array}$ \\
\hline Balboa & $3.5 \mathrm{~S}$ & $34.0 \mathrm{~W}$ & 20 & SA & PM & CAPRI & I1026 & 5 & 1976 & 36 & AA & $\begin{array}{l}\text { Town in the Panama Canal } \\
\text { zone. }\end{array}$ \\
\hline Baldet & $23.0 \mathrm{~N}$ & $294.5 \mathrm{~W}$ & 195 & EU & FR & MC13 & 1967 & 5 & 1973 & 68 & AA & $\begin{array}{l}\text { Ferdnand; French } \\
\text { astronomer (1885-1964). }\end{array}$ \\
\hline $\begin{array}{l}\text { Balta } \\
\text { Baltisk } \\
\text { Balvicar } \\
\text { Bamba } \\
\text { Bamberg } \\
\text { Banff } \\
\text { Banh }\end{array}$ & $\begin{array}{l}24.1 \mathrm{~S} \\
42.6 \mathrm{~S} \\
16.5 \mathrm{~N} \\
3.5 \mathrm{~S} \\
40.0 \mathrm{~N} \\
17.4 \mathrm{~N} \\
19.4 \mathrm{~N}\end{array}$ & $\begin{array}{l}26.4 \mathrm{~W} \\
54.5 \mathrm{~W} \\
53.1 \mathrm{~W} \\
41.7 \mathrm{~W} \\
3.0 \mathrm{~W} \\
30.5 \mathrm{~W} \\
55.2 \mathrm{~W}\end{array}$ & $\begin{array}{r}15 \\
48 \\
0 \\
21 \\
57 \\
3 \\
13\end{array}$ & $\begin{array}{l}\text { UR } \\
\text { UR } \\
\text { EU } \\
\text { AF } \\
\text { EU } \\
\text { NA } \\
\text { AF }\end{array}$ & $\begin{array}{l}\text { UK } \\
\text { RU } \\
\text { SC } \\
\text { ZA } \\
\text { GE } \\
\text { CA } \\
\text { BF }\end{array}$ & $\begin{array}{l}\text { CAPRI } \\
\text { CYDONI } \\
\text { CHRYHR } \\
\text { CHRY W }\end{array}$ & $\begin{array}{l}\mathrm{I} 1026 \\
\mathrm{I} 946 \\
\mathrm{I} 991 \\
\mathrm{I} 1068\end{array}$ & $\begin{array}{l}5 \\
5 \\
5 \\
5 \\
5 \\
5 \\
5\end{array}$ & $\begin{array}{l}1976 \\
1976 \\
1988 \\
1976 \\
1976 \\
1976 \\
1976\end{array}$ & $\begin{array}{r}36 \\
36 \\
0 \\
36 \\
36 \\
36 \\
36\end{array}$ & $\begin{array}{l}\text { AA } \\
\text { AA } \\
\text { AA } \\
\text { AA } \\
\text { AA } \\
\text { AA } \\
\text { AA }\end{array}$ & $\begin{array}{l}\text { Town in Ukraine. } \\
\text { Town in Russia. } \\
\text { Town in Scotland. } \\
\text { Town in Zaire. } \\
\text { Town in Germany. } \\
\text { Town in Alberta, Canada. } \\
\text { Town in Burkina Faso } \\
\text { (formerly Upper Volta). }\end{array}$ \\
\hline $\begin{array}{l}\text { Bar } \\
\text { Barabashov }\end{array}$ & $\begin{array}{l}25.6 \mathrm{~S} \\
47.6 \mathrm{~N}\end{array}$ & $\begin{array}{l}19.3 \mathrm{~W} \\
68.5 \mathrm{~W}\end{array}$ & $\begin{array}{r}2 \\
126\end{array}$ & $\begin{array}{l}\text { UR } \\
\text { UR }\end{array}$ & $\begin{array}{l}\text { UK } \\
\text { RU }\end{array}$ & $\begin{array}{l}\text { ERYTHR } \\
\text { MC03 }\end{array}$ & $\begin{array}{l}1947 \\
1963\end{array}$ & $\begin{array}{l}5 \\
5\end{array}$ & $\begin{array}{l}1976 \\
1973\end{array}$ & $\begin{array}{l}36 \\
68\end{array}$ & $\begin{array}{l}\mathrm{AA} \\
\mathrm{AA}\end{array}$ & $\begin{array}{l}\text { Town in Ukraine. } \\
\text { Nilolay P.; Russian } \\
\text { astronomer (1894-1971). }\end{array}$ \\
\hline Barnard & $61.3 \mathrm{~S}$ & 298.4W & 128 & NA & $\mathrm{AM}$ & MC28 & I1 169 & 5 & 1973 & 68 & AA & $\begin{array}{l}\text { Edward E.; American } \\
\text { astronomer (1857-1923). }\end{array}$ \\
\hline Dato & $24.9 \mathrm{~S}$ & $249.3 W$ & 0 & EU & IC & & I2064 & 5 & 1989 & 74 & AA & Town in Iceland. \\
\hline
\end{tabular}

lat: latitude of feature center.

long: longitude of feature center.

diam: diameter or long dimension of feature. et: ethnicity of name origin (see page $284 \mathrm{ff}$.)

quad: map quadrangle or informal name (see page xvii ff.). as: name approval status (see page $x$ vii)

ad: name approval date (year).

ref: reference source for name (see page $287 \mathrm{ff}$.).

ct: continent of name origin (see page $284 \mathrm{ff}$.) map: map name or USGS map number (see page xvii ff.). ft: feature type (see page 290 ). 
MARS

\begin{tabular}{|c|c|c|c|c|c|c|c|c|c|c|c|c|}
\hline Name & lat & long & diam & ct & et & quad & map & as & ad & ref & $\mathrm{ft}$ & origin \\
\hline $\begin{array}{l}\text { Basin } \\
\text { Batoka } \\
\text { Batoş } \\
\text { Baykonyr } \\
\text { Bazas } \\
\text { Becquerel }\end{array}$ & $\begin{array}{l}18.1 \mathrm{~N} \\
7.6 \mathrm{~S} \\
21.7 \mathrm{~N} \\
46.7 \mathrm{~N} \\
28.0 \mathrm{~S} \\
22.3 \mathrm{~N}\end{array}$ & $\begin{array}{r}253.2 \mathrm{~W} \\
36.8 \mathrm{~W} \\
29.5 \mathrm{~W} \\
227.2 \mathrm{~W} \\
266.5 \mathrm{~W} \\
7.9 \mathrm{~W}\end{array}$ & $\begin{array}{r}16 \\
14 \\
16 \\
4 \\
0 \\
167\end{array}$ & $\begin{array}{l}\text { US } \\
\text { AF } \\
\text { EU } \\
\text { UR } \\
\text { EU } \\
\text { EU }\end{array}$ & $\begin{array}{l}\text { WY } \\
\text { ZM } \\
\text { RO } \\
\text { SO } \\
\text { FR } \\
\text { FR }\end{array}$ & $\begin{array}{l}\text { TRITON } \\
\text { CAPRI } \\
\text { CHRYSE } \\
\text { CANBER } \\
\text { MC11 }\end{array}$ & $\begin{array}{l}11055 \\
\mathrm{I} 1026 \\
\mathrm{I} 939 \\
\mathrm{I} 1060 \\
\mathrm{I} 2039 \\
\mathrm{I} 1555\end{array}$ & $\begin{array}{l}5 \\
5 \\
5 \\
5 \\
5 \\
5\end{array}$ & $\begin{array}{l}1976 \\
1976 \\
1976 \\
1979 \\
1989 \\
1973\end{array}$ & $\begin{array}{l}36 \\
36 \\
36 \\
64 \\
74 \\
68\end{array}$ & $\begin{array}{l}\text { AA } \\
\text { AA } \\
\text { AA } \\
\text { AA } \\
\text { AA } \\
\text { AA }\end{array}$ & $\begin{array}{l}\text { Town in Wyoming, USA. } \\
\text { Town in Zambia. } \\
\text { Town in Romania. } \\
\text { Soviet launch site. } \\
\text { Town in France. } \\
\text { Antoine H.; French } \\
\text { physicist (1852-1908). }\end{array}$ \\
\hline Beer & $14.6 \mathrm{~S}$ & $8.2 \mathrm{~W}$ & 80 & EU & GE & MC19 & I1293 & 5 & 1973 & 68 & $\mathrm{AA}$ & $\begin{array}{l}\text { Wilhelm; German } \\
\text { astronomer (1797-1850). }\end{array}$ \\
\hline $\begin{array}{l}\text { Beltra } \\
\text { Belz } \\
\text { Bend } \\
\text { Bentham } \\
\text { Bentong } \\
\text { Bernard }\end{array}$ & $\begin{array}{l}18.3 \mathrm{~N} \\
22.0 \mathrm{~N} \\
22.6 \mathrm{~S} \\
56.0 \mathrm{~S} \\
22.6 \mathrm{~S} \\
23.8 \mathrm{~S}\end{array}$ & $\begin{array}{r}257.7 \mathrm{~W} \\
43.1 \mathrm{~W} \\
27.5 \mathrm{~W} \\
40.3 \mathrm{~W} \\
18.9 \mathrm{~W} \\
154.2 \mathrm{~W}\end{array}$ & $\begin{array}{r}0 \\
10 \\
2 \\
0 \\
10 \\
129\end{array}$ & $\begin{array}{l}\text { EU } \\
\text { UR } \\
\text { US } \\
\text { EU } \\
\text { AS } \\
\text { EU }\end{array}$ & $\begin{array}{l}\text { IR } \\
\text { UK } \\
\text { OR } \\
\text { EN } \\
\text { MA } \\
\text { FR }\end{array}$ & $\begin{array}{l}\text { CHRY W } \\
\text { ERYTHR } \\
-55043 \\
\text { ERYTHR } \\
1: 15 \mathrm{M}\end{array}$ & $\begin{array}{l}\mathrm{I} 1068 \\
\mathrm{I} 947 \\
\mathrm{I} 947 \\
\mathrm{I} 1535\end{array}$ & $\begin{array}{l}5 \\
5 \\
5 \\
5 \\
5 \\
5\end{array}$ & $\begin{array}{l}1988 \\
1976 \\
1976 \\
1991 \\
1976 \\
1985\end{array}$ & $\begin{array}{l}36 \\
36 \\
36 \\
36 \\
36 \\
68\end{array}$ & $\begin{array}{l}\text { AA } \\
\text { AA } \\
\text { AA } \\
\text { AA } \\
\text { AA } \\
\text { AA }\end{array}$ & $\begin{array}{l}\text { Town in Ireland. } \\
\text { Town in Ukraine. } \\
\text { Town in Oregon, USA. } \\
\text { Town in England. } \\
\text { Town in Malaysia. } \\
\text { P.; French atmospheric } \\
\text { scientist. }\end{array}$ \\
\hline $\begin{array}{l}\text { Berseba } \\
\text { Bhor } \\
\text { Bianchini }\end{array}$ & $\begin{array}{l}4.4 \mathrm{~S} \\
42.0 \mathrm{~N} \\
64.2 \mathrm{~S}\end{array}$ & $\begin{array}{r}37.7 \mathrm{~W} \\
225.5 \mathrm{~W} \\
95.1 \mathrm{~W}\end{array}$ & $\begin{array}{r}36 \\
6 \\
77\end{array}$ & $\begin{array}{l}\text { AF } \\
\text { AS } \\
\text { EU }\end{array}$ & $\begin{array}{l}\text { NM } \\
\text { IN } \\
\text { IT }\end{array}$ & $\begin{array}{l}\text { CAPRI } \\
\text { UTOPIA } \\
\text { MC25 }\end{array}$ & $\begin{array}{l}\text { I1026 } \\
\text { I1061 } \\
\text { I11 } 165\end{array}$ & $\begin{array}{l}5 \\
5 \\
5\end{array}$ & $\begin{array}{l}1976 \\
1979 \\
1973\end{array}$ & $\begin{array}{l}36 \\
36 \\
68\end{array}$ & $\begin{array}{l}\text { AA } \\
\text { AA } \\
\text { AA }\end{array}$ & $\begin{array}{l}\text { Town in Namibia. } \\
\text { Town in India. } \\
\text { Francesco; Italian } \\
\text { astronomer (1662-1729). }\end{array}$ \\
\hline $\begin{array}{l}\text { Bigbee } \\
\text { Bira } \\
\text { Bise } \\
\text { Bison } \\
\text { Bjerknes }\end{array}$ & $\begin{array}{l}25.0 \mathrm{~S} \\
25.3 \mathrm{~N} \\
20.2 \mathrm{~N} \\
26.6 \mathrm{~S} \\
43.4 \mathrm{~S}\end{array}$ & $\begin{array}{r}34.6 \mathrm{~W} \\
45.9 \mathrm{~W} \\
56.5 \mathrm{~W} \\
29.0 \mathrm{~W} \\
188.7 \mathrm{~W}\end{array}$ & $\begin{array}{r}18 \\
0 \\
9 \\
15 \\
89\end{array}$ & $\begin{array}{l}\text { US } \\
\text { UR } \\
\text { AS } \\
\text { US } \\
\text { EU }\end{array}$ & $\begin{array}{l}\text { MS } \\
\text { RU } \\
\text { JA } \\
\text { KS } \\
\text { NO }\end{array}$ & $\begin{array}{l}\text { ERYTHR } \\
\text { CHRY W } \\
\text { ERYTHR } \\
\text { MC29 }\end{array}$ & $\begin{array}{l}\text { I947 } \\
\text { I1068 } \\
\text { I947 } \\
\text { I1 } 170\end{array}$ & $\begin{array}{l}5 \\
5 \\
5 \\
5 \\
5\end{array}$ & $\begin{array}{l}1976 \\
1988 \\
1976 \\
1976 \\
1973\end{array}$ & $\begin{array}{r}36 \\
0 \\
36 \\
36 \\
68\end{array}$ & $\begin{array}{l}\text { AA } \\
\text { AA } \\
\text { AA } \\
\text { AA } \\
\text { AA }\end{array}$ & $\begin{array}{l}\text { Town in Mississippi, USA. } \\
\text { Town in Russia. } \\
\text { Town in Okinawa. } \\
\text { Town in Kansas, USA. } \\
\text { Vilhelm F.; Norwegian } \\
\text { physicist (1862-1951). }\end{array}$ \\
\hline $\begin{array}{l}\text { Bland } \\
\text { Bled } \\
\text { Blitta } \\
\text { Blois } \\
\text { Bluff } \\
\text { Boeddicker }\end{array}$ & $\begin{array}{l}18.5 \mathrm{~N} \\
21.8 \mathrm{~N} \\
26.3 \mathrm{~S} \\
24.6 \mathrm{~N} \\
23.7 \mathrm{~N} \\
14.8 \mathrm{~S}\end{array}$ & $\begin{array}{r}251.5 \mathrm{~W} \\
31.4 \mathrm{~W} \\
20.8 \mathrm{~W} \\
55.6 \mathrm{~W} \\
250.1 \mathrm{~W} \\
197.6 \mathrm{~W}\end{array}$ & $\begin{array}{r}0 \\
7 \\
11 \\
11 \\
6 \\
107\end{array}$ & $\begin{array}{l}\text { US } \\
\text { EU } \\
\text { AF } \\
\text { EU } \\
\text { OC } \\
\text { EU }\end{array}$ & $\begin{array}{l}\text { MO } \\
\text { YU } \\
\text { TO } \\
\text { FR } \\
\text { NZ } \\
\text { GE }\end{array}$ & $\begin{array}{l}\text { CHRYSE } \\
\text { ERYTHR } \\
\text { CHRY W } \\
\text { TRITON } \\
\text { MC23 }\end{array}$ & $\begin{array}{l}\text { I939 } \\
\text { I947 } \\
\text { I1068 } \\
\text { I1055 } \\
\text { I1552 }\end{array}$ & $\begin{array}{l}5 \\
5 \\
5 \\
5 \\
5 \\
5\end{array}$ & $\begin{array}{l}1988 \\
1976 \\
1976 \\
1976 \\
1976 \\
1973\end{array}$ & $\begin{array}{l}36 \\
36 \\
36 \\
36 \\
36 \\
68\end{array}$ & $\begin{array}{l}\text { AA } \\
\text { AA } \\
\text { AA } \\
\text { AA } \\
\text { AA } \\
\text { AA }\end{array}$ & $\begin{array}{l}\text { Town in Missouri, USA. } \\
\text { Town in Yugoslavia. } \\
\text { Town in Togo. } \\
\text { Town in France. } \\
\text { Town in New Zealand. } \\
\text { Otto; German astronomer } \\
(1853-1937) \text {. }\end{array}$ \\
\hline $\begin{array}{l}\text { Bogra } \\
\text { Bok } \\
\text { Bole } \\
\text { Bombala }\end{array}$ & $\begin{array}{l}24.4 \mathrm{~S} \\
20.8 \mathrm{~N} \\
25.4 \mathrm{~N} \\
30.3 \mathrm{~S}\end{array}$ & $\begin{array}{r}28.6 \mathrm{~W} \\
31.6 \mathrm{~W} \\
53.7 \mathrm{~W} \\
253.9 \mathrm{~W}\end{array}$ & $\begin{array}{r}18 \\
7 \\
8 \\
0\end{array}$ & $\begin{array}{l}\text { AS } \\
\text { OC } \\
\text { AF } \\
\text { OC }\end{array}$ & $\begin{array}{l}\text { BA } \\
\text { GU } \\
\text { GH } \\
\mathrm{AU}\end{array}$ & $\begin{array}{l}\text { ERYTHR } \\
\text { CHRYSE } \\
\text { CHRY W }\end{array}$ & $\begin{array}{l}\mathrm{I} 947 \\
\mathrm{I} 939 \\
\mathrm{I} 1068 \\
\mathrm{I} 2063\end{array}$ & $\begin{array}{l}5 \\
5 \\
5 \\
5\end{array}$ & $\begin{array}{l}1976 \\
1976 \\
1976 \\
1989\end{array}$ & $\begin{array}{l}36 \\
36 \\
36 \\
74\end{array}$ & $\begin{array}{l}\text { AA } \\
\text { AA } \\
\text { AA } \\
\text { AA }\end{array}$ & $\begin{array}{l}\text { Town in Bangladesh. } \\
\text { Town in New Guinea. } \\
\text { Town in Ghana. } \\
\text { Town in New S. Wales, } \\
\text { Australia. }\end{array}$ \\
\hline Bond & $33.3 \mathrm{~S}$ & $35.7 \mathrm{~W}$ & 104 & NA & $\mathrm{AM}$ & MC26 & I985 & 5 & 1973 & 68 & AA & $\begin{array}{l}\text { George P.; American } \\
\text { astronomer (1825-1865). }\end{array}$ \\
\hline $\begin{array}{l}\text { Bor } \\
\text { Bordeaux } \\
\text { Boru } \\
\text { Bouguer }\end{array}$ & $\begin{array}{l}18.1 \mathrm{~N} \\
23.4 \mathrm{~N} \\
24.6 \mathrm{~S} \\
18.6 \mathrm{~S}\end{array}$ & $\begin{array}{r}33.8 \mathrm{~W} \\
48.9 \mathrm{~W} \\
27.7 \mathrm{~W} \\
332.8 \mathrm{~W}\end{array}$ & $\begin{array}{r}4 \\
2 \\
11 \\
106\end{array}$ & $\begin{array}{l}\text { UR } \\
\text { EU } \\
\text { UR } \\
\text { EU }\end{array}$ & $\begin{array}{l}\text { RU } \\
\text { FR } \\
\text { RU } \\
\text { FR }\end{array}$ & $\begin{array}{l}\text { CHRYHR } \\
\text { YORKTN } \\
\text { ERYTHR } \\
\text { MC20 }\end{array}$ & $\begin{array}{l}\text { I991 } \\
\text { I1059 } \\
1947 \\
\text { I1296 }\end{array}$ & $\begin{array}{l}5 \\
5 \\
5 \\
5\end{array}$ & $\begin{array}{l}1976 \\
1979 \\
1976 \\
1973\end{array}$ & $\begin{array}{l}36 \\
36 \\
36 \\
68\end{array}$ & $\begin{array}{l}\text { AA } \\
\text { AA } \\
\text { AA } \\
\text { AA }\end{array}$ & $\begin{array}{l}\text { Town in Russia. } \\
\text { French port. } \\
\text { Town in Russia. } \\
\text { Pierre; French } \\
\text { physicist-hydrographer } \\
(1698-1758) \text {. }\end{array}$ \\
\hline Boulia & $23.1 \mathrm{~S}$ & $248.6 \mathrm{~W}$ & 0 & $\mathrm{OC}$ & $\mathrm{AU}$ & I2064 & & 5 & 1989 & 74 & AA & $\begin{array}{l}\text { Town in Queensland, } \\
\text { Australia. }\end{array}$ \\
\hline $\begin{array}{l}\text { Bozkir } \\
\text { Brashear }\end{array}$ & $\begin{array}{l}44.4 \mathrm{~S} \\
54.1 \mathrm{~S}\end{array}$ & $\begin{aligned} 32.0 \mathrm{~W} \\
119.2 \mathrm{~W}\end{aligned}$ & $\begin{array}{r}89 \\
126\end{array}$ & $\begin{array}{l}\text { AS } \\
\text { NA }\end{array}$ & $\begin{array}{l}\text { TU } \\
\text { AM }\end{array}$ & $\begin{array}{l}\text { NEREID } \\
\text { MC25 }\end{array}$ & $\begin{array}{l}1957 \\
11165\end{array}$ & $\begin{array}{l}5 \\
5\end{array}$ & $\begin{array}{l}1976 \\
1973\end{array}$ & $\begin{array}{l}36 \\
68\end{array}$ & $\begin{array}{l}\text { AA } \\
\text { AA }\end{array}$ & $\begin{array}{l}\text { Town in Turkey. } \\
\text { John A.; American } \\
\text { physicist (1840-1920). }\end{array}$ \\
\hline $\begin{array}{l}\text { Bremerhaven } \\
\text { Briault }\end{array}$ & $\begin{array}{l}23.5 \mathrm{~N} \\
10.1 \mathrm{~S}\end{array}$ & $\begin{array}{r}48.6 \mathrm{~W} \\
270.2 \mathrm{~W}\end{array}$ & $\begin{array}{r}2 \\
100\end{array}$ & $\begin{array}{l}\text { EU } \\
\text { EU }\end{array}$ & $\begin{array}{l}\text { GE } \\
\text { FR }\end{array}$ & $\begin{array}{l}\text { YORKTN } \\
\text { MC21 }\end{array}$ & $\begin{array}{l}\text { I1059 } \\
\text { I11118 }\end{array}$ & $\begin{array}{l}5 \\
5\end{array}$ & $\begin{array}{l}1979 \\
1973\end{array}$ & $\begin{array}{l}36 \\
68\end{array}$ & $\begin{array}{l}\text { AA } \\
\text { AA }\end{array}$ & $\begin{array}{l}\text { German port. } \\
\text { P.; French astronomer (d. } \\
\text { 1922). }\end{array}$ \\
\hline $\begin{array}{l}\text { Bridgetown } \\
\text { Bristol } \\
\text { Broach } \\
\text { Brush } \\
\text { Bulhar } \\
\text { Bunge }\end{array}$ & $\begin{array}{l}22.2 \mathrm{~N} \\
22.4 \mathrm{~N} \\
23.5 \mathrm{~N} \\
21.9 \mathrm{~N} \\
50.6 \mathrm{~N} \\
34.2 \mathrm{~S}\end{array}$ & $\begin{array}{r}47.1 \mathrm{~W} \\
47.0 \mathrm{~W} \\
56.6 \mathrm{~W} \\
248.8 \mathrm{~W} \\
225.6 \mathrm{~W} \\
48.4 \mathrm{~W}\end{array}$ & $\begin{array}{r}2 \\
3 \\
11 \\
5 \\
19 \\
78\end{array}$ & $\begin{array}{l}\text { SA } \\
\text { EU } \\
\text { AS } \\
\text { US } \\
\text { AF } \\
\text { UR }\end{array}$ & $\begin{array}{l}\text { BB } \\
\text { GB } \\
\text { IN } \\
\text { CO } \\
\text { SO } \\
\text { RU }\end{array}$ & $\begin{array}{l}\text { YORKTN } \\
\text { YORKTN } \\
\text { CHRY W } \\
\text { TRITON } \\
\text { UTOPIA } \\
\text { MC26 }\end{array}$ & $\begin{array}{l}\text { I1059 } \\
\text { I1059 } \\
\text { I1068 } \\
\text { I1055 } \\
\text { I1061 } \\
\text { I1535 }\end{array}$ & $\begin{array}{l}5 \\
5 \\
5 \\
5 \\
5 \\
5\end{array}$ & $\begin{array}{l}1979 \\
1979 \\
1976 \\
1976 \\
1979 \\
1979\end{array}$ & $\begin{array}{l}36 \\
36 \\
36 \\
36 \\
36 \\
68\end{array}$ & $\begin{array}{l}\text { AA } \\
\text { AA } \\
\text { AA } \\
\text { AA } \\
\text { AA } \\
\text { AA }\end{array}$ & $\begin{array}{l}\text { Port of Barbados. } \\
\text { English port. } \\
\text { Town in India. } \\
\text { Town in Colorado, USA. } \\
\text { Town in Somalia. } \\
\text { Nicolai A.; Russian } \\
\text { chemist; same crater as } \\
\text { A. A. Bunge. }\end{array}$ \\
\hline Bunge & $34.2 \mathrm{~S}$ & $49.4 W$ & 78 & UR & RU & & & 5 & 1979 & 64 & AA & $\begin{array}{l}\text { Andrey Aleksandrovich; } \\
\text { Russian zoologist, } \\
\text { permafrost investigator } \\
\text { (Late 19th century). }\end{array}$ \\
\hline Burroughs & $72.5 \mathrm{~S}$ & $243.1 \mathrm{~W}$ & 104 & NA & AM & $\mathrm{MC} 30$ & I970 & 5 & 1973 & 68 & AA & $\begin{array}{l}\text { Edgar R.; American } \\
\text { novelist (1875-1950). }\end{array}$ \\
\hline Burton & $14.5 \mathrm{~S}$ & $156.3 W$ & 137 & EU & GB & MC16 & I1554 & 5 & 1973 & 68 & AA & $\begin{array}{l}\text { Charles E.; British } \\
\text { astronomer (1846-1882). }\end{array}$ \\
\hline Buta & $23.5 \mathrm{~S}$ & $32.2 \mathrm{~W}$ & 12 & AF & $\mathrm{ZA}$ & ERYTHR & 1947 & 5 & 1979 & 36 & AA & Town in Zaire. \\
\hline
\end{tabular}

lat: latitude of feature center.

long: longitude of feature center.

diam: diameter or long dimension of feature. et: ethnicity of name origin (see page $284 \mathrm{ff}$.)

quad: map quadrangle or informal name

(see page xvii ff.). as: name approval status (see page xvii).

ad: name approval date (year)

ref: reference source for name (see page $287 \mathrm{ff}$.). 
MARS

\begin{tabular}{|c|c|c|c|c|c|c|c|c|c|c|c|c|}
\hline Name & lat & long & diam & ct & et & quad & map & as & ad & ref & $\mathbf{f t}$ & origin \\
\hline Butte & $5.1 \mathrm{~S}$ & $39.1 \mathrm{~W}$ & 12 & US & MT & CAPRI & I1026 & 5 & 1976 & 36 & AA & Town in Montana, USA. \\
\hline Byrd & $65.6 \mathrm{~S}$ & $231.9 \mathrm{~W}$ & 122 & NA & AM & MC29SW & 11170 & 5 & 1976 & 68 & $\mathrm{AA}$ & $\begin{array}{l}\text { Richard E.; American } \\
\text { aviator-explorer } \\
(1888-1975) \text {. }\end{array}$ \\
\hline Byske & $4.9 \mathrm{~S}$ & $31.1 \mathrm{~W}$ & 5 & EU & SW & CAPRI & I1026 & 5 & 1976 & 36 & AA & Town in Sweden. \\
\hline Cádiz & $23.4 \mathrm{~N}$ & $49.1 \mathrm{~W}$ & 1 & $\mathrm{EU}$ & SP & YORKTN & I1059 & 5 & 1979 & 36 & $\mathrm{AA}$ & Spanish port. \\
\hline Cairns & $24.0 \mathrm{~N}$ & $47.3 \mathrm{~W}$ & 10 & OC & $\mathrm{AU}$ & CHRY W & 11068 & 5 & 1976 & 36 & AA & Town in Australia. \\
\hline Calamar & $18.4 \mathrm{~N}$ & $54.9 \mathrm{~W}$ & 0 & SA & $\mathrm{CO}$ & & & 5 & 1988 & 36 & $\mathrm{AA}$ & Town in Colombia. \\
\hline Calbe & $25.5 \mathrm{~S}$ & $28.7 \mathrm{~W}$ & 14 & EU & GE & ERYTHR & I947 & 5 & 1976 & 36 & AA & Town in Germany. \\
\hline Camargo & $17.9 \mathrm{~N}$ & $250.6 \mathrm{~W}$ & 0 & SA & BO & & & 5 & 1988 & 36 & AA & Town in Bolivia. \\
\hline Camiling & $0.8 \mathrm{~S}$ & $38.1 \mathrm{~W}$ & 21 & AS & PH & CAPRI & 11026 & 5 & 1976 & 36 & $\mathrm{AA}$ & Town in the Philippines. \\
\hline Camiri & $45.1 \mathrm{~S}$ & $41.9 \mathrm{~W}$ & 20 & SA & BO & NEREID & 1957 & 5 & 1976 & 36 & AA & Town in Bolivia. \\
\hline Campbell & $54.0 \mathrm{~S}$ & $195.0 \mathrm{~W}$ & 123 & NA & AM & MC29 & I1170 & 5 & 1973 & 68 & AA & $\begin{array}{l}\text { John W.; Canadian } \\
\text { physicist (1889-1955). } \\
\text { William W.; American } \\
\text { astronomer (1862-1938). }\end{array}$ \\
\hline Campos & $22.0 \mathrm{~S}$ & $27.6 \mathrm{~W}$ & 7 & SA & BR & ERYTHR & I947 & 5 & 1976 & 36 & AA & Town in Brazil. \\
\hline & $48.4 \mathrm{~N}$ & $14.6 \mathrm{~W}$ & 6 & AS & TU & CYDONI & I946 & 5 & 1976 & 36 & AA & Town in Turkey. \\
\hline Canas & $31.4 \mathrm{~S}$ & $270.1 \mathrm{~W}$ & 0 & SA & PR & & I2038 & 5 & 1989 & 74 & AA & Town in Puerto Rico. \\
\hline Canaveral & $47.1 \mathrm{~N}$ & $224.0 \mathrm{~W}$ & 3 & $\mathrm{NA}$ & $\mathrm{AM}$ & CANBER & I1060 & 5 & 1979 & 36 & AA & American launch site. \\
\hline Canberra & $47.5 \mathrm{~N}$ & $227.3 \mathrm{~W}$ & 3 & OC & $\mathrm{AU}$ & CANBER & I1060 & 5 & 1979 & 36 & $\mathrm{AA}$ & Australian tracking site. \\
\hline Cangwu & $42.1 \mathrm{~N}$ & $89.5 \mathrm{~W}$ & 0 & AS & $\mathrm{CH}$ & 40087 & & 5 & 1991 & 36 & AA & Town in China. \\
\hline Canso & $21.5 \mathrm{~N}$ & $60.6 \mathrm{~W}$ & 0 & NA & $\mathrm{CA}$ & 20062 & I1868 & 5 & 1988 & 36 & AA & $\begin{array}{l}\text { Town in Nova Scotia, } \\
\text { Canada. }\end{array}$ \\
\hline Cantoura & $15.0 \mathrm{~N}$ & $51.5 \mathrm{~W}$ & 0 & SA & VE & & & 5 & 1988 & 36 & AA & Town in Venezuela. \\
\hline Cartago & $23.6 \mathrm{~S}$ & $17.8 \mathrm{~W}$ & 33 & SA & CR & ERYTHR & I947 & 5 & 1976 & 36 & AA & Town in Costa Rica. \\
\hline Cassini & $23.8 \mathrm{~N}$ & $327.9 \mathrm{~W}$ & 415 & $\mathrm{EU}$ & IT & $\mathrm{MC} 12$ & I 1651 & 5 & 1973 & 68 & $\mathrm{AA}$ & $\begin{array}{l}\text { Giovanni; Italian } \\
\text { astronomer }(1625-1712) \text {. }\end{array}$ \\
\hline Cave & $21.9 \mathrm{~N}$ & $35.6 \mathrm{~W}$ & 8 & OC & $\mathrm{NZ}$ & CHRYSE & 1939 & 5 & 1976 & 36 & AA & Town in New Zealand. \\
\hline Caxias & $29.5 \mathrm{~S}$ & $100.6 \mathrm{~W}$ & 0 & SA & BR & & I2061 & 5 & 1989 & 74 & AA & Town in Brazil. \\
\hline Cerulli & $32.6 \mathrm{~N}$ & $337.9 \mathrm{~W}$ & 120 & $\mathrm{EU}$ & IT & $\mathrm{MCO5}$ & I1495 & 5 & 1973 & 68 & AA & $\begin{array}{l}\text { Vicenzo; Italian } \\
\text { astronomer }(1859-1927) \text {. }\end{array}$ \\
\hline Chafe & $15.4 \mathrm{~N}$ & $257.9 \mathrm{~W}$ & 0 & $\mathrm{AF}$ & NI & & & 5 & 1988 & 36 & AA & Town in Nigeria. \\
\hline Chamberlin & $66.1 \mathrm{~S}$ & $124.3 \mathrm{~W}$ & 125 & NA & AM & $\mathrm{MC} 30$ & 1970 & 5 & 1973 & 68 & $\mathrm{AA}$ & $\begin{array}{l}\text { Thomas C.; American } \\
\text { geologist (1843-1928). }\end{array}$ \\
\hline Changsǒng & $23.5 \mathrm{~N}$ & $57.1 \mathrm{~W}$ & 32 & AS & KR & CHRY W & 11068 & 5 & 1976 & 36 & AA & $\begin{array}{l}\text { Town in the Republic of } \\
\text { Korea. }\end{array}$ \\
\hline Chapais & $22.6 \mathrm{~S}$ & $20.4 \mathrm{~W}$ & 33 & NA & $\mathrm{CA}$ & ERYTHR & I947 & 5 & 1976 & 36 & AA & Town in Quebec, Canada. \\
\hline Charleston & $22.9 \mathrm{~N}$ & $47.8 \mathrm{~W}$ & 2 & US & SC & YORKTN & I1059 & 5 & 1979 & 36 & AA & $\begin{array}{l}\text { American colonial town } \\
\text { (South Carolina). }\end{array}$ \\
\hline Charlier & $68.6 \mathrm{~S}$ & $168.4 \mathrm{~W}$ & 100 & EU & SW & MC30 & 1970 & 5 & 1973 & 68 & AA & $\begin{array}{l}\text { Carl V.; Swedish } \\
\text { astronomer (1862-1934). }\end{array}$ \\
\hline Charlieu & $38.3 \mathrm{~N}$ & $84.1 \mathrm{~W}$ & 0 & $\mathrm{EU}$ & FR & 40082 & & 5 & 1991 & 36 & AA & Town in France. \\
\hline Chatturat & $35.7 \mathrm{~N}$ & $94.8 \mathrm{~W}$ & 0 & AS & $\mathrm{TH}$ & 35092 & & 5 & 1991 & 36 & AA & Town in Thailand. \\
\hline Chauk & $23.3 \mathrm{~N}$ & $55.6 \mathrm{~W}$ & 9 & AS & BU & CHRY W & 11068 & 5 & 1976 & 36 & AA & Town in Burma. \\
\hline Cheb & $24.5 \mathrm{~S}$ & $19.3 \mathrm{~W}$ & 8 & $\mathrm{EU}$ & $\mathrm{CZ}$ & ERYTHR & 1947 & 5 & 1976 & 36 & AA & Town in Czechoslovakia. \\
\hline Chefu & $23.1 \mathrm{~N}$ & $247.7 \mathrm{~W}$ & 0 & $\mathrm{AF}$ & $\mathrm{MZ}$ & & 12064 & 5 & 1989 & 74 & $\mathrm{AA}$ & Town in Mozambique. \\
\hline Chekalin & $24.7 \mathrm{~S}$ & $26.6 \mathrm{~W}$ & 87 & UR & TK & ERYTHR & 1947 & 5 & 1976 & 36 & AA & Town in Turkmenistan. \\
\hline Chia & $1.6 \mathrm{~N}$ & $59.5 \mathrm{~W}$ & 94 & $\mathrm{EU}$ & SP & 00057 & 11675 & 5 & 1985 & 0 & AA & Town in Spain. \\
\hline Chimbote & $1.5 \mathrm{~S}$ & $39.8 \mathrm{~W}$ & 65 & SA & PE & CAPRI & 11026 & 5 & 1976 & 36 & AA & Town in Peru. \\
\hline Chincoteague & $41.5 \mathrm{~N}$ & $236.0 \mathrm{~W}$ & 35 & US & VA & UTOPIA & 11061 & 5 & 1979 & 36 & $\mathrm{AA}$ & Town in Virginia, USA. \\
\hline Chinju & $4.7 \mathrm{~S}$ & $42.3 \mathrm{~W}$ & 69 & AS & $\mathrm{KR}$ & CAPRI & I1026 & 5 & 1976 & 36 & AA & $\begin{array}{l}\text { Town in the Republic of } \\
\text { Korea. }\end{array}$ \\
\hline Chinook & $22.5 \mathrm{~N}$ & $55.2 \mathrm{~W}$ & 17 & NA & $\mathrm{CA}$ & CHRY W & I1068 & 5 & 1976 & 36 & AA & Town in Alberta, Canada. \\
\hline Chive & $21.6 \mathrm{~N}$ & $55.7 \mathrm{~W}$ & 8 & SA & BO & CHRY W & I1068 & 5 & 1976 & 36 & AA & Town in Bolivia. \\
\hline Choctaw & $41.5 \mathrm{~S}$ & $37.0 \mathrm{~W}$ & 20 & US & $\mathrm{OH}$ & NEREID & 1957 & 5 & 1976 & 36 & AA & Town in Ohio, USA. \\
\hline Chom & $38.7 \mathrm{~N}$ & $2.2 \mathrm{~W}$ & 6 & AS & TB & CYDONI & 1990 & 5 & 1976 & 36 & $\mathrm{AA}$ & Town in China (Tibet). \\
\hline Chur & $16.7 \mathrm{~N}$ & $29.2 \mathrm{~W}$ & 4 & UR & RU & CHRYHR & 1991 & 5 & 1976 & 36 & AA & Town in Russia. \\
\hline Circle & $22.4 \mathrm{~S}$ & $25.4 \mathrm{~W}$ & 11 & US & MT & ERYTHR & 1947 & 5 & 1976 & 36 & AA & Town in Montana, USA. \\
\hline Clark & $55.7 \mathrm{~S}$ & $133.2 \mathrm{~W}$ & 93 & NA & $\mathrm{AM}$ & MC24 & I1 166 & 5 & 1973 & 68 & $\mathrm{AA}$ & $\begin{array}{l}\text { Alvan; American } \\
\text { optician-astronomer } \\
\text { (1804-1887). }\end{array}$ \\
\hline Clogh & $20.8 \mathrm{~N}$ & $47.5 \mathrm{~W}$ & 10 & EU & IR & CHRY W & I1068 & 5 & 1976 & 36 & AA & Town in Ireland. \\
\hline Clova & $21.7 \mathrm{~N}$ & $52.0 \mathrm{~W}$ & 0 & NA & $\mathrm{CA}$ & & & 5 & 1988 & 36 & AA & Town in Quebec, Canada. \\
\hline Cluny & $24.1 \mathrm{~S}$ & $27.1 \mathrm{~W}$ & 9 & $\mathrm{EU}$ & FR & ERYTHR & 1947 & 5 & 1976 & 36 & AA & Town in France. \\
\hline Cobalt & $26.1 S$ & $26.8 \mathrm{~W}$ & 10 & US & CT & ERYTHR & 1947 & 5 & 1976 & 36 & $\mathrm{AA}$ & Town in Connecticut, USA \\
\hline Coblentz & $55.3 \mathrm{~S}$ & $90.2 \mathrm{~W}$ & 111 & NA & AM & MC25 & I1 165 & 5 & 1973 & 68 & AA & $\begin{array}{l}\text { William W; American } \\
\text { physicist }(1873-1962) \text {. }\end{array}$ \\
\hline Cobres & $12.1 \mathrm{~S}$ & $\begin{aligned} 153.7 \mathrm{~W} \\
491 \mathrm{~W}\end{aligned}$ & $\begin{array}{r}89 \\
?\end{array}$ & SA & $\mathrm{AR}$ & $\begin{array}{l}-10152 \\
\text { YOP }\end{array}$ & 11652 & 5 & 1985 & 36 & AA & Village in Argentina. \\
\hline Colón & $23.4 \mathrm{~N}$ & $49.1 \mathrm{~W}$ & 2 & SA & PM & YORKTN & 11059 & 5 & 1979 & 36 & AA & Port of Panama. \\
\hline
\end{tabular}

\footnotetext{
lat: latitude of feature center.

et: ethnicity of name origin (see page $284 \mathrm{ff}$.) long: longitude of feature center.

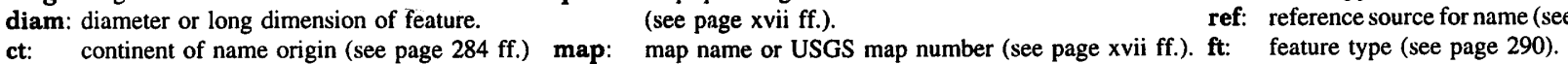

as: name approval status (see page xvii)

ad: name approval date (year).

ref: reference source for name (see page $287 \mathrm{ff}$.).
} 
MARS

\begin{tabular}{|c|c|c|c|c|c|c|c|c|c|c|c|c|}
\hline Name & lat & long & diam & ct & et & quad & map & as & ad & ref & ft & origin \\
\hline Columbus & $29.7 \mathrm{~S}$ & $165.8 \mathrm{~W}$ & 114 & EU & IT & MC16 & I1554 & 5 & 1976 & 68 & AA & Christopher; Italian \\
\hline Comas Sola & $20.1 S$ & $158.4 \mathrm{~W}$ & 132 & EU & SP & $\mathrm{MC} 16$ & I1554 & 5 & 1973 & 68 & AA & $\begin{array}{l}\text { Jose; Spanish astronomer } \\
(1868-1937) \text {. }\end{array}$ \\
\hline Conches & $4.2 \mathrm{~S}$ & $34.3 \mathrm{~W}$ & 18 & EU & FR & CAPRI & 11026 & 5 & 1976 & 36 & AA & Town in France. \\
\hline Concord & $16.6 \mathrm{~N}$ & $34.1 \mathrm{~W}$ & 20 & US & MA & CHRYSE & I939 & 5 & 1976 & 36 & AA & $\begin{array}{l}\text { Town in Massachusetts, } \\
\text { USA. }\end{array}$ \\
\hline Cooma & $24.0 \mathrm{~S}$ & $108.5 \mathrm{~W}$ & 0 & $\mathrm{OC}$ & $\mathrm{AU}$ & & I 2060 & 5 & 1989 & 74 & AA & $\begin{array}{l}\text { Town in New South Wales, } \\
\text { Australia. }\end{array}$ \\
\hline Copernicus & $50.0 \mathrm{~S}$ & $168.6 \mathrm{~W}$ & 292 & $\mathrm{EU}$ & $\mathrm{PO}$ & MC24 & I1 166 & 5 & 1973 & 68 & AA & $\begin{array}{l}\text { Nicolaus; Polish } \\
\text { astronomer (1473-1543). }\end{array}$ \\
\hline Corby & $43.1 \mathrm{~N}$ & $222.4 \mathrm{~W}$ & 7 & EU & EN & UTOPIA & I1061 & 5 & 1979 & 36 & AA & Town in England. \\
\hline Cost & $15.3 \mathrm{~N}$ & $256.1 \mathrm{~W}$ & 10 & US & TX & TRITON & I1055 & 5 & 1976 & 36 & AA & Town in Texas, USA. \\
\hline Cray & $44.4 \mathrm{~N}$ & $16.2 \mathrm{~W}$ & 5 & EU & EN & CYDONI & I946 & 5 & 1976 & 36 & AA & Town in England. \\
\hline Creel & $6.1 \mathrm{~S}$ & $39.0 \mathrm{~W}$ & 8 & NA & ME & CAPRI & I1026 & 5 & 1976 & 36 & AA & Town in Mexico. \\
\hline Crewe & $25.2 \mathrm{~S}$ & $19.4 \mathrm{~W}$ & 3 & EU & GB & ERYTHR & I947 & 5 & 1976 & 36 & AA & Town in England. \\
\hline Crommelin & $5.3 \mathrm{~N}$ & $10.2 \mathrm{~W}$ & 111 & $\overline{E U}$ & GB & $\mathrm{MC} 11$ & I978 & 5 & 1973 & 68 & AA & $\begin{array}{l}\text { Andrew C.; British } \\
\text { astronomer (1865-1939). }\end{array}$ \\
\hline Cruls & $43.2 \mathrm{~S}$ & $196.9 \mathrm{~W}$ & 83 & SA & BR & MC29 & I1170 & 5 & 1973 & 68 & AA & $\begin{array}{l}\text { Luiz; Brazilian } \\
\text { astronomer (1848-1908). }\end{array}$ \\
\hline Cruz & $38.6 \mathrm{~N}$ & $1.7 \mathrm{~W}$ & 6 & SA & VE & CYDONI & I990 & 5 & 1976 & 36 & AA & Town in Venezuela. \\
\hline Cue & $36.1 \mathrm{~S}$ & $266.8 \mathrm{~W}$ & 0 & $\mathrm{OC}$ & $\mathrm{AU}$ & & I2036 & 5 & 1989 & 74 & AA & $\begin{array}{l}\text { Town in Western } \\
\text { Australia. }\end{array}$ \\
\hline Curie & $29.2 \mathrm{~N}$ & $4.9 \mathrm{~W}$ & 98 & EU & FR & MC11 & I1551 & 5 & 1973 & 68 & AA & $\begin{array}{l}\text { Pierre; French } \\
\text { physicist-chemist } \\
(1859-1906) .\end{array}$ \\
\hline Cypress & $47.6 \mathrm{~S}$ & $47.0 \mathrm{~W}$ & 11 & US & IL & NEREID & 1957 & 5 & 1976 & 36 & AA & Town in Illinois, USA. \\
\hline Da Vinci & $1.5 \mathrm{~N}$ & $39.1 \mathrm{~W}$ & 98 & EU & IT & $\mathrm{MC11}$ & 1978 & 5 & 1973 & 68 & AA & $\begin{array}{l}\text { Leonardo; Italian } \\
\text { artist, scientist } \\
\text { (1452-1519). }\end{array}$ \\
\hline $\begin{array}{l}\text { Daan } \\
\text { Daet }\end{array}$ & $\begin{array}{r}40.9 \mathrm{~S} \\
7.4 \mathrm{~S}\end{array}$ & $\begin{array}{r}267.6 \mathrm{~W} \\
41.9 \mathrm{~W}\end{array}$ & $\begin{array}{r}0 \\
12\end{array}$ & $\begin{array}{l}\text { AS } \\
\text { AS }\end{array}$ & $\begin{array}{l}\mathrm{CH} \\
\mathrm{PH}\end{array}$ & CAPRI & $\begin{array}{l}\text { I2043 } \\
\text { I1026 }\end{array}$ & 5 & $\begin{array}{l}1989 \\
1976\end{array}$ & $\begin{array}{l}74 \\
36\end{array}$ & AA & $\begin{array}{l}\text { Town in China. } \\
\text { Town in the Philinnines. }\end{array}$ \\
\hline Daly & $66.4 \mathrm{~S}$ & $23.0 \mathrm{~W}$ & 99 & NA & $\mathrm{CA}$ & MC30 & $\begin{array}{l}11020 \\
1970\end{array}$ & 5 & 1973 & 68 & $\mathrm{AA}$ & $\begin{array}{l}\text { Reginald A.; Canadian } \\
\text { geologist (1871-1975). }\end{array}$ \\
\hline Dana & $72.6 \mathrm{~S}$ & $33.1 \mathrm{~W}$ & 95 & NA & AM & MC30 & 1970 & 5 & 1973 & 68 & AA & $\begin{array}{l}\text { James D.; American } \\
\text { geologist (1813-1895). }\end{array}$ \\
\hline Dank & $22.2 \mathrm{~N}$ & $253.2 \mathrm{~W}$ & 7 & AS & OM & TRITON & I1055 & 5 & 1976 & 36 & AA & Town in Oman. \\
\hline Darvel & $17.9 \mathrm{~N}$ & $51.0 \mathrm{~W}$ & 0 & EU & SC & & & 5 & 1988 & 36 & AA & Town in Scotland. \\
\hline Darwin & $57.2 \mathrm{~S}$ & $19.2 \mathrm{~W}$ & 166 & EU & GB & MC26 & I985 & 5 & 1973 & 68 & AA & $\begin{array}{l}\text { Charles R.; British } \\
\text { naturalist (1809-1882); } \\
\text { same crater as G. H. } \\
\text { Darwin. }\end{array}$ \\
\hline Darwin & $57.2 \mathrm{~S}$ & $19.2 \mathrm{~W}$ & 166 & $\mathrm{EU}$ & GB & & & 5 & 1973 & 68 & AA & $\begin{array}{l}\text { George H.; British } \\
\text { astronomer (1845-1912). }\end{array}$ \\
\hline Dawes & $9.3 \mathrm{~S}$ & $322.3 \mathrm{~W}$ & 191 & EU & GB & MC20 & 11296 & 5 & 1973 & 68 & AA & $\begin{array}{l}\text { William R.; British } \\
\text { astronomer (1799-1868). }\end{array}$ \\
\hline Deba & $24.3 S$ & $17.1 \mathrm{~W}$ & 7 & $\mathrm{AF}$ & NI & ERYTHR & I947 & 5 & 1976 & 36 & AA & Town in Nigeria. \\
\hline Dein & $38.5 \mathrm{~N}$ & $2.4 \mathrm{~W}$ & 24 & $\mathrm{OC}$ & GU & CYDONI & I990 & 5 & 1976 & 36 & AA & Town in New Guinea. \\
\hline Dejnev & $25.6 \mathrm{~S}$ & $164.5 \mathrm{~W}$ & 156 & UR & RU & $1: 15 \mathrm{M}$ & I535 & 5 & 1985 & 58 & AA & $\begin{array}{l}\text { Semen Ivanovich; Russian } \\
\text { geographer, explorer, and } \\
\text { navigator (1605-1673). }\end{array}$ \\
\hline $\begin{array}{l}\text { Delta } \\
\text { Denning }\end{array}$ & $46.3 \mathrm{~S}$ & $39.0 \mathrm{~W}$ & 5 & US & LA & NEREID & 1957 & 5 & 1976 & 36 & AA & Town in Louisiana, USA. \\
\hline Denning & $17.5 \mathrm{~S}$ & $326.6 \mathrm{~W}$ & 165 & EU & GB & $\mathrm{MC20}$ & 11296 & 5 & 1973 & 68 & AA & $\begin{array}{l}\text { William F.; British } \\
\text { astronomer (1848-1931). }\end{array}$ \\
\hline $\begin{array}{l}\text { Dersu } \\
\text { Dese }\end{array}$ & $25.8 \mathrm{~N}$ & $51.9 \mathrm{~W}$ & 0 & UR & RU & & & 5 & 1988 & 36 & AA & Town in Russia. \\
\hline Dessau & $\begin{array}{l}4 J .05 \\
43.1 \mathrm{~S}\end{array}$ & $\begin{array}{l}30.3 \mathrm{~W} \\
53.0 \mathrm{~W}\end{array}$ & $\begin{array}{r}12 \\
9\end{array}$ & $\begin{array}{l}\text { AF } \\
\text { EU }\end{array}$ & $\begin{array}{l}\text { E1 } \\
\text { GE }\end{array}$ & $\begin{array}{l}\text { NEREID } \\
\text { NEREID }\end{array}$ & & $\begin{array}{l}5 \\
5\end{array}$ & $\begin{array}{l}1976 \\
1976\end{array}$ & $\begin{array}{l}36 \\
36\end{array}$ & AA & $\begin{array}{l}\text { Town in Ethiopa. } \\
\text { Town in Germany. }\end{array}$ \\
\hline Dia-Cau & $0.3 \mathrm{~S}$ & $42.8 \mathrm{~W}$ & 28 & AS & $\mathrm{VT}$ & CAPRI & $\mathrm{I} 1026$ & 5 & 1976 & 36 & AA & $\begin{array}{l}\text { Town in the Socialist } \\
\text { Republic of Vietnam. }\end{array}$ \\
\hline Dingo & $24.0 \mathrm{~S}$ & $17.3 \mathrm{~W}$ & 13 & $\mathrm{OC}$ & $\mathrm{AU}$ & ERYTHR & 1947 & 5 & 1976 & 36 & $\mathrm{AA}$ & Town in Australia. \\
\hline Dinorwic & $30.5 \mathrm{~S}$ & $101.4 \mathrm{~W}$ & 0 & NA & $\mathrm{CA}$ & & 12061 & 5 & 198 & 74 & AA & Town in Ontario, Canada. \\
\hline Dison & $25.4 \mathrm{~S}$ & $16.3 \mathrm{~W}$ & 20 & EU & BE & ERYTHR & I947 & 5 & 1976 & 36 & AA & Town in Belgium. \\
\hline Dixie & $19.9 \mathrm{~N}$ & $55.9 \mathrm{~W}$ & 0 & NA & AM & 20057 & & 5 & 1988 & 36 & AA & Town in Georgia, USA. \\
\hline Dokuchaev & $60.8 \mathrm{~S}$ & $127.1 \mathrm{~W}$ & 73 & UR & RU & $1: 15 \mathrm{M}$ & I1535 & 5 & 1982 & 58 & AA & $\begin{array}{l}\text { Vasily Vasil'evich; } \\
\text { Russian soil scientist; } \\
\text { founded modern genetical } \\
\text { soil science (1840-1903). }\end{array}$ \\
\hline Doon & $23.8 \mathrm{~N}$ & $250.8 \mathrm{~W}$ & 0 & NA & CA & & & 5 & 1988 & 36 & AA & Town in Ontario, Canada. \\
\hline & & & 97 & & AM & MC25 & 11165 & 5 & 1973 & 68 & AA & $\begin{array}{l}\text { Andrew E.; American } \\
\text { astronomer (1867-1962). }\end{array}$ \\
\hline Dromore & $20.2 \mathrm{~N}$ & $49.3 \mathrm{~W}$ & 14 & EU & IR & CHRY W & I1068 & 5 & 1976 & 36 & AA & Town in Northern Ireland. \\
\hline
\end{tabular}

lat: latitude of feature center.

long: longitude of feature center.

diam: diameter or long dimension of feature. et: ethnicity of name origin (see page $284 \mathrm{ff}$.)

quad: map quadrangle or informal name

(see page xvii ff.). as: name approval status (see page $x$ vii).

ad: name approval date (year).

ref: reference source for name (see page $287 \mathrm{ff}$.).

ct. continent of name origin (see page $284 \mathrm{ff}$.) map: map name or USGS map number (see page xvii ff.). ft: feature type (see page 290 ). 
MARS

\begin{tabular}{|c|c|c|c|c|c|c|c|c|c|c|c|c|}
\hline Name & lat & long & diam & ct & et & quad & map & as & ad & ref & ft & origin \\
\hline Du Martheray & $5.8 \mathrm{~N}$ & $266.4 \mathrm{~W}$ & 94 & EU & SZ & MC14 & I1023 & 5 & 1973 & 68 & AA & $\begin{array}{l}\text { Maurice; Swiss astronomer } \\
(1892-1955) \text {. }\end{array}$ \\
\hline Du Toit & $71.8 \mathrm{~S}$ & $49.7 \mathrm{~W}$ & 75 & AF & SA & MC30 & I970 & 5 & 1973 & 68 & AA & $\begin{array}{l}\text { Alexander L.; South } \\
\text { African geologist } \\
(1878-1948) \text {. }\end{array}$ \\
\hline Dubki & $37.0 \mathrm{~S}$ & $55.0 \mathrm{~W}$ & 0 & UR & RU & SOVIET & & 5 & 1979 & 58 & AA & Town in Russia. \\
\hline Dunhuang & $80.9 S$ & $48.7 \mathrm{~W}$ & 0 & AS & $\mathrm{CH}$ & -80050 & & 5 & 1991 & 36 & AA & Town in China. \\
\hline Dush & $22.6 \mathrm{~N}$ & $54.0 \mathrm{~W}$ & 0 & $\mathrm{AF}$ & EG & & & 5 & 1988 & 36 & AA & Town in Egypt. \\
\hline Dzeng & $80.6 \mathrm{~S}$ & $70.5 \mathrm{~W}$ & 0 & AF & CR & -80070 & & 5 & 1991 & 36 & AA & Town in Cameroon. \\
\hline Eads & $28.9 \mathrm{~S}$ & $29.8 \mathrm{~W}$ & 2 & US & $\mathrm{CO}$ & ERYTHR & 1947 & 5 & 1976 & 36 & AA & Town in Colorado, USA. \\
\hline Eagle & $44.0 \mathrm{~N}$ & $8.2 \mathrm{~W}$ & 12 & US & ID & CYDONI & 1946 & 5 & 1976 & 36 & AA & Town in Idaho, USA. \\
\hline Echt & $22.2 S$ & $28.0 \mathrm{~W}$ & 2 & EU & $\mathrm{SC}$ & ERYTHR & 1947 & 5 & 1976 & 36 & AA & Town in Scotland. \\
\hline Edam & $26.6 S$ & $19.9 \mathrm{~W}$ & 20 & $\mathrm{EU}$ & DU & ERYTHR & 1947 & 5 & 1976 & 36 & AA & Town in the Netherlands. \\
\hline Eddie & $12.5 \mathrm{~N}$ & $217.8 \mathrm{~W}$ & 90 & AF & SA & MC15 & I1 135 & 5 & 1973 & 68 & AA & $\begin{array}{l}\text { Lindsay A.; South African } \\
\text { astronomer (1845-1913). }\end{array}$ \\
\hline $\begin{array}{l}\text { Eger } \\
\text { Eil }\end{array}$ & $\begin{array}{l}48.7 \mathrm{~S} \\
42.0 \mathrm{~N}\end{array}$ & $\begin{array}{r}51.8 \mathrm{~W} \\
9.7 \mathrm{~W}\end{array}$ & $\begin{array}{r}12 \\
4\end{array}$ & $\begin{array}{l}\text { EU } \\
\mathrm{AF}\end{array}$ & $\begin{array}{l}\mathrm{HU} \\
\mathrm{SO}\end{array}$ & $\begin{array}{l}\text { NEREID } \\
\text { CYDONI }\end{array}$ & $\begin{array}{l}\text { I957 } \\
\text { I946 }\end{array}$ & $\begin{array}{l}5 \\
5\end{array}$ & $\begin{array}{l}1976 \\
1976\end{array}$ & $\begin{array}{l}36 \\
36\end{array}$ & $\begin{array}{l}\text { AA } \\
\text { AA }\end{array}$ & $\begin{array}{l}\text { Town in Hungary. } \\
\text { Town in Somalia. }\end{array}$ \\
\hline Ejriksson & $19.6 \mathrm{~S}$ & $173.7 \mathrm{~W}$ & 56 & $\mathrm{EU}$ & NS & MC16 & I1554 & 5 & 1967 & 68 & $\mathrm{AA}$ & $\begin{array}{l}\text { Leif; Norse explorer (c. } \\
1000 \text { ). }\end{array}$ \\
\hline Elath & $46.1 \mathrm{~N}$ & $13.7 \mathrm{~W}$ & 13 & AS & IS & CYDONI & I946 & 5 & 1976 & 36 & AA & Town in Israel. \\
\hline Ellsley & $36.5 \mathrm{~N}$ & $83.0 \mathrm{~W}$ & 0 & EU & EN & 35082 & & 5 & 1991 & 36 & AA & Town in England. \\
\hline Ely & $23.9 \mathrm{~S}$ & $27.1 \mathrm{~W}$ & 10 & US & NV & ERYTHR & I947 & 5 & 1976 & 36 & AA & Town in Nevada, USA. \\
\hline Escalante & $0.3 \mathrm{~N}$ & $244.8 \mathrm{~W}$ & 83 & NA & ME & $\mathrm{MC} 14$ & I1024 & 5 & 1973 & 68 & AA & $\begin{array}{l}\text { F.; Mexican astronomer } \\
\text { (c. 1930). }\end{array}$ \\
\hline Escorial & $77.0 \mathrm{~N}$ & $54.3 \mathrm{~W}$ & 0 & EU & SP & MC 1D & & 5 & 1991 & 36 & AA & Town in Spain. \\
\hline Esk & $45.4 \mathrm{~N}$ & 7.0W & 4 & $\mathrm{OC}$ & $\mathrm{AU}$ & CYDONI & 1946 & 5 & 1976 & 36 & AA & Town in Australia. \\
\hline Espino & $19.9 \mathrm{~S}$ & $254.7 \mathrm{~W}$ & 0 & SA & VE & & 12065 & 5 & 1989 & 74 & AA & Town in Venezuela. \\
\hline Eudoxus & $45.0 \mathrm{~S}$ & $147.2 \mathrm{~W}$ & 92 & EU & GR & MC24NE & I1 166 & 5 & 1973 & 68 & AA & $\begin{array}{l}\text { Greek astronomer (c. } \\
\text { 408-355 B.C.). }\end{array}$ \\
\hline Evpatoriya & $47.3 \mathrm{~N}$ & $225.5 \mathrm{~W}$ & 6 & UR & SO & CANBER & I1060 & 5 & 1979 & 64 & AA & $\begin{array}{l}\text { Soviet tracking site } \\
\text { (Soviet spelling used). }\end{array}$ \\
\hline Faith & $43.2 \mathrm{~N}$ & $11.9 \mathrm{~W}$ & 5 & US & ND & CYDONI & I946 & 5 & 1976 & 36 & AA & $\begin{array}{l}\text { Town in North Dakota, } \\
\text { USA. }\end{array}$ \\
\hline $\begin{array}{l}\text { Falun } \\
\text { Faqu }\end{array}$ & $24.2 \mathrm{~S}$ & $24.4 \mathrm{~W}$ & 10 & EU & SW & ERYTHR & 1947 & 5 & 1976 & 36 & AA & Town in Sweden. \\
\hline $\begin{array}{l}\text { Faqu } \\
\text { Fastov }\end{array}$ & $24.7 \mathrm{~S}$ & $253.6 \mathrm{~W}$ & $\mathbf{0}$ & AS & JO & & I2063 & 5 & 198 & 74 & AA & Town in Jordan. \\
\hline Fenagh & $\begin{array}{l}25.4 \mathrm{~S} \\
34.6 \mathrm{~N}\end{array}$ & $\begin{array}{r}20.2 \mathrm{~W} \\
215.7 \mathrm{~W}\end{array}$ & $\begin{array}{r}12 \\
0\end{array}$ & $\begin{array}{l}\text { UR } \\
\text { EU }\end{array}$ & $\begin{array}{l}\text { UK } \\
\text { IR }\end{array}$ & $\begin{array}{l}\text { ERYTHR } \\
35217\end{array}$ & 1947 & $\begin{array}{l}5 \\
5\end{array}$ & 1976 & $\begin{array}{l}36 \\
36\end{array}$ & AA & Town in Ukraine. \\
\hline Fesenkov & $21.9 \mathrm{~N}$ & $86.4 \mathrm{~W}$ & 86 & UR & RU & MC10 & I971 & 5 & 1991 & 68 & $\mathrm{AA}$ & $\begin{array}{l}\text { Vasilii G.; Russian } \\
\text { astronomer (1889-1972). }\end{array}$ \\
\hline Flammarion & $25.7 \mathrm{~N}$ & $311.7 \mathrm{~W}$ & 160 & $\mathrm{EU}$ & FR & MC13 & 1967 & 5 & 1973 & 68 & AA & $\begin{array}{l}\text { Camille; French } \\
\text { astronomer (1842-1925). }\end{array}$ \\
\hline & $25.8 \mathrm{~S}$ & $\begin{array}{r}19.4 \mathrm{~W} \\
340 \mathrm{~W}\end{array}$ & 3 & US & $\mathrm{AL}$ & ERYTHR & 1947 & 5 & 1976 & 36 & AA & Town in Alabama, USA. \\
\hline Flaugergues & $17.0 \mathrm{~S}$ & $340.9 \mathrm{~W}$ & 235 & EU & FR & MC20 & I1296 & 5 & 1973 & 68 & AA & $\begin{array}{l}\text { Honore; French astronomer } \\
(1755-1835) .\end{array}$ \\
\hline $\begin{array}{l}\text { Floq } \\
\text { Flora }\end{array}$ & $\begin{array}{l}15.2 \mathrm{~N} \\
45.0 \mathrm{~S}\end{array}$ & 253.0W & 0 & EU & AL & & & 5 & 1988 & 36 & AA & Town in Albania. \\
\hline $\begin{array}{l}\text { Flora } \\
\text { Focas }\end{array}$ & $\begin{array}{l}45.0 \mathrm{~S} \\
33.9 \mathrm{~N}\end{array}$ & $\begin{aligned} 51.2 \mathrm{~W} \\
347.2 \mathrm{~W}\end{aligned}$ & $\begin{array}{l}15 \\
82\end{array}$ & $\begin{array}{l}\text { US } \\
\text { EU }\end{array}$ & $\begin{array}{l}\text { MS } \\
\text { FR }\end{array}$ & $\begin{array}{l}\text { NEREID } \\
\text { MC05 }\end{array}$ & $\begin{array}{l}1957 \\
\text { I1495 }\end{array}$ & $\begin{array}{l}5 \\
5\end{array}$ & $\begin{array}{l}1976 \\
1973\end{array}$ & $\begin{array}{l}36 \\
68\end{array}$ & $\begin{array}{l}\text { AA } \\
\text { AA }\end{array}$ & Town in Mississippi, USA. \\
\hline Fontana & $63.2 \mathrm{~S}$ & $71.9 \mathrm{~W}$ & 78 & EU & IT & MC25 & 11165 & 5 & 1973 & 68 & AA & astronomer (1909-1969). \\
\hline Foros & $34.0 \mathrm{~S}$ & $28.0 \mathrm{~W}$ & & UR & & & 1100 & & . & & & astronomer (1585-1685). \\
\hline Fournier & $4.2 \mathrm{~S}$ & $287.5 \mathrm{~W}$ & 112 & $\mathrm{EU}$ & FR & MC21 & I1118 & $\begin{array}{l}5 \\
5\end{array}$ & $\begin{array}{l}19 / 9 \\
1973\end{array}$ & $\begin{array}{l}30 \\
68\end{array}$ & AA & $\begin{array}{l}\text { Town in Ukraine. } \\
\text { Georges; French } \\
\text { astronomer (1881-1954). }\end{array}$ \\
\hline Freedom & $43.6 \mathrm{~N}$ & $9.0 \mathrm{~W}$ & 12 & US & OK & CYDONI & 1946 & 5 & 1976 & 36 & AA & Town in Oklahoma, USA. \\
\hline Funchal & $23.2 \mathrm{~N}$ & $49.5 \mathrm{~W}$ & 2 & EU & SP & YORKTN & I1059 & 5 & 197 & 36 & AA & Port of Madeira Islands. \\
\hline Gaan & $38.5 \mathrm{~N}$ & $3.2 \mathrm{~W}$ & 3 & $\mathrm{AF}$ & So & CYDOHR & 1990 & 5 & 19 & 36 & AA & Town in Somalia. \\
\hline Gagra & $20.9 \mathrm{~S}$ & $21.9 \mathrm{~W}$ & 14 & UR & GE & ERYTHR & I947 & 5 & 1976 & 36 & AA & $\begin{array}{l}\text { Town in the Republic } \\
\text { of Georgia. }\end{array}$ \\
\hline Gah & $45.1 \mathrm{~S}$ & $32.3 \mathrm{~W}$ & 2 & AS & ID & NEREID & I957 & 5 & 1976 & 36 & AA & Town in Indonesia. \\
\hline Gale & $5.3 \mathrm{~S}$ & $222.3 \mathrm{~W}$ & 172 & $\mathrm{OC}$ & $\mathrm{AU}$ & $\mathrm{MC23}$ & I1001 & 5 & 1973 & 68 & AA & $\begin{array}{l}\text { Walter F.; Australian } \\
\text { astronomer (1865-1945). }\end{array}$ \\
\hline Gali & $44.1 \mathrm{~S}$ & $36.9 \mathrm{~W}$ & 24 & UR & GE & NEREID & I957 & 5 & 1976 & 36 & AA & $\begin{array}{l}\text { Town in the Republic of } \\
\text { Georgia. }\end{array}$ \\
\hline Galilaei & $5.8 \mathrm{~N}$ & $26.9 \mathrm{~W}$ & 124 & EU & IT & MC11 & 11551 & 5 & 1973 & 68 & $\mathrm{AA}$ & $\begin{array}{l}\text { Galileo; Italian } \\
\text { astronomer and physicist } \\
(1564-1642) \text {. }\end{array}$ \\
\hline Galle & $50.8 \mathrm{~S}$ & $30.7 \mathrm{~W}$ & 230 & EU & GE & MC26 & 1985 & 5 & 1973 & 68 & $\mathrm{AA}$ & $\begin{array}{l}\text { Johann G.; German } \\
\text { astronomer (1812-1910). }\end{array}$ \\
\hline $\begin{array}{l}\text { Galu } \\
\text { Gander }\end{array}$ & $\begin{array}{l}22.3 \mathrm{~S} \\
31.5 \mathrm{~S}\end{array}$ & $\begin{array}{r}21.5 \mathrm{~W} \\
265.7 \mathrm{~W}\end{array}$ & $\begin{array}{l}9 \\
0\end{array}$ & $\begin{array}{l}\mathrm{AF} \\
\mathrm{NA}\end{array}$ & $\begin{array}{l}\mathrm{ZA} \\
\mathrm{CA}\end{array}$ & ERYTHR & $\begin{array}{l}1947 \\
12039\end{array}$ & $\begin{array}{l}5 \\
5\end{array}$ & $\begin{array}{l}1976 \\
1989\end{array}$ & $\begin{array}{l}36 \\
74\end{array}$ & $\begin{array}{l}\text { AA } \\
\text { AA }\end{array}$ & $\begin{array}{l}\text { Town in Zaire. } \\
\text { Town in Newfoundland, } \\
\text { Canada. }\end{array}$ \\
\hline
\end{tabular}

lat: latitude of feature center.

long: longitude of feature center.

diam: diameter or long dimension of feature.

ct: continent of name origin (see page $284 \mathrm{ff}$ ) map: map name or USGS map number (see page xvii ff.). ft: feature type (see page 290 ).

et: $\quad$ ethnicity of name origin (see page $284 \mathrm{ff}$.)

quad: map quadrangle or informal name

(see page $x$ vii ff.). as: name approval status (see page xvii)

ad: name approval date (year).

ref: reference source for name (see page $287 \mathrm{ff}$.). 
MARS

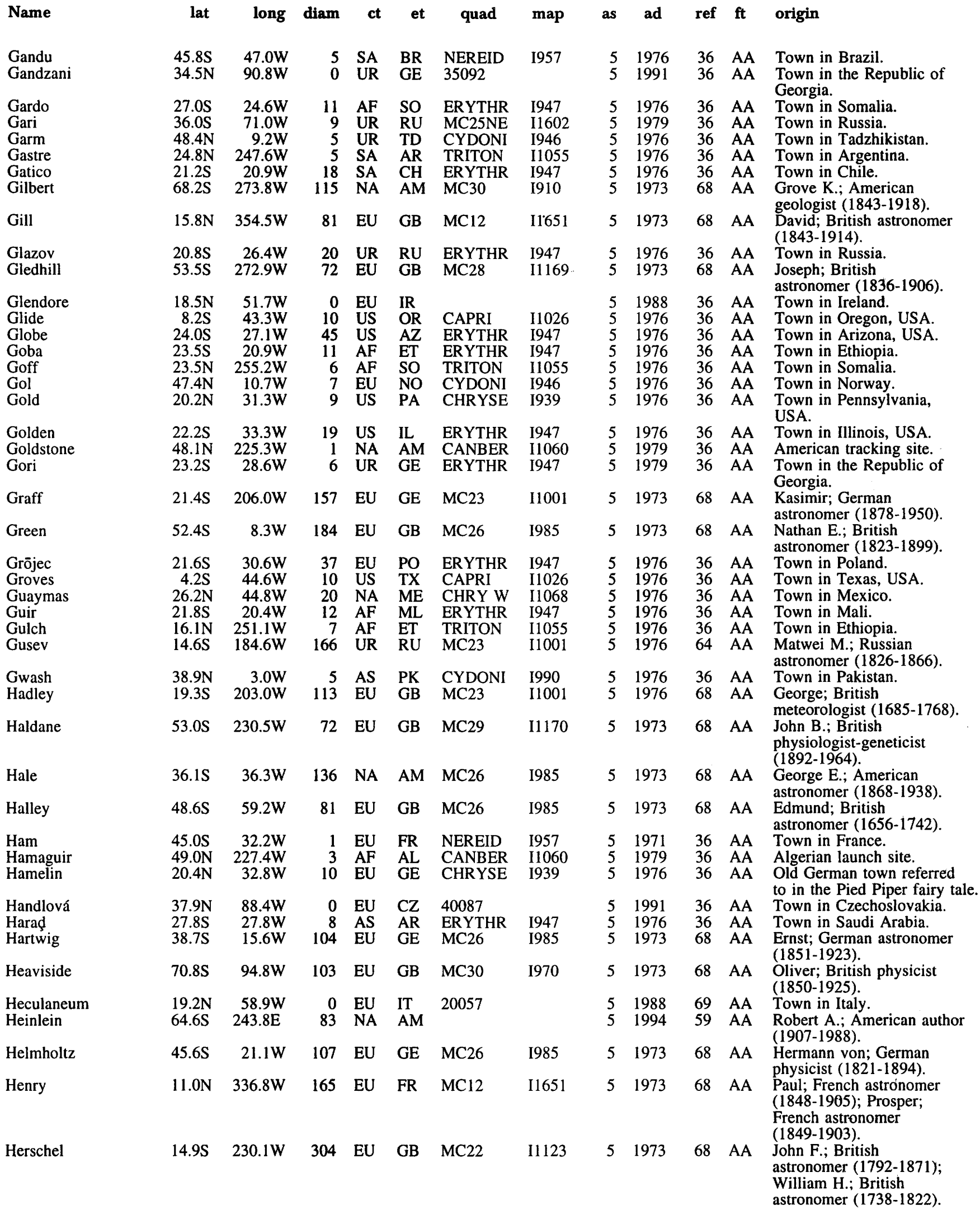

lat: latitude of feature center.

long: longitude of feature center.

diam: diameter or long dimension of feature.

et: ethnicity of name origin (see page $284 \mathrm{ff}$.)

quad: map quadrangle or informal name

(see page xvii ff.).

ct: continent of name origin (see page $284 \mathrm{ff}$.) map: map name or USGS map number (see page xvii ff.). ft: feature type (see page 290).

as: name approval status (see page xvii)

ad: name approval date (year).

ref: reference source for name (see page $287 \mathrm{ff}$.). 
MARS

\begin{tabular}{|c|c|c|c|c|c|c|c|c|c|c|c|c|}
\hline Name & lat & long & diam & ct & et & quad & map & as & ad & ref & $\mathbf{f t}$ & origin \\
\hline Hilo & $44.8 \mathrm{~S}$ & $35.5 \mathrm{~W}$ & 20 & US & HI & NEREID & 1957 & 5 & 1976 & 36 & AA & Town in Hawaii, USA. \\
\hline Hipparchus & $45.0 \mathrm{~S}$ & $151.1 \mathrm{~W}$ & 104 & $\mathrm{EU}$ & GR & $\mathrm{MC} 24$ & I1 166 & 5 & 1973 & 68 & $\mathrm{AA}$ & $\begin{array}{l}\text { Greek astronomer (c. } \\
160-125 \text { B.C.). }\end{array}$ \\
\hline & $47.3 \mathrm{~N}$ & $221.5 \mathrm{~W}$ & 7 & AS & IQ & UTOPIA & I1061 & 5 & 1979 & 36 & AA & Town in Iraq. \\
\hline Holden & $26.5 S$ & $33.9 \mathrm{~W}$ & 141 & NA & AM & MC 19 & 1975 & 5 & 1973 & 68 & AA & $\begin{array}{l}\text { Edward S.; American } \\
\text { astronomer (1846-1914). }\end{array}$ \\
\hline Holmes & $75.0 \mathrm{~S}$ & $293.9 \mathrm{~W}$ & 109 & EU & GB & MC30 & 1970 & 5 & 1973 & 68 & AA & $\begin{array}{l}\text { Arthur; British geologist } \\
(1890-1965) .\end{array}$ \\
\hline Honda & $22.7 \mathrm{~S}$ & $16.2 \mathrm{~W}$ & 6 & SA & $\mathrm{CO}$ & ERYTHR & 1947 & 5 & 1976 & 36 & AA & Town in Colombia. \\
\hline Hooke & $45.0 \mathrm{~S}$ & $44.4 \mathrm{~W}$ & 145 & EU & GB & MC26 & $\mathrm{I} 985$ & 5 & 1973 & 68 & AA & $\begin{array}{l}\text { Robert; British } \\
\text { physicist-astronomer } \\
\text { (1635-1703). }\end{array}$ \\
\hline Hope & $45.1 \mathrm{~N}$ & $10.3 \mathrm{~W}$ & 6 & NA & $\mathrm{CA}$ & CYDONI & 1946 & 5 & 1976 & 36 & AA & $\begin{array}{l}\text { Town in British Columbia, } \\
\text { Canada. }\end{array}$ \\
\hline Houston & $48.5 \mathrm{~N}$ & $223.9 \mathrm{~W}$ & 2 & NA & AM & CANBER & $\mathrm{I1} 060$ & 5 & 1979 & 36 & AA & $\begin{array}{l}\text { American mission control } \\
\text { site. }\end{array}$ \\
\hline Hsūanch'eng & $47.0 \mathrm{~N}$ & $227.2 \mathrm{~W}$ & 2 & AS & $\mathrm{CH}$ & CANBER & 11060 & 5 & 1979 & 36 & AA & Chinese launch site. \\
\hline Huancayo & $3.7 \mathrm{~S}$ & $39.8 \mathrm{~W}$ & 25 & SA & $\mathrm{PE}$ & CAPRI & I1026 & 5 & 1976 & 36 & AA & Town in Peru. \\
\hline Huggins & $49.3 \mathrm{~S}$ & $204.3 \mathrm{~W}$ & 82 & EU & GB & MC29SE & I1170 & 5 & 1973 & 68 & AA & $\begin{array}{l}\text { William; British } \\
\text { astronomer (1824-1910). }\end{array}$ \\
\hline Hussey & $53.8 \mathrm{~S}$ & $126.5 \mathrm{~W}$ & 100 & NA & $\mathrm{AM}$ & MC24 & I1166 & 5 & 1973 & 68 & AA & $\begin{array}{l}\text { William J.; American } \\
\text { astronomer (1862-1926). }\end{array}$ \\
\hline Hutton & $71.9 \mathrm{~S}$ & $255.5 \mathrm{~W}$ & 99 & EU & GB & MC30 & I970 & 5 & 1973 & 68 & AA & $\begin{array}{l}\text { James; British geologist } \\
(1726-1797) .\end{array}$ \\
\hline Huxley & $62.9 \mathrm{~S}$ & $259.2 \mathrm{~W}$ & 108 & EU & GB & MC28 & I1 169 & 5 & 1973 & 68 & AA & $\begin{array}{l}\text { Thomas H.; British } \\
\text { biologist (1825-1895). }\end{array}$ \\
\hline Huygens & $14.0 \mathrm{~S}$ & $304.4 \mathrm{~W}$ & 456 & EU & DU & MC21 & I1118 & 5 & 1973 & 68 & AA & $\begin{array}{l}\text { Christian; Dutch } \\
\text { physicist, astronomer } \\
(1629-1695) \text {. }\end{array}$ \\
\hline Ibragimov & $25.9 \mathrm{~S}$ & $59.5 \mathrm{~W}$ & 89 & UR & SO & $\mathrm{MC} 18$ & $\mathrm{I} 1535$ & 5 & 1982 & 64 & AA & $\begin{array}{l}\text { Nadir Baba Ogly; Soviet } \\
\text { astronomer (1932-1977). }\end{array}$ \\
\hline Igol & $20.4 S$ & $254.0 \mathrm{~W}$ & 0 & EU & HU & & I2065 & 5 & 1989 & 74 & AA & Town in Hungary. \\
\hline Ikej & $21.1 \mathrm{~N}$ & $247.6 \mathrm{~W}$ & 0 & UR & RU & & & 5 & 1988 & 36 & AA & Town in Russia. \\
\hline Imgr & $19.3 \mathrm{~N}$ & $249.0 \mathrm{~W}$ & 0 & UR & RU & & & 5 & 1988 & 36 & AA & Town in Russia. \\
\hline Innsbruck & $6.5 \mathrm{~S}$ & $40.0 \mathrm{~W}$ & 64 & $\mathrm{EU}$ & AS & CAPRI & I1026 & 5 & 197 & 36 & AA & Town in Austria. \\
\hline Ins & $24.8 \mathrm{~N}$ & $251.2 \mathrm{~W}$ & 0 & $\mathrm{EU}$ & $\mathrm{SZ}$ & & & 5 & 1988 & 36 & AA & Town in Switzerland. \\
\hline Inta & $24.6 \mathrm{~S}$ & $24.9 \mathrm{~W}$ & 14 & UR & RU & ERYTHR & I947 & 5 & 197 & 36 & AA & Town in Russia. \\
\hline Inuvik & $79.1 \mathrm{~N}$ & $39.7 \mathrm{~W}$ & 0 & NA & $\mathrm{CA}$ & 80030 & I1812 & 5 & 1988 & 36 & AA & $\begin{array}{l}\text { Town in Northwest } \\
\text { Territories, Canada. }\end{array}$ \\
\hline Irbit & $24.6 \mathrm{~S}$ & $24.7 \mathrm{~W}$ & 13 & UR & RU & ERYTHR & I947 & 5 & 1976 & 36 & AA & Town in Russia. \\
\hline Irharen & $34.8 \mathrm{~N}$ & $219.2 \mathrm{~W}$ & 0 & $\mathrm{AF}$ & $\mathrm{AL}$ & 35217 & & 5 & 195 & 36 & AA & Town in Algeria. \\
\hline & $27.1 \mathrm{~S}$ & $272.0 \mathrm{~W}$ & 0 & EU & SP & & I 2038 & 5 & 198 & 74 & AA & Town in Spain. \\
\hline Izendy & $29.4 \mathrm{~S}$ & $101.3 \mathrm{~W}$ & 0 & UR & RU & & I2061 & 5 & 198 & 74 & AA & Town in Russia. \\
\hline & $26.6 \mathrm{~S}$ & $28.6 \mathrm{~W}$ & 5 & US & NM & ERYTHR & I947 & 5 & 197 & 36 & $\mathrm{AA}$ & Town in New Mexico, USA \\
\hline Jama & $21.6 \mathrm{~N}$ & $53.1 \mathrm{~W}$ & 0 & $\mathrm{AF}$ & TN & & & 5 & 198 & 36 & $\mathrm{AA}$ & Town in Tunisia. \\
\hline Jampur & $38.8 \mathrm{~N}$ & $81.0 \mathrm{~W}$ & 0 & AS & PK & 40082 & & 5 & 199 & 36 & AA & Town in Pakistan. \\
\hline Janssen & $2.8 \mathrm{~N}$ & $322.4 \mathrm{~W}$ & 166 & $\mathrm{EU}$ & FR & $\mathrm{MC} 12$ & I1651 & 5 & 1973 & 68 & AA & $\begin{array}{l}\text { Pierre J.; French } \\
\text { astronomer (1824-1907). }\end{array}$ \\
\hline Jarry-Desloges & $9.5 \mathrm{~S}$ & $276.1 \mathrm{~W}$ & 97 & EU & FR & MC21 & 11118 & 5 & 1973 & 68 & AA & $\begin{array}{l}\text { Rene; French astronomer } \\
(1868-1951) \text {. }\end{array}$ \\
\hline Jeans & $69.9 \mathrm{~S}$ & $205.5 \mathrm{~W}$ & 71 & $\mathrm{EU}$ & GB & MC30 & $\mathrm{I} 970$ & 5 & 1973 & 68 & AA & $\begin{array}{l}\text { James H.; British } \\
\text { physicist, astronomer } \\
(1877-1946) .\end{array}$ \\
\hline Jeki & $23.9 \mathrm{~N}$ & $52.3 \mathrm{~W}$ & 0 & $\mathrm{AF}$ & ET & 25052 & I1869 & 5 & 1988 & 36 & AA & Town in Ethiopia. \\
\hline Jen & $40.1 \mathrm{~N}$ & $10.6 \mathrm{~W}$ & 8 & $\mathrm{AF}$ & NI & CYDONI & I946 & 5 & & 36 & AA & Town in Nigeria. \\
\hline Jezža & $48.8 \mathrm{~S}$ & $37.7 \mathrm{~W}$ & 6 & UR & RU & NEREID & 1957 & 5 & 197 & 3 & AA & Town in Russia. \\
\hline Jijiga & $25.2 \mathrm{~N}$ & $53.7 \mathrm{~W}$ & 14 & $\mathrm{AF}$ & ET & CHRY W & $\mathrm{I} 1068$ & 5 & 1976 & 36 & AA & Town in Ethiopia. \\
\hline Jodrell & $47.8 \mathrm{~N}$ & $227.6 \mathrm{~W}$ & 3 & $\mathrm{EU}$ & GB & CAMBER & $\mathrm{I} 1060$ & 5 & 1979 & 36 & AA & $\begin{array}{l}\text { United Kingdom tracking } \\
\text { site. }\end{array}$ \\
\hline Johannesburg & $48.2 \mathrm{~N}$ & $226.7 \mathrm{~W}$ & 1 & $\mathrm{AF}$ & SA & CANBER & $\mathrm{I} 1060$ & 5 & 1979 & 36 & AA & $\begin{array}{l}\text { Republic of South Africa } \\
\text { tracking site. }\end{array}$ \\
\hline Joly & $74.6 \mathrm{~S}$ & $42.5 \mathrm{~W}$ & 81 & EU & IR & MC30 & 1970 & 5 & 1973 & 68 & AA & $\begin{array}{l}\text { John; Irish geologist } \\
(1857-1933) \text {. }\end{array}$ \\
\hline Jones & $19.1 \mathrm{~S}$ & $19.8 \mathrm{~W}$ & 85 & EU & GB & MC19 & I1293 & 5 & 1973 & 68 & AA & $\begin{array}{l}\text { Harold S.; British } \\
\text { astronomer (1890-1960). }\end{array}$ \\
\hline Kachug & $18.4 \mathrm{~N}$ & $252.5 \mathrm{~W}$ & 0 & UR & RU & & & 5 & 1988 & 36 & AA & Town in Russia. \\
\hline Kagoshima & $47.6 \mathrm{~N}$ & $224.1 \mathrm{~W}$ & 1 & AS & JA & CANBER & I1060 & 5 & & 3 & AA & Japanese launch site. \\
\hline Kagul & $24.0 \mathrm{~S}$ & $18.9 \mathrm{~W}$ & 8 & UR & $\mathrm{MD}$ & ERYTHR & I947 & 5 & 1976 & 36 & AA & Town in Moldova. \\
\hline Kāid & $4.6 \mathrm{~S}$ & $44.8 \mathrm{~W}$ & 7 & AS & IQ & CAPRI & I1026 & 5 & 1976 & 36 & AA & Town in Iraq. \\
\hline Kaiser & $46.6 \mathrm{~S}$ & $340.9 \mathrm{~W}$ & 201 & $\mathrm{EU}$ & DU & MC27 & 11168 & 5 & 1973 & 68 & AA & $\begin{array}{l}\text { Frederick; Dutch } \\
\text { astronomer (1808-1872). }\end{array}$ \\
\hline
\end{tabular}

\footnotetext{
lat: latitude of feature center.

et: $\quad$ ethnicity of name origin (see page $284 \mathrm{ff}$.)

as: name approval status (see page xvii).

long: longitude of feature center.

quad: map quadrangle or informal name

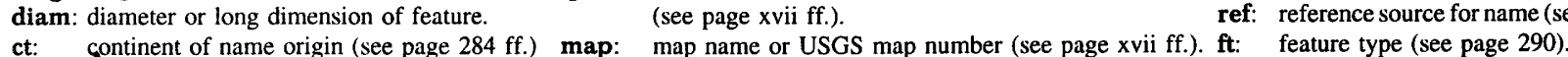

ad: name approval date (year).

ref: reference source for name (see page $287 \mathrm{ff}$.)

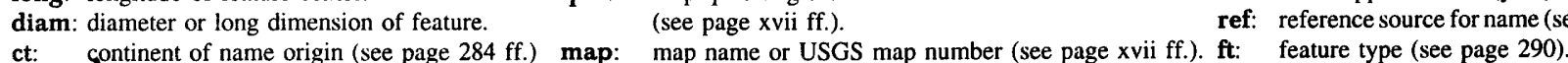

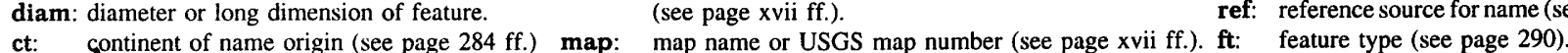


MARS

\begin{tabular}{|c|c|c|c|c|c|c|c|c|c|c|c|c|}
\hline Name & lat & long & diam & ct & et & quad & map & as & ad & ref & $\mathrm{ft}$ & origin \\
\hline Kaj & $27.4 S$ & $29.2 \mathrm{~W}$ & 2 & UR & RU & ERYTHR & 1947 & 5 & 1976 & 36 & AA & Town in Russia. \\
\hline Kakori & $41.9 \mathrm{~S}$ & $29.6 \mathrm{~W}$ & 25 & AS & IN & NEREID & 1957 & 5 & 1976 & 36 & AA & Town in India. \\
\hline Kaliningrad & $48.8 \mathrm{~N}$ & $224.9 W$ & 2 & UR & SO & CANBER & 11060 & 5 & 1979 & 36 & AA & $\begin{array}{l}\text { Soviet mission control } \\
\text { site. }\end{array}$ \\
\hline Kamativi & $20.7 \mathrm{~S}$ & $260.0 \mathrm{~W}$ & 0 & $\mathrm{AF}$ & $\mathrm{ZI}$ & -20257 & & 5 & 1991 & 36 & AA & Town in Zimbabwe. \\
\hline Kamloops & $53.9 S$ & $32.1 \mathrm{~W}$ & 0 & NA & $\mathrm{CA}$ & -55036 & $\mathrm{I} 2255$ & 5 & 1991 & 36 & AA & Town in Canada. \\
\hline Kampot & $42.1 \mathrm{~S}$ & $45.4 \mathrm{~W}$ & 9 & AS & $\mathrm{CM}$ & NEREID & 1957 & 5 & 1976 & 36 & AA & $\begin{array}{l}\text { Town in Democratic } \\
\text { Kampuchea (Cambodia). }\end{array}$ \\
\hline Kanab & $27.6 \mathrm{~S}$ & $18.8 \mathrm{~W}$ & 14 & US & UT & ERYTHR & I947 & 5 & 1976 & 36 & AA & Town in Utah, USA. \\
\hline Kansk & $20.8 \mathrm{~S}$ & $17.1 \mathrm{~W}$ & 34 & UR & RU & ERYTHR & I947 & 5 & 1976 & 36 & AA & Town in Russia. \\
\hline Kantang & $24.8 \mathrm{~S}$ & $17.5 \mathrm{~W}$ & 64 & AS & TH & ERYTHR & I947 & 5 & 1976 & 36 & AA & Town in Thailand. \\
\hline Karpinsk & $46.0 \mathrm{~S}$ & $31.8 \mathrm{~W}$ & 28 & UR & RU & NEREID & I957 & 5 & 1976 & 36 & AA & Town in Russia. \\
\hline Karshi & $23.6 \mathrm{~S}$ & $19.2 \mathrm{~W}$ & 22 & UR & $\mathrm{UZ}$ & ERYTHR & I947 & 5 & 1976 & 36 & $\mathrm{AA}$ & Town in Uzbekhistan. \\
\hline Kartabo & $41.2 \mathrm{~S}$ & $52.3 \mathrm{~W}$ & 17 & SA & GY & NEREID & 1957 & 5 & 1976 & 36 & AA & Town in Guyana. \\
\hline Kasabi & $28.0 \mathrm{~S}$ & $270.9 \mathrm{~W}$ & 0 & $\mathrm{AF}$ & $\mathrm{ZM}$ & & $\mathrm{I} 2038$ & 5 & 1989 & 74 & AA & Town in Zambia. \\
\hline Kashira & $27.5 S$ & $18.3 \mathrm{~W}$ & 68 & UR & RU & ERYTHR & $\mathrm{I} 947$ & 5 & 1976 & 36 & AA & Town in Russia. \\
\hline Kasimov & $25.0 \mathrm{~S}$ & $22.8 \mathrm{~W}$ & 92 & UR & RU & ERYTHR & I947 & 5 & 1976 & 36 & AA & Town in Russia. \\
\hline Kasra & $22.2 \mathrm{~N}$ & $256.4 \mathrm{~W}$ & 0 & $\mathrm{AF}$ & TN & & & 5 & 1988 & 36 & AA & Town in Tunisia. \\
\hline Kaup & $22.5 \mathrm{~N}$ & $33.6 \mathrm{~W}$ & 3 & OC & GU & CHRYHR & I991 & 5 & 1976 & 36 & AA & Town in New Guinea. \\
\hline Kaw & $16.7 \mathrm{~N}$ & $255.9 \mathrm{~W}$ & 10 & SA & FG & TRITON & 11055 & 5 & 1976 & 36 & $\mathrm{AA}$ & Town in French Guiana. \\
\hline Keeler & $60.7 S$ & $151.2 \mathrm{~W}$ & 92 & NA & $\mathrm{AM}$ & $\mathrm{MC} 24$ & 11166 & 5 & 1973 & 68 & AA & $\begin{array}{l}\text { James E.; American } \\
\text { astronomer }(1857-1900) \text {. }\end{array}$ \\
\hline Kem' & $45.3 \mathrm{~S}$ & $32.7 \mathrm{~W}$ & 2 & UR & RU & NEREID & I957 & 5 & 1976 & 36 & AA & Town in Russia. \\
\hline Kepler & $47.2 S$ & $218.7 \mathrm{~W}$ & 219 & $\mathrm{EU}$ & GE & MC29 & 11170 & 5 & 1973 & 68 & AA & $\begin{array}{l}\text { Johannes; German } \\
\text { astronomer }(1571-1630) \text {. }\end{array}$ \\
\hline Keul' & $46.3 \mathrm{~N}$ & $237.7 \mathrm{~W}$ & 6 & UR & RU & UTOPIA & I1061 & 5 & 1979 & 36 & AA & Town in Russia. \\
\hline Khanpur & $20.8 \mathrm{~N}$ & $257.5 \mathrm{~W}$ & 0 & AS & PK & & & 5 & 1988 & 36 & AA & Town in Pakistan. \\
\hline Kholm & $7.3 \mathrm{~S}$ & $42.1 \mathrm{~W}$ & 10 & UR & RU & CAPRI & I1026 & 5 & 1976 & 36 & AA & Town in Russia. \\
\hline Khurli & $21.2 \mathrm{~S}$ & $252.0 \mathrm{~W}$ & 0 & AS & PK & & I 2065 & 5 & 1989 & 74 & AA & Town in Pakistan. \\
\hline Kifr̄̄ & $46.0 \mathrm{~S}$ & $54.1 \mathrm{~W}$ & 12 & AS & IQ & NEREID & I957 & 5 & 1976 & 36 & AA & Town in Iraq. \\
\hline Kimry & $20.5 S$ & $16.2 \mathrm{~W}$ & 17 & UR & RU & ERYTHR & I947 & 5 & 1976 & 36 & AA & Town in Russia. \\
\hline Kin & $20.4 \mathrm{~N}$ & $33.4 \mathrm{~W}$ & 7 & AS & JA & CHRYSE & 1939 & 5 & 1976 & 36 & $\mathrm{AA}$ & Town in Japan. \\
\hline Kinda & $26.0 \mathrm{~S}$ & $105.0 \mathrm{~W}$ & 0 & $\mathrm{AF}$ & $\mathrm{ZA}$ & & I2060 & 5 & 1989 & 74 & AA & Town in Zaire. \\
\hline Kingston & $22.4 \mathrm{~N}$ & $47.0 \mathrm{~W}$ & 2 & SA & $\mathrm{JM}$ & YORKTN & 11059 & 5 & 1979 & 36 & AA & Jamaican port. \\
\hline Kinkora & $25.3 \mathrm{~S}$ & $247.0 \mathrm{~W}$ & 0 & NA & $\mathrm{CA}$ & & $\mathrm{I} 2064$ & 5 & 1989 & 74 & $\mathrm{AA}$ & Town in Prince Edward \\
\hline Kipini & $26.0 \mathrm{~N}$ & $31.5 \mathrm{~W}$ & 75 & $\mathrm{AF}$ & KY & CHRYHR & I991 & 5 & 1976 & 36 & AA & Town in Kenya. \\
\hline Kirs & $26.7 \mathrm{~S}$ & $19.3 \mathrm{~W}$ & 3 & UR & RU & ERYTHR & I947 & 5 & 1976 & 36 & $\mathrm{AA}$ & Town in Russia. \\
\hline Kirsanov & $22.4 \mathrm{~S}$ & $25.0 \mathrm{~W}$ & 15 & UR & RU & ERYTHR & 1947 & 5 & 1976 & 36 & AA & Town in Russia. \\
\hline Kisambo & $34.3 \mathrm{~N}$ & $89.0 \mathrm{~W}$ & 0 & $\mathrm{AF}$ & $\mathrm{ZA}$ & 35087 & I 2191 & 5 & 1991 & 36 & AA & Town in Zaire. \\
\hline Kita & $23.1 \mathrm{~S}$ & $17.0 \mathrm{~W}$ & 11 & $\mathrm{AF}$ & ML & ERYTHR & 1947 & 5 & 1976 & 36 & AA & Town in Mali. \\
\hline Knobel & $6.6 \mathrm{~S}$ & $226.9 \mathrm{~W}$ & 127 & $\mathrm{EU}$ & GB & $\mathrm{MC} 22$ & 11123 & 5 & 1973 & 68 & $\mathrm{AA}$ & $\begin{array}{l}\text { Edward B.; British } \\
\text { astronomer (1841-1930). }\end{array}$ \\
\hline Koga & $29.3 \mathrm{~S}$ & $103.6 \mathrm{~W}$ & 0 & $\mathrm{AF}$ & TA & & 12061 & 5 & 1989 & 74 & AA & Town in Tanzania. \\
\hline Kok & $15.7 \mathrm{~N}$ & $28.1 \mathrm{~W}$ & 6 & AS & MA & CHRYSE & 1939 & 5 & 1976 & 36 & AA & $\begin{array}{l}\text { Town in Malaysia } \\
\text { (Sarawak). }\end{array}$ \\
\hline Kong & $5.4 \mathrm{~S}$ & $38.7 \mathrm{~W}$ & 10 & $\mathrm{AF}$ & IC & CAPRI & I1026 & 5 & 1976 & 36 & AA & Town in Ivory Coast. \\
\hline Korolev & $72.9 \mathrm{~N}$ & $195.8 \mathrm{~W}$ & 84 & UR & RU & MC01 & I969 & 5 & 1973 & 68 & AA & $\begin{array}{l}\text { Sergey P.; Russian } \\
\text { engineer (1906-1966). }\end{array}$ \\
\hline Korph & $19.6 \mathrm{~N}$ & $254.7 \mathrm{~W}$ & 0 & UR & RU & & & 5 & 1988 & 36 & AA & Town in Russia. \\
\hline Kourou & $47.0 \mathrm{~N}$ & $227.1 \mathrm{~W}$ & 2 & $\mathrm{SA}$ & FG & CANBER & $\mathrm{I} 1060$ & 5 & 1979 & 36 & AA & $\begin{array}{l}\text { French Guianan launch } \\
\text { site. }\end{array}$ \\
\hline Koval'sky & $30.0 \mathrm{~S}$ & $141.4 \mathrm{~W}$ & 299 & UR & RU & MC24NC & I1555 & 5 & 1985 & 64 & AA & $\begin{array}{l}\text { M. A.; Russian astronomer } \\
\text { (1821-1884). }\end{array}$ \\
\hline Koy & $21.6 \mathrm{~N}$ & $50.4 \mathrm{~W}$ & 0 & UR & RU & & & 5 & 1988 & 36 & AA & Town in Russia. \\
\hline Krasnoye & $36.1 \mathrm{~N}$ & $216.2 \mathrm{~W}$ & 0 & UR & RU & 35217 & 11750 & 5 & 1991 & 36 & AA & Town in Russia. \\
\hline Kribi & $43.4 \mathrm{~S}$ & $43.4 \mathrm{~W}$ & 8 & AF & $\mathrm{CR}$ & NEREID & 1957 & 5 & 1976 & 36 & AA & $\begin{array}{l}\text { Town in the United } \\
\text { Republic of Cameroon. }\end{array}$ \\
\hline Krishtofovich & $48.6 \mathrm{~S}$ & $262.6 \mathrm{~W}$ & 111 & UR & SO & MC28 & I1 169 & 5 & 1982 & 64 & AA & $\begin{array}{l}\text { Afrikan Nikolaevich; } \\
\text { Soviet paleobotanist } \\
\text { (1885-1953). }\end{array}$ \\
\hline Kuba & $25.6 \mathrm{~S}$ & $19.5 \mathrm{~W}$ & 25 & UR & $\mathrm{AZ}$ & ERYTHR & I947 & 5 & 1976 & 36 & AA & Town in Azerbaijan. \\
\hline Kufra & $40.6 \mathrm{~N}$ & $239.7 \mathrm{~W}$ & 32 & AS & LB & UTOPIA & I1061 & 5 & 1979 & 36 & AA & Town in Libya. \\
\hline Kuiper & $57.3 \mathrm{~S}$ & $157.1 \mathrm{~W}$ & 86 & NA & $\mathrm{AM}$ & MC24 & I1 166 & 5 & 1976 & 68 & AA & $\begin{array}{l}\text { Gerard P.; American } \\
\text { astronomer (1905-1973). }\end{array}$ \\
\hline Kular & $16.6 \mathrm{~N}$ & $252.0 \mathrm{~W}$ & 0 & UR & RU & & & 5 & 1988 & 36 & AA & Town in Russia. \\
\hline Kumak & $36.0 \mathrm{~S}$ & $67.5 \mathrm{~W}$ & 15 & UR & RU & SOVIET & & 5 & 1979 & 36 & AA & Town in Russia. \\
\hline Kumara & $43.3 \mathrm{~N}$ & $231.4 \mathrm{~W}$ & 12 & $\mathrm{OC}$ & $\mathrm{NZ}$ & UTOPIA & I1061 & 5 & 1979 & 36 & AA & Town in New Zealand. \\
\hline Kunes & $25.5 \mathrm{~S}$ & $252.0 \mathrm{~W}$ & 0 & $\mathrm{EU}$ & NO & & I2063 & 5 & 1989 & 74 & AA & Town in Norway. \\
\hline Kunowsky & $57.0 \mathrm{~N}$ & $9.0 \mathrm{~W}$ & 60 & EU & GE & MC04NE & I1348 & 5 & 1973 & 68 & AA & $\begin{array}{l}\text { George K.; German } \\
\text { astronomer (1786-1846). }\end{array}$ \\
\hline
\end{tabular}

lat: latitude of feature center.

long: longitude of feature center.

diam: diameter or long dimension of feature. et: ethnicity of name origin (see page $284 \mathrm{ff}$.)

quad: map quadrangle or informal name

(see page xvii ff.). as: name approval status (see page xvii).

ad: name approval date (year).

ref: reference source for name (see page $287 \mathrm{ff}$.).

ct. continent of name origin (see page $284 \mathrm{ff}$.) map: map name or USGS map number (see page xvii ff.). ft: feature type (see page 290 ). 
MARS

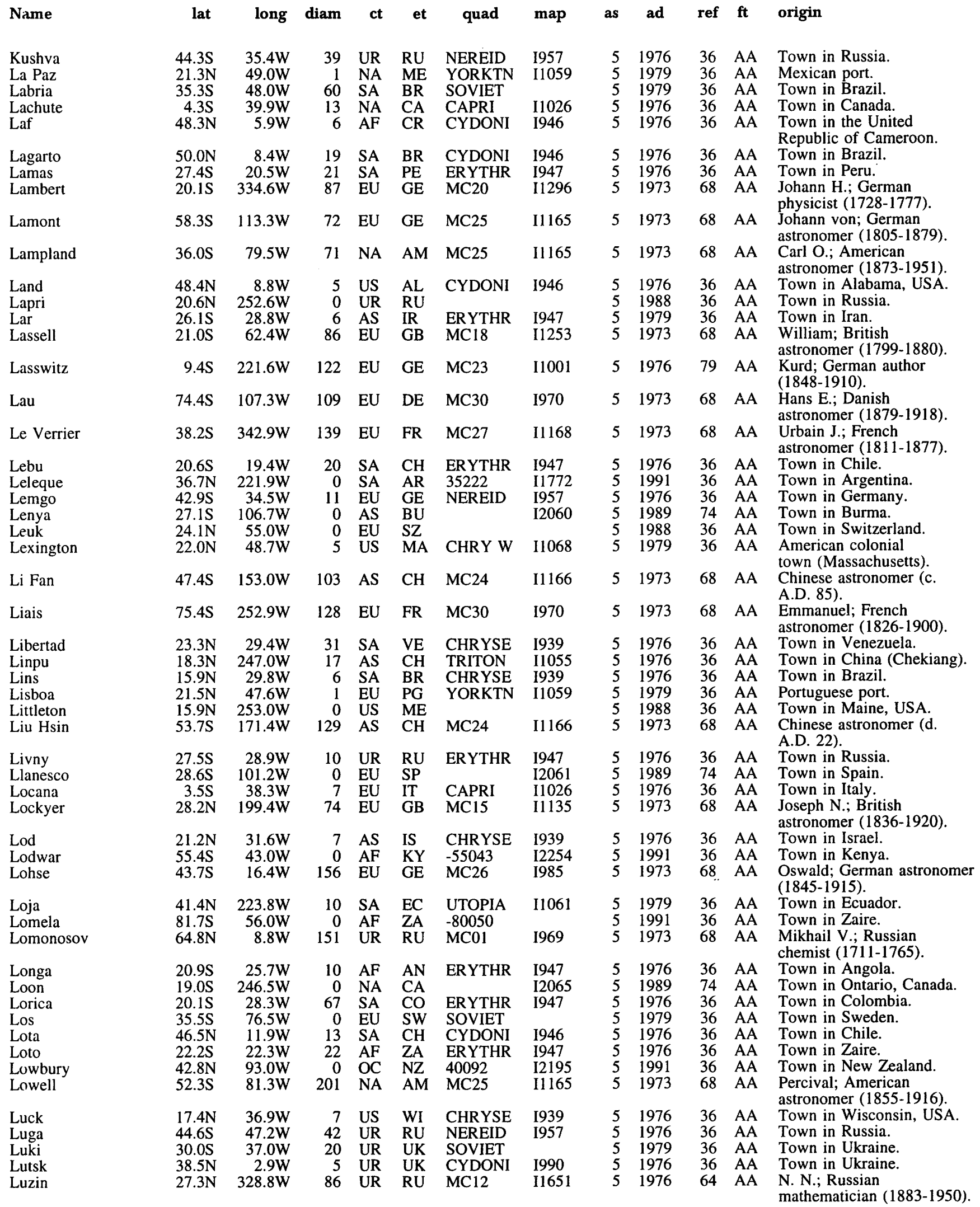

lat: latitude of feature center.

long: longitude of feature center.

diam: diameter or long dimension of feature. et: ethnicity of name origin (see page $284 \mathrm{ff}$.)

quad: map quadrangle or informal name

(see page xvii ff.) as: name approval status (see page xvii)

ad: name approval date (year).

ref: reference source for name (see page $287 \mathrm{ff}$.).

ct: continent of name origin (see page $284 \mathrm{ff}$.) map: map name or USGS map number (see page xvii ff.). ft: feature type (see page 290). 
MARS

\begin{tabular}{|c|c|c|c|c|c|c|c|c|c|c|c|c|}
\hline Name & lat & long & diam & ct & et & quad & map & as & ad & ref & $\mathrm{ft}$ & origin \\
\hline Lyell & $70.0 \mathrm{~S}$ & $15.6 \mathrm{~W}$ & 134 & EU & GB & MC30 & 1970 & 5 & 1973 & 68 & AA & Charles; British \\
\hline Lyot & $50.7 \mathrm{~N}$ & $330.7 \mathrm{~W}$ & 220 & EU & FR & MC05 & 11495 & 5 & 1973 & 68 & AA & Bernard; French \\
\hline Mädler & $10.8 \mathrm{~S}$ & $357.3 \mathrm{~W}$ & 100 & EU & GE & MC20 & 11296 & 5 & 1973 & 68 & AA & $\begin{array}{l}\text { Johann H. von; German } \\
\text { astronomer (1794-1874). }\end{array}$ \\
\hline Madrid & $48.7 \mathrm{~N}$ & $224.4 \mathrm{~W}$ & 3 & $\mathrm{EU}$ & SP & CANBER & I1060 & 5 & 1979 & 36 & AA & Spanish tracking site. \\
\hline Mafra & $44.4 \mathrm{~S}$ & $53.0 \mathrm{~W}$ & 12 & SA & BR & NEREID & 1957 & 5 & 1976 & 36 & AA & Town in Brazil. \\
\hline Magadi & $34.8 \mathrm{~S}$ & $46.0 \mathrm{~W}$ & 57 & AF & KY & SOVIET & I1 164 & 5 & 1979 & 36 & AA & Town in Kenya. \\
\hline Magelhaens & $32.9 \mathrm{~S}$ & $174.5 \mathrm{~W}$ & 102 & EU & PG & $\mathrm{MC} 24$ & I1166 & 5 & 1976 & 68 & AA & $\begin{array}{l}\text { Fernao de; Portuguese } \\
\text { navigator }(1480-1521)\end{array}$ \\
\hline Maggini & $28.0 \mathrm{~N}$ & $350.4 \mathrm{~W}$ & 146 & EU & IT & $\mathrm{MC} 12$ & I1651 & 5 & 1973 & 68 & AA & $\begin{array}{l}\text { Mentore; Italian } \\
\text { astronomer (1890-1941). }\end{array}$ \\
\hline $\begin{array}{l}\text { Mago } \\
\text { Maidstone }\end{array}$ & $\begin{array}{l}16.2 \mathrm{~N} \\
41.9 \mathrm{~S}\end{array}$ & $\begin{array}{r}254.8 \mathrm{~W} \\
54.1 \mathrm{~W}\end{array}$ & $\begin{array}{l}0 \\
9\end{array}$ & $\begin{array}{l}\text { UR } \\
\text { EU }\end{array}$ & $\begin{array}{l}\text { RU } \\
\text { GB }\end{array}$ & NEREID & I957 & $\begin{array}{l}5 \\
5\end{array}$ & $\begin{array}{l}1988 \\
1976\end{array}$ & $\begin{array}{l}36 \\
36\end{array}$ & $\begin{array}{l}\text { AA } \\
\text { AA }\end{array}$ & $\begin{array}{l}\text { Town in Russia. } \\
\text { Town in England. }\end{array}$ \\
\hline Main & $76.8 \mathrm{~S}$ & $310.9 \mathrm{~W}$ & 102 & $\mathrm{EU}$ & GB & $\mathrm{MC} 30$ & 1970 & 5 & 1973 & 68 & $\mathrm{AA}$ & $\begin{array}{l}\text { Robert; British } \\
\text { astronomer (1808-1878). }\end{array}$ \\
\hline $\begin{array}{l}\text { Manah } \\
\text { Mandora }\end{array}$ & $\begin{array}{r}4.7 \mathrm{~S} \\
12.4 \mathrm{~N}\end{array}$ & $\begin{array}{l}33.8 \mathrm{~W} \\
53.6 \mathrm{~W}\end{array}$ & $\begin{array}{l}9 \\
0\end{array}$ & $\begin{array}{l}\text { AS } \\
\text { OC }\end{array}$ & $\begin{array}{l}\mathrm{OM} \\
\mathrm{AU}\end{array}$ & CAPRI & I1026 & $\begin{array}{l}5 \\
5\end{array}$ & $\begin{array}{l}1976 \\
1988\end{array}$ & $\begin{array}{l}36 \\
36\end{array}$ & $\begin{array}{l}\text { AA } \\
\text { AA }\end{array}$ & $\begin{array}{l}\text { Town in Oman. } \\
\text { Town in Australia. }\end{array}$ \\
\hline Manti & $3.7 \mathrm{~S}$ & $37.7 \mathrm{~W}$ & 0 & US & UT & CAPRI & I1026 & 5 & 1976 & 36 & $\mathrm{AA}$ & Town in Utah, USA. \\
\hline Manzī & $22.3 \mathrm{~S}$ & $27.3 \mathrm{~W}$ & 7 & AS & BU & ERYTHR & 1947 & 5 & 197 & 36 & AA & Town in Burma. \\
\hline Maraldi & $62.2 \mathrm{~S}$ & $32.1 \mathrm{~W}$ & 119 & EU & FR & MC26 & 1970 & 5 & 1973 & 68 & AA & $\begin{array}{l}\text { Giacomo F.; French } \\
\text { astronomer }(1665-1729) .\end{array}$ \\
\hline Maraldi & $62.2 S$ & $32.1 \mathrm{~W}$ & 119 & EU & FR & MC26 & 1970 & 5 & 1973 & 68 & AA & $\begin{array}{l}\text { Giacomo F.; French } \\
\text { astronomer (1665-1729). }\end{array}$ \\
\hline $\begin{array}{l}\text { Marbach } \\
\text { Marca }\end{array}$ & $\begin{array}{l}17.9 \mathrm{~N} \\
10.4 \mathrm{~S}\end{array}$ & $\begin{array}{l}249.2 \mathrm{~W} \\
158.2 \mathrm{~W}\end{array}$ & $\begin{array}{l}20 \\
83\end{array}$ & EU & SZ & $\begin{array}{l}\text { TRITON } \\
-10157\end{array}$ & $\begin{array}{l}11055 \\
11653\end{array}$ & 5 & 1976 & 36 & AA & Town in Switzerland. \\
\hline Mari & $52.4 S$ & $45.7 \mathrm{~W}$ & 0 & $\begin{array}{l}\text { SA } \\
\text { AS }\end{array}$ & $\begin{array}{l}\mathrm{PE} \\
\mathrm{SY}\end{array}$ & -55043 & $\begin{array}{l}11053 \\
12254\end{array}$ & $\begin{array}{l}5 \\
5\end{array}$ & $\begin{array}{l}1985 \\
1991\end{array}$ & $\begin{array}{l}0 \\
0\end{array}$ & $\begin{array}{l}\text { AA } \\
\text { AA }\end{array}$ & $\begin{array}{l}\text { Village in Peru. } \\
\text { Ruined city in Syria. }\end{array}$ \\
\hline Mariner & $35.2 \mathrm{~S}$ & $164.3 \mathrm{~W}$ & 151 & NA & $\mathrm{AM}$ & MC24 & I1166 & 5 & 1967 & 68 & $\mathrm{AA}$ & $\begin{array}{l}\text { Named for Mariner IV } \\
\text { spacecraft. }\end{array}$ \\
\hline Marth & $13.1 \mathrm{~N}$ & $3.6 \mathrm{~W}$ & 104 & EU & GB & MC11SE & $\mathrm{I} 1342$ & 5 & 1973 & 68 & AA & $\begin{array}{l}\text { Albert; British } \\
\text { astronomer (1828-1897). }\end{array}$ \\
\hline Martz & $35.2 \mathrm{~S}$ & $215.8 \mathrm{~W}$ & 91 & NA & $\mathrm{AM}$ & MC29 & 11170 & 5 & 1973 & 68 & AA & $\begin{array}{l}\text { Edwin P.; American } \\
\text { physicist (1916-1967). }\end{array}$ \\
\hline Maunder & $50.0 \mathrm{~S}$ & $358.1 \mathrm{~W}$ & 93 & EU & GB & MC27 & 11168 & 5 & 1973 & 68 & AA & $\begin{array}{l}\text { Edward W.; British } \\
\text { astronomer (1851-1928). }\end{array}$ \\
\hline McLaughlin & $22.1 \mathrm{~N}$ & $22.5 \mathrm{~W}$ & 90 & NA & AM & MC11 & I1551 & 5 & 1973 & 68 & AA & $\begin{array}{l}\text { Dean B.; American } \\
\text { astronomer (1901-1965). }\end{array}$ \\
\hline $\begin{array}{l}\text { Medrissa } \\
\text { Mega }\end{array}$ & $\begin{array}{l}18.8 \mathrm{~N} \\
1.5 \mathrm{~S}\end{array}$ & $\begin{array}{l}56.6 \mathrm{~W} \\
37.0 \mathrm{~W}\end{array}$ & $\begin{array}{r}0 \\
13\end{array}$ & $\begin{array}{l}\mathrm{AF} \\
\mathrm{AF}\end{array}$ & $\begin{array}{l}\text { AL } \\
\text { ET }\end{array}$ & $\begin{array}{l}20057 \\
\text { CAPRI }\end{array}$ & I1026 & $\begin{array}{l}5 \\
5\end{array}$ & $\begin{array}{l}1988 \\
1976\end{array}$ & $\begin{array}{l}36 \\
36\end{array}$ & $\begin{array}{l}\text { AA } \\
\text { AA }\end{array}$ & $\begin{array}{l}\text { Town in Algeria. } \\
\text { Town in Ethiopia. }\end{array}$ \\
\hline Meget & $19.1 \mathrm{~N}$ & $252.9 \mathrm{~W}$ & 0 & UR & RU & & & 5 & 1988 & 36 & AA & Town in Russia. \\
\hline Mellish & $72.9 \mathrm{~S}$ & $24.0 \mathrm{E}$ & 99 & NA & $\mathrm{AM}$ & & & 5 & 1994 & & AA & $\begin{array}{l}\text { John E.; American amateur } \\
\text { astronomer (1886-1970). }\end{array}$ \\
\hline $\begin{array}{l}\text { Mena } \\
\text { Mendel }\end{array}$ & $\begin{array}{l}32.5 S \\
59.0 S\end{array}$ & $\begin{array}{r}18.5 \mathrm{~W} \\
198.5 \mathrm{~W}\end{array}$ & $\begin{array}{l}31 \\
82\end{array}$ & $\begin{array}{l}\text { UR } \\
\text { EU }\end{array}$ & $\begin{array}{l}\text { RU } \\
\text { AS }\end{array}$ & $\begin{array}{l}\text { SOVIET } \\
\text { MC11 }\end{array}$ & $\begin{array}{l}I 1264 \\
I 1170\end{array}$ & $\begin{array}{l}5 \\
5\end{array}$ & $\begin{array}{l}1979 \\
1973\end{array}$ & $\begin{array}{l}36 \\
68\end{array}$ & $\begin{array}{l}\text { AA } \\
\text { AA }\end{array}$ & $\begin{array}{l}\text { Town in Russia. } \\
\text { Gregor J.; Austrian } \\
\text { biologist (1822-1884). }\end{array}$ \\
\hline Mendota & $36.1 \mathrm{~N}$ & $221.8 \mathrm{~W}$ & 0 & NA & $\mathrm{AM}$ & 35222 & & 5 & 1991 & 36 & AA & Town in Illinois, USA. \\
\hline Mie & $48.6 \mathrm{~N}$ & $220.4 \mathrm{~W}$ & 93 & $\mathrm{EU}$ & GE & $\mathrm{MC07}$ & I1475 & 5 & 1973 & 68 & AA & $\begin{array}{l}\text { Gustav; German physicist } \\
(1868-1957) \text {. }\end{array}$ \\
\hline $\begin{array}{l}\text { Mila } \\
\text { Milankovið }\end{array}$ & $27.5 \mathrm{~S}$ & $20.6 \mathrm{~W}$ & 10 & AF & AL & ERYTHR & I947 & 5 & 1976 & 36 & AA & Town in Algeria. \\
\hline Milankovic & & $146.6 \mathrm{~W}$ & 113 & EU & YU & $\mathrm{MCO} 2$ & 11392 & 5 & 1973 & 68 & AA & $\begin{array}{l}\text { Milutin; Yugoslav } \\
\text { geophysicist, astrophysicist } \\
(1879-1958) \text {. }\end{array}$ \\
\hline $\begin{array}{l}\text { Milford } \\
\text { Millman }\end{array}$ & $\begin{array}{l}52.6 \mathrm{~S} \\
54.4 \mathrm{~S}\end{array}$ & $\begin{array}{l}45.1 \mathrm{~W} \\
1496 \mathrm{E}\end{array}$ & $\begin{array}{r}0 \\
82\end{array}$ & NA & $\begin{array}{l}\mathrm{AM} \\
\mathrm{CA}\end{array}$ & -55043 & I2254 & 5 & 1991 & 36 & AA & Town in Utah, USA. \\
\hline & & & & & & & & 3 & & & & (1906-1990). \\
\hline Millocnau & $21.5 \mathrm{~s}$ & $274.7 \mathrm{~W}$ & 102 & EU & FR & $\mathrm{MC} 21$ & 11118 & 5 & 1973 & 68 & AA & $\begin{array}{l}\text { Gaston; French astronomer } \\
\text { (b. 1866). }\end{array}$ \\
\hline $\begin{array}{l}\text { Mirtos } \\
\text { Misk }\end{array}$ & $22.3 \mathrm{~N}$ & $51.7 \mathrm{~W}$ & 0 & EU & GR & & & 5 & 1988 & 36 & AA & Town in Greece (Crete). \\
\hline Mistretta & $\begin{array}{r}0.9 \mathrm{~S} \\
25.0 \mathrm{~S}\end{array}$ & $135.5 \mathrm{~W}$ & $\begin{array}{r}10 \\
0\end{array}$ & AS & TU & CAPRI & 11026 & $\begin{array}{l}5 \\
5\end{array}$ & & 36 & & \\
\hline Mitchel & $67.8 \mathrm{~S}$ & $284.0 \mathrm{~W}$ & 141 & NA & AM & MC30 & I970 & 5 & 1973 & 68 & $\mathrm{AA}$ & $\begin{array}{l}\text { Ormsby M.; American } \\
\text { astronomer (1809-1862). }\end{array}$ \\
\hline $\begin{array}{l}\text { Mliba } \\
\text { Mohawk }\end{array}$ & $\begin{array}{l}39.9 \mathrm{~S} \\
43.2 \mathrm{~N}\end{array}$ & $\begin{array}{r}272.1 \mathrm{~W} \\
54 \mathrm{~W}\end{array}$ & & AF & SW & & I2038 & 5 & 1989 & 74 & AA & Town in Swaziland. \\
\hline $\begin{array}{l}\text { Mohawk } \\
\text { Molesworth }\end{array}$ & $\begin{array}{l}43.2 \mathrm{~N} \\
27.8 \mathrm{~S}\end{array}$ & $\begin{array}{r}5.4 \mathrm{~W} \\
210.6 \mathrm{~W}\end{array}$ & $\begin{array}{r}11 \\
175\end{array}$ & EU & $\begin{array}{l}\text { NY } \\
\text { GB }\end{array}$ & $\begin{array}{l}\text { CYDONI } \\
\text { MC23 }\end{array}$ & $\begin{array}{l}1946 \\
\mathrm{I} 1001\end{array}$ & 5 & & 36 & & Town in New York, USA. \\
\hline & & & & & & $1 \mathrm{~V} C 2 \mathrm{~J}$ & 11001 & 3 & & & AA & astronomer (1867-1908). \\
\hline $\begin{array}{l}\text { Montevallo } \\
\text { Moreux }\end{array}$ & $\begin{array}{l}15.3 \mathrm{~N} \\
42.2 \mathrm{~N}\end{array}$ & $\begin{array}{r}54.2 \mathrm{~W} \\
315.5 \mathrm{~W}\end{array}$ & $\begin{array}{r}0 \\
138\end{array}$ & $\begin{array}{l}\text { US } \\
\text { EU }\end{array}$ & $\begin{array}{l}\text { AL } \\
\text { FR }\end{array}$ & MC05 & I1495 & $\begin{array}{l}5 \\
5\end{array}$ & $\begin{array}{l}1988 \\
1973\end{array}$ & $\begin{array}{l}36 \\
68\end{array}$ & AA & Town in Alabama, USA. \\
\hline & & & & & & & & & & & & $\begin{array}{l}\text { astronomer and } \\
\text { meteorologist (1867-1954). }\end{array}$ \\
\hline
\end{tabular}

lat: latitude of feature center.

long: longitude of feature center

diam: diameter or long dimension of feature. et: ethnicity of name origin (see page $284 \mathrm{ff}$.)

quad: map quadrangle or informal name

(see page xvii ff.). as: name approval status (see page xvii).

ad: name approval date (year).

ref: reference source for name (see page $287 \mathrm{ff}$.).

ct. continent of name origin (see page $284 \mathrm{ff}$.) map: map name or USGS map number (see page $x$ vii ff.). $\mathrm{ft}$ : feature type (see page 290 ). 
MARS

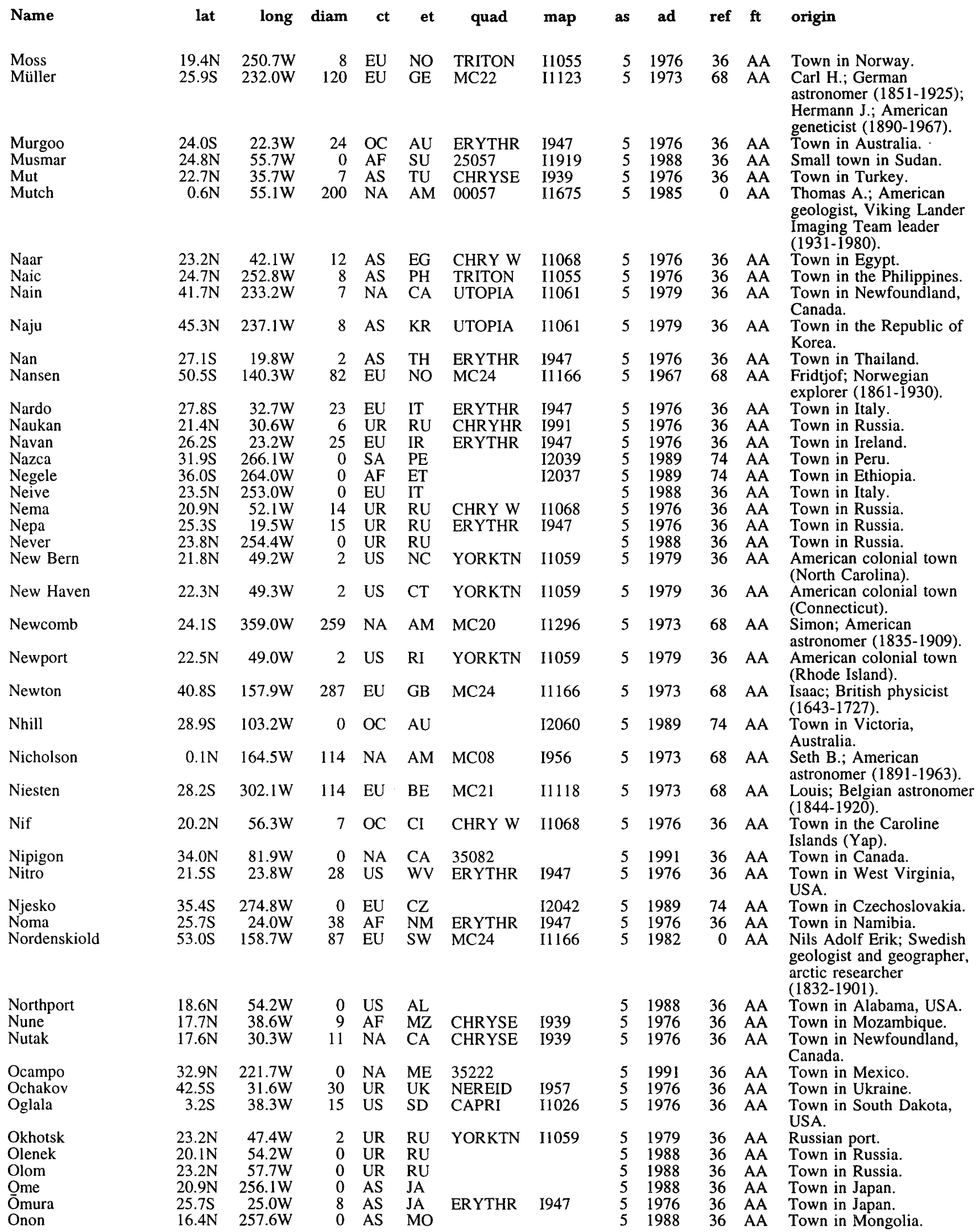

\footnotetext{
lat: latitude of feature center. et: ethnicity of name origin (see page $284 \mathrm{ff}$.) long: longitude of feature center.

quad: map quadrangle or informal name (see page xvii ff.).

as: name approval status (see page xvii). ad: name approval date (year). ref: reference source for name (see page $287 \mathrm{ff}$.) diam: diameter or long dimension of feature. map name or USGS map number (see page xvii ff.). ft: feature type (see page 290).
} 
MARS

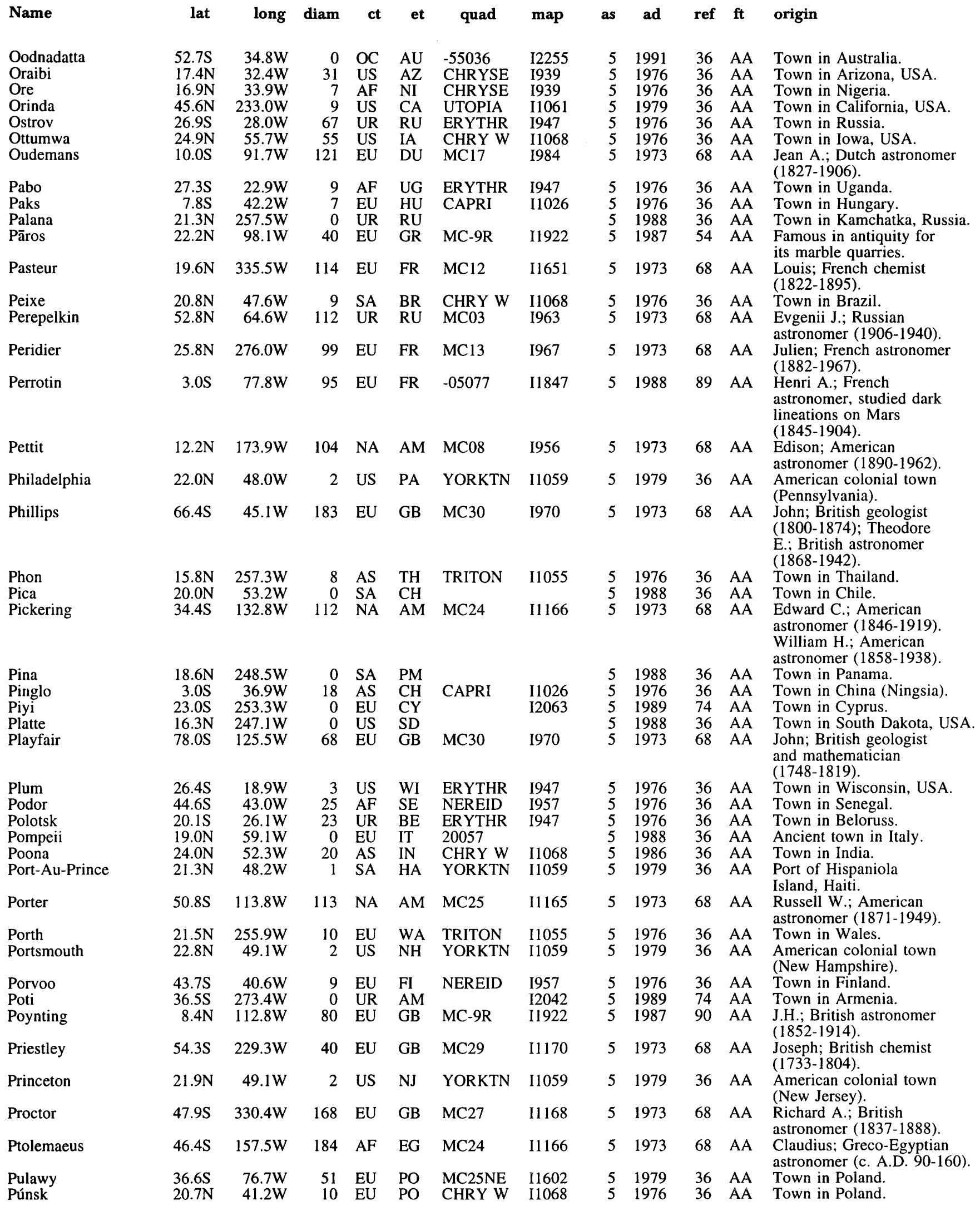

lat: latitude of feature center.

long: longitude of feature center.

diam: diameter or long dimension of feature. et: ethnicity of name origin (see page $284 \mathrm{ff}$.)

quad: map quadrangle or informal name

(see page xvil ff.). as: name approval status (see page $x$ vii).

ad: name approval date (year).

ref: reference source for name (see page $287 \mathrm{ff}$.).

ct: continent of name origin (see page $284 \mathrm{ff}$.) map: map name or USGS map number (see page xvii ff.). ft: feature type (see page 290 ). 
MARS

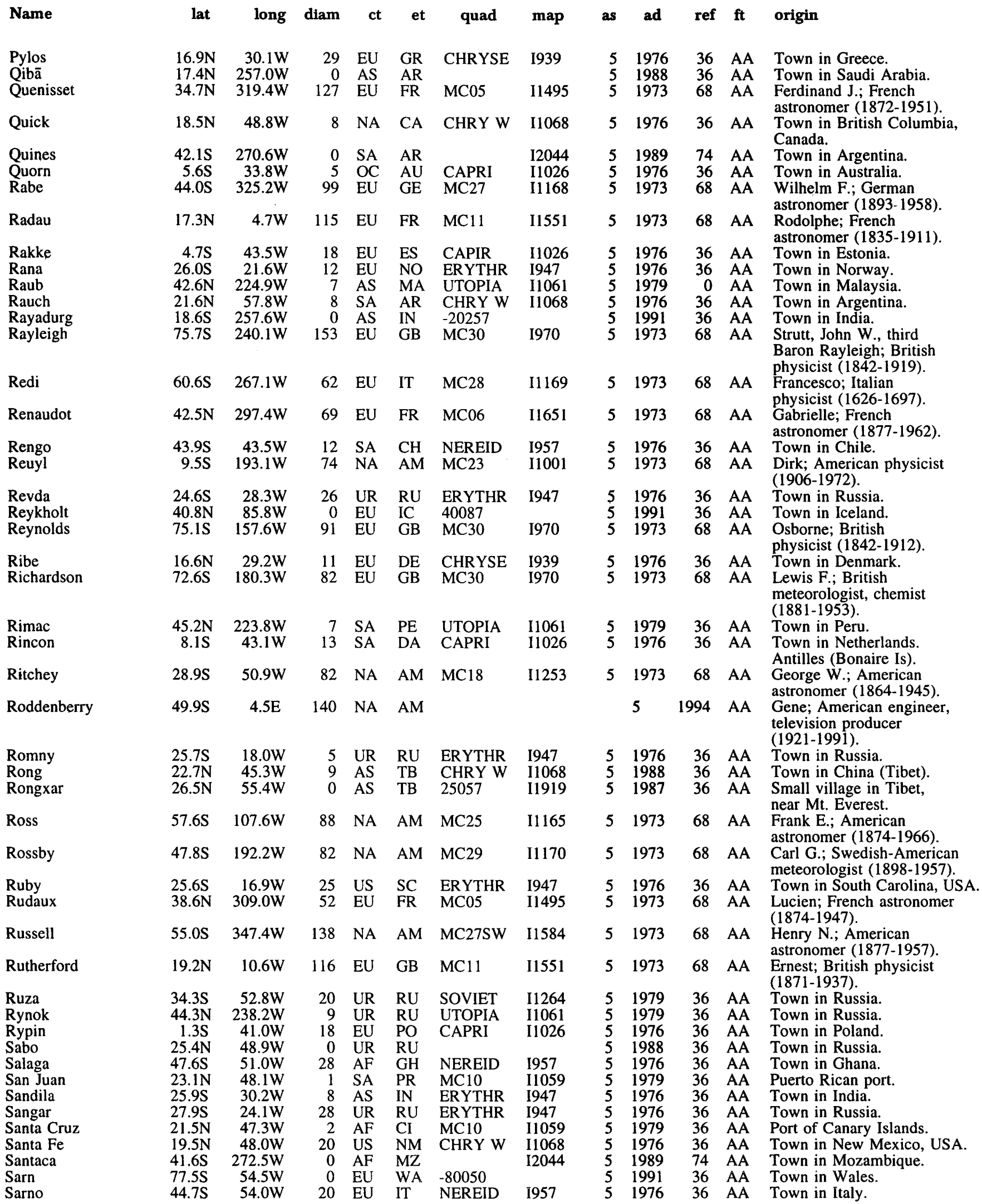

lat: latitude of feature center. long: longitude of feature center

diam: diameter or long dimension of feature. et: ethnicity of name origin (see page $284 \mathrm{ff}$.)

quad: map quadrangle or informal name (see page xvii ff.). as: name approval status (see page $\mathrm{xvii}$ )

ad: name approval date (year).

ref: reference source for name (see page $287 \mathrm{ff}$.). 
MARS

\begin{tabular}{|c|c|c|c|c|c|c|c|c|c|c|c|c|}
\hline Name & lat & long & diam & ct & et & quad & map & as & ad & ref & $\mathbf{f t}$ & origin \\
\hline $\begin{array}{l}\text { Satka } \\
\text { Sauk } \\
\text { Savannah }\end{array}$ & $\begin{array}{l}43.0 \mathrm{~S} \\
45.0 \mathrm{~S} \\
22.3 \mathrm{~N}\end{array}$ & $\begin{array}{l}36.7 \mathrm{~W} \\
32.3 \mathrm{~W} \\
47.8 \mathrm{~W}\end{array}$ & $\begin{array}{r}14 \\
2 \\
1\end{array}$ & $\begin{array}{l}\text { UR } \\
\text { US } \\
\text { US }\end{array}$ & $\begin{array}{l}\text { RU } \\
\text { WI } \\
\text { GA }\end{array}$ & $\begin{array}{l}\text { NEREID } \\
\text { NEREID } \\
\text { YORKTN }\end{array}$ & $\begin{array}{l}1957 \\
1957 \\
\text { I1059 }\end{array}$ & $\begin{array}{l}5 \\
5 \\
5\end{array}$ & $\begin{array}{l}1976 \\
1976 \\
1979\end{array}$ & $\begin{array}{l}36 \\
36 \\
36\end{array}$ & $\begin{array}{l}\text { AA } \\
\text { AA } \\
\text { AA }\end{array}$ & $\begin{array}{l}\text { Town in Russia. } \\
\text { Town in Wisconsin, USA. } \\
\text { American colonial town } \\
\text { (Georgia). }\end{array}$ \\
\hline $\begin{array}{l}\text { Savich } \\
\text { Say } \\
\text { Schaeberle }\end{array}$ & $\begin{array}{l}27.8 S \\
28.5 S \\
24.7 S\end{array}$ & $\begin{array}{r}263.5 \mathrm{~W} \\
29.5 \mathrm{~W} \\
309.8 \mathrm{~W}\end{array}$ & $\begin{array}{r}0 \\
15 \\
160\end{array}$ & $\begin{array}{l}\text { UR } \\
\text { AF } \\
\text { NA }\end{array}$ & $\begin{array}{l}\text { RU } \\
\text { NG } \\
\text { AM }\end{array}$ & $\begin{array}{l}\text { ERYTHR } \\
\text { MC21 }\end{array}$ & $\begin{array}{l}\mathrm{I} 2040 \\
\mathrm{I} 947 \\
\mathrm{I} 978\end{array}$ & $\begin{array}{l}5 \\
5 \\
5\end{array}$ & $\begin{array}{l}1989 \\
1976 \\
1973\end{array}$ & $\begin{array}{r}0 \\
36 \\
68\end{array}$ & $\begin{array}{l}\text { AA } \\
\text { AA } \\
\text { AA }\end{array}$ & $\begin{array}{l}\text { A.M.; Russian astronomer. } \\
\text { Town in Niger. } \\
\text { John M.; American } \\
\text { astronomer (1853-1924). }\end{array}$ \\
\hline Schiaparelli & $2.5 \mathrm{~S}$ & $343.4 \mathrm{~W}$ & 461 & EU & IT & $\mathrm{MC} 12$ & I1651 & 5 & 1973 & 68 & AA & $\begin{array}{l}\text { Giovanni V.; Italian } \\
\text { astronomer (1835-1910). }\end{array}$ \\
\hline Schmidt & $72.2 \mathrm{~S}$ & $77.5 \mathrm{~W}$ & 194 & EU & GE & MC30 & $\mathrm{I} 970$ & 5 & 1973 & 68 & $\mathrm{AA}$ & $\begin{array}{l}\text { Johann F.; German } \\
\text { astronomer (1825-1884); } \\
\text { Otto Y.; Russian } \\
\text { geophysicist (1891-1956). }\end{array}$ \\
\hline Schöner & $20.4 \mathrm{~N}$ & $309.5 \mathrm{~W}$ & 185 & EU & GE & $\mathrm{MC13}$ & I1118 & 5 & 1976 & 68 & AA & $\begin{array}{l}\text { Johannes; German } \\
\text { geographer (1477-1547). }\end{array}$ \\
\hline Schroeter & $1.8 \mathrm{~S}$ & $303.6 \mathrm{~W}$ & 337 & EU & GE & MC21 & I1 118 & 5 & 1976 & 68 & $\mathrm{AA}$ & $\begin{array}{l}\text { Johann H.; German } \\
\text { astronomer (1745-1816). }\end{array}$ \\
\hline $\begin{array}{l}\text { Sebec } \\
\text { Secchi }\end{array}$ & $\begin{array}{l}40.0 S \\
57.9 S\end{array}$ & $\begin{array}{l}260.5 \mathrm{~W} \\
257.7 \mathrm{~W}\end{array}$ & $\begin{array}{r}0 \\
218\end{array}$ & $\begin{array}{l}\text { US } \\
\text { EU }\end{array}$ & $\begin{array}{l}\text { ME } \\
\text { IT }\end{array}$ & MC28 & $\begin{array}{l}\mathrm{I} 2045 \\
\text { I1 } 169\end{array}$ & $\begin{array}{l}5 \\
5\end{array}$ & $\begin{array}{l}1989 \\
1973\end{array}$ & $\begin{array}{l}74 \\
68\end{array}$ & $\begin{array}{l}\text { AA } \\
\text { AA }\end{array}$ & $\begin{array}{l}\text { Town in Maine , USA. } \\
\text { Angelo; Italian } \\
\text { astronomer (1818-1878). }\end{array}$ \\
\hline Semeykin & $41.8 \mathrm{~N}$ & $351.2 \mathrm{~W}$ & 71 & UR & SO & MC05 & I1535 & 5 & 1982 & 64 & AA & $\begin{array}{l}\text { Boris Evgen'evich; Soviet } \\
\text { astronomer (1900-1937). }\end{array}$ \\
\hline $\begin{array}{l}\text { Seminole } \\
\text { Sevel } \\
\text { Sevi } \\
\text { Sfax } \\
\text { Shambe } \\
\text { Sharonov }\end{array}$ & $\begin{array}{l}24.5 \mathrm{~S} \\
78.2 \mathrm{~N} \\
19.1 \mathrm{~N} \\
7.8 \mathrm{~S} \\
20.7 \mathrm{~S} \\
27.3 \mathrm{~N}\end{array}$ & $\begin{array}{r}18.9 \mathrm{~W} \\
39.5 \mathrm{~W} \\
257.1 \mathrm{~W} \\
43.5 \mathrm{~W} \\
30.5 \mathrm{~W} \\
58.3 \mathrm{~W}\end{array}$ & $\begin{array}{r}21 \\
0 \\
0 \\
7 \\
29 \\
95\end{array}$ & $\begin{array}{l}\text { US } \\
\text { EU } \\
\text { UR } \\
\text { AF } \\
\text { AF } \\
\text { UR }\end{array}$ & $\begin{array}{l}\text { FL } \\
\text { DE } \\
\text { RU } \\
\text { TN } \\
\text { SU } \\
\text { RU }\end{array}$ & $\begin{array}{l}\text { ERYTHR } \\
80030 \\
\\
\text { CAPRI } \\
\text { ERYTHR } \\
\text { MC10 }\end{array}$ & $\begin{array}{l}\text { I947 } \\
\text { I1812 } \\
\\
\text { I1026 } \\
\text { I947 } \\
\text { I971 }\end{array}$ & $\begin{array}{l}5 \\
5 \\
5 \\
5 \\
5 \\
5\end{array}$ & $\begin{array}{l}1976 \\
1988 \\
1988 \\
1976 \\
1976 \\
1973\end{array}$ & $\begin{array}{l}36 \\
36 \\
36 \\
36 \\
36 \\
68\end{array}$ & $\begin{array}{l}\text { AA } \\
\text { AA } \\
\text { AA } \\
\text { AA } \\
\text { AA } \\
\text { AA }\end{array}$ & $\begin{array}{l}\text { Town in Florida, USA. } \\
\text { Town in Denmark. } \\
\text { Town in Russia. } \\
\text { Town in Tunisia. } \\
\text { Town in Sudan. } \\
\text { Vsevolod V.; Russian } \\
\text { astronomer (1901-1964). }\end{array}$ \\
\hline $\begin{array}{l}\text { Shatskiy } \\
\text { Shawnee } \\
\text { Sian } \\
\text { Sibu } \\
\text { Sigli } \\
\text { Sinda } \\
\text { Singa } \\
\text { Sinop } \\
\text { Sitka } \\
\text { Sklodowska }\end{array}$ & $\begin{array}{l}32.4 \mathrm{~S} \\
22.7 \mathrm{~N} \\
20.1 \mathrm{~N} \\
23.3 \mathrm{~S} \\
20.5 \mathrm{~S} \\
16.0 \mathrm{~N} \\
22.8 \mathrm{~S} \\
23.5 \mathrm{~S} \\
4.3 \mathrm{~S} \\
33.8 \mathrm{~N}\end{array}$ & $\begin{array}{r}14.7 \mathrm{~W} \\
31.5 \mathrm{~W} \\
48.0 \mathrm{~W} \\
19.6 \mathrm{~W} \\
30.6 \mathrm{~W} \\
248.9 \mathrm{~W} \\
17.2 \mathrm{~W} \\
249.3 \mathrm{~W} \\
39.3 \mathrm{~W} \\
2.8 \mathrm{~W}\end{array}$ & $\begin{array}{r}69 \\
16 \\
0 \\
31 \\
31 \\
0 \\
11 \\
0 \\
11 \\
116\end{array}$ & $\begin{array}{l}\text { UR } \\
\text { US } \\
\text { UR } \\
\text { AS } \\
\text { AS } \\
\text { UR } \\
\text { AF } \\
\text { AS } \\
\text { US } \\
\text { EU }\end{array}$ & $\begin{array}{l}\text { RU } \\
\text { OH } \\
\text { RU } \\
\text { MA } \\
\text { ID } \\
\text { RU } \\
\text { SU } \\
\text { TU } \\
\text { AL } \\
\text { PO }\end{array}$ & $\begin{array}{l}\text { MC26 } \\
\text { CHRYSE } \\
\text { ERYTHR } \\
\text { ERYTHR } \\
\text { ERYTHR } \\
\text { CAPRI } \\
\text { MC04 }\end{array}$ & $\begin{array}{l}I 1535 \\
I 939 \\
1947 \\
I 947 \\
\\
I 947 \\
I 2064 \\
I 1026 \\
I 979\end{array}$ & $\begin{array}{l}5 \\
5 \\
5 \\
5 \\
5 \\
5 \\
5 \\
5 \\
5 \\
5\end{array}$ & $\begin{array}{l}1979 \\
1976 \\
1988 \\
1976 \\
1976 \\
1988 \\
1976 \\
1989 \\
1976 \\
1973\end{array}$ & $\begin{array}{l}68 \\
36 \\
36 \\
36 \\
36 \\
36 \\
36 \\
74 \\
36 \\
68\end{array}$ & $\begin{array}{l}\text { AA } \\
\text { AA } \\
\text { AA } \\
\text { AA } \\
\text { AA } \\
\text { AA } \\
\text { AA } \\
\text { AA } \\
\text { AA } \\
\text { AA }\end{array}$ & $\begin{array}{l}\text { N. S.; Russian geologist. } \\
\text { Town in Ohio, USA. } \\
\text { Town in Russia. } \\
\text { Town in Malaysia. } \\
\text { Town in Indonesia. } \\
\text { Town in Russia. } \\
\text { Town in Sudan. } \\
\text { Town in Turkey. } \\
\text { Town in Alaska, USA. } \\
\text { Marie; Polish-born French } \\
\text { chemist (Mme P. Curie) } \\
\text { (1867-1934). }\end{array}$ \\
\hline Slipher & $47.7 \mathrm{~S}$ & $84.5 \mathrm{~W}$ & 129 & NA & AM & MC25 & I1165 & 5 & 1973 & 68 & AA & $\begin{array}{l}\text { Vesto M.; American } \\
\text { astronomer (1875-1969). }\end{array}$ \\
\hline Smith & $66.1 S$ & $102.8 \mathrm{~W}$ & 71 & EU & GB & MC30 & 1970 & 5 & 1973 & 68 & AA & $\begin{array}{l}\text { William; British geologist- } \\
\text { engineer (1769-1839). }\end{array}$ \\
\hline $\begin{array}{l}\text { Sögel } \\
\text { Sokol } \\
\text { Solano } \\
\text { Soochow } \\
\text { Souris } \\
\text { South }\end{array}$ & $\begin{array}{l}21.7 \mathrm{~N} \\
42.8 \mathrm{~S} \\
27.0 \mathrm{~S} \\
16.8 \mathrm{~N} \\
19.7 \mathrm{~N} \\
77.0 \mathrm{~S}\end{array}$ & $\begin{array}{r}55.1 \mathrm{~W} \\
40.5 \mathrm{~W} \\
251.0 \mathrm{~W} \\
28.9 \mathrm{~W} \\
246.9 \mathrm{~W} \\
338.0 \mathrm{~W}\end{array}$ & $\begin{array}{r}29 \\
20 \\
0 \\
30 \\
0 \\
111\end{array}$ & $\begin{array}{l}\text { EU } \\
\text { UR } \\
\text { AS } \\
\text { AS } \\
\text { NA } \\
\text { EU }\end{array}$ & $\begin{array}{l}\text { GE } \\
\text { RU } \\
\text { PH } \\
\text { CH } \\
\text { CA } \\
\text { GB }\end{array}$ & $\begin{array}{l}\text { CHRY W } \\
\text { NEREID } \\
\text { CHRYSE } \\
\text { MC30 }\end{array}$ & $\begin{array}{l}\text { I1068 } \\
\text { I } 957 \\
\text { I2063 } \\
\text { I939 }\end{array}$ & $\begin{array}{l}5 \\
5 \\
5 \\
5 \\
5 \\
5\end{array}$ & $\begin{array}{l}1976 \\
1976 \\
1989 \\
1976 \\
1988 \\
1973\end{array}$ & $\begin{array}{l}36 \\
36 \\
74 \\
36 \\
36 \\
68\end{array}$ & $\begin{array}{l}\text { AA } \\
\text { AA } \\
\text { AA } \\
\text { AA } \\
\text { AA } \\
\text { AA }\end{array}$ & $\begin{array}{l}\text { Town in Germany. } \\
\text { Town in Russia. } \\
\text { Town in Phillipines. } \\
\text { Town in China (Kiangsu), } \\
\text { Town in Manitoba, Canada. } \\
\text { James; British astronomer } \\
(1785-1867) \text {. }\end{array}$ \\
\hline Spallanzani & $58.4 S$ & $273.5 \mathrm{~W}$ & 72 & $\mathrm{EU}$ & IT & MC28 & 11169 & 5 & 1973 & 68 & AA & $\begin{array}{l}\text { Lazzaro; Italian } \\
\text { biologist (1729-1799). }\end{array}$ \\
\hline $\begin{array}{l}\text { Spry } \\
\text { Spur } \\
\text { Sripur } \\
\text { Stege } \\
\text { Steno }\end{array}$ & $\begin{array}{r}3.8 \mathrm{~S} \\
22.2 \mathrm{~N} \\
31.1 \mathrm{~S} \\
2.6 \mathrm{~N} \\
68.0 \mathrm{~S}\end{array}$ & $\begin{array}{r}38.6 \mathrm{~W} \\
52.3 \mathrm{~W} \\
100.6 \mathrm{~W} \\
58.4 \mathrm{~W} \\
115.3 \mathrm{~W}\end{array}$ & $\begin{array}{r}6 \\
7 \\
0 \\
72 \\
105\end{array}$ & $\begin{array}{l}\text { US } \\
\text { US } \\
\text { AS } \\
\text { EU } \\
\text { EU }\end{array}$ & $\begin{array}{l}\text { UT } \\
\text { TX } \\
\text { BA } \\
\text { DE } \\
\text { DE }\end{array}$ & $\begin{array}{l}\text { CAPRI } \\
\text { CHRY W } \\
05057 \\
\text { MC30 }\end{array}$ & $\begin{array}{l}\mathrm{I} 1026 \\
\mathrm{I} 1068 \\
\mathrm{I} 2061 \\
\mathrm{I} 1676 \\
\mathrm{I} 970\end{array}$ & $\begin{array}{l}5 \\
5 \\
5 \\
5 \\
5\end{array}$ & $\begin{array}{l}1976 \\
1976 \\
1989 \\
1985 \\
1973\end{array}$ & $\begin{array}{l}36 \\
36 \\
74 \\
36 \\
68\end{array}$ & $\begin{array}{l}\text { AA } \\
\text { AA } \\
\text { AA } \\
\text { AA } \\
\text { AA }\end{array}$ & $\begin{array}{l}\text { Town in Utah, USA. } \\
\text { Town in Texas, USA. } \\
\text { Town in Bangladesh. } \\
\text { Town in Denmark. } \\
\text { Nicolaus; Danish } \\
\text { geologist (1638-1686). }\end{array}$ \\
\hline $\begin{array}{l}\text { Stobs } \\
\text { Stokes }\end{array}$ & $\begin{array}{r}5.0 \mathrm{~S} \\
56.0 \mathrm{~N}\end{array}$ & $\begin{array}{r}38.4 \mathrm{~W} \\
189.0 \mathrm{~W}\end{array}$ & $\begin{array}{l}10 \\
70\end{array}$ & $\begin{array}{l}\text { EU } \\
\text { EU }\end{array}$ & $\begin{array}{l}\text { SC } \\
\text { GB }\end{array}$ & $\begin{array}{l}\text { CAPRI } \\
\text { MC07 }\end{array}$ & $\begin{array}{l}\mathrm{I} 1026 \\
\mathrm{I} 1475\end{array}$ & $\begin{array}{l}5 \\
5\end{array}$ & $\begin{array}{l}1976 \\
1973\end{array}$ & $\begin{array}{l}36 \\
68\end{array}$ & $\begin{array}{l}\text { AA } \\
\text { AA }\end{array}$ & $\begin{array}{l}\text { Town in Scotland. } \\
\text { George G.; British } \\
\text { physicist (1819-1903). }\end{array}$ \\
\hline $\begin{array}{l}\text { Ston } \\
\text { Stoney }\end{array}$ & $\begin{array}{l}47.2 \mathrm{~N} \\
69.8 \mathrm{~S}\end{array}$ & $\begin{array}{l}237.4 \mathrm{~W} \\
138.4 \mathrm{~W}\end{array}$ & $\begin{array}{r}7 \\
177\end{array}$ & $\begin{array}{l}\text { EU } \\
\text { EU }\end{array}$ & $\begin{array}{l}\text { YU } \\
\text { IR }\end{array}$ & $\begin{array}{l}\text { UTOPIA } \\
\text { MC30 }\end{array}$ & $\begin{array}{l}\text { I1061 } \\
\text { I970 }\end{array}$ & $\begin{array}{l}5 \\
5\end{array}$ & $\begin{array}{l}1979 \\
1973\end{array}$ & $\begin{array}{l}36 \\
68\end{array}$ & $\begin{array}{l}\mathrm{AA} \\
\mathrm{AA}\end{array}$ & $\begin{array}{l}\text { Town in Yugoslavia. } \\
\text { George J.; Irish } \\
\text { physicist }(1826-1911) \text {. }\end{array}$ \\
\hline $\begin{array}{l}\text { Suata } \\
\text { Sucre } \\
\text { Suess }\end{array}$ & $\begin{array}{l}19.2 S \\
24.0 \mathrm{~N} \\
67.1 \mathrm{~S}\end{array}$ & $\begin{array}{r}253.3 \mathrm{~W} \\
54.5 \mathrm{~W} \\
178.4 \mathrm{~W}\end{array}$ & $\begin{array}{r}0 \\
9 \\
72\end{array}$ & $\begin{array}{l}\text { SA } \\
\text { SA } \\
\text { EU }\end{array}$ & $\begin{array}{l}\text { VE } \\
\text { CO } \\
\text { AS }\end{array}$ & $\begin{array}{l}-20252 \\
\text { CHRY W } \\
\text { MC30 }\end{array}$ & $\begin{array}{l}\mathrm{I} 1068 \\
\mathrm{I} 970\end{array}$ & $\begin{array}{l}5 \\
5 \\
5\end{array}$ & $\begin{array}{l}1991 \\
1976 \\
1973\end{array}$ & $\begin{array}{l}36 \\
36 \\
68\end{array}$ & $\begin{array}{l}\text { AA } \\
\text { AA } \\
\text { AA }\end{array}$ & $\begin{array}{l}\text { Town in Venezuela. } \\
\text { Town in Colombia. } \\
\text { Eduard; Austrian geologist, } \\
\text { engineer (1831-1914). }\end{array}$ \\
\hline
\end{tabular}

\footnotetext{
lat: latitude of feature center.

long: longitude of feature center.

diam: diameter or long dimension of feature.

et: ethnicity of name origin (see page $284 \mathrm{ff}$.)

quad: map quadrangle or informal name

(see page xvii ff.).

as: name approval status (see page xvii).

ad: name approval date (year).

ref: reference source for name (see page $287 \mathrm{ff}$.)
} 
MARS

\begin{tabular}{|c|c|c|c|c|c|c|c|c|c|c|c|c|}
\hline Name & lat & long & diam & ct & et & quad & map & as & ad & ref & $\mathbf{f t}$ & origin \\
\hline $\begin{array}{l}\text { Sūf } \\
\text { Sulak } \\
\text { Sumgin }\end{array}$ & $\begin{array}{l}16.6 \mathrm{~N} \\
18.3 \mathrm{~N} \\
37.0 \mathrm{~S}\end{array}$ & $\begin{array}{l}38.2 \mathrm{~W} \\
78.6 \mathrm{~W} \\
48.6 \mathrm{~W}\end{array}$ & $\begin{array}{r}9 \\
0 \\
83\end{array}$ & $\begin{array}{l}\text { AS } \\
\text { UR } \\
\text { UR }\end{array}$ & $\begin{array}{l}\text { JO } \\
\text { RU } \\
\text { RU }\end{array}$ & $\begin{array}{l}\text { CHRYSE } \\
20077 \\
\text { MC26 }\end{array}$ & $\begin{array}{l}\text { I939 } \\
\text { I1782 } \\
\text { I1535 }\end{array}$ & $\begin{array}{l}5 \\
5 \\
5\end{array}$ & $\begin{array}{l}1976 \\
1985 \\
1979\end{array}$ & $\begin{array}{l}36 \\
36 \\
68\end{array}$ & $\begin{array}{l}\text { AA } \\
\text { AA } \\
\text { AA }\end{array}$ & $\begin{array}{l}\text { Town in Jordan. } \\
\text { Town in Russia. } \\
\text { M. I.; Russian } \\
\text { cryopedologist. }\end{array}$ \\
\hline $\begin{array}{l}\text { Surt } \\
\text { Suzhi } \\
\text { Sytinskaya }\end{array}$ & $\begin{array}{l}17.0 \mathrm{~N} \\
27.6 \mathrm{~S} \\
42.8 \mathrm{~N}\end{array}$ & $\begin{array}{r}30.6 \mathrm{~W} \\
273.1 \mathrm{~W} \\
52.8 \mathrm{~W}\end{array}$ & $\begin{array}{r}9 \\
0 \\
90\end{array}$ & $\begin{array}{l}\text { AF } \\
\text { AS } \\
\text { UR }\end{array}$ & $\begin{array}{l}\mathrm{LB} \\
\mathrm{CH} \\
\text { SO }\end{array}$ & $\begin{array}{l}\text { CHRYSE } \\
\text { MC04 }\end{array}$ & $\begin{array}{l}\mathrm{I} 939 \\
\mathrm{I} 2038 \\
\mathrm{I} 1476\end{array}$ & $\begin{array}{l}5 \\
5 \\
5\end{array}$ & $\begin{array}{l}1976 \\
1989 \\
1982\end{array}$ & $\begin{array}{l}36 \\
74 \\
64\end{array}$ & $\begin{array}{l}\text { AA } \\
\text { AA } \\
\text { AA }\end{array}$ & $\begin{array}{l}\text { Town in Libya. } \\
\text { Town in China. } \\
\text { Nadezhda Nikolaevna; } \\
\text { Soviet astronomer } \\
(1906-1974) \text {. }\end{array}$ \\
\hline $\begin{array}{l}\text { Tabor } \\
\text { Tabou } \\
\text { Taejin } \\
\text { Tak } \\
\text { Tala } \\
\text { Talsi } \\
\text { Tame } \\
\text { Tara } \\
\text { Tarakan }\end{array}$ & $\begin{array}{l}36.0 S \\
45.5 S \\
35.6 S \\
26.4 S \\
20.6 S \\
41.9 S \\
23.0 S \\
44.4 S \\
41.6 S\end{array}$ & $\begin{array}{r}58.5 \mathrm{~W} \\
34.8 \mathrm{~W} \\
274.3 \mathrm{~W} \\
28.5 \mathrm{~W} \\
252.2 \mathrm{~W} \\
49.1 \mathrm{~W} \\
107.8 \mathrm{~W} \\
52.7 \mathrm{~W} \\
30.1 \mathrm{~W}\end{array}$ & $\begin{array}{r}20 \\
8 \\
0 \\
5 \\
0 \\
9 \\
0 \\
27 \\
37\end{array}$ & $\begin{array}{l}\text { EU } \\
\text { AF } \\
\text { AS } \\
\text { AS } \\
\text { AF } \\
\text { EU } \\
\text { SA } \\
\text { EU } \\
\text { AS }\end{array}$ & $\begin{array}{l}\mathrm{CZ} \\
\text { IC } \\
\mathrm{KR} \\
\text { TH } \\
\text { TN } \\
\text { LV } \\
\text { CO } \\
\text { IR } \\
\text { ID }\end{array}$ & $\begin{array}{l}\text { SOVIET } \\
\text { NEREID } \\
\text { ERYTHR } \\
\text { NEREID } \\
\text { NEREID } \\
\text { NEREID }\end{array}$ & $\begin{array}{l}\mathrm{I} 957 \\
\mathrm{I} 2042 \\
\mathrm{I} 947 \\
\mathrm{I} 2065 \\
\mathrm{I} 957 \\
\mathrm{I} 2060 \\
\mathrm{I} 957 \\
\mathrm{I} 957\end{array}$ & $\begin{array}{l}5 \\
5 \\
5 \\
5 \\
5 \\
5 \\
5 \\
5 \\
5\end{array}$ & $\begin{array}{l}1979 \\
1976 \\
1989 \\
1976 \\
1989 \\
1976 \\
1989 \\
1976 \\
1976\end{array}$ & $\begin{array}{l}36 \\
36 \\
74 \\
36 \\
74 \\
36 \\
74 \\
36 \\
36\end{array}$ & $\begin{array}{l}\text { AA } \\
\text { AA } \\
\text { AA } \\
\text { AA } \\
\text { AA } \\
\text { AA } \\
\text { AA } \\
\text { AA } \\
\text { AA }\end{array}$ & $\begin{array}{l}\text { Town in Czechoslovakia. } \\
\text { Town in Ivory Coast. } \\
\text { Town in Korea. } \\
\text { Town in Thailand. } \\
\text { Town in Tunisia. } \\
\text { Town in Latvia. } \\
\text { Town in Colombia. } \\
\text { Town in Ireland. } \\
\text { Town in Indonesia } \\
\text { (Borneo). }\end{array}$ \\
\hline $\begin{array}{l}\text { Tarata } \\
\text { Tarma } \\
\text { Tarsus } \\
\text { Taxco } \\
\text { Taza } \\
\text { Tecolote } \\
\text { Teisserenc de } \\
\text { Bort }\end{array}$ & $\begin{array}{c}3.8 \mathrm{~S} \\
16.8 \mathrm{~N} \\
23.5 \mathrm{~N} \\
20.8 \mathrm{~N} \\
44.0 \mathrm{~S} \\
24.9 \mathrm{~S} \\
0.6 \mathrm{~N}\end{array}$ & $\begin{array}{r}41.3 \mathrm{~W} \\
250.3 \mathrm{~W} \\
40.2 \mathrm{~W} \\
40.0 \mathrm{~W} \\
45.1 \mathrm{~W} \\
106.7 \mathrm{~W} \\
315.0 \mathrm{~W}\end{array}$ & $\begin{array}{r}12 \\
0 \\
19 \\
17 \\
22 \\
0 \\
118\end{array}$ & $\begin{array}{l}\text { SA } \\
\text { SA } \\
\text { AS } \\
\text { NA } \\
\text { AF } \\
\text { US } \\
\text { EU }\end{array}$ & $\begin{array}{l}\text { BO } \\
\text { PE } \\
\text { TU } \\
\text { ME } \\
\text { MR } \\
\text { NM } \\
\text { FR }\end{array}$ & $\begin{array}{l}\text { CAPRI } \\
\text { CHRY W } \\
\text { CHRYSE } \\
\text { NEREID } \\
\text { MC12 }\end{array}$ & $\begin{array}{l}11068 \\
1939 \\
1957 \\
12060 \\
\mathrm{I} 1675\end{array}$ & $\begin{array}{l}5 \\
5 \\
5 \\
5 \\
5 \\
5 \\
5\end{array}$ & $\begin{array}{l}1976 \\
1988 \\
1976 \\
1976 \\
1976 \\
1989 \\
1973\end{array}$ & $\begin{array}{l}36 \\
36 \\
36 \\
36 \\
36 \\
74 \\
68\end{array}$ & $\begin{array}{l}\text { AA } \\
\text { AA } \\
\text { AA } \\
\text { AA } \\
\text { AA } \\
\text { AA } \\
\text { AA }\end{array}$ & $\begin{array}{l}\text { Town in Bolivia. } \\
\text { Town in Peru. } \\
\text { Town in Turkey. } \\
\text { Town in Mexico. } \\
\text { Town in Morocco. } \\
\text { Town in N. Mexico, USA. } \\
\text { Leon P.; French } \\
\text { meteorologist (1855-1913). }\end{array}$ \\
\hline $\begin{array}{l}\text { Tejn } \\
\text { Telz } \\
\text { Tem' } \\
\text { Tepko } \\
\text { Terby }\end{array}$ & $\begin{array}{l}15.7 \mathrm{~N} \\
21.4 \mathrm{~N} \\
42.1 \mathrm{~N} \\
15.5 \mathrm{~N} \\
28.2 \mathrm{~S}\end{array}$ & $\begin{array}{r}253.8 \mathrm{~W} \\
249.1 \mathrm{~W} \\
9.5 \mathrm{~W} \\
256.5 \mathrm{~W} \\
286.0 \mathrm{~W}\end{array}$ & $\begin{array}{r}0 \\
0 \\
4 \\
0 \\
135\end{array}$ & $\begin{array}{l}\text { EU } \\
\text { EU } \\
\text { UR } \\
\text { OC } \\
\text { EU }\end{array}$ & $\begin{array}{l}\text { DE } \\
\text { GE } \\
\text { RU } \\
\text { AU } \\
\text { BE }\end{array}$ & $\begin{array}{l}\text { CYDONI } \\
\text { MC21 }\end{array}$ & $\begin{array}{l}\mathrm{I} 946 \\
\mathrm{I} 1118\end{array}$ & $\begin{array}{l}5 \\
5 \\
5 \\
5 \\
5\end{array}$ & $\begin{array}{l}1988 \\
1988 \\
1976 \\
1988 \\
1973\end{array}$ & $\begin{array}{r}0 \\
36 \\
36 \\
36 \\
68\end{array}$ & $\begin{array}{l}\text { AA } \\
\text { AA } \\
\text { AA } \\
\text { AA } \\
\text { AA }\end{array}$ & $\begin{array}{l}\text { Town in Denmark. } \\
\text { Town in Germany. } \\
\text { Town in Russia. } \\
\text { Town in Australia. } \\
\text { Francois J.; Belgian } \\
\text { astronomer (1846-1911). }\end{array}$ \\
\hline $\begin{array}{l}\text { Thermia } \\
\text { Thom } \\
\text { Thule } \\
\text { Tibrikot } \\
\text { Tignish }\end{array}$ & $\begin{array}{l}19.9 \mathrm{~N} \\
41.5 \mathrm{~S} \\
23.6 \mathrm{~S} \\
12.7 \mathrm{~N} \\
31.1 \mathrm{~S}\end{array}$ & $\begin{array}{r}250.9 \mathrm{~W} \\
267.5 \mathrm{~W} \\
25.5 \mathrm{~W} \\
54.9 \mathrm{~W} \\
273.0 \mathrm{~W}\end{array}$ & $\begin{array}{r}0 \\
0 \\
14 \\
0 \\
0\end{array}$ & $\begin{array}{l}\text { EU } \\
\text { AS } \\
\text { EU } \\
\text { AS } \\
\text { NA }\end{array}$ & $\begin{array}{l}\text { GR } \\
\text { TH } \\
\text { GL } \\
\text { NE } \\
\text { CA }\end{array}$ & ERYTHR & $\begin{array}{l}\mathrm{I} 2043 \\
\mathrm{I} 947 \\
\mathrm{I} 2038\end{array}$ & $\begin{array}{l}5 \\
5 \\
5 \\
5 \\
5\end{array}$ & $\begin{array}{l}1988 \\
1989 \\
1976 \\
1988 \\
1989\end{array}$ & $\begin{array}{l}36 \\
74 \\
36 \\
36 \\
74\end{array}$ & $\begin{array}{l}\text { AA } \\
\text { AA } \\
\text { AA } \\
\text { AA } \\
\text { AA }\end{array}$ & $\begin{array}{l}\text { Town in Greece. } \\
\text { Town in Thailand. } \\
\text { Town in Greenland. } \\
\text { Town in Nepal. } \\
\text { Town in Prince Edward } \\
\text { Island, Canada. }\end{array}$ \\
\hline Tikhonravov & $13.7 \mathrm{~N}$ & $324.1 \mathrm{~W}$ & 390 & UR & RU & $\mathrm{MC} 12$ & $\mathrm{I} 1535$ & 5 & 1985 & 0 & AA & $\begin{array}{l}\text { M. K.; Russian rocket } \\
\text { scientist (1851-1916). }\end{array}$ \\
\hline Tikhov & $51.2 \mathrm{~S}$ & $254.1 \mathrm{~W}$ & 107 & UR & RU & MC28 & I1 169 & 5 & 1973 & 68 & AA & $\begin{array}{l}\text { Gavril A.; Russian } \\
\text { astronomer (1875-1960). }\end{array}$ \\
\hline $\begin{array}{l}\text { Tile } \\
\text { Timaru } \\
\text { Timbuktu } \\
\text { Timoshenko }\end{array}$ & $\begin{array}{r}17.8 \mathrm{~N} \\
25.6 \mathrm{~S} \\
5.7 \mathrm{~S} \\
42.1 \mathrm{~N}\end{array}$ & $\begin{array}{l}28.6 \mathrm{~W} \\
22.2 \mathrm{~W} \\
37.7 \mathrm{~W} \\
63.9 \mathrm{~W}\end{array}$ & $\begin{array}{r}8 \\
8 \\
63 \\
84\end{array}$ & $\begin{array}{l}\text { AF } \\
\text { OC } \\
\text { AS } \\
\text { UR }\end{array}$ & $\begin{array}{l}\text { SO } \\
\text { NZ } \\
\text { ML } \\
\text { SO }\end{array}$ & $\begin{array}{l}\text { CHRYSE } \\
\text { ERYTHR } \\
\text { CAPRI } \\
\text { MC03 }\end{array}$ & $\begin{array}{l}\mathrm{I} 939 \\
\mathrm{I} 947 \\
\mathrm{I} 1026 \\
\mathrm{I} 1535\end{array}$ & $\begin{array}{l}5 \\
5 \\
5 \\
5\end{array}$ & $\begin{array}{l}1976 \\
1976 \\
1976 \\
1982\end{array}$ & $\begin{array}{l}36 \\
36 \\
36 \\
64\end{array}$ & $\begin{array}{l}\text { AA } \\
\text { AA } \\
\text { AA } \\
\text { AA }\end{array}$ & $\begin{array}{l}\text { Town in Somalia. } \\
\text { Town in New Zealand. } \\
\text { Town in Mali. } \\
\text { Ivan Fedorovich; Soviet } \\
\text { astronomer (1918-1941). }\end{array}$ \\
\hline $\begin{array}{l}\text { Tiwi } \\
\text { Tokko } \\
\text { Tokma } \\
\text { Tolon } \\
\text { Alexey Tolstoy } \\
\text { Tomari } \\
\text { Tombe } \\
\text { Tōno } \\
\text { Torbay } \\
\text { Torsö } \\
\text { Torup } \\
\text { Trinidad } \\
\text { Troika } \\
\text { Trouvelot }\end{array}$ & $\begin{array}{l}27.9 \mathrm{~S} \\
22.7 \mathrm{~N} \\
21.6 \mathrm{~N} \\
18.5 \mathrm{~N} \\
47.6 \mathrm{~S} \\
20.1 \mathrm{~N} \\
42.8 \mathrm{~S} \\
45.2 \mathrm{~S} \\
18.1 \mathrm{~N} \\
44.7 \mathrm{~S} \\
28.1 \mathrm{~S} \\
23.9 \mathrm{~S} \\
17.1 \mathrm{~N} \\
16.3 \mathrm{~N}\end{array}$ & $\begin{array}{r}24.6 \mathrm{~W} \\
250.8 \mathrm{~W} \\
251.6 \mathrm{~W} \\
255.1 \mathrm{~W} \\
234.6 \mathrm{~W} \\
246.4 \mathrm{~W} \\
44.4 \mathrm{~W} \\
52.2 \mathrm{~W} \\
246.1 \mathrm{~W} \\
51.0 \mathrm{~W} \\
262.2 \mathrm{~W} \\
251.0 \mathrm{~W} \\
255.0 \mathrm{~W} \\
13.0 \mathrm{~W}\end{array}$ & $\begin{array}{r}17 \\
0 \\
0 \\
0 \\
94 \\
0 \\
12 \\
10 \\
0 \\
14 \\
0 \\
0 \\
13 \\
168\end{array}$ & $\begin{array}{l}\text { AS } \\
\text { UR } \\
\text { UR } \\
\text { UR } \\
\text { UR } \\
\text { UR } \\
\text { AF } \\
\text { AS } \\
\text { OC } \\
\text { EU } \\
\text { EU } \\
\text { SA } \\
\text { UR } \\
\text { NA }\end{array}$ & $\begin{array}{l}\text { OM } \\
\text { RU } \\
\text { RU } \\
\text { RU } \\
\text { SO } \\
\text { RU } \\
\text { SU } \\
\text { JA } \\
\text { AU } \\
\text { SW } \\
\text { SW } \\
\text { PE } \\
\text { RU } \\
\text { AM }\end{array}$ & $\begin{array}{l}\text { MC29 } \\
\text { NEREID } \\
\text { NEREID } \\
\text { NEREID }\end{array}$ & $\begin{array}{l}\mathrm{I} 1170 \\
\mathrm{I} 957 \\
\mathrm{I} 957 \\
\mathrm{I} 957 \\
\mathrm{I} 2040 \\
\\
\mathrm{I} 1055 \\
\mathrm{I} 1551\end{array}$ & $\begin{array}{l}5 \\
5 \\
5 \\
5 \\
5 \\
5 \\
5 \\
5 \\
5 \\
5 \\
5 \\
5 \\
5 \\
5\end{array}$ & $\begin{array}{l}1976 \\
1988 \\
1988 \\
1988 \\
1982 \\
1988 \\
1976 \\
1976 \\
1988 \\
1976 \\
1989 \\
1989 \\
1976 \\
1973\end{array}$ & $\begin{array}{r}36 \\
36 \\
36 \\
36 \\
0 \\
36 \\
36 \\
36 \\
36 \\
36 \\
74 \\
74 \\
36 \\
68\end{array}$ & $\begin{array}{l}\text { AA } \\
\text { AA } \\
\text { AA } \\
\text { AA } \\
\text { AA } \\
\text { AA } \\
\text { AA } \\
\text { AA } \\
\text { AA } \\
\text { AA } \\
\text { AA } \\
\text { AA } \\
\text { AA } \\
\text { AA }\end{array}$ & $\begin{array}{l}\text { Town in Oman. } \\
\text { Town in Russia. } \\
\text { Town in Russia. } \\
\text { Town in Russia. } \\
\text { Soviet writer (1882-1945). } \\
\text { Town in Russia. } \\
\text { Town in Sudan. } \\
\text { Town in Japan. } \\
\text { Town in Australia. } \\
\text { Town in Sweden. } \\
\text { Town in Sweden. } \\
\text { Town in Peru. } \\
\text { Town in Russia. } \\
\text { Etienne L.; } \\
\text { French-American } \\
\text { astronomer (1827-1895). }\end{array}$ \\
\hline $\begin{array}{l}\text { Troy } \\
\text { Trud } \\
\text { Trumpler }\end{array}$ & $\begin{array}{l}23.3 \mathrm{~N} \\
17.7 \mathrm{~N} \\
61.7 \mathrm{~S}\end{array}$ & $\begin{array}{r}52.4 \mathrm{~W} \\
30.8 \mathrm{~W} \\
150.6 \mathrm{~W}\end{array}$ & $\begin{array}{r}10 \\
5 \\
77\end{array}$ & $\begin{array}{l}\text { US } \\
\text { UR } \\
\text { NA }\end{array}$ & $\begin{array}{l}\text { ID } \\
\text { RU } \\
\text { AM }\end{array}$ & $\begin{array}{l}\text { CHRY W } \\
\text { CHRYHR } \\
\text { MC24 }\end{array}$ & $\begin{array}{l}\text { I1068 } \\
\text { I991 } \\
\text { I1166 }\end{array}$ & $\begin{array}{l}5 \\
5 \\
5\end{array}$ & $\begin{array}{l}1976 \\
1976 \\
1973\end{array}$ & $\begin{array}{l}36 \\
36 \\
68\end{array}$ & $\begin{array}{l}\text { AA } \\
\text { AA } \\
\text { AA }\end{array}$ & $\begin{array}{l}\text { Town in Idaho, USA. } \\
\text { Town in Russia. } \\
\text { Robert J.; American } \\
\text { astronomer (1886-1956). }\end{array}$ \\
\hline $\begin{array}{l}\text { Tsau } \\
\text { Tsukuba }\end{array}$ & $\begin{array}{l}49.8 \mathrm{~N} \\
48.9 \mathrm{~N}\end{array}$ & $\begin{array}{l}238.9 \mathrm{~W} \\
225.9 \mathrm{~W}\end{array}$ & $\begin{array}{l}6 \\
2\end{array}$ & $\begin{array}{l}\text { AF } \\
\text { AS }\end{array}$ & $\begin{array}{l}\text { BT } \\
\text { JA }\end{array}$ & $\begin{array}{l}\text { UTOPIA } \\
\text { CANBER }\end{array}$ & $\begin{array}{l}\text { I1061 } \\
\text { I1060 }\end{array}$ & $\begin{array}{l}5 \\
5\end{array}$ & $\begin{array}{l}1979 \\
1979\end{array}$ & $\begin{array}{l}36 \\
36\end{array}$ & $\begin{array}{l}\text { AA } \\
\text { AA }\end{array}$ & $\begin{array}{l}\text { Town in Botswana. } \\
\text { Japanese mission control } \\
\text { site. }\end{array}$ \\
\hline
\end{tabular}

lat: latitude of feature center.

long: longitude of feature center.

diam: diameter or long dimension of feature. et: ethnicity of name origin (see page $284 \mathrm{ff}$.)

quad: map quadrangle or informal name (see page xvii ff.). as: name approval status (see page $\mathrm{xvii}$.

ad: name approval date (year).

ref: reference source for name (see page $287 \mathrm{ff}$.).

ct: continent of name origin (see page $284 \mathrm{ff}$.) map: map name or USGS map number (see page xvii ff.). ft: feature type (see page 290 ). 
MARS

\begin{tabular}{|c|c|c|c|c|c|c|c|c|c|c|c|c|}
\hline Name & lat & long & diam & ct & et & quad & map & as & ad & ref & $\mathbf{f t}$ & origin \\
\hline $\begin{array}{l}\text { Tuapi } \\
\text { Tugaske } \\
\text { Tumul } \\
\text { Tungla } \\
\text { Tura } \\
\text { Turbi } \\
\text { Turma } \\
\text { Tuskegee } \\
\text { Tycho Brahe }\end{array}$ & $\begin{array}{l}17.3 \mathrm{~N} \\
32.2 \mathrm{~S} \\
15.0 \mathrm{~N} \\
41.1 \mathrm{~S} \\
27.0 \mathrm{~S} \\
40.9 \mathrm{~S} \\
17.5 \mathrm{~N} \\
2.9 \mathrm{~S} \\
49.5 \mathrm{~S}\end{array}$ & $\begin{array}{r}255.8 \mathrm{~W} \\
101.0 \mathrm{~W} \\
255.5 \mathrm{~W} \\
270.1 \mathrm{~W} \\
21.8 \mathrm{~W} \\
51.2 \mathrm{~W} \\
252.1 \mathrm{~W} \\
36.2 \mathrm{~W} \\
213.8 \mathrm{~W}\end{array}$ & $\begin{array}{r}0 \\
0 \\
0 \\
0 \\
14 \\
25 \\
0 \\
69 \\
108\end{array}$ & $\begin{array}{l}\text { SA } \\
\text { NA } \\
\text { UR } \\
\text { SA } \\
\text { UR } \\
\text { AF } \\
\text { UR } \\
\text { US } \\
\text { EU }\end{array}$ & $\begin{array}{l}\text { NI } \\
\text { CA } \\
\text { RU } \\
\text { NI } \\
\text { RU } \\
\text { KY } \\
\text { RU } \\
\text { AL } \\
\text { DE }\end{array}$ & $\begin{array}{l}\text { ERYTHR } \\
\text { NEREID }\end{array}$ & $\begin{array}{l}\mathrm{I} 2044 \\
1947 \\
\mathrm{I} 957 \\
\\
\mathrm{I} 1026 \\
\mathrm{I} 1170\end{array}$ & $\begin{array}{l}5 \\
5 \\
5 \\
5 \\
5 \\
5 \\
5 \\
5 \\
5\end{array}$ & $\begin{array}{l}1988 \\
1989 \\
1988 \\
1989 \\
1976 \\
1976 \\
1988 \\
1976 \\
1973\end{array}$ & $\begin{array}{l}36 \\
74 \\
36 \\
74 \\
36 \\
36 \\
36 \\
36 \\
68\end{array}$ & $\begin{array}{l}\text { AA } \\
\text { AA } \\
\text { AA } \\
\text { AA } \\
\text { AA } \\
\text { AA } \\
\text { AA } \\
\text { AA } \\
\text { AA }\end{array}$ & $\begin{array}{l}\text { Town in Nicaragua. } \\
\text { Town in Saskatchewan. } \\
\text { Town in Russia. } \\
\text { Town in Nicaragua. } \\
\text { Town in Russia. } \\
\text { Town in Kenya. } \\
\text { Town in Russia. } \\
\text { Town in Alabama, USA. } \\
\text { Danish astronomer } \\
(1546-1601)\end{array}$ \\
\hline Tyndall & $40.1 \mathrm{~N}$ & $190.4 W$ & 79 & EU & GB & MC07 & I1475 & 5 & 1973 & 68 & AA & $\begin{array}{l}\text { John; British physicist } \\
\text { (1820-1893). }\end{array}$ \\
\hline $\begin{array}{l}\text { Ulu } \\
\text { Ulya } \\
\text { Umatac } \\
\text { Urk } \\
\text { Utan } \\
\text { Vaals } \\
\text { Valga } \\
\text { Valverde }\end{array}$ & $\begin{array}{r}22.8 \mathrm{~N} \\
18.0 \mathrm{~S} \\
42.7 \mathrm{~N} \\
23.4 \mathrm{~N} \\
24.4 \mathrm{~N} \\
4.0 \mathrm{~S} \\
44.6 \mathrm{~S} \\
20.3 \mathrm{~N}\end{array}$ & $\begin{array}{r}252.9 \mathrm{~W} \\
253.1 \mathrm{~W} \\
222.8 \mathrm{~W} \\
248.7 \mathrm{~W} \\
246.3 \mathrm{~W} \\
33.1 \mathrm{~W} \\
36.3 \mathrm{~W} \\
55.8 \mathrm{~W}\end{array}$ & $\begin{array}{r}0 \\
0 \\
12 \\
0 \\
0 \\
8 \\
16 \\
35\end{array}$ & $\begin{array}{l}\text { UR } \\
\text { UR } \\
\text { OC } \\
\text { EU } \\
\text { UR } \\
\text { EU } \\
\text { EU } \\
\text { SA }\end{array}$ & $\begin{array}{l}\text { RU } \\
\text { RU } \\
\text { GM } \\
\text { DU } \\
\text { RU } \\
\text { DU } \\
\text { ES } \\
\text { DR }\end{array}$ & $\begin{array}{l}\text { CAPRI } \\
\text { NEREID } \\
\text { CHRY W }\end{array}$ & $\begin{array}{l}\mathrm{I} 1026 \\
\mathrm{I} 957 \\
\mathrm{I} 1068\end{array}$ & $\begin{array}{l}5 \\
5 \\
5 \\
5 \\
5 \\
5 \\
5 \\
5\end{array}$ & $\begin{array}{l}1988 \\
1989 \\
1979 \\
1988 \\
1988 \\
1976 \\
1976 \\
1976\end{array}$ & $\begin{array}{l}36 \\
74 \\
36 \\
36 \\
36 \\
36 \\
36 \\
36\end{array}$ & $\begin{array}{l}\text { AA } \\
\text { AA } \\
\text { AA } \\
\text { AA } \\
\text { AA } \\
\text { AA } \\
\text { AA } \\
\text { AA }\end{array}$ & $\begin{array}{l}\text { Town in Russia. } \\
\text { Town in Russia. } \\
\text { Town in Guam, USA. } \\
\text { Town in Netherlands. } \\
\text { Town in Russia. } \\
\text { Town in the Netherlands. } \\
\text { Town in Estonia. } \\
\text { Town in the Dominican } \\
\text { Republic. }\end{array}$ \\
\hline $\begin{array}{l}\text { Vätö } \\
\text { Vaux } \\
\text { Verlaine } \\
\text { Very }\end{array}$ & $\begin{array}{r}43.9 \mathrm{~S} \\
18.1 \mathrm{~N} \\
9.4 \mathrm{~S} \\
49.8 \mathrm{~S}\end{array}$ & $\begin{array}{c}53.2 \mathrm{~W} \\
42.8 \mathrm{~W} \\
295.9 \mathrm{E} \\
176.8 \mathrm{~W}\end{array}$ & $\begin{array}{r}10 \\
0 \\
42 \\
127\end{array}$ & $\begin{array}{l}\text { EU } \\
\text { EU } \\
\text { EU } \\
\text { NA }\end{array}$ & $\begin{array}{l}\text { SW } \\
\text { FR } \\
\text { FR } \\
\text { AM }\end{array}$ & $\begin{array}{l}\text { NEREID } \\
\text { CHRYSE } \\
\text { MC24 }\end{array}$ & $\begin{array}{l}\text { I957 } \\
\text { I939 } \\
\text { I1166 }\end{array}$ & $\begin{array}{l}5 \\
5 \\
5 \\
5\end{array}$ & $\begin{array}{l}1976 \\
1976 \\
1994 \\
1973\end{array}$ & $\begin{array}{l}36 \\
36 \\
91 \\
68\end{array}$ & $\begin{array}{l}\text { AA } \\
\text { AA } \\
\text { AA } \\
\text { AA }\end{array}$ & $\begin{array}{l}\text { Town in Sweden. } \\
\text { Town in France. } \\
\text { Town in France. } \\
\text { Frank W.; American } \\
\text { astronomer (1852-1927). }\end{array}$ \\
\hline $\begin{array}{l}\text { Viana } \\
\text { Vik } \\
\text { Vils } \\
\text { Vinogradov }\end{array}$ & $\begin{array}{l}19.5 \mathrm{~N} \\
36.0 \mathrm{~S} \\
39.2 \mathrm{~N} \\
20.3 \mathrm{~S}\end{array}$ & $\begin{array}{r}255.3 \mathrm{~W} \\
64.0 \mathrm{~W} \\
11.7 \mathrm{~W} \\
38.0 \mathrm{~W}\end{array}$ & $\begin{array}{r}29 \\
25 \\
6 \\
191\end{array}$ & $\begin{array}{l}\text { SA } \\
\text { EU } \\
\text { EU } \\
\text { UR }\end{array}$ & $\begin{array}{l}\text { BR } \\
\text { IC } \\
\text { AS } \\
\text { SO }\end{array}$ & $\begin{array}{l}\text { TRITON } \\
\text { SOVIET } \\
\text { CYDONI } \\
\text { MC19 }\end{array}$ & $\begin{array}{l}\text { I1055 } \\
\text { I946 } \\
\text { I1 } 293\end{array}$ & $\begin{array}{l}5 \\
5 \\
5 \\
5\end{array}$ & $\begin{array}{l}1976 \\
1979 \\
1976 \\
1979\end{array}$ & $\begin{array}{l}36 \\
36 \\
36 \\
68\end{array}$ & $\begin{array}{l}\text { AA } \\
\text { AA } \\
\text { AA } \\
\text { AA }\end{array}$ & $\begin{array}{l}\text { Town in Brazil. } \\
\text { Town in Iceland. } \\
\text { Town in Austria. } \\
\text { Aleksander P.; Soviet } \\
\text { geochemist (1895-1975). }\end{array}$ \\
\hline Vinogradsky & $56.3 \mathrm{~S}$ & $216.0 \mathrm{~W}$ & 61 & UR & RU & MC29 & I1170 & 5 & 1973 & 68 & AA & $\begin{array}{l}\text { Sergei N.; Russian } \\
\text { microbiologist (1856-1953). }\end{array}$ \\
\hline $\begin{array}{l}\text { Virrat } \\
\text { Vishniac }\end{array}$ & $\begin{array}{l}31.1 \mathrm{~S} \\
76.7 \mathrm{~S}\end{array}$ & $\begin{array}{l}102.9 \mathrm{~W} \\
276.1 \mathrm{~W}\end{array}$ & $\begin{array}{r}0 \\
76\end{array}$ & $\begin{array}{l}\text { EU } \\
\text { NA }\end{array}$ & $\begin{array}{l}\text { SW } \\
\text { AM }\end{array}$ & MC30 & $\begin{array}{l}\text { I2061 } \\
\text { I970 }\end{array}$ & $\begin{array}{l}5 \\
5\end{array}$ & $\begin{array}{l}1989 \\
1976\end{array}$ & $\begin{array}{l}74 \\
68\end{array}$ & $\begin{array}{l}\text { AA } \\
\text { AA }\end{array}$ & $\begin{array}{l}\text { Town in Sweden. } \\
\text { Wolf V.; American } \\
\text { microbiologist } \\
(1922-1974) .\end{array}$ \\
\hline $\begin{array}{l}\text { Vivero } \\
\text { Voeykov }\end{array}$ & $\begin{array}{l}49.4 \mathrm{~N} \\
32.5 \mathrm{~S}\end{array}$ & $\begin{array}{r}241.3 \mathrm{~W} \\
76.1 \mathrm{~W}\end{array}$ & $\begin{array}{l}64 \\
67\end{array}$ & $\begin{array}{l}\text { EU } \\
\text { UR }\end{array}$ & $\begin{array}{l}\text { SP } \\
\text { SO }\end{array}$ & $\begin{array}{l}\text { UTOPIA } \\
\text { MC25NE }\end{array}$ & $\begin{array}{l}\mathrm{I} 1061 \\
\mathrm{I} 1602\end{array}$ & $\begin{array}{l}5 \\
5\end{array}$ & $\begin{array}{l}1979 \\
1979\end{array}$ & $\begin{array}{l}36 \\
68\end{array}$ & $\begin{array}{l}\text { AA } \\
\text { AA }\end{array}$ & $\begin{array}{l}\text { Town in Spain. } \\
\text { A. I.; Russian } \\
\text { climatologist and } \\
\text { geographer }(1842-1916) .\end{array}$ \\
\hline Vogel & $37.0 \mathrm{~S}$ & $13.2 \mathrm{~W}$ & 124 & EU & GE & MC26 & $\mathrm{I} 985$ & 5 & 1973 & 68 & AA & $\begin{array}{l}\text { Hermann; German } \\
\text { astronomer (1841-1907). }\end{array}$ \\
\hline $\begin{array}{l}\text { Volgograd } \\
\text { Vol'sk } \\
\text { Von Karman }\end{array}$ & $\begin{array}{l}48.4 \mathrm{~N} \\
23.2 \mathrm{~N} \\
64.3 \mathrm{~S}\end{array}$ & $\begin{array}{r}224.8 \mathrm{~W} \\
51.3 \mathrm{~W} \\
58.4 \mathrm{~W}\end{array}$ & $\begin{array}{r}2 \\
8 \\
100\end{array}$ & $\begin{array}{l}\text { UR } \\
\text { UR } \\
\text { NA }\end{array}$ & $\begin{array}{l}\text { SO } \\
\text { RU } \\
\text { AM }\end{array}$ & $\begin{array}{l}\text { CANBER } \\
\text { CHRY W } \\
\text { MC26 }\end{array}$ & $\begin{array}{l}\text { I1060 } \\
\text { I1068 } \\
\text { I970 }\end{array}$ & $\begin{array}{l}5 \\
5 \\
5\end{array}$ & $\begin{array}{l}1979 \\
1976 \\
1973\end{array}$ & $\begin{array}{l}36 \\
36 \\
68\end{array}$ & $\begin{array}{l}\text { AA } \\
\text { AA } \\
\text { AA }\end{array}$ & $\begin{array}{l}\text { Soviet launch site. } \\
\text { Town in Russia. } \\
\text { Theodore; American } \\
\text { aeronautical engineer } \\
\text { (1881-1963). }\end{array}$ \\
\hline $\begin{array}{l}\text { Voo } \\
\text { Voza } \\
\text { Wabash } \\
\text { Wahoo } \\
\text { Wajir } \\
\text { Wallace }\end{array}$ & $\begin{array}{l}27.3 \mathrm{~S} \\
23.5 \mathrm{~N} \\
21.5 \mathrm{~N} \\
23.5 \mathrm{~N} \\
27.2 \mathrm{~S} \\
52.8 \mathrm{~S}\end{array}$ & $\begin{array}{r}19.8 \mathrm{~W} \\
53.5 \mathrm{~W} \\
33.7 \mathrm{~W} \\
33.6 \mathrm{~W} \\
254.5 \mathrm{~W} \\
249.2 \mathrm{~W}\end{array}$ & $\begin{array}{r}2 \\
0 \\
42 \\
66 \\
0 \\
159\end{array}$ & $\begin{array}{l}\text { AF } \\
\text { OC } \\
\text { US } \\
\text { US } \\
\text { AF } \\
\text { EU }\end{array}$ & $\begin{array}{l}\text { KY } \\
\text { SO } \\
\text { IN } \\
\text { NE } \\
\text { KY } \\
\text { GB }\end{array}$ & $\begin{array}{l}\text { ERYTHR } \\
\text { CHRYSE } \\
\text { CHRYSE } \\
\text { MC28 }\end{array}$ & $\begin{array}{l}\text { I947 } \\
\text { I939 } \\
\text { I939 } \\
\text { I2063 } \\
\text { I1169 }\end{array}$ & $\begin{array}{l}5 \\
5 \\
5 \\
5 \\
5 \\
5\end{array}$ & $\begin{array}{l}1976 \\
1988 \\
1976 \\
1976 \\
1989 \\
1973\end{array}$ & $\begin{array}{l}36 \\
36 \\
36 \\
36 \\
74 \\
68\end{array}$ & $\begin{array}{l}\text { AA } \\
\text { AA } \\
\text { AA } \\
\text { AA } \\
\text { AA } \\
\text { AA }\end{array}$ & $\begin{array}{l}\text { Town in Kenya. } \\
\text { Town in Solomon Islands. } \\
\text { Town in Indiana, USA. } \\
\text { Town in Nebraska, USA. } \\
\text { Town in Kenya. } \\
\text { Alfred R.; British } \\
\text { biologist (1823-1913). }\end{array}$ \\
\hline $\begin{array}{l}\text { Wallops } \\
\text { Warra } \\
\text { Waspam } \\
\text { Wassamu } \\
\text { Wau } \\
\text { Weer } \\
\text { Wegener }\end{array}$ & $\begin{array}{l}46.9 \mathrm{~N} \\
20.8 \mathrm{~N} \\
20.7 \mathrm{~N} \\
25.5 \mathrm{~N} \\
45.2 \mathrm{~S} \\
19.8 \mathrm{~N} \\
64.3 \mathrm{~S}\end{array}$ & $\begin{array}{r}227.2 \mathrm{~W} \\
37.5 \mathrm{~W} \\
56.6 \mathrm{~W} \\
53.0 \mathrm{~W} \\
42.4 \mathrm{~W} \\
51.4 \mathrm{~W} \\
4.0 \mathrm{~W}\end{array}$ & $\begin{array}{r}2 \\
11 \\
40 \\
17 \\
3 \\
9 \\
70\end{array}$ & $\begin{array}{l}\text { NA } \\
\text { OC } \\
\text { SA } \\
\text { AS } \\
\text { OC } \\
\text { EU } \\
\text { EU }\end{array}$ & $\begin{array}{l}\text { AM } \\
\text { AU } \\
\text { NI } \\
\text { JA } \\
\text { GU } \\
\text { DU } \\
\text { GE }\end{array}$ & $\begin{array}{l}\text { CANBER } \\
\text { CHRYSE } \\
\text { CHRY W } \\
\text { CHRY W } \\
\text { NEREID } \\
\text { CHRY W } \\
\text { MC26 }\end{array}$ & $\begin{array}{l}\mathrm{I} 1060 \\
\mathrm{I} 939 \\
\mathrm{I} 1068 \\
\mathrm{I} 1068 \\
\mathrm{I} 957 \\
\mathrm{I} 1068 \\
\mathrm{I} 985\end{array}$ & $\begin{array}{l}5 \\
5 \\
5 \\
5 \\
5 \\
5 \\
5\end{array}$ & $\begin{array}{l}1979 \\
1976 \\
1976 \\
1976 \\
1976 \\
1976 \\
1973\end{array}$ & $\begin{array}{l}36 \\
36 \\
36 \\
36 \\
36 \\
36 \\
68\end{array}$ & $\begin{array}{l}\text { AA } \\
\text { AA } \\
\text { AA } \\
\text { AA } \\
\text { AA } \\
\text { AA } \\
\text { AA }\end{array}$ & $\begin{array}{l}\text { American launch site. } \\
\text { Town in Australia. } \\
\text { Town in Nicaragua. } \\
\text { Town in Japan. } \\
\text { Town in New Guinea. } \\
\text { Town in the Netherlands. } \\
\text { Alfred L.; German }\end{array}$ \\
\hline Weinbaum & $65.9 \mathrm{~S}$ & $245.5 \mathrm{~W}$ & 86 & NA & AM & MC30 & I970 & 5 & 1973 & 68 & AA & $\begin{array}{l}\text { Stanley G.; American } \\
\text { novelist (1902-1935). }\end{array}$ \\
\hline Wells & $60.1 \mathrm{~S}$ & $237.4 \mathrm{~W}$ & 94 & $\mathrm{EU}$ & GB & MC29 & I1170 & 5 & 1973 & 68 & AA & $\begin{array}{l}\text { Herbert G.; British } \\
\text { novelist (1866-1946). }\end{array}$ \\
\hline $\begin{array}{l}\text { Wer } \\
\text { Wicklow } \\
\text { Wien }\end{array}$ & $\begin{array}{r}45.6 \mathrm{~N} \\
2.0 \mathrm{~S} \\
10.5 \mathrm{~S}\end{array}$ & $\begin{array}{r}6.2 \mathrm{~W} \\
40.7 \mathrm{~W} \\
220.1 \mathrm{~W}\end{array}$ & $\begin{array}{r}3 \\
21 \\
105\end{array}$ & $\begin{array}{l}\text { AS } \\
\text { EU } \\
\text { EU }\end{array}$ & $\begin{array}{l}\text { IN } \\
\text { IR } \\
\text { GE }\end{array}$ & $\begin{array}{l}\text { CYDOHR } \\
\text { CAPRI } \\
\text { MC23 }\end{array}$ & $\begin{array}{l}\text { I990 } \\
\text { I1026 } \\
\text { I1001 }\end{array}$ & $\begin{array}{l}5 \\
5 \\
5\end{array}$ & $\begin{array}{l}1976 \\
1976 \\
1976\end{array}$ & $\begin{array}{l}36 \\
36 \\
68\end{array}$ & $\begin{array}{l}\text { AA } \\
\text { AA } \\
\text { AA }\end{array}$ & $\begin{array}{l}\text { Town in India. } \\
\text { Town in Ireland. } \\
\text { Wilhelm; German physicist } \\
(1864-1928) \text {. }\end{array}$ \\
\hline
\end{tabular}

\footnotetext{
lat: latitude of feature center. et: ethnicity of name origin (see page $284 \mathrm{ff}$.) long: longitude of feature center. quad: map quadrangle or informal name (see page xvii ff.).

ct: continent of name origin (see page $284 \mathrm{ff}$.) map: map name or USGS map number (see page xvii ff.). ft: feature type (see page 290).
} 


\section{MARS}

\begin{tabular}{|c|c|c|c|c|c|c|c|c|c|c|c|c|}
\hline Name & lat & long & diam & ct & et & quad & map & as & ad & ref & $\mathbf{f t}$ & origin \\
\hline Williams & $18.8 \mathrm{~S}$ & $164.1 \mathrm{~W}$ & 125 & EU & GB & $\mathrm{MCl} 16$ & I1554 & 5 & 1973 & 68 & AA & Arthur S.; British \\
\hline Wilmington & $21.9 \mathrm{~N}$ & $47.5 \mathrm{~W}$ & 1 & US & $\mathrm{DE}$ & $\mathrm{MCl} 10$ & I1059 & 5 & 1979 & 36 & AA & $\begin{array}{l}\text { American colonial town } \\
\text { (Delaware). }\end{array}$ \\
\hline $\begin{array}{l}\text { Windfall } \\
\text { Wink } \\
\text { Wirtz }\end{array}$ & $\begin{array}{r}2.1 S \\
6.6 S \\
48.7 S\end{array}$ & $\begin{array}{l}43.5 \mathrm{~W} \\
41.5 \mathrm{~W} \\
25.8 \mathrm{~W}\end{array}$ & $\begin{array}{r}20 \\
8 \\
128\end{array}$ & $\begin{array}{l}\text { NA } \\
\text { US } \\
\text { EU }\end{array}$ & $\begin{array}{l}\text { CA } \\
\text { TX } \\
\text { GE }\end{array}$ & $\begin{array}{l}\text { CAPRI } \\
\text { CAPRI } \\
\text { MC26 }\end{array}$ & $\begin{array}{l}\mathrm{I} 1026 \\
\mathrm{I} 1026 \\
\mathrm{I} 985\end{array}$ & $\begin{array}{l}5 \\
5 \\
5\end{array}$ & $\begin{array}{l}1976 \\
1976 \\
1973\end{array}$ & $\begin{array}{l}36 \\
36 \\
68\end{array}$ & $\begin{array}{l}\text { AA } \\
\text { AA } \\
\text { AA }\end{array}$ & $\begin{array}{l}\text { Town in Álberta, Canada. } \\
\text { Town in Texas, USA. } \\
\text { Carl Wilhelm; German } \\
\text { astronomer (1876-1939). }\end{array}$ \\
\hline Wislicenus & $18.3 \mathrm{~S}$ & $348.7 W$ & 138 & EU & GE & MC20 & I1296 & 5 & 1973 & 68 & AA & $\begin{array}{l}\text { Walter; German astronomer } \\
(1859-1905) .\end{array}$ \\
\hline $\begin{array}{l}\text { Woolgar } \\
\text { Woomera } \\
\text { Worcester } \\
\text { Wright }\end{array}$ & $\begin{array}{l}34.8 \mathrm{~N} \\
48.4 \mathrm{~N} \\
26.8 \mathrm{~N} \\
58.6 \mathrm{~S}\end{array}$ & $\begin{array}{r}85.5 \mathrm{~W} \\
227.3 \mathrm{~W} \\
50.3 \mathrm{~W} \\
150.8 \mathrm{~W}\end{array}$ & $\begin{array}{r}0 \\
3 \\
0 \\
106\end{array}$ & $\begin{array}{l}\text { OC } \\
\text { OC } \\
\text { US } \\
\text { NA }\end{array}$ & $\begin{array}{l}\text { AU } \\
\text { AU } \\
\text { NY } \\
\text { AM }\end{array}$ & $\begin{array}{l}35087 \\
\text { CANBER } \\
25052 \\
\text { MC24 }\end{array}$ & $\begin{array}{l}\text { I1060 } \\
\text { I1869 } \\
\text { I1166 }\end{array}$ & $\begin{array}{l}5 \\
5 \\
5 \\
5\end{array}$ & $\begin{array}{l}1991 \\
1979 \\
1988 \\
1973\end{array}$ & $\begin{array}{l}36 \\
36 \\
91 \\
68\end{array}$ & $\begin{array}{l}\text { AA } \\
\text { AA } \\
\text { AA } \\
\text { AA }\end{array}$ & $\begin{array}{l}\text { Town in Australia. } \\
\text { Australian launch site. } \\
\text { Town in New York, USA. } \\
\text { William H.; American } \\
\text { astronomer (1871-1959). }\end{array}$ \\
\hline $\begin{array}{l}\text { Wukari } \\
\text { Wum } \\
\text { Xui } \\
\text { Yakima } \\
\text { Yala } \\
\text { Yalata } \\
\text { Yar } \\
\text { Yat } \\
\text { Yebra } \\
\text { Yegros } \\
\text { Yorktown }\end{array}$ & $\begin{array}{l}32.2 \mathrm{~S} \\
16.1 \mathrm{~N} \\
15.3 \mathrm{~N} \\
43.3 \mathrm{~N} \\
17.5 \mathrm{~N} \\
22.1 \mathrm{~N} \\
22.5 \mathrm{~N} \\
18.3 \mathrm{~N} \\
21.1 \mathrm{~N} \\
22.5 \mathrm{~S} \\
23.1 \mathrm{~N}\end{array}$ & $\begin{array}{r}102.8 \mathrm{~W} \\
246.2 \mathrm{~W} \\
247.6 \mathrm{~W} \\
3.2 \mathrm{~W} \\
38.5 \mathrm{~W} \\
254.0 \mathrm{~W} \\
39.1 \mathrm{~W} \\
29.1 \mathrm{~W} \\
254.4 \mathrm{~W} \\
23.5 \mathrm{~W} \\
48.7 \mathrm{~W}\end{array}$ & $\begin{array}{r}0 \\
0 \\
0 \\
12 \\
19 \\
0 \\
5 \\
8 \\
0 \\
14 \\
8\end{array}$ & $\begin{array}{l}\text { AF } \\
\text { AF } \\
\text { SA } \\
\text { US } \\
\text { AS } \\
\text { OC } \\
\text { UR } \\
\text { AF } \\
\text { EU } \\
\text { SA } \\
\text { US }\end{array}$ & $\begin{array}{l}\text { NI } \\
\text { CR } \\
\text { BR } \\
\text { WA } \\
\text { TH } \\
\text { AU } \\
\text { RU } \\
\text { NG } \\
\text { SP } \\
\text { PA } \\
\text { VA }\end{array}$ & $\begin{array}{l}\text { CYDONI } \\
\text { CHRYSE } \\
\text { CHRYSE } \\
\text { CHRYSE } \\
\text { ERYTHR } \\
\text { CHRY W }\end{array}$ & $\begin{array}{l}\text { I946 } \\
\text { I939 } \\
\text { I939 } \\
\text { I939 } \\
\\
\text { I947 } \\
\text { I1068 }\end{array}$ & $\begin{array}{l}5 \\
5 \\
5 \\
5 \\
5 \\
5 \\
5 \\
5 \\
5 \\
5 \\
5\end{array}$ & $\begin{array}{l}1989 \\
1988 \\
1988 \\
1976 \\
1976 \\
1988 \\
1976 \\
1976 \\
1988 \\
1976 \\
1976\end{array}$ & $\begin{array}{l}74 \\
36 \\
36 \\
36 \\
36 \\
36 \\
36 \\
36 \\
36 \\
36 \\
36\end{array}$ & $\begin{array}{l}\text { AA } \\
\text { AA } \\
\text { AA } \\
\text { AA } \\
\text { AA } \\
\text { AA } \\
\text { AA } \\
\text { AA } \\
\text { AA } \\
\text { AA } \\
\text { AA }\end{array}$ & $\begin{array}{l}\text { Town in Nigeria. } \\
\text { Town in Cameroon. } \\
\text { Town in Brazil. } \\
\text { Town in Washington, USA. } \\
\text { Town in Thailand. } \\
\text { Town in Australia. } \\
\text { Town in Russia. } \\
\text { Town in Niger. } \\
\text { Town in Spain. } \\
\text { Town in Paraguay. } \\
\text { American colonial town } \\
\text { (Virginia). }\end{array}$ \\
\hline $\begin{array}{l}\text { Yoro } \\
\text { Yungay } \\
\text { Yuty } \\
\text { Zhigou } \\
\text { Zilair } \\
\text { Zir } \\
\text { Zongo } \\
\text { Zulanka } \\
\text { Zuni }\end{array}$ & $\begin{array}{l}23.0 \mathrm{~N} \\
44.3 \mathrm{~S} \\
22.4 \mathrm{~N} \\
29.5 \mathrm{~S} \\
32.0 \mathrm{~S} \\
18.7 \mathrm{~N} \\
32.0 \mathrm{~S} \\
2.3 \mathrm{~S} \\
19.3 \mathrm{~N}\end{array}$ & $\begin{array}{c}28.0 \mathrm{~W} \\
44.6 \mathrm{~W} \\
34.1 \mathrm{~W} \\
102.5 \mathrm{~W} \\
33.0 \mathrm{~W} \\
36.6 \mathrm{~W} \\
42.0 \mathrm{~W} \\
42.3 \mathrm{~W} \\
29.6 \mathrm{~W}\end{array}$ & $\begin{array}{r}8 \\
17 \\
18 \\
0 \\
43 \\
6 \\
23 \\
47 \\
25\end{array}$ & $\begin{array}{l}\text { SA } \\
\text { SA } \\
\text { SA } \\
\text { AS } \\
\text { UR } \\
\text { AS } \\
\text { AF } \\
\text { UR } \\
\text { US }\end{array}$ & $\begin{array}{l}\text { HO } \\
\text { PE } \\
\text { PA } \\
\text { CH } \\
\text { RU } \\
\text { TU } \\
\text { ZA } \\
\text { RU } \\
\text { NM }\end{array}$ & $\begin{array}{l}\text { CHRYSE } \\
\text { NEREID } \\
\text { CHRYSE } \\
\text { SOVIET } \\
\text { CHRYSE } \\
\text { SOVIET } \\
\text { CAPRI } \\
\text { CHRYSE }\end{array}$ & $\begin{array}{l}\text { I939 } \\
\text { I957 } \\
I 939 \\
I 2061 \\
11264 \\
1939 \\
I 1264 \\
I 1026 \\
1939\end{array}$ & $\begin{array}{l}5 \\
5 \\
5 \\
5 \\
5 \\
5 \\
5 \\
5 \\
5\end{array}$ & $\begin{array}{l}1976 \\
1976 \\
1976 \\
1989 \\
1979 \\
1976 \\
1979 \\
1976 \\
1976\end{array}$ & $\begin{array}{l}36 \\
36 \\
36 \\
74 \\
36 \\
36 \\
36 \\
36 \\
36\end{array}$ & $\begin{array}{l}\text { AA } \\
\text { AA } \\
\text { AA } \\
\text { AA } \\
\text { AA } \\
\text { AA } \\
\text { AA } \\
\text { AA } \\
\text { AA }\end{array}$ & $\begin{array}{l}\text { Town in Honduras. } \\
\text { Town in Peru. } \\
\text { Town in Paraguay. } \\
\text { Town in China. } \\
\text { Town in Russia. } \\
\text { Town in Turkey. } \\
\text { Town in Zaire. } \\
\text { Town in Russia. } \\
\text { Town in New Mexico, USA }\end{array}$ \\
\hline \multicolumn{13}{|c|}{ ALBEDO FEATURE } \\
\hline Mare Acidalium & $45.0 \mathrm{~N}$ & $30.0 \mathrm{~W}$ & 0 & EU & GR & MC-4 & & 5 & 1958 & 60 & $\mathrm{AL}$ & $\begin{array}{l}\text { Name for Acidalian } \\
\text { (Venusian) fountain in } \\
\text { Boeotia where the Graces } \\
\text { bathed. }\end{array}$ \\
\hline Aeolis & $5.0 \mathrm{~S}$ & $215.0 \mathrm{~W}$ & 0 & EU & GR & MC-23 & & 5 & 1958 & 60 & $\mathrm{AL}$ & $\begin{array}{l}\text { Floating island where } \\
\text { winds were kept in a cave. }\end{array}$ \\
\hline Aeria & $10.0 \mathrm{~N}$ & $310.0 \mathrm{~W}$ & 0 & EU & GR & & & 5 & 1958 & 60 & $\mathrm{AL}$ & $\begin{array}{l}\text { Greek name for Egypt; } \\
\text { "far land of mist." }\end{array}$ \\
\hline Aetheria & $40.0 \mathrm{~N}$ & $230.0 \mathrm{~W}$ & 0 & EU & GR & & & 5 & 1958 & 60 & $\mathrm{AL}$ & $\begin{array}{l}\text { Upper world; land of } \\
\text { living. }\end{array}$ \\
\hline Aethiopis & $10.0 \mathrm{~N}$ & $230.0 \mathrm{~W}$ & 0 & EU & GR & & & 5 & 1958 & 60 & $\mathrm{AL}$ & $\begin{array}{l}\text { Countries of the } \\
\text { Ethiopians on southern } \\
\text { edge of the Earth. }\end{array}$ \\
\hline Amazonis & $0.0 \mathrm{~N}$ & $140.0 \mathrm{~W}$ & 0 & EU & GR & & & 5 & 1958 & 60 & $\mathrm{AL}$ & $\begin{array}{l}\text { Land of the Amazons; on } \\
\text { the island Hesperia. }\end{array}$ \\
\hline Amenthes & $5.0 \mathrm{~N}$ & $250.0 \mathrm{~W}$ & 0 & AF & EG & MC-14 & & 5 & 1958 & 60 & $\mathrm{AL}$ & $\begin{array}{l}\text { Egyptian name for place } \\
\text { where souls of the dead go. }\end{array}$ \\
\hline Aonium Sinus & $45.0 \mathrm{~S}$ & $105.0 \mathrm{~W}$ & 0 & EU & LA & & & 5 & 1958 & 60 & $\mathrm{AL}$ & $\begin{array}{l}\text { Named for Aonides, or } \\
\text { Muses. }\end{array}$ \\
\hline Arabia & $20.0 \mathrm{~N}$ & $330.0 \mathrm{~W}$ & 0 & EU & LA & MC-12 & & 5 & 1958 & 60 & $\mathrm{AL}$ & $\begin{array}{l}\text { Country bordering on } \\
\text { Aeria (Egypt). }\end{array}$ \\
\hline Arcadia & $45.0 \mathrm{~N}$ & $100.0 \mathrm{~W}$ & 0 & EU & GR & MC-3 & & 5 & 1958 & 60 & $\mathrm{AL}$ & $\begin{array}{l}\text { Mountainous region in } \\
\text { southern Greece. }\end{array}$ \\
\hline Argyre & $45.0 \mathrm{~S}$ & $25.0 \mathrm{~W}$ & 0 & EU & GR & MC-26 & & 5 & 1958 & 60 & $\mathrm{AL}$ & $\begin{array}{l}\text { "Silver" Island at mouth } \\
\text { of Ganges River; } \\
\text { present-day Arakan, Burma. }\end{array}$ \\
\hline Arnon & $48.0 \mathrm{~N}$ & $335.0 \mathrm{~W}$ & 0 & EU & GR & & & 5 & 1958 & 60 & $\mathrm{AL}$ & $\begin{array}{l}\text { Classical name for present } \\
\text { El-Mojib River, Jordan. }\end{array}$ \\
\hline Aurorae Sinus & $15.0 \mathrm{~S}$ & $50.0 \mathrm{~W}$ & 0 & EU & LA & & & 5 & 1958 & 60 & $\mathrm{AL}$ & $\begin{array}{l}\text { "Bay of Rosy Dawn"; } \\
\text { Aurora held back the dawn. }\end{array}$ \\
\hline Ausonia & $40.0 \mathrm{~S}$ & $250.0 \mathrm{~W}$ & 0 & $\mathrm{EU}$ & GR & & & 5 & 1958 & 60 & $\mathrm{AL}$ & $\begin{array}{l}\text { Country of the Aruncii } \\
\text { (Ausones in Greek). }\end{array}$ \\
\hline Mare Australe & $60.0 \mathrm{~S}$ & $10.0 \mathrm{~W}$ & 0 & EU & LA & & & 5 & 1958 & 60 & $\mathrm{AL}$ & "South Sea" \\
\hline
\end{tabular}

\footnotetext{
lat: latitude of feature center.

et: ethnicity of name origin (see page $284 \mathrm{ff}$.)

as: name approval status (see page xvii).

long: longitude of feature center.

quad: map quadrangle or informal name

ad: name approval date (year).

diam: diameter or long dimension of feature.

(see page xvii ff.).

ref: reference source for name (see page $287 \mathrm{ff}$.).

ct: continent of name origin (see page $284 \mathrm{ff}$.) map: map name or USGS map number (see page xvii ff.). ft: feature type (see page 290 ).
} 
MARS

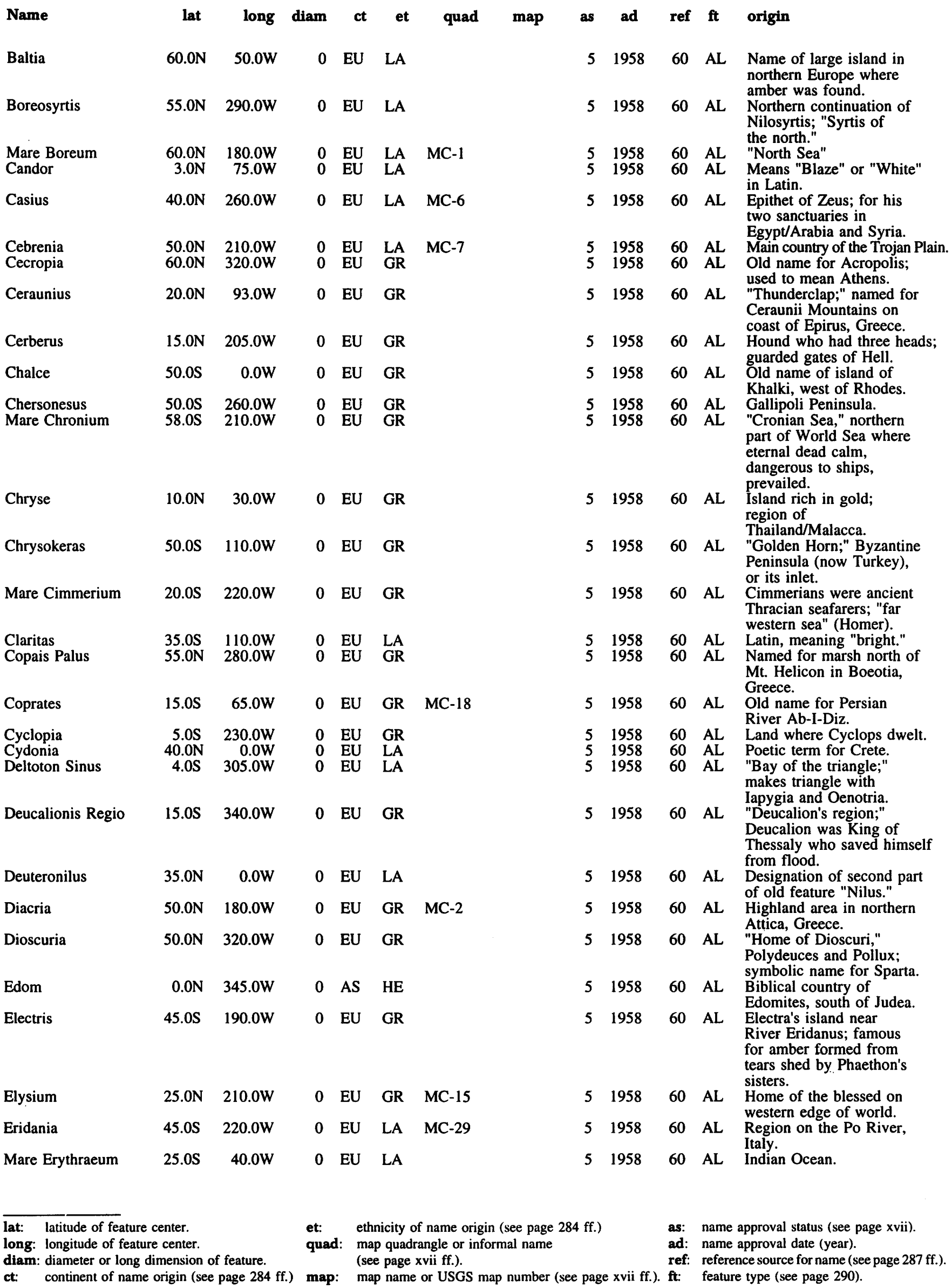


MARS

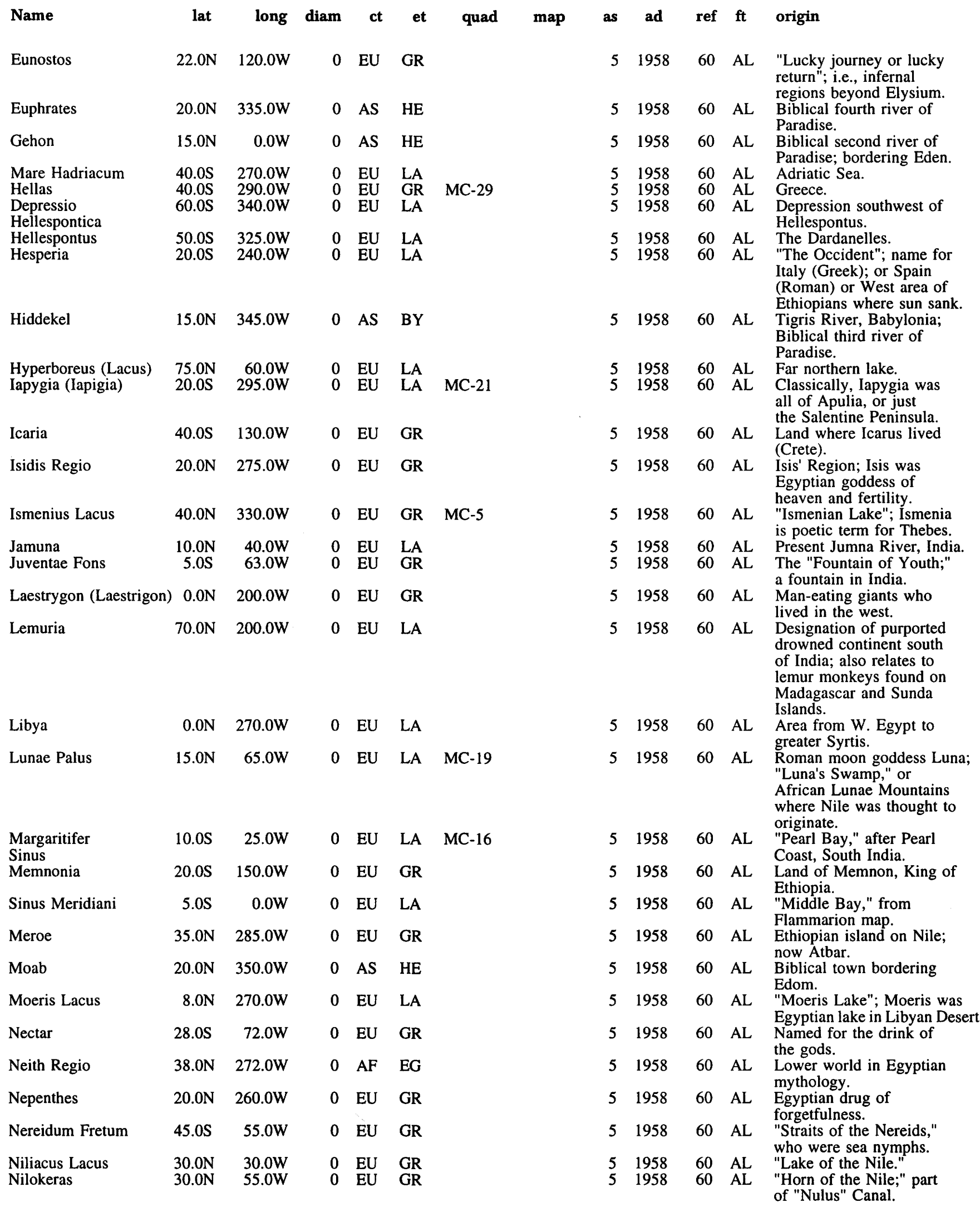

lat: latitude of feature center.

long: longitude of feature center.

diam: diameter or long dimension of feature.

ct: continent of name origin (see page $284 \mathrm{ff}$ ) et: ethnicity of name origin (see page $284 \mathrm{ff}$.)

quad: map quadrangle or informal name

(see page $x$ vii ff.).

map name or USGS map number (see page xvii ff.). ft: feature type (see page 290). as: name approval status (see page $x v i i$ )

ad: name approval date (year).

ref: reference source for name (see page $287 \mathrm{ff}$.). 


\section{MARS}

Name

Nilosyrtis

Nix Olympica

Noachis

Ogygis Regio

Olympia

Ophir

Ortygia

Oxia Palus

Oxus

Panchaia

$20.0 \mathrm{~N} \quad 12.0 \mathrm{~W}$

$60.0 \mathrm{~N} \quad 200.0 \mathrm{~W}$

$25.0 \mathrm{~S} \quad 316.0 \mathrm{~W}$

$0 \quad$ EU LA

Pandorae Fretum

$50.0 \mathrm{~S} \quad 155.0 \mathrm{~W}$

0 EU

LA

MC-24

Phison

Phlegra

$20.0 \mathrm{~N} \quad 320.0 \mathrm{~W}$

0 AS HE

$31.3 \mathrm{~N} \quad 187.8 \mathrm{~W}$

134 EU GR

Phoenicis Lacus

$12.0 \mathrm{~S} \quad 110.0 \mathrm{~W}$

0 EU LA MC-17

Phrixi Regio

40.0S $\quad 70.0 \mathrm{~W}$

0 EU LA

Promethei Sinus

65.0S 280.0W

0 EU GR

Propontis

Protei Regio

$45.0 \mathrm{~N} \quad 185.0 \mathrm{~W}$

0 EU GR

Protonilus

23.0

$50.0 \mathrm{~W}$

0 EU LA

$42.0 \mathrm{~N} \quad 315.0 \mathrm{~W}$

0 EU LA

Pyrrhae Regio

$15.0 \mathrm{~S} \quad 38.0 \mathrm{~W}$

0 EU LA

Sinus Sabaeus

$8.0 \mathrm{~S} \quad 340.0 \mathrm{~W}$

0 EU LA MC-20

Scandia

Mare Serpentis

$60.5 \mathrm{~N} \quad 147.9 \mathrm{~W}$

30.0S 320.0W

0 EU LA

Sinai

$20.0 \mathrm{~S} \quad 70.0 \mathrm{~W}$

0 AF $\mathrm{EG}$

Mare Sirenum

$30.0 \mathrm{~S} \quad 155.0 \mathrm{~W}$
0 EU GR quad map as ad ref $f t$ origin

$5 \quad 1958 \quad 60 \quad$ AL $\quad$ Syrtis of the Nile; part

of Nilus (Nile) Canal.

$51958 \quad 60$ AL "Snows of Olympus;"

Olympus was mountain home of gods in Greece.

$51958 \quad 60 \quad \mathrm{AL} \quad$ Biblical; "Noah's (Region)."

$51958 \quad 60 \quad \mathrm{AL} \quad$ "Region of Ogygos;" Ogygos was ancient King of Thebes or Athens, Greece.

$5 \quad 1958 \quad 60 \quad \mathrm{AL} \quad$ Ancient and modern Greek

$5 \quad 1958 \quad 60$ city.

Land to which King

Solomon sent naval a expedition; probably India.

$5 \quad 1958 \quad 60 \quad \mathrm{AL} \quad$ Floating island (present Delos) where Leto bore Apollo and Artemis.

$51958 \quad 60 \quad$ AL Lake (swamp into which Oxus River flows; i.e., Sea of Aral).

$51958 \quad 60 \quad$ AL Present Amu-Darya River.

$\begin{array}{lllll}5 & 1958 & 60 & \mathrm{AL} & \text { Situated near }\end{array}$

Heliopolis, Egypt; or island in Red Sea rich in frankincense, gold, silver, thus Utopia.

$5 \quad 1958 \quad 60 \quad \mathrm{AL}$ Woman who let loose evils of world when she opened a box.

5195860 AL "Of Phaethon"; who recklessly drove Chariot of the Sun.

$\begin{array}{llll}5 & 1958 \quad 60 & \mathrm{AL} & \text { Biblical first river of }\end{array}$

5195860 Paradise.

$51958 \quad 60$ AL "Burning plain"; in Chalcidian Peninsula of Greece where Zeus hurled thunderbolts at Titans to support Hercules.

5195860 AL "Lake of the Phoenix"; Arabia or India.

5195860 AL "Phrixus' Region"; Phrixus and sister Helle escaped sacrifice in Boeotia on back of ram with golden fleece.

5195860 AL Prometheus' Bay; Greek mythological character.

$51958 \quad 60 \quad$ AL The Sea of Marmora, Asia Minor.

$51958 \quad 60$ AL "Proteus' Region"; Proteus was a sea god with the gift of prophecy.

$51958 \quad 60 \quad$ AL Designation of first (Eastern) part of "Nilus" or Nile Canal.

$51958 \quad 60 \quad \mathrm{AL} \quad$ "Pyrrha's Region"; named for Pyrrha, wife of Deucalion.

$51958 \quad 60 \quad$ AL Today's Red Sea; Saba was part of southern Arabian peninsula.

$5 \quad 1958 \quad 60 \quad$ AL Southern Scandinavia.

$5 \quad 1958 \quad 60 \quad \mathrm{AL} \quad$ Named for Constellation

$5 \quad 1958 \quad 60 \quad$ AL $\quad \begin{aligned} & \text { Serpens (the snake). } \\ & \text { Biblical; named for area }\end{aligned}$ on Mars next to Mare Erythraeum (Indian Ocean).

5195860 AL "Sea of the Sirens." lat: latitude of feature center.

long: longitude of feature center.

diam: diameter or long dimension of feature.

ct: continent of name origin (see page 284 et: ethnicity of name origin (see page $284 \mathrm{ff}$.)

quad: map quadrangle or informal name

(see page xvii ff.).

map: map name or USGS map number (see page xvii ff.). ft: feature type (see page 290).

as: name approval status (see page $\mathrm{xvii}$ ).

ad: name approval date (year).

ref: reference source for name (see page $287 \mathrm{ff}$.) 
MARS

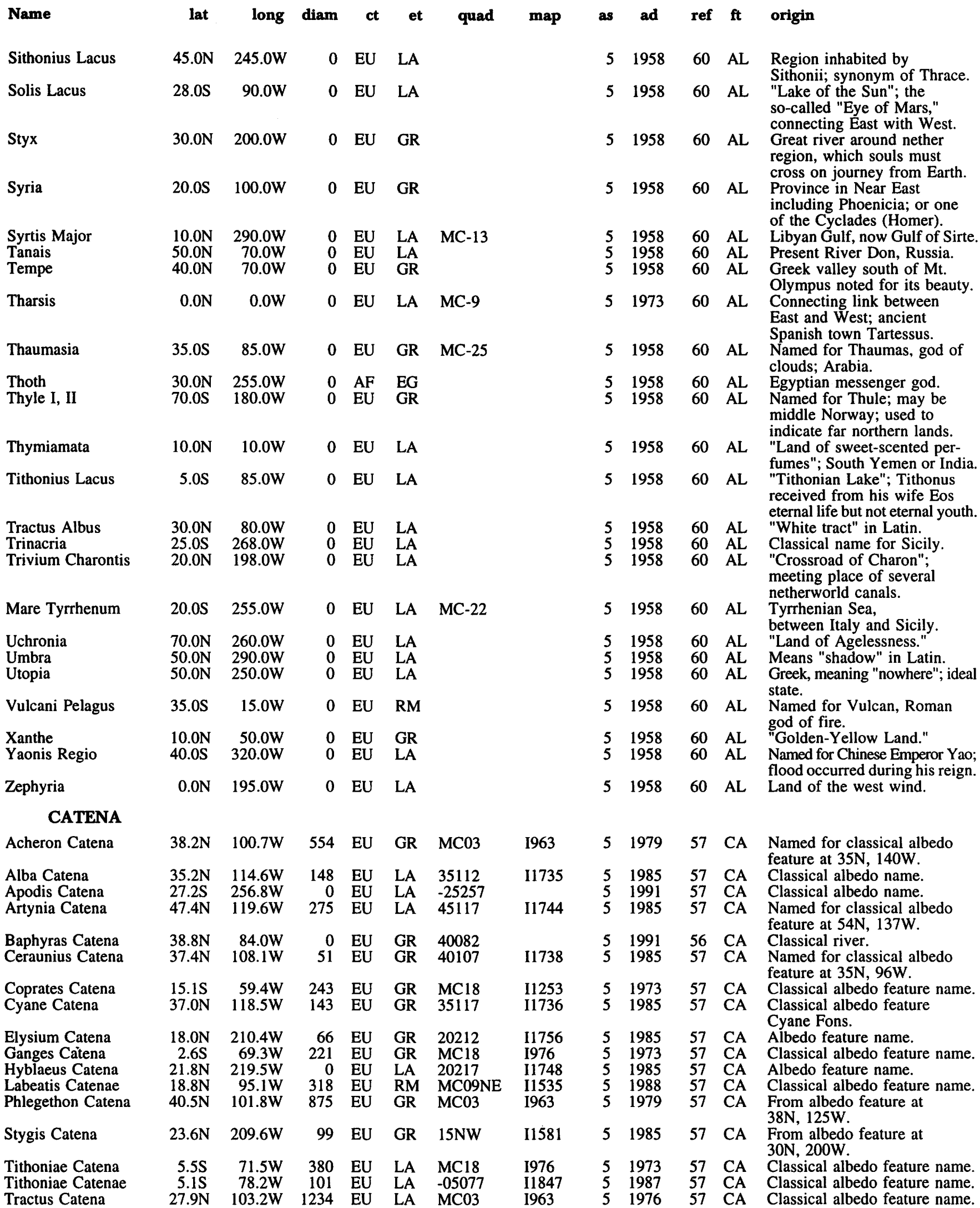

lat: latitude of feature center.

long: longitude of feature center.

diam: diameter or long dimension of feature.

ct: continent of name origin (see page $284 \mathrm{ff}$.) map: map name or USGS map number (see page xvii ff.). ft: feature type (see page 290).

et: ethnicity of name origin (see page $284 \mathrm{ff}$.)

quad: map quadrangle or informal name

(see page xvii ff.). as: name approval status (see page $x$ vii).

ad: name approval date (year).

ref: reference source for name (see page $287 \mathrm{ff}$.). 
MARS

Name

lat

long diam ct et quad

map

\section{CAVUS}

Argyre Cavi

Ausonia Cavus

Avernus Cavi

Cavi Angusti

Cavi Frigorēs

[Cavi Novi]

Hyperborei Cavi

Octantis Cavi

Peraea Cavus

Sisyphi Cavi

[Ultimi Cavi]

\section{CHAOS}

Aram Chaos

Aromatum Chaos

Arsinoes Chaos

Atlantis Chaos

Aureum Chaos

Aurorae Chaos

Candor Chaos

Echus Chaos

Eos Chaos

Galaxias Chaos

Gorgonum Chaos

$30.0 \mathrm{~S}$

264.5E

35 EU LA

$49.1 \mathrm{~S} \quad 40.2 \mathrm{~W}$

$32.2 \mathrm{~S} \quad 262.5 \mathrm{~W}$

$187.4 \mathrm{~W}$

0 EU GR -50036

0 EU LA I2040

$75.9 \mathrm{~S} \quad 71.9 \mathrm{~W}$

60 EU LA -05187

I-2253

$5 \quad 1994$

$71 \mathrm{CB}$

$5 \quad 1991$

51989

I1785

51985

$57 \mathrm{CB}$

57 CB

57 CB

51976

$80.1 \mathrm{~S} \quad 67.9 \mathrm{~W}$

294 EU

GR MC30AB

I1647

I1858

$\begin{array}{ll}5 & 1988\end{array}$

$\begin{array}{ll}57 & \text { CB } \\ 57 & \mathrm{CB}\end{array}$

\section{$67.5 \mathrm{~S} \quad 330.0 \mathrm{~W}$}

535 EU GR MC30J

1970

61976

57 CB

$79.6 \mathrm{~N} 52.5 \mathrm{~W}$

$52.7 \mathrm{~S}$

29.9S

$71.9 \mathrm{~S}$

$45.6 \mathrm{~W}$

$74.5 \mathrm{~S}$

$264.7 \mathrm{~W}$

$3.0 \mathrm{~W}$

$\begin{array}{rr}0 & \mathrm{EU} \\ 0 & \mathrm{EU} \\ 0 & \mathrm{EU} \\ 362 & \mathrm{EU}\end{array}$

RM 80050

I1836

I-2252

204.5W
LA $\quad-55043$

GR I2040

GR MC30

I1633

1970 $\begin{array}{ll}5 & 1988\end{array}$

$\begin{array}{ll}5 & 1991\end{array}$

51989

51976

$\begin{array}{ll}6 & 1984\end{array}$
57 CB

$57 \mathrm{CB}$

57 CB

57 CB

57 CB
$2.7 \mathrm{~N} \quad 21.0 \mathrm{~W}$

$7.9 \mathrm{~S}$

$28.1 \mathrm{~W}$

34.8S $\quad 176.7 \mathrm{~W}$

388 EU

203 EU

GR

MC19

11551

I1293

I1293

$141 \mathrm{EU}$

GR MC24NW

I1166

4.1S $27.1 \mathrm{~W}$

$80.6 \mathrm{~S} \quad 34.5 \mathrm{~W}$

$7.2 \mathrm{~S} \quad 72.7 \mathrm{~W}$

468 EU

0 EU

LA

$45 \mathrm{EU}$

LA

CHRYPL

I1448

$9.9 \mathrm{~N} \quad 74.6 \mathrm{~W}$

371 EU

LA

05072

11592

51976

51979

$57 \mathrm{CH}$

$57 \mathrm{CH}$

51982

$57 \mathrm{CH}$

51985

$57 \mathrm{CH}$

51976

51991

$57 \mathrm{CH}$

$57 \mathrm{CH}$

51985

$57 \mathrm{CH}$

11722

$46.5 \mathrm{~N} \quad 105.5 \mathrm{~W}$

335 EU GR MC19

I1293

51985

$57 \mathrm{CH}$

51982

$57 \mathrm{CH}$

$34.0 \mathrm{~N} \quad 212.4 \mathrm{~W}$

0 EU

LA 35212

11771

51985

$57 \mathrm{CH}$

$57 \mathrm{CH}$
Named for albedo feature

Anseris Fons.

Albedo name.

Albedo feature Ausonia.

Named for albedo feature at $10 \mathrm{~S}, 195 \mathrm{~W}$.

Classical albedo feature name.

From classical albedo

feature Polus Frigoris, at $84 \mathrm{~S}, 30 \mathrm{~W}$.

Classical albedo feature

name; (dropped 1984).

Classical albedo feature name.

Albedo name.

Albedo feature name.

Classical albedo feature name.

See Thyles Rupes; name dropped 1984.
Hellas Chaos

Hydaspis Chaos

Hydraotes Chaos

Iani Chaos

Ister Chaos

Margaritifer Chaos

Nilus Chaos

Pyrrhae Chaos

\section{CHASMA}

Arsia Chasmata

Ascraeus Chasmata

Chasma Australe

Baetis Chasma

Chasma Boreale

Candor Chasma

Capri Chasma

Ceti Chasma

Coprates Chasma

Echus Chasma

Elysium Chasma

Eos Chasma

Gangis Chasma

Hebes Chasma

Hyblaeus Chasma

Hydrae Chasma
46.0S 293.0E 900 EU GR

1994

$71 \mathrm{CH}$

$3.4 \mathrm{~N} \quad 27.7 \mathrm{~W}$

$0.9 \mathrm{~N} \quad 34.3 \mathrm{~W}$

$2.3 \mathrm{~S} \quad 16.6 \mathrm{~W}$

$12.5 \mathrm{~N} \quad 56.5 \mathrm{~W}$

363 EU

290 EU

GR MCl1

MC11

11551

I1551

I975

57 EU LA 10057

I1695

9.1S $\quad 21.4 \mathrm{~W}$

500 EU LA MC19

280 EU GR 25077

I975

11786

I1293

$10.5 \mathrm{~S} \quad 28.6 \mathrm{~W}$

174 EU LA MC19

7.9S $119.4 \mathrm{~W}$

$8.7 \mathrm{~N} \quad 105.5 \mathrm{~W}$

$82.9 \mathrm{~S} \quad 273.8 \mathrm{~W}$

$4.3 \mathrm{~S} \quad 64.8 \mathrm{~W}$

$\begin{aligned} 0 & \text { EU } \\ 0 & \text { EU } \\ 491 & \text { EU }\end{aligned}$

LA

$-05117$

LA 10107

95 EU LA -05062

1970

I1648

51991

51991

$5 \quad 1976$

51976

51976

$\begin{array}{ll}5 & 1985\end{array}$

$57 \mathrm{CH}$

$57 \mathrm{CH}$

$57 \mathrm{CH}$

$57 \mathrm{CH}$

$\begin{array}{llll}5 & 1976 \quad 57 & \mathrm{CH}\end{array}$

$\begin{array}{ll}57 & \mathrm{CH} \\ 57 & \mathrm{CH}\end{array}$

1985

51982

$57 \mathrm{CH}$

$57 \mathrm{CM}$

$57 \mathrm{CM}$

57 CM

$57 \mathrm{CM}$

$\begin{array}{cc}83.2 \mathrm{~N} & 21.3 \mathrm{~W} \\ 6.5 \mathrm{~S} & 71.0 \mathrm{~W} \\ 8.7 \mathrm{~S} & 42.6 \mathrm{~W} \\ 5.2 \mathrm{~S} & 68.3 \mathrm{~W} \\ & \\ 13.6 \mathrm{~S} & 60.7 \mathrm{~W} \\ 3.7 \mathrm{~N} & 79.1 \mathrm{~W} \\ 22.2 \mathrm{~N} & 218.0 \mathrm{~W} \\ 12.6 \mathrm{~S} & 45.1 \mathrm{~W} \\ 8.4 \mathrm{~S} & 48.1 \mathrm{~W} \\ 1.1 \mathrm{~S} & 76.1 \mathrm{~W} \\ 22.1 \mathrm{~N} & 218.9 \mathrm{~W} \\ 6.9 \mathrm{~S} & 61.9 \mathrm{~W}\end{array}$

$318 \mathrm{EU}$

816 EU

LA MC01

1969

I1253

1498 EU LA MC18

45 EU GR -05067

1976

I1590

962 EU GR MC18

I1253

1971

613 EU LA MC10

92 EU GR 20217

963 EU GR MC19

I1748

1975

I1253

1976

I1748

285 EU GR MC18

71 EU LA 20217

49 EU LA -05062

I1678

et: ethnicity of name origin (see page $284 \mathrm{ff}$.) 


\section{MARS}

Name

Ius Chasma

Juventae Chasma

Melas Chasma

Ophir Chasma

Pavonis Chasma

[Thyles Chasma]

Tithonium Chasma

COLIES

Alpheus Colles

Arena Colles

Ariadnes Colles

Astapus Colles

Avernus Colles

Colles Nili

Deuteronilus Colles

Galaxias Colles

Oxia Colles

Scandia Colles

Tartarus Colles

Tempe Colles

\section{DORSUM}

Aesacus Dorsum

Arena Dorsum

Dorsa Argentea

Atrax Dorsum

Auxo Dorsum

Avernus Dorsa

Dorsa Brevia

Cerberus Dorsa

Charis Dorsum

Cleia Dorsum

Eumenides Dorsum

Felis Dorsa

Gordii Dorsum

Hegemone Dorsum

Hesperia Dorsa

Iamuna Dorsa

Juventae Dorsa

Melas Dorsa

Nilus Dorsa

Pasithea Dorsum

Phaenna Dorsum

[Pityusa Dorsa]

Sacra Dorsa

Sinai Dorsa

Solis Dorsa

Styx Dorsum

Tyrrhena Dorsa

Uranius Dorsum

Xanthe Dorsa

Zea Dorsa lat

\section{$7.2 \mathrm{~S}$}

$1.9 \mathrm{~S}$

$10.5 \mathrm{~S}$

$4.0 \mathrm{~S}$

$3.8 \mathrm{~N}$

$71.0 \mathrm{~S}$

4.6S

38.5

$300.6 \mathrm{~W}$

$24.3 \mathrm{~N} \quad 278.7 \mathrm{~W}$

$34.8 \mathrm{~S} \quad 188.0 \mathrm{~W}$

$33.7 \mathrm{~N} \quad 271.1 \mathrm{~W}$

$2.0 \mathrm{~S} \quad 188.5 \mathrm{~W}$

$39.6 \mathrm{~N} \quad 295.3 \mathrm{~W}$

RM 40297

42.2N 338.3W

$40.7 \mathrm{~N} \quad 209.0 \mathrm{~W}$

$20.2 \mathrm{~N}$

27.0W

$66.0 \mathrm{~N}-135.4 \mathrm{~W}$

$17.2 \mathrm{~N} \quad 188.2 \mathrm{~W}$

79 EU

291 EU

$433 \mathrm{EU}$

LA

A 35207

IC05SW I1495

1232 EU

GR MC11

I1770

I1551

$17.2 \mathrm{~N}$

$82.7 \mathrm{~W}$

EU

LA 1:15M

$0 \mathrm{EU}$

GR 35082

I1535

$33.9 \mathrm{~N}$

$38.5 \mathrm{~N} \quad 206.6 \mathrm{~W}$

$\begin{array}{llll}0 & \text { EU } & \text { GR } & 35207\end{array}$

$13.0 \mathrm{~N}$

$291.5 \mathrm{~W}$

$77.6 \mathrm{~S}$

$38.3 \mathrm{~N}$

$56.1 \mathrm{~S}$

$4.7 \mathrm{~S}$

$33.3 \mathrm{~W}$

$89.1 \mathrm{~W}$
$41.9 \mathrm{~W}$

$70.8 \mathrm{~S}$

$13.8 \mathrm{~S}$

189.1W

$55.8 \mathrm{~S}$

$300.3 \mathrm{~W}$

$55.3 \mathrm{~S}$

$254.4 \mathrm{~W}$

$41.2 \mathrm{~W}$

$5.7 \mathrm{~N} \quad 156.3 \mathrm{~W}$

$46.3 \mathrm{~W}$

$22.3 \mathrm{~S}$

$65.5 \mathrm{~W}$

$5.3 \mathrm{~N}$

$55.3 \mathrm{~S}$

23.0S

$144.5 \mathrm{~W}$

$44.9 \mathrm{~W}$

$245.0 \mathrm{~W}$

$50.3 \mathrm{~W}$

$1.3 \mathrm{~N}$

$72.6 \mathrm{~W}$

$17.8 \mathrm{~S}$

$70.9 \mathrm{~W}$

$22.3 \mathrm{~N}$

$79.4 \mathrm{~W}$

$55.7 \mathrm{~S} \quad 41.8 \mathrm{~W}$

$54.3 \mathrm{~S} \quad 43.2 \mathrm{~W}$

$64.5 \mathrm{~S} \quad 330.5 \mathrm{~W}$

205 EU

749 EU

0 EU

0 EU

$130 \mathrm{EU}$

LA $\mathrm{MC} 13$

LA MC30

GR 40087

$748 \quad \mathrm{EU}$

407 EU

0 EU

$\begin{array}{lll}\text { GR } & -55043 & \text { I2252 } \\ \text { LA } & \text { MC23NE } & \text { I1497 }\end{array}$

11770

1967

1970

0 EU

LA

MC30

I1497

I 970

599 EU

486 EU

624 EU

0 EU

0 EU

0 EU

GR

MC22NW I1468

I1 468

I 2252

$-55043$

I956

GR MC08

LA MC18

GR MC08

I1 253

I956

12252

I2065

325 EU

LA

$-55043$

I1599

$501 \mathrm{EU}$

GR $\quad 00072$

188 EU

GR MC18

I1253

I1 787

0 EU

GR 20077

I 2252

$0 \quad$ EU GR $\quad-55043 \quad$ I2252

397 EU

I1584

$18.6 \mathrm{~N} \quad 67.0 \mathrm{~W}$

535 EU

13.1S $\quad 81.4 \mathrm{~W}$

23.4S 78.9W

$31.4 \mathrm{~N} \quad 208.3 \mathrm{~W}$

$20.5 \mathrm{~S} \quad 243.7 \mathrm{~W}$

$23.2 \mathrm{~N} \quad 76.3 \mathrm{~W}$

$21.3 \mathrm{~N} \quad 45.5 \mathrm{~W}$

49.8S $280.5 \mathrm{~W}$
$189 \mathrm{AF}$

771 EU

0 EU

0 EU

352 EU

664 EU

320 EU

\section{LA MC10 I1511}

EG $\quad-10077$

GR 30207

LA

GR MC10
I1842

I976

I1779

12065

I1787

I1068

I1169
LA MC18

GR 20077

LA MC28

as ad ref $\mathbf{f t}$ origin

$5 \quad 1973$

57 CM Classical albedo feature name.

$57 \mathrm{CM}$ Classical albedo feature name.

$5 \quad 1973 \quad 57$ CM Classical albedo feature name.

$5 \quad 1973 \quad 57 \quad \mathrm{CM}$ Classical albedo feature name.

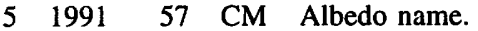

$6 \quad 1976 \quad 57 \quad$ CM Classical albedo feature; name changed to Thyles Rupes.

$5 \quad 1973 \quad 57 \quad$ CM Classical albedo feature name.

$\begin{array}{lllll}5 & 1985 & 55 & \mathrm{CO} & \text { From albedo feature at }\end{array}$

$\begin{array}{lllll}5 & 1985 \quad 57 \quad \mathrm{CO} \text { Classical albedo feature }\end{array}$ at $13 \mathrm{~N}, 294 \mathrm{~W}$.

$\begin{array}{lllll}5 & 1982 & 57 & \mathrm{CO} & \text { Classical albedo feature name }\end{array}$

$\begin{array}{lllll}5 & 1985 & 57 & \mathrm{CO} & \text { From albedo feature at }\end{array}$ $35 \mathrm{~N}, 269 \mathrm{~W}$

$51985 \quad 57$ CO Named for albedo feature

at 10S, 195W.

$\begin{array}{lllll}5 & 1987 & 57 & \mathrm{CO} & \text { From classical albedo }\end{array}$

feature Portus Nili, at

$38 \mathrm{~N}, 295 \mathrm{~W}$

$\begin{array}{lllll}5 & 1982 & 57 & \mathrm{CO} & \text { Classical albedo feature name. }\end{array}$

$5 \quad 1985 \quad 57 \quad \mathrm{CO}$ Albedo feature name.

5198557 CO From albedo feature at $25 \mathrm{~N}, 24 \mathrm{~W}$.

$5 \quad 1985 \quad 57 \quad \mathrm{CO} \quad$ From albedo feature name.

$5 \quad 1985 \quad 57$ CO From albedo feature at

$2 \mathrm{~N}, 183 \mathrm{~W}$.

$5 \quad 199157$ CO Classical albedo name.

$5 \quad 1985 \quad 57 \quad$ DO From albedo feature at $45 \mathrm{~N}, 205 \mathrm{~W}$

$5 \quad 1976$

$57 \quad \mathrm{DO}$

Classical albedo feature name.

51976

$\begin{array}{ll}5 & 1991\end{array}$

$57 \mathrm{DO}$

Classical albedo feature name.

56 DO Classical town.

1 DO One of the Graces.

$\begin{array}{ll}5 & 1985\end{array}$

57 DO

From albedo feature a 4S, 190W

51976

$\begin{array}{ll}5 & 1982\end{array}$

$\begin{array}{ll}5 & 1991\end{array}$

57 DO

$57 \mathrm{DO}$

Classical albedo feature name.

$\begin{array}{ll}5 & 1991\end{array}$

1 DO

Classical albedo feature name.

51976

$\begin{array}{ll}5 & 1976 \\ 5 & 1976\end{array}$ 
MARS

Name

lat

long diam ct et quad

origin

\section{FLUCTUS}

Tantalus

$32.3 \mathrm{~N} \quad 217.6 \mathrm{~W}$

$0.0 \mathrm{~N} \quad 0.0 \mathrm{~W}$

0 EU LA 30217

11758

12060

51985

51989

$57 \quad$

68 FL

FOSSA

Acheron Fossae

Aganippe Fossa

Alba Fossae

Albor Fossae

Amenthes Fossae

Calydon Fossa

Ceraunius Fossae

Chalce Fossa

Claritas Fossae

Coloe Fossae

Coracis Fossae

Cyane Fossae

Echus Fossae

Elysium Fossae

Erythraea Fossa

Fortuna Fossae

Galaxias Fossae

Gigas Fossae

Halex Fossae

Hephaestus Fossae

Hyblaeus Fossae

Icaria Fossae

Ismeniae Fossae

Jovis Fossae

Labeatis Fossae

38.

$\begin{array}{llll}8.7 \mathrm{~S} & 126.0 \mathrm{~W} & 329 & \mathrm{EU}\end{array}$

$43.4 \mathrm{~N} \quad 103.6 \mathrm{~W} \quad 2077$ EU

$18.6 \mathrm{~N} \quad 209.4 \mathrm{~W} \quad 113$ EU

$10.2 \mathrm{~N} \quad 259.3 \mathrm{~W} \quad 1592 \quad \mathrm{AF}$

$7.5 \mathrm{~S} \quad 88.8 \mathrm{~W}$

$24.8 \mathrm{~N} \quad 110.5 \mathrm{~W}$

$51.9 \mathrm{~S} \quad 40.0 \mathrm{~W}$

$34.8 \mathrm{~S}$

$37.1 \mathrm{~N}$

$34.8 \mathrm{~S}$

$99.1 \mathrm{~W}$

$303.9 \mathrm{~W}$

$36.0 \mathrm{~N}$

$2.2 \mathrm{~N}$

$27.5 \mathrm{~N}$

$27.8 \mathrm{~S}$

$5.1 \mathrm{~N}$

$38.3 \mathrm{~N}$

$4.5 \mathrm{~N}$

$27.3 \mathrm{~N}$

$121.4 \mathrm{~W}$

$77.1 \mathrm{~W}$

$219.5 \mathrm{~W}$

$30.5 \mathrm{~W}$

$92.5 \mathrm{~W}$

$218.8 \mathrm{~W}$

$129.7 \mathrm{~W}$

265 EU

711 EU

0 EU

2033 EU

930 EU

292 EU

236
1114
EU

1114
248 EU

309 EU

200 EU

328 EU

$126.1 \mathrm{~W}$

224 EU

$20.5 \mathrm{~N} \quad 237.5 \mathrm{~W}$

$528 \quad \mathrm{EU}$

$21.7 \mathrm{~N} \quad 223.2 \mathrm{~W}$

$53.7 \mathrm{~S}$

$135.0 \mathrm{~W}$

405 EU

$38.9 \mathrm{~N} \quad 326.1 \mathrm{~W}$

858 EU

$19.5 \mathrm{~N} \quad 116.1 \mathrm{~W}$

412 EU

$29.5 \mathrm{~N}$

$80.0 \mathrm{~W}$

388 EU

20.9

Labeatis Fossae

Mangala Fossa

Mareotis Fossae

Medusae Fossae

Melas Fossae

Memnonia Fossae

Nectaris Fossae

Nia Fossae

Nili Fossae

Nilokeras Fossa

Noctis Fossae

Oceanidum Fossa

Olympica Fossae

Oti Fossae

Pavonis Fossae

Pyramus Fossae

Sacra Fossae

Sirenum Fossae

Stygis Fossae

Tanais Fossae

Tantalus Fossae

\section{5}

$45.0 \mathrm{~N}$

24.8

$24.8 \mathrm{~S}$

$24.7 \mathrm{~S}$

$14.4 \mathrm{~S}$

$24.0 \mathrm{~N} \quad 283.0 \mathrm{~W}$

$25.4 \mathrm{~N} \quad 56.9 \mathrm{~W}$

$3.3 \mathrm{~S} \quad 99.0 \mathrm{~W}$

$616 \mathrm{EU}$

LA 25077

$\begin{array}{llll}0 & \text { AS } & \text { SA } & -20147\end{array}$

$148.8 \mathrm{~W}$

795 EU

GR MC03

61.7S 29.6W

291 EU

LA MC16

$72.4 \mathrm{~W}$

$154.4 \mathrm{~W}$

313 EU

$1370 \mathrm{EU}$

GR MC18

I392

51979

GR $\mathrm{MC} 17$

LA $\quad$ MCO3

1984

I477
I1746

$5 \quad 1976$

$\begin{array}{ll}5 & 1973 \\ 5 & 1985\end{array}$

EG MC14

GR MC18

GR $\quad-50036$

LA $\mathrm{MC17}$

I1024

I1253

1926

I 2253

I 984

I1495

GR MC25NE I1602

GR 35117

LA 00077

I1736

GR MC19

I1135

MC-9SE I1535

LA 35217

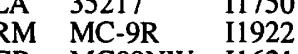

GR MC09NW

I1621

51976

$5 \quad 1982$

51973

51991

51973

$\begin{array}{ll}5 & 1982 \\ 5 & 1985\end{array}$

GR $\quad \mathrm{MC14} \quad \mathrm{I024}$

$\begin{array}{lll}\text { GR } & \text { MC24NE } & \text { I1751 }\end{array}$

$5 \quad 1985$

5 1988

51973

51976

51985

51985

$\begin{array}{ll}5 & 1987\end{array}$

51985

\section{$5 \quad 1973$}

$\begin{array}{ll}5 & 1985\end{array}$

$\begin{array}{ll}5 & 1985\end{array}$

$\mathrm{FO}$

57 FO

$\begin{array}{ll}57 & \text { FO } \\ 57 & \text { FO }\end{array}$

feature at $35 \mathrm{~N}, 140 \mathrm{~W}$.

57

57 FO

57 FO

57 FO

57 FO

57 FO

57 FO

57 FO

Classical albedo name.

From albedo feature at 20N, 205W.

The son of Ares and Astynome.

Classical albedo feature name.

Albedo name.

Classical albedo feature name.

Classical albedo feature name

From albedo feature at $46 \mathrm{~S}, 87 \mathrm{~W}$.

57 FO

57 FO

Classical albedo feature name.

57 FO

57 FO

$57 \mathrm{FO}$

57 FO

57 FO

57 FO

Classical albedo feature name.

Classical albedo feature name.

Classical albedo feature name.

Classical albedo feature name.

Albedo feature name.

Albedo feature name.

From albedo feature at $40 \mathrm{~N}, 110 \mathrm{~W}$.

57 FO

57 FO

Classical albedo feature name.

51982

Albedo feature name.

From albedo feature at 44S, 130W.

51985

From albedo feature at $40 \mathrm{~N}, 333 \mathrm{~W}$.

From albedo feature at $16 \mathrm{~N}, 111 \mathrm{~W}$.

$\begin{array}{llll}5 & 1985 & 56 & \mathrm{FO}\end{array}$

( at $30 \mathrm{~N}, 75 \mathrm{~W}$; expanded coordinates.

I1511

51985

$56 \quad \mathrm{FO}$

Previously named feature at $30 \mathrm{~N}$ $75 \mathrm{~W}$; expanded coordinates.

$5 \quad 1991 \quad 62$ FO Named for nearby valles.

$5 \quad 1973 \quad 57 \quad$ FO From albedo feature at $32 \mathrm{~N}, 96 \mathrm{~W}$.

51973

51985

51973

51985

MCl6NW I1554

664 EU LA MC18 I1253

333 EU LA MC18 11535

51982

57 FO

57 FO

57 FO

$57 \mathrm{FO}$

57 FO

Classical albedo feature name.

Classical albedo feature name.

Classical albedo feature name.

Classical albedo feature name.

Classical name for River

Gambia, West Africa.

57 FO Classical albedo feature name.

$\begin{array}{lllll}148 & \text { EU } & \text { LA } & \text { MC13 } & \text { I967 } \\ 25057 & \text { I1919 }\end{array}$

51973

Classical albedo feature name.

692 EU LA MC17NE I1535

51985

57 FO

57 FO

From classical albedo feature at $10 \mathrm{~S}, 96 \mathrm{~W}$. 
MARS

Name

Tithoniae Fossae

Tithoniae Fossae

Tractus Fossae

Tyrrhena Fossae

Ulysses Fossae

Uranius Fossae

Zephyrus Fossae

\section{LABES}

Candor Labes

Coprates Labes

Ius Labes

Melas Labes

Ophir Labes

$\begin{array}{cr}\text { lat } & \text { long } \\ & \\ 1.7 \mathrm{~S} & 81.9 \mathrm{~W} \\ 6.4 \mathrm{~S} & 82.5 \mathrm{~W} \\ 27.1 \mathrm{~N} & 101.1 \mathrm{~W} \\ 22.1 \mathrm{~S} & 254.5 \mathrm{~W} \\ 11.4 \mathrm{~N} & 123.3 \mathrm{~W} \\ 25.8 \mathrm{~N} & 90.1 \mathrm{~W} \\ 23.9 \mathrm{~N} & 214.4 \mathrm{~W}\end{array}$

diam

ct et

quad

map

\begin{tabular}{rrlllll} 
diam & ct & et & \multicolumn{1}{c}{ quad } & map & as & ad \\
& & & & & & \\
549 & EU & LA & MC18 & I1253 & 5 & 1982 \\
492 & EU & LA & -05077 & I1841 & 5 & 1988 \\
535 & EU & LA & MC09NE & I1535 & 5 & 1988 \\
0 & EU & LA & -20252 & & 5 & 1991 \\
720 & EU & GR & MC09SW & I1622 & 5 & 1985 \\
438 & EU & GR & MC-9NE & I1535 & 5 & 1985 \\
452 & EU & LA & 25217 & I1749 & 5 & 1985
\end{tabular}

ref $\mathbf{f t}$ origin

57 FO Classical albedo name.

57 FO

57 FO

57 FO

57 FO

57 FO

57 FO
Classical albedo feature name. Classical albedo feature name.

Classical albedo name.

From albedo feature name.

Classical albedo feature name. Albedo feature name.

\section{LABYRINTHUS}

Adamas

Labyrinthus

$36.5 \mathrm{~N}$

4.9S

$13.5 \mathrm{~S}$

7.35

$9.1 \mathrm{~S}$

$11.1 \mathrm{~S}$

$76.0 \mathrm{~W}$

165 EU LA -05077

$67.3 \mathrm{~W}$

$\begin{array}{llll}115 & \text { EU } & \text { GR } & -10067\end{array}$

79.0W 129 EU LA -10072

$71.2 \mathrm{~W}$

128 EU GR -10072

$\begin{array}{llll}0 & \text { EU } & \text { GR } & -10067\end{array}$
I1841

I1591

I1593

I1591
$5 \quad 1988$

51985

$5 \quad 1988$

$\begin{array}{ll}5 & 1985\end{array}$

51985
57 LA

57 LA

57 LA

57 LA

57 LA
From classical albedo feature at $5 \mathrm{~N}, 75 \mathrm{~W}$.

From albedo feature at $14 \mathrm{~S}, 65 \mathrm{~W}$.

Classical albedo feature name.

From albedo feature at 10S, 74W.

From albedo feature at $10 \mathrm{~S}, 65 \mathrm{~W}$.
Hyperboreus Labyrinthus9.8N

Noctis Labyrinthus
$55.5 \mathrm{~W}$

$101.3 \mathrm{~W}$
0 EU RO 80050

976 EU LA MC17
1646

I1836

1924

Aeolis Mensae

Ascraeus Mensa

Ausonia Mensa

Baetis Mensa

Candor Mensa

Ceti Mensa

Cydonia Mensae

Deuteronilus

Mensae

Galaxias Mensae

Hebes Mensa

Labeatis Mensa

Lunae Mensa

Nepenthes Mensae

Nilokeras Mensae

Nilosyrtis Mensae

Nilus Mensae

Protonilus Mensae

Sacra Mensa

Tempe Mensa

Zephyria Mensae

$$
\text { 3.7S }
$$

$30.2 \mathrm{~S} \quad 262.5 \mathrm{~W}$

$5.4 \mathrm{~S} \quad 72.4 \mathrm{~W}$

841 EU GR MC23

0 EU LA 10107

0 EU LA

181 EU GR -05072

6.0S 73.0W

$5.9 \mathrm{~S} \quad 76.3 \mathrm{~W}$

$37.0 \mathrm{~N}$

$12.8 \mathrm{~W}$

$50 \mathrm{E}$

55 EU

854 EU

LA $\mathrm{MC18}$

$45.7 \mathrm{~N} \quad 337.9 \mathrm{~W}$

$36.5 \mathrm{~N} \quad 212.7 \mathrm{~W}$

$1.0 \mathrm{~S} \quad 32.0 \mathrm{~W}$

627 EU

LA

MCO4

327 EU LA 35212

0 EU GR MC18

$25.6 \mathrm{~N} \quad 74.9 \mathrm{~W}$

$24.1 \mathrm{~N}$

$9.3 \mathrm{~N}$

$32.9 \mathrm{~N}$

$35.4 \mathrm{~N}$

$22.1 \mathrm{~N}$

$44.2 \mathrm{~N}$

$25.0 \mathrm{~N}$

$28.1 \mathrm{~N}$

10.15

$62.3 \mathrm{~W}$

$241.7 \mathrm{~W}$

$51.1 \mathrm{~W}$

293.6W

$71.8 \mathrm{~W}$

120 EU

$106 \mathrm{EU}$

1704 EU

$\begin{array}{lll}327 & \text { EU } & \text { GR } \\ 693 & \text { EU } & \text { GR }\end{array}$

196 EU

$309.4 \mathrm{~W}$

592 EU

$69.2 \mathrm{~W}$

602 EU

$71.5 \mathrm{~W}$

84 EU

230 EU

LA

RM

GR

MC14SE I1428

$\mathrm{MCO6}$

I1651

I1789

$\mathrm{MC05}$

$5 \quad 1982$

$57 \quad$ LB

Classical albedo feature

name; "A River of

Diamonds"; today's River

Sarbarnarekha in India.

1988

57 LB

Classical albedo feature name.

Classical albedo feature name.
I1001

12040

I1592

I1495

I1618

1979

11495

I1771

I1618

51976

$\begin{array}{ll}5 & 1991\end{array}$

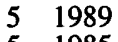

1985

$5 \quad 1982$

$\begin{array}{ll}5 & 1994\end{array}$

1976

51973

$5 \quad 1985$

$5 \quad 1982$

$5 \quad 1988$

$5 \quad 1988$

$5 \quad 1976$

$\begin{array}{ll}5 & 1988\end{array}$

$5 \quad 1973$

51985

51973

$5 \quad 1988$

0 1988

11783
57 LB

57 MN

$57 \mathrm{MN}$

$57 \mathrm{MN}$

Classical albedo feature name.

$57 \mathrm{MN}$

57 MN

71 MN

$57 \mathrm{MN}$

$57 \mathrm{MN}$

Classical albedo name.

Albedo feature name.

$7 \mathrm{~S}, 63 \mathrm{~W}$

$7 \mathrm{~S}, 63 \mathrm{~W}$.

Classical albedo feature

Albedo feature Ceti Lacus. $50 \mathrm{~N}, 355 \mathrm{~W}$.

From albedo feature at $35 \mathrm{~N}, 355 \mathrm{~W}$.

57 MN Albedo feature name.

57 MN Classical albedo feature; name of goddess of youth.

56 MN Albedo feature name.

57 MN Albedo feature name.

57 MN Classical albedo feature name.

56 MN Albedo feature name.

57 MN Classical albedo feature name.

57 MN Named for albedo feature at $20 \mathrm{~N}, 65 \mathrm{~W}$.

57 MN From albedo feature at

$42 \mathrm{~N}, 315 \mathrm{~W}$.

\section{MONS}

Anseris Mons

30.15

Arsia Mons

Ascraeus Mons

Ausonia Montes

Centauri Montes

Chalce Montes

Charitum Montes

Coronae Mons

$\begin{array}{cc}30.1 \mathrm{~S} & 263.2 \mathrm{~W} \\ & \\ 9.4 \mathrm{~S} & 120.5 \mathrm{~W} \\ & \\ 11.3 \mathrm{~N} & 104.5 \mathrm{~W} \\ & \\ 28.5 \mathrm{~S} & 260.0 \mathrm{~W} \\ 38.5 \mathrm{~S} & 263.1 \mathrm{~W} \\ 53.8 \mathrm{~S} & 37.0 \mathrm{~W} \\ 58.3 \mathrm{~S} & 44.2 \mathrm{~W} \\ 34.5 \mathrm{~S} & 271.5 \mathrm{~W}\end{array}$

0 EU LA

I2038

$5 \quad 1989$

485 EU LA $\mathrm{MCl} 7$

462 EU

LA MC09

0 EU

0 EU

LA

0 EU GR

1412 EU GR

0 EU GR 


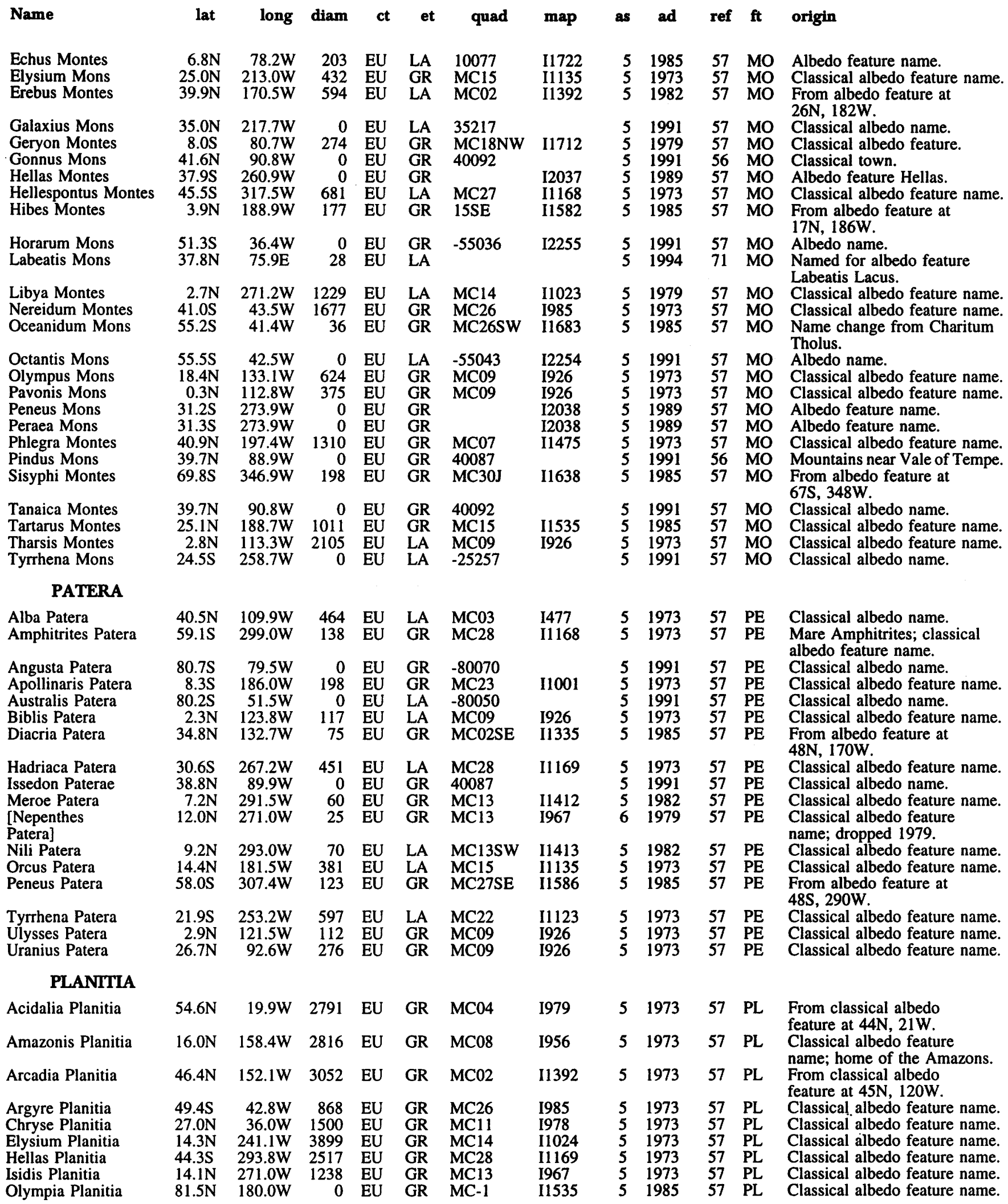

lat: latitude of feature center.

long: longitude of feature center.

diam: diameter or long dimension of feature.

et: ethnicity of name origin (see page $284 \mathrm{ff}$.)

quad: map quadrangle or informal name

(see page xvii ff.)

ct: continent of name origin (see page $284 \mathrm{ff}$.) map: map name or USGS map number (see page xvii ff.). ft: feature type (see page 290 ).

as: name approval status (see page xvii).

ad: name approval date (year).

ref: reference source for name (see page $287 \mathrm{ff}$.). 


\section{MARS}

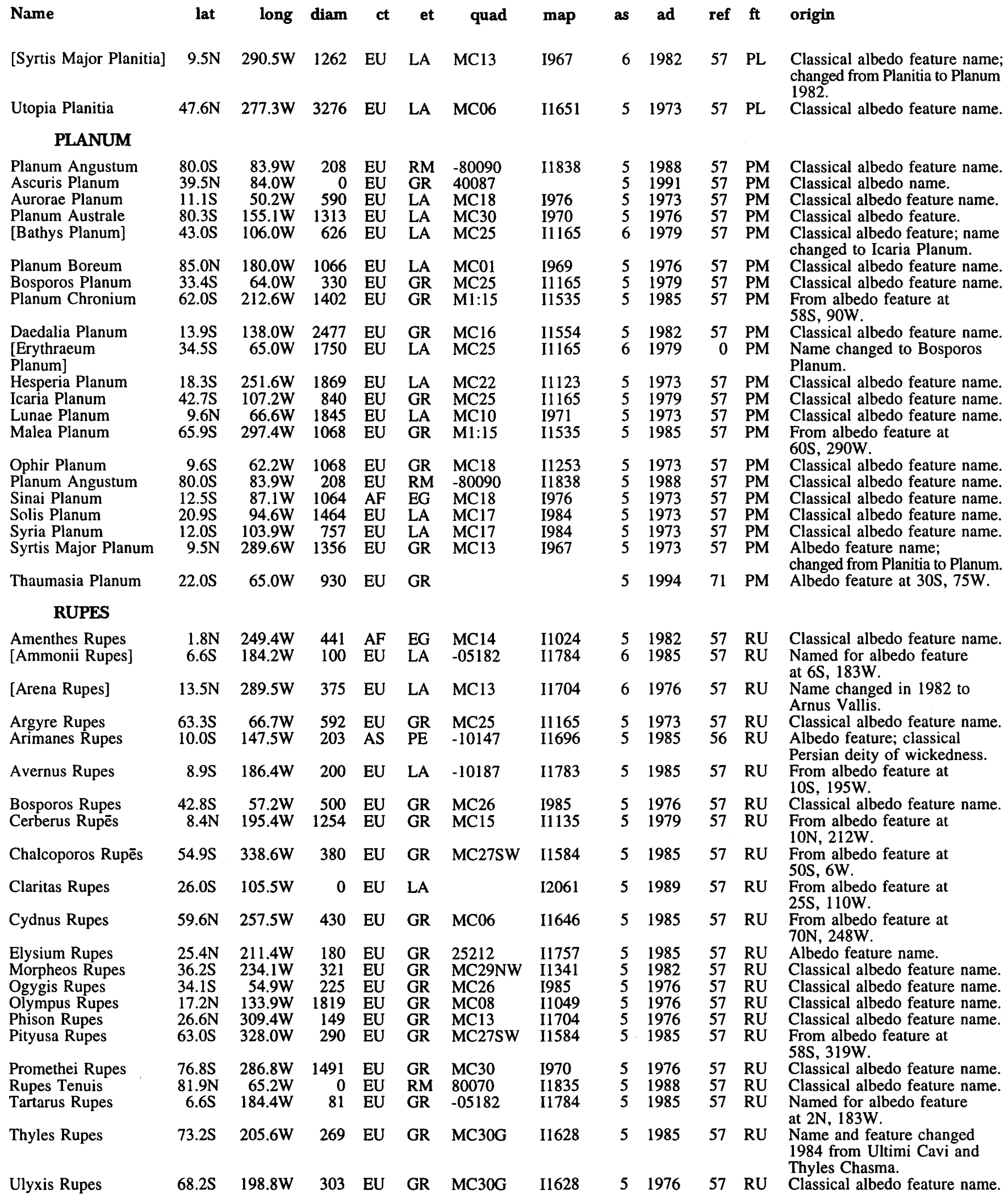

lat: latitude of feature center.

long: longitude of feature center.

diam: diameter or long dimension of feature. et: ethnicity of name origin (see page $284 \mathrm{ff}$.)

quad: map quadrangle or informal name

(see page xvii ff.). as: name approval status (see page $\mathrm{xvii}$.

ad: name approval date (year).

ref: reference source for name (see page $287 \mathrm{ff}$.).

ct: continent of name origin (see page $284 \mathrm{ff}$.) map: map name or USGS map number (see page xvii ff.). ft: feature type (see page 290 ). 
MARS

Name

\section{SCOPULUS}

Charybdis Scopulus

Coronae Scopulus

24:9S

Eridania Scopulus

33.9S

$294.1 \mathrm{~W}$

$53.4 \mathrm{~S} \quad 217.9 \mathrm{~W}$

$79.5 \mathrm{~N} \quad 184.0 \mathrm{~W}$

510 EU

0 EU

$0 \mathrm{EU}$

[Hyperboreus Scopulus] 82.5N

$160.0 \mathrm{~W}$

[Hypernotius Scopulus] 78.0S

Nilokeras Scopulus

$31.5 \mathrm{~N}$

$272.0 \mathrm{~W}$

$0 \mathrm{EU}$

57.2W 1064 EU

Oenotria Scopulus

Scylla Scopulus

$11.2 \mathrm{~S}$
$25.2 \mathrm{~S}$

$283.2 \mathrm{~W}$

$342.0 \mathrm{~W}$

Tartarus Scopulus

$6.4 \mathrm{~S}$

$181.7 \mathrm{~W}$

$19.6 \mathrm{~N}$

$52.1 \mathrm{~W}$

LA
Amazonis Sulci

Apollinaris Sulci

Arsia Sulci

Ascraeus Sulci

Cyane Sulci

Gigas Sulci

Gordii, Sulci

Lycus Sulci

Medusae Sulci

Memnonia Sulci

Pavonis Sulci

Sacra Sulci

\section{TERRA}

Aonia Terra

Arabia Terra

Terra Cimmeria

Margaritifer Terra

Terra Meridiani

Noachis Terra

Promethei Terra

Terra Sabaea

Terra Sirenum

Tempe Terra

Xanthe Terra

\section{THOLUS}

Albor Tholus

[Australis Tholus]

$3.3 \mathrm{~S}$

$11.3 \mathrm{~S}$

$6.4 \mathrm{~S}$

$11.9 \mathrm{~N}$

$25.5 \mathrm{~N}$

$9.9 \mathrm{~N} \quad 127.6 \mathrm{~W}$

$17.9 \mathrm{~N} \quad 125.6 \mathrm{~W}$

$29.2 \mathrm{~N} \quad 139.8 \mathrm{~W}$

$5.3 \mathrm{~S} \quad 159.8 \mathrm{~W}$

$6.5 \mathrm{~S} \quad 175.6 \mathrm{~W}$

$3.9 \mathrm{~N} \quad 117.6 \mathrm{~W}$

$25.0 \mathrm{~N} \quad 71.5 \mathrm{~W}$

\section{$25.0 \mathrm{~N}$}

34.0S

$16.2 \mathrm{~S}$

$7.2 \mathrm{~S}$

$45.0 \mathrm{~S}$

$52.9 \mathrm{~S}$

$10.4 \mathrm{~S}$

37.0S

$41.3 \mathrm{~N}$

$94.8 \mathrm{~W}$

$330.0 \mathrm{~W}$

$215.0 \mathrm{~W}$

$21.3 \mathrm{~W}$

$356.0 \mathrm{~W}$

$350.0 \mathrm{~W}$

262.2W

$70.5 \mathrm{~W}$

14.3S

278.5W

$4.2 \mathrm{~N}$

46.3W

$59.0 \mathrm{~N}$

$209.8 \mathrm{~W}$

$323.0 \mathrm{~W}$

$\begin{array}{rl}243 & \mathrm{EU} \\ 300 & \mathrm{EU} \\ 0 & \mathrm{EU} \\ 0 & \mathrm{EU} \\ 286 & \mathrm{EU} \\ 467 & \mathrm{EU} \\ 316 & \mathrm{EU} \\ 1639 & \mathrm{EU} \\ 91 & \mathrm{EU} \\ 361 & \mathrm{EU} \\ 0 & \mathrm{EU} \\ 0 & \mathrm{EU}\end{array}$

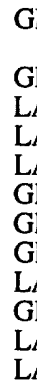

$\begin{array}{ll}\text { GR } & -05147 \\ \text { GR } & -10182 \\ \text { LA } & -05127 \\ \text { LA } & 10107 \\ \text { LA } & \text { MC09 } \\ \text { GR } & \text { MC09 } \\ \text { GR } & \text { MC09 } \\ \text { GR } & \text { MC08 } \\ \text { LA } & -05157 \\ \text { GR } & \text { MC16NW } \\ \text { LA } & 05117 \\ \text { LA } & 25067\end{array}$

I1664

I1782

I1535

I1535

I1621

I1535

I1666

I1710

I2250

I1587
$5 \quad 1985$

51985

1979

$\begin{array}{ll}6 & 1984\end{array}$

1984

$6 \quad 1984$

51976

$5 \quad 1982$

51985

51985

$5 \quad 1988$

51985

$5 \quad 1985$

5199

$\begin{array}{ll}5 & 1991\end{array}$

51976

51976

51976

51976

51985

$\begin{array}{ll}5 & 1985\end{array}$

$\begin{array}{ll}5 & 1991 \\ 5 & 1985\end{array}$
$57 \mathrm{SC}$

57 SC

$57 \mathrm{SC}$

57 SC

$57 \mathrm{SC}$

57 SC

57 SC

$57 \mathrm{SC}$

57 SC

57 SC

$71 \mathrm{SC}$

$57 \mathrm{SU}$

0 SU

$57 \mathrm{SU}$

57 SU

57 SU

57 SU

57 SU

$57 \mathrm{SU}$

$57 \mathrm{SU}$

57 SU

$57 \mathrm{SU}$

57 SU
From albedo feature at

19S, 320W.

From albedo feature at 26S, 276W.

Classical albedo feature name. Classical albedo feature; name dropped 1984.

Classical albedo feature name; dropped 1984.

Classical albedo feature name; dropped 1984.

From albedo feature at $30 \mathrm{~N}, 55 \mathrm{~W}$

Classical albedo feature name.

rrom albedo feature at 19S, 320W.

Named for albedo feature at $12 \mathrm{~S}, 182 \mathrm{~W}$

Classical albedo feature name.
Tyrrhena Terra

Ceraunius Tholus

[Charitum Tholus]

1367 EU

$160.0 \mathrm{~W} 2165$ EU

165 EU

40 EU

$24.2 \mathrm{~N} 97.2 \mathrm{~W}$

$108 \mathrm{EU}$

0 E

$36.1 \mathrm{~N} \quad 85.0 \mathrm{~W}$

$\begin{array}{llll}0 & \text { EU GR } & 35087\end{array}$

E. Mareotis Tholis

$32.7 \mathrm{~N} \quad 209.8 \mathrm{~W}$

$72.0 \mathrm{~N} \quad 15.0 \mathrm{~W}$

183 EU GR MC07

53 EU GR MC01

$36.3 \mathrm{~N} \quad 94.6 \mathrm{~W}$

$18.4 \mathrm{~N} \quad 117.5 \mathrm{~W}$

73.0N $358.0 \mathrm{~W}$

0 EU

$61 \mathrm{EU}$

GR 35092

GR MC09

[Kison Tholus]

$36.8 \mathrm{~N} \quad 86.0 \mathrm{~W}$

$\begin{array}{llll}0 & \text { EU } & \text { GR } & 35087\end{array}$

I1165

I1651

I1170

I1293

I1293

I1 168

I1 169

I1296

I1166

I1477

I1118

I1551

$\begin{array}{ll}5 & 1979\end{array}$

$\begin{array}{ll}5 & 1979\end{array}$

$\begin{array}{ll}5 & 1979\end{array}$

$\begin{array}{ll}5 & 1979\end{array}$

51979

$\begin{array}{ll}5 & 1979\end{array}$

51979

$\begin{array}{ll}5 & 1979\end{array}$

$\begin{array}{ll}5 & 1979\end{array}$

$\begin{array}{ll}5 & 1979\end{array}$

$\begin{array}{ll}5 & 1979\end{array}$

51979
57 TA

57 TA

57 TA

57 TA

57 TA

57 TA

57 TA

57 TA

57 TA

57 TA

57 TA

57 TA
Albedo feature name; home of the Amazons.

Albedo feature at 5S, $187 \mathrm{~W}$.

Albedo name.

Classical albedo name.

Classical albedo feature name.

Classical albedo feature name.

Classical albedo feature name.

Classical albedo feature name.

Albedo feature name.

Albedo feature name.

Albedo name.

From albedo feature at $20 \mathrm{~N}, 67 \mathrm{~W}$.

I1135
I1168
I926
I1165
I1475
I969
I926
I969

\section{$\begin{array}{llll}5 & 1973 & 57 & \text { TH }\end{array}$}

61978

$57 \mathrm{TH}$

51973

61976

$57 \mathrm{TH}$

$57 \mathrm{TH}$

51991 
MARS

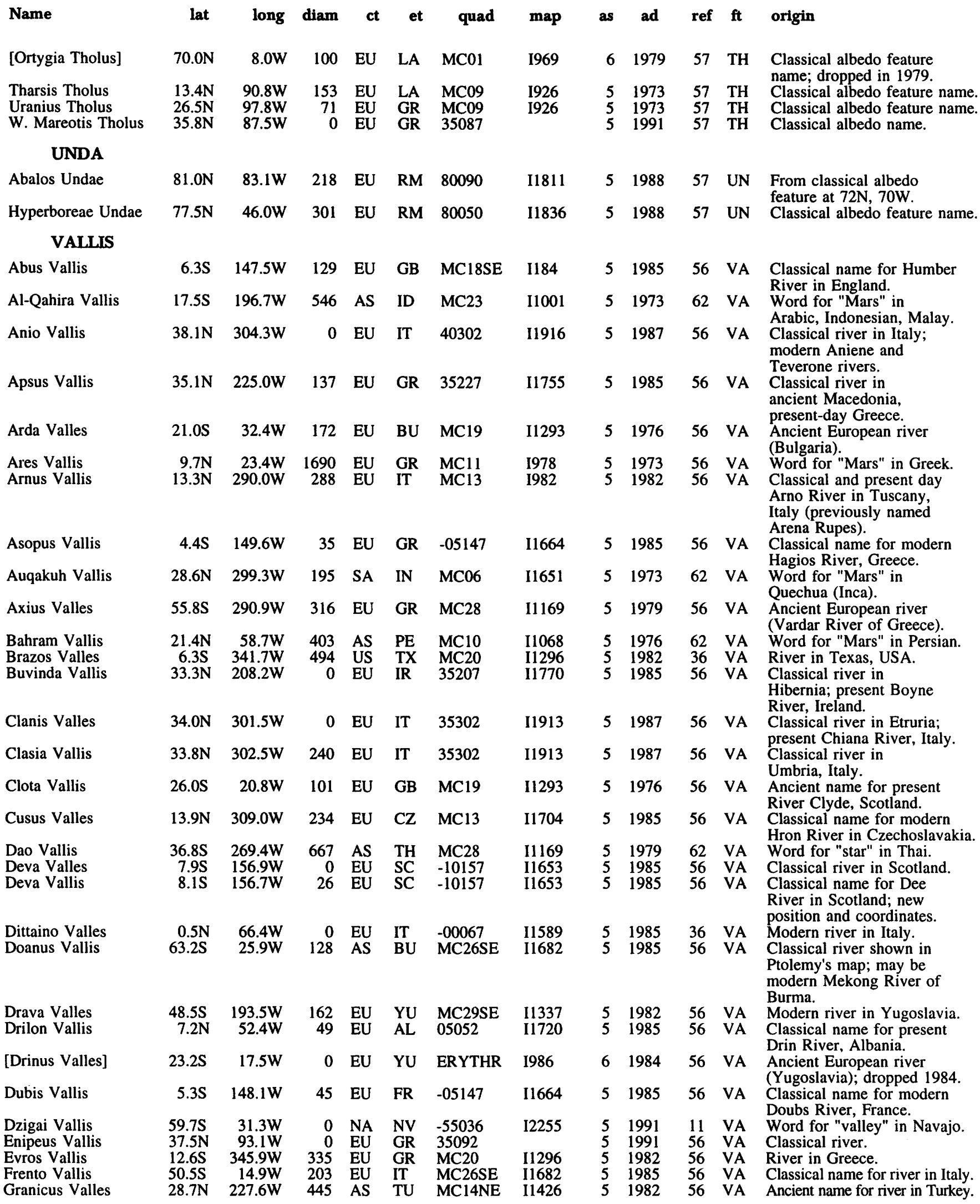

lat: latitude of feature center.

long: longitude of feature center.

diam: diameter or long dimension of feature.

ct: continent of name origin (see page 284 ff: et: ethnicity of name origin (see page $284 \mathrm{ff}$.)

quad: map quadrangle or informal name

(see page xvii ff.).

map name or USGS map number (see page xvii ff.). as: name approval status (see page xvii).

ad: name approval date (year).

ref: reference source for name (see page $287 \mathrm{ff}$.). 


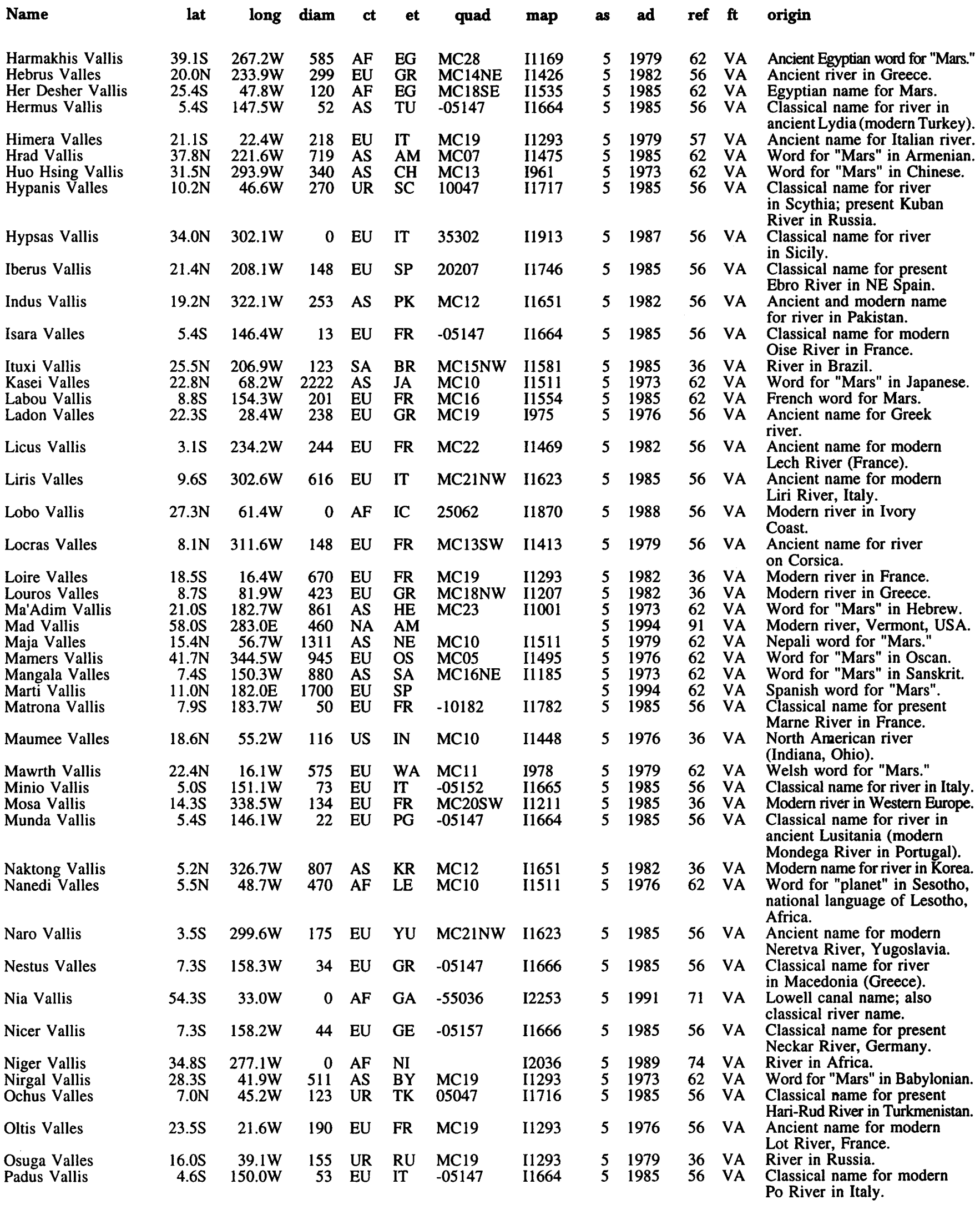

lat: latitude of feature center.

long: longitude of feature center.

diam: diameter or long dimension of feature. et: ethnicity of name origin (see page $284 \mathrm{ff}$.)

quad: map quadrangle or informal name

(see page xvii ff.).

ref: reference source for name (see

ref: reference source for name (see page $287 \mathrm{ff}$.). 


\section{MARS}

Name

Pallacopas Valli

Paraná Valles

Patapsco Vallis

Protva Valles

Ravi Vallis

Ravius Valles

Reull Vallis

Rhabon Valles

Rubicon Valles

Runa Vallis

Sabis Vallis

Sabrina Vallis

Samara Valles

Scamander Vallis

Senus Vallis

Sepik Vallis

Shalbatana Vallis

Simud Vallis

Stura Vallis

Subur Vallis

Surinda Valles

Surius Vallis

Tader Valles

Tagus Valles

Taus Vallis

Termes Vallis

Tinia Valles

Tinjar Valles

Tisia Valles

Tiu Vallis

Trebia Valles

Tyras Vallis

Uzboi Vallis

Valles Marineris

\begin{tabular}{|c|c|c|c|c|c|c|}
\hline $23.9 \mathrm{~N}$ & $208.0 \mathrm{~W}$ & 149 & US & MD & MC15NW & I1581 \\
\hline $\begin{array}{r}30.1 \mathrm{~S} \\
0.4 \mathrm{~S} \\
47.0 \mathrm{~N}\end{array}$ & $\begin{array}{r}60.0 \mathrm{~W} \\
40.7 \mathrm{~W} \\
111.6 \mathrm{~W}\end{array}$ & $\begin{array}{l}213 \\
169 \\
243\end{array}$ & $\begin{array}{l}\text { UR } \\
\text { AS } \\
\text { EU }\end{array}$ & $\begin{array}{l}\text { RU } \\
\text { PK } \\
\text { IR }\end{array}$ & $\begin{array}{l}1: 15 \mathrm{M} \\
\mathrm{MC} 19 \\
45112\end{array}$ & $\begin{array}{l}\text { I1 } 535 \\
\text { I1 } 068 \\
\text { I1 } 357\end{array}$ \\
\hline $\begin{array}{l}41.6 \mathrm{~S} \\
21.7 \mathrm{~N}\end{array}$ & $\begin{array}{r}258.3 \mathrm{~W} \\
90.3 \mathrm{~W}\end{array}$ & $\begin{array}{l}972 \\
204\end{array}$ & $\begin{array}{l}\text { EU } \\
\text { EU }\end{array}$ & $\begin{array}{l}\text { IR } \\
\text { RO }\end{array}$ & $\begin{array}{l}\text { MC28 } \\
\text { MC09NE }\end{array}$ & $\begin{array}{l}\text { I1169 } \\
1977\end{array}$ \\
\hline $\begin{array}{r}44.7 \mathrm{~N} \\
28.6 \mathrm{~S} \\
5.8 \mathrm{~S}\end{array}$ & $\begin{array}{r}116.8 \mathrm{~W} \\
36.7 \mathrm{~W} \\
152.7 \mathrm{~W}\end{array}$ & $\begin{array}{r}228 \\
36 \\
286\end{array}$ & $\begin{array}{l}\text { EU } \\
\text { UR } \\
\text { EU }\end{array}$ & $\begin{array}{l}\text { IT } \\
\text { SO } \\
\text { FR }\end{array}$ & $\begin{array}{l}45117 \\
\text { MC19SW } \\
\text { MC16NE }\end{array}$ & $\begin{array}{l}\text { I1 } 1357 \\
\text { I1 } 209 \\
\text { I1 } 185\end{array}$ \\
\hline $11.1 \mathrm{~N}$ & $48.9 \mathrm{~W}$ & 294 & $\mathrm{EU}$ & GB & 10047 & I1717 \\
\hline $24.2 \mathrm{~S}$ & $18.9 \mathrm{~W}$ & 575 & $\mathrm{EU}$ & FR & MC19 & I1293 \\
\hline $16.1 \mathrm{~N}$ & $331.1 \mathrm{~W}$ & 272 & AS & $\mathrm{TU}$ & $\mathrm{MC} 12$ & I1651 \\
\hline $\begin{array}{r}5.3 \mathrm{~S} \\
1.0 \mathrm{~S} \\
5.6 \mathrm{~N} \\
11.5 \mathrm{~N} \\
22.9 \mathrm{~N}\end{array}$ & $\begin{array}{r}146.9 \mathrm{~W} \\
65.8 \mathrm{~W} \\
43.1 \mathrm{~W} \\
38.5 \mathrm{~W} \\
217.6 \mathrm{~W}\end{array}$ & $\begin{array}{r}27 \\
0 \\
687 \\
1074 \\
0\end{array}$ & $\begin{array}{l}\text { EU } \\
\text { OC } \\
\text { AS } \\
\text { AS } \\
\text { EU }\end{array}$ & $\begin{array}{l}\text { IR } \\
\text { GU } \\
\text { AK } \\
\text { SU } \\
\text { RM }\end{array}$ & $\begin{array}{l}-05147 \\
-00067 \\
\text { MC11 } \\
\text { MC11 } \\
25217\end{array}$ & $\begin{array}{l}\mathbf{I} 1664 \\
\\
\mathrm{I} 1551 \\
\mathrm{I} 978 \\
11749\end{array}$ \\
\hline $\begin{array}{l}11.6 \mathrm{~N} \\
29.3 \mathrm{~S}\end{array}$ & $\begin{array}{l}53.1 \mathrm{~W} \\
34.8 \mathrm{~W}\end{array}$ & $\begin{array}{r}30 \\
101\end{array}$ & $\begin{array}{l}\text { AF } \\
\text { UR }\end{array}$ & $\begin{array}{l}\text { MA } \\
\text { SO }\end{array}$ & $\begin{array}{l}10152 \\
\text { MC19SW }\end{array}$ & I1209 \\
\hline $\begin{array}{l}60.3 S \\
50.0 S\end{array}$ & $\begin{array}{r}51.0 \mathrm{~W} \\
152.5 \mathrm{~W}\end{array}$ & $\begin{array}{r}0 \\
275\end{array}$ & EU & SP & $\begin{array}{l}-55043 \\
\mathrm{MC} 24 \mathrm{SW}\end{array}$ & $\begin{array}{l}\mathrm{I} 2254 \\
\mathrm{I} 1681\end{array}$ \\
\hline $7.3 \mathrm{~S}$ & $246.0 \mathrm{~W}$ & 35 & EU & SP & $\mathrm{MC} 22$ & I1123 \\
\hline $4.9 \mathrm{~S}$ & $148.3 \mathrm{~W}$ & 0 & EU & $\mathrm{SC}$ & -05147 & I1664 \\
\hline & $157.1 \mathrm{~W}$ & 0 & EU & SP & -10157 & II653 \\
\hline
\end{tabular}

$54.5 \mathrm{~S}$

$21.2 \mathrm{~W}$

0 AS BY MC 26S

$21.6 \mathrm{~S}$

$10.7 \mathrm{~W}$

219 SA

B

MC19

I1293

$5 \quad 1991$

$5 \quad 1979$

51985

$5 \quad 1979$

51979

51985

51979

51988

51985

$\begin{array}{ll}5 & 1979 \\ 5 & 1985\end{array}$

51985

51985

51976

51982

$5 \quad 1985$

$5 \quad 1985$

$\begin{array}{ll}5 & 1973\end{array}$

51973

51985

$5 \quad 1985$

$\begin{array}{ll}5 & 1979\end{array}$

51991

51985

51976

$5 \quad 1985$

4.7S $148.9 \mathrm{~W}$

20 EU IT -15047

I1664

$38.2 \mathrm{~N} \quad 235.7 \mathrm{~W}$

390 AS

11.6S $313.9 \mathrm{~W}$

390 UR

MA MC07

I1475

$8.6 \mathrm{~N}$

$34.8 \mathrm{~W}$

970 EU

UK MC21NW I1623

11551

32.4N 209.9W

0 EU

$8.3 \mathrm{~N}$

$50.1 \mathrm{~W}$

140

GB MC11

I1771

$30.9 \mathrm{~S}$

$35.6 \mathrm{~W}$

$11.6 \mathrm{~S}$
340 UR RU MC19

4128 EU LA MC18
I1707

I1293

I976 $\begin{array}{llll}5 & 1988 & 56 & \text { VA }\end{array}$

51985

51985

$5 \quad 1985$

$5 \quad 1973$

$5 \quad 1985$

$5 \quad 1985$

$5 \quad 1976$

$5 \quad 1973$
Varus Valles

Vedra Valles

Vistula Valles

Warrego Valles

\section{VASTITAS}

Vastitas Borealis

\subsection{S 155.9W}

$\begin{array}{llll}0 & \text { EU } & \text { FR } & -10157\end{array}$

I1653

$19.8 \mathrm{~N} \quad 55.7 \mathrm{~W}$

155 EU GB MC10

$14.2 \mathrm{~N}$

$52.1 \mathrm{~W}$

190 EU PO 15052

I1068

I1721

$43.0 \mathrm{~S}$

$93.1 \mathrm{~W}$

164 OC AU 1:15M

I1535
$5 \quad 1988 \quad 56 \quad$ VA

51976

51985

51979 ref $\mathbf{f t}$ origin

71 VA Lowell canal name; also classical river name.

Ancient and modern name for South American river (Brazil, Argentina).

Modern river in Maryland, USA.

36 VA River in Russia.

VA Ancient Pakistani river.

Classical name for river in N.W. Ireland.

62 VA Word for "planet" in Gaelic.

Classical river in Dacia (Romania).

Ancient river in Italy.

Name proposed by Soviets.

Classical name for present Sambre River in France and Belgium.

Classical name for present Severn River, England.

Ancient name for modern

Somme River, France.

Ancient name of river at

Troy (modern Turkey).

56 VA Classical river in Ireland.

56 VA River in New Guinea.

62 VA Word for "Mars" in Akkadian.

62 VA Word for "Mars" in Sumerian.

56 VA Classical river east of

Rome, Italy.

56 VA Classical river in Mauritania.

56 VA Name proposed by Soviets; found on Mars-5 map.

71 VA Lowell canal name.

56 VA Ancient name for present

Segura River, Spain.

56 VA Ancient and modern river in Spain, Portugal.

6 VA Classical river in

Caledonia (Scotland).

Classical river in ancient

Lusitania, present

Tormes River, Spain.

56 VA Classical river in Italy.

36 VA Modern river in Sarawak, Malaysia.

56 VA Ancient nmae for modern

Tisza River, Ukraine.

62 VA Word for "Mars" in old

English (West Germanic).

56 VA Classical name for modern

Trebbia River, Italy.

56 VA Classical name for present Dniester River, Ukraine.

36 VA Dry riverbed in Russia.

60 VA General name of the system of canyons honoring the scientific the team of the Mariner 9 program.

Classical name for present

Var River, France.

56 VA Ancient European river

(Great Britain).

Classical name for modern

Wistla River in Poland.

Modern Australian River. lat: latitude of feature center.

long: longitude of feature center.

diam: diameter or long dimension of feature.

ct: continent of name origin (see page et: ethnicity of name origin (see page $284 \mathrm{ff}$.)

quad: map quadrangle or informal name

(see page $x$ vii $f f$.). as: name approval status (see page xvii).

ad: name approval date (year).

ref: reference source for name (see page $287 \mathrm{ff}$.). 
MARS

Name

long diam ct et quad map as ad ref $f$ origin

DEIMOS

CRATER

Swift

Voltaire

EU GB

51973

60 AA Jonathan; British writer

EU FR

51973

60 AA (1667-1745)

Francios M.; French writer (1694-1778).

\section{PHOBOS}

\section{CRATER}

$\begin{array}{lrrrr}\text { D'Arrest } & 35.0 \mathrm{~S} & 185.0 \mathrm{~W} & \mathrm{EU} & \mathrm{GM} \\ \text { Hall } & 75.0 \mathrm{~S} & 225.0 \mathrm{~W} & \text { NA } & \text { AM } \\ \text { Roche } & 60.0 \mathrm{~N} & 185.0 \mathrm{~W} & \text { EU } & \text { FR } \\ \text { Sharpless } & 25.0 \mathrm{~S} & 165.0 \mathrm{~W} & \text { NA } & \text { AM } \\ \text { Stickney } & 5.0 \mathrm{~S} & 55.0 \mathrm{~W} & \text { NA } & \text { AM } \\ \text { Todd } & 5.0 \mathrm{~S} & 160.0 \mathrm{~W} & \text { NA } & \text { AM } \\ \text { Wendall } & 0.0 \mathrm{~N} & 140.0 \mathrm{~W} & \text { NA } & \text { AM }\end{array}$

\section{DORSUM}

Kepler Dorsum

EU GM

$5 \quad 1987$

5197360 AA Heinrich L.; German

astronomer (1822-1875).

5197360 AA Asaph; American astronomer

5197360 AA $\quad \begin{aligned} & (1829-1907) . \\ & \text { Edourd; French astronomer }\end{aligned}$

(1820-1883).

5197360 AA Bevan P.; American astronomer

$5 \quad 197360$ (1904-1950)

5197360 AA Angeline; American wife of

5197360 AA $\begin{aligned} & \text { astronomer A. Hall (d. 1938). } \\ & \text { David; American astronomer }\end{aligned}$

(1855-1939).

5197360 AA Oliver C.; American astronomer (1845-1912).

DO Johannes; German astronomer (1571-1630). lat: latitude of feature center.

long: longitude of feature center.

diam: diameter or long dimension of feature.

ct: continent of name origin (see page $284 \mathrm{ff}$.) et: ethnicity of name origin (see page $284 \mathrm{ff}$.)

quad: map quadrangle or informal name

(see page xvii ff.). as: name approval status (see page xvii).

ad: name approval date (year).

ref: reference source for name (see page $287 \mathrm{ff}$.).

map: map name or USGS map number (see page xvii ff.). ft: feature type (see page 290). 


\section{ASTEROIDS}

Name

lat

long

diam ct et quad

map

as ad

ref $\mathbf{f t}$ origin

\section{GASPRA}

\section{CRATER}

\begin{tabular}{|c|c|c|c|c|}
\hline Aix & $47.9 \mathrm{~N}$ & $160.3 \mathrm{~W}$ & 6 & EU \\
\hline Alupka & $65.0 \mathrm{~N}$ & $65.0 \mathrm{~W}$ & & UR \\
\hline Baden-Baden & $46.0 \mathrm{~N}$ & $55.0 \mathrm{~W}$ & & EU \\
\hline Badgastein & $25.0 \mathrm{~N}$ & $3.0 \mathrm{~W}$ & & EU \\
\hline Bagnoles & $55.0 \mathrm{~N}$ & $122.0 \mathrm{~W}$ & & EU \\
\hline Bath & $13.4 \mathrm{~N}$ & $9.7 \mathrm{~W}$ & 10 & EU \\
\hline Beppu & $3.9 \mathrm{~N}$ & $58.4 \mathrm{~W}$ & 5 & AS \\
\hline Brookton & $27.7 \mathrm{~N}$ & $103.3 \mathrm{~W}$ & 6 & US \\
\hline Calistoga & $30.0 \mathrm{~N}$ & $2.0 \mathrm{~W}$ & & US \\
\hline Carlsbad & $29.7 \mathrm{~N}$ & $88.8 \mathrm{~W}$ & 5 & EU \\
\hline Charax & $8.6 \mathrm{~N}$ & $0.0 \mathrm{~W}$ & 11 & UR \\
\hline Helwan & $22.4 \mathrm{~N}$ & $118.9 \mathrm{~W}$ & 6 & $\mathrm{AF}$ \\
\hline Ixtapan & $11.9 \mathrm{~N}$ & $86.9 \mathrm{~W}$ & 5 & NA \\
\hline Katsiveli & $55.0 \mathrm{~N}$ & $65.0 \mathrm{~N}$ & & UR \\
\hline Krynica & $49.0 \mathrm{~N}$ & $35.0 \mathrm{~W}$ & & $\mathrm{EU}$ \\
\hline Lisdoonvarna & $16.5 \mathrm{~N}$ & $358.1 \mathrm{~W}$ & 10 & EU \\
\hline Loutraki & $42.0 \mathrm{~N}$ & $140.0 \mathrm{~W}$ & & EU \\
\hline Mandal & $23.5 \mathrm{~N}$ & $46.5 \mathrm{~W}$ & 5 & $\mathrm{EU}$ \\
\hline Manikaran & $62.0 \mathrm{~N}$ & $155.0 \mathrm{~W}$ & & AS \\
\hline Marienbad & $35.4 \mathrm{~N}$ & $81.8 \mathrm{~W}$ & 5 & $\mathrm{EU}$ \\
\hline Miskhor & $15.0 \mathrm{~N}$ & $65.9 \mathrm{~W}$ & 5 & UR \\
\hline Moree & $15.1 \mathrm{~N}$ & $164.4 \mathrm{~W}$ & 6 & OC \\
\hline Ramlösa & $15.0 \mathrm{~N}$ & $4.9 \mathrm{~W}$ & 10 & EU \\
\hline Rio Hondo & $31.7 \mathrm{~N}$ & $20.7 \mathrm{~W}$ & 7 & SA \\
\hline Rotorua & $18.8 \mathrm{~N}$ & $30.7 \mathrm{~W}$ & 6 & OC \\
\hline Saratoga & $50.0 \mathrm{~N}$ & $270.0 \mathrm{~W}$ & & US \\
\hline Spa & $51.5 \mathrm{~N}$ & $152.0 \mathrm{~W}$ & 6 & EU \\
\hline Tang-Shan & $59.0 \mathrm{~N}$ & $256.0 \mathrm{~W}$ & & AS \\
\hline Yalova & $29.0 \mathrm{~N}$ & $10.0 \mathrm{~W}$ & & AS \\
\hline Yalta & $57.6 \mathrm{~N}$ & $261.3 W$ & 5 & UR \\
\hline Zohar & $23.0 \mathrm{~N}$ & $118.0 \mathrm{~W}$ & & AS \\
\hline
\end{tabular}

$\begin{array}{llll}5 & 1994 & 113 & \text { AA } \\ 5 & 1994 & 113 & \text { AA } \\ 5 & 1994 & 113 & \text { AA } \\ 5 & 1994 & 113 & \text { AA } \\ 5 & 1994 & 113 & \text { AA } \\ 5 & 1994 & 113 & \text { AA } \\ 5 & 1994 & 113 & \text { AA } \\ 5 & 1994 & 113 & \text { AA } \\ 5 & 1994 & 113 & \text { AA } \\ 5 & 1994 & 113 & \text { AA } \\ 5 & 1994 & & \text { AA } \\ & & & \\ 5 & 1994 & 113 & \text { AA } \\ 5 & 1994 & 113 & \text { AA } \\ 5 & 1994 & 113 & \text { AA } \\ 5 & 1994 & 113 & \text { AA } \\ 5 & 1994 & 113 & \text { AA } \\ 5 & 1994 & 113 & \text { AA } \\ 5 & 1994 & 113 & \text { AA } \\ 5 & 1994 & 113 & \text { AA } \\ 5 & 1994 & 113 & \text { AA } \\ 5 & 1994 & 113 & \text { AA } \\ 5 & 1994 & 113 & \text { AA } \\ 5 & 1994 & 113 & \text { AA } \\ 5 & 1994 & 113 & \text { AA } \\ 5 & 1994 & 113 & \text { AA } \\ 5 & 1994 & 113 & \text { AA } \\ 5 & 1994 & 113 & \text { AA } \\ 5 & 1994 & 113 & \text { AA } \\ 5 & 1994 & 113 & \text { AA } \\ 5 & 1994 & 113 & \text { AA } \\ 5 & 1994 & 113 & \text { AA }\end{array}$

Spa in France.

Spa in Crimea, Ukraine.

Spa in Germany.

Spa in Austria.

Spa in France.

Spa in England.

Spa on Kyushu, Japan.

Spa in New York, USA.

Resort in California, USA.

Spa in Czech Republic.

Roman fortress in Gaspra,

Crimea, Ukraine.

Spa in Egypt.

Spa in Mexico.

Spa in Crimea, Ukraine.

Health resort in Poland.

Spa in Ireland.

Spa in Greece.

Spa in Norway.

Spa in India.

Spa in Czech Republic.

Spa in Crimea, Ukraine.

Spa in Australia.

Spa in Sweden.

Spa in Argentina.

Spa in New Zealand.

Spa in New York, USA.

Health resort in Belgium.

Spa in China.

Health resort in Turkey.

Spa in Crimea, Ukraine.

Spa in Israel.

\section{REGIO}

Dunne Regio

Neujmin Regio

$15.0 \mathrm{~N} \quad 15.0 \mathrm{~W}$

$2.0 \mathrm{~N} \quad 80.0 \mathrm{~W}$

$65.0 \mathrm{~N} \quad 75.0 \mathrm{~W}$

NA AM

UR RU

NA AM
$5 \quad 1994$

51994

$5 \quad 1994$
RE James; Galileo Project planner.

RE Grigorij N.; Russian astronomer;

discoverer of Gaspra (1885-1946).

RE Clayne; Galileo Project manager.

IDA

CRATER

Afon

6.5S $\quad 0.0 \mathrm{~W}$

26 UR RU

1994 AA Cave in Russia.

lat: latitude of feature center.

long: longitude of feature center.

diam: diameter or long dimension of feature.

ct: continent of name origin (see page $284 \mathrm{ff}$.) et: ethnicity of name origin (see page $284 \mathrm{ff}$.)

quad: map quadrangle or informal name

(see page ix ff.). as: name approval status (see page $x$ vii).

ad: name approval date (year).

ref: reference source for name (see page $287 \mathrm{ff}$.). 
Name

lat long diam ct et quad

map

as ad

\section{AMALTHEA}

CRATER

Gaea

Pan

Ida Facula

Lyctos Facula

\section{CALISTO}

\section{CRATER}

Adal

Ägröi

Ahti

Ajleke

Akycha

Alfr

Âli

Aningan

Askr

Aziren

Balkr

Bavörr

Beli

Bragi

Brami

Bran

Buga

Buri

Burr

Dag

Danr

Dia

Dryops

Durinn

Egdir

Egres

Erlik

Fadir

Fili

Finnr

Freki

Frodi

Fulla

Fulnir

Geri

Gisl

$\begin{array}{lllllllllll}80.0 \mathrm{~S} & 90.0 \mathrm{~W} & 0 & \text { EU } & \text { GR } & \text { TRANS } & 1979 & 5 & 1979 & 19 & \text { AA } \\ 55.0 \mathrm{~N} & 35.0 \mathrm{~W} & 0 & \text { EU } & \text { GR } & \text { TRANS } & 1979 & 5 & 1979 & 19 & \text { AA }\end{array}$

20.0N $175.0 \mathrm{~W}$

20.0S $\quad 120.0 \mathrm{~W}$
0 EU GR TRANS 1979

0 EU GR TRANS 1979
51979

51979
Greek mother earth goddess who brought Zeus to Crete. Greek; goat-god, son of Amalthea and Hermes in some legends, also Zeus' foster brother.

\begin{tabular}{|c|c|c|c|c|c|c|c|c|c|c|c|}
\hline $\begin{array}{l}75.4 \mathrm{~N} \\
43.3 \mathrm{~N} \\
41.8 \mathrm{~N}\end{array}$ & $\begin{array}{r}80.8 \mathrm{~W} \\
11.0 \mathrm{~W} \\
103.1 \mathrm{~W}\end{array}$ & $\begin{array}{l}40 \\
55 \\
52\end{array}$ & $\begin{array}{l}\text { EU } \\
\text { EU } \\
\text { EU }\end{array}$ & $\begin{array}{l}\text { NS } \\
\text { FI } \\
\text { FI }\end{array}$ & $\begin{array}{l}\text { PRELIM } \\
\text { PRELIM } \\
\text { JC-3 }\end{array}$ & $\begin{array}{l}\text { I1239 } \\
\text { I1239 } \\
\text { I1 } 1888\end{array}$ & $\begin{array}{l}5 \\
5 \\
5\end{array}$ & $\begin{array}{l}1979 \\
1979 \\
1987\end{array}$ & $\begin{array}{l}17 \\
27 \\
64\end{array}$ & $\begin{array}{l}\text { AA } \\
\text { AA } \\
\text { AA }\end{array}$ & $\begin{array}{l}\text { Norse; son of Karl and Erna. } \\
\text { Finno-Ugric god of twins. } \\
\text { Finnish god of water; } \\
\text { sends fish to the fisherman }\end{array}$ \\
\hline $\begin{array}{r}22.4 \mathrm{~N} \\
72.5 \mathrm{~N} \\
9.7 \mathrm{~S} \\
59.3 \mathrm{~N} \\
44.1 \mathrm{~N} \\
50.5 \mathrm{~N}\end{array}$ & $\begin{array}{r}101.3 \mathrm{~W} \\
318.6 \mathrm{~W} \\
222.8 \mathrm{~W} \\
56.2 \mathrm{~W} \\
0.6 \mathrm{~W} \\
8.2 \mathrm{~W}\end{array}$ & $\begin{array}{r}46 \\
67 \\
60 \\
61 \\
47 \\
287\end{array}$ & $\begin{array}{l}\text { EU } \\
\text { NA } \\
\text { EU } \\
\text { EU } \\
\text { EU } \\
\text { NP }\end{array}$ & $\begin{array}{l}\text { LP } \\
\text { ES } \\
\text { NS } \\
\text { NS } \\
\text { NS } \\
\text { ES }\end{array}$ & $\begin{array}{l}\text { JC-3 } \\
\text { PRELIM } \\
\text { PRELIM } \\
\text { PRELIM } \\
\text { PRELIM } \\
\text { PRELIM }\end{array}$ & $\begin{array}{l}\text { I1888 } \\
\text { I1 } 239 \\
\text { I1239 } \\
\text { I1239 } \\
\text { I1239 } \\
\text { I1239 }\end{array}$ & 5 & $\begin{array}{l}1987 \\
1979 \\
1979 \\
1979 \\
1979 \\
1979\end{array}$ & $\begin{array}{l}64 \\
26 \\
17 \\
17 \\
17 \\
26\end{array}$ & $\begin{array}{l}\text { AA } \\
\text { AA } \\
\text { AA } \\
\text { AA } \\
\text { AA } \\
\text { AA }\end{array}$ & $\begin{array}{l}\text { Saami god of holidays. } \\
\text { Alaskan name of the sun. } \\
\text { Norse dwarf. } \\
\text { Norse; strongest of men. } \\
\text { Norse dwarf. } \\
\text { Moon god of Greenland } \\
\text { Eskimos. }\end{array}$ \\
\hline $51.7 \mathrm{~N}$ & $324.1 \mathrm{~W}$ & 64 & EU & NS & PRELIM & I1239 & 5 & 1979 & 17 & AA & $\begin{array}{l}\text { Norse; first man, created } \\
\text { from a log drifted ashore } \\
\text { on a beach. }\end{array}$ \\
\hline $\begin{array}{l}35.4 \mathrm{~N} \\
29.1 \mathrm{~N} \\
49.2 \mathrm{~N} \\
62.6 \mathrm{~N} \\
75.7 \mathrm{~N} \\
28.9 \mathrm{~N} \\
24.3 \mathrm{~S}\end{array}$ & $\begin{array}{r}178.3 \mathrm{~W} \\
11.9 \mathrm{~W} \\
20.3 \mathrm{~W} \\
81.7 \mathrm{~W} \\
61.7 \mathrm{~W} \\
19.2 \mathrm{~W} \\
207.7 \mathrm{~W}\end{array}$ & $\begin{array}{l}64 \\
64 \\
84 \\
50 \\
65 \\
67 \\
89\end{array}$ & $\begin{array}{l}\text { UR } \\
\text { EU } \\
\text { EU } \\
\text { EU } \\
\text { EU } \\
\text { EU } \\
\text { EU }\end{array}$ & $\begin{array}{l}\text { ES } \\
\text { NS } \\
\text { NS } \\
\text { CE } \\
\text { NS } \\
\text { NS } \\
\text { CE }\end{array}$ & $\begin{array}{l}\text { JC-3 } \\
\text { PRELIM } \\
\text { PRELIM } \\
\text { PRELIM } \\
\text { PRELIM } \\
\text { PRELIM } \\
\text { PRELIM }\end{array}$ & $\begin{array}{l}\text { I1 } 1888 \\
\text { I1239 } \\
\text { I1239 } \\
\text { I1239 } \\
\text { I1239 } \\
\text { I1239 } \\
\text { I1239 }\end{array}$ & 5 & $\begin{array}{l}1987 \\
1979 \\
1979 \\
1979 \\
1979 \\
1979 \\
1979\end{array}$ & $\begin{array}{r}64 \\
17 \\
17 \\
27 \\
17 \\
17 \\
1\end{array}$ & $\begin{array}{l}\text { AA } \\
\text { AA } \\
\text { AA } \\
\text { AA } \\
\text { AA } \\
\text { AA } \\
\text { AA }\end{array}$ & $\begin{array}{l}\text { Estonian spirit of death. } \\
\text { Norse; Ottar's ancestor. } \\
\text { Norse dwarf. } \\
\text { Celtic; father of Caswallawn. } \\
\text { Skaldic; god of poetry. } \\
\text { Norse; Ottar's ancestor. } \\
\text { Celtic; omnipotent god }\end{array}$ \\
\hline $\begin{array}{l}2.2 \mathrm{~N} \\
8.7 \mathrm{~S} \\
2.5 \mathrm{~N}\end{array}$ & $\begin{array}{r}323.9 \mathrm{~W} \\
46.2 \mathrm{~W} \\
135.5 \mathrm{~W}\end{array}$ & $\begin{array}{l}54 \\
98 \\
74\end{array}$ & $\begin{array}{l}\text { UR } \\
\text { EU } \\
\text { EU }\end{array}$ & $\begin{array}{l}\text { TU } \\
\text { NS } \\
\text { NS }\end{array}$ & $\begin{array}{l}\text { PRELIM } \\
\text { PRELIM } \\
\text { PRELIM }\end{array}$ & $\begin{array}{l}I 1239 \\
\text { I1239 } \\
\text { I1 } 1239\end{array}$ & & $\begin{array}{l}1979 \\
1979 \\
1979\end{array}$ & $\begin{array}{l}27 \\
17 \\
17\end{array}$ & $\begin{array}{l}\text { AA } \\
\text { AA } \\
\text { AA }\end{array}$ & $\begin{array}{l}\text { Tungu heaven god. } \\
\text { Norse dwarf. } \\
\text { Norse giant; his sons } \\
\text { raised up heaven's vault }\end{array}$ \\
\hline $\begin{array}{l}8.6 \mathrm{~N} \\
2.5 \mathrm{~N}\end{array}$ & $\begin{array}{l}74.2 \mathrm{~W} \\
77.8 \mathrm{~W}\end{array}$ & $\begin{array}{l}40 \\
48\end{array}$ & $\begin{array}{l}\text { EU } \\
\text { EU }\end{array}$ & $\begin{array}{l}\text { NS } \\
\text { NS }\end{array}$ & $\begin{array}{l}\text { PRELIM } \\
\text { PRELIM }\end{array}$ & & & $\begin{array}{l}1979 \\
1979\end{array}$ & $\begin{array}{l}17 \\
17\end{array}$ & & $\begin{array}{l}\text { Norse; Ottar's ancestor. } \\
\text { Norse; king against whom } \\
\text { Konr marched. }\end{array}$ \\
\hline $\begin{array}{l}73.0 \mathrm{~N} \\
77.6 \mathrm{~N} \\
67.0 \mathrm{~N} \\
33.9 \mathrm{~N} \\
42.5 \mathrm{~N}\end{array}$ & $\begin{array}{r}50.4 \mathrm{~W} \\
21.3 \mathrm{~W} \\
90.1 \mathrm{~W} \\
35.9 \mathrm{~W} \\
176.6 \mathrm{~W}\end{array}$ & $\begin{array}{l}35 \\
42 \\
49 \\
58 \\
38\end{array}$ & $\begin{array}{l}\text { EU } \\
\text { EU } \\
\text { EU } \\
\text { EU } \\
\text { UR }\end{array}$ & $\begin{array}{l}\text { GR } \\
\text { GR } \\
\text { NS } \\
\text { NS } \\
\text { KA }\end{array}$ & $\begin{array}{l}\text { PRELIM } \\
\text { PRELIM } \\
\text { PRELIM } \\
\text { PRELIM } \\
\text { JC-3 }\end{array}$ & $\begin{array}{l}\text { I1239 } \\
\text { I1239 } \\
\text { I1239 } \\
\text { I1239 } \\
\text { I1 } 1888\end{array}$ & 5 & $\begin{array}{l}1979 \\
1979 \\
1979 \\
1979 \\
1987\end{array}$ & $\begin{array}{l}19 \\
19 \\
17 \\
17 \\
64\end{array}$ & $\begin{array}{l}\text { A } \\
\text { A } \\
\text { A } \\
\text { A } \\
\text { A }\end{array}$ & $\begin{array}{l}\text { isto's sister. } \\
\text { of Dia by Apollo. } \\
\text { f. } \\
\text { herd for the giants. } \\
\text { ity of the }\end{array}$ \\
\hline $66.8 \mathrm{~N}$ & $1.3 \mathrm{~W}$ & 39 & UR & RU & PRELIM & I1239 & 5 & 1979 & 17 & AA & $\begin{array}{l}\text { nan who } \\
\text { l. }\end{array}$ \\
\hline $\begin{array}{l}56.4 \mathrm{~N} \\
64.3 \mathrm{~N} \\
15.5 \mathrm{~N} \\
79.9 \mathrm{~N}\end{array}$ & $\begin{array}{r}12.7 \mathrm{~W} \\
349.5 \mathrm{~W} \\
4.3 \mathrm{~W} \\
352.0 \mathrm{~W}\end{array}$ & $\begin{array}{l}81 \\
42 \\
65 \\
48\end{array}$ & $\begin{array}{l}\text { EU } \\
\text { EU } \\
\text { EU } \\
\text { EU }\end{array}$ & $\begin{array}{l}\text { NS } \\
\text { NS } \\
\text { NS } \\
\text { NS }\end{array}$ & $\begin{array}{l}\text { PRELIM } \\
\text { PRELIM } \\
\text { PRELIM } \\
\text { PRELIM }\end{array}$ & $\begin{array}{l}\text { I1239 } \\
\text { I1239 }\end{array}$ & $\begin{array}{l}5 \\
5 \\
5 \\
5\end{array}$ & $\begin{array}{l}1979 \\
1979 \\
1979 \\
1979\end{array}$ & $\begin{array}{l}17 \\
17 \\
17 \\
17\end{array}$ & $\begin{array}{l}\text { AA } \\
\text { AA } \\
\text { AA } \\
\text { AA }\end{array}$ & $\begin{array}{l}\text { farmer. } \\
\text { dwarf. } \\
\text { dwarf. } \\
\text { wolf's name } \\
\text { ing "insatiable." }\end{array}$ \\
\hline $\begin{array}{l}68.3 \mathrm{~N} \\
73.5 \mathrm{~N}\end{array}$ & $\begin{array}{l}139.1 \mathrm{~W} \\
103.7 \mathrm{~W}\end{array}$ & $\begin{array}{l}44 \\
45\end{array}$ & $\begin{array}{l}\text { EU } \\
\text { EU }\end{array}$ & $\begin{array}{l}\text { NS } \\
\text { NS }\end{array}$ & $\begin{array}{l}\text { PRELIM } \\
\text { PRELIM }\end{array}$ & $\begin{array}{l}\text { I1239 } \\
\text { I1239 }\end{array}$ & $\begin{array}{l}5 \\
5\end{array}$ & $\begin{array}{l}1979 \\
1979\end{array}$ & $\begin{array}{l}17 \\
17\end{array}$ & $\begin{array}{l}\text { AA } \\
\text { AA }\end{array}$ & $\begin{array}{l}\text { Norse; Hledis' father. } \\
\text { Norse; maid to Frigg, queen of } \\
\text { the gods. }\end{array}$ \\
\hline $\begin{array}{l}60.3 \mathrm{~N} \\
66.7 \mathrm{~N}\end{array}$ & $\begin{array}{r}35.5 \mathrm{~W} \\
354.2 \mathrm{~W}\end{array}$ & $\begin{array}{l}46 \\
38\end{array}$ & $\begin{array}{l}\text { EU } \\
\text { EU }\end{array}$ & $\begin{array}{l}\text { NS } \\
\text { NS }\end{array}$ & $\begin{array}{l}\text { PRELIM } \\
\text { PRELIM }\end{array}$ & $\begin{array}{l}\text { I1239 } \\
\text { I1239 }\end{array}$ & $\begin{array}{l}5 \\
5\end{array}$ & $\begin{array}{l}1979 \\
1979\end{array}$ & $\begin{array}{l}17 \\
17\end{array}$ & $\begin{array}{l}\text { AA } \\
\text { AA }\end{array}$ & $\begin{array}{l}\text { Norse; son of Thrael and Thyr. } \\
\text { Norse; wolf's name } \\
\text { meaning "greedy" }\end{array}$ \\
\hline $7.2 \mathrm{~N}$ & $34.8 \mathrm{~W}$ & 39 & EU & NS & PRELIM & I1239 & 5 & 1979 & 17 & AA & Norse; steed ridden by Aesir. \\
\hline
\end{tabular}

lat: latitude of feature center. long: longitude of feature center.

diam: diameter or long dimension of feature.

ct: continent of name origin (see page $284 \mathrm{ff}$.) et: ethnicity of name origin (see page $284 \mathrm{ff}$.)

quad: map quadrangle or informal name (see page xvii ff.).
Greek; mountain where Zeus played as a child.

Greek; area in Crete where Zeus was raised. 


\section{JOVIAN SYSTEM}

\begin{tabular}{|c|c|c|c|c|c|c|c|c|c|c|c|c|}
\hline Name & lat & long & diam & ct & et & quad & map & as & ad & ref & $\mathrm{ft}$ & origin \\
\hline Hoenir & $33.9 \mathrm{~S}$ & $261.2 \mathrm{~W}$ & 84 & EU & NS & PRELIM & I1239 & 5 & 1979 & 17 & AA & $\begin{array}{l}\text { Norse; god who gave souls } \\
\text { to first humans. }\end{array}$ \\
\hline $\begin{array}{l}\text { Högni } \\
\text { Höldr }\end{array}$ & $\begin{array}{l}13.5 \mathrm{~S} \\
44.1 \mathrm{~N}\end{array}$ & $\begin{array}{r}4.5 \mathrm{~W} \\
109.2 \mathrm{~W}\end{array}$ & $\begin{array}{l}65 \\
61\end{array}$ & $\begin{array}{l}\text { EU } \\
\text { EU }\end{array}$ & $\begin{array}{l}\text { NS } \\
\text { NS }\end{array}$ & $\begin{array}{l}\text { PRELIM } \\
\text { JC-3 }\end{array}$ & $\begin{array}{l}\text { I1 } 239 \\
\text { I1 } 1888\end{array}$ & $\begin{array}{l}5 \\
5\end{array}$ & $\begin{array}{l}1979 \\
1988\end{array}$ & $\begin{array}{l}17 \\
17\end{array}$ & $\begin{array}{l}\text { AA } \\
\text { AA }\end{array}$ & $\begin{array}{l}\text { Norse; Ottar's ancestor. } \\
\text { Son of Karl and Snor in } \\
\text { Rigdismal. }\end{array}$ \\
\hline Lodurr & $51.2 \mathrm{~S}$ & $270.8 \mathrm{~W}$ & 76 & EU & NS & PRELIM & I1 239 & 5 & 1979 & 17 & AA & $\begin{array}{l}\text { Norse; god who gave first } \\
\text { humans goodly color. }\end{array}$ \\
\hline $\begin{array}{l}\text { Loni } \\
\text { Losy }\end{array}$ & $\begin{array}{r}3.6 \mathrm{~S} \\
65.3 \mathrm{~N}\end{array}$ & $\begin{array}{l}214.9 \mathrm{~W} \\
323.1 \mathrm{~W}\end{array}$ & $\begin{array}{l}86 \\
62\end{array}$ & $\begin{array}{l}\text { EU } \\
\text { AS }\end{array}$ & $\begin{array}{l}\text { NS } \\
\text { MO }\end{array}$ & $\begin{array}{l}\text { PRELIM } \\
\text { PRELIM }\end{array}$ & $\begin{array}{l}I 1239 \\
\text { I1239 }\end{array}$ & $\begin{array}{l}5 \\
5\end{array}$ & $\begin{array}{l}1979 \\
1979\end{array}$ & $\begin{array}{l}17 \\
27\end{array}$ & $\begin{array}{l}\text { AA } \\
\text { AA }\end{array}$ & $\begin{array}{l}\text { Norse dwarf. } \\
\text { Mongolian; Mongol evil snake; } \\
\text { tried to kill all living things. }\end{array}$ \\
\hline $\begin{array}{l}\text { Maderatcha } \\
\text { Mera }\end{array}$ & $\begin{array}{l}30.5 \mathrm{~N} \\
64.1 \mathrm{~N}\end{array}$ & $\begin{array}{l}95.9 \mathrm{~W} \\
75.8 \mathrm{~W}\end{array}$ & $\begin{array}{l}57 \\
36\end{array}$ & $\begin{array}{l}\mathrm{EU} \\
\mathrm{EU}\end{array}$ & $\begin{array}{l}\text { LP } \\
\text { GR }\end{array}$ & $\begin{array}{l}\text { JC-3 } \\
\text { PRELIM }\end{array}$ & $\begin{array}{l}\text { I1888 } \\
\text { I1239 }\end{array}$ & $\begin{array}{l}5 \\
5\end{array}$ & $\begin{array}{l}1987 \\
1979\end{array}$ & $\begin{array}{l}64 \\
19\end{array}$ & $\begin{array}{l}\text { AA } \\
\text { AA }\end{array}$ & $\begin{array}{l}\text { Saami sky god. } \\
\text { Greek; another nymph of } \\
\text { Artemis seduced by Zeus. }\end{array}$ \\
\hline $\begin{array}{l}\text { Nori } \\
\text { Norov-Ava } \\
\text { Nuada } \\
\text { Omol' } \\
\text { Oski } \\
\text { Ottar } \\
\text { Pekko } \\
\text { Reginn } \\
\text { Rigr }\end{array}$ & $\begin{array}{l}45.4 \mathrm{~N} \\
54.6 \mathrm{~N} \\
62.1 \mathrm{~N} \\
42.2 \mathrm{~N} \\
57.2 \mathrm{~N} \\
61.5 \mathrm{~N} \\
18.3 \mathrm{~N} \\
39.7 \mathrm{~N} \\
70.9 \mathrm{~N}\end{array}$ & $\begin{array}{r}343.5 \mathrm{~W} \\
113.7 \mathrm{~W} \\
273.2 \mathrm{~W} \\
118.4 \mathrm{~W} \\
269.3 \mathrm{~W} \\
104.8 \mathrm{~W} \\
5.4 \mathrm{~W} \\
90.8 \mathrm{~W} \\
245.0 \mathrm{~W}\end{array}$ & $\begin{array}{l}86 \\
47 \\
66 \\
60 \\
57 \\
50 \\
61 \\
51 \\
54\end{array}$ & $\begin{array}{l}\text { EU } \\
\text { UR } \\
\text { EU } \\
\text { UR } \\
\text { EU } \\
\text { EU } \\
\text { EU } \\
\text { EU } \\
\text { EU }\end{array}$ & $\begin{array}{l}\text { NS } \\
\text { MO } \\
\text { CE } \\
\text { RU } \\
\text { NS } \\
\text { NS } \\
\text { FI } \\
\text { NS } \\
\text { NS }\end{array}$ & $\begin{array}{l}\text { PRELIM } \\
\text { JC-3 } \\
\text { PRELIM } \\
\text { JC-3 } \\
\text { PRELIM } \\
\text { PRELIM } \\
\text { PRELIM } \\
\text { JC-3 } \\
\text { PRELIM }\end{array}$ & $\begin{array}{l}\text { I1 } 239 \\
I 1888 \\
I 1239 \\
I 1888 \\
I 1239 \\
\text { I1888 } \\
\text { I1239 } \\
\text { I1888 } \\
\text { I1 } 239\end{array}$ & $\begin{array}{l}5 \\
5 \\
5 \\
5 \\
5 \\
5 \\
5 \\
5 \\
5\end{array}$ & $\begin{array}{l}1979 \\
1987 \\
1979 \\
1987 \\
1979 \\
1979 \\
1979 \\
1979 \\
1979\end{array}$ & $\begin{array}{r}17 \\
64 \\
1 \\
64 \\
17 \\
17 \\
27 \\
17 \\
17\end{array}$ & $\begin{array}{l}\text { AA } \\
\text { AA } \\
\text { AA } \\
\text { AA } \\
\text { AA } \\
\text { AA } \\
\text { AA } \\
\text { AA } \\
\text { AA }\end{array}$ & $\begin{array}{l}\text { Norse dwarf. } \\
\text { Mordvinian mistress of the field. } \\
\text { Irish chieftain god. } \\
\text { Komi wood spirit. } \\
\text { Norse; a name for Odin. } \\
\text { Innsteinn's son and Freyja's favorite. } \\
\text { Finno-Ugric god of barley. } \\
\text { Norse dwarf. } \\
\text { Norse; another name for the god } \\
\text { Heimdall. }\end{array}$ \\
\hline Rongoteus & $53.5 \mathrm{~N}$ & $106.8 \mathrm{~W}$ & 35 & UR & KA & JC-3 & I1888 & 5 & 1987 & 64 & $\mathrm{AA}$ & $\begin{array}{l}\text { Karelian deity of the harvest of } \\
\text { rye. }\end{array}$ \\
\hline $\begin{array}{l}\text { Rota } \\
\text { Saga }\end{array}$ & $\begin{array}{r}27.8 \mathrm{~N} \\
0.0 \mathrm{~N}\end{array}$ & $\begin{array}{l}\text { 109.8W } \\
326.0 \mathrm{~W}\end{array}$ & $\begin{array}{r}55 \\
0\end{array}$ & $\begin{array}{l}\text { EU } \\
\text { EU }\end{array}$ & $\begin{array}{l}\text { LP } \\
\text { SC }\end{array}$ & $\begin{array}{l}\text { JC-3 } \\
\text { PRELIM }\end{array}$ & $\begin{array}{l}\text { I1 } 1888 \\
\text { I1 } 239\end{array}$ & $\begin{array}{l}5 \\
5\end{array}$ & $\begin{array}{l}1987 \\
1979\end{array}$ & $\begin{array}{l}64 \\
17\end{array}$ & $\begin{array}{l}\mathrm{AA} \\
\mathrm{AA}\end{array}$ & $\begin{array}{l}\text { Deity of the underground world. } \\
\text { Scandinavian goddess, } \\
\text { wife of Odin; Rand } \\
\text { control point crater. }\end{array}$ \\
\hline $\begin{array}{l}\text { Sarakka } \\
\text { Seqinek } \\
\text { Sholmo } \\
\text { Sigyn }\end{array}$ & $\begin{array}{r}3.7 \mathrm{~S} \\
55.5 \mathrm{~N} \\
53.9 \mathrm{~N} \\
35.8 \mathrm{~N}\end{array}$ & $\begin{array}{l}53.7 \mathrm{~W} \\
25.5 \mathrm{~W} \\
16.4 \mathrm{~W} \\
29.2 \mathrm{~W}\end{array}$ & $\begin{array}{l}56 \\
80 \\
58 \\
44\end{array}$ & $\begin{array}{l}\text { EU } \\
\text { NA } \\
\text { EU } \\
\text { EU }\end{array}$ & $\begin{array}{l}\text { FI } \\
\text { ES } \\
\text { FI } \\
\text { NS }\end{array}$ & $\begin{array}{l}\text { PRELIM } \\
\text { PRELIM } \\
\text { PRELIM } \\
\text { PRELIM }\end{array}$ & $\begin{array}{l}\mathrm{I} 1239 \\
\mathrm{I} 1239 \\
\mathrm{I} 1239 \\
\mathrm{I} 1239\end{array}$ & $\begin{array}{l}5 \\
5 \\
5 \\
5\end{array}$ & $\begin{array}{l}1979 \\
1979 \\
1979 \\
1979\end{array}$ & $\begin{array}{l}27 \\
26 \\
27 \\
17\end{array}$ & $\begin{array}{l}\text { AA } \\
\text { AA } \\
\text { AA } \\
\text { AA }\end{array}$ & $\begin{array}{l}\text { Finno-Ugric goddess of childbirth. } \\
\text { Eskimo; the sun. } \\
\text { Finno-Ugric heaven god. } \\
\text { Norse; Loki's wife. }\end{array}$ \\
\hline
\end{tabular}

lat: latitude of feature center. long: longitude of feature center.

diam: diameter or long dimension of feature.

ct: continent of name origin (see page $284 \mathrm{ff}$.) et: ethnicity of name origin (see page $284 \mathrm{ff}$.)

quad: map quadrangle or informal name (see page xvii ff.). as: name approval status (see page xvii)

ad: name approval date (year).

ref: reference source for name (see page $287 \mathrm{ff}$.).

map: map name or USGS map number (see page xvii ff.). ft: feature type (see page 290). 


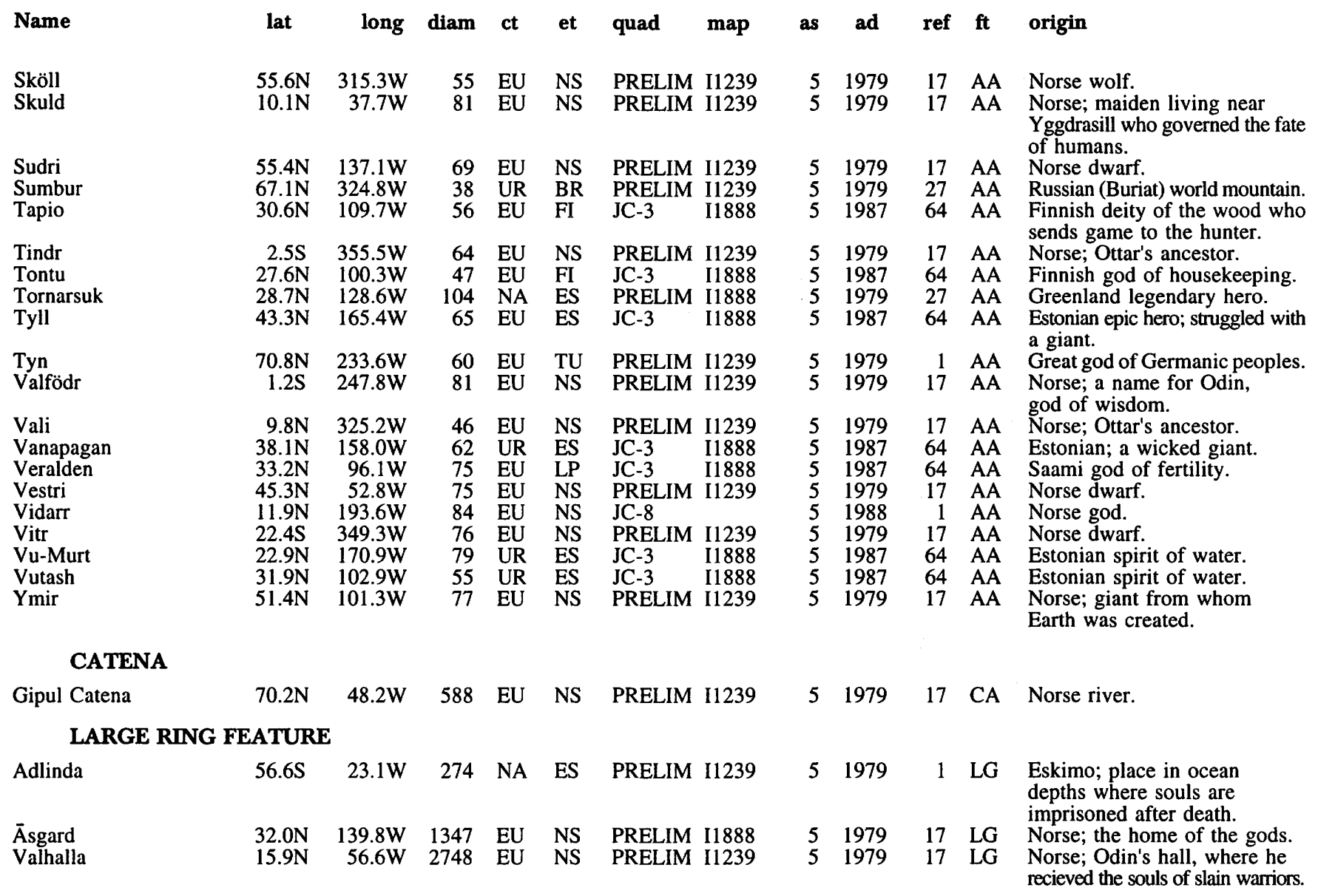

\section{GANYMEDE}

\section{CRATER}

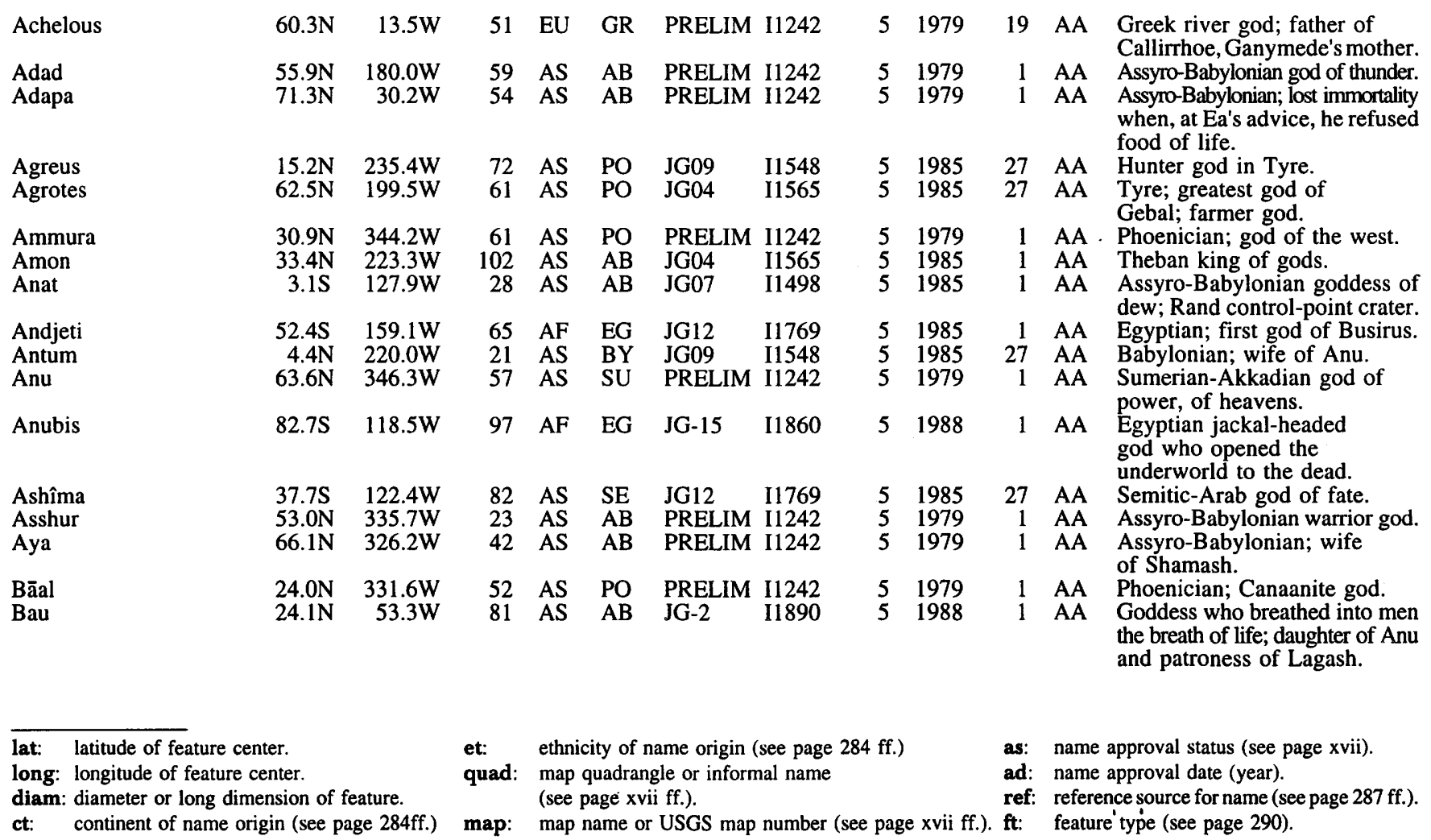


JOVIAN SYSTEM

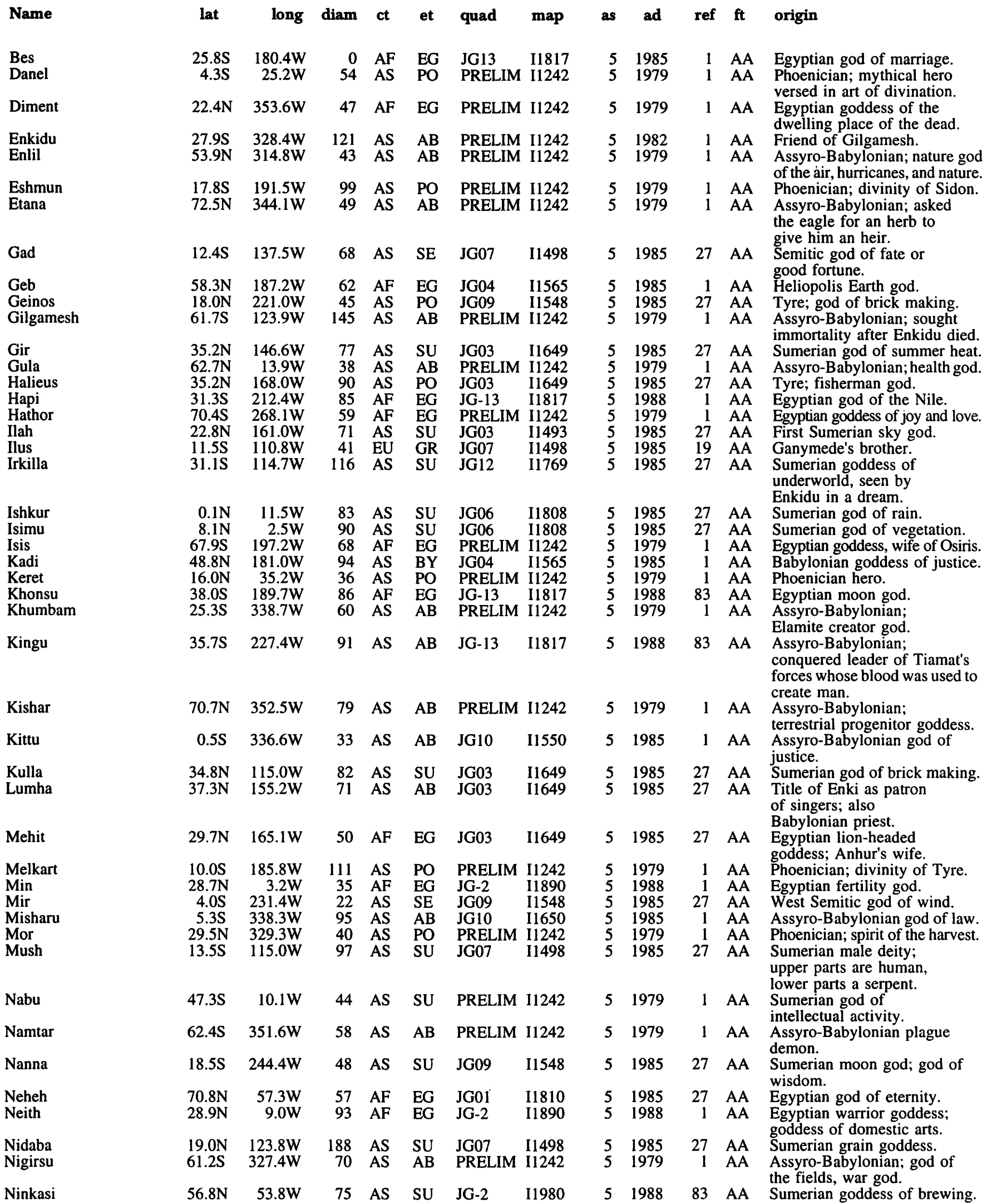

lat: latitude of feature center.

long: longitude of feature center.

diam: diameter or long dimension of feature.

ct: continent of name origin (see page $284 \mathrm{ff}$ ) et: ethnicity of name origin (see page $284 \mathrm{ff}$.)

quad: map quadrangle or informal name

(see page xvii ff.). as: name approval status (see page xvii).

ad: name approval date (year).

ref: reference source for name (see page $287 \mathrm{ff}$.)

map: map name or USGS map number (see page xvii ff.). $\mathrm{ft}$ : feature type (see page 290). 


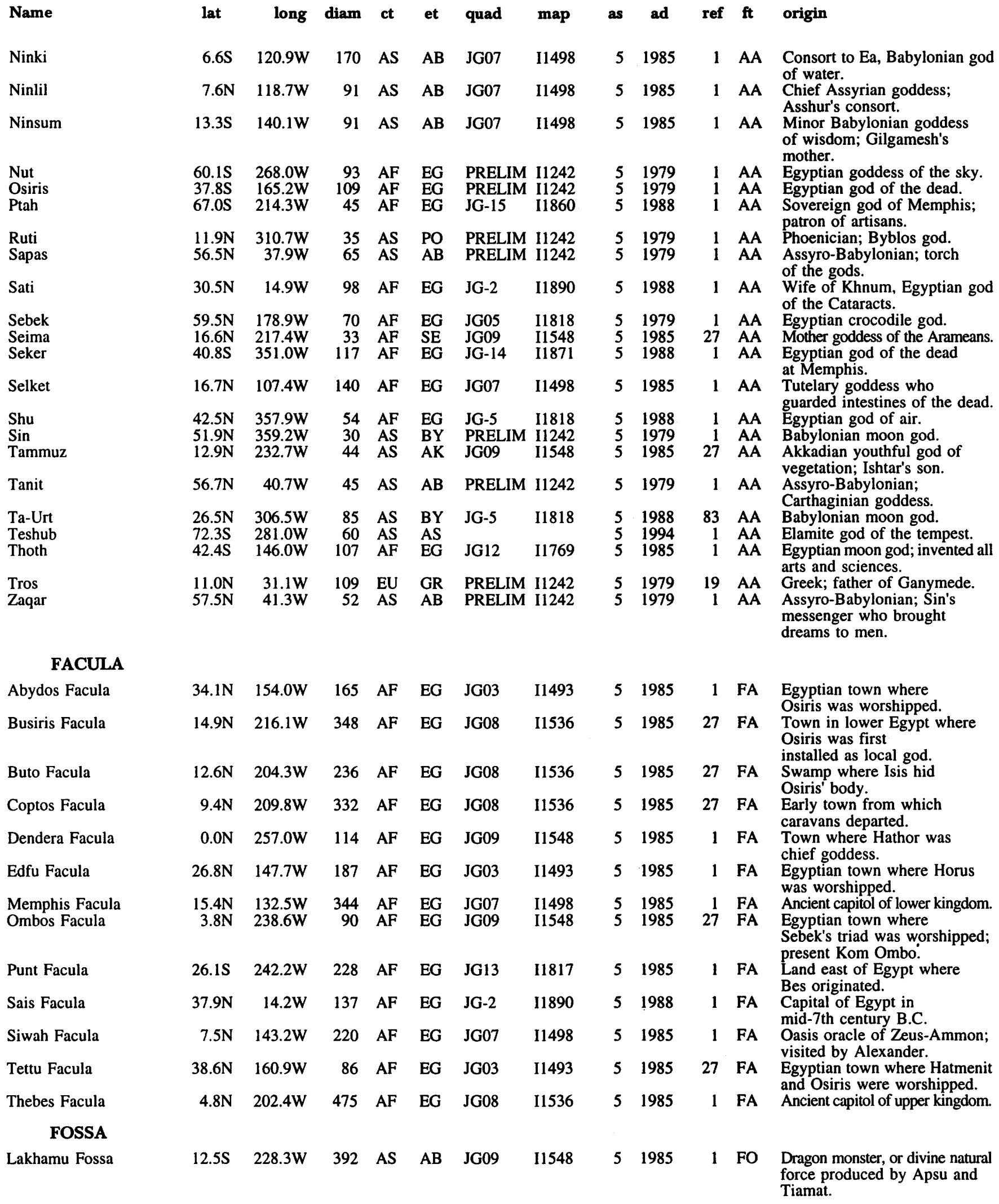

lat: latitude of feature center. long: longitude of feature center.

diam: diameter or long dimension of feature.

ct: continent of name origin (see page 284ff.) et: ethnicity of name origin (see page $284 \mathrm{ff}$.)

quad: map quadrangle or informal name

(see page xvii ff.). as: name approval status (see page xvii).

ad: name approval date (year).

ref: reference source for name (see page $287 \mathrm{ff}$.). 
JOVIAN SYSTEM

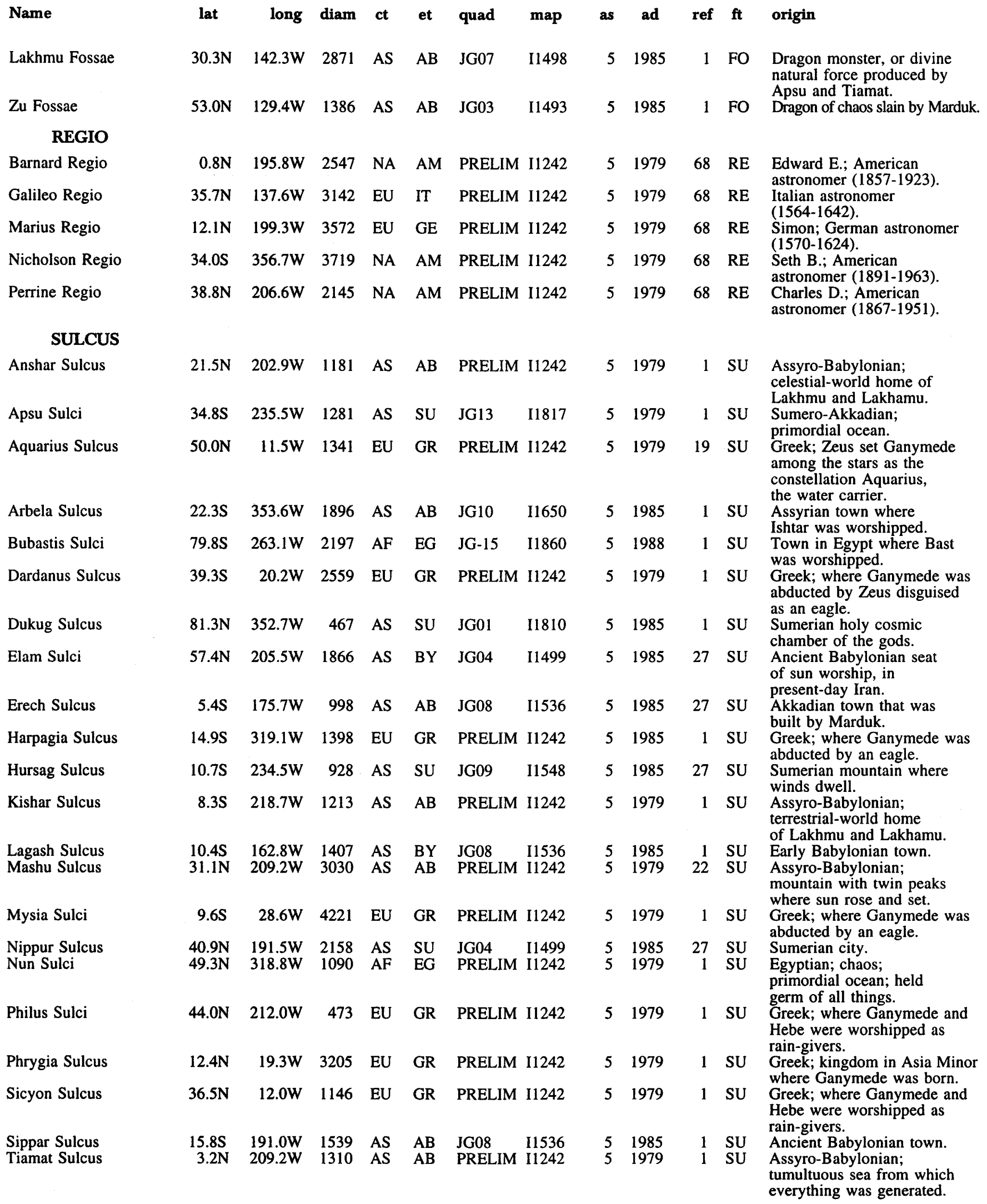

\footnotetext{
lat: latitude of feature center.

et: ethnicity of name origin (see page $284 \mathrm{ff}$.)

long: longitude of feature center.

quad: map quadrangle or informal name

(see page xvii ff.).

as: name approval status (see page xvii).

ad: name approval date (year).

diam: diameter or long dimension of feature.

ref: reference source for name (see page $287 \mathrm{ff}$.)

ct: continent of name origin (see page 284ff.) map: map name or USGS map number (see page xvii ff.). ft: feature type (see page 290).
} 
JOVIAN SYSTEM

$\begin{array}{lrrrrrrrrrrrr}\text { Name } & \text { lat } & \text { long } & \text { diam } & \text { ct } & \text { et } & \text { quad } & \text { map } & \text { as } & \text { ad } & \text { ref } & \text { origin } \\ \text { Ur Sulcus } & 56.5 \mathrm{~N} & 177.3 \mathrm{~W} & 1774 & \text { AS } & \text { SU } & \text { JG04 } & 11499 & 5 & 1985 & 27 & \text { SU } & \begin{array}{l}\text { Ancient Sumerian seat of } \\ \text { moon worship. }\end{array} \\ \text { Uruk Sulcus } & 8.4 \mathrm{~N} & 169.0 \mathrm{~W} & 2456 & \text { AS } & \text { BY } & \text { PRELIM } & \text { I1242 } & 5 & 1979 & 1 & \text { SU } & \begin{array}{l}\text { Babylonian city ruled by } \\ \text { Gilgamesh. }\end{array}\end{array}$

EUROPA

CRATER

Morvran

Rhiannon

Taliesin

Tegid

$\begin{array}{cccccc}1.2 \mathrm{~N} & 181.9 \mathrm{~W} & 23 & \mathrm{EU} & \mathrm{GR} & \mathrm{JE} 03 \\ 5.7 \mathrm{~S} & 152.2 \mathrm{~W} & 25 & \mathrm{EU} & \mathrm{CE} & \text { JE03 } \\ 81.8 \mathrm{~S} & 199.7 \mathrm{~W} & 25 & \mathrm{EU} & \mathrm{CE} & \text { JE04 } \\ 23.2 \mathrm{~S} & 137.4 \mathrm{~W} & 48 & \mathrm{EU} & \mathrm{CE} & \mathrm{JE} 03 \\ 0.6 \mathrm{~S} & 164.0 \mathrm{~W} & 29 & \mathrm{EU} & \mathrm{CE} & \text { JE03 }\end{array}$

I1493

I1493

I1499

I1493

I1493

$\begin{array}{ll}5 & 1985 \\ 5 & 1985 \\ 5 & 1985 \\ 5 & 1985 \\ 5 & 1985\end{array}$

19 AA

27 AA

19 AA

27 AA

27 AA

Babylonian

\section{FLEXUS}

Cilicia Flexus

Delphi Flexus

Gortyna Flexus

Phocis Flexus

Sidon Flexus

\section{LINEA}

Adonis Line

Agenor Linea

Alphesiboea Linea

Argiope Linea

Asterius Linea

Belus Linea

Cadmus Linea

Echion Linea

Libya Linea

Minos Linea

Pelorus Linea

Phineus Linea

Phoenix Linea

Rhadamanthys Linea

Sarpedon Linea

Tectamus Linea

Telephassa Linea

Thasus Linea

Thynia Linea
$69.7 \mathrm{~S}$

$42.4 \mathrm{~S}$

$48.6 \mathrm{~S}$

$64.5 S$

$170.4 W$

1216

639

EU

GR

PRELIM I1241

51979

$19 \mathrm{FE}$

$19 \mathrm{FE}$

51985

19.

$5 \quad 1979$

$19 \mathrm{FE}$

51985

$19 \mathrm{FE}$

$\begin{array}{lll}\text { GR } & \text { JE04 I1499 }\end{array}$

51979
22 FE
Brother of Europa; Rand control-point crater.

Celtic; ugly son of Tegid.

Celtic heroine.

Celtic, son of Bran; magician.

Celtic hero who lived in

Bula Lake.

Land named for Cilix on his search for Europa. Where the cow led Cadmus before it stopped at the site of Thebes.

Place on Crete where Zeus brought Europa.

Where the cow lead Cadmus before it stopped at the site of Thebes.

Another name for Tyre; where Europa was born.

\begin{tabular}{|c|c|c|c|c|c|c|c|c|c|c|}
\hline $51.8 \mathrm{~S}$ & $113.2 \mathrm{~W}$ & 758 & EU & GR & PRELIM & I1241 & 5 & 1979 & 19 & LI \\
\hline & $208.2 \mathrm{~W}$ & 1326 & EU & GR & PRELIM & I1241 & 5 & 1979 & & \\
\hline $28.0 \mathrm{~S}$ & $182.6 \mathrm{~W}$ & 1642 & EU & GR & JE03 & I1493 & 5 & 1985 & 9 & LI \\
\hline $8.2 \mathrm{~S}$ & $202.2 \mathrm{~W}$ & 934 & EU & GR & PRELIM & 11241 & 5 & 1979 & 19 & LI \\
\hline $17.7 \mathrm{~N}$ & $265.6 \mathrm{~W}$ & 2753 & EU & GR & PRELIM & I1241 & 5 & 1979 & 19 & LI \\
\hline $\begin{array}{l}11.8 \mathrm{~N} \\
27.8 \mathrm{~N} \\
13.1 \mathrm{~S}\end{array}$ & $\begin{array}{l}228.3 \mathrm{~W} \\
173.1 \mathrm{~W} \\
184.3 \mathrm{~W}\end{array}$ & $\begin{array}{l}2580 \\
1212 \\
1217\end{array}$ & $\begin{array}{l}\text { EU } \\
\text { EU } \\
\text { EU }\end{array}$ & $\begin{array}{l}\text { GR } \\
\text { GR } \\
\text { GR }\end{array}$ & $\begin{array}{l}\text { PRELIM } \\
\text { PRELIM } \\
\text { JE03 }\end{array}$ & $\begin{array}{l}\text { I1241 } \\
\text { I1241 } \\
\text { I1493 }\end{array}$ & $\begin{array}{l}5 \\
5 \\
5\end{array}$ & $\begin{array}{l}1979 \\
1979 \\
1985\end{array}$ & $\begin{array}{l}19 \\
19 \\
19\end{array}$ & $\begin{array}{l}\text { LI } \\
\text { LI } \\
\text { LI }\end{array}$ \\
\hline $\begin{array}{l}56.2 \mathrm{~S} \\
45.3 \mathrm{~N} \\
17.1 \mathrm{~S}\end{array}$ & $\begin{array}{l}183.3 \mathrm{~W} \\
195.7 \mathrm{~W} \\
175.9 \mathrm{~W}\end{array}$ & $\begin{array}{r}452 \\
2134 \\
1770\end{array}$ & $\begin{array}{l}\text { EU } \\
\text { EU } \\
\text { EU }\end{array}$ & $\begin{array}{l}\text { GR } \\
\text { GR } \\
\text { GR }\end{array}$ & $\begin{array}{l}\text { PRELIM } \\
\text { PRELIM } \\
\text { PRELIM }\end{array}$ & $\begin{array}{l}\text { I1241 } \\
\text { I1241 } \\
\text { I1 } 241\end{array}$ & $\begin{array}{l}5 \\
5 \\
5\end{array}$ & $\begin{array}{l}1979 \\
1979 \\
1979\end{array}$ & $\begin{array}{l}19 \\
19 \\
19\end{array}$ & $\begin{array}{l}\mathrm{LI} \\
\mathrm{LI} \\
\mathrm{LI}\end{array}$ \\
\hline $\begin{array}{l}33.0 \mathrm{~S} \\
14.5 \mathrm{~N} \\
18.5 \mathrm{~N} \\
42.2 \mathrm{~S} \\
17.9 \mathrm{~N} \\
2.8 \mathrm{~S} \\
68.7 \mathrm{~S} \\
57.9 \mathrm{~S}\end{array}$ & $\begin{array}{r}269.2 \mathrm{~W} \\
184.7 \mathrm{~W} \\
200.8 \mathrm{~W} \\
89.4 \mathrm{~W} \\
181.9 \mathrm{~W} \\
178.8 \mathrm{~W} \\
187.4 \mathrm{~W} \\
148.6 \mathrm{~W}\end{array}$ & $\begin{array}{r}1984 \\
732 \\
1780 \\
940 \\
719 \\
800 \\
1027 \\
398\end{array}$ & $\begin{array}{l}\text { EU } \\
\text { EU } \\
\text { EU } \\
\text { EU } \\
\text { EU } \\
\text { EU } \\
\text { EU } \\
\text { EU }\end{array}$ & $\begin{array}{l}\text { GR } \\
\text { GR } \\
\text { GR } \\
\text { GR } \\
\text { GR } \\
\text { GR } \\
\text { GR } \\
\text { GR }\end{array}$ & $\begin{array}{l}\text { PRELIM } \\
\text { JE03 } \\
\text { JE03 } \\
\text { PRELIM } \\
\text { JE03 } \\
\text { JE03 } \\
\text { PRELIM } \\
\text { JE04 }\end{array}$ & $\begin{array}{l}\text { I1241 } \\
\text { I1493 } \\
\text { I1493 } \\
\text { I1241 } \\
\text { I1493 } \\
\text { I1493 } \\
\text { I1241 } \\
\text { I1499 }\end{array}$ & $\begin{array}{l}5 \\
5 \\
5 \\
5 \\
5 \\
5 \\
5 \\
5\end{array}$ & $\begin{array}{l}1979 \\
1985 \\
1985 \\
1979 \\
1985 \\
1985 \\
1979 \\
1985\end{array}$ & $\begin{array}{l}19 \\
19 \\
19 \\
19 \\
10\end{array}$ & $\begin{array}{l}\text { LI } \\
\text { LI } \\
\text { LI } \\
\text { LI } \\
\text { LI } \\
\text { LI } \\
\text { LI } \\
\text { LI }\end{array}$ \\
\hline
\end{tabular}

Greek; son of Phoenix, nephew of Europa.

Greek; Europa's father.

Son of Phoenix, nephew of

Europa.

Greek; another name for

Telephassa.

Greek; Europa's husband after Zeus.

Greek; Agenor's twin brother.

Greek; brother of Europa.

Survivor of the men Cadmus sowed with the dragon's teeth; a founder of Thebes.

Greek; Agenor's mother.

Greek; son of Europa and Zeus.

Greek; survivor of the men Cadmus sowed with the dragon's teeth; a founder Thebes.

Greek; brother of Europa.

Brother of Europa.

Son of Europa and Zeus.

Greek; son of Europa and Zeus.

Father of Asterius.

Europa's mother.

Greek; brother of Europa.

Peninsula between Black and Marmara Seas, where Phineus sought Europa.

\section{MACULA}

Thera Macula

Thrace Macula $\begin{array}{llllll}47.7 S & 180.9 \mathrm{~W} & 78 & \mathrm{EU} & \mathrm{GR} & \text { PRELIM I1241 }\end{array}$

46.6S 171.2W 173 EU GR PRELIM I1241
51979

51979
19 MA

19
Greek; place where Cadmus stopped in his search for Europa.

Place in northern Greece where Cadmus stopped in his search for Europa. lat: latitude of feature center.

long: longitude of feature center.

diam: diameter or long dimension of feature.

ct: continent of name origin (see page $284 \mathrm{ff}$.) et: ethnicity of name origin (see page $284 \mathrm{ff}$.)

quad: map quadrangle or informal name

(see page xvii $\mathrm{ff}$.).

map: map name or USGS map number (see page xvii ff.). as: name approval status (see page $x v i i$ ).

ad: name approval date (year).

ref: reference source for name (see page $287 \mathrm{ff}$.). 


\section{JOVIAN SYSTEM}

$\begin{array}{llllllllllllll}\text { Name } & \text { lat } & \text { long } & \text { diam } & \text { ct } & \text { et } & \text { quad } & \text { map } & \text { as } & \text { ad } & \text { ref } & \text { origin } \\ \text { Tyre Macula } & 31.7 \mathrm{~N} & 147.0 \mathrm{~W} & 148 & \mathrm{EU} & \mathrm{GR} & \text { PRELIM } 11241 & 5 & 1979 & 19 & \text { MA } & \begin{array}{l}\text { Greek; the seashore from } \\ \text { which Zeus abducted Europa. }\end{array}\end{array}$

IO

CATENA

$\begin{array}{lllllllllllll}\text { Mazda Catena } & 8.6 \mathrm{~S} & 313.5 \mathrm{~W} & 0 & \text { AS } & \text { BY } & \text { JI02 } & \text { I1491 } & 5 & 1979 & 1 & \text { CA } & \text { Babylonian sun god. } \\ \text { Reshet Catena } & 0.8 \mathrm{~N} & 305.6 \mathrm{~W} & 0 & \text { AS } & \text { SE } & \text { JI02 } & \text { I1491 } & 5 & 1985 & 27 & \text { CA } & \text { Aramaic sun god. }\end{array}$

\section{ERUPTIVE CENTER}

Amirani
Loki
Marduk
Masubi
Maui
Pele
Prometheus
Surt
Volund

\section{FLUCTUS}

Euboea Fluctūs

Tung Yo Fluctus

Uta Fluctūs

\section{MENSA}

Echo Mensa

Epaphus Mensa

Iynx Mensa

Pan Mensa

\section{MONS}

Boösaule Montes

Crimea Mons

Euboea Montes

Haemus Montes

Silpium Mons

\section{PATERA}

Agni Patera

Amaterasu Patera

Angpetu Patera

Aramazd Patera

Asha Patera.

Atar Patera

Aten Patera

Babbar Patera

Bochica Patera

Carancho Patera

$\begin{array}{rr}45.1 \mathrm{~S} & 351.3 \mathrm{~W} \\ & \\ 16.4 \mathrm{~S} & 357.8 \mathrm{~W} \\ 32.6 \mathrm{~S} & 19.2 \mathrm{~W}\end{array}$

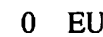

GR JI02

0 AS $\mathrm{CH} \quad \mathrm{JIO} 2$

0 AS SU JI02

I1491

I1491

I1491

I1550
I1491
I1550
I1491
I 1550
I1550
I1550
I1491
II550

$\begin{array}{rrrr}5 & 1979 & 18 & \text { ER } \\ 5 & 1979 & 1 & \text { ER } \\ 5 & 1979 & 1 & \text { ER } \\ 5 & 1979 & 1 & \text { ER } \\ 5 & 1979 & 1 & \text { ER } \\ & & & \\ 5 & 1979 & 1 & \text { ER } \\ 5 & 1979 & 1 & \text { ER } \\ 5 & 1979 & 27 & \text { ER } \\ 5 & 1979 & 27 & \text { ER }\end{array}$

Georgian god of fire.

Norse blacksmith, trickster god.

Sumero-Âkkadian fire god.

Japanese fire god.

Hawaiian demigod who sought fire from Mafuike.

Hawaiian goddess of the volcano.

Greek fire god.

Icelandic volcano god.

Germanic supreme smith of the gods.

$\begin{array}{rr}79.6 \mathrm{~S} & 357.4 \mathrm{~W} \\ 53.5 \mathrm{~S} & 241.3 \mathrm{~W} \\ & \\ 61.1 \mathrm{~S} & 304.6 \mathrm{~W} \\ 49.5 \mathrm{~S} & 35.4 \mathrm{~W}\end{array}$
76.1

46.3S $244.8 \mathrm{~W}$

590 EU GR J103

68.9S 46.6W

$52.6 \mathrm{~S} \quad 272.9 \mathrm{~W}$
$270.1 \mathrm{~W}$
0 EU GR JI04

$\begin{array}{llll}0 & \text { EU } & \text { GR } & \mathrm{JI02}\end{array}$

0 EU GR JI04

$\begin{array}{llll}0 & \text { EU } & \text { GR } & \mathrm{JI} 04\end{array}$

$0 \quad$ EU GR JI04

$\begin{array}{llll}0 & \text { EU } & \text { GR } & \mathrm{JI} 04\end{array}$

0 EU GR JI04

\begin{tabular}{|c|c|c|c|c|c|}
\hline $\begin{array}{l}\text { I1549 } \\
\text { I1 } 549\end{array}$ & $\begin{array}{l}5 \\
5\end{array}$ & $\begin{array}{l}1985 \\
1985\end{array}$ & $\begin{array}{l}19 \\
19\end{array}$ & $\begin{array}{l}\text { MN } \\
\text { MN }\end{array}$ & $\begin{array}{l}\text { Mother of Iynx. } \\
\text { "Child of touch," son of } \\
\text { Io and Zeus. }\end{array}$ \\
\hline I1549 & 5 & 1985 & 19 & $\mathrm{MN}$ & $\begin{array}{l}\text { Cast a spell on Zeus so } \\
\text { he fell in love with Io. }\end{array}$ \\
\hline 11549 & e & 1985 & 19 & MN & Father of Iynx. \\
\hline
\end{tabular}

$\begin{array}{llllll}11550 & 5 & 1985 & 19 & \text { MO } & \text { Cave where Io bore Epaphus. }\end{array}$

$\begin{array}{llll}11549 & 5 & 1985 & 19\end{array}$ MO Where Io passed by in

I1491 $5 \quad 1985$ her wanderings. Where Io passed by in her wanderings.

I1549 $51979 \quad 19$ MO Where Io passed by in I1549 $51979 \quad 19$ MO $\begin{aligned} & \text { her wanderings. } \\ & \text { Greek; where lo dies of }\end{aligned}$ grief in some legends.

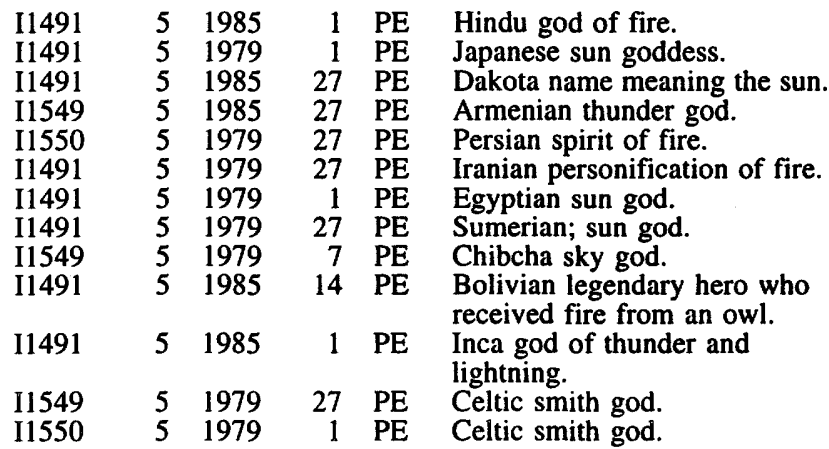

Cataquil Patera

Creidne Patera

Culann Patera lat: latitude of feature center. long: longitude of feature center.

diam: diameter or long dimension of feature.

ct: continent of name origin (see page 284ff.) et: ethnicity of name origin (see page $284 \mathrm{ff}$.)

quad: map quadrangle or informal name (see page xvii ff.).

as: name approval status (see page xvii)

ad: name approval date (year).

ref: reference source for name (see page $287 \mathrm{ff}$.).

map: map name or USGS map number (see page xvii ff.). $\mathrm{ft}$ : feature type (see page 290). 
JOVIAN SYSTEM

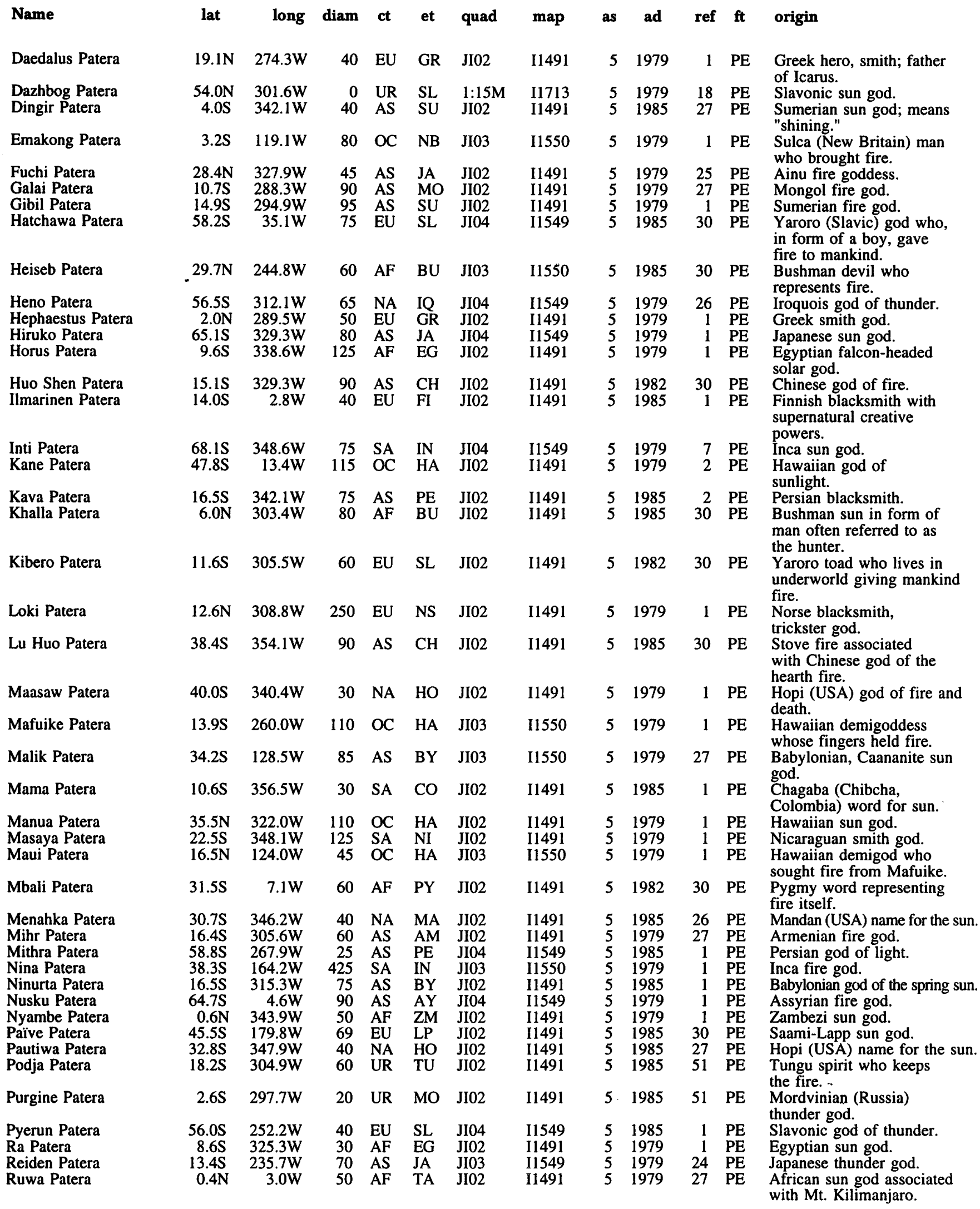

lat: latitude of feature center.

long: longitude of feature center.

diam: diameter or long dimension of feature.

ct: continent of name origin (see page $284 \mathrm{ff}$.) et: ethnicity of name origin (see page $284 \mathrm{ff}$.)

quad: map quadrangle or informal name

(see page xvii ff.)

as: name approval status (see page xvii)

ad: name approval date (year).

ref: reference source for name (see page $287 \mathrm{ff}$.).

map: map name or USGS map number (see page xvii ff.). ft: feature type (see page 290). 


\section{JOVIAN SYSTEM}

Name

Sengen Patera

Shakuru Patera

Shamash Patera

Shoshu Patera

Siun Patera

Sui Jen Patera

Svarog Patera

Talos Patera

Taranis Patera

Taw Patera

Tohil Patera

Tol-Ava Patera

Tung Yo Patera

Ülgen Patera

Uta Patera

Vahagn Patera

Viracocha Patera

\section{PLANUM}

Argos Planum

Danube Planum

Dodona Planum

Ethiopia Planum

Hybristes Planum

Iopolis Planum

Lyrcea Planum

Nemea Planum

\section{REGIO}

Bactria Regio

Chalybes Regio

Colchis Regio

Lerna Regio

Media Regio

Mycenae Regio

Tarsus Regio

\section{THOLUS}

Apis Tholus

Inachus Tholus lat

$2.8 \mathrm{~S}$

$32.8 \mathrm{~S}$

303.8W

$23.6 \mathrm{~N} \quad 266.4 \mathrm{~W}$

70 NA PW JI03

$33.7 \mathrm{~S}$

$152.1 \mathrm{~W}$

$19.6 \mathrm{~S} \quad 324.1 \mathrm{~W}$

$\begin{array}{ll}49.8 \mathrm{~S} & 1.4 \mathrm{~W} \\ 19.2 \mathrm{~S} & 4.3 \mathrm{~W}\end{array}$

48.3S 267.5W

$26.1 \mathrm{~S} \quad 356.5 \mathrm{~W}$

$70.8 \mathrm{~S}$

$33.3 \mathrm{~S}$

$28.6 \mathrm{~W}$

$180.0 \mathrm{~W}$

26.3S 156.5W

$1.7 \mathrm{~N} \quad 322.0 \mathrm{~W}$

$18.7 \mathrm{~S}$

$40.4 \mathrm{~S}$

$2.5 \mathrm{~W}$

$288.0 \mathrm{~W}$

$35.3 \mathrm{~S}$

$23.8 \mathrm{~S}$

24.9W

$61.2 \mathrm{~S}$

$351.7 \mathrm{~W}$

$281.7 \mathrm{~W}$

$47.0 \mathrm{~S}$

$318.2 \mathrm{~W}$

140 EU GR JI02

20.9S

$258.7 \mathrm{~W}$

$56.8 \mathrm{~S}$

$352.9 \mathrm{~W}$

44.9S

27.0W

$54.0 \mathrm{~S}$

$21.1 \mathrm{~W}$

$34.5 \mathrm{~S}$

$333.5 \mathrm{~W}$

40.3S

$73.3 \mathrm{~S}$

269.3W $275.5 \mathrm{~W}$

$\begin{array}{lll}\mathrm{EU} & \mathrm{CE} & \mathrm{JI} 04\end{array}$

\section{AS AB $\mathrm{J} 03$}

50 UR CC JI02

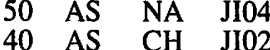

$\begin{array}{llll}70 & \text { UR } & \text { RU } & \text { JI04 }\end{array}$

20 EU GR JI02

20 SA MY JI03

70 UR MO JI02

20 AS $\mathrm{CH} \quad \mathrm{JI02}$

49 UR SI JI02

30 AS SU $\mathrm{JI02}$

70 AS AM JI02

55 SA IN JI04

I1491

I1491

I1549

I1491

I1549
I1491

I1550

I1491

I1491

I1491

I1491

I1491
I1549 as ad

$5 \quad 1985$

51979

51979

$5 \quad 1979$

$\begin{array}{ll}5 & 1982\end{array}$

$\begin{array}{ll}5 & 1985 \\ 5 & 1985\end{array}$

$5 \quad 1985$

$\begin{array}{ll}5 & 1979 \\ 5 & 1982\end{array}$

51985

51982

51979

51985

$5 \quad 1985$

$\begin{array}{ll}5 & 1979\end{array}$

$5 \quad 1979$

51979

51979 ref $\mathrm{ft}$ origin

27 PE

1 PE

1 PE

$1 \mathrm{PE}$

30 PE

54 PE

30 PE

83 PE

1 PE

1 PE

30 PE

$1 \mathrm{PE}$

51 PE

30 PE

27 PE

28 PE

$1 \mathrm{PE}$

7 PE

hearth.
Phoenician chariot rider of the Sun.

Japanese; deity of Mt.

Fugiyama.

Pawnee (USA) sun god of

the East; gives light and heat.

Assyro-Babylonian sun god.

Caucasian patron of fire.

Nanai (Siberia) sun god.

Chinese hero who

discovered fire.

Russian smith god.

Nephew of Daedalus; also

a blacksmith.

Celtic thunder god.

Monguor word for fire or

Central American god who

gave fire to man.

Mordvinian (Russia)

goddess of fire.

Chinese fire God.

Siberian progenitor god

who struck first fire.

Sumerian sun god.

Armenian fire god.

Qechua sun god.
$150 \mathrm{EU}$

GR JI03

$390 \mathrm{EU}$

GR JI04

$105 \mathrm{AF}$

150 EU

125 EU GR JI02

I1491

I1491

I1550

I1549

I1491

I1549

310 EU GR JI03

$\begin{array}{llll}500 & \text { EU } & \text { GR } & \text { JI04 }\end{array}$
I1550

I1549
1985

19 PM

1985

51979

19 PM

19 PM

1985

51985

19 PM

19 PM

51985

Where Io was captured by Zeus.

Where Io passed by in her wanderings. after the death of Argus. Where Io passed by in her wanderings.

Where Io passed by in her wanderings.

19 PM Town where Io was worshipped as moon goddess (present-day Antioch).

198519 PM Plain where Io was born. $\begin{array}{llll}5 & 1979 & 19 & \text { PM }\end{array}$

Greek; where Io was turned into a cow by Zeus and given to Hera.

\begin{tabular}{|c|c|c|c|c|c|c|c|c|c|c|}
\hline $45.8 \mathrm{~S}$ & $123.4 \mathrm{~W}$ & 0 & $\mathrm{EU}$ & GR & $1: 15 \mathrm{M}$ & I 1550 & 5 & 1979 & 19 & RE \\
\hline $45.5 \mathrm{~N}$ & $83.2 \mathrm{~W}$ & 0 & EU & GR & $1: 15 \mathrm{M}$ & 11713 & 5 & 1979 & 19 & RE \\
\hline $5.3 \mathrm{~N}$ & $199.8 \mathrm{~W}$ & 0 & EU & GR & $1: 15 \mathrm{M}$ & 11550 & 5 & 1979 & 19 & RE \\
\hline $\begin{array}{r}64.0 \mathrm{~S} \\
4.6 \mathrm{~N}\end{array}$ & $\begin{array}{r}292.6 \mathrm{~W} \\
58.8 \mathrm{~W}\end{array}$ & $\begin{array}{l}0 \\
0\end{array}$ & EU & GR & $\begin{array}{l}1: 15 \mathrm{M} \\
1: 15 \mathrm{M}\end{array}$ & I1549 & 5 & $\begin{array}{l}1979 \\
1979\end{array}$ & $\begin{array}{l}21 \\
19\end{array}$ & $\begin{array}{l}\text { RE } \\
\text { RE }\end{array}$ \\
\hline
\end{tabular}

$\begin{array}{lr}37.3 \mathrm{~S} & 165.9 \mathrm{~W} \\ 43.7 \mathrm{~S} & 61.4 \mathrm{~W}\end{array}$

0 EU GR 1:15M I1550

51979

19 RE

$\begin{array}{ll}5 & 1979\end{array}$

$19 \mathrm{RE}$
Io passed through this area of ancient Iran in her wanderings.

Greek; Io passed through here in her wanderings. Greek; Io passed through this part of Asia Minor in her wanderings.

Greek; meadows of Lyrcea. Greek; Io passed through this part of Iran in her wanderings.

Greek; in some legends, Io was transformed there.

Io passed through here in her wanderings.
$11.2 \mathrm{~S} \quad 348.8 \mathrm{~W}$

$15.9 \mathrm{~S} \quad 348.9 \mathrm{~W}$ $\begin{array}{lllll}0 & \text { EU } & \text { GR } & 1: 15 M & \text { I1491 }\end{array}$

$\begin{array}{lllll}0 & \text { EU } & \text { GR } & 1: 15 M & \text { I1491 }\end{array}$
51979

$5 \quad 1979$
19 TH

$19 \mathrm{TH}$
Greek; name for Epaphus, son of Io and Zeus.

Greek; river god, father of Io. 


\section{SATURNIAN SYSTEM}

Name

DIONE

\section{CRATER}

Adrastus

$61.7 \mathrm{~S}$

45.9W

$31 \mathrm{EU}$

GR PRELIM I1488

$26.1 \mathrm{~N} \quad 46.3 \mathrm{~W}$

Aeneas

Amata

Anchises

Antenor

$$
\begin{array}{r}
7.7 \\
33.7
\end{array}
$$

$7.7 \mathrm{~N}$

33.7

$285.3 \mathrm{~W}$

$66.1 \mathrm{~W}$

$10.4 W$

82 EU

RM PRELIM I1488

RM PRELIM I1488

RM PRELIM I1488

$5 \quad 1982$

44 AA

King of Argos, one of the seven against Thebes, and the only one to return alive.

Hero of the Aeneid. The

son of Anchises and Venus and a member of the royal family of Troy.

Butes

$\begin{array}{crrlll}64.2 \mathrm{~N} & 48.8 \mathrm{~W} & 26 & \text { EU } & \text { GR } & \text { PRELIM I1488 } \\ 23.3 \mathrm{~S} & 80.5 \mathrm{~W} & 70 & \text { EU } & \text { GR } & \text { PRELIM I1488 } \\ 39.5 \mathrm{~S} & 244.1 \mathrm{~W} & 36 & \mathrm{EU} & \text { RM } & \text { PRELIM I1488 } \\ 1.6 \mathrm{~S} & 273.0 \mathrm{~W} & 35 & \mathrm{EU} & \text { RM } & \text { PRELIM I1488 } \\ 0.6 \mathrm{~N} & 266.4 \mathrm{~W} & 37 & \text { EU } & \text { RM } & \text { PRELIM I1488 }\end{array}$

$5 \quad 1982$

1982

$5 \quad 1982$

51982

44 AA

44 AA

Caieta
Cassandra
Catillus
Coras

\section{Creusa}

Dido

Halys

Ilia

Italus

Latagus

Lausus

Magus

Massicus

Palinurus

Remus

Ripheus

Romulus

Sabinus

Turnus

$\begin{array}{llrlll}48.0 \mathrm{~N} & 76.9 \mathrm{~W} & 35 & \mathrm{EU} & \mathrm{RM} & \text { PRELIM I1488 } \\ 23.7 \mathrm{~S} & 18.5 \mathrm{~W} & 118 & \mathrm{EU} & \mathrm{RM} & \text { PRELIM I1488 } \\ 59.1 \mathrm{~S} & 53.6 \mathrm{~W} & 29 & \text { EU } & \text { RM } & \text { PRELIM I1488 }\end{array}$

$0.1 \mathrm{~N} 346.0 \mathrm{~W}$

51

18.1S 77.5W

$15.7 \mathrm{~N}$

$36.2 \mathrm{~N}$

26.4W

$23.2 \mathrm{~W}$

$19.3 N$

24.4W

$34.8 S$

$56.0 \mathrm{~W}$

$4.0 \mathrm{~S}$

$13.2 S$

$61.4 \mathrm{~W}$

$31.1 \mathrm{~W}$

$56.1 \mathrm{~S} \quad 35.5 \mathrm{~W}$

40 EU

RM PRELIM I1488

$5 \quad 1982$

$5 \quad 1982$

51982

$5 \quad 1982$

$5 \quad 1982$

$37 \mathrm{EU}$

RM PRELIM I1488

28 EU

RM PRELIM I1488

$41 \mathrm{EU}$

RM PRELIM I1488

$43 \mathrm{EU}$

RM PRELIM I1488

33 EU

$69 \mathrm{EU}$

RM PRELIM I1488

RM PRELIM I1488

$32 \mathrm{EU}$

RM PRELIM I1488

$7.3 S \quad 26.5 W$

RM PRELIM I1488

47.8S $175.6 \mathrm{~W}$

$16.2 \mathrm{~N} \quad 344.6 \mathrm{~W}$
79 EU RM PRELIM I1488

97 EU RM PRELIM I1488
$5 \quad 1982$
81 EU $\begin{array}{ll}5 & 1982 \\ 5 & 1982\end{array}$

$5 \quad 1982$

$5 \quad 1982$
Mother of Lavinia (wife of Aeneas).

Aeneas' father.

Nephew of Priam. He escaped the fall of Troy and reached Italy before Aeneas, where he founded Padua.

A famous boxer who had been defeated by Dares.

A nurse of Aeneas.

Daughter of Priam; she could

44 AA Daughter of Priam;

Brother of Tiburtus and

twin brother of Coras.

Brother of Tiburtus and

twin brother of Catillus.

He was founder of Tibur and an ally of Turnus against Aeneas.

Daughter of Priam; first wife of Aeneas.

Tyrian princess who

founded Carthage.

A Trojan defending

Aeneas' camp against the

Rutulian attack. He was killed by Turnus.

Also known as Rhea

Silvia; mother by Mars of

Romulus and Remus, the

founders of Rome.

Ancient hero, eponymous

ancestor of the

Italians.

$5 \quad 1982 \quad 44$ AA Soldier of Aeneas.

5198244 AA Son of Mezentius, killed by Aeneas.

$5 \quad 1982 \quad 44$ AA A soldier of Turnus

killed by Aeneas.

$51982 \quad 44$ AA An Etruscan ally of

Aeneás.

5198244 AA Pilot of Aeneas' fleet.

44 AA

$\mathrm{He}$ and his brother

$5 \quad 1982 \quad 44 \quad$ AA

Romulus founded Rome.

A Trojan. He fought at

the side of Aeneas during

Troy's last night.

$51982 \quad 44$ AA Mythical founder of Rome

in 754 or 753 B.C., son

of Mars by Ilia (Rhea Silvia).

$5 \quad 1982 \quad 44 \quad \mathrm{AA}$

Rutililan king; Aeneas' rival for hand of Lavinia.

\section{CHASMA}

Larissa Chasma

Latium Chasma $\begin{array}{llllll}30.2 \mathrm{~N} & 71.1 \mathrm{~W} & 315 & \mathrm{EU} & \mathrm{GR} & \text { PRELIM I1488 } \\ 21.2 \mathrm{~N} & 69.5 \mathrm{~W} & 381 & \text { EU } & \text { RM } & \text { PRELIM I1488 }\end{array}$ $\begin{array}{lll}5 & 1982 \\ 5 & 1982\end{array}$

44
A town in Thessaly, Achilles' native region. The Trojans' promised land in Italy. lat: latitude of feature center.

long: longitude of feature center.

diam: diameter or long dimension of feature.

ct: continent of name origin (see page $284 \mathrm{ff}$.) et: ethnicity of name origin (see page $284 \mathrm{ff}$.)

quad: map quadrangle or informal name

(see page xvii ff.).

map: map name or USGS map number (see page xvii ff.). as: name approval status (see page xvii).

ad: name approval date (year).

ref: reference source for name (see page $287 \mathrm{ff}$.). 


\section{SATURNIAN SYSTEM}

Name

Palatine Chasma

Tibur Chasmata

$75.6 \mathrm{~S}$

$57.2 \mathrm{~N}$ long diam ct et quad map

$25.1 \mathrm{~W}$ 394 EU

$69.1 \mathrm{~W}$
$156 \mathrm{EU}$
$12.7 \mathrm{~N}$

$20.0 S$

$40.6 \mathrm{~S}$
321.9W

$210.7 \mathrm{~W}$

Padua Linea

Palatine Linea

\section{ENCELADUS}

\section{CRATER}

Ahmad

Aladdin

Ali Baba

Dalilah

Duban

Dunyazad

Gharib

Julnar

Musa

Peri-Banu

Salih

Samad

Shahrazad

Shahryar

Sindbad

\section{FOSSA}

Bassorah Fossa

Daryabar Fossa

Isbanir Fossa

\section{PLANITIA}

Diyar Planitia

Sarandib Planitia
$57.4 \mathrm{~N}$

$63.1 \mathrm{~N}$

$57.2 \mathrm{~N}$

$12.0 \mathrm{~W}$

$52.9 \mathrm{~N}$

246.4 W

$58.3 \mathrm{~N}$

277.5W

$42.6 \mathrm{~N}$

$196.5 \mathrm{~W}$

$81.3 \mathrm{~N} \quad 251.7 \mathrm{~W}$

$54.2 \mathrm{~N}$

$342.0 \mathrm{~W}$

$73.8 \mathrm{~N}$

$6.5 \mathrm{~W}$

$63.1 \mathrm{~N} \quad 317.9 \mathrm{~W}$

$6.5 S$

$62.3 \mathrm{~N}$

$0.0 \mathrm{~W}$ $355.1 \mathrm{~W}$

$48.2 \mathrm{~N} \quad 195.1 \mathrm{~W}$

20 AS

AR

PRELIM I1485

$59.7 \mathrm{~N} \quad 225.0 \mathrm{~W}$

21 AS

AR PRELIM 11485

$68.9 \mathrm{~N} \quad 211.4 \mathrm{~W}$

23 AS

PRELIM I1485 $\begin{array}{llll}5 & 1982 & 44 & \text { LI }\end{array}$

51982

51982

\section{LI}

44 LI
A Punic (Phoenician) city in North Africa.

City in northern Italy founded by Antenor.

One of the Seven Hills of Rome.
Rome.

Ancient town of Italy not far from Rome on the river Arno. 


\section{SATURNIAN SYSTEM}

Name

\section{SULCUS}

Harran Sulci

Samarkand Sulci

EPIMETHEUS

CRATER

Hilairea

Pollux

\section{HYPERION}

\section{CRATER}

Bahloo

Helios

Jarilo

Meri

\section{DORSUM}

Bond-Lassell

Dorsum

$48.0 \mathrm{~N} \quad 143.5 \mathrm{~W}$

$\begin{array}{llllll}26.7 \mathrm{~N} & 237.6 \mathrm{~W} & 276 & \text { AS } & \text { AR } & \text { PRELIM I1485 } \\ 30.5 \mathrm{~N} & 326.8 \mathrm{~W} & 383 & \text { AS } & \text { AR } & \text { PRELIM I1485 }\end{array}$

$5 \quad 1982$

$5 \quad 1982$
1 AA

$\begin{array}{llll}5 & 1982 & 21 & \text { AA }\end{array}$
Greek; sister of Phoibe, daughter of Leukippos.

Latin name for Polydeukes, Castor's twin. father ruled.

Country ruled over by

Zaman, brother of Shahryar.
$36.0 \mathrm{~N} \quad 196.0 \mathrm{~W}$

$71.0 \mathrm{~N} \quad 132.0 \mathrm{~W}$

$61.0 \mathrm{~N} \quad 183.0 \mathrm{~W}$

$3.0 \mathrm{~N} \quad 171.0 \mathrm{~W}$

$0 \quad O C$

0 EU GR

0 UR RU

PRELIM TRANS

PRELIM TRANS

0 SA BO PRELIM TRANS

$5 \quad 1982$

$5 \quad 1982$

$5 \quad 1982$

4 AA

$1 \mathrm{AA}$

51 AA

51982

7 AA Greek sun god; son of Hyperion.

East Slavic god of the

sun, fertility and love.

Bororo folk hero; the sun.

\section{IAPETUS}

\section{CRATER}

\begin{tabular}{|c|c|c|c|c|c|c|c|c|c|c|c|c|}
\hline Almeric & $53.4 \mathrm{~N}$ & $276.6 \mathrm{~W}$ & 43 & EU & FR & PRELIM & I1486 & 5 & 1982 & 29 & AA & $\begin{array}{l}\text { One of } 12 \text { peers, killed } \\
\text { by Marsilion. }\end{array}$ \\
\hline $\begin{array}{l}\text { Baligant } \\
\text { enlisted }\end{array}$ & $16.4 \mathrm{~N}$ & $224.9 W$ & 66 & EU & FR & PRELIM & I1486 & 5 & 1982 & 29 & AA & Emir of Babylon; Marsilion \\
\hline Basan & $33.3 \mathrm{~N}$ & $194.7 W$ & 76 & EU & FR & PRELIM & I1486 & 5 & 1982 & 29 & AA & $\begin{array}{l}\text { his help against Charlemagne. } \\
\text { French baron; Murdered while } \\
\text { serving as Ambassador of Marsilon. }\end{array}$ \\
\hline Berenger & $62.1 \mathrm{~N}$ & $219.7 \mathrm{~W}$ & 84 & EU & FR & PRELIM & I1486 & 5 & 1982 & 29 & AA & $\begin{array}{l}\text { One of twelve peers; killed } \\
\text { Estramarin; killed by Grandoyne. }\end{array}$ \\
\hline Besgun & $76.0 \mathrm{~N}$ & $309.8 \mathrm{~W}$ & 56 & EU & FR & PRELIM & I1486 & 5 & 1982 & 29 & AA & $\begin{array}{l}\text { Chief cook for Charlemagne's army; } \\
\text { heguarded Ganelon after Ganelon's } \\
\text { treachery was discovered. }\end{array}$ \\
\hline Charlemagne & $55.0 \mathrm{~N}$ & $258.8 \mathrm{~W}$ & 95 & EU & FR & PRELIM & I1486 & 5 & 1982 & 29 & AA & $\begin{array}{l}\text { Emperor of France and Germanic } \\
\text { nations; his forces fought the } \\
\text { Saracens in Spain. }\end{array}$ \\
\hline Geboin & $58.6 \mathrm{~N}$ & $173.4 \mathrm{~W}$ & 81 & EU & FR & PRELIM & I1486 & 5 & 1982 & 29 & AA & $\begin{array}{l}\text { Guarded French dead; became } \\
\text { leader of Charlemagne's second } \\
\text { column. }\end{array}$ \\
\hline Godefroy & $71.9 \mathrm{~N}$ & $249.1 \mathrm{~W}$ & 63 & EU & FR & PRELIM & I1486 & 5 & 1982 & 29 & AA & $\begin{array}{l}\text { Standard bearer of Charlemagne; } \\
\text { brother of Tierri, Charlemagne's } \\
\text { defender against Pinabel. }\end{array}$ \\
\hline Hamon & $10.6 \mathrm{~N}$ & $270.0 \mathrm{~W}$ & 96 & EU & FR & PRELIM & 11486 & 5 & 1982 & 29 & AA & $\begin{array}{l}\text { Joint Commander of Charlemagne's } \\
\text { Eighth Division. }\end{array}$ \\
\hline Lorant & $65.2 \mathrm{~N}$ & $159.8 \mathrm{~W}$ & 44 & EU & FR & PRELIM & I1486 & 5 & 1982 & 29 & AA & $\begin{array}{l}\text { French commander of one of first } \\
\text { divisions against Baligant; killed } \\
\text { by Baligant. }\end{array}$ \\
\hline Marsilion & $39.2 \mathrm{~N}$ & $176.1 \mathrm{~W}$ & 136 & EU & FR & PRELIM & I1486 & 5 & 1982 & 29 & AA & $\begin{array}{l}\text { Saracen king of Spain; Roland } \\
\text { wounded him and he died of wound } \\
\text { later. }\end{array}$ \\
\hline $\begin{array}{l}\text { lat: latitude o } \\
\text { long: longitude } \\
\text { diam: diameter } \\
\text { ct: continent }\end{array}$ & $\begin{array}{l}\text { nter. } \\
\text { nsion of } f \\
\text { in (see } p\end{array}$ & ge $284 \mathrm{ff}$.) & $\begin{array}{l}\text { et: } \\
\text { quad: } \\
\text { map: }\end{array}$ & \multicolumn{6}{|c|}{$\begin{array}{l}\text { ethnicity of name origin (see page } 284 \mathrm{ff} \text {.) } \\
\text { map quadrangle or informal name } \\
\text { (see page xvii ff.). } \\
\text { map name or USGS map number (see page xvii }\end{array}$} & $\begin{array}{l}\text { as: } \\
\text { ad: } \\
\text { ref: } \\
\text { ft: }\end{array}$ & \multicolumn{2}{|c|}{$\begin{array}{l}\text { name approval status (see page xvii). } \\
\text { name approval date (year). } \\
\text { reference source for name (see page } 287 \mathrm{ff} \text {.). } \\
\text { feature type (see page } 290 \text { ). }\end{array}$} \\
\hline
\end{tabular}




\section{SATURNIAN SYSTEM}

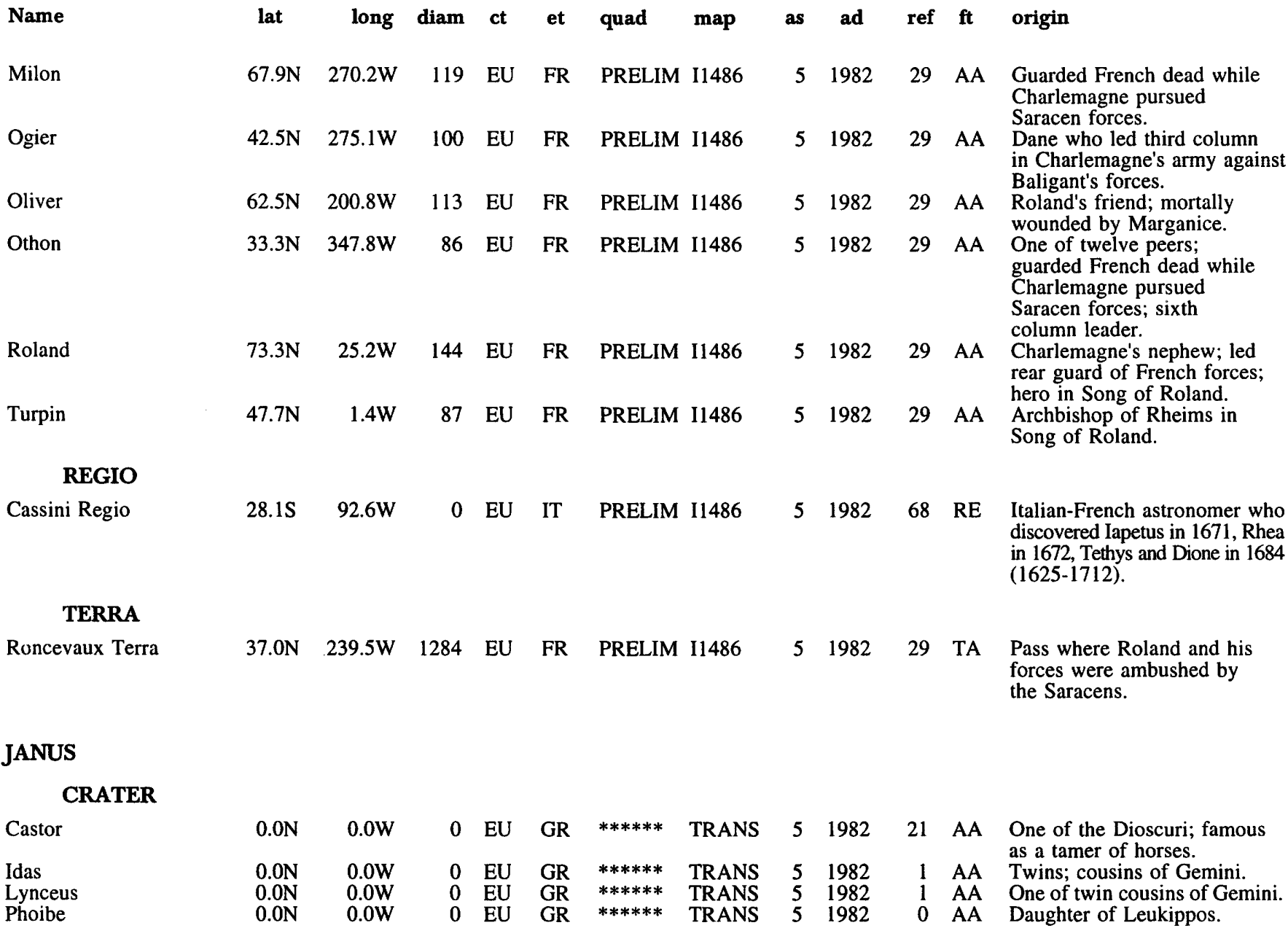

\section{MIMAS}

\section{CRATER}

\begin{tabular}{|c|c|c|c|c|c|c|c|c|c|c|c|}
\hline Accolon & $65.7 \mathrm{~S}$ & $184.7 \mathrm{~W}$ & 0 & EU & GB & I1489 & 5 & 1982 & 37 & AA & $\begin{array}{l}\text { Companion of Arthur's; he was } \\
\text { tricked into jousting with Arthur. }\end{array}$ \\
\hline Arthur & $33.2 \mathrm{~S}$ & $195.6 \mathrm{~W}$ & 0 & EU & GB & I1489 & 5 & 1982 & 37 & AA & $\begin{array}{l}\text { King of the Round Table } \\
\text { assemblage. }\end{array}$ \\
\hline Balin & $17.1 \mathrm{~N}$ & $86.8 \mathrm{~W}$ & 0 & $\mathrm{EU}$ & GB & I1489 & 5 & 1982 & 37 & AA & $\begin{array}{l}\text { Knight of "matchless } \\
\text { courage and virtue." }\end{array}$ \\
\hline Ban & $39.2 \mathrm{~N}$ & $156.4 \mathrm{~W}$ & 0 & EU & GB & I1489 & 5 & 1982 & 37 & AA & $\begin{array}{l}\text { King of Benwick; father of Sir } \\
\text { Launcelot, ally of Arthur in the } \\
\text { battle of Bedgrayne. }\end{array}$ \\
\hline Bedivere & $9.5 \mathrm{~N}$ & $152.3 \mathrm{~W}$ & 0 & EU & GB & I1489 & 5 & 1982 & 37 & AA & Arthurian knight. \\
\hline $\begin{array}{l}\text { Dynas } \\
\text { Elaine }\end{array}$ & $\begin{array}{r}3.8 \mathrm{~N} \\
44.5 \mathrm{~N}\end{array}$ & $\begin{array}{r}82.9 \mathrm{~W} \\
108.2 \mathrm{~W}\end{array}$ & $\begin{array}{l}0 \\
0\end{array}$ & $\begin{array}{l}\text { EU } \\
\text { EU }\end{array}$ & $\begin{array}{l}\text { GB } \\
\text { GB }\end{array}$ & $\begin{array}{l}\text { I1489 } \\
\text { I1489 }\end{array}$ & $\begin{array}{l}5 \\
5\end{array}$ & $\begin{array}{l}1982 \\
1982\end{array}$ & $\begin{array}{l}37 \\
37\end{array}$ & $\begin{array}{l}\text { AA } \\
\text { AA }\end{array}$ & $\begin{array}{l}\text { A knight of the Round Table. } \\
\text { Daughter of King Pelles, } \\
\text { lover of Sir Launcelot } \\
\text { and mother, by him, of } \\
\text { Sir Galahad. }\end{array}$ \\
\hline Gaheris & 40.9S & $298.4 W$ & 0 & EU & GB & I1489 & 5 & 1982 & 37 & AA & $\begin{array}{l}\text { Older son of King Lot; } \\
\text { killed by Sir Launcelot } \\
\text { in his rescue of } \\
\text { Gwynevere from burning. }\end{array}$ \\
\hline
\end{tabular}

lat: latitude of feature center.

long: longitude of feature center.

diam: diameter or long dimension of feature.

ct: continent of name origin (see page $284 \mathrm{ff}$.) et: ethnicity of name origin (see page $284 \mathrm{ff}$.)

quad: map quadrangle or informal name

(see page xvii ff.).

as: name approval status (see page xvii).

ad: name approval date (year).

ref: reference source for name (see page $287 \mathrm{ff}$.).

map: map name or USGS map number (see page xvii ff.). ft: feature type (see page 290). 


\section{SATURNIAN SYSTEM}

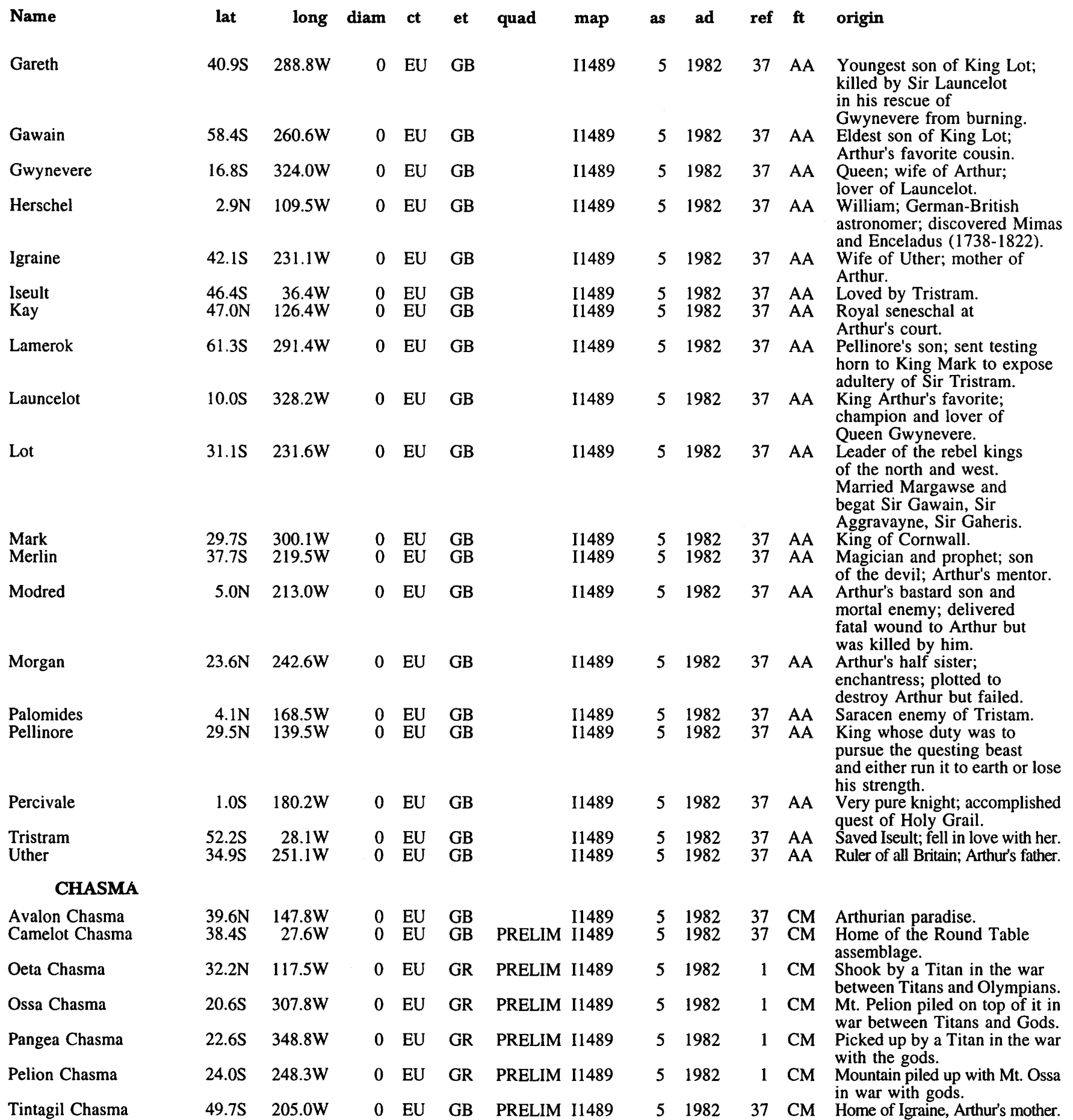

RHEA

\section{CRATER}

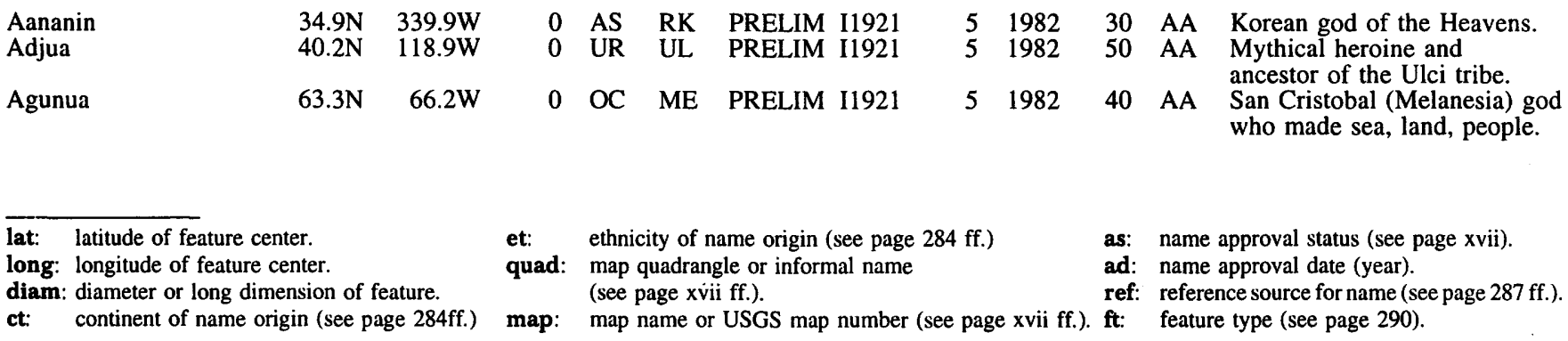


SATURNIAN SYSTEM

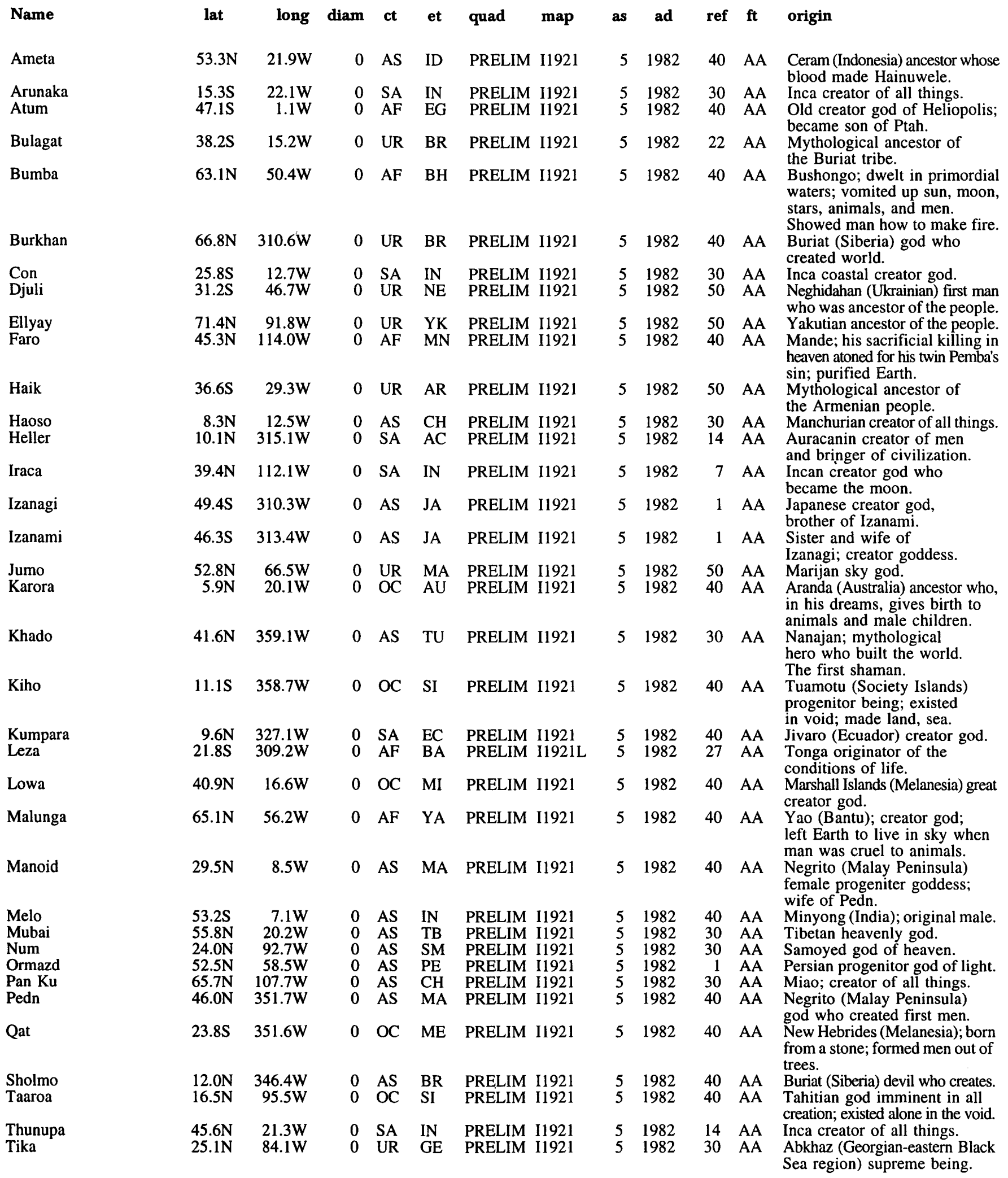

lat: latitude of feature center.

long: longitude of feature center.

diam: diameter or long dimension of feature.

ct: continent of name origin (see page $284 \mathrm{ff}$.) et: ethnicity of name origin (see page $284 \mathrm{ff}$.)

quad: map quadrangle or informal name

(see page xvii ff.).

map: map name or USGS map number (see page xvii ff.). as: name approval status (see page $\mathrm{xvii}$.

ad: name approval date (year).

ref: reference source for name (see page $287 \mathrm{ff}$.) 


\section{SATURNIAN SYSTEM}

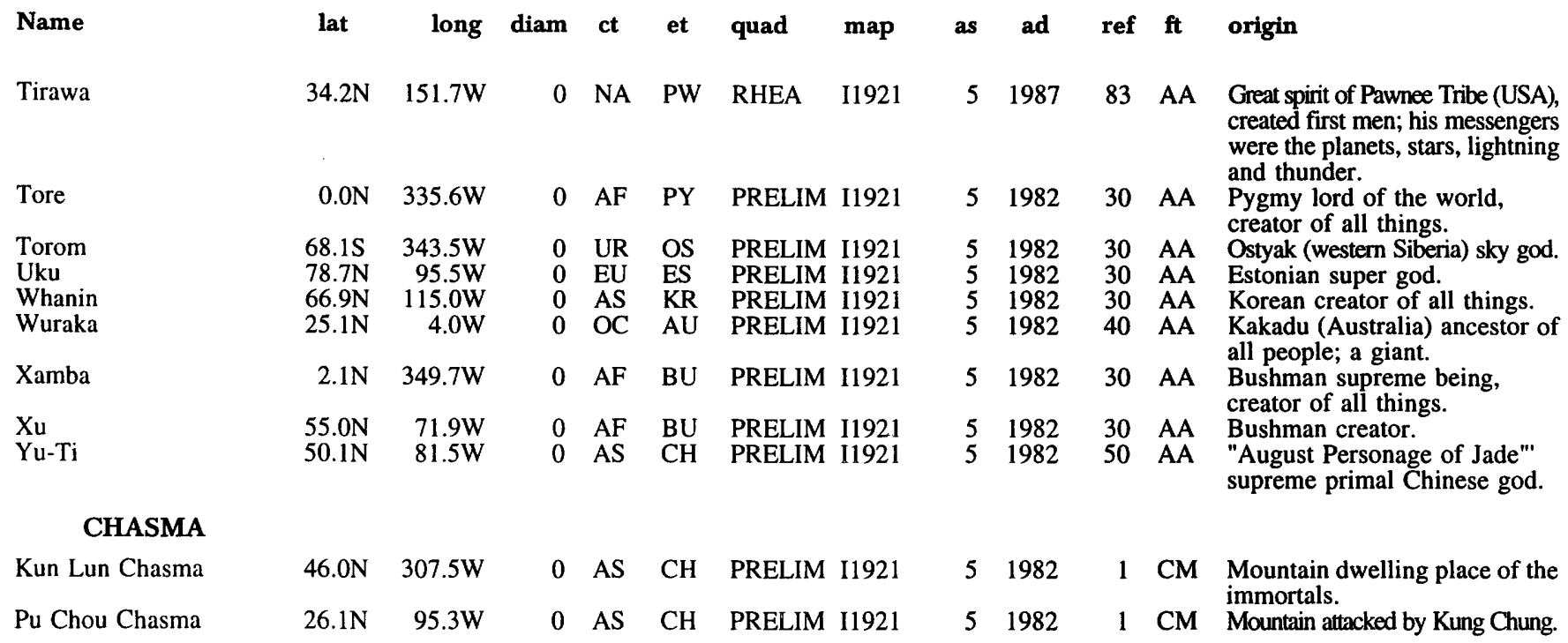

\section{TETHYS}

\section{CRATER}

Ajax

Anticleia

Antinous

Arete

Circe

Elpenor

Eumaeus

Eurycleia

Laertes

Melanthius

Mentor

Nausicaa

Nestor

Odysseus

Penelope

Phemius

Polyphemus

Teiresias

Telemachus

CHASMA

Ithaca Chasma
29.1S 282.0W

$52.3 \mathrm{~N} \quad 34.4 \mathrm{~W}$

62.0S 275.0W

$4.6 \mathrm{~S} \quad 299.7 \mathrm{~W}$

12.1S $\quad 53.7 \mathrm{~W}$

$54.8 \mathrm{~N} \quad 263.3 \mathrm{~W}$

$22.8 \mathrm{~N} \quad 53.4 \mathrm{~W}$

$52.7 \mathrm{~N} \quad 245.9 \mathrm{~W}$

$\begin{array}{lr}47.6 \mathrm{~S} & 66.4 \mathrm{~W} \\ 62.0 \mathrm{~S} & 204.0 \mathrm{~W}\end{array}$

$1.3 \mathrm{~S} \quad 45.0 \mathrm{~W}$

$82.3 \mathrm{~N} \quad 357.3 \mathrm{~W}$

$54.6 \mathrm{~S} \quad 61.7 \mathrm{~W}$

$30.0 \mathrm{~N} \quad 130.0 \mathrm{~W}$

$11.5 \mathrm{~S} \quad 248.0 \mathrm{~W}$

$12.0 \mathrm{~N} \quad 285.8 \mathrm{~W}$

$4.6 \mathrm{~S} \quad 282.8 \mathrm{~W}$

$59.6 \mathrm{~N} \quad 5.7 \mathrm{~W}$

$54.0 \mathrm{~N} \quad 338.7 \mathrm{~W}$

$10.3 \mathrm{~S} \quad 3.0 \mathrm{~W}$
0 EU GR

0 EU GR PRELIM I1487

0 EU GR

0 EU GR

PRELIM I1487

PRELIM I1487

0 EU GR

PRELIM I1487

0 EU GR PRELIM I1487

0 EU GR PRELIM I1487

0 EU GR

$\begin{array}{lll}0 & \mathrm{EU} & \mathrm{GR} \\ 0 & \mathrm{EU} & \mathrm{GR}\end{array}$

PRELIM I1487

PRELIM I1487

PRELIM I1487

0 EU GR PRELIM I1487

0 EU GR PRELIM I1487

0 EU GR PRELIM I1487

0 EU GR

0 EU GR

0 EU GR

PRELIM I1487

PRELIM I1487

PRELIM I1487

0 EU GR PRELIM I1487

0 EU GR PRELIM I1487

0 EU GR PRELIM I1487

$0 \mathrm{EU}$

PRELIM I1487

$\begin{array}{llll}5 & 1982 & 28 & \text { AA } \\ 5 & 1982 & 28 & \text { AA } \\ 5 & 1982 & 28 & \text { AA } \\ 5 & 1982 & 28 & \text { AA } \\ 5 & 1982 & 28 & \text { AA } \\ 5 & 1982 & 28 & \text { AA } \\ 5 & 1982 & 28 & \text { AA }\end{array}$

Greek hero second only to Achilles. Mother of Odysseus.

Chief of the wooers; slain by Odysseus.

Wife of Alcinous, mother of Nausicaa.

Changed Odysseus' companions into swine.

Follower of Odysseus.

Faithful swineherd who greeted Odysseus, gave him warm cloak and guides him to palace.

$\begin{array}{llll}5 & 1982 & 28 & \text { AA }\end{array}$

$5 \quad 1982 \quad 28$ AA Father of Odysseus.

$51982 \quad 28$ AA Disloyal goatherd; insults

Odysseus; is slain.

$\begin{array}{llll}5 & 1982 & 28 & \text { AA }\end{array}$

$51982 \quad 28$ AA Daughter of Alcinous who

advised Odysseus.

$5 \quad 1982 \quad 28$ AA A wise old king.

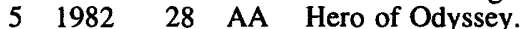

$5 \quad 1982 \quad 28$ AA Faithful wife of Odysseus.

$51982 \quad 28$ AA Minstrel to the wooers;

spared by Odysseus.

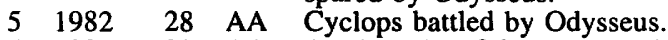

$\begin{array}{llll}5 & 1982 & 28 & \text { AA }\end{array}$

$5 \quad 1982 \quad 28 \quad$ AA $\quad \begin{aligned} & \text { Son of Odysseus. } \\ & 5\end{aligned}$ Aged prophet; Odysseus consults

$51982 \quad 28 \mathrm{CM}$ An Ionian island, home of Odysseus.

lat: latitude of feature center.

long: longitude of feature center.

diam: diameter or long dimension of feature.

ct: continent of name origin (see page $284 \mathrm{ff}$ ) et: ethnicity of name origin (see page $284 \mathrm{ff}$.)

quad: map quadrangle or informal name

(see page $x$ vii ff.). as: name approval status (see page $\mathrm{xvii)}$.

ad: name approval date (year).

ref: reference source for name (see page $287 \mathrm{ff}$.). 
URANIAN SYSTEM

Name

OBERON

CRATER

Antony
Caesar
Coriolanus
Falstaff
Hamlet
Lear
Macbeth
Othello
Romeo

CHASMA

Mommur Chasma

$\begin{array}{rrrllllll}27.5 \mathrm{~S} & 65.4 \mathrm{E} & 47 & \text { EU } & \text { GB } & \text { OBERON } & \text { O1920 } & 5 & 1988 \\ & & & & & & & & \\ 26.6 \mathrm{~S} & 61.1 \mathrm{E} & 76 & \text { EU } & \text { GB } & \text { OBERON } & \text { I1920 } & 5 & 1988 \\ & & & & & & & & \\ 11.4 \mathrm{~S} & 345.2 \mathrm{E} & 120 & \text { EU } & \text { GB } & \text { OBERON } & \text { I1920 } & 5 & 1988 \\ 22.1 \mathrm{~S} & 19.0 \mathrm{E} & 124 & \text { EU } & \text { GB } & \text { OBERON } & \mathrm{I} 1920 & 5 & 1988 \\ & & & & & & & & \\ 46.1 \mathrm{~S} & 44.4 \mathrm{E} & 206 & \text { EU } & \text { GB } & \text { OBERON } & \text { I1920 } & 5 & 1988 \\ 5.4 \mathrm{~S} & 31.5 \mathrm{E} & 126 & \text { EU } & \text { GB } & \text { OBERON } & \mathrm{I} 1920 & 5 & 1988 \\ & & & & & & & & \\ 58.4 \mathrm{~S} & 112.5 \mathrm{E} & 203 & \text { EU } & \text { GB } & \text { OBERON } & \text { I1920 } & 5 & 1988 \\ 66.0 \mathrm{~S} & 42.9 \mathrm{E} & 114 & \text { EU } & \text { GB } & \text { OBERON } & \mathrm{I} 1920 & 5 & 1988 \\ 28.7 \mathrm{~S} & 89.4 \mathrm{E} & 159 & \text { EU } & \text { GB } & \text { OBERON } & \text { I1920 } & 5 & 1988\end{array}$

$16.3 \mathrm{~S}$ long diam

\begin{abstract}
ct et quad
\end{abstract}

\begin{abstract}
map
\end{abstract}
as ad ref $\mathrm{ft}$ origin 


\section{URANIAN SYSTEM}

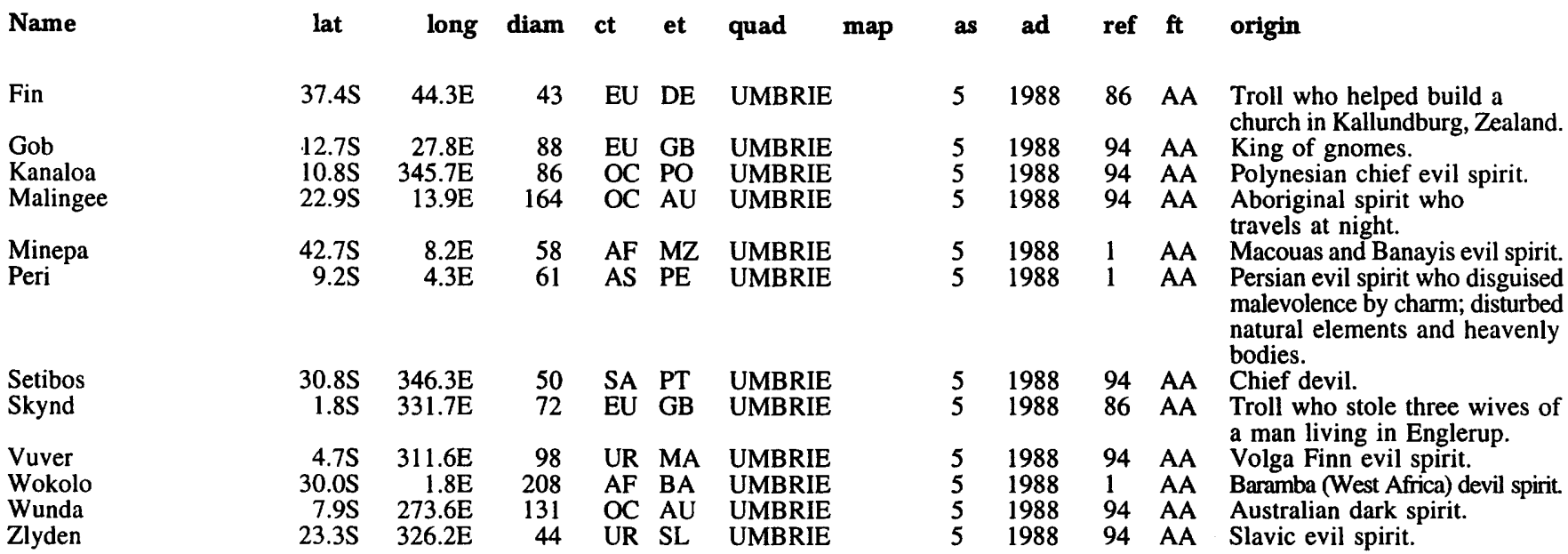

ARIEL

\section{CRATER}

\begin{tabular}{l} 
Abans \\
Agape \\
Ataksak \\
Befana \\
Berylune \\
Deive \\
Djadek \\
Domovoy \\
Finvara \\
Gwyn \\
Huon \\
Laica \\
Mab \\
Melusine \\
Oonagh \\
Rima \\
Yangoor \\
\multicolumn{1}{c}{ CHASMA } \\
Brownie Chasma \\
Kachina Chasmata \\
Kewpie Chasma \\
Korrigan Chasma \\
Kra Chasma \\
Pixie Chasma \\
Sylph Chasma \\
\\
Leprechaun Valli
\end{tabular}

Leprechaun Vallis Sprite Vallis

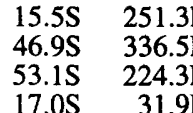

17.0S $\quad 31.9 \mathrm{E}$

22.5S $\quad 327.9 \mathrm{E}$

22.3S $23.0 \mathrm{E}$

12.0S 251.1E

71.5S $\quad 339.7 \mathrm{E}$

15.8S 19.0E

$77.5 \mathrm{~S} \quad 22.5 \mathrm{E}$

37.8S $\quad 33.7 \mathrm{E}$

21.3S $\quad 44.4 \mathrm{E}$

$38.8 \mathrm{~S} \quad 352.2 \mathrm{E}$

$52.9 \mathrm{~S} \quad 8.9 \mathrm{E}$

21.9S 244.4E

18.3S 260.8E

68.7S 279.7E

16.0S $337.6 \mathrm{E}$

$33.7 \mathrm{~S} \quad 246.0 \mathrm{E}$

343

EU GE ARIEL

I1920

NA HO ARIEL

I1920

$28.3 \mathrm{~S} \quad 326.9 \mathrm{E}$

467

27.6S $\quad 347.5 \mathrm{E}$

365

$32.1 \mathrm{~S}$

20.4 S

354.2E

$48.6 \mathrm{~S} \quad 353.0 \mathrm{E}$

EU GB ARIEL

EU FR ARIEL

AF GC ARIEL

EU GB ARIEL
AS PE ARIEL

EU GB ARIEL

NA ES ARIEL

ARIEL

I1920

I1920

I1920

I1920

5
5
5

1988

1988

1988

1988

\section{9}

20
22

71

31

34

40

30

SA IN

EU CE ARIEL

I1920

1988

UR LI ARIEL

$\begin{array}{lll}\mathrm{I} 1920 & 5 & 1988\end{array}$

$\begin{array}{lll}11920 & 5 & 1988\end{array}$

$\begin{array}{lll}\text { I1920 } & 5 & 1988\end{array}$

$\begin{array}{lll}I 1920 & 5 & 1988\end{array}$

$\begin{array}{lll}11920 & 5 & 1988\end{array}$

$\begin{array}{lll}\text { I1920 } & 5 & 1988\end{array}$

$\begin{array}{lll}\text { I1920 } & 5 & 1988\end{array}$

$\begin{array}{lll}11920 & 5 & 1988\end{array}$

$\begin{array}{lll}\text { I1920 } & 5 & 1988\end{array}$

EU FR ARIEL

I1920

I1920

1988

1988

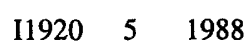

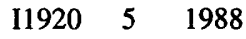

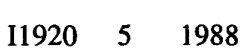

$\begin{array}{lll}11920 & 5 & 1988\end{array}$

$\begin{array}{lll}11920 & 5 & 1988\end{array}$

$\begin{array}{lll}11920 & 5 & 1988\end{array}$

83 AA Spirit of the iron mines.

83 AA Spirit in Spenser's "Fairy Queene."

94 AA Eskimo benevolent spirit.

83 AA Good spirit who fills Italian children's stockings with toys on Twelfth Night.

97 AA Good spirit in Maeterlinck's "The Bluebird."

94 AA Spirit of beautiful maiden.

83 AA Czech ancestral benevolent spirit and household guardian.

83 AA Slavic spirit protector of home.

83 AA Irish king of spirits; provided horses and wine to men.

83 AA Irish god of battle; leads mens' souls to Annwn.

83 AA Replaced Oberon as King of Spirits when Oberon died.

94 AA Inca good spirit.

86 AA Queen of Spirits, dethroned Titania.

86 AA Spirit heroine of

92 medieval French story.

92 AA Irish Queen of Fairies.

96 AA Spirit in Hudson's "Green

Mansions."

94 AA Spirit that brings day. $\begin{array}{lll}10.4 \mathrm{~S} & 10.2 \mathrm{E} \quad 328\end{array}$

14.9S $\quad 340.0 \mathrm{E} \quad 305$
EU IR ARIEL

EU GB ARIEL $\begin{array}{lll}11920 & 5 & 1988\end{array}$

$\begin{array}{lll}11920 & 5 & 1988\end{array}$
$86 \mathrm{CM}$ German good spirits who live in woods.

87 CM Pueblo (USA) good spirits who bring rain or other blessings.

$94 \mathrm{CM}$ British race of quaint spirit babies.

$86 \mathrm{CM}$ French wind spirits who cure diseases.

94 CM Vital spirits (Gold Coast).

86 CM British spirits that live in rocks.

94 CM British air spirits who influence the temperaments of man. lat: latitude of feature center.

long: longitude of feature center.

diam: diameter or long dimension of feature.

ct: continent of name origin (see page $284 \mathrm{f}$ ) et: ethnicity of name origin (see page $284 \mathrm{ff}$.)

quad: map quadrangle or informal name

(see page xvii ff.).

as: name approval status (see page xvii)

ad: name approval date (year).

ref: reference source for name (see page $287 \mathrm{ff}$.). map name or USGS map number (see page xvii ff.). ft: feature type (see page 290). 


\section{URANIAN SYSTEM}

Name

lat

MIRANDA

\section{CRATER}

\begin{tabular}{|c|c|c|c|c|c|c|c|c|c|c|c|}
\hline Alonso & $44.0 \mathrm{~S}$ & $352.6 \mathrm{E}$ & 25 & EU & GB & MIRAND & 5 & 1988 & 85 & AA & $\begin{array}{l}\text { King of Naples in "The } \\
\text { Tempest." }\end{array}$ \\
\hline Ferdinand & $34.8 \mathrm{~S}$ & $202.1 \mathrm{E}$ & 17 & $\mathrm{EU}$ & GB & MIRAND & 5 & 1988 & 85 & AA & \multirow{5}{*}{$\begin{array}{l}\text { Son of King of Naples; loves } \\
\text { Miranda in "The Tempest." } \\
\text { A lord of Naples in "The } \\
\text { Tempest." } \\
\text { Honest old counsellor of } \\
\text { Naples in "The Tempest." } \\
\text { Rightful Duke of Milan } \\
\text { in "The Tempest." } \\
\text { A drunken butler in "The } \\
\text { Tempest." } \\
\text { A jester in "The Tempest." }\end{array}$} \\
\hline Francisco & $73.2 S$ & $236.0 \mathrm{E}$ & 14 & $\mathrm{EU}$ & GB & MIRAND & 5 & 1988 & 85 & AA & \\
\hline Gonzalo & $11.4 \mathrm{~S}$ & $77.0 \mathrm{E}$ & 11 & $\mathrm{EU}$ & GB & MIRAND & 5 & 1988 & 85 & AA & \\
\hline Prospero & $32.9 \mathrm{~S}$ & $329.9 \mathrm{E}$ & 21 & EU & GB & MIRAND & 5 & 1988 & 85 & AA & \\
\hline Trinculo & $63.7 \mathrm{~S}$ & $163.4 \mathrm{E}$ & 11 & EU & GB & MIRAND & 5 & 1988 & 85 & AA & \\
\hline \multicolumn{12}{|l|}{ CORONA } \\
\hline Arden Corona & $29.1 S$ & $73.7 \mathrm{E}$ & 318 & EU & GB & MIRAND & 5 & 1988 & 85 & CR & \multirow{2}{*}{$\begin{array}{l}\text { Forest, location of "As } \\
\text { You Like It." } \\
\text { Location of Hamlet's castle. } \\
\text { Location of Macbeth's castle. }\end{array}$} \\
\hline $\begin{array}{l}\text { Elsinore Corona } \\
\text { Inverness Corona }\end{array}$ & $\begin{array}{l}24.8 S \\
66.9 S\end{array}$ & $\begin{array}{l}257.1 \mathrm{E} \\
325.7 \mathrm{E}\end{array}$ & $\begin{array}{l}323 \\
234\end{array}$ & $\begin{array}{l}\text { EU } \\
\text { EU }\end{array}$ & $\begin{array}{l}\text { DE } \\
\text { SC }\end{array}$ & $\begin{array}{l}\text { MIRAND } \\
\text { MIRAND }\end{array}$ & $\begin{array}{l}5 \\
5\end{array}$ & $\begin{array}{l}1988 \\
1988\end{array}$ & $\begin{array}{l}85 \\
85\end{array}$ & $\begin{array}{l}\text { CR } \\
\text { CR }\end{array}$ & \\
\hline Mantua Regio & $39.6 \mathrm{~S}$ & $180.2 \mathrm{E}$ & 399 & $\mathrm{EU}$ & IT & MIRAND & 5 & 1988 & 85 & RE & \multirow{2}{*}{$\begin{array}{l}\text { Location of castle where } \\
\text { Macbeth was defeated. } \\
\text { Location of part of "Two } \\
\text { Gentlemen From Verona." } \\
\text { Location of "Winter's Tale." }\end{array}$} \\
\hline Sicilia Regio & $30.0 \mathrm{~S}$ & $317.2 \mathrm{E}$ & 174 & EU & IT & MIRAND & 5 & 1988 & 85 & RE & \\
\hline \multicolumn{12}{|l|}{ RUPES } \\
\hline Argier Rupes & $43.2 \mathrm{~S}$ & $322.8 \mathrm{E}$ & 141 & EU & FR & MIRAND & 5 & 1988 & 85 & RU & \multirow{2}{*}{$\begin{array}{l}\text { Location of earlier } \\
\text { action in "The Tempest." } \\
\text { Where Romeo and Juliet lived. }\end{array}$} \\
\hline Verona Rupes & $18.3 \mathrm{~S}$ & $347.8 \mathrm{E}$ & 116 & EU & IT & MIRAND & 5 & 1988 & 85 & RU & \\
\hline
\end{tabular}

PUCK

CRATER

Bogle

Butz

EU SC

EU GE

EU GB

1988

1988

1988
94 AA Scottish mischievous spirits.

94 AA German roguish or evil spirits.

94 AA British mischievous spirits. lat: latitude of feature center.

long: longitude of feature center.

diam: diameter or long dimension of feature.

ct: continent of name origin (see page $284 \mathrm{ff}$.) et: ethnicity of name origin (see page $284 \mathrm{ff}$.)

quad: map quadrangle or informal name

$\begin{array}{lll}\text { map: } & \text { ref: } & \text { reference source for name (se } \\ \text { map name or USGS map number (see page xvii ff.). } \mathrm{ft}: & \text { feature type (see page } 290 \text { ). }\end{array}$

$\begin{array}{lll}\text { map: } & \text { ref: } & \text { reference source for name (se } \\ \text { map name or USGS map number (see page xvii ff.). } \mathrm{ft}: & \text { feature type (see page } 290 \text { ). }\end{array}$

as: name approval status (see page $x$ vii)

ad: name approval date (year).

$\begin{array}{lll}\text { (see page xvii ff.). } & \text { ref: } & \text { reference source for name (see page } 287 \mathrm{ff} \text {.). } \\ \text { map: } & \text { map name or USGS map number (see page xvii ff.). } \mathrm{ft} \text { feature type (see page 290). }\end{array}$ 
Name

\section{CRATER}

$\begin{array}{lrllll}\text { Amarum } & 26.0 \mathrm{~N} & 24.5 \mathrm{E} & 0 & \mathrm{SA} & \mathrm{EC} \\ \text { Andvari } & 20.5 \mathrm{~N} & 34.0 \mathrm{E} & 0 & \mathrm{EU} & \mathrm{NS} \\ \text { Cay } & 12.0 \mathrm{~S} & 44.0 \mathrm{E} & 0 & \mathrm{NA} & \mathrm{ME} \\ \text { Ilomba } & 14.5 \mathrm{~S} & 57.0 \mathrm{E} & 0 & \mathrm{AF} & \mathrm{ZM} \\ & & & & & \\ \text { Kurma } & 16.5 \mathrm{~S} & 61.0 \mathrm{E} & 0 & \mathrm{AS} & \mathrm{IN} \\ \text { Mazomba } & 18.5 \mathrm{~S} & 63.5 \mathrm{E} & 0 & \mathrm{AF} & \mathrm{TA} \\ & & & & & \\ \text { Ravgga } & 3.0 \mathrm{~S} & 71.5 \mathrm{E} & 0 & \mathrm{EU} & \mathrm{FI} \\ \text { Tangaroa } & 25.0 \mathrm{~S} & 65.5 \mathrm{E} & 0 & \mathrm{OC} & \mathrm{NZ} \\ \text { Vodyanoy } & 17.0 \mathrm{~S} & 28.5 \mathrm{E} & 0 & \mathrm{EU} & \mathrm{SL}\end{array}$

CATENAG1
Kraken Catena

Set Catena

\section{CAVUS}

\section{Apep Cavus}

Bheki Cavus

Dagon Cavus

Hekt Cavus

Hirugo Cavus

Kasyapa Cavus

Kulilu Cavus

Mah Cavus

Mangwe Cavus

Ukupanio Cavus

\section{DORSUM}

Awib Dorsa

FOSSA

Jumna Fossae

Raz Fossae

Yenisey Fossa

\section{MACULA}

Akupara Maculae

Doro Macula

Kikimora Maculae

Namazu Macula

Rem Maculae

Viviane Macula

Zin Maculae

\section{PATERA}

Gandvik Patera

Kasu Patera

Kibu Patera
Dilolo Patera lat map ref $\mathbf{f t}$ origin
12153

$\begin{array}{lll}12153 & 5 & 91\end{array}$ $\begin{array}{rr}83 & \mathrm{CA} \\ 1 & \mathrm{CA}\end{array}$

\begin{tabular}{|c|c|c|c|c|c|}
\hline $\begin{array}{l}12153 \\
\mathrm{I} 2153 \\
\mathrm{I} 2153 \\
\mathrm{I} 2153\end{array}$ & $\begin{array}{l}5 \\
5 \\
5 \\
5\end{array}$ & $\begin{array}{l}91 \\
91 \\
91 \\
91\end{array}$ & $\begin{array}{l}83 \\
83 \\
83 \\
85\end{array}$ & $\begin{array}{l}\text { AA } \\
\text { AA } \\
\text { AA } \\
\text { AA }\end{array}$ & $\begin{array}{l}\text { Quecha (Ecuador) water boa. } \\
\text { Norse fish shaped dwarf. } \\
\text { Mayan deity. } \\
\text { Lozi (Zambia) water snake }\end{array}$ \\
\hline $\begin{array}{l}\mathrm{I} 2153 \\
\mathrm{I} 2153\end{array}$ & $\begin{array}{l}5 \\
5\end{array}$ & $\begin{array}{l}91 \\
91\end{array}$ & $\begin{array}{l}83 \\
27\end{array}$ & $\begin{array}{l}\text { AA } \\
\text { AA }\end{array}$ & $\begin{array}{l}\text { Vishnu in the form of a tortoise. } \\
\text { Chaga (Tanzania) mythical } \\
\text { large fish. }\end{array}$ \\
\hline $\begin{array}{l}\mathrm{I} 2153 \\
\mathrm{I} 2153 \\
\mathrm{I} 2153\end{array}$ & $\begin{array}{l}5 \\
5 \\
5\end{array}$ & $\begin{array}{l}91 \\
91 \\
91\end{array}$ & $\begin{array}{l}83 \\
83 \\
83\end{array}$ & $\begin{array}{l}\text { AA } \\
\text { AA } \\
\text { AA }\end{array}$ & $\begin{array}{l}\text { Finnish fortune-telling fish god. } \\
\text { Maori fishing and sea god. } \\
\text { Slavic water spirit. }\end{array}$ \\
\hline
\end{tabular}

$14.0 \mathrm{~N} \quad 35.5 \mathrm{E} \quad 0 \quad \mathrm{EU} \quad \mathrm{NS}$

$22.0 \mathrm{~N} \quad 33.5 \mathrm{E} \quad 0$ AF $\mathrm{EG}$
20.0N 301.5E $\quad 0 \quad$ AF $\quad$ EG

$16.0 \mathrm{~N} \quad 308.0 \mathrm{E} \quad 0$ AS IN

$\begin{array}{lllll}29.0 \mathrm{~N} & 345.0 \mathrm{E} & 0 & \text { AS } & \text { BY }\end{array}$

$26.0 \mathrm{~N} \quad 342.0 \mathrm{E} \quad 0 \quad \mathrm{AF} \quad \mathrm{EG}$

$\begin{array}{lllll}14.5 \mathrm{~N} & 345.0 \mathrm{E} & 0 & \mathrm{AS} & \mathrm{JA}\end{array}$

$\begin{array}{lllll}7.5 \mathrm{~N} & 358.0 \mathrm{E} & 0 & \text { AS } & \text { IN }\end{array}$

$41.0 \mathrm{~N} \quad 4.0 \mathrm{E} \quad 0 \quad \mathrm{EU} \quad \mathrm{BY}$

38.0N $6.0 \mathrm{E} \quad 0 \quad \mathrm{AS} \quad \mathrm{PE}$

$\begin{array}{lllll}7.0 \mathrm{~S} & 343.0 \mathrm{E} & 0 & \mathrm{AF} & \mathrm{ZM}\end{array}$

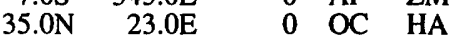

I215

1215

12153

I2153

$12153 \quad 5 \quad 9$

$12153 \quad 5 \quad 91$

I2153 591

$12153 \quad 5 \quad 91$

I2153 591

2153
83 CB

83 CB

83 CB

83 CB

83 CB

83 CB

$83 \mathrm{CB}$

$83 \mathrm{CB}$

$85 \mathrm{CB}$

83 CB
Norse giant sea monster.

Egypian water monster; personification of evil.

Egyptian dragon of darkness. Frog symbolizing the sun on the horizon (India). Babylonian fertility god represented as a fish. Egyptian frog goddess. Japanese deity born in shape of a jellyfish. The god Prajapati as a tortoise (India).

Babylonian destructive fish-man spirit.

Fish that holds up the universe (Persian). Ila (Zambia) "the flooder." Hawaiian shark god.
12153

I2153

12153

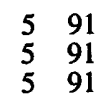

12153

12153

12153

12153

12153

0 AF EG

13.0N $\quad 349.5 \mathrm{E}$

31.0S $\quad 36.5 \mathrm{E}$

$24.5 \mathrm{~S} \quad 68.0 \mathrm{E}$

I 2153

12153

0 AF NG

591

591

591

591

591

$5 \quad 91$

$\begin{array}{ll}5 & 91\end{array}$

$\begin{array}{lrlll}26.0 \mathrm{~N} & 24.5 \mathrm{E} & 0 & \text { AF } & \text { AN } \\ 28.0 \mathrm{~N} & 5.5 \mathrm{E} & 0 & \text { EU } & \text { NS } \\ 39.0 \mathrm{~N} & 14.0 \mathrm{E} & 0 & \text { AS } & \text { PE } \\ 10.5 \mathrm{~N} & 43.0 \mathrm{E} & 0 & \text { OC } & \text { ME }\end{array}$

$\begin{array}{lll}12153 & 5 & 91 \\ \mathrm{I} 2153 & 5 & 91 \\ \mathrm{I} 2153 & 5 & 91 \\ \mathrm{I} 2153 & 5 & 91\end{array}$

83 FO Hindu river goddess.

83 FO Breton bay of souls.
83 FO Siberian mythical holy river.
83 MA Tortoise upholding the world (India).

50 MA Nanay mistress of fishing, Sea of Okhotsk.

27 MA Slavic spirit of swamps, household spirit.

83 MA Japanese mythic fish; maker of earthquakes.

27 MA Egyptian fish who wept fertilizing tears.

27 MA Amour of Merlin (Welsh).

13 MA Niger water spirits. lat: latitude of feature center.

long: longitude of feature center.

diam: diameter or long dimension of feature.

ct: continent of name origin (see page $284 \mathrm{ff}$ ) et: ethnicity of name origin (see page $284 \mathrm{ff}$.)

quad: map quadrangle or informal name

$\begin{array}{lll} & \text { (see page xvii ff.). } & \text { reference source for name (se } \\ \text { map: map name or USGS map number (see page xvii ff.). } \mathrm{ft} \text { : } & \text { feature type (see page } 290 \text { ). }\end{array}$

as: name approval status (see page xvii).

ad: name approval date (year).

ref: reference source for name (see page $287 \mathrm{ff}$.). 


\section{NEPTUNIAN SYSTEM}

Name

Leviathan Patera

28.5E

PLANITIA

Ruach Planitia

Ryugu Planitia

Sipapu Planitia

Tuonela Planitia

\section{PLANUM}

Abatos Planum

Cipango Planum

Medamothi Planum

$\begin{array}{rllll}28.0 \mathrm{~N} & 24.0 \mathrm{E} & 0 & \mathrm{EU} & \mathrm{FR} \\ 5.0 \mathrm{~S} & 27.0 \mathrm{E} & 0 & \text { AS } & \text { JA } \\ 4.0 \mathrm{~S} & 36.0 \mathrm{E} & 0 & \text { NA } & \text { PU } \\ 34.0 \mathrm{~N} & 14.5 \mathrm{E} & 0 & \mathrm{EU} & \text { FI }\end{array}$

$\begin{array}{rllll}21.5 \mathrm{~S} & 58.0 \mathrm{E} & 0 & \mathrm{AF} & \mathrm{EG} \\ 11.5 \mathrm{~N} & 34.0 \mathrm{E} & 0 & \mathrm{EU} & \text { IT } \\ 3.5 \mathrm{~N} & 69.0 \mathrm{E} & 0 & \mathrm{EU} & \text { FR }\end{array}$

\section{PLUME}

Hili

Mahilani

\section{REGIO}

Bubembe Regio

Monad Regio

Uhlanga Regio

$50.5 \mathrm{~S} \quad 359.5 \mathrm{E}$

$\begin{array}{lll}0 & \text { AF } & \text { SA } \\ 0 & \text { OC } & \text { TG }\end{array}$

$18.0 \mathrm{~N} \quad 335.0 \mathrm{E} \quad 0 \quad \mathrm{AF} \quad \mathrm{UG}$

$20.0 \mathrm{~N} \quad 37.0 \mathrm{E}$

0 AS $\mathrm{CH}$

$37.0 \mathrm{~S} \quad 357.0 \mathrm{E}$

0 AF AS

\section{SULCUS}

Boynne Sulci

Ho Sulci

Kormet Sulci

Leipter Sulci

Lo Sulci

Ob Sulci

Ormet Sulci

Slidr Sulci

Tano Sulci

Vimur Sulci

Yasu Sulci

$\begin{array}{rrrrr}38.0 \mathrm{~S} & 3.0 \mathrm{E} & 0 & \mathrm{AF} & \mathrm{NI} \\ 13.0 \mathrm{~S} & 350.0 \mathrm{E} & 0 & \mathrm{EU} & \mathrm{CE} \\ 2.0 \mathrm{~N} & 305.0 \mathrm{E} & 0 & \mathrm{AS} & \mathrm{CH} \\ 23.0 \mathrm{~N} & 335.5 \mathrm{E} & 0 & \mathrm{EU} & \mathrm{NS} \\ 7.0 \mathrm{~N} & 9.0 \mathrm{E} & 0 & \mathrm{EU} & \mathrm{NS} \\ 3.8 \mathrm{~N} & 321.0 \mathrm{E} & 0 & \mathrm{AS} & \mathrm{CH} \\ 6.0 \mathrm{~S} & 328.0 \mathrm{E} & 0 & \mathrm{UR} & \mathrm{OS} \\ 17.0 \mathrm{~N} & 337.0 \mathrm{E} & 0 & \mathrm{EU} & \mathrm{NS} \\ 23.5 \mathrm{~N} & 350.0 \mathrm{E} & 0 & \mathrm{EU} & \mathrm{NS} \\ 33.5 \mathrm{~N} & 337.0 \mathrm{E} & 0 & \mathrm{AF} & \mathrm{NI} \\ 11.0 \mathrm{~S} & 59.0 \mathrm{E} & 0 & \mathrm{EU} & \mathrm{NS} \\ 2.0 \mathrm{~N} & 347.0 \mathrm{E} & 0 & \mathrm{AS} & \mathrm{JA}\end{array}$

$\begin{array}{llllll}\operatorname{map} & \text { as } & \text { ad } & \text { ref } & \text { ft } \\ \mathrm{I} 2153 & 5 & 91 & 83 & \mathrm{PE}\end{array}$

Hebrew sea monster upholding earth.

\begin{tabular}{|c|c|c|c|c|c|}
\hline $\begin{array}{l}\mathrm{I} 2153 \\
\mathrm{I} 2153\end{array}$ & $\begin{array}{l}5 \\
5\end{array}$ & $\begin{array}{l}91 \\
91\end{array}$ & $\begin{array}{l}83 \\
27\end{array}$ & $\begin{array}{l}\text { PL } \\
\text { PL }\end{array}$ & $\begin{array}{l}\text { French isle of winds. } \\
\text { Japanese undersea dragon }\end{array}$ \\
\hline 12153 & 5 & 91 & 83 & PL & $\begin{array}{l}\text { Pueblo (USA) hole or lake } \\
\text { of emergence from underworld. }\end{array}$ \\
\hline I 2153 & 5 & 91 & 83 & PL & $\begin{array}{l}\text { Underground realm across } \\
\text { Black River (Finnish). }\end{array}$ \\
\hline
\end{tabular}

$\begin{array}{lllrll}\text { I2153 } & 5 & 91 & 27 & \text { PU } & \text { Zulu water-sprite. } \\ \text { I2153 } & 5 & 91 & 0 & \text { PU } & \text { Tonga sea spirit. }\end{array}$

$\begin{array}{llllll}\text { I2153 } & 5 & 91 & 27 & \text { RE } & \begin{array}{l}\text { Island location of temple } \\ \text { of Mukasa (Uganda). }\end{array} \\ \mathrm{I} 2153 & 5 & 91 & 83 & \mathrm{RE} & \begin{array}{l}\text { Chinese symbol of duality } \\ \text { in nature. }\end{array} \\ \mathrm{I} 2153 & 5 & 91 & 27 & \mathrm{RE} & \begin{array}{l}\text { Zulu reed from which } \\ \text { humanity sprang. }\end{array}\end{array}$

\section{PROTEUS}

\section{CRATER}

N pr Pharos

\begin{tabular}{|c|c|c|c|c|c|}
\hline 12153 & 5 & 91 & 13 & SU & Yoruba; river named for \\
\hline $\begin{array}{l}\text { I2153 } \\
\text { I } 2153 \\
\text { I } 2153\end{array}$ & $\begin{array}{l}5 \\
5 \\
5\end{array}$ & $\begin{array}{l}91 \\
91 \\
91\end{array}$ & $\begin{array}{l}83 \\
83 \\
83\end{array}$ & $\begin{array}{l}\text { SU } \\
\text { SU } \\
\text { SU }\end{array}$ & $\begin{array}{l}\text { Celtic mythological river. } \\
\text { Chinese sacred river. } \\
\text { Norse river through which } \\
\text { dead must pass. }\end{array}$ \\
\hline $\begin{array}{l}\text { I2153 } \\
\text { I } 2153 \\
\text { I } 2153\end{array}$ & $\begin{array}{l}5 \\
5 \\
5\end{array}$ & $\begin{array}{l}91 \\
91 \\
91\end{array}$ & $\begin{array}{l}83 \\
83 \\
83\end{array}$ & $\begin{array}{l}\text { SU } \\
\text { SU } \\
\text { SU }\end{array}$ & $\begin{array}{l}\text { Norse sacred river. } \\
\text { Chinese sacred river. } \\
\text { Mouth of this river is Ostiak } \\
\text { entrance to underworld. }\end{array}$ \\
\hline 12153 & 5 & 91 & 83 & SU & $\begin{array}{l}\text { Norse river through which } \\
\text { dead pass. }\end{array}$ \\
\hline 12153 & 5 & 91 & 83 & SU & $\begin{array}{l}\text { Norse river of daggers } \\
\text { and spears. }\end{array}$ \\
\hline I2153 & 5 & 91 & 85 & SU & $\begin{array}{l}\text { Yoruba; river named for } \\
\text { willful son of god. }\end{array}$ \\
\hline I 2153 & 5 & 91 & 83 & SU & $\begin{array}{l}\text { Greatest of Elivagar } \\
\text { rivers, a stream of ice. }\end{array}$ \\
\hline 12153 & 5 & 91 & 83 & SU & $\begin{array}{l}\text { Japanese heavenly river; } \\
\text { literally, "peace." }\end{array}$ \\
\hline
\end{tabular}

lat: latitude of feature center.

long: longitude of feature center.

diam: diameter or long dimension of feature.

ct: continent of name origin (see page $284 \mathrm{ff}$ ) et: $\quad$ ethnicity of name origin (see page $284 \mathrm{ff}$.)

quad: map quadrangle or informal name

(see page xvii ff.). map: map name or USGS map number (see page xvii ff.). ft: feature type (see page 290).

\footnotetext{
as: name approval status (see page xvii).

ad: name approval date (year).

ref: reference source for name (see page $287 \mathrm{ff}$.)
} 
SECTION 2-ALPHABETICAL LIST OF NAMES 


\section{ALPHABETICAL LIST OF NAMES}

\section{P Sa Name}

$S$ rh Aananin

V V Abaka

M M Abalos Undae

M M Aban

$\mathrm{U}$ ar Abans

$\mathrm{N}$ tr Abatos Planum

L L Abbe

L L Abbot

L L [Abduh]

L L Abel

L L Abenezra

L L Abetti

V V Abigail

$\mathrm{V} V$ Abington

H H Abu Nuwas

L L Abul Wáfa

L L Abulfeda

V V Abundia Corona

M M Abus Vallis

J ga Abydos Facula

$\mathrm{S}$ mi Accolon

M M Achar

$\mathrm{J}$ ga Achelous

M M Acheron Catena

M M Acheron Fossae

M M Acidalia Planitia

L L Acosta

J ga Adad

$\mathrm{V} V$ Adaiah

J ca Adal

M M Adamas Labyrinthus

L L Adams lat

$34.9 \mathrm{~N}$

$52.6 \mathrm{~S} \quad 104.2 \mathrm{E}$

$81.0 \mathrm{~N} \quad 83.1 \mathrm{~W}$

$16.2 \mathrm{~N} \quad 249.1 \mathrm{~W}$

$15.5 \mathrm{~S} \quad 251.3 \mathrm{E}$

$21.5 \mathrm{~S} \quad 58.0 \mathrm{E}$

57.3S $\quad 175.2 \mathrm{E}$

$5.6 \mathrm{~N} \quad 54.8 \mathrm{E}$

$14.7 \mathrm{~N}$

$34.5 \mathrm{~S}$

$39.0 \mathrm{E}$

$21.0 \mathrm{~S}$

87.3E

$21.0 \mathrm{~S}$

$11.9 \mathrm{E}$

$19.9 \mathrm{~N}$

27.7E

$52.2 S$

$47.8 \mathrm{~S}$

$111.1 \mathrm{E}$

$17.4 \mathrm{~N}$

$1.0 \mathrm{~N} \quad 116.6 \mathrm{E}$

$13.8 \mathrm{~S}$

$13.9 \mathrm{E}$

$18.5 \mathrm{~N}$

$6.3 \mathrm{~S}$

$125.0 \mathrm{E}$

$34.1 \mathrm{~N}$

$65.7 \mathrm{~S}$

$45.7 \mathrm{~N}$

$60.3 \mathrm{~N}$

$147.5 \mathrm{~W}$

$154.0 \mathrm{~W}$

$184.7 \mathrm{~W}$

$38.2 N$

$38.7 \mathrm{~N}$

$236.8 \mathrm{~W}$

$13.5 \mathrm{~W}$

$100.7 \mathrm{~W}$

$136.6 \mathrm{~W}$

1120

$54.6 \mathrm{~N}$

$19.9 \mathrm{~W}$

2791

$5.6 \mathrm{~S}$

$60.1 \mathrm{E}$

$55.9 \mathrm{~N}$

$47.3 \mathrm{~S}$

$75.4 \mathrm{~N}$

$36.5 \mathrm{~N}$

$180.0 \mathrm{~W}$

253.3E

$80.8 \mathrm{~W}$

13

65

19

23

AS HE

EU EN

1994

116

AS SY

AS PE

$5 \quad 1976$

65

AS SY

$5 \quad 1935$

EU NS

EU GB

$\begin{array}{ll}5 & 1994 \\ 5 & 1985\end{array}$

165

AF EG

51985

51982

6 SA UR

51979

51979

51979

EU GR

51979

51973

51976

59

59
19
40
664

EU PG

51979

AS AB

AE HE

EU NS

$\begin{array}{ll}5 & 1994\end{array}$

$\begin{array}{ll}5 & 1979\end{array}$

$\begin{array}{ll}5 & 1982\end{array}$

$31.9 \mathrm{~S}$

68.2E
66

NA

AM

$\begin{array}{ll}5 & 1970\end{array}$

$\begin{array}{rrrrrrrr}31.3 \mathrm{~N} & 197.1 \mathrm{~W} & 100 & \text { NA } & \text { AM } & 5 & 1973 & 68 \\ 14.8 \mathrm{~S} & 29.6 \mathrm{E} & 20 & \text { EU } & \text { AS } & 5 & 1994 & 87 \\ 71.3 \mathrm{~N} & 30.2 \mathrm{~W} & 54 & \text { AS } & \text { AB } & 5 & 1979 & 1 \\ & & & & & & & \\ 56.1 \mathrm{~S} & 98.9 \mathrm{E} & 90 & \text { NA } & \text { AM } & 5 & 1994 & 87 \\ 8.9 \mathrm{~N} & 76.1 \mathrm{E} & 31 & \text { AS } & \text { TU } & 5 & 1991 & 99\end{array}$

\section{ref $f t$ origin}

AA Korean god of the Heavens.

AA Mari first name.

UN Classical albedo feature at $72 \mathrm{~N}$, 70W.

AA Town in Russia.

AA Spirit of the iron mines.

PM Egyptian sacred island in Nile, "paradise."

AA Ernst K.; German optician, physician, astronomer (1840-1905).

AA Charles Greeley; American astrophysicist (1872-1973).

AA Mohammed; Egyptian writer (1849-1905).

AA Niels H.; Norwegian mathematician (1802-1829).

AA Abraham Bar Rabbi Ben Ezra; Spanish Jewish mathematician, astronomer (1092-1167).

AA Antonio; Italian astronomer (1846-1928); Georgio; Italian astronomer (1882-1982).

AA First name from Hebrew.

AA Frances; English actress (1737-1815).

AA Arab poet (c. 1756-1810).

AA Persian mathematician, astronomer (940-998)

AA Abu'L-fida, Ismail (1273-1331); Syrian geographer.

CR Norse goddess of giving.

VA Classical name for Humber River in England.

FA Egyptian town where Osiris was worshipped.

AA Companion of Arthur's; he was tricked into jousting with Arthur.

AA Town in Uruguay.

AA Greek river god; father of Callirrhoe, Ganymede's mother.

CA Named for classical albedo feature at $35 \mathrm{~N}, 140 \mathrm{~W}$.

FO From classical albedo feature at $35 \mathrm{~N}, 140 \mathrm{~W}$

PL From classical albedo feature at $44 \mathrm{~N}, 21 \mathrm{~W}$.

AA Cristobal; Portuguese doctor, natural historian (1515-1580).

AA Assyro-Babylonian god of thunder.

AA Hebrew first name.

AA Norse; son of Karl and Erna.

LB Classical albedo feature name; "A River of Diamonds"; today's River Sarbarnarekha in India.

AA John Couch; British astronomer (1819-1892); Charles H.; American astronomer (1868-1951); Walter S.; American astronomer (1876-1956).

AA Walter S.; American astronomer (1876-1956).

AA Joy; Austrian author, animal expert (1910-1980).

AA Assyro-Babylonian; lost immortality when, at Ea's advice, he refused food of life.

AA Jane; American social reformer (1860-1935).

AA Halide; Turkish educator, author (1883-1964).
P: $\quad$ planetary system (see page $x v i)$.

Sa: $\quad$ satellite (see page $x v i$ ).

lat: latitude of feature center.

long: longitude of feature center. diam: diameter or long dimension of feature.

ct: continent of name origin (see page $284 \mathrm{ff}$.)

et: ethnicity of name origin (see page $284 \mathrm{ff}$.)

as: name approval status (see page xvii). ad: name approval date (year).

ref: reference source for name (see page $287 \mathrm{ff}$.).

ft: feature type (see page 290). 


\section{ALPHABETICAL LIST OF NAMES}

\section{P Sa Name}

S rh Adjua

$\mathrm{J}$ ca Adlinda

J eu Adonis Linea

$S$ di Adrastus

$\mathrm{U}$ ti Adriana

H H Adventure Rupes

V V Aegina Farrum

$S$ di Aeneas

M M Aeolis

M M Aeolis Mensae

V V Aeracura Corona

M M Aeria

M M Aesacus Dorsum

V V Aethelflaed

M M Aetheria

M M Aethiopis

$A$ id Afon

$\mathrm{H} \quad \mathrm{H}$ Africanus Horton

M M Aganippe Fossa

$\mathrm{U}$ ar Agape

M M Agassiz

L L Agatharchides

$J$ eu Agenor Linea

$\mathrm{V} V$ Aglaonice

$V \quad V$ Agnesi

$J$ io Agni Patera

V V Agraulos Corona

$J$ ga Agreus

L L Agrippa

V V Agrippina

J ca Ägröi

J ga Agrotes

$S$ rh Agunua

$S$ en Ahmad

H H Ahmad Baba

V V Ahsonnutli Dorsa

J ca Ahti

V V Aimee

$\checkmark \quad \mathrm{V}$ Aino Planitia

\section{lat long diam}

$\begin{array}{rrrrrrrr}40.2 \mathrm{~N} & 118.9 \mathrm{~W} & 0 & \text { UR } & \text { UL } & 5 & 1982 & 50 \\ 56.6 \mathrm{~S} & 23.1 \mathrm{~W} & 274 & \text { NA } & \text { ES } & 5 & 1979 & 1 \\ & & & & & & & \\ 51.8 \mathrm{~S} & 113.2 \mathrm{~W} & 758 & \mathrm{EU} & \text { GR } & 5 & 1979 & 19 \\ 61.7 \mathrm{~S} & 45.9 \mathrm{~W} & 31 & \text { EU } & \text { GR } & 5 & 1982 & 44 \\ & & & & & & & \\ 20.1 \mathrm{~S} & 3.9 \mathrm{E} & 50 & \text { EU } & \text { GB } & 5 & 1988 & 85 \\ 65.1 \mathrm{~S} & 65.5 \mathrm{~W} & 0 & \text { EU } & \text { EN } & 5 & 1976 & 59\end{array}$

$35.5 \mathrm{~N} \quad 20.9 \mathrm{E}$

$26.1 \mathrm{~N} \quad 46.3 \mathrm{~W}$

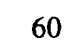

EU GR

$5 \quad 1994$

166

EU RM

$5 \quad 1982$

5.0

3.7
19.0

$19.0 \mathrm{~S}$

215.0W

0 EU GR

$5 \quad 1958$

841

$218.5 \mathrm{~W}$

$238.5 \mathrm{E}$

250

EU

GR

38.5

18.2

206.6W

250
0

$\mathrm{EU}$

GR

51976

$\begin{array}{ll}5 & 1994 \\ 5 & 1958\end{array}$

51985

$18.2 \mathrm{~S} \quad 196.5 \mathrm{E}$

$40.0 \mathrm{~N} \quad 230.0 \mathrm{~W}$

$10.0 \mathrm{~N} \quad 230.0 \mathrm{~W}$

$6.5 \mathrm{~S}$

$51.5 \mathrm{~S}$

8.7S

$46.9 \mathrm{~S}$

$70.1 \mathrm{~S}$

$19.8 \mathrm{~S}$

$43.6 \mathrm{~S}$

$26.5 \mathrm{~S}$

$39.5 \mathrm{~S}$

40.5

27.7

$15.2 \mathrm{~N}$

$4.1 \mathrm{~N}$

$33.2 \mathrm{~S}$

$43.3 \mathrm{~N}$

$62.5 \mathrm{~N}$

$63.3 \mathrm{~N}$

$0.0 \mathrm{~W}$

$41.2 \mathrm{~W}$

$\mathrm{EU}$

GR

51994

17 EU EN

51958

$\begin{array}{lll}0 & \text { EU } & \text { GR } \\ 0 & \text { EU } & \text { GR }\end{array}$

$\begin{array}{ll}5 & 1958\end{array}$

$\begin{array}{lllll}25 & \text { UR } & \text { RU } & 5 & 1994\end{array}$

135 AF $\quad$ SL

$5 \quad 1976$

$126.0 \mathrm{~W}$

$336.5 \mathrm{E}$

$88.4 \mathrm{~W}$

329

34

EU GR

EU GB

NA AM

$30.9 \mathrm{~W}$

48

EU GR

51976

51988

51973

51935

208.2W

$339.9 \mathrm{E}$

1326

EU GR

51979

$37.8 \mathrm{E}$

40

$\begin{array}{ll}5 & 1991\end{array}$

51991

$334.2 \mathrm{~W}$

$165.8 \mathrm{E}$

$235.4 \mathrm{~W}$

20

170

AS IN

AS IN

AS PO

1985

$5 \quad 1994$

$5 \quad 1985$

51935

$65.6 \mathrm{E}$

37

EU RM

51991

$11.0 \mathrm{~W}$

$199.5 \mathrm{~W}$

55

EU

RM

$5 \quad 1979$

$66.2 \mathrm{~W}$

$57.4 \mathrm{~N} \quad 305.4 \mathrm{~W}$

0

AS

PO

51985

$5 \quad 1982$

51982

$58.5 \mathrm{~N} \quad 126.8 \mathrm{~W}$

17

AS

AR

1979

$47.9 \mathrm{~N}$

$41.8 \mathrm{~N}$

$16.2 \mathrm{~N}$

$40.5 \mathrm{~S}$
196.5E

127

AF SU

$5 \quad 1979$

1708

NA NV

51985

$5 \quad 1987$

EU FI

$5 \quad 1994$

18

EU FR ref

ft origin

AA Mythical heroine and ancestor of the Ulci tribe.

LG Eskimo; place in ocean depths where souls are imprisoned after death.

LI Greek; son of Phoenix, nephew of Europa.

AA King of Argos, one of the seven against Thebes, and the only one to return alive.

AA Wife of Antipholus of Ephesus in "The Comedy of Errors."

RU English; one of Cook's ships on second voyage to the Pacific, 1772-75.

FR Greek river nymph.

AA Hero of the Aeneid. The son of Anchises and Venus and a member of the royal family of Troy.

AL Floating island where winds were kept in a cave.

MN Classical albedo feature name.

CR Celtic earth goddess.

AL Greek name for Egypt; "far land of mist".

DO From albedo feature at $45 \mathrm{~N}, 205 \mathrm{~W}$.

AA English leader of the Mercians (c. 884-918).

AL Upper world; land of living.

AL Countries of the Ethiopians on southern edge of the Earth.

AA Cave in Russia.

AA (James Beale); Sierra Leonean author, folklorist (1835-1883).

FO Classical albedo feature name.

AA Spirit in Spenser's "Fairy Queene."

AA Jean L.; American naturalist (1807-1873).

AA Greek geographer (unkn-c. 150 B.C.)

LI Greek; Europa's father.

AA Ancient Greek astronomer.

AA Maria; Italian mathemetician (1718-1799).

PE Hindu god of fire.

CR Greek fertility goddess.

AA Hunter god in Tyre.

AA Greek astronomer (unkn-fl. A.D. 92).

AA Roman empress (c. 13 B.C.-A.D. 33)

AA Finno-Ugric god of twins.

AA Tyre; greatest god of Gebal; farmer god

AA San Cristobal (Melanesia) god who made sea, land, people.

AA Youngest son; brings father a magic apple; marries the Genie Peri Banu.

AA Abu-a;-Abbas Aj,Ad Obm Aj,Ad a;-Takruri Al-Massufi; Sudanese writer (1556-1627)

DO Navajo (N. America) spirit of light and sky.

AA Finnish god of water; sends fish to the fisherman.

AA French first name.

PL Finnish heroine who became water spirit.
P: $\quad$ planetary system (see page xvi).

Sa: satellite (see page xvi).

lat: latitude of feature center.

long: longitude of feature center. diam: diameter or long dimension of feature.

ct: $\quad$ continent of name origin (see page $284 \mathrm{ff}$.)

et: ethnicity of name origin (see page $284 \mathrm{ff}$.)

as: name approval status (see page xvii). ad: name approval date (year).

ref: reference source for name (see page $287 \mathrm{ff}$.).

$\mathrm{ft}$ : feature type (see page 290). 


\section{ALPHABETICAL LIST OF NAMES}

\section{P Sa Name}

M M Airy

L L Airy

V V Aita

V V Aitchison Patera

L L Aitken

A gs Aix

$S$ te Ajax

J ca Ajleke

M M Ajon

V V Akeley

V V Akhmatova

M M Aki

V V Akiko

L L Akis

V V Akkruva Colles

V V Akna Montes

V V Aksentyeva

M M Aktaj

$\mathrm{N}$ tr Akupara Maculae

J ca Akycha

$S$ en Aladdin

$\mathrm{H} \quad \mathrm{H}$ Al-Akhtal

L L Alan

M M Alba Catena

M M Alba Fossae

M M Alba Patera

L L Al-Bakri

M M Albany

L L Albategnius

U um Alberich

M M Albi

L L Al-Biruni

M M Albor Fossae

M M Albor Tholus

V V Alcott

L L Alden

L L Alder

L L Aldrin

V V Ale Tholus

L L Alekhin

H H Alencar

L L Alexander

M M Alexey Tolstoy

J ca Alfr lat

lon

$\begin{array}{rrrrrrrr}5.2 \mathrm{~S} & 0.0 \mathrm{~W} & 56 & \text { EU } & \text { GB } & 5 & 1973 & 68 \\ 18.1 \mathrm{~S} & 5.7 \mathrm{E} & 36 & \text { EU } & \text { GB } & 5 & 1935 & 66 \\ & & & & & & & \\ 8.9 \mathrm{~N} & 270.7 \mathrm{E} & 14 & \text { UR } & \text { ES } & 5 & 1994 & 112 \\ 16.7 \mathrm{~S} & 349.4 \mathrm{E} & 28 & \text { NA } & \text { AM } & 5 & 1994 & 0 \\ 16.8 \mathrm{~S} & 173.4 \mathrm{E} & 135 & \text { NA } & \text { AM } & 5 & 1970 & 0 \\ & & & & & & & \\ 47.9 \mathrm{~N} & 160.3 \mathrm{~W} & 6 & \text { EU } & \text { FR } & 5 & 1994 & 113 \\ 29.1 \mathrm{~S} & 282.0 \mathrm{~W} & 0 & \text { EU } & \text { GR } & 5 & 1982 & 28 \\ & & & & & & & \\ 22.4 \mathrm{~N} & 101.3 \mathrm{~W} & 46 & \text { EU } & \text { LP } & 5 & 1987 & 64 \\ 16.8 \mathrm{~N} & 257.9 \mathrm{~W} & 0 & \text { UR } & \text { RU } & 5 & 1988 & 36 \\ 8.0 \mathrm{~N} & 244.4 \mathrm{E} & 25 & \text { NA } & \text { AM } & 5 & 1994 & 87\end{array}$

$61.3 \mathrm{~N}$

$36.7 \mathrm{~N}$

$\begin{array}{lllllll}20.0 \mathrm{~N} & 31.8 \mathrm{~W} & 2 & \mathrm{EU} & \mathrm{GR} & 5 & 1976\end{array}$

$\begin{array}{llllllll}46.1 \mathrm{~N} & 115.5 \mathrm{E} & 1059 & \mathrm{EU} & \mathrm{LA} & 5 & 1985 & 65\end{array}$

$\begin{array}{rrrrrrrr}68.9 \mathrm{~N} & 318.2 \mathrm{E} & 830 & \mathrm{CA} & \mathrm{MY} & 5 & 1979 & 1 \\ 42.0 \mathrm{~S} & 271.9 \mathrm{E} & 43 & \text { UR } & \text { SO } & 5 & 1994 & 107\end{array}$

$\begin{array}{llllllll}20.7 \mathrm{~N} & 46.5 \mathrm{~W} & 0 & \mathrm{UR} & \mathrm{RU} & 5 & 1988 & 36\end{array}$

$27.5 \mathrm{~S} \quad 63.0 \mathrm{E}$

$72.5 \mathrm{~N} \quad 318.6 \mathrm{~W}$

63.1N $\quad 16.9 \mathrm{~W}$

59.2N $97.0 \mathrm{~W}$

$10.9 \mathrm{~S}$

$35.2 \mathrm{~N} \quad 114.6 \mathrm{~W}$

$43.4 \mathrm{~N} \quad 103.6 \mathrm{~W}$

$40.5 \mathrm{~N} \quad 109.9 \mathrm{~W}$

$14.3 \mathrm{~N} \quad 20.2 \mathrm{E}$

$23.3 \mathrm{~N} \quad 49.0 \mathrm{~W}$

$11.7 \mathrm{~S}$

$4.3 \mathrm{E}$

$33.6 \mathrm{~S}$

42.2E

41.9

17.9

18.

$19.3 N$

59.5

23.6

48.6

$1.4 \mathrm{~N}$

$68.2 \mathrm{~N} \quad 247.0 \mathrm{E}$

$68.2 \mathrm{~S} \quad 131.3 \mathrm{~W}$

63.5S 103.5W

$40.3 \mathrm{~N} \quad 13.5 \mathrm{E}$

47.6S 234.6W

$9.7 \mathrm{~S} \quad 222.8 \mathrm{~W}$

$\begin{array}{lll}0 & \text { UR } & \text { RU } \\ 0 & \text { AS } & \text { IN }\end{array}$

67
34

NA ES

AS AR

102 AS AR

148

2077

EU LA

EU SP

US NY

114 AS IQ$$
52
$$

EU

GE

51988

1979
1982

$5 \quad 1985$

$5 \quad 1976$

1985

51973

$5 \quad 1973$

51976

51979

51935

66

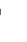




\begin{tabular}{|c|c|c|c|c|c|c|c|c|c|}
\hline $\mathbf{P}$ & $\mathbf{S a}$ & Name & lat & long & diam & ct & et & as & ad \\
\hline L & $\mathrm{L}$ & Alfraganus & $5.4 \mathrm{~S}$ & $19.0 \mathrm{E}$ & 20 & AS & PE & 5 & 1935 \\
\hline $\begin{array}{l}\mathrm{M} \\
\mathrm{H} \\
\mathrm{L}\end{array}$ & $\begin{array}{l}\mathrm{M} \\
\mathrm{H} \\
\mathrm{L}\end{array}$ & $\begin{array}{l}\text { Alga } \\
\text { Al-Hamadhani } \\
\text { Alhazen }\end{array}$ & $\begin{array}{l}24.6 \mathrm{~S} \\
38.8 \mathrm{~N} \\
15.9 \mathrm{~N}\end{array}$ & $\begin{array}{l}26.5 \mathrm{~W} \\
89.7 \mathrm{~W} \\
71.8 \mathrm{E}\end{array}$ & $\begin{array}{r}19 \\
186 \\
32\end{array}$ & $\begin{array}{l}\text { UR } \\
\text { AS } \\
\text { AS }\end{array}$ & $\begin{array}{r}\mathrm{KZ} \\
\mathrm{AR} \\
\mathrm{IQ}\end{array}$ & $\begin{array}{l}5 \\
5 \\
5\end{array}$ & $\begin{array}{l}1976 \\
1979 \\
1935\end{array}$ \\
\hline $\begin{array}{l}\mathrm{J} \\
\mathrm{S}\end{array}$ & $\begin{array}{l}\text { ca } \\
\text { en }\end{array}$ & $\begin{array}{l}\text { Āli } \\
\text { Ali Baba }\end{array}$ & $\begin{array}{l}59.3 \mathrm{~N} \\
57.2 \mathrm{~N}\end{array}$ & $\begin{array}{l}56.2 \mathrm{~W} \\
12.0 \mathrm{~W}\end{array}$ & $\begin{array}{l}61 \\
35\end{array}$ & $\begin{array}{l}\text { EU } \\
\text { AS }\end{array}$ & $\begin{array}{l}\text { NS } \\
\text { AR }\end{array}$ & $\begin{array}{l}5 \\
5\end{array}$ & $\begin{array}{l}1979 \\
1982\end{array}$ \\
\hline $\mathbf{L}$ & $\mathrm{L}$ & Aliacensis & $30.6 \mathrm{~S}$ & $5.2 \mathrm{E}$ & 79 & $\mathrm{EU}$ & FR & 5 & 1935 \\
\hline $\begin{array}{l}V \\
V \\
V \\
M \\
H \\
L\end{array}$ & $\begin{array}{l}\text { V } \\
V \\
\text { V } \\
M \\
H \\
\text { L }\end{array}$ & $\begin{array}{l}\text { Alima } \\
\text { Alimat } \\
\text { Alison } \\
\text { Alitus } \\
\text { Al-Jāhiz } \\
\text { Al-Khwarizmi }\end{array}$ & $\begin{array}{r}45.9 \mathrm{~S} \\
29.6 \mathrm{~S} \\
4.0 \mathrm{~S} \\
34.5 \mathrm{~S} \\
1.2 \mathrm{~N} \\
7.1 \mathrm{~N}\end{array}$ & $\begin{array}{c}229.2 \mathrm{E} \\
205.9 \mathrm{E} \\
165.6 \mathrm{E} \\
49.0 \mathrm{~W} \\
21.5 \mathrm{~W} \\
106.4 \mathrm{E}\end{array}$ & $\begin{array}{l}13 \\
14 \\
16 \\
29 \\
91 \\
65\end{array}$ & $\begin{array}{l}\text { UR } \\
\text { UR } \\
\text { EU } \\
\text { UR } \\
\text { AS } \\
\text { AS }\end{array}$ & $\begin{array}{c}\text { RU } \\
\text { GE } \\
\text { IR } \\
\text { EU } \\
\text { AR } \\
\text { IQ }\end{array}$ & $\begin{array}{l}5 \\
5 \\
5 \\
5 \\
5 \\
5\end{array}$ & $\begin{array}{l}1994 \\
1994 \\
1994 \\
1979 \\
1976 \\
1973\end{array}$ \\
\hline $\begin{array}{l}\text { V } \\
\text { V } \\
\text { V } \\
\text { L }\end{array}$ & $\begin{array}{l}\text { V } \\
\text { V } \\
\text { V } \\
\text { L }\end{array}$ & $\begin{array}{l}\text { Allat Dorsa } \\
\text { Allatu Corona } \\
\text { Alma } \\
\text { Almanon }\end{array}$ & $\begin{array}{r}63.3 \mathrm{~N} \\
15.5 \mathrm{~N} \\
2.4 \mathrm{~S} \\
16.8 \mathrm{~S}\end{array}$ & $\begin{array}{r}71.3 \mathrm{E} \\
114.0 \mathrm{E} \\
228.7 \mathrm{E} \\
15.2 \mathrm{E}\end{array}$ & $\begin{array}{r}302 \\
125 \\
17 \\
49\end{array}$ & $\begin{array}{l}\text { AS } \\
\text { AS } \\
\text { UR } \\
\text { AS }\end{array}$ & $\begin{array}{l}\text { AR } \\
\text { AK } \\
\text { KZ } \\
\text { PE }\end{array}$ & $\begin{array}{l}5 \\
5 \\
5 \\
5\end{array}$ & $\begin{array}{l}1985 \\
1994 \\
1994 \\
1935\end{array}$ \\
\hline L & L & Al-Marrakushi & $10.4 S$ & $55.8 \mathrm{E}$ & 8 & $\mathrm{AF}$ & MR & 5 & 1976 \\
\hline $\begin{array}{l}\mathrm{V} \\
\mathrm{S}\end{array}$ & $\begin{array}{l}\text { V } \\
\text { ia }\end{array}$ & $\begin{array}{l}\text { Almeida } \\
\text { Almeric }\end{array}$ & $\begin{array}{l}46.7 \mathrm{~N} \\
53.4 \mathrm{~N}\end{array}$ & $\begin{array}{l}123.2 \mathrm{E} \\
276.6 \mathrm{~W}\end{array}$ & $\begin{array}{l}14 \\
43\end{array}$ & $\begin{array}{l}\mathrm{EU} \\
\mathrm{EU}\end{array}$ & $\begin{array}{l}\text { PG } \\
\text { FR }\end{array}$ & $\begin{array}{l}5 \\
5\end{array}$ & $\begin{array}{l}1985 \\
1982\end{array}$ \\
\hline $\begin{array}{l}\mathrm{L} \\
\mathrm{U}\end{array}$ & $\begin{array}{l}\mathrm{L} \\
\mathrm{mr} \\
\mathrm{L}\end{array}$ & $\begin{array}{l}\text { Aloha } \\
\text { Alonso } \\
\text { Alpetragius }\end{array}$ & $\begin{array}{l}29.8 \mathrm{~N} \\
44.0 \mathrm{~S} \\
16.0 \mathrm{~S}\end{array}$ & $\begin{array}{c}53.9 \mathrm{~W} \\
352.6 \mathrm{E} \\
4.5 \mathrm{~W}\end{array}$ & $\begin{array}{r}3 \\
25 \\
39\end{array}$ & $\begin{array}{l}\text { OC } \\
\text { EU } \\
\text { AF }\end{array}$ & $\begin{array}{l}\text { HA } \\
\text { GB } \\
\text { MR }\end{array}$ & $\begin{array}{l}5 \\
5 \\
5\end{array}$ & $\begin{array}{l}1976 \\
1988 \\
1935\end{array}$ \\
\hline $\begin{array}{l}\text { V } \\
\mathbf{J} \\
\mathrm{M} \\
\mathrm{L}\end{array}$ & $\begin{array}{l}\mathrm{V} \\
\mathrm{eu} \\
\mathrm{M} \\
\mathrm{L}\end{array}$ & $\begin{array}{l}\text { Alpha Regio } \\
\text { Alphesiboea Linea } \\
\text { Alpheus Colles } \\
\text { Alphonsus }\end{array}$ & $\begin{array}{l}25.5 S \\
28.0 S \\
38.5 S \\
13.7 S\end{array}$ & $\begin{array}{c}0.3 \mathrm{E} \\
182.6 \mathrm{~W} \\
300.6 \mathrm{~W} \\
3.2 \mathrm{~W}\end{array}$ & $\begin{array}{r}1897 \\
1642 \\
514 \\
108\end{array}$ & $\begin{array}{l}\text { EU } \\
\text { EU } \\
\text { EU } \\
\text { EU }\end{array}$ & $\begin{array}{r}\text { GR } \\
\text { GR } \\
\text { GR } \\
\text { SP }\end{array}$ & $\begin{array}{l}5 \\
5 \\
5 \\
5\end{array}$ & $\begin{array}{l}1979 \\
1985 \\
1985 \\
1935\end{array}$ \\
\hline $\mathbf{M}$ & $\mathbf{M}$ & Al-Qahira Vallis & $17.5 \mathrm{~S}$ & $196.7 \mathrm{~W}$ & 546 & AS & ID & 5 & 1973 \\
\hline V & $\mathrm{V}$ & Al-Taymuriyya & $32.9 \mathrm{~N}$ & $336.2 \mathrm{E}$ & 22 & $\mathrm{AF}$ & EG & 5 & 1991 \\
\hline L & L & Alter & $18.7 \mathrm{~N}$ & $107.5 \mathrm{~W}$ & 64 & NA & $\mathrm{AM}$ & 5 & 1970 \\
\hline $\begin{array}{l}A \\
\text { V } \\
\text { V }\end{array}$ & $\begin{array}{l}\text { gs } \\
\mathrm{V} \\
\mathrm{V}\end{array}$ & $\begin{array}{l}\text { Alupka } \\
\text { Al-Uzza Undae } \\
\text { Amalasthuna }\end{array}$ & $\begin{array}{l}65.0 \mathrm{~N} \\
67.7 \mathrm{~N} \\
11.5 \mathrm{~S}\end{array}$ & $\begin{array}{c}65.0 \mathrm{~W} \\
90.5 \mathrm{E} \\
342.4 \mathrm{E}\end{array}$ & $\begin{array}{r}0 \\
150 \\
16\end{array}$ & $\begin{array}{l}\text { UR } \\
\text { AS } \\
\text { UR }\end{array}$ & $\begin{array}{l}\text { UK } \\
\text { AR } \\
\text { RU }\end{array}$ & $\begin{array}{l}5 \\
5 \\
5\end{array}$ & $\begin{array}{l}1994 \\
1994 \\
1991\end{array}$ \\
\hline $\begin{array}{l}N \\
S\end{array}$ & $\begin{array}{l}\text { tr } \\
\mathrm{di}\end{array}$ & $\begin{array}{l}\text { Amarum } \\
\text { Amata }\end{array}$ & $\begin{array}{r}26.0 \mathrm{~N} \\
7.7 \mathrm{~N}\end{array}$ & $\begin{array}{c}24.5 \mathrm{E} \\
285.3 \mathrm{~W}\end{array}$ & $\begin{array}{r}0 \\
231\end{array}$ & $\begin{array}{l}\text { SA } \\
\text { EU }\end{array}$ & $\begin{array}{r}\mathrm{EC} \\
\mathrm{RM}\end{array}$ & $\begin{array}{l}5 \\
5\end{array}$ & $\begin{array}{r}91 \\
1982\end{array}$ \\
\hline J & io & $\begin{array}{l}\text { Amaterasu Patera } \\
\text { Amaya }\end{array}$ & $\begin{array}{l}37.7 \mathrm{~N} \\
11.3 \mathrm{~N}\end{array}$ & $\begin{array}{c}306.6 \mathrm{~W} \\
89.3 \mathrm{E}\end{array}$ & $\begin{array}{r}100 \\
33\end{array}$ & $\begin{array}{l}\text { AS } \\
\text { EU }\end{array}$ & $\begin{array}{l}\text { JA } \\
\text { SP }\end{array}$ & $\begin{array}{l}5 \\
5\end{array}$ & $\begin{array}{l}1979 \\
1991\end{array}$ \\
\hline M & M & Amazonis & $0.0 \mathrm{~N}$ & $140.0 \mathrm{~W}$ & 0 & $\mathrm{EU}$ & GR & 5 & 1958 \\
\hline $\mathbf{M}$ & $\mathbf{M}$ & Amazonis Planitia & $16.0 \mathrm{~N}$ & $158.4 \mathrm{~W}$ & 2816 & EU & GR & 5 & 1973 \\
\hline M & $\mathbf{M}$ & Amazonis Sulci & $3.3 \mathrm{~S}$ & $145.1 \mathrm{~W}$ & 243 & EU & GR & 5 & 1985 \\
\hline $\mathrm{L}$ & $\mathrm{L}$ & Ameghino & $3.3 \mathrm{~N}$ & $57.0 \mathrm{E}$ & 9 & EU & IT & 5 & 1976 \\
\hline $\begin{array}{l}\mathrm{V} \\
\mathrm{V} \\
\mathrm{M}\end{array}$ & $\begin{array}{l}\mathrm{V} \\
\mathrm{V} \\
\mathrm{M}\end{array}$ & $\begin{array}{l}\text { Amenardes } \\
\text { Ament Corona } \\
\text { Amenthes }\end{array}$ & $\begin{array}{c}15.0 \mathrm{~N} \\
67.2 \mathrm{~S} \\
5.0 \mathrm{~N}\end{array}$ & $\begin{array}{l}54.1 \mathrm{E} \\
217.9 \mathrm{E} \\
250.0 \mathrm{~W}\end{array}$ & $\begin{array}{r}25 \\
115 \\
0\end{array}$ & $\begin{array}{l}\text { AF } \\
\text { AF } \\
\text { AF }\end{array}$ & $\begin{array}{l}\text { EG } \\
\text { EG } \\
\text { EG }\end{array}$ & $\begin{array}{l}5 \\
5 \\
5\end{array}$ & $\begin{array}{l}1991 \\
1994 \\
1958\end{array}$ \\
\hline $\begin{array}{l}\mathrm{M} \\
\mathrm{M} \\
\mathrm{M} \\
\mathrm{S}\end{array}$ & $\begin{array}{l}\mathrm{M} \\
\mathrm{M} \\
\mathrm{M} \\
\mathrm{rh}\end{array}$ & $\begin{array}{l}\text { Amenthes Fossae } \\
\text { Amenthes Rupes } \\
\text { Amet } \\
\text { Ameta }\end{array}$ & $\begin{array}{r}10.2 \mathrm{~N} \\
1.8 \mathrm{~N} \\
23.7 \mathrm{~N} \\
53.3 \mathrm{~N}\end{array}$ & $\begin{array}{r}259.3 \mathrm{~W} \\
249.4 \mathrm{~W} \\
57.4 \mathrm{~W} \\
21.9 \mathrm{~W}\end{array}$ & $\begin{array}{r}1592 \\
441 \\
0 \\
0\end{array}$ & $\begin{array}{l}\text { AF } \\
\text { AF } \\
\text { AS } \\
\text { AS }\end{array}$ & $\begin{array}{l}\text { EG } \\
\text { EG } \\
\text { IN } \\
\text { ID }\end{array}$ & $\begin{array}{l}5 \\
5 \\
5 \\
5\end{array}$ & $\begin{array}{l}1976 \\
1982 \\
1988 \\
1982\end{array}$ \\
\hline
\end{tabular}

ft origin

AA Al Fargani, Muhammed Ebn Ketir; Persian astronomer (unkn-c. 840).

AA Town in Kazakhstan.

AA Arab writer (d. 1007).

AA Abu Ali Al-Hasan Ibn Al Haitham; Iraqi mathematician (987-1038).

AA Norse; strongest of men.

AA Hero of tale who found a great treasure owned by 40 thieves.

AA D'Ailly, Pierre; French geographer (1350-1420)

AA Tatar first name.

AA Osset first name.

AA Irish first name.

AA Town in Lithuania.

AA Arab author (d. 869).

AA Iraqi mathematician (unkn-c. 825)

DO Arab sky goddess.

CR Akkadian earth goddess.

AA Kazakh first name.

AA Abdalla Al Mamun; Persian astronomer (786-833).

AA Moroccan astronomer, mathematician (fl. c. 1262).

AA Portuguese first name.

AA One of 12 peers, killed by Marsilion.

AA Hawaiian greeting.

AA King of Naples in "The Tempest."

AA Nur Ed-Din Al Betrugi; Moroccan astronomer (unkn-c. 1100).

RE First letter in Greek alphabet.

LI Son of Phoenix, nephew of Europa.

CO From albedo feature at 45S, $292 \mathrm{~W}$.

AA Alfonso X; Spanish astronomer (1223-1284)

VA Word for 'Mars' in Arabic Indonesian, Malay.

AA Ayesha; Egyptian author, feminist (1840-1902).

AA Dinsmore; American astronomer, meteorologist (1888-1968).

AA Spa in Crimea, Ukraine.

UN Arabian desert goddess.

AA Ostrogoth queen (c. 498-535 A.D.)

AA Quecha (Ecuador) water boa.

AA Mother of Lavinia (wife of Aeneas).

PE Japanese sun goddess.

AA Carmen; Spanish Gypsy dancer (1913-1963).

AL Land of the Amazons; on the island Hesperia.

PL Classical albedo feature name; home of the Amazons.

SU Albedo feature name; home of the Amazons.

AA Fiorino (or Florentino); Italian natural historian (c. 1854-1911)

AA Egyptian princess (718-655 B.C.).

CR Egyptian earth goddess.

AL Egyptian name for place where souls of the dead go.

FO Classical albedo feature name.

RU Classical albedo feature name.

AA Town in India.

AA Ceram (Indonesia) ancestor whose blood made Hainuwele.

\footnotetext{
P: $\quad$ planetary system (see page xvi).

Sa: $\quad$ satellite (see page xvi).

lat: latitude of feature center.

long: longitude of feature center.
}

diam: diameter or long dimension of feature.

ct: continent of name origin (see page $284 \mathrm{ff}$.)

et: ethnicity of name origin (see page $284 \mathrm{ff}$.)

as: name approval status (see page xvii). ad: name approval date (year).

ref: reference source for name (see page $287 \mathrm{ff}$.)

ft: feature type (see page 290). 
ALPHABETICAL LIST OF NAMES

\begin{tabular}{|c|c|c|c|c|c|c|c|c|c|}
\hline $\mathbf{P}$ & Sa & Name & lat & long & diam & ct & et & as & ad \\
\hline $\mathbf{L}$ & $\mathrm{L}$ & Amici & $9.9 \mathrm{~S}$ & $172.1 \mathrm{~W}$ & 54 & EU & IT & 5 & 1970 \\
\hline I & $\begin{array}{l}\text { io } \\
\mathrm{M}\end{array}$ & $\begin{array}{l}\text { Amirani } \\
\text { [Ammonii Rupes] }\end{array}$ & $\begin{array}{r}25.9 \mathrm{~N} \\
6.6 \mathrm{~S}\end{array}$ & $\begin{array}{l}114.5 \mathrm{~W} \\
184.2 \mathrm{~W}\end{array}$ & $\begin{array}{r}0 \\
100\end{array}$ & $\begin{array}{l}\text { UR } \\
\text { EU }\end{array}$ & $\begin{array}{l}\text { GE } \\
\text { LA }\end{array}$ & $\begin{array}{l}5 \\
6\end{array}$ & $\begin{array}{l}1979 \\
1985\end{array}$ \\
\hline L & $\begin{array}{l}\text { L } \\
\text { ga } \\
\text { ga } \\
\text { L }\end{array}$ & $\begin{array}{l}\text { Ammonius } \\
\text { Ammura } \\
\text { Amon } \\
\text { Amontons }\end{array}$ & $\begin{array}{r}8.5 \mathrm{~S} \\
30.9 \mathrm{~N} \\
33.4 \mathrm{~N} \\
5.3 \mathrm{~S}\end{array}$ & $\begin{array}{c}0.8 \mathrm{~W} \\
344.2 \mathrm{~W} \\
223.3 \mathrm{~W} \\
46.8 \mathrm{E}\end{array}$ & $\begin{array}{r}8 \\
61 \\
102 \\
2\end{array}$ & $\begin{array}{l}\text { EU } \\
\text { AS } \\
\text { AS } \\
\text { EU }\end{array}$ & $\begin{array}{l}\text { GR } \\
\text { PO } \\
\text { AB } \\
\text { FR }\end{array}$ & $\begin{array}{l}5 \\
5 \\
5 \\
5\end{array}$ & $\begin{array}{l}1976 \\
1979 \\
1985 \\
1976\end{array}$ \\
\hline $\mathbf{M}$ & $\mathbf{M}$ & Amphitrites Patera & $59.1 \mathrm{~S}$ & $299.0 \mathrm{~W}$ & 138 & $\mathrm{EU}$ & GR & 5 & 1973 \\
\hline $\begin{array}{l}\mathrm{H} \\
\mathbf{M}\end{array}$ & $\begin{array}{l}\mathrm{H} \\
\mathbf{M} \\
\mathrm{L}\end{array}$ & $\begin{array}{l}\text { Amru Al-Qays } \\
\text { Amsterdam } \\
\text { Amundsen }\end{array}$ & $\begin{array}{l}12.3 \mathrm{~N} \\
23.3 \mathrm{~N} \\
84.3 \mathrm{~S}\end{array}$ & $\begin{array}{c}175.6 \mathrm{~W} \\
47.0 \mathrm{~W} \\
85.6 \mathrm{E}\end{array}$ & $\begin{array}{r}50 \\
2 \\
101\end{array}$ & $\begin{array}{l}\text { AS } \\
\text { EU } \\
\text { EU }\end{array}$ & $\begin{array}{l}\text { AR } \\
\text { DU } \\
\text { NO }\end{array}$ & $\begin{array}{l}5 \\
5 \\
5\end{array}$ & $\begin{array}{l}1976 \\
1979 \\
1964\end{array}$ \\
\hline $\begin{array}{l}V \\
V \\
V \\
J \\
J\end{array}$ & $\begin{array}{l}V \\
V \\
V \\
\text { ca } \\
\text { ga }\end{array}$ & $\begin{array}{l}\text { Anahit Corona } \\
\text { Anala Corona } \\
\text { Ananke Tessera } \\
\overline{\text { Änarr }} \\
\text { Anat }\end{array}$ & $\begin{array}{r}77.1 \mathrm{~N} \\
11.0 \mathrm{~N} \\
53.3 \mathrm{~N} \\
44.1 \mathrm{~N} \\
3.1 \mathrm{~S}\end{array}$ & $\begin{array}{r}277.3 \mathrm{E} \\
14.0 \mathrm{E} \\
133.3 \mathrm{E} \\
0.6 \mathrm{~W} \\
127.9 \mathrm{~W}\end{array}$ & $\begin{array}{r}324 \\
240 \\
1060 \\
47 \\
28\end{array}$ & $\begin{array}{l}\text { AS } \\
\text { AS } \\
\text { EU } \\
\text { EU } \\
\text { AS }\end{array}$ & $\begin{array}{r}\mathrm{AM} \\
\mathrm{IN} \\
\mathrm{GR} \\
\mathrm{NS} \\
\mathrm{AB}\end{array}$ & $\begin{array}{l}5 \\
5 \\
5 \\
5 \\
5\end{array}$ & $\begin{array}{l}1985 \\
1994 \\
1985 \\
1979 \\
1985\end{array}$ \\
\hline $\begin{array}{l}\text { L } \\
\text { V } \\
\text { L }\end{array}$ & $\begin{array}{l}\text { L } \\
\text { V } \\
\text { L }\end{array}$ & $\begin{array}{l}\text { Anaxagoras } \\
\text { Anaxandra } \\
\text { Anaximander }\end{array}$ & $\begin{array}{l}73.4 \mathrm{~N} \\
44.2 \mathrm{~N} \\
66.9 \mathrm{~N}\end{array}$ & $\begin{array}{c}10.1 \mathrm{~W} \\
162.1 \mathrm{E} \\
51.3 \mathrm{~W}\end{array}$ & $\begin{array}{l}50 \\
21 \\
67\end{array}$ & $\begin{array}{l}\text { EU } \\
\text { EU } \\
\text { EU }\end{array}$ & $\begin{array}{l}\text { GR } \\
\text { GR } \\
\text { GR }\end{array}$ & $\begin{array}{l}5 \\
5 \\
5\end{array}$ & $\begin{array}{l}1935 \\
1994 \\
1935\end{array}$ \\
\hline $\begin{array}{l}\text { L } \\
S \\
H \\
\text { V } \\
\text { L }\end{array}$ & $\begin{array}{l}\text { L } \\
\text { di } \\
\text { H } \\
\text { V } \\
\text { L }\end{array}$ & $\begin{array}{l}\text { Anaximenes } \\
\text { Anchises } \\
\text { Andal } \\
\text { Andami } \\
\text { Anděl }\end{array}$ & $\begin{array}{l}72.5 \mathrm{~N} \\
33.7 \mathrm{~S} \\
47.7 \mathrm{~S} \\
17.5 \mathrm{~S} \\
10.4 \mathrm{~S}\end{array}$ & $\begin{array}{l}44.5 \mathrm{~W} \\
66.1 \mathrm{~W} \\
37.7 \mathrm{~W} \\
26.5 \mathrm{E} \\
12.4 \mathrm{E}\end{array}$ & $\begin{array}{r}80 \\
42 \\
108 \\
30 \\
35\end{array}$ & $\begin{array}{l}\text { EU } \\
\text { EU } \\
\text { UN } \\
\text { AS } \\
\text { EU }\end{array}$ & $\begin{array}{l}\text { GR } \\
\text { RM } \\
\text { UN } \\
\text { IR } \\
\text { CZ }\end{array}$ & $\begin{array}{l}5 \\
5 \\
5 \\
5 \\
5\end{array}$ & $\begin{array}{l}1935 \\
1982 \\
1976 \\
1991 \\
1935\end{array}$ \\
\hline L & $L$ & Anders & $41.3 \mathrm{~S}$ & $142.9 \mathrm{~W}$ & 40 & NA & AM & 5 & 1970 \\
\hline & L & Anderson & $15.8 \mathrm{~N}$ & 171.1E & 109 & NA & $\mathrm{AM}$ & 5 & 1970 \\
\hline $\mathbf{L}$ & L & Andersson & $49.7 \mathrm{~S}$ & $95.3 \mathrm{~W}$ & 13 & NA & $\mathrm{AM}$ & 5 & 1985 \\
\hline J & $\stackrel{g a}{V}$ & $\begin{array}{l}\text { Andjeti } \\
\text { Andreianova }\end{array}$ & $\begin{array}{r}52.4 \mathrm{~S} \\
3.0 \mathrm{~S}\end{array}$ & $\begin{array}{c}159.1 \mathrm{~W} \\
68.7 \mathrm{E}\end{array}$ & $\begin{array}{l}65 \\
70\end{array}$ & $\begin{array}{l}\text { AF } \\
\text { UR }\end{array}$ & $\begin{array}{l}\text { EG } \\
\text { RU }\end{array}$ & $\begin{array}{l}5 \\
5\end{array}$ & $\begin{array}{l}1985 \\
1994\end{array}$ \\
\hline $\mathrm{L}$ & $\mathrm{L}$ & Andronov & $22.7 \mathrm{~S}$ & $146.1 \mathrm{E}$ & 16 & UR & So & 5 & 1976 \\
\hline $\begin{array}{l}\mathbf{N} \\
\mathbf{L} \\
\text { J } \\
\text { L }\end{array}$ & $\begin{array}{l}\text { tr } \\
\mathrm{L} \\
\text { io } \\
\mathrm{L}\end{array}$ & $\begin{array}{l}\text { Andvari } \\
\text { Ango } \\
\text { Angpetu Patera } \\
\text { Angström }\end{array}$ & $\begin{array}{l}20.5 \mathrm{~N} \\
20.5 \mathrm{~N} \\
21.2 \mathrm{~S} \\
29.9 \mathrm{~N}\end{array}$ & $\begin{array}{l}34.0 \mathrm{E} \\
32.3 \mathrm{~W} \\
10.6 \mathrm{~W} \\
41.6 \mathrm{~W}\end{array}$ & $\begin{array}{r}0 \\
1 \\
45 \\
9\end{array}$ & $\begin{array}{l}\text { EU } \\
\text { AF } \\
\text { NA } \\
\text { EU }\end{array}$ & $\begin{array}{l}\text { NS } \\
\text { UN } \\
\text { DA } \\
\text { SW }\end{array}$ & $\begin{array}{l}5 \\
5 \\
5 \\
5\end{array}$ & $\begin{array}{r}91 \\
1976 \\
1985 \\
1935\end{array}$ \\
\hline $\begin{array}{l}\mathrm{M} \\
\mathrm{M} \\
\mathrm{M} \\
\mathrm{V}\end{array}$ & $\begin{array}{l}\mathbf{M} \\
\mathbf{M} \\
\mathbf{M} \\
\mathrm{V}\end{array}$ & $\begin{array}{l}\text { Angu } \\
\text { Angusta Patera } \\
\text { Aniak } \\
\text { Anicia }\end{array}$ & $\begin{array}{l}20.3 \mathrm{~N} \\
80.7 \mathrm{~S} \\
32.1 \mathrm{~S} \\
26.3 \mathrm{~S}\end{array}$ & $\begin{array}{c}254.5 \mathrm{~W} \\
79.5 \mathrm{~W} \\
69.6 \mathrm{~W} \\
31.3 \mathrm{E}\end{array}$ & $\begin{array}{r}0 \\
0 \\
58 \\
38\end{array}$ & $\begin{array}{l}\text { AF } \\
\text { EU } \\
\text { US } \\
\text { EU }\end{array}$ & $\begin{array}{l}\text { ZA } \\
\text { GR } \\
\text { AK } \\
\text { GR }\end{array}$ & $\begin{array}{l}5 \\
5 \\
5 \\
5\end{array}$ & $\begin{array}{l}1988 \\
1991 \\
1979 \\
1991\end{array}$ \\
\hline $\begin{array}{l}\mathbf{J} \\
\mathbf{M}\end{array}$ & $\begin{array}{l}\text { ca } \\
\mathbf{M}\end{array}$ & $\begin{array}{l}\text { Aningan } \\
\text { Anio Vallis }\end{array}$ & $\begin{array}{l}50.5 \mathrm{~N} \\
38.1 \mathrm{~N}\end{array}$ & $\begin{array}{r}8.2 \mathrm{~W} \\
304.3 \mathrm{~W}\end{array}$ & $\begin{array}{r}287 \\
0\end{array}$ & $\begin{array}{l}\text { NP } \\
\text { EU }\end{array}$ & IT & $\begin{array}{l}5 \\
5\end{array}$ & $\begin{array}{l}1979 \\
1987\end{array}$ \\
\hline $\begin{array}{l}\mathbf{L} \\
\mathbf{M}\end{array}$ & $\begin{array}{l}\mathrm{L} \\
\mathrm{M}\end{array}$ & $\begin{array}{l}\text { Ann } \\
\text { Annapolis }\end{array}$ & $\begin{array}{l}25.1 \mathrm{~N} \\
23.4 \mathrm{~N}\end{array}$ & $\begin{array}{r}0.1 \mathrm{~W} \\
47.8 \mathrm{~W}\end{array}$ & $\begin{array}{l}3 \\
1\end{array}$ & $\begin{array}{l}\text { AS } \\
\text { US }\end{array}$ & $\begin{array}{l}\mathrm{HE} \\
\mathrm{MD}\end{array}$ & $\begin{array}{l}5 \\
5\end{array}$ & $\begin{array}{l}1976 \\
1979\end{array}$ \\
\hline $\begin{array}{l}\text { V } \\
\text { L } \\
\text { V }\end{array}$ & $\begin{array}{l}\text { V } \\
\text { L } \\
\text { V }\end{array}$ & $\begin{array}{l}\text { Annapurna Corona } \\
\text { Annegrit } \\
\text { Annia Faustina }\end{array}$ & $\begin{array}{l}35.5 \mathrm{~S} \\
29.4 \mathrm{~N} \\
22.1 \mathrm{~N}\end{array}$ & $\begin{array}{c}152.0 \mathrm{E} \\
25.6 \mathrm{~W} \\
4.3 \mathrm{E}\end{array}$ & $\begin{array}{r}300 \\
1 \\
23\end{array}$ & $\begin{array}{l}\mathrm{AS} \\
\mathrm{EU} \\
\mathrm{EU}\end{array}$ & $\begin{array}{r}\text { IN } \\
\text { GE } \\
\text { RM }\end{array}$ & $\begin{array}{l}5 \\
5 \\
5\end{array}$ & $\begin{array}{l}1994 \\
1976 \\
1991\end{array}$ \\
\hline V & V & Anning Paterae & $66.5 \mathrm{~N}$ & $57.8 \mathrm{E}$ & 0 & EU & GB & 5 & 91 \\
\hline $\begin{array}{l}\text { V } \\
\mathbf{M}\end{array}$ & $\begin{array}{l}\mathrm{V} \\
\mathrm{M}\end{array}$ & $\begin{array}{l}\text { Anqet Farrum } \\
\text { Anseris Cavus }\end{array}$ & $\begin{array}{l}33.6 \mathrm{~N} \\
30.0 \mathrm{~S}\end{array}$ & $\begin{array}{l}311.5 \mathrm{E} \\
264.5 \mathrm{~W}\end{array}$ & $\begin{array}{r}125 \\
35\end{array}$ & $\begin{array}{l}\mathrm{AF} \\
\mathrm{EU}\end{array}$ & $\begin{array}{l}\text { EG } \\
\text { LA }\end{array}$ & $\begin{array}{l}5 \\
5\end{array}$ & $\begin{array}{l}1994 \\
1994\end{array}$ \\
\hline M & $\begin{array}{l}\mathbf{M} \\
\mathrm{L}\end{array}$ & $\begin{array}{l}\text { Anseris Mons } \\
\text { Ansgarius }\end{array}$ & $\begin{array}{l}30.1 \mathrm{~S} \\
12.7 \mathrm{~S}\end{array}$ & $\begin{array}{c}263.2 \mathrm{~W} \\
79.7 \mathrm{E}\end{array}$ & $\begin{array}{r}0 \\
94\end{array}$ & $\begin{array}{l}\mathrm{EU} \\
\mathrm{EU}\end{array}$ & $\begin{array}{l}\text { LA } \\
\text { GE }\end{array}$ & $\begin{array}{l}5 \\
5\end{array}$ & $\begin{array}{l}1989 \\
1935\end{array}$ \\
\hline
\end{tabular}

$\mathrm{ft}$ origin

AA Giovanni B.; Italian astronomer, botanist (1786-1863).

ER Georgian god of fire.

RU Named for albedo feature at $6 \mathrm{~S}, 183 \mathrm{~W}$.

AA Greek philosopher (unkn-c. 517)

AA Phoenician; god of the west.

AA Theban king of gods.

AA Guillaume; French physicist (1663-1705)

PE Mare Amphitrites; classical albedo feature name.

AA Arab poet (pre-Islamic).

AA Dutch port.

AA Roald E.; Norwegian explorer (1872-1928).

CR Armenian goddess of fertility.

CR Hindu fertility goddess.

TE Greek goddess of necessity.

AA Norse dwarf.

AA Assyro-Babylonian goddess of dew; Rand control-point crater.

AA Greek astronomer (500-428 B.C.)

AA Greek artist (fl. c. 228 B.C.).

AA Greek astronomer (c. 611-547 B.C.).

AA Greek astronomer (585-528 B.C.).

AA Aeneas' father.

AA (18th century).

AA Iranian doctor.

AA Karel; Czechoslovakian astronomer (1884-1947)

AA William A.; American astronaut (1933-Live)

AA John A.; American astronaut (1876-1959).

AA Leif Erland; American astronomer (1943-1979)

AA Egyptian; first god of Busirus.

AA Elena; Russian ballerina (c. 1821-c. 1855).

AA Aleksandr Aleksandrovich; Soviet physicist (1901-1952).

AA Norse fish shaped dwarf.

AA African male name.

PE Dakota name meaning the sun.

AA Anders Jonas; Swedish physicist (1814-1874).

AA Town in Zaire.

PE Classical albedo name.

AA Town in Alaska, USA.

AA Greek physician, poet (fl. c.300 B.C.).

AA Moon god of Greenland Eskimos.

VA Classical river in Italy; modern Aniene and Teverone rivers.

AA Hebrew female name.

AA American colonial town (Maryland).

CR Indian goddess of wealth.

AA German female name.

AA Roman empress, wife of Marcus Aurelius (125-175 A.D.).

PE Mary; English paleontologist (1799-1847).

FR Egyptian goddess of fertile waters.

CB Named for albedo feature Anseris Fons.

MO From albedo feature Anseris Fons.

AA St. Ansgar; German theologian (801-864).

\footnotetext{
P: $\quad$ planetary system (see page xvi).

Sa: satellite (see page $x v i$ ).

lat: latitude of feature center.

long: longitude of feature center.
}

diam: diameter or long dimension of feature.

ct: continent of name origin (see page $284 \mathrm{ff}$.)

et: ethnicity of name origin (see page $284 \mathrm{ff}$.)

as: name approval status (see page xvii). ad: name approval date (year).

ref: reference source for name (see page $287 \mathrm{ff}$.). ft: feature type (see page 290) 


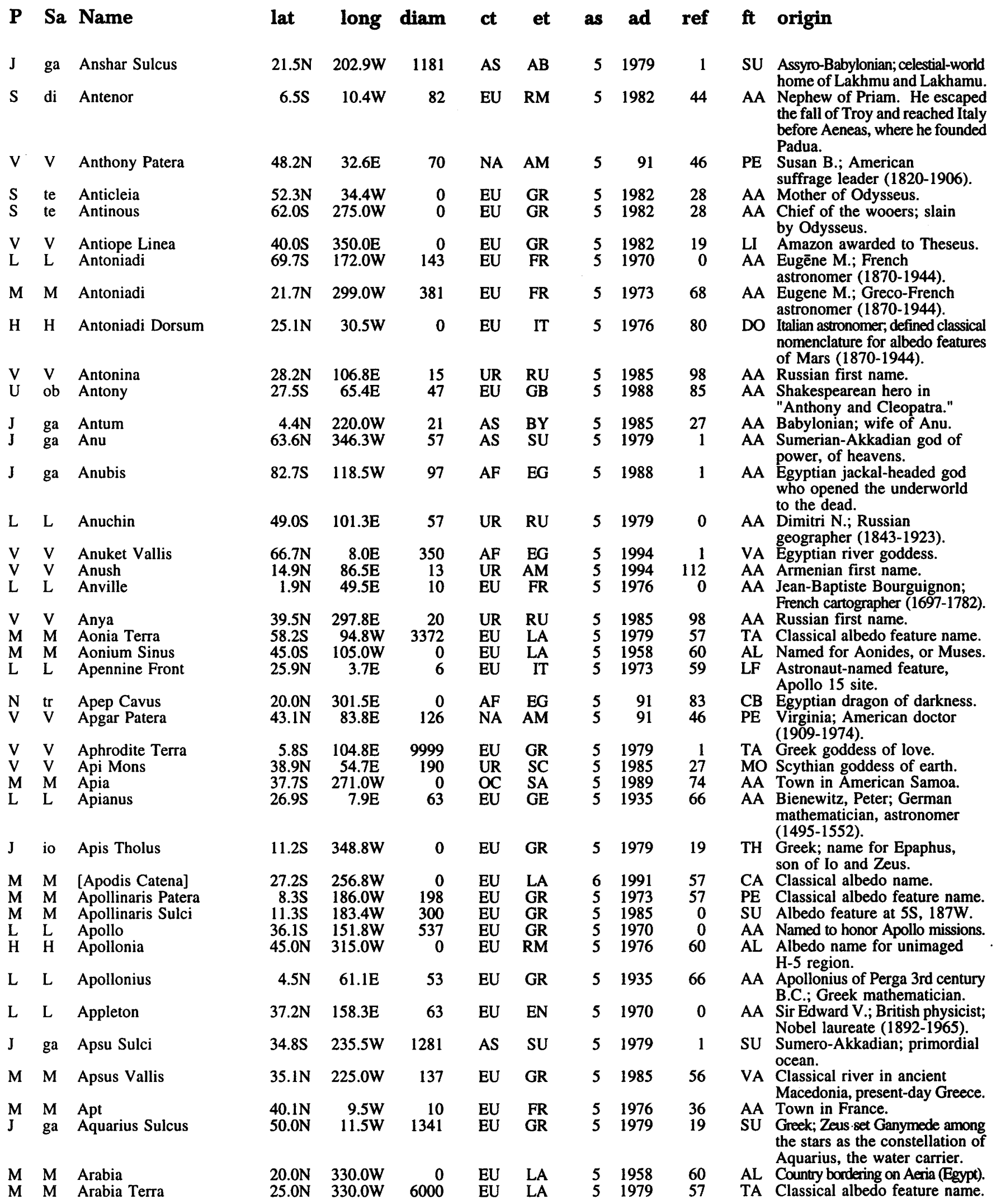

\footnotetext{
P: $\quad$ planetary system (see page $x v i)$.

Sa: satellite (see page $x v i)$.

lat: latitude of feature center.

long: longitude of feature center.
}

diam: diameter or long dimension of feature.

ct: continent of name origin (see page $284 \mathrm{ff}$.)

et: ethnicity of name origin (see page $284 \mathrm{ff}$.)

as: name approval status (see page $x$ vii). ad: name approval date (year).

ref: reference source for name (see page $287 \mathrm{ff}$.).

ft: feature type (see page 290). 


\section{ALPHABETICAL LIST OF NAMES}

\section{P Sa Name}

L L Arago

M M Arago

M M Aram Chaos

V V Aramaiti Corona

J io Aramazd Patera

M M Arandas

V V Aranyani Chasma

L L Aratus

J ga Arbela Sulcus

M M Arcadia

M M Arcadia Planitia

L L Archimedes

L L Archytas

M M Arda Valles

U mr Arden Corona

$\mathrm{H} \quad \mathrm{H}$ Arecibo Vallis

M M Arena Colles

M M Arena Dorsum

M M [Arena Rupes]

M M Ares Vallis

$S$ te Arete

M M Argas

L L Argelander

U mr Argier Rupes

J eu Argiope Linea

$J$ io Argos Planum

M M Argyre

M M Argyre Cavi

M M Argyre Planitia

M M Argyre Rupes

L L Ariadaeus

V V Ariadne

M M Ariadnes Colles

V V Arianrod Fossae

M M Arica

M M Arimanes Rupes

L L [Ariosto]

L L Aristarchus

L L Aristillus

L L Aristoteles

$\mathrm{H} \quad \mathrm{H}$ Aristoxenus

M M Arkhangelsky

L L Armínski

L L Armstrong lat long diar

diam

ct et

as ad

ref

$\begin{array}{rrrlrrrr}6.2 \mathrm{~N} & 21.4 \mathrm{E} & 26 & \text { EU } & \text { FR } & 5 & 1935 & 66 \\ & & & & & & & \\ 10.5 \mathrm{~N} & 330.2 \mathrm{~W} & 154 & \text { EU } & \text { FR } & 5 & 1973 & 68 \\ & & & & & & & \\ 2.7 \mathrm{~N} & 21.0 \mathrm{~W} & 388 & \text { EU } & \text { GR } & 5 & 1976 & 57 \\ 26.3 \mathrm{~S} & 82.0 \mathrm{E} & 350 & \text { AS } & \text { PE } & 5 & 1994 & 1 \\ 73.4 \mathrm{~S} & 337.7 \mathrm{~W} & 38 & \text { AS } & \text { AM } & 5 & 1985 & 27 \\ 42.6 \mathrm{~N} & 15.1 \mathrm{~W} & 22 & \text { NA } & \text { ME } & 5 & 1976 & 36 \\ 69.3 \mathrm{~N} & 74.4 \mathrm{E} & 718 & \text { AS } & \text { IN } & 5 & 1985 & 65 \\ 23.6 \mathrm{~N} & 4.5 \mathrm{E} & 10 & \text { EU } & \text { GR } & 5 & 1935 & 66\end{array}$

$22.3 \mathrm{~S} \quad 353.6 \mathrm{~W} \quad 1896 \quad$ AS $\mathrm{AB}$

51985

$\begin{array}{lllll}45.0 \mathrm{~N} & 100.0 \mathrm{~W} & 0 & \mathrm{EU} & \mathrm{GR}\end{array}$

$5 \quad 1958$

$46.4 \mathrm{~N}$

152.1W 3052

EU GR

51973

$29.7 \mathrm{~N}$

$58.7 \mathrm{~N}$

$4.0 \mathrm{~W}$

82

EU

GR

51935

$21.0 \mathrm{~S}$

$5.0 \mathrm{E}$

31

EU

GR

51935

$29.1 \mathrm{~S}$

$32.4 \mathrm{~W}$

172

EU

BU

51976

$5 \quad 1988$

$27.5 \mathrm{~S}$

73.7E

318

EU

$24.3 \mathrm{~N} \quad 278.7 \mathrm{~W}$

$13.0 \mathrm{~N} \quad 291.5 \mathrm{~W}$

$13.5 \mathrm{~N} \quad 289.5 \mathrm{~W}$

$9.7 \mathrm{~N} \quad 23.4 \mathrm{~W}$

4.6S $299.7 \mathrm{~W}$

23.5

$16.5 \mathrm{~S}$

43.2S $322.8 \mathrm{E}$

$50.1 \mathrm{~W}$

$8.2 \mathrm{~S}$

$202.2 \mathrm{~W}$

47.0S $318.2 \mathrm{~W}$

$45.0 \mathrm{~S}$

$25.0 \mathrm{~W}$

$49.1 \mathrm{~S}$

49.4S

$63.3 \mathrm{~S}$

$4.6 \mathrm{~N}$

$40.2 \mathrm{~W}$

$42.8 \mathrm{~W}$

$66.7 \mathrm{~W}$

17.3E

$43.9 \mathrm{~N}$

$0.0 \mathrm{E}$

34.8S 188.0W

$37.0 \mathrm{~N} \quad 239.9 \mathrm{E}$

24.0S $249.7 \mathrm{~W}$

$10.0 \mathrm{~S}$

$147.5 \mathrm{~W}$

$3.6 \mathrm{~S}$

95.6E

$23.7 \mathrm{~N}$

$33.9 \mathrm{~N}$

$50.2 \mathrm{~N}$

47.4W

$1.2 \mathrm{E}$

$17.4 \mathrm{E}$

$82.0 \mathrm{~N}$

$11.4 \mathrm{~W}$

41.3S 24.6W

16.4S 154.2E

$1.4 \mathrm{~N} \quad 25.0 \mathrm{E}$

0
578

SA PR

205

375

EU LA

1690

0

$5 \quad 1976$

51985

$5 \quad 1976$

$\begin{array}{ll}6 & 1976\end{array}$

$\begin{array}{llll}\text { EU } & \text { GR } & 5 & 1973\end{array}$

EU GR

$5 \quad 1982$

0 UR RU

34 EU GE

$5 \quad 1988$

51935

141

EU

FR

$\begin{array}{ll}5 & 1988\end{array}$

934 EU GR

51979

140 EU GR

EU GR

$5 \quad 1985$

$\begin{array}{ll}5 & 1958\end{array}$

0
868
592

EU GR

EU GR

EU GR

$5 \quad 1991$

$5 \quad 1973$

51973

11

AS
AA Dominique Francois Jean; French astronomer (1786-1853).

AA Dominique F.; French astronomer (1786-1853).

$\mathrm{CH}$ Classical albedo feature name

CR Persian fertility goddess.

PE Armenian thunder god.

AA Town in Mexico.

CM Indian forest goddess.

AA Greek astronomer (315-245 B.C. ?).

SU Assyrian town where Ishtar was worshipped.

AL Mountainous region in southern Greece.

PL From classical albedo feature at $45 \mathrm{~N}, 120 \mathrm{~W}$.

AA Greek physicist, mathematician (c. 287-212 B.C.)

AA Greek mathematician (428-347 B.C. ?).

VA Ancient European river (Bulgaria).

CR Forest, location of "As You Like It."

VA Radio telescope in Puerto Rico.

$\mathrm{CO}$ Classical albedo feature at $13 \mathrm{~N}, 294 \mathrm{~W}$.

DO Classical albedo feature name.

RU Name changed in 1982 to Arnus Vallis.

VA Word for 'Mars' in Greek.

AA Wife of Alcinous, mother of Nausicaa.

AA Town in Russia.

AA Friedrich Wilhelm August; German astronomer (1799-1875)

RU Location of earlier action in "The Tempest."

LI Greek; another name for Telephassa.

PM Where Io was captured by Zeus.

$\mathrm{AL}$ "Silver" Island at mouth of Ganges River; present-day Arakan, Burma.

CB Albedo name.

PL Classical albedo feature name.

RU Classical albedo feature name.

AA Arrhidaeus, Philipus; King of Babylon chronologer (unkn.-317 B.C.).

AA Greek first name; crater defines longitude.

CO Classical albedo feature name.

FO Celtic warrior queen.

AA Town in Colombia.

RU Albedo feature; classical Persian deity of wickedness.

AA Ludovico; Italian writer (1474-1533).

AA Greek astronomer (310-230 B.C. ?).

AA Greek astronomer (fl. c. 280 B.C.)

AA Greek astronomer, philosopher (383-322 B.C.)

AA Greek philosopher and musical theorist (fl. 4th century B.C.).

AA A.D.; Russian geologist.

AA Franciszek; Polish astronomer (1789-1848).

AA Neil A.; American astronaut (1930-Live).
P: $\quad$ planetary system (see page xvi).

Sa: satellite (see page $x v i$ ).

lat: latitude of feature center.

long: longitude of feature center. diam: diameter or long dimension of feature.

ct: continent of name origin (see page $284 \mathrm{ff}$.)

et: ethnicity of name origin (see page $284 \mathrm{ff}$.)

as: name approval status (see page $x v i i$ ). ad: name approval date (year).

ref: reference source for name (see page $287 \mathrm{ff}$.).

ft: feature type (see page 290). 


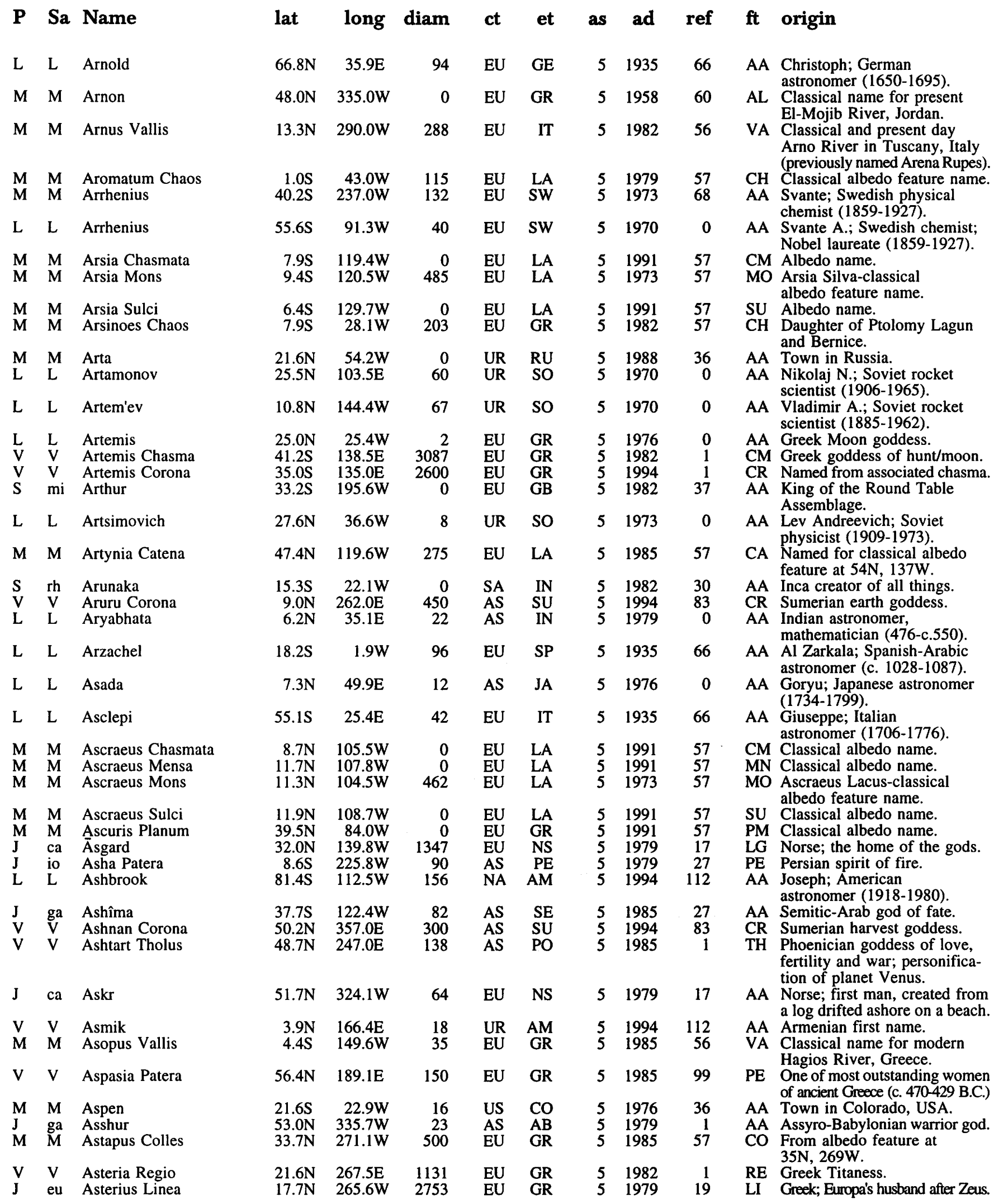

\footnotetext{
P: $\quad$ planetary system (see page xvi).

Sa: $\quad$ satellite (see page $x v i$ ).

lat: latitude of feature center.

long: longitude of feature center.
}

$9.0 \mathrm{~N} \quad 262.0 \mathrm{E}$

$35.1 \mathrm{E}$

$19 \mathrm{~W}$

$49.9 \mathrm{E}$

$8.7 \mathrm{~N} \quad 105.5 \mathrm{~W}$

$11.7 \mathrm{~N} \quad 107.8 \mathrm{~W}$

$11.3 \mathrm{~N} \quad 104.5 \mathrm{~W}$

$108.7 W$

2.0N $139.8 \mathrm{~W}$

$8.6 \mathrm{~S} \quad 225.8 \mathrm{~W}$

$81.4 \mathrm{~S} \quad 112.5 \mathrm{~W}$

$122.4 \mathrm{~W}$

$50.2 \mathrm{~N} \quad 357.0 \mathrm{E}$ astronomer (1650-1695)

Classical name for present El-Mojib River, Jordan. Arno River in Tuscany, Italy (Previously named Arena Rupes)

Svante; Swedish physical Albedo name.

Daughter of Ptolomy Lagun

AA Nikolaj N.; Soviet rocket (1906-1965).

scientist (1885-1962).

CM Greek goddess of hunt/moon

CR Named from associated chasma.

King of the Round Table physicist (1909-1973).

A Inca creator of all thing. mathematician (476-c.550). Goryu; Japanese astronome

CM Classical albedo name.

MO Ascraeus Lacus-classical albedo feature name.

PE Persian spirit of fire.

astronomer (1918-1980).

AA Semitic-Arab god of fate.

CR Sumerian harvest goddess. fertility and war; personificaArmenian first name.

Classical name for modern Hagios River, Greece.

One of most outstanding women

A Town in Colorado, USA.

AA Assyro-Babylonian warrior god. From albedo

LI Greek; Europa's husband after Zeus. 


\section{ALPHABETICAL LIST OF NAMES}

\section{P Sa Name}

L L Aston

V V Astrid

H H Astrolabe Rupes

H H Aśvaghosa

$\mathrm{U}$ ar Ataksak

V V Atalanta Planitia

J io Atar Patera

V V Atargatis Corona

$\begin{array}{lll}\text { J } & \text { io Aten Patera } \\ \text { V V Atete Corona }\end{array}$

V V Atira Mons

V V Atla Regio

M M Atlantis Chaos

L L Atlas

M M Atrax Dorsum

V V Atropos Tessera

V V Atse Estsan Corona

$S$ rh Atum

L L Atwood

V V Audhumla Corona

V V Audra Planitia

V V Audrey

M M Auqakuh Vallis

V V Aurelia

M M Aureum Chaos

$\mathrm{H}$ H Aurora

M M Aurorae Chaos

M M Aurorae Planum

M M Aurorae Sinus

V V Auska Dorsum

M M Ausonia

M M Ausonia Cavus

M M Ausonia Mensa

M M Ausonia Montes

V V Aušrā Dorsa

V V Austen

$\mathrm{L}$ L [Austen]

H H Australia

M M Australis Patera

M M [Australis Tholus]

L L Autolycus

L L Auwers

M M Auxo Dorsum

L L Auzout

S mi Avalon Chasma

M M Aveiro

M M Avernus Cavi

M M Avernus Colles

M M Avernus Dorsa lat

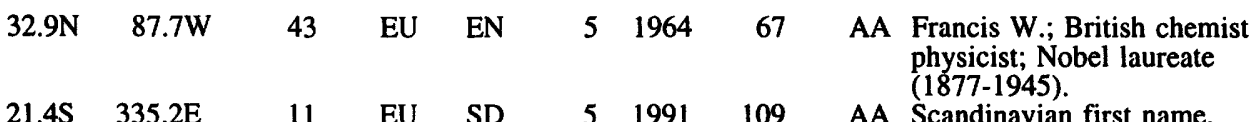

$\begin{array}{rrrrrrrr}21.4 S & 335.2 \mathrm{E} & 11 & \text { EU } & \text { SD } & 5 & 1991 & 109 \\ 42.6 \mathrm{~S} & 70.7 \mathrm{~W} & 0 & \text { EU } & \text { FR } & 5 & 1976 & 80 \\ & & & & & & & \\ 10.4 \mathrm{~N} & 21.0 \mathrm{~W} & 90 & \text { AS } & \text { IN } & 5 & 1976 & 59 \\ & & & & & & & \\ 53.1 \mathrm{~S} & 224.3 \mathrm{E} & 22 & \text { NA } & \text { ES } & 5 & 1988 & 94 \\ 45.6 \mathrm{~N} & 165.8 \mathrm{E} & 2048 & \text { EU } & \text { GR } & 5 & 1982 & \\ 30.2 \mathrm{~N} & 278.9 \mathrm{~W} & 125 & \text { AS } & \text { IR } & 5 & 1979 & 27 \\ 8.0 \mathrm{~S} & 8.6 \mathrm{E} & 220 & \text { AS } & \text { TU } & 5 & 1994 & 83 \\ 47.9 \mathrm{~S} & 310.1 \mathrm{~W} & 40 & \text { AF } & \text { EG } & 5 & 1979 & \\ 16.0 \mathrm{~S} & 243.5 \mathrm{E} & 600 & \text { AF } & \text { ET } & 5 & 1994 & 107\end{array}$

$52.2 \mathrm{~N} \quad 267.6 \mathrm{E} \quad 152$

$9.2 \mathrm{~N}$

34.8

$46.7 \mathrm{~N}$

$38.3 \mathrm{~N}$

$71.5 \mathrm{~N}$

$8.5 \mathrm{~N}$

$47.1 \mathrm{~S}$

5.8

$45.5 \mathrm{~N}$

$59.8 \mathrm{~N}$

$23.8 \mathrm{~N}$

$28.6 \mathrm{~N}$

$20.3 N$

45.0

80.6

$11.1 \mathrm{~S}$

$15.0 \mathrm{~S}$

$59.9 \mathrm{~N}$

$40.0 \mathrm{~S}$

32.25

$30.2 \mathrm{~S}$

$28.5 \mathrm{~S}$

$49.4 \mathrm{~N}$

$25.0 \mathrm{~S}$

$9.0 \mathrm{~S}$
$72.5 \mathrm{~S}$

80.25

59.0

$30.7 \mathrm{~N}$

$15.1 \mathrm{~N}$

$56.1 \mathrm{~S}$

$10.3 \mathrm{~N}$

$200.1 \mathrm{E}$

3200

NA PW

51985

51982

EU NO

$5 \quad 1985$

51935

$44.4 \mathrm{E}$

$89.1 \mathrm{~W}$

304.0E

$92.0 \mathrm{E}$

$1.1 \mathrm{~W}$

141 EU GR

87 EU GR

0 EU GR

469 EU GR

150 NA NV

57.7E

29

EU EN

$12.0 \mathrm{E}$

92.3E

$299.3 \mathrm{~W}$

225

1861

15

EU NS

AS LI

195

SA IN

31

468

EU RM

$27.1 \mathrm{~W}$

90.0W

$34.5 \mathrm{~W}$

$50.2 \mathrm{~W}$

$50.0 \mathrm{~W}$

590

EU

LA

EU LA

EU

LA

$357.8 \mathrm{E}$

$250.0 \mathrm{~W}$

361
0

$262.5 \mathrm{~W}$

$262.5 \mathrm{~W}$

$260.0 \mathrm{~W}$

25.3E

$168.3 \mathrm{E}$

$0.0 \mathrm{E}$

$0.0 \mathrm{~W}$

$51.5 \mathrm{~W}$

$323.0 \mathrm{~W}$

$1.5 \mathrm{E}$

17.2E

$41.9 \mathrm{~W}$

64.1E

$39.6 \mathrm{~N} \quad 147.8 \mathrm{~W}$

$21.5 \mathrm{~N} \quad 80.0 \mathrm{~W}$

3.8S $187.4 \mathrm{~W}$

$2.0 \mathrm{~S} \quad 188.5 \mathrm{~W}$

4.7S $189.1 \mathrm{~W}$
UR LI

EU GR

EU LA

EU LA

EU LA

UR LI

EU EN

EU GB

EU RM

0 EU LA

40 EU LA

39 EU GR

20 EU GE

51935

0 EU GR

51991

32

$\begin{array}{ll}5 & 1961\end{array}$

$5 \quad 1982$

51985

$\begin{array}{rll}0 & \text { EU } & \text { PG } \\ 60 & \text { EU } & \text { LA }\end{array}$

51985

51985

51985
80

59

94

1
27

83

107

$$
1
$$

27

57

66

56

1

83

40$$
0
$$

83

83
64

64
82

62

99

57

60

57
57

60

65

60

57

57

57

65

87

0

60

57

57

66

66

1

37

36
57

57

57 ft origin

RU French; d'Urville's ship to explore Antarctica, 1838-40.

AA Indian philosopher and poet (fl. A.D. 80-150).

AA Eskimo benevolent spirit.

PL Greek mythical heroine.

$P E$ Iranian personification of fire.

CR Hittite fertility goddess.

PE Egyptian sun god.

CR Oromo (Ethiopia) fertility goddess.

MO Pawnee (N. America) wife of Great Spirit Tirawa.

RE Norse giantess, mother of Heimdall.

$\mathrm{CH}$ From albedo feature at $30 \mathrm{~N}, 173 \mathrm{~W}$.

AA Mythological Greek Titan.

DO Classical town.

TE Greek; one of three Fates.

CR Navajo fertility goddess.

AA Old creator God of Heliopolis; became son of Ptah.

AA G.; British mathematician, physicist (1745-1807).

CR Norse primordial nourisher.

PL Lithuanian sea mistress.

AA English first name.

VA Word for 'Mars' in Quechua (Inca).

AA Mother of Julius Caesar.

$\mathrm{CH}$ Classical albedo feature name.

AL Albedo name for $\mathrm{H}-2$, Victoria region.

$\mathrm{CH}$ Classical albedo name.

PM Classical albedo feature name.

AL "Bay of Rosy Dawn"; Aurora held back the dawn.

DO Lithuanian goddess of sun rays.

AL Country of the Aruncii (Ausones in Greek).

CB Albedo feature Ausonia.

MN Albedo feature name.

MO Albedo feature name.

DO Lithuanian dawn goddess.

AA Jane; English novelist (1775-1817).

AA Jane; British author (1775-1817).

AL Albedo name for $\mathrm{H}-15$, Bach region.

PE Classical albedo name.

TH Classical albedo feature name; dropped in 1978.

AA Greek astronomer (unkn-c. 330 B.C.).

AA Georg Friedrich Julius Arthur; German astronomer (1838-1915).

DO One of the Graces.

AA Adrien; French astronomer, physicist (1622-1691).

CM Arthurian paradise. 


\section{ALPHABETICAL LIST OF NAMES}

\section{P Sa Name}

M M Avernus Rupes

L L Avery

V V Avfruvva Vallis

L L Avicenna

L L Avogadro

V V Avviyar

$N$ tr Awib Dorsa

M M Axius Valles

J ga Aya

M M Ayacucho

V V Ayana

M M Ayr

V V Ayrton Patera

J ca Aziren

L L Azophi

M M Azul

M M Azusa

L L Baade

J ga Bāal

V V Baba-Jaga Chasma

M M Babakin

L L Babakin

L L Babbage

J io Babbar Patera

L L Babcock

L L Baby Ray

$\mathrm{H} \quad \mathrm{H}$ Bach

M M Bacht

V V Bachue Corona

L L Back

L L Backlund

L L Bacon

J io Bactria Regio

M M Bada

V V Badarzewska

A gs Baden-Baden

A gs Badgastein

M M Baetis Chasma

M M Baetis Mensa

A gs Bagnoles

V V Ba'het Patera

$S$ hy Bahloo

M M Bahn

M M Bahram Vallis

$\mathrm{L}$ L Baillaud

L L Bailly lat long diam

$\begin{array}{rrrrrrrr}8.9 \mathrm{~S} & 186.4 \mathrm{~W} & 200 & \text { EU } & \text { LA } & 5 & 1985 & 57 \\ 1.4 \mathrm{~S} & 81.4 \mathrm{E} & 9 & \text { NA } & \text { CA } & 5 & 1976 & 0 \\ & & & & & & & \\ 2.0 \mathrm{~N} & 70.0 \mathrm{E} & 70 & \text { EU } & \text { FI } & 5 & 1994 & 111 \\ 39.7 \mathrm{~N} & 97.2 \mathrm{~W} & 74 & \text { AS } & \text { PE } & 5 & 1970 & 0 \\ 63.1 \mathrm{~N} & 164.9 \mathrm{E} & 139 & \text { EU } & \text { IT } & 5 & 1970 & 0 \\ & & & & & & & \\ 18.0 \mathrm{~S} & 353.6 \mathrm{E} & 21 & \text { AS } & \text { IN } & 5 & 1991 & 84 \\ 7.0 \mathrm{~S} & 80.0 \mathrm{E} & 0 & \text { AF } & \text { NM } & 5 & 91 & 85 \\ 55.8 \mathrm{~S} & 290.9 \mathrm{~W} & 316 & \text { EU } & \text { GR } & 5 & 1979 & 56\end{array}$

$\begin{array}{lllll}66.1 \mathrm{~N} & 326.2 \mathrm{~W} & 42 & \mathrm{AS} & \mathrm{AB}\end{array}$

51979

$38.5 \mathrm{~N} 92.0 \mathrm{~W}$

29.1S $\quad 175.5 \mathrm{E}$

39.5S 268.2W

6.0N 228.3E

$35.4 \mathrm{~N} \quad 178.3 \mathrm{~W}$

$22.1 \mathrm{~S} \quad 12.7 \mathrm{E}$

$42.5 S$

44.8

$24.0 \mathrm{~N} \quad 331.6 \mathrm{~W}$

$53.2 \mathrm{~N} \quad 49.5 \mathrm{E}$

$36.4 \mathrm{~S} \quad 71.4 \mathrm{~W}$

20.8S 123.3E

$59.7 \mathrm{~N}$

39.5S 272.1W

$\begin{array}{rl}4.2 \mathrm{~N} & 93.9 \mathrm{E}\end{array}$

9.1S $\quad 15.4 \mathrm{E}$

$68.5 S$

18.9N

$73.3 \mathrm{~N}$

$1.1 \mathrm{~N}$

$16.0 \mathrm{~S}$

$51.0 \mathrm{~S}$

$45.8 \mathrm{~S}$

$20.5 \mathrm{~N}$

22.6

$46.0 \mathrm{~N}$

$25.0 \mathrm{~N}$

$4.3 \mathrm{~S}$

5.4

$55.0 \mathrm{~N}$

$48.4 \mathrm{~N}$

$36.0 \mathrm{~N}$

$3.5 \mathrm{~S}$

$21.4 \mathrm{~N}$

$74.6 \mathrm{~N}$

$66.5 S$
$42.3 \mathrm{~W}$

$40.5 \mathrm{~W}$

$57.1 \mathrm{~W}$

SA BO

UR RU

OC AU

51991

51994

$\begin{array}{ll}5 & 1989 \\ 5 & 1994\end{array}$

85

UR ES

47

AS PE

51987

1935

41 US CA

55 NA AM

1976

51976

$\begin{array}{ll}5 & 1964\end{array}$

52 AS PO

580
78

UR SL

1979

51985

20

UR SO

51973

143

EU GB

51935

95
99

AS SU

NA AM

$\begin{array}{ll}5 & 1979 \\ 5 & 1970\end{array}$

NA AM

51973

51976

214 EU GE

UR UZ

$257.5 \mathrm{~W}$

261.4E

$\begin{array}{rll}7 & \text { UR } & \text { UZ } \\ 463 & \text { SA } & \text { CO }\end{array}$

51976

$5 \quad 1985$

51976

51970

51935

19.1E

75

UR RU

$123.4 \mathrm{~W}$

$$
69
$$

EU GB

51979

$50.7 \mathrm{~W}$

137.2E

0 UR RU

5198

$55.0 \mathrm{~W}$

$3.0 \mathrm{~W}$

$64.8 \mathrm{~W}$

0 EU GE

51994

$5 \quad 1994$

113

$72.4 \mathrm{~W}$

$122.0 \mathrm{~W}$

$0.1 \mathrm{E}$

$196.0 \mathrm{~W}$

43.5W

$58.7 \mathrm{~W}$

37.5E

181 EU GR

$\begin{array}{rll}181 & \text { EU } & \text { GR } \\ 0 & \text { EU } & \text { FR } \\ 145 & \text { AF } & \text { EG } \\ 0 & \text { OC } & \text { AS }\end{array}$

0 OC AS

403

AF

AS

LI
PE
FR

$5 \quad 1985$

69.1W
287
EU
20 SA AR

$5 \quad 1985$

0 EU AS

$\begin{array}{rr}1985 & 57 \\ 1994 & 113 \\ 91 & 0 \\ 1982 & 4 \\ 1976 & 36 \\ 1976 & 62 \\ 1935 & 66 \\ & \\ 1935 & 66\end{array}$

RU From albedo feature Avernus at 10S, 195W.

AA Oswald Theodore; Canadian doctor (1877-1955).

VA Saami (Lapp) river goddess.

AA Abu Ali Ibn Sina; Persian doctor (980-1037).

AA Amedeo (Conte Di Quarengna); Italian physicist (1776-1856).

AA Tamil poet (c. 100 B.C.).

DO Nama Bushman word for rain.

VA Ancient European River (Vardar River of Greece).

AA Assyro-Babylonian; wife of Shamash.

AA Town in Bolivia.

AA Altai first name.

AA Town in Queensland, Australia.

PE Hertha M.; English physicist (1854-1923).

AA Estonian spirit of death.

AA Al-Sufi, Abderrahman; Persian astronomer (903-986).

AA Town in Argentina.

AA Town in California, USA.

AA Walter; American astronomer (1893-1960).

AA Phoenician; Canaanite god.

CM Slavic forest witch.

AA Soviet builder of unmanned space stations (1914-1970).

AA Georgij N.; Soviet space scientist (1914-1971).

AA Charles; British mathematician (1792-1871)

PE Sumerian; sun god.

AA Harold D.; American astronomer, physicist (1882-1968)

LF Astronaut-named feature, Apollo 16 site.

AA J. S.; German composer (1685-1750).

AA Town in Uzbekistan.

CR Chibcha (Colombia) goddess of fertility.

AA Ernst E. A.; German physicist (1881-1959).

AA Oscar A.; Russian astronomer (1846-1916).

AA Roger, British natural philosopher, optician (1214-1294)

RE Io passed through this area of ancient Iran in her wanderings.

AA Town in Russia.

AA Thekla; Polish composer (1834-1861).

AA Spa in Germany.

AA Spa in Austria.

CM From classical albedo feature at $5 \mathrm{~S}, 60 \mathrm{~W}$; changed from Iamunae Chasma.

MN From albedo feature at $7 \mathrm{~S}, 63 \mathrm{~W}$.

AA Spa in France.

PE Egyptian who defeated Portugese.

AA The Moon; maker of girl babies.

AA Town in Liberia.

VA Word for 'Mars' in Persian.

AA Benjamin; French astronomer (1848-1934).

AA Jean Sylvain; French astronomer (1736-1793).

\footnotetext{
P: $\quad$ planetary system (see page $x v i)$

Sa: satellite (see page xvi).

lat: latitude of feature center.

long: longitude of feature center.
}

diam: diameter or long dimension of feature.

ct: continent of name origin (see page $284 \mathrm{ff}$.)

et: ethnicity of name origin (see page $284 \mathrm{ff}$.)

as: name approval status (see page $x v i i$ ). ad: name approval date (year).

ref: reference source for name (see page $287 \mathrm{ff}$.).

ft: feature type (see page 290). 


\section{ALPHABETICAL LIST OF NAMES}

\section{P Sa Name}

L L Baily

M M Bak

V V Baker

M M Bakhuysen

H H Balagtas

L L Balandin

M M Balboa

L L Balboa

V V Balch

M M Baldet

L L Baldet

$S$ ia Baligant

$S$ mi Balin

J ca Balkr

L L Ball

L L Balmer

M M Balta

M M Baltia

$\mathrm{V} V$ Baltis Vallis

M M Baltisk

M M Balvicar

L $\quad$ L $\quad$ [Balzac]

H H Balzac

M M Bamba

M M Bamberg

$\mathrm{S}$ mi Ban

V V Ban Zhao

L L Banachiewicz

L L Bancroft

M M Banff

M M Banh

L L Banting

M M Baphyras Catena

M M Bar

M M Barabashov

V V Baranamtarra

L L Barbier

L L Barkla

H H Barma

L L Barnard

M M Barnard

J ga Barnard Regio

\section{lat long}

diam

ct et

\begin{tabular}{|c|c|c|c|c|c|}
\hline $30.4 \mathrm{E}$ & 26 & $\mathrm{EU}$ & GB & 5 & 1935 \\
\hline $\begin{array}{c}256.3 \mathrm{~W} \\
40.5 \mathrm{E}\end{array}$ & $\begin{array}{r}0 \\
105\end{array}$ & $\begin{array}{l}\text { EU } \\
\text { NA }\end{array}$ & $\begin{array}{l}\mathrm{HU} \\
\mathrm{AM}\end{array}$ & 5 & $\begin{array}{l}1988 \\
1994\end{array}$ \\
\hline $344.3 \mathrm{~W}$ & 162 & $\mathrm{EU}$ & DU & 5 & 1973 \\
\hline $\begin{array}{c}13.7 \mathrm{~W} \\
152.6 \mathrm{E} \\
34.0 \mathrm{~W} \\
83.2 \mathrm{~W}\end{array}$ & $\begin{array}{l}98 \\
12 \\
20 \\
69\end{array}$ & $\begin{array}{l}\text { AS } \\
\text { UR } \\
\text { SA } \\
\text { EU }\end{array}$ & $\begin{array}{l}\text { PH } \\
\text { SO } \\
\text { PM } \\
\text { SP }\end{array}$ & 5 & $\begin{array}{l}1976 \\
1976 \\
1976 \\
1964\end{array}$ \\
\hline 282.9E & 37 & NA & $\mathrm{AM}$ & 5 & 1994 \\
\hline $294.5 \mathrm{~W}$ & 195 & $\mathrm{EU}$ & FR & 5 & 1973 \\
\hline $151.1 \mathrm{~W}$ & 55 & EU & FR & 5 & 1970 \\
\hline $224.9 \mathrm{~W}$ & 66 & $\mathrm{EU}$ & FR & 5 & 1982 \\
\hline $86.8 \mathrm{~W}$ & 0 & EU & GB & 5 & 1982 \\
\hline $\begin{array}{r}11.9 \mathrm{~W} \\
8.4 \mathrm{~W}\end{array}$ & $\begin{array}{l}64 \\
41\end{array}$ & $\begin{array}{l}\mathrm{EU} \\
\mathrm{EU}\end{array}$ & $\begin{array}{l}\text { NS } \\
\text { GB }\end{array}$ & 5 & $\begin{array}{l}1979 \\
1935\end{array}$ \\
\hline $69.8 \mathrm{E}$ & 138 & EU & SZ & 5 & 1964 \\
\hline $\begin{array}{l}26.4 \mathrm{~W} \\
50.0 \mathrm{~W}\end{array}$ & $\begin{array}{r}15 \\
0\end{array}$ & $\begin{array}{l}\text { UR } \\
\text { EU }\end{array}$ & $\begin{array}{l}\text { UK } \\
\text { LA }\end{array}$ & & $\begin{array}{l}1976 \\
1958\end{array}$ \\
\hline $\begin{array}{c}161.4 \mathrm{E} \\
54.5 \mathrm{~W} \\
53.1 \mathrm{~W} \\
95.0 \mathrm{E}\end{array}$ & $\begin{array}{r}6000 \\
48 \\
0 \\
39\end{array}$ & $\begin{array}{l}\text { AS } \\
\text { UR } \\
\text { EU } \\
\text { EU }\end{array}$ & $\begin{array}{l}\text { SY } \\
\text { RU } \\
\text { SC } \\
\text { FR }\end{array}$ & 0 & $\begin{array}{r}1994 \\
1976 \\
1988 \\
0\end{array}$ \\
\hline $144.1 \mathrm{~W}$ & 80 & EU & FR & 5 & 1976 \\
\hline $\begin{array}{r}41.7 \mathrm{~W} \\
3.0 \mathrm{~W} \\
156.4 \mathrm{~W}\end{array}$ & $\begin{array}{r}21 \\
57 \\
0\end{array}$ & $\begin{array}{l}\mathrm{AF} \\
\mathrm{EU} \\
\mathrm{EU}\end{array}$ & $\begin{array}{l}\mathrm{ZA} \\
\mathrm{GE} \\
\mathrm{GB}\end{array}$ & 5 & $\begin{array}{l}1976 \\
1976 \\
1982\end{array}$ \\
\hline
\end{tabular}

$17.2 \mathrm{~N} \quad 146.9 \mathrm{E}$

$5.2 \mathrm{~N}$

$80.1 \mathrm{E}$

$28.0 \mathrm{~N}$

$17.4 \mathrm{~N}$

$19.4 \mathrm{~N}$

$6.4 \mathrm{~W}$

$30.5 \mathrm{~W}$

$55.2 \mathrm{~W}$

$26.6 \mathrm{~N}$

16.4E

$38.8 \mathrm{~N}$

$25.6 \mathrm{~S}$

$47.6 \mathrm{~N}$

84.0W

84. $19.3 \mathrm{~W}$

$68.5 \mathrm{~W}$

$17.9 \mathrm{~N} \quad 267.8 \mathrm{E}$

$23.8 \mathrm{~S} \quad 157.9 \mathrm{E}$

$10.7 \mathrm{~S}$

67.2E

41.3S

$162.8 \mathrm{~W}$

$29.5 \mathrm{~S}$

$85.6 \mathrm{E}$

$61.3 \mathrm{~S} \quad 298.4 \mathrm{~W}$

$0.8 \mathrm{~N} \quad 195.8 \mathrm{~W}$
128

42 AS $\mathrm{CH}$

$\begin{array}{ll}5 & 1991\end{array}$

$5 \quad 1964$

51976

$$
13 \text { NA AM }
$$

$\begin{array}{ll}5 & 1976 \\ 5 & 1976\end{array}$

3 NA CA

$\mathrm{AF} \quad \mathrm{BF}$

$5 \quad 1973$

5 NA CA

$5 \quad 1991$

0 EU GR

$\begin{array}{rrr}2 & \text { UR } & \text { UK } \\ 126 & \text { UR } & \text { RU }\end{array}$

$\begin{array}{ll}5 & 1991 \\ 5 & 1976 \\ 5 & 1973\end{array}$

25

AS $A B$

51994

66 EU FR

51970

42

EU GB

51979

128

UR RU

51982

$5 \quad 1964$

NA AM

51973

$\begin{array}{ll}5 & 1979\end{array}$ ref

ft origin

AA Francis; British astronomer (1774-1844).

AA Town in Hungary.

AA Josephine; American expatriate dancer, singer (1906-1975).

AA Henricus G.; Dutch astronomer (1838-1923).

AA F.; Philippino writer (1788-1862).

AA A. A.; Soviet chemist (1898-1967).

AA Town in the Panama Canal zone.

AA Vasco N. de; Spanish explorer (1475-1517).

AA Emily; American economist, Nobel laureate (1867-1961).

AA Ferdnand; French astronomer (1885-1964).

AA Francois; French astronomer (1885-1964).

AA Emir of Babylon; Marsilion enlisted his help against Charlemagne.

AA Knight of "matchless courage and virtue."

AA Norse; Ottar's ancestor.

AA William; British astonomer (unkn-1690)

AA Johann J.; Swiss mathematician, physician (1825-1898).

AA Town in Ukraine.

AL Name of large island in northern Europe where amber was found

VA Syrian word for planet Venus.

AA Town in Russia.

AA Town in Scotland

AA Honore de; French author (1799-1850).

AA Honore de; French novelist (1799-1850).

AA Town in Zaire.

AA Town in Germany.

AA King of Benwick; father of Sir Launcelot, ally of Arthur in the battle of Bedgrayne.

AA Chinese historian (c. A.D. 35-100).

AA Tadeusz; Polish astronomer, mathematician (1882-1954).

AA W. D.; American chemist (1867-1953).

AA Town in Alberta, Canada.

AA Town in Burkina Faso (formerly Upper Volta).

AA Sir Frederick Grant; Canadian doctor, Nobel laureate (1891-1941)

CA Classical river.

AA Town in Ukraine.

AA Nilolay P.; Russian astronomer (1894-1971).

AA Mesopotamian queen (c. 2500 B.C.).

AA Daniel; French astronomer (1907-1965).

AA C. G.; British physicist; Nobel laureate (1877-1944).

AA Yakovlev; 16th Century Russian architect.

AA Edward E.; American astronomer (1857-1923).

AA Edward E.; American astronomer (1857-1923).

RE Edward E.; American astronomer (1857-1923).

\footnotetext{
P: $\quad$ planetary system (see page $x v i)$.

Sa: $\quad$ satellite (see page $x v i)$.

lat: latitude of feature center.

long: longitude of feature center.
}

diam: diameter or long dimension of feature.

ct: continent of name origin (see page $284 \mathrm{ff}$.)

et: ethnicity of name origin (see page $284 \mathrm{ff}$.)

as: name approval status (see page xvii). ad: name approval date (year).

ref: reference source for name (see page $287 \mathrm{ff}$.).

ft: feature type (see page 290). 


\section{ALPHABETICAL LIST OF NAMES}

\begin{tabular}{|c|c|c|c|c|c|c|c|c|c|c|}
\hline $\mathbf{P}$ & $\mathbf{S a}$ & Name & lat & long & diam & ct & et & as & ad & ref \\
\hline $\begin{array}{l}M \\
L\end{array}$ & $\begin{array}{l}\mathrm{M} \\
\mathrm{L}\end{array}$ & $\begin{array}{l}\text { Baro } \\
\text { Barocius }\end{array}$ & $\begin{array}{l}24.9 \mathrm{~S} \\
44.9 \mathrm{~S}\end{array}$ & $\begin{array}{c}249.3 \mathrm{~W} \\
16.8 \mathrm{E}\end{array}$ & $\begin{array}{r}0 \\
82\end{array}$ & $\begin{array}{l}\mathrm{EU} \\
\mathrm{EU}\end{array}$ & $\begin{array}{l}\text { IC } \\
\text { IT }\end{array}$ & $\begin{array}{l}5 \\
5\end{array}$ & $\begin{array}{l}1989 \\
1935\end{array}$ & $\begin{array}{l}74 \\
66\end{array}$ \\
\hline V & V & Barrera & $16.6 \mathrm{~N}$ & 109.3E & 27 & $\mathrm{EU}$ & SP & 5 & 1991 & 100 \\
\hline $\mathbf{L}$ & $\mathrm{L}$ & Barringer & $28.0 \mathrm{~S}$ & $149.7 \mathrm{~W}$ & 68 & NA & $\mathrm{AM}$ & 5 & 1970 & 0 \\
\hline L & $\mathbf{L}$ & Barrow & $71.3 \mathrm{~N}$ & $7.7 \mathrm{E}$ & 92 & EU & GB & 5 & 1935 & 66 \\
\hline V & V & Barrymore & $52.3 \mathrm{~S}$ & $195.6 \mathrm{E}$ & 50 & NA & AM & 5 & 1994 & 87 \\
\hline $\begin{array}{l}\text { V } \\
\text { L }\end{array}$ & $\begin{array}{l}\mathrm{V} \\
\mathrm{L}\end{array}$ & $\begin{array}{l}\text { Barsova } \\
\text { Bartels }\end{array}$ & $\begin{array}{l}61.3 \mathrm{~N} \\
24.5 \mathrm{~N}\end{array}$ & $\begin{array}{c}223.0 \mathrm{E} \\
89.8 \mathrm{~W}\end{array}$ & $\begin{array}{l}79 \\
55\end{array}$ & $\begin{array}{l}\text { UR } \\
\text { EU }\end{array}$ & $\begin{array}{l}\text { SO } \\
\text { GE }\end{array}$ & $\begin{array}{l}5 \\
5\end{array}$ & $\begin{array}{l}1985 \\
1970\end{array}$ & $\begin{array}{r}64 \\
0\end{array}$ \\
\hline $\begin{array}{l}\mathrm{V} \\
\mathrm{H}\end{array}$ & $\begin{array}{l}\mathrm{V} \\
\mathrm{H}\end{array}$ & $\begin{array}{l}\text { Barto } \\
\text { Bartók }\end{array}$ & $\begin{array}{l}45.3 \mathrm{~N} \\
29.6 \mathrm{~S}\end{array}$ & $\begin{array}{l}146.2 \mathrm{E} \\
134.6 \mathrm{~W}\end{array}$ & $\begin{array}{r}49 \\
112\end{array}$ & $\begin{array}{l}\text { UR } \\
\text { EU }\end{array}$ & $\begin{array}{l}\text { SO } \\
\mathrm{HU}\end{array}$ & $\begin{array}{l}5 \\
5\end{array}$ & $\begin{array}{l}1985 \\
1979\end{array}$ & $\begin{array}{l}64 \\
59\end{array}$ \\
\hline V & V & Barton & $27.4 \mathrm{~N}$ & $337.5 \mathrm{E}$ & 54 & NA & $\mathrm{AM}$ & 5 & 1991 & 99 \\
\hline $\mathbf{S}$ & ia & Basan & $33.3 \mathrm{~N}$ & $194.7 \mathrm{~W}$ & 76 & EU & FR & 5 & 1982 & 29 \\
\hline V & V & Bascom & $10.3 \mathrm{~S}$ & $302.2 \mathrm{E}$ & 36 & NA & AM & 5 & 1994 & 99 \\
\hline V & V & Bashkirtseff & $14.7 \mathrm{~N}$ & $194.0 \mathrm{E}$ & 38 & UR & RU & 5 & 1994 & 87 \\
\hline $\begin{array}{l}\mathrm{H} \\
\mathrm{M} \\
\mathrm{V}\end{array}$ & $\begin{array}{l}\mathrm{H} \\
\mathrm{M} \\
\mathrm{V}\end{array}$ & $\begin{array}{l}\text { Bashō } \\
\text { Basin } \\
\text { Bassi }\end{array}$ & $\begin{array}{l}32.7 \mathrm{~S} \\
18.1 \mathrm{~N} \\
19.0 \mathrm{~S}\end{array}$ & $\begin{array}{c}169.7 \mathrm{~W} \\
253.2 \mathrm{~W} \\
64.7 \mathrm{E}\end{array}$ & $\begin{array}{l}80 \\
16 \\
33\end{array}$ & $\begin{array}{l}\text { AS } \\
\text { US } \\
\text { EU }\end{array}$ & $\begin{array}{r}\text { JA } \\
\text { WY } \\
\text { IT }\end{array}$ & $\begin{array}{l}5 \\
5 \\
5\end{array}$ & $\begin{array}{l}1979 \\
1976 \\
1991\end{array}$ & $\begin{array}{r}80 \\
36 \\
100\end{array}$ \\
\hline $\mathbf{S}$ & en & Bassorah Fossa & $45.4 \mathrm{~N}$ & $6.3 W$ & 131 & AS & $\mathrm{AR}$ & 5 & 1982 & 52 \\
\hline $\begin{array}{l}\text { V } \\
\text { A } \\
\text { V } \\
\text { M }\end{array}$ & $\begin{array}{l}\text { V } \\
\text { gs } \\
\text { V } \\
\text { M }\end{array}$ & $\begin{array}{l}\text { Bast Tholus } \\
\text { Bath } \\
\text { Bathsheba } \\
\text { [Bathys Planum] }\end{array}$ & $\begin{array}{l}57.8 \mathrm{~N} \\
13.4 \mathrm{~N} \\
15.1 \mathrm{~S} \\
43.0 \mathrm{~S}\end{array}$ & $\begin{array}{c}130.3 \mathrm{E} \\
9.7 \mathrm{~W} \\
49.3 \mathrm{E} \\
106.0 \mathrm{~W}\end{array}$ & $\begin{array}{r}83 \\
10 \\
36 \\
626\end{array}$ & $\begin{array}{l}\text { AF } \\
\text { EU } \\
\text { AS } \\
\text { EU }\end{array}$ & $\begin{array}{l}\text { EG } \\
\text { EN } \\
\text { HE } \\
\text { LA }\end{array}$ & $\begin{array}{l}5 \\
5 \\
5 \\
6\end{array}$ & $\begin{array}{l}1985 \\
1994 \\
1994 \\
1979\end{array}$ & $\begin{array}{r}1 \\
113 \\
99 \\
57\end{array}$ \\
\hline $\begin{array}{l}\mathbf{M} \\
\mathbf{M}\end{array}$ & $\begin{array}{l}\text { M } \\
\text { M } \\
\text { ga }\end{array}$ & $\begin{array}{l}\text { Batoka } \\
\text { Batoş } \\
\text { Bau }\end{array}$ & $\begin{array}{c}7.6 \mathrm{~S} \\
21.7 \mathrm{~N} \\
24.1 \mathrm{~N}\end{array}$ & $\begin{array}{l}36.8 \mathrm{~W} \\
29.5 \mathrm{~W} \\
53.3 \mathrm{~W}\end{array}$ & $\begin{array}{l}14 \\
16 \\
81\end{array}$ & $\begin{array}{l}\text { AF } \\
\text { EU } \\
\text { AS }\end{array}$ & $\begin{array}{l}\mathrm{ZM} \\
\mathrm{RO} \\
\mathrm{AB}\end{array}$ & $\begin{array}{l}5 \\
5 \\
5\end{array}$ & $\begin{array}{l}1976 \\
1976 \\
1988\end{array}$ & $\begin{array}{r}36 \\
36 \\
1\end{array}$ \\
\hline $\begin{array}{l}\mathrm{V} \\
\mathrm{L}\end{array}$ & $\begin{array}{l}\text { V } \\
\text { L }\end{array}$ & $\begin{array}{l}\text { Bau Corona } \\
\text { [Baudelaire] }\end{array}$ & $\begin{array}{l}52.8 \mathrm{~N} \\
23.2 \mathrm{~S}\end{array}$ & $\begin{array}{l}259.3 \mathrm{E} \\
123.1 \mathrm{E}\end{array}$ & $\begin{array}{r}355 \\
15\end{array}$ & $\begin{array}{l}\mathrm{AS} \\
\mathrm{EU}\end{array}$ & $\begin{array}{l}\text { SU } \\
\text { FR }\end{array}$ & $\begin{array}{l}5 \\
6\end{array}$ & $\begin{array}{r}91 \\
0\end{array}$ & $\begin{array}{r}64 \\
0\end{array}$ \\
\hline $\begin{array}{l}J \\
\text { L } \\
\text { V }\end{array}$ & $\begin{array}{l}\mathrm{ca} \\
\mathrm{L} \\
\mathrm{V}\end{array}$ & $\begin{array}{l}\text { Bavörr } \\
\text { Bawa } \\
\text { Bayara Vallis }\end{array}$ & $\begin{array}{l}49.2 \mathrm{~N} \\
25.3 \mathrm{~S} \\
45.6 \mathrm{~N}\end{array}$ & $\begin{array}{c}20.3 \mathrm{~W} \\
102.6 \mathrm{E} \\
16.5 \mathrm{E}\end{array}$ & $\begin{array}{r}84 \\
1 \\
500\end{array}$ & $\begin{array}{l}\mathrm{EU} \\
\mathrm{AF} \\
\mathrm{AF}\end{array}$ & $\begin{array}{l}\text { NS } \\
\text { UN } \\
\text { ML }\end{array}$ & $\begin{array}{l}5 \\
5 \\
5\end{array}$ & $\begin{array}{l}1979 \\
1976 \\
1994\end{array}$ & $\begin{array}{r}17 \\
0 \\
107\end{array}$ \\
\hline L & $\mathrm{L}$ & Bayer & $51.6 \mathrm{~S}$ & $35.0 \mathrm{~W}$ & 47 & EU & GE & 5 & 1935 & 66 \\
\hline $\begin{array}{l}\mathrm{M} \\
\mathrm{M} \\
\mathrm{L}\end{array}$ & $\begin{array}{l}\mathbf{M} \\
\mathbf{M} \\
\mathrm{L}\end{array}$ & $\begin{array}{l}\text { Baykonyr } \\
\text { Bazas } \\
\text { Beals }\end{array}$ & $\begin{array}{l}46.7 \mathrm{~N} \\
28.0 \mathrm{~S} \\
37.3 \mathrm{~N}\end{array}$ & $\begin{array}{c}227.2 \mathrm{~W} \\
266.5 \mathrm{~W} \\
86.5 \mathrm{E}\end{array}$ & $\begin{array}{r}4 \\
0 \\
48\end{array}$ & $\begin{array}{l}\text { UR } \\
\text { EU } \\
\text { NA }\end{array}$ & $\begin{array}{l}\text { SO } \\
\text { FR } \\
\text { CA }\end{array}$ & $\begin{array}{l}5 \\
5 \\
5\end{array}$ & $\begin{array}{l}1979 \\
1989 \\
1982\end{array}$ & $\begin{array}{r}64 \\
74 \\
0\end{array}$ \\
\hline L & $\mathbf{L}$ & Bear Mountain & $20.0 \mathrm{~N}$ & $30.7 \mathrm{E}$ & 0 & NA & $\mathrm{AM}$ & 5 & 1973 & 59 \\
\hline L & L & Beaumont & $18.0 \mathrm{~S}$ & $28.8 \mathrm{E}$ & 53 & EU & FR & 5 & 1935 & 66 \\
\hline M & $\mathbf{M}$ & Becquerel & $22.3 \mathrm{~N}$ & $7.9 \mathrm{~W}$ & 167 & $\mathrm{EU}$ & FR & 5 & 1973 & 68 \\
\hline L & $\mathrm{L}$ & Becquerel & $40.7 \mathrm{~N}$ & $129.7 \mathrm{E}$ & 65 & EU & FR & 5 & 1970 & 0 \\
\hline $\begin{array}{l}\mathrm{V} \\
\mathrm{L}\end{array}$ & $\begin{array}{l}\text { V } \\
\text { L }\end{array}$ & $\begin{array}{l}\text { Bécuma Mons } \\
\text { Bečvár }\end{array}$ & $\begin{array}{c}34.0 \mathrm{~N} \\
1.9 \mathrm{~S}\end{array}$ & $\begin{array}{r}21.9 \mathrm{E} \\
125.2 \mathrm{E}\end{array}$ & $\begin{array}{r}0 \\
67\end{array}$ & $\begin{array}{l}\mathrm{EU} \\
\mathrm{EU}\end{array}$ & $\begin{array}{l}\text { IR } \\
\text { CZ }\end{array}$ & $\begin{array}{l}5 \\
5\end{array}$ & $\begin{array}{r}91 \\
1970\end{array}$ & $\begin{array}{r}27 \\
0\end{array}$ \\
\hline $\begin{array}{l}S \\
V\end{array}$ & $\mathrm{mi}$ & $\begin{array}{l}\text { Bedivere } \\
\text { Beecher }\end{array}$ & $\begin{array}{r}9.5 \mathrm{~N} \\
13.1 \mathrm{~N}\end{array}$ & $\begin{array}{l}152.3 \mathrm{~W} \\
253.5 \mathrm{E}\end{array}$ & $\begin{array}{r}0 \\
35\end{array}$ & $\begin{array}{l}\text { EU } \\
\text { NA }\end{array}$ & $\begin{array}{l}\text { GB } \\
\text { AM }\end{array}$ & $\begin{array}{l}5 \\
5\end{array}$ & $\begin{array}{l}1982 \\
1994\end{array}$ & $\begin{array}{l}37 \\
87\end{array}$ \\
\hline L & $\mathrm{L}$ & Beer & $27.1 \mathrm{~N}$ & $9.1 \mathrm{~W}$ & 9 & $\mathrm{EU}$ & GE & 5 & 1935 & 66 \\
\hline
\end{tabular}

\section{$\mathrm{ft}$ origin}

AA Town in Iceland.

AA Francesco; Italian mathematician (unkn-fl. 1570).

AA Olivia; Spanish medical writer (b. 1562).

AA Daniel M.; American engineer, geologist (1860-1929).

AA Isaac; British mathematician (1630-1677).

AA Ethel; American actress (1879-1959).

AA Valeria; Soviet singer (1892-1967).

AA Julius; German geophysicist (1899-1964)

AA Agniya; Soviet poetess (1906-1981).

AA Bela; Hungarian composer (1881-1945).

AA Clara; American Red Cross founder (1821-1912).

AA French baron; Murdered while serving as Ambassador of Marsilon.

AA Florence; American geologist (1862-1945).

AA Marie; Russian painter, diarist (c. 1859-1884).

AA Matsuo; Japanese poet (16441694).

AA Town in Wyoming, USA.

AA Laura; Italian physicist, mathemetician (1711-1778).

FO Town from which Sindbad embarked on his 3rd voyage.

TH Egyptian goddess of joy.

AA Spa in England.

AA Hebrew queen (c. 1030 B.C.).

PM Classical albedo feature; name changed to Icaria Planum.

AA Town in Zambia.

AA Town in Romania.

AA Goddess who breathed into men the breath of life; daughter of Anu and patroness of Lagash.

CR Sumerian fertility goddess.

AA Pierre Charles; French poet 1821-1867

AA Norse dwarf.

AA African male name

VA Dogon (Mali) word for planet Venus.

AA Johann; German astronomer (1572-1625).

AA Soviet launch site.

AA Town in France.

AA .Carlyle F.; Canadian astronomer (1899-1979)

LF Astronaut-named feature, Apollo 17 site.

AA Leonce Elie de; French geologist (1798-1874).

AA Antoine H.; French physicist (1852-1908).

AA Antoine H.; French physicist; Nobel laureate (1852-1908).

MO Irish dawn goddess.

AA Antonin; Czechoslovakian astronomer (1901-1965).

AA Arthurian knight.

AA Catherine; American educator, author (1800-1878)

AA Wilhelm; German astronomer (1797-1850).

\footnotetext{
P: $\quad$ planetary system (see page $x v i)$.

Sa: satellite (see page $\mathrm{xvi}$ ).

lat: latitude of feature center.

long: longitude of feature center.
}

diam: diameter or long dimension of feature.

ct: continent of name origin (see page $284 \mathrm{ff}$.)

et: ethnicity of name origin (see page $284 \mathrm{ff}$.)

as: name approval status (see page xvii). ad: name approval date (year).

ref: reference source for name (see page $287 \mathrm{ff}$.).

ft: feature type (see page 290) 


\section{ALPHABETICAL LIST OF NAMES}

\begin{tabular}{|c|c|c|c|c|c|c|c|c|c|c|}
\hline $\mathbf{P}$ & $\mathbf{S a}$ & Name & lat & long & diam & ct & et & as & ad & ref \\
\hline M & $\mathbf{M}$ & Beer & $14.6 \mathrm{~S}$ & $8.2 \mathrm{~W}$ & 80 & EU & GE & 5 & 1973 & 68 \\
\hline $\mathbf{H}$ & $\mathbf{H}$ & Beethoven & $20.8 \mathrm{~S}$ & $123.6 \mathrm{~W}$ & 643 & EU & GE & 5 & 1976 & 59 \\
\hline $\mathbf{U}$ & ar & Befana & $17.0 \mathrm{~S}$ & $31.9 \mathrm{E}$ & 21 & EU & IT & 5 & 1988 & 3 \\
\hline L & $\mathbf{L}$ & Behaim & $16.5 S$ & $79.4 \mathrm{E}$ & 55 & EU & GE & 5 & 1935 & 66 \\
\hline V & V & Behn & $32.4 \mathrm{~S}$ & $142.0 \mathrm{E}$ & 25 & EU & EN & 5 & 1991 & 99 \\
\hline L & L & Beijerinck & $13.5 \mathrm{~S}$ & $151.8 \mathrm{E}$ & 70 & EU & DU & 5 & 1970 & 0 \\
\hline $\begin{array}{l}\text { V } \\
\text { L }\end{array}$ & $\begin{array}{l}\text { V } \\
\text { L }\end{array}$ & $\begin{array}{l}\text { Beiwe Corona } \\
\text { Beketov }\end{array}$ & $\begin{array}{l}52.6 \mathrm{~N} \\
16.3 \mathrm{~N}\end{array}$ & $\begin{array}{r}306.5 \mathrm{E} \\
29.2 \mathrm{E}\end{array}$ & $\begin{array}{r}600 \\
8\end{array}$ & $\begin{array}{l}\text { EU } \\
\text { UR }\end{array}$ & $\begin{array}{r}\text { FI } \\
\mathbf{R U}\end{array}$ & $\begin{array}{l}5 \\
5\end{array}$ & $\begin{array}{l}1994 \\
1976\end{array}$ & $\begin{array}{r}111 \\
0\end{array}$ \\
\hline $\begin{array}{l}\mathbf{L} \\
\mathbf{V}\end{array}$ & $\begin{array}{l}\mathrm{L} \\
\mathrm{V}\end{array}$ & $\begin{array}{l}\text { Béla } \\
\text { Belet-Ili Corona }\end{array}$ & $\begin{array}{r}24.7 \mathrm{~N} \\
6.0 \mathrm{~N}\end{array}$ & $\begin{array}{r}2.3 \mathrm{E} \\
20.0 \mathrm{E}\end{array}$ & $\begin{array}{r}11 \\
300\end{array}$ & $\begin{array}{l}\text { EU } \\
\text { AS }\end{array}$ & $\begin{array}{l}\text { SL } \\
\mathrm{AB}\end{array}$ & $\begin{array}{l}5 \\
5\end{array}$ & $\begin{array}{l}1976 \\
1994\end{array}$ & $\begin{array}{r}0 \\
27\end{array}$ \\
\hline $\begin{array}{l}\mathbf{J} \\
\mathbf{U}\end{array}$ & $\begin{array}{l}\text { ca } \\
\text { ss }\end{array}$ & $\begin{array}{l}\text { Beli } \\
\text { Belinda }\end{array}$ & $\begin{array}{r}62.6 \mathrm{~N} \\
0.0 \mathrm{~N}\end{array}$ & $\begin{array}{c}81.7 \mathrm{~W} \\
0.0 \mathrm{X}\end{array}$ & $\begin{array}{r}50 \\
0\end{array}$ & $\begin{array}{l}\text { EU } \\
\text { EU }\end{array}$ & $\begin{array}{l}\text { CE } \\
\text { GB }\end{array}$ & $\begin{array}{l}5 \\
5\end{array}$ & $\begin{array}{l}1979 \\
1988\end{array}$ & $\begin{array}{l}27 \\
94\end{array}$ \\
\hline H & $\mathbf{H}$ & Belinskij & $76.0 \mathrm{~S}$ & $103.4 \mathrm{~W}$ & 70 & UR & RU & 5 & 1985 & 59 \\
\hline $\begin{array}{l}\text { V } \\
\mathbf{L}\end{array}$ & $\begin{array}{l}\text { V } \\
\text { L }\end{array}$ & $\begin{array}{l}\text { Belisama Vallis } \\
\text { Bel'kovich }\end{array}$ & $\begin{array}{l}50.0 \mathrm{~N} \\
61.1 \mathrm{~N}\end{array}$ & $\begin{array}{l}22.5 \mathrm{E} \\
90.2 \mathrm{E}\end{array}$ & $\begin{array}{l}220 \\
214\end{array}$ & $\begin{array}{l}\text { EU } \\
\text { UR }\end{array}$ & $\begin{array}{l}\text { EN } \\
\text { SO }\end{array}$ & $\begin{array}{l}5 \\
5\end{array}$ & $\begin{array}{l}1994 \\
1964\end{array}$ & $\begin{array}{r}111 \\
67\end{array}$ \\
\hline L & L & Bell & $21.8 \mathrm{~N}$ & $96.4 \mathrm{~W}$ & 86 & EU & SC & 5 & 1970 & 0 \\
\hline $\begin{array}{l}\text { V } \\
\text { L }\end{array}$ & $\begin{array}{l}\text { V } \\
\mathbf{L}\end{array}$ & $\begin{array}{l}\text { Bell Regio } \\
\text { Bellinsgauzen }\end{array}$ & $\begin{array}{l}32.8 \mathrm{~N} \\
60.6 \mathrm{~S}\end{array}$ & $\begin{array}{l}51.4 \mathrm{E} \\
164.6 \mathrm{~W}\end{array}$ & $\begin{array}{r}1778 \\
63\end{array}$ & $\begin{array}{l}\text { EU } \\
\text { UR }\end{array}$ & $\begin{array}{l}\text { GB } \\
\text { RU }\end{array}$ & $\begin{array}{l}5 \\
5\end{array}$ & $\begin{array}{l}1982 \\
1970\end{array}$ & $\begin{array}{r}47 \\
0\end{array}$ \\
\hline H & $\mathbf{H}$ & Bello & $18.9 \mathrm{~S}$ & $120.0 \mathrm{~W}$ & 129 & SA & VE & 5 & 1976 & 80 \\
\hline $\begin{array}{l}\text { V } \\
\text { L }\end{array}$ & $\begin{array}{l}\text { V } \\
\text { L }\end{array}$ & $\begin{array}{l}\text { Bellona Fossae } \\
\text { Bellot }\end{array}$ & $\begin{array}{l}38.0 \mathrm{~N} \\
12.4 \mathrm{~S}\end{array}$ & $\begin{array}{r}222.1 \mathrm{E} \\
48.2 \mathrm{E}\end{array}$ & $\begin{array}{r}855 \\
17\end{array}$ & $\begin{array}{l}\text { EU } \\
\text { EU }\end{array}$ & $\begin{array}{l}\text { RO } \\
\text { FR }\end{array}$ & $\begin{array}{l}5 \\
5\end{array}$ & $\begin{array}{l}1985 \\
1935\end{array}$ & $\begin{array}{r}1 \\
66\end{array}$ \\
\hline $\begin{array}{l}\mathrm{U} \\
\mathrm{L}\end{array}$ & $\begin{array}{l}\mathrm{ti} \\
\mathrm{L}\end{array}$ & $\begin{array}{l}\text { Belmont Chasma } \\
\text { Belopol'skiy }\end{array}$ & $\begin{array}{r}8.5 S \\
17.2 S\end{array}$ & $\begin{array}{c}32.6 \mathrm{E} \\
128.1 \mathrm{~W}\end{array}$ & $\begin{array}{r}258 \\
59\end{array}$ & $\begin{array}{l}\text { EU } \\
\text { UR }\end{array}$ & $\begin{array}{l}\text { GB } \\
\text { RU }\end{array}$ & $\begin{array}{l}5 \\
5\end{array}$ & $\begin{array}{l}1988 \\
1970\end{array}$ & $\begin{array}{r}85 \\
0\end{array}$ \\
\hline $\begin{array}{l}\mathbf{M} \\
\mathbf{J} \\
\mathbf{L}\end{array}$ & $\begin{array}{l}\text { M } \\
\text { eu } \\
\text { L }\end{array}$ & $\begin{array}{l}\text { Beltra } \\
\text { Belus Linea } \\
\text { Belyaev }\end{array}$ & $\begin{array}{l}18.3 \mathrm{~N} \\
11.8 \mathrm{~N} \\
23.3 \mathrm{~N}\end{array}$ & $\begin{array}{l}257.7 \mathrm{~W} \\
228.3 \mathrm{~W} \\
143.5 \mathrm{E}\end{array}$ & $\begin{array}{r}0 \\
2580 \\
54\end{array}$ & $\begin{array}{l}\text { EU } \\
\text { EU } \\
\text { UR }\end{array}$ & $\begin{array}{l}\text { IR } \\
\text { GR } \\
\text { SO }\end{array}$ & $\begin{array}{l}5 \\
5 \\
5\end{array}$ & $\begin{array}{l}1988 \\
1979 \\
1970\end{array}$ & $\begin{array}{r}36 \\
19 \\
0\end{array}$ \\
\hline $\begin{array}{l}\text { M } \\
\text { V } \\
\text { L }\end{array}$ & $\begin{array}{l}\text { M } \\
\text { V } \\
\text { L }\end{array}$ & $\begin{array}{l}\text { Belz } \\
\text { Ben Dorsa } \\
\text { Bench }\end{array}$ & $\begin{array}{r}22.0 \mathrm{~N} \\
71.2 \mathrm{~N} \\
3.2 \mathrm{~S}\end{array}$ & $\begin{array}{c}43.1 \mathrm{~W} \\
284.1 \mathrm{E} \\
23.4 \mathrm{~W}\end{array}$ & $\begin{array}{r}10 \\
628 \\
0\end{array}$ & $\begin{array}{l}\text { UR } \\
\text { AS } \\
\text { NA }\end{array}$ & $\begin{array}{l}\text { UK } \\
\text { VT } \\
\text { AM }\end{array}$ & $\begin{array}{l}5 \\
5 \\
5\end{array}$ & $\begin{array}{l}1976 \\
1985 \\
1973\end{array}$ & $\begin{array}{l}36 \\
65 \\
60\end{array}$ \\
\hline $\begin{array}{l}\text { M } \\
\mathrm{L}\end{array}$ & $\begin{array}{l}\text { M } \\
\mathbf{L}\end{array}$ & $\begin{array}{l}\text { Bend } \\
\text { Benedict }\end{array}$ & $\begin{array}{r}22.6 \mathrm{~S} \\
4.4 \mathrm{~N}\end{array}$ & $\begin{array}{l}27.5 \mathrm{~W} \\
141.5 \mathrm{E}\end{array}$ & $\begin{array}{r}2 \\
14\end{array}$ & $\begin{array}{l}\text { US } \\
\text { NA }\end{array}$ & $\begin{array}{l}\text { OR } \\
\text { AM }\end{array}$ & $\begin{array}{l}5 \\
5\end{array}$ & $\begin{array}{l}1976 \\
1976\end{array}$ & $\begin{array}{r}36 \\
0\end{array}$ \\
\hline $\begin{array}{l}\text { V } \\
\text { V } \\
M \\
M \\
\text { A } \\
\text { V } \\
\text { S }\end{array}$ & $\begin{array}{l}\text { V } \\
\text { V } \\
M \\
M \\
\text { gs } \\
\text { V } \\
\text { ia }\end{array}$ & $\begin{array}{l}\text { Bennu Vallis } \\
\text { Benten Corona } \\
\text { Bentham } \\
\text { Bentong } \\
\text { Beppu } \\
\text { Bereghinya Planitia } \\
\text { Berenger }\end{array}$ & $\begin{array}{r}1.3 \mathrm{~N} \\
16.0 \mathrm{~N} \\
56.0 \mathrm{~S} \\
22.6 \mathrm{~S} \\
3.9 \mathrm{~N} \\
28.6 \mathrm{~N} \\
62.1 \mathrm{~N}\end{array}$ & $\begin{array}{c}341.2 \mathrm{E} \\
340.0 \mathrm{E} \\
40.3 \mathrm{~W} \\
18.9 \mathrm{~W} \\
58.4 \mathrm{~W} \\
23.6 \mathrm{E} \\
219.7 \mathrm{~W}\end{array}$ & $\begin{array}{r}710 \\
310 \\
0 \\
10 \\
5 \\
3902 \\
84\end{array}$ & $\begin{array}{l}\text { AF } \\
\text { AS } \\
\text { EU } \\
\text { AS } \\
\text { AS } \\
\text { EU } \\
\text { EU }\end{array}$ & $\begin{array}{c}\text { EG } \\
\text { JA } \\
\text { EN } \\
\text { MA } \\
\text { JA } \\
\text { SL } \\
\text { FR }\end{array}$ & $\begin{array}{l}5 \\
5 \\
5 \\
5 \\
5 \\
5 \\
5\end{array}$ & $\begin{array}{l}1994 \\
1994 \\
1991 \\
1976 \\
1994 \\
1985 \\
1982\end{array}$ & $\begin{array}{r}107 \\
83 \\
36 \\
36 \\
113 \\
64 \\
29\end{array}$ \\
\hline $\begin{array}{l}\text { V } \\
\text { L }\end{array}$ & $\begin{array}{l}\text { V } \\
\text { L }\end{array}$ & $\begin{array}{l}\text { Berggolts } \\
\text { Bergman }\end{array}$ & $\begin{array}{r}63.4 \mathrm{~S} \\
7.0 \mathrm{~N}\end{array}$ & $\begin{array}{r}53.0 \mathrm{E} \\
137.5 \mathrm{E}\end{array}$ & $\begin{array}{l}31 \\
21\end{array}$ & $\begin{array}{l}\text { UR } \\
\text { EU }\end{array}$ & $\begin{array}{l}\text { RU } \\
\text { SW }\end{array}$ & $\begin{array}{l}5 \\
5\end{array}$ & $\begin{array}{l}1994 \\
1976\end{array}$ & $\begin{array}{r}107 \\
0\end{array}$ \\
\hline L & L & Bergstrand & $18.8 \mathrm{~S}$ & $176.3 \mathrm{E}$ & 43 & EU & SW & 5 & 1970 & 0 \\
\hline L & L & Berkner & $25.2 \mathrm{~N}$ & $105.2 W$ & 86 & NA & $\mathrm{AM}$ & 5 & 1970 & 0 \\
\hline L & L & Berlage & $63.2 \mathrm{~S}$ & $162.8 \mathrm{~W}$ & 92 & EU & DU & 5 & 1970 & \\
\hline
\end{tabular}

\section{ft origin}

AA Wilhelm; German astronomer (1797-1850).

AA Ludwig van; German composer of Flemish descent (1770-1827).

AA Good spirit who fills Italian children's stockings with toys on twelfth night.

AA Martin; German navigator, cartographer (1436-1506).

AA Aphra; English novelist, poet, playwright (1640-1689).

AA Martinus W.; Dutch botanist (1851-1931).

CR Saami (Lapp) fertility goddess.

AA N. N.; Russian chemist (1827-1911).

AA Slavic female name.

CR Mesopotamian nature/fertility goddess.

AA Celtic; father of Caswallawn.

SS 1986 U5; one whose hair was stolen in Pope's "Rape of the Lock"; $75,258 \mathrm{~km}$ semi-major axis

AA Vissarion Grigoryevich; Russian literary critic and journalist (1811-1848).

VA English Celtic river goddess.

AA Igor V.; Soviet astronomer (1904-1949)

AA Alexander G.; ScottishAmerican inventor (1847-1922).

RE English giantess.

AA Faddey F.; Russian explorer (1778-1852).

AA Andres; Venezuelan poet and scholar (1781-1865).

FO Roman war goddess, wife of Mars.

AA Joseph Rene; French explorer (1826-1853).

CM Location in "As You Like It."

AA Aristarch A.; Russian astronomer (1854-1934)

AA Town in Ireland.

LI Greek; Agenor's twin brother.

AA Pavel I. Soviet cosmonaut (1925-1970).

AA Town in Ukraine.

DO Vietnamese sky goddess.

LF Astronaut-named feature, Apollo 12 site.

AA Town in Oregon, USA.

AA F. G.; American chemist, physiologist (1870-1957).

VA Egyptian word for planet Venus.

CR Japanese love/fertility goddess.

AA Town in England.

AA Town in Malaysia.

AA Spa on Kyushu, Japan.

PL Slavic water spirit.

AA One of twelve peers; killed Estramarin; killed by Grandoyne.

AA Olga; Russian poet (1910-1975).

AA Torbern Olof; Swedish chemist, mineralogist, astronomer (1735-1784).

AA Carl O. E.; Swedish astronomer (1873-1948).

AA Lloyd V.; American geophysicist (1905-1967)

AA Hendrik P.; Dutch geophysicist, meteorologist (1896-1968).

\footnotetext{
P: $\quad$ planetary system (see page $x v i)$

Sa: $\quad$ satellite (see page $x v i)$

lat: latitude of feature center.

long: longitude of feature center.
}

diam: diameter or long dimension of feature.

ct: continent of name origin (see page $284 \mathrm{ff}$.)

et: ethnicity of name origin (see page $284 \mathrm{ff}$.)

as: name approval status (see page xvii). ad: name approval date (year).

ref: reference source for name (see page $287 \mathrm{ff}$.).

ft: feature type (see page 290). 


\section{ALPHABETICAL LIST OF NAMES}

\section{P Sa Name}

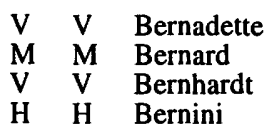

L L [Bernini]

L L Bernouilli

L L Berosus

M M Berseba

V V Berta

V V Beruth Corona

$\mathrm{U}$ ar Berylune

L L Berzelius

J ga Bes

$S$ ia Besgun

L L Bessarion

L L Bessel

V V Beta Regio

V V Bethune Patera

V V Bette

L L Bettinus

V V Beyla Corona

L L Bhabha

$\mathrm{N}$ tr Bheki Cavus

M M Bhor

V V Bhumidevi Corona

V V Bhumiya Corona

$N$ tr Bia Sulci

$\mathrm{U}$ ss Bianca lat long diam

46.75

$23.8 \mathrm{~S}$

$79.2 \mathrm{~S}$

$14.9 \mathrm{~N}$

$35.0 \mathrm{~N}$

$33.5 \mathrm{~N}$

4.45

$62.0 \mathrm{~N}$

$19.0 \mathrm{~S}$

$22.5 \mathrm{~S}$

$285.6 \mathrm{E}$

$154.2 \mathrm{~W}$

84.5E

$136.5 \mathrm{~W}$

diam

ct et

$30.0 \mathrm{E}$

15
129
25
146

EU FR

EU FR

EU IT

as ad

7

EU

IT

$60.7 \mathrm{E}$

47

EU SZ

61984

51985

$36.6 \mathrm{~N}$

$69.9 \mathrm{E}$

74

AS BY

51935

$37.7 \mathrm{~W}$

$322.0 \mathrm{E}$

233.5E

36

36
20
350

AF NM

EU $\quad$ FI

AS PO

51976

51985

$\begin{array}{ll}5 & 1994\end{array}$

29

EU FR

1988

25.8S $180.4 \mathrm{~W}$

$76.0 \mathrm{~N} \quad 309.8 \mathrm{~W}$

50 EU SW

51935

0 AF EG

51985

56 EU FR

$\begin{array}{ll}5 & 1982\end{array}$

$14.9 \mathrm{~N} \quad 37.3 \mathrm{~W}$

$21.8 \mathrm{~N} \quad 17.9 \mathrm{E}$

10

EU GR

51935

15

EU GE

51935

$25.3 \mathrm{~N} \quad 282.8 \mathrm{E}$

$46.5 \mathrm{~N} \quad 321.3 \mathrm{E}$

2869

EU GR

51979

24.6S 347.9E

63.4

$44.8 \mathrm{~W}$

6

EU GE

51994

$25.0 \mathrm{~N}$

$15.5 \mathrm{E}$

$30.4 \mathrm{~N} \quad 36.5 \mathrm{E}$

$55.1 \mathrm{~S} \quad 164.5 \mathrm{~W}$

71

EU IT

51935

400

807

EU NS

UR LI

$\begin{array}{lr}5 & 91 \\ 5 & 1985\end{array}$

51985

$16.0 \mathrm{~N} \quad 308.0 \mathrm{E}$

$42.0 \mathrm{~N} 225.5 \mathrm{~W}$

17.2S $343.6 \mathrm{E}$

$15.0 \mathrm{~N} \quad 118.0 \mathrm{E}$

38.0S

$3.0 \mathrm{E}$

$0.0 \mathrm{~N} \quad 0.0 \mathrm{X}$

$$
\begin{array}{r}
0 \\
200 \\
100
\end{array}
$$

AS

$$
\text { IN }
$$$$
591
$$

6

AS

AS

$$
\text { IN }
$$

$\begin{array}{ll}5 & 1979\end{array}$

$\begin{array}{ll}5 & 1994\end{array}$

100 AS

1994

0

EU

$5 \quad 1988$

$\begin{array}{rrrrrrrr}64.2 \mathrm{~S} & 95.1 \mathrm{~W} & 77 & \text { EU } & \text { IT } & 5 & 1973 & 68 \\ 48.7 \mathrm{~N} & 34.3 \mathrm{~W} & 38 & \text { EU } & \text { IT } & 5 & 1935 & 66 \\ 2.3 \mathrm{~N} & 123.8 \mathrm{~W} & 117 & \text { EU } & \text { LA } & 5 & 1973 & 57 \\ 82.0 \mathrm{~S} & 170.8 \mathrm{E} & 39 & \text { NA } & \text { AM } & 5 & 1994 & 99 \\ 54.9 \mathrm{~S} & 51.3 \mathrm{E} & 76 & \text { EU } & \text { AS } & 5 & 1935 & 66 \\ 25.0 \mathrm{~S} & 34.6 \mathrm{~W} & 18 & \text { US } & \text { MS } & 5 & 1976 & 36 \\ 5.8 \mathrm{~S} & 56.3 \mathrm{E} & 43 & \text { EU } & \text { GE } & 5 & 1976 & 0 \\ 13.8 \mathrm{~S} & 50.1 \mathrm{~W} & 45 & \text { EU } & \text { FR } & 5 & 1935 & 66 \\ 8.1 \mathrm{~N} & 115.1 \mathrm{E} & 33 & \text { NA } & \text { AM } & 5 & 1976 & 0 \\ 22.6 \mathrm{~S} & 51.1 \mathrm{E} & 12 & \text { EU } & \text { FR } & 5 & 1935 & 66 \\ 25.3 \mathrm{~N} & 45.9 \mathrm{~W} & 0 & \text { UR } & \text { RU } & 5 & 1988 & 0 \\ 30.2 \mathrm{~S} & 173.9 \mathrm{E} & 82 & \text { EU } & \text { NO } & 5 & 1970 & 0 \\ 58.7 \mathrm{~N} & 146.1 \mathrm{~W} & 345 & \text { NA } & \text { AM } & 5 & 1970 & 0 \\ 65.1 \mathrm{~N} & 10.5 \mathrm{~W} & 92 & \text { EU } & \text { IR } & 5 & 1935 & 66\end{array}$

\section{ft origin}

AA French first name.

AA P.; French atmospheric scientist.

AA Sarah; French actress (1844-1923).

AA Gian Lorenzo; Italian sculptor and architect (1598-1680).

AA Gianlorenzo; Italian artist (1598-1680).

AA Jacques; Swiss mathematician (1654-1705); Jean; Swiss mathematician (1667-1748).

AA Berosus the Chaldean; Babylonian astronomer (unkn-c. 250 B.C.)

AA Town in Namibia.

AA Finnish first name.

CR Phoenician earth goddess.

AA Good spirit in Maeterlinck's "The Bluebird."

AA Jons Jakob; Swedish chemist (1779-1848).

AA Egyptian god of marriage.

AA Chief cook for Charlemagne's army; he guarded Ganelon after Ganelon's treachery was discovered.

AA Johannes; Greek scholar (c. 1369-1472).

AA Friedrich Wilhelm; German astronomer (1784-1846).

RE Second letter in Greek alphabet.

PE Mary; American educator (1875-1955).

AA German first name (form of Elizabeth).

AA Mario; Italian mathematician, astronomer (1582-1657).

CR Norse earth goddess.

DO Lithuanian evening light goddess.

AA Homi J.; Indian physicist (1909-1966).

CB Frog symbolizing the sun on the horizon (India).

AA Town in India.

CR Hindu earth goddess.

CR Hindu earth goddess.

SU Yoruba; river named for obedient son of god.

SS 1986 U9; other daughter to Baptista in "The Taming of the Shrew"; $59,173 \mathrm{~km}$ semi-major axis

AA Francesco; Italian astronomer (1662-1729).

AA Francesco; Italian astronomer (1662-1729).

PE Classical albedo feature name.

AA Mary; American Civil War nurse (1817-1901).

AA Wilhelm von; Austrian astronomer (1782-1856).

AA Town in Mississippi, USA.

AA T.; German doctor (1825-1862).

AA Jacques de; French mathematician (1602-1679)

AA H.; American explorer (1875-1956).

AA Jean-Baptiste; French astronomer (1774-1862)

AA Town in Russia.

AA Olaf K.; Norwegian physicist (1867-1917).

AA George D.; American mathematician (1884-1944)

AA John; Irish astronomer (1829-1884).
P: $\quad$ planetary system (see page xvi).

Sa: $\quad$ satellite (see page $\mathrm{xvi}$ ).

lat: latitude of feature center.

long: longitude of feature center. diam: diameter or long dimension of feature. ct: continent of name origin (see page $284 \mathrm{ff}$.)

et: ethnicity of name origin (see page $284 \mathrm{ff}$.)

as: name approval status (see page xvii). ad: name approval date (year).

ref: reference source for name (see page $287 \mathrm{ff}$.).

ft: feature type (see page 290 ). 


\section{ALPHABETICAL LIST OF NAMES}

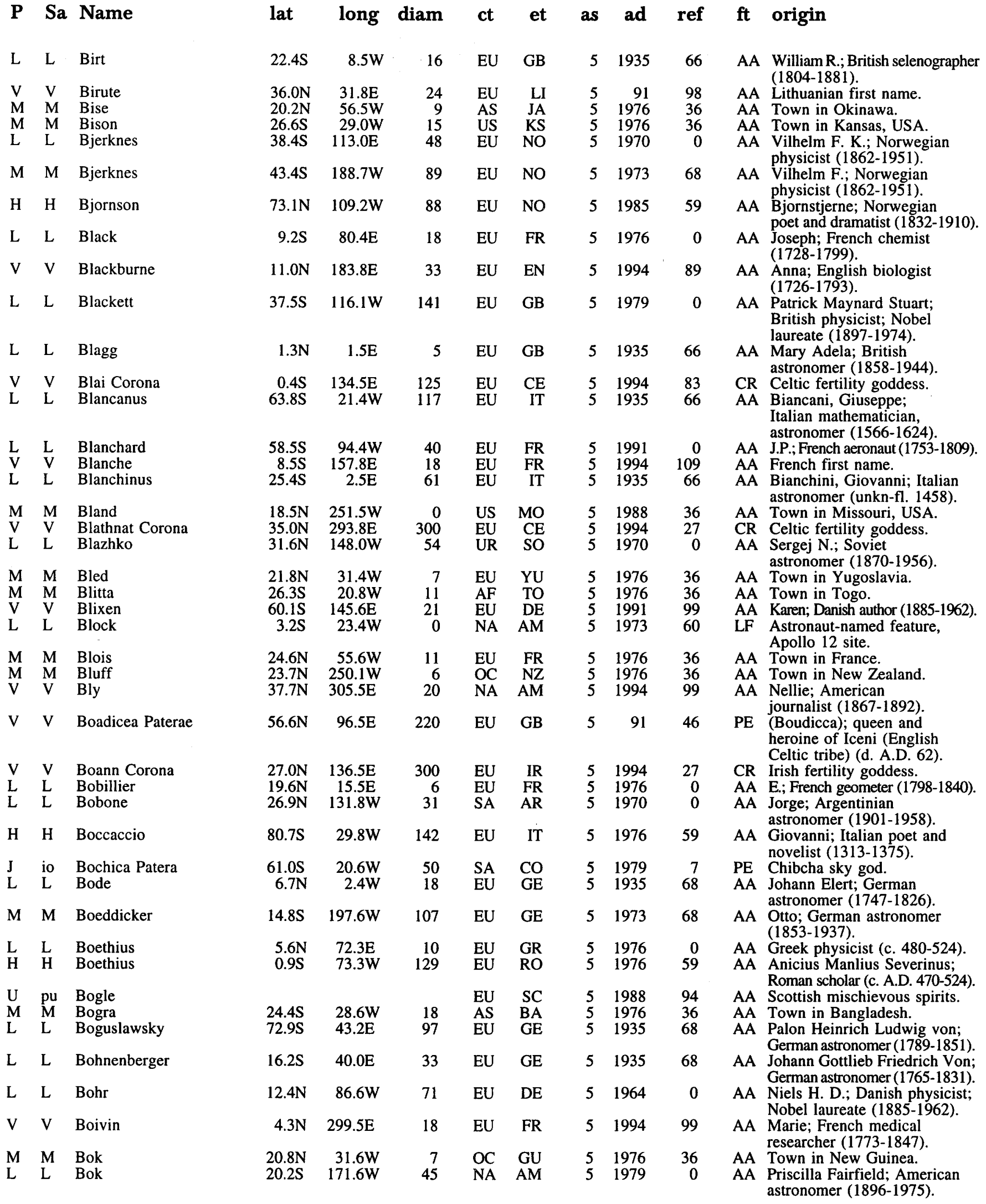

\footnotetext{
P: $\quad$ planetary system (see page $x v i$ ).

Sa: satellite (see page xvi)

lat: latitude of feature center.

long: longitude of feature center.
}

diam: diameter or long dimension of feature.

$\begin{array}{ll}\text { ct: } & \text { continent of name origin (see page } 284 \mathrm{ff} \text {.) } \\ \text { et: } & \text { ethnicity of name origin (see page } 284 \mathrm{ff} \text {.) }\end{array}$

as: name approval status (see page xvii). ad: name approval date (year).

ref: reference source for name (see page $287 \mathrm{ff}$.)

ft: feature type (see page 290). 


\begin{tabular}{|c|c|c|c|c|c|c|c|c|c|}
\hline $\mathbf{P}$ & $\mathbf{S a}$ & Name & lat & long & diam & ct & et & as & ad \\
\hline $\begin{array}{l}\mathrm{M} \\
\mathrm{V} \\
\mathrm{L}\end{array}$ & $\begin{array}{l}\mathrm{M} \\
\mathrm{V} \\
\mathrm{L}\end{array}$ & $\begin{array}{l}\text { Bole } \\
\text { Boleyn } \\
\text { Boltzmann }\end{array}$ & $\begin{array}{l}25.4 \mathrm{~N} \\
24.4 \mathrm{~N} \\
74.9 \mathrm{~S}\end{array}$ & $\begin{array}{c}53.7 \mathrm{~W} \\
219.9 \mathrm{E} \\
90.7 \mathrm{~W}\end{array}$ & $\begin{array}{r}8 \\
69 \\
76\end{array}$ & $\begin{array}{l}\mathrm{AF} \\
\mathrm{EU} \\
\mathrm{EU}\end{array}$ & $\begin{array}{l}\text { GH } \\
\text { EN } \\
\text { AS }\end{array}$ & $\begin{array}{l}5 \\
5 \\
5\end{array}$ & $\begin{array}{l}1976 \\
1994 \\
1964\end{array}$ \\
\hline L & $\mathbf{L}$ & Bolyai & $33.6 \mathrm{~S}$ & $125.9 \mathrm{E}$ & 135 & EU & $\mathrm{HU}$ & 5 & 1970 \\
\hline $\begin{array}{l}\mathrm{M} \\
\mathrm{L}\end{array}$ & $\begin{array}{l}\mathrm{M} \\
\mathrm{L}\end{array}$ & $\begin{array}{l}\text { Bombala } \\
\text { Bombelli }\end{array}$ & $\begin{array}{r}30.3 \mathrm{~S} \\
5.3 \mathrm{~N}\end{array}$ & $\begin{array}{c}253.9 \mathrm{~W} \\
56.2 \mathrm{E}\end{array}$ & $\begin{array}{r}0 \\
10\end{array}$ & $\begin{array}{l}\text { OC } \\
\text { EU }\end{array}$ & AU & $\begin{array}{l}5 \\
5\end{array}$ & $\begin{array}{l}1989 \\
1976\end{array}$ \\
\hline $\mathrm{U}$ & $\mathrm{ti}$ & Bona & $55.8 \mathrm{~S}$ & $351.2 \mathrm{E}$ & 51 & $\mathrm{EU}$ & GB & 5 & 1988 \\
\hline $\begin{array}{l}\mathrm{V} \\
\mathrm{M}\end{array}$ & $\begin{array}{l}\mathrm{V} \\
\mathrm{M}\end{array}$ & $\begin{array}{l}\text { Bona Corona } \\
\text { Bond }\end{array}$ & $\begin{array}{l}24.0 S \\
33.3 S\end{array}$ & $\begin{array}{c}157.5 \mathrm{E} \\
35.7 \mathrm{~W}\end{array}$ & $\begin{array}{l}275 \\
104\end{array}$ & $\begin{array}{l}\text { EU } \\
\text { NA }\end{array}$ & $\begin{array}{l}\text { RM } \\
\text { AM }\end{array}$ & $\begin{array}{l}5 \\
5\end{array}$ & $\begin{array}{l}1994 \\
1973\end{array}$ \\
\hline $\mathbf{L}$ & $\mathrm{L}$ & Bondarenko & $17.8 \mathrm{~S}$ & $136.3 \mathrm{E}$ & 30 & UR & so & 5 & 1991 \\
\hline$S$ & hy & Bond-Lassell Dorsum & $48.0 \mathrm{~N}$ & $143.5 \mathrm{~W}$ & 0 & NA & AM & 5 & 1982 \\
\hline $\begin{array}{l}\mathrm{V} \\
\mathrm{V}\end{array}$ & $\begin{array}{l}\mathrm{V} \\
\mathrm{V}\end{array}$ & $\begin{array}{l}\text { Bonnevie } \\
\text { Bonnin }\end{array}$ & $\begin{array}{r}36.1 \mathrm{~S} \\
6.2 \mathrm{~S}\end{array}$ & $\begin{array}{l}127.0 \mathrm{E} \\
117.6 \mathrm{E}\end{array}$ & $\begin{array}{l}91 \\
40\end{array}$ & $\begin{array}{l}\text { EU } \\
\text { NA }\end{array}$ & $\begin{array}{l}\text { NO } \\
\text { AM }\end{array}$ & $\begin{array}{l}5 \\
5\end{array}$ & $\begin{array}{l}1991 \\
1994\end{array}$ \\
\hline $\mathbf{L}$ & $\mathrm{L}$ & Bonpland & $8.3 \mathrm{~S}$ & $17.4 \mathrm{~W}$ & 60 & EU & FR & 5 & 1935 \\
\hline $\mathbf{L}$ & $\mathrm{L}$ & Boole & $63.7 \mathrm{~N}$ & $87.4 \mathrm{~W}$ & 63 & EU & GB & 5 & 1964 \\
\hline $\begin{array}{l}\mathrm{J} \\
\mathrm{M} \\
\mathrm{L}\end{array}$ & $\begin{array}{l}\text { io } \\
\mathrm{M} \\
\mathrm{L}\end{array}$ & $\begin{array}{l}\text { Boösaule Montes } \\
\text { Bor } \\
\text { Borda }\end{array}$ & $\begin{array}{l}4.4 \mathrm{~S} \\
18.1 \mathrm{~N} \\
25.1 \mathrm{~S}\end{array}$ & $\begin{array}{c}270.1 \mathrm{~W} \\
33.8 \mathrm{~W} \\
46.6 \mathrm{E}\end{array}$ & $\begin{array}{r}590 \\
4 \\
44\end{array}$ & $\begin{array}{l}\text { EU } \\
\text { UR } \\
\text { EU }\end{array}$ & $\begin{array}{l}\text { GR } \\
\text { RU } \\
\text { FR }\end{array}$ & $\begin{array}{l}5 \\
5 \\
5\end{array}$ & $\begin{array}{l}1985 \\
1976 \\
1935\end{array}$ \\
\hline $\begin{array}{l}\mathrm{M} \\
\mathrm{H}\end{array}$ & $\begin{array}{l}\mathrm{M} \\
\mathrm{H}\end{array}$ & $\begin{array}{l}\text { Bordeaux } \\
\text { Borea }\end{array}$ & $\begin{array}{l}23.4 \mathrm{~N} \\
75.0 \mathrm{~N}\end{array}$ & $\begin{array}{r}48.9 \mathrm{~W} \\
0.0 \mathrm{~W}\end{array}$ & $\begin{array}{l}2 \\
0\end{array}$ & $\begin{array}{l}\text { EU } \\
\text { EU }\end{array}$ & $\begin{array}{l}\text { FR } \\
\text { RM }\end{array}$ & $\begin{array}{l}5 \\
5\end{array}$ & $\begin{array}{l}1979 \\
1976\end{array}$ \\
\hline H & $\mathrm{H}$ & Borealis Planitia & $73.4 \mathrm{~N}$ & $79.5 \mathrm{~W}$ & 0 & EU & LA & 5 & 1976 \\
\hline $\mathrm{L}$ & $\mathrm{L}$ & Borel & $22.3 \mathrm{~N}$ & $26.4 \mathrm{E}$ & 4 . & $\mathrm{EU}$ & FR & 5 & 1976 \\
\hline $\mathbf{M}$ & M & Boreosyrtis & $55.0 \mathrm{~N}$ & $290.0 \mathrm{~W}$ & 0 & EU & LA & 5 & 1958 \\
\hline $\begin{array}{l}\mathrm{L} \\
\mathrm{L}\end{array}$ & $\begin{array}{l}\mathrm{L} \\
\mathrm{L}\end{array}$ & $\begin{array}{l}\text { Boris } \\
\text { Borman }\end{array}$ & $\begin{array}{l}30.6 \mathrm{~N} \\
38.8 \mathrm{~S}\end{array}$ & $\begin{array}{r}33.5 \mathrm{~W} \\
147.7 \mathrm{~W}\end{array}$ & $\begin{array}{l}10 \\
50\end{array}$ & $\begin{array}{l}\text { UR } \\
\text { NA }\end{array}$ & $\begin{array}{l}\text { RU } \\
\text { AM }\end{array}$ & $\begin{array}{l}5 \\
5\end{array}$ & $\begin{array}{l}1979 \\
1970\end{array}$ \\
\hline L & L & Born & $6.0 \mathrm{~S}$ & $66.8 \mathrm{E}$ & 14 & $\mathrm{EU}$ & GE & 5 & 1979 \\
\hline $\mathbf{S}$ & $\mathrm{mi}$ & Bors & $39.0 \mathrm{~N}$ & $166.0 \mathrm{~W}$ & 0 & $\mathrm{EU}$ & GB & 5 & 1982 \\
\hline $\begin{array}{l}\mathrm{M} \\
\mathrm{L}\end{array}$ & $\begin{array}{l}\mathrm{M} \\
\mathrm{L}\end{array}$ & $\begin{array}{l}\text { Boru } \\
\text { Boscovich }\end{array}$ & $\begin{array}{c}24.6 \mathrm{~S} \\
9.8 \mathrm{~N}\end{array}$ & $\begin{array}{l}27.7 \mathrm{~W} \\
11.1 \mathrm{E}\end{array}$ & $\begin{array}{l}11 \\
46\end{array}$ & $\begin{array}{l}\text { UR } \\
\text { EU }\end{array}$ & $\underset{\text { IT }}{\text { RU }}$ & $\begin{array}{l}5 \\
5\end{array}$ & $\begin{array}{l}1976 \\
1935\end{array}$ \\
\hline $\mathrm{L}$ & L & Bose & $53.5 \mathrm{~S}$ & $168.6 \mathrm{~W}$ & 91 & AS & IN & 5 & 1970 \\
\hline $\begin{array}{l}M \\
M \\
L\end{array}$ & $\begin{array}{l}M \\
M \\
L\end{array}$ & $\begin{array}{l}\text { Bosporos Planum } \\
\text { Bosporos Rupes } \\
\text { Boss }\end{array}$ & $\begin{array}{l}33.4 \mathrm{~S} \\
42.8 \mathrm{~S} \\
45.8 \mathrm{~N}\end{array}$ & $\begin{array}{l}64.0 \mathrm{~W} \\
57.2 \mathrm{~W} \\
89.2 \mathrm{E}\end{array}$ & $\begin{array}{r}330 \\
500 \\
47\end{array}$ & $\begin{array}{l}\text { EU } \\
\text { EU } \\
\text { NA }\end{array}$ & $\begin{array}{l}\text { GR } \\
\text { GR } \\
\text { AM }\end{array}$ & $\begin{array}{l}5 \\
5 \\
5\end{array}$ & $\begin{array}{l}1979 \\
1976 \\
1964\end{array}$ \\
\hline $\begin{array}{l}\mathrm{H} \\
\mathrm{L}\end{array}$ & $\begin{array}{l}\mathrm{H} \\
\mathrm{L}\end{array}$ & $\begin{array}{l}\text { Botticelli } \\
\text { Bouguer }\end{array}$ & $\begin{array}{l}63.7 \mathrm{~N} \\
52.3 \mathrm{~N}\end{array}$ & $\begin{array}{r}109.6 \mathrm{~W} \\
35.8 \mathrm{~W}\end{array}$ & $\begin{array}{r}143 \\
22\end{array}$ & $\begin{array}{l}\mathrm{EU} \\
\mathrm{EU}\end{array}$ & $\begin{array}{l}\text { IT } \\
\text { FR }\end{array}$ & $\begin{array}{l}5 \\
5\end{array}$ & $\begin{array}{l}1979 \\
1935\end{array}$ \\
\hline M & $\mathbf{M}$ & Bouguer & $18.6 \mathrm{~S}$ & $332.8 \mathrm{~W}$ & 106 & $\mathrm{EU}$ & FR & 5 & 1973 \\
\hline V & V & Boulanger & $26.5 S$ & $99.3 \mathrm{E}$ & 62 & $\mathrm{EU}$ & FR & 5 & 1991 \\
\hline $\begin{array}{l}M \\
V\end{array}$ & $\begin{array}{l}\mathrm{M} \\
\mathrm{V}\end{array}$ & $\begin{array}{l}\text { Boulia } \\
\text { Bourke-White }\end{array}$ & $\begin{array}{l}23.1 \mathrm{~S} \\
21.2 \mathrm{~N}\end{array}$ & $\begin{array}{l}248.6 \mathrm{~W} \\
147.9 \mathrm{E}\end{array}$ & $\begin{array}{r}0 \\
35\end{array}$ & $\begin{array}{l}\text { OC } \\
\text { NA }\end{array}$ & $\begin{array}{l}\mathrm{AU} \\
\mathrm{AM}\end{array}$ & $\begin{array}{l}5 \\
5\end{array}$ & $\begin{array}{l}1989 \\
1991\end{array}$ \\
\hline L & $\mathrm{L}$ & Boussingault & $70.2 S$ & $54.6 \mathrm{E}$ & 142 & EU & FR & 5 & 1935 \\
\hline $\mathbf{L}$ & L & Bowditch & $25.0 \mathrm{~S}$ & $103.1 \mathrm{E}$ & 40 & NA & $\mathrm{AM}$ & 5 & 1976 \\
\hline
\end{tabular}

\section{$\mathrm{ft}$ origin}

AA Town in Ghana.

AA Ann; English queen (1507-1536).

AA Ludwig E.; Austrian physicist (1844-1906).

AA Janos; Hungarian mathematician (1802-1860).

AA Town in New S. Wales, Australia.

AA R.; Italian mathematician (1526-1572).

AA Sister of the French queen in "Henry VI, part 3."

CR Roman virgin/fertility goddess.

AA George P.; American astronomer (1825-1865)

AA V. V.; Soviet student-cosmonaut (1937-1961)

DO G.P. Bond (American) and William Lassell (British); discovered Hyperion on the same night in 1848 .

AA Norwegian biologist.

AA Gertrude (Zitkala-sa) Dakota reformer, writer (1875-1938).

AA Aime; French botanist (1773-1858).

AA George; British mathematician (1815-1864).

MO Cave where Io bore Epaphus.

AA Town in Russia.

AA Jean Charles; French astronomer (1733-1799).

AA French port.

AL Albedo name for $\mathrm{H}-1$, Borealis region.

PL "Northern plain"; from classical albedo name for quadrangle.

AA Felix Edouard Emile; French mathematician (1871-1956).

AL Northern continuation of Nilosyrtis; "Syrtis of the north."

AA Russian male name.

AA Frank; American astronaut, engineer (1928-Live).

AA Max; German physicist (1882-1970)

AA King of Gaul; father of Sir Ector de Marys, Sir Bors, Sir Lyonel.

AA Town in Russia.

AA Ruggiero Giuseppe; Italian physicist (1711-1787).

AA Jagadis C.; Indian botanist, physicist (1858-1937).

PM Classical albedo feature name.

RU Classical albedo feature name.

AA Lewis; American astronomer (1846-1912).

AA Sandro; Italian painter (1445-1510).

AA Pierre; French hydrographer (1698-1758)

AA Pierre; French physicist-hydrographer (1698-1758).

AA Nadia; French pianist, composer (1881-1979)

AA Town in Queensland, Australia.

AA Margaret; American photo-journalist (1905-1971).

AA Jean Baptiste Dieudonne; French chemist (1802-1887).

AA Nathaniel; American astronomer, mathematician (1773-1848).

\footnotetext{
P: $\quad$ planetary system (see page $\mathrm{xvi}$ )

Sa: $\quad$ satellite (see page $\mathrm{xvi}$ ).

lat: latitude of feature center.

long: longitude of feature center.
}

diam: diameter or long dimension of feature.

ct: continent of name origin (see page $284 \mathrm{ff}$.)

et: ethnicity of name origin (see page $284 \mathrm{ff}$.)

as: name approval status (see page xvii). ad: name approval date (year).

ref: reference source for name (see page $287 \mathrm{ff}$.). ft: feature type (see page 290). 


\section{ALPHABETICAL LIST OF NAMES}

\begin{tabular}{|c|c|c|c|c|c|c|c|c|c|}
\hline $\mathbf{P}$ & $\mathbf{S a}$ & Name & lat & long & diam & ct & et & as & ad \\
\hline $\mathrm{L}$ & L & Bowen & $17.6 \mathrm{~N}$ & $9.1 \mathrm{E}$ & 8 & NA & $\mathrm{AM}$ & 5 & 1973 \\
\hline $\mathrm{L}$ & $\mathbf{L}$ & Bowen-Apollo & $20.3 \mathrm{~N}$ & $30.9 \mathrm{E}$ & 0 & NA & $\mathrm{AM}$ & 5 & 1973 \\
\hline V & V & Boyd & $39.3 \mathrm{~S}$ & $221.3 \mathrm{E}$ & 25 & NA & AM & 5 & 1994 \\
\hline V & V & Boye & $9.6 \mathrm{~S}$ & $292.3 \mathrm{E}$ & 30 & $\mathrm{EU}$ & SW & 5 & 1994 \\
\hline $\mathrm{L}$ & $\mathbf{L}$ & Boyle & $53.1 \mathrm{~S}$ & $178.1 \mathrm{E}$ & 57 & EU & GB & 5 & 1970 \\
\hline $\begin{array}{l}\mathrm{N} \\
\mathrm{M} \\
\mathrm{L}\end{array}$ & $\begin{array}{l}\mathrm{tr} \\
\mathrm{M} \\
\mathrm{L}\end{array}$ & $\begin{array}{l}\text { Boynne Sulci } \\
\text { Bozkir } \\
\text { Brackett }\end{array}$ & $\begin{array}{l}13.0 \mathrm{~S} \\
44.4 \mathrm{~S} \\
17.9 \mathrm{~N}\end{array}$ & $\begin{array}{l}350.0 \mathrm{E} \\
32.0 \mathrm{~W} \\
23.6 \mathrm{E}\end{array}$ & $\begin{array}{r}0 \\
89 \\
8\end{array}$ & $\begin{array}{l}\text { EU } \\
\text { AS } \\
\text { NA }\end{array}$ & $\begin{array}{l}\text { CE } \\
\text { TU } \\
\text { AM }\end{array}$ & $\begin{array}{l}5 \\
5 \\
5\end{array}$ & $\begin{array}{r}91 \\
1976 \\
1973\end{array}$ \\
\hline V & V & Bradstreet & $16.5 \mathrm{~N}$ & $47.6 \mathrm{E}$ & 39 & NA & AM & 5 & 1994 \\
\hline L & $\mathrm{L}$ & Bragg & $42.5 \mathrm{~N}$ & $102.9 \mathrm{~W}$ & 84 & OC & $\mathrm{AU}$ & 5 & 1970 \\
\hline $\begin{array}{l}\text { J } \\
\mathbf{H}\end{array}$ & $\begin{array}{l}\mathrm{ca} \\
\mathrm{H}\end{array}$ & $\begin{array}{l}\text { Bragi } \\
\text { Brahms }\end{array}$ & $\begin{array}{l}75.7 \mathrm{~N} \\
58.5 \mathrm{~N}\end{array}$ & $\begin{array}{r}61.7 \mathrm{~W} \\
176.2 \mathrm{~W}\end{array}$ & $\begin{array}{l}65 \\
96\end{array}$ & $\begin{array}{l}\text { EU } \\
\text { EU }\end{array}$ & $\begin{array}{l}\text { NS } \\
\text { GE }\end{array}$ & $\begin{array}{l}5 \\
5\end{array}$ & $\begin{array}{l}1979 \\
1979\end{array}$ \\
\hline H & $\mathrm{H}$ & Bramante & $47.5 \mathrm{~S}$ & $61.8 \mathrm{~W}$ & 159 & $\mathrm{EU}$ & IT & 5 & 1976 \\
\hline J & $\begin{array}{l}\text { ca } \\
\text { ca }\end{array}$ & $\begin{array}{l}\text { Brami } \\
\text { Bran }\end{array}$ & $\begin{array}{l}28.9 \mathrm{~N} \\
24.3 \mathrm{~S}\end{array}$ & $\begin{array}{r}19.2 \mathrm{~W} \\
207.7 \mathrm{~W}\end{array}$ & $\begin{array}{l}67 \\
89\end{array}$ & $\begin{array}{l}\mathrm{EU} \\
\mathrm{EU}\end{array}$ & $\begin{array}{l}\mathrm{NS} \\
\mathrm{CE}\end{array}$ & $\begin{array}{l}5 \\
5\end{array}$ & $\begin{array}{l}1979 \\
1979\end{array}$ \\
\hline $\mathbf{L}$ & $\mathrm{L}$ & Brashear & $73.8 \mathrm{~S}$ & $170.7 \mathrm{~W}$ & 55 & NA & $\mathrm{AM}$ & 5 & 1970 \\
\hline $\mathbf{M}$ & $\mathbf{M}$ & Brashear & $54.1 \mathrm{~S}$ & $119.2 \mathrm{~W}$ & 126 & NA & $\mathrm{AM}$ & 5 & 1973 \\
\hline $\mathrm{L}$ & $\mathrm{L}$ & Brayley & $20.9 \mathrm{~N}$ & $36.9 \mathrm{~W}$ & 14 & EU & GB & 5 & 1935 \\
\hline $\begin{array}{l}\mathrm{M} \\
\mathrm{L}\end{array}$ & $\begin{array}{l}\mathrm{M} \\
\mathrm{L}\end{array}$ & $\begin{array}{l}\text { Brazos Valles } \\
\text { Bredikhin }\end{array}$ & $\begin{array}{c}6.3 \mathrm{~S} \\
17.3 \mathrm{~N}\end{array}$ & $\begin{array}{l}341.7 W \\
158.2 W\end{array}$ & $\begin{array}{r}494 \\
59\end{array}$ & $\begin{array}{l}\text { US } \\
\text { UR }\end{array}$ & $\begin{array}{l}\text { TX } \\
\text { RU }\end{array}$ & $\begin{array}{l}5 \\
5\end{array}$ & $\begin{array}{l}1982 \\
1970\end{array}$ \\
\hline L & $\mathrm{L}$ & Breislak & $48.2 \mathrm{~S}$ & $18.3 \mathrm{E}$ & 49 & $\mathrm{EU}$ & IT & 5 & 1935 \\
\hline $\begin{array}{l}\mathrm{V} \\
\mathrm{V}\end{array}$ & $\begin{array}{l}\mathrm{V} \\
\mathrm{V}\end{array}$ & $\begin{array}{l}\text { Breksta Dorsa } \\
\text { Bremer Patera }\end{array}$ & $\begin{array}{l}35.9 \mathrm{~N} \\
66.8 \mathrm{~N}\end{array}$ & $\begin{array}{r}304.0 \mathrm{E} \\
63.7 \mathrm{E}\end{array}$ & $\begin{array}{r}700 \\
91\end{array}$ & $\begin{array}{l}\text { UR } \\
\text { EU }\end{array}$ & LI & $\begin{array}{l}5 \\
5\end{array}$ & $\begin{array}{r}1985 \\
91\end{array}$ \\
\hline$\underset{L}{M}$ & $\begin{array}{l}\mathrm{M} \\
\mathrm{L}\end{array}$ & $\begin{array}{l}\text { Bremerhaven } \\
\text { Brenner }\end{array}$ & $\begin{array}{l}23.5 \mathrm{~N} \\
39.0 \mathrm{~S}\end{array}$ & $\begin{array}{l}48.6 \mathrm{~W} \\
39.3 \mathrm{E}\end{array}$ & $\begin{array}{r}2 \\
97\end{array}$ & $\begin{array}{l}\text { EU } \\
\text { EU }\end{array}$ & $\begin{array}{l}\text { GE } \\
\text { AS }\end{array}$ & $\begin{array}{l}5 \\
5\end{array}$ & $\begin{array}{l}1979 \\
1935\end{array}$ \\
\hline L & $\mathrm{L}$ & Brewster & $23.3 \mathrm{~N}$ & $34.7 \mathrm{E}$ & 10 & $\mathrm{EU}$ & $\mathrm{SC}$ & 5 & 1976 \\
\hline L & $\mathbf{L}$ & Brianchon & $75.0 \mathrm{~N}$ & $86.2 W$ & 134 & EU & FR & 5 & 1964 \\
\hline $\begin{array}{l}\mathrm{M} \\
\mathrm{L}\end{array}$ & $\begin{array}{l}\mathrm{M} \\
\mathrm{L}\end{array}$ & $\begin{array}{l}\text { Briault } \\
\text { Bridge }\end{array}$ & $\begin{array}{l}10.1 S \\
26.0 \mathrm{~N}\end{array}$ & $\begin{array}{c}270.2 \mathrm{~W} \\
3.6 \mathrm{E}\end{array}$ & $\begin{array}{r}100 \\
1\end{array}$ & $\begin{array}{l}\text { EU } \\
\text { NA }\end{array}$ & $\begin{array}{r}\text { FR } \\
\text { AM }\end{array}$ & $\begin{array}{l}5 \\
5\end{array}$ & $\begin{array}{l}1973 \\
1973\end{array}$ \\
\hline $\begin{array}{l}\text { M } \\
\text { V } \\
\mathrm{L}\end{array}$ & $\begin{array}{l}\mathrm{M} \\
\mathrm{V} \\
\mathrm{L}\end{array}$ & $\begin{array}{l}\text { Bridgetown } \\
\text { Bridgit } \\
\text { Bridgman }\end{array}$ & $\begin{array}{l}22.2 \mathrm{~N} \\
45.3 \mathrm{~S} \\
43.5 \mathrm{~N}\end{array}$ & $\begin{array}{l}47.1 \mathrm{~W} \\
348.8 \mathrm{E} \\
137.1 \mathrm{E}\end{array}$ & $\begin{array}{r}2 \\
11 \\
80\end{array}$ & $\begin{array}{l}\text { SA } \\
\text { EU } \\
\text { NA }\end{array}$ & $\begin{array}{r}\text { BB } \\
\text { IR } \\
\text { AM }\end{array}$ & $\begin{array}{l}5 \\
5 \\
5\end{array}$ & $\begin{array}{l}1979 \\
1991 \\
1970\end{array}$ \\
\hline L & $\mathrm{L}$ & Briggs & $26.5 \mathrm{~N}$ & $69.1 \mathrm{~W}$ & 37 & $\mathrm{EU}$ & GB & 5 & 1935 \\
\hline V & V & Brigit Tholus & $49.0 \mathrm{~N}$ & $246.0 \mathrm{E}$ & 0 & $\mathrm{EU}$ & $\mathrm{CE}$ & 5 & 1985 \\
\hline $\mathbf{L}$ & $\mathrm{L}$ & Brisbane & $49.1 \mathrm{~S}$ & $68.5 \mathrm{E}$ & 44 & $\mathrm{EU}$ & SC & 5 & 1935 \\
\hline $\begin{array}{l}\mathrm{M} \\
\mathrm{V} \\
\mathrm{M} \\
\mathrm{L}\end{array}$ & $\begin{array}{l}M \\
V \\
M \\
L\end{array}$ & $\begin{array}{l}\text { Bristol } \\
\text { Britomartis Chasma } \\
\text { Broach } \\
\text { Bronk }\end{array}$ & $\begin{array}{l}22.4 \mathrm{~N} \\
33.0 \mathrm{~S} \\
23.5 \mathrm{~N} \\
26.1 \mathrm{~N}\end{array}$ & $\begin{array}{c}47.0 \mathrm{~W} \\
130.0 \mathrm{E} \\
56.6 \mathrm{~W} \\
134.5 \mathrm{~W}\end{array}$ & $\begin{array}{r}3 \\
0 \\
11 \\
64\end{array}$ & $\begin{array}{l}\text { EU } \\
\text { EU } \\
\text { AS } \\
\text { NA }\end{array}$ & $\begin{array}{r}\text { GB } \\
\text { GR } \\
\text { IN } \\
\text { AM }\end{array}$ & $\begin{array}{l}5 \\
5 \\
5 \\
5\end{array}$ & $\begin{array}{l}1979 \\
1994 \\
1976 \\
1979\end{array}$ \\
\hline $\mathbf{H}$ & $\mathrm{H}$ & Brontë & $38.7 \mathrm{~N}$ & $125.9 \mathrm{~W}$ & 60 & $\mathrm{EU}$ & EN & 5 & 1976 \\
\hline L & $\mathrm{L}$ & Brontè & $20.2 \mathrm{~N}$ & $30.7 \mathrm{E}$ & 0 & NA & $\mathrm{AM}$ & 5 & 1973 \\
\hline V & V & Brooke & $48.4 \mathrm{~N}$ & $296.6 \mathrm{E}$ & 22 & NA & $\mathrm{CA}$ & 5 & 1985 \\
\hline
\end{tabular}

ft origin

AA Ira Sprague; American astronomer (1898-1973)

LF Astronaut-named feature, Apollo 17 site.

AA Louise; American explorer (1887-1972).

AA Karen; Swedish poet, novelist (1900-1941)

AA Robert; British natural philosopher, chemist (1627-1691).

SU Celtic mythological river.

AA Town in Turkey.

AA Frederick Sumner; American physicist (1896-Live).

AA Anne; American poet (c. 1612-1672).

AA William H.; Australian physicist, Nobel laureate (1862-1942).

AA Skaldic; god of poetry.

AA Johannes; German composer and pianist (1883-1897).

AA Donato; Italian architect (1444-1514)

AA Norse; Ottar's ancestor.

AA Celtic; omnipotent god who watched over people.

AA John A.; American astronomer (1840-1920).

AA John A.; American physicist (1840-1920).

AA Edward William; British geographer (1801-1870)

VA River in Texas, USA.

AA Fedor A.; Russian astronomer (1831-1904).

AA Scipione; Italian chemist, geologist, mathematician (1748-1826).

DO Lithuanian night darkness goddess.

PE Frederika; Swedish writer, reformer, feminist (1801-1865).

AA German port.

AA Leo; Austrian astronomer (1855-1928)

AA David; Scottish optician (1781-1868)

AA Charles J.; French mathematician (1783-1864)

AA P.; French astronomer (d. 1922).

LF Astronaut-named feature, Apollo 15 site.

AA Port of Barbados.

AA Irish first name.

AA Percy W.; American physicist, Nobel laureate (1882-1961).

AA Henry; British mathematician (1556-1630).

TH Celtic goddess of wisdom, doctoring, smithing.

AA Sir Thomas; Scottish astronomer (1770-1860).

AA English port.

CM Greek/Cretan goddess of the hunt.

AA Town in India.

AA Detlev Wulf; American neurophysiologist(1897-1975).

AA Charlotte (1816-1855) and Emily (1818-1848); English novelists.

LF Astronaut-named feature, Apollo 17 site.

AA Frances; Canadian novelist (1724-1789).

\footnotetext{
P: $\quad$ planetary system (see page xvi)

Sa: $\quad$ satellite (see page $x v i$ ).

lat: latitude of feature center.

long: longitude of feature center.
}

diam: diameter or long dimension of feature. ct: continent of name origin (see page $284 \mathrm{ff}$.) et: ethnicity of name origin (see page $284 \mathrm{ff}$.)

as: name approval status (see page $\mathrm{xvii}$. ad: name approval date (year). ref: reference source for name (see page $287 \mathrm{ff}$.) ft: feature type (see page 290). 


\section{ALPHABETICAL LIST OF NAMES}

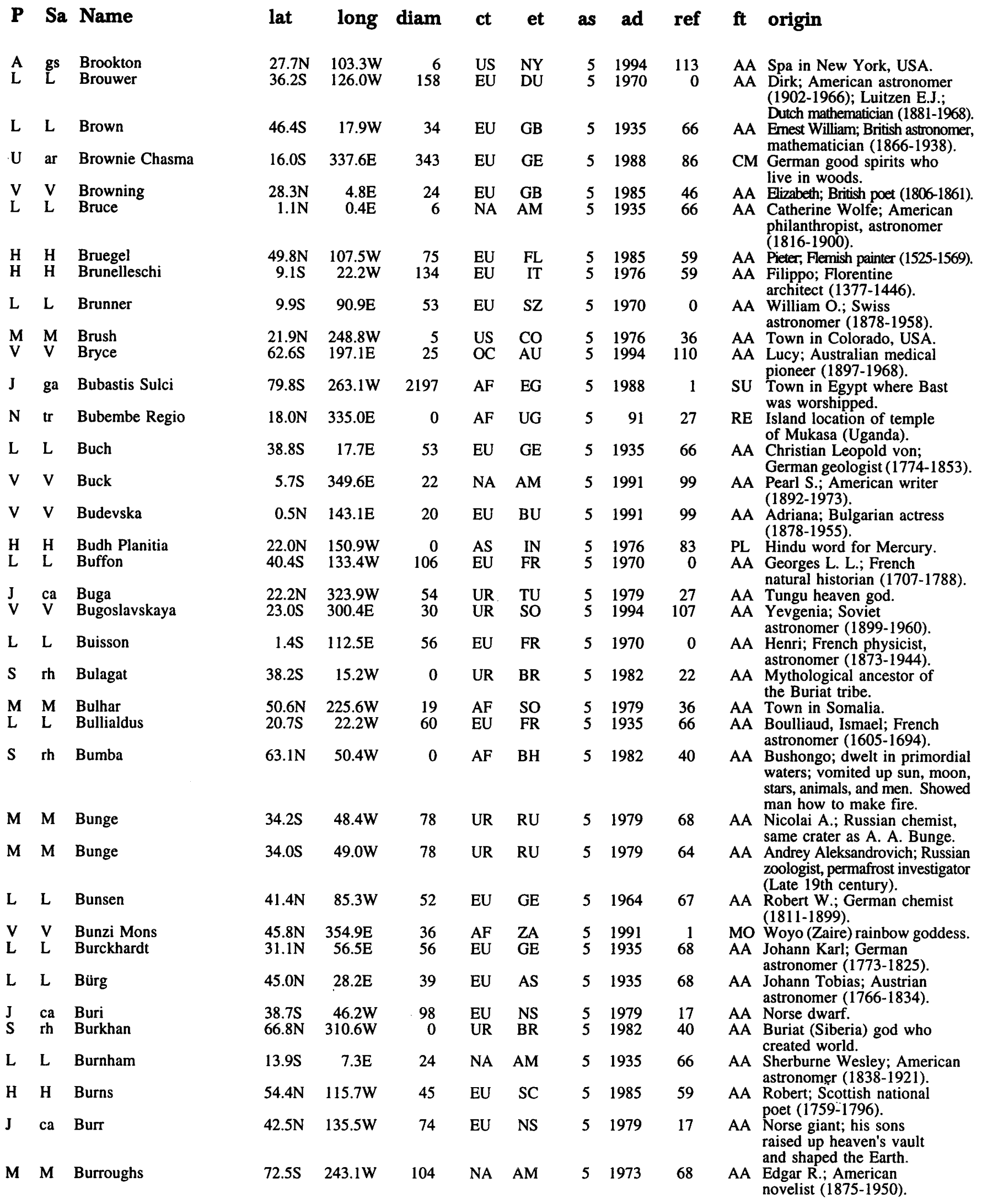

P: planetary system (see page xvi).

Sa: satellite (see page $\mathrm{xvi}$ ).

lat: latitude of feature center.

long: longitude of feature center. diam: diameter or long dimension of feature.

ct: continent of name origin (see page $284 \mathrm{ff}$.)

et: ethnicity of name origin (see page $284 \mathrm{ff}$.)

as: name approval status (see page $x v i i$ ). ad: name approval date (year).

ref: reference source for name (see page $287 \mathrm{ff}$.).

ft: feature type (see page 290). 


\section{ALPHABETICAL LIST OF NAMES}

\begin{tabular}{|c|c|c|c|c|c|c|c|c|c|c|}
\hline $\mathbf{P}$ & $\mathbf{S a}$ & Name & lat & long & diam & ct & et & as & ad & ref \\
\hline $\mathbf{M}$ & $\mathbf{M}$ & Burton & $14.5 \mathrm{~S}$ & $156.3 \mathrm{~W}$ & 137 & $\mathrm{EU}$ & GB & 5 & 1973 & 68 \\
\hline $\mathrm{L}$ & $\mathrm{L}$ & Büsching & $38.0 \mathrm{~S}$ & $20.0 \mathrm{E}$ & 52 & EU & GE & 5 & 1935 & 66 \\
\hline J & ga & Busiris Facula & $14.9 \mathrm{~N}$ & $216.1 \mathrm{~W}$ & 348 & $\mathrm{AF}$ & EG & 5 & 1985 & 27 \\
\hline $\begin{array}{l}M \\
S\end{array}$ & $\begin{array}{l}\mathrm{M} \\
\mathrm{di}\end{array}$ & $\begin{array}{l}\text { Buta } \\
\text { Butes }\end{array}$ & $\begin{array}{l}23.5 \mathrm{~S} \\
64.2 \mathrm{~N}\end{array}$ & $\begin{array}{l}32.2 \mathrm{~W} \\
48.8 \mathrm{~W}\end{array}$ & $\begin{array}{l}12 \\
26\end{array}$ & $\begin{array}{l}\mathrm{AF} \\
\mathrm{EU}\end{array}$ & $\begin{array}{l}\text { ZA } \\
\text { GR }\end{array}$ & $\begin{array}{l}5 \\
5\end{array}$ & $\begin{array}{l}1979 \\
1982\end{array}$ & $\begin{array}{l}36 \\
44\end{array}$ \\
\hline L & $\mathrm{L}$ & Butlerov & $12.5 \mathrm{~N}$ & $108.7 \mathrm{~W}$ & 40 & UR & RU & 5 & 1970 & 0 \\
\hline J & ga & Buto Facula & $12.6 \mathrm{~N}$ & $204.3 W$ & 236 & $\mathrm{AF}$ & EG & 5 & 1985 & 27 \\
\hline $\begin{array}{l}\mathrm{M} \\
\mathrm{U} \\
\mathrm{M}\end{array}$ & $\begin{array}{l}\mathrm{M} \\
\mathrm{pu} \\
\mathrm{M}\end{array}$ & $\begin{array}{l}\text { Butte } \\
\text { Butz } \\
\text { Buvinda Vallis }\end{array}$ & $\begin{array}{r}5.1 \mathrm{~S} \\
33.3 \mathrm{~N}\end{array}$ & $\begin{array}{r}39.1 \mathrm{~W} \\
208.2 \mathrm{~W}\end{array}$ & 12 & $\begin{array}{l}\text { US } \\
\text { EU } \\
\text { EU }\end{array}$ & $\begin{array}{l}\text { MT } \\
\text { GE } \\
\text { IR }\end{array}$ & $\begin{array}{l}5 \\
5 \\
5\end{array}$ & $\begin{array}{l}1976 \\
1988 \\
1985\end{array}$ & $\begin{array}{l}36 \\
94 \\
56\end{array}$ \\
\hline L & L & Buys-Ballot & $20.8 \mathrm{~N}$ & 174.5E & 55 & EU & DU & 5 & 1970 & 0 \\
\hline L & $\mathrm{L}$ & Byrd & $85.3 \mathrm{~N}$ & $9.8 \mathrm{E}$ & 93 & NA & AM & 5 & 1964 & 67 \\
\hline M & M & Byrd & $65.6 \mathrm{~S}$ & $231.9 \mathrm{~W}$ & 122 & NA & AM & 5 & 1976 & 68 \\
\hline $\mathbf{L}$ & $\mathrm{L}$ & Byrgius & $24.7 \mathrm{~S}$ & $65.3 \mathrm{~W}$ & 87 & $\mathrm{EU}$ & $\mathrm{SZ}$ & 5 & 1935 & 66 \\
\hline $\begin{array}{l}\mathrm{H} \\
\mathrm{M} \\
\mathrm{L}\end{array}$ & $\begin{array}{l}\mathrm{H} \\
\mathrm{M} \\
\mathrm{L}\end{array}$ & $\begin{array}{l}\text { Byron } \\
\text { Byske } \\
\text { C. Herschel }\end{array}$ & $\begin{array}{r}8.5 \mathrm{~S} \\
4.9 \mathrm{~S} \\
34.5 \mathrm{~N}\end{array}$ & $\begin{array}{l}32.7 \mathrm{~W} \\
31.1 \mathrm{~W} \\
31.2 \mathrm{~W}\end{array}$ & $\begin{array}{r}105 \\
5 \\
13\end{array}$ & $\begin{array}{l}\text { EU } \\
\text { EU } \\
\text { EU }\end{array}$ & $\begin{array}{l}\text { EN } \\
\text { SW } \\
\text { GB }\end{array}$ & $\begin{array}{l}5 \\
5 \\
5\end{array}$ & $\begin{array}{l}1976 \\
1976 \\
1935\end{array}$ & $\begin{array}{l}59 \\
36 \\
66\end{array}$ \\
\hline $\mathrm{L}$ & $\mathrm{L}$ & C. Mayer & $63.2 \mathrm{~N}$ & $17.3 \mathrm{E}$ & 38 & $\mathrm{EU}$ & GE & 5 & 1935 & 66 \\
\hline $\begin{array}{l}\mathrm{L} \\
\mathrm{L}\end{array}$ & $\begin{array}{l}\mathrm{L} \\
\mathrm{L}\end{array}$ & $\begin{array}{l}\text { Cabannes } \\
\text { Cabeus }\end{array}$ & $\begin{array}{l}60.9 \mathrm{~S} \\
84.9 \mathrm{~S}\end{array}$ & $\begin{array}{r}169.6 \mathrm{~W} \\
35.5 \mathrm{~W}\end{array}$ & $\begin{array}{l}80 \\
98\end{array}$ & $\begin{array}{l}\text { EU } \\
\text { EU }\end{array}$ & $\begin{array}{c}\text { FR } \\
\text { IT }\end{array}$ & $\begin{array}{l}5 \\
5\end{array}$ & $\begin{array}{l}1970 \\
1935\end{array}$ & $\begin{array}{r}0 \\
66\end{array}$ \\
\hline V & V & Caccini & $17.4 \mathrm{~N}$ & $170.4 \mathrm{E}$ & 38 & EU & IT & 5 & 1994 & 99 \\
\hline $\begin{array}{l}\mathbf{M} \\
\mathbf{J} \\
\mathrm{H}\end{array}$ & $\begin{array}{l}\mathrm{M} \\
\mathrm{eu} \\
\mathrm{H}\end{array}$ & $\begin{array}{l}\text { Cádiz } \\
\text { Cadmus Linea } \\
\text { Caduceata }\end{array}$ & $\begin{array}{l}23.4 \mathrm{~N} \\
27.8 \mathrm{~N} \\
45.0 \mathrm{~N}\end{array}$ & $\begin{array}{l}49.1 \mathrm{~W} \\
173.1 \mathrm{~W} \\
135.0 \mathrm{~W}\end{array}$ & $\begin{array}{r}1 \\
1212 \\
0\end{array}$ & $\begin{array}{l}\mathrm{EU} \\
\mathrm{EU} \\
\mathrm{EU}\end{array}$ & $\begin{array}{l}\text { SP } \\
\text { GR } \\
\text { RM }\end{array}$ & $\begin{array}{l}5 \\
5 \\
5\end{array}$ & $\begin{array}{l}1979 \\
1979 \\
1976\end{array}$ & $\begin{array}{l}36 \\
19 \\
60\end{array}$ \\
\hline U & $\mathrm{ob}$ & Caesar & $26.6 \mathrm{~S}$ & $61.1 \mathrm{E}$ & 76 & $\mathrm{EU}$ & GB & 5 & 1988 & 85 \\
\hline $\begin{array}{l}S \\
V \\
M \\
V \\
V\end{array}$ & $\begin{array}{l}\text { di } \\
\text { V } \\
M \\
\text { V } \\
\text { V }\end{array}$ & $\begin{array}{l}\text { Caieta } \\
\text { Cailleach Corona } \\
\text { Cairns } \\
\text { Caitlin } \\
\text { Caiwenji }\end{array}$ & $\begin{array}{l}23.3 \mathrm{~S} \\
48.0 \mathrm{~S} \\
24.0 \mathrm{~N} \\
65.3 \mathrm{~S} \\
12.4 \mathrm{~S}\end{array}$ & $\begin{array}{l}80.5 \mathrm{~W} \\
88.3 \mathrm{E} \\
47.3 \mathrm{~W} \\
12.1 \mathrm{E} \\
287.5 \mathrm{E}\end{array}$ & $\begin{array}{r}70 \\
125 \\
10 \\
14 \\
22\end{array}$ & $\begin{array}{l}\text { EU } \\
\text { EU } \\
\text { OC } \\
\text { EU } \\
\text { AS }\end{array}$ & $\begin{array}{l}\text { GR } \\
\text { SC } \\
\text { AU } \\
\text { WA } \\
\text { CH }\end{array}$ & $\begin{array}{l}5 \\
5 \\
5 \\
5 \\
5\end{array}$ & $\begin{array}{l}1982 \\
1994 \\
1976 \\
1994 \\
1994\end{array}$ & $\begin{array}{r}44 \\
83 \\
36 \\
109 \\
87\end{array}$ \\
\hline L & $\mathrm{L}$ & Cajal & $12.6 \mathrm{~N}$ & $31.1 \mathrm{E}$ & 9 & $\mathrm{EU}$ & SP & 5 & 1973 & 0 \\
\hline L & $\mathrm{L}$ & Cajori & $47.4 \mathrm{~S}$ & $168.8 \mathrm{E}$ & 70 & NA & $\mathrm{AM}$ & 5 & 1970 & 0 \\
\hline $\begin{array}{l}\mathrm{V} \\
\mathrm{M} \\
\mathrm{M} \\
\mathrm{V}\end{array}$ & $\begin{array}{l}\mathrm{V} \\
\mathrm{M} \\
\mathrm{M} \\
\mathrm{V}\end{array}$ & $\begin{array}{l}\text { Calakomana Corona } \\
\text { Calamar } \\
\text { Calbe } \\
\text { Caldwell }\end{array}$ & $\begin{array}{r}6.5 \mathrm{~N} \\
18.4 \mathrm{~N} \\
25.5 \mathrm{~S} \\
23.6 \mathrm{~N}\end{array}$ & $\begin{array}{c}43.5 \mathrm{E} \\
54.9 \mathrm{~W} \\
28.7 \mathrm{~W} \\
112.1 \mathrm{E}\end{array}$ & $\begin{array}{r}575 \\
0 \\
14 \\
44\end{array}$ & $\begin{array}{l}\text { NA } \\
\text { SA } \\
\text { EU } \\
\text { NA }\end{array}$ & $\begin{array}{l}\text { PU } \\
\text { CO } \\
\text { GE } \\
\text { AM }\end{array}$ & $\begin{array}{l}5 \\
5 \\
5 \\
5\end{array}$ & $\begin{array}{l}1994 \\
1988 \\
1976 \\
1994\end{array}$ & $\begin{array}{l}83 \\
36 \\
36 \\
87\end{array}$ \\
\hline L & $\mathrm{L}$ & Calippus & $38.9 \mathrm{~N}$ & $10.7 \mathrm{E}$ & 32 & EU & GR & 5 & 1935 & 66 \\
\hline $\begin{array}{l}\text { A } \\
\text { V }\end{array}$ & gs & $\begin{array}{l}\text { Calistoga } \\
\text { Callas }\end{array}$ & $\begin{array}{r}30.0 \mathrm{~N} \\
2.4 \mathrm{~N}\end{array}$ & $\begin{array}{c}2.0 \mathrm{~W} \\
27.0 \mathrm{E}\end{array}$ & $\begin{array}{r}0 \\
31\end{array}$ & $\begin{array}{l}\text { US } \\
\text { NA }\end{array}$ & $\begin{array}{l}\text { CA } \\
\text { AM }\end{array}$ & $\begin{array}{l}5 \\
5\end{array}$ & $\begin{array}{l}1994 \\
1991\end{array}$ & $\begin{array}{r}113 \\
99\end{array}$ \\
\hline $\begin{array}{l}\mathrm{H} \\
\mathrm{V} \\
\mathrm{H}\end{array}$ & $\begin{array}{l}\mathrm{H} \\
\mathrm{V} \\
\mathrm{H}\end{array}$ & $\begin{array}{l}\text { Callicrates } \\
\text { Callirhoe } \\
\text { Caloris Montes }\end{array}$ & $\begin{array}{l}66.3 \mathrm{~S} \\
21.3 \mathrm{~N} \\
39.4 \mathrm{~N}\end{array}$ & $\begin{array}{l}32.6 \mathrm{~W} \\
140.6 \mathrm{E} \\
187.2 \mathrm{~W}\end{array}$ & $\begin{array}{r}70 \\
35 \\
0\end{array}$ & $\begin{array}{l}\text { EU } \\
\text { EU } \\
\text { EU }\end{array}$ & $\begin{array}{l}\text { GR } \\
\text { GR } \\
\text { LA }\end{array}$ & $\begin{array}{l}5 \\
5 \\
5\end{array}$ & $\begin{array}{l}1976 \\
1991 \\
1976\end{array}$ & $\begin{array}{l}59 \\
99 \\
80\end{array}$ \\
\hline $\mathrm{H}$ & $\mathrm{H}$ & Caloris Planitia & $30.5 \mathrm{~N}$ & $189.8 \mathrm{~W}$ & 0 & EU & LA & 5 & 1976 & 80 \\
\hline U & ti & Calphurnia & $42.4 \mathrm{~S}$ & 291.4E & 100 & EU & GB & 5 & 1988 & 85 \\
\hline
\end{tabular}

\section{ft origin}

AA Charles E.; British astronomer (1846-1882).

AA Anton Friedrich; German geographer (1724-1793).

FA Town in lower Egypt where Osiris was first installed as local god.

AA Town in Zaire.

AA A famous boxer who had been defeated by Dares.

AA Aleksandr M.; Russian chemist (1828-1886).

FA Swamp where Isis hid Osiris' body.

AA Town in Montana, USA.

AA German roguish or evil spirits.

VA Classical river in Hibernia; present Boyne River, Ireland.

AA C. H. D.; Dutch meteorologist (1817-1890).

AA Richard E.; American explorer, aviator, navigator (1888-1957).

AA Richard E.; American aviator-explorer (1888-1975).

AA Burgi, Joost; Swiss horologist (1552-1632).

AA G. G.; English poet (1788-1824).

AA Town in Sweden.

AA Caroline; British astronomer (1750-1848)

AA Christian; German astronomer, mathematician, physicist (1719-1783)

AA Jean; French physicist (1885-1959).

AA Cabeo, Niccolo; Italian astronomer (1586-1650).

AA Francesca; Italian poet, composer (c. 1581-c. 1640).

AA Spanish port.

LI Greek; brother of Europa.

AL Albedo name for $\mathrm{H}-3$, Shakespeare region.

AA Shakespearean hero in "Julius Caesar."

AA A nurse of Aeneas.

CR Scottish Celtic fertility goddess.

AA Town in Australia.

AA Welsh first name.

AA Chinese painter, calligrapher (907-960).

AA Santiago Ramon Y; Spanish doctor, Nobel laureate (1852-1934).

AA Florian; American mathematician (1859-1930).

CR Pueblo Indian corn goddess.

AA Town in Colombia.

AA Town in Germany.

AA Taylor; American author (1900-1985).

AA Calippus of Cyzicus; Greek astronomer (c. 330 B.C.).

AA Resort in California, USA.

AA Maria; American opera singer (1923-1977).

AA Greek architect (5th century B.C.).

AA Greek sculptor (c. 600 B.C.).

MO "Hot mountains"; surface temperature hottest near this position.

PL "Hot plain"; surface temperature hottest near this position.

AA Wife of Julius Caesar.

\footnotetext{
P: $\quad$ planetary system (see page $x v i$ ).

Sa: satellite (see page $x v i$ ).

lat: latitude of feature center.

long: longitude of feature center.
}

diam: diameter or long dimension of feature.

ct: continent of name origin (see page $284 \mathrm{ff}$.)

et: ethnicity of name origin (see page $284 \mathrm{ff}$.)

as: name approval status (see page xvii). ad: name approval date (year).

ref: reference source for name (see page $287 \mathrm{ff}$.).

ft: feature type (see page 290). 
ALPHABETICAL LIST OF NAMES

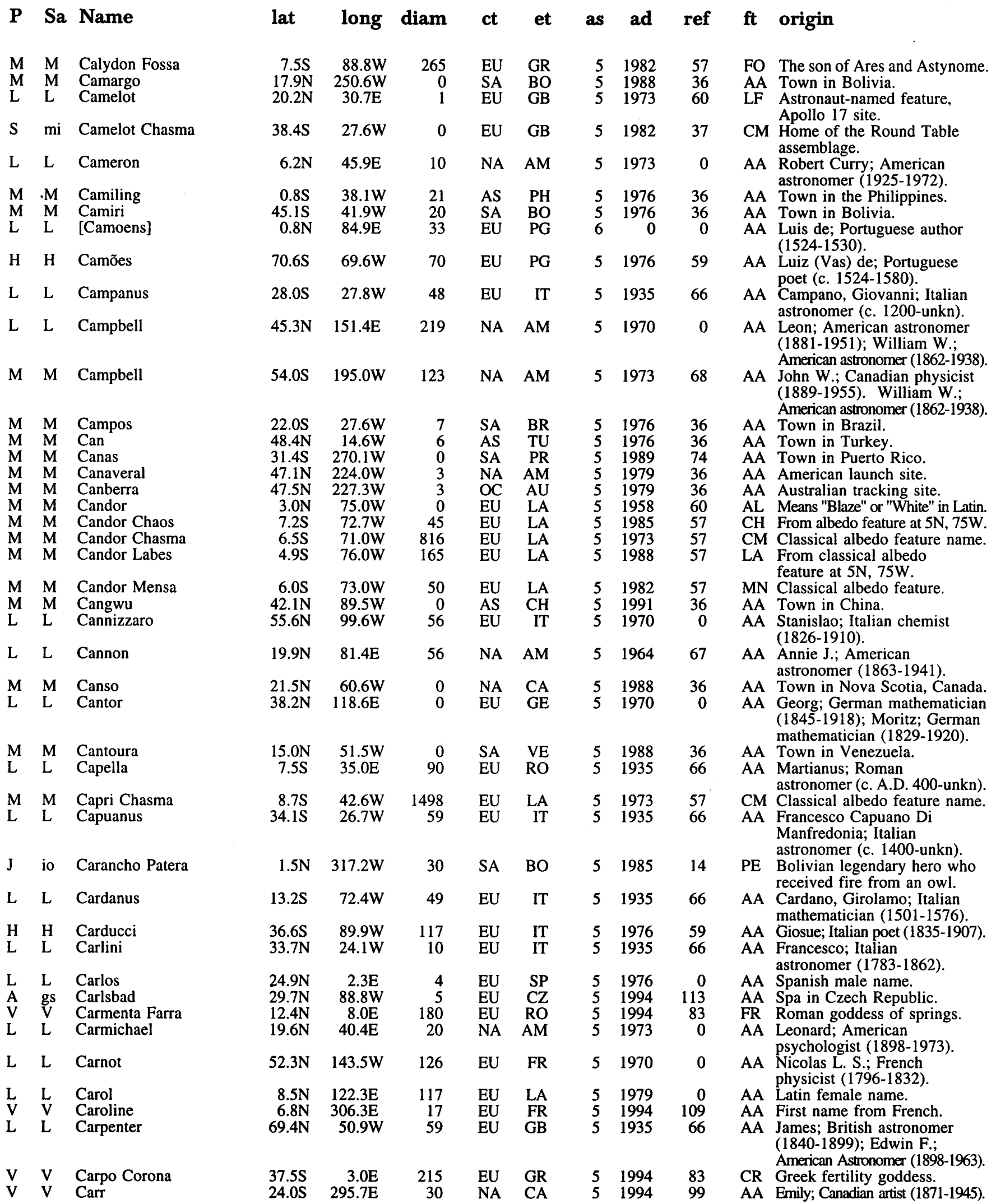

\footnotetext{
P: $\quad$ planetary system (see page $\mathrm{xvi}$ ).

Sa: $\quad$ satellite (see page $x v i$ ).

lat: latitude of feature center.

long: longitude of feature center.
}

diam: diameter or long dimension of feature. ct: continent of name origin (see page $284 \mathrm{ff}$.) et: ethnicity of name origin (see page $284 \mathrm{ff}$.) as: name approval status (see page $\mathrm{xvii}$ ). ad: name approval date (year) ref: reference source for name (see page $287 \mathrm{ff}$.). $\mathrm{ft}$ : feature type (see page 290). 
ALPHABETICAL LIST OF NAMES

\begin{tabular}{|c|c|c|c|c|c|c|c|c|c|}
\hline $\mathbf{P}$ & Sa Name & lat & long & diam & ct & et & as & ad & ref \\
\hline L & Carrel & $10.7 \mathrm{~N}$ & 26.7E & 15 & EU & FR & 5 & 1979 & 0 \\
\hline V & Carreno & $3.9 \mathrm{~S}$ & $16.1 \mathrm{E}$ & 59 & SA & VE & 5 & 1991 & 99 \\
\hline V & Carriera Patera & $48.6 \mathrm{~N}$ & $48.8 \mathrm{E}$ & 97 & EU & IT & 5 & 91 & 46 \\
\hline L & Carrillo & $2.2 \mathrm{~S}$ & $80.9 \mathrm{E}$ & 16 & NA & ME & 5 & 1979 & 0 \\
\hline L & Carrington & $44.0 \mathrm{~N}$ & $62.1 \mathrm{E}$ & 30 & EU & GB & 5 & 1935 & 66 \\
\hline V & Carson & $24.2 \mathrm{~S}$ & $344.1 \mathrm{E}$ & 41 & NA & AM & 5 & 1991 & 99 \\
\hline $\begin{array}{l}\mathbf{M} \\
\mathbf{L}\end{array}$ & $\begin{array}{l}\text { Cartago } \\
\text { Cartan }\end{array}$ & $\begin{array}{r}23.6 \mathrm{~S} \\
4.2 \mathrm{~N}\end{array}$ & $\begin{array}{l}17.8 \mathrm{~W} \\
59.3 \mathrm{E}\end{array}$ & $\begin{array}{l}33 \\
15\end{array}$ & $\begin{array}{l}\text { SA } \\
\text { EU }\end{array}$ & $\begin{array}{l}\text { CR } \\
\text { FR }\end{array}$ & $\begin{array}{l}5 \\
5\end{array}$ & $\begin{array}{l}1976 \\
1976\end{array}$ & $\begin{array}{r}36 \\
0\end{array}$ \\
\hline V & Carter & $5.3 \mathrm{~N}$ & $67.2 \mathrm{E}$ & 18 & NA & $\mathrm{AM}$ & 5 & 1994 & 87 \\
\hline $\mathbf{S}$ & Carthage Linea & $12.7 \mathrm{~N}$ & $321.9 \mathrm{~W}$ & 318 & EU & RM & 5 & 1982 & 44 \\
\hline L & Carver & $43.0 \mathrm{~S}$ & $126.9 \mathrm{E}$ & 59 & NA & AM & 5 & 1970 & 0 \\
\hline L & Casatus & $72.8 \mathrm{~S}$ & $29.5 \mathrm{~W}$ & 108 & EU & IT & 5 & 1935 & 66 \\
\hline $\mathbf{M}$ & Casius & $40.0 \mathrm{~N}$ & $260.0 \mathrm{~W}$ & 0 & EU & LA & 5 & 1958 & 60 \\
\hline $\mathbf{S}$ & Cassandra & $39.5 \mathrm{~S}$ & $244.1 W$ & 36 & EU & RM & 5 & 1982 & 44 \\
\hline V & Cassatt Patera & $65.6 \mathrm{~N}$ & $207.6 \mathrm{E}$ & 130 & NA & $\mathrm{AM}$ & 5 & 91 & 46 \\
\hline L & Cassegrain & $52.4 \mathrm{~S}$ & $113.5 \mathrm{E}$ & 55 & EU & FR & 5 & 1970 & 0 \\
\hline $\mathbf{M}$ & Cassini & $23.8 \mathrm{~N}$ & $327.9 \mathrm{~W}$ & 415 & $\mathrm{EU}$ & IT & 5 & 1973 & 68 \\
\hline L & Cassini & $40.2 \mathrm{~N}$ & $4.6 \mathrm{E}$ & 56 & EU & IT & 5 & 1935 & 66 \\
\hline $\mathbf{S}$ & Cassini Regio & $28.1 S$ & $92.6 \mathrm{~W}$ & $\mathbf{0}$ & EU & IT & 5 & 1982 & 68 \\
\hline 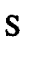 & Castor & $0.0 \mathrm{~N}$ & $0.0 \mathrm{~W}$ & 0 & $\mathrm{EU}$ & GR & 5 & 1982 & 21 \\
\hline V & Castro & $3.3 \mathrm{~N}$ & $233.9 \mathrm{E}$ & 23 & EU & SP & 5 & 1994 & 99 \\
\hline L & Catalán & $45.7 \mathrm{~S}$ & $87.3 \mathrm{~W}$ & 25 & EU & $\mathbf{S P}$ & 5 & 1970 & 0 \\
\hline $\begin{array}{l}\mathrm{L} \\
\mathrm{L} \\
\mathrm{L} \\
\mathrm{L} \\
\mathrm{L} \\
\mathrm{L} \\
\mathrm{L} \\
\mathrm{L} \\
\mathrm{L} \\
\mathrm{L}\end{array}$ & $\begin{array}{l}\text { Cataquil Patera } \\
\text { Catena Abulfeda } \\
\text { Catena Artamonov } \\
\text { Catena Brigitte } \\
\text { Catena Davy } \\
\text { Catena Dziewulski } \\
\text { Catena Gregory } \\
\text { Catena Humboldt } \\
\text { Catena Krafft } \\
\text { Catena Kurchatov } \\
\text { Catena Leuschner (GDL) }\end{array}$ & $\begin{array}{l}24.2 \mathrm{~S} \\
16.9 \mathrm{~S} \\
26.0 \mathrm{~N} \\
18.5 \mathrm{~N} \\
11.0 \mathrm{~S} \\
19.0 \mathrm{~N} \\
0.6 \mathrm{~S} \\
21.5 \mathrm{~S} \\
15.0 \mathrm{~N} \\
37.2 \mathrm{~N} \\
4.7 \mathrm{~N}\end{array}$ & $\begin{array}{c}18.7 \mathrm{~W} \\
17.2 \mathrm{E} \\
105.9 \mathrm{E} \\
27.5 \mathrm{E} \\
7.0 \mathrm{~W} \\
100.0 \mathrm{E} \\
129.9 \mathrm{E} \\
84.6 \mathrm{E} \\
72.0 \mathrm{~W} \\
136.3 \mathrm{E} \\
110.1 \mathrm{~W}\end{array}$ & $\begin{array}{r}125 \\
219 \\
134 \\
5 \\
50 \\
80 \\
152 \\
165 \\
60 \\
226 \\
364\end{array}$ & $\begin{array}{l}\text { SA } \\
\text { AS } \\
\text { UR } \\
\text { EU } \\
\text { EU } \\
\text { EU } \\
\text { EU } \\
\text { EU } \\
\text { EU } \\
\text { UR } \\
\text { NA }\end{array}$ & $\begin{array}{l}\text { IN } \\
\text { SY } \\
\text { SO } \\
\text { FR } \\
\text { GB } \\
\text { PO } \\
\text { SC } \\
\text { GE } \\
\text { GE } \\
\text { SO } \\
\text { AM }\end{array}$ & $\begin{array}{l}5 \\
5 \\
5 \\
5 \\
5 \\
5 \\
5 \\
5 \\
5 \\
5 \\
5\end{array}$ & $\begin{array}{l}1985 \\
1976 \\
1976 \\
1976 \\
1976 \\
1976 \\
1976 \\
1976 \\
1976 \\
1976 \\
1979\end{array}$ & $\begin{array}{l}1 \\
0 \\
0 \\
0 \\
0 \\
0 \\
0 \\
0 \\
0 \\
0 \\
0\end{array}$ \\
\hline $\begin{array}{l}\mathrm{L} \\
\mathrm{L}\end{array}$ & $\begin{array}{l}\text { Catena Littrow } \\
\text { Catena Lucretius (RNII) }\end{array}$ & $\begin{array}{r}22.2 \mathrm{~N} \\
3.4 \mathrm{~S}\end{array}$ & $\begin{array}{l}29.5 \mathrm{E} \\
126.1 \mathrm{~W}\end{array}$ & $\begin{array}{r}10 \\
271\end{array}$ & $\begin{array}{l}\text { EU } \\
\text { EU }\end{array}$ & $\begin{array}{r}\text { CZ } \\
\text { RM }\end{array}$ & $\begin{array}{l}5 \\
5\end{array}$ & $\begin{array}{l}1985 \\
1979\end{array}$ & $\begin{array}{l}0 \\
0\end{array}$ \\
\hline $\begin{array}{l}\mathrm{L} \\
\mathrm{L}\end{array}$ & $\begin{array}{l}\text { Catena Mendeleev } \\
\text { Catena Michelson (GIRD) }\end{array}$ & $\begin{array}{l}6.3 \mathrm{~N} \\
1.4 \mathrm{~N}\end{array}$ & $\begin{array}{l}139.4 \mathrm{E} \\
113.4 \mathrm{~W}\end{array}$ & $\begin{array}{l}188 \\
456\end{array}$ & $\begin{array}{l}\text { UR } \\
\text { EU }\end{array}$ & $\begin{array}{l}\text { RU } \\
\text { GE }\end{array}$ & $\begin{array}{l}5 \\
5\end{array}$ & $\begin{array}{l}1976 \\
1979\end{array}$ & $\begin{array}{l}0 \\
0\end{array}$ \\
\hline $\begin{array}{l}\mathrm{L} \\
\mathrm{L} \\
\mathrm{L}\end{array}$ & $\begin{array}{ll}\text { L } & \text { Catena Pierre } \\
\text { L } & \text { Catena Sumner } \\
\text { L } & \text { Catena Sylvester } \\
\text { L } & \text { Catena Taruntius }\end{array}$ & $\begin{array}{r}19.8 \mathrm{~N} \\
37.3 \mathrm{~N} \\
81.4 \mathrm{~N} \\
3.0 \mathrm{~N}\end{array}$ & $\begin{array}{c}31.8 \mathrm{~W} \\
112.3 \mathrm{E} \\
86.2 \mathrm{~W} \\
48.0 \mathrm{E}\end{array}$ & $\begin{array}{r}9 \\
247 \\
173 \\
100\end{array}$ & $\begin{array}{l}\text { EU } \\
\text { NA } \\
\text { EU } \\
\text { EU }\end{array}$ & $\begin{array}{l}\text { FR } \\
\text { AM } \\
\text { GB } \\
\text { RM }\end{array}$ & $\begin{array}{l}5 \\
5 \\
5 \\
5\end{array}$ & $\begin{array}{l}1976 \\
1976 \\
1976 \\
1985\end{array}$ & $\begin{array}{l}0 \\
0 \\
0 \\
0\end{array}$ \\
\hline
\end{tabular}

ft origin

AA Alexis; French doctor, physiologist; Nobel laureate (1873-1944)

AA Teresa; Venezuelan pianist, composer (1853-1917)

PE Rosalba; Italian portrait painter (1675-1757).

AA Flores Nabor; Mexican soil engineer (1911-1967).

AA Richard Christopher; British astronomer (1826-1875).

AA Rachel; American biologist, author (1907-1964).

AA Town in Costa Rica.

AA E. J.; French mathematician (1869-1951)

AA Maybelle; American singer, songwriter (1909-1978).

LI A Punic (Phoenician) city in North Africa.

AA George W.; American. botanist (c. 1864-1943)

AA Casati, Paolo; Italian mathematician (1617-1707)

AL Epithet of Zeus; for his two sanctuaries in Egypt/Arabia and Syria.

AA Daughter of Priam; she could fortell the future.

PE Mary; American Impressionist painter (1844-1926)

AA Giovanni D.; French astronomer, doctor (1625-1712).

AA Giovanni; Italian astronomer (1625-1712).

AA Giovanni Domenico; Italian-French astronomer (1625-1712); Jacques J.; French astronomer (1677-1756).

RE Italian-French astronomer who discovered Iapetus in 1671 , Rhea in 1672, Tethys and Dione in 1684 (1625-1712)

AA One of the Dioscuri; famous as a tamer of horses.

AA Rosalie; Spanish poet, novelist (1837-1885).

AA Miguel A.; Spanish spectroscopist (1894-1957)

PE Inca god of thunder and lightning.

CA Named from nearby crater.

CA Named from nearby crater.

CA French female name.

CA Named from nearby crater.

CA Named from nearby crater.

CA Named from nearby crater.

CA Named from nearby crater.

CA Named from nearby crater.

CA Named from nearby crater.

CA Named from nearby crater; GDL $=$ Gas Dynamics Laboratory

CA Named from nearby crater.

CA Named from nearby crater; RNII=Rocket Research Institute.

CA Named from nearby crater.

CA Named from nearby crater; GIRD=Group for the Study of Reaction Motion.

CA French male name.

CA Named from nearby crater.

CA Named from nearby crater.

CA Named from nearby crater.

\footnotetext{
P: $\quad$ planetary system (see page $\mathrm{xvi}$ ).

Sa: satellite (see page $\mathrm{xvi}$ ).

lat: latitude of feature center.

long: longitude of feature center.
}

\footnotetext{
diam: diameter or long dimension of feature. ct: continent of name origin (see page $284 \mathrm{ff}$.) et: ethnicity of name origin (see page $284 \mathrm{ff}$.) as: name approval status (see page xvii).
}

ad: name approval date (year).

ref: reference source for name (see page $287 \mathrm{ff}$.).

ft: feature type (see page 290). 


\section{P Sa Name}

L L Catena Timocharis

L L Catena Yuri

L L Catharina

V V Cather

S di Catillus

L L Cauchy

V V Cauteovan Corona

L L Cavalerius

M M Cave

V V Cavell Patera

L L Cavendish

L L Caventou

M M Cavi Angusti

M M Cavi Frigorēs

M M [Cavi Novi]

M M Caxias

$N$ tr Cay

L L Cayley

M M Cebrenia

M M Cecropia

L L [Cellini]

L L Celsius

L L Censorinus

M M Centauri Montes

V V Centlivre

L L Cepheus

M M Ceraunius

M M Ceraunius Catena

M M Ceraunius Fossae

M M Ceraunius Tholus

M M Cerberus

M M Cerberus Dorsa

M M Cerberus Rupēs

V V Ceres Corona

M M Cerulli

L L [Cervantes]

H H Cervantes

M M Ceti Chasma

M M Ceti Mensa

H H Cézanne

L L Chacornac lat long diam ct et as ad ref $f t$ origin

$\begin{array}{rrrrrrrr}29.0 \mathrm{~N} & 13.0 \mathrm{~W} & 50 & \text { EU } & \text { GR } & 5 & 1985 & 0 \\ 24.4 \mathrm{~N} & 30.4 \mathrm{~W} & 5 & \text { UR } & \text { RU } & 5 & 1976 & 0 \\ 18.1 \mathrm{~S} & 23.4 \mathrm{E} & 104 & \text { EU } & \text { GR } & 5 & 1935 & 66 \\ & & & & & & & \\ 47.1 \mathrm{~N} & 106.8 \mathrm{E} & 27 & \text { NA } & \text { AM } & 5 & 1985 & 46 \\ 1.6 \mathrm{~S} & 273.0 \mathrm{~W} & 35 & \text { EU } & \text { RM } & 5 & 1982 & 44 \\ 9.6 \mathrm{~N} & 38.6 \mathrm{E} & 12 & \text { EU } & \text { FR } & 5 & 1935 & 66 \\ 31.5 \mathrm{~N} & 142.9 \mathrm{E} & 553 & \text { SA } & \text { CO } & 5 & 91 & 64 \\ 5.1 \mathrm{~N} & 66.8 \mathrm{~W} & 57 & \text { EU } & \text { IT } & 5 & 1935 & 66 \\ 21.9 \mathrm{~N} & 35.6 \mathrm{~W} & 8 & \text { OC } & \text { NZ } & 5 & 1976 & 36 \\ 37.9 \mathrm{~N} & 18.7 \mathrm{E} & 76 & \text { EU } & \text { GB } & 5 & 91 & 46 \\ 24.5 \mathrm{~S} & 53.7 \mathrm{~W} & 56 & \text { EU } & \text { GB } & 5 & 1935 & 66 \\ 29.8 \mathrm{~N} & 29.4 \mathrm{~W} & 3 & \text { EU } & \text { FR } & 5 & 1976 & 68\end{array}$

75.9

80.15

$71.9 \mathrm{~W}$

$67.9 \mathrm{~W}$

294

199

EU GR

1976

67.5S $330.0 \mathrm{~W}$

535 EU GR

61976

29.5S

$12.0 \mathrm{~S}$

$100.6 \mathrm{~W}$

$4.0 \mathrm{~N}$

$44.0 \mathrm{E}$

$50.0 \mathrm{~N} \quad 210.0 \mathrm{~W}$

$60.0 \mathrm{~N} \quad 320.0 \mathrm{~W}$

$7.8 \mathrm{~S} \quad 3.0 \mathrm{E}$

$34.1 S$

$0.4 S$

$38.5 \mathrm{~S}$

$19.1 \mathrm{~N}$

$40.8 \mathrm{~N}$

$20.0 \mathrm{~N}$

20.1E

32.7E

$263.1 \mathrm{~W}$

290.4E

$45.8 \mathrm{E}$

93.0W

$37.4 \mathrm{~N} \quad 108.1 \mathrm{~W}$

$24.8 \mathrm{~N} \quad 110.5 \mathrm{~W}$

$24.2 \mathrm{~N}$

$15.0 \mathrm{~N}$

97.2W

205.0W

13.8S $254.4 \mathrm{~W}$

$8.4 \mathrm{~N}$

$195.4 \mathrm{~W}$

0 SA

0 NA

51989

$\begin{array}{ll}\text { NA } & \text { ME } \\ \text { EU } & \text { GB }\end{array}$

591

51935

0 EU LA

51958

$\begin{array}{ll}5 & 1958\end{array}$

34

EU IT

60

36

EU SW

51935

3 EU RM

$\begin{array}{ll}\text { EU } & \text { LA } \\ \text { EU } & \text { EN }\end{array}$

$5 \quad 1935$

51989

26

EU GR

51994

51935

51958

EU GR

51958

51

EU GR

51985

711 EU GR

108 EU GR

51973

0

EU GR

$\begin{array}{ll}5 & 1973 \\ 5 & 1958\end{array}$

407

EU GR

$5 \quad 1982$

1254

EU GR

51979

$16.0 \mathrm{~S} \quad 151.5 \mathrm{E}$

675

EU RM

51994

$32.6 \mathrm{~N}$

$337.9 \mathrm{~W}$$$
\text { EU }
$$

3.4S $99.2 \mathrm{E}$

25

EU

$74.6 \mathrm{~S}$

122.0W

181

EU

SP

60

51976
$5.2 \mathrm{~S} \quad 68.3 \mathrm{~W}$

45
55
75
51

$\begin{array}{ll}\text { EU } & \text { GR } \\ & \\ \text { EU } & \text { LA } \\ \text { EU } & \text { FR }\end{array}$

51985

$5.9 \mathrm{~S} \quad 76.3 \mathrm{~W}$

$8.5 \mathrm{~S} \quad 123.4 \mathrm{~W}$

31.7E

EU FR

CA Named from nearby crater.

CA Russian male name.

AA St. Catherine of Alexandria; Greek theologian, philosopher (unkn-c. 307).

AA Willa; American novelist (1876-1947)

AA Brother of Tiburtus and twin brother of Coras.

AA Augustin Louis; French mathematician (1789-1857).

CR Kataba (Columbia) fertility goddess.

AA Cavalieri, Buonaventura; Italian mathematician (1598-1647)

AA Town in New Zealand.

PE Edith; British nurse, heroine (1865-1915).

AA Henry; British chemist, physicist (1731-1810).

AA Joseph Bienaime; French chemist, pharmacologist (1795-1877)

CB Classical albedo feature name.

CB From classical albedo feature Polus Frigoris, at $84 \mathrm{~S}, 30 \mathrm{~W}$.

CB Classical albedo feature name; (dropped 1984).

AA Town in Brazil.

AA Mayan diety.

AA Arthur; British astronomer, mathematician (1821-1895).

AL Main country of the Trojan Plain.

AL Old name for Acropolis; used to mean Athens.

AA Benvenuto; Italian artist, writer (1500-1571).

AA Anders; Swedish astronomer (1701-1744).

AA Roman astronomer (fl. 238-unkn).

MO Albedo feature Centauri Lacus.

AA Susannah; English actress, playwright (c. 1667-1723)

AA Mythological astronomer, father of Andromeda.

AL "Thunderclap"; named for Ceraunii Mountains on coast of Epirus, Greece.

CA Named for classical albedo feature at $35 \mathrm{~N} 96 \mathrm{~W}$.

FO Classical albedo feature name

TH Classical albedo feature name.

AL Hound who had 3 heads; guarded gates of hell.

DO Classical albedo feature name.

RU From albedo feature at $10 \mathrm{n}, 212 \mathrm{~W}$.

CR Roman harvest goddess.

AA Vicenzo; Italian astronomer (1859-1927).

AA Miguel De; Spanish writer (1547-1616).

AA Miguel de; Spanish novelist, playwright and poet (1547-1616).

CM From albedo feature at $10 \mathrm{~S}, 74 \mathrm{~W}$.

MN Albedo feature Ceti Lacus.

AA Paul; French painter (1839-1906).

AA Jean; French astronomer (1823-1873).
P: $\quad$ planetary system (see page $x v i)$.

Sa: satellite (see page xvi).

lat: latitude of feature center.

long: longitude of feature center. diam: diameter or long dimension of feature.

ct: continent of name origin (see page $284 \mathrm{ff}$.)

et: ethnicity of name origin (see page $284 \mathrm{ff}$.)

as: name approval status (see page xvii). ad: name approval date (year).

ref: reference source for name (see page $287 \mathrm{ff}$.).

ft: feature type (see page 290). 


\section{ALPHABETICAL LIST OF NAMES}

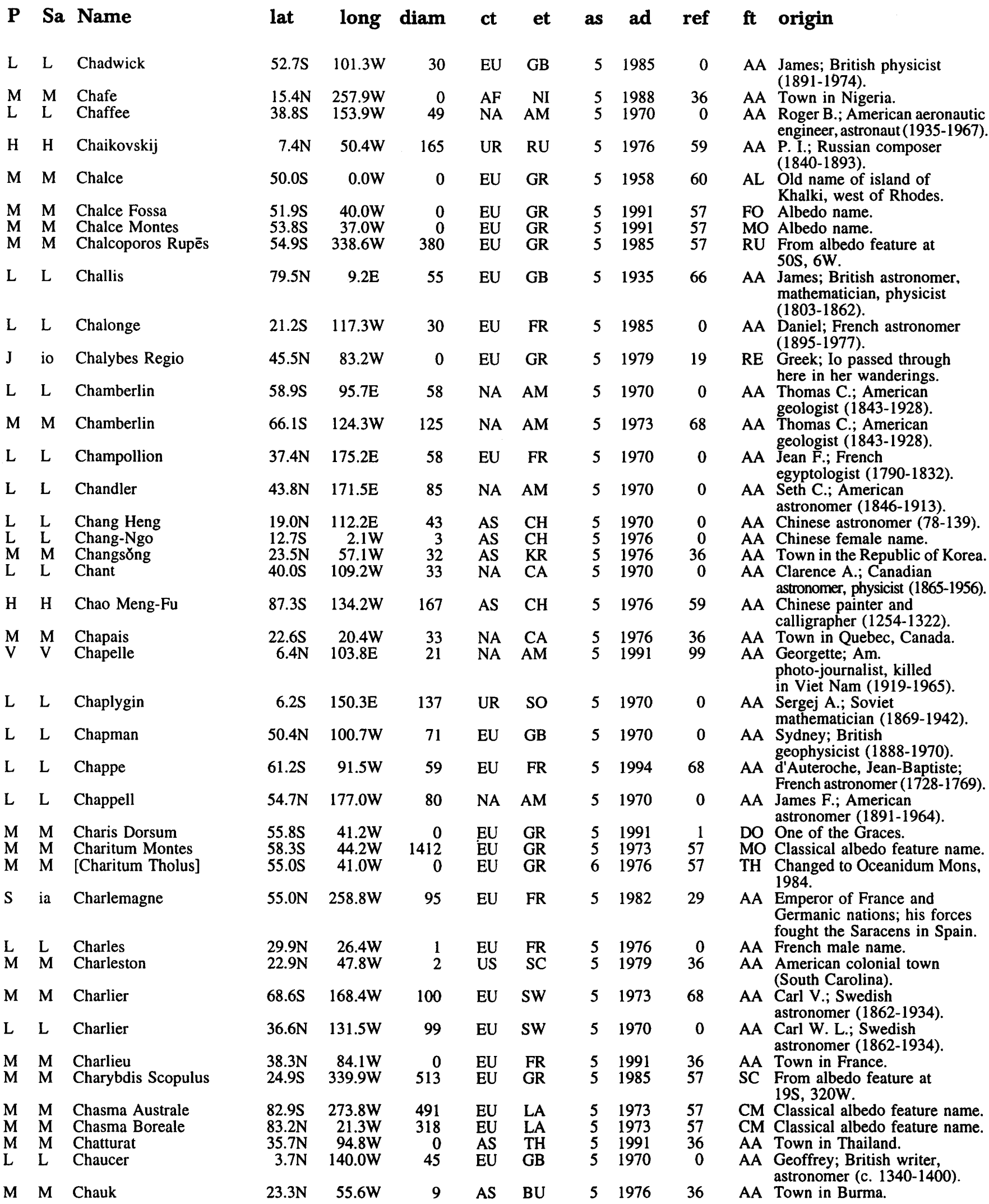

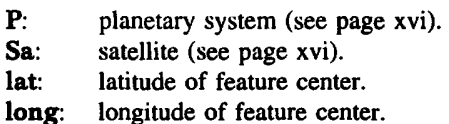

diam: diameter or long dimension of feature.

ct: continent of name origin (see page $284 \mathrm{ff}$.

et: ethnicity of name origin (see page $284 \mathrm{ff}$.)

as: name approval status (see page $x v i i$ ) ad: name approval date (year).

ref: reference source for name (see page $287 \mathrm{ff}$.). ft: feature type (see page 290). 


\section{P Sa Name}

$\begin{array}{lll}\text { L } & \text { L } & \text { Chauvenet } \\ \text { M } & \text { M } & \text { Cheb } \\ \text { L } & \text { L } & \text { Chebyshev } \\ \text { M } & \text { M } & \text { Chefu } \\ \text { M } & \text { M } & \text { Chekalin } \\ \text { H } & \text { H } & \text { Chekhov } \\ \text { L } & \text { L } & \text { [Chekov] } \\ \text { L } & \text { L } & \text { [Chenier] } \\ \text { L } & \text { L } & \text { Chernyshev } \\ & & \\ \text { M } & \text { M } & \text { Chersonesus } \\ \text { L } & \text { L } & \text { Chevallier } \\ & & \\ \text { M } & \text { M } & \text { Chia } \\ \text { H } & \text { H } & \text { Chiang K'ui } \\ \text { V } & \text { V } & \text { Chih Nu Dorsum } \\ \text { M } & \text { M } & \text { Chimbote } \\ \text { M } & \text { M } & \text { Chincoteague } \\ \text { L } & \text { L } & \text { Ching-Te } \\ \text { M } & \text { M } & \text { Chinju } \\ \text { M } & \text { M } & \text { Chinook } \\ \text { V } & \text { V } & \text { Chiun Corona } \\ \text { M } & \text { M } & \text { Chive } \\ \text { V } & \text { V } & \text { Chiyojo } \\ \text { L } & \text { L } & \text { Chladni } \\ \text { V } & \text { V } & \text { Chloe } \\ \text { M } & \text { M } & \text { Choctaw } \\ \text { M } & \text { M } & \text { Chom } \\ \text { H } & \text { H } & \text { Chŏng Ch'ŏl } \\ \text { H } & \text { H } & \text { Chopin } \\ & & \\ & \end{array}$

L L Chrétien

$\begin{array}{lll}\text { L } & \text { L } & \text { Christel } \\ \text { V } & \text { V } & \text { Christie }\end{array}$

M M Chryse

M M Chryse Planitia

M M Chrysokeras

\section{$\mathrm{H} \quad \mathrm{H}$ Chu Ta \\ M M Chur \\ L L Cichus}

J eu Cilicia Flexus

J eu Cilix

L L Cinco

$\mathrm{N}$ tr Cipango Planum

$S$ te Circe

M M Circle

V V Citlalpul Valles

V V Ciuacoatl Corona

L L Clairaut lat long

\begin{tabular}{|c|c|c|c|c|c|c|}
\hline $1.5 \mathrm{~S}$ & $137.0 \mathrm{E}$ & 81 & NA & AM & 5 & 1970 \\
\hline $\begin{array}{l}24.5 \mathrm{~S} \\
33.7 \mathrm{~S}\end{array}$ & $\begin{array}{c}19.3 \mathrm{~W} \\
133.1 \mathrm{~W}\end{array}$ & $\begin{array}{r}8 \\
178\end{array}$ & $\begin{array}{l}\text { EU } \\
\text { UR }\end{array}$ & $\begin{array}{l}\mathrm{CZ} \\
\mathrm{RU}\end{array}$ & 5 & $\begin{array}{l}1976 \\
1970\end{array}$ \\
\hline $\begin{array}{l}23.1 \mathrm{~N} \\
24.7 \mathrm{~S} \\
36.2 \mathrm{~S}\end{array}$ & $\begin{array}{r}247.7 \mathrm{~W} \\
26.6 \mathrm{~W} \\
61.5 \mathrm{~W}\end{array}$ & $\begin{array}{r}0 \\
87 \\
199\end{array}$ & $\begin{array}{l}\mathrm{AF} \\
\mathrm{UR} \\
\mathrm{UR}\end{array}$ & $\begin{array}{l}\mathrm{MZ} \\
\text { TK } \\
\text { RU }\end{array}$ & $\begin{array}{l}5 \\
5 \\
5\end{array}$ & $\begin{array}{l}198 \\
197 \\
197\end{array}$ \\
\hline $6.6 \mathrm{~S}$ & $82.0 \mathrm{E}$ & 0 & UR & RU & 6 & \\
\hline $17.7 \mathrm{~S}$ & $132.4 \mathrm{E}$ & 33 & EU & FR & 6 & \\
\hline $7.3 \mathrm{~N}$ & $174.2 \mathrm{E}$ & 58 & UR & So & 5 & \\
\hline $\begin{array}{l}50.0 \mathrm{~S} \\
44.9 \mathrm{~N}\end{array}$ & $\begin{array}{c}260.0 \mathrm{~W} \\
51.2 \mathrm{E}\end{array}$ & $\begin{array}{r}0 \\
52\end{array}$ & $\begin{array}{l}\text { EU } \\
\text { EU }\end{array}$ & $\begin{array}{l}\text { GR } \\
\text { GB }\end{array}$ & 5 & \\
\hline $\begin{array}{r}1.6 \mathrm{~N} \\
13.8 \mathrm{~N} \\
73.0 \mathrm{~S} \\
1.5 \mathrm{~S} \\
41.5 \mathrm{~N} \\
20.0 \mathrm{~N} \\
4.7 \mathrm{~S} \\
22.5 \mathrm{~N} \\
18.3 \mathrm{~N} \\
21.6 \mathrm{~N} \\
47.8 \mathrm{~S} \\
4.0 \mathrm{~N}\end{array}$ & $\begin{array}{c}59.5 \mathrm{~W} \\
102.7 \mathrm{~W} \\
195.0 \mathrm{E} \\
39.8 \mathrm{~W} \\
236.0 \mathrm{~W} \\
30.0 \mathrm{E} \\
42.3 \mathrm{~W} \\
55.2 \mathrm{~W} \\
340.5 \mathrm{E} \\
55.7 \mathrm{~W} \\
95.7 \mathrm{E} \\
1.1 \mathrm{E}\end{array}$ & $\begin{array}{r}94 \\
35 \\
625 \\
65 \\
35 \\
4 \\
69 \\
17 \\
150 \\
8 \\
43 \\
13\end{array}$ & $\begin{array}{l}\text { EU } \\
\text { AS } \\
\text { AS } \\
\text { SA } \\
\text { US } \\
\text { AS } \\
\text { AS } \\
\text { NA } \\
\text { AS } \\
\text { SA } \\
\text { AS } \\
\text { EU }\end{array}$ & $\begin{array}{l}\text { SP } \\
\text { CH } \\
\text { CH } \\
\text { PE } \\
\text { VA } \\
\text { CH } \\
\text { KR } \\
\text { CA } \\
\text { HE } \\
\text { BO } \\
\text { JA } \\
\text { GE }\end{array}$ & $\begin{array}{l}5 \\
5 \\
5 \\
5 \\
5 \\
5 \\
5 \\
5 \\
5 \\
5 \\
5\end{array}$ & \\
\hline $\begin{array}{r}7.4 \mathrm{~S} \\
41.5 \mathrm{~S} \\
38.7 \mathrm{~N} \\
46.4 \mathrm{~N} \\
65.1 \mathrm{~S}\end{array}$ & $\begin{array}{r}98.6 \mathrm{E} \\
37.0 \mathrm{~W} \\
2.2 \mathrm{~W} \\
116.2 \mathrm{~W} \\
123.1 \mathrm{~W}\end{array}$ & $\begin{array}{r}19 \\
20 \\
6 \\
162 \\
129\end{array}$ & $\begin{array}{l}\text { EU } \\
\text { US } \\
\text { AS } \\
\text { AS } \\
\text { EU }\end{array}$ & $\begin{array}{l}\text { GR } \\
\text { OH } \\
\text { TB } \\
\text { KR } \\
\text { PO }\end{array}$ & $\begin{array}{l}3 \\
5 \\
5\end{array}$ & \\
\hline
\end{tabular}

$162.9 \mathrm{E}$

$24.5 \mathrm{~N}$

$28.3 \mathrm{~N}$

$11.0 \mathrm{E}$

$10.0 \mathrm{~N}$

$72.6 \mathrm{E}$

$27.0 \mathrm{~N}$

$50.0 \mathrm{~S}$

88

EU

FR

$5 \quad 1970$

\section{$2.2 \mathrm{~N}$}

$16.7 \mathrm{~N}$

33.3S

$105.1 \mathrm{~W}$

$29.2 \mathrm{~W}$

$21.1 \mathrm{~W}$

47.6S $142.6 \mathrm{~W}$

$1.2 \mathrm{~N} \quad 181.9 \mathrm{~W}$

EU GE

2
25

EU

0 EU GR

$5 \quad 1976$

$5 \quad 1985$

$\begin{array}{ll}5 & 1958\end{array}$

$\begin{array}{lllll}1500 & \text { EU } & \text { GR } & 5 & 1973\end{array}$

$\begin{array}{lllll}0 & \text { EU } & \text { GR } & 5 & 1958\end{array}$

$\begin{array}{rrrrr}110 & \text { AS } & \text { CH } & 5 & 1976 \\ 4 & \text { UR } & \text { RU } & 5 & 1976 \\ 40 & \text { EU } & \text { IT } & 5 & 1935\end{array}$

19.1S $15.5 \mathrm{E}$

$11.5 \mathrm{~N} \quad 34.0 \mathrm{E}$

$12.1 \mathrm{~S}$

$53.7 \mathrm{~W}$

$22.4 \mathrm{~S}$

$57.4 \mathrm{~S}$

$53.0 \mathrm{~N}$

$25.4 \mathrm{~W}$

$185.0 \mathrm{E}$

$150.9 \mathrm{E}$

$13.9 \mathrm{E}$

$\begin{array}{lllll}23 & \text { EU } & \text { GR } & 5 & 1985\end{array}$

51973

$5 \quad 91$

$5 \quad 1982$

$5 \quad 1976$

$\begin{array}{ll}5 & 1994\end{array}$ 2350 2350
100
US MT

NA ME

NA AZ $\begin{array}{ll}5 & 1994 \\ 5 & 1935\end{array}$ ref $\mathrm{ft}$ origin

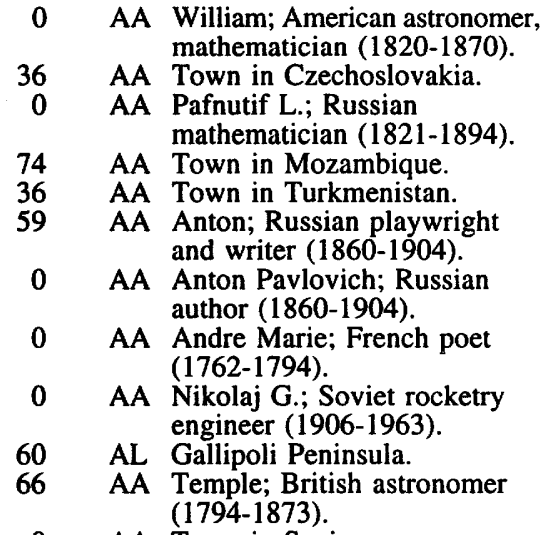

AA Town in Spain.

80

111

AA Chinese composer (12th century).

DO Chinese sky goddess.

AA Town in Peru.

AA Town in Virginia, USA.

AA Chinese male name.

AA Town in the Republic of Korea.

AA Town in Alberta, Canada.

CR Hebrew fertility goddess.

AA Town in Bolivia.

AA Japanese poet.

AA Ernst Florens Friedrich; German physicist (1756-1827).

AA First name from Greek.

AA Town in Ohio, USA.

AA Town in China (Tibet).

AA Korean poet (1536-1593).

AA Frederic; Polish-born French composer and pianist (1810-1849).

AA Chrétien, Henri; French mathematician, astronomer (1870-1956).

AA German female name.

AA Agatha; British novelist (1891-1976)

AL Island rich in gold: region of Thailand/Malacca.

57

PL Classical albedo feature name.

AL "Golden Horn"; Byzantine Peninsula (now Turkey), or its inlet.

AA Chinese painter (c. 1625-1705).

AA Town in Russia.

AA . Franceso Degli Stabili (Cecco D'Ascoli); Italian astronomer (1257-1327).

FE Land named for Cilix on his search for Europa.

AA Brother of Europa; Rand control-point crater.

60

LF Astronaut-named feature, Apollo 16 site.

PM Legendary island described by Marco Polo.

AA Changed Odysseus' companions into swines.

AA Town in Montana, USA

VA Aztec name for planet Venus.

CR Aztec earth goddess.

AA Alexis Claude; French mathematician (1713-1765).

\footnotetext{
P: planetary system (see page $x v i)$.

Sa: $\quad$ satellite (see page xvi).

lat: latitude of feature center.

long: longitude of feature center.
}

diam: diameter or long dimension of feature.

ct: continent of name origin (see page $284 \mathrm{ff}$.)

et: ethnicity of name origin (see page $284 \mathrm{ff}$.)

as: name approval status (see page xvii). ad: name approval date (year).

ref: reference source for name (see page $287 \mathrm{ff}$.).

ft: feature type (see page 290). 


\section{ALPHABETICAL LIST OF NAMES}

\begin{tabular}{|c|c|c|c|c|c|c|c|c|c|}
\hline $\mathbf{P}$ & $\mathbf{S a}$ & Name & lat & long & diam & ct & et & as & ad \\
\hline $\mathbf{M}$ & $\mathbf{M}$ & Clanis Valles & $34.0 \mathrm{~N}$ & $301.5 \mathrm{~W}$ & 0 & EU & IT & 5 & 1987 \\
\hline $\begin{array}{l}\mathrm{M} \\
\mathrm{M} \\
\mathrm{M}\end{array}$ & $\begin{array}{l}\mathbf{M} \\
\mathbf{M} \\
\mathbf{M}\end{array}$ & $\begin{array}{l}\text { Claritas } \\
\text { Claritas Fossae } \\
\text { Claritas Rupes }\end{array}$ & $\begin{array}{l}35.0 S \\
34.8 S \\
26.0 S\end{array}$ & $\begin{array}{r}110.0 \mathrm{~W} \\
99.1 \mathrm{~W} \\
105.5 \mathrm{~W}\end{array}$ & $\begin{array}{r}0 \\
2033 \\
0\end{array}$ & $\begin{array}{l}\text { EU } \\
\text { EU } \\
\text { EU }\end{array}$ & $\begin{array}{l}\text { LA } \\
\text { LA } \\
\text { LA }\end{array}$ & $\begin{array}{l}5 \\
5 \\
5\end{array}$ & $\begin{array}{l}1958 \\
1973 \\
1989\end{array}$ \\
\hline $\mathbf{L}$ & $\mathbf{L}$ & Clark & $38.4 \mathrm{~S}$ & $118.9 \mathrm{E}$ & 49 & NA & $\mathrm{AM}$ & 5 & 1970 \\
\hline $\mathbf{M}$ & $\mathbf{M}$ & Clark & $55.7 \mathrm{~S}$ & $133.2 \mathrm{~W}$ & 93 & NA & AM & 5 & 1973 \\
\hline$\underset{\mathrm{L}}{\mathrm{M}}$ & $\begin{array}{l}M \\
L\end{array}$ & $\begin{array}{l}\text { Clasia Vallis } \\
\text { Clausius }\end{array}$ & $\begin{array}{l}33.8 \mathrm{~N} \\
36.9 \mathrm{~S}\end{array}$ & $\begin{array}{r}302.5 \mathrm{~W} \\
43.8 \mathrm{~W}\end{array}$ & $\begin{array}{r}240 \\
24\end{array}$ & $\begin{array}{l}\mathrm{EU} \\
\mathrm{EU}\end{array}$ & IT & $\begin{array}{l}5 \\
5\end{array}$ & $\begin{array}{l}1987 \\
1935\end{array}$ \\
\hline $\mathrm{L}$ & $\mathrm{L}$ & Clavius & $58.8 \mathrm{~S}$ & $14.1 \mathrm{~W}$ & 245 & EU & GE & 5 & 1935 \\
\hline $\begin{array}{l}M \\
L\end{array}$ & $\begin{array}{l}M \\
L\end{array}$ & $\begin{array}{l}\text { Cleia Dorsum } \\
\text { Cleomedes }\end{array}$ & $\begin{array}{l}55.3 \mathrm{~S} \\
27.7 \mathrm{~N}\end{array}$ & $\begin{array}{l}46.3 \mathrm{~W} \\
56.0 \mathrm{E}\end{array}$ & $\begin{array}{r}0 \\
125\end{array}$ & $\begin{array}{l}\mathrm{EU} \\
\mathrm{EU}\end{array}$ & $\begin{array}{l}\text { GR } \\
\text { GR }\end{array}$ & $\begin{array}{l}5 \\
5\end{array}$ & $\begin{array}{l}1991 \\
1935\end{array}$ \\
\hline V & $\mathrm{V}$ & Cleopatra & $65.9 \mathrm{~N}$ & $7.0 \mathrm{E}$ & 105 & $\mathrm{AF}$ & EG & 5 & 1992 \\
\hline $\begin{array}{l}\mathrm{V} \\
\mathrm{L}\end{array}$ & $\begin{array}{l}\mathrm{V} \\
\mathrm{L}\end{array}$ & $\begin{array}{l}\text { [Cleopatra Patera] } \\
\text { Cleostratus }\end{array}$ & $\begin{array}{l}66.0 \mathrm{~N} \\
60.4 \mathrm{~N}\end{array}$ & $\begin{array}{c}6.9 \mathrm{E} \\
77.0 \mathrm{~W}\end{array}$ & $\begin{array}{r}119 \\
62\end{array}$ & $\begin{array}{l}\text { AF } \\
\text { EU }\end{array}$ & $\begin{array}{l}\text { EG } \\
\text { GR }\end{array}$ & $\begin{array}{l}6 \\
5\end{array}$ & $\begin{array}{l}1992 \\
1935\end{array}$ \\
\hline $\mathbf{L}$ & $\mathbf{L}$ & Clerke & $21.7 \mathrm{~N}$ & $29.8 \mathrm{E}$ & 6 & $\mathrm{EU}$ & GB & 5 & 1973 \\
\hline V & V & Cline & $21.8 \mathrm{~S}$ & $317.0 \mathrm{E}$ & 40 & NA & $\mathrm{AM}$ & 5 & 1994 \\
\hline $\begin{array}{l}\mathbf{M} \\
\mathbf{M}\end{array}$ & $\begin{array}{l}\mathbf{M} \\
\mathbf{M}\end{array}$ & $\begin{array}{l}\text { Clogh } \\
\text { Clota Vallis }\end{array}$ & $\begin{array}{l}20.8 \mathrm{~N} \\
26.0 \mathrm{~S}\end{array}$ & $\begin{array}{l}47.5 \mathrm{~W} \\
20.8 \mathrm{~W}\end{array}$ & $\begin{array}{r}10 \\
101\end{array}$ & $\begin{array}{l}\mathrm{EU} \\
\mathrm{EU}\end{array}$ & $\underset{\mathrm{GB}}{\mathrm{IR}}$ & $\begin{array}{l}5 \\
5\end{array}$ & $\begin{array}{l}1976 \\
1976\end{array}$ \\
\hline $\begin{array}{l}\mathrm{V} \\
\mathrm{M} \\
\mathrm{M} \\
\mathrm{V} \\
\mathrm{M} \\
\mathrm{L}\end{array}$ & $\begin{array}{l}\mathrm{V} \\
\mathrm{M} \\
\mathrm{M} \\
\mathrm{V} \\
\mathrm{M} \\
\mathrm{L}\end{array}$ & $\begin{array}{l}\text { Clotho Tessera } \\
\text { Clova } \\
\text { Cluny } \\
\text { Coatlicue Corona } \\
\text { Cobalt } \\
\text { Coblentz }\end{array}$ & $\begin{array}{l}56.4 \mathrm{~N} \\
21.7 \mathrm{~N} \\
24.1 \mathrm{~S} \\
63.2 \mathrm{~N} \\
26.1 \mathrm{~S} \\
37.9 \mathrm{~S}\end{array}$ & $\begin{array}{c}334.9 \mathrm{E} \\
52.0 \mathrm{~W} \\
27.1 \mathrm{~W} \\
273.0 \mathrm{E} \\
26.8 \mathrm{~W} \\
126.1 \mathrm{E}\end{array}$ & $\begin{array}{r}289 \\
0 \\
9 \\
199 \\
10 \\
33\end{array}$ & $\begin{array}{l}\text { EU } \\
\text { NA } \\
\text { EU } \\
\text { CA } \\
\text { US } \\
\text { NA }\end{array}$ & $\begin{array}{l}\text { GR } \\
\text { CA } \\
\text { FR } \\
\text { AZ } \\
\text { CT } \\
\text { AM }\end{array}$ & $\begin{array}{l}5 \\
5 \\
5 \\
5 \\
5 \\
5\end{array}$ & $\begin{array}{l}1985 \\
1988 \\
1976 \\
1985 \\
1976 \\
1970\end{array}$ \\
\hline $\mathbf{M}$ & $\mathbf{M}$ & Coblentz & $55.3 \mathrm{~S}$ & $90.2 \mathrm{~W}$ & 111 & NA & $\mathrm{AM}$ & 5 & 1973 \\
\hline $\begin{array}{l}M \\
L\end{array}$ & $\begin{array}{l}\mathbf{M} \\
\mathbf{L}\end{array}$ & $\begin{array}{l}\text { Cobres } \\
\text { Cochise }\end{array}$ & $\begin{array}{l}12.1 S \\
20.2 S\end{array}$ & $\begin{array}{c}153.7 \mathrm{~W} \\
30.8 \mathrm{E}\end{array}$ & $\begin{array}{r}89 \\
1\end{array}$ & $\begin{array}{l}\text { SA } \\
\text { NA }\end{array}$ & $\begin{array}{l}\text { AR } \\
\text { AM }\end{array}$ & $\begin{array}{l}5 \\
5\end{array}$ & $\begin{array}{l}1985 \\
1973\end{array}$ \\
\hline V & V & Cochran & $51.8 \mathrm{~N}$ & $143.2 \mathrm{E}$ & 100 & NA & $\mathrm{AM}$ & 5 & 1985 \\
\hline L & L & Cockcroft & $31.3 \mathrm{~N}$ & $162.6 \mathrm{~W}$ & 93 & EU & GB & 5 & 1970 \\
\hline V & V & Cohn & $33.2 \mathrm{~S}$ & $208.1 \mathrm{E}$ & 21 & OC & $\mathrm{AU}$ & 5 & 1994 \\
\hline J & io & Colchis Regio & $5.3 \mathrm{~N}$ & $199.8 \mathrm{~W}$ & 0 & EU & GR & 5 & 1979 \\
\hline H & $\mathbf{H}$ & Coleridge & $55.9 \mathrm{~S}$ & $66.7 \mathrm{~W}$ & 110 & EU & EN & 5 & 1976 \\
\hline $\begin{array}{l}\mathrm{V} \\
\mathrm{V}\end{array}$ & $\begin{array}{l}\mathrm{V} \\
\mathrm{V}\end{array}$ & $\begin{array}{l}\text { [Colette] } \\
\text { Colette Patera }\end{array}$ & $\begin{array}{l}65.0 \mathrm{~N} \\
66.3 \mathrm{~N}\end{array}$ & $\begin{array}{l}322.0 \mathrm{E} \\
322.8 \mathrm{E}\end{array}$ & $\begin{array}{r}0 \\
149\end{array}$ & $\begin{array}{l}\mathrm{EU} \\
\mathrm{EU}\end{array}$ & $\begin{array}{l}\text { FR } \\
\text { FR }\end{array}$ & $\begin{array}{l}6 \\
5\end{array}$ & $\begin{array}{l}1982 \\
1982\end{array}$ \\
\hline $\begin{array}{l}\mathrm{V} \\
\mathrm{V} \\
\mathrm{M}\end{array}$ & $\begin{array}{l}\mathrm{V} \\
\mathrm{V} \\
\mathrm{M}\end{array}$ & $\begin{array}{l}\text { Colijnsplaat Corona } \\
\text { Colleen } \\
\text { Colles Nili }\end{array}$ & $\begin{array}{l}32.0 \mathrm{~S} \\
60.8 \mathrm{~S} \\
39.6 \mathrm{~N}\end{array}$ & $\begin{array}{l}151.0 \mathrm{E} \\
162.2 \mathrm{E} \\
295.3 \mathrm{~W}\end{array}$ & $\begin{array}{r}350 \\
14 \\
551\end{array}$ & $\begin{array}{l}\mathrm{EU} \\
\mathrm{EU} \\
\mathrm{EU}\end{array}$ & $\begin{array}{r}\text { TU } \\
\text { IR } \\
\text { RM }\end{array}$ & $\begin{array}{l}5 \\
5 \\
5\end{array}$ & $\begin{array}{l}1994 \\
1994 \\
1987\end{array}$ \\
\hline $\mathrm{L}$ & L & Collins & $1.3 \mathrm{~N}$ & $23.7 \mathrm{E}$ & 2 & NA & $\mathrm{AM}$ & 5 & 1970 \\
\hline $\begin{array}{l}\mathrm{M} \\
\mathrm{L}\end{array}$ & $\begin{array}{l}\mathbf{M} \\
\mathbf{L}\end{array}$ & $\begin{array}{l}\text { Coloe Fossae } \\
\text { Colombo }\end{array}$ & $\begin{array}{l}37.1 \mathrm{~N} \\
15.1 \mathrm{~S}\end{array}$ & $\begin{array}{c}303.9 \mathrm{~W} \\
45.8 \mathrm{E}\end{array}$ & $\begin{array}{r}930 \\
76\end{array}$ & $\begin{array}{l}\text { EU } \\
\text { EU }\end{array}$ & $\begin{array}{r}\text { GR } \\
\text { SP }\end{array}$ & $\begin{array}{l}5 \\
5\end{array}$ & $\begin{array}{l}1982 \\
1935\end{array}$ \\
\hline $\begin{array}{l}\mathrm{M} \\
\mathrm{V} \\
\mathrm{M}\end{array}$ & $\begin{array}{l}\mathrm{M} \\
\mathrm{V} \\
\mathrm{M}\end{array}$ & $\begin{array}{l}\text { Colōn } \\
\text { Colonna } \\
\text { Columbus }\end{array}$ & $\begin{array}{l}23.4 \mathrm{~N} \\
64.7 \mathrm{~N} \\
29.7 \mathrm{~S}\end{array}$ & $\begin{array}{l}49.1 \mathrm{~W} \\
216.8 \mathrm{E} \\
165.8 \mathrm{~W}\end{array}$ & $\begin{array}{r}2 \\
28 \\
114\end{array}$ & $\begin{array}{l}\text { SA } \\
\text { EU } \\
\text { EU }\end{array}$ & $\begin{array}{l}\text { PM } \\
\text { IT } \\
\text { IT }\end{array}$ & $\begin{array}{l}5 \\
5 \\
5\end{array}$ & $\begin{array}{l}1979 \\
1994 \\
1976\end{array}$ \\
\hline
\end{tabular}

\section{ft origin}

56 VA Classical river in Etruria; present Chiana River, Italy.

AL Latin, meaning "bright."

FO Classical albedo feature name.

RU From albedo feature at 25S, 110W.

AA Alvan; American astronomer (1804-1887); Alvan G.; American astronomer, optician (1832-1897).

AA Alvan; American opticianastronomer (1804-1887)

VA Classical river in Umbria, Italy.

AA Rudolf Julius Emmanuel; German physicist (1822-1888)

AA Christopher Klau; German mathematician (1537-1612).

DO One of the Graces.

AA Greek astronomer (unkn-c. 50 B.C.).

AA Egyptian queen, notable for love affairs with Julius Caesar and Mark Anthony (69-30 B.C.)

PE Egyptian queen c. 69-30 B.C.

AA Greek astronomer (unkn-c. 500 B.C.).

AA Agnes Mary; British astronomer (1842-1907)

AA Patsy; American singer (1932-1963)

AA Town in Ireland.

VA Ancient name for present River Clyde, Scotland.

TE Greek; one of three Fates

AA Town in Quebec, Canada.

AA Town in France.

CR Aztec earth goddess.

AA Town in Connecticut, USA

AA William W.; American physicist, astronomer (1873-1962).

AA William W.; American physicist (1873-1962)

AA Village in Argentina.

LF Astronaut-named feature, Apollo 17 site.

AA Jacqueline; American aviator (c. 1906-1980)

AA Sir John D.; British nuclear physicist; Nobel laureate (1897-1967).

AA Carola; Australian artist (1892-1964)

RE Greek; lo passed through this part of Asia Minor in her wanderings.

AA Samuel Taylor; English poet (1772-1834).

AA Changed to Colette Patera

PE Claudine; French novelist (1873-1954).

CR Teutonic fertility goddess.

AA Irish first name.

CO From classical albedo feature Portus Nili, at 38N, 295W.

AA Michael; American astronaut (1930-Live).

FO Classical albedo feature name.

AA Columbus, Christopher; Spanish explorer (c. 1446-1506). AA Port of Panama.

AA Vittoria; Italian poet (c. 1490-1547).

AA Christopher; Italian explorer (1451-1506).

\footnotetext{
P: $\quad$ planetary system (see page xvi).

Sa: $\quad$ satellite (see page $x v i$ ).

lat: latitude of feature center.

long: longitude of feature center.
}

diam: diameter or long dimension of feature.

ct: continent of name origin (see page $284 \mathrm{ff}$.)

et: ethnicity of name origin (see page $284 \mathrm{ff}$.)

as: name approval status (see page $\mathrm{xvii}$ ). ad: name approval date (year).

ref: reference source for name (see page $287 \mathrm{ff}$.).

ft: feature type (see page 290). 


\section{ALPHABETICAL LIST OF NAMES}

\section{P Sa Name \\ M M Comas Sola \\ V V Comnena \\ L L Compton}

L L Comrie

L L Comstock

$S$ rh Con

M M Conches

M M Concord

L L Condon

L L Condorcet

L L Cone

L L Congreve

L L Conon

V V Conway

L L Cook

M M Cooma

L L Cooper

M M Copais Palus

L L Copernicus

M M Copernicus

V V Copia Corona

H H Copley

M M Coprates

M M Coprates Catena

M M Coprates Chasma

M M Coprates Labes

J ga Coptos Facula

M M Coracis Fossae

$S$ di Coras

$\begin{array}{ccl}\text { M } & \text { M } & \begin{array}{l}\text { Corby } \\ \text { V }\end{array} \\ \text { V } & \text { Corday Patera } \\ \text { U } & \text { ss } & \text { Cordelia } \\ & & \\ \text { L } & \text { L } & \text { Cori } \\ \text { V } & \text { V } & \text { Cori } \\ \text { V } & \text { V } & \text { Corinna } \\ \text { U } & \text { ob } & \text { Coriolanus } \\ \text { L } & \text { L } & \text { Coriolis } \\ \text { L } & \text { L } & \text { [Corneille] }\end{array}$

lat long

$\begin{array}{rrrrrrrr}20.1 \mathrm{~S} & 158.4 \mathrm{~W} & 132 & \text { EU } & \text { SP } & 5 & 1973 & 68 \\ 1.2 \mathrm{~N} & 343.7 \mathrm{E} & 20 & \text { EU } & \text { BZ } & 5 & 1994 & 87 \\ 55.3 \mathrm{~N} & 103.8 \mathrm{E} & 182 & \text { NA } & \text { AM } & 5 & 1970 & 0\end{array}$

\begin{abstract}
$23.3 \mathrm{~N} \quad 112.7 \mathrm{~W}$
\end{abstract}
59

$21.8 \mathrm{~N} \quad 121.5 \mathrm{~W}$

EU GB

$5 \quad 1970$

25.8S $12.7 \mathrm{~W}$

4.2S $34.3 \mathrm{~W}$

$16.6 \mathrm{~N} \quad 34.1 \mathrm{~W}$

$1.9 \mathrm{~N} \quad 60.4 \mathrm{E}$

$12.1 \mathrm{~N}$

$69.6 \mathrm{E}$

3.7S

$0.2 \mathrm{~S}$

$17.4 \mathrm{~W}$

2. $167.3 \mathrm{~W}$

$21.6 \mathrm{~N}$

$2.0 \mathrm{E}$

$48.3 \mathrm{~N}$

$39.1 \mathrm{E}$

$17.5 \mathrm{~S}$

24.0S

$48.9 \mathrm{E}$
$108.5 \mathrm{~W}$

$52.9 \mathrm{~N}$

$175.6 \mathrm{E}$

$55.0 \mathrm{~N} 280.0 \mathrm{~W}$

$9.7 \mathrm{~N}$

$20.1 \mathrm{~W}$

50.0S $168.6 \mathrm{~W}$

72 NA AM

51970

0

SA IN

EU FR

US MA

NA AM

$5 \quad 1982$

$5 \quad 1976$

$\begin{array}{ll}5 & 1976 \\ 5 & 1976\end{array}$

$5 \quad 1935$

74

EU FR

51973

0 NA AM

$5 \quad 1970$

57

EU GB

51935

21

EU GR

51994

46

EU EN

EU GB

$\begin{array}{ll}5 & 1935 \\ 5 & 1989\end{array}$

46
0

C AU

$5 \quad 1970$

0

EU GR

51958

107

EU PO

$5 \quad 1935$

42.5

$42.5 \mathrm{~S}$
$38.4 \mathrm{~S}$

$15.0 \mathrm{~S}$

$$
75.5 \mathrm{E}
$$

292

EU PO

51973

500

EU RM

$5 \quad 1994$

15.15

$65.0 \mathrm{~W}$

30

NA AM

51976

$15.1 S$

$59.4 \mathrm{~W}$

$13.5 \mathrm{~S}$

$60.7 \mathrm{~W}$

67.3W

0 EU GR

51958

243

962

115

EU GR

EU GR

EU GR

51973

9.4N 209.8W

332

AF EG

$5 \quad 1985$

34.8S 78.6W

747

EU GR

$5 \quad 1985$

$0.6 \mathrm{~N} 266.4 \mathrm{~W}$

37

EU

\begin{tabular}{rr}
$43.1 \mathrm{~N}$ & $222.4 \mathrm{~W}$ \\
& $40.2 \mathrm{E}$ \\
& \\
\hline &
\end{tabular}

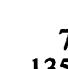

7
135

EU EN

$$
0
$$

FR

$\begin{array}{rr}5 & 1979 \\ 5 & 91\end{array}$

1985

51982

$\begin{array}{rrrrrrrr}0.0 \mathrm{~N} & 0.0 \mathrm{X} & 0 & \mathrm{EU} & \mathrm{GB} & 5 & 1988 & 85 \\ & & & & & & & \\ 50.6 \mathrm{~S} & 151.9 \mathrm{~W} & 65 & \mathrm{EU} & \mathrm{CZ} & 5 & 1979 & 0 \\ & & & & & & & \\ 25.4 \mathrm{~N} & 72.7 \mathrm{E} & 55 & \mathrm{EU} & \mathrm{CZ} & 5 & 1991 & 99 \\ & & & & & & & \\ 22.8 \mathrm{~N} & 40.5 \mathrm{E} & 21 & \mathrm{EU} & \mathrm{GR} & 5 & 1994 & 99 \\ 11.4 \mathrm{~S} & 345.2 \mathrm{E} & 120 & \mathrm{EU} & \mathrm{GB} & 5 & 1988 & 85 \\ 0.1 \mathrm{~N} & 171.8 \mathrm{E} & 78 & \mathrm{EU} & \mathrm{FR} & 5 & 1970 & 0 \\ 12.3 \mathrm{~N} & 134.8 \mathrm{E} & 35 & \text { EU } & \text { FR } & 6 & 0 & 0\end{array}$

\section{ft origin}

AA Jose; Spanish astronomer (1868-1937).

AA Anna; Byzantine princess, physician, writer (1083-1148).

AA Arthur H.; American physicist, Nobel laureate (1892-1962); Karl T.; American physicist (1887-1954).

AA Leslie J.; British astronomer (1893-1950).

AA George C.; American astronomer (1855-1934).

AA Inca coastal creator god.

AA Town in France.

AA Town in Massachusetts, USA.

AA Edward U.; American physicist (1902-1974).

AA Jean de; French mathematician (1743-1794).

60

LF Astronaut-named feature, Apollo 14 site.

AA Sir William; British rocket engineer, inventor (1772-1828).

66

AA Conon of Samos; Greek astronomer (c. 260 B.C.).

AA Anne Finch; English natural scientist (1631-1679).

66 AA James; British explorer (1728-1779).

74 AA Town in New South Wales, Australia.

0 AA John C.; American humanitarian (1887-1967).

60 AL Named for marsh north of Mt. Helicon in Boeotia, Greece.

66

AA Nicholas; Polish astronomer (1473-1543).

68

AA Nicolaus; Polish astronomer (1473-1543).

CR Roman goddess of plenty.

AA John Singleton; American painter (1738-1815).

AL Old name for Persian River Ab-I-Diz.

CA Classical albedo feature name.

CM Classical albedo feature name.

LA From albedo feature at $14 \mathrm{~S}, 65 \mathrm{~W}$.

FA Early town from which caravans departed.

FO From albedo feature at $46 \mathrm{~S}, 87 \mathrm{~W}$.

44

AA Brother of Tiburtus and twin brother of Catillus. He was founder of Tibur and an ally of Turnus against Aeneas.

AA Town in England.

PE Charlotte; French patriot (1768-1798).

SS 1986 U7; Daughter to Lear in "KIng Lear" 49,771 km semi-major axis

AA Gerty Theresa Radnitz; Czech-American physiologist; Nobel laureate (1896-1957).

AA Gerty; Czech biochemist, Nobel laureate (1896-1957)

AA Greek poet (fl. c. 490 B.C.).

AA Shakespearean hero.

AA Gaspard G. De; French physicist (1792-1843).

AA Piere; French lieraure (1606-1684).

\footnotetext{
P: $\quad$ planetary system (see page xvi).

Sa: $\quad$ satellite (see page xvi).

lat: latitude of feature center.

long: longitude of feature center.
}

diam: diameter or long dimension of feature.

ct: continent of name origin (see page $284 \mathrm{ff}$.)

et: ethnicity of name origin (see page $284 \mathrm{ff}$.)

as: name approval status (see page xvii). ad: name approval date (year).

ref: reference source for name (see page $287 \mathrm{ff}$.).

ft: feature type (see page 290). 


\section{ALPHABETICAL LIST OF NAMES}

\begin{tabular}{|c|c|c|c|c|c|c|c|c|c|}
\hline $\mathbf{P}$ & $\mathrm{Sa}$ & Name & lat & long & diam & ct & et & as & ad \\
\hline $\begin{array}{l}\mathbf{M} \\
\mathbf{M}\end{array}$ & $\begin{array}{l}M \\
M\end{array}$ & $\begin{array}{l}\text { Coronae Mons } \\
\text { Coronae Scopulus }\end{array}$ & $\begin{array}{l}34.5 \mathrm{~S} \\
33.9 \mathrm{~S}\end{array}$ & $\begin{array}{l}271.5 W \\
294.1 W\end{array}$ & $\begin{array}{r}0 \\
366\end{array}$ & $\begin{array}{l}\mathrm{EU} \\
\mathrm{EU}\end{array}$ & $\begin{array}{l}\text { GR } \\
\text { GR }\end{array}$ & $\begin{array}{l}5 \\
5\end{array}$ & $\begin{array}{l}1989 \\
1985\end{array}$ \\
\hline V & $\mathrm{V}$ & Corpman & $0.3 \mathrm{~N}$ & $151.8 \mathrm{E}$ & 52 & $\mathrm{EU}$ & PO & 5 & 1994 \\
\hline V & V & Cortese & $11.4 \mathrm{~S}$ & $218.3 \mathrm{E}$ & 28 & EU & IT & 5 & 1994 \\
\hline $\begin{array}{l}\mathrm{M} \\
\mathrm{V}\end{array}$ & $\begin{array}{l}\mathrm{M} \\
\mathrm{V}\end{array}$ & $\begin{array}{l}\text { Cost } \\
\text { Cotis Mons }\end{array}$ & $\begin{array}{l}15.3 \mathrm{~N} \\
44.1 \mathrm{~N}\end{array}$ & $\begin{array}{l}256.1 \mathrm{~W} \\
233.1 \mathrm{E}\end{array}$ & $\begin{array}{l}10 \\
62\end{array}$ & $\begin{array}{l}\text { US } \\
\text { EU }\end{array}$ & $\begin{array}{l}\text { TX } \\
\text { GR }\end{array}$ & $\begin{array}{l}5 \\
5\end{array}$ & $\begin{array}{l}1976 \\
1985\end{array}$ \\
\hline V & $\mathrm{V}$ & Cotton & $70.7 \mathrm{~N}$ & $300.3 \mathrm{E}$ & 47 & EU & FR & 5 & 1985 \\
\hline $\mathrm{L}$ & $\mathrm{L}$ & Couder & $4.8 \mathrm{~S}$ & $92.4 \mathrm{~W}$ & 21 & EU & FR & 5 & 1985 \\
\hline L & L & Coulomb & $54.7 \mathrm{~N}$ & $114.6 \mathrm{~W}$ & 89 & EU & FR & 5 & 1970 \\
\hline $\mathrm{H}$ & $\mathrm{H}$ & Couperin & $29.8 \mathrm{~N}$ & $151.4 \mathrm{~W}$ & 80 & EU & FR & 5 & 1979 \\
\hline $\begin{array}{l}\mathrm{L} \\
\mathrm{M} \\
\mathrm{M} \\
\mathrm{J} \\
\mathrm{L}\end{array}$ & $\begin{array}{l}\mathrm{L} \\
\mathrm{M} \\
\mathrm{M} \\
\text { io } \\
\mathrm{L}\end{array}$ & $\begin{array}{l}\text { Courtney } \\
\text { Cray } \\
\text { Creel } \\
\text { Creidne Patera } \\
\text { Cremona }\end{array}$ & $\begin{array}{c}25.1 \mathrm{~N} \\
44.4 \mathrm{~N} \\
6.1 \mathrm{~S} \\
52.4 \mathrm{~S} \\
67.5 \mathrm{~N}\end{array}$ & $\begin{array}{r}30.8 \mathrm{~W} \\
16.2 \mathrm{~W} \\
39.0 \mathrm{~W} \\
343.5 \mathrm{~W} \\
90.6 \mathrm{~W}\end{array}$ & $\begin{array}{r}1 \\
5 \\
8 \\
125 \\
85\end{array}$ & $\begin{array}{l}\text { EU } \\
\text { EU } \\
\text { NA } \\
\text { EU } \\
\text { EU }\end{array}$ & $\begin{array}{c}\mathrm{EN} \\
\mathrm{EN} \\
\mathrm{ME} \\
\mathrm{CE} \\
\mathrm{IT}\end{array}$ & $\begin{array}{l}5 \\
5 \\
5 \\
5 \\
5\end{array}$ & $\begin{array}{l}1976 \\
1976 \\
1976 \\
1979 \\
1964\end{array}$ \\
\hline $\mathrm{L}$ & $\mathrm{L}$ & Crescent & $2.9 \mathrm{~S}$ & $23.4 \mathrm{~W}$ & 1 & NA & $\mathrm{AM}$ & 5 & 1973 \\
\hline $\mathrm{U}$ & ss & Cressida & $0.0 \mathrm{~N}$ & $0.0 \mathrm{X}$ & 0 & EU & GB & 5 & 1988 \\
\hline $\mathbf{S}$ & di & Creusa & $48.0 \mathrm{~N}$ & $76.9 w$ & 35 & EU & $\mathbf{R M}$ & 5 & 1982 \\
\hline $\begin{array}{l}M \\
L\end{array}$ & $\begin{array}{l}\mathrm{M} \\
\mathrm{L}\end{array}$ & $\begin{array}{l}\text { Crewe } \\
\text { Crile }\end{array}$ & $\begin{array}{l}25.2 \mathrm{~S} \\
14.2 \mathrm{~N}\end{array}$ & $\begin{array}{l}19.4 \mathrm{~W} \\
46.0 \mathrm{E}\end{array}$ & $\begin{array}{l}3 \\
9\end{array}$ & $\begin{array}{l}\text { EU } \\
\text { NA }\end{array}$ & $\begin{array}{r}\mathrm{GB} \\
\mathrm{AM}\end{array}$ & $\begin{array}{l}5 \\
5\end{array}$ & $\begin{array}{l}1976 \\
1976\end{array}$ \\
\hline $\mathbf{J}$ & io & Crimea Mons & $76.1 S$ & $244.8 W$ & 0 & EU & GR & 5 & 1985 \\
\hline L & $\mathrm{L}$ & Crocco & $47.5 \mathrm{~S}$ & $150.2 \mathrm{E}$ & 75 & EU & IT & 5 & 1970 \\
\hline $\mathbf{M}$ & $\mathbf{M}$ & Crommelin & $5.3 \mathrm{~N}$ & $10.2 \mathrm{~W}$ & 111 & $\mathrm{EU}$ & GB & 5 & 1973 \\
\hline $\mathrm{L}$ & $\mathrm{L}$ & Crommelin & $68.1 S$ & $146.9 \mathrm{~W}$ & 94 & EU & GB & 5 & 1970 \\
\hline $\mathrm{L}$ & $\mathrm{L}$ & Crookes & $10.3 \mathrm{~S}$ & $164.5 \mathrm{~W}$ & 49 & EU & GB & 5 & 1970 \\
\hline L & $\mathrm{L}$ & Crozier & $13.5 \mathrm{~S}$ & $50.8 \mathrm{E}$ & 22 & EU & GB & 5 & 1935 \\
\hline L & $\mathrm{L}$ & Crüger & $16.7 \mathrm{~S}$ & $66.8 \mathrm{~W}$ & 45 & $\mathrm{EU}$ & $\mathrm{GE}$ & 5 & 1935 \\
\hline $\mathbf{M}$ & M & Cruls & $43.2 \mathrm{~S}$ & $196.9 W$ & 83 & SA & BR & 5 & 1973 \\
\hline $\begin{array}{l}\mathrm{M} \\
\mathrm{L}\end{array}$ & $\begin{array}{l}\mathrm{M} \\
\mathrm{L}\end{array}$ & $\begin{array}{l}\text { Cruz } \\
\text { Ctesibius }\end{array}$ & $\begin{array}{r}38.6 \mathrm{~N} \\
0.8 \mathrm{~N}\end{array}$ & $\begin{array}{l}1.7 W \\
118.7 \mathrm{E}\end{array}$ & $\begin{array}{r}6 \\
36\end{array}$ & $\begin{array}{l}\text { SA } \\
\text { AF }\end{array}$ & $\begin{array}{l}\text { VE } \\
\text { EG }\end{array}$ & $\begin{array}{l}5 \\
5\end{array}$ & $\begin{array}{l}1976 \\
1976\end{array}$ \\
\hline $\begin{array}{l}\mathrm{M} \\
\mathrm{J} \\
\mathrm{V}\end{array}$ & $\begin{array}{l}M \\
\text { io } \\
\text { V }\end{array}$ & $\begin{array}{l}\text { Cue } \\
\text { Culann Patera } \\
\text { Cunitz }\end{array}$ & $\begin{array}{l}36.1 \mathrm{~S} \\
19.9 \mathrm{~S} \\
14.5 \mathrm{~N}\end{array}$ & $\begin{array}{l}266.8 \mathrm{~W} \\
158.7 \mathrm{~W} \\
350.9 \mathrm{E}\end{array}$ & $\begin{array}{r}0 \\
100 \\
53\end{array}$ & $\begin{array}{l}\text { OC } \\
\text { EU } \\
\text { EU }\end{array}$ & $\begin{array}{l}\text { AU } \\
\text { CE } \\
\text { PO }\end{array}$ & $\begin{array}{l}5 \\
5 \\
5\end{array}$ & $\begin{array}{l}1989 \\
1979 \\
1991\end{array}$ \\
\hline L & $\mathbf{L}$ & Curie & $22.9 \mathrm{~S}$ & $91.0 \mathrm{E}$ & 151 & EU & FR & 5 & 1970 \\
\hline $\mathbf{M}$ & $\mathbf{M}$ & Curie & $29.2 \mathrm{~N}$ & $4.9 \mathrm{~W}$ & 98 & EU & FR & 5 & 1973 \\
\hline L & $\mathrm{L}$ & Curtis & $14.6 \mathrm{~N}$ & $56.6 \mathrm{E}$ & 2 & NA & $\mathrm{AM}$ & 5 & 1973 \\
\hline L & L & Curtius & $67.2 \mathrm{~S}$ & $4.4 \mathrm{E}$ & 95 & $\mathrm{EU}$ & GE & 5 & 1935 \\
\hline L & L & Cusanus & $72.0 \mathrm{~N}$ & $70.8 \mathrm{E}$ & 63 & $\mathrm{EU}$ & GE & 5 & 1935 \\
\hline $\mathbf{M}$ & M & Cusus Valles & $13.9 \mathrm{~N}$ & $309.0 \mathrm{~W}$ & 234 & EU & $\mathrm{CZ}$ & 5 & 1985 \\
\hline
\end{tabular}

$\mathrm{ft}$ origin

MO Albedo feature name.

SC From albedo feature at 26S, 276W.

AA Elizabeth; Polish astronomer, wife of Hevelius ( 17 th century).

AA Isabella; Italian physician. medical writer (d. 1561).

AA Town in Texas, USA.

MO Thracian goddess, mother of gods, similar to Cybele.

AA Egenni; French physicist (1881-1967)

AA Andre; French astronomer (1897-1978)

AA Charles A. De; French physicist (1736-1806).

AA Francois; French composer (1688-1733).

AA English male name.

AA Town in England.

AA Town in Mexico.

PE Celtic smith god.

AA Luigi; Italian mathematician (1830-1903)

LF Astronaut-named feature, Apollo 12 site.

SS $1986 \mathrm{U3}$; daughter to Calchus in "Troilus and Cressida" $61,777 \mathrm{~km}$ semi-major axis

AA Daughter of Priam; first wife of Aeneas.

AA Town in England.

AA G.; American doctor (1864-1943)

MO Where Io passed by in her wanderings.

AA Gaetano A.; Italian aeronautical engineer (1877-1968).

AA Andrew C.; British astronomer (1865-1939).

AA Andrew Claude De La Cherois; British astronomer (1865-1939)

AA Sir William; British physicist, chemist (1832-1919).

AA Francis Rawdon Moira; British explorer (1796-1848).

AA Peter; German mathematician (1580-1639)

AA Luiz; Brazilian astronomer (1848-1908)

AA Town in Venezuela.

AA Egyptian physicist (unkn-c. 100 B.C.)

AA Town in Western Australia.

PE Celtic smith god.

AA Maria; Polish astronomermathemetician (1610-1664).

AA Pierre; French physicist, chemist; Nobel laureate (1859-1906).

AA Pierre; French physicist-chemist (1859-1906).

AA Heber Doust; American astronomer (1872-1942)

AA Curtz, Albert; German astronomer (1600-1671).

AA Nikolaus Krebs; German mathematician, philosopher (1401-1464).

VA Classical name for modern Hron River in Czechoslavakia.

\footnotetext{
P: planetary system (see page xvi).

Sa: satellite (see page $x v i$ ).

lat: latitude of feature center.

long: longitude of feature center.
}

diam: diameter or long dimension of feature.

ct: continent of name origin (see page $284 \mathrm{ff}$.)

et: ethnicity of name origin (see page $284 \mathrm{ff}$.)

as: name approval status (see page xvii). ad: name approval date (year).

ref: reference source for name (see page $287 \mathrm{ff}$.)

$\mathrm{ft}$ : feature type (see page 290). 


\section{ALPHABETICAL LIST OF NAMES}

\section{P Sa Name}

L L Cuvier

M M Cyane Catena

M M Cyane Fossae

M M Cyane Sulci

V V Cybele Corona

M M Cyclopia

M M Cydnus Rupes

M M Cydonia

M M Cydonia Mensae

H H Cyllene

V V Cynthia

M M Cypress

L L Cyrano

L L Cyrillus

L L Cysatus

L L da Vinci

M M Da Vinci

M M Daan

M M Daedalia Planum

L L Daedalus

J io Daedalus Patera

M M Daet

J ca Dag

L L Dag

$\mathrm{N}$ tr Dagon Cavus

L L Daguerre

L L Dale

L L D'Alembert

V V Dali Chasma

$S$ en Dalilah

L L Dalton

M M Daly

L L Daly

L L Damoiseau

M M Dana

J ga Danel

L L Daniell

V V Danilova

L L Danjon

M M Dank lat long diam ct et as ad ref $\mathrm{ft}$ origin

$\begin{array}{llllllll}50.3 \mathrm{~S} & 9.9 \mathrm{E} & 75 & \mathrm{EU} & \mathrm{FR} & 5 & 1935 \quad 66\end{array}$

$\begin{array}{rrrrrrrr}37.0 \mathrm{~N} & 118.5 \mathrm{~W} & 143 & \text { EU } & \text { GR } & 5 & 1985 & 57 \\ 36.0 \mathrm{~N} & 121.4 \mathrm{~W} & 292 & \text { EU } & \text { GR } & 5 & 1985 & 57 \\ 25.5 \mathrm{~N} & 128.4 \mathrm{~W} & 286 & \text { EU } & \text { LA } & 5 & 1976 & 57 \\ 7.5 \mathrm{~S} & 20.7 \mathrm{E} & 480 & \text { AS } & \text { TU } & 5 & 1994 & 83 \\ 5.0 \mathrm{~S} & 230.0 \mathrm{~W} & 0 & \text { EU } & \text { GR } & 5 & 1958 & 60 \\ 59.6 \mathrm{~N} & 257.5 \mathrm{~W} & 430 & \text { EU } & \text { GR } & 5 & 1985 & 57 \\ 40.0 \mathrm{~N} & 0.0 \mathrm{~W} & 0 & \text { EU } & \text { LA } & 5 & 1958 & 60 \\ 37.0 \mathrm{~N} & 12.8 \mathrm{~W} & 854 & \text { EU } & \text { LA } & 5 & 1976 & 57 \\ 41.0 \mathrm{~S} & 270.0 \mathrm{~W} & 0 & \text { EU } & \text { RM } & 5 & 1976 & 60 \\ 16.7 \mathrm{~S} & 347.4 \mathrm{E} & 16 & \text { EU } & \text { GR } & 5 & 1991 & 109 \\ 47.6 \mathrm{~S} & 47.0 \mathrm{~W} & 11 & \text { US } & \text { IL } & 5 & 1976 & 36 \\ 20.5 \mathrm{~S} & 157.7 \mathrm{E} & 80 & \text { EU } & \text { FR } & 5 & 1970 & 0 \\ 13.2 \mathrm{~S} & 24.0 \mathrm{E} & 98 & \text { AF } & \text { EG } & 5 & 1935 & 66 \\ 66.2 \mathrm{~S} & 6.1 \mathrm{~W} & 48 & \text { EU } & \text { SZ } & 5 & 1935 & 66\end{array}$

$9.1 \mathrm{~N} \quad 45.0 \mathrm{E}$

$1.5 \mathrm{~N} \quad 39.1 \mathrm{~W}$

$37 \quad \mathrm{EU}$

51935

51973

40.9S 267.6W

13.9S $138.0 \mathrm{~W}$

$\begin{array}{lll}98 & \mathrm{EU} & \mathrm{IT}\end{array}$

1989

$\begin{array}{rll}0 & \text { AS } & \text { CH } \\ 2477 & \text { EU } & \text { GR }\end{array}$

93 EU GR

$19.1 \mathrm{~N} \quad 274.3 \mathrm{~W}$

40

EU GR

$7.4 \mathrm{~S} \quad 41.9 \mathrm{~W}$

$58.6 \mathrm{~N} \quad 74.2 \mathrm{~W}$

$18.7 \mathrm{~N} \quad 5.3 \mathrm{E}$

$29.0 \mathrm{~N} \quad 345.0 \mathrm{E}$

$11.9 \mathrm{~S} \quad 33.6 \mathrm{E}$

$9.6 \mathrm{~S}$

$82.9 \mathrm{E}$

12 AS PH

40 EU NS

EU SD

0 AS BY

$\begin{array}{ll}5 & 1982\end{array}$

51970

51979

51976

$\begin{array}{ll}5 & 1979\end{array}$ 51976

$\begin{array}{rr}5 & 1976 \\ 5 & 91\end{array}$

51935

46 EU FR

$5 \quad 1976$

$50.8 \mathrm{~N} \quad 163.9 \mathrm{E}$

248

EU FR

$5 \quad 1970$

$\begin{array}{lllllll}17.6 \mathrm{~S} & 167.0 \mathrm{E} & 2077 & \text { UR } & \text { GE } & 5 & 1985 \\ 52.9 \mathrm{~N} & 246.4 \mathrm{~W} & 14 & \mathrm{AS} & \mathrm{AR} & 5 & 1982\end{array}$

$52.9 \mathrm{~N} \quad 246.4 \mathrm{~W}$

$17.1 \mathrm{~N} \quad 84.3 \mathrm{~W}$

60 EU GB

$5 \quad 1964$

$66.4 \mathrm{~S} \quad 23.0 \mathrm{~W}$

99

NA CA

51973

$5.7 \mathrm{~N} \quad 59.6 \mathrm{E}$

17

NA CA

$5 \quad 1973$

$4.8 \mathrm{~S}$

$61.1 \mathrm{~W}$

36

EU FR

51935

$72.6 \mathrm{~S}$

$33.1 \mathrm{~W}$

95

NA AM

51973

4.3S $25.2 \mathrm{~W}$

54 AS PO

51979

35.3N $31.1 \mathrm{E}$

29

EU GB

$5 \quad 1935$

26.4S 337.3E

49

UR RU

$5 \quad 1991$

$11.4 \mathrm{~S} \quad 124.0 \mathrm{E}$

71

EU FR

51970

$22.2 \mathrm{~N} \quad 253.2 \mathrm{~W}$
7 AS OM
51976
66

57

57

57

83
60

57

60

57

60

109

36
0

66

66

66

68

74

57

0

$$
36
$$

0

27
52

52

67

68

0

66

68

1

66

99

0

36
AA Georges; French natural scientist, paleontologist (1769-1832).

CA Classical albedo feature Cyane Fons.

FO Classical albedo feature name.

SU Classical albedo feature name.

CR Phrygian fertility goddess.

AL Land where cyclops dwelt.

RU From albedo feature at $70 \mathrm{~N}, 248 \mathrm{~W}$.

AL Poetic term for Crete.

MN From albedo feature at $50 \mathrm{~N}, 355 \mathrm{~W}$.

AL Albedo name for unimaged $\mathrm{H}-14$ region.

AA First name from Greek.

AA Town in Illinois, USA

AA Cyrano De Bergerac Savinien French writer (1615-1655).

AA Saint Cyril; Egyptian theologian, chronologist (unkn-A.D. 444)

AA Cysat, Jean-Baptiste; Swiss mathematician, astronomer (1588-1657).

AA Leonardo; Italian artist, inventor, mathematician (1452-1519).

AA Leonardo; Italian artist-scientist (1452-1519)

AA Town in China.

PM Classical albedo feature name.

AA Greek mythological character.

PE Greek hero, smith; father of Icarus.

AA Town in the Philippines.

AA Norse; Ottar's ancestor.

AA Scandinavian male name.

CB Babylonian fertility god represented as a fish.

AA Louis; French artist, chemist, photographer (1789-1851)

AA Sir Henry Hallett; British physiologist; Nobel laureate (1875-1968)

AA Jean-Le-Rond; French mathematician, physicist (1717-1783).

CM Georgian; goddess of hunt.

AA Crafty old crone who fools several men.

AA John; British chemist, physicist (1766-1844).

AA Reginald A.; Canadian geologist (1871-1975).

AA Reginald Aldworth; Canadian geologist (1871-1957).

AA Marie Charles Theodor De; French astronomer (1768-1846).

AA James D.; American geologist (1813-1895)

AA Phoenician; mythical hero versed in art of divination.

AA John Frederick; British physicist, chemist, meteorologist (1790-1845).

AA Maria; Russian ballet dancer (b. 1793)

AA Andre; French astronomer (1890-1967).

AA Town in Oman

\footnotetext{
P: $\quad$ planetary system (see page xvi).

Sa: $\quad$ satellite (see page $x v i$ ).

lat: latitude of feature center.

long: longitude of feature center.
}

diam: diameter or long dimension of feature.

ct: continent of name origin (see page $284 \mathrm{ff}$.)

et: ethnicity of name origin (see page $284 \mathrm{ff}$.)

as: name approval status (see page xvii). ad: name approval date (year)

ref: reference source for name (see page $287 \mathrm{ff}$.).

ft: feature type (see page 290). 


\section{P Sa Name}

$\begin{array}{lll}\text { J } & \text { ca } & \text { Danr } \\ \text { L } & \text { L } & \text { Dante } \\ \text { V } & \text { V } & \text { Danu Montes } \\ \text { J } & \text { io } & \text { Danube Planum } \\ & & \\ \text { V } & \text { V } & \text { Danute } \\ \text { M } & \text { M } & \text { Dao Vallis } \\ \text { V } & \text { V } & \text { Daphne } \\ \text { V } & \text { V } & \text { Darago Fluctus } \\ \text { J } & \text { ga } & \text { Dardanus Sulcus }\end{array}$

H H Darío

L L [Dario]

V V Darline

L L Darney

L L D'Arrest

M ph D'Arrest

L L D'Arsonval

M M Darvel

M M Darwin

L L Darwin

M M Darwin

S en Daryabar Fossa

L L Das

V V Dashkova

V V Datsolalee

L L Daubrée

V V Daura Chasma

V V Davies Patera

L L Davisson

L L Davy

L L Dawes

M M Dawes

L L Dawson

J io Dazhbog Patera

V V de Ayala

V V 'de Beausoleil

V V de Beauvoir

L L De Forest

L L de Gasparis

L L De La Rue lat long diar

$\begin{array}{lrrlrrrr}62.5 \mathrm{~N} & 77.8 \mathrm{~W} & 48 & \text { EU } & \text { NS } & 5 & 1979 & 17 \\ 25.5 \mathrm{~N} & 180.0 \mathrm{E} & 54 & \text { EU } & \text { IT } & 5 & 1970 & 0 \\ & & & & & & & \\ 58.5 \mathrm{~N} & 334.0 \mathrm{E} & 808 & \text { EU } & \text { CE } & 5 & 1985 & 27 \\ 20.9 \mathrm{~S} & 258.7 \mathrm{~W} & 150 & \text { EU } & \text { GR } & 5 & 1985 & 19 \\ & & & & & & & \\ 63.5 \mathrm{~S} & 56.5 \mathrm{E} & 14 & \text { EU } & \text { LI } & 5 & 1994 & 107 \\ 36.8 \mathrm{~S} & 269.4 \mathrm{~W} & 667 & \text { AS } & \text { TH } & 5 & 1979 & 62 \\ 41.3 \mathrm{~N} & 280.4 \mathrm{E} & 14 & \text { EU } & \text { GR } & 5 & 1994 & 109 \\ 11.5 S & 313.5 \mathrm{E} & 775 & \text { AS } & \text { PH } & 5 & 1994 & 111 \\ 39.3 \mathrm{~S} & 20.2 \mathrm{~W} & 2559 & \text { EU } & \text { GR } & 5 & 1979 & 1\end{array}$

$\begin{array}{llllllll}11.3 \mathrm{~S} & 90.7 \mathrm{E} & 19 & \text { SA } & \text { NI } & 6 & 0 & 0\end{array}$

$\begin{array}{llllllll}19.3 S & 232.6 \mathrm{E} & 13 & \text { EU } & \text { EN } & 5 & 1994 & 109\end{array}$

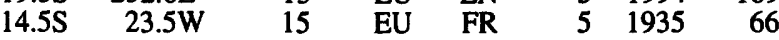

$\begin{array}{llllllll}2.3 \mathrm{~N} & 14.7 \mathrm{E} & 30 & \mathrm{EU} & \mathrm{GE} & 5 & 1935 & 66\end{array}$

$\begin{array}{lllllll}35.0 S & 185.0 \mathrm{~W} & 0 & \mathrm{EU} & \mathrm{GM} & 5 & 1973\end{array}$

$10.3 \mathrm{~S} \quad 124.6 \mathrm{E} \quad 28$

$17.9 \mathrm{~N}$

51.0W

57.25

$19.2 \mathrm{~W}$

$57.0 \mathrm{~S}$

6.5W

9.7N $359.1 \mathrm{~W}$

201

26.6S $136.8 \mathrm{~W}$

$78.3 \mathrm{~N} \quad 306.2 \mathrm{E}$

$38.3 \mathrm{~N} \quad 171.6 \mathrm{E}$

$15.7 \mathrm{~N}$

14.7E

72.4

$47.2 \mathrm{~N}$

$53.8 \mathrm{E}$

$37.5 S$

$11.8 \mathrm{~S}$

$17.2 \mathrm{~N}$

9.

67.4

$54.0 \mathrm{~N} \quad 301.6 \mathrm{~W}$

$12.3 \mathrm{~N} \quad 31.9 \mathrm{E}$

\begin{tabular}{ll}
$5.0 \mathrm{~S} \quad 102.9 \mathrm{E}$ \\
\hline
\end{tabular}

$2.0 \mathrm{~N} \quad 96.1 \mathrm{E}$

77.3

25.9

59.1
$7.3 \mathrm{~S}$

$162.1 \mathrm{~W}$

$50.7 \mathrm{~W}$

52.3E

38

$$
\text { AS IN }
$$$$
\text { UR RU }
$$

NA AM

51994

51973

14
729

EU FR

AF SU

51985

EU GB

$5 \quad 1970$

51935

EU GB

51935

51973

EU GB

51970

SA AR

$\begin{array}{ll}5 & 1979 \\ 5 & 1994\end{array}$

$\begin{array}{ll}\text { UR } & \text { SL } \\ \text { EU } & \text { SP }\end{array}$

$5 \quad 1994$

$5 \quad 1991$

$5 \quad 1970$

51935

51935

134 EU GB

EU FR

$5 \quad 1976$

0

EU SC

$\begin{array}{ll}5 & 1988 \\ 5 & 1973\end{array}$

166

EU GB

51935

120

EU GB

51973

AS AR

60

68

36

68

66

68

52

$$
0
$$

64

101

$$
0
$$

65

46

0

55

66

68

$$
0
$$

18 99

89

99

0

66

66
AA Norse; king against whom Konr marched.

AA Alighieri; Italian poet (1265-1321).

MO Celtic mother of gods.

PM Where Io passed by in her wanderings.

AA Lithuanian first name.

VA Word for "star" in Thai.

AA First name from Greek.

FL Philippine volcano goddess.

SU Greek; where Ganymede was abducted by Zeus disguised as an eagle.

AA Ruben; Nicaraguan poet, journalist (1867-1916).

AA Ruben; Nicaraguan author 1867-1916

AA Anglo-Saxon first name.

AA Maurice; French astronomer (1882-1958).

AA Heinrich Ludwig; German astronomer (1822-1875).

AA Heinrich L.; German astronomer 1822-1875

AA Jacques Arsène; French physicist (1851-1940).

AA Town in Scotland.

AA George H.; British astronomer (1845-1912).

AA Charles; British natural scientist (1809-1882).

AA Charles R.; British naturalist 1809-1882; same crater as G. H. Darwin.

FO "Ocean region"; land from which Princess Daryabar came.

AA Amil K.; Indian astronomer (1902-1961).

AA Yekaterina; Russian philologist (1743-1810).

AA Washo Indian artist, basketmaker (1835-1925).

AA Gabriel-Auguste; French geologist (1814-1896).

CM Hausa (W. Sudan) great huntress.

PE Emily Sarah; British educator; college founder (1830-1921).

AA Clinton J.; American physicist; Nobel laureate (1881-1958)

AA Humphry; British physicist (1778-1829)

AA William Rutter; British astronomer (1799-1868).

AA William R.; British astronomer (1799-1868).

AA Bernhard H.; Argentinian astronomer (1890-1960).

PE Slavonic sun god.

AA Josefa; Spanish painter (1630-1684)

AA Martine; French earth science researcher (17th century).

AA Simone; French writer (1908-1986).

AA Lee; American inventor (1873-1961).

AA Annibale; Italian astronomer (1819-1892)
AA Warren; British astronomer (1815-1889).

\footnotetext{
P: $\quad$ planetary system (see page $x v i$ )

Sa: satellite (see page xvi).

lat: latitude of feature center.

long: longitude of feature center.
}

diam: diameter or long dimension of feature.

ct: continent of name origin (see page $284 \mathrm{ff}$.)

et: ethnicity of name origin (see page $284 \mathrm{ff}$.)

as: name approval status (see page xvii). ad: name approval date (year).

ref: reference source for name (see page $287 \mathrm{ff}$.).

ft: feature type (see page 290). 


\begin{tabular}{|c|c|c|c|c|c|c|c|c|c|c|}
\hline $\mathbf{P}$ & $\mathbf{S a}$ & Name & lat & long & diam & ct & et & as & ad & ref \\
\hline V & V & de Lalande & $20.4 \mathrm{~N}$ & $355.0 \mathrm{E}$ & 21 & EU & FR & 5 & 1991 & 100 \\
\hline $\mathbf{L}$ & $\mathrm{L}$ & De Moraes & $49.5 \mathrm{~N}$ & $143.2 \mathrm{E}$ & 53 & SA & BR & 5 & 1979 & 0 \\
\hline L & L & De Morgan & $3.3 \mathrm{~N}$ & $14.9 \mathrm{E}$ & 10 & EU & GB & 5 & 1935 & 66 \\
\hline L & L & De Roy & $55.3 \mathrm{~S}$ & $99.1 \mathrm{~W}$ & 43 & EU & $\mathrm{BE}$ & 5 & 1970 & 0 \\
\hline $\mathbf{L}$ & $\mathbf{L}$ & De Sitter & $80.1 \mathrm{~N}$ & $39.6 \mathrm{E}$ & 64 & EU & DU & 5 & 1964 & 67 \\
\hline $\begin{array}{l}\text { V } \\
\text { L }\end{array}$ & $\begin{array}{l}\text { V } \\
\text { L }\end{array}$ & $\begin{array}{l}\text { de Staël } \\
\text { De Vico }\end{array}$ & $\begin{array}{l}37.4 \mathrm{~N} \\
19.7 \mathrm{~S}\end{array}$ & $\begin{array}{l}324.2 \mathrm{E} \\
60.2 \mathrm{~W}\end{array}$ & $\begin{array}{l}25 \\
20\end{array}$ & $\begin{array}{l}\mathrm{EU} \\
\mathrm{EU}\end{array}$ & $\begin{array}{r}\text { FR } \\
\text { IT }\end{array}$ & $\begin{array}{l}5 \\
5\end{array}$ & $\begin{array}{l}1994 \\
1935\end{array}$ & $\begin{array}{l}99 \\
66\end{array}$ \\
\hline L & $\mathbf{L}$ & De Vries & $19.9 \mathrm{~S}$ & $176.7 \mathrm{~W}$ & 59 & $\mathrm{EU}$ & DU & 5 & 1970 & J \\
\hline V & V & De Witt & $6.5 \mathrm{~S}$ & $275.6 \mathrm{E}$ & 21 & NA & AM & 5 & 1994 & 99 \\
\hline $\begin{array}{l}\text { M } \\
\mathrm{L}\end{array}$ & $\begin{array}{l}\mathrm{M} \\
\mathrm{L}\end{array}$ & $\begin{array}{l}\text { Deba } \\
\text { Debes }\end{array}$ & $\begin{array}{l}24.3 S \\
29.5 \mathrm{~N}\end{array}$ & $\begin{array}{l}17.1 \mathrm{~W} \\
51.7 \mathrm{E}\end{array}$ & $\begin{array}{r}7 \\
30\end{array}$ & $\begin{array}{l}\mathrm{AF} \\
\mathrm{EU}\end{array}$ & $\stackrel{\text { NI }}{\mathrm{GE}}$ & $\begin{array}{l}5 \\
5\end{array}$ & $\begin{array}{l}1976 \\
1961\end{array}$ & $\begin{array}{l}36 \\
67\end{array}$ \\
\hline L & L & Debye & $49.6 \mathrm{~N}$ & $176.2 W$ & 142 & EU & DU & 5 & 1970 & \\
\hline L & L & Dechen & $46.1 \mathrm{~N}$ & $68.2 \mathrm{~W}$ & 12 & EU & GE & 5 & 1935 & 66 \\
\hline L & L & [Defoe] & $6.0 \mathrm{~S}$ & $80.5 \mathrm{E}$ & 18 & EU & GB & 6 & 0 & 0 \\
\hline $\begin{array}{l}\mathbf{H} \\
\mathrm{M} \\
\mathrm{U} \\
\mathrm{M}\end{array}$ & $\begin{array}{l}\mathrm{H} \\
\mathrm{M} \\
\mathrm{ar} \\
\mathrm{M}\end{array}$ & $\begin{array}{l}\text { Degas } \\
\text { Dein } \\
\text { Deive } \\
\text { Dejnev }\end{array}$ & $\begin{array}{l}37.4 \mathrm{~N} \\
38.5 \mathrm{~N} \\
22.3 \mathrm{~S} \\
25.6 \mathrm{~S}\end{array}$ & $\begin{array}{c}126.4 \mathrm{~W} \\
2.4 \mathrm{~W} \\
23.0 \mathrm{E} \\
164.5 \mathrm{~W}\end{array}$ & $\begin{array}{r}60 \\
24 \\
20 \\
156\end{array}$ & $\begin{array}{l}\text { EU } \\
\text { OC } \\
\text { UR } \\
\text { UR }\end{array}$ & $\begin{array}{r}\text { FR } \\
\text { GU } \\
\text { LI } \\
\text { RU }\end{array}$ & $\begin{array}{l}5 \\
5 \\
5 \\
5\end{array}$ & $\begin{array}{l}1979 \\
1976 \\
1988 \\
1985\end{array}$ & $\begin{array}{l}59 \\
36 \\
94 \\
58\end{array}$ \\
\hline V & V & Deken & $47.1 \mathrm{~N}$ & $288.3 \mathrm{E}$ & 48 & EU & DU & 5 & 1985 & 46 \\
\hline $\begin{array}{l}\text { V } \\
H\end{array}$ & $\begin{array}{l}\mathrm{V} \\
\mathrm{H}\end{array}$ & $\begin{array}{l}\text { Dekla Tessera } \\
\text { Delacroix }\end{array}$ & $\begin{array}{l}57.4 \mathrm{~N} \\
44.7 \mathrm{~S}\end{array}$ & $\begin{array}{c}71.8 \mathrm{E} \\
129.0 \mathrm{~W}\end{array}$ & $\begin{array}{r}1363 \\
146\end{array}$ & $\begin{array}{l}\text { UR } \\
\text { EU }\end{array}$ & $\begin{array}{l}\text { LV } \\
\text { FR }\end{array}$ & $\begin{array}{l}5 \\
5\end{array}$ & $\begin{array}{l}1985 \\
1979\end{array}$ & $\begin{array}{l}64 \\
59\end{array}$ \\
\hline L & $\mathrm{L}$ & Delambre & $1.9 \mathrm{~S}$ & $17.5 \mathrm{E}$ & 51 & EU & FR & 5 & 1935 & 66 \\
\hline L & $\mathrm{L}$ & Delaunay & $22.2 \mathrm{~S}$ & $2.5 \mathrm{E}$ & 46 & EU & FR & 5 & 1935 & 66 \\
\hline V & V & Deledda & $76.0 \mathrm{~N}$ & $127.5 \mathrm{E}$ & 32 & EU & IT & 5 & 1985 & 46 \\
\hline $\begin{array}{l}\mathrm{L} \\
\mathrm{V} \\
\mathrm{L}\end{array}$ & $\begin{array}{l}\mathrm{L} \\
\mathrm{V} \\
\mathrm{L}\end{array}$ & $\begin{array}{l}\text { Delia } \\
\text { Delilah } \\
\text { Delisle }\end{array}$ & $\begin{array}{l}10.9 \mathrm{~S} \\
57.9 \mathrm{~S} \\
29.9 \mathrm{~N}\end{array}$ & $\begin{array}{c}6.1 \mathrm{~W} \\
250.5 \mathrm{E} \\
34.6 \mathrm{~W}\end{array}$ & $\begin{array}{r}2 \\
18 \\
25\end{array}$ & $\begin{array}{l}\mathrm{EU} \\
\mathrm{AS} \\
\mathrm{EU}\end{array}$ & $\begin{array}{l}\text { GR } \\
\text { HE } \\
\text { FR }\end{array}$ & $\begin{array}{l}5 \\
5 \\
5\end{array}$ & $\begin{array}{l}1976 \\
1994 \\
1935\end{array}$ & $\begin{array}{r}0 \\
82 \\
66\end{array}$ \\
\hline L & $\mathrm{L}$ & Dellinger & $6.8 \mathrm{~S}$ & $140.6 \mathrm{E}$ & 81 & NA & AM & 5 & 1970 & 0 \\
\hline L & $\mathrm{L}$ & Delmotte & $27.1 \mathrm{~N}$ & $60.2 \mathrm{E}$ & 32 & EU & FR & 5 & 1935 & 66 \\
\hline V & V & Deloria & $32.0 \mathrm{~S}$ & $97.0 \mathrm{E}$ & 37 & NA & DA & 5 & 1991 & 100 \\
\hline J & eu & Delphi Flexus & $69.7 \mathrm{~S}$ & $172.3 \mathrm{~W}$ & 1125 & EU & GR & 5 & 1985 & 19 \\
\hline L & $\mathbf{L}$ & Delporte & $16.0 \mathrm{~S}$ & $121.6 \mathrm{E}$ & 45 & EU & $\mathrm{BE}$ & 5 & 1970 & 0 \\
\hline $\begin{array}{l}\mathbf{M} \\
\mathbf{M}\end{array}$ & $\begin{array}{l}\mathbf{M} \\
\mathbf{M}\end{array}$ & $\begin{array}{l}\text { Delta } \\
\text { Deltoton Sinus }\end{array}$ & $\begin{array}{r}46.3 \mathrm{~S} \\
4.0 \mathrm{~S}\end{array}$ & $\begin{array}{r}39.0 \mathrm{~W} \\
305.0 \mathrm{~W}\end{array}$ & $\begin{array}{l}5 \\
0\end{array}$ & $\begin{array}{l}\text { US } \\
\text { EU }\end{array}$ & $\begin{array}{l}\text { LA } \\
\text { LA }\end{array}$ & $\begin{array}{l}5 \\
5\end{array}$ & $\begin{array}{l}1976 \\
1958\end{array}$ & $\begin{array}{l}36 \\
60\end{array}$ \\
\hline $\mathbf{L}$ & $\mathbf{L}$ & Deluc & $55.0 \mathrm{~S}$ & $2.8 \mathrm{~W}$ & 46 & EU & SZ & 5 & 1935 & 66 \\
\hline L & L & Dembowski & $2.9 \mathrm{~N}$ & $7.2 \mathrm{E}$ & 26 & EU & IT & 5 & 1935 & 66 \\
\hline $\begin{array}{l}\text { V } \\
\mathrm{L}\end{array}$ & $\begin{array}{l}\text { V } \\
\mathrm{L}\end{array}$ & $\begin{array}{l}\text { Demeter Corona } \\
\text { Democritus }\end{array}$ & $\begin{array}{l}53.9 \mathrm{~N} \\
62.3 \mathrm{~N}\end{array}$ & $\begin{array}{r}294.8 \mathrm{E} \\
35.0 \mathrm{E}\end{array}$ & $\begin{array}{r}560 \\
39\end{array}$ & $\begin{array}{l}\text { EU } \\
\text { EU }\end{array}$ & $\begin{array}{l}\text { GR } \\
\text { GR }\end{array}$ & $\begin{array}{l}5 \\
5\end{array}$ & $\begin{array}{l}1985 \\
1935\end{array}$ & $\begin{array}{r}1 \\
66\end{array}$ \\
\hline $\mathbf{L}$ & $\mathrm{L}$ & Demonax & $77.9 \mathrm{~S}$ & $60.8 \mathrm{E}$ & 128 & EU & GR & 5 & 1935 & 66 \\
\hline
\end{tabular}

ft origin

AA Marie-Jeanne; French astronomer (1768-1832)

AA A.; Brazilian astronomer (1916-1970).

AA Augustus; British mathematician (1806-1871).

AA Felix; Belgian astronomer (1883-1942)

AA Willem; Dutch astronomer (1872-1934).

AA Anne; French writer (1766-1817).

AA Francesco; Italian astronomer (1805-1848)

AA Hugo M.; Dutch botanist (1848-1935).

AA Lydia; American pathologist (1859-1928).

AA Town in Nigeria.

AA Ernest; German cartographer (1840-1923)

AA Peter J. W; Dutch physicist, chemist; Nobel laureate (1884-1966).

AA Ernst Heinrich Karl von; German geologist, mineralogist (1800-1889)

AA Daniel; British author (c. 1661-1731).

AA Edgar, French painter (1834-1917).

AA Town in New Guinea.

AA Spirit of beautiful maiden.

AA Semen Ivanovich; Russian geographer, explorer, and navigator (1605-1673)

AA Agatha; Dutch novelist (1741-1804).

TE Latvian goddess of fate

AA Eugene; French painter (1798-1863)

AA Jean-Baptiste Joseph; French astronomer (1749-1822)

AA Charles Eugene; French astronomer (1816-1872)

AA Grazia; Italian novelist (1871-1936)

AA Greek female name.

AA First name from Hebrew.

AA Joseph Nicolas; French astronomer (1688-1768).

AA John H.; American physicist (1886-1962).

AA Gabriel; French astronomer (1876-1950)

AA Ella; Dakota (Sioux) anthropologist (1888-1971).

FE Where the cow led Cadmus before it stopped at the site of Thebes.

AA Eugene J.; Belgian

. astronomer (1882-1955).

AA Town in Louisiana, USA.

AL "Bay of the triangle"; makes triangle with lapygia and Oenotria

AA Jean Andre; Swiss geologist, physicist (1727-1817).

AA Baron Ercole; Italian astronomer (1815-1881).

CR Greek goddess of fertility.

AA Greek astronomer, philosopher (c. 460-360 B.C.)

AA Greek philosopher (unkn-c. 100 B.C.)

\footnotetext{
P: $\quad$ planetary system (see page $x v i)$

Sa: $\quad$ satellite (see page $x v i$ ).

lat: latitude of feature center.

long: longitude of feature center
}

diam: diameter or long dimension of feature. ct: continent of name origin (see page $284 \mathrm{ff}$.) et: ethnicity of name origin (see page $284 \mathrm{ff}$.) as: name approval status (see page xvii) ad: name approval date (year).

ref: reference source for name (see page $287 \mathrm{ff}$.). ft: feature type (see page 290). 


\section{ALPHABETICAL LIST OF NAMES}

\section{P Sa Name}

$\begin{array}{lll}\text { J } & \text { ga } & \text { Dendera Facula } \\ \text { M } & \text { M } & \text { Denning } \\ \text { L } & \text { L } & \text { Denning } \\ \text { V } & \text { V } & \text { Dennitsa Dorsa } \\ \text { M } & \text { M } & \text { Depressio Hellespontica } \\ \text { V } & \text { V } & \text { Derceto Corona } \\ \text { M } & \text { M } & \text { Dersu } \\ \text { H } & \text { H } & \text { Derzhavin } \\ \text { L } & \text { L } & \text { Desargues } \\ \text { L } & \text { L } & \text { Descartes } \\ \text { U } & \text { ss } & \text { Desdemona } \\ & & \\ \text { M } & \text { M } & \text { Dese }\end{array}$

L L Deseilligny

L L Deslandres

H H Despréz

M M Dessau

V V d'Este

M M Deucalionis Regio

M M Deuteronilus

M M Deuteronilus Colles

M M Deuteronilus Mensae

L L Deutsch

M M Deva Valles

M M Deva Vallis

V V Devana Chasma

V V Devorah

V V Devorguilla

L L Dewar

V V Dhisana Corona

J ca Dia

M M Dia-Cau

M M Diacria

M M Diacria Patera

L L Diana

V V Diana Chasma

$\mathrm{H} H$ Dickens

V V Dickinson

L L Diderot

$S$ di Dido

$\mathrm{N}$ tr Dilolo Patera

J ga Diment lat long diam

$\begin{array}{rrrrrrrr}0.0 \mathrm{~N} & 257.0 \mathrm{~W} & 114 & \text { AF } & \text { EG } & 5 & 1985 & 1 \\ 17.5 \mathrm{~S} & 326.6 \mathrm{~W} & 165 & \text { EU } & \text { GB } & 5 & 1973 & 68 \\ 16.4 \mathrm{~S} & 142.6 \mathrm{E} & 44 & \text { EU } & \text { GB } & 5 & 1970 & 0 \\ & & & & & & & \\ 85.6 \mathrm{~N} & 205.9 \mathrm{E} & 872 & \text { EU } & \text { SL } & 5 & 1985 & 65 \\ 60.0 \mathrm{~S} & 340.0 \mathrm{~W} & 0 & \text { EU } & \text { LA } & 5 & 1958 & 60 \\ 46.8 \mathrm{~S} & 20.2 \mathrm{E} & 200 & \text { AS } & \text { SE } & 5 & 1994 & 83 \\ 25.8 \mathrm{~N} & 51.9 \mathrm{~W} & 0 & \text { UR } & \text { RU } & 5 & 1988 & 36 \\ 44.9 \mathrm{~N} & 35.3 \mathrm{~W} & 159 & \text { UR } & \text { RU } & 5 & 1979 & 59 \\ 70.2 \mathrm{~N} & 73.3 \mathrm{~W} & 85 & \text { EU } & \text { FR } & 5 & 1964 & 67 \\ 11.7 \mathrm{~S} & 15.7 \mathrm{E} & 48 & \text { EU } & \text { FR } & 5 & 1935 & 66 \\ 0.0 \mathrm{~N} & 0.0 \mathrm{X} & 0 & \text { EU } & \text { GB } & 5 & 1988 & 85\end{array}$

$\begin{array}{llllllll}45.8 \mathrm{~S} & 30.3 \mathrm{~W} & 12 & \text { AF } & \text { ET } & 5 & 1976 & 36\end{array}$

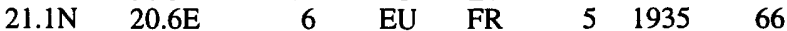

$\begin{array}{llllllll}33.1 \mathrm{~S} & 4.8 \mathrm{~W} & 256 & \mathrm{EU} & \mathrm{FR} & 5 & 1948 & 67\end{array}$

$\begin{array}{llllllll}80.8 \mathrm{~N} & 90.7 \mathrm{~W} & 50 & \mathrm{EU} & \text { FR } & 5 & 1979 & 59\end{array}$

43.1S

$53.0 \mathrm{~W}$

$34.2 \mathrm{~S}$

238.7E

21

$15.0 \mathrm{~S}$

$340.0 \mathrm{~W}$

$35.0 \mathrm{~N}$

$0.0 \mathrm{~W}$

$42.2 \mathrm{~N} \quad 338.3 \mathrm{~W}$

$45.7 \mathrm{~N} \quad 337.9 \mathrm{~W}$

$24.1 \mathrm{~N} \quad 110.5 \mathrm{E}$

7.9S $156.9 \mathrm{~W}$

$8.1 \mathrm{~S} \quad 156.7 \mathrm{~W}$

EU GE

$\begin{array}{ll}5 & 1976 \\ 5 & 1994\end{array}$

EU IT

$5 \quad 1958$

36

99

60

EU GR

$5 \quad 1958$

60

79

EU LA

$\begin{array}{ll}5 & 1982 \\ 5 & 1973\end{array}$

EU LA

57
57

66

NA AM

$5 \quad 1970$

51985

0 EU SC

EU SC

51985

$9.6 \mathrm{~N} \quad 284.4 \mathrm{E}$

1616

EU

$\mathrm{CZ}$

$5 \quad 1982$

27

22.5S 343.4E

$15.3 \mathrm{~N}$

2.7

$14.5 \mathrm{~N} \quad 111.7 \mathrm{E}$

$73.0 \mathrm{~N} \quad 50.4 \mathrm{~W}$

$0.3 \mathrm{~S}$

$42.8 \mathrm{~W}$

6
23

AS HE

$5 \quad 1994$

$\begin{array}{ll}5 & 1991\end{array}$

51970

EU GB

$5 \quad 1994$

100

AS IN

EU GR

$\begin{array}{ll}5 & 1994 \\ 5 & 1979\end{array}$

51976

$50.0 \mathrm{~N} \quad 180.0 \mathrm{~W}$

EU GR

51958

EU GR

$5 \quad 1985$

$34.8 \mathrm{~N} \quad 132.7 \mathrm{~W}$

75

EU LA

$14.3 \mathrm{~N} \quad 35.7 \mathrm{E}$

$14.8 \mathrm{~S} \quad 154.8 \mathrm{E}$

50
938

$5 \quad 1979$

$72.9 \mathrm{~S}$

EU EN

74.3N $\quad 177.3 \mathrm{E}$

69

NA AM

$\begin{array}{ll}5 & 1982 \\ 5 & 1976\end{array}$

$20.4 \mathrm{~S}$

$121.5 \mathrm{E}$

20

EU

$5 \quad 1985$

23.7S

$18.5 \mathrm{~W}$

118

EU RM

$5 \quad 1979$

$26.0 \mathrm{~N} \quad 24.5 \mathrm{E}$

$22.4 \mathrm{~N} \quad 353.6 \mathrm{~W}$

$\begin{array}{rrr}0 & \text { AF } & \text { AN } \\ 47 & \text { AF } & \text { EG }\end{array}$

$\begin{array}{lll}5 & 1982 & 44\end{array}$

$\begin{array}{lrr}5 & 91 & 27\end{array}$

FA Town where Hathor was chief goddess.

AA William F.; British astronomer (1848-1931)

AA William F.; British astronomer (1848-1931)

DO Slavic goddess of day, light.

AL Depression southwest of Hellespontus.

CR Phillistine fertility goddess.

AA Town in Russia.

AA Gavrila Romanovich; Russian poet (1743-1816).

AA Gerard; French mathematician, engineer (1593-1662).

AA Rene; French mathematician, philosopher (1596-1650).

SS 1986 U6; wife to Othello in "Othello, The Moor of Venice" $62,676 \mathrm{~km}$ semi-major axis

AA Town in Ethiopa.

AA Jules Alfred Pierrot; French selenographer (1868-1918).

AA Henri Alexandre; French astrophysicist (1853-1948).

AA Josquin; French composer (c. 1440-1521).

AA Town in Germany.

AA Isabella; Italian archaeologist, businesswoman (1474-1539).

AL "Deucalion's region;" Deucalion was King of Thessaly who saved himself from flood.

AL Designation of 2 nd part of old feature "Nilus."

CO Classical albedo feature name.

MN From albedo feature at $35 \mathrm{~N}, 355 \mathrm{~W}$

AA Armin J.; American astronomer (1918-1969).

VA Classical river in Scotland.

VA Classical name for Dee River in Scotland; new position and coordinates.

CM Czechoslovakian goddess of hunting.

AA Hebrew first name.

AA Irish heroine (d. 1193)

AA Sir James; British chemist (1842-1923).

CR Vedic goddess of plenty.

AA Greek; Callisto's sister.

AA Town in the Socialist

Republic of Vietnam.

AL Highland area in northern Attica, Greece.

PE From albedo feature at $48 \mathrm{~N}, 170 \mathrm{~W}$.

AA Latin female name.

CM Roman goddess of hunt/moon.

AA Charles; English novelist (1812-1870)

AA Emily; American poet (1830-1886)

AA Denis; French philosopher (1713-1784).

AA Tyrian princess who founded Carthage.

PE Angolan sacred lake.

AA Egyptian goddess of the dwelling place of the dead.

\footnotetext{
P: $\quad$ planetary system (see page xvi)

Sa: $\quad$ satellite (see page xvi).

lat: latitude of feature center.

long: longitude of feature center.
}

diam: diameter or long dimension of feature.

ct: continent of name origin (see page $284 \mathrm{ff}$.)

et: ethnicity of name origin (see page $284 \mathrm{ff}$.)

as: name approval status (see page xvii). ad: name approval date (year).

ref: reference source for name (see page $287 \mathrm{ff}$.).

ft: feature type (see page 290). 
P Sa Name

$\begin{array}{lll}\text { V } & \text { V } & \text { Dinah } \\ \text { J } & \text { io } & \text { Dingir Patera } \\ \text { M } & \text { M } & \text { Dingo } \\ \text { M } & \text { M } & \text { Dinorwic } \\ \text { V } & \text { V } & \text { Dione Regio } \\ \text { L } & \text { L } & \text { Dionysius } \\ \text { L } & \text { L } & \text { Diophantus } \\ \text { M } & \text { M } & \text { Dioscuria }\end{array}$

\section{L Dirichlet \\ H H Discovery Rupes \\ M M Dison \\ M M Dittaino Valles \\ V V Dix \\ M M Dixie \\ $S$ en Diyar Planitia \\ $\mathrm{U}$ ar Djadek \\ $S$ rh Djuli \\ M M Doanus Vallis}

L L Dobrovol'skiy

V V Dodola Dorsa

$\mathrm{J}$ io Dodona Planum

L L Doerfel

M M Dokuchaev

L L Dollond

U ar Domovoy

L L Donati

L L Donna

H H Donne

L L Donner

M M Doon

L L Doppelmayer

L L Doppler

V V Doris

$\mathrm{N}$ tr Doro Macula

L L Dorsa Aldrovandi

L L Dorsa Andrusov

L L Dorsa Argand

M M Dorsa Argentea

L L Dorsa Barlow

M M Dorsa Brevia

L L Dorsa Burnet lat

long diam

ct et

as ad

$62.8 \mathrm{~S} \quad 37.0 \mathrm{E}$

$4.0 \mathrm{~S} \quad 342.1 \mathrm{~W}$

24.0S 17.3W

$30.5 \mathrm{~S} \quad 101.4 \mathrm{~W}$

$31.5 \mathrm{~S} \quad 328.0 \mathrm{E}$

$2.8 \mathrm{~N} \quad 17.3 \mathrm{E}$

$27.6 \mathrm{~N} \quad 34.3 \mathrm{~W}$

$50.0 \mathrm{~N} \quad 320.0 \mathrm{~W}$

$\begin{array}{rll}19 & \text { AS } & \text { HE } \\ 40 & \text { AS } & \text { SU } \\ 13 & \text { OC } & \text { AU } \\ 0 & \text { NA } & \text { CA } \\ 2300 & \text { EU } & \text { GR } \\ 18 & \text { EU } & \text { GR }\end{array}$

51994

$5 \quad 1985$

51976

$\begin{array}{ll}5 & 1989\end{array}$

$5 \quad 91$

1935

17

EU GR

51935

0 EU GR

$\begin{array}{ll}5 & 1958\end{array}$

$11.1 \mathrm{~N} \quad 151.4 \mathrm{~W}$

$56.3 \mathrm{~S} \quad 38.3 \mathrm{~W}$

47

EU GE

$5 \quad 1970$

$25.4 \mathrm{~S}$

$16.3 \mathrm{~W}$

$0.5 \mathrm{~N}$

$66.4 \mathrm{~W}$

36.9S 329.1E

$19.9 \mathrm{~N} \quad 55.9 \mathrm{~W}$

$0.5 \mathrm{~N} \quad 239.7 \mathrm{~W}$

12.0S 251.1E

$31.2 \mathrm{~S}$

$46.7 \mathrm{~W}$

63.2S

$25.9 \mathrm{~W}$$$
0
$$

EU

51976

20

EU

$$
68
$$

$$
\text { NA }
$$

1976

51985

0
311

NA
AS

1988

22

EU CZ

51982

$\begin{array}{ll}5 & 1988\end{array}$

0

UR NE

$5 \quad 1982$

128

AS

51985

$12.8 \mathrm{~S} \quad 129.7 \mathrm{E}$

$46.8 \mathrm{~N} \quad 272.6 \mathrm{E}$

38

UR SO

$5 \quad 1973$

$56.8 \mathrm{~S}$

607

390

EU SL

$5 \quad 1985$

69.1

107.9W

68

EU

$60.8 \mathrm{~S}$

$127.1 \mathrm{~W}$

73

EU GE

51985

UR RU

51982

10.4S 14.4E

$51.5 \mathrm{~N} \quad 201.4 \mathrm{E}$

$\begin{array}{ll}71.5 \mathrm{~S} & 339.7 \mathrm{E}\end{array}$

$20.7 \mathrm{~S}$

$5.2 \mathrm{E}$

$7.2 \mathrm{~N}$

38.3E

$2.8 \mathrm{~N} \quad 13.8 \mathrm{~W}$

31.4S $98.0 \mathrm{E}$

$23.8 \mathrm{~N} \quad 250.8 \mathrm{~W}$

28.5S $41.4 \mathrm{~W}$

$12.6 \mathrm{~S} \quad 159.6 \mathrm{~W}$

$2.3 \mathrm{~N}$

$27.5 S$

$89.9 \mathrm{E}$

$24.0 \mathrm{~N} 28.5 \mathrm{E}$

$1.0 \mathrm{~S} \quad 57.0 \mathrm{E}$

$28.1 \mathrm{~N} \quad 40.6 \mathrm{~W} \quad 109$

$77.6 \mathrm{~S}$

$15.0 \mathrm{~N}$

$70.8 \mathrm{~S} \quad 300.3 \mathrm{~W}$

$28.4 \mathrm{~N} \quad 57.0 \mathrm{~W}$
11

12

71
36

EU GB

EU SP

EU SL

$5 \quad 1935$

51985

$\begin{array}{ll}5 & 1988\end{array}$

$5 \quad 1935$

2

EU IT

EU EN

$5 \quad 1979$

$5 \quad 1976$

0

NA CA

$5 \quad 1988$

63

EU

EU

AS

$5 \quad 1970$

16 EU GR

$5 \quad 1994$

$\begin{array}{llrrr}136 & \text { EU } & \text { IT } & 5 & 1976 \\ 160 & \text { UR } & \text { SO } & 5 & 1976\end{array}$

EU SZ

$5 \quad 1976$

749

120

EU LA

$\begin{array}{llll}\text { EU } & \text { LA } & 5 & 1976 \\ \text { EU } & \text { GB } & 5 & 1976\end{array}$

748

194 $\begin{array}{llll}\text { EU } & \text { LA } & 5 & 1976 \\ \text { EU } & \text { GB } & 5 & 1976\end{array}$
UR NA ref

83

27

36

74

66

66

60

0

59

36

36

99

36

52

66

\section{ft origin}

AA Hebrew first name.

PE Sumerian sun god; means "shining".

AA Town in Australia.

AA Town in Ontario, Canada.

RE Greek Titanness; 1st wife of Zeus.

AA St. Dionysius the Areopagite; Greek astronomer (A.D. 9-120).

AA Greek mathematician (unkn-c. A.D. 300)

AL "Home of Dioscuri," Polydeuces and Pollux; symbolic name for Sparta.

AA Peter G. L.; German mathematician (1805-1859)

RU English; Cook's ship on last voyage to Pacific, 1776-80.

AA Town in Belgium.

VA Modern river in Italy.

AA Dorothea; American nurse, reformer (1802-1887).

AA Town in Georgia, USA.

PL Country where Khudadad's father ruled.

AA Czech ancestral benevolent spirit and household guardian.

AA Neghidahan (Ukrainian) first man who was ancestor of the people.

VA Classical river shown in Ptolemy's map; may be modern Mekong River of Burma.

AA Georgiy T.; Soviet aeronautical engineer (1928-1971).

DO South Slavic rain goddess.

PM Greek; where Io went after the death of Argus.

AA Georg Samuel; German astronomer (1643-1688).

AA Vasily Vasil'evich; Russian soil scientist; founded modern genetical soil science (1840-1903).

AA John; British optician (1706-1761).

AA Spanish first name.

AA Slavic spirit protector of home.

AA Giovanni Battista; Italian astronomer (1826-1873).

AA Italian female name.

AA John; English poet (1572-1631).

AA Anders; Finnish astronomer (1873-1949).

AA Town in Ontario, Canada.

AA Johann Gabriel; German mathematician, astronomer (1671-1750). 
ALPHABETICAL LIST OF NAMES

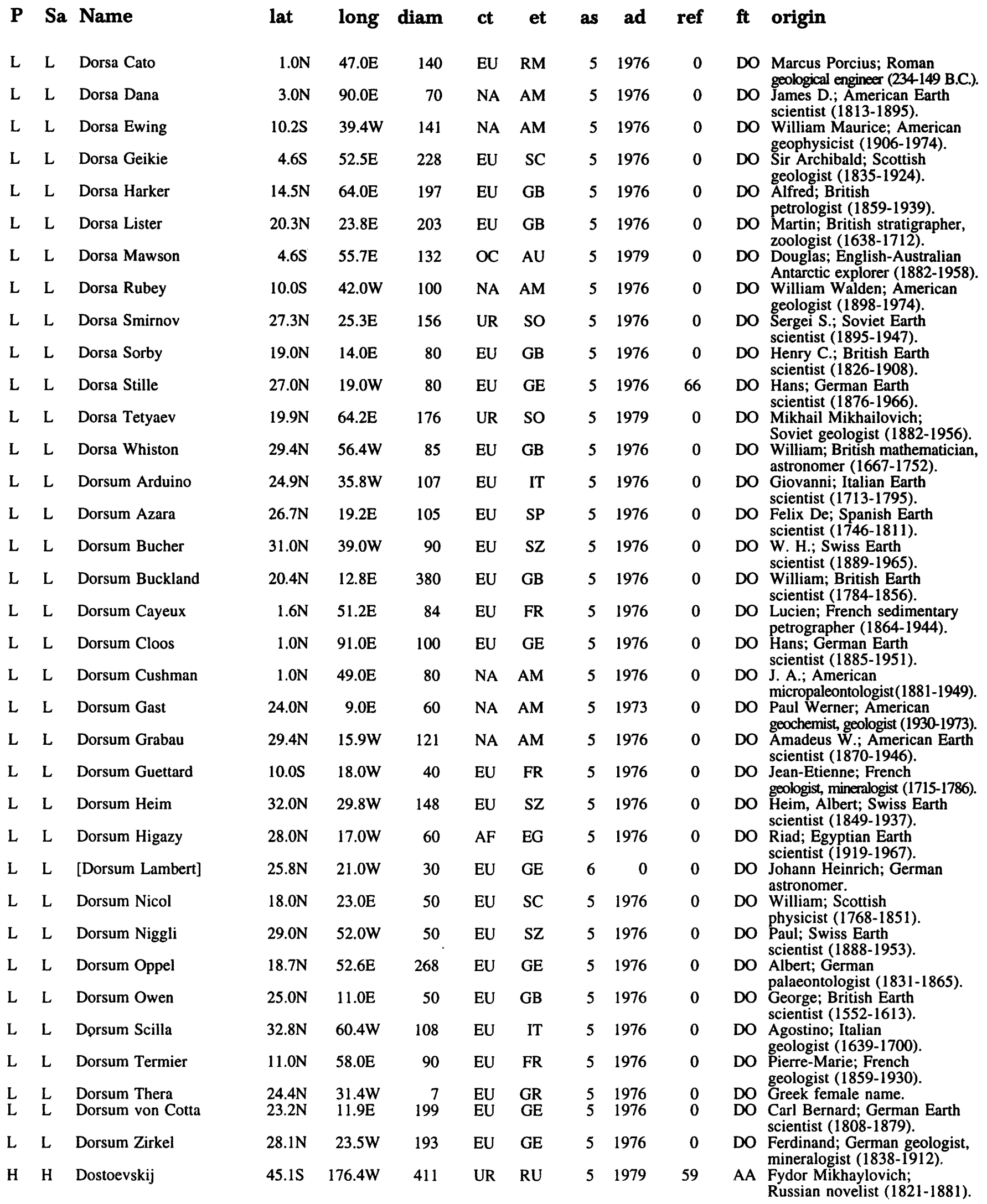

\footnotetext{
P: $\quad$ planetary system (see page $x v i$ ).

Sa: satellite (see page $x v i$ )

lat: latitude of feature center.

long: longitude of feature center.
}

diam: diameter or long dimension of feature

ct: continent of name origin (see page $284 \mathrm{ff}$.)

et: ethnicity of name origin (see page $284 \mathrm{ff}$.)

as: name approval status (see page xvii). ad: name approval date (year).

ref: reference source for name (see page $287 \mathrm{ff}$.).

ft: feature type (see page 290 ). 


\section{ALPHABETICAL LIST OF NAMES}

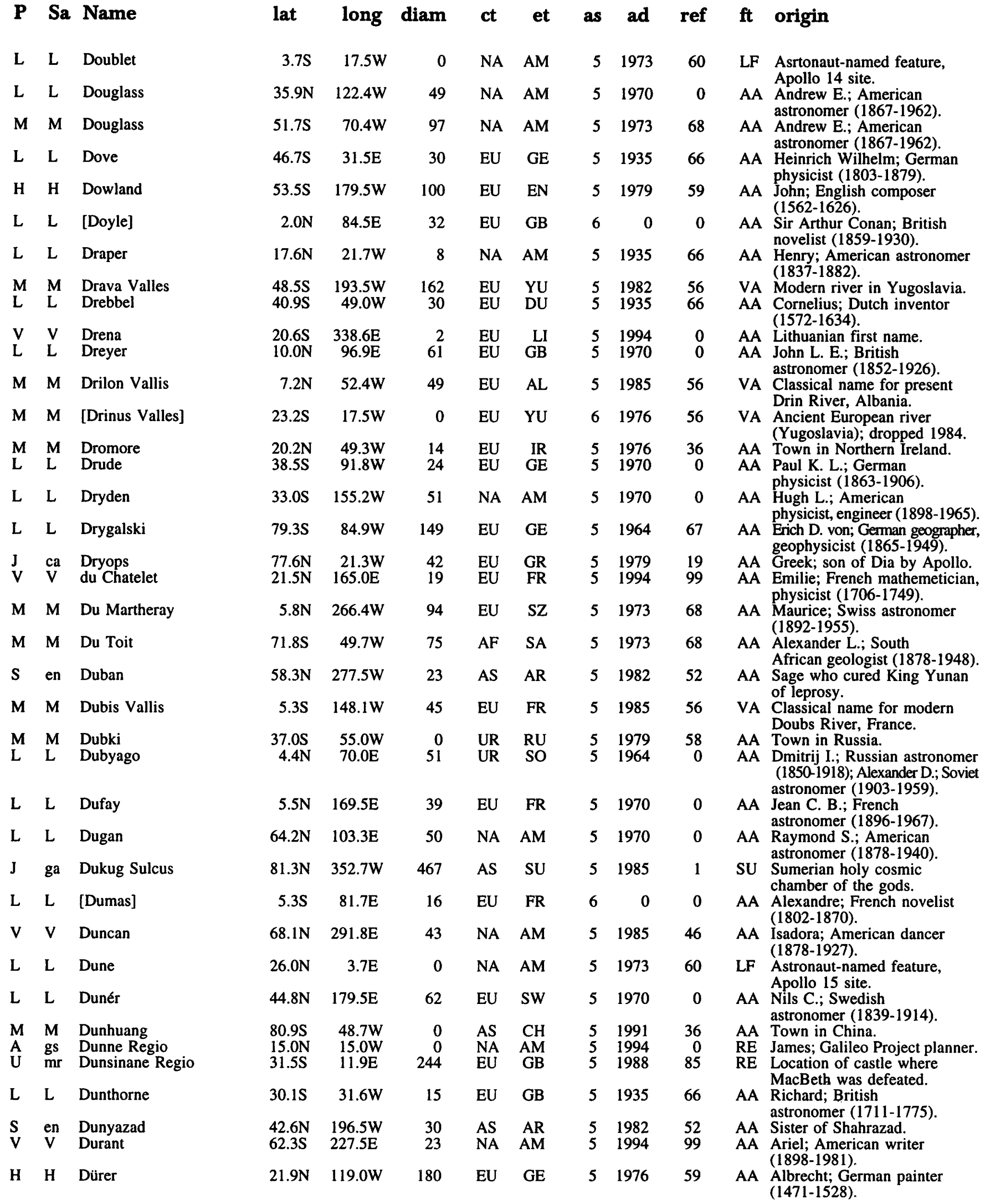

P: $\quad$ planetary system (see page $x v i)$.

Sa: satellite (see page xvi).

lat: latitude of feature center.

long: longitude of feature center. diam: diameter or long dimension of feature. ct: continent of name origin (see page $284 \mathrm{ff}$.) et: ethnicity of name origin (see page $284 \mathrm{ff}$.) as: name approval status (see page xvii). ad: name approval date (year).

ref: reference source for name (see page $287 \mathrm{ff}$.).

$\mathrm{ft}$ : feature type (see page 290). 


\section{ALPHABETICAL LIST OF NAMES}

\begin{tabular}{|c|c|c|c|c|c|c|c|c|c|}
\hline $\mathbf{P}$ & Sa & Name & lat & long & diam & ct & et & as & ad \\
\hline $\begin{array}{l}J \\
V\end{array}$ & $\begin{array}{l}\mathrm{ca} \\
\mathrm{V}\end{array}$ & $\begin{array}{l}\text { Durinn } \\
\text { Duse }\end{array}$ & $\begin{array}{l}67.0 \mathrm{~N} \\
82.5 \mathrm{~S}\end{array}$ & $\begin{array}{c}90.1 \mathrm{~W} \\
358.0 \mathrm{E}\end{array}$ & $\begin{array}{l}49 \\
27\end{array}$ & $\begin{array}{l}\text { EU } \\
\text { EU }\end{array}$ & $\begin{array}{l}\text { NS } \\
\text { IT }\end{array}$ & $\begin{array}{l}5 \\
5\end{array}$ & $\begin{array}{l}1979 \\
1994\end{array}$ \\
\hline $\begin{array}{l}\mathrm{M} \\
\mathrm{H}\end{array}$ & $\underset{\mathrm{H}}{\mathrm{M}}$ & $\begin{array}{l}\text { Dush } \\
\text { Dvorák }\end{array}$ & $\begin{array}{r}22.6 \mathrm{~N} \\
9.6 \mathrm{~S}\end{array}$ & $\begin{array}{l}54.0 \mathrm{~W} \\
11.9 \mathrm{~W}\end{array}$ & $\begin{array}{r}0 \\
82\end{array}$ & $\begin{array}{l}\mathrm{AF} \\
\mathrm{EU}\end{array}$ & $\begin{array}{l}\mathrm{EG} \\
\mathrm{CZ}\end{array}$ & $\begin{array}{l}5 \\
5\end{array}$ & $\begin{array}{l}1988 \\
1976\end{array}$ \\
\hline $\begin{array}{l}\text { V } \\
\text { S } \\
\text { L }\end{array}$ & $\begin{array}{l}\mathrm{V} \\
\mathrm{mi} \\
\mathrm{L}\end{array}$ & $\begin{array}{l}\text { Dyan-Mu Dorsa } \\
\text { Dynas } \\
\text { Dyson }\end{array}$ & $\begin{array}{r}78.2 \mathrm{~N} \\
3.8 \mathrm{~N} \\
61.3 \mathrm{~N}\end{array}$ & $\begin{array}{c}31.9 \mathrm{E} \\
82.9 \mathrm{~W} \\
121.2 \mathrm{~W}\end{array}$ & $\begin{array}{r}687 \\
0 \\
63\end{array}$ & $\begin{array}{l}\text { AS } \\
\text { EU } \\
\text { EU }\end{array}$ & $\begin{array}{l}\text { CH } \\
\text { GB } \\
\text { GB }\end{array}$ & $\begin{array}{l}5 \\
5 \\
5\end{array}$ & $\begin{array}{l}1985 \\
1982 \\
1970\end{array}$ \\
\hline $\begin{array}{l}\mathbf{M} \\
\mathbf{L}\end{array}$ & $\begin{array}{l}\mathrm{M} \\
\mathrm{L}\end{array}$ & $\begin{array}{l}\text { Dzeng } \\
\text { Dziewulski }\end{array}$ & $\begin{array}{l}80.6 \mathrm{~S} \\
21.2 \mathrm{~N}\end{array}$ & $\begin{array}{l}70.5 \mathrm{~W} \\
98.9 \mathrm{E}\end{array}$ & $\begin{array}{r}0 \\
63\end{array}$ & $\begin{array}{l}\mathrm{AF} \\
\mathrm{EU}\end{array}$ & $\begin{array}{l}\text { CR } \\
\text { PO }\end{array}$ & $\begin{array}{l}5 \\
5\end{array}$ & $\begin{array}{l}1991 \\
1970\end{array}$ \\
\hline $\begin{array}{l}\mathrm{M} \\
\mathrm{M} \\
\mathrm{M} \\
\mathrm{M} \\
\mathrm{V}\end{array}$ & $\begin{array}{l}\mathbf{M} \\
\mathbf{M} \\
\mathbf{M} \\
\mathrm{M} \\
\mathrm{V}\end{array}$ & $\begin{array}{l}\text { Dzigai Vallis } \\
\text { E. Mareotis Tholis } \\
\text { Eads } \\
\text { Eagle } \\
\text { [Earhart] }\end{array}$ & $\begin{array}{l}59.7 \mathrm{~S} \\
36.1 \mathrm{~N} \\
28.9 \mathrm{~S} \\
44.0 \mathrm{~N} \\
72.0 \mathrm{~N}\end{array}$ & $\begin{array}{c}31.3 \mathrm{~W} \\
85.0 \mathrm{~W} \\
29.8 \mathrm{~W} \\
8.2 \mathrm{~W} \\
136.0 \mathrm{E}\end{array}$ & $\begin{array}{r}0 \\
0 \\
2 \\
12 \\
0\end{array}$ & $\begin{array}{l}\text { NA } \\
\text { EU } \\
\text { US } \\
\text { US } \\
\text { NA }\end{array}$ & $\begin{array}{r}\text { NV } \\
\text { GR } \\
\text { CO } \\
\text { ID } \\
\text { AM }\end{array}$ & $\begin{array}{l}5 \\
5 \\
5 \\
5 \\
6\end{array}$ & $\begin{array}{l}1991 \\
1991 \\
1976 \\
1976 \\
1982\end{array}$ \\
\hline V & V & Earhart Corona & $70.1 \mathrm{~N}$ & $136.2 \mathrm{E}$ & 414 & NA & $\mathrm{AM}$ & 5 & 1985 \\
\hline L & $\mathrm{L}$ & Earthlight & $26.1 \mathrm{~N}$ & $3.7 \mathrm{E}$ & 0 & NA & AM & 5 & 1973 \\
\hline H & $\mathbf{H}$ & Echegaray & $42.7 \mathrm{~N}$ & $19.2 \mathrm{~W}$ & 75 & EU & SP & 5 & 1985 \\
\hline $\mathbf{J}$ & eu & Echion Linea & $13.1 \mathrm{~S}$ & $184.3 W$ & 1217 & EU & GR & 5 & 1985 \\
\hline $\begin{array}{l}\mathrm{J} \\
\mathrm{M} \\
\mathrm{M}\end{array}$ & $\begin{array}{l}\text { io } \\
\mathrm{M} \\
\mathrm{M}\end{array}$ & $\begin{array}{l}\text { Echo Mensa } \\
\text { Echt } \\
\text { Echus Chaos }\end{array}$ & $\begin{array}{r}79.6 \mathrm{~S} \\
22.2 \mathrm{~S} \\
9.9 \mathrm{~N}\end{array}$ & $\begin{array}{r}357.4 \mathrm{~W} \\
28.0 \mathrm{~W} \\
74.6 \mathrm{~W}\end{array}$ & $\begin{array}{r}0 \\
2 \\
371\end{array}$ & $\begin{array}{l}\text { EU } \\
\text { EU } \\
\text { EU }\end{array}$ & $\begin{array}{l}\text { GR } \\
\text { SC } \\
\text { LA }\end{array}$ & $\begin{array}{l}5 \\
5 \\
5\end{array}$ & $\begin{array}{l}1985 \\
1976 \\
1985\end{array}$ \\
\hline $\begin{array}{l}\mathrm{M} \\
\mathrm{M} \\
\mathrm{M} \\
\mathrm{L}\end{array}$ & $\begin{array}{l}\mathrm{M} \\
\mathrm{M} \\
\mathrm{M} \\
\mathrm{L}\end{array}$ & $\begin{array}{l}\text { Echus Chasma } \\
\text { Echus Fossae } \\
\text { Echus Montes } \\
\text { Eckert }\end{array}$ & $\begin{array}{r}3.7 \mathrm{~N} \\
2.2 \mathrm{~N} \\
6.8 \mathrm{~N} \\
17.3 \mathrm{~N}\end{array}$ & $\begin{array}{l}79.1 \mathrm{~W} \\
77.1 \mathrm{~W} \\
78.2 \mathrm{~W} \\
58.3 \mathrm{E}\end{array}$ & $\begin{array}{r}613 \\
236 \\
203 \\
2\end{array}$ & $\begin{array}{l}\text { EU } \\
\text { EU } \\
\text { EU } \\
\text { NA }\end{array}$ & $\begin{array}{l}\text { LA } \\
\text { LA } \\
\text { LA } \\
\text { AM }\end{array}$ & $\begin{array}{l}5 \\
5 \\
5 \\
5\end{array}$ & $\begin{array}{l}1976 \\
1988 \\
1985 \\
1973\end{array}$ \\
\hline $\begin{array}{l}\mathbf{M} \\
\mathbf{M}\end{array}$ & $\begin{array}{l}\mathrm{M} \\
\mathrm{M}\end{array}$ & $\begin{array}{l}\text { Edam } \\
\text { Eddie }\end{array}$ & $\begin{array}{l}26.6 \mathrm{~S} \\
12.5 \mathrm{~N}\end{array}$ & $\begin{array}{r}19.9 \mathrm{~W} \\
217.8 \mathrm{~W}\end{array}$ & $\begin{array}{l}20 \\
90\end{array}$ & $\begin{array}{l}\mathrm{EU} \\
\mathrm{AF}\end{array}$ & $\begin{array}{l}\text { DU } \\
\text { SA }\end{array}$ & $\begin{array}{l}5 \\
5\end{array}$ & $\begin{array}{l}1976 \\
1973\end{array}$ \\
\hline L & $\mathrm{L}$ & Eddington & $21.3 \mathrm{~N}$ & $72.2 \mathrm{~W}$ & 118 & EU & GB & 5 & 1964 \\
\hline J & ga & Edfu Facula & $26.8 \mathrm{~N}$ & $147.7 \mathrm{~W}$ & 187 & $\mathrm{AF}$ & EG & 5 & 1985 \\
\hline $\begin{array}{l}\text { V } \\
\text { V }\end{array}$ & $\begin{array}{l}\mathrm{V} \\
\mathrm{V}\end{array}$ & $\begin{array}{l}\text { Edgeworth } \\
\text { Edinger }\end{array}$ & $\begin{array}{l}32.2 \mathrm{~N} \\
68.8 \mathrm{~S}\end{array}$ & $\begin{array}{r}22.7 \mathrm{E} \\
208.3 \mathrm{E}\end{array}$ & $\begin{array}{l}31 \\
34\end{array}$ & $\begin{array}{l}\text { EU } \\
\text { NA }\end{array}$ & $\begin{array}{r}\text { IR } \\
\text { AM }\end{array}$ & $\begin{array}{l}5 \\
5\end{array}$ & $\begin{array}{l}1985 \\
1994\end{array}$ \\
\hline L & $\mathrm{L}$ & Edison & $25.0 \mathrm{~N}$ & $99.1 \mathrm{E}$ & 62 & NA & $\mathrm{AM}$ & 5 & 1961 \\
\hline $\begin{array}{l}\mathrm{L} \\
\mathrm{M}\end{array}$ & $\begin{array}{l}\mathrm{L} \\
\mathrm{M}\end{array}$ & $\begin{array}{l}\text { Edith } \\
\text { Edom }\end{array}$ & $\begin{array}{r}25.8 \mathrm{~S} \\
0.0 \mathrm{~N}\end{array}$ & $\begin{array}{l}102.3 \mathrm{E} \\
345.0 \mathrm{~W}\end{array}$ & $\begin{array}{l}8 \\
0\end{array}$ & $\begin{array}{l}\text { EU } \\
\text { AS }\end{array}$ & $\begin{array}{l}\text { GB } \\
\mathrm{HE}\end{array}$ & $\begin{array}{l}5 \\
5\end{array}$ & $\begin{array}{l}1976 \\
1958\end{array}$ \\
\hline V & $\mathrm{V}$ & Efimova & $81.0 \mathrm{~N}$ & $222.5 \mathrm{E}$ & 29 & UR & So & 5 & 1985 \\
\hline $\begin{array}{l}\mathrm{J} \\
\mathrm{L}\end{array}$ & $\begin{array}{l}\text { ca } \\
\mathrm{L}\end{array}$ & $\begin{array}{l}\text { Egdir } \\
\text { Egede }\end{array}$ & $\begin{array}{l}33.9 \mathrm{~N} \\
48.7 \mathrm{~N}\end{array}$ & $\begin{array}{l}35.9 \mathrm{~W} \\
10.6 \mathrm{E}\end{array}$ & $\begin{array}{l}58 \\
37\end{array}$ & $\begin{array}{l}\mathrm{EU} \\
\mathrm{EU}\end{array}$ & $\begin{array}{l}\text { NS } \\
\text { DE }\end{array}$ & $\begin{array}{l}5 \\
5\end{array}$ & $\begin{array}{l}1979 \\
1935\end{array}$ \\
\hline $\begin{array}{l}\mathrm{M} \\
\mathrm{V} \\
\mathrm{J}\end{array}$ & $\begin{array}{l}\mathrm{M} \\
\mathrm{V} \\
\mathrm{ca}\end{array}$ & $\begin{array}{l}\text { Eger } \\
\text { Egeria Farrum } \\
\text { Egres }\end{array}$ & $\begin{array}{l}48.7 \mathrm{~S} \\
43.6 \mathrm{~N} \\
42.5 \mathrm{~N}\end{array}$ & $\begin{array}{c}51.8 \mathrm{~W} \\
7.5 \mathrm{E} \\
176.6 \mathrm{~W}\end{array}$ & $\begin{array}{l}12 \\
40 \\
38\end{array}$ & $\begin{array}{l}\text { EU } \\
\text { EU } \\
\text { UR }\end{array}$ & $\begin{array}{l}\text { HU } \\
\text { RO } \\
\text { KA }\end{array}$ & $\begin{array}{l}5 \\
5 \\
5\end{array}$ & $\begin{array}{l}1976 \\
1994 \\
1987\end{array}$ \\
\hline $\mathrm{L}$ & $\mathrm{L}$ & Ehrlich & $40.9 \mathrm{~N}$ & $172.4 \mathrm{~W}$ & 30 & EU & GE & 5 & 1970 \\
\hline $\mathrm{L}$ & $\mathrm{L}$ & Eichstadt & $22.6 \mathrm{~S}$ & $78.3 \mathrm{~W}$ & 49 & $\mathrm{EU}$ & GE & 5 & 1935 \\
\hline $\begin{array}{l}\mathrm{V} \\
\mathrm{L}\end{array}$ & $\begin{array}{l}\mathrm{V} \\
\mathrm{L}\end{array}$ & $\begin{array}{l}\text { Eigin Corona } \\
\text { Eijkman }\end{array}$ & $\begin{array}{r}5.0 \mathrm{~S} \\
63.1 \mathrm{~S}\end{array}$ & $\begin{array}{l}175.0 \mathrm{E} \\
143.0 \mathrm{~W}\end{array}$ & $\begin{array}{r}200 \\
54\end{array}$ & $\begin{array}{l}\text { EU } \\
\text { EU }\end{array}$ & $\begin{array}{l}\text { CE } \\
\text { DU }\end{array}$ & $\begin{array}{l}5 \\
5\end{array}$ & $\begin{array}{l}1994 \\
1970\end{array}$ \\
\hline M & $\mathbf{M}$ & Eil & $42.0 \mathrm{~N}$ & $9.7 \mathrm{~W}$ & 4 & $\mathrm{AF}$ & SO & 5 & 1976 \\
\hline
\end{tabular}

AA Norse dwarf.

AA Eleonora; Italian actress (1858-1924)

AA Town in Egypt.

AA Anton; Bohemian composer (1841-1904).

DO Chinese lightning goddess.

AA A knight of the Round Table.

AA Sir Frank W.; British astronomer (1868-1939).

AA Town in Cameroon.

AA Wladyslaw; Polish astronomer (1878-1962).

VA Word for "valley" in Navajo.

TH Classical albedo name.

AA Town in Colorado, USA.

AA Town in Idaho, USA.

AA Name changed to Earhart Corona.

CR Amelia; American aviator (1897-1937).

LF Astronaut-named feature, Apollo 15 site

AA Jose; Spanish dramatist, Nobel laureate (1832-1916).

LI Survivor of the men Cadmus sowed with the dragon's teeth; a founder of Thebes.

MN Mother of Iynx.

AA Town in Scotland.

$\mathrm{CH}$ From albedo feature Echus Lacus at $1 \mathrm{~N}, 90 \mathrm{~W}$.

CM Classical albedo feature name.

FO Classical albedo feature name.

MO Albedo feature name.

AA Wallace J.; American astronomer (1902-1971).

AA Town in the Netherlands.

AA Lindsay A.; South African astronomer (1845-1913).

AA Sir Arthur S.; British astrophysicist, mathematician (1882-1944).

FA Egyptian town where Horus was worshipped.

AA Maria; Irish writer (1767-1849).

AA Tilly; American geologist (1897-1967).

AA Thomas A.; American inventor (1847-1931)

AA English female name.

AL Biblical country of Edomites, south of Judea.

AA (Simonovich-Efimova) Nina; Soviet painter and puppet-theatre designer (1877-1948).

AA Norse; shepherd for the giants.

AA Hans; Danish natural historian (1686-1758).

AA Town in Hungary.

FR Roman water nymph.

AA Karelian deity of the harvest of beans.

AA Paul; German doctor; Nobel laureate (1854-1915).

AA Lorentz; German mathematician, astronomer (1596-1660).

CR Celtic fertility goddess.

AA Christiaan H.; Dutch doctor; Nobel laureate (1858-1930).

AA Town in Somalia.

diam: diameter or long dimension of feature. ct: continent of name origin (see page $284 \mathrm{ff}$.)

et: ethnicity of name origin (see page $284 \mathrm{ff}$.)

as: name approval status (see page $x v i i$ ). ad: name approval date (year).

ref: reference source for name (see page $287 \mathrm{ff}$.).

ft: feature type (see page 290). 


\section{P Sa Name}

V V Eileen

L L Eimmart

L L Einstein

L L Einthoven

V V Eistla Regio

V V Eithinoha Corona

$\mathrm{H}$ H Eitoku

M M Ejriksson

L L [El Greco]

$\mathrm{S}$ mi Elaine

J ga Elam Sulci

M M Elath

L L Elbow

M M Electris

V V Elena

L L Elger

$\mathrm{U} \quad \mathrm{ti}$ Elinor

V V Eliot Patera

L L Ellerman

L L Ellison

M M Ellsley

$S$ rh Ellyay

L L Elmer

$S$ te Elpenor

U mr Elsinore Corona

L L Elvey

M M Ely

M M Elysium

M M Elysium Catena

M M Elysium Chasma

M M Elysium Fossae

M M Elysium Mons

M M Elysium Planitia

M M Elysium Rupes

V V Elza

$\mathrm{J}$ io Emakong Patera

L L Emden

L L Emory

L L Encke

L L End

H H Endeavour Rupes

L L Endymion lat long

$\begin{array}{lrrrrrrr}22.8 \mathrm{~S} & 232.6 \mathrm{E} & 15 & \mathrm{EU} & \mathrm{IR} & 5 & 1994 & 82 \\ 24.0 \mathrm{~N} & 64.8 \mathrm{E} & 46 & \mathrm{EU} & \mathrm{GE} & 5 & 1935 & 66 \\ 16.3 \mathrm{~N} & 88.7 \mathrm{~W} & 198 & \text { NA } & \text { AM } & 5 & 1964 & 67\end{array}$

$$
4
$$

10.5

22.1S

$22.1 \mathrm{~S}$

$19.6 \mathrm{~S}$
$14.0 \mathrm{~N}$

$44.5 \mathrm{~N}$

57.4

$46.1 \mathrm{~N}$

$26.0 \mathrm{~N}$

$45.0 \mathrm{~S}$

18.35

35.3

44.8S $333.6 \mathrm{E}$

$39.1 \mathrm{~N}$

25.35

$55.1 \mathrm{~N}$

$36.5 \mathrm{~N}$

$71.4 \mathrm{~N}$

$10.1 \mathrm{~S}$

$54.8 \mathrm{~N} 263.3 \mathrm{~W}$

$24.8 \mathrm{~S}$

109.6E

69

EU DU

$5 \quad 1970$

$21.5 \mathrm{E}$

7.5E

8015

$156.9 \mathrm{~W} \quad 100$

EU NS

NA AM

$5 \quad 1982$

$\begin{array}{lr}5 & 1982 \\ 5 & 91 \\ 5 & 1976\end{array}$

173.7W

56

EU NS

EU GR

$5 \quad 1967$

34.7E

108.2W

0

EU G

51982

51985

AS BY

$\begin{array}{ll}5 & 1976 \\ 5 & 1973\end{array}$

$13.7 \mathrm{~W}$

13

AS IS

190.0W

NA AM

51958

EU GR

$5 \quad 1994$

$73.3 \mathrm{E}$

$29.8 \mathrm{~W}$

18

EU IT

EU GB

$5 \quad 1935$

74

EU GB

$5 \quad 1988$

$79.0 \mathrm{E}$

116

EU GB

$5 \quad 1970$

107.5W

36

EU GB

$5 \quad 1970$

83.0W

$91.8 \mathrm{~W}$

84.1E

0

EU EN

$5 \quad 1991$

$\begin{array}{rrrrr}0 & \text { UR } & \text { YK } & 5 & 1982\end{array}$

16 NA AM

51976

0
323

EU GR

EU DE

$\begin{array}{ll}5 & 1982\end{array}$

$\begin{array}{ll}5 & 1988\end{array}$

$\begin{array}{ll}5 & 1970\end{array}$

23.9S 27.1W

$25.0 \mathrm{~N} 210.0 \mathrm{~W}$

10 US NV

$18.0 \mathrm{~N} \quad 210.4 \mathrm{~W}$

$22.2 \mathrm{~N} \quad 218.0 \mathrm{~W}$

$27.5 \mathrm{~N} \quad 219.5 \mathrm{~W}$

$25.0 \mathrm{~N} \quad 213.0 \mathrm{~W}$

$14.3 \mathrm{~N} \quad 241.1 \mathrm{~W}$

$25.4 \mathrm{~N} \quad 211.4 \mathrm{~W}$

$34.4 \mathrm{~S} \quad 275.8 \mathrm{E}$

3.2S $119.1 \mathrm{~W}$

63.3N $177.3 \mathrm{~W}$

$20.1 \mathrm{~N} \quad 30.8 \mathrm{E}$

$4.6 \mathrm{~N} \quad 36.6 \mathrm{~W}$

66

92
1114

1114
432

3899

180

17

80

$\begin{array}{ll}\text { US } & \text { NV } \\ \text { EU } & \text { GR }\end{array}$

$5 \quad 1976$

EU GR

EU GR

EU GR

EU GR

EU GR

EU GR

UR LV

OC NB

111 EU SZ

51958

$5 \quad 1985$

$\begin{array}{ll}5 & 1985\end{array}$

51973

51973

$5 \quad 1973$

$5 \quad 1985$

$5 \quad 1994$

51979

$5 \quad 1970$

$5 \quad 1973$

NA AM

$5 \quad 1935$

8.9S $15.6 \mathrm{E}$

$37.5 \mathrm{~N} \quad 31.3 \mathrm{~W}$

$\begin{array}{llllll}0 & \text { EU } & \text { EN } & 5 & 1976 & 59\end{array}$

$53.9 \mathrm{~N} \quad 57.0 \mathrm{E}$
123
EU GR

$5 \quad 1935$
AA Irish first name.

AA Georg Christoph; German astronomer (1638-1705). physicist; Nobel laureate (1879-1955).

Dutch physiologist; Nobel (1879-1955).

goddes Kuninobu; painter (1543-1590).

AA Leif; Norse explorer (c. 1000). Spanish artist, born in
Crete (c. 1541-1614). Daughter of King Pelles, lover of Sir Launcelot and mother, by him, of Sir Galahad.

in Israel. Apollo 15 site.

sisland near River Eridanus;

AA Thomas Gwyn; British astronomer (1838-1897)

( King John. ; (Mary Ann Evans) English writer (1819-1880) astronomer (1869-1940) Mervyn A.; British astronomer (1909-1963).

AA Town in England.

AA Yakutian ancestor of the people. W.; American

AA Follower of Odysseus.

CR Location of Hamlet's castle. Christian T.; American (1899-1970).

AA Town in Nevada, USA. Home of the blessed on

Classical albedo feature name.

Clssical albedo feature name.

RU Albedo feature name.

AA Latvian first name.

Sulca (New Britain) man meteorologist (1862-1940). Apollo 17 site. (1791-1865)

Apollo 16 site.

RU English; Cook's ship to explore Tahiti, New Zealand, Australia, 1768-71.

66

AA Greek mythological character.

\footnotetext{
P: $\quad$ planetary system (see page xvi).

Sa: satellite (see page $x v i$ ).

lat: latitude of feature center.

long: longitude of feature center.
}

diam: diameter or long dimension of feature.

ct: $\quad$ continent of name origin (see page $284 \mathrm{ff}$.)

et: ethnicity of name origin (see page $284 \mathrm{ff}$.)

as: name approval status (see page $x v i i$ ). ad: name approval date (year).

ref: reference source for name (see page $287 \mathrm{ff}$.).

ft: feature type (see page 290 ). 
ALPHABETICAL LIST OF NAMES

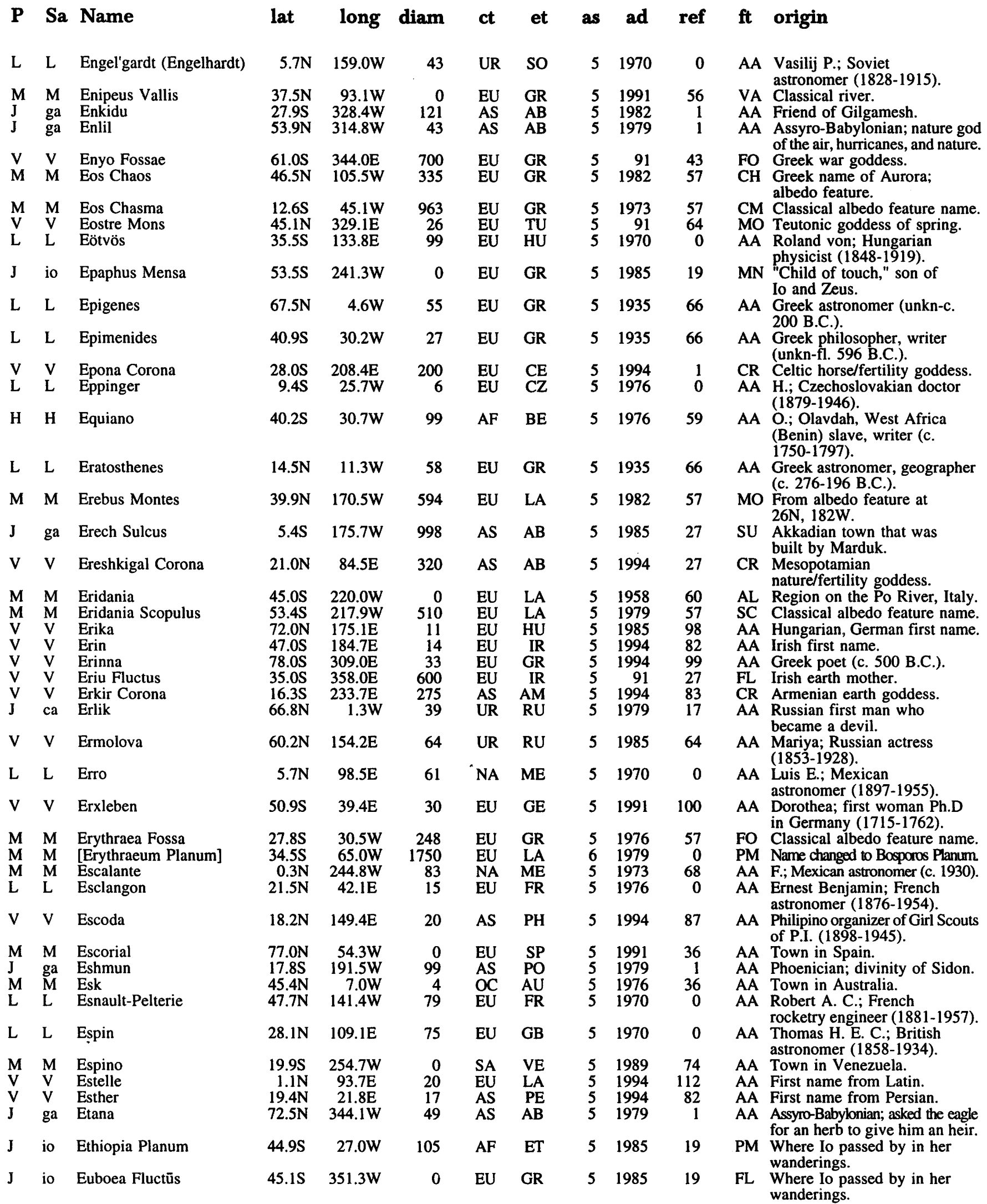

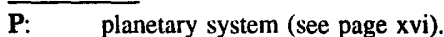

Sa: $\quad$ satellite (see page xvi).

lat: latitude of feature center.

long: longitude of feature center. diam: diameter or long dimension of feature.

ct: continent of name origin (see page $284 \mathrm{ff}$.)

et: ethnicity of name origin (see page $284 \mathrm{ff}$.)

as: name approval status (see page xvii). ad: name approval date (year).

ref: reference source for name (see page $287 \mathrm{ff}$.).

ft: feature type (see page 290). 


\section{ALPHABETICAL LIST OF NAMES}

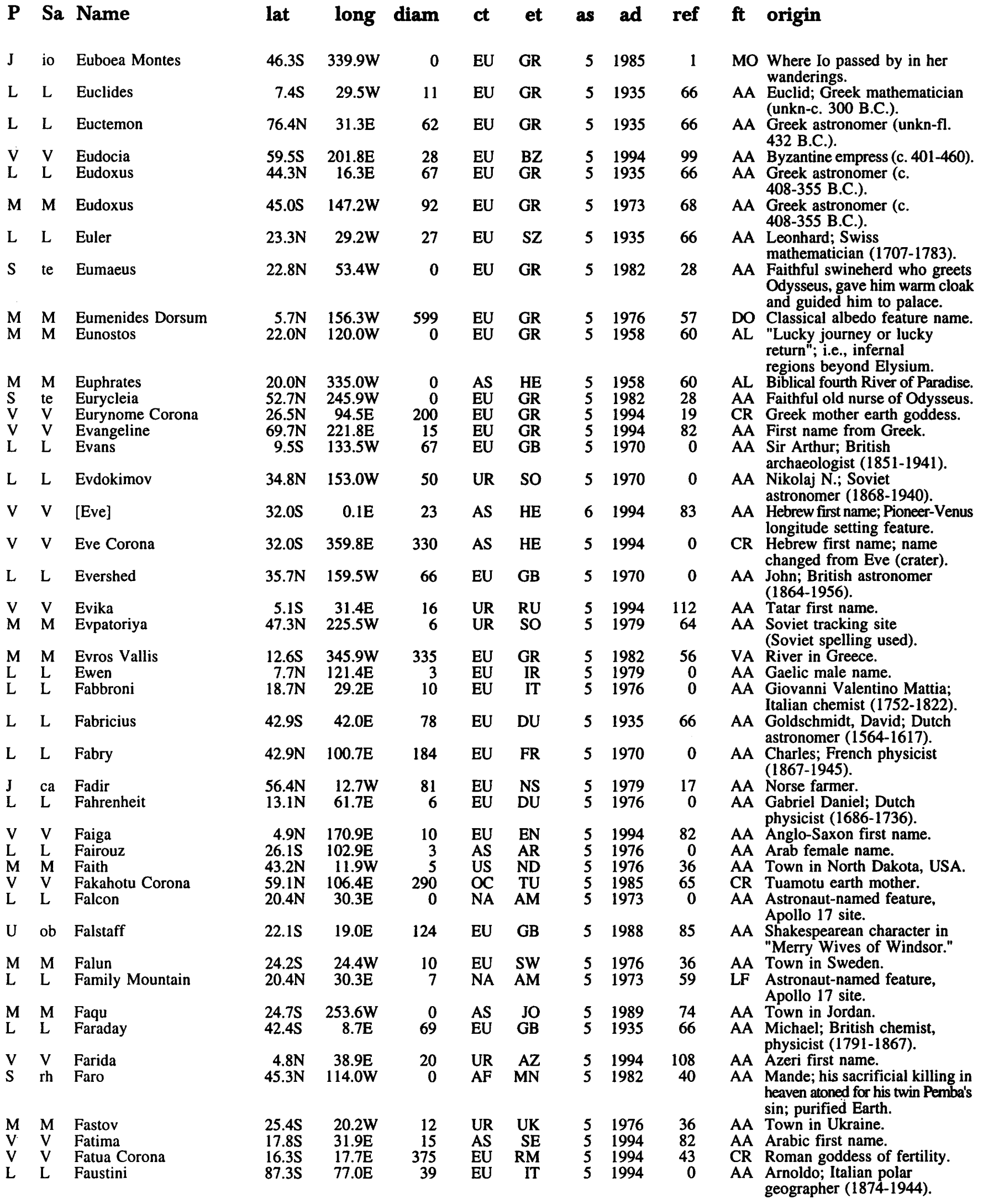

\footnotetext{
P: $\quad$ planetary system (see page $x v i)$.

Sa: $\quad$ satellite (see page $x v i$ ).

lat: latitude of feature center.

long: longitude of feature center.
}

diam: diameter or long dimension of feature.
ct: continent of name origin (see page $284 \mathrm{ff}$.)
et: ethnicity of name origin (see page $284 \mathrm{ff}$.)
as: name approval status (see page $x v i i)$.

ad: name approval date (year).

ref: reference source for name (see page $287 \mathrm{ff}$.).

ft: feature type (see page 290 ). 
ALPHABETICAL LIST OF NAMES

\begin{tabular}{|c|c|c|c|c|c|c|c|c|c|}
\hline $\mathbf{P}$ & $\mathbf{S a}$ & Name & lat & long & diam & ct & et & as & ad \\
\hline L & $\mathrm{L}$ & Fauth & $6.3 \mathrm{~N}$ & $20.1 \mathrm{~W}$ & 12 & $\mathrm{EU}$ & $\mathrm{GE}$ & 5 & 1935 \\
\hline $\mathrm{L}$ & $\mathrm{L}$ & Faye & $21.4 S$ & $3.9 \mathrm{E}$ & 36 & EU & FR & 5 & 1935 \\
\hline $\mathrm{L}$ & $\mathrm{L}$ & Fechner & $59.0 \mathrm{~S}$ & $124.9 \mathrm{E}$ & 63 & $\mathrm{EU}$ & GE & 5 & 1970 \\
\hline V & $\mathrm{V}$ & Fedorets & $59.6 \mathrm{~N}$ & $65.1 \mathrm{E}$ & 54 & UR & So & 5 & 1985 \\
\hline $\mathbf{L}$ & $\mathrm{L}$ & Fedorov & $28.2 \mathrm{~N}$ & $37.0 \mathrm{~W}$ & 6 & UR & RU & 5 & 1979 \\
\hline $\mathrm{V}$ & $\mathrm{V}$ & [Fedosova] & $45.0 \mathrm{~N}$ & $171.8 \mathrm{E}$ & 24 & UR & RU & 6 & 1992 \\
\hline $\mathrm{V}$ & V & Felesta Fossae & $35.0 \mathrm{~N}$ & $46.5 \mathrm{E}$ & 0 & AS & $\mathrm{SC}$ & 5 & 91 \\
\hline $\begin{array}{l}\mathrm{V} \\
\mathrm{M} \\
\mathrm{L} \\
\mathrm{M} \\
\mathrm{L}\end{array}$ & $\begin{array}{l}\mathrm{V} \\
\mathrm{M} \\
\mathrm{L} \\
\mathrm{M} \\
\mathrm{L}\end{array}$ & $\begin{array}{l}\text { Felicia } \\
\text { Felis Dorsa } \\
\text { Felix } \\
\text { Fenagh } \\
\text { Fényi }\end{array}$ & $\begin{array}{l}19.8 \mathrm{~S} \\
22.3 \mathrm{~S} \\
28.2 \mathrm{~N} \\
34.6 \mathrm{~N} \\
44.9 \mathrm{~S}\end{array}$ & $\begin{array}{r}226.4 \mathrm{E} \\
65.5 \mathrm{~W} \\
37.0 \mathrm{~W} \\
215.7 \mathrm{~W} \\
105.1 \mathrm{~W}\end{array}$ & $\begin{array}{r}12 \\
486 \\
6 \\
0 \\
38\end{array}$ & $\begin{array}{l}\text { EU } \\
\text { EU } \\
\text { EU } \\
\text { EU } \\
\text { EU }\end{array}$ & $\begin{array}{r}\text { LA } \\
\text { LA } \\
\text { LA } \\
\text { IR } \\
\text { HU }\end{array}$ & $\begin{array}{l}5 \\
5 \\
5 \\
5 \\
5\end{array}$ & $\begin{array}{l}1994 \\
1976 \\
1976 \\
1991 \\
1970\end{array}$ \\
\hline $\mathbf{L}$ & $\mathrm{L}$ & Feoktistov & $30.9 \mathrm{~N}$ & $140.7 \mathrm{E}$ & 23 & UR & so & 5 & 1970 \\
\hline V & $\mathrm{V}$ & Ferber & $26.4 \mathrm{~N}$ & $13.0 \mathrm{E}$ & 23 & NA & $\mathrm{AM}$ & 5 & 1991 \\
\hline $\mathrm{U}$ & $\mathrm{mr}$ & Ferdinand & $34.8 \mathrm{~S}$ & $202.1 \mathrm{E}$ & 17 & EU & GB & 5 & 1988 \\
\hline L & $\mathrm{L}$ & Fermat & $22.6 \mathrm{~S}$ & $19.8 \mathrm{E}$ & 38 & $\mathrm{EU}$ & FR & 5 & 1935 \\
\hline L & $\mathrm{L}$ & Fermi & $19.3 \mathrm{~S}$ & $122.6 \mathrm{E}$ & 183 & $\mathrm{EU}$ & IT & 5 & 1970 \\
\hline V & V & Fernandez & $76.3 \mathrm{~N}$ & $17.1 \mathrm{E}$ & 21 & $\mathrm{EU}$ & SP & 5 & 1985 \\
\hline $\mathrm{L}$ & $\mathrm{L}$ & Fernelius & $38.1 \mathrm{~S}$ & $4.9 \mathrm{E}$ & 65 & EU & FR & 5 & 1935 \\
\hline V & $\mathrm{V}$ & Feronia Corona & $68.0 \mathrm{~N}$ & $281.7 \mathrm{E}$ & 360 & EU & IT & 5 & 1985 \\
\hline $\mathrm{V}$ & $\mathrm{V}$ & Ferrier & $15.8 \mathrm{~N}$ & $111.3 \mathrm{E}$ & 33 & $\mathrm{EU}$ & EN & 5 & 1991 \\
\hline L & $\mathrm{L}$ & Fersman & $18.7 \mathrm{~N}$ & $126.0 \mathrm{~W}$ & 151 & UR & so & 5 & 1970 \\
\hline M & $\mathbf{M}$ & Fesenkov & $21.9 \mathrm{~N}$ & $86.4 \mathrm{~W}$ & 86 & UR & $\mathrm{RU}$ & 5 & 1973 \\
\hline L & $\mathbf{L}$ & Fesenkov & $23.2 \mathrm{~S}$ & $135.1 \mathrm{E}$ & 35 & UR & RU & 5 & 1973 \\
\hline $\begin{array}{l}\text { V } \\
\mathrm{H}\end{array}$ & $\begin{array}{l}\mathrm{V} \\
\mathrm{H}\end{array}$ & $\begin{array}{l}\text { Festa } \\
\text { Fet }\end{array}$ & $\begin{array}{r}11.5 \mathrm{~N} \\
4.9 \mathrm{~S}\end{array}$ & $\begin{array}{c}27.2 \mathrm{E} \\
179.9 \mathrm{~W}\end{array}$ & $\begin{array}{l}25 \\
24\end{array}$ & $\begin{array}{l}\text { EU } \\
\text { UR }\end{array}$ & $\begin{array}{r}\text { IT } \\
\text { RU }\end{array}$ & $\begin{array}{l}5 \\
5\end{array}$ & $\begin{array}{l}1991 \\
1985\end{array}$ \\
\hline L & $\mathrm{L}$ & Feuillée & $27.4 \mathrm{~N}$ & $9.4 \mathrm{~W}$ & 9 & EU & FR & 5 & 1935 \\
\hline $\begin{array}{l}\text { J } \\
U\end{array}$ & $\begin{array}{l}\text { ca } \\
\text { um }\end{array}$ & $\begin{array}{l}\text { Fili } \\
\text { Fin }\end{array}$ & $\begin{array}{l}64.3 \mathrm{~N} \\
37.4 \mathrm{~S}\end{array}$ & $\begin{array}{c}349.5 \mathrm{~W} \\
44.3 \mathrm{E}\end{array}$ & $\begin{array}{l}42 \\
43\end{array}$ & $\begin{array}{l}\mathrm{EU} \\
\mathrm{EU}\end{array}$ & $\begin{array}{l}\mathrm{NS} \\
\mathrm{DE}\end{array}$ & $\begin{array}{l}5 \\
5\end{array}$ & $\begin{array}{l}1979 \\
1988\end{array}$ \\
\hline $\begin{array}{l}\mathbf{J} \\
\mathbf{L}\end{array}$ & $\begin{array}{l}\mathrm{ca} \\
\mathrm{L}\end{array}$ & $\begin{array}{l}\text { Finnr } \\
\text { Finsch }\end{array}$ & $\begin{array}{l}15.5 \mathrm{~N} \\
23.6 \mathrm{~N}\end{array}$ & $\begin{array}{l}4.3 \mathrm{~W} \\
21.3 \mathrm{E}\end{array}$ & $\begin{array}{r}65 \\
4\end{array}$ & $\begin{array}{l}\mathrm{EU} \\
\mathrm{EU}\end{array}$ & $\begin{array}{l}\text { NS } \\
\text { GE }\end{array}$ & $\begin{array}{l}5 \\
5\end{array}$ & $\begin{array}{l}1979 \\
1976\end{array}$ \\
\hline $\mathbf{L}$ & $\mathbf{L}$ & Finsen & $42.0 \mathrm{~S}$ & $177.9 \mathrm{~W}$ & 72 & EU & $\mathrm{DE}$ & 5 & 1979 \\
\hline $\mathbf{U}$ & ar & Finvara & $15.8 \mathrm{~S}$ & $19.0 \mathrm{E}$ & 31 & EU & IR & 5 & 1988 \\
\hline L & $\mathrm{L}$ & [Firdausi] & $24.8 \mathrm{~N}$ & $34.0 \mathrm{~W}$ & 6 & AS & PE & 6 & 0 \\
\hline $\mathrm{L}$ & $\mathrm{L}$ & Firmicus & $7.3 \mathrm{~N}$ & $63.4 \mathrm{E}$ & 56 & $\mathrm{EU}$ & IT & 5 & 1935 \\
\hline $\mathrm{L}$ & $\mathrm{L}$ & Firsov & $4.5 \mathrm{~N}$ & $112.2 \mathrm{E}$ & 51 & UR & so & 5 & 1970 \\
\hline $\mathrm{L}$ & $\mathrm{L}$ & Fischer & $8.0 \mathrm{~N}$ & $142.4 \mathrm{E}$ & 30 & $\mathrm{EU}$ & GE & 5 & 1976 \\
\hline
\end{tabular}

AA Philipp Johann Heinrich; German selenographer (1867-1941).

AA Herve; French astronomer (1814-1902)

AA Gustav T.; German physicist, psychologist (1801-1887)

AA Velentina; Soviet astronomer (1923-1976).

AA A.P.; Russian rocket scientist (1872-1920)

AA Irina; Russian folk poet (1831-1899).

FO Amazon queen in Scythian epic tales.

AA First name from Latin.

DO Classical albedo feature name.

AA Latin male name.

AA Town in Ireland.

AA Gyula: Hungarian astronomer (1845-1927).

AA Konstantin P.; Soviet cosmonaut (1926-Live)

AA Edna; American author (1887-1968).

AA Son of King of Naples: loves Miranda in "The Tempest."

AA Pierre De; French mathematician (1601-1665).

AA Enrico; Italian-American physicist; Nobel laureate (1901-1954).

AA M. A.; Spanish actress (18th century)

AA Jean; French doctor astronomer (1497-1558).

CR Ancient Italian goddess of spring and flowers.

AA Kathleen; English opera singer (1912-1953).

AA Aleksandr; Soviet geochemist (1883-1945).

AA Vasilii G.; Russian astronomer (1889-1972).

AA Vasiliy Grigor'evich; Soviet astrophysicist (1889-1972).

AA Italian painter.

AA Afanasy Afanasyevich; Russian poet (1820-1892)

AA Louis; French natural scientist (1660-1732)

AA Norse dwarf.

AA Troll who helped build a church in Kallundburg, Zealand

AA Norse dwarf.

AA O. F. H.; German zoologist (1839-1917).

AA Niels Ryberg; Danish phototherapist; Nobel laureate (1860-1904).

AA Irish king of spirits; provided horses and wine to men.

AA Hasan; Persian author (c. 940-1020).

AA Maternus; Italian astronomer (unkn-c. 330)

AA Georgij F.; Soviet rocketry engineer (1917-1960).

AA Emil; German chemist (1852-1919); Hans; German organic chemist (1881-1945).

\footnotetext{
P: $\quad$ planetary system (see page xvi).

Sa: satellite (see page xvi).

lat: latitude of feature center.

long: longitude of feature center.
}

diam: diameter or long dimension of feature.

ct: continent of name origin (see page $284 \mathrm{ff}$.)

et: ethnicity of name origin (see page $284 \mathrm{ff}$.)

as: name approval status (see page xvii). ad: name approval date (year)

ref: reference source for name (see page $287 \mathrm{ff}$.).

ft: feature type (see page 290). 


\section{ALPHABETICAL LIST OF NAMES}

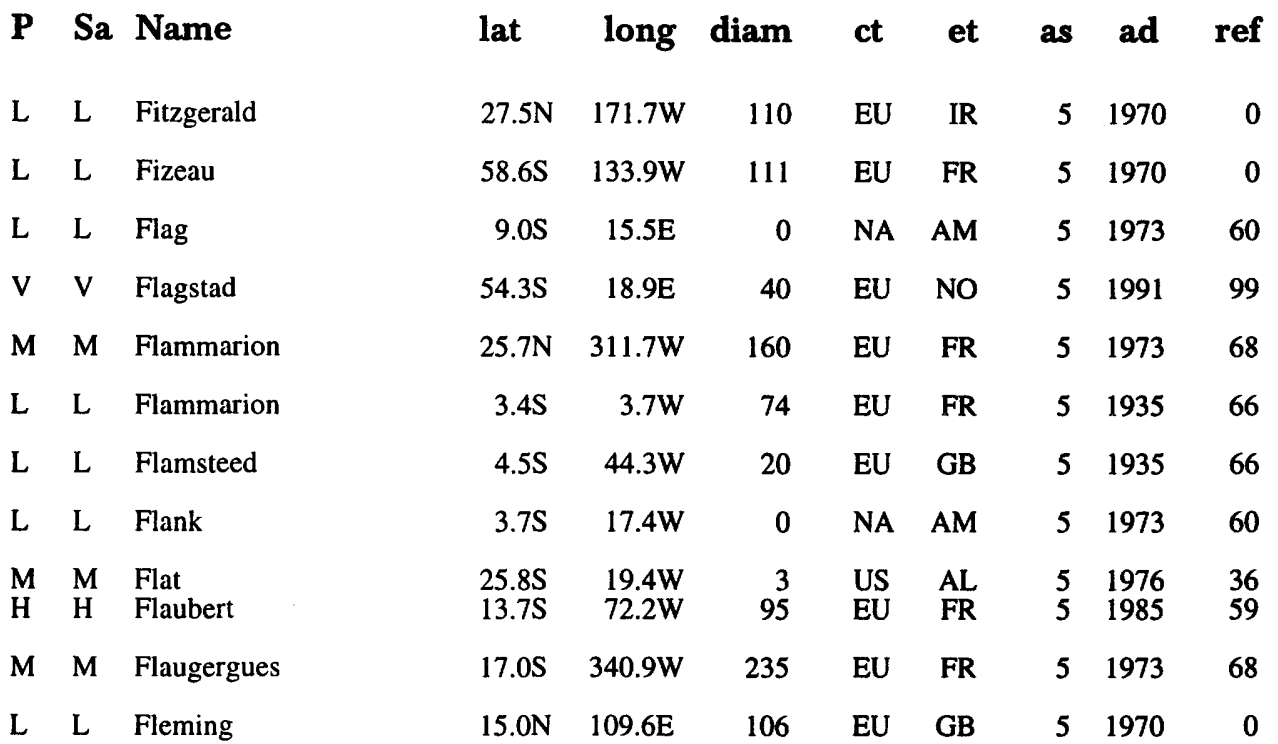

$\begin{array}{lll}\text { M } & \text { M } & \text { Floq } \\ \text { M } & \text { M } & \text { Flora }\end{array}$

L L Florensky

V V Flosshilde Farra

M M Focas

L L Focas

M M Fontana

L L Fontana

L L Fontenelle

$\mathrm{V} V$ Foquet

V V Fornax Rupes

M M Foros

M M Fortuna Fossae

V V Fortuna Tessera

L L [Fossa Casals]

$\begin{array}{lll}\text { L } & \text { L } & \text { [Fossa Cauchy] } \\ \text { V } & \text { V } & \text { Fossey }\end{array}$

L L Foster

V V Fotla Corona

L L Foucault

L L Fourier

M M Fournier

L L Fowler
L L Fox
L L Fra Mauro
L L Fracastorius

\begin{tabular}{|c|c|c|c|c|c|c|}
\hline $\begin{array}{l}15.2 \mathrm{~N} \\
45.0 \mathrm{~S} \\
25.3 \mathrm{~N}\end{array}$ & $\begin{array}{c}253.0 \mathrm{~W} \\
51.2 \mathrm{~W} \\
131.5 \mathrm{E}\end{array}$ & $\begin{array}{r}0 \\
15 \\
0\end{array}$ & $\begin{array}{l}\text { EU } \\
\text { US } \\
\text { UR }\end{array}$ & $\begin{array}{l}\text { AL } \\
\text { MS } \\
\text { SO }\end{array}$ & $\begin{array}{l}5 \\
5 \\
5\end{array}$ & $\begin{array}{l}1988 \\
1976 \\
1985\end{array}$ \\
\hline $\begin{array}{l}10.5 \mathrm{~N} \\
33.9 \mathrm{~N}\end{array}$ & $\begin{array}{l}279.4 \mathrm{E} \\
347.2 \mathrm{~W}\end{array}$ & $\begin{array}{l}75 \\
82\end{array}$ & $\begin{array}{l}\mathrm{EU} \\
\mathrm{EU}\end{array}$ & $\begin{array}{l}\text { GE } \\
\text { FR }\end{array}$ & $\begin{array}{l}5 \\
5\end{array}$ & $\begin{array}{l}1994 \\
1973 .\end{array}$ \\
\hline $33.7 \mathrm{~S}$ & $93.8 \mathrm{~W}$ & 22 & $\mathrm{EU}$ & FR & 5 & 1970 \\
\hline $63.2 \mathrm{~S}$ & $71.9 \mathrm{~W}$ & 78 & EU & IT & 5 & 1973 \\
\hline $16.1 \mathrm{~S}$ & $56.6 \mathrm{~W}$ & 31 & EU & IT & 5 & 1935 \\
\hline $63.4 \mathrm{~N}$ & $18.9 \mathrm{~W}$ & 38 & EU & FR & 5 & 1935 \\
\hline $15.1 \mathrm{~S}$ & 203.5E & 50 & EU & FR & 5 & 1994 \\
\hline $30.3 \mathrm{~N}$ & 201.1E & 729 & $\mathrm{EU}$ & RM & 5 & 1985 \\
\hline $\begin{array}{r}34.0 \mathrm{~S} \\
5.1 \mathrm{~N} \\
69.9 \mathrm{~N} \\
0.0 \mathrm{~N} \\
0.0 \mathrm{~N} \\
2.0 \mathrm{~N}\end{array}$ & $\begin{array}{c}28.0 \mathrm{~W} \\
92.5 \mathrm{~W} \\
45.1 \mathrm{E} \\
0.0 \mathrm{E} \\
0.0 \mathrm{E} \\
188.7 \mathrm{E}\end{array}$ & $\begin{array}{r}23 \\
309 \\
2801 \\
0 \\
0 \\
30\end{array}$ & $\begin{array}{l}\text { UR } \\
\text { EU } \\
\text { EU } \\
\text { UN } \\
\text { EU } \\
\text { NA }\end{array}$ & $\begin{array}{l}\text { UK } \\
\text { LA } \\
\text { RM } \\
\text { UN } \\
\text { FR } \\
\text { AM }\end{array}$ & $\begin{array}{l}5 \\
5 \\
5 \\
6 \\
6 \\
5\end{array}$ & $\begin{array}{r}1979 \\
1985 \\
1985 \\
0 \\
0 \\
0 \\
1994\end{array}$ \\
\hline $23.7 \mathrm{~N}$ & $141.5 \mathrm{~W}$ & 33 & NA & $\mathrm{CA}$ & 5 & 1970 \\
\hline $\begin{array}{l}58.5 \mathrm{~S} \\
50.4 \mathrm{~N}\end{array}$ & $\begin{array}{c}163.5 \mathrm{E} \\
39.7 \mathrm{~W}\end{array}$ & $\begin{array}{r}150 \\
23\end{array}$ & $\begin{array}{l}\text { EU } \\
\text { EU }\end{array}$ & $\begin{array}{l}\text { CE } \\
\text { FR }\end{array}$ & $\begin{array}{l}5 \\
5\end{array}$ & $\begin{array}{l}1994 \\
1935\end{array}$ \\
\hline $30.3 \mathrm{~S}$ & $53.0 \mathrm{~W}$ & 51 & EU & FR & 5 & 1935 \\
\hline $4.2 \mathrm{~S}$ & $287.5 \mathrm{~W}$ & 112 & EU & FR & 5 & 1973 \\
\hline $42.3 \mathrm{~N}$ & $145.0 \mathrm{~W}$ & 146 & EU & GB & 5 & 1970 \\
\hline $0.5 \mathrm{~N}$ & $98.2 \mathrm{E}$ & 24 & NA & $\mathrm{AM}$ & 5 & 1973 \\
\hline $6.1 S$ & $17.0 \mathrm{~W}$ & 101 & EU & IT & 5 & 1935 \\
\hline $21.5 S$ & $33.2 \mathrm{E}$ & 112 & EU & IT & 5 & 1935 \\
\hline
\end{tabular}

\section{ft origin}

AA George F.; Irish physicist (1851-1901).

AA Armand H. L.; French physicist (1819-1896)

LF Astronaut-named feature, Apollo 16 site.

AA Kirsten; Norwegian opera singer (1895-1962).

AA Camille; French astronomer (1842-1925).

AA Camille; French astronomer (1842-1925).

AA John; British astronomer (1646-1720).

LF Astronaut-named feature, Apollo 14 site.

AA Town in Alabama, USA.

AA Gustave; French novelist (1821-1880).

AA Honore; French astronomer (1755-1835).

AA Alexander; British doctor, Nobel laureate (1881-1955); Williamina P.; American astronomer (1857-1911).

AA Town in Albania.

AA Town in Mississippi, USA.

AA Kirill P.; Soviet geologist (1915-1982).

FR German water nymph.

AA Jean H.; Greco-French astronomer (1909-1969).

AA Ionnas; French astronomer (1908-1969).

AA Francesco; Italian astronomer (1585-1685).

AA Francesco; Italian astronomer (c. 1585-1656)

AA Bernard Le Bovier De; French astronomer (1657-1757).

AA Marie; French medical writer, charity worker (17th century).

RU Roman goddess of hearth and baking of bread.

AA Town in Ukraine.

FO Classical albedo feature name.

TE Roman goddess of chance.

FO Same as Rupes Cauchey.

FO Same as Rima Cauchy.

AA Dian; American zoologist, conservationist (1932-1985).

AA John S.; Canadian physicist (1890-1964).

CR Celtic fertility goddess.

AA Leon; French physicist (1819-1868).

AA Jean-Baptiste Joseph; French mathematician (1768-1830).

AA Georges; French astronomer (1881-1954).

AA Alfred; British astronomer (1868-1940); Ralph H.; British mathematician; physicist (1889-1944).

AA Philip; American astronomer (1878-1944).

AA Italian geographer (unkn-1459).

AA Fracastoro, Girolamo; Italian doctor, astronomer (1483-1553).

\footnotetext{
P: $\quad$ planetary system (see page $x v i)$.

Sa: satellite (see page $\mathrm{xvi}$ ).

lat: latitude of feature center.

long: longitude of feature center.
}

diam: diameter or long dimension of feature. ct: continent of name origin (see page $284 \mathrm{ff}$.) et: ethnicity of name origin (see page $284 \mathrm{ff}$.) as: name approval status (see page xvii). ad: name approval date (year).

ref: reference source for name (see page $287 \mathrm{ff}$.). ft: feature type (see page 290). 
ALPHABETICAL LIST OF NAMES

\begin{tabular}{|c|c|c|c|c|c|c|c|c|c|c|}
\hline $\mathbf{P}$ & $\mathbf{S a}$ & Name & lat & long & diam & ct & et & as & ad & ref \\
\hline H & $\mathbf{H}$ & Fram Rupes & $56.9 \mathrm{~S}$ & $93.3 \mathrm{~W}$ & 0 & EU & NO & 5 & 1976 & 80 \\
\hline $\begin{array}{l}\text { V } \\
U \\
\text { L }\end{array}$ & $\begin{array}{l}\mathrm{V} \\
\mathrm{mr} \\
\mathrm{L}\end{array}$ & $\begin{array}{l}\text { Francesca } \\
\text { Francisco } \\
\text { Franck }\end{array}$ & $\begin{array}{l}28.0 \mathrm{~S} \\
73.2 \mathrm{~S} \\
22.6 \mathrm{~N}\end{array}$ & $\begin{array}{r}57.7 \mathrm{E} \\
236.0 \mathrm{E} \\
35.5 \mathrm{E}\end{array}$ & $\begin{array}{l}18 \\
14 \\
12\end{array}$ & $\begin{array}{l}\text { EU } \\
\text { EU } \\
\text { EU }\end{array}$ & $\begin{array}{l}\text { IT } \\
\text { GB } \\
\text { GE }\end{array}$ & $\begin{array}{l}5 \\
5 \\
5\end{array}$ & $\begin{array}{l}1994 \\
1988 \\
1973\end{array}$ & $\begin{array}{r}83 \\
85 \\
0\end{array}$ \\
\hline V & V & Frank & $13.1 \mathrm{~S}$ & $12.9 \mathrm{E}$ & 23 & EU & DU & 5 & 1991 & 99 \\
\hline L & L & Franklin & $38.8 \mathrm{~N}$ & 47.7E & 56 & NA & AM & 5 & 1935 & 66 \\
\hline L & $\mathrm{L}$ & Franz & $16.6 \mathrm{~N}$ & $40.2 \mathrm{E}$ & 25 & $\mathrm{EU}$ & GE & 5 & 1935 & 66 \\
\hline $\mathbf{L}$ & L & Fraunhofer & $39.5 \mathrm{~S}$ & $59.1 \mathrm{E}$ & 56 & $\mathrm{EU}$ & GE & 5 & 1935 & 66 \\
\hline $\begin{array}{l}\text { V } \\
\text { L }\end{array}$ & $\begin{array}{l}\text { V } \\
\text { L }\end{array}$ & $\begin{array}{l}\text { Fredegonde } \\
\text { Fredholm }\end{array}$ & $\begin{array}{l}50.5 \mathrm{~S} \\
18.4 \mathrm{~N}\end{array}$ & $\begin{array}{l}93.1 \mathrm{E} \\
46.5 \mathrm{E}\end{array}$ & $\begin{array}{l}26 \\
14\end{array}$ & $\begin{array}{l}\mathrm{EU} \\
\mathrm{EU}\end{array}$ & $\begin{array}{l}\mathrm{GE} \\
\mathrm{SW}\end{array}$ & $\begin{array}{l}5 \\
5\end{array}$ & $\begin{array}{l}1991 \\
1976\end{array}$ & $\begin{array}{r}99 \\
0\end{array}$ \\
\hline $\begin{array}{l}\mathbf{M} \\
\mathbf{J}\end{array}$ & $\begin{array}{l}\mathrm{M} \\
\mathrm{ca}\end{array}$ & $\begin{array}{l}\text { Freedom } \\
\text { Freki }\end{array}$ & $\begin{array}{l}43.6 \mathrm{~N} \\
79.9 \mathrm{~N}\end{array}$ & $\begin{array}{r}9.0 \mathrm{~W} \\
352.0 \mathrm{~W}\end{array}$ & $\begin{array}{l}12 \\
48\end{array}$ & $\begin{array}{l}\text { US } \\
\text { EU }\end{array}$ & $\begin{array}{l}\text { OK } \\
\text { NS }\end{array}$ & $\begin{array}{l}5 \\
5\end{array}$ & $\begin{array}{l}1976 \\
1979\end{array}$ & $\begin{array}{l}36 \\
17\end{array}$ \\
\hline $\begin{array}{l}\mathrm{M} \\
\mathbf{L}\end{array}$ & $\begin{array}{l}\mathbf{M} \\
\mathbf{L}\end{array}$ & $\begin{array}{l}\text { Frento Vallis } \\
\text { Freud }\end{array}$ & $\begin{array}{l}50.5 \mathrm{~S} \\
25.8 \mathrm{~N}\end{array}$ & $\begin{array}{l}14.9 \mathrm{~W} \\
52.3 \mathrm{~W}\end{array}$ & $\begin{array}{r}203 \\
2\end{array}$ & $\begin{array}{l}\mathrm{EU} \\
\mathrm{EU}\end{array}$ & $\underset{\text { AS }}{\text { IT }}$ & $\begin{array}{l}5 \\
5\end{array}$ & $\begin{array}{l}1985 \\
1973\end{array}$ & $\begin{array}{r}56 \\
0\end{array}$ \\
\hline L & L & Freundlich & $25.0 \mathrm{~N}$ & $171.0 \mathrm{E}$ & 85 & EU & GE & 5 & 1970 & 0 \\
\hline $\begin{array}{l}\text { V } \\
\text { V }\end{array}$ & $\begin{array}{l}\mathrm{V} \\
\mathrm{V}\end{array}$ & $\begin{array}{l}\text { Freyja Montes } \\
\text { Friagabi Fossae }\end{array}$ & $\begin{array}{l}74.1 \mathrm{~N} \\
50.2 \mathrm{~N}\end{array}$ & $\begin{array}{l}333.8 \mathrm{E} \\
109.5 \mathrm{E}\end{array}$ & $\begin{array}{l}579 \\
141\end{array}$ & $\begin{array}{l}\mathrm{EU} \\
\mathrm{EU}\end{array}$ & $\begin{array}{l}\text { NS } \\
\text { GB }\end{array}$ & $\begin{array}{l}5 \\
5\end{array}$ & $\begin{array}{l}1979 \\
1985\end{array}$ & $\begin{array}{l}27 \\
27\end{array}$ \\
\hline $\begin{array}{l}\text { V } \\
\text { L }\end{array}$ & $\begin{array}{l}\mathrm{V} \\
\mathrm{L}\end{array}$ & $\begin{array}{l}\text { Frida } \\
\text { Fridman }\end{array}$ & $\begin{array}{l}68.1 \mathrm{~N} \\
12.6 \mathrm{~S}\end{array}$ & $\begin{array}{c}55.5 \mathrm{E} \\
126.0 \mathrm{~W}\end{array}$ & $\begin{array}{r}22 \\
102\end{array}$ & $\begin{array}{l}\text { EU } \\
\text { UR }\end{array}$ & $\begin{array}{l}\text { SW } \\
\text { SO }\end{array}$ & $\begin{array}{l}5 \\
5\end{array}$ & $\begin{array}{l}1985 \\
1970\end{array}$ & $\begin{array}{r}98 \\
0\end{array}$ \\
\hline $\begin{array}{l}\mathrm{V} \\
\mathrm{M}\end{array}$ & $\begin{array}{l}\mathrm{V} \\
\mathrm{M}\end{array}$ & $\begin{array}{l}\text { Frigg Dorsa } \\
\text { [Frigoris Scopulus] }\end{array}$ & $\begin{array}{l}51.2 \mathrm{~N} \\
79.5 \mathrm{~N}\end{array}$ & $\begin{array}{l}150.6 \mathrm{E} \\
184.0 \mathrm{~W}\end{array}$ & $\begin{array}{r}896 \\
0\end{array}$ & $\begin{array}{l}\text { EU } \\
\text { EU }\end{array}$ & $\begin{array}{l}\text { NS } \\
\text { LA }\end{array}$ & $\begin{array}{l}5 \\
6\end{array}$ & $\begin{array}{l}1985 \\
1984\end{array}$ & $\begin{array}{l}65 \\
57\end{array}$ \\
\hline $\begin{array}{l}\mathrm{J} \\
\mathrm{L}\end{array}$ & $\begin{array}{l}\mathrm{ca} \\
\mathrm{L}\end{array}$ & $\begin{array}{l}\text { Frodi } \\
\text { Froelich }\end{array}$ & $\begin{array}{l}68.3 \mathrm{~N} \\
80.3 \mathrm{~N}\end{array}$ & $\begin{array}{l}139.1 \mathrm{~W} \\
109.7 \mathrm{~W}\end{array}$ & $\begin{array}{l}44 \\
58\end{array}$ & $\begin{array}{l}\text { EU } \\
\text { NA }\end{array}$ & $\begin{array}{r}\text { NS } \\
\text { AM }\end{array}$ & $\begin{array}{l}5 \\
5\end{array}$ & $\begin{array}{l}1979 \\
1970\end{array}$ & $\begin{array}{r}17 \\
0\end{array}$ \\
\hline L & $\mathrm{L}$ & Frost & $37.7 \mathrm{~N}$ & $118.4 \mathrm{~W}$ & 75 & NA & $\mathrm{AM}$ & 5 & 1970 & 0 \\
\hline $\mathrm{L}$ & $\mathrm{L}$ & Fryxell & $21.3 \mathrm{~S}$ & $101.4 \mathrm{~W}$ & 18 & NA & $\mathrm{AM}$ & 5 & 1985 & 0 \\
\hline $\begin{array}{l}\text { J } \\
\text { V } \\
\text { J }\end{array}$ & $\begin{array}{l}\text { io } \\
\mathrm{V} \\
\text { ca }\end{array}$ & $\begin{array}{l}\text { Fuchi Patera } \\
\text { Fukiko } \\
\text { Fulla }\end{array}$ & $\begin{array}{l}28.4 \mathrm{~N} \\
23.2 \mathrm{~S} \\
73.5 \mathrm{~N}\end{array}$ & $\begin{array}{l}327.9 \mathrm{~W} \\
105.7 \mathrm{E} \\
103.7 \mathrm{~W}\end{array}$ & $\begin{array}{l}45 \\
15 \\
45\end{array}$ & $\begin{array}{l}\text { AS } \\
\text { AS } \\
\text { EU }\end{array}$ & $\begin{array}{l}\text { JA } \\
\text { JA } \\
\text { NS }\end{array}$ & $\begin{array}{l}5 \\
5 \\
5\end{array}$ & $\begin{array}{l}1979 \\
1994 \\
1979\end{array}$ & $\begin{array}{r}25 \\
0 \\
17\end{array}$ \\
\hline $\begin{array}{l}\mathrm{J} \\
\mathrm{M} \\
\mathrm{V}\end{array}$ & $\begin{array}{l}\mathrm{ca} \\
\mathrm{M} \\
\mathrm{V}\end{array}$ & $\begin{array}{l}\text { Fulnir } \\
\text { Funchal } \\
\text { Furki Mons }\end{array}$ & $\begin{array}{l}60.3 \mathrm{~N} \\
23.2 \mathrm{~N} \\
35.9 \mathrm{~N}\end{array}$ & $\begin{array}{c}35.5 \mathrm{~W} \\
49.5 \mathrm{~W} \\
236.4 \mathrm{E}\end{array}$ & $\begin{array}{r}46 \\
2 \\
79\end{array}$ & $\begin{array}{l}\text { EU } \\
\text { EU } \\
\text { UR }\end{array}$ & $\begin{array}{l}\text { NS } \\
\text { SP } \\
\text { CC }\end{array}$ & $\begin{array}{l}5 \\
5 \\
5\end{array}$ & $\begin{array}{l}1979 \\
1979 \\
1985\end{array}$ & $\begin{array}{l}17 \\
36 \\
65\end{array}$ \\
\hline L & $\mathrm{L}$ & Furnerius & $36.0 \mathrm{~S}$ & $60.6 \mathrm{E}$ & 135 & EU & FR & 5 & 1935 & 66 \\
\hline $\begin{array}{l}\mathrm{H} \\
\mathrm{L}\end{array}$ & $\begin{array}{l}\mathbf{H} \\
\mathbf{L}\end{array}$ & $\begin{array}{l}\text { Futabatei } \\
\text { G. Bond }\end{array}$ & $\begin{array}{l}16.2 \mathrm{~S} \\
32.4 \mathrm{~N}\end{array}$ & $\begin{array}{l}83.0 \mathrm{~W} \\
36.2 \mathrm{E}\end{array}$ & $\begin{array}{l}66 \\
20\end{array}$ & $\begin{array}{l}\text { AS } \\
\text { NA }\end{array}$ & $\begin{array}{r}\text { JA } \\
\text { AM }\end{array}$ & $\begin{array}{l}5 \\
5\end{array}$ & $\begin{array}{l}1976 \\
1935\end{array}$ & $\begin{array}{l}59 \\
66\end{array}$ \\
\hline $\begin{array}{l}\text { M } \\
\text { V }\end{array}$ & $\underset{\mathrm{V}}{\mathrm{M}}$ & $\begin{array}{l}\text { Gaan } \\
\text { Gabie Rupes }\end{array}$ & $\begin{array}{l}38.5 \mathrm{~N} \\
67.5 \mathrm{~N}\end{array}$ & $\begin{array}{c}3.2 \mathrm{~W} \\
109.9 \mathrm{E}\end{array}$ & $\begin{array}{r}3 \\
350\end{array}$ & $\begin{array}{l}\text { AF } \\
\text { UR }\end{array}$ & $\begin{array}{r}\text { SO } \\
\text { LI }\end{array}$ & $\begin{array}{l}5 \\
5\end{array}$ & $\begin{array}{l}1976 \\
1985\end{array}$ & $\begin{array}{l}36 \\
64\end{array}$ \\
\hline $\begin{array}{l}\mathrm{V} \\
\mathrm{J} \\
\mathrm{L} \\
\mathrm{J}\end{array}$ & $\begin{array}{l}\text { V } \\
\text { ga } \\
\text { L } \\
\text { am }\end{array}$ & $\begin{array}{l}\text { Gabriela } \\
\text { Gad } \\
\text { Gadomski } \\
\text { Gaea }\end{array}$ & $\begin{array}{l}17.9 \mathrm{~S} \\
12.4 \mathrm{~S} \\
36.4 \mathrm{~N} \\
80.0 \mathrm{~S}\end{array}$ & $\begin{array}{c}240.4 \mathrm{E} \\
137.5 \mathrm{~W} \\
147.3 \mathrm{~W} \\
90.0 \mathrm{~W}\end{array}$ & $\begin{array}{r}19 \\
68 \\
65 \\
0\end{array}$ & $\begin{array}{l}\text { AS } \\
\text { AS } \\
\text { EU } \\
\text { EU }\end{array}$ & $\begin{array}{l}\text { HE } \\
\text { SE } \\
\text { PO } \\
\text { GR }\end{array}$ & $\begin{array}{l}5 \\
5 \\
5 \\
5\end{array}$ & $\begin{array}{l}1994 \\
1985 \\
1970 \\
1979\end{array}$ & $\begin{array}{r}109 \\
27 \\
0 \\
19\end{array}$ \\
\hline $\mathrm{L}$ & $\mathrm{L}$ & Gagarin & $20.2 S$ & $149.2 \mathrm{E}$ & 265 & UR & so & 5 & 1970 & 0 \\
\hline $\begin{array}{l}M \\
M \\
S\end{array}$ & $\begin{array}{l}\mathrm{M} \\
\mathrm{M} \\
\mathrm{mi}\end{array}$ & $\begin{array}{l}\text { Gagra } \\
\text { Gah } \\
\text { Gaheris }\end{array}$ & $\begin{array}{l}20.9 \mathrm{~S} \\
45.1 \mathrm{~S} \\
40.9 \mathrm{~S}\end{array}$ & $\begin{array}{r}21.9 \mathrm{~W} \\
32.3 \mathrm{~W} \\
298.4 \mathrm{~W}\end{array}$ & $\begin{array}{r}14 \\
2 \\
0\end{array}$ & $\begin{array}{l}\text { UR } \\
\text { AS } \\
\text { EU }\end{array}$ & $\begin{array}{l}\text { GE } \\
\text { ID } \\
\text { GB }\end{array}$ & $\begin{array}{l}5 \\
5 \\
5\end{array}$ & $\begin{array}{l}1976 \\
1976 \\
1982\end{array}$ & $\begin{array}{l}36 \\
36 \\
37\end{array}$ \\
\hline
\end{tabular}

ft origin

80 RU Norwegian; ship used in Arctic by Nansen, $1892-96$, and by Sverdrup and Amundsen in Antarctica, 1909.

AA Italian first name.

AA A lord of Naples in "The Tempest."

AA James; German physicist; Nobel laureate (1882-1964).

AA Anne; Dutch heroine, diarist (1929-1945).

AA Benjamin; American inventor (1706-1790).

AA Julius Heinrich; German astronomer (1847-1913)

AA Joseph von; German astronomer, optician (1787-1826).

AA Frankish queen (d. A.D. 597).

AA Erik Ivar; Swedish mathematician (1866-1927).

AA Town in Oklahoma, USA.

AA Norse; wolf's name meaning "unsatiable."

VA Classical name for river in Italy.

AA Sigmund; Austrian psychoanalyst (1856-1939)

AA Erwin(Finlay-); German-British astronomer (1885-1964).

MO Norse, mother of Odin.

FO Old English goddess, connected with Mars

AA Swedish first name.

AA Aleksandr; Soviet physicist (1888-1925).

DO Norse, wife of supreme god Odin.

SC Classical albedo feature; name dropped 1984.

AA Norse; Hledis' father.

AA Jack E.; American rocket scientist (1921-1967).

AA Edwin B.; American astronomer (1866-1935)

AA R. H.; American geologist (1934-1974).

PE Ainu fire goddess.

AA Japanese first name.

AA Norse; maid to Frigg, queen of the gods.

AA Norse; son of Thrael and Thyr. AA Port of Madeira Islands.

MO Chechen and Ingush (Caucasus) goddess, wife of thunder god Sela.

AA Furner, Georges; French mathematician (unkn-fl. 1643).

AA S.; Japanese novelist (1864-1909).

AA George Philip; American astronomer (1826-1865).

AA Town in Somalia.

RU Lithuanian goddess of fire and hearth.

AA First name from Hebrew.

AA Semitic god of fate or good fortune.

AA Jan; Polish astronomer (1889-1966).

AA Greek mother earth goddess who brought Zeus to Crete.

AA Yurij A.; Soviet cosmonaut (1934-1968).

AA Town in the Republic of Georgia.

AA Town in Indonesia.

AA Older son of King Lot; killed by Sir Launcelot in his rescue of Gwynevere from burning.

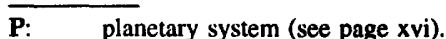

Sa: satellite (see page xvi).

lat: latitude of feature center.

long: longitude of feature center. diam: diameter or long dimension of feature.

ct: continent of name origin (see page $284 \mathrm{ff}$.)

et: ethnicity of name origin (see page $284 \mathrm{ff}$.)

as: name approval status (see page xvii). ad: name approval date (year).

ref: reference source for name (see page $287 \mathrm{ff}$.).

$\mathrm{ft}$ feature type (see page 290) 


\section{ALPHABETICAL LIST OF NAMES}

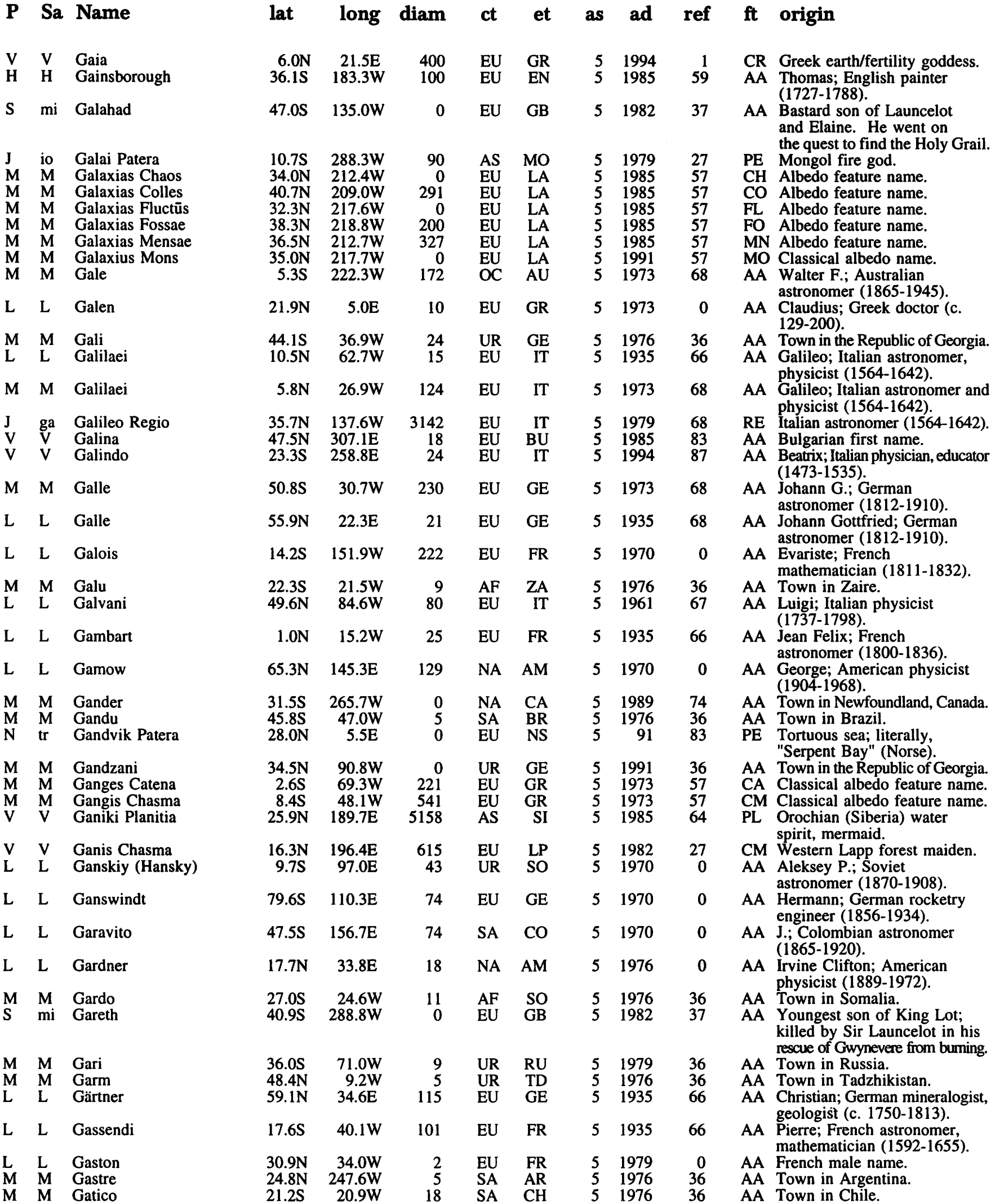

\footnotetext{
P: $\quad$ planetary system (see page xvi).

Sa: $\quad$ satellite (see page xvi).

lat: latitude of feature center.

long: longitude of feature center.
}

diam: diameter or long dimension of feature. ct: continent of name origin (see page $284 \mathrm{ff}$.)

et: ethnicity of name origin (see page $284 \mathrm{ff}$.)

as: name approval status (see page xvii). ad: name approval date (year).

ref: reference source for name (see page $287 \mathrm{ff}$.).

ft: feature type (see page 290). 


\section{ALPHABETICAL LIST OF NAMES}

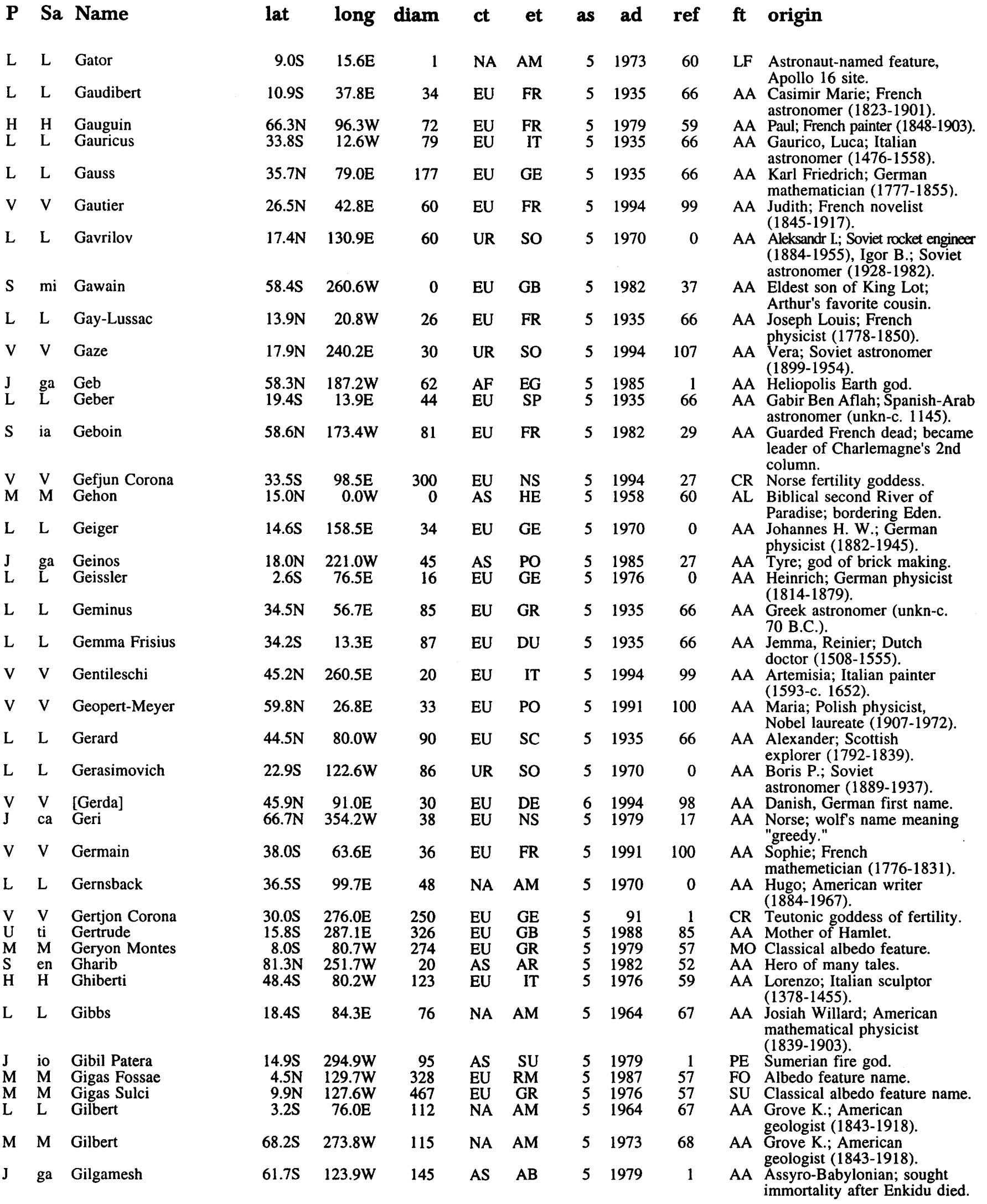

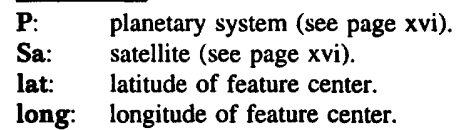

diam: diameter or long dimension of feature. ct: $\quad$ continent of name origin (see page $284 \mathrm{ff}$.) et: ethnicity of name origin (see page $284 \mathrm{ff}$.) as: name approval status (see page $x v i i$ ). ad: name approval date (year). ref: reference source for name (see page $287 \mathrm{ff}$.). ft: feature type (see page 290). 


\begin{tabular}{|c|c|c|c|c|c|c|c|c|c|}
\hline $\mathbf{P}$ & $\mathrm{Sa}$ & Name & lat & long & diam & ct & et & as & ad \\
\hline V & V & Giliani & $72.9 \mathrm{~S}$ & $142.0 \mathrm{E}$ & 27 & EU & IT & 5 & 1994 \\
\hline L & $\mathrm{L}$ & Gill & $63.9 \mathrm{~S}$ & $75.9 \mathrm{E}$ & 66 & $\mathrm{EU}$ & GB & 5 & 1964 \\
\hline $\mathbf{M}$ & $\mathbf{M}$ & Gill & $15.8 \mathrm{~N}$ & $354.5 \mathrm{~W}$ & 81 & $\mathrm{EU}$ & GB & 5 & 1973 \\
\hline $\begin{array}{l}\text { V } \\
\text { V } \\
\text { V } \\
\text { L }\end{array}$ & $\begin{array}{l}V \\
V \\
V \\
L\end{array}$ & $\begin{array}{l}\text { Gillian } \\
\text { Gilmore } \\
\text { Gina } \\
\text { Ginzel }\end{array}$ & $\begin{array}{c}15.2 \mathrm{~S} \\
6.6 \mathrm{~S} \\
78.1 \mathrm{~N} \\
14.3 \mathrm{~N}\end{array}$ & $\begin{array}{r}49.9 \mathrm{E} \\
132.8 \mathrm{E} \\
75.5 \mathrm{E} \\
97.4 \mathrm{E}\end{array}$ & $\begin{array}{l}16 \\
23 \\
14 \\
55\end{array}$ & $\begin{array}{l}\text { EU } \\
\text { OC } \\
\text { EU } \\
\text { EU }\end{array}$ & $\begin{array}{c}\text { LA } \\
\text { AU } \\
\text { IT } \\
\text { AS }\end{array}$ & $\begin{array}{l}5 \\
5 \\
5 \\
5\end{array}$ & $\begin{array}{l}1994 \\
1994 \\
1985 \\
1970\end{array}$ \\
\hline $\mathrm{L}$ & $\mathrm{L}$ & Gioja & $83.3 \mathrm{~N}$ & $2.0 \mathrm{E}$ & 41 & $\mathrm{EU}$ & IT & 5 & 1935 \\
\hline $\begin{array}{l}\mathrm{L} \\
\mathrm{H} \\
\mathrm{J} \\
\mathrm{J} \\
\mathrm{J}\end{array}$ & $\begin{array}{l}\mathrm{L} \\
\mathrm{H} \\
\mathrm{ca} \\
\mathrm{ga} \\
\mathrm{ca}\end{array}$ & $\begin{array}{l}\text { Giordano Bruno } \\
\text { Giotto } \\
\text { Gipul Catena } \\
\text { Gir } \\
\text { Gisl }\end{array}$ & $\begin{array}{l}35.9 \mathrm{~N} \\
12.0 \mathrm{~N} \\
70.2 \mathrm{~N} \\
35.2 \mathrm{~N} \\
57.2 \mathrm{~N}\end{array}$ & $\begin{array}{c}102.8 \mathrm{E} \\
55.8 \mathrm{~W} \\
48.2 \mathrm{~W} \\
146.6 \mathrm{~W} \\
34.8 \mathrm{~W}\end{array}$ & $\begin{array}{r}22 \\
150 \\
588 \\
77 \\
39\end{array}$ & $\begin{array}{l}\mathrm{EU} \\
\mathrm{EU} \\
\mathrm{EU} \\
\mathrm{AS} \\
\mathrm{EU}\end{array}$ & $\begin{array}{l}\text { IT } \\
\text { IT } \\
\text { NS } \\
\text { SU } \\
\text { NS }\end{array}$ & $\begin{array}{l}5 \\
5 \\
5 \\
5 \\
5\end{array}$ & $\begin{array}{l}1961 \\
1976 \\
1979 \\
1985 \\
1979\end{array}$ \\
\hline $\mathbf{H}$ & $\mathrm{H}$ & Gjöa Rupes & $66.7 \mathrm{~S}$ & $159.3 \mathrm{~W}$ & 0 & EU & NO & 5 & 1976 \\
\hline $\mathrm{L}$ & L & Glaisher & $13.2 \mathrm{~N}$ & $49.5 \mathrm{E}$ & 15 & $\mathrm{EU}$ & GB & 5 & 1935 \\
\hline V & V & Glaspell & $58.4 \mathrm{~S}$ & $269.6 \mathrm{E}$ & 26 & NA & $\mathrm{AM}$ & 5 & 1994 \\
\hline $\mathrm{L}$ & $\mathrm{L}$ & Glauber & $11.5 \mathrm{~N}$ & $142.6 \mathrm{E}$ & 15 & $\mathrm{EU}$ & GE & 5 & 1976 \\
\hline $\mathrm{L}$ & $\mathrm{L}$ & Glazenap & $1.6 \mathrm{~S}$ & $137.6 \mathrm{E}$ & 43 & UR & So & 5 & 1970 \\
\hline $\begin{array}{l}\mathbf{M} \\
\mathbf{M}\end{array}$ & $\begin{array}{l}\mathrm{M} \\
\mathbf{M}\end{array}$ & $\begin{array}{l}\text { Glazov } \\
\text { Gledhill }\end{array}$ & $\begin{array}{l}20.8 \mathrm{~S} \\
53.5 \mathrm{~S}\end{array}$ & $\begin{array}{r}26.4 W \\
272.9 W\end{array}$ & $\begin{array}{l}20 \\
72\end{array}$ & $\begin{array}{l}\text { UR } \\
\text { EU }\end{array}$ & $\begin{array}{l}\mathrm{RU} \\
\mathrm{GB}\end{array}$ & $\begin{array}{l}5 \\
5\end{array}$ & $\begin{array}{l}1976 \\
1973\end{array}$ \\
\hline $\begin{array}{l}M \\
M \\
M \\
J \\
V \\
H\end{array}$ & $\begin{array}{l}M \\
M \\
M \\
c \mathfrak{a} \\
\mathbf{V} \\
\mathrm{H}\end{array}$ & $\begin{array}{l}\text { Glendore } \\
\text { Glide } \\
\text { Globe } \\
\text { Gloi } \\
\text { Gloria } \\
\text { Gluck }\end{array}$ & $\begin{array}{l}18.5 \mathrm{~N} \\
8.2 \mathrm{~S} \\
24.0 \mathrm{~S} \\
49.0 \mathrm{~N} \\
68.5 \mathrm{~N} \\
37.3 \mathrm{~N}\end{array}$ & $\begin{array}{c}51.7 \mathrm{~W} \\
43.3 \mathrm{~W} \\
27.1 \mathrm{~W} \\
245.7 \mathrm{~W} \\
94.0 \mathrm{E} \\
18.1 \mathrm{~W}\end{array}$ & $\begin{array}{r}0 \\
10 \\
45 \\
112 \\
20 \\
105\end{array}$ & $\begin{array}{l}\text { EU } \\
\text { US } \\
\text { US } \\
\text { EU } \\
\text { EU } \\
\text { EU }\end{array}$ & $\begin{array}{l}\text { IR } \\
\text { OR } \\
\text { AZ } \\
\text { NS } \\
\text { PG } \\
\text { GE }\end{array}$ & $\begin{array}{l}5 \\
5 \\
5 \\
5 \\
5 \\
5\end{array}$ & $\begin{array}{l}1988 \\
1976 \\
1976 \\
1979 \\
1985 \\
1979\end{array}$ \\
\hline $\mathrm{L}$ & $\mathrm{L}$ & Glushko & $8.4 \mathrm{~N}$ & $77.6 \mathrm{~W}$ & 43 & UR & RU & 5 & 1994 \\
\hline $\begin{array}{l}\mathrm{U} \\
\mathrm{M} \\
\mathrm{L}\end{array}$ & $\begin{array}{l}\mathrm{um} \\
\mathrm{L}\end{array}$ & $\begin{array}{l}\text { Gob } \\
\text { Goba } \\
\text { Goclenius }\end{array}$ & $\begin{array}{l}12.7 \mathrm{~S} \\
23.5 \mathrm{~S} \\
10.0 \mathrm{~S}\end{array}$ & $\begin{array}{l}27.8 \mathrm{E} \\
20.9 \mathrm{~W} \\
45.0 \mathrm{E}\end{array}$ & $\begin{array}{l}88 \\
11 \\
72\end{array}$ & $\begin{array}{l}\mathrm{EU} \\
\mathrm{AF} \\
\mathrm{EU}\end{array}$ & $\begin{array}{l}\text { GB } \\
\text { ET } \\
\text { GE }\end{array}$ & $\begin{array}{l}5 \\
5 \\
5\end{array}$ & $\begin{array}{l}1988 \\
1976 \\
1935\end{array}$ \\
\hline L & $\mathrm{L}$ & Goddard & $14.8 \mathrm{~N}$ & $89.0 \mathrm{E}$ & 89 & NA & $\mathrm{AM}$ & 5 & 1964 \\
\hline$S$ & ia & Godefroy & $71.9 \mathrm{~N}$ & $249.1 \mathrm{~W}$ & 63 & $\mathrm{EU}$ & FR & 5 & 1982 \\
\hline L & L & Godin & $1.8 \mathrm{~N}$ & $10.2 \mathrm{E}$ & 34 & $\mathrm{EU}$ & FR & 5 & 1935 \\
\hline V & $\mathrm{V}$ & Godiva & $56.1 \mathrm{~S}$ & $251.5 \mathrm{E}$ & 32 & EU & EN & 5 & 1994 \\
\hline $\mathrm{H}$ & $\mathbf{H}$ & Goethe & $78.5 \mathrm{~N}$ & $44.5 \mathrm{~W}$ & 383 & $\mathrm{EU}$ & GE & 5 & 1979 \\
\hline $\begin{array}{l}\mathbf{M} \\
\mathbf{H}\end{array}$ & $\begin{array}{l}\mathbf{M} \\
\mathrm{H}\end{array}$ & $\begin{array}{l}\text { Goff } \\
\text { Gogol }\end{array}$ & $\begin{array}{l}23.5 \mathrm{~N} \\
28.1 \mathrm{~S}\end{array}$ & $\begin{array}{l}255.2 \mathrm{~W} \\
146.4 \mathrm{~W}\end{array}$ & $\begin{array}{r}6 \\
87\end{array}$ & $\begin{array}{l}\mathrm{AF} \\
\mathrm{UR}\end{array}$ & $\begin{array}{l}\text { SO } \\
\text { RU }\end{array}$ & $\begin{array}{l}5 \\
5\end{array}$ & $\begin{array}{l}1976 \\
1985\end{array}$ \\
\hline $\begin{array}{l}\mathrm{M} \\
\mathrm{M} \\
\mathrm{M} \\
\mathrm{L}\end{array}$ & $\begin{array}{l}\mathrm{M} \\
\mathrm{M} \\
\mathrm{M} \\
\mathrm{L}\end{array}$ & $\begin{array}{l}\text { Gol } \\
\text { Gold } \\
\text { Golden } \\
\text { Goldschmidt }\end{array}$ & $\begin{array}{l}47.4 \mathrm{~N} \\
20.2 \mathrm{~N} \\
22.2 \mathrm{~S} \\
73.2 \mathrm{~N}\end{array}$ & $\begin{array}{r}10.7 \mathrm{~W} \\
31.3 \mathrm{~W} \\
33.3 \mathrm{~W} \\
3.8 \mathrm{~W}\end{array}$ & $\begin{array}{r}7 \\
9 \\
19 \\
113\end{array}$ & $\begin{array}{l}\text { EU } \\
\text { US } \\
\text { EU }\end{array}$ & $\begin{array}{l}\text { NO } \\
\text { PA } \\
\text { IL } \\
\text { GE }\end{array}$ & $\begin{array}{l}5 \\
5 \\
5 \\
5\end{array}$ & $\begin{array}{l}1976 \\
1976 \\
1976 \\
1935\end{array}$ \\
\hline $\begin{array}{l}\mathbf{M} \\
\mathbf{H}\end{array}$ & $\begin{array}{l}\mathrm{M} \\
\mathrm{H}\end{array}$ & $\begin{array}{l}\text { Goldstone } \\
\text { Goldstone Vallis }\end{array}$ & $\begin{array}{l}48.1 \mathrm{~N} \\
15.8 \mathrm{~S}\end{array}$ & $\begin{array}{r}225.3 \mathrm{~W} \\
31.7 \mathrm{~W}\end{array}$ & $\begin{array}{l}1 \\
0\end{array}$ & $\begin{array}{l}\text { NA } \\
\text { NA }\end{array}$ & $\begin{array}{l}\mathrm{AM} \\
\mathrm{AM}\end{array}$ & $\begin{array}{l}5 \\
5\end{array}$ & $\begin{array}{l}1979 \\
1976\end{array}$ \\
\hline
\end{tabular}

\section{ft origin}

AA Alessandra; Italian anatomist (1307-1326)

AA Sir David; British astronomer (1843-1914).

AA David; British astronomer (1843-1914)

AA First name from Latin.

AA Mary; Australian poet (1865-1962).

AA Italian first name.

AA Friedrich K.; Austrian astronomer (1850-1926)

AA Flavio; Italian inventor (unkn-fl. 1302).

AA Italian astronomer (1548-1600).

AA Italian painter (c. 1271-1337)

CA Norse river.

AA Sumerian god of summer heat.

AA Norse; steed ridden by Aesir.

RU Norwegian; Amundsen's ship through Northwest passage, 1903-06.

AA James; British meteorologist (1809-1903)

AA Susan; American playwright, novelist (c. 1876-1948).

AA Johann Rudolph; German chemist (c. 1603-1670).

AA Sergej P.; Soviet astronomer (1848-1937)

AA Town in Russia.

AA Joseph; British astronomer (1836-1906)

AA Town in Ireland

AA Town in Oregon, USA.

AA Town in Arizona, USA.

AA Norse dwarf.

AA Portuguese first name.

AA Christoph Willibald; German composer (1714-1787).

AA V.P.; Russian space scientist (1908-1989).

AA King of gnomes.

AA Town in Ethiopia.

AA Gockel, Rudolf; German physicist, doctor, mathematician (1572-1621).

AA Robert H.; American rocketry scientist (1882-1945).

AA Standard bearer of Charlemagne; brother of Tierri, Charlemagne's defender against Pinabel.

AA Louis; French astronomer, mathematician (1704-1760)

AA (Godgifu); Mercian noblewoman (c. 1040-1085)

AA Johann Wolfgang von; German poet and dramatist (1749-1832).

AA Town in Somalia.

AA Nikolay; Russian dramatist and novelist (1809-1852).

AA Town in Norway.

AA Town in Pennsylvania, USA.

AA Town in Illinois, USA

AA Hermann; German astronomer (1802-1866)

AA American tracking site.

VA Radio telescope facility in California.

\footnotetext{
P: $\quad$ planetary system (see page xvi)

Sa: satellite (see page $\mathrm{xvi}$ ).

lat: latitude of feature center.

long: longitude of feature center.
}

diam: diameter or long dimension of feature.

ct: continent of name origin (see page $284 \mathrm{ff}$.)

et: ethnicity of name origin (see page $284 \mathrm{ff}$.)

as: name approval status (see page xvii). ad: name approval date (year).

ref: reference source for name (see page $287 \mathrm{ff}$.)

ft: feature type (see page 290). 


\section{ALPHABETICAL LIST OF NAMES}

\begin{tabular}{|c|c|c|c|c|c|c|c|c|c|c|}
\hline $\mathbf{P}$ & $\mathbf{S a}$ & Name & lat & long & diam & ct & et & as & ad & ref \\
\hline $\mathbf{L}$ & $\mathbf{L}$ & Golgi & $27.8 \mathrm{~N}$ & $60.0 \mathrm{~W}$ & 5 & EU & IT & 5 & 1976 & 0 \\
\hline L & $\mathrm{L}$ & Golitsyn & $25.1 S$ & $105.0 \mathrm{~W}$ & 36 & UR & RU & 5 & 1970 & 0 \\
\hline $\begin{array}{l}\mathbf{J} \\
\mathbf{L}\end{array}$ & $\begin{array}{l}\text { ca } \\
\mathrm{L}\end{array}$ & $\begin{array}{l}\text { Göll } \\
\text { Golovin }\end{array}$ & $\begin{array}{l}57.5 \mathrm{~N} \\
39.9 \mathrm{~N}\end{array}$ & $\begin{array}{l}319.5 \mathrm{~W} \\
161.1 \mathrm{E}\end{array}$ & $\begin{array}{l}55 \\
37\end{array}$ & $\begin{array}{l}\text { EU } \\
\text { NA }\end{array}$ & $\begin{array}{l}\text { NS } \\
\text { AM }\end{array}$ & $\begin{array}{l}5 \\
5\end{array}$ & $\begin{array}{l}1979 \\
1970\end{array}$ & $\begin{array}{r}17 \\
0\end{array}$ \\
\hline $\begin{array}{l}V \\
V \\
\mathbf{J} \\
\mathbf{M} \\
\mathbf{U}\end{array}$ & $\begin{array}{l}\mathrm{V} \\
\mathrm{V} \\
\mathrm{ca} \\
\mathrm{M} \\
\mathrm{mr}\end{array}$ & $\begin{array}{l}\text { Golubkina } \\
\text { Goncharova } \\
\text { Göndul } \\
\text { Gonnus Mons } \\
\text { Gonzalo }\end{array}$ & $\begin{array}{l}60.3 \mathrm{~N} \\
63.0 \mathrm{~S} \\
59.9 \mathrm{~N} \\
41.6 \mathrm{~N} \\
11.4 \mathrm{~S}\end{array}$ & $\begin{array}{c}286.5 \mathrm{E} \\
97.7 \mathrm{E} \\
115.5 \mathrm{~W} \\
90.8 \mathrm{~W} \\
77.0 \mathrm{E}\end{array}$ & $\begin{array}{r}31 \\
30 \\
50 \\
0 \\
11\end{array}$ & $\begin{array}{l}\text { UR } \\
\text { UR } \\
\text { EU } \\
\text { EU } \\
\text { EU }\end{array}$ & $\begin{array}{l}\text { SO } \\
\text { RU } \\
\text { NS } \\
\text { GR } \\
\text { GB }\end{array}$ & $\begin{array}{l}5 \\
5 \\
5 \\
5 \\
5\end{array}$ & $\begin{array}{l}1985 \\
1994 \\
1979 \\
1991 \\
1988\end{array}$ & $\begin{array}{l}64 \\
87 \\
17 \\
56 \\
85\end{array}$ \\
\hline L & L & Goodacre & $32.7 \mathrm{~S}$ & $14.1 \mathrm{E}$ & 46 & EU & GB & 5 & 1935 & 66 \\
\hline $\begin{array}{l}\mathbf{M} \\
\mathbf{M}\end{array}$ & $\begin{array}{l}\mathbf{M} \\
\mathbf{M}\end{array}$ & $\begin{array}{l}\text { Gordii Dorsum } \\
\text { Gorgonum Chaos }\end{array}$ & $\begin{array}{r}5.3 \mathrm{~N} \\
39.4 \mathrm{~S}\end{array}$ & $\begin{array}{l}144.5 \mathrm{~W} \\
170.8 \mathrm{~W}\end{array}$ & $\begin{array}{r}624 \\
83\end{array}$ & $\begin{array}{l}\text { EU } \\
\text { EU }\end{array}$ & $\begin{array}{l}\text { GR } \\
\text { GR }\end{array}$ & $\begin{array}{l}5 \\
5\end{array}$ & $\begin{array}{l}1976 \\
1985\end{array}$ & $\begin{array}{l}57 \\
57\end{array}$ \\
\hline $\begin{array}{l}\mathbf{M} \\
\mathbf{J}\end{array}$ & $\begin{array}{l}\mathrm{M} \\
\mathrm{eu}\end{array}$ & $\begin{array}{l}\text { Gori } \\
\text { Gortyna Flexus }\end{array}$ & $\begin{array}{l}23.2 S \\
42.4 S\end{array}$ & $\begin{array}{r}28.6 \mathrm{~W} \\
144.6 \mathrm{~W}\end{array}$ & $\begin{array}{r}6 \\
1261\end{array}$ & $\begin{array}{l}\text { UR } \\
\text { EU }\end{array}$ & $\begin{array}{l}\text { GE } \\
\text { GR }\end{array}$ & $\begin{array}{l}5 \\
5\end{array}$ & $\begin{array}{l}1979 \\
1979\end{array}$ & $\begin{array}{l}36 \\
19\end{array}$ \\
\hline L & $\mathrm{L}$ & Gould & $19.2 \mathrm{~S}$ & $17.2 \mathrm{~W}$ & 34 & NA & AM & 5 & 1935 & 66 \\
\hline $\mathbf{H}$ & $\mathbf{H}$ & Goya & $7.2 \mathrm{~S}$ & $152.0 \mathrm{~W}$ & 135 & EU & SP & 5 & 1976 & 59 \\
\hline $\begin{array}{l}\text { V } \\
\text { L } \\
\text { L }\end{array}$ & $\begin{array}{l}\text { V } \\
\text { L } \\
\text { L }\end{array}$ & $\begin{array}{l}\text { Grace } \\
\text { Grace } \\
\text { Grachev }\end{array}$ & $\begin{array}{c}13.9 \mathrm{~S} \\
14.2 \mathrm{~N} \\
3.7 \mathrm{~S}\end{array}$ & $\begin{array}{c}268.9 \mathrm{E} \\
35.9 \mathrm{E} \\
108.2 \mathrm{~W}\end{array}$ & $\begin{array}{r}19 \\
1 \\
35\end{array}$ & $\begin{array}{l}\text { EU } \\
\text { EU } \\
\text { UR }\end{array}$ & $\begin{array}{l}\text { GR } \\
\text { GB } \\
\text { SO }\end{array}$ & $\begin{array}{l}5 \\
5 \\
5\end{array}$ & $\begin{array}{l}1994 \\
1979 \\
1970\end{array}$ & $\begin{array}{r}82 \\
0 \\
0\end{array}$ \\
\hline M & $\mathbf{M}$ & Graff & $21.4 \mathrm{~S}$ & $206.0 \mathrm{~W}$ & 157 & EU & GE & 5 & 1973 & 68 \\
\hline L & $\mathbf{L}$ & Graff & $42.4 \mathrm{~S}$ & $88.6 \mathrm{~W}$ & 36 & EU & GE & 5 & 1970 & 0 \\
\hline V & V & Graham & $6.0 \mathrm{~S}$ & $6.0 \mathrm{E}$ & 75 & NA & $\mathbf{A M}$ & 5 & 1994 & 99 \\
\hline $\mathbf{S}$ & ia & Grandoyne & $17.7 \mathrm{~N}$ & $214.5 \mathrm{~W}$ & 65 & EU & FR & 5 & 1982 & 29 \\
\hline
\end{tabular}

M M Granicus Valles

L L Grave

$28.7 \mathrm{~N} \quad 227.6 \mathrm{~W}$

17.1S $\quad 150.3 \mathrm{E}$

445

AS TU

$\begin{array}{lrlllll}72.5 \mathrm{~N} & 337.5 \mathrm{E} & 16 & \mathrm{EU} & \mathrm{LI} & 5 & 1994 \\ 13.2 \mathrm{~N} & 52.7 \mathrm{E} & 13 & \mathrm{EU} & \mathrm{GB} & 5 & 1976\end{array}$

$\begin{array}{lll}\text { V } & \text { V } & \text { Grazina } \\ \text { L } & \text { L } & \text { Greaves }\end{array}$

L L Green

M M Green

V V Greenaway

L L Gregory

V V Gregory

V V Gretchen

V V Grey

H H Grieg

L L Grigg

L L Grimaldi

V V Grimke

L L [Grimm]

J ca Grimr

$\begin{array}{lllllll}4.1 \mathrm{~N} & 132.9 \mathrm{E} & 65 & \mathrm{EU} & \mathrm{GB} & 5 & 1970\end{array}$

$52.4 \mathrm{~S}$

8.3W

$22.9 \mathrm{~N} \quad 145.0 \mathrm{E}$

$2.2 \mathrm{~N} \quad 127.2 \mathrm{E}$

7.2N $\quad 95.8 \mathrm{E}$

$59.6 \mathrm{~S} \quad 213.2 \mathrm{E}$

$52.4 \mathrm{~S} \quad 329.2 \mathrm{E}$

$51.1 \mathrm{~N} \quad 14.0 \mathrm{~W}$

$12.9 \mathrm{~N} \quad 129.4 \mathrm{~W}$

5.5S $68.3 \mathrm{~W}$

17.3N 215.3E

15.0S $130.2 \mathrm{E}$

$41.6 \mathrm{~N} \quad 215.2 \mathrm{~W}$

184

EU GB

51973

51991

51970

67 EU SC

$5 \quad 1994$

21 EU IR

20

50
65

EU $\quad$ GE

51994

$\begin{array}{ll}5 & 1994 \\ 5 & 1985\end{array}$

36. OC NE 51970

172

EU IT

$5 \quad 1935$

37

NA AM

51994

15

EU GE

60

51979
AA Camillo; Italian doctor; Nobel laureate (c. 1843-1926).

AA Boris B.; Russian physicist (1862-1916).

AA Norse; servant to the gods.

AA Nicholas E.; American rocketry scientist (1912-1969)

AA Anna; Soviet sculptor (1864-1927).

AA Natalya; Russian artist (1881-1962).

AA Norse; a Valkyrie.

MO Classical town.

AA Honest old counsellor of Naples in "The Tempest."

AA Walter; British selenographer (1856-1938).

DO Classical albedo feature name.

$\mathrm{CH}$ From albedo feature at 24S, 154W.

AA town in the Republic of Georgia.

FE Place on Crete where Zeus brought Europa.

AA Benjamin Apthorp; American astronomer (1824-1896).

AA Francisco (Jose) de Goy (y Lucientes); Spanish painter (1746-1828).

AA First name from Greek.

AA English female name.

AA Andrej D.; Soviet rocketry scientist (1900-1964).

AA Kasimir; German astronomer (1878-1950).

AA Kasimir R.; Polish-German astronomer (1878-1950).

AA Martha; American dancer, choreographer (1894-1991).

AA Son of Cappadocian King Capuel; killed Gerin, Gerier, Berenger, Guy St. Antoine, Duke Astorge; killed by Roland.

VA Ancient name for river in Turkey.

AA Dmitriy A.; Soviet engineer (1863-1939); Ivan P.; Soviet engineer (1874-1960).

AA Lithuanian first name.

AA William Michael Herbert; British astronomer (1897-1955)

AA George; British mathematician (1793-1841).

AA Nathan E.; British astronomer (1823-1899).

AA Kate; English author, illustrator (1846-1901).

AA James; Scottish astronomer, mathematician (1638-1675)

AA Isabella; Irish playwright (1852-1932).

AA German first name.

AA Jane; English queen (1537-1555).

AA Edvard; Norwegian composer (1843-1907).

AA J.; New Zealander astronomer (1838-1920).

AA Francesco Maria; Italian astronomer, physicist (1618-1663).

AA Sarah; American abolishionist (1792-1873).

AA Wilhelm Karl; German story-teller (1786-1859).

AA Norse; a name for Odin.

\footnotetext{
P: $\quad$ planetary system (see page $x v i)$.

Sa: $\quad$ satellite (see page $x v i$ ).

lat: latitude of feature center.

long: longitude of feature center.
}

diam: diameter or long dimension of feature.

ct: continent of name origin (see page $284 \mathrm{ff}$.)

et: ethnicity of name origin (see page $284 \mathrm{ff}$.)

as: name approval status (see page xvii). ad: name approval date (year).

ref: reference source for name (see page $287 \mathrm{ff}$.). ft: feature type (see page 290). 


\section{ALPHABETICAL LIST OF NAMES}

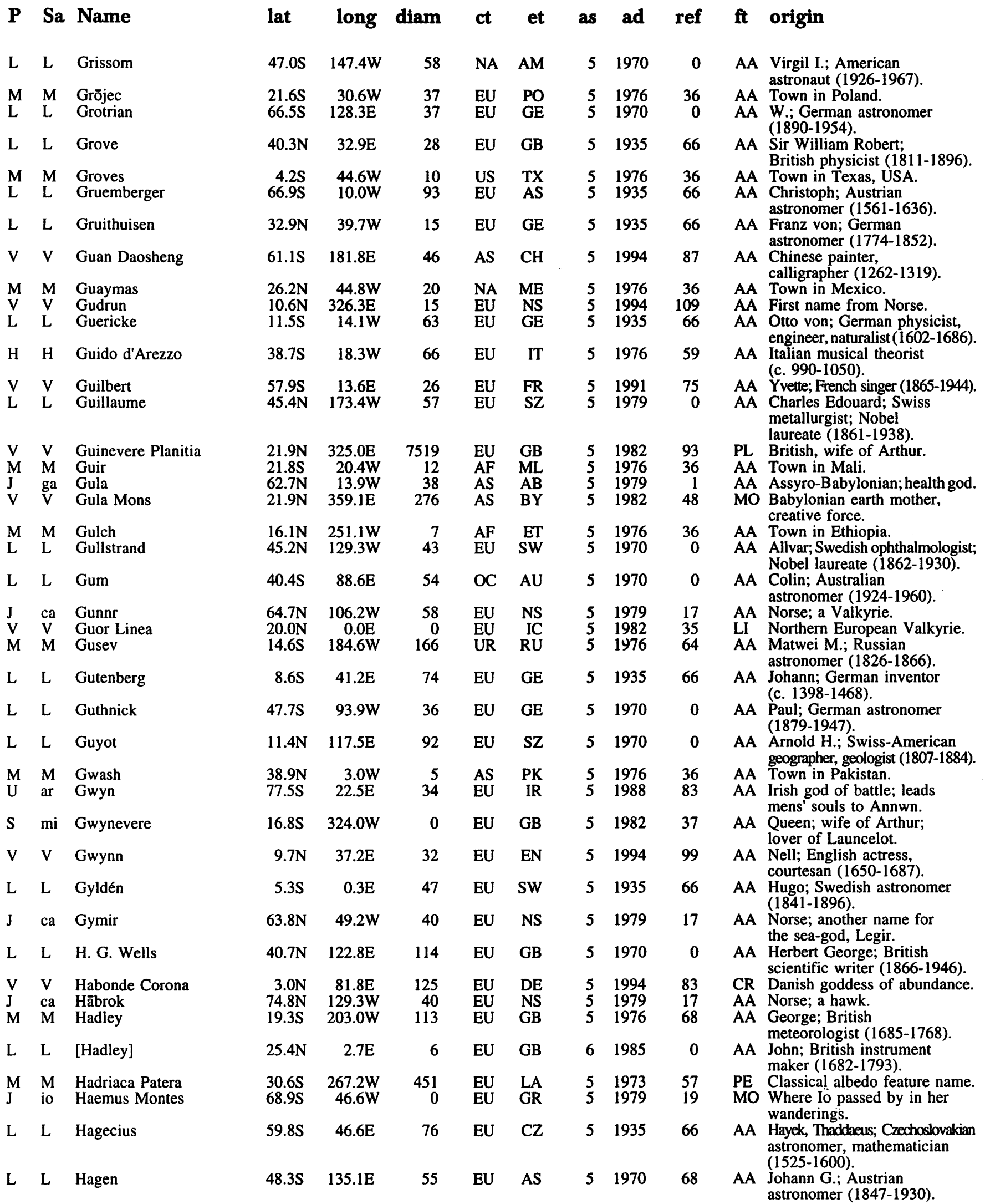

P: planetary system (see page xvi)

Sa: satellite (see page $x v i$ ).

lat: latitude of feature center.

long: longitude of feature center. diam: diameter or long dimension of feature.

ct: continent of name origin (see page $284 \mathrm{ff}$.)

et: ethnicity of name origin (see page $284 \mathrm{ff}$.)

as: name approval status (see page $x v i i$ ). ad: name approval date (year)

ref: reference source for name (see page $287 \mathrm{ff}$.).

ft: feature type (see page 290). 


\section{ALPHABETICAL LIST OF NAMES}

\section{P Sa Name}

L L Hahn

L L Haidinger

$S$ rh Haik

L L Hainzel

J ca Haki

M M Haldane

L L Haldane

L L Hale

M M Hale

M M Halex Fossae

L L Halfway

J ga Halieus

L L Hall

M ph Hall

V V Halle

M M Halley

L L Halley

V V Hallgerda Mons

L L Halo

H H Hals

$S$ di Halys

M M Ham

M M Hamaguir

M M Hamelin

L L Hamilton

U ob Hamlet

$S$ ia Hamon

H H Han Kan

$\mathrm{H} H$ Handel

M M Handlová

V V Hannah

L L Hanno

V V Hansberry

L L Hansen

L L Hansteen

$S$ rh Haoso

J ga Hapi

J ca Hār

M M Harad

L L Harden lat long diam

$\begin{array}{rrrrrrrr}31.3 \mathrm{~N} & 73.6 \mathrm{E} & 84 & \text { EU } & \text { GE } & 5 & 1935 & 66 \\ 39.2 \mathrm{~S} & 25.0 \mathrm{~W} & 22 & \text { EU } & \text { AS } & 5 & 1935 & 66 \\ 36.6 \mathrm{~S} & 29.3 \mathrm{~W} & 0 & \text { UR } & \text { AR } & 5 & 1982 & 50 \\ 41.3 \mathrm{~S} & 33.5 \mathrm{~W} & 70 & \text { EU } & \text { GE } & 5 & 1935 & 66 \\ 24.9 \mathrm{~N} & 315.1 \mathrm{~W} & 69 & \text { EU } & \text { NS } & 5 & 1979 & 17 \\ 53.0 \mathrm{~S} & 230.5 \mathrm{~W} & 72 & \text { EU } & \text { GB } & 5 & 1973 & 68 \\ 1.7 \mathrm{~S} & 84.1 \mathrm{E} & 37 & \text { EU } & \text { GB } & 5 & 1973 & 0 \\ 74.2 \mathrm{~S} & 90.8 \mathrm{E} & 83 & \text { NA } & \text { AM } & 5 & 1964 & 67\end{array}$

$\begin{array}{rrrrrrrr}36.1 \mathrm{~S} & 36.3 \mathrm{~W} & 136 & \text { NA } & \text { AM } & 5 & 1973 & 68 \\ 27.3 \mathrm{~N} & 126.1 \mathrm{~W} & 224 & \text { EU } & \text { GR } & 5 & 1985 & 57 \\ 9.0 \mathrm{~S} & 15.5 \mathrm{E} & 0 & \text { NA } & \text { AM } & 5 & 1973 & 59 \\ 35.2 \mathrm{~N} & 168.0 \mathrm{~W} & 90 & \text { AS } & \text { PO } & 5 & 1985 & 27 \\ 33.7 \mathrm{~N} & 37.0 \mathrm{E} & 35 & \text { NA } & \text { AM } & 5 & 1935 & 66 \\ 75.0 \mathrm{~S} & 225.0 \mathrm{~W} & 0 & \text { NA } & \text { AM } & 5 & 1973 & 60 \\ 19.8 \mathrm{~S} & 145.5 \mathrm{E} & 22 & \text { EU } & \text { AS } & 5 & 1991 & 99 \\ 48.6 \mathrm{~S} & 59.2 \mathrm{~W} & 81 & \text { EU } & \text { GB } & 5 & 1973 & 68 \\ 8.0 \mathrm{~S} & 5.7 \mathrm{E} & 36 & \text { EU } & \text { GB } & 5 & 1935 & 66 \\ 53.1 \mathrm{~N} & 198.3 \mathrm{E} & 57 & \text { EU } & \text { IC } & 5 & 1991 & 64 \\ 3.2 \mathrm{~S} & 23.4 \mathrm{~W} & 0 & \text { NA } & \text { AM } & 5 & 1973 & 60 \\ 54.8 \mathrm{~S} & 115.0 \mathrm{~W} & 100 & \text { EU } & \text { DU } & 5 & 1985 & 80 \\ 59.1 \mathrm{~S} & 53.6 \mathrm{~W} & 29 & \text { EU } & \text { RM } & 5 & 1982 & 44\end{array}$

\begin{tabular}{|c|c|c|c|c|c|c|}
\hline $\begin{array}{l}45.0 \mathrm{~S} \\
49.0 \mathrm{~N} \\
20.4 \mathrm{~N}\end{array}$ & $\begin{array}{r}32.2 \mathrm{~W} \\
227.4 \mathrm{~W} \\
32.8 \mathrm{~W}\end{array}$ & $\begin{array}{r}1 \\
3 \\
10\end{array}$ & $\begin{array}{l}\mathrm{EU} \\
\mathrm{AF} \\
\mathrm{EU}\end{array}$ & $\begin{array}{l}\text { FR } \\
\text { AL } \\
\text { GE }\end{array}$ & $\begin{array}{l}5 \\
5 \\
5\end{array}$ & $\begin{array}{l}1971 \\
1979 \\
1976\end{array}$ \\
\hline $42.8 \mathrm{~S}$ & 84.7E & 57 & EU & IR & 5 & 1964 \\
\hline $\begin{array}{l}46.1 \mathrm{~S} \\
10.6 \mathrm{~N}\end{array}$ & $\begin{array}{c}44.4 \mathrm{E} \\
270.0 \mathrm{~W}\end{array}$ & $\begin{array}{r}206 \\
96\end{array}$ & $\begin{array}{l}\text { EU } \\
\text { EU }\end{array}$ & $\begin{array}{l}\text { GB } \\
\text { FR }\end{array}$ & $\begin{array}{l}5 \\
5\end{array}$ & $\begin{array}{l}1988 \\
1982\end{array}$ \\
\hline $\begin{array}{r}71.6 \mathrm{~S} \\
3.4 \mathrm{~N}\end{array}$ & $\begin{array}{r}143.8 \mathrm{~W} \\
33.8 \mathrm{~W}\end{array}$ & $\begin{array}{r}50 \\
166\end{array}$ & $\begin{array}{l}\text { AS } \\
\text { EU }\end{array}$ & $\begin{array}{l}\mathrm{CH} \\
\mathrm{GE}\end{array}$ & $\begin{array}{l}5 \\
5\end{array}$ & $\begin{array}{l}1985 \\
1976\end{array}$ \\
\hline $\begin{array}{l}37.9 \mathrm{~N} \\
17.9 \mathrm{~N} \\
56.3 \mathrm{~S} \\
22.7 \mathrm{~S}\end{array}$ & $\begin{array}{c}88.4 \mathrm{~W} \\
102.6 \mathrm{E} \\
71.2 \mathrm{E} \\
324.1 \mathrm{E}\end{array}$ & $\begin{array}{r}0 \\
19 \\
56 \\
28\end{array}$ & $\begin{array}{l}\text { EU } \\
\text { AS } \\
\text { EU } \\
\text { NA }\end{array}$ & $\begin{array}{l}\text { CZ } \\
\text { HE } \\
\text { RM } \\
\text { MA }\end{array}$ & $\begin{array}{l}5 \\
5 \\
5 \\
5\end{array}$ & $\begin{array}{l}1991 \\
1994 \\
1935 \\
1994\end{array}$ \\
\hline $14.0 \mathrm{~N}$ & $72.5 \mathrm{E}$ & 39 & $\mathrm{EU}$ & $\mathrm{DE}$ & 5 & 1935 \\
\hline $11.5 \mathrm{~S}$ & $52.0 \mathrm{~W}$ & 44 & EU & NO & 5 & 1935 \\
\hline $\begin{array}{r}8.3 \mathrm{~N} \\
31.3 \mathrm{~S} \\
3.6 \mathrm{~S} \\
27.8 \mathrm{~S} \\
5.5 \mathrm{~N}\end{array}$ & $\begin{array}{c}12.5 \mathrm{~W} \\
212.4 \mathrm{~W} \\
357.9 \mathrm{~W} \\
27.8 \mathrm{~W} \\
143.5 \mathrm{E}\end{array}$ & $\begin{array}{r}0 \\
85 \\
44 \\
8 \\
15\end{array}$ & $\begin{array}{l}\text { AS } \\
\text { AF } \\
\text { EU } \\
\text { AS } \\
\text { EU }\end{array}$ & $\begin{array}{l}\text { CH } \\
\text { EG } \\
\text { NS } \\
\text { AR } \\
\text { GB }\end{array}$ & $\begin{array}{l}5 \\
5 \\
5 \\
5 \\
5\end{array}$ & $\begin{array}{l}1982 \\
1988 \\
1979 \\
1976 \\
1976\end{array}$ \\
\hline
\end{tabular}

AA Friedrich von; German astronomer (1741-1805); Otto; German chemist (1879-1968).

AA Wilhelm Karl von; Austrian geologist, physicist (1795-1871).

AA Mythological ancestor of the Armenian people.

AA Paul; German astronomer (unkn-fl. 1570)

AA Norse giant.

AA John B.; British physiologist-geneticist(1892-1964).

AA John Burdon Sanderson; British doctor (1892-1964).

AA George Ellery; American astronomer (1868-1938); William; British rocket scientist (1797-1870).

AA George E.; American astronomer (1868-1938)

FO From albedo feature at $40 \mathrm{~N}, 110 \mathrm{~W}$.

LF Astronaut-named feature, Apollo 16 site.

AA Tyre; fisherman god.

AA Asaph; American astronomer (1829-1907).

AA Asaph; American astronomer 1829-1907

AA Wilhelmina; Austrian violinist (1839-1911).

AA Edmund; British astronomer (1656-1742)

AA Edmond; British astronomer (1656-1742)

MO Icelandic goddess of vanity.

LF Astronaut-named feature, Apollo 12 site.

AA Frans; Dutch painter (1581/1585-1666).

AA A Trojan defending Aeneas camp against the Rutulian attack. He was killed by Turnus.

AA Town in France.

AA Algerian launch site.

AA Old German town referred to in the Pied Piper fairy tale.

AA Sir William R.; Irish mathematician (1805-1865)

AA Shakespearean hero.

AA Joint Commander of Charlemagne's Eighth Division.

AA Chinese painter (720-780).

AA G. F.; German-British composer (1685-1759)

AA Town in Czechoslovakia.

AA First name from Hebrew.

AA Roman explorer (unkn-c. 500 B.C.).

AA Lorraine; American playwright (1930-1965).

AA Peter Andreas; Danish astronomer (1795-1874).

AA Christopher; Norwegian astronomer (1784-1873).

AA Manchurian creator of all things.

AA Egyptian god of the Nile.

AA Norse; a name for Odin.

AA Town in Saudi Arabia.

AA Sir Arthur; British chemist; Nobel laureate (1865-1940).

\footnotetext{
P: $\quad$ planetary system (see page xvi).

Sa: $\quad$ satellite (see page $x v i$ ).

lat: latitude of feature center.

long: longitude of feature center.
}

diam: diameter or long dimension of feature.

ct: continent of name origin (see page $284 \mathrm{ff}$.)

et: ethnicity of name origin (see page $284 \mathrm{ff}$.)

as: name approval status (see page $\mathrm{xvii}$ ). ad: name approval date (year).

ref: reference source for name (see page $287 \mathrm{ff}$.).

$\mathrm{ft:}$ feature type (see page 290). 


\begin{tabular}{|c|c|c|c|c|c|c|c|c|c|c|}
\hline $\mathbf{P}$ & $\mathbf{S a}$ & Name & lat & long & diam & ct & et & as & ad & ref \\
\hline $\mathrm{L}$ & $\mathrm{L}$ & Harding & $43.5 \mathrm{~N}$ & $71.7 \mathrm{~W}$ & 22 & $\mathrm{EU}$ & GE & 5 & 1935 & 66 \\
\hline $\mathbf{L}$ & $\mathrm{L}$ & Haret & $58.4 \mathrm{~S}$ & $175.6 \mathrm{~W}$ & 29 & $\mathrm{EU}$ & RO & 5 & 1970 & 0 \\
\hline L & $\mathbf{L}$ & Hargreaves & $2.2 \mathrm{~S}$ & $64.0 \mathrm{E}$ & 16 & EU & GB & 5 & 1979 & 0 \\
\hline $\begin{array}{l}\text { V } \\
\text { L } \\
\text { M } \\
\text { L } \\
\text { J }\end{array}$ & $\begin{array}{l}V \\
L \\
M \\
L \\
\text { ga }\end{array}$ & $\begin{array}{l}\text { Hariasa Linea } \\
\text { Harkhebi } \\
\text { Harmakhis Vallis } \\
\text { Harold } \\
\text { Harpagia Sulcus }\end{array}$ & $\begin{array}{l}19.0 \mathrm{~N} \\
39.6 \mathrm{~N} \\
39.1 \mathrm{~S} \\
10.9 \mathrm{~S} \\
14.9 \mathrm{~S}\end{array}$ & $\begin{array}{r}15.0 \mathrm{E} \\
98.3 \mathrm{E} \\
267.2 \mathrm{~W} \\
6.0 \mathrm{~W} \\
319.1 \mathrm{~W}\end{array}$ & $\begin{array}{r}0 \\
237 \\
585 \\
2 \\
1398\end{array}$ & $\begin{array}{l}\mathrm{EU} \\
\mathrm{AF} \\
\mathrm{AF} \\
\mathrm{EU} \\
\mathrm{EU}\end{array}$ & $\begin{array}{l}\text { GE } \\
\text { EG } \\
\text { EG } \\
\text { SD } \\
\text { GR }\end{array}$ & $\begin{array}{l}5 \\
5 \\
5 \\
5 \\
5\end{array}$ & $\begin{array}{l}1982 \\
1979 \\
1979 \\
1976 \\
1985\end{array}$ & $\begin{array}{r}27 \\
0 \\
62 \\
0 \\
1\end{array}$ \\
\hline L & $\mathbf{L}$ & Harpalus & $52.6 \mathrm{~N}$ & $43.4 \mathrm{~W}$ & 39 & $\mathrm{EU}$ & GR & 5 & 1935 & 66 \\
\hline$S$ & en & Harran Sulci & $26.7 \mathrm{~N}$ & $237.6 \mathrm{~W}$ & 276 & AS & AR & 5 & 1982 & 52 \\
\hline L & $\mathbf{L}$ & Harriot & $33.1 \mathrm{~N}$ & $114.3 \mathrm{E}$ & 56 & EU & GB & 5 & 1970 & 0 \\
\hline L & $\mathrm{L}$ & Hartmann & $3.2 \mathrm{~N}$ & $135.3 \mathrm{E}$ & 61 & EU & GE & 5 & 1970 & 0 \\
\hline $\mathbf{M}$ & M & Hartwig & $38.7 \mathrm{~S}$ & $15.6 \mathrm{~W}$ & 104 & EU & GE & 5 & 1973 & 68 \\
\hline $\mathbf{L}$ & $\mathrm{L}$ & Hartwig & $6.1 S$ & $80.5 \mathrm{~W}$ & 79 & EU & GE & 5 & 1964 & 67 \\
\hline $\begin{array}{l}\mathrm{H} \\
\mathrm{L}\end{array}$ & $\begin{array}{l}\mathrm{H} \\
\mathrm{L}\end{array}$ & $\begin{array}{l}\text { Harunobu } \\
\text { Harvey }\end{array}$ & $\begin{array}{l}15.0 \mathrm{~N} \\
19.5 \mathrm{~N}\end{array}$ & $\begin{array}{l}140.7 W \\
146.5 W\end{array}$ & $\begin{array}{r}110 \\
60\end{array}$ & $\begin{array}{l}\text { AS } \\
\text { EU }\end{array}$ & $\begin{array}{l}\text { JA } \\
\text { GB }\end{array}$ & $\begin{array}{l}5 \\
5\end{array}$ & $\begin{array}{l}1976 \\
1970\end{array}$ & $\begin{array}{r}59 \\
0\end{array}$ \\
\hline L & $\mathrm{L}$ & Hase & $29.4 S$ & $62.5 \mathrm{E}$ & 83 & EU & GE & 5 & 1935 & 66 \\
\hline L & L & Hatanaka & $29.7 \mathrm{~N}$ & $121.5 \mathrm{~W}$ & 26 & AS & JA & 5 & 1970 & 0 \\
\hline J & io & Hatchawa Patera & $58.2 \mathrm{~S}$ & $35.1 \mathrm{~W}$ & 75 & EU & SL & 5 & 1985 & 30 \\
\hline $\begin{array}{l}J \\
V \\
V \\
V \\
H\end{array}$ & $\begin{array}{l}\text { ga } \\
\mathrm{V} \\
\mathrm{V} \\
\mathrm{V} \\
\mathrm{H}\end{array}$ & $\begin{array}{l}\text { Hathor } \\
\text { Hathor Mons } \\
\text { Hatshepsut Patera } \\
\text { Haumea Corona } \\
\text { Hauptmann }\end{array}$ & $\begin{array}{l}70.4 \mathrm{~S} \\
38.7 \mathrm{~S} \\
28.1 \mathrm{~N} \\
54.0 \mathrm{~N} \\
23.7 \mathrm{~S}\end{array}$ & $\begin{array}{c}268.1 \mathrm{~W} \\
324.7 \mathrm{E} \\
64.5 \mathrm{E} \\
21.8 \mathrm{E} \\
179.9 \mathrm{~W}\end{array}$ & $\begin{array}{r}59 \\
333 \\
118 \\
375 \\
120\end{array}$ & $\begin{array}{l}\text { AF } \\
\text { AF } \\
\text { AF } \\
\text { OC } \\
\text { EU }\end{array}$ & $\begin{array}{l}\text { EG } \\
\text { EG } \\
\text { EG } \\
\text { PO } \\
\text { GE }\end{array}$ & $\begin{array}{l}5 \\
5 \\
5 \\
5 \\
5\end{array}$ & $\begin{array}{l}1979 \\
1979 \\
1985 \\
1994 \\
1985\end{array}$ & $\begin{array}{r}1 \\
48 \\
99 \\
98 \\
59\end{array}$ \\
\hline $\mathbf{L}$ & $\mathrm{L}$ & Hausen & $65.0 \mathrm{~S}$ & $88.1 \mathrm{~W}$ & 167 & EU & GE & 5 & 1961 & 67 \\
\hline H & $\mathrm{H}$ & Hawthorne & $51.3 \mathrm{~S}$ & $115.1 \mathrm{~W}$ & 107 & NA & $\mathrm{AM}$ & 5 & 1979 & 59 \\
\hline V & V & Hayasi & $53.8 \mathrm{~N}$ & $243.6 \mathrm{E}$ & 47 & AS & JA & 5 & 1985 & 46 \\
\hline H & $\mathrm{H}$ & Haydn & $27.3 S$ & $71.6 \mathrm{~W}$ & 270 & EU & AS & 5 & 1976 & 59 \\
\hline L & $\mathrm{L}$ & Hayford & $12.7 \mathrm{~N}$ & $176.4 \mathrm{~W}$ & 27 & NA & $\mathrm{AM}$ & 5 & 1970 & 0 \\
\hline $\mathbf{L}$ & $\mathrm{L}$ & Hayn & $64.7 \mathrm{~N}$ & $85.2 \mathrm{E}$ & 87 & $\mathrm{EU}$ & GE & 5 & 1964 & 67 \\
\hline H & $\mathrm{H}$ & Haystack Vallis & $4.7 \mathrm{~N}$ & $46.2 \mathrm{~W}$ & 0 & NA & $\mathrm{AM}$ & 5 & 1976 & 80 \\
\hline L & L & Head & $3.0 \mathrm{~S}$ & $23.4 \mathrm{~W}$ & 0 & NA & $\mathrm{AM}$ & 5 & 1973 & 59 \\
\hline L & $\mathbf{L}$ & Healy & $32.8 \mathrm{~N}$ & $110.5 \mathrm{~W}$ & 38 & NA & $\mathrm{AM}$ & 5 & 1970 & 0 \\
\hline $\begin{array}{l}\mathrm{V} \\
\mathrm{M}\end{array}$ & $\begin{array}{l}\mathrm{V} \\
\mathrm{M}\end{array}$ & $\begin{array}{l}\text { Heather } \\
\text { Heaviside }\end{array}$ & $\begin{array}{r}6.7 \mathrm{~S} \\
70.8 \mathrm{~S}\end{array}$ & $\begin{array}{c}334.1 \mathrm{E} \\
94.8 \mathrm{~W}\end{array}$ & $\begin{array}{r}12 \\
103\end{array}$ & $\begin{array}{l}\mathrm{EU} \\
\mathrm{EU}\end{array}$ & $\begin{array}{l}\text { EN } \\
\text { GB }\end{array}$ & $\begin{array}{l}5 \\
5\end{array}$ & $\begin{array}{l}1994 \\
1973\end{array}$ & $\begin{array}{r}109 \\
68\end{array}$ \\
\hline $\mathrm{L}$ & $\mathbf{L}$ & Heaviside & $10.4 S$ & $167.1 \mathrm{E}$ & 165 & EU & GB & 5 & 1970 & 0 \\
\hline $\begin{array}{l}\mathbf{M} \\
\mathbf{M}\end{array}$ & $\begin{array}{l}\mathbf{M} \\
\mathbf{M}\end{array}$ & $\begin{array}{l}\text { Hebes Chasma } \\
\text { Hebes Mensa }\end{array}$ & $\begin{array}{l}1.1 \mathrm{~S} \\
1.0 \mathrm{~S}\end{array}$ & $\begin{array}{l}76.1 \mathrm{~W} \\
32.0 \mathrm{~W}\end{array}$ & $\begin{array}{r}285 \\
0\end{array}$ & $\begin{array}{l}\mathrm{EU} \\
\mathrm{EU}\end{array}$ & $\begin{array}{l}\text { GR } \\
\text { GR }\end{array}$ & $\begin{array}{l}5 \\
5\end{array}$ & $\begin{array}{l}1973 \\
1982\end{array}$ & $\begin{array}{l}57 \\
57\end{array}$ \\
\hline $\begin{array}{l}\mathrm{M} \\
\mathrm{L}\end{array}$ & $\begin{array}{l}\mathrm{M} \\
\mathrm{L}\end{array}$ & $\begin{array}{l}\text { Hebrus Valles } \\
\text { Hecataeus }\end{array}$ & $\begin{array}{l}20.0 \mathrm{~N} \\
21.8 \mathrm{~S}\end{array}$ & $\begin{array}{c}233.9 \mathrm{~W} \\
79.4 \mathrm{E}\end{array}$ & $\begin{array}{l}299 \\
167\end{array}$ & $\begin{array}{l}\text { EU } \\
\text { EU }\end{array}$ & $\begin{array}{l}\text { GR } \\
\text { GR }\end{array}$ & $\begin{array}{l}5 \\
5\end{array}$ & $\begin{array}{l}1982 \\
1935\end{array}$ & $\begin{array}{l}56 \\
66\end{array}$ \\
\hline $\begin{array}{l}\text { V } \\
M\end{array}$ & $\begin{array}{l}\text { V } \\
M\end{array}$ & $\begin{array}{l}\text { Hecate Chasma } \\
\text { Hecates Tholus }\end{array}$ & $\begin{array}{l}18.2 \mathrm{~N} \\
32.7 \mathrm{~N}\end{array}$ & $\begin{array}{l}254.3 \mathrm{E} \\
209.8 \mathrm{~W}\end{array}$ & $\begin{array}{r}3145 \\
183\end{array}$ & $\begin{array}{l}\text { EU } \\
\text { EU }\end{array}$ & $\begin{array}{l}\text { GR } \\
\text { GR }\end{array}$ & $\begin{array}{l}5 \\
5\end{array}$ & $\begin{array}{l}1982 \\
1973\end{array}$ & $\begin{array}{r}1 \\
57\end{array}$ \\
\hline
\end{tabular}

\section{ft origin}

AA Karl Ludwig; German astronomer (1765-1834).

AA Spiru; Rumanian astronomer (1851-1912).

AA Frederick James; British astronomer, optician (1891-1970).

LI German war goddess.

AA Egyptian astronomer (c. 300 B.C.)

VA Ancient Egyptian word for "Mars."

AA Scandinavian male name.

SU Greek; where Ganymede was abducted an eagle.

AA Greek astronomer (unkn-c. 460 B.C.).

SU City where Khudadad's father ruled.

AA Thomas; British mathematician, astronomer (1560-1621).

AA Johannes D.; German astronomer (1865-1936).

AA Ernst; German astronomer (1851-1923).

AA Carl E.; German astronomer (1851-1923).

AA Suzuki (1720/1724-1770).

AA William; British doctor (1578-1657).

AA Johann Matthias; German mathematician (1684-1742).

AA Takeo; Japanese astronomer (1914-1963).

PE Yaroro(Slavic) god who, in form of a boy, gave fire to mankind.

AA Egyptian goddess of joy and love.

MO Egyptian sky goddess.

PE Egyptian pharoah (1479 B.C.)

CR Polynesian fertility goddess.

AA Gerhart; German novelist and dramatist (1862-1946).

AA Christian August; German astronomer, mathematician, physicist (1693-1743).

AA Nathaniel; American novelist (1804-1864).

AA Fumiko; Japanese writer (1903-1951).

AA J.; Austrian composer (1732-1809).

AA John F.; American civil engineer (1868-1925).

AA Friedrich; German astronomer (1863-1928).

VA Radio telescope facility in Massachusetts.

LF Astronaut-named feature, Apollo 12 site.

AA Roy; American rocketry scientist (1915-1968).

AA English first name.

AA Oliver; British physicist (1850-1925).

AA Oliver; British mathematician, physicist (1850-1925).

CM Classical albedo feature name.

MN Classical albedo feature; name of goddess of youth.

VA Ancient river in Greece.

AA Greek geographer (unkn-c. 476 B.C.)

CM Greek moon goddess.

TH Classcial albedo feature name.

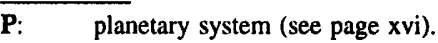

Sa: satellite (see page $\mathrm{xvi}$ ).

lat: latitude of feature center.

long: longitude of feature center.
}

diam: diameter or long dimension of feature ct: continent of name origin (see page $284 \mathrm{ff}$.)

et: ethnicity of name origin (see page $284 \mathrm{ff}$.) as: name approval status (see page xvii). ad: name approval date (year).

ref: reference source for name (see page $287 \mathrm{ff}$.). ft: feature type (see page 290). 


\section{ALPHABETICAL LIST OF NAMES}

\begin{tabular}{|c|c|c|c|c|c|c|c|c|c|}
\hline $\mathbf{P}$ & $\mathbf{S a}$ & Name & lat & long & diam & ct & et & as & ad \\
\hline $\begin{array}{l}\mathrm{M} \\
\mathrm{L}\end{array}$ & $\begin{array}{l}\mathrm{M} \\
\mathrm{L}\end{array}$ & $\begin{array}{l}\text { Heculaneum } \\
\text { Hédervári }\end{array}$ & $\begin{array}{l}19.2 \mathrm{~N} \\
81.8 \mathrm{~S}\end{array}$ & $\begin{array}{l}58.9 \mathrm{~W} \\
84.0 \mathrm{E}\end{array}$ & $\begin{array}{r}0 \\
69\end{array}$ & $\begin{array}{l}\mathrm{EU} \\
\mathrm{EU}\end{array}$ & $\underset{H U}{\text { IT }}$ & $\begin{array}{l}5 \\
5\end{array}$ & $\begin{array}{l}1988 \\
1994\end{array}$ \\
\hline $\mathrm{L}$ & $\mathbf{L}$ & Hedin & $2.0 \mathrm{~N}$ & $76.5 \mathrm{~W}$ & 150 & EU & SW & 5 & 1964 \\
\hline $\mathrm{H}$ & $\mathrm{H}$ & Heemskerck Rupes & $25.9 \mathrm{~N}$ & $125.3 \mathrm{~W}$ & 0 & EU & DU & 5 & 1976 \\
\hline $\begin{array}{l}M \\
V \\
L\end{array}$ & $\begin{array}{l}\mathrm{M} \\
\mathrm{V} \\
\mathrm{L}\end{array}$ & $\begin{array}{l}\text { Hegemone Dorsum } \\
\text { Heidi } \\
\text { [Heine] }\end{array}$ & $\begin{array}{l}55.3 \mathrm{~S} \\
23.6 \mathrm{~N} \\
14.4 \mathrm{~S}\end{array}$ & $\begin{array}{l}44.9 \mathrm{~W} \\
350.1 \mathrm{E} \\
132.0 \mathrm{E}\end{array}$ & $\begin{array}{r}0 \\
14 \\
28\end{array}$ & $\begin{array}{l}\text { EU } \\
\text { EU } \\
\text { EU }\end{array}$ & $\begin{array}{l}\text { GR } \\
\text { LA } \\
\text { GE }\end{array}$ & $\begin{array}{l}5 \\
5 \\
6\end{array}$ & $\begin{array}{r}1991 \\
1994 \\
0\end{array}$ \\
\hline $\begin{array}{l}\mathrm{H} \\
\mathrm{M}\end{array}$ & $\begin{array}{l}\mathrm{H} \\
\mathrm{M}\end{array}$ & $\begin{array}{l}\text { Heine } \\
\text { Heinlein }\end{array}$ & $\begin{array}{l}32.6 \mathrm{~N} \\
64.6 \mathrm{~S}\end{array}$ & $\begin{array}{l}124.1 \mathrm{~W} \\
243.8 \mathrm{~W}\end{array}$ & $\begin{array}{l}75 \\
83\end{array}$ & $\begin{array}{l}\text { EU } \\
\text { NA }\end{array}$ & $\begin{array}{l}\text { GE } \\
\mathrm{AM}\end{array}$ & $\begin{array}{l}5 \\
5\end{array}$ & $\begin{array}{l}1979 \\
1994\end{array}$ \\
\hline $\mathbf{L}$ & L & Heinrich & $24.8 \mathrm{~N}$ & $15.3 \mathrm{~W}$ & 6 & EU & $\mathrm{CZ}$ & 5 & 1979 \\
\hline L & $\mathbf{L}$ & Heinsius & $39.5 \mathrm{~S}$ & $17.7 \mathrm{~W}$ & 64 & $\mathrm{EU}$ & GE & 5 & 1935 \\
\hline L & L & Heis & $32.4 \mathrm{~N}$ & $31.9 \mathrm{~W}$ & 14 & EU & GE & 5 & 1935 \\
\hline $\mathbf{J}$ & io & Heiseb Patera & $29.7 \mathrm{~N}$ & $244.8 W$ & 60 & $\mathrm{AF}$ & BU & 5 & 1985 \\
\hline $\begin{array}{l}\mathrm{N} \\
\mathrm{L}\end{array}$ & $\begin{array}{l}\mathrm{tr} \\
\mathrm{L}\end{array}$ & $\begin{array}{l}\text { Hekt Cavus } \\
\text { Helberg }\end{array}$ & $\begin{array}{l}26.0 \mathrm{~N} \\
22.5 \mathrm{~N}\end{array}$ & $\begin{array}{l}342.0 \mathrm{E} \\
102.2 \mathrm{~W}\end{array}$ & $\begin{array}{r}0 \\
62\end{array}$ & $\begin{array}{l}\text { AF } \\
\text { NA }\end{array}$ & $\begin{array}{l}\text { EG } \\
\text { AM }\end{array}$ & $\begin{array}{l}5 \\
5\end{array}$ & $\begin{array}{r}91 \\
1970\end{array}$ \\
\hline V & V & Helen Planitia & $51.7 \mathrm{~S}$ & $263.9 \mathrm{E}$ & 4362 & EU & GR & 5 & 1982 \\
\hline L & L & Helicon & $40.4 \mathrm{~N}$ & $23.1 \mathrm{~W}$ & 24 & $\mathrm{EU}$ & GR & 5 & 1935 \\
\hline $\begin{array}{l}\text { H } \\
\text { S } \\
\mathrm{L}\end{array}$ & $\begin{array}{l}\mathrm{H} \\
\text { hy } \\
\mathrm{L}\end{array}$ & $\begin{array}{l}\text { Heliocaminus } \\
\text { Helios } \\
\text { Hell }\end{array}$ & $\begin{array}{l}40.0 \mathrm{~N} \\
71.0 \mathrm{~N} \\
32.4 \mathrm{~S}\end{array}$ & $\begin{array}{r}170.0 \mathrm{~W} \\
132.0 \mathrm{~W} \\
7.8 \mathrm{~W}\end{array}$ & $\begin{array}{r}0 \\
0 \\
33\end{array}$ & $\begin{array}{l}\text { EU } \\
\text { EU } \\
\text { EU }\end{array}$ & $\begin{array}{l}\text { RM } \\
\text { GR } \\
\text { HU }\end{array}$ & $\begin{array}{l}5 \\
5 \\
5\end{array}$ & $\begin{array}{l}1976 \\
1982 \\
1935\end{array}$ \\
\hline $\begin{array}{l}\mathrm{M} \\
\mathrm{M} \\
\mathrm{M} \\
\mathrm{M} \\
\mathrm{S}\end{array}$ & $\begin{array}{l}\mathrm{M} \\
\mathrm{M} \\
\mathrm{M} \\
\mathrm{M} \\
\mathrm{rh}\end{array}$ & $\begin{array}{l}\text { Hellas } \\
\text { Hellas Chaos } \\
\text { Hellas Montes } \\
\text { Hellas Planitia } \\
\text { Heller }\end{array}$ & $\begin{array}{l}40.0 \mathrm{~S} \\
46.0 \mathrm{~S} \\
37.9 \mathrm{~S} \\
44.3 \mathrm{~S} \\
10.1 \mathrm{~N}\end{array}$ & $\begin{array}{l}290.0 \mathrm{~W} \\
293.0 \mathrm{~W} \\
260.9 \mathrm{~W} \\
293.8 \mathrm{~W} \\
315.1 \mathrm{~W}\end{array}$ & $\begin{array}{r}0 \\
900 \\
0 \\
2517 \\
0\end{array}$ & $\begin{array}{l}\text { EU } \\
\text { EU } \\
\text { EU } \\
\text { EU } \\
\text { SA }\end{array}$ & $\begin{array}{l}\text { GR } \\
\text { GR } \\
\text { GR } \\
\text { GR } \\
\text { AC }\end{array}$ & $\begin{array}{l}5 \\
5 \\
5 \\
5 \\
5\end{array}$ & $\begin{array}{l}1958 \\
1994 \\
1989 \\
1973 \\
1982\end{array}$ \\
\hline $\begin{array}{l}\mathrm{M} \\
\mathrm{M} \\
\mathrm{V}\end{array}$ & $\begin{array}{l}\mathrm{M} \\
\mathrm{M} \\
\mathrm{V}\end{array}$ & $\begin{array}{l}\text { Hellespontus } \\
\text { Hellespontus Montes } \\
\text { Hellman }\end{array}$ & $\begin{array}{r}50.0 \mathrm{~S} \\
45.5 \mathrm{~S} \\
4.8 \mathrm{~N}\end{array}$ & $\begin{array}{l}325.0 \mathrm{~W} \\
317.5 \mathrm{~W} \\
356.3 \mathrm{E}\end{array}$ & $\begin{array}{r}0 \\
681 \\
35\end{array}$ & $\begin{array}{l}\mathrm{EU} \\
\mathrm{EU} \\
\mathrm{NA}\end{array}$ & $\begin{array}{l}\text { LA } \\
\text { LA } \\
\text { AM }\end{array}$ & $\begin{array}{l}5 \\
5 \\
5\end{array}$ & $\begin{array}{l}1958 \\
1973 \\
1991\end{array}$ \\
\hline $\mathrm{L}$ & $\mathrm{L}$ & Helmert & $7.6 \mathrm{~S}$ & $87.6 \mathrm{E}$ & 26 & EU & GE & 5 & 1973 \\
\hline L & L & Helmholtz & $68.1 S$ & $64.1 \mathrm{E}$ & 94 & $\mathrm{EU}$ & GE & 5 & 1935 \\
\hline $\mathbf{M}$ & $\mathbf{M}$ & Helmholtz & $45.6 \mathrm{~S}$ & $21.1 \mathrm{~W}$ & 107 & EU & GE & 5 & 1973 \\
\hline V & V & Heloise & $40.0 \mathrm{~N}$ & $51.9 \mathrm{E}$ & 40 & EU & FR & 5 & 1994 \\
\hline $\begin{array}{l}\mathrm{A} \\
\mathrm{V}\end{array}$ & gs & $\begin{array}{l}\text { Helwan } \\
\text { Hemera Dorsa }\end{array}$ & $\begin{array}{l}22.4 \mathrm{~N} \\
51.0 \mathrm{~N}\end{array}$ & $\begin{array}{l}118.9 \mathrm{~W} \\
243.4 \mathrm{E}\end{array}$ & $\begin{array}{r}6 \\
587\end{array}$ & $\begin{array}{l}\mathrm{AF} \\
\mathrm{EU}\end{array}$ & $\begin{array}{l}\text { EG } \\
\text { GR }\end{array}$ & $\begin{array}{l}5 \\
5\end{array}$ & $\begin{array}{l}1994 \\
1985\end{array}$ \\
\hline L & $\mathrm{L}$ & Henderson & $4.8 \mathrm{~N}$ & $152.1 \mathrm{E}$ & 47 & EU & SC & 5 & 1970 \\
\hline L & $\mathrm{L}$ & Hendrix & $46.6 \mathrm{~S}$ & $159.2 W$ & 18 & NA & $\mathrm{AM}$ & 5 & 1970 \\
\hline $\begin{array}{l}\mathrm{V} \\
\mathrm{V} \\
\mathrm{V}\end{array}$ & $\begin{array}{l}\mathrm{V} \\
\mathrm{V} \\
\mathrm{V}\end{array}$ & $\begin{array}{l}\text { Heng-o Chasma } \\
\text { Heng-o Corona } \\
\text { Henie. }\end{array}$ & $\begin{array}{r}6.6 \mathrm{~N} \\
2.0 \mathrm{~N} \\
51.9 \mathrm{~S}\end{array}$ & $\begin{array}{l}355.5 \mathrm{E} \\
355.0 \mathrm{E} \\
145.8 \mathrm{E}\end{array}$ & $\begin{array}{r}734 \\
1060 \\
70\end{array}$ & $\begin{array}{l}\text { AS } \\
\text { AS } \\
\text { EU }\end{array}$ & $\begin{array}{l}\mathrm{CH} \\
\mathrm{CH} \\
\mathrm{NO}\end{array}$ & $\begin{array}{l}5 \\
5 \\
5\end{array}$ & $\begin{array}{l}1982 \\
1994 \\
1991\end{array}$ \\
\hline $\begin{array}{l}\mathbf{J} \\
\mathbf{M}\end{array}$ & $\begin{array}{l}\text { io } \\
\mathrm{M}\end{array}$ & $\begin{array}{l}\text { Heno Patera } \\
\text { Henry }\end{array}$ & $\begin{array}{l}56.5 \mathrm{~S} \\
11.0 \mathrm{~N}\end{array}$ & $\begin{array}{l}312.1 \mathrm{~W} \\
336.8 \mathrm{~W}\end{array}$ & $\begin{array}{r}65 \\
165\end{array}$ & $\begin{array}{l}\text { NA } \\
\text { EU }\end{array}$ & $\begin{array}{l}\text { IQ } \\
\text { FR }\end{array}$ & $\begin{array}{l}5 \\
5\end{array}$ & $\begin{array}{l}1979 \\
1973\end{array}$ \\
\hline L & $\mathbf{L}$ & Henry & $24.0 \mathrm{~S}$ & $56.8 \mathrm{~W}$ & 41 & NA & AM & 5 & 1970 \\
\hline
\end{tabular}

\section{ft origin}

AA Town in Italy.

AA Peter; Hungarian geoscientist (1931-1984).

AA Sven A.; Swedish explorer (1865-1952)

RU Dutch; one of Tasman's ships to explore Australia, New Zealand, 1642-43.

DO One of the Graces.

AA First name; form of Hester.

AA Heinrich; German poet and critic (1797-1856).

AA Heinich; German poet (1797-1856).

AA Robert A.; American author (1907-1988)

AA Wladimir W.; Czechoslovakian astronomer (1884-1965).

AA Gottfried; German astronomer (1709-1769).

AA Eduard; German astronomer (1806-1877).

PE Bushman devil who represents fire.

CB Egyptian frog goddess.

AA Robert J.; American aeronautical engineer (1906-1967).

PL Greek; "the face that launched 1000 ships."

AA Greek astronomer, mathematician (unkn-c. 400 B.C.)

AL Albedo feature; Antoniadi map.

AA Greek sun god; son of Hyperion.

AA Maximilian; Hungarian astronomer (1720-1792)

AL Greece.

CH Named for albedo feature Hellas. MO Albedo feature Hellas.

PL Classical albedo feature name.

AA Auracanin creator of men and bringer of civilization.

AL The Dardanelles.

MO Classical albedo feature name.

AA Lillian; American playwright, author (1905-1984).

AA Friedrich Robert; German astronomer, geodesist (1843-1917).

AA Hermann Von; German doctor (1821-1894).

AA Hermann von; German physicist (1821-1894).

AA French physician, hospital founder (c. 1098-1164).

AA Spa in Egypt.

DO Greek goddess, personification of day.

AA Thomas; Scottish astronomer (1798-1844).

AA Don O.; American optician (1905-1961).

CM Chinese moon goddess.

CR Named for associated chasma.

AA Sonja; Norwegian skater (1912-1969).

$\mathrm{PE}$ Iroquois god of thunder.

AA Paul; French astronomer (1848-1905). Prosper; French astronomer (1849-1903).

AA Joseph; American physicist (1792-1878)

\footnotetext{
P: $\quad$ planetary system (see page $x v i)$

Sa: $\quad$ satellite (see page $x v i$ ).

lat: latitude of feature center.

long: longitude of feature center.
}

diam: diameter or long dimension of feature.

ct: continent of name origin (see page $284 \mathrm{ff}$.)

et: ethnicity of name origin (see page $284 \mathrm{ff}$.)

as: name approval status (see page xvii). ad: name approval date (year).

ref: reference source for name (see page $287 \mathrm{ff}$.).

$\mathrm{ft}$ : feature type (see page 290). 


\section{P Sa Name}

L L Henry Fréres

V V Henwen Fluctus

L L Henyey

V V Hepat Corona

M M Hephaestus Fossae

J io Hephaestus Patera

J ca Hepti

V V Hepworth

M M Her Desher Vallis

V V Hera Dorsa

L L Heraclitus

M M Herculaneum

L L Hercules

L L Herigonius

L L Hermann

L L Hermite

M M Hermus Vallis

H H Hero Rupes

L L Herodotus

L L Heron (Hero)

$S$ mi Herschel

L L Herschel

M M Herschel

L L Hertz

L L Hertzsprung

V V Hervor Corona

$\mathrm{H} \mathrm{H}$ Hesiod

L L Hesiodus

M M Hesperia

M M Hesperia Dorsa

M M Hesperia Planum

$\mathrm{H}$ H Hesperis

L L Hess

L L Hess-Apollo

V V Hestia Rupes

L L Hevelius

L L Heymans

L L Heyrovsky

M M Hibes Montes lat long diam ct et as ad ref $\mathrm{ft}$ origin

$\begin{array}{rrrrrrrr}23.5 \mathrm{~S} & 58.9 \mathrm{~W} & 42 & \text { EU } & \text { FR } & 5 & 1961 & 66 \\ & & & & & & & \\ 20.5 \mathrm{~S} & 179.9 \mathrm{E} & 485 & \text { EU } & \text { EN } & 5 & 1994 & 111 \\ 13.5 \mathrm{~N} & 151.6 \mathrm{~W} & 63 & \text { NA } & \text { AM } & 5 & 1970 & 0 \\ 2.0 \mathrm{~S} & 145.5 \mathrm{E} & 150 & \text { AS } & \text { TU } & 5 & 1994 & 83 \\ 20.5 \mathrm{~N} & 237.5 \mathrm{~W} & 528 & \text { EU } & \text { GR } & 5 & 1973 & 57 \\ 2.0 \mathrm{~N} & 289.5 \mathrm{~W} & 50 & \text { EU } & \text { GR } & 5 & 1979 & 1 \\ 64.5 \mathrm{~N} & 23.9 \mathrm{~W} & 41 & \text { EU } & \text { NS } & 5 & 1979 & 17 \\ 5.1 \mathrm{~N} & 94.6 \mathrm{E} & 61 & \text { EU } & \text { EN } & 5 & 1991 & 99 \\ & & & & & & & \\ 25.4 \mathrm{~S} & 47.8 \mathrm{~W} & 120 & \text { AF } & \text { EG } & 5 & 1985 & 62 \\ 36.4 \mathrm{~N} & 29.5 \mathrm{E} & 813 & \text { EU } & \text { GR } & 5 & 1985 & 1 \\ 49.2 \mathrm{~S} & 6.2 \mathrm{E} & 90 & \text { EU } & \text { GR } & 5 & 1961 & 67 \\ 19.2 \mathrm{~N} & 58.9 \mathrm{~W} & 0 & \text { EU } & \text { IT } & 5 & 1988 & 36 \\ 46.7 \mathrm{~N} & 39.1 \mathrm{E} & 69 & \text { EU } & \text { GR } & 5 & 1935 & 66 \\ 13.3 \mathrm{~S} & 33.9 \mathrm{~W} & 15 & \text { EU } & \text { FR } & 5 & 1935 & 66 \\ & & & & & & & \\ 0.9 \mathrm{~S} & 57.0 \mathrm{~W} & 15 & \text { EU } & \text { SZ } & 5 & 1935 & 66 \\ 86.0 \mathrm{~N} & 89.9 \mathrm{~W} & 104 & \text { EU } & \text { FR } & 5 & 1964 & 67 \\ 5.4 \mathrm{~S} & 147.5 \mathrm{~W} & 52 & \text { AS } & \text { TU } & 5 & 1985 & 56 \\ 58.4 \mathrm{~S} & 171.4 \mathrm{~W} & 0 & \text { NA } & \text { AM } & 5 & 1976 & 80 \\ 23.2 \mathrm{~N} & 49.7 \mathrm{~W} & 34 & \text { EU } & \text { GR } & 5 & 1935 & 66 \\ 0.7 \mathrm{~N} & 119.8 \mathrm{E} & 24 & \text { AF } & \text { EG } & 5 & 1976 & 0 \\ 2.9 \mathrm{~N} & 109.5 \mathrm{~W} & 0 & \text { EU } & \text { GB } & 5 & 1982 & 37\end{array}$

$5.7 \mathrm{~S}$

$2.1 \mathrm{~W}$

40

EU GB

$14.9 S$

$230.1 \mathrm{~W}$

304

EU GB

$5 \quad 1935$

66

13.4N 104.5E

90 EU GE

51973

68

$\begin{array}{rrrrrrrr}2.6 \mathrm{~N} & 129.2 \mathrm{~W} & 591 & \text { EU } & \text { DE } & 5 & 1970 & 0 \\ 25.5 \mathrm{~S} & 269.0 \mathrm{E} & 250 & \text { EU } & \text { NS } & 5 & 91 & 27 \\ 58.5 \mathrm{~S} & 35.0 \mathrm{~W} & 107 & \text { EU } & \text { GR } & 5 & 1976 & 59 \\ 29.4 \mathrm{~S} & 16.3 \mathrm{~W} & 42 & \text { EU } & \text { GR } & 5 & 1935 & 66 \\ & & & & & & & \\ 20.0 \mathrm{~S} & 240.0 \mathrm{~W} & 0 & \text { EU } & \text { LA } & 5 & 1958 & 60 \\ & & & & & & & \\ 23.0 \mathrm{~S} & 245.0 \mathrm{~W} & 0 & \text { EU } & \text { LA } & 5 & 1989 & 57 \\ 18.3 \mathrm{~S} & 251.6 \mathrm{~W} & 1869 & \text { EU } & \text { LA } & 5 & 1973 & 57 \\ 45.0 \mathrm{~S} & 355.0 \mathrm{~W} & 0 & \text { EU } & \text { RM } & 5 & 1976 & 60 \\ 54.3 \mathrm{~S} & 174.6 \mathrm{E} & 88 & \text { NA } & \text { AM } & 5 & 1970 & 0\end{array}$

20.1

$6.0 \mathrm{~N}$

30.7E

1 NA AM

51973

59

$2.2 \mathrm{~N} \quad 71.1 \mathrm{E}$

588

EU GR

51982

$67.6 \mathrm{~W}$

115

EU

GR
PO

51935

$75.3 \mathrm{~N} \quad 144.1 \mathrm{~W}$

50

EU

BE

51970

39.6S 95.3W

16

EU

$\mathrm{CZ}$

51985

$3.9 \mathrm{~N} \quad 188.9 \mathrm{~W}$
177 EU GR
AA Prosper; French astronomer (1849-1903); Paul; French astronomer (1848-1905).

FL British Celtic sow-goddess.

AA Louis G.; American astronomer (1910-1970)

CR Hittite mother goddess.

FO Classical albedo feature name.

PE Greek smith god.

AA Norse dwarf.

AA Barbara; English sculptor (1903-1975).

VA Egyptian name for Mars.

DO Greek sky goddess, wife of Zeus.

AA Greek philosopher (c. 540-480 B.C.)

AA Town in Italy.

AA Greek mythological hero.

AA Herigone, Pierre; French mathematician, astronomer (fl. 1644).

AA Jacob; Swiss mathematician (1678-1733)

AA Charles; French mathematician (1822-1901)

VA Classical name for river in ancient Lydia (modern Turkey).

RU American; Palmer's ship to explore Antarctic coast, 1820-21.

AA Of Halikarnassus; Greek historian (c. 484-408 B.C.).

AA Egyptian inventor (unkn-c. 100 B.C.).

AA William; German-British astronomer; discovered Mimas and Enceladus (1738-1822).

AA William; British astronomer (1738-1822).

AA John F.; British astronomer (1792-1871; William H.; British astronomer (1738-1822).

AA Heinrich R.; German physicist (1857-1894).

AA Hertzsprung, Ejnar; Danish astronomer (1873-1967).

CR Norse fertility goddess.

AA Greek poet (c. 800 B.C.).

AA Hesiod; Greek humanitarian (c. 735 B.C.).

AL "The Occiden"; name for Italy (Greek); or Spain (Roman) or West area of Ethiopians where sun sank

DO Albedo feature name.

PM Classical albedo feature name.

AL Albedo feature; Antoniadi map.

AA Victor F.; American physicist (1883-1964); Harry H.; American geologist (1906-1969).

LF Astronaut-named feature, Apollo 17 site.

RU Greek hearth goddess.

AA Howelcke, Johann; Polish astronomer.(1611-1687)

AA Corneille J. F.; Belgian physiologist; Nobel laureate (1892-1968).

AA Jaroslav; Czechoslovakian chemist (1890-1967).

MO From albedo feature at $17 \mathrm{~N}, 186 \mathrm{~W}$.

\footnotetext{
P: $\quad$ planetary system (see page $x v i$ )

Sa: $\quad$ satellite (see page $x v i$ ).

lat: latitude of feature center.

long: longitude of feature center.
}

diam: diameter or long dimension of feature.

ct: continent of name origin (see page $284 \mathrm{ff}$.)

et: ethnicity of name origin (see page $284 \mathrm{ff}$.)

as: name approval status (see page xvii). ad: name approval date (year).

ref: reference source for name (see page $287 \mathrm{ff}$.)

ft: feature type (see page 290). 
ALPHABETICAL LIST OF NAMES

P Sa Name

M M Hiddekel

V V Hiei Chu Patera

V V Higgins

J ca Hijsi

$\mathrm{S}$ ep Hilairea

L L Hilbert

V V Hildr Fossa

$N$ tr Hili

$\mathrm{L}$ L Hill

M M Hilo

M M Himera Valles

V V Himiko

V V Hina Chasma

L L Hind

L L Hippalus

M M Hipparchus

L L Hipparchus

L L Hippocrates

V V Hippolyta Linea

L L Hirayama

$\mathrm{H} \quad \mathrm{H}$ Hiroshige

$\mathrm{N}$ tr Hirugo Cavus

J io Hiruko Patera

M M Hít

$\mathrm{H}$ H Hitomaro

$\mathrm{N}$ tr Ho Sulci

J ca Hödr

J ca Hoenir

L L Hoffmeister

L L Hogg

J ca Högni

L L Hohmann

$\mathrm{H}$ H Holbein

H H Holberg

V V Holde Corona

M M Holden

L L Holden

J ca Höldr

L L Holetschek

V V Holiday

M M Holmes

H H Homer lat

long

$\begin{array}{rrrrrrrr}15.0 \mathrm{~N} & 345.0 \mathrm{~W} & 0 & \text { AS } & \text { BY } & 5 & 1958 & 60 \\ 48.2 \mathrm{~N} & 97.4 \mathrm{E} & 139 & \text { AS } & \text { CH } & 5 & 1985 & 80 \\ & & & & & & & \\ 7.6 \mathrm{~N} & 241.4 \mathrm{E} & 40 & \text { NA } & \text { AM } & 5 & 1994 & 99 \\ & & & & & & & \\ 61.6 \mathrm{~N} & 169.3 \mathrm{~W} & 51 & \text { UR } & \text { KA } & 5 & 1987 & 64 \\ 0.0 \mathrm{~N} & 0.0 \mathrm{~W} & 0 & \text { EU } & \text { GR } & 5 & 1982 & 1 \\ & & & & & & & \\ 17.9 \mathrm{~S} & 108.2 \mathrm{E} & 151 & \text { EU } & \text { GE } & 5 & 1970 & 0 \\ & & & & & & & \\ 45.4 \mathrm{~N} & 159.4 \mathrm{E} & 677 & \text { EU } & \text { NS } & 5 & 1985 & 17 \\ 57.0 \mathrm{~S} & 35.0 \mathrm{E} & 0 & \text { AF } & \text { SA } & 5 & 91 & 27 \\ 20.9 \mathrm{~N} & 40.8 \mathrm{E} & 16 & \text { NA } & \text { AM } & 5 & 1973 & 0\end{array}$

44.8S $\quad 35.5 \mathrm{~W}$

$21.1 \mathrm{~S} \quad 22.4 \mathrm{~W}$

$19.0 \mathrm{~N} \quad 124.2 \mathrm{E}$

$63.7 \mathrm{~N} \quad 20.0 \mathrm{E}$

$7.9 \mathrm{~S} \quad 7.4 \mathrm{E}$

24.8S

$45.0 \mathrm{~S}$

$30.2 \mathrm{~W}$

$151.1 \mathrm{~W}$

$5.1 \mathrm{~S}$

$70.7 \mathrm{~N}$

$42.0 \mathrm{~S}$

$6.1 \mathrm{~S}$

$5.2 \mathrm{E}$

$145.9 \mathrm{~W}$

$345.0 \mathrm{E}$

93.5E

$13.4 \mathrm{~S}$

$14.5 \mathrm{~N}$

$26.7 \mathrm{~W}$

$14.5 \mathrm{~N} \quad 345.0 \mathrm{E}$

65.1S 329.3W

$47.3 \mathrm{~N} \quad 221.5 \mathrm{~W}$

$16.2 \mathrm{~S} \quad 15.8 \mathrm{~W}$

$2.0 \mathrm{~N} \quad 305.0 \mathrm{E}$

$69.0 \mathrm{~N} \quad 87.7 \mathrm{~W}$

33.9S 261.2W

$15.2 \mathrm{~N}$

$136.9 \mathrm{E}$

$33.6 \mathrm{~N}$

121.9E

$13.5 \mathrm{~S}$

$17.9 \mathrm{~S}$

$35.6 \mathrm{~N}$

$4.5 \mathrm{~W}$

$94.1 \mathrm{~W}$

20
218

218
37

415

29

US HI

EU

AS

OC JA

EU GB

57 EU GR

104

EU GR

138 EU GR

60 EU GR

0 EU GR

51976

$\begin{array}{ll}5 & 1979\end{array}$

$\begin{array}{ll}5 & 1991\end{array}$

51991

1935

51935

$\begin{array}{ll}5 & 1935 \\ 5 & 1973\end{array}$

51935

51970

$5 \quad 1982$

$\begin{array}{lllll}132 & \text { AS } & \text { JA } & 5 & 1970\end{array}$

138 AS JA

0 AS JA

51976

AS

JA

JQ

1979

107

AS

JA

1979

$\begin{array}{rr}0 & \text { AS } \\ 76 & \text { EU }\end{array}$

$\mathrm{CH}$

$5 \quad 91$

51979

84

EU

NS

$5 \quad 1979$

45

EU

GE

51970

38

$\mathrm{OC} \quad \mathrm{AU}$

51970

$67.0 \mathrm{~S}$

$53.5 \mathrm{~N}$

26.5S

28.9W

65
16

EU NS

51979

51970

51979

113 EU GE

51976

61.1W

61

EU

NO

51994

19.1S

$155.8 \mathrm{E}$

200 EU GE

$\begin{array}{ll}5 & 1973\end{array}$

$44.1 \mathrm{~N} \quad 109.2 \mathrm{~W}$

27.6S

$150.9 \mathrm{E}$

46.7S

$12.8 \mathrm{E}$

75.0S 293.9W

$1.2 \mathrm{~S}$

$36.2 \mathrm{~W}$

141

NA AM

51935

51988

$5 \quad 1970$

51991

51973

51976 ref ft origin

AL Tigris River, Babylonia; Biblical third River of Paradise.

PE Chinese, converted silk worm product into thread and material (2698 B.C.).

AA Marguerite; American journalist (1920-1966).

AA Karelian deity of hunting.

AA Greek; sister of Phoibe, daughter of Leukippos.

AA David; German mathematician (1862-1943).

FO Norse mythological warrior.

PU Zulu water-sprite.

AA George William; American astronomer, mathematician (1838-1914)

AA Town in Hawaii, USA.

VA Ancient name for Italian river.

AA Japanese queen (4th century A.D.).

CM Hawaiian moon goddess.

AA John Russell; British astronomer (1823-1895).

AA Greek explorer (unkn-c. 120).

AA Greek astronomer (c. 160-125 B.C.)

AA Greek astronomer (unkn-fl. 140 B.C.).

AA Greek doctor (c. 460-377 B.C.).

LI Amazon queen.

AA Kiyotsugu; Japanese astronomer (1874-1943); Shin; Japanese astronomer (1867-1945).

AA A.; Japanese artist (1797-1858).

CB Japanese diety born in shape of a jellyfish.

PE Japanese sun god.

AA Town in Iraq.

AA Kakinomoto No; Japanese poet (c. 655-c. 700)

SU Chinese sacred river.

AA Norse; Baldr's blind brother who shot Baldr unknowingly.

AA Norse; god who gave souls to first humans.

AA Cuno; German astronomer (1892-1968)

AA Arthur R.; Australian astronomer (1903-1966); Frank S.; Canadian astronomer (1904-1951).

AA Norse; Ottar's ancestor.

AA Walter; German space flight engineer (1880-1945).

AA Hans (c. 1465-1524), and Hans (c. 1497-1543); German painters.

AA Ludvig; Norwegian-Danish writer (1684-1754).

CR German fertility goddess.

AA Edward S.; American astronomer (1846-1914).

AA Edward Singleton; American astronomer (1846-1914).

AA Son of Karl and Snor in Rigdismal.

AA Johann; Austrian astronomer (1846-1923).

AA Billie; American singer (1915-1959).

AA Arthur; British geologist (1890-1965)

AA Greek epic poet (8th or 9th century B.C.)

\footnotetext{
P: planetary system (see page xvi).

Sa: $\quad$ satellite (see page $\mathrm{xvi}$ ).

lat: latitude of feature center.

long: longitude of feature center.
}

diam: diameter or long dimension of feature.

ct: continent of name origin (see page $284 \mathrm{ff}$.)

et: ethnicity of name origin (see page $284 \mathrm{ff}$.)

as: name approval status (see page xvii). ad: name approval date (year).

ref: reference source for name (see page $287 \mathrm{ff}$.).

ft: feature type (see page 290). 


\section{ALPHABETICAL LIST OF NAMES}

\section{P Sa Name}

L L [Homer]

L L Hommel

M M Honda

M M Hooke

L L Hooke

M M Hope

L L Hopmann

$\mathrm{H} \quad \mathrm{H}$ Horace

M M Horarum Mons

L L Horatio

V V Horner

L L Hornsby

L L Horrebow

L L Horrocks

L L Hortensius

J io Horus Patera

M M Houston

L L Houtermans

L L Houzeau

V V Howe

M M Hrad Vallis

V V Hroswitha Patera

M M Hsūanch'eng

V V Hsueh T'ao

V V Hua Mulan

M M Huancayo

V V Huang Daopo

L L Hubble

M M Huggins

L L Huggins

L L [Hugo]

H H Hugo

V V Hull

L L Humason

L L Humboldt

L L Hume

$\mathrm{H} \quad \mathrm{H}$ Hun $\mathrm{Kal}$

M M Huo Hsing Vallis

J io Huo Shen Patera

$\mathrm{U}$ ar Huon

V V H'uraru Corona

J ga Hursag Sulcus lat long diam

ct et as

\begin{tabular}{|c|c|c|c|c|c|c|c|}
\hline $24.3 S$ & $133.6 \mathrm{E}$ & 0 & $\mathrm{EU}$ & GR & 6 & 0 & 0 \\
\hline $54.7 \mathrm{~S}$ & $33.8 \mathrm{E}$ & 126 & $\mathrm{EU}$ & $\mathrm{GE}$ & 5 & 1935 & 66 \\
\hline $\begin{array}{l}22.7 S \\
45.0 S\end{array}$ & $\begin{array}{l}16.2 \mathrm{~W} \\
44.4 \mathrm{~W}\end{array}$ & $\begin{array}{r}6 \\
145\end{array}$ & $\begin{array}{l}\text { SA } \\
\text { EU }\end{array}$ & $\begin{array}{l}\mathrm{CO} \\
\mathrm{GB}\end{array}$ & $\begin{array}{l}5 \\
5\end{array}$ & $\begin{array}{l}1976 \\
1973\end{array}$ & $\begin{array}{l}36 \\
68\end{array}$ \\
\hline $41.2 \mathrm{~N}$ & $54.9 \mathrm{E}$ & 36 & $\mathrm{EU}$ & GB & 5 & 1935 & 66 \\
\hline $\begin{array}{l}45.1 \mathrm{~N} \\
50.8 \mathrm{~S}\end{array}$ & $\begin{array}{c}10.3 \mathrm{~W} \\
160.3 \mathrm{E}\end{array}$ & $\begin{array}{r}6 \\
88\end{array}$ & $\begin{array}{l}\text { NA } \\
\text { EU }\end{array}$ & $\begin{array}{l}\text { CA } \\
\text { AS }\end{array}$ & $\begin{array}{l}5 \\
5\end{array}$ & $\begin{array}{l}1976 \\
1979\end{array}$ & $\begin{array}{r}36 \\
0\end{array}$ \\
\hline $\begin{array}{l}68.9 \mathrm{~S} \\
51.3 \mathrm{~S} \\
20.2 \mathrm{~N}\end{array}$ & $\begin{array}{l}52.0 \mathrm{~W} \\
36.4 \mathrm{~W} \\
30.7 \mathrm{E}\end{array}$ & $\begin{array}{r}58 \\
0 \\
0\end{array}$ & $\begin{array}{l}\text { EU } \\
\text { EU } \\
\text { NA }\end{array}$ & $\begin{array}{c}\text { RM } \\
\text { GR } \\
\text { AM }\end{array}$ & $\begin{array}{l}5 \\
5 \\
5\end{array}$ & $\begin{array}{l}1976 \\
1991 \\
1973\end{array}$ & $\begin{array}{l}80 \\
57 \\
59\end{array}$ \\
\hline $23.4 \mathrm{~N}$ & 97.7E & 28 & EU & EN & 5 & 1991 & 100 \\
\hline $23.8 \mathrm{~N}$ & $12.5 \mathrm{E}$ & 3 & EU & GB & 5 & 1973 & \\
\hline
\end{tabular}

58.7

$40.8 \mathrm{~W}$

24

EU DE

$5 \quad 1935$

$4.0 \mathrm{~S}$

$5.9 \mathrm{E}$

30

EU GB

$6.5 \mathrm{~N} \quad 28.0 \mathrm{~W}$

EU DU

9.6S $338.6 \mathrm{~W}$

125

AF EG

$48.5 \mathrm{~N} \quad 223.9 \mathrm{~W}$

9.4S 87.2E

$\begin{array}{rrr}2 & \text { NA } & \text { AM } \\ 29 & \text { EU } & \text { GE }\end{array}$

$17.1 \mathrm{~S} \quad 123.5 \mathrm{~W}$

71

EU BE

45.7S $174.6 \mathrm{E}$

39

NA AM

$37.8 \mathrm{~N}$

$35.8 \mathrm{~N}$

$47.0 \mathrm{~N}$

$52.9 \mathrm{~S}$

$86.8 \mathrm{~N}$

$3.7 \mathrm{~S}$

$54.2 \mathrm{~S}$

$22.1 \mathrm{~N}$

49.3

41.1

$0.7 S$

$38.9 \mathrm{~N}$

$221.6 \mathrm{~W}$

$34.8 \mathrm{E}$

$227.2 \mathrm{~W}$

$13.7 \mathrm{E}$

337.7E

$39.8 \mathrm{~W}$

$165.4 \mathrm{E}$

$86.9 \mathrm{E}$

719

163

AS AM

EU GE

AS CH

20 AS $\mathrm{CH}$

24

25

AS

AA

NA

PE

$\mathrm{CH}$

AM

51935

51935

51979

$5 \quad 1979$

51973

$5 \quad 1970$

51994

204.3W

$$
82
$$

EU GB

$1.4 \mathrm{~W}$

$$
65
$$

EU

GB

92.9E

$$
24
$$

EU

FR

59.4

30.7

$27.0 \mathrm{~S}$

4.7S

$1.6 \mathrm{~S}$

47.0W

198

EU FR

51979

51994

51973

$56.6 \mathrm{~W}$

48 NA AM

NA AM

51935

$80.9 \mathrm{E}$

189

EU GE

51976

$31.5 \mathrm{~N} \quad 293.9 \mathrm{~W}$

$15.1 \mathrm{~S} \quad 329.3 \mathrm{~W}$

37.8S 33.7E

340
90
40

$9.0 \mathrm{~N} \quad 68.0 \mathrm{E}$

$10.7 \mathrm{~S} \quad 234.5 \mathrm{~W}$
$5 \quad 1976 \quad 80$

23 EU SC

SA MY

51973

AS $\mathrm{CH}$

$\begin{array}{ll}\mathrm{AS} & \mathrm{CH} \\ \mathrm{EU} & \mathrm{FR}\end{array}$

$\begin{array}{ll}5 & 1982 \\ 5 & 1988\end{array}$

150

$\begin{array}{ll}\text { NA } & \text { PW } \\ \text { AS } & \text { SU }\end{array}$

83
27
$5 \quad 1994$

$5 \quad 1985$ ft origin

AA Greek epic poet (8th or 9th century B.C.).

AA Johann; Greek astronomer, mathematician (1518-1562)

AA Town in Colombia.

AA Robert; British physicist-astronomer (1635-1703)

AA Robert; British physicist, inventor (1635-1703)

AA Town in British Columbia, Canada

AA Josef; Austrian astronomer (1890-1975).

AA Roman poet (65-8 B.C.)

MO Albedo name.

LF Astronaut-named feature Apollo 17 site.

AA Mary; English naturalist, geologist (19th century).

AA Thomas; British astronomer (1733-1810).

AA Peder; Danish astronomer (1679-1764)

AA Jeremiah; British astronomer (1619-1641).

AA Hove, Martin van den; Dutch astronomer (1605-1639).

PE Egyptian falcon-headed solar god.

AA American mission control site.

AA Friedrich Georg; German physicist (1903-1966).

AA Jean C. (De Lehaie); Belgian astronomer (1820-1888).

AA Julia; American biographer, poet (1819-1910)

VA Word for "Mars" in Armenian.

PE German writer (c. A.D. 935-975).

AA Chinese launch site.

AA Chinese poet, artist (c. A.D.760).

AA Chinese warrior (c. A.D. 590)

AA Town in Peru.

AA Chinese engineer.

AA Edwin P.; American astronomer (1889-1953).

AA William; British astronomer (1824-1910)

AA Sir William; British astronomer (1824-1910)

AA Victor; French writer dramatist, poet (1802-1885).

AA Victor; French writer, dramatist and poet (1802-1885).

AA Peggy; American war correspondent (1889-1967).

AA Milton L.; American astronomer (1891-1972)

AA Wilhelm von; German philologist (1767-1835).

AA David; Scottish philosopher (1711-1776).

AA Means " 20 " in Mayan language; 20th meridian passes through this crater.

VA Word for "Mars" in Chinese.

PE Chinese god of fire.

AA Replaced Oberon as King of Spirits when Oberon died.

CR Pawnee earth mother.

SU Sumerian mountain where winds dwell.
P: $\quad$ planetary system (see page xvi).

Sa: satellite (see page xvi).

lat: latitude of feature center.

long: longitude of feature center. diam: diameter or long dimension of feature.

ct: continent of name origin (see page $284 \mathrm{ff}$.)

et: ethnicity of name origin (see page $284 \mathrm{ff}$.)

as: name approval status (see page xvii). ad: name approval date (year).

ref: reference source for name (see page $287 \mathrm{ff}$.).

ft: feature type (see page 290). 
ALPHABETICAL LIST OF NAMES

\begin{tabular}{|c|c|c|c|c|c|c|c|c|c|c|c|c|}
\hline $\mathbf{P}$ & $\mathbf{S a}$ & Name & lat & long & diam & ct & et & as & ad & ref & $\mathbf{f t}$ & origin \\
\hline V & V & Hurston & $77.7 \mathrm{~S}$ & $94.5 \mathrm{E}$ & 65 & NA & $\mathbf{A M}$ & 5 & 1994 & 99 & AA & $\begin{array}{l}\text { Zora; American anthropologist } \\
\text { writer (c. 1901-1960). }\end{array}$ \\
\hline $\begin{array}{l}\mathbf{L} \\
\mathbf{M}\end{array}$ & $\begin{array}{l}\mathrm{L} \\
\mathbf{M}\end{array}$ & $\begin{array}{l}\text { [Hussein] } \\
\text { Hussey }\end{array}$ & $\begin{array}{l}12.3 \mathrm{~N} \\
53.8 \mathrm{~S}\end{array}$ & $\begin{array}{l}38.0 \mathrm{E} \\
126.5 \mathrm{~W}\end{array}$ & $\begin{array}{r}6 \\
100\end{array}$ & $\begin{array}{l}\text { AF } \\
\text { NA }\end{array}$ & $\begin{array}{l}\text { EG } \\
\text { AM }\end{array}$ & $\begin{array}{l}6 \\
5\end{array}$ & $\begin{array}{r}0 \\
1973\end{array}$ & $\begin{array}{r}0 \\
68\end{array}$ & $\begin{array}{l}\mathrm{AA} \\
\mathrm{AA}\end{array}$ & $\begin{array}{l}\text { Taha; Egyptian author (1889-1973). } \\
\text { William J.; American }\end{array}$ \\
\hline L & $\mathrm{L}$ & Hutton & $37.3 \mathrm{~N}$ & $168.7 \mathrm{E}$ & 50 & EU & SC & 5 & 1970 & 0 & AA & $\begin{array}{l}\text { astronomer (1862-1926). } \\
\text { James; Scottish geologist } \\
\text { (1726-1797). }\end{array}$ \\
\hline M & $\mathbf{M}$ & Hutton & $71.9 \mathrm{~S}$ & $255.5 \mathrm{~W}$ & 99 & EU & GB & 5 & 1973 & 68 & AA & $\begin{array}{l}\text { James; British geologist } \\
\text { (1726-1797). }\end{array}$ \\
\hline L & $\mathrm{L}$ & Huxley & $20.2 \mathrm{~N}$ & $4.5 W$ & 4 & EU & GB & 5 & 1973 & 0 & AA & $\begin{array}{l}\text { Thomas Henry; British } \\
\text { biologist (1825-1895). }\end{array}$ \\
\hline M & $\mathbf{M}$ & Huxley & $62.9 \mathrm{~S}$ & $259.2 W$ & 108 & EU & GB & 5 & 1973 & 68 & AA & $\begin{array}{l}\text { Thomas } \mathrm{H} \text {;; British } \\
\text { biologist (1825-1895). }\end{array}$ \\
\hline M & $\mathbf{M}$ & Huygens & $14.0 \mathrm{~S}$ & $304.4 \mathrm{~W}$ & 456 & EU & DU & 5 & 1973 & 68 & AA & $\begin{array}{l}\text { Christian; Dutch } \\
\text { physicist-astronomer (1629-1695). }\end{array}$ \\
\hline $\begin{array}{l}\mathbf{V} \\
\mathbf{M} \\
\mathbf{M} \\
\mathbf{M} \\
\mathbf{J}\end{array}$ & $\begin{array}{l}\mathrm{V} \\
M \\
M \\
M \\
\text { io }\end{array}$ & $\begin{array}{l}\text { Hwangcini } \\
\text { Hyblaeus Catena } \\
\text { Hyblaeus Chasma } \\
\text { Hyblaeus Fossae } \\
\text { Hybristes Planum }\end{array}$ & $\begin{array}{r}6.3 \mathrm{~N} \\
21.8 \mathrm{~N} \\
22.1 \mathrm{~N} \\
21.7 \mathrm{~N} \\
54.0 \mathrm{~S}\end{array}$ & $\begin{array}{r}141.7 \mathrm{E} \\
219.5 \mathrm{~W} \\
218.9 \mathrm{~W} \\
223.2 \mathrm{~W} \\
21.1 \mathrm{~W}\end{array}$ & $\begin{array}{r}30 \\
0 \\
71 \\
405 \\
150\end{array}$ & $\begin{array}{l}\text { AS } \\
\text { EU } \\
\text { EU } \\
\text { EU } \\
\text { EU }\end{array}$ & $\begin{array}{l}\text { KR } \\
\text { LA } \\
\text { LA } \\
\text { LA } \\
\text { GR }\end{array}$ & $\begin{array}{l}5 \\
5 \\
5 \\
5 \\
5\end{array}$ & $\begin{array}{l}1991 \\
1985 \\
1985 \\
1985 \\
1985\end{array}$ & $\begin{array}{r}102 \\
57 \\
57 \\
57 \\
19\end{array}$ & $\begin{array}{l}\text { AA } \\
\text { CA } \\
\text { CM } \\
\text { FO } \\
\text { PM }\end{array}$ & $\begin{array}{l}\text { Korean poet (16th century A.D.). } \\
\text { Albedo feature name. } \\
\text { Albedo feature name. } \\
\text { Albedo feature name. } \\
\text { Where lo passed by in her } \\
\text { wanderings. }\end{array}$ \\
\hline $\begin{array}{l}\mathrm{M} \\
\mathrm{M} \\
\mathrm{M} \\
\mathrm{L}\end{array}$ & $\begin{array}{l}\mathbf{M} \\
\mathbf{M} \\
\mathrm{M} \\
\mathrm{L}\end{array}$ & $\begin{array}{l}\text { Hydaspis Chaos } \\
\text { Hydrae Chasma } \\
\text { Hydraotes Chaos } \\
\text { Hyginus }\end{array}$ & $\begin{array}{l}3.4 \mathrm{~N} \\
6.9 \mathrm{~S} \\
0.9 \mathrm{~N} \\
7.8 \mathrm{~N}\end{array}$ & $\begin{array}{c}27.7 \mathrm{~W} \\
61.9 \mathrm{~W} \\
34.3 \mathrm{~W} \\
6.3 \mathrm{E}\end{array}$ & $\begin{array}{r}363 \\
49 \\
290 \\
9\end{array}$ & $\begin{array}{l}\text { EU } \\
\text { EU } \\
\text { EU } \\
\text { EU }\end{array}$ & $\begin{array}{l}\text { GR } \\
\text { LA } \\
\text { GR } \\
\text { SP }\end{array}$ & $\begin{array}{l}5 \\
5 \\
5 \\
5\end{array}$ & $\begin{array}{l}1976 \\
1985 \\
1976 \\
1935\end{array}$ & $\begin{array}{l}57 \\
57 \\
57 \\
66\end{array}$ & $\begin{array}{l}\mathrm{CH} \\
\mathrm{CM} \\
\mathrm{CH} \\
\mathrm{AA}\end{array}$ & $\begin{array}{l}\text { Classical albedo feature name. } \\
\text { Classical albedo feature name. } \\
\text { Classical albedo feature name. } \\
\text { Caius Julius; Spanish } \\
\text { astronomer (unkn-c. A.D. 100). }\end{array}$ \\
\hline $\begin{array}{l}\text { V } \\
\text { M }\end{array}$ & $\begin{array}{l}\mathbf{V} \\
\mathbf{M}\end{array}$ & $\begin{array}{l}\text { Hyndla Regio } \\
\text { Hypanis Valles }\end{array}$ & $\begin{array}{l}22.5 \mathrm{~N} \\
10.2 \mathrm{~N}\end{array}$ & $\begin{array}{l}294.5 \mathrm{E} \\
46.6 \mathrm{~W}\end{array}$ & $\begin{array}{r}2300 \\
270\end{array}$ & $\begin{array}{l}\text { EU } \\
\text { UR }\end{array}$ & $\begin{array}{l}\text { NS } \\
\text { SC }\end{array}$ & $\begin{array}{l}5 \\
5\end{array}$ & $\begin{array}{r}91 \\
1985\end{array}$ & $\begin{array}{l}27 \\
56\end{array}$ & $\begin{array}{l}\text { RE } \\
\text { VA }\end{array}$ & $\begin{array}{l}\text { Norse wood giantess. } \\
\text { Classical name for river in Scythia; } \\
\text { present Kuban River in Russia. }\end{array}$ \\
\hline L & $\mathrm{L}$ & Hypatia & $4.3 \mathrm{~S}$ & $22.6 \mathrm{E}$ & 40 & $\mathrm{AF}$ & EG & 5 & 1935 & 66 & AA & $\begin{array}{l}\text { Egyptian mathematician } \\
\text { (unkn-A.D. 415). }\end{array}$ \\
\hline $\begin{array}{l}\text { M } \\
\mathbf{M} \\
\mathbf{M} \\
\mathbf{M} \\
\mathbf{M}\end{array}$ & $\begin{array}{l}\mathbf{M} \\
\mathbf{M} \\
\mathbf{M} \\
\mathbf{M} \\
\mathbf{M}\end{array}$ & $\begin{array}{l}\text { Hyperboreae Undae } \\
\text { Hyperborei Cavi } \\
\text { Hyperboreus Labyrinthus } \\
\text { Hyperboreus (Lacus) } \\
\text { [Hyperboreus Scopulus] }\end{array}$ & $\begin{array}{l}77.5 \mathrm{~N} \\
79.6 \mathrm{~N} \\
79.8 \mathrm{~N} \\
75.0 \mathrm{~N} \\
82.5 \mathrm{~N}\end{array}$ & $\begin{array}{r}46.0 \mathrm{~W} \\
52.5 \mathrm{~W} \\
55.5 \mathrm{~W} \\
60.0 \mathrm{~W} \\
160.0 \mathrm{~W}\end{array}$ & $\begin{array}{r}301 \\
0 \\
0 \\
0 \\
0\end{array}$ & $\begin{array}{l}\text { EU } \\
\text { EU } \\
\text { EU } \\
\text { EU }\end{array}$ & $\begin{array}{l}\text { RM } \\
\text { RM } \\
\text { RO } \\
\text { LA } \\
\text { GR }\end{array}$ & $\begin{array}{l}5 \\
5 \\
5 \\
5 \\
6\end{array}$ & $\begin{array}{l}1988 \\
1988 \\
1988 \\
1958 \\
1984\end{array}$ & $\begin{array}{l}57 \\
57 \\
57 \\
60 \\
57\end{array}$ & $\begin{array}{l}\text { UN } \\
\text { CB } \\
\text { LB } \\
\text { AL } \\
\text { SC }\end{array}$ & $\begin{array}{l}\text { Classical albedo feature name. } \\
\text { Classical albedo feature name. } \\
\text { Classical albedo feature name. } \\
\text { Far northern lake. } \\
\text { Classical albedo feature } \\
\text { name; dropped } 1984 \text {. }\end{array}$ \\
\hline M & $\mathbf{M}$ & [Hypernotius Scopulus] & $78.0 \mathrm{~S}$ & $272.0 \mathrm{~W}$ & 0 & EU & GR & 6 & 1984 & 57 & $\mathrm{SC}$ & $\begin{array}{l}\text { Classical albedo feature } \\
\text { name; dropped } 1984 .\end{array}$ \\
\hline $\begin{array}{l}\mathbf{M} \\
\mathbf{M}\end{array}$ & $\begin{array}{l}\mathbf{M} \\
\mathbf{M}\end{array}$ & $\begin{array}{l}\text { Hypsas Vallis } \\
\text { Iamuna Dorsa }\end{array}$ & $\begin{array}{l}34.0 \mathrm{~N} \\
21.0 \mathrm{~N}\end{array}$ & $\begin{array}{r}302.1 \mathrm{~W} \\
50.3 \mathrm{~W}\end{array}$ & $\begin{array}{l}0 \\
0\end{array}$ & $\begin{array}{l}\text { EU } \\
\text { EU }\end{array}$ & $\begin{array}{l}\text { IT } \\
\text { LA }\end{array}$ & $\begin{array}{l}5 \\
5\end{array}$ & $\begin{array}{l}1987 \\
1988\end{array}$ & $\begin{array}{l}56 \\
71\end{array}$ & $\begin{array}{l}\text { VA } \\
\text { DO }\end{array}$ & $\begin{array}{l}\text { Classical name for river in Sicily. } \\
\text { From classical albedo } \\
\text { feature Iamunae Sinus. }\end{array}$ \\
\hline $\begin{array}{l}\mathbf{L} \\
\mathbf{M} \\
\mathbf{M}\end{array}$ & $\begin{array}{l}\mathrm{L} \\
\mathrm{M} \\
\mathrm{M}\end{array}$ & $\begin{array}{l}\text { Ian } \\
\text { Iani Chaos } \\
\text { Iapygia (Iapigia) }\end{array}$ & $\begin{array}{l}25.7 \mathrm{~N} \\
2.3 \mathrm{~S} \\
20.0 \mathrm{~S}\end{array}$ & $\begin{array}{r}0.4 \mathrm{~W} \\
16.6 \mathrm{~W} \\
295.0 \mathrm{~W}\end{array}$ & $\begin{array}{r}1 \\
565 \\
0\end{array}$ & $\begin{array}{l}\text { EU } \\
\text { EU } \\
\text { EU }\end{array}$ & $\begin{array}{l}\text { SC } \\
\text { LA } \\
\text { LA }\end{array}$ & $\begin{array}{l}5 \\
5 \\
5\end{array}$ & $\begin{array}{l}1976 \\
1976 \\
1958\end{array}$ & $\begin{array}{r}0 \\
57 \\
60\end{array}$ & $\begin{array}{l}\mathrm{AA} \\
\mathrm{CH} \\
\mathrm{AL}\end{array}$ & $\begin{array}{l}\text { Scottish male name. } \\
\text { Classical albedo feature name. } \\
\text { Classically, Iapygia was } \\
\text { all of Apulia, or just the } \\
\text { Salentine Peninsula. }\end{array}$ \\
\hline $\mathbf{M}$ & $\mathbf{M}$ & [Iaxartes Tholus] & $72.0 \mathrm{~N}$ & $15.0 \mathrm{~W}$ & 53 & EU & GR & 6 & 1979 & 57 & TH & $\begin{array}{l}\text { Classical albedo feature } \\
\text { name; name dropped } 1979 .\end{array}$ \\
\hline $\mathbf{M}$ & $\mathbf{M}$ & Iberus Vallis & $21.4 \mathrm{~N}$ & $208.1 \mathrm{~W}$ & 148 & EU & SP & 5 & 1985 & 56 & VA & $\begin{array}{l}\text { Classical name for river } \\
\text { present Ebro River in NE Spain. }\end{array}$ \\
\hline L & $\mathrm{L}$ & Ibn Battuta & $6.9 \mathrm{~S}$ & $50.4 \mathrm{E}$ & 11 & $\mathrm{AF}$ & MR & 5 & 1976 & $\mathbf{0}$ & AA & $\begin{array}{l}\text { Abu Abd Allah Mohammed Ibn } \\
\text { Abd Allah; Moroccan } \\
\text { geographer (1304-1377). }\end{array}$ \\
\hline L & $\mathrm{L}$ & Ibn Firnas & $6.8 \mathrm{~N}$ & $122.3 \mathrm{E}$ & 89 & EU & SP & 5 & 1976 & 0 & $\mathbf{A A}$ & $\begin{array}{l}\text { Spanish-Arab humanitarian, } \\
\text { technologist (unkn-c. A.D. 887). }\end{array}$ \\
\hline L & $\mathrm{L}$ & Ibn Yunus & $14.1 \mathrm{~N}$ & $91.1 \mathrm{E}$ & 58 & AF & EG & 5 & 1970 & 0 & $\mathbf{A A}$ & $\begin{array}{l}\text { C. Abu Muhammad Ibn-Rushd } \\
\text { (Averrdes); Egyptian } \\
\text { astronomer (950-1009). }\end{array}$ \\
\hline $\mathbf{L}$ & $\mathbf{L}$ & Ibn-Rushd & $11.7 \mathrm{~S}$ & $21.7 \mathrm{E}$ & 32 & EU & SP & 5 & 1976 & 0 & AA & $\begin{array}{l}\text { Spanish-Arab philosopher, } \\
\text { doctor (1126-1198). }\end{array}$ \\
\hline M & $\mathbf{M}$ & İbragimov & $25.9 \mathrm{~S}$ & $59.5 \mathrm{~W}$ & 89 & UR & SO & 5 & 1982 & 64 & $\mathbf{A A}$ & $\begin{array}{l}\text { Nadir Baba Ogly; Soviet } \\
\text { astronomer (1932-1977). }\end{array}$ \\
\hline H & H & Ibsen & $24.1 S$ & $35.6 \mathrm{~W}$ & 159 & EU & NO & 5 & 1976 & 59 & $\mathrm{AA}$ & $\begin{array}{l}\text { Heinrich J.; Norwegian } \\
\text { poet and dramatist (1828-1906). }\end{array}$ \\
\hline $\begin{array}{l}M \\
M\end{array}$ & $\begin{array}{l}\mathbf{M} \\
\mathbf{M}\end{array}$ & $\begin{array}{l}\text { Icaria } \\
\text { Icaria Fossae }\end{array}$ & $\begin{array}{l}40.0 \mathrm{~S} \\
53.7 \mathrm{~S}\end{array}$ & $\begin{array}{l}130.0 \mathrm{~W} \\
135.0 \mathrm{~W}\end{array}$ & $\begin{array}{r}0 \\
2153\end{array}$ & $\begin{array}{l}\text { EU } \\
\text { EU }\end{array}$ & $\begin{array}{l}\text { GR } \\
\text { GR }\end{array}$ & $\begin{array}{l}5 \\
5\end{array}$ & $\begin{array}{l}1958 \\
1985\end{array}$ & $\begin{array}{l}60 \\
57\end{array}$ & $\begin{array}{l}\text { AL } \\
\text { FO }\end{array}$ & $\begin{array}{l}\text { Land where Icarus lived (Crete). } \\
\text { From albedo feature at } \\
44 \mathrm{~S}, 130 \mathrm{~W} \text {. }\end{array}$ \\
\hline $\begin{array}{l}\mathbf{M} \\
\mathbf{L}\end{array}$ & $\begin{array}{l}\mathbf{M} \\
\mathbf{L}\end{array}$ & $\begin{array}{l}\text { Icaria Planum } \\
\text { Icarus }\end{array}$ & $\begin{array}{r}42.7 \mathrm{~S} \\
5.3 \mathrm{~S}\end{array}$ & $\begin{array}{l}107.2 \mathrm{~W} \\
173.2 \mathrm{~W}\end{array}$ & $\begin{array}{r}840 \\
96\end{array}$ & $\begin{array}{l}\text { EU } \\
\text { EU }\end{array}$ & $\begin{array}{l}\text { GR } \\
\text { GR }\end{array}$ & $\begin{array}{l}5 \\
5\end{array}$ & $\begin{array}{l}1979 \\
1970\end{array}$ & $\begin{array}{r}57 \\
0\end{array}$ & $\begin{array}{l}\text { PM } \\
\text { AA }\end{array}$ & $\begin{array}{l}\text { Classical albedo feature name. } \\
\text { Greek mythical flyer. }\end{array}$ \\
\hline
\end{tabular}

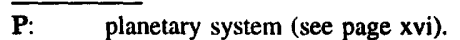

Sa: satellite (see page $x$ vi).

lat: latitude of feature center.

long: longitude of feature center. diam: diameter or long dimension of feature. ct: continent of name origin (see page $284 \mathrm{ff}$.)

et: ethnicity of name origin (see page $284 \mathrm{ff}$.)

as: name approval status (see page xvii). ad: name approval date (year).

ref: reference source for name (see page $287 \mathrm{ff}$.).

ft: feature type (see page 290). 


\section{ALPHABETICAL LIST OF NAMES}

\section{P Sa Name \\ V V Ichikawa \\ $\mathrm{H} \quad \mathrm{H}$ Ictinus \\ J am Ida Facula \\ $S$ ja Idas \\ L L Ideler \\ L L Idel'son \\ V V Idem-Kuva Corona \\ V V Idunn Mons \\ $\mathrm{J}$ ca Igaluk \\ M M Igol \\ $\mathrm{S}$ mi Igraine \\ M M II Thyle I}

M M Ikej

$\begin{array}{lll}\text { J } & \text { ga } & \text { Ilah } \\ \text { V } & \text { V } & \text { Ilbis Fossae }\end{array}$

$\begin{array}{lll}\text { V } & \text { V } & \text { Ilga } \\ \text { S } & \text { di } & \text { Ilia }\end{array}$

L L Il'in

V V Ilithyia Mons

J ca Ilma

$\mathrm{J}$ io Ilmarinen Patera

$\mathrm{N}$ tr Ilomba

J ga Ilus

$\begin{array}{lll}\text { V } & \text { ga } & \text { Ilus } \\ \text { Imdr Regio }\end{array}$

M M Imgr

H H Imhotep

$\mathrm{U}$ ti Imogen

L L Ina

$\mathrm{J}$ io Inachus Tholus

V V Inanna Corona

V V Inari Corona

$\mathrm{L} L$ Index

V V Indira

V V Indrani Corona

M M Indus Vallis

L L Ingalls

L L Inghirami

V V Ingrid

V V Inira

L L Innes

V V Innini Mons

M M Innsbruck

M M Ins

M M Inta

J io Inti Patera

M M Inuvik

U mr Inverness Corona

L L Ioffe lat long diam

$\begin{array}{rrrrrrrr}61.6 \mathrm{~S} & 156.4 \mathrm{E} & 36 & \text { AS } & \text { JA } & 5 & 1994 & 99 \\ 79.1 \mathrm{~S} & 165.2 \mathrm{~W} & 119 & \text { EU } & \text { GR } & 5 & 1976 & 59 \\ & & & & & & & \\ 20.0 \mathrm{~N} & 175.0 \mathrm{~W} & 0 & \text { EU } & \text { GR } & 5 & 1979 & 19 \\ & & & & & & & \\ 0.0 \mathrm{~N} & 0.0 \mathrm{~W} & 0 & \text { EU } & \text { GR } & 5 & 1982 & 1 \\ 49.2 \mathrm{~S} & 22.3 \mathrm{E} & 38 & \text { EU } & \text { GE } & 5 & 1935 & 66 \\ & & & & & & & \\ 81.5 \mathrm{~S} & 110.9 \mathrm{E} & 60 & \text { UR } & \text { SO } & 5 & 1970 & 0 \\ & & & & & & & \\ 25.0 \mathrm{~N} & 358.0 \mathrm{E} & 230 & \text { EU } & \text { FI } & 5 & 1994 & 27 \\ 46.5 \mathrm{~S} & 213.5 \mathrm{E} & 250 & \text { EU } & \text { NS } & 5 & 1994 & 111 \\ 5.6 \mathrm{~N} & 315.9 \mathrm{~W} & 105 & \text { NA } & \text { ES } & 5 & 1979 & 26 \\ 20.4 \mathrm{~S} & 254.0 \mathrm{~W} & 0 & \text { EU } & \text { HU } & 5 & 1989 & 74 \\ 42.1 \mathrm{~S} & 231.1 \mathrm{~W} & 0 & \text { EU } & \text { GB } & 5 & 1982 & 37 \\ 70.0 \mathrm{~S} & 180.0 \mathrm{~W} & 0 & \text { EU } & \text { GR } & 5 & 1958 & 60\end{array}$

21.1N 247.6W

$228 \mathrm{~N}$

$71.9 \mathrm{~N} \quad 254.6 \mathrm{E}$

UR RU

$\begin{array}{rll}71 & \text { AS } & \text { SU } \\ 512 & \text { UR } & \text { YK }\end{array}$

$5 \quad 1988$ $\begin{array}{ll}5 & 1985 \\ 5 & 1985\end{array}$

12.4S 307.4E

$0.1 \mathrm{~N} 346.0 \mathrm{~W}$

$\begin{array}{lll}5 & 1994 & 108\end{array}$

$17.8 \mathrm{~S} \quad 97.5 \mathrm{~W}$

13 UR SO

51985

$13.5 \mathrm{~S}$

315.5E

$30.0 \mathrm{~S} \quad 167.2 \mathrm{~W}$

14.0S $2.8 \mathrm{~W}$

90 EU GR

1994

14.5S $\quad 57.0 \mathrm{E}$

$11.5 \mathrm{~S}$

43.0S

$19.3 \mathrm{~N}$

$18.1 \mathrm{~S}$

$110.8 \mathrm{~W}$

$212.0 \mathrm{E}$

249.0W

$37.3 \mathrm{~W}$

23.8S $321.2 \mathrm{E}$

49.2S 22.3E

$15.9 \mathrm{~S} \quad 348.9 \mathrm{~W}$

$37.0 \mathrm{~S} \quad 35.9 \mathrm{E}$

18.0S $120.3 \mathrm{E}$

$26.1 \mathrm{~N} \quad 3.7 \mathrm{E}$

64.1N $\quad 289.8 \mathrm{E}$

$37.5 \mathrm{~S} \quad 70.5 \mathrm{E}$

$19.2 \mathrm{~N} \quad 322.1 \mathrm{~W}$

53 EU FI

$\begin{array}{ll}5 & 1988\end{array}$

40 EU FI

51985

0 AF $\mathrm{ZM}$

591

41 EU GR

1611 EU NS

0 UR RU

51985

51982

$\begin{array}{ll}5 & 1988\end{array}$

51976

28 EU GB

$\begin{array}{rlr}38 & \text { EU } & \text { LA } \\ 0 & \text { EU } & \text { GR }\end{array}$

51988

350

300
0

AS SE

AS JA

1976

51979

51994

1994

$\begin{array}{ll}5 & 1994 \\ 5 & 1973\end{array}$

$\begin{array}{rllll}17 & \text { AS } & \text { IN } & 5 & 1985 \\ 200 & \text { AS } & \text { IN } & 5 & 1994\end{array}$

253 AS PK

51982

$26.4 \mathrm{~N} \quad 153.1 \mathrm{~W}$

37

NA AM

51970

47.5S $68.8 \mathrm{~W}$

91

EU IT

51935

12.4S 308.8E

43.1S 239.2E

27.8N $119.2 \mathrm{E}$

15 EU SD

$\begin{array}{lll}17 & \text { UR } & \text { RU } \\ 42 & \text { EU } & \text { SC }\end{array}$

51994

51994

51970

34.6S $\quad 328.5 \mathrm{E}$

339

6.5S 40.0W

$24.8 \mathrm{~N} \quad 251.2 \mathrm{~W}$

24.6S 24.9W

68.1S $348.6 \mathrm{~W}$

$79.1 \mathrm{~N} \quad 39.7 \mathrm{~W}$

66.9S 325.7E

$14.4 \mathrm{~S}$

AS BY

51982

64 EU AS

EU SZ

UR RU

SA IN

51976

$\begin{array}{ll}5 & 1988\end{array}$

51976

$\begin{array}{ll}5 & 1979\end{array}$

$\begin{array}{ll}5 & 1988\end{array}$

234

EU SC

$5 \quad 1988$ ref

ft origin (1893-1981)

AA Greek architect (5th century B.C.).

FA Greek; mountain where Zeus played as a child.

AA Twins; cousins of Gemini.

AA Christian Ludwig; German astronomer (1766-1846).

AA Naum I.; Soviet astronomer (1885-1951)

CR Finno-Ugraic harvest spirit.

MO Norse goddess.

AA Alaskan name of the Moon.

AA Town in Hungary.

AA Wife of Uther; mother of Arthur.

AL Named for Thule; may be middle Norway; used to indicate far northern lands.

AA Town in Russia.

AA First Sumerian sky god.

FO Yakutian (Siberia) goddess of bloodshed.

AA Latvian first name.

AA Also known as Rhea Silvia; Mother by Mars of Romulus and Remus, the founders of Rome.

AA N.Ja.; Soviet rocketry scientist (1901-1937)

MO Greek goddess of childbirth.

AA A celestial divinity of air.

PE Finnish blacksmith with supernatural creative powers.

AA Lozi (Zambia) water snake linked with destruction.

AA Ganymede's brother.

RE Norse giantess.

AA Town in Russia.

AA Egyptian physician and sage (c. 2686-2613 B.C.)

AA Cymbelline's daughter.

AA Latin female name.

TH Greek; river god, father of Io.

CR Semitic fertility goddess.

CR Japanese rice goddess.

LF Astronaut-named feature, Apollo 15 site.

AA Hindu first name.

CR Hindu fertility goddess.

VA Ancient and modern name for river in Pakistan.

AA Albert L.; American optician (1888-1958).

AA Giovanni; Italian astronomer (1779-1851).

AA Scandanavian first name.

AA Eskimo first name.

AA Robert T. A.; Scottish astronomer (1861-1933).

MO Babylonian earth mother worshipped at Kish.

AA Town in Austria.

AA Town in Switzerland.

AA Town in Russia.

PE Inca sun god.

AA Town in Northwest Territories, Canada.

CR Location of MacBeth's castle.

AA Joffe, Abram F.; Soviet physicist (1880-1960).

\footnotetext{
P: $\quad$ planetary system (see page $x v i)$.

Sa: $\quad$ satellite (see page $\mathrm{xvi}$ ).

lat: latitude of feature center.

long: longitude of feature center.
}

diam: diameter or long dimension of feature.

ct: continent of name origin (see page $284 \mathrm{ff}$.)

et: ethnicity of name origin (see page $284 \mathrm{ff}$.)

as: name approval status (see page $x v i i)$. ad: name approval date (year).

ref: reference source for name (see page $287 \mathrm{ff}$.).

ft: feature type (see page 290). 
ALPHABETICAL LIST OF NAMES

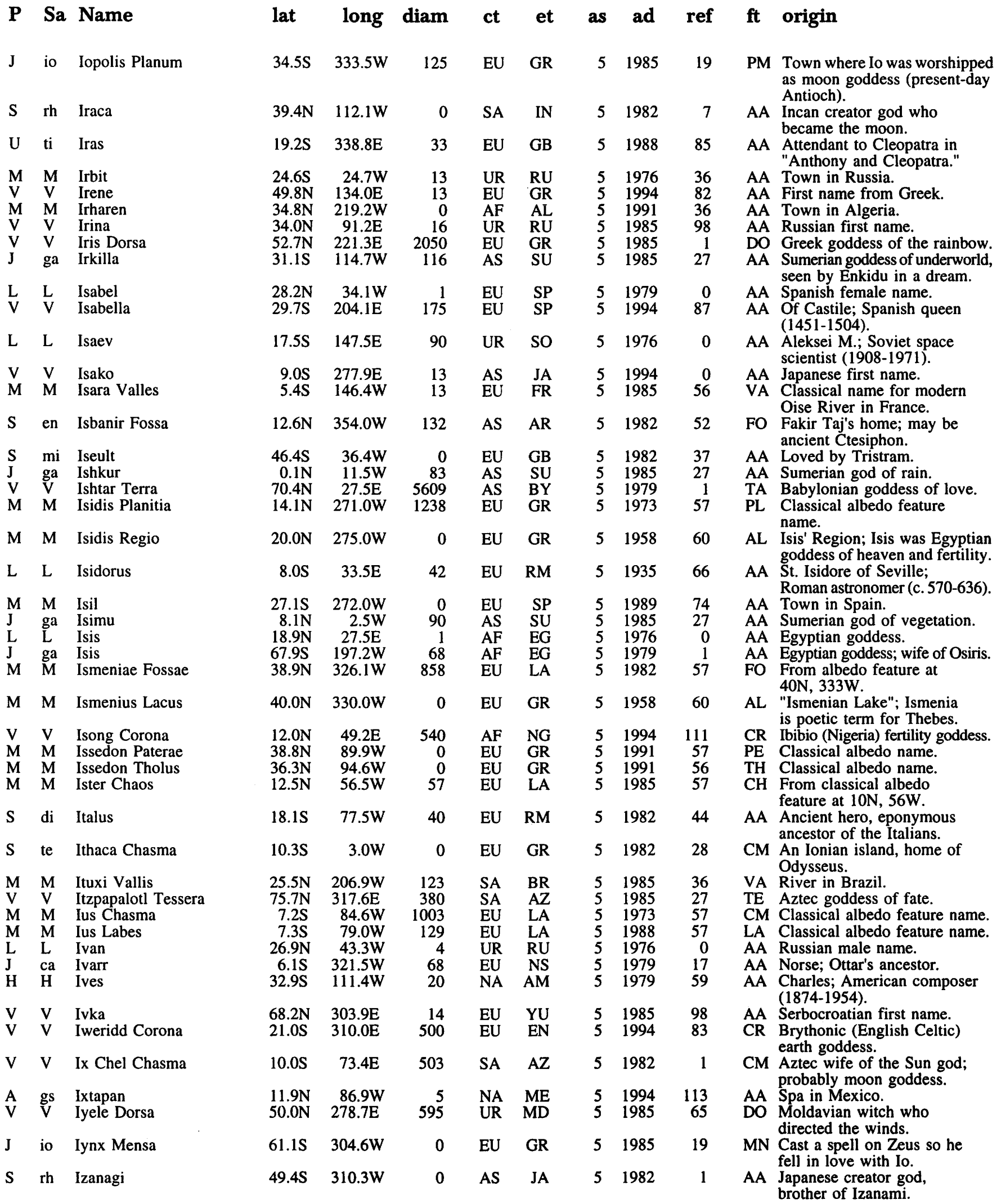

\footnotetext{
P: $\quad$ planetary system (see page xvi).

Sa: $\quad$ satellite (see page $x v i)$.

lat: latitude of feature center.

long: longitude of feature center.
}

diam: diameter or long dimension of feature.
ct: continent of name origin (see page $284 \mathrm{ff}$.)
et: ethnicity of name origin (see page $284 \mathrm{ff}$.)
as: name approval status (see page $x v i i$ ).

ad: name approval date (year).

ref: reference source for name (see page $287 \mathrm{ff}$.).

ft: feature type (see page 290). 


\section{ALPHABETICAL LIST OF NAMES}

\section{P Sa Name}

S rh Izanami

M M Izendy

L L Izsak

V V Izumi Patera

L L J. Hersche]

L L Jackson

L L Jacobi

$\mathrm{V} V$ Jacqueline

V V Jadwiga

V V Jael Mons

M M Jal

M M Jama

L L [James]

M M Jampur

M M Jamuna

H H Janáček

L L Jansen

L L Jansky

M M Janssen

L L Janssen

$S$ hy Jarilo

M M Jarry-Desloges

L L Jarvis

V V Jeanne

M M Jeans

L L Jeans

L L Jehan

M M Jeki

M M Jen

L L Jenkins

L L Jenner

V V Jennifer

L L Jerik

V V Jerusha

$\mathrm{U}$ ti Jessica

V V Jex-Blake

M M Jezža

V V Jhirad

M M Jijiga

V V Jocelyn

M M Jodrell

V V Johanna

M M Johannesburg

V V Johnson

L L [Johnson]

H H Jókai

V V Joliet-Curie lat long diam ct et as ad ref $f$ origin

\begin{tabular}{|c|c|c|c|c|c|c|}
\hline $46.3 S$ & $313.4 \mathrm{~W}$ & 0 & AS & JA & 5 & 1982 \\
\hline $\begin{array}{l}29.4 S \\
23.3 S\end{array}$ & $\begin{array}{l}101.3 \mathrm{~W} \\
117.1 \mathrm{E}\end{array}$ & $\begin{array}{r}0 \\
30\end{array}$ & $\begin{array}{l}\text { UR } \\
\text { NA }\end{array}$ & $\begin{array}{l}\text { RU } \\
\text { AM }\end{array}$ & $\begin{array}{l}5 \\
5\end{array}$ & $\begin{array}{l}1989 \\
1970\end{array}$ \\
\hline $\begin{array}{l}50.3 \mathrm{~N} \\
62.0 \mathrm{~N}\end{array}$ & $\begin{array}{c}193.6 \mathrm{E} \\
42.0 \mathrm{~W}\end{array}$ & $\begin{array}{r}74 \\
165\end{array}$ & $\begin{array}{l}\text { AS } \\
\text { EU }\end{array}$ & $\begin{array}{l}\text { JA } \\
\text { GB }\end{array}$ & $\begin{array}{l}5 \\
5\end{array}$ & $\begin{array}{l}1985 \\
1935\end{array}$ \\
\hline $22.4 \mathrm{~N}$ & $163.1 \mathrm{~W}$ & 71 & EU & SC & 5 & 1970 \\
\hline $56.7 \mathrm{~S}$ & $11.4 \mathrm{E}$ & 68 & EU & GE & 5 & 1935 \\
\hline $\begin{array}{l}70.0 \mathrm{~S} \\
68.4 \mathrm{~N} \\
51.2 \mathrm{~N} \\
26.6 \mathrm{~S} \\
21.6 \mathrm{~N} \\
10.2 \mathrm{~N}\end{array}$ & $\begin{array}{c}123.8 \mathrm{E} \\
91.0 \mathrm{E} \\
120.8 \mathrm{E} \\
28.6 \mathrm{~W} \\
53.1 \mathrm{~W} \\
50.4 \mathrm{E}\end{array}$ & $\begin{array}{r}17 \\
13 \\
36 \\
5 \\
0 \\
28\end{array}$ & $\begin{array}{l}\text { EU } \\
\text { EU } \\
\text { AS } \\
\text { US } \\
\text { AF } \\
\text { NA }\end{array}$ & $\begin{array}{r}\text { FR } \\
\text { PO } \\
\text { HE } \\
\text { NM } \\
\text { TN } \\
\text { AM }\end{array}$ & $\begin{array}{l}5 \\
5 \\
5 \\
5 \\
5 \\
6\end{array}$ & $\begin{array}{r}1994 \\
1985 \\
91 \\
1976 \\
1988 \\
0\end{array}$ \\
\hline $\begin{array}{l}38.8 \mathrm{~N} \\
10.0 \mathrm{~N} \\
56.0 \mathrm{~N}\end{array}$ & $\begin{array}{r}81.0 \mathrm{~W} \\
40.0 \mathrm{~W} \\
153.8 \mathrm{~W}\end{array}$ & $\begin{array}{r}0 \\
0 \\
47\end{array}$ & $\begin{array}{l}\text { AS } \\
\text { EU } \\
\text { EU }\end{array}$ & $\begin{array}{l}\text { PK } \\
\text { LA } \\
\text { CZ }\end{array}$ & $\begin{array}{l}5 \\
5 \\
5\end{array}$ & $\begin{array}{l}1991 \\
1958 \\
1985\end{array}$ \\
\hline $13.5 \mathrm{~N}$ & 28.7E & 23 & EU & DU & 5 & 1935 \\
\hline $8.5 \mathrm{~N}$ & $89.5 \mathrm{E}$ & 72 & NA & AM & 5 & 1964 \\
\hline $2.8 \mathrm{~N}$ & $322.4 \mathrm{~W}$ & 166 & $\mathrm{EU}$ & FR & 5 & 1973 \\
\hline $45.4 S$ & $40.3 \mathrm{E}$ & 199 & EU & FR & 5 & 1935 \\
\hline $61.0 \mathrm{~N}$ & $183.0 \mathrm{~W}$ & 0 & UR & RU & 5 & 1982 \\
\hline $9.5 \mathrm{~S}$ & $276.1 \mathrm{~W}$ & 97 & EU & FR & 5 & 1973 \\
\hline $34.9 \mathrm{~S}$ & $148.9 \mathrm{~W}$ & 38 & NA & AM & 5 & 1988 \\
\hline
\end{tabular}

$\begin{array}{llllllll}40.0 \mathrm{~N} & 331.5 \mathrm{E} & 20 & \mathrm{EU} & \text { FR } & 5 & 1985 & 83\end{array}$

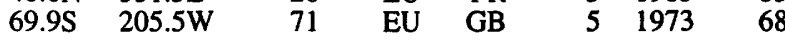

$\begin{array}{llllllll}55.8 \mathrm{~S} & 91.4 \mathrm{E} & 79 & \mathrm{EU} & \mathrm{GB} & 5 & 1964 & 67\end{array}$

$20.7 \mathrm{~N} \quad 31.9 \mathrm{~W}$

$23.9 \mathrm{~N} \quad 52.3 \mathrm{~W}$

$40.1 \mathrm{~N} \quad 10.6 \mathrm{~W}$

$\begin{array}{llll}\mathrm{AF} & \mathrm{NI} & 5 & 1976\end{array}$

$\begin{array}{lrrr}\text { AS } & \text { TU } & 5 & 1976 \\ \text { AF } & \text { ET } & 5 & 1988 \\ \text { AF } & \text { NI } & 5 & 1976 \\ \text { NA } & \text { AM } & 5 & 1982\end{array}$

42.1S $\quad 95.9 \mathrm{E}$

$4.6 \mathrm{~S} \quad 99.8 \mathrm{E}$

$18.5 \mathrm{~N} \quad 27.6 \mathrm{E}$

$22.0 \mathrm{~S} \quad 342.7 \mathrm{E}$

$55.3 \mathrm{~S} \quad 285.9 \mathrm{E}$

$65.3 \mathrm{~N} \quad 169.0 \mathrm{E}$

$48.8 \mathrm{~S} \quad 37.7 \mathrm{~W}$

$16.8 \mathrm{~S} \quad 105.6 \mathrm{E}$

$25.2 \mathrm{~N} \quad 53.7 \mathrm{~W}$

33.2S 276.4E

$47.8 \mathrm{~N} \quad 227.6 \mathrm{~W}$

$19.5 \mathrm{~N} \quad 247.2 \mathrm{E}$

$48.2 \mathrm{~N} \quad 226.7 \mathrm{~W}$

$51.9 \mathrm{~N} \quad 254.5 \mathrm{E}$

EU GB

1970

$\begin{array}{lllll}71 & \text { EU } & \text { GB } & 5 & 1970 \\ 9 & \text { EU } & \text { GR } & 5 & 1994\end{array}$

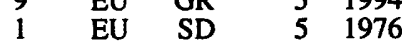

17 AS HE

64 EU GB

$\begin{array}{ll}5 & 1994 \\ 5 & 1988\end{array}$

33 EU GB

$\begin{array}{ll}5 & 1991\end{array}$

$\begin{array}{lllll}6 & \text { UR } & \text { RU } & 5 & 1976\end{array}$

$\begin{array}{rrrrr}50 & \text { AS } & \text { IN } & 5 & 1994 \\ 14 & \text { AF } & \text { ET } & 5 & 1976\end{array}$

$\begin{array}{lllll}14 & \text { AF } & \text { ET } & 5 & 1976 \\ 14 & \text { EU } & \text { GE } & 5 & 1994\end{array}$

EU GB

$\begin{array}{ll}\text { AS } & \text { HE } \\ \text { AF } & \text { SA }\end{array}$

$\begin{array}{ll}5 & 1979 \\ 5 & 1994\end{array}$

$5 \quad 1994$

51979

51994

\begin{tabular}{ll}
$8.7 \mathrm{~S} \quad 89.0 \mathrm{E}$ \\
\hline
\end{tabular}

OC AU

$\begin{array}{rr}6 & 0 \\ 5 & 1979\end{array}$

$72.4 \mathrm{~N}$

$135.3 \mathrm{~W}$

EU GB

$\begin{array}{llllllll}1.6 \mathrm{~S} & 62.5 \mathrm{E} & 100 & \mathrm{EU} & \text { FR } & 5 & 1991 & 99\end{array}$

AA Sister and wife of Izanagi; creator goddess.

AA Town in Russia.

AA Imre; Hungarian-American astronomer (1929-1965).

PE Sikibu; Japanese writer (974-1036)

AA John; British astronomer (1792-1871).

AA John; Scottish astronomer (1887-1958)

AA Karl Gustav Jacob; German mathematician (1804-1851).

AA First name from French.

AA Polish first name.

MO Hebrew goddess of dawn.

AA Town in New Mexico, USA.

AA Town in Tunisia.

AA Henry; American writer (1843-1916).

AA Town in Pakistan.

AL Present Jumna River, India.

AA Leos; Czechoslovakian composer (1854-1928).

AA Janszoon, Zacharias; Dutch optician (1580-c. 1638)

AA Karl; American radio engineer (1905-1950)

AA Pierre J.; French astronomer (1824-1907).

AA Pierre Jules; French astronomer (1824-1907).

AA East Slavic god of the sun, fertility and love.

AA Rene; French astronomer (1868-1951)

AA Gregory B.; Member of the Challenger crew (1944-1986); previous designation Borman Z

AA French first name.

AA James H.; British physicist, astronomer (1877-1946).

AA Sir James H.; British mathematical physicist (1877-1946).

AA Turkish female name.

AA Town in Ethiopia.

AA Town in Nigeria.

AA Louise F.; American astronomer (1888-1970).

AA Edward; British doctor (1749-1823).

AA First name from Greek.

AA Scandinavian male name.

AA Hebrew first name.

AA Shylock's daughter in "The Merchant of Venice."

AA Sophia; British pioneer woman physician (1840-1912).

AA Town in Russia.

AA Jerusha; Indian physician.

AA Town in Ethiopia.

AA German first name.

AA United Kingdom tracking site.

AA Hebrew first name.

AA Republic of South Africa tracking site.

AA Amy; Australian aviator (1903-1941).

AA Samuel; British writer (1709-1784).

AA Mor; Hungarian novelist (1825-1904)
AA Irene; French physicist, Nobel laureate (1897-1956).
P: $\quad$ planetary system (see page xvi).

Sa: $\quad$ satellite (see page $x v i$ ).

lat: latitude of feature center.

long: longitude of feature center. diam: diameter or long dimension of feature. ct: continent of name origin (see page $284 \mathrm{ff}$.)

et: ethnicity of name origin (see page $284 \mathrm{ff}$.)

as: name approval status (see page xvii). ad: name approval date (year).

ref: reference source for name (see page $287 \mathrm{ff}$.).

ft: feature type (see page 290). 
ALPHABETICAL LIST OF NAMES

\section{P Sa Name}

L L Joliot

M M Joly

$\begin{array}{lll}\mathrm{L} & \mathrm{L} & \text { Jomo } \\ \mathrm{M} & \mathbf{M} & \text { Jones }\end{array}$

V V Jord Corona

L L José

$\mathrm{V}$ V [Josefina]

V V Joshee

L L Joule

M M Jovis Fossae

M M Jovis Tholus

L L Joy

V V Juanita

H H Judah Ha-Levi

V V Judith

L L Jules Verne

V V Julie

L L Julienne

L L Julius Caesar

$S$ en Julnar

J ca Jumal

$N$ tr Jumna Fossae

$S$ rh Jumo

J ca Jumo

V V Junkgowa Corona

V V Juno Chasma

V V Juno Dorsum

V V Jurate Colles

M M Juventae Chasma

M M Juventae Dorsa

M M Juventae Fons

U ar Kachina Chasmata

M M Kachug

J ga Kadi

M M Kagoshima

M M Kagul

V V Kahlo

M M Kāid

V V Kaikilani

L L Kaiser

M M Kaiser

V V Kaiwan Fluctus

M M Kaj

M M Kakori

V V Kala

V V Kalaipahoa Linea

H H Kālidāsā

M M Kaliningrad lat

long

25

$\begin{array}{rrrrrrrr}25.8 \mathrm{~N} & 93.1 \mathrm{E} & 164 & \text { EU } & \text { FR } & 5 & 1970 & 0 \\ & & & & & & & \\ 74.6 \mathrm{~S} & 42.5 \mathrm{~W} & 81 & \text { EU } & \text { IR } & 5 & 1973 & 68 \\ 24.4 \mathrm{~N} & 1.6 \mathrm{~W} & 7 & \text { AF } & \text { UN } & 5 & 1976 & 0 \\ 19.1 \mathrm{~S} & 19.8 \mathrm{~W} & 85 & \text { EU } & \text { GB } & 5 & 1973 & 68 \\ & & & & & & & \\ 58.5 \mathrm{~S} & 349.5 \mathrm{E} & 130 & \text { EU } & \text { NS } & 5 & 1994 & 1 \\ 12.7 \mathrm{~S} & 1.6 \mathrm{~W} & 2 & \text { EU } & \text { SP } & 5 & 1976 & 0 \\ 44.9 \mathrm{~N} & 32.3 \mathrm{E} & 26 & \text { EU } & \text { PG } & 6 & 1994 & 83 \\ 5.5 \mathrm{~N} & 288.8 \mathrm{E} & 34 & \text { AS } & \text { IN } & 5 & 1994 & 99 \\ & & & & & & & \\ 27.3 \mathrm{~N} & 144.2 \mathrm{~W} & 96 & \text { EU } & \text { GB } & 5 & 1970 & 0 \\ & & & & & & & \\ 19.5 \mathrm{~N} & 116.1 \mathrm{~W} & 412 & \text { EU } & \text { GR } & 5 & 1985 & 57 \\ & & & & & & & \\ 18.4 \mathrm{~N} & 117.5 \mathrm{~W} & 61 & \text { EU } & \text { GR } & 5 & 1973 & 57 \\ 25.0 \mathrm{~N} & 6.6 \mathrm{E} & 5 & \text { NA } & \text { AM } & 5 & 1973 & 0 \\ 62.9 \mathrm{~S} & 89.9 \mathrm{E} & 19 & \text { EU } & \text { SP } & 5 & 1994 & 82 \\ 10.9 \mathrm{~N} & 107.7 \mathrm{~W} & 80 & \text { AS } & \text { JW } & 5 & 1976 & 59 \\ & & & & & & & \\ 29.1 \mathrm{~S} & 104.5 \mathrm{E} & 20 & \text { AS } & \text { HE } & 5 & 1994 & 82 \\ 35.0 \mathrm{~S} & 147.0 \mathrm{E} & 143 & \text { EU } & \text { FR } & 5 & 1961 & 67 \\ 50.9 \mathrm{~N} & 242.5 \mathrm{E} & 13 & \text { EU } & \text { CZ } & 5 & 1985 & 98 \\ 26.0 \mathrm{~N} & 3.2 \mathrm{E} & 2 & \text { EU } & \text { FR } & 5 & 1976 & 0 \\ 0.0 \mathrm{~N} & 0.0 \mathrm{X} & 0 & \text { EU } & \text { GB } & 5 & 1988 & 85\end{array}$

$9.0 \mathrm{~N} \quad 15.4 \mathrm{E}$

$54.2 \mathrm{~N} \quad 342.0 \mathrm{~W}$

$58.8 \mathrm{~N}$

$13.5 \mathrm{~S}$

$52.8 \mathrm{~N} \quad 66.5 \mathrm{~W}$

$65.9 \mathrm{~N} \quad 12.3 \mathrm{~W}$

$37.0 \mathrm{~N} \quad 257.0 \mathrm{E}$

30.5S 111.1E

$31.0 \mathrm{~S}$

95.6E

$56.8 \mathrm{~N} \quad 153.5 \mathrm{E}$

$1.9 \mathrm{~S} \quad 61.8 \mathrm{~W}$

$1.3 \mathrm{~N} \quad 72.6 \mathrm{~W}$

$5.0 \mathrm{~S}$

33.7S 246.0E

$63.0 \mathrm{~W}$

$18.4 \mathrm{~N} \quad 252.5 \mathrm{~W}$

$48.8 \mathrm{~N}$

$47.6 \mathrm{~N}$

$24.0 \mathrm{~S}$

$59.9 \mathrm{~S}$
$4.6 \mathrm{~S}$

32.7S

$181.0 \mathrm{~W}$

224.1W

$18.9 \mathrm{~W}$

$178.8 \mathrm{E}$

$44.8 \mathrm{~W}$

$163.1 \mathrm{E}$

$36.5 \mathrm{~S}$

$6.5 \mathrm{E}$

46.6S

$340.9 \mathrm{~W}$

48.0S

$27.4 \mathrm{~S}$

$41.9 \mathrm{~S}$

$1.5 \mathrm{~N}$
$60.5 \mathrm{~S}$

$1.5 \mathrm{E}$
$29.2 \mathrm{~W}$

$29.6 \mathrm{~W}$

$60.5 \mathrm{~S}$

314.2E

18.1S

179.2W

$48.8 \mathrm{~N} 224.9 \mathrm{~W}$
90

\section{EU RM}

AS AR

$5 \quad 1935$

$5 \quad 1982$

60

0
0
37

UR ES

EU FI

400 OC AU

915 EU RM

1652

EU RM

418

495

325

UR LI

$\begin{array}{ll}\text { EU } & \text { GR } \\ & \text { GR }\end{array}$

$\begin{array}{ll}5 & 1987\end{array}$

$\begin{array}{rr}5 & 91 \\ 5 & 1982\end{array}$

$\begin{array}{ll}5 & 1979\end{array}$

$\begin{array}{ll}5 & 1994\end{array}$

51982

$5 \quad 1982$

$\begin{array}{ll}5 & 1985\end{array}$

$\begin{array}{ll}5 & 1973 \\ 5 & 1985\end{array}$

0 UR RU

1 AS JA

8 UR MD

36 NA ME

19

OC HA

51988

51985

$\begin{array}{ll}5 & 1979\end{array}$

51976

$\begin{array}{ll}5 & 1994\end{array}$

$\begin{array}{ll}5 & 1994 \\ 5 & 1976\end{array}$

51994

52 EU DU

51935

51973

201 EU DU

$\begin{array}{rr}5 & 91 \\ 5 & 1976\end{array}$

$\begin{array}{rrr}1200 & \text { AF } & \text { ET } \\ 2 & \text { UR } & \text { RU }\end{array}$

25 AS IN

2400

107

2
AS IN

$\begin{array}{lllll}0 & \text { EU } & \text { GR } & 5 & 1958\end{array}$

$\begin{array}{lllll}622 & \text { NA } & \text { HO } & 5 & 1988\end{array}$

94 AS BY

7 AS IQ

17 EU RU
AA Frederic Joliot-Curie; French physicist; Nobel laureate (1900-1958).

AA John; Irish geologist (1857-1933).

AA African male name.

AA Harold S.; British astronomer (1890-1960).

CR Norse earth goddess.

AA Spanish male name.

AA Portuguese first name.

AA Anandibai; Indian pioneer physician (1865-1887).

AA James P.; British physicist (1818-1889).

FO From albedo feature at $16 \mathrm{~N}, 111 \mathrm{~W}$.

TH Classical albedo feature name.

AA Alfred H.; American astronomer (1882-1973).

AA Spanish first name.

AA Judah; Jewish poet and religious philosopher (c. 1075-1141).

AA Hebrew first name.

AA French writer (1828-1905).

AA Czech, German first name.

AA French female name.

SS 1986 U2; beloved of Romeo in "Romeo and Juliet" $64,352 \mathrm{~km}$ semi-major axis

AA Roman emperor (c. 102-44 B.C.).

AA The seaborn; heroine of nights 738 to 756 .

AA Estonian sky god.

FO Hindu river goddess.

AA Marijan sky god.

AA Finno-Ugric heaven god.

CR Yulengor (Australia) fertility goddess.

CM Roman sky goddess; sister and consort of Jupiter.

DO Roman sky goddess; sister and consort of Jupiter.

CO Lithuanian sea goddess.

CM Classical albedo feature name.

DO From albedo feature at $4 S$, 63W.

60

87

AL The "Fountain of Youth;" a fountain in India.

CM Pueblo (USA) good spirits who bring rain or other blessings.

AA Town in Russia.

AA Babylonian goddess of justice.

AA Japanese launch site.

AA Town in Moldova.

AA Frida; Mexican artist (1910-1954).

AA Town in Iraq.

AA First female ruler of Hawaii (c. 1555).

AA Frederick; Dutch astronomer (1808-1872).

AA Frederick; Dutch astronomer (1808-1872).

FL Ethiopian earth mother.

AA Town in Russia.

AA Town in India.

AA Kamchatka first name.

LI Hawaiian war goddess.

AA Indian poet and dramatist (c. 5th century).

AA Soviet mission control site

\footnotetext{
P: $\quad$ planetary system (see page $\mathrm{xvi}$ ).

Sa: satellite (see page $\mathrm{xvi}$ ).

lat: lattude of feature center.

long: longitude of feature center.
}

diam: diameter or long dimension of feature.

ct: continent of name origin (see page $284 \mathrm{ff}$.)

et: ethnicity of name origin (see page $284 \mathrm{ff}$.)

as: name approval status (see page xvii). ad: name approval date (year).

ref: reference source for name (see page $287 \mathrm{ff}$.). ft: feature type (see page 290). 


\section{ALPHABETICAL LIST OF NAMES}

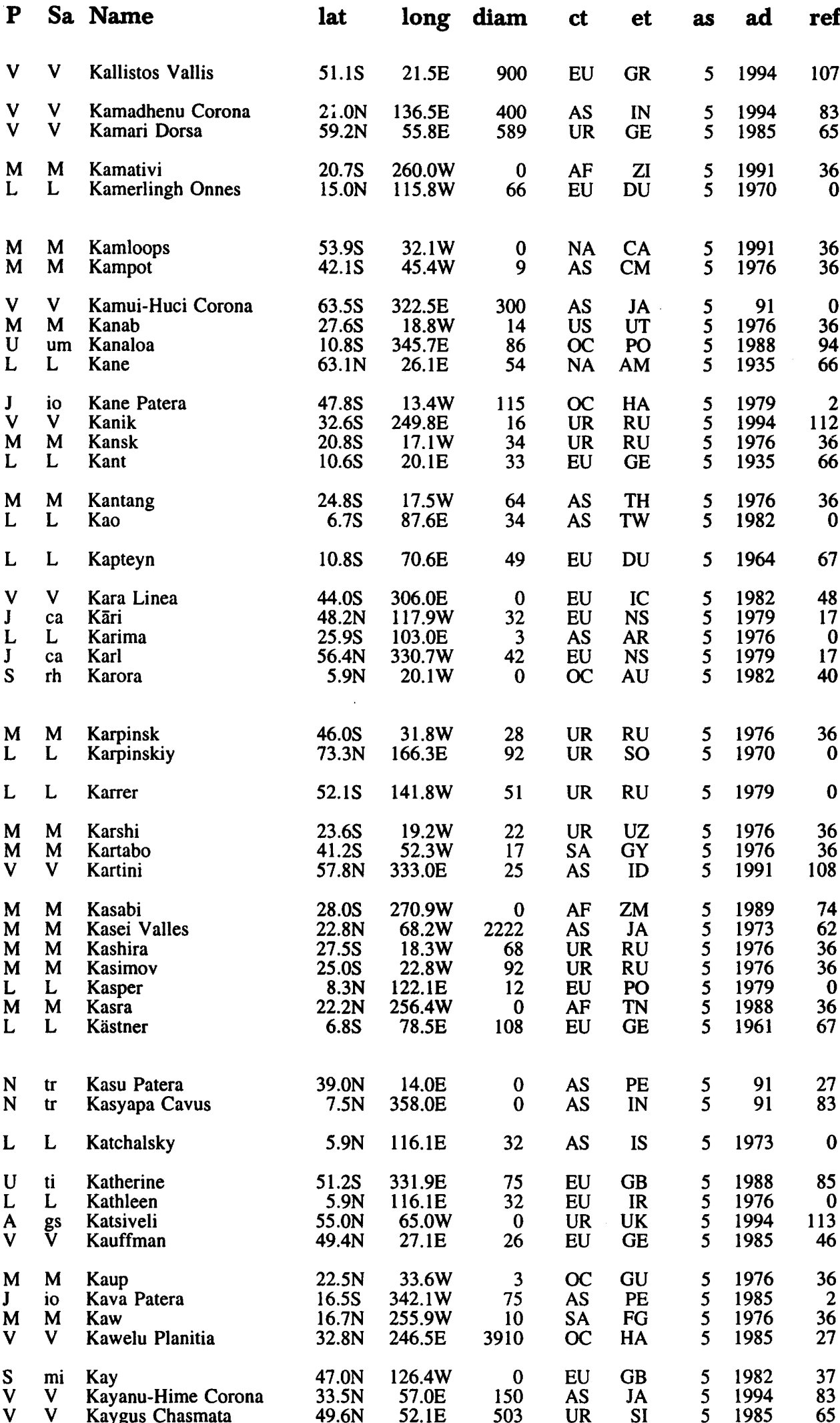

$\mathrm{ft}$ origin

VA Ancient Greek name for planet Venus.

CR Hindu goddess of plenty.

DO Georgian sky maiden, daughter of weather god.

AA Town in Zimbabwe.

AA Heike Kamerlingh Onnes; Dutch physicist; Nobel laureate (1853-1926).

AA Town in Canada.

AA Town in Democratic Kampuchea (Cambodia)

CR Ainu (Japan) earth goddess.

AA Town in Utah, USA.

AA Polynesian chief evil spirit.

AA Elisha Kent; American explorer (1820-1857).

PE Hawaiian god of sunlight.

AA Sakhalin first name.

AA Town in Russia.

AA Immanuel; German philosopher (1724-1804).

AA Town in Thailand.

AA Ping-Tse; Taiwanese astronomer (1888-1970).

AA Jacobus C.; Dutch astronomer (1851-1922).

LI Icelandic Valkryie.

AA Ottar's ancestor.

AA Arabic female name.

AA Norse; Rigr's son with Amma.

AA Aranda (Australia) ancestor who, in his dreams, gives birth to animals and male children.

AA Town in Russia.

AA Aleksey P.; Soviet geologist (1846-1936)

AA Paul;Russian/Swissbiochemist; Nobel laureate (1889-1971).

AA Town in Uzbekhistan.

AA Town in Guyana.

AA Raden Adjeng; Javanese educator (1879-1904).

AA Town in Zambia.

VA Word for "Mars" in Japanese.

AA Town in Russia.

AA Town in Russia.

AA Polish male name

AA Town in Tunisia.

AA Abraham Gotthelf; German mathematician, physicist (1719-1800)

PE Sacred lake of Zoroastrianism.

CB The god Prajapati as a tortoise (India).

AA Katzir-Katchalsky, Aharon; Polish-Israeli chemist (1914-1972).

AA Henry VIII's first queen.

AA Irish female name.

AA Spa in Crimea, Ukraine.

AA Angelica; German painter (1741-1807).

AA Town in New Guinea.

PE Persian blacksmith.

AA Town in French Guiana.

PL Hawaiian mythological heroine, died and brought back to life.

AA Royal seneschel at Arthur's court.

CR Shinto grain goddess.

CM Ketian (Siberia) ruler of forest animals.
P: $\quad$ planetary system (see page xvi).

Sa: satellite (see page $\mathrm{xvi}$ ).

lat: latitude of feature center.

long: longitude of feature center. diam: diameter or long dimension of feature. ct: continent of name origin (see page $284 \mathrm{ff}$.)

et: ethnicity of name origin (see page $284 \mathrm{ff}$.)

as: name approval status (see page xvii). ad: name approval date (year).

ref: reference source for name (see page $287 \mathrm{ff}$.)

ft: feature type (see page 290). 


\section{ALPHABETICAL LIST OF NAMES}

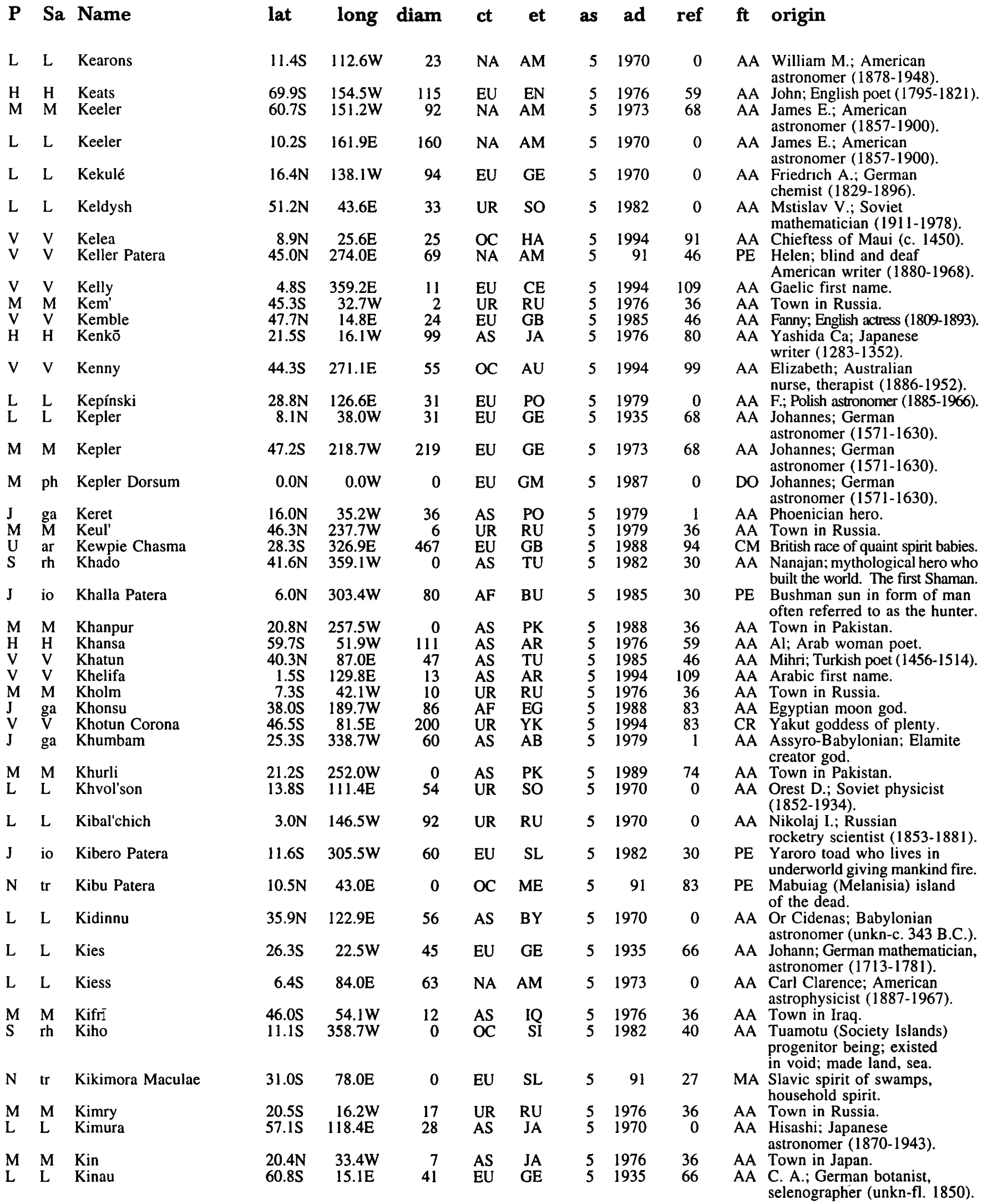

\footnotetext{
P: $\quad$ planetary system (see page $x$ vi).

Sa: $\quad$ satellite (see page $x v i$ ).

lat: latitude of feature center.

long: longitude of feature center.
}

diam: diameter or long dimension of feature.

ct: continent of name origin (see page $284 \mathrm{ff}$.)

et: ethnicity of name origin (see page $284 \mathrm{ff}$.)

as: name approval status (see page xvii). ad: name approval date (year).

ref: reference source for name (see page $287 \mathrm{ff}$.).

ft: feature type (see page 290). 
ALPHABETICAL LIST OF NAMES

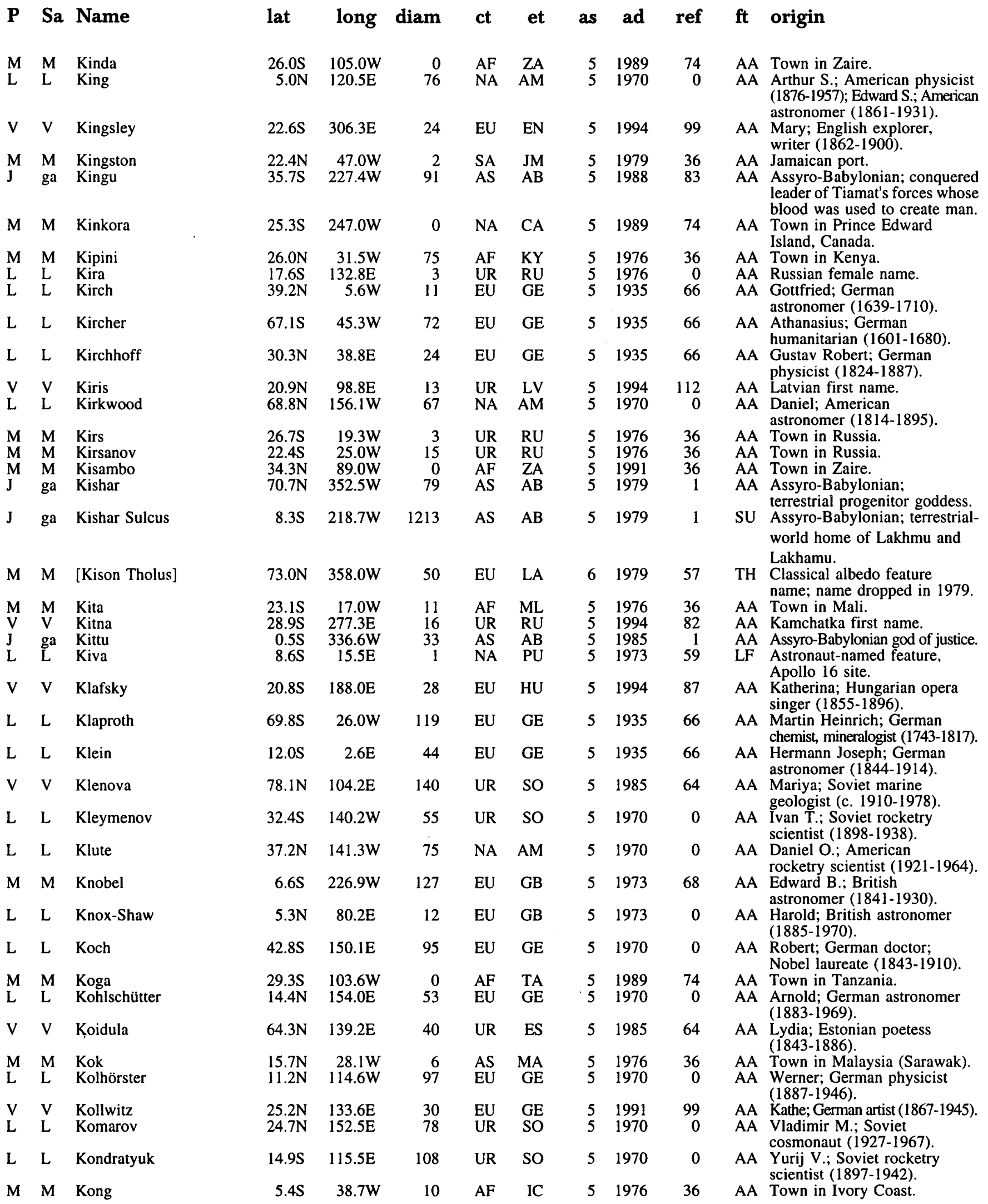

\footnotetext{
P: $\quad$ planetary system (see page $x v i)$.

Sa: $\quad$ satellite (see page xvi).

lat: latitude of feature center.

long: longitude of feature center.
}

diam: diameter or long dimension of feature.

ct: continent of name origin (see page $284 \mathrm{ff}$.)

et: ethnicity of name origin (see page $284 \mathrm{ff}$.)

as: name approval status (see page $x$ vii). ad: name approval date (year)

ref: reference source for name (see page $287 \mathrm{ff}$.).

ft: feature type (see page 290). 
ALPHABETICAL LIST OF NAMES

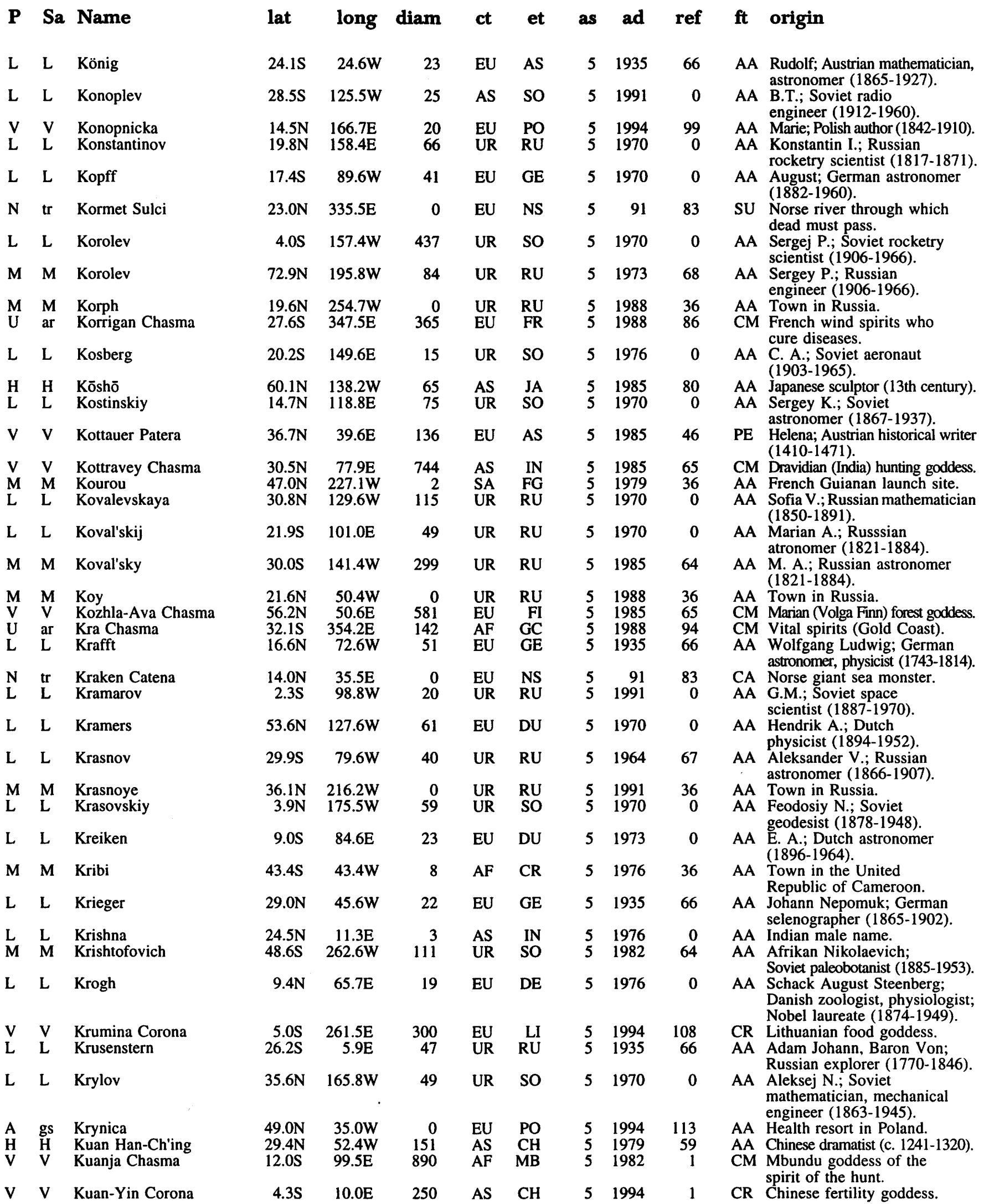

\footnotetext{
P: $\quad$ planetary system (see page $x v i)$.

Sa: satellite (see page $\mathrm{xvi}$ ).

lat: latitude of feature center.

long: longitude of feature center.
}

diam: diameter or long dimension of feature.

ct: continent of name origin (see page $284 \mathrm{ff}$.)

et: ethnicity of name origin (see page $284 \mathrm{ff}$.)

as: name approval status (see page $x$ vii). ad: name approval date (year)

ref: reference source for name (see page $287 \mathrm{ff}$.). ft: feature type (see page 290). 


\section{P Sa Name \\ M M Kuba \\ V V Kubebe Corona \\ M M Kufra \\ L L Kugler \\ H H Kuiper}

\begin{tabular}{|c|c|c|}
\hline & M & Kuiper \\
\hline & $\mathrm{L}$ & Kuiper \\
\hline & $\begin{array}{l}\mathrm{ca} \\
\mathrm{M} \\
\mathrm{L}\end{array}$ & $\begin{array}{l}\text { Kul' } \\
\text { Kular } \\
\text { Kulik }\end{array}$ \\
\hline & $\mathrm{tr}$ & Kulilu Cavus \\
\hline & $\begin{array}{l}\text { ga } \\
\mathrm{M} \\
\mathrm{M} \\
\mathrm{rh} \\
\mathrm{rh}\end{array}$ & $\begin{array}{l}\text { Kulla } \\
\text { Kumak } \\
\text { Kumara } \\
\text { Kumpara } \\
\text { Kun Lun Chasn }\end{array}$ \\
\hline & $\begin{array}{l}\text { V } \\
\mathrm{L}\end{array}$ & $\begin{array}{l}\text { Kunapipi Corona } \\
\text { Kundt }\end{array}$ \\
\hline & $\begin{array}{l}\mathrm{M} \\
\mathrm{V} \\
\mathrm{L}\end{array}$ & $\begin{array}{l}\text { Kunes } \\
\text { Kunhild Corona } \\
\text { Kunowsky }\end{array}$ \\
\hline & M & Kunowsky \\
\hline & $\begin{array}{l}\mathrm{L} \\
\mathrm{L}\end{array}$ & $\begin{array}{l}\text { Kuo Shou Ching } \\
\text { Kurchatov }\end{array}$ \\
\hline & $\begin{array}{l}\mathrm{tr} \\
\mathrm{H} \\
\mathrm{V} \\
\mathrm{M} \\
\mathrm{V}\end{array}$ & $\begin{array}{l}\text { Kurma } \\
\text { Kurosawa } \\
\text { Kurukulla Mons } \\
\text { Kushva } \\
\text { Kutue Tessera }\end{array}$ \\
\hline & $\mathrm{L}$ & La Caille \\
\hline & $\mathrm{L}$ & La Condamine \\
\hline & V & La Fayette \\
\hline & $\begin{array}{l}\mathrm{M} \\
\mathrm{L}\end{array}$ & $\begin{array}{l}\text { La Paz } \\
\text { La Pérouse }\end{array}$ \\
\hline & $\begin{array}{l}\mathrm{V} \\
\mathrm{M} \\
\mathrm{M}\end{array}$ & $\begin{array}{l}\text { Labé Patera } \\
\text { Labeatis Catenae } \\
\text { Labeatis Fossae }\end{array}$ \\
\hline & $\mathbf{M}$ & Labeatis Fossae \\
\hline & $\begin{array}{l}\mathbf{M} \\
\mathbf{M}\end{array}$ & $\begin{array}{l}\text { Labeatis Mensa } \\
\text { Labeatis Mons }\end{array}$ \\
\hline & $\begin{array}{l}\mathrm{M} \\
\mathbf{M} \\
\mathrm{L}\end{array}$ & $\begin{array}{l}\text { Labou Vallis } \\
\text { Labria } \\
\text { Lacchini }\end{array}$ \\
\hline & V & Lachappelle \\
\hline & $\begin{array}{l}\mathrm{V} \\
\mathrm{M}\end{array}$ & $\begin{array}{l}\text { Lachesis Tessera } \\
\text { Lachute }\end{array}$ \\
\hline
\end{tabular}

lat long diam ct et as ad ref $\mathrm{ft}$ origin

$\begin{array}{llrllllr}25.6 \mathrm{~S} & 19.5 \mathrm{~W} & 25 & \text { UR } & \text { AZ } & 5 & 1976 & 36 \\ 15.5 \mathrm{~N} & 132.5 \mathrm{E} & 125 & \text { AS } & \text { TU } & 5 & 1994 & 83 \\ 40.6 \mathrm{~N} & 239.7 \mathrm{~W} & 32 & \text { AS } & \text { LB } & 5 & 1979 & 36 \\ 53.8 \mathrm{~S} & 103.7 \mathrm{E} & 65 & \text { EU } & \text { GE } & 5 & 1970 & 0 \\ 11.3 \mathrm{~S} & 31.1 \mathrm{~W} & 62 & \text { NA } & \text { AM } & 5 & 1976 & 59\end{array}$

$\begin{array}{rrrrrrrr}57.3 \mathrm{~S} & 157.1 \mathrm{~W} & 86 & \text { NA } & \text { AM } & 5 & 1976 & 68 \\ 9.8 \mathrm{~S} & 22.7 \mathrm{~W} & 6 & \text { NA } & \text { AM } & 5 & 1976 & 0 \\ & & & & & & & \\ 62.7 \mathrm{~N} & 123.5 \mathrm{~W} & 43 & \text { UR } & \text { RU } & 5 & 1987 & 64 \\ 16.6 \mathrm{~N} & 252.0 \mathrm{~W} & 0 & \text { UR } & \text { RU } & 5 & 1988 & 36 \\ 42.4 \mathrm{~N} & 154.5 \mathrm{~W} & 58 & \text { UR } & \text { SO } & 5 & 1970 & 0\end{array}$

$41.0 \mathrm{~N} \quad 4.0 \mathrm{E}$

$34.8 \mathrm{~N} \quad 115.0 \mathrm{~W}$

36.0S $\quad 67.5 \mathrm{~W}$

$43.3 \mathrm{~N} \quad 231.4 \mathrm{~W}$

$9.6 \mathrm{~N} \quad 327.1 \mathrm{~W}$

$46.0 \mathrm{~N} \quad 307.5 \mathrm{~W}$

$\begin{array}{lllll}0 & \text { EU } & \text { BY } & 5 & 91\end{array}$

82

AS SU

UR RU

OC NZ

SA $\quad$ EC

1985

$5 \quad 1979$

$\begin{array}{ll}5 & 1979\end{array}$

$\begin{array}{ll}5 & 1982 \\ 5 & 1982\end{array}$

33.9S $\quad 86.0 \mathrm{E}$

$11.5 \mathrm{~S} \quad 11.5 \mathrm{~W}$

OC AU

$5 \quad 1994$

25.5S 252.0W

$19.3 \mathrm{~N} \quad 80.1 \mathrm{E}$

$3.2 \mathrm{~N} \quad 32.5 \mathrm{~W}$

10

EU GE

51976

$\begin{array}{lllll}0 & \text { EU } & \text { NO } & 5 & 1989\end{array}$

$\begin{array}{lllll}200 & \text { EU } & \text { GE } & 5 & 1994\end{array}$

$57.0 \mathrm{~N} \quad 9.0 \mathrm{~W}$

$8.4 \mathrm{~N} \quad 133.7 \mathrm{~W}$

$38.3 \mathrm{~N} \quad 142.1 \mathrm{E}$

16.5S $\quad 61.0 \mathrm{E}$

53.4S $21.8 \mathrm{~W}$

$48.7 \mathrm{~N} \quad 103.0 \mathrm{E}$

$44.3 \mathrm{~S} \quad 35.4 \mathrm{~W}$

$39.5 \mathrm{~N} \quad 108.8 \mathrm{E}$

60

EU GE

1935

51973

34

AS $\mathrm{CH}$

$5 \quad 1970$

$$
106
$$

UR SO

51970

0 AS IN

159

59

39
653

AS IN

AS TB

UR RU

$5 \quad 91$

$\begin{array}{rr}5 & 1976 \\ 5 & 91\end{array}$

$\begin{array}{ll}5 & 91 \\ 5 & 1976\end{array}$

$\begin{array}{ll}5 & 1976 \\ 5 & 1985\end{array}$

$\begin{array}{lllllll}23.8 S & 1.1 \mathrm{E} & 67 & \mathrm{EU} & \text { FR } & 5 & 1961\end{array}$

$53.4 \mathrm{~N}$

$28.2 \mathrm{~W}$

37

EU FR

1961

70.2N 107.5E

$21.3 \mathrm{~N} \quad 49.0 \mathrm{~W}$

$10.7 \mathrm{~S} \quad 76.3 \mathrm{E}$

40

EU FR

51985

1 NA ME

$5 \quad 1979$

77 EU FR

$5 \quad 1935$

52.1N 273.1E

$18.8 \mathrm{~N} \quad 95.1 \mathrm{~W}$

$20.9 \mathrm{~N} \quad 95.0 \mathrm{~W}$

\section{3}

318

EU

616

EU RM

$29.5 \mathrm{~N} \quad 80.0 \mathrm{~W}$

388

EU LA

$\begin{array}{rr}5 & 91 \\ 5 & 1988\end{array}$

51985

$25.6 \mathrm{~N} 74.9 \mathrm{~W}$

EU LA

51985

$37.8 \mathrm{~N} \quad 75.9 \mathrm{~W}$

120

EU LA

$5 \quad 1988$

8.8S 154.3W

35.3S 48.0W

28

EU LA

51994

$41.7 \mathrm{~N} \quad 107.5 \mathrm{~W}$

EU FR

51985

$\begin{array}{rrrrr}60 & \text { SA } & \text { BR } & 5 & 1979 \\ 58 & \text { EU } & \text { IT } & 5 & 1970\end{array}$

$26.7 \mathrm{~N} \quad 336.5 \mathrm{E}$

38

EU FR

$5 \quad 1991$

44.4N $300.1 \mathrm{E}$

$4.3 \mathrm{~S} \quad 39.9 \mathrm{~W}$
664

13 $\begin{array}{ll}\text { EU } & \text { GR } \\ \text { NA } & \text { CA }\end{array}$

36
83
36
0
59

AA Town in Azerbaijan.

CR Hittite mother earth goddess.

AA Town in Libya.

AA F. X.; German-Babylonian chronologist (1862-1929).

AA Gerard P.; Dutch-American astronomer, member of original Mariner Venus-Mercury Imaging Team (1905-1973).

AA Gerard P.; American astronomer (1905-1973).

0 AA Gerard Peter; Dutch-American astronomer (1905-1973).

AA Komi wood spirit.

AA Town in Russia.

AA Leonid A.; Soviet mineralogist (1883-1942).

83

CB Babylonian destructive fish-man spirit.

AA Sumerian god of brick making.

AA Town in Russia.

AA Town in New Zealand.

AA Jivaro (Ecuador) creator god.

CM Mountain dwelling place of the immortals.

83

CR Australian mother earth goddess.

AA August; German physicist (1839-1894).

AA Town in Norway.

CR German fertility maiden.

AA Georg Karl Friedrich; German astronomer (1786-1846).

AA George K.; German astronomer (1786-1846).

AA Chinese astronomer (1231-1316).

AA Igor' V.; Soviet nuclear physicist (1903-1960).

AA Vishnu in the form of a tortoise.

AA K.; (18th century).

MO Etan (Tibet) goddess of wealth.

AA Town in Russia.

TE Ulchian (Siberia) folklore toad that brings happiness.

67

67

AA Nicholas Louis De; French astronomer (1713-1762).

AA Charles Marie De; French astronomer, physicist (1701-1774).

46

36

AA Marie; French novelist (1634-1693).

66

AA Mexican port.

AA Jean Francois de Galoup, Comte De La Pérouse; French explorer (1741-1788).

46
57

PE Etan goddess of wealth

CA Classical albedo feature name.

FO Previously named feature at 30N, 75W; expanded coordinates.

FO Previously named feature at 30N, 75W; expanded coordinates.

56 MN Classical albedo feature name.

MO Named for albedo feature Labeatis Lacus.

VA French word for Mars.

36 AA Town in Brazil.

AA Giovanni; Italian astronomer (1884-1967)

100

AA Marie; French medical researcher (1769-1821).

TE Greek, one of three Fates.

AA Town in Canada.

\footnotetext{
P: $\quad$ planetary system (see page $\mathrm{xvi}$ ).

Sa: $\quad$ satellite (see page $x v i)$.

lat: latitude of feature center.

long: longitude of feature center.
}

diam: diameter or long dimension of feature.

ct: continent of name origin (see page $284 \mathrm{ff}$.)

et: ethnicity of name origin (see page $284 \mathrm{ff}$.)

as: name approval status (see page xvii). ad: name approval date (year).

ref: reference source for name (see page $287 \mathrm{ff}$.).

ft: feature type (see page 290). 


\section{ALPHABETICAL LIST OF NAMES}

\section{P Sa Name}

L L Lacroix

L L Lacus Aestatis

L L Lacus Autumni

L L Lacus Bonitatis

L L Lacus Doloris

L L Lacus Excellentiae

L L Lacus Felicitatis

L L Lacus Gaudii

L L Lacus Hiemalis

L L Lacus Lenitatis

L L Lacus Luxuriae

L L Lacus Mortis

L L Lacus Oblivionis

L L Lacus Odii

L L Lacus Perseverantiae

L L Lacus Solitudinis

L L Lacus Somniorum

L L Lacus Spei

L L Lacus Temporis

L L Lacus Timoris

L L Lacus Veris

Lada Terra

L L Lade

M M Ladon Valles

$S$ te Laertes

M M Laestrygon (Laestrigon)

M M Laf

L L Lagalla

M M Lagarto

$\mathrm{J}$ ga Lagash Sulcus

$\mathrm{V} V$ Lagerlöf

L L Lagrange

$\begin{array}{lll}\mathrm{U} & \text { ar } & \text { Laica } \\ \mathrm{V} & \mathrm{V} & \text { Laima Tessera }\end{array}$

J ga Lakhamu Fossa

J ga Lakhmu Fossae

V V Lakshmi Planum

L L Lalande

L L Lallemand

L L Lamarck

M M Lamas

L L Lamb

L L Lambert

M M Lambert

L L Lamé

L L Lamèch

S mi Lamerok

\begin{tabular}{|c|c|c|c|c|c|c|}
\hline lat & long & diam & ct & et & as & ad \\
\hline $37.9 \mathrm{~S}$ & $59.0 \mathrm{~W}$ & 37 & $\mathrm{EU}$ & FR & 5 & 1935 \\
\hline $\begin{array}{r}15.0 \mathrm{~S} \\
9.9 \mathrm{~S} \\
23.2 \mathrm{~N} \\
17.1 \mathrm{~N} \\
35.4 \mathrm{~S} \\
19.0 \mathrm{~N} \\
16.2 \mathrm{~N} \\
15.0 \mathrm{~N} \\
14.0 \mathrm{~N} \\
19.0 \mathrm{~N} \\
45.0 \mathrm{~N} \\
21.0 \mathrm{~S} \\
19.0 \mathrm{~N} \\
8.0 \mathrm{~N} \\
27.8 \mathrm{~S} \\
38.0 \mathrm{~N} \\
43.0 \mathrm{~N} \\
45.9 \mathrm{~N} \\
38.8 \mathrm{~S} \\
16.5 \mathrm{~S} \\
51.1 \mathrm{~S} \\
1.3 \mathrm{~S}\end{array}$ & $\begin{array}{c}69.0 \mathrm{~W} \\
83.9 \mathrm{~W} \\
43.7 \mathrm{E} \\
9.0 \mathrm{E} \\
44.0 \mathrm{~W} \\
5.0 \mathrm{E} \\
12.6 \mathrm{E} \\
14.0 \mathrm{E} \\
12.0 \mathrm{E} \\
176.0 \mathrm{E} \\
27.2 \mathrm{E} \\
168.0 \mathrm{~W} \\
7.0 \mathrm{E} \\
62.0 \mathrm{E} \\
104.3 \mathrm{E} \\
29.2 \mathrm{E} \\
65.0 \mathrm{E} \\
58.4 \mathrm{E} \\
27.3 \mathrm{~W} \\
86.1 \mathrm{~W} \\
342.5 \mathrm{E} \\
10.1 \mathrm{E}\end{array}$ & $\begin{array}{r}90 \\
183 \\
92 \\
110 \\
184 \\
90 \\
113 \\
50 \\
80 \\
50 \\
151 \\
50 \\
70 \\
70 \\
139 \\
384 \\
80 \\
117 \\
117 \\
396 \\
8614 \\
55\end{array}$ & $\begin{array}{l}\text { EU } \\
\text { EU } \\
\text { EU } \\
\text { EU } \\
\text { EU } \\
\text { EU } \\
\text { EU } \\
\text { EU } \\
\text { EU } \\
\text { EU } \\
\text { EU } \\
\text { EU } \\
\text { EU } \\
\text { EU } \\
\text { EU } \\
\text { EU } \\
\text { EU } \\
\text { EU } \\
\text { EU } \\
\text { EU }\end{array}$ & $\begin{array}{l}\text { LA } \\
\text { LA } \\
\text { LA } \\
\text { LA } \\
\text { LA } \\
\text { LA } \\
\text { LA } \\
\text { LA } \\
\text { LA } \\
\text { LA } \\
\text { LA } \\
\text { LA } \\
\text { LA } \\
\text { LA } \\
\text { LA } \\
\text { LA } \\
\text { LA } \\
\text { LA } \\
\text { LA } \\
\text { LA } \\
\text { SL } \\
\text { GE }\end{array}$ & $\begin{array}{l}5 \\
5 \\
5 \\
5 \\
5 \\
5 \\
5 \\
5 \\
5 \\
5 \\
5 \\
5 \\
5 \\
5 \\
5 \\
5 \\
5 \\
5 \\
5 \\
5 \\
5 \\
5\end{array}$ & $\begin{array}{l}1970 \\
1970 \\
1976 \\
1976 \\
1976 \\
1976 \\
1976 \\
1976 \\
1976 \\
1976 \\
1935 \\
1976 \\
1976 \\
1979 \\
1976 \\
1935 \\
1976 \\
1976 \\
1976 \\
1970 \\
1982 \\
1935\end{array}$ \\
\hline $\begin{array}{r}22.3 \mathrm{~S} \\
47.6 \mathrm{~S} \\
0.0 \mathrm{~N}\end{array}$ & $\begin{array}{r}28.4 \mathrm{~W} \\
66.4 \mathrm{~W} \\
200.0 \mathrm{~W}\end{array}$ & $\begin{array}{r}238 \\
0 \\
0\end{array}$ & $\begin{array}{l}\text { EU } \\
\text { EU } \\
\text { EU }\end{array}$ & $\begin{array}{l}\text { GR } \\
\text { GR } \\
\text { GR }\end{array}$ & $\begin{array}{l}5 \\
5 \\
5\end{array}$ & $\begin{array}{l}1976 \\
1982 \\
1958\end{array}$ \\
\hline $48.3 \mathrm{~N}$ & $5.9 \mathrm{~W}$ & 6 & $\mathrm{AF}$ & $\mathrm{CR}$ & 5 & 1976 \\
\hline $44.6 \mathrm{~S}$ & $22.5 \mathrm{~W}$ & 85 & EU & IT & 5 & 1935 \\
\hline $\begin{array}{l}50.0 \mathrm{~N} \\
10.4 \mathrm{~S} \\
81.1 \mathrm{~N}\end{array}$ & $\begin{array}{r}8.4 \mathrm{~W} \\
162.8 \mathrm{~W} \\
285.3 \mathrm{E}\end{array}$ & $\begin{array}{r}19 \\
1407 \\
59\end{array}$ & $\begin{array}{l}\text { SA } \\
\text { AS } \\
\text { EU }\end{array}$ & $\begin{array}{l}\text { BR } \\
\text { BY } \\
\text { SW }\end{array}$ & $\begin{array}{l}5 \\
5 \\
5\end{array}$ & $\begin{array}{l}1976 \\
1985 \\
1985\end{array}$ \\
\hline $32.3 \mathrm{~S}$ & $72.8 \mathrm{~W}$ & 225 & $\mathrm{EU}$ & IT & 5 & 1935 \\
\hline $\begin{array}{l}21.3 \mathrm{~S} \\
55.0 \mathrm{~N}\end{array}$ & $\begin{array}{l}44.4 \mathrm{E} \\
48.5 \mathrm{E}\end{array}$ & $\begin{array}{r}30 \\
971\end{array}$ & $\begin{array}{l}\text { SA } \\
\text { UR }\end{array}$ & $\begin{array}{l}\text { IN } \\
\text { LV }\end{array}$ & $\begin{array}{l}5 \\
5\end{array}$ & $\begin{array}{l}1988 \\
1985\end{array}$ \\
\hline $12.5 \mathrm{~S}$ & $228.3 \mathrm{~W}$ & 392 & AS & $\mathrm{AB}$ & 5 & 1985 \\
\hline $30.3 \mathrm{~N}$ & $142.3 \mathrm{~W}$ & 2871 & AS & $\mathrm{AB}$ & 5 & 1985 \\
\hline $\begin{array}{r}68.6 \mathrm{~N} \\
4.4 \mathrm{~S}\end{array}$ & $\begin{array}{c}339.3 \mathrm{E} \\
8.6 \mathrm{~W}\end{array}$ & $\begin{array}{r}2343 \\
24\end{array}$ & $\begin{array}{l}\text { AS } \\
\text { EU }\end{array}$ & $\begin{array}{l}\text { IN } \\
\text { FR }\end{array}$ & $\begin{array}{l}5 \\
5\end{array}$ & $\begin{array}{l}1979 \\
1935\end{array}$ \\
\hline $14.3 \mathrm{~S}$ & $84.1 \mathrm{~W}$ & 18 & $\mathrm{EU}$ & FR & 5 & 1985 \\
\hline $22.9 \mathrm{~S}$ & $69.8 \mathrm{~W}$ & 100 & $\mathrm{EU}$ & FR & 5 & 1964 \\
\hline $\begin{array}{l}27.4 \mathrm{~S} \\
42.9 \mathrm{~S}\end{array}$ & $\begin{array}{c}20.5 \mathrm{~W} \\
100.1 \mathrm{E}\end{array}$ & $\begin{array}{r}21 \\
106\end{array}$ & $\begin{array}{l}\text { SA } \\
\text { EU }\end{array}$ & $\begin{array}{l}\text { PE } \\
\text { GB }\end{array}$ & $\begin{array}{l}5 \\
5\end{array}$ & $\begin{array}{l}1976 \\
1970\end{array}$ \\
\hline $25.8 \mathrm{~N}$ & $21.0 \mathrm{~W}$ & 30 & EU & GE & 5 & 1935 \\
\hline $20.1 S$ & $334.6 \mathrm{~W}$ & 87 & $\mathrm{EU}$ & GE & 5 & 1973 \\
\hline $14.7 \mathrm{~S}$ & $64.5 \mathrm{E}$ & 84 & EU & FR & 5 & 1964 \\
\hline $42.7 \mathrm{~N}$ & $13.1 \mathrm{E}$ & 13 & EU & FR & 5 & 1935 \\
\hline $61.3 \mathrm{~S}$ & $291.4 \mathrm{~W}$ & 0 & EU & GB & 5 & 1982 \\
\hline
\end{tabular}

\section{ft origin}

AA Sylvestre Francois De; French mathematician (1765-1843).

LC "Lake of Summer".

LC "Lake of Autumn".

LC "Lake of Goodness".

LC "Lake of Sorrow".

LC "Lake of Excellence".

LC "Lake of Happiness".

LC "Lake of Joy".

LC "Wintry Lake".

LC "Lake of Softness".

LC "Lake of Luxury"

LC "Lake of Death".

LC "Lake of Forgetfulness".

LC "Lake of Hatred".

LC "Lake of Perseverance".

LC "Lake of Solitude".

LC "Lake of Dreams".

LC "Lake of Hope".

LC "Lake of Time".

LC "Lake of Fear".

LC "Lake of Spring".

TA Slavic goddess of love.

AA Heinrich Eduard von; German astronomer (1817-1904)

VA Ancient name for Greek river.

AA Father of Odysseus.

AL Man-eating giants who lived in the west.

AA Town in the United Republic of Cameroon.

AA Giulio Cesare; Italian philosopher (1571-1624).

AA Town in Brazil.

SU Early Babylonian town.

AA Selma; Swedish novelist (1858-1940)

AA Joseph Louis; Italian mathematician (1736-1813).

AA Inca good spirit.

TE Latvian and Lithuanian goddess of fate.

FO Dragon monster, or divine natural force produced by Apsu and Tiamat.

FO Dragon monster, or divine natural force produced by Apsu and Tiamat.

PM Indian goddess of love and war.

AA Joseph Jerome Le Francois De; French astronomer (1732-1807)

AA Andre; French astronomer (1904-1978).

AA Jean B. P. A. De M.; French natural historian (1744-1829).

AA Town in Peru.

AA Sir Horace; British mathematician, physicist (1849-1934).

AA Johann Heinrich; German astronomer, mathematician, physicist (1728-1777).

AA Johann H.; German physicist (1728-1777)

AA Gabriel; French mathematician (1795-1870).

AA Felix Chemla; French selenographer (1894-1962).

AA Pellinore's son; sent testing horn to King Mark to expose adultery of Sir Tristram.

\footnotetext{
P: $\quad$ planetary system (see page $x v i)$.

Sa: $\quad$ satellite (see page xvi).

lat: latitude of feature center.

long: longitude of feature center.
}

diam: diameter or long dimension of feature.

ct: continent of name origin (see page $284 \mathrm{ff}$.)

et: ethnicity of name origin (see page $284 \mathrm{ff}$.)

as: name approval status (see page xvii). ad: name approval date (year)

ref: reference source for name (see page $287 \mathrm{ff}$.)

ft: feature type (see page 290). 


\section{ALPHABETICAL LIST OF NAMES}

\section{P Sa Name}

L L Lamont

M M Lamont

V V Lampedo Linea

M M Lampland

L L Lampland

$M \quad M \quad$ Land

L L Landau

L L Lander

V V Landowska

L L Landsteiner

L L Lane

L L Langemak

L L Langevin

L L Langley

L L Langmuir

L L Langrenus

V V Langtry

L L Lansberg

M M Lapri

M M Lar

L L Lara

S di Larissa Chasma

L L Larmor

V V Lasdona Chasma

L L Lassell

M M Lassell

M M Lasswitz

L L Last

$S$ di Latagus

$S$ di Latium Chasma

M M Lau

L L Laue

V V Laulani

V V Laūma Dorsa

$S$ mi Launcelot

$\mathrm{V} \quad \mathrm{V}$ Laura

$\mathrm{V}$ V Laurencin

L L Lauritsen

$S$ di Lausus

V V Lavinia Planitia

L L Lavoisier lat long

diam

ct et

as ad

$\begin{array}{rrrrrrrr}4.4 \mathrm{~N} & 23.7 \mathrm{E} & 106 & \text { EU } & \text { SC } & 5 & 1935 & 68 \\ 58.3 \mathrm{~S} & 113.3 \mathrm{~W} & 72 & \text { EU } & \text { GE } & 5 & 1973 & 68 \\ 57.0 \mathrm{~N} & 295.0 \mathrm{E} & 0 & \text { UR } & \text { SC } & 5 & 1982 & 1 \\ 36.0 \mathrm{~S} & 79.5 \mathrm{~W} & 71 & \text { NA } & \text { AM } & 5 & 1973 & 68 \\ 31.0 \mathrm{~S} & 131.0 \mathrm{E} & 65 & \text { NA } & \text { AM } & 5 & 1970 & 0 \\ 48.4 \mathrm{~N} & 8.8 \mathrm{~W} & 5 & \text { US } & \text { AL } & 5 & 1976 & 36 \\ 41.6 \mathrm{~N} & 118.1 \mathrm{~W} & 214 & \text { UR } & \text { SO } & 5 & 1970 & 0 \\ 15.3 \mathrm{~S} & 131.8 \mathrm{E} & 40 & \text { EU } & \text { GB } & 5 & 1976 & 0 \\ 84.5 \mathrm{~N} & 74.2 \mathrm{E} & 33 & \text { EU } & \text { PO } & 5 & 1985 & 46 \\ 31.3 \mathrm{~N} & 14.8 \mathrm{~W} & 6 & \text { EU } & \text { AS } & 5 & 1976 & 0\end{array}$

$$
9.5 \mathrm{~S}
$$

$132.0 \mathrm{E}$

55

NA AM

$10.3 \mathrm{~S} \quad 118.7 \mathrm{E} \quad 97 \quad$ UR $\quad S O$

$\begin{array}{llll}44.3 \mathrm{~N} & 162.7 \mathrm{E} \quad 58\end{array}$

EU FR

$5 \quad 1970$

$51.1 \mathrm{~N} \quad 86.3 \mathrm{~W}$

59

NA AM

$5 \quad 1964$

$35.7 \mathrm{~S} \quad 128.4 \mathrm{~W}$

$8.9 \mathrm{~S}$

61.1E

91

NA AM

51970

17.05

$17.0 \mathrm{~S}$
$0.3 \mathrm{~S}$

$155.0 \mathrm{E}$

$20.6 \mathrm{~N} \quad 252.6 \mathrm{~W}$

$26.1 \mathrm{~S} \quad 28.8 \mathrm{~W}$

$20.4 \mathrm{~N} \quad 30.5 \mathrm{E}$

$30.2 \mathrm{~N} \quad 71.1 \mathrm{~W}$

127

EU BE

51935

$32.1 \mathrm{~N} \quad 179.7 \mathrm{~W}$

52

EU EN

38 EU BE

$5 \quad 1994$

$\begin{array}{ll}5 & 1935\end{array}$

0 UR RU

6 AS IR

$\begin{array}{ll}5 & 1988\end{array}$

$\begin{array}{ll}5 & 1988 \\ 5 & 1979\end{array}$

NA AM 51973

315

EU GR

$5 \quad 1982$

0

$36.8 \mathrm{E}$

$15.5 \mathrm{~S}$

$7.9 \mathrm{~W}$

97

EU GB

51970

697

UR LI

$5 \quad 1985$

$21.0 \mathrm{~S}$

$62.4 \mathrm{~W}$

86

EU G

$\begin{array}{ll}5 & 1935\end{array}$

9.4S

$26.1 \mathrm{~N}$

$221.6 \mathrm{~W}$

122

$15.7 \mathrm{~N}$

$0.0 \mathrm{E}$

EU GB

$5 \quad 1973$

$\begin{array}{llll}\text { EU } & \text { GE } & 5 & 1976 \\ \text { NA } & \text { AM } & 5 & 1973\end{array}$

$21.2 \mathrm{~N}$

$26.4 \mathrm{~W}$

$74.4 \mathrm{~S}$

$69.5 \mathrm{~W}$

$107.3 \mathrm{~W}$

37

$$
381
$$

EU RM

EU RM

$5 \quad 1982$

$28.0 \mathrm{~N}$

$96.7 \mathrm{~W}$

87

68.2S 121.3E

$64.8 \mathrm{~N} \quad 190.4 \mathrm{E}$

$10.0 \mathrm{~S}$

$328.2 \mathrm{~W}$

15

1517
0

EU GE

$5 \quad 1982$

49.0N 141.1E

$15.4 \mathrm{~S}$

$27.6 \mathrm{~S}$

$46.4 \mathrm{E}$

19
30

52

OC HA

$\begin{array}{ll}\text { UR } & \text { LV } \\ \text { EU } & \text { GB }\end{array}$

51970

$36.2 \mathrm{~N} \quad 23.2 \mathrm{~W} \quad 28$

47.3S $347.5 \mathrm{E} \quad 2820$

$38.2 \mathrm{~N}$ $\begin{array}{ll}\text { EU } & \text { SP } \\ \text { EU } & \text { FR }\end{array}$

EU DE

EU RM

$\begin{array}{ll}\text { EU } & \text { RM } \\ \text { EU } & \text { FR }\end{array}$

\section{ref $\mathbf{f t}$ origin}

AA John; Scottish astronomer (1805-1879).

AA Johann von; German astronomer (1805-1879).

LI Scythian Amazon queen.

AA Carl O.; American astronomer (1873-1951).

AA Carl O.; American astronomer (1873-1951).

AA Town in Alabama, USA.

AA Lev D.; Soviet physicist; Nobel laureate (1908-1968).

AA Richard Lemon; British explorer (1804-1834)

AA Wanda; Polish pianist (1877-1959).

AA Karl; Austrian-American pathologist; Nobel laureate (1868-1943).

AA Jonathan H.; American physicist, astrophysicist (1819-1880).

AA Georgij E.; Soviet rocketry scientist (1898-1938).

AA Paul; French physicist (1872-1946).

67 AA Samuel P.; American astronomer, physicist (18341906).

AA Irving; American physicist, chemist; Nobel laureate (1881-1957)

AA Langren, Michel Florent van; Belgian selenographer, engineer (c. 1600-1675).

AA Lillie; English actress (1853-1929).

AA Philippe van; Belgian astronomer (1561-1632).

AA Town in Russia.

AA Town in Iran.

LF Astronaut-named feature, Apollo 17 site.

CM A town in Thessaly, Achilles' native region.

AA Sir Joseph; British mathematician, physicist (1857-1942).

65

66

CM Lithuanian main forest goddess.

AA William; British astronomer (1799-1880).

68

AA William; British astronomer (1799-1880)

79
59

AA Kurd; German author (1848-1910).

LF Astronaut-named feature, Apollo 15 site.

44

44

AA Soldier of Aeneas.

CM The Trojans' promised land in Italy.

AA Hans E.; Danish astronomer (1879-1918).

AA Max T. F. von; German physicist; Nobel laureate (1879-1960).

AA Hawaiian first name.

DO Latvian witch, flies in the sky.

AA King Arthur's favorite; champion and lover of Queen Gwynevere.

AA Spanish, Italian first name.

AA Marie; French painter (1885-1956).

AA Charles C.; Danish-American physicist (1892-1968).

AA Son of Mezentius, killed by Aeneas.

PL Roman; wife of Aeneas.

AA Antoine Laurent; French chemist (1743-1794).

\footnotetext{
P: $\quad$ planetary system (see page $x v i)$.

Sa: $\quad$ satellite (see page $x v i$ ).

lat: latitude of feature center.

long: longitude of feature center.
}

diam: diameter or long dimension of feature.

ct: continent of name origin (see page $284 \mathrm{ff}$.)

et: ethnicity of name origin (see page $284 \mathrm{ff}$.)

as: name approval status (see page xvii). ad: name approval date (year).

ref: reference source for name (see page $287 \mathrm{ff}$.).

$\mathrm{ft}$ feature type (see page 290). 


\section{ALPHABETICAL LIST OF NAMES}

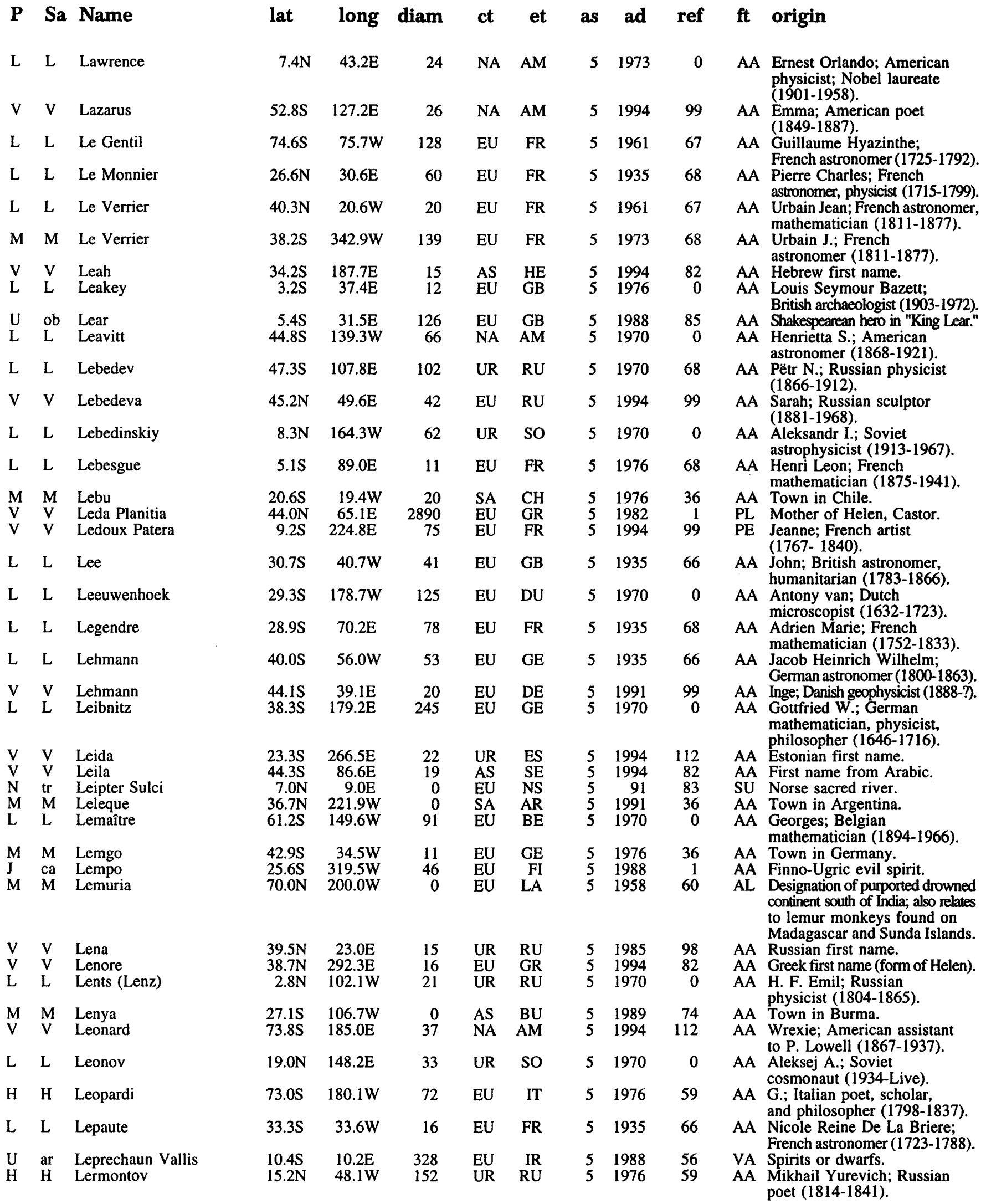

\footnotetext{
P: $\quad$ planetary system (see page xvi).

Sa: satellite (see page $x v i$ ).

lat: latitude of feature center.

long: longitude of feature center.
}

diam: diameter or long dimension of feature.

ct: continent of name origin (see page $284 \mathrm{ff}$.)

et: ethnicity of name origin (see page $284 \mathrm{ff}$.)

as: name approval status (see page xvii) ad: name approval date (year).

ref: reference source for name (see page $287 \mathrm{ff}$.)

ft: feature type (see page 290). 
ALPHABETICAL LIST OF NAMES

\begin{tabular}{|c|c|c|c|c|c|c|c|c|}
\hline $\mathbf{P}$ & Sa Name & lat & long & diam & ct & et & as & ad \\
\hline $\begin{array}{l}\text { J } \\
\mathbf{H}\end{array}$ & $\begin{array}{l}\text { io Lerna Regio } \\
\mathrm{H} \text { Lessing }\end{array}$ & $\begin{array}{l}64.0 \mathrm{~S} \\
28.7 \mathrm{~S}\end{array}$ & $\begin{array}{r}292.6 \mathrm{~W} \\
89.7 \mathrm{~W}\end{array}$ & $\begin{array}{r}0 \\
100\end{array}$ & $\begin{array}{l}\text { EU } \\
\text { EU }\end{array}$ & $\begin{array}{l}\text { GR } \\
\text { GE }\end{array}$ & $\begin{array}{l}5 \\
5\end{array}$ & $\begin{array}{l}1979 \\
1985\end{array}$ \\
\hline $\begin{array}{l}\text { V } \\
\text { L }\end{array}$ & $\begin{array}{l}\text { Letitia } \\
\text { Letronne }\end{array}$ & $\begin{array}{l}34.6 \mathrm{~N} \\
10.8 \mathrm{~S}\end{array}$ & $\begin{array}{l}288.6 \mathrm{E} \\
42.5 \mathrm{~W}\end{array}$ & $\begin{array}{r}16 \\
116\end{array}$ & $\begin{array}{l}\text { EU } \\
\text { EU }\end{array}$ & $\begin{array}{l}\text { LA } \\
\text { FR }\end{array}$ & $\begin{array}{l}5 \\
5\end{array}$ & $\begin{array}{l}1994 \\
1935\end{array}$ \\
\hline L & Leucippus & $29.1 \mathrm{~N}$ & $116.0 \mathrm{~W}$ & 56 & EU & GR & 5 & 1970 \\
\hline $\begin{array}{l}\mathbf{M} \\
\mathbf{L}\end{array}$ & $\begin{array}{l}\text { Leuk } \\
\text { Leuschner }\end{array}$ & $\begin{array}{r}24.1 \mathrm{~N} \\
1.8 \mathrm{~N}\end{array}$ & $\begin{array}{r}55.0 \mathrm{~W} \\
108.8 \mathrm{~W}\end{array}$ & $\begin{array}{r}0 \\
49\end{array}$ & $\begin{array}{l}\text { EU } \\
\text { NA }\end{array}$ & $\begin{array}{r}\text { SZ } \\
\text { AM }\end{array}$ & $\begin{array}{l}5 \\
5\end{array}$ & $\begin{array}{l}1988 \\
1970\end{array}$ \\
\hline $\mathbf{N}$ & Leviathan Patera & $17.0 \mathrm{~N}$ & $28.5 \mathrm{E}$ & 0 & AS & $\mathrm{HE}$ & 5 & 91 \\
\hline L & Levi-Civita & $23.7 \mathrm{~S}$ & $143.4 \mathrm{E}$ & 121 & EU & IT & 5 & 1970 \\
\hline L & Lewis & $18.5 S$ & $113.8 \mathrm{~W}$ & 42 & NA & $\mathrm{AM}$ & 5 & 1970 \\
\hline $\mathbf{L}$ & Lexell & $35.8 \mathrm{~S}$ & $4.2 \mathrm{~W}$ & 62 & EU & SW & 5 & 1935 \\
\hline M & Lexington & $22.0 \mathrm{~N}$ & $48.7 \mathrm{~W}$ & 5 & US & MA & 5 & 1979 \\
\hline $\mathbf{L}$ & Ley & $42.2 \mathrm{~N}$ & $154.9 \mathrm{E}$ & 79 & NA & $\mathrm{AM}$ & 5 & 1970 \\
\hline V & Leyster & $1.0 \mathrm{~N}$ & $259.9 \mathrm{E}$ & 45 & EU & DU & 5 & 1994 \\
\hline $\mathbf{S}$ & Leza & $21.8 \mathrm{~S}$ & $309.2 \mathrm{~W}$ & 0 & $\mathrm{AF}$ & BA & 5 & 1982 \\
\hline $\begin{array}{l}\mathrm{H} \\
\mathbf{M} \\
\mathrm{L} \\
\mathrm{H} \\
\mathrm{V}\end{array}$ & $\begin{array}{l}\text { Li Ch'ing-Chao } \\
\text { Li Fan } \\
\text { [Li Po] } \\
\text { Li Po } \\
\text { Li Qingzhao }\end{array}$ & $\begin{array}{r}77.1 \mathrm{~S} \\
47.4 \mathrm{~S} \\
3.5 \mathrm{~S} \\
16.9 \mathrm{~N} \\
23.7 \mathrm{~N}\end{array}$ & $\begin{array}{c}73.1 \mathrm{~W} \\
153.0 \mathrm{~W} \\
90.6 \mathrm{E} \\
35.0 \mathrm{~W} \\
94.5 \mathrm{E}\end{array}$ & $\begin{array}{r}61 \\
103 \\
15 \\
120 \\
22\end{array}$ & $\begin{array}{l}\text { AS } \\
\text { AS } \\
\text { AS } \\
\text { AS } \\
\text { AS }\end{array}$ & $\begin{array}{l}\mathrm{CH} \\
\mathrm{CH} \\
\mathrm{CH} \\
\mathrm{CH} \\
\mathrm{CH}\end{array}$ & $\begin{array}{l}5 \\
5 \\
6 \\
5 \\
5\end{array}$ & $\begin{array}{r}1976 \\
1973 \\
0 \\
1976 \\
1991\end{array}$ \\
\hline $\mathbf{M}$ & Liais & $75.4 \mathrm{~S}$ & $252.9 \mathrm{~W}$ & 128 & EU & FR & 5 & 1973 \\
\hline $\begin{array}{l}\mathbf{H} \\
\mathrm{V} \\
\mathrm{V} \\
\mathrm{M} \\
\mathrm{M}\end{array}$ & $\begin{array}{l}\text { Liang K'ai } \\
\text { Liban Farra } \\
\text { Libera Corona } \\
\text { Libertad } \\
\text { Libya }\end{array}$ & $\begin{array}{r}40.3 \mathrm{~S} \\
23.9 \mathrm{~S} \\
12.5 \mathrm{~N} \\
23.3 \mathrm{~N} \\
0.0 \mathrm{~N}\end{array}$ & $\begin{array}{c}182.8 \mathrm{~W} \\
353.5 \mathrm{E} \\
24.0 \mathrm{E} \\
29.4 \mathrm{~W} \\
270.0 \mathrm{~W}\end{array}$ & $\begin{array}{r}140 \\
125 \\
350 \\
31 \\
0\end{array}$ & $\begin{array}{l}\text { AS } \\
\text { EU } \\
\text { EU } \\
\text { SA } \\
\text { EU }\end{array}$ & $\begin{array}{l}\text { CH } \\
\text { IR } \\
\text { RM } \\
\text { VE } \\
\text { LA }\end{array}$ & $\begin{array}{l}5 \\
5 \\
5 \\
5 \\
5\end{array}$ & $\begin{array}{l}1979 \\
1994 \\
1994 \\
1976 \\
1958\end{array}$ \\
\hline $\begin{array}{l}\mathbf{J} \\
\mathbf{M} \\
\mathrm{L}\end{array}$ & $\begin{array}{ll}\text { eu } & \text { Libya Linea } \\
\text { M } & \text { Libya Montes } \\
\text { L } & \text { Licetus }\end{array}$ & $\begin{array}{r}56.2 \mathrm{~S} \\
2.7 \mathrm{~N} \\
47.1 \mathrm{~S}\end{array}$ & $\begin{array}{c}183.3 \mathrm{~W} \\
271.2 \mathrm{~W} \\
6.7 \mathrm{E}\end{array}$ & $\begin{array}{r}452 \\
1229 \\
74\end{array}$ & $\begin{array}{l}\text { EU } \\
\text { EU } \\
\text { EU }\end{array}$ & $\begin{array}{c}\text { GR } \\
\text { LA } \\
\text { IT }\end{array}$ & $\begin{array}{l}5 \\
5 \\
5\end{array}$ & $\begin{array}{l}1979 \\
1979 \\
1935\end{array}$ \\
\hline L & Lichtenberg & $31.8 \mathrm{~N}$ & $67.7 \mathrm{~W}$ & 20 & $\mathrm{EU}$ & $\mathrm{GE}$ & 5 & 1935 \\
\hline L & Lick & $12.4 \mathrm{~N}$ & $52.7 \mathrm{E}$ & 31 & NA & $\mathrm{AM}$ & 5 & 1935 \\
\hline M & Licus Vallis & $3.1 \mathrm{~S}$ & $234.2 \mathrm{~W}$ & 244 & EU & FR & 5 & 1982 \\
\hline V & $\begin{array}{l}\text { Lida } \\
\text { Liebig }\end{array}$ & $\begin{array}{l}29.1 S \\
24.3 S\end{array}$ & $\begin{array}{l}94.5 \mathrm{E} \\
48.2 \mathrm{~W}\end{array}$ & $\begin{array}{l}14 \\
37\end{array}$ & $\begin{array}{l}\mathrm{EU} \\
\mathrm{EU}\end{array}$ & $\begin{array}{l}\text { GR } \\
\text { GE }\end{array}$ & $\begin{array}{l}5 \\
5\end{array}$ & $\begin{array}{l}1994 \\
1935\end{array}$ \\
\hline 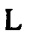 & Light Mantle & $20.2 \mathrm{~N}$ & $30.8 \mathrm{E}$ & 4 & NA & $\mathrm{AM}$ & 5 & 1973 \\
\hline $\mathrm{H}$ & Liguria & $45.0 \mathrm{~N}$ & $225.0 \mathrm{~W}$ & 0 & EU & RM & 5 & 1976 \\
\hline $\begin{array}{l}\text { V } \\
\text { V } \\
\text { L }\end{array}$ & $\begin{array}{l}\text { Lilian } \\
\text { Lilinau Corona } \\
\text { Lilius }\end{array}$ & $\begin{array}{l}25.6 \mathrm{~N} \\
34.0 \mathrm{~N} \\
54.5 \mathrm{~S}\end{array}$ & $\begin{array}{r}336.0 \mathrm{E} \\
22.0 \mathrm{E} \\
6.2 \mathrm{E}\end{array}$ & $\begin{array}{r}14 \\
200 \\
61\end{array}$ & $\begin{array}{l}\text { AS } \\
\text { NA } \\
\text { EU }\end{array}$ & $\begin{array}{r}\mathrm{HE} \\
\mathrm{AM} \\
\mathrm{IT}\end{array}$ & $\begin{array}{l}5 \\
5 \\
5\end{array}$ & $\begin{array}{l}1994 \\
1994 \\
1935\end{array}$ \\
\hline $\begin{array}{l}\text { V } \\
\text { V } \\
\text { V } \\
\text { L } \\
\text { L }\end{array}$ & $\begin{array}{l}\text { Liliya } \\
\text { Lilwani Corona } \\
\text { Lind } \\
\text { Linda } \\
\text { Lindbergh }\end{array}$ & $\begin{array}{c}30.2 \mathrm{~N} \\
29.5 \mathrm{~S} \\
50.3 \mathrm{~N} \\
30.7 \mathrm{~N} \\
5.4 \mathrm{~S}\end{array}$ & $\begin{array}{c}31.1 \mathrm{E} \\
271.5 \mathrm{E} \\
355.0 \mathrm{E} \\
33.4 \mathrm{~W} \\
52.9 \mathrm{E}\end{array}$ & $\begin{array}{r}16 \\
500 \\
28 \\
1 \\
12\end{array}$ & $\begin{array}{l}\text { UR } \\
\text { AS } \\
\text { EU } \\
\text { EU } \\
\text { NA }\end{array}$ & $\begin{array}{l}\text { RU } \\
\text { TU } \\
\text { SW } \\
\text { SP } \\
\text { AM }\end{array}$ & $\begin{array}{l}5 \\
5 \\
5 \\
5 \\
5\end{array}$ & $\begin{array}{l}1985 \\
1994 \\
1985 \\
1979 \\
1976\end{array}$ \\
\hline
\end{tabular}

\section{$\mathrm{ft}$ origin}

RE Greek; meadows of Lyrcea.

AA Gotthold Ephraim; German critic and dramatist (1729-1781).

AA First name from Latin.

AA Jean Antoine; French archaeologist (1787-1848).

AA Greek philosopher (unkn-fl. c. 440 B.C.).

AA Town in Switzerland.

AA Armin O.; American astronomer (1868-1953).

PE Hebrew sea monster upholding earth.

AA Tullio; Italian mathematician, physicist (1873-1941)

AA Gilbert N.; American chemist (1875-1946).

AA Anders Johann; Swedish mathematician, astronomer (1740-1784).

AA American colonial town (Massachusetts).

AA Willy; German-American rocketry scientist (1906-1969).

AA Judith; Dutch painter (1609-1660).

AA Tonga originator of the conditions of life.

AA Chinese poet (1081-c. 1141).

AA Chinese astronomer (c. A.D. 85).

AA Chinese writer (701-762).

AA Chinese poet (701-762).

AA Chinese essayist, scholar (1085-1151).

AA Emmanuel; French astronomer (1826-1900).

AA Chinese painter (c. 1140-1210).

FM Irish water goddess.

CR Roman fertility goddess

AA Town in Venezuela.

AL Area from W. Egypt to greater Syrtis.

LI Greek; Agenor's mother.

MO Classical albedo feature name.

AA Liceti, Fortunio; Italian physicist, philosopher. doctor (1577-1657).

AA Georg Christoph; German physicist (1742-1799).

AA James; American benefactor (1796-1876).

VA Ancient name for modern Lech River, France.

AA First name from Greek.

AA Justus, Baron von Liebig; German chemist (1803-1873)

LF Astronaut-named feature, Apollo 17 site.

AL Albedo name for unimaged H-4 region.

AA First name from Hebrew.

CR Native American fertility maiden.

AA Luigi Giglio; Italian doctor, philosopher, chronologist (unkn-1576).

AA Russian first name.

CR Hittite earth goddess.

AA Jenny; Swedish singer (1820-1887).

AA Spanish female name.

AA Charles Augustus; American aviator (1902-1974).

\footnotetext{
P: $\quad$ planetary system (see page $x v i)$.

Sa: satellite (see page $\mathrm{xvi}$ ).

lat: latitude of feature center.

long: longitude of feature center.
}

diam: diameter or long dimension of feature.

ct: continent of name origin (see page $284 \mathrm{ff}$.)

et: ethnicity of name origin (see page $284 \mathrm{ff}$.)

as: name approval status (see page xvii). ad: name approval date (year).

ref: reference source for name (see page $287 \mathrm{ff}$.).

ft: feature type (see page 290). 


\section{ALPHABETICAL LIST OF NAMES}

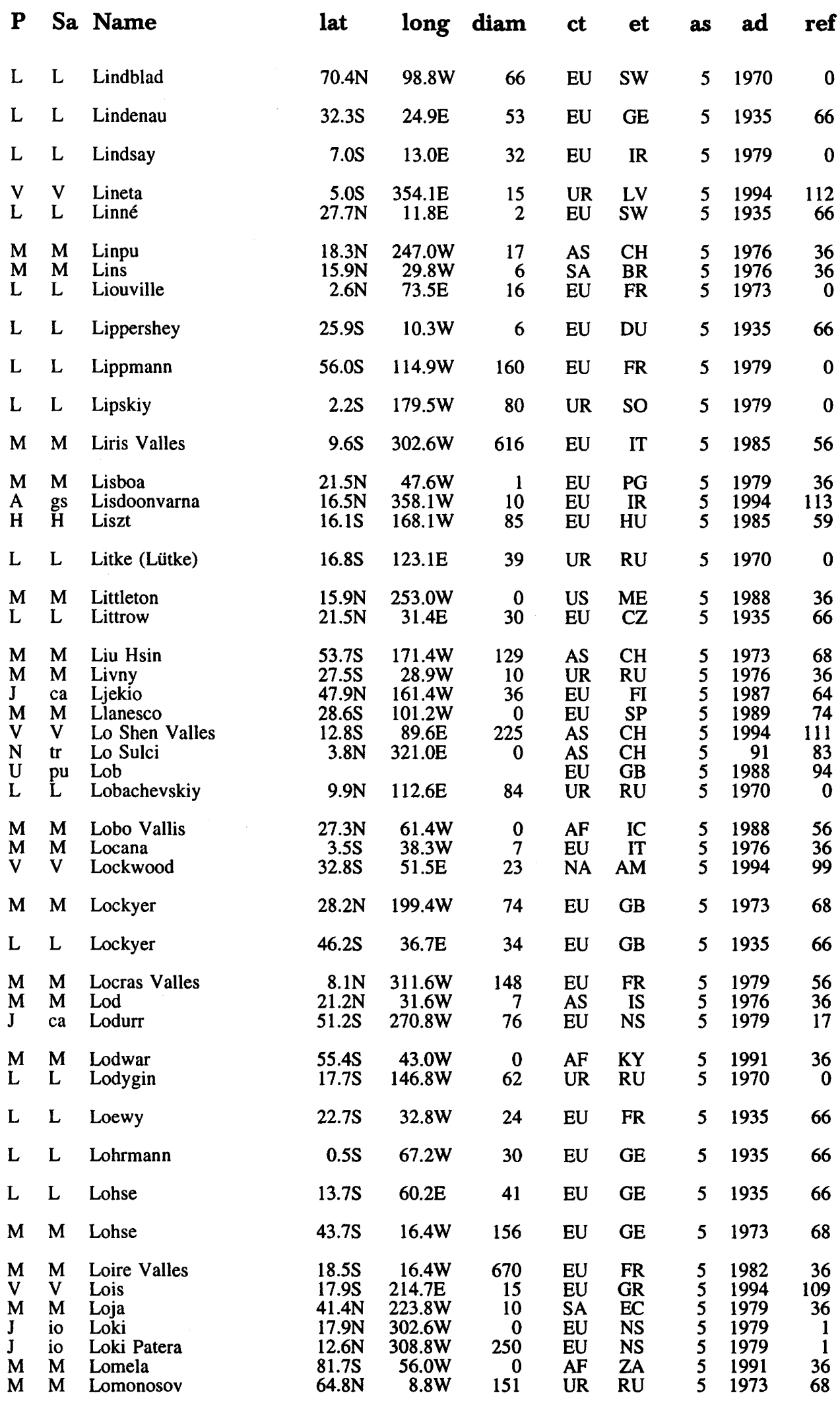

ft origin

AA Bertil; Swedish astronomer (1895-1965).

AA Bernhard von; German astronomer (1780-1854).

AA Eric M.; Irish astronomer (1907-1974).

AA Latvian first name.

AA Carl von; Swedish botanist (1707-1778).

AA Town in China (Chekiang).

AA Town in Brazil.

AA Joseph; French mathematician (1809-1882)

AA Hans (Jan Lapprey); Dutch optician (unkn-1619).

AA Gabriel; French physicist; Nobel laureate (1845-1921).

AA Yuriy N.; Soviet selenographer (1909-1978).

VA Ancient name for modern Liri River, Italy.

AA Portuguese port.

AA Spa in Ireland.

AA Franz; Hungarian piano virtuoso and composer (1811-1886).

AA Fedor P.; Russian geographer (1797-1882).

AA Town in Maine, USA.

AA Johann Josef von; Czechoslovakian astronomer (1781-1840).

AA Chinese astronomer (d. A.D. 22).

AA Town in Russia.

AA Finnish god of grass, roots of trees.

AA Town in Spain.

VA Chinese river goddess.

SU Chinese sacred river.

AA British mischievous spirits.

AA Nikolay I.; Russian mathematician (1793-1856).

VA Modern river in Ivory Coast.

AA Town in Italy.

AA Belva; American lawyer, feminist (1830-1917).

AA Joseph N.; British astronomer (1836-1920).

AA Norman; British astrophysicist (1836-1920).

VA Ancient name for river on Corsica.

AA Town in Israel.

AA Norse; god who gave first humans goodly color.

AA Town in Kenya.

AA Aleksandr N.; Russian inventor (1847-1923).

AA Moritz; French astronomer (1833-1907).

AA Wilhelm Gotthelf; German selenographer (1796-1840).

AA Oswald; German astronomer (1845-1915).

AA Oswald; German astronomer (1845-1915).

VA Modern river in France.

AA First name from Greek.

AA Town in Ecuador.

ER Norse blacksmith, trickster god.

PE Norse blacksmith, trickster god.

AA Town in Zaire.

AA Mikhail V.; Russian chemist (1711-1765).

\footnotetext{
P: $\quad$ planetary system (see page xvi).

Sa: $\quad$ satellite (see page $x v i$ ).

lat: latitude of feature center.

long: longitude of feature center.
}

diam: diameter or long dimension of feature.

ct: continent of name origin (see page $284 \mathrm{ff}$.)

et: ethnicity of name origin (see page $284 \mathrm{ff}$.)

as: name approval status (see page xvii). ad: name approval date (year).

ref: reference source for name (see page $287 \mathrm{ff}$.).

ft: feature type (see page 290). 


\section{ALPHABETICAL LIST OF NAMES}

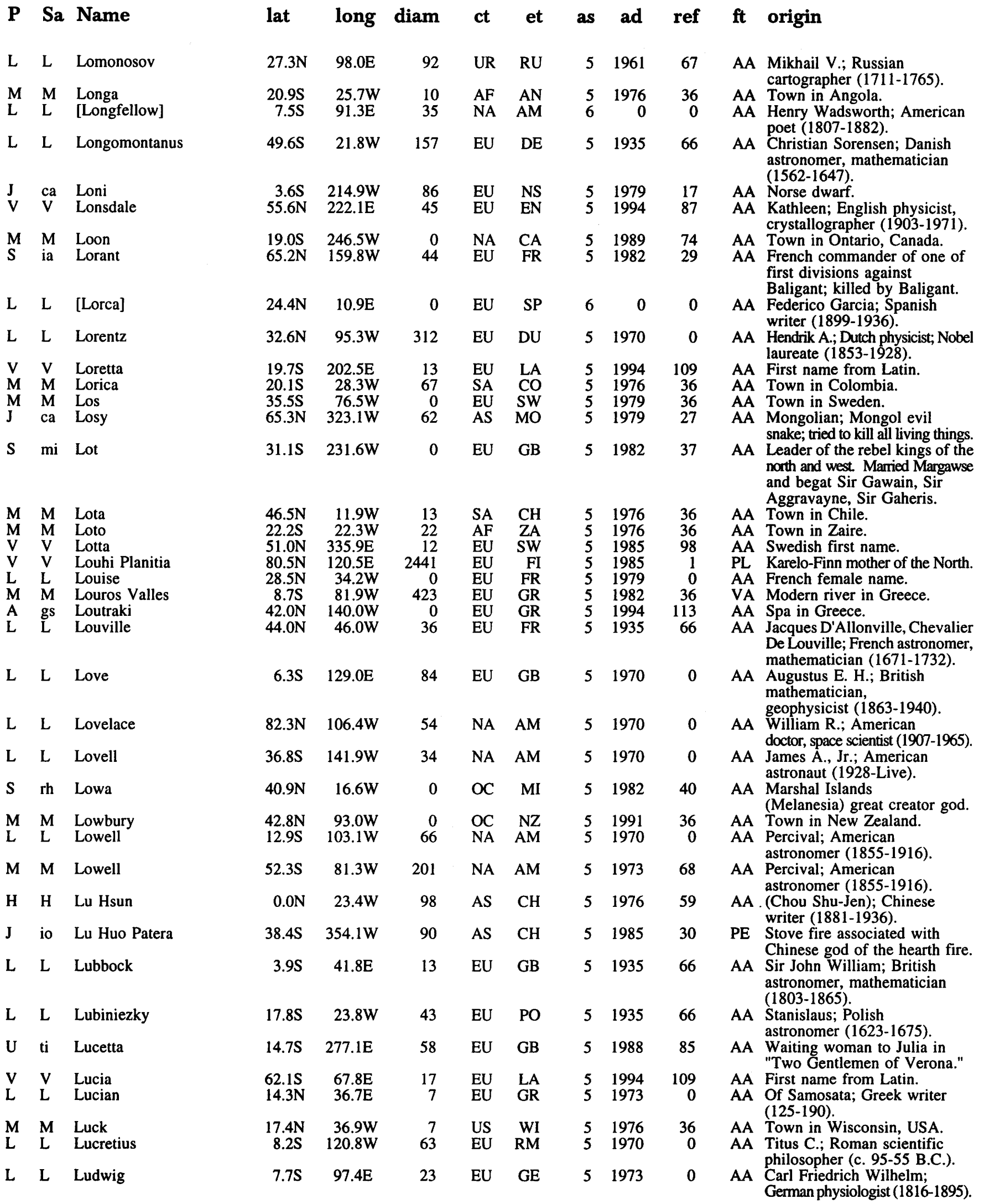

\footnotetext{
P: $\quad$ planetary system (see page xvi).

Sa: $\quad$ satellite (see page $x v i$ ).

lat: latitude of feature center.

long: longitude of feature center.
}

diam: diameter or long dimension of feature.

ct: continent of name origin (see page $284 \mathrm{ff}$.)

et: ethnicity of name origin (see page $284 \mathrm{ff}$.)

as: name approval status (see page xvii). ad: name approval date (year).

ref: reference source for name (see page $287 \mathrm{ff}$.). $\mathrm{ft}$ : feature type (see page 290). 


\section{ALPHABETICAL LIST OF NAMES}

\section{P Sa Name \\ M M Luga \\ V V Lukelong Dorsa \\ M M Luki \\ V V Lullin \\ J ga Lumha \\ M M Lunae Mensa \\ M M Lunae Palus}

lat

$\begin{array}{lcrcrrrr}44.6 \mathrm{~S} & 47.2 \mathrm{~W} & 42 & \text { UR } & \text { RU } & 5 & 1976 & 36 \\ 73.3 \mathrm{~N} & 178.8 \mathrm{E} & 1566 & \text { OC } & \text { CI } & 5 & 1985 & 1 \\ 30.0 \mathrm{~S} & 37.0 \mathrm{~W} & 20 & \text { UR } & \text { UK } & 5 & 1979 & 36 \\ 23.1 \mathrm{~N} & 81.2 \mathrm{E} & 25 & \text { EU } & \text { SZ } & 5 & 1991 & 100 \\ 37.3 \mathrm{~N} & 155.2 \mathrm{~W} & 71 & \text { AS } & \text { AB } & 5 & 1985 & 27 \\ & & & & & & & \\ 24.1 \mathrm{~N} & 62.3 \mathrm{~W} & 106 & \text { EU } & \text { RM } & 5 & 1988 & 57 \\ 15.0 \mathrm{~N} & 65.0 \mathrm{~W} & 0 & \text { EU } & \text { LA } & 5 & 1958 & 60\end{array}$
39.7

$33.2 \mathrm{~N}$

$38.5 N$

$27.3 \mathrm{~N}$

26.3

J am Lyctos Facula

M M Lycus Sulci

V V Lydia

M M Lyell

L L Lyell

L L Lyman

S ja Lynceus

L L Lyot

M M Lyot

J io Lyrcea Planum

H H Lysippus

V V Lyudmila

H H Ma Chih-Yuan

V V Ma Shouzhen

M M Ma'Adim Vallis

V V Maa-Ling

V V Maan-Eno Corona

$J$ io Maasaw Patera

V V Maat Mons

U ar Mab

U ob MacBeth

V V MacDonald

L L Mach

H H Machaut

L L Mackin-Apollo (Mackin)

L L MacLaurin

L L MacLear

L L MacMillan

L L Macrobius

M M Mad Vallis

$\begin{array}{llrlllll}20.0 \mathrm{~S} & 120.0 \mathrm{~W} & 0 & \mathrm{EU} & \text { GR } & 5 & 1979 & 19 \\ & & & & & & & \\ 29.2 \mathrm{~N} & 139.8 \mathrm{~W} & 1639 & \mathrm{EU} & \text { GR } & 5 & 1976 & 57 \\ 10.7 \mathrm{~N} & 340.8 \mathrm{E} & 15 & \text { EU } & \text { GR } & 5 & 1994 & 8 \\ 70.0 \mathrm{~S} & 15.6 \mathrm{~W} & 134 & \text { EU } & \text { GB } & 5 & 1973 & 68\end{array}$

$\begin{array}{llllllll}13.6 \mathrm{~N} & 40.6 \mathrm{E} & 32 & \mathrm{EU} & \mathrm{SC} & 5 & 1935 & 66\end{array}$

$\begin{array}{llllllll}64.8 \mathrm{~S} & 163.6 \mathrm{E} & 84 & \mathrm{NA} & \mathrm{AM} & 5 & 1970 & 0\end{array}$

$\begin{array}{lrllllll}0.0 \mathrm{~N} & 0.0 \mathrm{~W} & 0 & \mathrm{EU} & \mathrm{GR} & 5 & 1982 & 1\end{array}$

$\begin{array}{llllllll}49.8 \mathrm{~S} & 84.5 \mathrm{E} & 132 & \mathrm{EU} & \text { FR } & 5 & 1964 & 67\end{array}$

$\begin{array}{llllllll}50.7 \mathrm{~N} & 330.7 \mathrm{~W} & 220 & \mathrm{EU} & \mathrm{FR} & 5 & 1973 & 68\end{array}$

40.3S 269.3W 310 EU GR

$\begin{array}{rrrrr}0.8 \mathrm{~N} & 132.5 \mathrm{~W} & 140 & \mathrm{EU} & \mathrm{GR} \\ 62.1 \mathrm{~N} & 229.8 \mathrm{E} & 14 & \mathrm{UR} & \mathrm{RU}\end{array}$

$60.4 \mathrm{~S} \quad 78.0 \mathrm{~W} \quad 179$ AS $\mathrm{CH}$

$\begin{array}{lllll}35.7 S & 92.4 \mathrm{E} & 20 & \mathrm{AS} & \mathrm{CH}\end{array}$

21.0S $182.7 \mathrm{~W} \quad 861$ AS HE

14.7S 359.5E 6 AS CH

$40.8 \mathrm{~N} \quad 102.5 \mathrm{E}$

$40.0 \mathrm{~S} \quad 340.4 \mathrm{~W}$

$0.5 \mathrm{~N} \quad 194.6 \mathrm{E}$

300

UR ES

NA HO

395

AF

1985

1976

1985

1976

51994

1994

51994

51994

$\begin{array}{ll}5 & 1994 \\ 5 & 1979\end{array}$

51982

38.8S 352.2E

34 EU CE

$\begin{array}{rrr}203 & \text { EU } & \text { GB } \\ 19 & \text { EU } & \text { SC }\end{array}$

1988

$\begin{array}{ll}5 & 1988 \\ & \end{array}$

$\begin{array}{ll}58.4 \mathrm{~S} & 112.5 \mathrm{E} \\ 30.0 \mathrm{~N} & 120.7 \mathrm{E}\end{array}$

$18.5 \mathrm{~N} \quad 149.3 \mathrm{~W}$

180 EU AS

$5 \quad 1994$

$5 \quad 1970$

$1.9 \mathrm{~S}$

$82.1 \mathrm{~W}$

106

51976

51973

20.1N $30.7 \mathrm{E}$

0 NA AM

$5 \quad 1935$

50

EU SC

51961

$10.5 \mathrm{~N}$

$20.1 \mathrm{E}$

20

EU IR

51976

0

$24.2 \mathrm{~N} \quad 7.8 \mathrm{~W}$

NA AM

51935

66

$21.3 \mathrm{~N} \quad 46.0 \mathrm{E}$

64 EU RM

$\begin{array}{llllllll}58.0 S & 283.0 \mathrm{~W} & 460 & \text { NA } & \text { AM } & 5 & 1994 & 91\end{array}$

\section{long diam ct et as ad ref $\mathrm{ft}$ origin}

AA Town in Russia.

DO Polynesian goddess, creator of heavens.

AA Town in Ukraine.

AA Maria; Swiss entomologist (1750-1831)

AA Title of Enki as patron of singers; also Babylonian priest.

MN Albedo feature name.

AL Roman Moon goddess Luna; "Luna's Swamp," or African Lunae Mountains where Nile was thought to originate.

PM Classical albedo feature name.

AA Knut E.; Swedish astronomer (1889-1958)

AA Robert; German astronomer (1822-1900)

AA Town in Ukraine.

AA N. N.; Russian mathematician (1883-1950).

AA Aleksandr M.; Russian mathematician, engineer (1857-1918)

1.9S $68.0 \mathrm{E}$

FA Greek; area in Crete where Zeus was raised.

SU Classical albedo feature name.

AA First name from Greek.

AA Charles; British geologist (1797-1875).

AA Sir Charles; Scottish geologist (1797-1875)

AA Theodore; American physicist (1874-1954).

AA One of twin cousins of Gemini.

AA Mary; American educator, college president (1797-1849).

AA Bernard F.; French astronomer (1897-1952).

AA Bernard; French astronomer (1897-1952).

PM Plain where Io was born.

AA Greek sculptor (4th century B.C.).

AA Russian first name.

AA Chinese dramatist (fl. 1251)

AA Chinese poet, painter (1592-1628).

VA Word for "Mars" in Hebrew.

AA Chinese first name.

CR Estonian harvest goddess.

PE Hopi (USA) god of fire and death.

MO Ancient Egyptian goddess of truth and justice.

AA Queen of Spirits, dethroned Titania

AA Shakespearean hero.

AA Flora; Scottish heroine (1722-1790).

AA Ernst; Austrian physicist, philosopher (1838-1916).

AA Guillaume de; French poet and composer (c. 1300-1377).

LF Astronaut-named feature, Apollo 17 site.

AA Colin; Scottish mathematician (1698-1746).

AA Thomas; Irish astronomer (1794-1879).

AA William Duncan; American mathematician, astronomer (1871-1948).

AA Ambrosius Aurelius Theodosius; Roman writer (unkn-fl. c. 410)
P: $\quad$ planetary system (see page xvi)

Sa: $\quad$ satellite (see page $x v i)$.

lat: latitude of feature center.

long: longitude of feature center. diam: diameter or long dimension of feature.

ct: continent of name origin (see page $284 \mathrm{ff}$.)

et: ethnicity of name origin (see page $284 \mathrm{ff}$.)

as: name approval status (see page xvii). ad: name approval date (year).

ref: reference source for name (see page $287 \mathrm{ff}$.).

$\mathrm{ft}$ : feature type (see page 290). 


\section{ALPHABETICAL LIST OF NAMES}

\begin{tabular}{|c|c|c|c|c|c|c|c|c|c|c|}
\hline $\mathbf{P}$ & $\mathbf{S a}$ & Name & lat & long & diam & ct & et & as & ad & ref \\
\hline $\begin{array}{l}\text { V } \\
\text { J } \\
\text { M }\end{array}$ & $\begin{array}{l}\mathrm{V} \\
\mathrm{ca} \\
\mathrm{M}\end{array}$ & $\begin{array}{l}\text { Madeleine } \\
\text { Maderatcha } \\
\text { Mädler }\end{array}$ & $\begin{array}{l}4.7 \mathrm{~S} \\
30.5 \mathrm{~N} \\
10.8 \mathrm{~S}\end{array}$ & $\begin{array}{c}293.2 \mathrm{E} \\
95.9 \mathrm{~W} \\
357.3 \mathrm{~W}\end{array}$ & $\begin{array}{r}18 \\
57 \\
100\end{array}$ & $\begin{array}{l}\text { EU } \\
\text { EU } \\
\text { EU }\end{array}$ & $\begin{array}{l}\text { FR } \\
\text { LP } \\
\text { GE }\end{array}$ & $\begin{array}{l}5 \\
5 \\
5\end{array}$ & $\begin{array}{l}1994 \\
1987 \\
1973\end{array}$ & $\begin{array}{l}82 \\
64 \\
68\end{array}$ \\
\hline L & $\mathrm{L}$ & Mädler & $11.0 \mathrm{~S}$ & $29.8 \mathrm{E}$ & 27 & EU & GE & 5 & 1935 & 66 \\
\hline $\begin{array}{l}\mathrm{M} \\
\mathrm{L}\end{array}$ & $\begin{array}{l}M \\
L\end{array}$ & $\begin{array}{l}\text { Madrid } \\
\text { Maestlin }\end{array}$ & $\begin{array}{r}48.7 \mathrm{~N} \\
4.9 \mathrm{~N}\end{array}$ & $\begin{array}{r}224.4 \mathrm{~W} \\
40.6 \mathrm{~W}\end{array}$ & $\begin{array}{l}3 \\
7\end{array}$ & $\begin{array}{l}\text { EU } \\
\text { EU }\end{array}$ & $\begin{array}{l}\text { SP } \\
\text { GE }\end{array}$ & $\begin{array}{l}5 \\
5\end{array}$ & $\begin{array}{l}1979 \\
1961\end{array}$ & $\begin{array}{l}36 \\
67\end{array}$ \\
\hline $\begin{array}{l}\mathrm{M} \\
\mathrm{J}\end{array}$ & $\begin{array}{l}M \\
\text { io }\end{array}$ & $\begin{array}{l}\text { Mafra } \\
\text { Mafuike Patera }\end{array}$ & $\begin{array}{l}44.4 S \\
13.9 S\end{array}$ & $\begin{array}{r}53.0 \mathrm{~W} \\
260.0 \mathrm{~W}\end{array}$ & $\begin{array}{r}12 \\
110\end{array}$ & $\begin{array}{l}\text { SA } \\
\text { OC }\end{array}$ & $\begin{array}{l}\text { BR } \\
\text { HA }\end{array}$ & $\begin{array}{l}5 \\
5\end{array}$ & $\begin{array}{l}1976 \\
1979\end{array}$ & $\begin{array}{r}36 \\
1\end{array}$ \\
\hline $\begin{array}{l}M \\
V \\
M\end{array}$ & $\begin{array}{l}M \\
V \\
M\end{array}$ & $\begin{array}{l}\text { Magadi } \\
\text { Magda } \\
\text { Magelhaens }\end{array}$ & $\begin{array}{l}34.8 \mathrm{~S} \\
67.0 \mathrm{~N} \\
32.9 \mathrm{~S}\end{array}$ & $\begin{array}{l}46.0 \mathrm{~W} \\
329.6 \mathrm{E} \\
174.5 \mathrm{~W}\end{array}$ & $\begin{array}{r}57 \\
11 \\
102\end{array}$ & $\begin{array}{l}\text { AF } \\
\text { EU } \\
\text { EU }\end{array}$ & $\begin{array}{l}\text { KY } \\
\text { DE } \\
\text { PG }\end{array}$ & $\begin{array}{l}5 \\
5 \\
5\end{array}$ & $\begin{array}{l}1979 \\
1985 \\
1976\end{array}$ & $\begin{array}{l}36 \\
83 \\
68\end{array}$ \\
\hline $\mathrm{L}$ & $\mathbf{L}$ & Magelhaens & $11.9 \mathrm{~S}$ & $44.1 \mathrm{E}$ & 40 & EU & PG & 5 & 1935 & 66 \\
\hline $\mathbf{M}$ & M & Maggini & $28.0 \mathrm{~N}$ & $350.4 \mathrm{~W}$ & 146 & EU & IT & 5 & 1973 & 68 \\
\hline L & $\mathrm{L}$ & Maginus & $50.5 S$ & $6.3 W$ & 194 & EU & IT & 5 & 1935 & 66 \\
\hline $\begin{array}{l}V \\
M \\
S\end{array}$ & $\begin{array}{l}\mathrm{V} \\
\mathrm{M} \\
\mathrm{di}\end{array}$ & $\begin{array}{l}\text { Magnani } \\
\text { Mago } \\
\text { Magus }\end{array}$ & $\begin{array}{l}58.6 \mathrm{~N} \\
16.2 \mathrm{~N} \\
19.3 \mathrm{~N}\end{array}$ & $\begin{array}{r}337.0 \mathrm{E} \\
254.8 \mathrm{~W} \\
24.4 \mathrm{~W}\end{array}$ & $\begin{array}{r}27 \\
0 \\
41\end{array}$ & $\begin{array}{l}\text { EU } \\
\text { UR } \\
\text { EU }\end{array}$ & $\begin{array}{l}\text { IT } \\
\text { RU } \\
\text { RM }\end{array}$ & $\begin{array}{l}5 \\
5 \\
5\end{array}$ & $\begin{array}{l}1985 \\
1988 \\
1982\end{array}$ & $\begin{array}{l}99 \\
36 \\
44\end{array}$ \\
\hline $\mathbf{N}$ & $\operatorname{tr}$ & Mah Cavus & $38.0 \mathrm{~N}$ & $6.0 \mathrm{E}$ & 0 & PE & PE & 5 & 91 & 83 \\
\hline $\begin{array}{l}\mathrm{N} \\
\mathrm{V} \\
\mathrm{H}\end{array}$ & $\begin{array}{l}\text { tr } \\
\text { V } \\
\mathrm{H}\end{array}$ & $\begin{array}{l}\text { Mahilani } \\
\text { Mahina } \\
\text { Mahler }\end{array}$ & $\begin{array}{r}50.5 S \\
2.0 S \\
20.0 S\end{array}$ & $\begin{array}{c}359.5 \mathrm{E} \\
182.2 \mathrm{E} \\
18.7 \mathrm{~W}\end{array}$ & $\begin{array}{r}0 \\
16 \\
103\end{array}$ & $\begin{array}{l}\text { OC } \\
\text { OC } \\
\text { EU }\end{array}$ & $\begin{array}{l}\text { TG } \\
\text { HA } \\
\text { AS }\end{array}$ & $\begin{array}{l}5 \\
5 \\
5\end{array}$ & $\begin{array}{r}91 \\
1994 \\
1976\end{array}$ & $\begin{array}{r}0 \\
109 \\
59\end{array}$ \\
\hline $\begin{array}{l}\mathrm{V} \\
\mathrm{M} \\
\mathrm{M}\end{array}$ & $\begin{array}{l}\mathrm{V} \\
\mathrm{M} \\
\mathrm{M}\end{array}$ & $\begin{array}{l}\text { Mahuea Tholus } \\
\text { Maidstone } \\
\text { Main }\end{array}$ & $\begin{array}{l}37.5 S \\
41.9 S \\
76.8 S\end{array}$ & $\begin{array}{c}164.7 \mathrm{E} \\
54.1 \mathrm{~W} \\
310.9 \mathrm{~W}\end{array}$ & $\begin{array}{r}110 \\
9 \\
102\end{array}$ & $\begin{array}{l}\mathrm{OC} \\
\mathrm{EU} \\
\mathrm{EU}\end{array}$ & $\begin{array}{l}\mathrm{NZ} \\
\mathrm{GB} \\
\mathrm{GB}\end{array}$ & $\begin{array}{l}5 \\
5 \\
5\end{array}$ & $\begin{array}{l}1994 \\
1976 \\
1973\end{array}$ & $\begin{array}{r}111 \\
36 \\
68\end{array}$ \\
\hline L & $\mathrm{L}$ & Main & $80.8 \mathrm{~N}$ & $10.1 \mathrm{E}$ & 46 & EU & GB & 5 & 1935 & 66 \\
\hline $\mathrm{L}$ & $\mathbf{L}$ & Mairan & $41.6 \mathrm{~N}$ & $43.4 \mathrm{~W}$ & 40 & EU & FR & 5 & 1935 & 66 \\
\hline $\mathrm{M}$ & $\begin{array}{l}\mathrm{M} \\
\mathrm{V}\end{array}$ & $\begin{array}{l}\text { Maja Valles } \\
\text { Makh Corona }\end{array}$ & $\begin{array}{l}15.4 \mathrm{~N} \\
48.7 \mathrm{~S}\end{array}$ & $\begin{array}{l}56.7 \mathrm{~W} \\
85.0 \mathrm{E}\end{array}$ & $\begin{array}{r}1311 \\
200\end{array}$ & $\begin{array}{l}\text { AS } \\
\text { AS }\end{array}$ & $\begin{array}{l}\mathrm{NE} \\
\mathrm{AB}\end{array}$ & $\begin{array}{l}5 \\
5\end{array}$ & $\begin{array}{l}1979 \\
1994\end{array}$ & $\begin{array}{l}62 \\
83\end{array}$ \\
\hline $\begin{array}{l}\text { V } \\
\text { L }\end{array}$ & $\begin{array}{l}\mathrm{V} \\
\mathrm{L}\end{array}$ & $\begin{array}{l}\text { Makola } \\
\text { Maksutov }\end{array}$ & $\begin{array}{r}3.8 S \\
40.5 S\end{array}$ & $\begin{array}{l}106.7 \mathrm{E} \\
168.7 \mathrm{~W}\end{array}$ & $\begin{array}{l}18 \\
83\end{array}$ & $\begin{array}{l}\text { OC } \\
\text { UR }\end{array}$ & $\begin{array}{l}\text { HA } \\
\text { SO }\end{array}$ & $\begin{array}{l}5 \\
5\end{array}$ & $\begin{array}{l}1994 \\
1970\end{array}$ & $\begin{array}{r}109 \\
0\end{array}$ \\
\hline L & $\mathrm{L}$ & Malapert & $84.9 \mathrm{~S}$ & $12.9 \mathrm{E}$ & 69 & EU & $\mathrm{BE}$ & 5 & 1935 & 66 \\
\hline M & $\mathbf{M}$ & Malea Planum & $65.9 \mathrm{~S}$ & $297.4 \mathrm{~W}$ & 1068 & EU & GR & 5 & 1985 & 57 \\
\hline $\begin{array}{l}\text { J } \\
\mathrm{U}\end{array}$ & $\begin{array}{l}\text { io } \\
\text { um }\end{array}$ & $\begin{array}{l}\text { Malik Patera } \\
\text { Malingee }\end{array}$ & $\begin{array}{l}34.2 \mathrm{~S} \\
22.9 \mathrm{~S}\end{array}$ & $\begin{array}{c}128.5 \mathrm{~W} \\
13.9 \mathrm{E}\end{array}$ & $\begin{array}{r}85 \\
164\end{array}$ & $\begin{array}{l}\mathrm{AS} \\
\mathrm{OC}\end{array}$ & $\begin{array}{l}\text { BY } \\
\text { AU }\end{array}$ & $\begin{array}{l}5 \\
5\end{array}$ & $\begin{array}{l}1979 \\
1988\end{array}$ & $\begin{array}{l}27 \\
94\end{array}$ \\
\hline V & V & Malintzin Patera & $57.0 \mathrm{~N}$ & $81.5 \mathrm{E}$ & 60 & SA & $\mathrm{AZ}$ & 5 & 91 & 46 \\
\hline L & $\mathrm{L}$ & Mallet & $45.4 \mathrm{~S}$ & $54.2 \mathrm{E}$ & 58 & EU & IR & 5 & 1935 & 66 \\
\hline V & V & Maltby & $23.3 \mathrm{~S}$ & $119.8 \mathrm{E}$ & 40 & NA & $\mathrm{AM}$ & 5 & 1994 & 99 \\
\hline $\mathbf{S}$ & rh & Malunga & $65.1 \mathrm{~N}$ & $56.2 \mathrm{~W}$ & $\mathbf{0}$ & AF & YA & 5 & 1982 & 40 \\
\hline $\mathrm{L}$ & $\mathrm{L}$ & Malyy & $21.9 \mathrm{~N}$ & $105.3 \mathrm{E}$ & 41 & UR & So & 5 & 1970 & 0 \\
\hline J & io & Mama Patera & $10.6 \mathrm{~S}$ & $356.5 \mathrm{~W}$ & 30 & SA & $\mathrm{CO}$ & 5 & 1985 & 1 \\
\hline $\begin{array}{l}\mathrm{V} \\
\mathrm{M} \\
\mathrm{M}\end{array}$ & $\begin{array}{l}\mathrm{V} \\
\mathrm{M} \\
\mathrm{M}\end{array}$ & $\begin{array}{l}\text { Mama-Allpa Corona } \\
\text { Mamers Vallis } \\
\text { Manah }\end{array}$ & $\begin{array}{r}27.0 \mathrm{~S} \\
41.7 \mathrm{~N} \\
4.7 \mathrm{~S}\end{array}$ & $\begin{array}{c}31.0 \mathrm{E} \\
344.5 \mathrm{~W} \\
33.8 \mathrm{~W}\end{array}$ & $\begin{array}{r}300 \\
945 \\
9\end{array}$ & $\begin{array}{l}\text { SA } \\
\text { EU } \\
\text { AS }\end{array}$ & $\begin{array}{l}\text { PE } \\
\text { OS } \\
\text { OM }\end{array}$ & $\begin{array}{l}5 \\
5 \\
5\end{array}$ & $\begin{array}{l}1994 \\
1976 \\
1976\end{array}$ & $\begin{array}{l}83 \\
62 \\
36\end{array}$ \\
\hline
\end{tabular}

\section{$\mathrm{ft}$ origin}

AA French first name.

AA Saami sky god.

AA Johann H. von; German astronomer (1794-1874).

AA Johann Heinrich; German astronomer (1794-1874)

AA Spanish tracking site.

AA Michael; German mathematician (1550-1631)

AA Town in Brazil.

PE Hawaiian demigoddess whose fingers held fire.

AA Town in Kenya.

AA Danish first name.

AA Fernao de; Portuguese navigator (1480-1521)

AA Fernao De (Ferdinand Magellan); Portuguese explorer (1480-1521).

AA Mentore; Italian astronomer (1890-1941)

AA Magini, Giovanni Antonio; Italian astronomer, mathematician (1555-1617)

AA Anna; Italian actress (1908-1973).

AA Town in Russia.

AA A soldier of Turnus, killed by Aeneas.

CB Fish that holds up the universe (Persian).

PU Tonga sea spirit.

AA Hawaiian first name.

AA Gustave; Austrian composer (1860-1911).

TH Maori fire goddess.

AA Town in England.

AA Robert; British astronomer (1808-1878)

AA Robert; British astronomer (1808-1878).

AA Jean Jacques D'Ortous De; French geophysicist (1678-1771).

VA Nepali word for "Mars".

CR Assyro-Babylonian goddess of fecundity.

AA Hawaiian first name.

AA Dmitrij D.; Soviet optician (1896-1964)

AA Charles; Belgian astronomer, mathematician, philosopher (1581-1630).

PM From albedo feature at $60 \mathrm{~S}, 290 \mathrm{~W}$.

PE Babylonian, Caananite sun god.

AA Aboriginal spirit who travels at night.

PE (Malina); Aztec Indian guide, interpreter (1501-1550).

AA Robert; Irish seismologist, engineer (1810-1881)

AA Margaret; American physicist (1860-1944)

AA Yao (Bantu); creator god; left Earth to live in sky when man was cruel to animals.

AA Aleksandr L.; Soviet rocketry scientist (1907-1961).

PE Chagaba (Chibcha, Colombia) word for sun.

CR Peruvian harvest goddess.

VA Word for "Mars" in Oscan.

AA Town in Oman.

\footnotetext{
P: $\quad$ planetary system (see page xvi).

Sa: $\quad$ satellite (see page xvi).

lat: latitude of feature center.

long: longitude of feature center.
}

diam: diameter or long dimension of feature.

ct: continent of name origin (see page $284 \mathrm{ff}$.)

et: ethnicity of name origin (see page $284 \mathrm{ff}$.)

as: name approval status (see page xvii). ad: name approval date (year).

ref: reference source for name (see page $287 \mathrm{ff}$.).

ft: feature type (see page 290). 


\section{ALPHABETICAL LIST OF NAMES}

\section{P Sa Name}

A gs Mandal

L $\stackrel{\text { L }}{\text { Mandel'shtam }}$

M M Mandora

M M Mangala Fossa

M M Mangala Valles

$\mathrm{N}$ tr Mangwe Cavus

A gs Manikaran

L L Manilius

L L [Mann]

L L Manners

S rh Manoid

H H Mansart

H H Mansur

M M Manti

V V Manto Fossae

V V Manton

U mr Mantua Regio

J io Manua Patera

L L Manuel

V V Manzan-Gurme Tesserae

M M Manzī

L L Manzinus

V V Manzolini

L L Maraldi

M M Maraldi

V V Maram Corona

V V Maranda

M M Marbach

M M Marca

$\mathrm{H}$ H March

L L Marci

L L Marco Polo

L L Marconi

V V Mardezh-Ava Dorsa

J io Marduk

M M Mare Acidalium

L L Mare Anguis

M M Mare Australe

L L Mare Australe

M M Mare Boreum

M M Mare Chronium

M M Mare Cimmerium

L L Mare Cognitum lat long diam ct et as ad ref $\mathrm{ft}$ origin

$\begin{array}{rrrrrrrr}23.5 \mathrm{~N} & 46.5 \mathrm{~W} & 5 & \text { EU } & \text { NO } & 5 & 1994 & 113 \\ 5.4 \mathrm{~N} & 162.4 \mathrm{E} & 197 & \text { UR } & \text { SO } & 5 & 1970 & 0 \\ 12.4 \mathrm{~N} & 53.6 \mathrm{~W} & 0 & \text { OC } & \text { AU } & 5 & 1988 & 36 \\ 16.5 \mathrm{~S} & 148.8 \mathrm{~W} & 0 & \text { AS } & \text { SA } & 5 & 1991 & 62 \\ 7.4 \mathrm{~S} & 150.3 \mathrm{~W} & 880 & \text { AS } & \text { SA } & 5 & 1973 & 62 \\ 7.0 \mathrm{~S} & 343.0 \mathrm{E} & 0 & \text { AF } & \text { ZM } & 5 & 91 & 85 \\ 62.0 \mathrm{~N} & 155.0 \mathrm{~W} & 0 & \text { AS } & \text { IN } & 5 & 1994 & 113 \\ 14.5 \mathrm{~N} & 9.1 \mathrm{E} & 38 & \text { EU } & \text { RM } & 5 & 1935 & 66 \\ 23.1 \mathrm{~S} & 120.1 \mathrm{E} & 23 & \text { EU } & \text { GE } & 6 & 0 & 0 \\ 4.6 \mathrm{~N} & 20.0 \mathrm{E} & 15 & \text { EU } & \text { GB } & 5 & 1935 & 66 \\ 29.5 \mathrm{~N} & 8.5 \mathrm{~W} & 0 & \text { AS } & \text { MA } & 5 & 1982 & 40 \\ 73.2 \mathrm{~N} & 118.7 \mathrm{~W} & 95 & \text { EU } & \text { FR } & 5 & 1979 & 59 \\ & & & & & & & \\ 47.8 \mathrm{~N} & 162.6 \mathrm{~W} & 100 & \text { AS } & \text { IN } & 5 & 1979 & 59 \\ 3.7 \mathrm{~S} & 37.7 \mathrm{~W} & 0 & \text { US } & \text { UT } & 5 & 1976 & 36 \\ 63.6 \mathrm{~N} & 64.9 \mathrm{E} & 536 & \text { EU } & \text { GR } & 5 & 91 & 1 \\ 9.3 \mathrm{~N} & 26.8 \mathrm{E} & 18 & \text { EU } & \text { EN } & 5 & 1994 & 87 \\ 39.6 \mathrm{~S} & 180.2 \mathrm{E} & 399 & \text { EU } & \text { IT } & 5 & 1988 & 85 \\ & & & & & & & \\ 35.5 \mathrm{~N} & 322.0 \mathrm{~W} & 110 & \text { OC } & \text { HA } & 5 & 1979 & 1 \\ 24.5 \mathrm{~N} & 11.3 \mathrm{E} & 0 & \text { EU } & \text { SP } & 5 & 1976 & 0 \\ 39.0 \mathrm{~N} & 359.5 \mathrm{E} & 1354 & \text { AS } & \text { TB } & 5 & 1985 & 27\end{array}$

$22.3 \mathrm{~S}$

27.3W

$26.8 \mathrm{E}$

AS BU

1976

$\begin{array}{rr}7 & \text { AS } \\ 98 & \text { EU }\end{array}$

BU

$\begin{array}{ll}5 & 1935\end{array}$

36

$25.7 \mathrm{~N} \quad 91.2 \mathrm{E}$

49 EU

51991

$19.4 \mathrm{~N} \quad 34.9 \mathrm{E}$

39

EU

IT

51935

100

$62.2 \mathrm{~S}$

$32.1 \mathrm{~W}$

119 EU FR

$5 \quad 1973$

68

$7.5 \mathrm{~S} \quad 221.5 \mathrm{E}$

$4.9 \mathrm{~N} \quad 169.8 \mathrm{E}$

$17.9 \mathrm{~N} \quad 249.2 \mathrm{~W}$

$10.4 \mathrm{~S} \quad 158.2 \mathrm{~W}$

$31.1 \mathrm{~N} \quad 175.5 \mathrm{~W}$

600 AF ET

51994

600
14
20

UR

LV

51994

51976

$\begin{array}{lllll}83 & \text { SA } & \text { PE } & 5 & 1985 \\ 70 & \text { EU } & \text { SP } & 5 & 1979\end{array}$

$22.6 \mathrm{~N} \quad 167.0 \mathrm{~W}$

25

EU

CZ

$5 \quad 1970$

$15.4 \mathrm{~N} \quad 2.0 \mathrm{~W}$

$9.6 \mathrm{~S}$

145.1E

28

EU

IT

$\begin{array}{ll}5 & 1961\end{array}$

$32.4 \mathrm{~N}$

$68.6 \mathrm{E}$

27.1S 207.5W

$\begin{array}{rlrrr}906 & \text { EU } & \text { FI } & 5 & 1985 \\ 0 & \text { AS } & \text { AK } & 5 & 1979 \\ 0 & \text { EU } & \text { GR } & 5 & 1958\end{array}$

107

112

36
0

$45.0 \mathrm{~N} \quad 30.0 \mathrm{~W}$

51958

$22.6 \mathrm{~N}$

$67.7 \mathrm{E}$

$60.0 \mathrm{~S}$

$10.0 \mathrm{~W}$

$38.9 \mathrm{~S}$

$60.0 \mathrm{~N}$

$58.0 \mathrm{~S}$

93.0E

$180.0 \mathrm{~W}$

210.0W

$\begin{array}{rll}150 & \text { EU } & \text { LA } \\ 0 & \text { EU } & \text { LA } \\ 603 & \text { EU } & \text { LA } \\ 0 & \text { EU } & \text { LA } \\ 0 & \text { EU } & \text { GR }\end{array}$

51935

$\begin{array}{ll}5 & 1958 \\ 5 & 1935\end{array}$

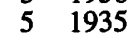

$\begin{array}{ll}5 & 1935 \\ 5 & 1958 \\ 5 & 1958\end{array}$

20.0S 220.0W

0

EU GR

$5 \quad 1958$

60

$10.0 \mathrm{~S}$

$23.1 \mathrm{~W}$
EU LA
66

0
59

AA Spa in Norway.

AA Leonid I.; Soviet physicist (1879-1944).

AA Town in Australia.

FO Named for nearby valles.

VA Word for "Mars" in Sanskrit.

CB Ila (Zambia) "the flooder."

AA Spa in India.

AA Marcus; Roman writer (unkn-c. 50 B.C.).

AA Thomas; German writer (1875-1955).

AA Russell Henry; British astronomer (1800-1870).

AA Negrito (Malay Peninsula) female progeniter goddess; wife of Pedn

AA Jules Hardouin; French architect (c. 1646-1708)

AA Ustad; Indian painter (17th century).

AA Town in Utah, USA.

FO Greek war goddess.

AA Sidnie; English zoologist (1902-1980).

RE Location of part of "Two Gentlemen From Verona."

PE Hawaiian sun god.

AA Spanish male name.

TE Ancestress who possesses the book of fate in Mongol, Tibetan, Buriat mythologies.

AA Town in Burma.

AA Manzini, Carlo Antonio; Italian astronomer (1599-1677)

AA Anna; Italian anatomist, teacher (1716-1774)

AA Giovanni Domenico; Italian astronomer, geodesist (1709-1788); Jacques Philippe; French astronomer (1665-1729).

AA Giacomo F.; French astronomer (1665-1729).

CR Oromo (Ethiopia) fertility goddess.

AA Latvian first name.

AA Town in Switzerland.

AA Village in Peru.

AA Ausias; Spanish (Catalan) poet (1397-1459).

AA Jan Marek Marci von Kronland; Czechoslovakian physicist (1595-1667)

AA Italian explorer (1254-1324).

AA Guglielmo; Italian physicist, inventor; Nobel laureate (1874-1937).

DO Marian (Volga Finn) wind goddess.

ER Sumero-Akkadian fire god.

AL Name for Acidalian (Venusian) fountain in Boeotia where the Graces bathed.

ME "Serpent Sea".

AL "South Sea"

ME "Southern Sea".

AL "North Sea"

AL "Cronian Sea", northern part of World Sea where eternal dead calm, dangerous to ships, prevailed.

AL Cimmerians were ancient Thracian seafarers; "far western sea" (Homer).

ME "Sea that has become known".

\footnotetext{
P: $\quad$ planetary system (see page xvi).

Sa: satellite (see page $x v i$ ).

lat: latitude of feature center.

long: longitude of feature center.
}

diam: diameter or long dimension of feature.

$\begin{array}{ll}\text { ct: } & \text { continent of name origin (see page } 284 \mathrm{ff} \text {.) } \\ \text { et: } & \text { ethnicity of name origin (see page } 284 \mathrm{ff} \text {.) } \\ \text { as: } & \text { name approval status (see page xvii). }\end{array}$

ad: name approval date (year).

ref: reference source for name (see page $287 \mathrm{ff}$.).

ft: feature type (see page 290). 


\section{ALPHABETICAL LIST OF NAMES}

P Sa Name

L L Mare Crisium

M M Mare Erythraeum

L L Mare Fecunditatis

L L Mare Frigoris

M M Mare Hadriacum

L L Mare Humboldtianum

L L Mare Humorum

L L Mare Imbrium

L L Mare Ingenii

L L Mare Insularum

L L Mare Marginis

L L Mare Moscoviense

L L Mare Nectaris

L L Mare Nubium

L L Mare Orientale

L L Mare Serenitatis

M M Mare Serpentis

M M Mare Sirenum

L L Mare Smythii

L L Mare Spumans

L L Mare Tranquillitatis

M M Mare Tyrrhenum

L L Mare Undarum

L L Mare Vaporum

M M Mareotis Fossae

V V Margarita

M M Margaritifer Chaos

M M Margaritifer Sinus

M M Margaritifer Terra

V V Margit

M M Mari

V V Mari Corona

V V Marie

V V Marie Celeste

A gs Marienbad

U ti Marina

M M Mariner

L L Marinus

L L Mariotte

L L Marius

J ga Marius Regio

$\mathrm{S} \quad \mathrm{mi}$ Mark

H $\quad$ H Mark Twain

V V Markham

L L Markov

V V Marsh

$S$ ia Marsilion

M M Marth

L L Marth lat

long

$17.0 \mathrm{~N}$

$25.0 \mathrm{~S}$

$7.8 \mathrm{~S}$

$56.0 \mathrm{~N}$

$40.0 \mathrm{~S}$

$56.8 \mathrm{~N}$

$24.4 \mathrm{~S}$

$32.8 \mathrm{~N}$

33.7S

$7.5 \mathrm{~N}$

$13.3 \mathrm{~N}$

$27.3 \mathrm{~N}$
$15.2 \mathrm{~S}$

$15.2 \mathrm{~S}$

21.3S

$28.0 \mathrm{~N}$

$30.0 \mathrm{~S}$

$30.0 \mathrm{~S}$

$1.3 \mathrm{~N}$

$1.1 \mathrm{~N}$

$8.5 \mathrm{~N}$
$20.0 \mathrm{~S}$

$6.8 \mathrm{~N}$

$13.3 \mathrm{~N}$

$45.0 \mathrm{~N}$

$12.8 \mathrm{~N}$

$9.1 \mathrm{~S}$

$10.0 \mathrm{~S}$

$16.2 \mathrm{~S}$

$60.1 \mathrm{~N}$

$52.4 \mathrm{~S}$

$54.0 \mathrm{~N}$

$21.7 \mathrm{~S}$

$23.5 \mathrm{~N}$
$35.4 \mathrm{~N}$

$15.5 \mathrm{~S}$

35.25

$39.4 \mathrm{~S}$

$28.5 \mathrm{~S}$

$11.9 \mathrm{~N}$

$12.1 \mathrm{~N}$

29.7S

$11.2 \mathrm{~S}$

4.1S

$53.4 \mathrm{~N}$

$59.1 \mathrm{E}$

$40.0 \mathrm{~W}$

$51.3 \mathrm{E}$

$1.4 \mathrm{E}$

$270.0 \mathrm{~W}$

81.5E

diam

ct

et

as ad

$\begin{array}{rll}418 & \text { EU } & \text { LA } \\ 0 & \text { EU } & \text { LA } \\ 909 & \text { EU } & \text { LA } \\ 1596 & \text { EU } & \text { LA } \\ 0 & \text { EU } & \text { LA } \\ 273 & \text { EU } & \text { GE }\end{array}$

$81.5 \mathrm{E} 273$

$38.6 \mathrm{~W} \quad 389$

$15.6 \mathrm{~W} \quad 1123$

$163.5 \mathrm{E}$

$30.9 \mathrm{~W}$

86.1E

$147.9 \mathrm{E}$

$35.5 \mathrm{E}$

$16.6 \mathrm{~W}$

$92.8 \mathrm{~W}$

$17.5 \mathrm{E}$

$320.0 \mathrm{~W}$

318
513

420

277

333

715

327

327
707
0

$155.0 \mathrm{~W}$

$87.5 \mathrm{E}$

$65.1 \mathrm{E}$

$31.4 \mathrm{E}$

$255.0 \mathrm{~W}$

$68.4 \mathrm{E}$

$3.6 \mathrm{E}$

$79.3 \mathrm{~W}$

0

EU LA

EU LA

EU LA

EU LA

EU LA

EU LA

EU LA

EU LA

EU LA

EU LA

EU LA

0
373

EU GR

139

139
873

0

243

245
795

9.2E

$21.4 \mathrm{~W}$

$25.0 \mathrm{~W}$

13

500

$21.3 \mathrm{~W}$

2049

73.0E

$45.7 \mathrm{~W}$

$151.0 \mathrm{E}$

232.4E

$140.2 \mathrm{E}$

$81.8 \mathrm{~W}$

$316.0 \mathrm{E}$

18

200

15

95

5
40

164.3W

76.5E

151

58

$139.1 \mathrm{~W}$

65

$50.8 \mathrm{~W}$

41

199.3W

3572

$300.1 \mathrm{~W}$

$137.9 \mathrm{~W}$

$\begin{array}{rrrrr}0 & \text { EU } & \text { GB } & 5 & 1982 \\ 149 & \text { NA } & \text { AM } & 5 & 1976\end{array}$

$155.6 \mathrm{E}$

$62.7 \mathrm{~W}$

69

EU EN

$\begin{array}{llllllll}63.7 \mathrm{~S} & 46.6 \mathrm{E} & 49 & \mathrm{OC} & \mathrm{NZ} & 5 & 1991 & 100\end{array}$

$39.2 \mathrm{~N} \quad 176.1 \mathrm{~W} \quad 136$

EU FR

13.1N 3.6W $104 \quad \mathrm{EU} \quad \mathrm{GB}$

31.1S 29.3W
51935

$5 \quad 1958$

51935

$5 \quad 1935$

$\begin{array}{ll}5 & 1958\end{array}$

$5 \quad 1935$

1979

51976

$5 \quad 1994$

$\begin{array}{ll}5 & 1964\end{array}$

$\begin{array}{ll}5 & 1982\end{array}$

29

$5 \quad 1973$

68

ref $f t$ origin

66 ME "Sea of Crises".

60 AL Indian Ocean.

ME "Sea of Fecundity".

66 ME "Sea of Cold".

60 AL Adriatic Sea.

66 ME Humboldt, Alexander von; German natural historian (1769-1859).

ME "Sea of Moisture".

ME "Sea of Showers".

ME "Sea of Cleverness".

ME "Sea of Islands".

ME "Sea of the Edge".

ME "Sea of Muscovy".

ME "Sea of Nectar".

ME "Sea of Clouds".

ME "Eastern sea"

ME "Sea of Serenity".

AL Named for Constellation Serpens (the snake).

AL "Sea of the Sirens."

ME Smyth, William Henry; British astronomer (1788-1865).

ME "Foaming Sea".

ME "Sea of Tranquility".

AL Tyrrhenian Sea, between Italy and Sicily.

ME "Sea of Waves".

ME "Sea of Vapors".

FO From albedo feature at $32 \mathrm{~N}, 96 \mathrm{~W}$.

AA Greek first name.

CH Classical albedo feature name.

AL "Pearl Bay," after Pearl Coast, South India.

TA Classical albedo feature name.

AA Hungarian first name.

AA Ruined city in Syria.

CR Cretan goddess of plenty.

AA French first name.

AA Daughter of Galileo (d. 1634).

AA Spa in Czech Republic.

AA Daughter to Pericles in "Pericles, Prince of Tyre."

AA Named for Mariner IV spacecraft.

AA Of Tyre; Greek geographer (unkn-c. 100).

AA Edme; French physicist (1620-1684).

AA Mayer, Simon; German astronomer (1570-1624).

RE Simon; German astronomer (1570-1624).

AA King of Cornwall.

AA (Samuel Clemens); American novelist, satirist (1835-1910).

AA Beryl; English aviator (1902-1986).

AA Aleksandr V.; Soviet astrophysicist (1897-1968); Andrei A.; Russian mathematician (1856-1922).

AA Ngaio; New Zealand playwright, novelist (1899-1982).

AA Saracen king of Spain; oland wounds him and he died of wound later.

AA Albert; British astronomer (1828-1897).

$51935 \quad 66$

AA Albert; German astronomer (1828-1897).

\footnotetext{
P: $\quad$ planetary system (see page $x v i)$.

Sa: $\quad$ satellite (see page $x v i$ ).

lat: latitude of feature center.

long: longitude of feature center.
} 


\section{ALPHABETICAL LIST OF NAMES}

\section{P Sa Name}

H H Martí

M M Marti Vallis

H H Martial

V V Martinez

M M Martz

L L Mary

V V Marzhan

V V Masako

J io Masaya Patera

V V [Masha]

J ga Mashu Sulcus

L L Maskelyne

V V Maslenitsa Corona

L L Mason

S di Massicus

J io Masubi

H H Matisse

M M Matrona Vallis

J io Maui

J io Maui Patera

M M Maumee Valles

M M Maunder

L L Maunder

L L Maupertuis

L L Maurolycus

L L Maury

L L Mavis

M M Mawrth Vallis

V V Mawu Corona

L L Maxwell

V V Maxwell Montes

V V Maya Corona

V V Mayaeul Corona

J io Mazda Catena

$\mathrm{N}$ tr Mazomba

$\mathrm{J}$ io Mbali Patera

V V Mbokomu Mons

L L McAdie

L L McAuliffe lat long diam

$\begin{array}{lrrrrrrr}75.6 \mathrm{~S} & 164.6 \mathrm{~W} & 68 & \text { SA } & \text { CU } & 5 & 1976 & 59 \\ & & & & & & & \\ 11.0 \mathrm{~N} & 182.0 \mathrm{~W} & 1700 & \text { EU } & \text { SP } & 5 & 1994 & 62 \\ 69.1 \mathrm{~N} & 177.1 \mathrm{~W} & 51 & \text { EU } & \text { RM } & 5 & 1979 & 59 \\ 11.7 \mathrm{~S} & 174.7 \mathrm{E} & 25 & \text { NA } & \text { PU } & 5 & 1994 & 101 \\ & & & & & & & \\ 35.2 \mathrm{~S} & 215.8 \mathrm{~W} & 91 & \text { NA } & \text { AM } & 5 & 1973 & 68 \\ & & & & & & & \\ 18.9 \mathrm{~N} & 27.4 \mathrm{E} & 1 & \text { AS } & \text { HE } & 5 & 1976 & 0 \\ & & & & & & & \\ 58.9 \mathrm{~S} & 248.5 \mathrm{E} & 20 & \text { UR } & \text { UZ } & 5 & 1994 & 112 \\ 30.2 \mathrm{~S} & 53.1 \mathrm{E} & 26 & \text { AS } & \text { JA } & 5 & 1994 & 0 \\ 22.5 \mathrm{~S} & 348.1 \mathrm{~W} & 125 & \text { SA } & \text { NI } & 5 & 1979 & 1 \\ 63.0 \mathrm{~N} & 88.0 \mathrm{E} & 16 & \text { UR } & \text { RU } & 6 & 1994 & 98 \\ 31.1 \mathrm{~N} & 209.2 \mathrm{~W} & 3030 & \text { AS } & \text { AB } & 5 & 1979 & 22\end{array}$

$\begin{array}{rrrrrrrr}2.2 \mathrm{~N} & 30.1 \mathrm{E} & 23 & \text { EU } & \text { GB } & 5 & 1935 & 66 \\ 77.0 \mathrm{~N} & 202.5 \mathrm{E} & 0 & \text { EU } & \text { SL } & 5 & 91 & 64 \\ 42.6 \mathrm{~N} & 30.5 \mathrm{E} & 33 & \text { EU } & \text { GB } & 5 & 1935 & 66 \\ & & & & & & & \\ 34.8 \mathrm{~S} & 56.0 \mathrm{~W} & 43 & \text { EU } & \text { RM } & 5 & 1982 & 44 \\ 43.6 \mathrm{~S} & 54.7 \mathrm{~W} & 0 & \text { AS } & \text { JA } & 5 & 1979 & 1 \\ 24.0 \mathrm{~S} & 89.8 \mathrm{~W} & 186 & \text { EU } & \text { FR } & 5 & 1976 & 59 \\ 7.9 \mathrm{~S} & 183.7 \mathrm{~W} & 50 & \text { EU } & \text { FR } & 5 & 1985 & 56 \\ 20.0 \mathrm{~N} & 122.0 \mathrm{~W} & 0 & \text { OC } & \text { HA } & 5 & 1979 & 1 \\ 16.5 \mathrm{~N} & 124.0 \mathrm{~W} & 45 & \text { OC } & \text { HA } & 5 & 1979 & 1 \\ 18.6 \mathrm{~N} & 55.2 \mathrm{~W} & 116 & \text { US } & \text { IN } & 5 & 1976 & 36 \\ 50.0 \mathrm{~S} & 358.1 \mathrm{~W} & 93 & \text { EU } & \text { GB } & 5 & 1973 & 68 \\ 14.6 \mathrm{~S} & 93.8 \mathrm{~W} & 55 & \text { EU } & \text { GB } & 5 & 1970 & 0\end{array}$

$\begin{array}{lllll}49.6 \mathrm{~N} & 27.3 \mathrm{~W} & 45 & \mathrm{EU} & \mathrm{FR}\end{array}$

51935

66

42.0

$37.1 \mathrm{~N}$

$14.0 \mathrm{E}$

114

EU IT

$5 \quad 1935$

17

NA AM

51935

$\begin{array}{rrrrrrrr}29.8 \mathrm{~N} & 26.4 \mathrm{~W} & 1 & \text { EU } & \text { SC } & 5 & 1976 & 0 \\ 22.4 \mathrm{~N} & 16.1 \mathrm{~W} & 575 & \text { EU } & \text { WA } & 5 & 1979 & 62 \\ 31.7 \mathrm{~N} & 241.3 \mathrm{E} & 295 & \text { AF } & \text { BE } & 5 & 1991 & 64 \\ 30.2 \mathrm{~N} & 98.9 \mathrm{E} & 107 & \text { EU } & \text { GB } & 5 & 1961 & 67 \\ & & & & & & & \\ 65.2 \mathrm{~N} & 3.3 \mathrm{E} & 797 & \text { EU } & \text { GB } & 5 & 1979 & 61 \\ 23.0 \mathrm{~N} & 98.0 \mathrm{E} & 225 & \text { AS } & \text { IN } & 5 & 1994 & 83 \\ 27.5 \mathrm{~S} & 154.0 \mathrm{E} & 200 & \text { NA } & \text { ME } & 5 & 1994 & 83 \\ 8.6 \mathrm{~S} & 313.5 \mathrm{~W} & 0 & \text { AS } & \text { BY } & 5 & 1979 & 1 \\ 18.5 \mathrm{~S} & 63.5 \mathrm{E} & 0 & \text { AF } & \text { TA } & 5 & 91 & 27 \\ 31.5 \mathrm{~S} & 7.1 \mathrm{~W} & 60 & \text { AF } & \text { PY } & 5 & 1982 & 30 \\ 15.1 \mathrm{~S} & 215.2 \mathrm{E} & 460 & \text { AF } & \text { ZA } & 5 & 1994 & 40 \\ 2.1 \mathrm{~N} & 92.1 \mathrm{E} & 45 & \text { NA } & \text { AM } & 5 & 1973 & 0 \\ 33.0 \mathrm{~S} & 148.9 \mathrm{~W} & 19 & \text { NA } & \text { AM } & 5 & 1988 & 88\end{array}$

AA Jose Julian Marti y Perez; Cuban poet and essayist (1853-1895).

VA Spanish word for "Mars".

AA Marcus Valerius; Roman epigrammist (c. A.D. 40-c. 103).

AA Maria; Pueblo artist, potter (1886-1980).

AA Edwin P.; American physicist (1916-1967).

AA English form of Hebrew female name.

AA Karakal first name.

AA Hozyo; Japanese ruler (1157-1225).

PE Nicaraguan smith god.

AA Russian first name.

SU Assyro-Babylonian; mountain with twin peaks where sun rose and set.

AA Nevil; British astronomer (1732-1811).

CR Slavonic personification of fertility.

AA Charles; British astronomer (1730-1787)

AA An Etruscan ally of Aeneas.

ER Japanese fire god.

AA Henri; French painter and sculptor (1869-1954)

VA Classical name for present Marne River, France.

ER Hawaiian demigod who sought fire from Mafuike.

PE Hawaiian demigod who sought fire from Mafuike.

VA North American river (Indiana, Ohio).

AA Edward W.; British astronomer (1851-1928).

AA Annie S. D. R.; British astronomer (1868-1947); Edward Walter; British astronomer (1851-1928).

AA Pierre Louis De; French mathematician (1698-1759).

AA Maurolico, Francesco; Italian mathematician (1494-1575).

AA Matthew Fontaine; American oceanographer (1806-1873); Antonia C.; American astronomer (1866-1952).

AA Scottish female name.

VA Welsh word for "Mars".

CR Fon (Benin) goddess of fertility.

AA James Clerk; British physicist (1831-1879).

MO James C.; British physicist (1831-1879)

CR Hindu mother earth goddess.

CR Mexican goddess of plenty.

CA Babylonian sun god.

AA Chaga (Tazania) mythical large fish.

PE Pygmy word representing fire itself. MO Ngombe (Zaire) ancestor goddess.

AA Alexander George; American meteorologist (1863-1943).

AA Sharon Christa; civilian school teacher member of the Challenger crew (1948-1986); previous designation Borman Y.

\footnotetext{
P: $\quad$ planetary system (see page xvi)

Sa: $\quad$ satellite (see page $x v i)$.

lat: latitude of feature center.

long: longitude of feature center.
}

diam: diameter or long dimension of feature.

ct: continent of name origin (see page $284 \mathrm{ff}$.)

et: ethnicity of name origin (see page $284 \mathrm{ff}$.)

as: name approval status (see page xvii). ad: name approval date (year)

ref: reference source for name (see page $287 \mathrm{ff}$.)

ft: feature type (see page 290) 


\section{ALPHABETICAL LIST OF NAMES}

\section{P Sa Name}

\section{L McClure \\ L L McDonald}

$\begin{array}{lll}\text { L } & \text { L } & \text { McKellar } \\ \text { M } & \text { M } & \text { McLaughlin } \\ \text { L } & \text { L } & \text { McLaughlin } \\ \text { L } & \text { L } & \text { McMath }\end{array}$

L L McNair

L L McNally

V V Mead

L L Mechnikov

N tr Medamothi Planum

V V Medeina Chasma

V V Medhavi

J io Media Regio

M M Medrissa

M M Medusae Fossae

M M Medusae Sulci

L L Mee

L L Mees

M M Mega

V V Megan

M M Meget

L L Meggers

J ga Mehit

L L Meitner

V V Meitner

V V Melanie

$S$ te Melanthius

M M Melas Chasma

M M Melas Dorsa

M M Melas Fossae

M M Melas Labes

V V Melba

V V Melia Mons

L L Melissa

J ga Melkart

M M Mellish

$S$ rh Melo

$\mathrm{U}$ ar Melusine

H H Melville lat long diam ct et as ad ref $f t$ origin

$\begin{array}{lrrrrrrr}15.3 \mathrm{~S} & 50.3 \mathrm{E} & 23 & \text { EU } & \text { GB } & 5 & 1935 & 66 \\ 30.4 \mathrm{~N} & 20.9 \mathrm{~W} & 7 & \text { NA } & \text { AM } & 5 & 1973 & 0 \\ & & & & & & & \\ 15.7 \mathrm{~S} & 170.8 \mathrm{~W} & 51 & \text { NA } & \text { CA } & 5 & 1970 & 0 \\ 22.1 \mathrm{~N} & 22.5 \mathrm{~W} & 90 & \text { NA } & \text { AM } & 5 & 1973 & 68 \\ 47.1 \mathrm{~N} & 92.9 \mathrm{~W} & 79 & \text { NA } & \text { AM } & 5 & 1970 & 0 \\ 17.3 \mathrm{~N} & 165.6 \mathrm{~W} & 86 & \text { NA } & \text { AM } & 5 & 1970 & 0\end{array}$

$\begin{array}{rrrrrrrr}35.7 \mathrm{~S} & 147.3 \mathrm{~W} & 29 & \text { NA } & \text { AM } & 5 & 1988 & 88 \\ 22.6 \mathrm{~N} & 127.2 \mathrm{~W} & 47 & \text { NA } & \text { AM } & 5 & 1970 & 0 \\ 12.5 \mathrm{~N} & 57.2 \mathrm{E} & 280 & \text { NA } & \text { AM } & 5 & 1991 & 100 \\ 11.0 \mathrm{~S} & 149.0 \mathrm{~W} & 60 & \text { UR } & \text { RU } & 5 & 1970 & 0\end{array}$

$\begin{array}{llllllll}3.5 \mathrm{~N} & 69.0 \mathrm{E} & 0 & \mathrm{EU} & \mathrm{FR} & 5 & 91 & 83\end{array}$

$\begin{array}{llllllll}46.2 \mathrm{~N} & 89.3 \mathrm{E} & 606 & \text { UR } & \text { LI } & 5 & 1985 & 65\end{array}$

$\begin{array}{llllllll}19.5 \mathrm{~S} & 40.6 \mathrm{E} & 30 & \text { AS } & \text { IN } & 5 & 1994 & 99\end{array}$

$\begin{array}{llllllll}4.6 \mathrm{~N} & 58.8 \mathrm{~W} & 0 & \text { EU } & \text { GR } & 5 & 1979 & 19\end{array}$

$18.8 \mathrm{~N} \quad 56.6 \mathrm{~W}$

$2.0 \mathrm{~S} \quad 162.1 \mathrm{~W}$

5.3S $159.8 \mathrm{~W}$

43.7S $35.3 \mathrm{~W}$

0 AF $\mathrm{AL}$

$5 \quad 1988$

291

EU LA

EU LA

1973

51985

126

NA AM

51935

$13.6 \mathrm{~N}$

$1.5 \mathrm{~S}$

96.1W

50

51970

61.7S

$37.0 \mathrm{~W}$

$19.1 \mathrm{~N}$

$29.7 \mathrm{~N} \quad 165.1 \mathrm{~W}$

AF ET

EU WA

UR RU

51976

$\begin{array}{ll}5 & 1976 \\ 5 & 1994\end{array}$

$5 \quad 1988$

51970

51985

AF EG

$5 \quad 1970$

10.5S $\quad 112.7 \mathrm{E}$

EU AS

51979

$55.6 \mathrm{~S}$

$321.6 \mathrm{E}$

150

EU AS

$5 \quad 1994$

$\begin{array}{rll}16 & \text { EU } & \text { GR } \\ 0 & \text { EU } & \text { GR }\end{array}$

$\begin{array}{ll}5 & 1994 \\ 5 & 1982\end{array}$

204.0W

51973

$10.5 \mathrm{~S}$

$17.8 \mathrm{~S}$

$72.9 \mathrm{~W}$

$70.9 \mathrm{~W}$

526 EU GR

501

EU GR

EU GR

51976

$5 \quad 1985$

$9.1 \mathrm{~S}$

71.2W

128

EU GR

51985

4.7N $\quad 193.4 \mathrm{E}$

$62.8 \mathrm{~N}$

$8.1 \mathrm{~N}$

10.0S

$119.3 \mathrm{E}$
$121.8 \mathrm{E}$

24 OC AU

51994

311 EU GR

$\begin{array}{lll}311 & \text { EU } & \text { GR } \\ 18 & \text { EU } & \text { GR }\end{array}$

111 AS PO

51985

$\begin{array}{ll}5 & 1985 \\ 5 & 1979\end{array}$

51979

51994

$53.2 \mathrm{~S} \quad 7.1 \mathrm{~W}$

$52.9 \mathrm{~S}$

$24.0 \mathrm{~W}$

NA AM

$5 \quad 1982$

$\begin{array}{rllll}0 & \text { AS } & \text { IN } & 5 & 1982 \\ 50 & \text { EU } & \text { FR } & 5 & 1988\end{array}$

$21.5 \mathrm{~N} \quad 10.1 \mathrm{~W}$

154

NA AM
AA Robert Le Mesurier;

British explorer (1807-1873).

AA William Johnson; American benefactor (1844-1926); Thomas Logie; Scottish selenographer (unkn.-1973).

AA Andrew; Canadian astronomer (1910-1960).

AA Dean B.; American astronomer (1901-1965).

AA Dean B.; American astronomer (1901-1965).

AA Francis C.; American engineer, astronomer (1867-1938); Robert R.; American astronomer (1891-1962).

AA Ronald Erwin; member of the Challenger crew (1950-1986); previous designation Borman A

AA Paul A.; American astronomer (1890-1955).

AA Margaret; American anthropologist (1901-1978).

AA Il'ya I.; Russian-French bacteriologist; Nobel laureate (1845-1916).

PM French fictional island, meaning "nowhere."

CM Lithuanian forest goddess.

AA Ramabai; East Indian author, humanitarian (1858-1922).

RE Greek; Io passed through this part of Iran in her wanderings.

AA Town in Algeria.

FO Classical albedo feature name.

SU Albedo feature name.

AA Arthur Butler Phillips; Scottish astronomer (1860-1926).

AA C. E. Kenneth; Anglo-American photographer (1882-1960).

AA Town in Ethiopia.

AA Welsh first name.

AA Town in Russia.

AA William F.; American physicist (1888-1968).

AA Egyptian lion-headed goddess; Anhur's wife.

AA Lise; Austrian physicist (1878-1968).

AA Lise; Austrian physicist (1878-1968).

AA First name from Greek.

AA Disloyal goatherd; insults Odysseus; is slain.

CM Classical albedo feature name.

DO Classical albedo feature name.

FO Classical albedo feature name.

LA From albedo feature at $10 \mathrm{~S}, 74 \mathrm{~W}$.

AA Nellie; Australian opera singer (1861-1931).

MO Greek nymph.

AA Greek female name.

AA Phoenician; divinity of Tyre.

AA John E:; American amateur astronomer (1886-1970).

AA Minyong (India); original male.

AA Spirit heroine of medieval French story.

AA Herman; American novelist (1819-1891).
P: $\quad$ planetary system (see page xvi)

Sa: satellite (see page $x v i$ ).

lat: latitude of feature center.

long: longitude of feature center. diam: diameter or long dimension of feature.

ct: continent of name origin (see page $284 \mathrm{ff}$.)

et: ethnicity of name origin (see page $284 \mathrm{ff}$.)

as: name approval status (see page xvii). ad: name approval date (year).

ref: reference source for name (see page $287 \mathrm{ff}$.).

ft: feature type (see page 290). 
ALPHABETICAL LIST OF NAMES

$\begin{array}{lll}\text { P } & \text { Sa } & \text { Name } \\ & & \\ \text { M } & \text { M } & \text { Memnonia } \\ \text { M } & \text { M } & \text { Memnonia Fossae } \\ \text { M } & \text { M } & \text { Memnonia Sulci } \\ \text { J } & \text { ga } & \text { Memphis Facula } \\ \text { M } & \text { M } & \text { Mena } \\ \text { H } & \text { H } & \text { Mena } \\ \text { V } & \text { V } & \text { Mena Colles } \\ \text { J } & \text { io } & \text { Menahka Patera } \\ \text { V } & \text { V } & \text { Menat Undae } \\ \text { M } & \text { M } & \text { Mendel } \\ & & \\ \text { L } & \text { L } & \text { Mendel } \\ \text { L } & \text { L } & \text { Mendeleev } \\ \text { H } & \text { H } & \text { Mendes Pinto } \\ \text { M } & \text { M } & \text { Mendota } \\ \text { L } & \text { L } & \text { Menelaus } \\ \text { V } & \text { V } & \text { Meni Tessera } \\ \text { V } & \text { V } & \text { Mentha Mons } \\ \text { S } & \text { te } & \text { Mentor } \\ \text { L } & \text { L } & \text { Menzel } \\ & & \\ \text { J } & \text { ca } & \text { Mera } \\ \text { L } & \text { L } & \text { Mercator } \\ & & \end{array}$

$$
s
$$


ALPHABETICAL LIST OF NAMES

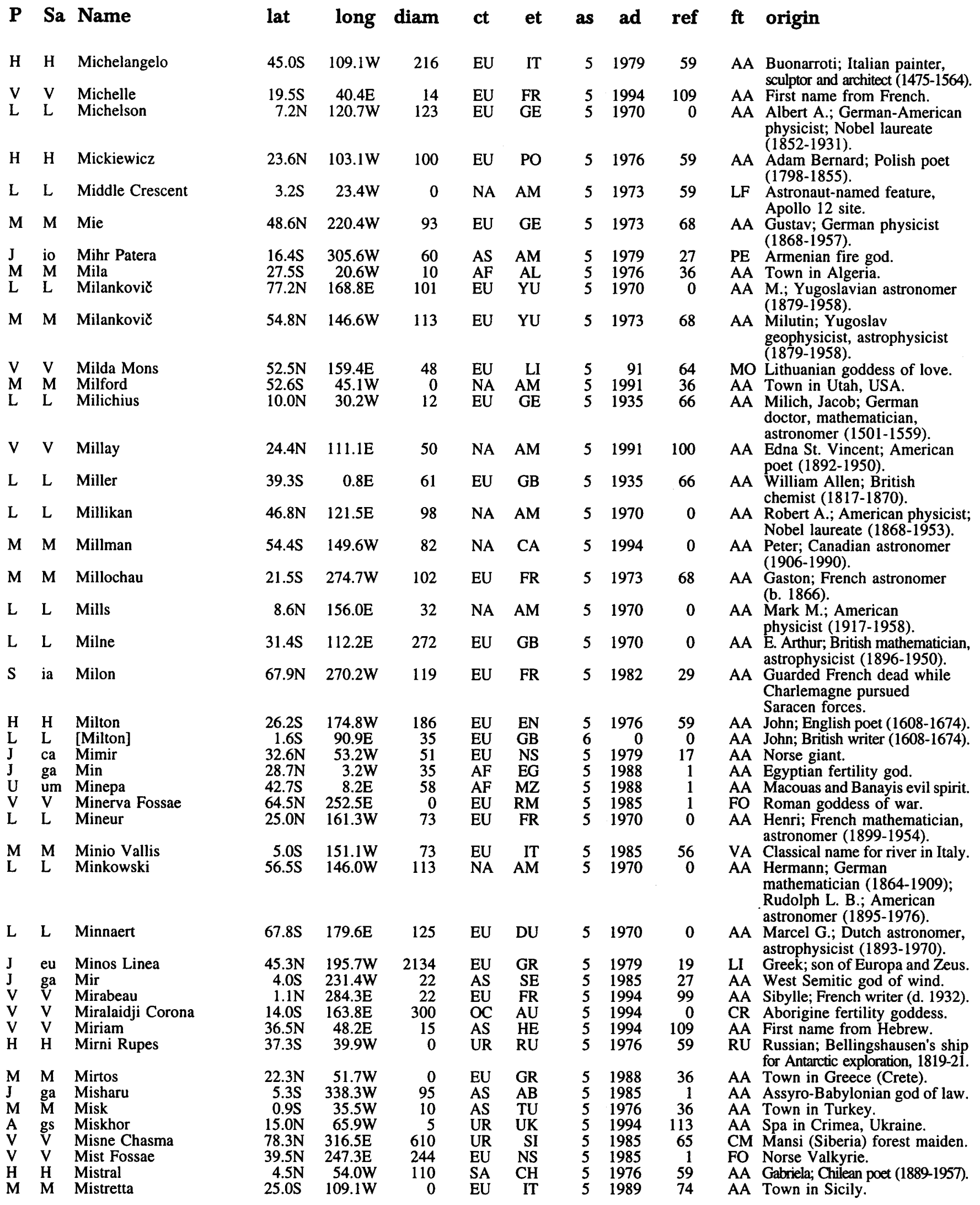

\footnotetext{
P: $\quad$ planetary system (see page $x v i)$.

Sa: $\quad$ satellite (see page $x v i$ ).

lat: latitude of feature center.

long: longitude of feature center.
}

diam: diameter or long dimension of feature.

ct: continent of name origin (see page $284 \mathrm{ff}$.)
et: ethnicity of name origin (see page $284 \mathrm{ff}$.)

as: name approval status (see page xvii). ad: name approval date (year).

ref: reference source for name (see page $287 \mathrm{ff}$.). ft: feature type (see page 290). 


\section{ALPHABETICAL LIST OF NAMES}

\section{P Sa Name}

$\begin{array}{lll}\text { M } & \text { M } & \text { Mitchel } \\ \text { L } & \text { L } & \text { Mitchell } \\ \text { J } & \text { io } & \text { Mithra Patera } \\ \text { L } & \text { L } & \text { Mitra } \\ \text { J } & \text { ca } & \text { Mitsina } \\ \text { M } & \text { M } & \text { Mliba } \\ \text { V } & \text { V } & \text { Mnemosyne Regio } \\ \text { M } & \text { M } & \text { Moab } \\ \text { L } & \text { L } & \text { Möbius } \\ & & \\ \text { J } & \text { ca } & \text { Modi } \\ \text { S } & \text { mi } & \text { Modred }\end{array}$

M M Moeris Lacus

H H Mofolo

M M Mohawk

L L Mohoróvičic

L L Moigno

V V Moira Tessera

L L Moiseev

L L Moissan

V V Mokosha Mons

M M Molesworth

H H Molière

V V Molpadia Linea

V V Molpe Colles

L L Moltke

U ob Mommur Chasma

V V Mona Lisa

$N$ tr Monad Regio

$\mathrm{H} H$ Monet

L L Monge

V V Monika

L L Monira

L L Mons Agnes

L L Mons Ampère

L L Mons André

L L Mons Ardeshir

L L Mons Argaeus

L L Mons Bradley

L L Mons Delisle

L L Mons Dieter

L L Mons Dilip

L L Mons Esam

L L [Mons Euler]

L L Mons Ganau lat long cian

diam

$\begin{array}{rrrrrrrr}67.8 \mathrm{~S} & 284.0 \mathrm{~W} & 141 & \text { NA } & \text { AM } & 5 & 1973 & 68 \\ 49.7 \mathrm{~N} & 20.2 \mathrm{E} & 30 & \text { NA } & \text { AM } & 5 & 1935 & 66 \\ & & & & & & & \\ 58.8 \mathrm{~S} & 267.9 \mathrm{~W} & 25 & \text { AS } & \text { PE } & 5 & 1985 & 1 \\ 18.0 \mathrm{~N} & 154.7 \mathrm{~W} & 92 & \text { AS } & \text { IN } & 5 & 1970 & 0 \\ 57.5 \mathrm{~N} & 104.7 \mathrm{~W} & 43 & \text { NA } & \text { ES } & 5 & 1979 & 26 \\ & & & & & & & \\ 39.9 \mathrm{~S} & 272.1 \mathrm{~W} & 0 & \text { AF } & \text { SW } & 5 & 1989 & 74 \\ 65.8 \mathrm{~N} & 277.9 \mathrm{E} & 0 & \text { EU } & \text { GR } & 5 & 1982 & 1 \\ 20.0 \mathrm{~N} & 350.0 \mathrm{~W} & 0 & \text { AS } & \text { HE } & 5 & 1958 & 60 \\ 15.8 \mathrm{~N} & 101.2 \mathrm{E} & 50 & \text { EU } & \text { GE } & 5 & 1970 & 0 \\ & & & & & & & \\ 66.4 \mathrm{~N} & 120.9 \mathrm{~W} & 43 & \text { EU } & \text { NS } & 5 & 1979 & 17 \\ 5.0 \mathrm{~N} & 213.0 \mathrm{~W} & 0 & \text { EU } & \text { GB } & 5 & 1982 & 37\end{array}$

$8.0 \mathrm{~N} \quad 270.0 \mathrm{~W}$

37.7

$28.2 \mathrm{~W}$

$43.2 \mathrm{~N} \quad 5.4 \mathrm{~W}$

19.0S 165.0W

$66.4 \mathrm{~N} \quad 28.9 \mathrm{E}$

$58.7 \mathrm{~N} \quad 310.5 \mathrm{E}$

$9.5 \mathrm{~N} \quad 103.3 \mathrm{E}$

361

$4.8 \mathrm{~N}$

137.4E

$57.7 \mathrm{~N}$

$27.8 \mathrm{~S}$

$15.6 \mathrm{~N}$

$255.0 \mathrm{E}$

$210.6 \mathrm{~W}$

$16.9 \mathrm{~W}$

48.0S

$74.7 \mathrm{~N}$

$0.6 \mathrm{~S}$

$359.0 \mathrm{E}$

(6.6S

$24.2 \mathrm{E}$

$16.3 \mathrm{~S} \quad 323.5 \mathrm{E}$

$25.5 \mathrm{~N}$

$20.0 \mathrm{~N}$

$44.4 \mathrm{~N}$

$19.2 \mathrm{~S}$

$72.3 \mathrm{~N}$

$12.6 \mathrm{~S}$

$18.6 \mathrm{~N}$
$19.0 \mathrm{~N}$

\section{$5.2 \mathrm{~N}$}

$5.0 \mathrm{~N}$

$63.6 \mathrm{~S}$

$22.0 \mathrm{~N}$

$29.5 \mathrm{~N}$

$5.6 \mathrm{~N}$

$14.6 \mathrm{~N}$

$23.3 \mathrm{~N}$

25.1E

37.0E

$10.3 \mathrm{~W}$

47.6E

$122.3 \mathrm{E}$

$1.7 \mathrm{~W}$

$5.3 \mathrm{E}$

$4.0 \mathrm{~W}$

EU FR

51976

270

175

EU SL

$$
\text { EU }
$$

132

EU

FR

$5 \quad 1985$

$\begin{array}{ll}5 & 1973\end{array}$

51976

0 EU GR

548 EU GR

$5 \quad 1982$

$\begin{array}{ll}5 & 1991 \\ 5 & 1935\end{array}$

537 EU GB

51988

$\begin{array}{lllll}86 & \text { EU } & \text { IT } & 5 & 1991\end{array}$

0
303

0
303
36

AS CH

EU FR

$5 \quad 91$

$\begin{array}{ll}5 & 1979 \\ 5 & 1935\end{array}$

26

EU GE

AS AR

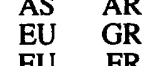

51985

$\begin{array}{ll}5 & 1976\end{array}$

$\begin{array}{ll}5 & 1979 \\ 5 & 1935\end{array}$

30

EU FR

$5 \quad 1976$

$120.6 \mathrm{E}$

$121.0 \mathrm{E}$

$30.1 \mathrm{E}$

$\begin{array}{rll}10 & \text { EU } & \text { FR } \\ 8 & \text { AS } & \text { PE } \\ 77 & \text { EU } & \text { SP }\end{array}$

$\begin{array}{ll}5 & 1976 \\ 5 & 1935\end{array}$

30

EU GB

51961

51985

$35.8 \mathrm{~W}$

120.2E

$120.8 \mathrm{E}$

35.7E

$29.2 \mathrm{~W}$

30
20

EU FR

EU GE

AS IN

$\begin{array}{ll}\text { AS } & \text { AR } \\ \text { EU } & \text { SZ }\end{array}$

$5 \quad 1976$

51976

$\begin{array}{ll}5 & 1979\end{array}$

$\begin{array}{ll}6 & 1979\end{array}$

$4.8 \mathrm{~N} \quad 120.6 \mathrm{E}$

14 AF UN

51976 diam: diameter or long dimension of feature.

ct: continent of name origin (see page $284 \mathrm{ff}$.)

et: ethnicity of name origin (see page $284 \mathrm{ff}$.)

as: name approval status (see page xvii).
AA Ormsby M.; American astronomer (1809-1862).

AA Maria; American astronomer (1818-1889)

PE Persian god of light.

AA S. K.; Indian physicist (1890-1963).

AA Alaskan old man who perished while hunting on ice.

AA Town in Swaziland.

RE Greek Titaness.

AL Biblical town bordering dom.

AA August F.; German mathematician, astronomer (1790-1868).

AA Norse; son of Thor and Sif.

AA Arthur's bastard son and mortal enemy; delivered fatal wound to Arthur but was killed by him.

AL "Moeris Lake;" Moeris was Egyptian lake in Libyan Desert.

AA Thomas; South African (Lesotho) novelist (1876/77-1948).

AA Town in New York, USA.

AA Andrija; Croatian geophysicist (1857-1936)

AA Francois Napoleon Marie; French mathematician, physicist (1804-1884)

TE Greek fate goddess.

AA Nikolaj D.; Soviet astronomer (1902-1955).

AA Ferdinand Frederic Henri; French chemist; Nobel laureate (1852-1907).

64

MO East Slavic main goddess.

AA Percy B.; British astronomer (1867-1908).

AA Jean Baptiste Poquelin; French dramatist and satirist (1622-1673).

LI Amazon.

CO Greek; mother of Sirens.

AA Helmuth Karl, Graf von; German benefactor (1800-1891).

CM Spirit place, forest home of Oberon in "Midsummer Night's Dream."

99

AA (Lisa Giacondo); Leonardo da Vinci's model (b. c. 1474).

83

59

RE Chinese symbol of duality in nature.

AA Claude; French painter (1840-1926).

AA Gaspard; French mathematician (1746-1818).

AA German first name.

AA Arabic female name.

MO Greek female name.

MO Andre Marie; French physicist (1775-1836).

MO French male name.

MO Persian (Iranian) king's name.

AA Vincente Mut, or Muth; Spanish astronomer (unkn-1673).

MO James; British astronomer (1692-1762).

MO Named from nearby crater.

MO German male name.

MO Indian male name.

MO Arabic male name.

MO Leonhard; Swiss mathematician (1707-1783); now called Mons Vinogradov.

MO African male name.

\footnotetext{
P: $\quad$ planetary system (see page xvi).

satellite (see page xvi).

long: longitude of feature center.
} 


\section{P Sa Name}

L L Mons Gruithuisen Delta 36.0N

L L Mons Gruithuisen Gamma $36.6 \mathrm{~N}$

L L Mons Hadley 26.5N

L L Mons Hadley Delta

L L Mons Hansteen

L L Mons Herodotus

L L Mons Huygens

L L Mons La Hire

L L Mons Maraldi

L L Mons Moro

L L Mons Penck

L L Mons Pico

L L Mons Piton

L L Mons Rümker

L L Mons Usov

L L Mons Vinogradov

L L Mons Vitruvius

L L Mons Wolff

L L Mont Blanc

V V Montagu

L L [Montaigne]

L L Montanari

L L Montes Agricola

L L Montes Alpes

L L Montes Apenninus

L L Montes Archimedes

L L Montes Carpatus

L L Montes Caucasus

L L Montes Cordillera

L L Montes Haemus

L L Montes Harbinger

L L Montes Jura

L L Montes Pyrenaeus

L L Montes Recti

L L Montes Riphaeus

L L Montes Rook

L L Montes Secchi

L L Montes Spitzbergen

L L Montes Taurus

L L Montes Teneriffe

L . L [Montesquieu]

V V Montessori

M M Montevallo

$19.4 \mathrm{~N}$

$17.0 \mathrm{~N}$

$45.0 \mathrm{~N}$

$36.9 \mathrm{~N}$

4.4S

$45.8 \mathrm{~S}$

$29.1 \mathrm{~N}$

$46.4 \mathrm{~N}$

$18.9 \mathrm{~N}$

$25.3 \mathrm{~N}$

$14.5 \mathrm{~N}$

$38.4 \mathrm{~N}$

$17.5 \mathrm{~S}$

$19.9 \mathrm{~N}$

$27.0 \mathrm{~N}$

$47.1 \mathrm{~N}$

$15.6 \mathrm{~S}$

$48.0 \mathrm{~N}$

$7.7 \mathrm{~S}$

$20.6 S$

$3.0 \mathrm{~N}$

$35.0 \mathrm{~N}$

$28.4 \mathrm{~N}$

$47.1 \mathrm{~N}$

$6.2 \mathrm{~S}$
$25.8 \mathrm{~N}$

$12.1 \mathrm{~S}$

$27.5 \mathrm{~N}$

$20.0 \mathrm{~N}$

$27.8 \mathrm{~N}$

\section{long \\ diam}

$40.5 \mathrm{~W}$

4.7E

$3.8 \mathrm{E}$

$50.0 \mathrm{~W}$

$53.0 \mathrm{~W}$

$2.9 \mathrm{~W}$

$25.5 \mathrm{~W}$

$20.3 \mathrm{~N}$

$12.0 \mathrm{~S}$

35.3E

$19.7 \mathrm{~W}$

$10.0 \mathrm{~S}$

$45.7 \mathrm{~N}$

21.6E

$40.6 \mathrm{~N}$

$8.9 \mathrm{~W}$

$40.8 \mathrm{~N}$

$12.0 \mathrm{~N}$

$22.4 \mathrm{~N}$

20
20
25
15
30
5
40

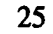

15

30

25

$58.1 \mathrm{~W}$

70

$63.0 \mathrm{E}$

$32.4 \mathrm{~W}$

15

25
$\begin{array}{ll}E U & G E \\ E U & G E\end{array}$

EU GB

EU GB

EU SW

EU GR

EU DU

EU

FR

51961

$\begin{array}{llll}\text { EU } & \text { IT } & 5 & 1976 \\ \text { EU } & \text { IT } & 5 & 1976\end{array}$

EU GE

51976

EU SP

51935

EU GE

UR SO

51935

51979

UR SO

$5 \quad 1979$

$30.8 \mathrm{E}$

$1.0 \mathrm{E}$

15
35

EU RM

EU GE

51976

99.5E

$20.6 \mathrm{~W}$

$54.2 \mathrm{~W}$

$0.8 \mathrm{~W}$

$3.7 \mathrm{~W}$

$4.6 \mathrm{~W}$

$24.4 \mathrm{~W}$

$10.0 \mathrm{E}$

$81.6 \mathrm{~W}$

$9.2 \mathrm{E}$

$41.0 \mathrm{~W}$

$34.0 \mathrm{~W}$

41.2E

20.0W

$28.1 \mathrm{~W}$

$82.5 \mathrm{~W}$

43.0E

$5.0 \mathrm{~W}$

41.1E

$11.8 \mathrm{~W}$

92.3E

$59.4 \mathrm{~N} \quad 280.2 \mathrm{E}$

$\begin{array}{ll}15.3 \mathrm{~N} & 54.2 \mathrm{~W}\end{array}$
EU FR

EU EN

$$
25
$$

EU FR

76

EU

141

EU GE

281

401
163

361
445

EU SZ

EU IT

EU RO

574

560

EU SP

EU BU

EU LA

422

EU GE

164

90

EU SP

EU LA

791

EU GB

50

EU IT

$\begin{array}{ll}\text { EU } & \text { IT }\end{array}$

$\begin{array}{ll}5 & 1976 \\ 5 & 1961\end{array}$

172 AS TU 51935

182

AF $\quad C$

$5 \quad 1935$

$\begin{array}{rrr}182 & \text { AF } & \text { CI } \\ 0 & \text { EU } & \text { FR }\end{array}$

(2)

44
0 $\begin{array}{llll}\text { EU } & \text { IT } & 5 & 1985 \\ \text { US } & \text { AL } & 5 & 1988\end{array}$ ref $\mathbf{f t}$ origin

0
0
66
66
0
0
67

MO Named from nearby crater.

MO Named from nearby crater.

MO Hadley, John; British instrument maker (1682-1743).

MO Named from nearby mountain.

MO Named from nearby crater.

MO Named from nearby crater.

MO Christian; Dutch astronomer, mathematician, physicist (1629-1695).

67

MO Philippe De; French mathematician, astronomer (1640-1718).

MO Named from nearby crater.

MO Antonio Lazzaro; Italian Earth scientist (1687-1764)

MO Albrecht; German geographer (1858-1945).

MO Spanish for "peak".

MO Named from Mt. Piton on Tenerife Islands.

MO Karl Ludwig Christian; German astronomer (1788-1862).

MO Mikhail A.; Soviet geologist (1883-1933).

MO Aleksandr Pavlovich; Soviet geochemist and cosmochemist (1895-1975); formerly called Mons Euler.

MO Named from nearby crater.

MO Christian, Baron von; German philosopher (1679-1754).

MO Named for terrestrial mountain in Alps.

AA Mary; English medical pioneer, poet, writer (1689-1762).

AA Michel De; French writer (1533-1592)

AA Geminiano; Italian astronomer, mathematician (1633-1687).

MO Georgius; German Earth scientist (1494-1555).

MO Named from terrestrial Alps.

MO Named from terrestrial Apennines.

MO Named from nearby crater.

MO Named from terrestrial Carpathians.

MO Named from terrestrial Caucasus Mountains.

MO Spanish for "mountain chain".

MO Named for range in the Balkans.

MO Harbingers of dawn on crater Aristarchus.

MO Named from terrestrial Jura Mountains.

MO Named from terrestrial Pyrenees.

MO Latin for "straight range".

MO Named from range in Asia (now Ural Mountains).

MO Lawrence; British astronomer (1622-1666).

MO Named from nearby crater.

MO German for "sharp peaks", and named for resemblance to the terrestrial island group.

66

MO Named from terrestrial Taurus Mts.

66
0

MO Named from terrestrial island.

AA Charles De Secondat, Baron De; French writer (1689-1755).

46
36
AA Maria; Italian educator (1870-1952).

AA Town in Alabama, USA.

\footnotetext{
P: $\quad$ planetary system (see page $x v i)$.
} 


\section{ALPHABETICAL LIST OF NAMES}

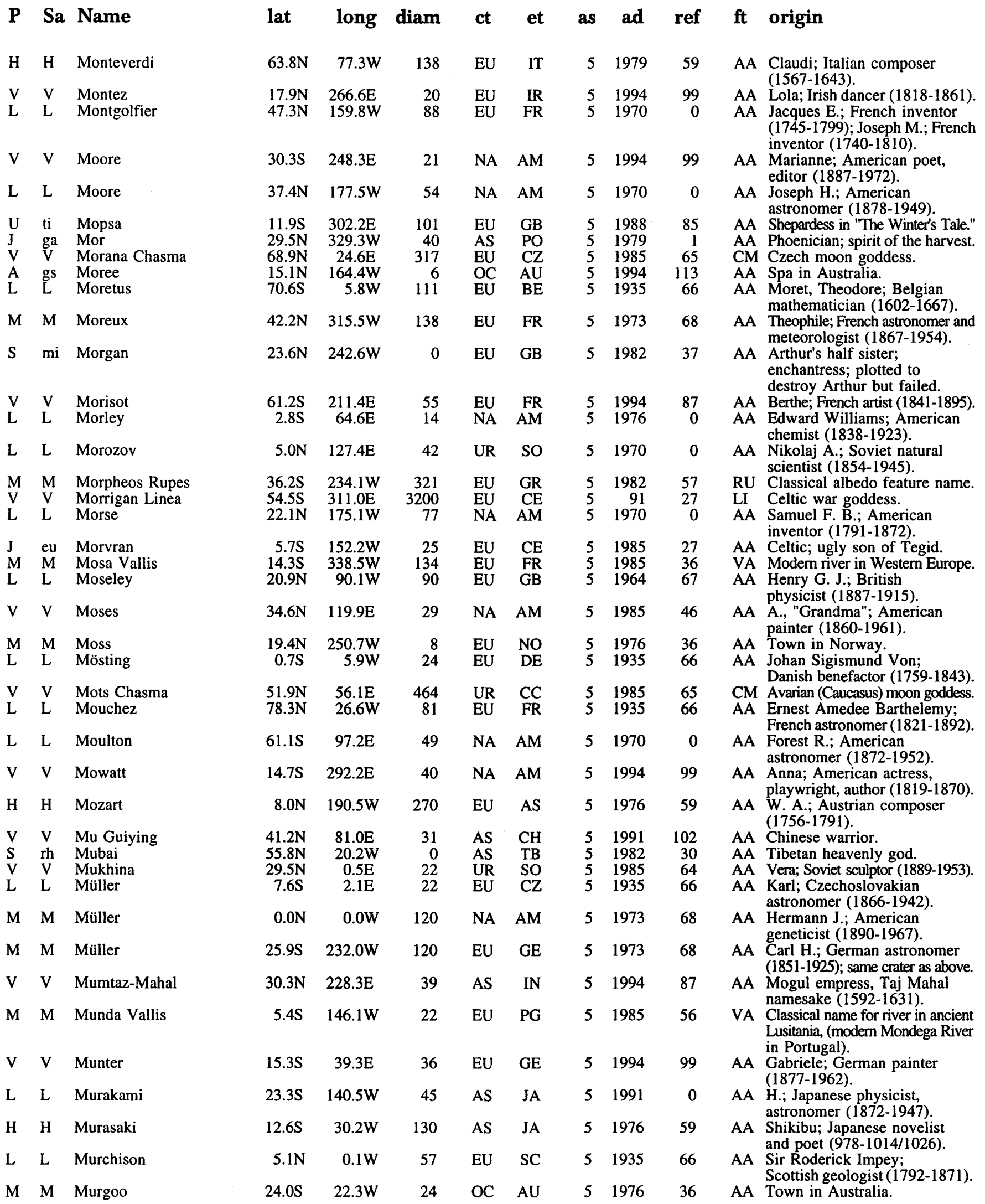

P: $\quad$ planetary system (see page $\mathrm{xvi}$ ).

Sa: satellite (see page xvi).

lat: latitude of feature center.

long: longitude of feature center. diam: diameter or long dimension of feature.

ct: continent of name origin (see page $284 \mathrm{ff}$.)

et: ethnicity of name origin (see page $284 \mathrm{ff}$.)

as: name approval status (see page xvii). ad: name approval date (year)

ref: reference source for name (see page $287 \mathrm{ff}$.).

$\mathrm{ft}$ : feature type (see page 290). 


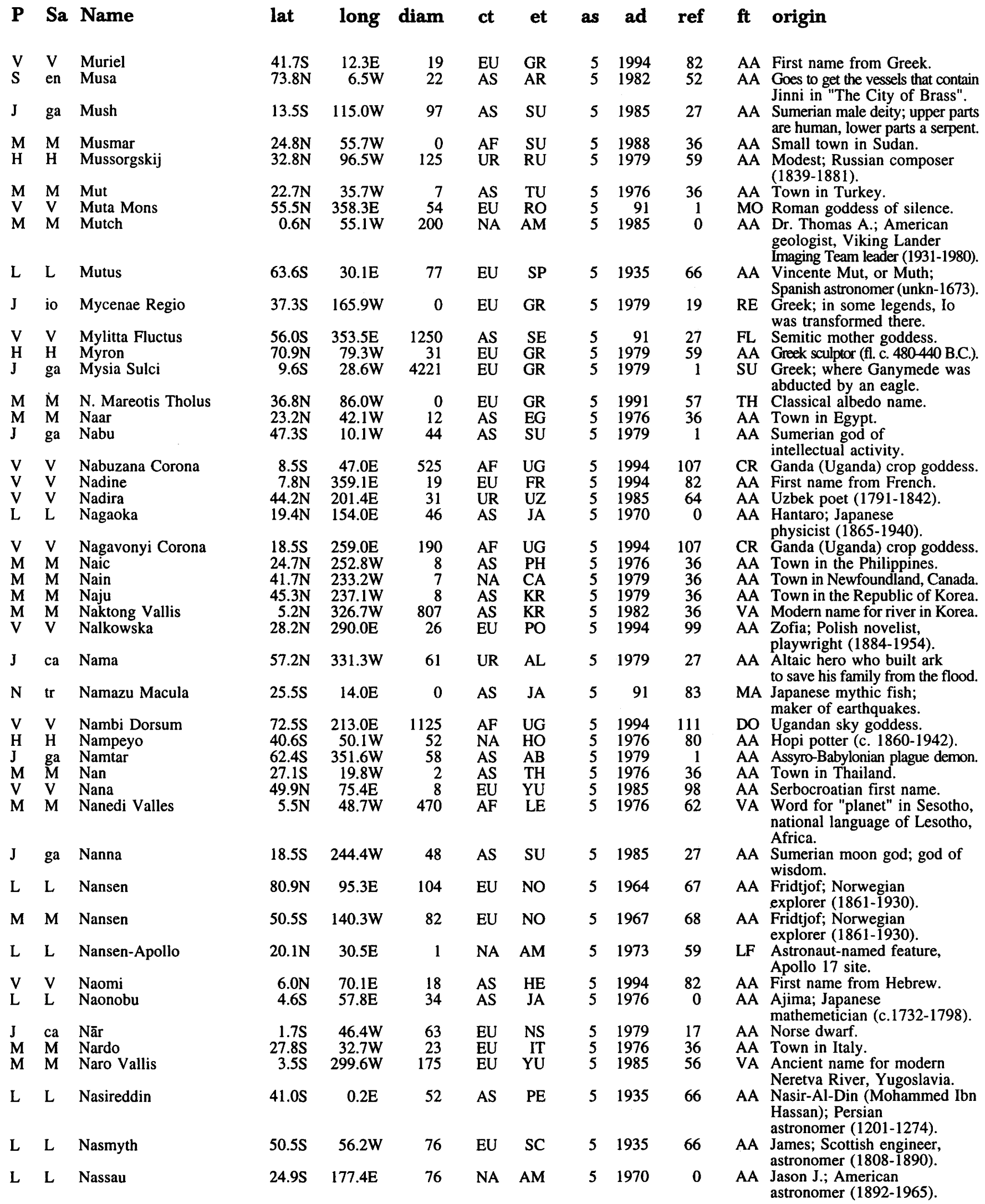

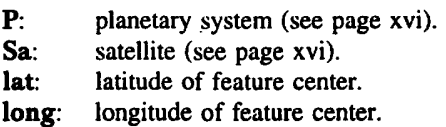

diam: diameter or long dimension of feature. ct: continent of name origin (see page $284 \mathrm{ff}$.) et: ethnicity of name origin (see page $284 \mathrm{ff}$.) as: name approval status (see page xvii) ad: name approval date (year).

ref: reference source for name (see page $287 \mathrm{ff}$.). ft: feature type (see page 290). 
ALPHABETICAL LIST OF NAMES

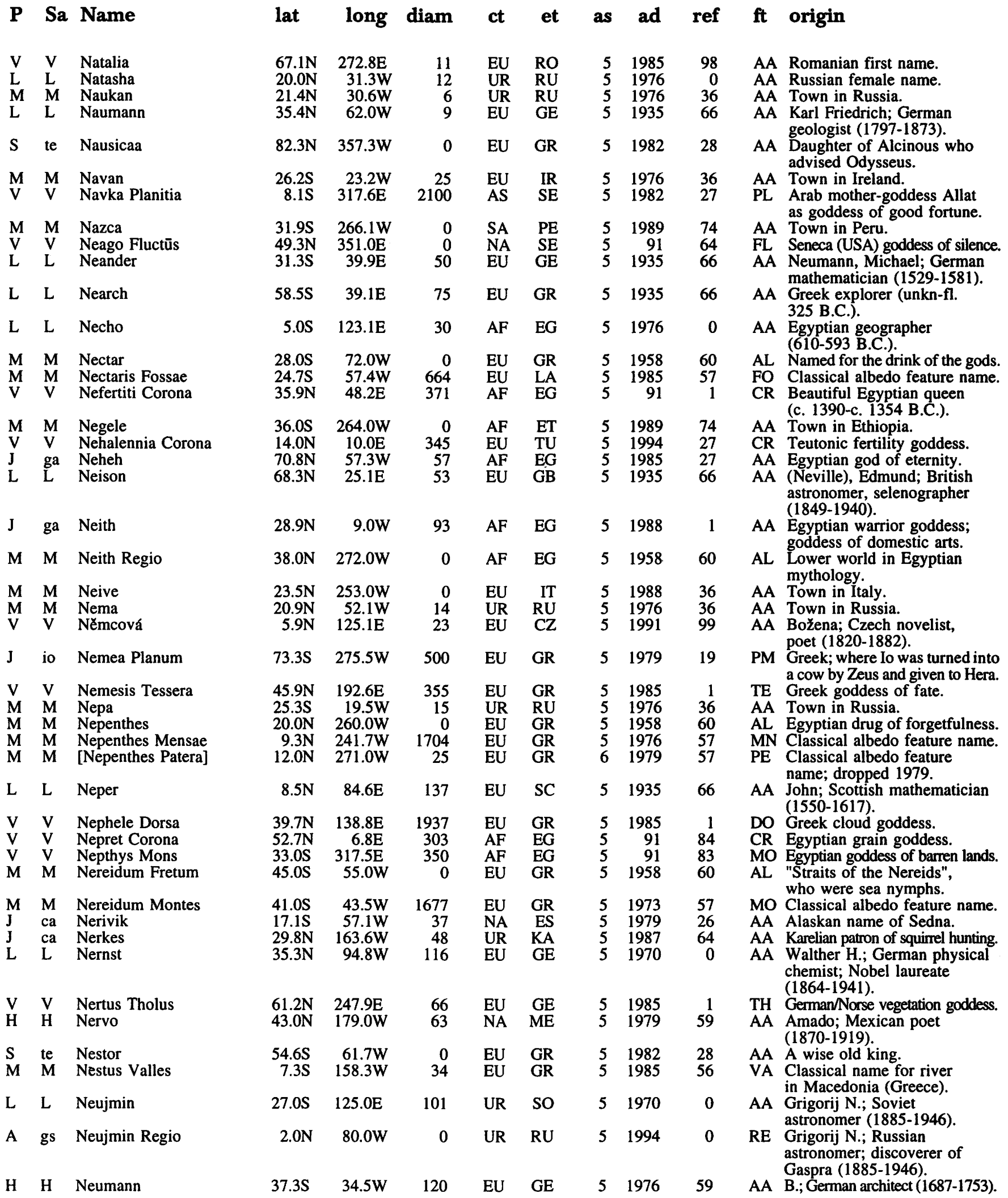

\footnotetext{
P: $\quad$ planetary system (see page $\mathrm{xvi}$ ).

Sa: $\quad$ satellite (see page $x v i$ )

lat: latitude of feature center.

long: longitude of feature center.
}

diam: diameter or long dimension of feature.

ct: continent of name origin (see page $284 \mathrm{ff}$.)

et: ethnicity of name origin (see page $284 \mathrm{ff}$.)

as: name approval status (see page xvii). ad: name approval date (year).

ref: reference source for name (see page $287 \mathrm{ff}$.). ft: feature type (see page 290). 


\section{ALPHABETICAL LIST OF NAMES}

\section{P Sa Name}

L L Neumayer

V V Nevelson

M M Never

M M New Bern

M M New Haven

L L Newcomb

M M Newcomb

M M Newport

M M Newton

L L Newton

V V Neyterkob Corona

M M Nhill

M M Nia Fossae

M M Nia Vallis

M M Nicer Vallis

L L Nicholson

M M Nicholson

J ga Nicholson Regio

L L Nicolai

L L Nicollet

$\mathrm{J}$ ga Nidaba

J ca Nidi

L L Nielsen

L L Niépce

M M Niesten

M M Nif

M M Niger Vallis

V V [Nightingale]

V V Nightingale Corona

J ga Nigirsu

V V Nijinskaya

L L Nijland

V V Nike Fossae

L L Nikolaev

M M Nili Fossae

M M Nili Patera

M M Niliacus Lacus

M M Nilokeras

M M Nilokeras Fossa

M M Nilokeras Mensae lat

$\begin{array}{lrrrrrrr}71.1 \mathrm{~S} & 70.7 \mathrm{E} & 76 & \text { EU } & \text { GE } & 5 & 1935 & 66 \\ & & & & & & & \\ 35.3 \mathrm{~S} & 307.8 \mathrm{E} & 75 & \text { NA } & \text { AM } & 5 & 1994 & 99 \\ & & & & & & & \\ 23.8 \mathrm{~N} & 254.4 \mathrm{~W} & 0 & \text { UR } & \text { RU } & 5 & 1988 & 36 \\ 21.8 \mathrm{~N} & 49.2 \mathrm{~W} & 2 & \text { US } & \text { NC } & 5 & 1979 & 36 \\ 22.3 \mathrm{~N} & 49.3 \mathrm{~W} & 2 & \text { US } & \text { CT } & 5 & 1979 & 36 \\ & & & & & & & \\ 29.9 \mathrm{~N} & 43.8 \mathrm{E} & 41 & \text { NA } & \text { CA } & 5 & 1935 & 66 \\ & & & & & & & \\ 24.1 \mathrm{~S} & 359.0 \mathrm{~W} & 259 & \text { NA } & \text { AM } & 5 & 1973 & 68 \\ & & & & & & & \\ 22.5 \mathrm{~N} & 49.0 \mathrm{~W} & 2 & \text { US } & \text { RI } & 5 & 1979 & 36 \\ 40.8 \mathrm{~S} & 157.9 \mathrm{~W} & 287 & \text { EU } & \text { GB } & 5 & 1973 & 68 \\ 76.7 \mathrm{~S} & 16.9 \mathrm{~W} & 78 & \text { EU } & \text { GB } & 5 & 1935 & 66\end{array}$

49.7N $\quad 204.7 \mathrm{E}$

$28.9 \mathrm{~S} \quad 103.2 \mathrm{~W}$

14.4S $72.4 \mathrm{~W}$

54.3S 33.0W

211
0
333

AF KY

EU AU

$5 \quad 91$

1989

0 AF $\mathrm{GA}$

$\begin{array}{ll}5 & 1991\end{array}$

$7.3 \mathrm{~S}$

158.2W

$26.2 S$

85.1W

$0.1 \mathrm{~N}$

34.0S

$42.4 \mathrm{~S}$

$164.5 \mathrm{~W}$

44

EU GE

51985

NA AM

51970

114

NA AM

51973

3719

NA AM

$5 \quad 1979$

$25.9 \mathrm{E}$$$
42
$$

EU GE

51935

21.9S $12.5 \mathrm{~W}$

$19.0 \mathrm{~N}$

$66.7 \mathrm{~N}$

$123.8 \mathrm{~W}$

$51.8 \mathrm{~W}$

$72.7 \mathrm{~N}$

$28.2 \mathrm{~S}$

$20.2 \mathrm{~N}$

$34.8 \mathrm{~S}$

$62.0 \mathrm{~N}$

$63.6 \mathrm{~N}$

$61.2 S$

$25.8 \mathrm{~N}$

$33.0 \mathrm{~N}$

$62.0 \mathrm{~S}$

$35.2 \mathrm{~N}$

$24.0 \mathrm{~N}$

$9.2 \mathrm{~N}$

$30.0 \mathrm{~N}$

$30.0 \mathrm{~N}$

$25.4 \mathrm{~N}$

$32.9 \mathrm{~N}$
$119.1 \mathrm{~W}$

$302.1 \mathrm{~W}$

$56.3 \mathrm{~W}$

$277.1 \mathrm{~W}$

$132.0 \mathrm{E}$

$129.5 \mathrm{E}$

$327.4 \mathrm{~W}$

$122.5 \mathrm{E}$

134.1E

$347.0 \mathrm{E}$

151.3E

283.0W

$293.0 \mathrm{~W}$

$30.0 \mathrm{~W}$

$55.0 \mathrm{~W}$

$56.9 \mathrm{~W}$

$51.1 \mathrm{~W}$

$\begin{array}{rrrrrr}15 & \text { EU } & \text { FR } & 5 & 1935 & 66 \\ 188 & \text { AS } & \text { SU } & 5 & 1985 & 27 \\ 43 & \text { EU } & \text { NS } & 5 & 1979 & 17 \\ 9 & \text { NA } & \text { AM } & 5 & 1973 & 0\end{array}$

57

114 EU BE

51970

$5 \quad 1973$

51976

51989

$5 \quad 1985$

51979

51991

51970

$\begin{array}{lr}5 & 91 \\ 5 & 1970\end{array}$

800 EU GR

UR SO

709 EU LA

51973

$5 \quad 1982$

$\begin{array}{ll}5 & 1958 \\ 5 & 1958\end{array}$

$\begin{array}{lll}0 & \text { EU } & \text { GR } \\ 0 & \text { EU } & \text { GR }\end{array}$

148

327
EU GR $\begin{array}{ll}5 & 1988\end{array}$

$\begin{array}{ll}5 & 1988\end{array}$
$6 \quad 1982$

35 EU DU
AA Georg Balthasar von; German meteorologist, hydrographer (1826-1909).

AA Louise; American artist (1899-1988).

AA Town in Russia.

AA American colonial town (North Carolina).

AA American colonial town (Connecticut).

AA Newcomb, Simon; CanadianAmerican astronomer (1835-1909)

AA Simon; American astronomer (1835-1909).

AA American colonial town (Rhode Island).

AA Isaac; British physicist (1643-1727).

AA Isaac; British mathematician, physicist, astronomer (1643-1727).

CR Masai earth/fertility goddess.

AA Town in Victoria, Australia.

FO Classical name for river Gambia, West Africa.

VA Lowell canal name; also classical river name.

VA Classical name for present Neckar River, Germany.

AA Seth B.; American astronomer (1891-1963).

AA Seth B.; American astronomer (1891-1963).

RE Seth B.; American astronomer (1891-1963).

AA Friedrich Bernhard Gottfried; German astronomer (1793-1846).

AA Jean Nicholas; French astronomer (1788-1843).

AA Sumerian grain goddess.

AA Norse dwarf.

AA Axel V.; Danish astronomer (1902-1970); Harald Herborg; American physicist (1903-1973)

AA Joseph N.; French physicist, photographer (1765-1833).

AA Louis; Belgian astronomer (1844-1920).

AA Town in the Caroline Islands (Yap).

VA River in Africa.

AA Name changed to Nightingale Corona.

CR Florence; English nurse (1820-1910).

AA Assyro-Babylonian; god of the fields, war god.

AA Bronislava; Russian dancer (1891-1972).

AA Albertus A.; Dutch astronomer (1868-1936).

FO Greek goddess of victory.

AA Andriyan G.; Soviet cosmonaut (1929-Live).

FO Classical albedo feature name.

PE Classical albedo feature name.

AL "Lake of the Nile."

AL "Horn of the Nile;" part of "Nulus" Canal.

FO Classical albedo feature name.

MN Albedo feature name.
P: $\quad$ planetary system (see page $x v i$ ).

Sa: satellite (see page $x v i$ ).

lat: latitude of feature center.

long: longitude of feature center. diam: diameter or long dimension of feature. ct: continent of name origin (see page $284 \mathrm{ff}$.)

et: ethnicity of name origin (see page $284 \mathrm{ff}$.)

as: name approval status (see page xvii). ad: name approval date (year).

ref: reference source for name (see page $287 \mathrm{ff}$.).

ft: feature type (see page 290). 


\section{ALPHABETICAL LIST OF NAMES}

\begin{tabular}{|c|c|c|c|c|c|c|c|c|c|c|}
\hline $\mathbf{P}$ & $\mathbf{S a}$ & Name & lat & long & diam & ct & et & as & ad & ref \\
\hline M & $\mathbf{M}$ & Nilokeras Scopulus & $31.5 \mathrm{~N}$ & $57.2 \mathrm{~W}$ & 1064 & EU & GR & 5 & 1976 & 57 \\
\hline M & M & Nilosyrtis & $42.0 \mathrm{~N}$ & $290.0 \mathrm{~W}$ & 0 & EU & GR & 5 & 1958 & 60 \\
\hline $\begin{array}{l}\mathrm{M} \\
\mathrm{V}\end{array}$ & $\begin{array}{l}\mathrm{M} \\
\mathrm{V}\end{array}$ & $\begin{array}{l}\text { Nilosyrtis Mensae } \\
\text { Nilsson }\end{array}$ & $\begin{array}{l}35.4 \mathrm{~N} \\
76.0 \mathrm{~S}\end{array}$ & $\begin{array}{l}293.6 \mathrm{~W} \\
277.7 \mathrm{E}\end{array}$ & $\begin{array}{r}693 \\
26\end{array}$ & $\begin{array}{l}\mathrm{EU} \\
\mathrm{EU}\end{array}$ & $\begin{array}{l}\text { GR } \\
\text { SW }\end{array}$ & $\begin{array}{l}5 \\
5\end{array}$ & $\begin{array}{l}1973 \\
1994\end{array}$ & $\begin{array}{l}57 \\
87\end{array}$ \\
\hline M & $\mathbf{M}$ & Nilus Chaos & $25.5 \mathrm{~N}$ & $77.6 \mathrm{~W}$ & 280 & EU & GR & 5 & 1985 & 57 \\
\hline M & M & Nilus Dorsa & $22.3 \mathrm{~N}$ & $79.4 \mathrm{~W}$ & 188 & $\mathrm{EU}$ & GR & 5 & 1985 & 57 \\
\hline M & $\mathbf{M}$ & Nilus Mensae & $22.1 \mathrm{~N}$ & $71.8 \mathrm{~W}$ & 196 & $\mathrm{EU}$ & GR & 5 & 1985 & 57 \\
\hline V & V & Nin & $3.9 \mathrm{~S}$ & $266.4 \mathrm{E}$ & 27 & EU & FR & 5 & 1994 & 99 \\
\hline $\begin{array}{l}\text { V } \\
\text { J } \\
\text { V } \\
\text { V } \\
\text { V } \\
\text { J } \\
\text { J }\end{array}$ & $\begin{array}{l}\mathrm{V} \\
\text { io } \\
\mathrm{V} \\
\mathrm{V} \\
\mathrm{V} \\
\mathrm{ga} \\
\text { ga }\end{array}$ & $\begin{array}{l}\text { Nina } \\
\text { Nina Patera } \\
\text { Ningal Undae } \\
\text { Ningyo Fluctus } \\
\text { Ninhursag Corona } \\
\text { Ninkasi } \\
\text { Ninki }\end{array}$ & $\begin{array}{r}55.5 \mathrm{~S} \\
38.3 \mathrm{~S} \\
9.0 \mathrm{~N} \\
5.5 \mathrm{~S} \\
38.0 \mathrm{~S} \\
56.8 \mathrm{~N} \\
6.6 \mathrm{~S}\end{array}$ & $\begin{array}{c}238.7 \mathrm{E} \\
164.2 \mathrm{~W} \\
60.7 \mathrm{E} \\
206.0 \mathrm{E} \\
23.5 \mathrm{E} \\
53.8 \mathrm{~W} \\
120.9 \mathrm{~W}\end{array}$ & $\begin{array}{r}23 \\
425 \\
225 \\
970 \\
125 \\
75 \\
170\end{array}$ & $\begin{array}{l}\text { UR } \\
\text { SA } \\
\text { AS } \\
\text { AS } \\
\text { AS } \\
\text { AS } \\
\text { AS }\end{array}$ & $\begin{array}{l}\text { RU } \\
\text { IN } \\
\text { SA } \\
\text { JA } \\
\text { BY } \\
\text { SU } \\
\text { AB }\end{array}$ & $\begin{array}{l}5 \\
5 \\
5 \\
5 \\
5 \\
5 \\
5\end{array}$ & $\begin{array}{l}1994 \\
1979 \\
1994 \\
1994 \\
1994 \\
1988 \\
1985\end{array}$ & $\begin{array}{r}82 \\
1 \\
111 \\
111 \\
88 \\
83 \\
1\end{array}$ \\
\hline $\mathbf{J}$ & ga & Ninlil & $7.6 \mathrm{~N}$ & $118.7 \mathrm{~W}$ & 91 & AS & $\mathrm{AB}$ & 5 & 1985 & 1 \\
\hline $\mathbf{J}$ & ga & Ninsum & $13.3 \mathrm{~S}$ & $140.1 \mathrm{~W}$ & 91 & AS & $\mathrm{AB}$ & 5 & 1985 & 1 \\
\hline $\begin{array}{l}\text { V } \\
\text { J } \\
\text { V }\end{array}$ & $\begin{array}{l}\mathrm{V} \\
\text { io } \\
\mathrm{V}\end{array}$ & $\begin{array}{l}\text { Nintu Corona } \\
\text { Ninurta Patera } \\
\text { Niobe Planitia }\end{array}$ & $\begin{array}{l}19.2 \mathrm{~N} \\
16.5 \mathrm{~S} \\
21.0 \mathrm{~N}\end{array}$ & $\begin{array}{l}123.5 \mathrm{E} \\
315.3 \mathrm{~W} \\
112.3 \mathrm{E}\end{array}$ & $\begin{array}{r}75 \\
75 \\
5008\end{array}$ & $\begin{array}{l}\text { AS } \\
\text { AS } \\
\text { EU }\end{array}$ & $\begin{array}{l}\text { AK } \\
\text { BY } \\
\text { GR }\end{array}$ & $\begin{array}{l}5 \\
5 \\
5\end{array}$ & $\begin{array}{l}1994 \\
1985 \\
1982\end{array}$ & $\begin{array}{r}88 \\
1 \\
1\end{array}$ \\
\hline $\begin{array}{l}\mathbf{M} \\
\mathbf{J} \\
\mathbf{M} \\
\mathrm{L}\end{array}$ & $\begin{array}{l}\mathrm{M} \\
\text { ga } \\
\mathrm{M} \\
\mathrm{L}\end{array}$ & $\begin{array}{l}\text { Nipigon } \\
\text { Nippur Sulcus } \\
\text { Nirgal Vallis } \\
\text { Nishina }\end{array}$ & $\begin{array}{l}34.0 \mathrm{~N} \\
40.9 \mathrm{~N} \\
28.3 \mathrm{~S} \\
44.6 \mathrm{~S}\end{array}$ & $\begin{array}{r}81.9 \mathrm{~W} \\
191.5 \mathrm{~W} \\
41.9 \mathrm{~W} \\
170.4 \mathrm{~W}\end{array}$ & $\begin{array}{r}0 \\
2158 \\
511 \\
65\end{array}$ & $\begin{array}{l}\text { NA } \\
\text { AS } \\
\text { AS } \\
\text { AS }\end{array}$ & $\begin{array}{r}\text { CA } \\
\text { SU } \\
\text { BY } \\
\text { JA }\end{array}$ & $\begin{array}{l}5 \\
5 \\
5 \\
5\end{array}$ & $\begin{array}{l}1991 \\
1985 \\
1973 \\
1970\end{array}$ & $\begin{array}{r}36 \\
27 \\
62 \\
0\end{array}$ \\
\hline $\begin{array}{l}\mathrm{V} \\
\mathrm{V}\end{array}$ & $\begin{array}{l}\mathrm{V} \\
\mathrm{V}\end{array}$ & $\begin{array}{l}\text { Nishtigri Corona } \\
\text { Nissaba Corona }\end{array}$ & $\begin{array}{l}24.5 \mathrm{~S} \\
25.5 \mathrm{~N}\end{array}$ & $\begin{array}{r}72.0 \mathrm{E} \\
355.5 \mathrm{E}\end{array}$ & $\begin{array}{l}275 \\
300\end{array}$ & $\begin{array}{l}\text { AS } \\
\text { AS }\end{array}$ & $\begin{array}{l}\text { IN } \\
\text { AS }\end{array}$ & $\begin{array}{l}5 \\
5\end{array}$ & $\begin{array}{l}1994 \\
1994\end{array}$ & $\begin{array}{r}83 \\
0\end{array}$ \\
\hline $\begin{array}{l}M \\
M\end{array}$ & $\begin{array}{l}\mathbf{M} \\
\mathbf{M}\end{array}$ & $\begin{array}{l}\text { Nitro } \\
\text { Nix Olympica }\end{array}$ & $\begin{array}{l}21.5 \mathrm{~S} \\
20.0 \mathrm{~N}\end{array}$ & $\begin{array}{r}23.8 \mathrm{~W} \\
130.0 \mathrm{~W}\end{array}$ & $\begin{array}{r}28 \\
0\end{array}$ & $\begin{array}{l}\text { US } \\
\text { EU }\end{array}$ & $\begin{array}{l}\text { WV } \\
\text { LA }\end{array}$ & $\begin{array}{l}5 \\
5\end{array}$ & $\begin{array}{l}1976 \\
1958\end{array}$ & $\begin{array}{l}36 \\
60\end{array}$ \\
\hline $\mathrm{H}$ & $\mathbf{H}$ & Nizāmīi & $71.5 \mathrm{~N}$ & $165.0 \mathrm{~W}$ & 76 & AS & PE & 5 & 1979 & 59 \\
\hline$\underset{J}{M}$ & $\begin{array}{l}\mathrm{M} \\
\mathrm{ca}\end{array}$ & $\begin{array}{l}\text { Njesko } \\
\text { Njord }\end{array}$ & $\begin{array}{l}35.4 \mathrm{~S} \\
16.5 \mathrm{~N}\end{array}$ & $\begin{array}{l}274.8 \mathrm{~W} \\
132.8 \mathrm{~W}\end{array}$ & $\begin{array}{r}0 \\
34\end{array}$ & $\begin{array}{l}\mathrm{EU} \\
\mathrm{EU}\end{array}$ & $\begin{array}{l}\text { CZ } \\
\text { NS }\end{array}$ & $\begin{array}{l}5 \\
5\end{array}$ & $\begin{array}{l}1989 \\
1988\end{array}$ & $\begin{array}{r}74 \\
1\end{array}$ \\
\hline $\begin{array}{l}\mathrm{M} \\
\mathrm{M} \\
\mathrm{L}\end{array}$ & $\begin{array}{l}\mathrm{M} \\
\mathrm{M} \\
\mathrm{L}\end{array}$ & $\begin{array}{l}\text { Noachis } \\
\text { Noachis Terra } \\
\text { Nobel }\end{array}$ & $\begin{array}{l}45.0 \mathrm{~S} \\
45.0 \mathrm{~S} \\
15.0 \mathrm{~N}\end{array}$ & $\begin{array}{l}330.0 \mathrm{~W} \\
350.0 \mathrm{~W} \\
101.3 \mathrm{~W}\end{array}$ & $\begin{array}{r}0 \\
3500 \\
48\end{array}$ & $\begin{array}{l}E U \\
\text { EU } \\
\text { EU }\end{array}$ & $\begin{array}{l}\text { GR } \\
\text { GR } \\
\text { SW }\end{array}$ & $\begin{array}{l}5 \\
5 \\
5\end{array}$ & $\begin{array}{l}1958 \\
1979 \\
1970\end{array}$ & $\begin{array}{r}60 \\
57 \\
0\end{array}$ \\
\hline $\mathrm{L}$ & $\mathrm{L}$ & Nobile & $85.2 S$ & $53.5 \mathrm{E}$ & 73 & $\mathrm{EU}$ & IT & 5 & 1994 & 68 \\
\hline L & $\mathrm{L}$ & Nobili & $0.2 \mathrm{~N}$ & $75.9 \mathrm{E}$ & 42 & EU & IT & 5 & 1976 & 0 \\
\hline M & $\mathbf{M}$ & Noctis Fossae & $3.3 \mathrm{~S}$ & $99.0 \mathrm{~W}$ & 692 & EU & LA & 5 & 1985 & 57 \\
\hline $\begin{array}{l}\text { M } \\
\text { V } \\
L\end{array}$ & $\begin{array}{l}\text { M } \\
\text { V } \\
\text { L }\end{array}$ & $\begin{array}{l}\text { Noctis Labyrinthus } \\
\text { Nofret } \\
\text { Nöggerath }\end{array}$ & $\begin{array}{r}7.2 S \\
58.7 S \\
48.8 S\end{array}$ & $\begin{array}{l}101.3 \mathrm{~W} \\
252.0 \mathrm{E} \\
45.7 \mathrm{~W}\end{array}$ & $\begin{array}{r}976 \\
22 \\
30\end{array}$ & $\begin{array}{l}\mathrm{EU} \\
\mathrm{AF} \\
\mathrm{EU}\end{array}$ & $\begin{array}{l}\text { LA } \\
\text { EG } \\
\text { GE }\end{array}$ & $\begin{array}{l}5 \\
5 \\
5\end{array}$ & $\begin{array}{l}1973 \\
1994 \\
1935\end{array}$ & $\begin{array}{l}57 \\
99 \\
66\end{array}$ \\
\hline V & $\mathrm{V}$ & Nokomis Montes & $18.9 \mathrm{~N}$ & $189.9 \mathrm{E}$ & 486 & NA & $\mathrm{AL}$ & 5 & 1982 & 48 \\
\hline$M$ & $\begin{array}{l}\mathrm{M} \\
\mathrm{L}\end{array}$ & $\begin{array}{l}\text { Noma } \\
\text { Nonius }\end{array}$ & $\begin{array}{l}25.7 \mathrm{~S} \\
34.8 \mathrm{~S}\end{array}$ & $\begin{array}{c}24.0 \mathrm{~W} \\
3.8 \mathrm{E}\end{array}$ & $\begin{array}{l}38 \\
69\end{array}$ & $\begin{array}{l}\mathrm{AF} \\
\mathrm{EU}\end{array}$ & $\begin{array}{r}\text { NM } \\
\text { PG }\end{array}$ & $\begin{array}{l}5 \\
5\end{array}$ & $\begin{array}{l}1976 \\
1935\end{array}$ & $\begin{array}{l}36 \\
66\end{array}$ \\
\hline
\end{tabular}

\section{ft origin}

SC From albedo feature at $30 \mathrm{~N}, 55 \mathrm{~W}$.

AL Syrtis of the Nile; part of Nilus (Nile) Canal.

MN Classical albedo feature name.

AA Christine; Swedish opera singer, violinist (1843-1921).

$\mathrm{CH}$ Named for albedo feature at $20 \mathrm{~N}, 65 \mathrm{~W}$.

DO Named for albedo feature at $20 \mathrm{~N}, 65 \mathrm{~W}$.

MN Named for albedo feature at $20 \mathrm{~N}, 65 \mathrm{~W}$.

AA Anais; French novelist (1903-1977).

AA First name from Russian.

PE Inca fire god.

UN Sumerian desert goddess.

FL Japanese fish goddess.

CR Babylonian earth goddess.

AA Sumerian goddess of brewing.

AA Consort to $\mathrm{Ea}$, Babylonian god of water.

AA Chief Assyrian goddess; Asshur's consort.

AA Minor Babylonian goddess of wisdom; Gilgamesh's mother.

CR Akkadian earth goddess.

PE Babylonian god of the spring sun.

PL Greek; her 12 children killed by Artemis and Apollo.

AA Town in Canada.

SU Sumerian city.

VA Word for "Mars" in Babylonian.

AA Yoshio; Japanese physicist (1890-1951).

CR Hindu earth mother.

CR Mesopotamian wisdom/fetility goddess.

AA Town in West Virginia, USA.

AL "Snows of Olympus;" Olympus was mountain home of gods in Greece.

AA Elyas Yusof Ganjavi; Persian epic poet (c. 1141-1209).

AA Town in Czechoslovakia.

AA Nordic gods called the Vanir; pacific, benevolent, guardians of man.

AL Biblical; "Noah's (Region)."

TA Classical albedo feature name.

AA Alfred B.; Swedish inventor (1833-1896)

AA Umberto; Italian artic explorer (1885-1978)

AA Leopoldo; Italian physicist (1784-1835)

FO Classical albedo feature at $10 \mathrm{~S}, 96 \mathrm{~W}$.

LB Classical albedo feature name.

AA Egyptian queen (c. 1900 B.C.).

AA Johann Jakob; German geologist, mineralogist, seismologist (1788-1877).

MO Algonquin (N. America) earth mother.

AA Town in Namibia.

AA Nunez, Pedro; Portuguese mathematician (1492(?)-1578)

\footnotetext{
P: $\quad$ planetary system (see page $x v i)$.

Sa: $\quad$ satellite (see page $x v i$ ).

lat: latitude of feature center.

long: longitude of feature center.
}

diam: diameter or long dimension of feature.

ct: continent of name origin (see page $284 \mathrm{ff}$.)

et: ethnicity of name origin (see page $284 \mathrm{ff}$.)

as: name approval status (see page xvii). ad: name approval date (year).

ref: reference source for name (see page $287 \mathrm{ff}$.).

ft: feature type (see page 290). 


\section{ALPHABETICAL LIST OF NAMES}

\begin{tabular}{|c|c|c|c|c|c|c|c|c|c|}
\hline $\mathbf{P}$ & Sa & Name & lat & long & diam & ct & et & as & ad \\
\hline M & M & Nordenskiold & $53.0 \mathrm{~S}$ & $158.7 \mathrm{~W}$ & 87 & EU & SW & 5 & 1982 \\
\hline $\begin{array}{l}\mathrm{J} \\
\mathrm{V} \\
\mathrm{L}\end{array}$ & $\begin{array}{l}\mathrm{V} \\
\mathrm{ca} \\
\mathrm{V} \\
\mathrm{L}\end{array}$ & $\begin{array}{l}\text { Noreen } \\
\text { Nori } \\
\text { Noriko } \\
\text { Norman }\end{array}$ & $\begin{array}{r}33.5 \mathrm{~N} \\
45.4 \mathrm{~N} \\
5.3 \mathrm{~S} \\
11.8 \mathrm{~S}\end{array}$ & $\begin{array}{c}22.7 \mathrm{E} \\
343.5 \mathrm{~W} \\
358.3 \mathrm{E} \\
30.4 \mathrm{~W}\end{array}$ & $\begin{array}{r}20 \\
86 \\
7 \\
10\end{array}$ & $\begin{array}{l}\text { EU } \\
\text { EU } \\
\text { AS } \\
\text { EU }\end{array}$ & $\begin{array}{r}\text { IR } \\
\text { NS } \\
\text { JA } \\
\text { GB }\end{array}$ & $\begin{array}{l}5 \\
5 \\
5 \\
5\end{array}$ & $\begin{array}{l}1994 \\
1979 \\
1994 \\
1976\end{array}$ \\
\hline $\begin{array}{l}\mathrm{J} \\
\mathrm{L}\end{array}$ & $\begin{array}{l}\mathrm{ca} \\
\mathrm{L}\end{array}$ & $\begin{array}{l}\text { Norov-Ava } \\
\text { North Complex }\end{array}$ & $\begin{array}{l}54.6 \mathrm{~N} \\
26.2 \mathrm{~N}\end{array}$ & $\begin{array}{c}113.7 \mathrm{~W} \\
3.6 \mathrm{E}\end{array}$ & $\begin{array}{r}47 \\
2\end{array}$ & $\begin{array}{l}\text { UR } \\
\text { NA }\end{array}$ & $\begin{array}{l}\text { MO } \\
\text { AM }\end{array}$ & $\begin{array}{l}5 \\
5\end{array}$ & $\begin{array}{l}1987 \\
1973\end{array}$ \\
\hline L & L & North Massif & $20.4 \mathrm{~N}$ & $30.8 \mathrm{E}$ & 14 & EU & FR & 5 & 1973 \\
\hline L & $\mathrm{L}$ & North Ray & $8.8 \mathrm{~S}$ & $15.5 \mathrm{E}$ & 1 & NA & AM & 5 & 1973 \\
\hline $\begin{array}{l}M \\
L\end{array}$ & $\begin{array}{l}M \\
L\end{array}$ & $\begin{array}{l}\text { Northport } \\
\text { Nöther (Noether) }\end{array}$ & $\begin{array}{l}18.6 \mathrm{~N} \\
66.6 \mathrm{~N}\end{array}$ & $\begin{array}{r}54.2 W \\
113.5 W\end{array}$ & $\begin{array}{r}0 \\
67\end{array}$ & $\begin{array}{l}\text { US } \\
\text { EU }\end{array}$ & $\begin{array}{l}\mathrm{AL} \\
\mathrm{GE}\end{array}$ & $\begin{array}{l}5 \\
5\end{array}$ & $\begin{array}{l}1988 \\
1970\end{array}$ \\
\hline $\mathrm{L}$ & L & [Novalis] & $11.7 \mathrm{~S}$ & 84.7E & 8 & EU & GE & 6 & 0 \\
\hline $\begin{array}{l}\text { V } \\
\text { J } \\
\text { S } \\
\mathrm{L}\end{array}$ & $\begin{array}{l}\mathrm{V} \\
\mathrm{ca} \\
\mathrm{rh} \\
\mathrm{L}\end{array}$ & $\begin{array}{l}\text { Nsomeka Planitia } \\
\text { Nuada } \\
\text { Num } \\
\text { Numerov }\end{array}$ & $\begin{array}{l}55.0 \mathrm{~S} \\
62.1 \mathrm{~N} \\
24.0 \mathrm{~N} \\
70.7 \mathrm{~S}\end{array}$ & $\begin{array}{c}170.0 \mathrm{E} \\
273.2 \mathrm{~W} \\
92.7 \mathrm{~W} \\
160.7 \mathrm{~W}\end{array}$ & $\begin{array}{r}7000 \\
66 \\
0 \\
113\end{array}$ & $\begin{array}{l}\text { AF } \\
\text { EU } \\
\text { AS } \\
\text { UR }\end{array}$ & $\begin{array}{l}\text { BA } \\
\text { CE } \\
\text { SM } \\
\text { SO }\end{array}$ & $\begin{array}{l}5 \\
5 \\
5 \\
5\end{array}$ & $\begin{array}{l}1994 \\
1979 \\
1982 \\
1970\end{array}$ \\
\hline & ga & Nun Sulci & $49.3 \mathrm{~N}$ & $318.8 \mathrm{~W}$ & 1090 & AF & EG & 5 & 1979 \\
\hline $\begin{array}{l}\mathrm{M} \\
\mathrm{L}\end{array}$ & $\begin{array}{l}\mathrm{M} \\
\mathrm{L}\end{array}$ & $\begin{array}{l}\text { Nune } \\
\text { Nunn }\end{array}$ & $\begin{array}{r}17.7 \mathrm{~N} \\
4.6 \mathrm{~N}\end{array}$ & $\begin{array}{l}38.6 \mathrm{~W} \\
91.1 \mathrm{E}\end{array}$ & $\begin{array}{r}9 \\
19\end{array}$ & $\begin{array}{l}\mathrm{AF} \\
\mathrm{NA}\end{array}$ & $\begin{array}{l}\text { MZ } \\
\text { AM }\end{array}$ & $\begin{array}{l}5 \\
5\end{array}$ & $\begin{array}{l}1976 \\
1973\end{array}$ \\
\hline $\mathbf{J}$ & $\begin{array}{l}\text { io } \\
\mathrm{L}\end{array}$ & $\begin{array}{l}\text { Nusku Patera } \\
\text { Nüsl }\end{array}$ & $\begin{array}{l}64.7 \mathrm{~S} \\
32.3 \mathrm{~N}\end{array}$ & $\begin{array}{r}4.6 \mathrm{~W} \\
167.6 \mathrm{E}\end{array}$ & $\begin{array}{l}90 \\
61\end{array}$ & $\begin{array}{l}\text { AS } \\
\text { EU }\end{array}$ & $\begin{array}{l}\mathrm{AY} \\
\mathrm{CZ}\end{array}$ & $\begin{array}{l}5 \\
5\end{array}$ & $\begin{array}{l}1979 \\
1970\end{array}$ \\
\hline $\begin{array}{l}\mathrm{M} \\
\mathrm{J} \\
\mathrm{V}\end{array}$ & $\begin{array}{l}\text { ga } \\
\text { M } \\
\text { io } \\
\text { V }\end{array}$ & $\begin{array}{l}\text { Nut } \\
\text { Nutak } \\
\text { Nyambe Patera } \\
\text { Nzingha Patera }\end{array}$ & $\begin{array}{r}60.1 \mathrm{~S} \\
17.6 \mathrm{~N} \\
0.6 \mathrm{~N} \\
68.7 \mathrm{~N}\end{array}$ & $\begin{array}{l}268.0 \mathrm{~W} \\
30.3 \mathrm{~W} \\
343.9 \mathrm{~W} \\
205.7 \mathrm{E}\end{array}$ & $\begin{array}{r}93 \\
11 \\
50 \\
143\end{array}$ & $\begin{array}{l}\mathrm{AF} \\
\mathrm{NA} \\
\mathrm{AF} \\
\mathrm{AF}\end{array}$ & $\begin{array}{l}\text { EG } \\
\text { CA } \\
\text { ZM } \\
\text { AN }\end{array}$ & $\begin{array}{l}5 \\
5 \\
5 \\
5\end{array}$ & $\begin{array}{r}1979 \\
1976 \\
1979 \\
91\end{array}$ \\
\hline V & V & Oakley & $29.3 S$ & $310.5 \mathrm{E}$ & 22 & NA & $\mathrm{AM}$ & 5 & 1994 \\
\hline $\begin{array}{l}\mathrm{V} \\
\mathrm{N}\end{array}$ & $\begin{array}{l}\mathrm{V} \\
\mathrm{tr}\end{array}$ & $\begin{array}{l}\text { Oanuava Corona } \\
\text { Ob Sulci }\end{array}$ & $\begin{array}{r}32.5 \mathrm{~S} \\
6.0 \mathrm{~S}\end{array}$ & $\begin{array}{l}255.5 \mathrm{E} \\
328.0 \mathrm{E}\end{array}$ & $\begin{array}{r}375 \\
0\end{array}$ & $\begin{array}{l}\text { EU } \\
\text { UR }\end{array}$ & $\begin{array}{l}\text { FR } \\
\text { OS }\end{array}$ & $\begin{array}{l}5 \\
5\end{array}$ & $\begin{array}{r}1994 \\
91\end{array}$ \\
\hline & $\mathrm{L}$ & Obruchev & $38.9 \mathrm{~S}$ & $162.1 \mathrm{E}$ & 71 & UR & SO & 5 & 1970 \\
\hline V & V & Obukhova & $70.7 \mathrm{~N}$ & $287.0 \mathrm{E}$ & 46 & UR & SO & 5 & 1985 \\
\hline $\begin{array}{l}M \\
M \\
M\end{array}$ & $\begin{array}{l}\mathrm{M} \\
\mathbf{M} \\
\mathbf{M}\end{array}$ & $\begin{array}{l}\text { Ocampo } \\
\text { Oceanidum Fossa } \\
\text { Oceanidum Mons }\end{array}$ & $\begin{array}{l}32.9 \mathrm{~N} \\
61.7 \mathrm{~S} \\
55.2 \mathrm{~S}\end{array}$ & $\begin{array}{r}221.7 \mathrm{~W} \\
29.6 \mathrm{~W} \\
41.4 \mathrm{~W}\end{array}$ & $\begin{array}{r}0 \\
165 \\
36\end{array}$ & $\begin{array}{l}\text { NA } \\
\text { EU } \\
\text { EU }\end{array}$ & $\begin{array}{l}\text { ME } \\
\text { GR } \\
\text { GR }\end{array}$ & $\begin{array}{l}5 \\
5 \\
5\end{array}$ & $\begin{array}{l}1991 \\
1976 \\
1985\end{array}$ \\
\hline $\begin{array}{l}\mathrm{L} \\
\mathbf{M} \\
\mathbf{M}\end{array}$ & $\begin{array}{l}\mathrm{L} \\
\mathrm{M} \\
\mathrm{M}\end{array}$ & $\begin{array}{l}\text { Oceanus Procellarum } \\
\text { Ochakov } \\
\text { Ochus Valles }\end{array}$ & $\begin{array}{r}18.4 \mathrm{~N} \\
42.5 \mathrm{~S} \\
7.0 \mathrm{~N}\end{array}$ & $\begin{array}{l}57.4 \mathrm{~W} \\
31.6 \mathrm{~W} \\
45.2 \mathrm{~W}\end{array}$ & $\begin{array}{r}2568 \\
30 \\
123\end{array}$ & $\begin{array}{l}\text { EU } \\
\text { UR } \\
\text { UR }\end{array}$ & $\begin{array}{l}\text { LA } \\
\text { UK } \\
\text { TK }\end{array}$ & $\begin{array}{l}5 \\
5 \\
5\end{array}$ & $\begin{array}{l}1935 \\
1976 \\
1985\end{array}$ \\
\hline & V & O'Connor & $26.0 S$ & $143.8 \mathrm{E}$ & 31 & NA & AM & 5 & 1991 \\
\hline $\begin{array}{l}M \\
M \\
L\end{array}$ & $\begin{array}{l}\mathrm{M} \\
\mathbf{M} \\
\mathrm{L}\end{array}$ & $\begin{array}{l}\text { Octantis Cavi } \\
\text { Octantis Mons } \\
\text { O'Day }\end{array}$ & $\begin{array}{l}52.7 \mathrm{~S} \\
55.5 \mathrm{~S} \\
30.6 \mathrm{~S}\end{array}$ & $\begin{array}{l}45.6 \mathrm{~W} \\
42.5 \mathrm{~W} \\
157.5 \mathrm{E}\end{array}$ & $\begin{array}{r}0 \\
0 \\
71\end{array}$ & $\begin{array}{l}\text { EU } \\
\text { EU } \\
\text { NA }\end{array}$ & $\begin{array}{l}\text { LA } \\
\text { LA } \\
\text { AM }\end{array}$ & $\begin{array}{l}5 \\
5 \\
5\end{array}$ & $\begin{array}{l}1991 \\
1991 \\
1970\end{array}$ \\
\hline $\begin{array}{l}\mathrm{V} \\
\mathrm{H} \\
\mathrm{V} \\
\mathrm{S}\end{array}$ & $\begin{array}{l}\mathrm{V} \\
\mathrm{H} \\
\mathrm{V} \\
\mathrm{te} \\
\mathrm{L}\end{array}$ & $\begin{array}{l}\text { Odilia } \\
\text { Odin Planitia } \\
\text { Oduduva Corona } \\
\text { Odysseus } \\
\text { Oenopides }\end{array}$ & $\begin{array}{l}81.2 \mathrm{~N} \\
23.3 \mathrm{~N} \\
11.0 \mathrm{~S} \\
30.0 \mathrm{~N} \\
57.0 \mathrm{~N}\end{array}$ & $\begin{array}{c}200.0 \mathrm{E} \\
171.6 \mathrm{~W} \\
211.5 \mathrm{E} \\
130.0 \mathrm{~W} \\
64.1 \mathrm{~W}\end{array}$ & $\begin{array}{r}18 \\
0 \\
150 \\
0 \\
67\end{array}$ & $\begin{array}{l}\text { EU } \\
\text { EU } \\
\text { AF } \\
\text { EU } \\
\text { EU }\end{array}$ & $\begin{array}{l}\text { PG } \\
\text { NO } \\
\text { NI } \\
\text { GR } \\
\text { GR }\end{array}$ & $\begin{array}{l}5 \\
5 \\
5 \\
5 \\
5\end{array}$ & $\begin{array}{l}1985 \\
1976 \\
1994 \\
1982 \\
1935\end{array}$ \\
\hline & $\begin{array}{l}\mathbf{M} \\
\mathbf{L}\end{array}$ & $\begin{array}{l}\text { Oenotria Scopulus } \\
\text { Oersted }\end{array}$ & $\begin{array}{l}11.2 \mathrm{~S} \\
43.1 \mathrm{~N}\end{array}$ & $\begin{array}{c}283.2 \mathrm{~W} \\
47.2 \mathrm{E}\end{array}$ & $\begin{array}{r}1438 \\
42\end{array}$ & $\begin{array}{l}\text { EU } \\
\text { EU }\end{array}$ & $\begin{array}{l}\text { LA } \\
\text { DE }\end{array}$ & $\begin{array}{l}5 \\
5\end{array}$ & $\begin{array}{l}1982 \\
1935\end{array}$ \\
\hline
\end{tabular}

\section{ft origin}

AA Nils Adolf Erik; Swedish geologist and geographer, arctic researcher (1832-1901).

AA Irish first name.

AA Norse dwarf.

AA Japanese first name.

AA Robert; British natural scientist (unkn-fl. c. 1590)

AA Mordvinian mistress of the field.

LF Astronaut-named feature, Apollo 15 site.

LF Astronaut-named feature, Apollo 17 site.

LF Astronaut-named feature, Apollo 16 site.

AA Town in Alabama, USA.

AA Emmy; German mathematician (1882-1935).

AA Noval, Friedrich von Hardenberg. German writer (1772-1801).

PL Bantu culture heroine.

AA Irish chieftan god.

AA Samoyed god of heaven.

AA Boris V.; Soviet astronomer (1891-1941).

SU Egyptian; chaos; primordial ocean; held germ of all things.

AA Town in Mozambique.

AA Joseph; American engineer (1905-1968).

PE Assyrian fire god.

AA Frantisek; Czechoslovakian astronomer (1867-1925).

AA Egyptian goddess of the sky.

AA Town in Nefoundland, Canada.

PE Zambezi sun god.

PE (Ann Zingha) queen, head of Amazon band (1582-1663).

AA Annie; American sharpshooter, entertainer (1860-1926).

CR Gaulish Celtic earth goddess.

SU Mouth of this river is Ostiak entrance to underworld.

AA Vladimir A.; Soviet geologist (1863-1956).

AA Nadezhda; Soviet singer (1886-1961).

AA Town in Mexico.

FO Classical albedo feature name.

MO Name change from Charitum Tholus.

OC "Ocean of Storms".

AA Town in Ukraine.

VA Classical name for present Hari-Rud River in Turkmenistan

AA Flannery; American novelist (1925-1964).

CB Albedo name.

MO Albedo name.

AA Marcus; American physicist (1897-1961).

AA Portuguese first name.

PL Norse god.

CR Yoruba (Nigeria) fertility goddess.

AA Hero of Odyssey.

AA Of Chios; Greek astronomer, geometrician (500(?)-430 B.C.)

SC Classical albedo feature name.

AA Hans Christian; Danish physicist, chemist (1777-1851).

\footnotetext{
P: $\quad$ planetary system (see page xvi).

Sa: satellite (see page $\mathrm{xvi}$ ).

lat: latitude of feature center.

long: longitude of feature center.
}

diam: diameter or long dimension of feature.

ct: continent of name origin (see page $284 \mathrm{ff}$.)

et: ethnicity of name origin (see page $284 \mathrm{ff}$.)

as: name approval status (see page xvii). ad: name approval date (year).

ref: reference source for name (see page $287 \mathrm{ff}$.).

ft: feature type (see page 290). 
ALPHABETICAL LIST OF NAMES

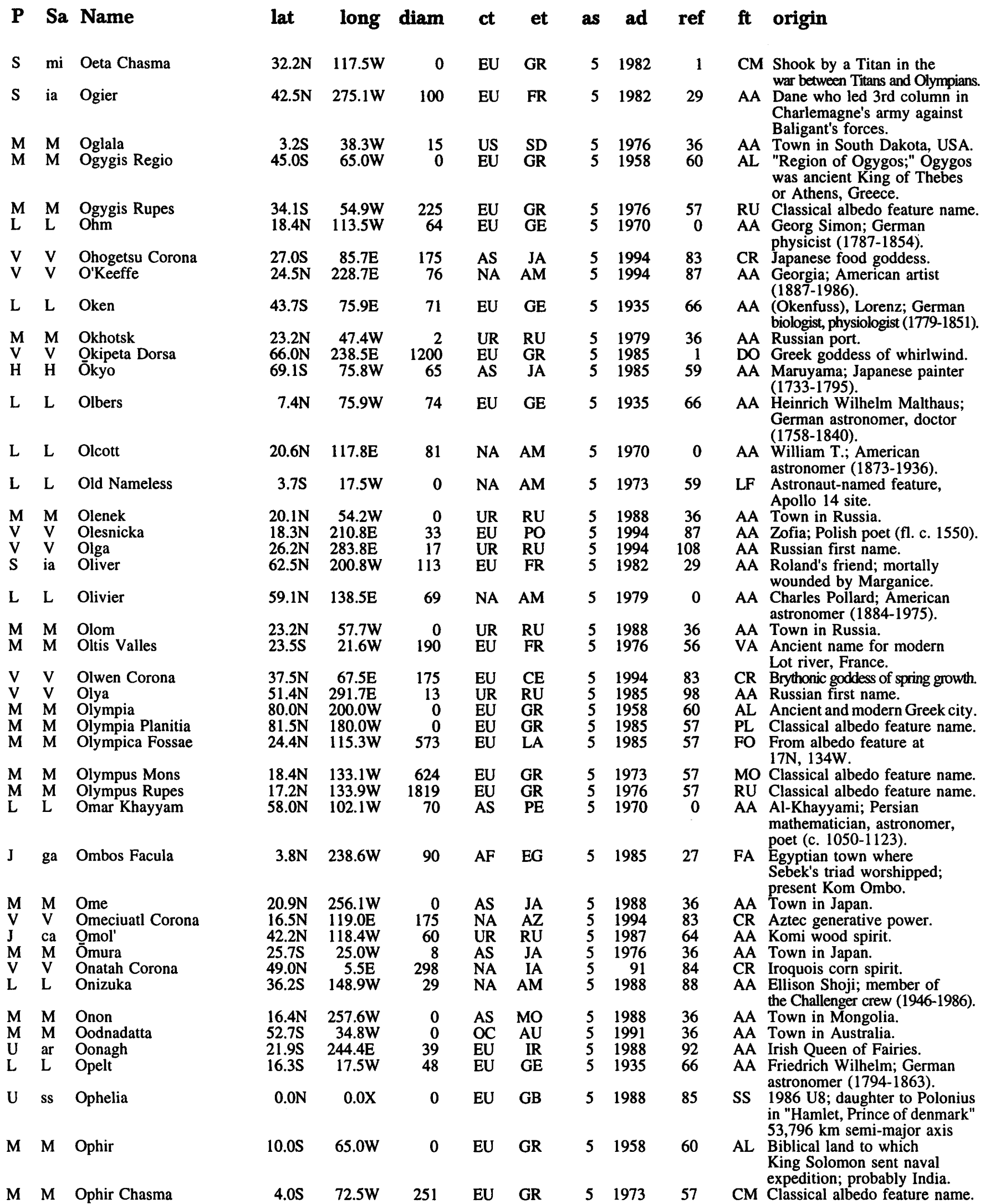

P: $\quad$ planetary system (see page $x v i$ ).

Sa: $\quad$ satellite (see page $x v i$ ).

lat: latitude of feature center.

long: longitude of feature center. diam: diameter or long dimension of feature.

ct: continent of name origin (see page $284 \mathrm{ff}$.)

et: ethnicity of name origin (see page $284 \mathrm{ff}$.)

as: name approval status.(see page $x v i i$ ). ad: name approval date (year)

ref: reference source for name (see page $287 \mathrm{ff}$.).

ft: feature type (see page 290). 


\section{ALPHABETICAL LIST OF NAMES}

\begin{tabular}{|c|c|c|c|c|c|c|c|c|c|}
\hline $\mathbf{P}$ & $\mathbf{S a}$ & Name & lat & long & diam & ct & et & as & ad \\
\hline $\mathbf{M}$ & $\mathbf{M}$ & Ophir Labes & $11.1 \mathrm{~S}$ & $68.3 W$ & 0 & $\mathrm{EU}$ & GR & 5 & 1985 \\
\hline $\begin{array}{l}\mathbf{M} \\
\mathbf{L}\end{array}$ & $\begin{array}{l}\mathbf{M} \\
\mathrm{L}\end{array}$ & $\begin{array}{l}\text { Ophir Planum } \\
\text { Oppenheimer }\end{array}$ & $\begin{array}{r}9.6 \mathrm{~S} \\
35.2 \mathrm{~S}\end{array}$ & $\begin{array}{r}62.2 W \\
166.3 W\end{array}$ & $\begin{array}{r}1068 \\
208\end{array}$ & $\begin{array}{l}\text { EU } \\
\text { NA }\end{array}$ & $\begin{array}{l}\text { GR } \\
\text { AM }\end{array}$ & $\begin{array}{l}5 \\
5\end{array}$ & $\begin{array}{l}1973 \\
1970\end{array}$ \\
\hline L & $\mathrm{L}$ & Oppolzer & $1.5 \mathrm{~S}$ & $0.5 \mathrm{~W}$ & 40 & EU & $\mathrm{CZ}$ & 5 & 1935 \\
\hline $\begin{array}{l}\mathrm{V} \\
\mathrm{M} \\
\mathrm{M} \\
\mathrm{V}\end{array}$ & $\begin{array}{l}\mathrm{V} \\
\mathrm{M} \\
\mathrm{M} \\
\mathrm{V}\end{array}$ & $\begin{array}{l}\text { Ops Corona } \\
\text { Oraibi } \\
\text { Orcus Patera } \\
\text { Orczy }\end{array}$ & $\begin{array}{r}68.8 \mathrm{~N} \\
17.4 \mathrm{~N} \\
14.4 \mathrm{~N} \\
3.6 \mathrm{~N}\end{array}$ & $\begin{array}{c}89.0 \mathrm{E} \\
32.4 \mathrm{~W} \\
181.5 \mathrm{~W} \\
52.2 \mathrm{E}\end{array}$ & $\begin{array}{r}183 \\
31 \\
381 \\
29\end{array}$ & $\begin{array}{l}\text { EU } \\
\text { US } \\
\text { EU } \\
\text { EU }\end{array}$ & $\begin{array}{l}\text { GR } \\
\text { AZ } \\
\text { LA } \\
\text { HU }\end{array}$ & $\begin{array}{l}5 \\
5 \\
5 \\
5\end{array}$ & $\begin{array}{r}91 \\
1976 \\
1973 \\
1994\end{array}$ \\
\hline $\begin{array}{l}\mathrm{M} \\
\mathrm{L}\end{array}$ & $\begin{array}{l}\mathrm{M} \\
\mathrm{L}\end{array}$ & $\begin{array}{l}\text { Ore } \\
\text { Oresme }\end{array}$ & $\begin{array}{l}16.9 \mathrm{~N} \\
42.4 \mathrm{~S}\end{array}$ & $\begin{array}{l}33.9 \mathrm{~W} \\
169.2 \mathrm{E}\end{array}$ & $\begin{array}{r}7 \\
76\end{array}$ & $\begin{array}{l}\mathrm{AF} \\
\mathrm{EU}\end{array}$ & $\underset{\text { FR }}{\text { NI }}$ & $\begin{array}{l}5 \\
5\end{array}$ & $\begin{array}{l}1976 \\
1970\end{array}$ \\
\hline $\begin{array}{l}\mathrm{M} \\
\mathrm{L}\end{array}$ & $\begin{array}{l}\mathrm{M} \\
\mathrm{L}\end{array}$ & $\begin{array}{l}\text { Orinda } \\
\text { Orlov }\end{array}$ & $\begin{array}{l}45.6 \mathrm{~N} \\
25.7 \mathrm{~S}\end{array}$ & $\begin{array}{l}233.0 \mathrm{~W} \\
175.0 \mathrm{~W}\end{array}$ & $\begin{array}{r}9 \\
81\end{array}$ & $\begin{array}{l}\text { US } \\
\text { UR }\end{array}$ & $\begin{array}{l}\text { CA } \\
\text { SO }\end{array}$ & $\begin{array}{l}5 \\
5\end{array}$ & $\begin{array}{l}1979 \\
1970\end{array}$ \\
\hline $\begin{array}{l}\mathrm{V} \\
\mathrm{S} \\
\mathrm{N}\end{array}$ & $\begin{array}{l}\mathrm{V} \\
\mathrm{rh} \\
\mathrm{tr}\end{array}$ & $\begin{array}{l}\text { Orlova } \\
\text { Ormazd } \\
\text { Ormet Sulci }\end{array}$ & $\begin{array}{l}56.5 \mathrm{~N} \\
52.5 \mathrm{~N} \\
17.0 \mathrm{~N}\end{array}$ & $\begin{array}{c}235.0 \mathrm{E} \\
58.5 \mathrm{~W} \\
337.0 \mathrm{E}\end{array}$ & $\begin{array}{r}21 \\
0 \\
0\end{array}$ & $\begin{array}{l}\text { UR } \\
\text { AS } \\
\text { EU }\end{array}$ & $\begin{array}{l}\text { SO } \\
\text { PE } \\
\text { NS }\end{array}$ & $\begin{array}{l}5 \\
5 \\
5\end{array}$ & $\begin{array}{r}1985 \\
1982 \\
91\end{array}$ \\
\hline $\mathrm{L}$ & $\mathrm{L}$ & Orontius & $40.6 S$ & $4.6 \mathrm{~W}$ & 105 & $\mathrm{EU}$ & FR & 5 & 1935 \\
\hline M & $\mathbf{M}$ & Ortygia & $60.0 \mathrm{~N}$ & $0.0 \mathrm{~W}$ & 0 & $\mathrm{EU}$ & GR & 5 & 1958 \\
\hline M & $\mathbf{M}$ & [Ortygia Tholus] & $70.0 \mathrm{~N}$ & $8.0 \mathrm{~W}$ & 100 & EU & LA & 6 & 1979 \\
\hline $\begin{array}{l}\mathrm{L} \\
\mathrm{V}\end{array}$ & L & $\begin{array}{l}\text { Osama } \\
\text { Oshun Farra }\end{array}$ & $\begin{array}{r}18.6 \mathrm{~N} \\
4.2 \mathrm{~N}\end{array}$ & $\begin{array}{r}5.2 \mathrm{E} \\
19.3 \mathrm{E}\end{array}$ & $\begin{array}{r}0 \\
80\end{array}$ & $\begin{array}{l}\text { AS } \\
\text { AF }\end{array}$ & $\begin{array}{l}\text { JA } \\
\text { NI }\end{array}$ & $\begin{array}{l}5 \\
5\end{array}$ & $\begin{array}{l}1976 \\
1994\end{array}$ \\
\hline $\begin{array}{l}\text { V } \\
\text { L } \\
\text { J } \\
\text { J } \\
\text { L } \\
\text { S }\end{array}$ & $\begin{array}{l}\text { V } \\
\mathrm{L} \\
\mathrm{ga} \\
\mathrm{ca} \\
\mathrm{L} \\
\mathrm{mi}\end{array}$ & $\begin{array}{l}\text { Osipenko } \\
\text { Osiris } \\
\text { Osiris } \\
\text { Oski } \\
\text { Osman } \\
\text { Ossa Chasma }\end{array}$ & $\begin{array}{l}71.0 \mathrm{~N} \\
18.6 \mathrm{~N} \\
37.8 \mathrm{~S} \\
57.2 \mathrm{~N} \\
11.0 \mathrm{~S} \\
20.6 \mathrm{~S}\end{array}$ & $\begin{array}{c}321.0 \mathrm{E} \\
27.6 \mathrm{E} \\
165.2 \mathrm{~W} \\
269.3 \mathrm{~W} \\
6.2 \mathrm{~W} \\
307.8 \mathrm{~W}\end{array}$ & $\begin{array}{r}30 \\
1 \\
109 \\
57 \\
2 \\
0\end{array}$ & $\begin{array}{l}\text { UR } \\
\text { AF } \\
\text { AF } \\
\text { EU } \\
\text { AS } \\
\text { EU }\end{array}$ & $\begin{array}{l}\text { SO } \\
\text { EG } \\
\text { EG } \\
\text { NS } \\
\text { TU } \\
\text { GR }\end{array}$ & $\begin{array}{l}5 \\
5 \\
5 \\
5 \\
5 \\
5\end{array}$ & $\begin{array}{l}1985 \\
1976 \\
1979 \\
1979 \\
1976 \\
1982\end{array}$ \\
\hline $\begin{array}{l}\mathrm{M} \\
\mathrm{L}\end{array}$ & $\begin{array}{l}\mathbf{M} \\
\mathbf{L}\end{array}$ & $\begin{array}{l}\text { Ostrov } \\
\text { Ostwald }\end{array}$ & $\begin{array}{l}26.9 \mathrm{~S} \\
10.4 \mathrm{~N}\end{array}$ & $\begin{array}{l}28.0 \mathrm{~W} \\
121.9 \mathrm{E}\end{array}$ & $\begin{array}{r}67 \\
104\end{array}$ & $\begin{array}{l}\text { UR } \\
\text { EU }\end{array}$ & $\begin{array}{l}\mathrm{RU} \\
\mathrm{GE}\end{array}$ & $\begin{array}{l}5 \\
5\end{array}$ & $\begin{array}{l}1976 \\
1970\end{array}$ \\
\hline $\begin{array}{l}\mathrm{M} \\
\mathrm{V}\end{array}$ & $\begin{array}{l}\mathrm{M} \\
\mathrm{V}\end{array}$ & $\begin{array}{l}\text { Osuga Valles } \\
\text { Otau Corona }\end{array}$ & $\begin{array}{l}16.0 \mathrm{~S} \\
67.8 \mathrm{~N}\end{array}$ & $\begin{array}{c}39.1 \mathrm{~W} \\
298.7 \mathrm{E}\end{array}$ & $\begin{array}{l}155 \\
172\end{array}$ & $\begin{array}{l}\text { UR } \\
\text { AF }\end{array}$ & $\underset{\mathrm{NI}}{\mathrm{RU}}$ & $\begin{array}{l}5 \\
5\end{array}$ & $\begin{array}{l}1979 \\
1985\end{array}$ \\
\hline $\begin{array}{l}\mathrm{U} \\
\mathrm{S}\end{array}$ & $\begin{array}{l}\text { ob } \\
\text { ia }\end{array}$ & $\begin{array}{l}\text { Othello } \\
\text { Othon }\end{array}$ & $\begin{array}{l}66.0 \mathrm{~S} \\
33.3 \mathrm{~N}\end{array}$ & $\begin{array}{c}42.9 \mathrm{E} \\
347.8 \mathrm{~W}\end{array}$ & $\begin{array}{r}114 \\
86\end{array}$ & $\begin{array}{l}\text { EU } \\
\text { EU }\end{array}$ & $\begin{array}{l}\text { GB } \\
\text { FR }\end{array}$ & $\begin{array}{l}5 \\
5\end{array}$ & $\begin{array}{l}1988 \\
1982\end{array}$ \\
\hline $\begin{array}{l}\mathrm{M} \\
\mathrm{J} \\
\mathrm{M} \\
\mathrm{V} \\
\mathrm{M}\end{array}$ & $\begin{array}{l}M \\
\text { ca } \\
M \\
V \\
M\end{array}$ & $\begin{array}{l}\text { Oti Fossae } \\
\text { Ottar } \\
\text { Ottumwa } \\
\text { Otygen Corona } \\
\text { Oudemans }\end{array}$ & $\begin{array}{l}10.0 \mathrm{~S} \\
61.5 \mathrm{~N} \\
24.9 \mathrm{~N} \\
57.0 \mathrm{~S} \\
10.0 \mathrm{~S}\end{array}$ & $\begin{array}{c}117.7 \mathrm{~W} \\
104.8 \mathrm{~W} \\
55.7 \mathrm{~W} \\
30.5 \mathrm{E} \\
91.7 \mathrm{~W}\end{array}$ & $\begin{array}{r}231 \\
50 \\
55 \\
400 \\
121\end{array}$ & $\begin{array}{l}\text { EU } \\
\text { EU } \\
\text { US } \\
\text { AS } \\
\text { EU }\end{array}$ & $\begin{array}{l}\text { GR } \\
\text { NS } \\
\text { IA } \\
\text { MO } \\
\text { DU }\end{array}$ & $\begin{array}{l}5 \\
5 \\
5 \\
5 \\
5\end{array}$ & $\begin{array}{r}1982 \\
1979 \\
1976 \\
91 \\
1973\end{array}$ \\
\hline V & V & Ovda Fluctus & $6.1 \mathrm{~S}$ & $95.5 \mathrm{E}$ & 310 & UR & MJ & 5 & 1994 \\
\hline V & V & Ovda Regio & $2.8 \mathrm{~S}$ & $85.6 \mathrm{E}$ & 5280 & UR & MJ & 5 & 1982 \\
\hline $\begin{array}{l}\mathrm{H} \\
\mathrm{M}\end{array}$ & $\begin{array}{l}\mathrm{H} \\
\mathrm{M}\end{array}$ & $\begin{array}{l}\text { Ovid } \\
\text { Oxia Colles }\end{array}$ & $\begin{array}{l}69.5 \mathrm{~S} \\
20.2 \mathrm{~N}\end{array}$ & $\begin{array}{l}22.5 \mathrm{~W} \\
27.0 \mathrm{~W}\end{array}$ & $\begin{array}{r}44 \\
433\end{array}$ & $\begin{array}{l}\text { EU } \\
\text { EU }\end{array}$ & $\begin{array}{l}\text { RM } \\
\text { GR }\end{array}$ & $\begin{array}{l}5 \\
5\end{array}$ & $\begin{array}{l}1976 \\
1985\end{array}$ \\
\hline M & $\mathbf{M}$ & Oxia Palus & $8.0 \mathrm{~N}$ & $180.0 \mathrm{~W}$ & 0 & $\mathrm{EU}$ & LA & 5 & 1958 \\
\hline $\begin{array}{l}\text { M } \\
\text { V }\end{array}$ & $\begin{array}{l}\mathrm{M} \\
\mathrm{V}\end{array}$ & $\begin{array}{l}\text { Oxus } \\
\text { Ozza Mons }\end{array}$ & $\begin{array}{r}20.0 \mathrm{~N} \\
4.5 \mathrm{~N}\end{array}$ & $\begin{array}{c}12.0 \mathrm{~W} \\
201.0 \mathrm{E}\end{array}$ & $\begin{array}{r}0 \\
507\end{array}$ & $\begin{array}{l}\text { EU } \\
\text { AS }\end{array}$ & $\begin{array}{l}\text { LA } \\
\text { PE }\end{array}$ & $\begin{array}{l}5 \\
5\end{array}$ & $\begin{array}{l}1958 \\
1982\end{array}$ \\
\hline
\end{tabular}

ft origin

LA From albedo feature at $10 \mathrm{~S}, 65 \mathrm{~W}$.

PM Classical albedo feature name.

AA J. Robert; American physicist (1904-1967).

AA Theodor Egon von; Czechoslovakian astronomer (1841-1886).

CR Greek fertility goddess.

AA Town in Arizona, USA.

PE Classical albedo feature name.

AA Emmuska; Hungarian novelist, playwright (1865-1947).

AA Town in Nigeria.

AA Oresme, Nicole; French mathematician (1323(?)-1382).

AA Town in California, USA.

AA Aleksandr V.; Soviet astronomer (1880-1954); Sergei V.; Soviet astronomer (1880-1958).

AA Lyubov; Soviet actress (1902-1975)

AA Persian progenitor god of light.

SU Norse river through which dead pass.

AA Finnaeus (Oronce Fine); French mathemetician, cartographer 1494-1555

AL Floating island (present Delos) where Leto bore Apollo and Artemis.

TH Classical albedo feature name; dropped in 1979.

AA Japanese male name

FR Yoruba (Nigeria) fresh water goddess.

AA Polina; Soviet aviator (1907-1939).

AA Egyptian god of the dead

AA Egyptian god of the dead.

AA Norse; a name for Odin.

AA Turkish male name.

CM Mt. Pelion piled on top of it in war between Titans and Gods.

AA Town in Russia.

AA Wilhelm; German chemist; Nobel laureate (1853-1932).

VA River in Russia.

CR Bini (S. Nigeria) goddess of fertility.

AA Shakespearean character.

AA One of twelve peers; guarded French dead while Charlemagne pursued Saracen forces; sixth column leader.

FO Classical albedo feature.

AA Innsteinn's son and Freyja's favorite

AA Town in Iowa, USA.

CR Mongolian earth mother.

AA Jean A.; Dutch astronomer (1827-1906).

FL Named from regio where feature is located.

RE Marijian; Titaness having supernatural power.

AA Roman poet (43 B.C.-A.D. 17).

CO From albedo feature at $25 \mathrm{~N}, 24 \mathrm{~W}$.

AL Lake (swamp into which Oxus River flows; i.e. Sea of Aral).

AL Present Amoo-Darya River.

MO Persian goddess honored by the Koreishies.
P: $\quad$ planetary system (see page xvi).

Sa: satellite (see page $\mathrm{xvi}$ ).

lat: latitude of feature center.

long: longitude of feature center. diam: diameter or long dimension of feature. ct: continent of name origin (see page $284 \mathrm{ff}$.)

et: ethnicity of name origin (see page $284 \mathrm{ff}$.)

as: name approval status (see page xvii). ad: name approval date (year).

ref: reference source for name (see page $287 \mathrm{ff}$.).

ft: feature type (see page 290). 
ALPHABETICAL LIST OF NAMES

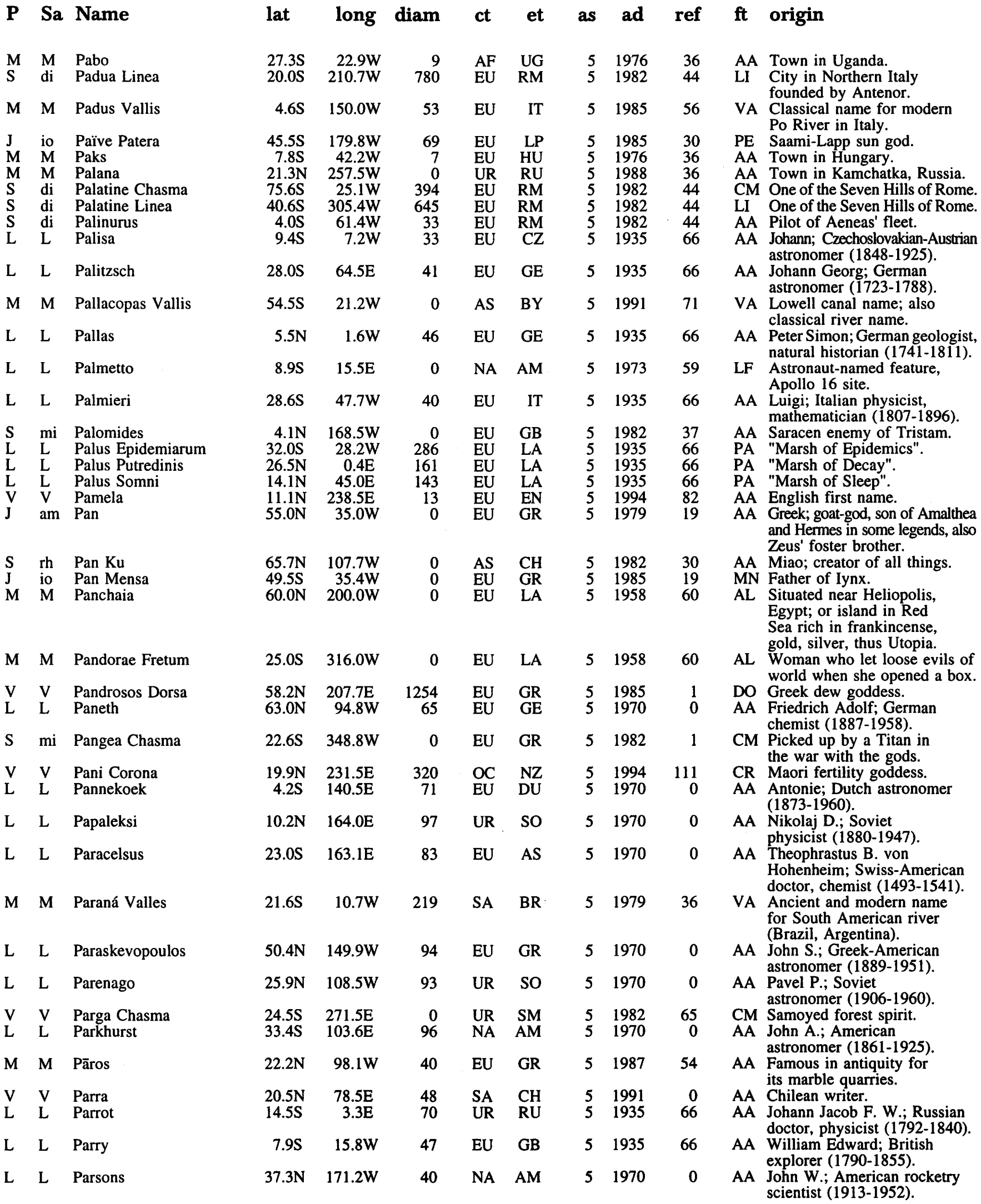

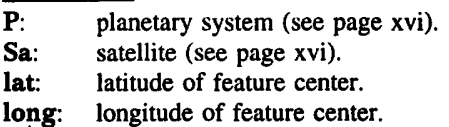

diam: diameter or long dimension of feature. ct: $\quad$ continent of name origin (see page $284 \mathrm{ff}$.) et: ethnicity of name origin (see page $284 \mathrm{ff}$.) as: name approval status (see page xvii) ad: name approval date (year).

ref: reference source for name (see page $287 \mathrm{ff}$.). ft: feature type (see page 290). 
ALPHABETICAL LIST OF NAMES

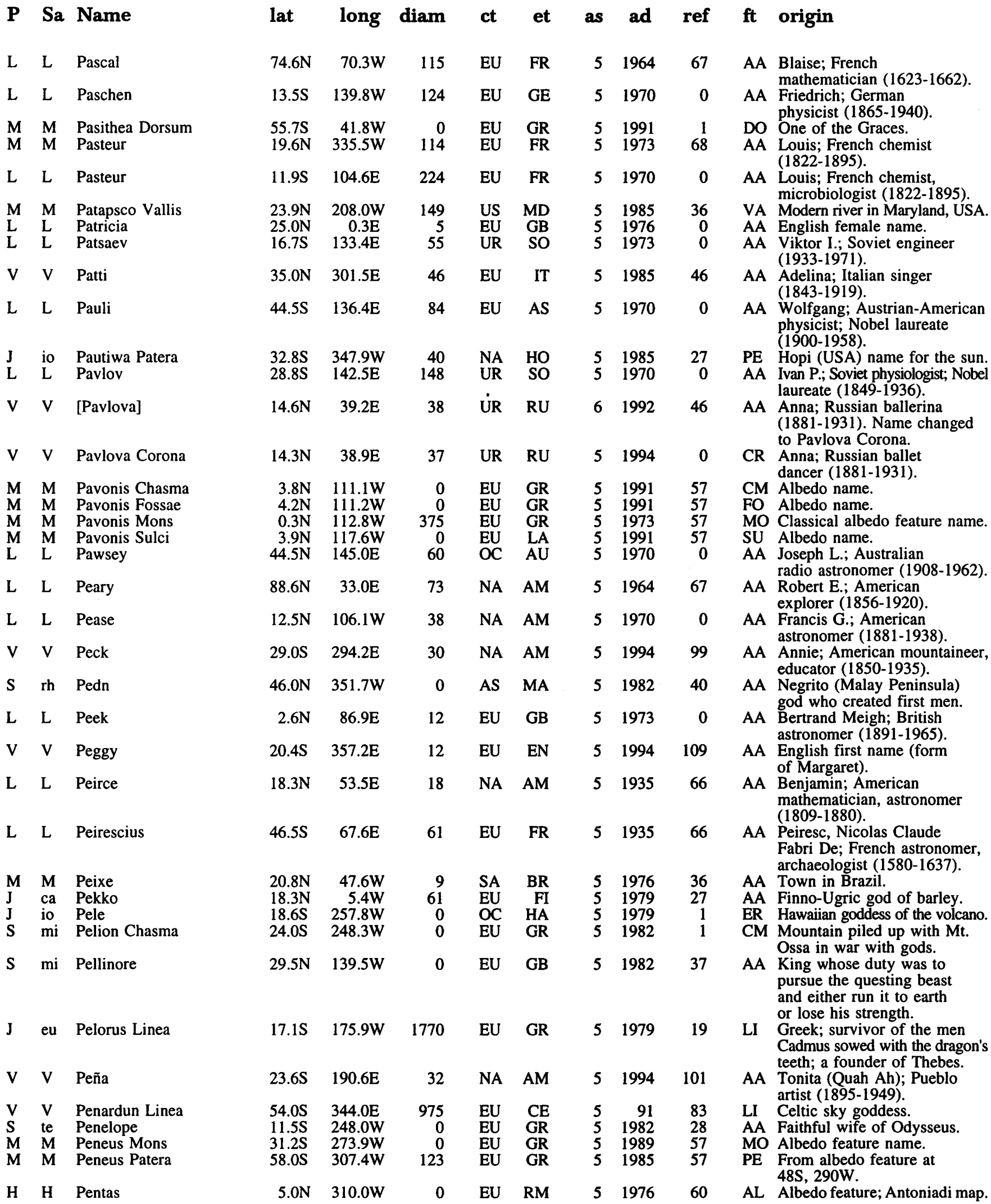

\footnotetext{
P: $\quad$ planetary system (see page $\mathrm{xvi}$ ).

Sa: $\quad$ satellite (see page $\mathrm{xvi}$ ).

lat: latitude of feature center.

long. longitude of feature center.
}

diam: diameter or long dimension of feature.

ct: continent of name origin (see page $284 \mathrm{ff}$.)

et: ethnicity of name origin (see page $284 \mathrm{ff}$.)

as: name approval status (see page xvii). ad: name approval date (year).

ref: reference source for name (see page $287 \mathrm{ff}$.). ft: feature type (see page 290). 


\section{ALPHABETICAL LIST OF NAMES}

\section{P Sa Name}

L L Pentland

M M Peraea Cavus

M M Peraea Mons

V V Perchta Corona

$\mathrm{S}$ mi Percivale

L L Perel'man

M M Perepelkin

L L Perepelkin

U um Peri

$S$ en Peri-Banu

M M Peridier

L L Perkin

L L Perrine

J ga Perrine Regio

M M Perrotin

L L Petavius

L L Petermann

L L Peters

L L Petit

H H Petrarch

L L Petrie

L L Petropavlovskiy

L L Petrov

M M Pettit

L L Pettit

L L Petzval

V V Phaedra

M M Phaenna Dorsum

H H Phaethontias

M M Phaethontis

$\mathrm{N}$ pr Pharos

$S$ te Phemius

H H Phidias

M M Philadelphia

L L Phillips

M M Phillips lat long diam ct et as ad ref $f t$ origin

$\begin{array}{rrrrrrrr}64.6 \mathrm{~S} & 11.5 \mathrm{E} & 56 & \mathrm{EU} & \text { IR } & 5 & 1935 & 66 \\ 29.9 \mathrm{~S} & 264.7 \mathrm{~W} & 0 & \mathrm{EU} & \text { GR } & 5 & 1989 & 57 \\ 31.3 \mathrm{~S} & 273.9 \mathrm{~W} & 0 & \mathrm{EU} & \text { GR } & 5 & 1989 & 57 \\ 17.0 \mathrm{~N} & 234.5 \mathrm{E} & 500 & \mathrm{EU} & \text { GE } & 5 & 1994 & 111 \\ 1.0 \mathrm{~S} & 180.2 \mathrm{~W} & 0 & \text { EU } & \text { GB } & 5 & 1982 & 37 \\ 24.0 \mathrm{~S} & 106.0 \mathrm{E} & 46 & \text { UR } & \text { SO } & 5 & 1970 & 0 \\ 52.8 \mathrm{~N} & 64.6 \mathrm{~W} & 112 & \text { UR } & \text { RU } & 5 & 1973 & 68 \\ 10.0 \mathrm{~S} & 129.0 \mathrm{E} & 97 & \text { UR } & \text { SO } & 5 & 1970 & 0 \\ 9.2 \mathrm{~S} & 4.3 \mathrm{E} & 61 & \text { AS } & \text { PE } & 5 & 1988 & 1\end{array}$

$\begin{array}{llllllll}63.1 \mathrm{~N} & 317.9 \mathrm{~W} & 18 & \text { AS } & \text { AR } & 5 & 1982 & 52\end{array}$

25.8

47.2N $175.9 \mathrm{~W}$

$42.5 \mathrm{~N} \quad 127.8 \mathrm{~W}$

$38.8 \mathrm{~N} \quad 206.6 \mathrm{~W}$

$3.0 \mathrm{~S}$

$77.8 \mathrm{~W}$

$25.1 \mathrm{~S}$

$60.4 \mathrm{E}$

$74.2 \mathrm{~N}$

$68.1 \mathrm{~N}$

66.3E

$2.3 \mathrm{~N}$

29.5E

$30.6 \mathrm{~S}$

63.5E

$45.3 \mathrm{~N}$

$26.2 \mathrm{~W}$

108.4E

$37.2 \mathrm{~N} \quad 114.8 \mathrm{~W}$

61.4S 88.0E

$12.2 \mathrm{~N}$

$173.9 \mathrm{~W}$

$27.5 \mathrm{~S}$

$86.6 \mathrm{~W}$

$62.7 \mathrm{~S}$

$110.4 \mathrm{~W}$

$35.9 \mathrm{~N} \quad 252.7 \mathrm{E}$

$54.3 \mathrm{~S}$

$0.0 \mathrm{~N}$

$50.0 \mathrm{~S}$

$0.0 \mathrm{~N}$

$12.0 \mathrm{~N}$

$8.7 \mathrm{~N}$

$22.0 \mathrm{~N}$

$26.6 S$

43.2W

$167.0 \mathrm{~W}$

$155.0 \mathrm{~W}$

$0.0 \mathrm{~W}$

$285.8 \mathrm{~W}$

$149.3 \mathrm{~W}$

48.0W

75.3E

$66.4 \mathrm{~S}$

$45.1 \mathrm{~W}$

$\begin{array}{rll}99 & \text { EU } & \text { FR } \\ 62 & \text { NA } & \text { AM } \\ 86 & \text { NA } & \text { AM } \\ 2145 & \text { NA } & \text { AM }\end{array}$

$1973 \quad 68$

$5 \quad 1970$

51970

$5 \quad 1979$

$5 \quad 1988$

$5 \quad 1935$

51935

51935

51976

51976

51970

51970

$5 \quad 1970$

$5 \quad 1973$

51970

$5 \quad 1970$

90 EU AS

51994

EU GR

$\begin{array}{ll}\text { EU } & \text { GR } \\ \text { EU } & \text { RM }\end{array}$

51991

51976

$5 \quad 1958$

EU LA

$5 \quad 1994$

$\begin{array}{ll}\text { EU } & \text { GR } \\ \text { EU } & \text { GR }\end{array}$

$5 \quad 1982$

51976

51979

$5 \quad 1935$

$5 \quad 1973$
AA Joseph Barclay; Irish geographer (1797-1873).

CB Albedo feature name.

MO Albedo feature name.

CR German fertility goddess.

AA Very pure knight; accomplished quest of Holy Grail.

AA Yakov I.; Soviet rocketry scientist (1882-1942).

AA Evgenii J.; Russian astronomer (1906-1940).

AA Evgenij J.; Soviet astrophysicist (1906-1940).

AA Persian evil spirit who disguised malevolence by charm; disturbed natural elements and heavenly bodies.

AA Genie who marries Ahmad and helps him fulfill the demands of his father.

AA Julien; French astronomer (1882-1967).

AA Richard S.; American telescope manufacturer (1906-1969).

AA Charles D.; American astronomer (1867-1951).

RE Charles D.; American astronomer (1867-1951).

AA Henri A.; French astronomer, studied dark lineations on Mars (1845-1904).

AA Petau, Denis; French chronologist, astronomer (1583-1652).

AA August Heinrich; German geographer (1822-1878).

AA Christian August Friectrich; German astronomer (1806-1880).

AA Alexis Therese; French physicist (1771-1820).

AA Francesco; Italian poet (1304-1374).

AA Robert M.; Canadian astronomer (1906-1966).

AA Boris S.; Soviet rocketry engineer (1898-1933).

AA Evgenij S.; Soviet rocketry scientist (1900-1942).

AA Edison; American astronomer (1890-1962).

AA Edison; American astronomer (1889-1962).

AA Joseph von; Austrian optician (1807-1891).

AA First name from Greek.

DO One of the Graces.

AL Albedo name for $\mathrm{H}-8$, Tolstoj region.

AL "Of Phaethon;" who recklessly drove Chariot of Sun.

AA Island where Proteus reigned.

AA Minstrel to the wooers; spared by Odysseus.

AA Greek sculptor (fl. c. 490-430 B.C.).

AA American colonial town (Pennsylvania).

AA John; British geologist, astronomer (1800-1874).

AA John; British geologist (1800-1874). Theodore E. British astronomer (1868-1942).

\footnotetext{
P: $\quad$ planetary system (see page $x v i)$.

Sa: satellite (see page xvi).

lat: latitude of feature center.

long: longitude of feature center.
}

diam: diameter or long dimension of feature.

ct: continent of name origin (see page $284 \mathrm{ff}$.)

et: ethnicity of name origin (see page $284 \mathrm{ff}$.)

as: name approval status (see page xvii). ad: name approval date (year).

ref: reference source for name (see page $287 \mathrm{ff}$.).

ft: feature type (see page 290). 


\section{ALPHABETICAL LIST OF NAMES}

\section{P Sa Name}

L L Ptilolaus

H H Ptiloxenus

J ga Philus Sulci

J eu Ptineus Linea

M M Prison

M M Ptison Rupes

M M Pr legethon Catena

M M Prlegra

M M Pr legra Montes

J eu Procis Flexus

L L Procylides

\section{V Proebe Regio}

M M Proenicis Lacus

J eu Proenix Linea

$S$ ja Proibe

M M Pton

V V Prra Naret Corona

M M Prrixi Regio

\section{J ga Prrygia Sulcus}

V V Prryne

$U$ ti Prrynia

$\begin{array}{lll}\text { V } & \text { V } & \text { Phyllis } \\ \text { V } & \text { V } & \text { Pi if }\end{array}$

L L Piızzi

L L Pi.zzi Smyth

M M Piia

L L Pi scolomini

M M Piikering

L L Piikering
L L Pistet
H $\quad \mathrm{H} \quad$ Pißria
H H Pijgalle
L L Pikel'ner

L L Pi âtre

M M Pi ra

M M Piıdus Mons

\section{lat long diam}

$\begin{array}{rrrrrrrr}72.1 \mathrm{~N} & 32.4 \mathrm{~W} & 70 & \text { EU } & \text { GR } & 5 & 1935 & 66 \\ & & & & & & & \\ 8.7 \mathrm{~S} & 111.5 \mathrm{~W} & 90 & \text { EU } & \text { GR } & 5 & 1976 & 59 \\ 44.0 \mathrm{~N} & 212.0 \mathrm{~W} & 473 & \text { EU } & \text { GR } & 5 & 1979 & 1 \\ & & & & & & & \\ 33.0 \mathrm{~S} & 269.2 \mathrm{~W} & 1984 & \text { EU } & \text { GR } & 5 & 1979 & 19 \\ 20.0 \mathrm{~N} & 320.0 \mathrm{~W} & 0 & \text { AS } & \text { HE } & 5 & 1958 & 60 \\ 26.6 \mathrm{~N} & 309.4 \mathrm{~W} & 149 & \text { EU } & \text { GR } & 5 & 1976 & 57 \\ 40.5 \mathrm{~N} & 101.8 \mathrm{~W} & 875 & \text { EU } & \text { GR } & 5 & 1979 & 57 \\ 31.3 \mathrm{~N} & 187.8 \mathrm{~W} & 134 & \text { EU } & \text { GR } & 5 & 1958 & 60\end{array}$

$\begin{array}{rrrrrrrr}40.9 \mathrm{~N} & 197.4 \mathrm{~W} & 1310 & \text { EU } & \text { GR } & 5 & 1973 & 57 \\ 48.6 \mathrm{~S} & 197.2 \mathrm{~W} & 298 & \text { EU } & \text { GR } & 5 & 1985 & 19 \\ 52.7 \mathrm{~S} & 57.0 \mathrm{~W} & 121 & \text { EU } & \text { DU } & 5 & 1935 & 66 \\ & & & & & & & \\ 6.0 \mathrm{~S} & 282.8 \mathrm{E} & 2852 & \text { EU } & \text { GR } & 5 & 1982 & 1 \\ 12.0 \mathrm{~S} & 110.0 \mathrm{~W} & 0 & \text { EU } & \text { LA } & 5 & 1958 & 60 \\ & & & & & & & \\ 14.5 \mathrm{~N} & 184.7 \mathrm{~W} & 732 & \text { EU } & \text { GR } & 5 & 1985 & 19 \\ 0.0 \mathrm{~N} & 0.0 \mathrm{~W} & 0 & \text { EU } & \text { GR } & 5 & 1982 & 0 \\ 15.8 \mathrm{~N} & 257.3 \mathrm{~W} & 8 & \text { AS } & \text { TH } & 5 & 1976 & 36 \\ 66.6 \mathrm{~S} & 209.6 \mathrm{E} & 150 & \text { AS } & \text { TH } & 5 & 1994 & 83 \\ 40.0 \mathrm{~S} & 70.0 \mathrm{~W} & 0 & \text { EU } & \text { LA } & 5 & 1958 & 60\end{array}$

L L Pi;ard

$12.4 \mathrm{~N}$

19.3W

$46.2 \mathrm{~S}$

24.3S

$12.3 \mathrm{~N}$

$0.8 \mathrm{~N}$

$36.6 \mathrm{~S}$

$41.9 \mathrm{~N}$

$20.0 \mathrm{~N}$

$14.6 \mathrm{~N}$

29.7S

$34.4 \mathrm{~S}$

314.8E

309.2E

$132.4 \mathrm{E}$

$5.2 \mathrm{E}$

67.9W

$3.2 \mathrm{~W}$

$53.2 \mathrm{~W}$

54.7E

$32.2 \mathrm{E}$

$132.8 \mathrm{~W}$

$2.9 \mathrm{~S}$

7.0E

43.6S

$7.4 \mathrm{~W}$

$0.0 \mathrm{~N}$

$270.0 \mathrm{~W}$

$38.5 \mathrm{~S}$

$9.5 \mathrm{~W}$

47.9S

123.3E

$60.2 S$

$86.9 \mathrm{~W}$

$18.6 \mathrm{~N} \quad 248.5 \mathrm{~W}$

$39.7 \mathrm{~N} \quad 88.9 \mathrm{~W}$

$\begin{array}{lllll}3205 & \text { EU } & \text { GR } & 5 & 1979\end{array}$

51994

$\begin{array}{ll}5 & 1988\end{array}$

$5 \quad 1994$

51991

$5 \quad 1935$

51935

$\begin{array}{rrrrr}0 & \text { SA } & \text { CH } & 5 & 1988 \\ 22 & \text { EU } & \text { FR } & 5 & 1935\end{array}$

87 EU IT $\quad 5 \quad 1935$

112 NA AM

$5 \quad 1973$

66

\footnotetext{
P: plane ary system (see page $\mathrm{xvi}$ ).

Sa: $\quad$ satellite (see page xvi).

lat: latitucle of feature center.

long: longitude of feature center.
}

diam: diameter or long dimension of feature.

ct: continent of name origin (see page $284 \mathrm{ff}$.)

et: ethnicity of name origin (see page $284 \mathrm{ff}$.)

as: name approval status (see page xvii).

\section{ft origin}

AA Of Croton; Greek mathematician, astronomer, philosopher (unkn-fl. 400 B.C.).

AA Greek lyric poet (436-380 B.C.).

SU Greek; where Ganymede and Hebe were worshipped as rain-givers.

LI Greek; brother of Europa.

AL Biblical first river of Paradise.

RU Classical albedo feature name.

CA From albedo feature at $38 \mathrm{~N}, 125 \mathrm{~W}$.

AL "Burning plain;" in Chalcidian Peninsula of Greece where Zeus hurled thunderbolts at Titans to support Hercules.

MO Classical albedo feature name.

FE Where the cow lead Cadmus before it stopped at the site of Thebes.

AA Johannes Phocylides Holwarda (Jan Fokker); Dutch astronomer (1618-1651).

RE Greek Titaness.

AL "Lake of the Phoenix;" Arabia or India.

LI Brother of Europa.

AA Daughter of Leukippos.

AA Town in Thailand

CR Thai fertility goddess.

AL "Phrixus' Region;" Phrixus and sister Helle escaped sacrifice in Boeotia on back of ram with golden fleece.

SU Greek; kingdom in Asia Minor where Ganymede was born.

AA Greek model, courtesan (fourth century B.C.).

AA Alcibiades' mistress in "Timon of Athens."

AA First name from Greek.

AA Edith; French singer, songwriter (1915-1963).

AA Giuseppe; Italian astronomer (1746-1826).

AA Charles; Scottish astronomer (1819-1900).

AA Town in Chile.

AA Jean; French astronomer (1620-1682).

AA Alessandro; Italian astronomer (1508-1578).

AA Edward C.; American astronomer (1846-1919). William H.; American astronomer (1858-1938).

AA Edward Charles; American astronomer (1846-1919); Willaim H.; American astronomer (1858-1938).

AA Pictet-Turretin, Marc-Auguste; Swiss physicist (1752-1825).

AL Albedo name for unimaged H-10 region.

AA Jean Baptiste; French sculptor (1714-1785).

AA Solomon Börisovich; Soviet astronomer, cosmologist (1921-1975).

AA Rozier, F. De; French aeronaut (1753-1785)

AA Town in Panama.

MO Mountains near Vale of Tempe. ad: name approval date (year).

ref: reference source for name (see page $287 \mathrm{ff}$.).

ft: feature type (see page 290). 
ALPHABETICAL LIST OF NAMES

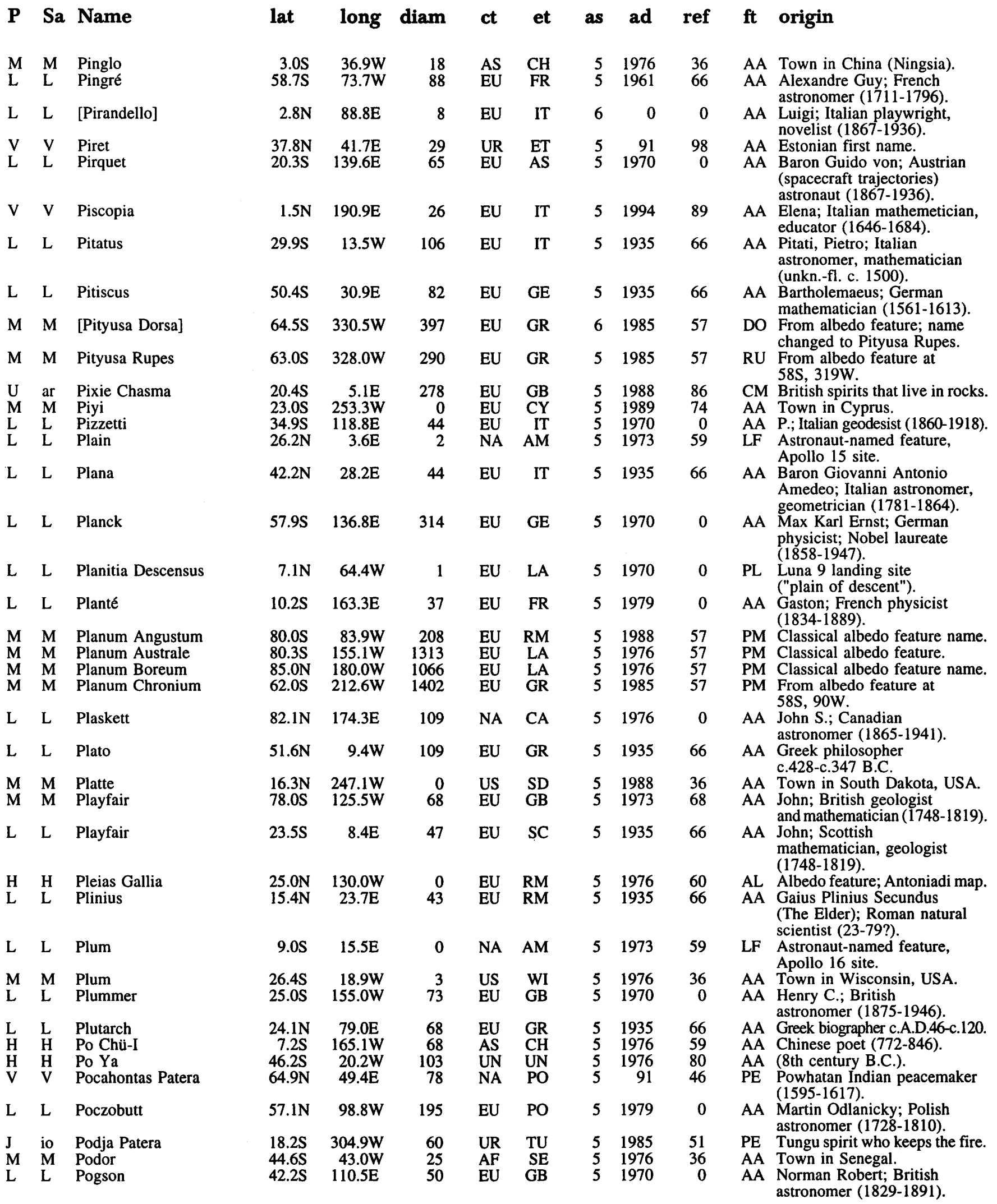

\footnotetext{
P: $\quad$ planetary system (see page $\mathrm{xvi}$ ).

Sa: satellite (see page xvi).

lat: latitude of feature center.

long: longitude of feature center.
}

diam: diameter or long dimension of feature.

ct: continent of name origin (see page $284 \mathrm{ff}$.)

et: ethnicity of name origin (see page $284 \mathrm{ff}$.)

as: name approval status (see page xvii). ad: name approval date (year)

ref: reference source for name (see page $287 \mathrm{ff}$.).

$\mathrm{ft}$ feature type (see page 290). 


\section{ALPHABETICAL LIST OF NAMES}

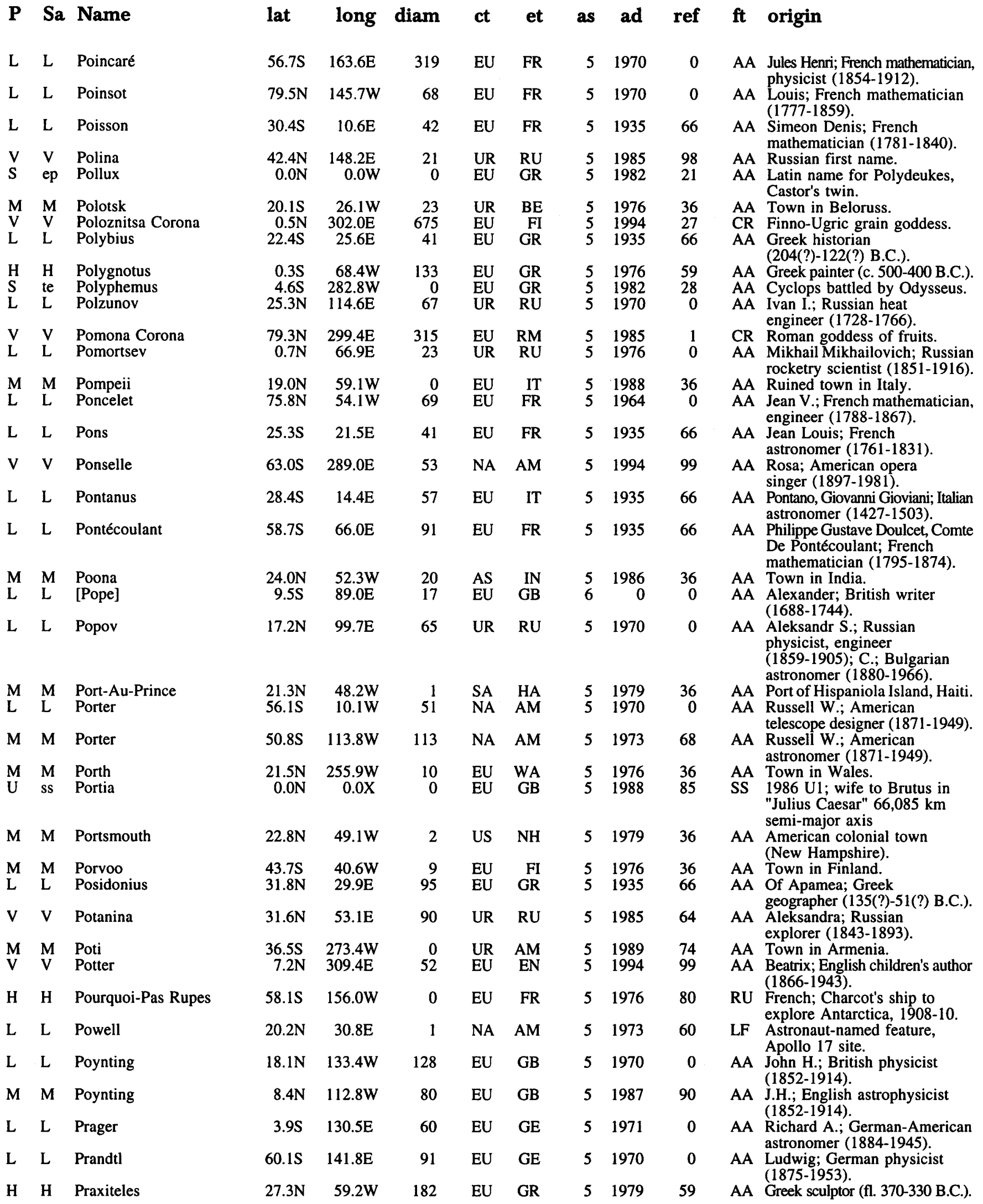

\footnotetext{
P: $\quad$ planetary system (see page xvi).

Sa: $\quad$ satellite (see page $\mathrm{xvi}$ )

lat: latitude of feature center.

long: longitude of feature center.
}

diam: diameter or long dimension of feature. ct: continent of name origin (see page $284 \mathrm{ff}$.) et: ethnicity of name origin (see page $284 \mathrm{ff}$.)

as: name approval status (see page xvii). ad: name approval date (year).

ref: reference source for name (see page $287 \mathrm{ff}$.). $\mathrm{ft}$ : feature type (see page 290). 
ALPHABETICAL LIST OF NAMES

\section{P Sa Name \\ V V Prichard \\ L L Priestley \\ M M Priestley \\ M M Princeton \\ L L Prinz \\ $\begin{array}{lll}\text { L } & \text { L } & \text { Priscilla } \\ \text { L } & \text { L } & \text { Proclus }\end{array}$}

M M Proctor

L L Proctor

M M Promethei Rupes

M M Promethei Sinus

M M Promethei Terra

J io Prometheus

L L Promontorium Agarum

L L Promontorium Agassi

L L Promontorium Archerusia

L L Promontorium Deville

L L Promontorium Fresnel

L L Promontorium Heraclides

L L Promontorium Kelvin

L L Promontorium Laplace

L L Promontorium Taenarium

M M Propontis

U mr Prospero

L L Protagoras

M M Protei Regio

M M Protonilus

M M Protonilus Mensae

M M Protva Valles

H H Proust

J ga Ptah

M M Ptolemaeus

L L Ptolemaeus

S rh Pu Chou Chasma

$\mathrm{H} \quad \mathrm{H}$ Puccini

U ss Puck lat

long

\begin{tabular}{|c|c|c|c|c|c|c|}
\hline $44.0 \mathrm{~N}$ & $11.5 \mathrm{E}$ & 22 & $O C$ & $\mathrm{AU}$ & 5 & 1985 \\
\hline $57.3 \mathrm{~S}$ & $108.4 \mathrm{E}$ & 52 & $\mathrm{EU}$ & GB & 5 & 1970 \\
\hline $54.3 \mathrm{~S}$ & $229.3 W$ & 40 & EU & GB & 5 & 1973 \\
\hline $21.9 \mathrm{~N}$ & $49.1 \mathrm{~W}$ & 2 & US & NJ & 5 & 1979 \\
\hline $25.5 \mathrm{~N}$ & $44.1 \mathrm{~W}$ & 46 & $\mathrm{EU}$ & GE & 5 & 1935 \\
\hline $\begin{array}{r}0.0 \mathrm{~N} \\
16.1 \mathrm{~N}\end{array}$ & $\begin{array}{r}0.0 \mathrm{E} \\
46.8 \mathrm{E}\end{array}$ & $\begin{array}{r}0 \\
28\end{array}$ & $\begin{array}{l}\mathrm{EU} \\
\mathrm{EU}\end{array}$ & $\begin{array}{l}\text { LA } \\
\text { GR }\end{array}$ & 5 & $\begin{array}{l}1976 \\
1935\end{array}$ \\
\hline
\end{tabular}

47.9

$46.4 \mathrm{~S}$

$330.4 \mathrm{~W}$

\section{$76.8 \mathrm{~S}$}

65.0S

$52.9 \mathrm{~S}$

$14.0 \mathrm{~N}$

$42.0 \mathrm{~N}$

$16.7 \mathrm{~N}$

$43.2 \mathrm{~N}$

$29.0 \mathrm{~N}$

$40.3 \mathrm{~N}$

$27.0 \mathrm{~S}$

$46.0 \mathrm{~N}$

$25.8 \mathrm{~W}$

$19.0 S$

8.0W

$45.0 \mathrm{~N} \quad 185.0 \mathrm{~W}$

$32.9 \mathrm{~S}$

$56.0 \mathrm{~N}$

$329.9 \mathrm{E}$

23.0

$42.0 \mathrm{~N}$

$44.2 \mathrm{~N}$

30.1

$19.7 \mathrm{~N}$

$67.0 \mathrm{~S}$

$46.4 S$

9.3S

7.3E

$50.0 \mathrm{~W}$

168

EU GB

$5 \quad 1973$

$86.8 \mathrm{~W}$

1491

NA AM

51935

EU GR

51976

$262.2 \mathrm{~W}$

153.0W

$1.8 \mathrm{E}$

2761

0

70
20

EU GR

EU GR

EU GR

UR RU

EU SZ

UR RU

51961

EU FR

$5 \quad 1935$

51935

EU FR

51935

EU GR

51935

51935

EU FR

$5 \quad 1961$

67

0 EU GR

51958

$\begin{array}{ll}5 & 1988\end{array}$

51935

EU GR

$5 \quad 1958$

$5 \quad 1958$

51973

$309.4 \mathrm{~W}$

592

$60.0 \mathrm{~W}$

213

$46.7 \mathrm{~W}$

157

51979

51976

51988

51973

51935

$26.1 \mathrm{~N}$

65.3S

95.3W

$46.8 \mathrm{~W}$

$0.0 \mathrm{~N}$

$0.0 \mathrm{X}$
$5 \quad 1982$

51976

$\begin{array}{ll}5 & 1988\end{array}$
68

66

67

60

ref $f t$ origin

AA Catharina; Australian writer (1884-1969)

AA Joseph; British chemist (1733-1804)

AA Joseph; British chemist (1733-1804).

AA American colonial town (New Jersey).

AA Wilhelm; German-Belgian astronomer (1857-1910).

AA Latin female name.

AA Diadochos (The Successor); Greek mathematician astronomer, philosopher (410-485).

AA Richard A.; British astronomer (1837-1888).

AA Mary; American astronomer (1862-1957)

RU Classical albedo feature name.

AL Prometheus' Bay; Greek mythological character.

TA Classical albedo feature name.

ER Greek fire god.

PR Named from cape in Sea of Azov.

PR Jean Louis Rodolphe; Swiss zoologist, geologist (1807-1873).

PR Named from cape on the Black Sea.

PR Sainte-Claire Charles; French geologist (1814-1876).

PR Augustin Jean; French optician (1788-1827).

PR Ponticus; Greek astronomer (c. 388-310 B.C.).

PR William Thomson, Lord Kelvin; Scottish natural philosopher (1824-1907).

PR Pierre Simon; French mathematician, astronomer (1749-1827).

PR Named from cape in Greece; now Matapan or Tainaron.

AL The Sea of Marmora, Asia Minor.

AA Rightful Duke of Mila in "The Tempest."

AA Greek philosopher (481(?)-411(?) B.C.)

AL "Proteus' Region;" Proteus was a sea god with a gift of prophecy.

AL Designation of first (Eastern) part of "Nilus" or Nile Canal.

MN From albedo feature at $42 \mathrm{~N}, 315 \mathrm{~W}$.

VA River in Russia.

AA Marcel; French novelist (1871-1922).

AA Sovereign god of Memphis; patron of artisans.

AA Claudius; Greco-Egyptian astronomer (c. A.D. 90-160).

AA Ptolemy, Claudius; Greek astrononer, mathematician, geographer (c. A.D. 87-150).

CM Mountain attacked by Kung Chung.

AA Giacomo; Italian composer (1858-1924).

SS $1985 \mathrm{U1}$; mischievous spirit in "Midsummer Night's Dream" 86,000 $\mathrm{km}$ semi-major axis.

\footnotetext{
P: $\quad$ planetary system (see page xvi)

Sa: $\quad$ satellite (see page xvi).

lat: latitude of feature center.

long: longitude of feature center.
}

diam: diameter or long dimension of feature.

ct: $\quad$ continent of name origin (see page $284 \mathrm{ff}$.)

et: ethnicity of name origin (see page $284 \mathrm{ff}$.)

as: name approval status (see page xvii). ad: name approval date (year).

ref: reference source for name (see page $287 \mathrm{ff}$.).

ft: feature type (see page 290). 


\section{ALPHABETICAL LIST OF NAMES}

\section{P Sa Name}

M M Pulawy

M M Púnsk

J ga Punt Facula

L L Pupin

V V Purandhi Corona

L L Purbach

H H Purcell

J io Purgine Patera

L L Purkyně

H H Pushkin

$J$ io Pyerun Patera

M M Pylos

M M Pyramus Fossae

M M Pyrrhae Chaos

M M Pyrrhae Regio

L L Pythagoras

L L Pytheas

S rh Qat

$\begin{array}{lll}\text { V } & \text { V } & \text { Qetesh Corona } \\ \text { M } & \text { M } & \text { Qibā }\end{array}$

M M Quenisset

L L Quetelet

V V Quetzalpetlatl Corona

M M Quick

V V Quilla Chasma

M M Quines

M M Quorn

J io Ra Patera

L L Rabbi Levi

M M Rabe

H H Rabelais

L L Racah

V V Rachel

$\mathrm{L}$ [Racine]

M M Radau

V V Radka

L L Raimond

V V Raisa

H H Rajnis

M M Rakke

L L Raman lat

long

diam

ct

et

as ad

$\begin{array}{lrrrrrrr}27.8 S & 39.0 \mathrm{~W} & 24 & \text { EU } & \text { FR } & 5 & 1935 & 66 \\ 36.6 \mathrm{~S} & 76.7 \mathrm{~W} & 51 & \text { EU } & \text { PO } & 5 & 1979 & 36 \\ 20.7 \mathrm{~N} & 41.2 \mathrm{~W} & 10 & \text { EU } & \text { PO } & 5 & 1976 & 36 \\ 26.1 \mathrm{~S} & 242.2 \mathrm{~W} & 228 & \text { AF } & \text { EG } & 5 & 1985 & 1 \\ 23.8 \mathrm{~N} & 11.0 \mathrm{~W} & 2 & \text { EU } & \text { YU } & 5 & 1976 & 0 \\ & & & & & & & \\ 26.1 \mathrm{~N} & 343.5 \mathrm{E} & 170 & \text { AS } & \text { IN } & 5 & 1994 & 83 \\ 25.5 \mathrm{~S} & 2.3 \mathrm{~W} & 115 & \text { EU } & \text { AS } & 5 & 1935 & 66\end{array}$

$81.3 \mathrm{~N} \quad 146.8 \mathrm{~W}$

91

$2.6 \mathrm{~S}$

$1.6 \mathrm{~S}$

297.7W

$66.3 \mathrm{~S}$

94.9E

20

EU EN

51979

UR MO

$5 \quad 1985$

$56.0 \mathrm{~S}$

$22.4 \mathrm{~W}$

231

$56.0 S \quad 252.2 \mathrm{~W}$

$16.9 \mathrm{~N} \quad 30.1 \mathrm{~W}$

$52.6 \mathrm{~N} \quad 293.5 \mathrm{~W}$

10.5S 28.6W

$15.0 \mathrm{~S} \quad 38.0 \mathrm{~W}$

$63.5 \mathrm{~N} \quad 63.0 \mathrm{~W}$

$20.5 \mathrm{~N} \quad 20.6 \mathrm{~W}$

$23.8 \mathrm{~S} \quad 351.6 \mathrm{~W}$

20.5S 343.5E

$17.4 \mathrm{~N} \quad 257.0 \mathrm{~W}$

$34.7 \mathrm{~N} \quad 319.4 \mathrm{~W}$

150

0
127

AS

43.1N $134.9 \mathrm{~W}$

55

E

64.0S $\quad 354.5 \mathrm{E}$

$18.5 \mathrm{~N}$

23.7S

$42.1 \mathrm{~S}$

$5.6 \mathrm{~S}$

$8.6 \mathrm{~S}$
$34.7 \mathrm{~S}$

$48.8 \mathrm{~W}$

127.3E

$270.6 \mathrm{~W}$

$33.8 \mathrm{~W}$

$325.3 \mathrm{~W}$

23.6E

$$
\begin{array}{r}
400 \\
8 \\
973
\end{array}
$$

400
8
973
0

0
5
30

30
81

EU CZ

1970

UR RU

51976

$\begin{array}{llll}\text { EU SL } & 5 & 1985\end{array}$

EU GR

29
165

EU GR

51985

$\begin{array}{llll}\text { EU } & \text { LA } & 5 & 1982\end{array}$

$\begin{array}{llll}\text { EU } & \text { LA } & 5 & 1958\end{array}$

51935

EU GR

51935

EU GR

$5 \quad 1982$

OC ME

\section{$5 \quad 1994$}

EG

AS AR

$\begin{array}{ll}5 & 1988 \\ 5 & 1973\end{array}$

EU BE 51970

SA AZ

NA CA

SA IN

SA AR

OC AU

51970

$\begin{array}{lr}5 & 9 \\ 5 & 1976\end{array}$

$\begin{array}{ll}5 & 1976 \\ 5 & 1982\end{array}$

$\begin{array}{ll}5 & 1989\end{array}$

51976

$\begin{array}{ll}5 & 1979\end{array}$

EU

1935

44.0S $325.2 \mathrm{~W}$

99

EU

$$
\text { GE }
$$

$5 \quad 1973$

$61.0 \mathrm{~S} \quad 62.4 \mathrm{~W}$

141

EU FR

51976

13.8S $179.8 \mathrm{~W}$

48.7S $\quad 13.5 \mathrm{E}$

$8.3 \mathrm{~S}$

99.0E

63

AS

12

12
30

AS HE

$17.3 \mathrm{~N}$

$4.7 \mathrm{~W}$

115

EU FR

$5 \quad 1970$

$75.5 \mathrm{~N}$

$14.6 \mathrm{~N}$

96.4E

$159.3 \mathrm{~W}$

12
70

$\begin{array}{ll}\text { EU } & \text { BU } \\ \text { EU } & \text { DU }\end{array}$

$27.5 \mathrm{~N} \quad 280.3 \mathrm{E}$

$\begin{array}{ll}4.5 \mathrm{~N} & 95.8 \mathrm{~W}\end{array}$

$4.7 \mathrm{~S} \quad 43.5 \mathrm{~W}$

$27.0 \mathrm{~N} \quad 55.1 \mathrm{~W}$

$\begin{array}{ll}\text { UR } & \text { RU } \\ \text { EU } & \text { LV } \\ \text { EU } & \text { ES } \\ \text { AS } & \text { IN }\end{array}$

ref $\mathbf{f t}$ origin

AA Pierre; French astronomer (1855-1928).

AA Town in Poland

AA Town in Poland.

FA Land east of Egypt where Bes originated.

AA Michael Idvorsky; Yugoslavian-American physicist (1858-1935).

CR Hindu goddess of plenty.

AA Georg von; Austrian mathematician, astronomer (1423-1461).

AA Henry; English composer (c. 1659-1695).

PE Mordvinian (Russia) thunder god.

AA Jan Evangelista; Czechoslovakian doctor, physiologist (1787-1869).

59

AA Alecksandr Sergeyevich; Russian poet (1799-1837)

PE Slavonic god of thunder.

AA Town in Greece.

FO From albedo feature at $65 \mathrm{~N}, 300 \mathrm{~W}$.

$\mathrm{CH}$ Albedo feature name.

AL "Pyrrha's Region;" named for Pyrrha, wife of Deucalion.

AA Of Samos; Greek philosopher, mathematician (unkn-fl. c. 532 B.C.).

68

AA Of Marseilles; Greek navigator, geographer (b. c. 308 B.C.).

AA New Hebrides (Melanesia); born from a stone; formed men out of trees.

CR Egyptian fertility goddess.

AA Town in Saudi Arabia.

AA Ferdinand J.; French astronomer (1872-1951).

AA Lambert A. J.; Belgian statistician, astronomer (1796-1874).

CR Aztec fertility goddess.

AA Town in British Columbia, Canada.

CM Inca moon goddess.

AA Town in Argentina.

AA Town in Australia.

PE Egyptian sun god.

AA Gershon, Levi Ben; Spanish-Jewish philosopher, mathematician, astronomer (1288-1344).

68

AA Wilhelm F.; German astronomer (1893-1958).

AA Francois; French writer (c. 1483-1553).

AA Giulio; Italian-Israeli physicist (1909-1965).

AA First name from Hebrew.

AA Jean Baptiste; French playwright (1639-1699).

AA Rodolphe; French astronomer (1835-1911).

AA Bulgarian first name.

AA J. J., Jr.; Dutch astronomer (1903-1961).

AA Russian first name.

AA Ya; Latvian poet (1865-1925).

AA Town in Estonia.

AA Chandrasekhara V.; Indian physicist; Nobel laureate (1888-1970).

\footnotetext{
P: $\quad$ planetary system (see page xvi).

Sa: $\quad$ satellite (see page $x v i)$.

lat: latitude of feature center.

long: longitude of feature center.
}

diam: diameter or long dimension of feature. ct: continent of name origin (see page $284 \mathrm{ff}$.)

et: ethnicity of name origin (see page $284 \mathrm{ff}$.)

as: name approval status (see page xvii). ad: name approval date (year).

ref: reference source for name (see page $287 \mathrm{ff}$.).

ft: feature type (see page 290). 


\section{ALPHABETICAL LIST OF NAMES}

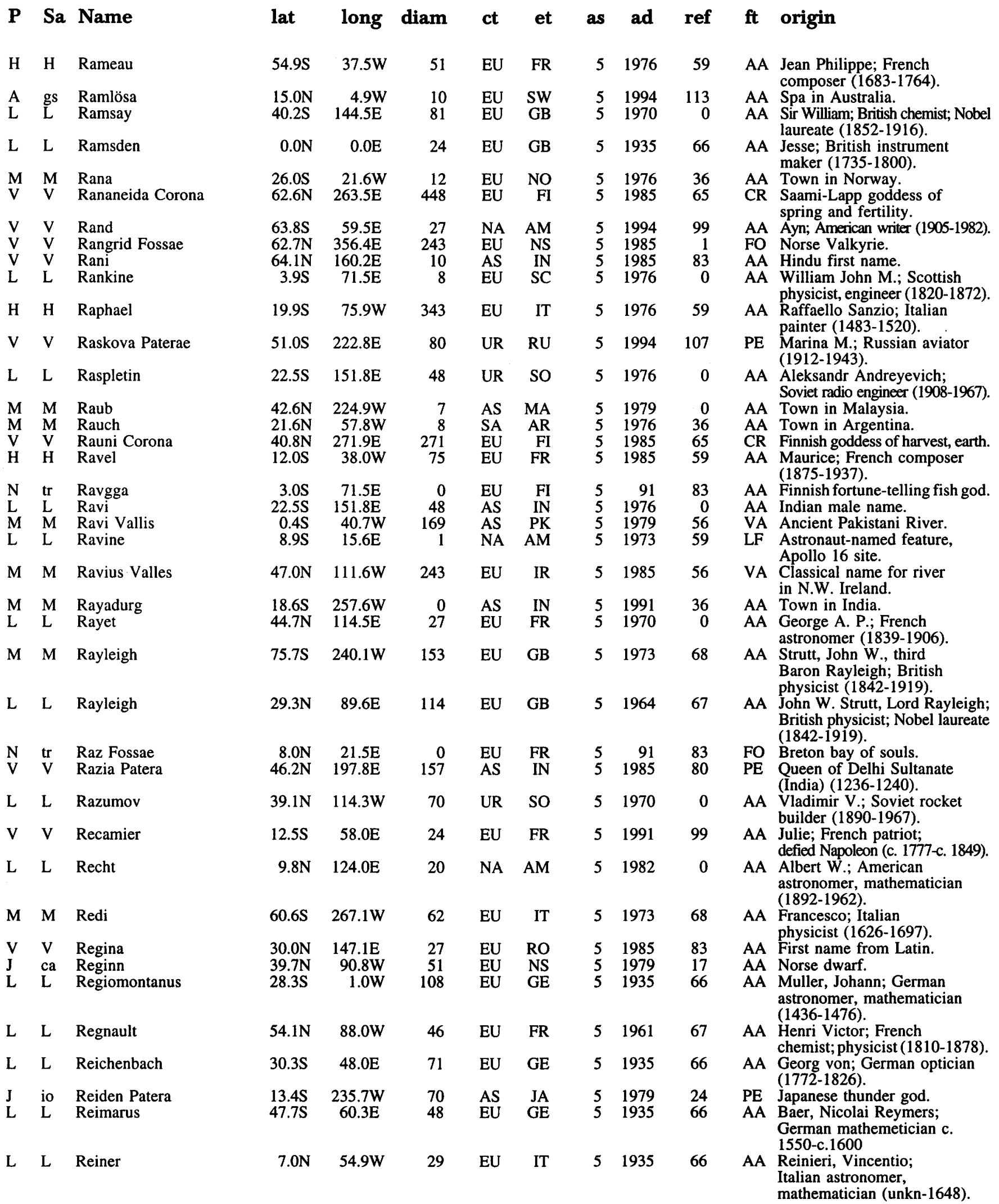

$\overline{\mathbf{P}} \quad$ planetary system (see page $\mathrm{xvi}$ )

Sa: $\quad$ satellite (see page $\mathrm{xvi}$ ).

lat: latitude of feature center.

long: longitude of feature center. diam: diameter or long dimension of feature. ct: continent of name origin (see page $284 \mathrm{ff}$.) et: ethnicity of name origin (see page $284 \mathrm{ff}$.) as: name approval status (see page xvii). ad: name approval date (year)

ref: reference source for name (see page $287 \mathrm{ff}$.).

$\mathrm{ft}$ : feature type (see page 290). 


\section{ALPHABETICAL LIST OF NAMES}

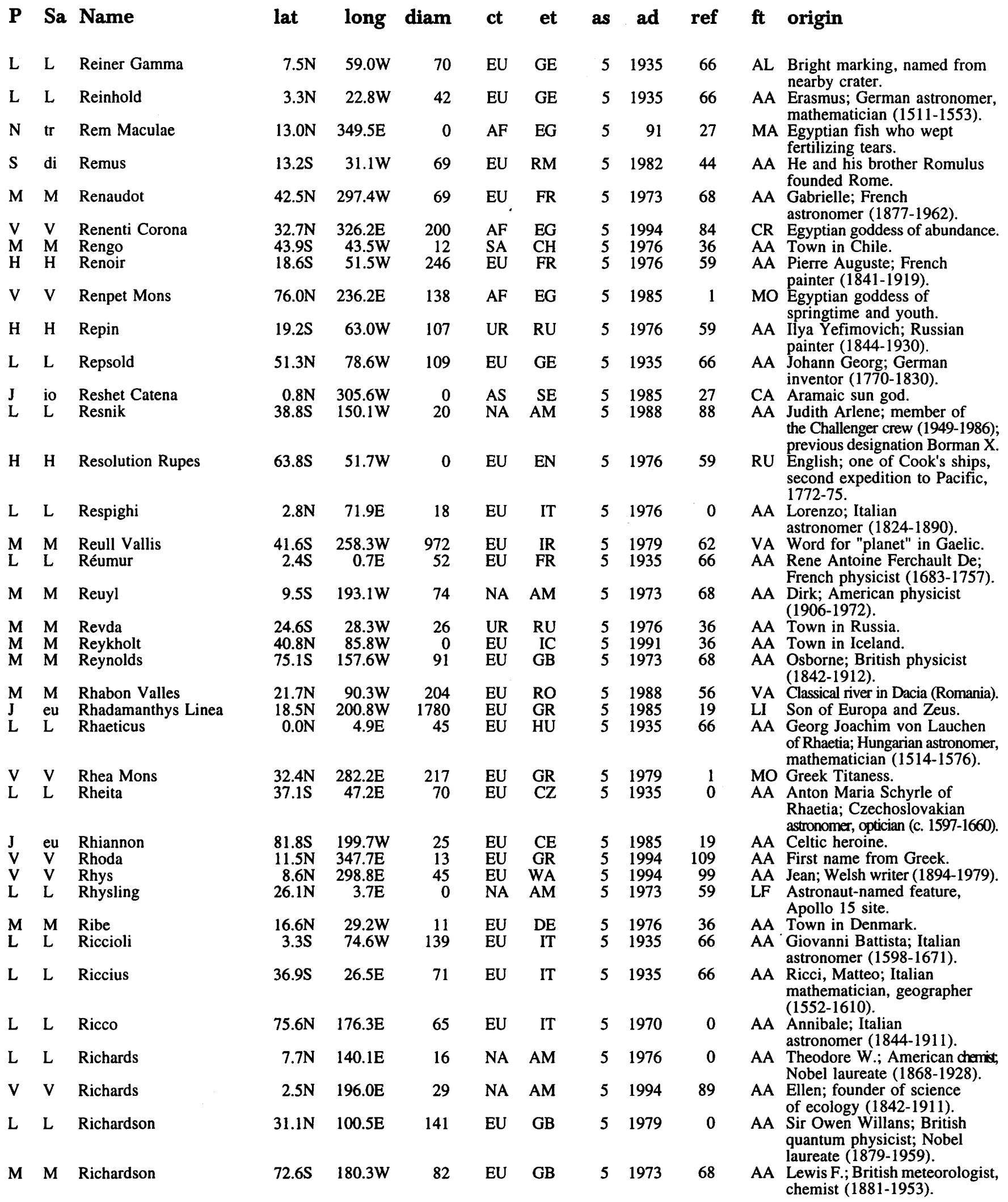

\footnotetext{
P: $\quad$ planetary system (see page xvi).

Sa: $\quad$ satellite (see page $x v i)$.

lat: latitude of feature center.

long: longitude of feature center.
}

diam: diameter or long dimension of feature.

ct: continent of name origin (see page $284 \mathrm{ff}$.)

et: ethnicity of name origin (see page $284 \mathrm{ff}$.)

as: name approval status (see page xvii) ad: name approval date (year).

ref: reference source for name (see page $287 \mathrm{ff}$.).

ft: feature type (see page 290). 


\section{ALPHABETICAL LIST OF NAMES}

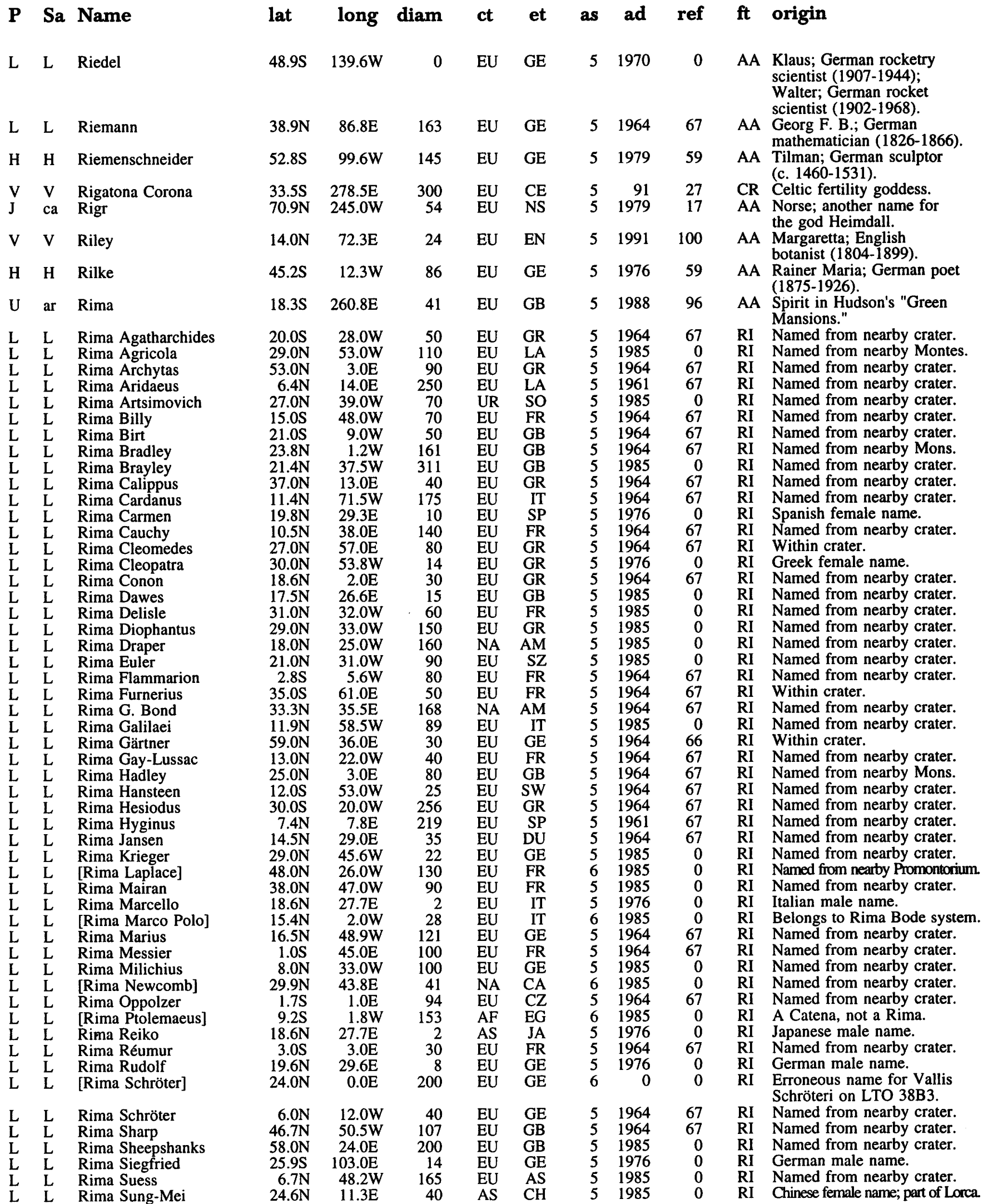

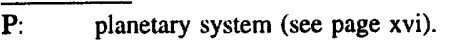

Sa: $\quad$ satellite (see page xvi).

lat: latitude of feature center.

long: longitude of feature center. diam: diameter or long dimension of feature. ct: continent of name origin (see page $284 \mathrm{ff}$.) et: ethnicity of name origin (see page $284 \mathrm{ff}$.) as: name approval status (see page xvii) ad: name approval date (year).

ref: reference source for name (see page $287 \mathrm{ff}$.). ft: feature type (see page 290). 
P Sa Name.

$\begin{array}{lll}\text { L } & \text { L } & \text { Rima T. Mayer } \\ \text { L } & \text { L } & \text { Rima Wan-Yu } \\ \text { L } & \text { L } & \text { [Rima Widmannstatten] } \\ \text { L } & \text { L } & \text { Rima Yangel' } \\ \text { L } & \text { L } & \text { Rima Zahia } \\ \text { M } & \text { M } & \text { Rimac } \\ \text { L } & \text { L } & \text { Rimae Alphonsus } \\ \text { L } & \text { L } & \text { Rimae Apollonius } \\ \text { L } & \text { L } & \text { Rimae Archimedes } \\ \text { L } & \text { L } & \text { Rimae Aristarchus } \\ \text { L } & \text { L } & \text { Rimae Arzachel } \\ \text { L } & \text { L } & \text { Rimae Atlas } \\ \text { L } & \text { L } & \text { Rimae Bode } \\ \text { L } & \text { L } & \text { Rimae Boscovich } \\ \text { L } & \text { L } & \text { Rimae Bürg } \\ \text { L } & \text { L } & \text { Rimae Chacornac }\end{array}$

L L Rimae Darwin

L L Rimae de Gasparis

L L Rimae Doppelmayer

L L Rimae Focas

L L Rimae Fresnel

L L Rimae Gassendi

L L Rimae Gerard

L L Rimae Goclenius

L L [Rimae Golitsyn]

L L Rimae Grimaldi

L L Rimae Gutenberg

L L Rimae Hase

L L Rimae Herigonius

L L Rimae Hevelius

L L Rimae Hippalus

L L Rimae Hypatia

L L Rimae Janssen

L L Rimae Kopff

L L Rimae Liebig

L L Rimae Littrow

L L Rimae MacLear

L L Rimae Maestlin

L L Rimae Maupertuis

L L Rimae Menelaus

L L Rimae Mersenius

L L Rimae Opelt

L L Rimae Palmieri

L L Rimae Parry

L L Rimae Petavius

L L Rimae Pettit

L L Rimae Pitatus

L L Rimae Plato

L L Rimae Plinius

L L Rimae Posidonius

L L Rimae Prinz

L L Rimae Ramsden

L L Rimae Repsold

L L Rimae Riccioli

L L Rimae Ritter

L L Rimae Römer

L L Rimae Secchi

L L Rimae Sirsalis

L L Rimae Sosigenes

L L [Rimae Stadius]

L L Rimae Sulpicius Gallus

L L Rimae Taruntius

L L Rimae Theaetetus

L L Rimae Triesnecker

L L Rimae Vasco da Gama

L L Rimae Zupus

H H Rimbaud

M M Rincon

A gs Rio Hondo lat long diam

$\begin{array}{rcr}13.0 \mathrm{~N} & 31.0 \mathrm{~W} & 50 \\ 20.0 \mathrm{~N} & 31.5 \mathrm{~W} & 12 \\ 6.1 \mathrm{~S} & 85.5 \mathrm{E} & 46 \\ 16.7 \mathrm{~N} & 4.6 \mathrm{E} & 30 \\ 25.0 \mathrm{~N} & 29.5 \mathrm{~W} & 16 \\ 45.2 \mathrm{~N} & 223.8 \mathrm{~W} & \\ 14.0 \mathrm{~S} & 2.0 \mathrm{~W} & 80 \\ 5.0 \mathrm{~N} & 53.0 \mathrm{E} & 23 \\ 26.6 \mathrm{~N} & 4.1 \mathrm{~W} & 169 \\ 26.9 \mathrm{~N} & 47.5 \mathrm{~W} & 12 \\ 18.0 \mathrm{~S} & 2.0 \mathrm{~W} & 5 \\ 47.5 \mathrm{~N} & 43.6 \mathrm{E} & 60 \\ 10.0 \mathrm{~N} & 4.0 \mathrm{~W} & 7 \\ 9.8 \mathrm{~N} & 11.1 \mathrm{E} & 40 \\ 44.5 \mathrm{~N} & 23.8 \mathrm{E} & 147 \\ 29.0 \mathrm{~N} & 32.0 \mathrm{E} & 120 \\ 37.0 \mathrm{~N} & 26.0 \mathrm{E} & 200 \\ 19.3 \mathrm{~S} & 69.5 \mathrm{~W} & 143 \\ 24.6 \mathrm{~S} & 51.1 \mathrm{~W} & 93 \\ 25.9 \mathrm{~S} & 45.1 \mathrm{~W} & 162 \\ 28.0 \mathrm{~S} & 98.0 \mathrm{~W} & 100 \\ 28.0 \mathrm{~N} & 4.0 \mathrm{E} & 90\end{array}$

$\begin{array}{lll}28.0 \mathrm{~N} & 4.0 \mathrm{E} & 90 \\ 18.0 \mathrm{~S} & 40.0 \mathrm{~W} & 70\end{array}$

$46.0 \mathrm{~N} \quad 84.0 \mathrm{~W} \quad 100$

$\begin{array}{rrr}8.0 \mathrm{~S} & 43.0 \mathrm{E} & 240 \\ 25.1 \mathrm{~N} & 105.0 \mathrm{~W} & 36\end{array}$

$\begin{array}{rrr}9.0 \mathrm{~N} & 64.0 \mathrm{~W} & 230\end{array}$

$\begin{array}{lll}5.0 \mathrm{~S} & 38.0 \mathrm{E} & 330\end{array}$

$29.4 \mathrm{~S} \quad 62.5 \mathrm{E}$

$13.0 \mathrm{~S} \quad 37.0 \mathrm{~W}$

$\begin{array}{lll}1.0 \mathrm{~N} & 68.0 \mathrm{~W} \quad 182\end{array}$

$\begin{array}{rll}25.5 \mathrm{~S} & 29.2 \mathrm{~W} & 191 \\ 0.4 \mathrm{~S} & 22.4 \mathrm{E} & 206\end{array}$

$\begin{array}{lll}45.6 \mathrm{~S} & 40.0 \mathrm{E} \quad 114\end{array}$

$\begin{array}{llr}17.4 \mathrm{~S} & 89.6 \mathrm{~W} & 41 \\ 20.0 \mathrm{~S} & 45.0 \mathrm{~W} & 140\end{array}$

$22.1 \mathrm{~N} \quad 29.9 \mathrm{E} \quad 115$

$\begin{array}{rrr}13.0 \mathrm{~N} & 20.0 \mathrm{E} & 110 \\ 2.0 \mathrm{~N} & 40.0 \mathrm{~W} & 80\end{array}$

$52.0 \mathrm{~N} \quad 23.0 \mathrm{~W} \quad 60$

$\begin{array}{lll}17.2 \mathrm{~N} & 17.9 \mathrm{E} & 131\end{array}$

$\begin{array}{lll}21.5 \mathrm{~S} & 49.2 \mathrm{~W} & 84 \\ 13.0 \mathrm{~S} & 18.0 \mathrm{~W} & 70\end{array}$

$\begin{array}{rrr}13.0 \mathrm{~S} & 18.0 \mathrm{~W} & 70 \\ 28.0 \mathrm{~S} & 47.0 \mathrm{~W} & 150\end{array}$

$6.1 \mathrm{~S} \quad 16.8 \mathrm{~W}$

$25.9 \mathrm{~S} \quad 58.9 \mathrm{E}$

23.0S $92.0 \mathrm{~W}$

$\begin{array}{ll}28.5 \mathrm{~S} & 13.8 \mathrm{~W} \\ 52.9 \mathrm{~N} & 3.2 \mathrm{~W}\end{array}$

$17.9 \mathrm{~N}$

$32.0 \mathrm{~N}$

$33.9 \mathrm{~S}$

$50.6 \mathrm{~N}$

$2.0 \mathrm{~N}$

$27.0 \mathrm{~N}$

$27.0 \mathrm{~N}$

$15.7 \mathrm{~S}$

$8.6 \mathrm{~N}$

$10.5 \mathrm{~N}$

$5.5 \mathrm{~N}$

$33.0 \mathrm{~N}$

$4.3 \mathrm{~N}$

$10.0 \mathrm{~N}$

$15.0 \mathrm{~S}$

$62.0 \mathrm{~S}$

$8.1 \mathrm{~S}$

$23.6 \mathrm{E}$

28.7E

$43.0 \mathrm{~W}$

$31.4 \mathrm{~W}$

$81.7 \mathrm{~W}$

$74.0 \mathrm{~W}$

$18.0 \mathrm{E}$

$35.0 \mathrm{E}$

$44.0 \mathrm{E}$

$61.7 \mathrm{~W}$

$18.7 \mathrm{E}$

$13.7 \mathrm{~W}$

$10.0 \mathrm{E}$

$46.5 \mathrm{E}$

$6.0 \mathrm{E}$

$4.6 \mathrm{E}$

$82.0 \mathrm{~W}$

$53.0 \mathrm{~W}$

$148.0 \mathrm{~W}$

$31.7 \mathrm{~N}$

80
450

87

24

70
80

108

166

400

100

35

426
190

69
90

25
50

215

60

120
85

85
13

ct et as ad ref $\mathrm{ft}$ origin

$\begin{array}{lllllll}\text { EU } & \text { GE } & 5 & 1985 & 0 & \text { RI } & \begin{array}{l}\text { Named from nearby crater. } \\ \text { AS }\end{array} \\ \text { CH } & 5 & 1976 & 0 & \text { RI } & \text { Chinese female name. } \\ \text { EU } & \text { GE } & 6 & 1985 & 0 & \text { RI } & \text { Named from nearby crater. } \\ \text { UR } & \text { SO } & 5 & 1978 & 0 & \text { RI } & \text { Named from nearby crater. } \\ \text { AS } & \text { AR } & 5 & 1976 & 0 & \text { RI } & \text { Arabic female name. }\end{array}$

36

67

67

67

67

67

67

67

67

67

66
67

67

EU GE

EU FR

$5 \quad 1964$

51985

$\begin{array}{ll}5 & 1964 \\ 5 & 1964\end{array}$

51985

EU GB

EU GE

EU IT

$\begin{array}{ll}5 & 1964 \\ 6 & 1985\end{array}$

$\begin{array}{ll}5 & 1964\end{array}$

$\begin{array}{ll}5 & 1964\end{array}$

51985

EU GE

EU FR

EU GR

AF EG

EU GE

EU GE

EU CZ

EU IR

EU FR

EU GR

EU FR

EU IT

EU GB

NA AM

EU IT

EU RM

EU GR

EU GB

EU GE

EU GE

EU DE

EU IT

EU BE

EU RM

EU RM

$\begin{array}{ll}\text { EU } & \text { GR } \\ \text { EU } & \text { AS }\end{array}$

EU PG

EU IT

SA DA

$\begin{array}{ll}5 & 1964 \\ 5 & 1964\end{array}$

$\begin{array}{ll}5 & 1964\end{array}$

51964

$\begin{array}{ll}5 & 1964\end{array}$

51985

51985

$5 \quad 1964$

$\begin{array}{ll}5 & 1964 \\ 5 & 1985\end{array}$

$5 \quad 1985$

$5 \quad 1985$

$\begin{array}{ll}5 & 1978\end{array}$

51938

$5 \quad 1964$

51964

$5 \quad 1964$

51964

51985

$\begin{array}{ll}5 & 1964\end{array}$

$\begin{array}{ll}5 & 1964\end{array}$

$5 \quad 1964$

51964

$\begin{array}{ll}5 & 1964\end{array}$

$\begin{array}{ll}5 & 1964\end{array}$

51985

51985

$5 \quad 1964$

51964

$5 \quad 1985$

51964

$5 \quad 1964$

61985

$\begin{array}{ll}5 & 1964\end{array}$

51985

51964

$\begin{array}{ll}5 & 1964\end{array}$

51985

51976

51985

51976

AA Town in Peru.

RI Within crater of same name.

RI Named from nearby crater.

RI Named from nearby crater.

RI Named from nearby crater.

Within crater.

RI Within crater.

RI Named from nearby crater.

RI Within crater.

RI Named from nearby crater.

RI Named from nearby crater.

RI Named from nearby crater.

RI Named from nearby crater.

RI Named from nearby crater.

RI Named from nearby crater.

RI Named from nearby crater.

RI Named from nearby promontorium.

RI Within crater.

RI Named from nearby crater. 


\section{ALPHABETICAL LIST OF NAMES}

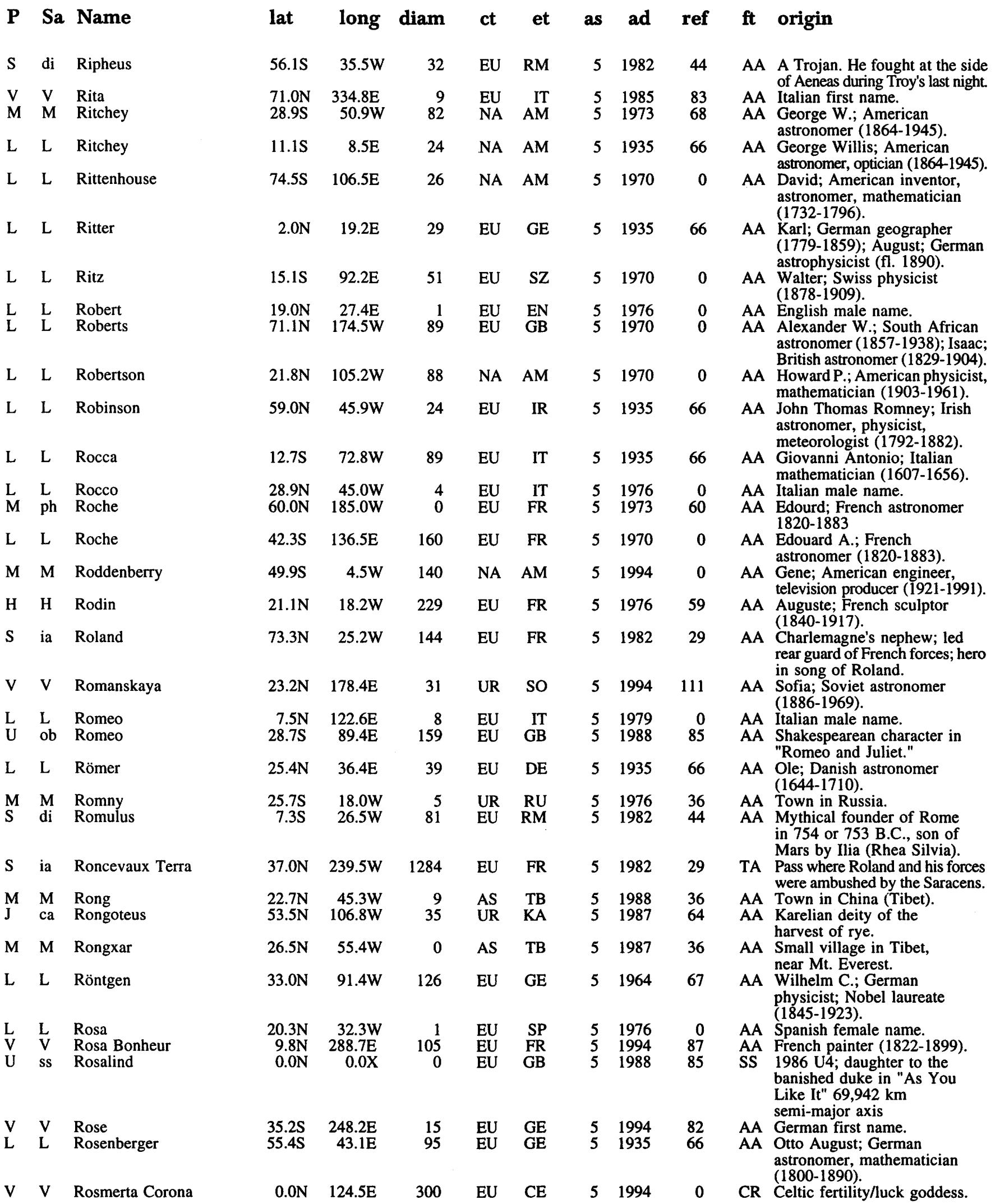

\footnotetext{
P: $\quad$ planetary system (see page xvi).

Sa: satellite (see page $x v i$ ).

lat: latitude of feature center.

long: longitude of feature center.
}

diam: diameter or long dimension of feature. ct: continent of name origin (see page $284 \mathrm{ff}$.) et: ethnicity of name origin (see page $284 \mathrm{ff}$.) as: name approval status (see page xvii). ad: name approval date (year).

ref: reference source for name (see page $287 \mathrm{ff}$.).

ft: feature type (see page 290). 


\section{ALPHABETICAL LIST OF NAMES}

\section{P Sa Name}

M M Ross

L L Ross

M M Rossby

L L Rosse

L L Rosseland

V V Rossetti

L L Rost

J ca Rota

L L Rothmann

A gs Rotorua

$\mathrm{U}$ ti Rousillon Rupes

V V Rowena

L L Rowland

V V Roxanna

L L Rozhdestvenskiy

$\mathrm{N}$ tr Ruach Planitia

$\mathrm{H} H$ Rubens

M M Rubicon Valles

H H Rublev

M M Ruby

H H Rūdaki

M M Rudaux

H H Rude

V V Rudneva

L L Rumford

H H Rumi

M M Runa Vallis

L L Runge

L L Rupes Altai

L L Rupes Boris

L L Rupes Cauchy

L L Rupes Kelvin

L L Rupes Liebig

L L Rupes Mercator

L L Rupes Recta

M M Rupes Tenuis

$\begin{array}{lll}\text { L } & \text { L } & \text { Rupes Toscanelli } \\ \text { V } & \text { V } & \text { Rusalka Planitia }\end{array}$

V V Ruslanova

L L Russell lat long diam

$\begin{array}{lccccccc}57.6 \mathrm{~S} & 107.6 \mathrm{~W} & 88 & \text { NA } & \text { AM } & 5 & 1973 & 68 \\ 11.7 \mathrm{~N} & 21.7 \mathrm{E} & 24 & \text { EU } & \text { GB } & 5 & 1935 & 66 \\ & & & & & & & \\ 47.8 \mathrm{~S} & 192.2 \mathrm{~W} & 82 & \text { NA } & \text { AM } & 5 & 1973 & 68 \\ 17.9 \mathrm{~S} & 35.0 \mathrm{E} & 11 & \text { EU } & \text { IR } & 5 & 1935 & 66\end{array}$

$\begin{array}{lllllll}41.0 \mathrm{~S} & 131.0 \mathrm{E} & 75 & \mathrm{EU} & \mathrm{NO} & 5 & 1994\end{array}$

$\begin{array}{llllllll}57.0 \mathrm{~N} & 6.4 \mathrm{E} & 22 & \mathrm{EU} & \mathrm{GB} & 5 & 1985 & 46\end{array}$

$\begin{array}{llllllll}56.4 \mathrm{~S} & 33.7 \mathrm{~W} & 48 & \mathrm{EU} & \mathrm{GE} & 5 & 1935 & 66\end{array}$

$\begin{array}{llllllll}27.8 \mathrm{~N} & 109.8 \mathrm{~W} & 55 & \text { EU } & \text { LP } & 5 & 1987 & 64\end{array}$

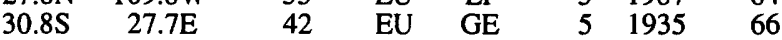

$\begin{array}{llrllllr}18.8 \mathrm{~N} & 30.7 \mathrm{~W} & 6 & \mathrm{OC} & \mathrm{NZ} & 5 & 1994 & 113 \\ 14.7 \mathrm{~S} & 26.5 \mathrm{E} & 402 & \text { EU } & \text { FR } & 5 & 1988 & 85\end{array}$

$\begin{array}{rrrrrrrr}10.4 \mathrm{~N} & 171.3 \mathrm{E} & 18 & \mathrm{EU} & \mathrm{CE} & 5 & 1994 & 82\end{array}$

$57.4 \mathrm{~N} \quad 162.5 \mathrm{~W} \quad 171 \quad$ NA $\mathrm{AM}$

$26.5 \mathrm{~N} \quad 334.6 \mathrm{E}$

$\begin{array}{ll}85.2 \mathrm{~N} & 155.4 \mathrm{~W}\end{array}$

51970

$28.0 \mathrm{~N} \quad 24.0 \mathrm{E}$

59.8

$44.7 \mathrm{~N} \quad 116.8 \mathrm{~W}$

$15.1 \mathrm{~S} \quad 156.8 \mathrm{~W}$

$25.6 \mathrm{~S} \quad 16.9 \mathrm{~W}$

$\begin{array}{cr}4.0 \mathrm{~S} & 51.1 \mathrm{~W} \\ 38.6 \mathrm{~N} & 309.0 \mathrm{~W}\end{array}$

$32.8 \mathrm{~S} \quad 79.6 \mathrm{~W}$

$78.4 \mathrm{~N} \quad 174.0 \mathrm{E}$

$28.8 \mathrm{~S} \quad 169.8 \mathrm{~W}$

24.1

$104.7 \mathrm{~W}$

$28.6 \mathrm{~S}$

$2.5 \mathrm{~S}$

$36.7 \mathrm{~W}$

86.7E

$24.3 S$

$30.5 \mathrm{~N}$

$9.0 \mathrm{~N}$

$27.3 \mathrm{~S}$

$24.4 \mathrm{~S}$

$31.0 \mathrm{~S}$

$22.1 \mathrm{~S}$

$22.6 \mathrm{E}$

$33.5 \mathrm{~W}$

$37.0 \mathrm{E}$

$33.1 \mathrm{~W}$

$48.5 \mathrm{~W}$

$22.3 \mathrm{~W}$

$7.8 \mathrm{~W}$

$81.9 \mathrm{~N}$

$27.4 \mathrm{~N}$

$9.8 \mathrm{~N}$

$84.0 \mathrm{~N}$

$26.5 \mathrm{~N}$

$65.2 \mathrm{~W}$

$47.5 \mathrm{~W}$

$170.1 \mathrm{E}$

16.5E

$75.4 \mathrm{~W}$

177 UR SO

0 EU FR

175

EU

FL

228

EU IT

UN UN

25 US SC

120
52

AS $\quad$ PE

$5 \quad 1991$

$\begin{array}{ll}5 & 1970\end{array}$

$5 \quad 91$

51979

$5 \quad 1985$

51976

51976

$\begin{array}{ll}5 & 1976\end{array}$

$5 \quad 1973$

51985

EU FR

51985

30 UR RU

61

EU GB

51970

75

AS PE

51985

59
10 AS PE

$$
\begin{array}{lll}
\text { M } & \text { M } & \text { Russell } \\
\text { V } & \text { V } & \text { Ruth }
\end{array}
$$

$\begin{array}{rrrrrrrr}55.0 \mathrm{~S} & 347.4 \mathrm{~W} & 138 & \text { NA } & \text { AM } & 5 & 1973 & 68 \\ 43.2 \mathrm{~N} & 20.0 \mathrm{E} & 18 & \text { AS } & \text { HE } & 5 & 1985 & 83\end{array}$

\section{ft origin}

AA Frank E.; American astronomer (1874-1966).

AA James Clark; British explorer (1800-1862); Frank E.; American astronomer, optician (1874-1966).

AA Carl G.; Swedish-American meteorologist (1898-1957).

AA William Parsons, Earl of

Rosse; Irish astronomer (1800-1867).

AA Svein; Norwegian astrophysicist (1894-1985).

AA Christina; English poet (1830-1894).

AA Leonhard; German astronomer (1688-1727).

AA Deity of the underground world.

AA Christopher; German astronomer (unkn-1600).

AA Spa in New Zealand.

RU Location in "All's Well That Ends Well."

AA Celtic first name.

AA Henry A.; American physicist (1848-1901).

AA First name from Persian.

AA Dmitriy S.; Soviet physicist (1876-1940)

PL French isle of winds.

AA Peter Paul; Flemish painter (1577-1640)

VA Ancient river in Italy.

AA Andrey; Russian painter (c. 1370-1430).

AA Town in South Carolina, USA.

AA Persian poet (c. 859-940/941).

AA Lucien; French astronomer (1874-1947).

AA Francois; French sculptor (1784-1855).

AA Varvara; Russian physician (1844-1899).

AA Benjamin Thompson, Count Rumford; British physicist (1753-1814).

AA Jalal Ad-Din, also Mawlana; Persian poet and Sufi mystic (1207-1273).

VA Name proposed by Soviets.

AA Carl David Tolme; German mathematician (1856-1927).

RU Named from terrestrial Altai Mountains.

RU Named from nearby crater.

RU Named from nearby crater.

RU Named from nearby promontorium

RU Named from nearby crater.

RU Named from nearby crater.

RU Latin for "straight cliff" (The straight wall).

RU Classical albedo feature name.

RU Named from nearby crater.

PL Russian mermaid.

AA Lidiya; Soviet singer (1900-1973).

AA Henry Norris; American astronomer (1877-1957); John; British selenographer (1745-1806)

AA Henry N.; American astronomer (1877-1957).

AA Hebrew first name.

\footnotetext{
P: $\quad$ planetary system (see page xvi)

Sa: $\quad$ satellite (see page $x v i$ ).

lat: latitude of feature center.

long: longitude of feature center.
}

diam: diameter or long dimension of feature.

ct: continent of name origin (see page $284 \mathrm{ff}$.)

et: ethnicity of name origin (see page $284 \mathrm{ff}$.)

as: name approval status (see page xvii). ad: name approval date (year).

ref: reference source for name (see page $287 \mathrm{ff}$.)

ft: feature type (see page 290). 


\section{ALPHABETICAL LIST OF NAMES}

\section{P Sa Name}

L L Ruth

M M Rutherford

L L Rutherford

L L Rutherfurd

J ga Ruti

J io Ruwa Patera

M M Ruza

L L Rydberg

L L Rynin

M M Rynok

M M Rypin

$\mathrm{N}$ tr Ryugu Planitia

L L Sabatier

V V Sabin

L L Sabine

$S$ di Sabinus

V V Sabira

M M Sabis Vallis

M M Sabo

M M Sabrina Vallis

V V Sacajawea Patera

V V Sachs Patera

M M Sacra Dorsa

M M Sacra Fossae

M M Sacra Mensa

M M Sacra Sulci

L L Sacrobosco la

$\begin{array}{rrrrrrrr}28.7 \mathrm{~N} & 45.1 \mathrm{~W} & 3 & \text { AS } & \text { HE } & 5 & 1976 & 0 \\ 19.2 \mathrm{~N} & 10.6 \mathrm{~W} & 116 & \text { EU } & \text { GB } & 5 & 1973 & 68 \\ 10.7 \mathrm{~N} & 137.0 \mathrm{E} & 13 & \text { EU } & \text { GB } & 5 & 1976 & 0 \\ 60.9 \mathrm{~S} & 12.1 \mathrm{~W} & 48 & \text { NA } & \text { AM } & 5 & 1935 & 66 \\ & & & & & & & \\ 11.9 \mathrm{~N} & 310.7 \mathrm{~W} & 35 & \text { AS } & \text { PO } & 5 & 1979 & 1 \\ 0.4 \mathrm{~N} & 3.0 \mathrm{~W} & 50 & \text { AF } & \text { TA } & 5 & 1979 & 27 \\ 34.3 \mathrm{~S} & 52.8 \mathrm{~W} & 20 & \text { UR } & \text { RU } & 5 & 1979 & 36 \\ 46.5 \mathrm{~S} & 96.3 \mathrm{~W} & 49 & \text { EU } & \text { SW } & 5 & 1970 & 0 \\ & & & & & & & \\ 47.0 \mathrm{~N} & 103.5 \mathrm{~W} & 75 & \text { UR } & \text { SO } & 5 & 1970 & 0 \\ 44.3 \mathrm{~N} & 238.2 \mathrm{~W} & 9 & \text { UR } & \text { RU } & 5 & 1979 & 36 \\ 1.3 \mathrm{~S} & 41.0 \mathrm{~W} & 18 & \text { EU } & \text { PO } & 5 & 1976 & 36 \\ 5.0 \mathrm{~S} & 27.0 \mathrm{E} & 0 & \text { AS } & \text { JA } & 5 & 91 & 27 \\ 13.2 \mathrm{~N} & 79.0 \mathrm{E} & 10 & \text { EU } & \text { FR } & 5 & 1979 & 0 \\ 38.5 S & 274.6 \mathrm{E} & 36 & \text { NA } & \text { AM } & 5 & 1994 & 99 \\ & & & & & & & \\ 1.4 \mathrm{~N} & 20.1 \mathrm{E} & 30 & \text { EU } & \text { IR } & 5 & 1935 & 66 \\ 47.8 S & 175.6 \mathrm{~W} & 79 & \text { EU } & \text { RM } & 5 & 1982 & 44 \\ 5.8 S & 239.9 \mathrm{E} & 14 & \text { UR } & \text { RU } & 5 & 1994 & 112 \\ 5.8 S & 152.7 \mathrm{~W} & 286 & \text { EU } & \text { FR } & 5 & 1985 & 56 \\ 25.4 \mathrm{~N} & 48.9 \mathrm{~W} & 0 & \text { UR } & \text { RU } & 5 & 1988 & 36 \\ 11.1 \mathrm{~N} & 48.9 \mathrm{~W} & 294 & \text { EU } & \text { GB } & 5 & 1985 & 56 \\ 64.3 \mathrm{~N} & 335.4 \mathrm{E} & 233 & \text { NA } & \text { DA } & 5 & 1982 & 99\end{array}$

49.1N 334.2E

65

EU SW

$5 \quad 91$

$18.6 \mathrm{~N}$

67.0W

535

EU

LA

51985

$16.5 \mathrm{~N}$

$25.0 \mathrm{~N}$

$72.0 \mathrm{~W}$

$69.2 \mathrm{~W}$

$71.5 \mathrm{~W}$

23.7S

16.7E

461

602

EU LA

51976

$\begin{array}{ll}5 & 1976 \\ 5 & 1988\end{array}$

$\begin{array}{ll}5 & 1985\end{array}$

51935
EU LA

ref

\section{ft origin}

AA Hebrew female name.

AA Ernest; British physicist (1871-1937).

AA Sir Emest; British physicist; Nobel laureate (1871-1937)

AA Lewis Morris; American astronomer (1816-1892).

AA Phoenician; Byblos god.

PE African sun god associated Mt. Kilimanjaro.

AA Town in Russia.

AA Johannes R.; Swedish physicist (1854-1919).

AA Nikolaj A.; Soviet rocketry scientist (1877-1942)

AA Town in Russia.

AA Town in Poland.

PL Japanese undersea dragon palace.

AA Paul; French chemist; Nobel laureate (1854-1941)

AA Florence; American medical researcher (1871-1953)

AA Sir Edward; Irish physicist, astronomer (1788-1883).

AA Fabled ancestor of the Sabines

AA Tatar first name.

VA Classical name for present Sambre River in France and Belgium.

AA Town in Russia.

VA Classical name for present Severn River, England.

PE Blackfoot Indian woman who guided Lewis \& Clark expedition to the Pacific Northwest (1786-1812).

PE Nelly; German-born Swedish playwright, poet (1891-1970).

DO From albedo feature at $20 \mathrm{~N}, 67 \mathrm{~W}$.

FO Classical albedo feature name.

MN Albedo feature name.

SU From albedo feature at $20 \mathrm{~N}, 67 \mathrm{~W}$.

AA John of Holywood, Johannes Sacrobuschus; British astronomer, mathematician (c. 1200-1256)

$\begin{array}{rrrrrrrr}78.6 \mathrm{~S} & 56.0 \mathrm{~W} & 68 & \text { AS } & \text { PE } & 5 & 1976 & 59 \\ 4.3 \mathrm{~N} & 102.4 \mathrm{E} & 75 & \text { EU } & \text { GE } & 5 & 1970 & 0 \\ 10.6 \mathrm{~N} & 176.9 \mathrm{E} & 27 & \text { EU } & \text { CZ } & 5 & 1970 & 0 \\ 0.0 \mathrm{~N} & 326.0 \mathrm{~W} & 0 & \text { EU } & \text { SC } & 5 & 1979 & 17 \\ & & & & & & & \\ 76.1 \mathrm{~N} & 340.6 \mathrm{E} & 450 & \text { EU } & \text { NS } & 5 & 1994 & 111 \\ 1.6 \mathrm{~S} & 102.7 \mathrm{E} & 99 & \text { AS } & \text { IN } & 5 & 1970 & 0 \\ & & & & & & & \\ 72.9 \mathrm{~N} & 176.3 \mathrm{~W} & 88 & \text { AS } & \text { JA } & 5 & 1979 & 59 \\ 37.9 \mathrm{~N} & 14.2 \mathrm{~W} & 137 & \text { AF } & \text { EG } & 5 & 1988 & 1 \\ & & & & & & & \\ 47.6 \mathrm{~S} & 51.0 \mathrm{~W} & 28 & \text { AF } & \text { GH } & 5 & 1976 & 36 \\ 6.5 \mathrm{~S} & 0.0 \mathrm{~W} & 4 & \text { AS } & \text { AR } & 5 & 1982 & 52 \\ 5.0 \mathrm{~S} & 97.7 \mathrm{E} & 14 & \text { UR } & \text { RU } & 5 & 1994 & 112 \\ 58.0 \mathrm{~N} & 25.2 \mathrm{E} & 447 & \text { UR } & \text { ET } & 5 & 1991 & 64 \\ 62.3 \mathrm{~N} & 355.1 \mathrm{~W} & 16 & \text { AS } & \text { AR } & 5 & 1982 & 52\end{array}$

$45.5 \mathrm{~N} \quad 281.4 \mathrm{E}$
16 AS SE
$5 \quad 1994$
AA Persian poet (c. 1213-1291/1292).

AA Eugen; German rocketry scientist (1905-1964).

AA Vojtech; Czechoslovakian astronomer (1829-1902).

AA Scandinavian goddess, wife of Odin; Rand control point crater

VA Norse goddess in the form of a waterfall.

AA Meghnad; Indian astrophysicist (1893-1956).

AA Ihara; Japanese novelist and poet (1642-1693)

FA Capital of Egypt in mid-7th century B.C.

AA Town in Ghana.

AA Brother of Julnar.

AA Mari first name.

DO Estonian sky maiden.

AA Shayk who guides Musa and Talib to the mountains in "The City of Brass".

AA First name from Aramaic.
P: planetary system (see page xvi).

Sa: $\quad$ satellite (see page $\mathrm{xvi}$ ).

lat: latitude of feature center.

long: longitude of feature center diam: diameter or long dimension of feature.

ct: continent of name origin (see page $284 \mathrm{ff}$.)

et: ethnicity of name origin (see page $284 \mathrm{ff}$.)

as: name approval status (see page xvii). ad: name approval date (year).

ref: reference source for name (see page $287 \mathrm{ff}$.)

ft: feature type (see page 290). 


\section{ALPHABETICAL LIST OF NAMES}

\section{P Sa Name}

M M Samara Valles

S en Samarkand Sulci

V V Samintang

L L Samir

V V Samundra Vallis

M M San Juan

V V Sand Patera

V V Sandel

M M Sandila

L L Sanford

M M Sangar

V V Sanija

M M Santa Cruz

M M Santa Fe

M M Santaca

L L Santbech

L L Santos-Dumont

J ga Sapas

V V Sapas Mons

L L $\quad$ [Sappho]

V V Sappho Patera

L L Sarabhai

V V Sarah

J ca Sarakka

$S$ en Sarandib Planitia
L L Sampson

V V Sanger

H H Santa María Rupes

lat

24.2S

$30.5 \mathrm{~N}$

$39.0 \mathrm{~S}$

$28.5 \mathrm{~N}$

$29.7 \mathrm{~N}$

24.15

347.1E

$41.7 \mathrm{~N} \quad 15.5 \mathrm{E}$

45.7S 211.6E

$25.9 \mathrm{~S}$

$32.6 \mathrm{~N}$

$30.2 \mathrm{~W}$

$138.9 \mathrm{~W}$

27.9S 24.1W

$33.8 \mathrm{~N} \quad 288.5 \mathrm{E}$

$33.1 \mathrm{~N} \quad 250.0 \mathrm{E}$

$21.5 \mathrm{~N}$

$19.5 \mathrm{~N}$

$5.5 \mathrm{~N}$

$47.3 \mathrm{~W}$

$48.0 \mathrm{~W}$

$19.7 \mathrm{~W}$

41.6S 272.5W

$20.9 \mathrm{~S}$

44.0E

$27.7 \mathrm{~N}$

$4.8 \mathrm{E}$

$56.5 \mathrm{~N}$

$8.5 \mathrm{~N}$

$25.0 \mathrm{~S}$

$14.1 \mathrm{~N}$

$24.7 \mathrm{~N}$

42.4S

$3.7 \mathrm{~S}$

$4.4 \mathrm{~N}$

$37.9 \mathrm{~W}$

$188.3 \mathrm{E}$

133.2E

$16.5 \mathrm{E}$

21.0E

$1.7 \mathrm{E}$

$53.7 \mathrm{~W}$

$298.0 \mathrm{~W}$

$50.0 \mathrm{~N} \quad 270.0 \mathrm{~W}$

$29.8 \mathrm{~S} \quad 187.7 \mathrm{~W}$

$77.5 \mathrm{~S}$

$44.7 \mathrm{~S}$

$54.5 \mathrm{~W}$

$52.3 \mathrm{~S}$

$42.2 \mathrm{~S}$

$63.4 \mathrm{~S}$

$54.0 \mathrm{~W}$

$14.6 \mathrm{E}$

$89.4 \mathrm{~W}$

67.1E

$49.3 \mathrm{~N} \quad 121.1 \mathrm{~W}$

28.6

39.1

337.2E

L L $\quad$\begin{tabular}{ll} 
Sasserides \\
\hline
\end{tabular}

J ga Sati

V V Sati Vallis

M M Satka

M M Sauk

V V Saule Dorsa

L L Saunder

$\begin{array}{lll}\text { L } & \text { L } & \text { Saussure } \\ \text { M } & \text { M } & \text { Savannah }\end{array}$
$30.5 \mathrm{~N}$

$3.2 \mathrm{~N} \quad 334.4 \mathrm{E}$

$43.0 \mathrm{~S}$

$45.0 \mathrm{~S}$

$58.0 \mathrm{~S}$

$4.2 \mathrm{~S}$

$32.3 \mathrm{~W}$

206.0E

$8.8 \mathrm{E}$

43.4S

$3.8 \mathrm{~W}$

$22.3 \mathrm{~N}$

$47.8 \mathrm{~W}$
1375 EU WI

0
145

US NY

ct et as ad

EU FR

$5 \quad 1976$

51982

AS AR

AS $\mathrm{KR}$

AS AB

$\begin{array}{ll}5 & 1991\end{array}$

$\begin{array}{ll}5 & 1979 \\ 5 & 1976\end{array}$

AS IN

$\begin{array}{rll}1 & \text { SA } & \text { PR } \\ 181 & \text { EU } & \text { FR }\end{array}$

1994

$5 \quad 1979$

20

EU NO

$5 \quad 1994$

8
55

AS IN

NA AM

51976

51970

28
84

UR RU

NA AM

51976

$5 \quad 1994$

18

UR RU

AF CI

EU SP

1994

$\begin{array}{ll}5 & 1979\end{array}$

$5 \quad 1976$

51976

AF $\quad \mathrm{MZ}$

64

EU

$\begin{array}{llll}\text { SA } & \text { BR } & 5 & 1976\end{array}$

65

AS $\mathrm{AB}$

51979

217

28
92

AS PO

EU GR

$5 \quad 1982$

51979

7 AS IN

$5 \quad 1973$

19
56

200

AS

EU

HE
FI

$5 \quad 1994$

$\begin{array}{ll}5 & 1979\end{array}$

1982

SA AR

$\begin{array}{ll}5 & 1994 \\ 5 & 1979\end{array}$

0

EU WA

20

EU

AS BY

AS BY

$5 \quad 1991$

$5 \quad 1976$

51994

$\begin{array}{ll}5 & 1979\end{array}$

1994

69

EU B

51970

$\begin{array}{lllll}37 & E U & D U & 5 & 1991\end{array}$

90 EU DE

$5 \quad 1935$

$5 \quad 1988$

98 AF EG

$5 \quad 1994$

$\begin{array}{rll}225 & \text { AF } & \text { EG } \\ 14 & \text { UR } & \text { RU } \\ 2 & \text { US } & \text { WI }\end{array}$

44 EU GB

1976

1994

1935

$\begin{array}{rrrrrr}54 & \text { EU } & \text { SZ } & 5 & 1935 & 66 \\ 1 & \text { US } & \text { GA } & 5 & 1979 & 36\end{array}$

ft origin

56 VA Ancient name for modern Somme River, France.

52 SU Country ruled over by Zaman, brother of Shahryar.

102 AA 16th century Korean poet.

0 AA Arabic male name.

0 AA Ralph Allen; British astronomer, mathematician (1866-1939).

VA Indian river goddess.

AA Puerto Rican port.

PE George (Aurore Dupin); French novelist (1804-1876).

AA Cora; Norwegian author (1880-1974).

AA Town in India.

AA Roscoe F.; American astronomer (1883-1958).

AA Town in Russia.

AA Margaret; American medical researcher (1883-1966).

AA Tatar first name.

AA Port of Canary Islands.

AA Town in New Mexico, USA.

RU Spanish; Columbus' flagship, first voyage to America, 1492.

AA Town in Mozambique.

AA Daniel Santbech Noviomagus; Dutch mathematician, astronomer (unkn-fl. 1561).

AA Alberto; Brazilian aeronautical engineer (1873-1932).

AA Assyro-Babylonian; torch of the gods.

MO Phoenician goddess.

AA Greek poetess (unkn-c. 600 B.C.).

PE Lyric poetess, Lesbos, Asia Minor (f1. between 160-580 B.C.).

AA Vikram Ambalal; Indian astrophysicist (1919-1971).

AA Hebrew first name.

AA Finno-Ugric goddess of childbirth.

PL Ceylon (Sri Lanka); the island visited by Sindbad on his 6th voyage.

AA Spa in New York, USA.

AA Domingo Faustino; Argentine writer (1811-1888).

AA Town in Wales.

AA Town in Italy.

CR Babylonian fertility goddess.

LI Greek; son of Europa and Zeus.

AA Ibu Dewi; Indonesian educator (1884-1942).

AA George (A. L.); Belgian-American historian of science (1844-1956).

AA Artist's model, wife of Rembrandt.

AA Sasceride, Gellio; Danish astronomer, doctor (1562-1612).

AA Wife of Khnum, Egyptian god of the Cataracts.

VA Egyptian river goddess.

AA Town in Russia.

AA Town in Wisconsin, USA.

DO Lithuanian sun goddess.

AA Samuel Ar̈thur; British mathematician, selenographer (1852-1912).

AA Horace Benedict De; Swiss geologist (1740-1799).

AA American colonial town (Georgia).

\footnotetext{
P: $\quad$ planetary system (see page xvi).

Sa: satellite (see page $x v i$ ).

lat: latitude of feature center.

long: longitude of feature center.
}

diam: diameter or long dimension of feature.

ct: continent of name origin (see page $284 \mathrm{ff}$.)

et: ethnicity of name origin (see page $284 \mathrm{ff}$.) 


\section{ALPHABETICAL LIST OF NAMES}

\section{P Sa Name}

M M Savich

M M Say

$\mathrm{H} \quad \mathrm{H}$ Sayat-Nova

V V Sayers

L L Scaliger

M M Scamander Vallis

M M Scandia

M M Scandia Colles

H H Scarlatti

L L Scarp

V V Scarpellini

M M Schaeberle

L L Schaeberle

L L Scheele

L L Scheiner

M M Schiaparelli

L L Schiaparelli

H H Schiaparelli Dorsum

L L Schickard

L L Schiller

L L Schjellerup

L L Schlesinger

L L Schliemann

L L Schlüter

M M Schmidt

L L Schmidt lat

long

$\begin{array}{lr}27.8 S & 263.5 \mathrm{~W} \\ 28.5 \mathrm{~S} & 29.5 \mathrm{~W} \\ 28.4 \mathrm{~S} & 122.1 \mathrm{~W}\end{array}$

diam

ct et

as ad

ref

ft origin

$28.4 \mathrm{~S}$

22.1W

$\begin{array}{rlr}0 & \text { UR } & \text { RU } \\ 15 & \text { AF } & \text { NG } \\ 158 & \text { AS } & \text { AM }\end{array}$

$\begin{array}{ll}5 & 1989 \\ 5 & 1976 \\ 5 & 1979\end{array}$

67.5S $230.0 \mathrm{E}$

90

EU EN

$5 \quad 1994$

27.1S 108.9E

84

$16.1 \mathrm{~N} \quad 331.1 \mathrm{~W}$

272

$60.5 \mathrm{~N} \quad 147.9 \mathrm{~W}$

$66.0 \mathrm{~N} \quad 135.4 \mathrm{~W}$

$40.5 \mathrm{~N} \quad 100.0 \mathrm{~W}$

0
1232
129

EU

FR

$5 \quad 1970$

$5 \quad 1982$

51958

$\begin{array}{ll}5 & 1985\end{array}$

$\begin{array}{lrrr}\text { EU } & \text { LA } & 5 & 1958 \\ \text { EU } & \text { LA } & 5 & 1985 \\ \text { EU } & \text { IT } & 5 & 1979\end{array}$

$20.3 \mathrm{~N} \quad 30.6 \mathrm{E}$

$5 \quad 1973$

$23.2 S$

$34.6 \mathrm{E}$

NA AM

$5 \quad 1991$

24.7S $309.8 \mathrm{~W}$

26

EU IT

$5 \quad 1973$

26.2S 117.2E

62

NA AM

51970

$9.4 \mathrm{~S}$

$37.8 \mathrm{~W}$

$60.5 \mathrm{~S}$

$27.5 \mathrm{~W}$

$2.5 \mathrm{~S} \quad 343.4 \mathrm{~W}$

23.4N

$23.0 \mathrm{~N}$

$58.8 \mathrm{~W}$

62 NA AM

51976

110 EU GE

51935

461 EU IT

51973

51935

44.3S

$164.1 \mathrm{~W}$

24

EU

IT

51976

$51.9 \mathrm{~S}$

$55.3 \mathrm{~W}$

EU IT

$5 \quad 1935$

51935

$69.7 \mathrm{~N} \quad 157.1 \mathrm{E} \quad 62$

$47.4 \mathrm{~N} \quad 138.6 \mathrm{~W}$

51970

2.1S 155.2E

97

NA AM

$5 \quad 1970$

$5.9 \mathrm{~S}$

83.3W

$72.2 \mathrm{~S}$

$77.5 \mathrm{~W}$

80

EU GE

$5 \quad 1970$

51964

51973

$1.0 \mathrm{~N}$

$18.8 \mathrm{E}$

11

EU

GE

51935

66

$\begin{array}{lrrrrrrr}41.8 \mathrm{~N} & 163.6 \mathrm{~W} & 54 & \mathrm{EU} & \mathrm{GE} & 5 & 1970 & 0 \\ 16.0 \mathrm{~S} & 135.7 \mathrm{~W} & 29 & \text { EU } & \text { AS } & 5 & 1976 & 59 \\ 76.7 \mathrm{~S} & 24.9 \mathrm{E} & 85 & \mathrm{EU} & \mathrm{AS} & 5 & 1935 & 66 \\ 20.4 \mathrm{~N} & 309.5 \mathrm{~W} & 185 & \mathrm{EU} & \mathrm{GE} & 5 & 1976 & 68 \\ 44.8 \mathrm{~N} & 98.1 \mathrm{~W} & 25 & \mathrm{EU} & \mathrm{GE} & 5 & 1970 & 0 \\ 19.5 \mathrm{~S} & 89.7 \mathrm{E} & 53 & \text { EU } & \text { GE } & 5 & 1970 & 0 \\ 67.0 \mathrm{~S} & 132.4 \mathrm{E} & 312 & \text { EU } & \text { AS } & 5 & 1970 & 0\end{array}$

AA A.M. Savich; Russian astronomer.

AA Town in Niger.

AA Aruthin Sayadian; Armenian/Georgian song writer (1712-1795).

AA Dorothy L.; English novelist, playwright (1893-1957).

AA Joseph J.; French chronologist (1540-1609)

VA Ancient name of river at Troy (modern Turkey).

AL Southern Scandinavia.

CO From albedo feature name.

AA Domenico (1685-1757) and Alessandro (1660-1725); Italian composers.

LF Astronaut-named feature, Apollo 17 site.

AA Caterina; 19th century Italian astronomer.

AA John M.; American astronomer (1853-1924).

AA John M.; American astronomer (1853-1924).

AA Carl Wilhelm; Swedish chemist (1742-1786).

AA Christopher; German astronomer (1575-1650).

AA Giovanni V.; Italian astronomer (1835-1910).

AA Giovanni Virginio; Italian astronomer (1835-1910).

DO Giovanni V.; Italian astronomer (1835-1910).

AA Wilhelm; German astronomer, mathematician (1592-1635).

AA Julius; German astronomer (unkn-fl. 1627).

AA H. C.; Danish astronomer (1827-1887).

AA Frank; American astronomer (1871-1943).

AA Heinrich; German archaeologist (1822-1890).

AA Heinrich; German astronomer (1815-1844).

AA Johann F.; German astronomer (1825-1884); Otto Y.; Russian geophysicist (1891-1956).

AA Johann Friedrich Julius; German astronomer (1825-1884); Bernhard; German optician (1879-1935); Otto Y.; Soviet astronomer (1891-1956).

AA Schneller, Herbert; German astronomer (1901-1967).

AA Arnold; Austrian-American composer (1874-1951).

AA Georg; Austrian astronomer, mathematician (1597-1645).

AA Johannes; German geographer (1477-1547)

AA Eduard; German astronomer (1828-1891).

AA Richard; German astronomer (1867-1951).

AA Schrödinger, Erwin; Austrian physicist; Nobel laureate (1887-1961).
P: $\quad$ planetary system (see page $\mathrm{xvi}$ ).

Sa: $\quad$ satellite (see page $x v i$ ).

lat: latitude of feature center.

long: longitude of feature center. diam: diameter or long dimension of feature.

ct: continent of name origin (see page $284 \mathrm{ff}$.)

et: ethnicity of name origin (see page $284 \mathrm{ff}$.)

as: name approval status (see page xvii). ad: name approval date (year).

ref: reference source for name (see page $287 \mathrm{ff}$.).

ft: feature type (see page 290). 


\section{ALPHABETICAL LIST OF NAMES}

$\begin{array}{lll}\text { P } & \text { Sa } & \text { Name } \\ \text { M } & \text { M } & \text { Schroeter } \\ \text { L } & \text { L } & \text { Schröter } \\ \text { L } & \text { L } & \text { Schubert } \\ \text { H } & \text { H } & \text { Schubert } \\ \text { L } & \text { L } & \text { Schumacher } \\ \text { V } & \text { V } & \text { Schumann-Heink Patera } \\ \text { L } & \text { L } & \text { Schuster } \\ \text { L } & \text { L } & \text { Schwabe } \\ \text { L } & \text { L } & \text { Schwarzschild } \\ \text { L } & \text { L } & \text { Scobee }\end{array}$

H H Scopas

L L Scoresby

L L Scott

L L Sculptured Hills

M M Scylla Scopulus

L L Seares

M M Sebec

J ga Sebek

L L Secchi

M M Secchi

L L Sechenov

J io Sêd Patera

V V Sedna Planitia

L L Seeliger

L L Segers

L L Segner

$\mathrm{H} \quad \mathrm{H} \quad$ Sei

V V Seia Corona

L L Seidel

$\begin{array}{lll}\text { J } & \text { ga } & \text { Seima } \\ \text { J } & \text { ga } & \text { Seker }\end{array}$

V V Sekmet Mons

V V Sel-Anya Dorsa

L L Seleucus

J ga Selket

V V Selma lat long diam ct et as ad ref $f t$ origin

$\begin{array}{rrrrrrrr}1.8 \mathrm{~S} & 303.6 \mathrm{~W} & 337 & \mathrm{EU} & \mathrm{GE} & 5 & 1976 & 68 \\ 2.6 \mathrm{~N} & 7.0 \mathrm{~W} & 35 & \mathrm{EU} & \mathrm{GE} & 5 & 1935 & 66 \\ 2.8 \mathrm{~N} & 81.0 \mathrm{E} & 54 & \mathrm{UR} & \mathrm{RU} & 5 & 1935 & 66 \\ 43.4 \mathrm{~S} & 54.3 \mathrm{~W} & 185 & \mathrm{EU} & \mathrm{AS} & 5 & 1976 & 59 \\ 42.4 \mathrm{~N} & 60.7 \mathrm{E} & 60 & \mathrm{EU} & \mathrm{GE} & 5 & 1935 & 66 \\ 74.0 \mathrm{~N} & 215.0 \mathrm{E} & 0 & \mathrm{EU} & \mathrm{GM} & 5 & 91 & 46 \\ 4.2 \mathrm{~N} & 146.5 \mathrm{E} & 108 & \mathrm{EU} & \mathrm{GB} & 5 & 1970 & 0 \\ 65.1 \mathrm{~N} & 45.6 \mathrm{E} & 25 & \mathrm{EU} & \mathrm{GE} & 5 & 1935 & 66 \\ 70.1 \mathrm{~N} & 121.2 \mathrm{E} & 212 & \mathrm{EU} & \mathrm{GE} & 5 & 1970 & 0 \\ 31.1 \mathrm{~S} & 148.9 \mathrm{~W} & 40 & \mathrm{NA} & \text { AM } & 5 & 1988 & 88\end{array}$

$\begin{array}{rrrrrrrr}81.1 \mathrm{~S} & 172.9 \mathrm{~W} & 105 & \text { EU } & \text { GR } & 5 & 1976 & 59 \\ 77.7 \mathrm{~N} & 14.1 \mathrm{E} & 55 & \text { EU } & \text { GB } & 5 & 1935 & 66 \\ 82.1 \mathrm{~S} & 48.5 \mathrm{E} & 103 & \text { EU } & \text { GB } & 5 & 1964 & 67 \\ 20.3 \mathrm{~N} & 31.0 \mathrm{E} & 8 & \text { NA } & \text { AM } & 5 & 1973 & 59 \\ 25.2 \mathrm{~S} & 342.0 \mathrm{~W} & 474 & \text { EU } & \text { GR } & 5 & 1985 & 57 \\ & & & & & 5 & 1970 & 0 \\ 73.5 \mathrm{~N} & 145.8 \mathrm{E} & 110 & \text { NA } & \text { AM } & 5 & \\ 40.0 \mathrm{~S} & 260.5 \mathrm{~W} & 0 & \text { US } & \text { ME } & 5 & 1989 & 74 \\ 59.5 \mathrm{~N} & 178.9 \mathrm{~W} & 70 & \text { AF } & \text { EG } & 5 & 1979 & 1 \\ 2.4 \mathrm{~N} & 43.5 \mathrm{E} & 22 & \text { EU } & \text { IT } & 5 & 1935 & 68\end{array}$

$\begin{array}{llllllll}57.9 S & 257.7 \mathrm{~W} & 218 & \mathrm{EU} & \mathrm{IT} & 5 & 1973 & 68\end{array}$

$\begin{array}{llllllll}7.1 \mathrm{~S} & 142.6 \mathrm{~W} & 62 & \mathrm{UR} & \mathrm{RU} & 5 & 1970 & 0\end{array}$

$\begin{array}{llllllll}2.8 \mathrm{~S} & 303.8 \mathrm{~W} & 70 & \text { AS } & \text { PO } & 5 & 1985 & 27\end{array}$

$\begin{array}{llllllll}42.7 \mathrm{~N} & 340.7 \mathrm{E} & 3572 & \mathrm{NA} & \mathrm{ES} & 5 & 1982 & 1\end{array}$

$\begin{array}{llllllll}2.2 \mathrm{~S} & 3.0 \mathrm{E} & 8 & \mathrm{EU} & \mathrm{GE} & 5 & 1935 & 66\end{array}$

$\begin{array}{llllllll}47.1 \mathrm{~N} & 127.7 \mathrm{E} & 17 & \mathrm{SA} & \text { AR } & 5 & 1970 & 0\end{array}$

$\begin{array}{llllllll}58.9 \mathrm{~S} & 48.3 \mathrm{~W} & 67 & \mathrm{EU} & \mathrm{GE} & 5 & 1935 & 66\end{array}$

$\begin{array}{llllllll}64.3 S & 89.1 \mathrm{~W} & 113 & \text { AS } & \text { JA } & 5 & 1976 & 59\end{array}$

$\begin{array}{llllllll}3.0 \mathrm{~S} & 153.0 \mathrm{E} & 225 & \mathrm{EU} & \mathrm{RM} & 5 & 1994 & 27\end{array}$

$\begin{array}{llllllll}16.6 \mathrm{~N} & 217.4 \mathrm{~W} & 33 & \mathrm{AF} & \mathrm{SE} & 5 & 1985 & 27\end{array}$

$\begin{array}{lllllll}40.8 \mathrm{~S} & 351.0 \mathrm{~W} & 117 & \mathrm{AF} & \mathrm{EG} & 5 & 1988\end{array}$

44.2N 240.1E 338 AF EG

$\begin{array}{llllllll}79.4 \mathrm{~N} & 81.3 \mathrm{E} & 975 & \mathrm{EU} & \mathrm{HU} & 5 & 1985 & 65\end{array}$

$\begin{array}{llllllll}21.0 \mathrm{~N} & 66.6 \mathrm{~W} & 43 & \text { AS } & \text { BY } & 5 & 1935 & 66\end{array}$

$16.7 \mathrm{~N} \quad 107.4 \mathrm{~W} \quad 140 \quad$ AF $\quad$ EG

51985

$\begin{array}{lllll}68.5 \mathrm{~N} & 155.6 \mathrm{E} & 10 & \mathrm{EU} & \mathrm{CE}\end{array}$
$5 \quad 1985$

1

83

AA Johann H.; German astronomer (1745-1816)

AA Johann Hieronymus; German astronomer (1745-1816)

AA Theodor Friedrich Von; Russian cartographer (1789-1865).

AA Franz S.; Austrian composer (1797-1828)

AA Heinrich Christian; German astronomer (1780-1850).

PE Ernestine; German singer (1861-1936).

AA Sir Arthur; British mathematician, physicist (1851-1934).

AA Heinrich; German astronomer (1789-1875).

AA Karl; German astronomer (1873-1916)

AA Francis Richard; member of the Challenger crew 1939-1986); previous designation Barringer L.

AA Greek sculptor and architect (fi. 4th century B.C.).

AA William; British explorer (1789-1857)

AA Robert F.; British explorer (1868-1912)

LF Astronaut-named feature, Apollo 17 site.

SC From albedo feature at 19S, 320W.

AA Frederick H.; American astronomer (1873-1964)

AA Town in Maine, USA.

AA Egyptian crocodile god.

AA Pietro Angelo; Italian astronomer, astrophysicist (1818-1878).

AA Angelo; Italian astronomer (1818-1878).

AA Ivan M.; Russian physiologist (1829-1905).

PE Phoenician chariot rider of the Sun.

PL Eskimo; her fingers became seals and whales.

AA Hugo von; German astronomer (1849-1924)

AA Carlos; Argentinian astronomer 1900-1967

AA Johann Andreas von; German (1704-1777)

AA Shonagun; Japanese diarist and poet (c. 966-1013).

CR Roman grain goddess.

AA Ludwig P. Von; German astronomer, mathematician (1821-1896)

AA Mother goddess of the Arameans.

AA Egyptian god of the dead at Memphis.

MO Ancient Egyptian goddess of war and battle.

DO Hungarian wind goddess.

AA Babylonian astronomer (unkn-fl. c. 150 B.C.).

AA Tutelary goddess who guarded intestines of the dead.

$51985 \quad 83 \quad$ AA First name from Celtic.
P: $\quad$ planetary system (see page xvi).

Sa: satellite (see page xvi).

lat: latitude of feature center.

long: longitude of feature center. diam: diameter or long dimension of feature. ct: continent of name origin (see page $284 \mathrm{ff}$.)

et: ethnicity of name origin (see page $284 \mathrm{ff}$.)

as: name approval status (see page xvii). ad: name approval date (year).

ref: reference source for name (see page $287 \mathrm{ff}$.).

ft: feature type (see page 290). 


\section{ALPHABETICAL LIST OF NAMES}

\begin{tabular}{|c|c|c|c|c|c|c|c|c|c|c|}
\hline $\mathbf{P}$ & $\mathbf{S a}$ & Name & lat & long & diam & ct & et & as & ad & ref \\
\hline $\begin{array}{l}\text { V } \\
\text { V } \\
M\end{array}$ & $\begin{array}{l}\mathrm{V} \\
\mathrm{V} \\
\mathrm{M}\end{array}$ & $\begin{array}{l}\text { Selu Corona } \\
\text { Semele Tholi } \\
\text { Semeykin }\end{array}$ & $\begin{array}{l}42.5 \mathrm{~S} \\
64.3 \mathrm{~N} \\
41.8 \mathrm{~N}\end{array}$ & $\begin{array}{c}6.0 \mathrm{E} \\
202.9 \mathrm{E} \\
351.2 \mathrm{~W}\end{array}$ & $\begin{array}{r}300 \\
194 \\
71\end{array}$ & $\begin{array}{l}\text { NA } \\
\text { AS } \\
\text { UR }\end{array}$ & $\begin{array}{l}\text { AM } \\
\text { PO } \\
\text { SO }\end{array}$ & $\begin{array}{l}5 \\
5 \\
5\end{array}$ & $\begin{array}{l}1994 \\
1985 \\
1982\end{array}$ & $\begin{array}{r}83 \\
1 \\
64\end{array}$ \\
\hline $\begin{array}{l}\text { M } \\
\text { V } \\
\text { L }\end{array}$ & $\begin{array}{l}M \\
V \\
L\end{array}$ & $\begin{array}{l}\text { Seminole } \\
\text { Semuni Dorsa } \\
\text { Seneca }\end{array}$ & $\begin{array}{l}24.5 \mathrm{~S} \\
75.9 \mathrm{~N} \\
26.6 \mathrm{~N}\end{array}$ & $\begin{array}{c}18.9 \mathrm{~W} \\
8.0 \mathrm{E} \\
80.2 \mathrm{E}\end{array}$ & $\begin{array}{r}21 \\
514 \\
46\end{array}$ & $\begin{array}{l}\text { US } \\
\text { UR } \\
\text { EU }\end{array}$ & $\begin{array}{r}\text { FL } \\
\text { SI } \\
\text { RM }\end{array}$ & $\begin{array}{l}5 \\
5 \\
5\end{array}$ & $\begin{array}{l}1976 \\
1985 \\
1961\end{array}$ & $\begin{array}{l}36 \\
65 \\
67\end{array}$ \\
\hline $\begin{array}{l}\text { J } \\
M \\
\text { V } \\
M \\
\text { J } \\
\text { V } \\
N\end{array}$ & $\begin{array}{l}\text { io } \\
\mathrm{M} \\
\mathrm{V} \\
\mathrm{M} \\
\mathrm{ca} \\
\mathrm{V} \\
\mathrm{tr}\end{array}$ & $\begin{array}{l}\text { Sengen Patera } \\
\text { Senus Vallis } \\
\text { Seoritsu Farra } \\
\text { Sepik Vallis } \\
\text { Seqinek } \\
\text { Seshat Mons } \\
\text { Set Catena }\end{array}$ & $\begin{array}{r}32.8 \mathrm{~S} \\
5.3 \mathrm{~S} \\
30.0 \mathrm{~S} \\
1.0 \mathrm{~S} \\
55.5 \mathrm{~N} \\
26.3 \mathrm{~N} \\
22.0 \mathrm{~N}\end{array}$ & $\begin{array}{c}304.3 \mathrm{~W} \\
146.9 \mathrm{~W} \\
11.0 \mathrm{E} \\
65.8 \mathrm{~W} \\
25.5 \mathrm{~W} \\
33.2 \mathrm{E} \\
33.5 \mathrm{E}\end{array}$ & $\begin{array}{r}55 \\
27 \\
230 \\
0 \\
80 \\
62 \\
0\end{array}$ & $\begin{array}{l}\text { AS } \\
\text { EU } \\
\text { AS } \\
\text { OC } \\
\text { NA } \\
\text { AF } \\
\text { AF }\end{array}$ & $\begin{array}{l}\text { JA } \\
\text { IR } \\
\text { JA } \\
\text { GU } \\
\text { ES } \\
\text { EG } \\
\text { EG }\end{array}$ & $\begin{array}{l}5 \\
5 \\
5 \\
5 \\
5 \\
5 \\
5\end{array}$ & $\begin{array}{r}1979 \\
1985 \\
1994 \\
1985 \\
1979 \\
91 \\
91\end{array}$ & $\begin{array}{r}1 \\
56 \\
83 \\
56 \\
26 \\
1 \\
1\end{array}$ \\
\hline $\begin{array}{l}\mathrm{U} \\
\mathrm{M} \\
\mathrm{M} \\
\mathrm{V} \\
\mathrm{L}\end{array}$ & $\begin{array}{l}u m \\
M \\
M \\
V \\
L\end{array}$ & $\begin{array}{l}\text { Setibos } \\
\text { Sevel } \\
\text { Sevi } \\
\text { Sévigné } \\
\text { Seyfert }\end{array}$ & $\begin{array}{l}30.8 \mathrm{~S} \\
78.2 \mathrm{~N} \\
19.1 \mathrm{~N} \\
56.6 \mathrm{~N} \\
29.1 \mathrm{~N}\end{array}$ & $\begin{array}{c}346.3 \mathrm{E} \\
39.5 \mathrm{~W} \\
257.1 \mathrm{~W} \\
326.5 \mathrm{E} \\
114.6 \mathrm{E}\end{array}$ & $\begin{array}{r}50 \\
0 \\
0 \\
28 \\
110\end{array}$ & $\begin{array}{l}\text { SA } \\
\text { EU } \\
\text { UR } \\
\text { EU } \\
\text { NA }\end{array}$ & $\begin{array}{r}\text { PT } \\
\text { DE } \\
\text { RU } \\
\text { FR } \\
\text { AM }\end{array}$ & $\begin{array}{l}5 \\
5 \\
5 \\
5 \\
5\end{array}$ & $\begin{array}{l}1988 \\
1988 \\
1988 \\
1985 \\
1970\end{array}$ & $\begin{array}{r}94 \\
36 \\
36 \\
46 \\
0\end{array}$ \\
\hline $\begin{array}{l}\mathrm{V} \\
\mathrm{M} \\
\mathrm{L} \\
\mathrm{S}\end{array}$ & $\begin{array}{l}V \\
M \\
L \\
\text { en }\end{array}$ & $\begin{array}{l}\text { Seymour } \\
\text { Sfax } \\
\text { Shahinaz } \\
\text { Shahrazad }\end{array}$ & $\begin{array}{r}18.2 \mathrm{~N} \\
7.8 \mathrm{~S} \\
7.5 \mathrm{~N} \\
48.2 \mathrm{~N}\end{array}$ & $\begin{array}{c}326.5 \mathrm{E} \\
43.5 \mathrm{~W} \\
122.4 \mathrm{E} \\
195.1 \mathrm{~W}\end{array}$ & $\begin{array}{r}65 \\
7 \\
15 \\
20\end{array}$ & $\begin{array}{l}\text { EU } \\
\text { AF } \\
\text { AS } \\
\text { AS }\end{array}$ & $\begin{array}{l}\text { EN } \\
\text { TN } \\
\text { TU } \\
\text { AR }\end{array}$ & $\begin{array}{l}5 \\
5 \\
5 \\
5\end{array}$ & $\begin{array}{l}1994 \\
1976 \\
1979 \\
1982\end{array}$ & $\begin{array}{r}99 \\
36 \\
0 \\
52\end{array}$ \\
\hline $\mathbf{S}$ & en & Shahryar & $59.7 \mathrm{~N}$ & $225.0 \mathrm{~W}$ & 21 & AS & $\mathrm{AR}$ & 5 & 1982 & 52 \\
\hline $\mathbf{L}$ & $\mathrm{L}$ & Shakespeare & $20.2 \mathrm{~N}$ & $30.8 \mathrm{E}$ & 1 & EU & GB & 5 & 1973 & 59 \\
\hline H & $\mathbf{H}$ & Shakespeare & $49.7 \mathrm{~N}$ & $150.9 \mathrm{~W}$ & 370 & EU & EN & 5 & 1979 & 59 \\
\hline $\begin{array}{l}\text { V } \\
\text { L }\end{array}$ & $\begin{array}{l}\mathrm{V} \\
\mathrm{L}\end{array}$ & $\begin{array}{l}\text { Shakira } \\
\text { Shakleton }\end{array}$ & $\begin{array}{l}3.0 \mathrm{~N} \\
89.9 \mathrm{~S}\end{array}$ & $\begin{array}{r}213.7 \mathrm{E} \\
0.0 \mathrm{E}\end{array}$ & $\begin{array}{l}19 \\
19\end{array}$ & $\begin{array}{l}\text { UR } \\
\text { EU }\end{array}$ & $\begin{array}{l}\text { RU } \\
\text { EN }\end{array}$ & $\begin{array}{l}5 \\
5\end{array}$ & $\begin{array}{l}1994 \\
1994\end{array}$ & $\begin{array}{r}112 \\
68\end{array}$ \\
\hline $\mathrm{J}$ & io & Shakuru Patera & $23.6 \mathrm{~N}$ & $266.4 \mathrm{~W}$ & 70 & NA & PW & 5 & 1979 & 1 \\
\hline $\begin{array}{l}\mathrm{M} \\
\mathrm{L}\end{array}$ & $\begin{array}{l}\mathrm{M} \\
\mathrm{L}\end{array}$ & $\begin{array}{l}\text { Shalbatana Vallis } \\
\text { Shaler }\end{array}$ & $\begin{array}{r}5.6 \mathrm{~N} \\
32.9 \mathrm{~S}\end{array}$ & $\begin{array}{l}43.1 \mathrm{~W} \\
85.2 \mathrm{~W}\end{array}$ & $\begin{array}{r}687 \\
48\end{array}$ & $\begin{array}{l}\text { AS } \\
\text { NA }\end{array}$ & $\begin{array}{l}\mathrm{AK} \\
\mathrm{AM}\end{array}$ & $\begin{array}{l}5 \\
5\end{array}$ & $\begin{array}{l}1973 \\
1964\end{array}$ & $\begin{array}{l}62 \\
67\end{array}$ \\
\hline $\begin{array}{l}\mathrm{J} \\
\mathrm{M} \\
\mathrm{L}\end{array}$ & $\begin{array}{l}\text { io } \\
\mathrm{M} \\
\mathrm{L}\end{array}$ & $\begin{array}{l}\text { Shamash Patera } \\
\text { Shambe } \\
\text { Shapley }\end{array}$ & $\begin{array}{c}33.7 \mathrm{~S} \\
20.7 \mathrm{~S} \\
9.4 \mathrm{~N}\end{array}$ & $\begin{array}{c}152.1 \mathrm{~W} \\
30.5 \mathrm{~W} \\
56.9 \mathrm{E}\end{array}$ & $\begin{array}{r}110 \\
29 \\
23\end{array}$ & $\begin{array}{l}\text { AS } \\
\text { AF } \\
\text { NA }\end{array}$ & $\begin{array}{l}\mathrm{AB} \\
\mathrm{SU} \\
\mathrm{AM}\end{array}$ & $\begin{array}{l}5 \\
5 \\
5\end{array}$ & $\begin{array}{l}1979 \\
1976 \\
1973\end{array}$ & $\begin{array}{r}1 \\
36 \\
0\end{array}$ \\
\hline M & $\mathbf{M}$ & Sharonov & $27.3 \mathrm{~N}$ & $58.3 \mathrm{~W}$ & 95 & UR & RU & 5 & 1973 & 68 \\
\hline $\mathrm{L}$ & $\mathbf{L}$ & Sharonov & $12.4 \mathrm{~N}$ & $173.3 \mathrm{E}$ & 74 & UR & SO & 5 & 1970 & 0 \\
\hline $\mathrm{L}$ & $\mathrm{L}$ & Sharp & $45.7 \mathrm{~N}$ & $40.2 \mathrm{~W}$ & 39 & $\mathrm{EU}$ & GB & 5 & 1935 & 66 \\
\hline $\mathbf{L}$ & $\mathbf{L}$ & Sharp-Apollo & $3.2 \mathrm{~S}$ & $23.4 \mathrm{~W}$ & 0 & NA & $\mathrm{AM}$ & 5 & 1973 & 59 \\
\hline $\mathbf{M}$ & ph & Sharpless & $25.0 \mathrm{~S}$ & $165.0 \mathrm{~W}$ & 0 & NA & $\mathrm{AM}$ & 5 & 1973 & 60 \\
\hline L & $\mathbf{L}$ & Shatalov & $24.3 \mathrm{~N}$ & $141.5 \mathrm{E}$ & 21 & UR & SO & 5 & 1970 & 0 \\
\hline $\begin{array}{l}\mathrm{M} \\
\mathbf{M} \\
\mathrm{L}\end{array}$ & $\begin{array}{l}\mathbf{M} \\
\mathbf{M} \\
\mathbf{L}\end{array}$ & $\begin{array}{l}\text { Shatskiy } \\
\text { Shawnee } \\
\text { Shayn }\end{array}$ & $\begin{array}{l}32.4 \mathrm{~S} \\
22.7 \mathrm{~N} \\
32.6 \mathrm{~N}\end{array}$ & $\begin{array}{c}14.7 \mathrm{~W} \\
31.5 \mathrm{~W} \\
172.5 \mathrm{E}\end{array}$ & $\begin{array}{l}69 \\
16 \\
93\end{array}$ & $\begin{array}{l}\text { UR } \\
\text { US } \\
\text { UR }\end{array}$ & $\begin{array}{l}\text { RU } \\
\text { OH } \\
\text { SO }\end{array}$ & $\begin{array}{l}5 \\
5 \\
5\end{array}$ & $\begin{array}{l}1979 \\
1976 \\
1970\end{array}$ & $\begin{array}{r}68 \\
36 \\
0\end{array}$ \\
\hline $\mathrm{L}$ & L & Sheepshanks & $59.2 \mathrm{~N}$ & $16.9 \mathrm{E}$ & 25 & $\mathrm{EU}$ & GB & 5 & 1935 & 66 \\
\hline $\mathbf{L}$ & $\mathbf{L}$ & [Shekhov (Chekhov)] & $6.6 S$ & $82.0 \mathrm{E}$ & 19 & UR & RU & 6 & 0 & 0 \\
\hline $\mathrm{H}$ & H & Shelley & $47.8 \mathrm{~S}$ & $127.8 \mathrm{~W}$ & 164 & EU & EN & 5 & 1979 & 59 \\
\hline $\mathrm{L}$ & L & Sherlock & $20.2 \mathrm{~N}$ & $30.8 \mathrm{E}$ & 0 & EU & GB & 5 & 1973 & 59 \\
\hline
\end{tabular}

\section{$\mathrm{ft}$ origin}

CR Cherokee corn goddess.

TH Frygian (Phoenician) Earth goddess.

AA Boris Evgen'evich; Soviet astronomer (1900-1937).

AA Town in Florida, USA.

DO Ulchian (Siberia) sky goddess.

AA Lucius Annaeus; Roman philosopher, natural scientist (4 B.C.- A.D. 65)

PE Japanese; deity of Mt. Fugiyama.

VA Classical river in Ireland.

FR Japanese stream goddess.

VA River in New Guinea.

AA Eskimo; the sun.

MO Egyptian goddess of writing.

CA Egypian water monster; personification of evil.

AA Chief devil.

AA Town in Denmark.

AA Town in Russia.

AA Marie; French writer (1626-1696).

AA Carl K.; American astronomer (1911-1960).

AA Jane; English queen (c. 1509-1537).

AA Town in Tunisia.

AA Turkish female name.

AA Heroine who tells King Shahryar "The Tales of a Thousand Nights".

AA King whom Shahrazad beguiles with the tales of a thousand nights and a night.

LF Astronaut-named feature, Apollo 17 site.

AA William; English poet and dramatist (1564-1616).

AA Bashkir first name.

AA Earnest H.; English antarctic explorer (1874-1922).

PE Pawnee (USA) sun god of the East; gives light and heat.

VA Word for "Mars" in Akkadian.

AA Nathaniel S.; American geologist, paleontologist (1841-1906).

PE Assyro-Babylonian sun god.

AA Town in Sudan.

AA Harlow; American astronomer (1885-1972).

AA Vsevolod V.; Russian astronomer (1901-1964).

AA Vsevolod V.; Soviet astronomer (1901-1964).

AA Abraham; British astronomer, mathematician (1651-1742)

LF Astronaut-named feature, Apollo 12 site.

AA Bevan P.; American astronomer 1904-1950

AA Vladimir A.; Soviet cosmonaut (1927-Live).

AA N. S.; Russian geologist.

AA Town in Ohio, USA.

AA Grigoriy A.; Soviet astrophysicist (1892-1956).

AA Anne; British benefactor (1789-1876).

AA Anton Pavlovich; Russian writer (1860-1904).

AA Percy Bysshe; English poet (1792-1822).

LF Astronaut-named feature, Apollo 17 site.

\footnotetext{
P: $\quad$ planetary system (see page xvi).

Sa: satellite (see page $\mathrm{xvi}$ ).

lat: latitude of feature center.

long: longitude of feature center.
}

diam: diameter or long dimension of feature. ct: continent of name origin (see page $284 \mathrm{ff}$.) et: ethnicity of name origin (see page $284 \mathrm{ff}$.) as: name approval status (see page xvii). ad: name approval date (year).

ref: reference source for name (see page $287 \mathrm{ff}$.).

ft: feature type (see page 290). 


\section{ALPHABETICAL LIST OF NAMES}

\section{P Sa Name}

L L Sherrington

H H Shevchenko

L L Shi Shen

V $\quad \mathrm{V}$ Shih Mai-Yu

V V Shimti Tessera

L L Shirakatsi

V V Shiwanokia Corona

H H Sholem Aleichem

J ca Sholmo

$S$ rh Sholmo

L L Short

L L Shorty

J io Shoshu Patera

L L Shternberg (Sternberg)

J ga Shu

L L Shuckburgh

L L Shulejkin

M M Sian

$\mathrm{H} \quad \mathrm{H}$ Sibelius

M M Sibu

V V Sicasica Fluctus

U mr Sicilia Regio

J ga Sicyon Sulcus

$\mathrm{V} \quad \mathrm{V}$ [Siddons]

V V Sidney

J eu Sidon Flexus

L L Siedentopf

L L Sierpinski

$\mathrm{V} V$ Sif Mons

$\mathrm{V} V$ Sige Dorsa

M M Sigli

$\mathrm{V} V$ Sigrid

V V Sigrun Fossae

$J$ ca Sigyn

L L Sikorsky

L L Silberschlag

J io Silpium Mons

H H Simeiz Vallis

$\mathrm{V} V$ [Simone]

V V Simonenko

$\mathrm{H}$ H Simonides

L L Simpelius

M M Simud Vallis

J ga Sin lat long diam

$\begin{array}{lrrlrrrr}11.1 \mathrm{~S} & 118.0 \mathrm{E} & 18 & \text { EU } & \text { GB } & 5 & 1976 & 0 \\ & & & & & & & \\ 53.8 \mathrm{~S} & 46.5 \mathrm{~W} & 137 & \text { UR } & \text { UK } & 5 & 1976 & 59 \\ 76.0 \mathrm{~N} & 104.1 \mathrm{E} & 43 & \text { AS } & \text { CH } & 5 & 1970 & 0 \\ 18.4 \mathrm{~N} & 318.9 \mathrm{E} & 25 & \text { AS } & \text { CH } & 5 & 1994 & 99 \\ 31.9 \mathrm{~N} & 97.7 \mathrm{E} & 1275 & \text { AS } & \text { BY } & 5 & 1985 & 27 \\ 12.1 \mathrm{~S} & 128.6 \mathrm{E} & 51 & \text { AS } & \text { AM } & 5 & 1979 & 0 \\ 42.0 \mathrm{~S} & 279.8 \mathrm{E} & 500 & \mathrm{NA} & \mathrm{PU} & 5 & 1994 & 27 \\ 50.4 \mathrm{~N} & 87.7 \mathrm{~W} & 200 & \text { AS } & \text { JW } & 5 & 1979 & 59 \\ 53.9 \mathrm{~N} & 16.4 \mathrm{~W} & 58 & \text { EU } & \text { FI } & 5 & 1979 & 27 \\ 12.0 \mathrm{~N} & 346.4 \mathrm{~W} & 0 & \text { AS } & \text { BR } & 5 & 1982 & 40 \\ 74.6 \mathrm{~S} & 7.3 \mathrm{~W} & 70 & \text { EU } & \text { SC } & 5 & 1935 & 66 \\ 20.2 \mathrm{~N} & 30.6 \mathrm{E} & 0 & \text { NA } & \text { AM } & 5 & 1973 & 59 \\ 19.6 \mathrm{~S} & 324.1 \mathrm{~W} & 50 & \text { UR } & \text { CC } & 5 & 1982 & 30 \\ 19.5 \mathrm{~N} & 116.3 \mathrm{~W} & 70 & \text { UR } & \text { RU } & 5 & 1970 & 0 \\ 42.5 \mathrm{~N} & 357.9 \mathrm{~W} & 54 & \text { AF } & \text { EG } & 5 & 1988 & 1 \\ 42.6 \mathrm{~N} & 52.8 \mathrm{E} & 38 & \text { EU } & \text { GB } & 5 & 1935 & 66 \\ 27.1 \mathrm{~S} & 92.5 \mathrm{~W} & 15 & \text { UR } & \text { SO } & 5 & 1985 & 0 \\ 20.1 \mathrm{~N} & 48.0 \mathrm{~W} & 0 & \text { UR } & \text { RU } & 5 & 1988 & 36 \\ 49.6 \mathrm{~S} & 144.7 \mathrm{~W} & 90 & \text { EU } & \text { FI } & 5 & 1985 & 59 \\ 23.3 \mathrm{~S} & 19.6 \mathrm{~W} & 31 & \text { AS } & \text { MA } & 5 & 1976 & 36 \\ 52.0 \mathrm{~S} & 180.4 \mathrm{E} & 175 & \text { SA } & \text { BO } & 5 & 1994 & 111 \\ 30.0 \mathrm{~S} & 317.2 \mathrm{E} & 174 & \text { EU } & \text { IT } & 5 & 1988 & 85 \\ 36.5 \mathrm{~N} & 12.0 \mathrm{~W} & 1146 & \text { EU } & \text { GR } & 5 & 1979 & 1 \\ & & & & & & & \end{array}$

$61.6 \mathrm{~N} \quad 340.6 \mathrm{E}$

$47 \quad$ EU $\quad$ GB

61992

$13.4 \mathrm{~N} \quad 199.6 \mathrm{E}$

21 EU EN

51994

$64.5 \mathrm{~S} \quad 170.4 \mathrm{~W}$

1216

EU GR

$22.0 \mathrm{~N} \quad 135.5 \mathrm{E}$

$27.2 \mathrm{~S} \quad 154.5 \mathrm{E}$

61

EU GE

51979

51970

$22.0 \mathrm{~N} \quad 352.4 \mathrm{E}$

$31.2 \mathrm{~N}$

$20.5 \mathrm{~S}$

$63.6 \mathrm{~N}$

$52.3 \mathrm{~N}$

$35.8 \mathrm{~N}$

$66.1 \mathrm{~S}$

$6.2 \mathrm{~N}$

$52.6 \mathrm{~S}$

$13.2 \mathrm{~S}$

$59.5 \mathrm{~N}$

$26.8 \mathrm{~S}$

$29.1 \mathrm{~S}$

$73.0 \mathrm{~S}$

$11.5 \mathrm{~N}$ $51.9 \mathrm{~N}$
$106.9 \mathrm{E}$

$30.6 \mathrm{~W}$

$314.4 \mathrm{E}$

$19.9 \mathrm{E}$

$29.2 \mathrm{~W}$

103.2E

$$
69
$$

EU PO

51970

200 EU GE

478 AF $\quad \mathrm{BY}$

31

15

971

AS

EU SD

EU NS

EU NS

UR RU

$12.5 \mathrm{E}$

13

EU GE

$272.9 \mathrm{~W}$

0

EU GR

64.3W

\section{$82.0 \mathrm{E}$}

$97.5 \mathrm{E}$

$45.0 \mathrm{~W}$

$15.2 \mathrm{E}$

0

UR UK

1982

$5 \quad 91$

51976

51985

51985

$5 \quad 1979$

51979

51935

$5 \quad 1979$

51976

14 EU FR

61994

34
95
70

UR RU

EU GR

$\begin{array}{ll}6 & 1994 \\ 5 & 1998\end{array}$

$\begin{array}{llll}\mathrm{EU} & \mathrm{SC} & 5 & 1935\end{array}$

$38.5 \mathrm{~W}$

1074

AS

SU

51973

$\begin{array}{ll}5 & 1979\end{array}$ AA Sir Charles Scott; British
neurophysiologist; Nobel laureate (1856-1952).

AA Taras Hryhorovych; Ukrainian poet (1814-1861).

AA Shi(H) Shen; Chinese astronomer (unkn-c. 300 B.C.).

AA Chinese physician (1873-1954).

TE Babylonian; Ishtar as the goddess of fate.

AA Anania; Armenian geographer (620(?)-685(?)).

CR Zuni fertility goddess.

AA (Yakov Rabinowitz); Yiddish writer (1859-1916).

AA Finno-Ugric heaven god.

AA Buriat (Siberia) devil who creates.

AA James; Scottish mathematician, optician (1710-1768)

LF Astronaut-named feature Apollo 17 site.

PE Caucasian patron of fire.

AA Pavel K.; Russian astronomer (1865-1920).

AA Egyptian god of air.

AA Sir George; British geographer, benefactor (1751-1804).

AA M.V.; Soviet radio engineer (1884-1939).

AA Town in Russia.

AA Jean; Finnish composer (1865-1957).

AA Town in Malaysia.

FL Aymara (Bolivia) mountain goddess.

RE Location of "Winter's Tale."

SU Greek; where Ganymede and Hebe were worshipped as rain-givers.

AA Sarah; English actress (1755-1831)

AA Mary; Elizabethan dramatist (1561-1621).

FE Another name for Tyre; where Europa was born.

AA H.; German astronomer (1906-1963)

AA Waclaw; Polish mathematician (1882-1969).

MO Teutonic goddess; Thor's wife.

DO Babylonian sky goddess.

AA Town in Indonesia.

AA Scandinavian first name.

FO Norse Valkyrie.

AA Norse; Loki's wife.

AA Igor Ivanovich; Russian-American aeronautical engineer (1889-1972).

AA Johann Essaias; German astronomer (1721-1791)

MO Greek; where Io dies of grief in some legends.

VA Radio telescope facility, Crimea, Ukraine.

AA French first name.

AA Soviet astronomer

AA Greek lyric poet (556-468 B.C.).

AA Sempill, Hugh; Scottish mathematician (1596-1654).

VA Word for "Mars" in Sumerian.

AA Babylonian moon god.

\footnotetext{
P: $\quad$ planetary system (see page xvi)

Sa: satellite (see page $x v i$ ).

lat: latitude of feature center.

long: longitude of feature center.
}

diam: diameter or long dimension of feature.

ct: continent of name origin (see page $284 \mathrm{ff}$.)

et: ethnicity of name origin (see page $284 \mathrm{ff}$.)

as: name approval status (see page xvii). ad: name approval date (year).

ref: reference source for name (see page $287 \mathrm{ff}$.)

ft: feature type (see page 290). 


\section{ALPHABETICAL LIST OF NAMES}

\begin{tabular}{|c|c|c|c|c|c|c|c|c|c|}
\hline $\mathbf{P}$ & $\mathbf{S a}$ & Name & lat & long & diam & ct & et & as & ad \\
\hline $\mathrm{M}$ & M & Sinai & $20.0 \mathrm{~S}$ & $70.0 \mathrm{~W}$ & 0 & $\mathrm{AF}$ & EG & 5 & 1958 \\
\hline $\begin{array}{l}\mathrm{M} \\
\mathrm{M} \\
\mathrm{H}\end{array}$ & $\begin{array}{l}\mathrm{M} \\
\mathrm{M} \\
\mathrm{H}\end{array}$ & $\begin{array}{l}\text { Sinai Dorsa } \\
\text { Sinai Planum } \\
\text { Sinan }\end{array}$ & $\begin{array}{l}13.1 \mathrm{~S} \\
12.5 \mathrm{~S} \\
15.5 \mathrm{~N}\end{array}$ & $\begin{array}{l}81.4 \mathrm{~W} \\
87.1 \mathrm{~W} \\
29.8 \mathrm{~W}\end{array}$ & $\begin{array}{r}189 \\
1064 \\
147\end{array}$ & $\begin{array}{l}\text { AF } \\
\text { AF } \\
\text { AS }\end{array}$ & $\begin{array}{l}\text { EG } \\
\text { EG } \\
\text { TU }\end{array}$ & $\begin{array}{l}5 \\
5 \\
5\end{array}$ & $\begin{array}{l}1988 \\
1973 \\
1976\end{array}$ \\
\hline $\begin{array}{l}\text { V } \\
\mathrm{L}\end{array}$ & $\begin{array}{l}\mathrm{V} \\
\mathrm{L}\end{array}$ & $\begin{array}{l}\text { Sinann Vallis } \\
\text { Sinas }\end{array}$ & $\begin{array}{r}49.0 \mathrm{~S} \\
8.8 \mathrm{~N}\end{array}$ & $\begin{array}{r}270.0 \mathrm{E} \\
31.6 \mathrm{E}\end{array}$ & $\begin{array}{r}425 \\
11\end{array}$ & $\begin{array}{l}\text { EU } \\
\text { EU }\end{array}$ & $\begin{array}{r}\text { IR } \\
\text { GR }\end{array}$ & $\begin{array}{l}5 \\
5\end{array}$ & $\begin{array}{l}1994 \\
1935\end{array}$ \\
\hline $\begin{array}{l}M \\
S\end{array}$ & $\begin{array}{l}M \\
\text { en }\end{array}$ & $\begin{array}{l}\text { Sinda } \\
\text { Sindbad }\end{array}$ & $\begin{array}{l}16.0 \mathrm{~N} \\
68.9 \mathrm{~N}\end{array}$ & $\begin{array}{l}248.9 \mathrm{~W} \\
211.4 \mathrm{~W}\end{array}$ & $\begin{array}{r}0 \\
23\end{array}$ & $\begin{array}{l}\text { UR } \\
\text { AS }\end{array}$ & $\begin{array}{l}\text { RU } \\
\text { AR }\end{array}$ & $\begin{array}{l}5 \\
5\end{array}$ & $\begin{array}{l}1988 \\
1982\end{array}$ \\
\hline $\begin{array}{l}M \\
M \\
L \\
L \\
H \\
L \\
L \\
L \\
L \\
L \\
L\end{array}$ & $\begin{array}{l}\text { M } \\
\text { M } \\
\text { L } \\
\text { L } \\
\text { H } \\
\text { L } \\
\text { L } \\
\text { L } \\
\text { L } \\
\text { L } \\
\text { L }\end{array}$ & $\begin{array}{l}\text { Singa } \\
\text { Sinop } \\
\text { Sinus Aestuun } \\
\text { Sinus Amoris } \\
\text { Sinus Argiphontae } \\
\text { Sinus Asperitatis } \\
\text { Sinus Concordiae } \\
\text { Sinus Fidei } \\
\text { Sinus Honoris } \\
\text { Sinus Iridum } \\
\text { Sinus Lunicus }\end{array}$ & $\begin{array}{l}22.8 \mathrm{~S} \\
23.5 \mathrm{~S} \\
10.9 \mathrm{~N} \\
18.1 \mathrm{~N} \\
10.0 \mathrm{~S} \\
3.8 \mathrm{~S} \\
10.8 \mathrm{~N} \\
18.0 \mathrm{~N} \\
11.7 \mathrm{~N} \\
44.1 \mathrm{~N} \\
31.8 \mathrm{~N}\end{array}$ & $\begin{array}{c}17.2 \mathrm{~W} \\
249.3 \mathrm{~W} \\
8.8 \mathrm{~W} \\
39.1 \mathrm{E} \\
335.0 \mathrm{~W} \\
27.4 \mathrm{E} \\
43.2 \mathrm{E} \\
2.0 \mathrm{E} \\
18.1 \mathrm{E} \\
31.5 \mathrm{~W} \\
1.4 \mathrm{~W}\end{array}$ & $\begin{array}{r}11 \\
0 \\
290 \\
130 \\
0 \\
206 \\
142 \\
70 \\
109 \\
236 \\
126\end{array}$ & $\begin{array}{l}\text { AF } \\
\text { AS } \\
\text { EU } \\
\text { EU } \\
\text { EU } \\
\text { EU } \\
\text { EU } \\
\text { EU } \\
\text { EU } \\
\text { EU } \\
\text { EU }\end{array}$ & $\begin{array}{l}\text { SU } \\
\text { TU } \\
\text { LA } \\
\text { LA } \\
\text { RM } \\
\text { LA } \\
\text { LA } \\
\text { LA } \\
\text { LA } \\
\text { LA } \\
\text { LA }\end{array}$ & $\begin{array}{l}5 \\
5 \\
5 \\
5 \\
5 \\
5 \\
5 \\
5 \\
5 \\
5 \\
5\end{array}$ & $\begin{array}{l}1976 \\
1989 \\
1935 \\
1976 \\
1976 \\
1976 \\
1976 \\
1976 \\
1976 \\
1935 \\
1970\end{array}$ \\
\hline $\begin{array}{l}\mathrm{L} \\
\mathrm{M}\end{array}$ & $\begin{array}{l}\mathrm{L} \\
\mathrm{M}\end{array}$ & $\begin{array}{l}\text { Sinus Medii } \\
\text { Sinus Meridiani }\end{array}$ & $\begin{array}{l}2.4 \mathrm{~N} \\
5.0 \mathrm{~S}\end{array}$ & $\begin{array}{l}1.7 \mathrm{E} \\
0.0 \mathrm{~W}\end{array}$ & $\begin{array}{r}335 \\
0\end{array}$ & $\begin{array}{l}\text { EU } \\
\text { EU }\end{array}$ & $\begin{array}{l}\text { LA } \\
\text { LA }\end{array}$ & $\begin{array}{l}5 \\
5\end{array}$ & $\begin{array}{l}1935 \\
1958\end{array}$ \\
\hline $\begin{array}{l}\mathrm{L} \\
\mathrm{M}\end{array}$ & $\stackrel{\mathrm{L}}{\mathrm{M}}$ & $\begin{array}{l}\text { Sinus Roris } \\
\text { Sinus Sabaeus }\end{array}$ & $\begin{array}{r}54.0 \mathrm{~N} \\
8.0 \mathrm{~S}\end{array}$ & $\begin{array}{r}56.6 \mathrm{~W} \\
340.0 \mathrm{~W}\end{array}$ & $\begin{array}{r}202 \\
0\end{array}$ & $\begin{array}{l}\text { EU } \\
\text { EU }\end{array}$ & $\begin{array}{l}\text { LA } \\
\text { LA }\end{array}$ & $\begin{array}{l}5 \\
5\end{array}$ & $\begin{array}{l}1935 \\
1958\end{array}$ \\
\hline $\begin{array}{l}\mathrm{L} \\
\mathrm{N}\end{array}$ & $\begin{array}{l}\mathrm{L} \\
\mathrm{tr}\end{array}$ & $\begin{array}{l}\text { Sinus Successus } \\
\text { Sipapu Planitia }\end{array}$ & $\begin{array}{l}0.9 \mathrm{~N} \\
4.0 \mathrm{~S}\end{array}$ & $\begin{array}{l}59.0 \mathrm{E} \\
36.0 \mathrm{E}\end{array}$ & $\begin{array}{r}132 \\
0\end{array}$ & $\begin{array}{l}\text { EU } \\
\text { NA }\end{array}$ & $\begin{array}{l}\text { LA } \\
\text { PU }\end{array}$ & $\begin{array}{l}5 \\
5\end{array}$ & $\begin{array}{r}1979 \\
91\end{array}$ \\
\hline $\begin{array}{l}J \\
V\end{array}$ & ga & $\begin{array}{l}\text { Sippar Sulcus } \\
\text { Sirani }\end{array}$ & $\begin{array}{l}15.8 \mathrm{~S} \\
31.5 \mathrm{~S}\end{array}$ & $\begin{array}{l}191.0 \mathrm{~W} \\
230.4 \mathrm{E}\end{array}$ & $\begin{array}{r}1539 \\
28\end{array}$ & $\begin{array}{l}\text { AS } \\
\text { EU }\end{array}$ & AB & $\begin{array}{l}5 \\
5\end{array}$ & $\begin{array}{l}1985 \\
1994\end{array}$ \\
\hline $\begin{array}{l}\mathrm{M} \\
\mathrm{L}\end{array}$ & $\begin{array}{l}\mathrm{M} \\
\mathrm{L}\end{array}$ & $\begin{array}{l}\text { Sirenum Fossae } \\
\text { Sirsalis }\end{array}$ & $\begin{array}{l}34.5 \mathrm{~S} \\
12.5 \mathrm{~S}\end{array}$ & $\begin{array}{r}158.2 \mathrm{~W} \\
60.4 \mathrm{~W}\end{array}$ & $\begin{array}{r}2712 \\
42\end{array}$ & $\begin{array}{l}\text { EU } \\
\text { EU }\end{array}$ & $\begin{array}{r}\text { GR } \\
\text { IT }\end{array}$ & $\begin{array}{l}5 \\
5\end{array}$ & $\begin{array}{l}1973 \\
1935\end{array}$ \\
\hline $\mathrm{L}$ & $\mathrm{L}$ & Sisakyan & $41.2 \mathrm{~N}$ & $109.0 \mathrm{E}$ & 34 & UR & SO & 5 & 1970 \\
\hline $\begin{array}{l}\text { M } \\
M\end{array}$ & $\begin{array}{l}\mathrm{M} \\
\mathbf{M}\end{array}$ & $\begin{array}{l}\text { Sisyphi Cavi } \\
\text { Sisyphi Montes }\end{array}$ & $\begin{array}{l}71.9 \mathrm{~S} \\
69.8 \mathrm{~S}\end{array}$ & $\begin{array}{r}3.0 \mathrm{~W} \\
346.9 \mathrm{~W}\end{array}$ & $\begin{array}{l}362 \\
198\end{array}$ & $\begin{array}{l}\text { EU } \\
\text { EU }\end{array}$ & $\begin{array}{l}\text { GR } \\
\text { GR }\end{array}$ & $\begin{array}{l}5 \\
5\end{array}$ & $\begin{array}{l}1976 \\
1985\end{array}$ \\
\hline $\begin{array}{l}\mathrm{L} \\
\mathrm{V} \\
\mathrm{M}\end{array}$ & $\begin{array}{l}\mathrm{L} \\
\mathrm{V} \\
\mathrm{M}\end{array}$ & $\begin{array}{l}\text { Sita } \\
\text { Sith Corona } \\
\text { Sithonius Lacus }\end{array}$ & $\begin{array}{l}4.6 \mathrm{~N} \\
10.2 \mathrm{~S} \\
45.0 \mathrm{~N}\end{array}$ & $\begin{array}{l}120.8 \mathrm{E} \\
176.5 \mathrm{E} \\
245.0 \mathrm{~W}\end{array}$ & $\begin{array}{r}2 \\
350 \\
0\end{array}$ & $\begin{array}{l}\text { AS } \\
\text { EU } \\
\text { EU }\end{array}$ & $\begin{array}{l}\text { IN } \\
\text { NS } \\
\text { LA }\end{array}$ & $\begin{array}{l}5 \\
5 \\
5\end{array}$ & $\begin{array}{l}1976 \\
1994 \\
1958\end{array}$ \\
\hline $\begin{array}{l}\mathrm{M} \\
\mathrm{V}\end{array}$ & $\begin{array}{l}\mathrm{M} \\
\mathrm{V}\end{array}$ & $\begin{array}{l}\text { Sitka } \\
\text { Sitwell }\end{array}$ & $\begin{array}{r}4.3 \mathrm{~S} \\
16.7 \mathrm{~N}\end{array}$ & $\begin{array}{l}39.3 \mathrm{~W} \\
190.3 \mathrm{E}\end{array}$ & $\begin{array}{l}11 \\
35\end{array}$ & $\begin{array}{l}\text { US } \\
\text { EU }\end{array}$ & $\begin{array}{l}\mathrm{AL} \\
\mathrm{EN}\end{array}$ & $\begin{array}{l}5 \\
5\end{array}$ & $\begin{array}{l}1976 \\
1994\end{array}$ \\
\hline $\begin{array}{l}\mathbf{J} \\
\mathbf{J}\end{array}$ & $\begin{array}{l}\text { io } \\
\text { ga }\end{array}$ & $\begin{array}{l}\text { Siun Patera } \\
\text { Siwah Facula }\end{array}$ & $\begin{array}{r}49.8 \mathrm{~S} \\
7.5 \mathrm{~N}\end{array}$ & $\begin{array}{r}1.4 \mathrm{~W} \\
143.2 \mathrm{~W}\end{array}$ & $\begin{array}{r}50 \\
220\end{array}$ & $\begin{array}{l}\text { AS } \\
\text { AF }\end{array}$ & $\begin{array}{l}\text { NA } \\
\text { EG }\end{array}$ & $\begin{array}{l}5 \\
5\end{array}$ & $\begin{array}{l}1985 \\
1985\end{array}$ \\
\hline $\begin{array}{l}\text { V } \\
\text { L }\end{array}$ & $\begin{array}{l}\text { V } \\
\text { L }\end{array}$ & $\begin{array}{l}\text { Skadi Mons } \\
\text { Sklodowska }\end{array}$ & $\begin{array}{l}64.0 \mathrm{~N} \\
18.2 \mathrm{~S}\end{array}$ & $\begin{array}{r}4.0 \mathrm{E} \\
95.5 \mathrm{E}\end{array}$ & $\begin{array}{r}40 \\
127\end{array}$ & $\begin{array}{l}\text { EU } \\
\text { EU }\end{array}$ & $\begin{array}{l}\text { NS } \\
\text { PO }\end{array}$ & $\begin{array}{l}5 \\
5\end{array}$ & $\begin{array}{l}1994 \\
1970\end{array}$ \\
\hline M & M & Sklodowska & $33.8 \mathrm{~N}$ & $2.8 \mathrm{~W}$ & 116 & EU & $\mathrm{PO}$ & 5 & 1973 \\
\hline $\begin{array}{l}\text { J } \\
\text { J }\end{array}$ & $\begin{array}{l}\mathrm{ca} \\
\mathrm{ca}\end{array}$ & $\begin{array}{l}\text { Sköll } \\
\text { Skuld }\end{array}$ & $\begin{array}{l}55.6 \mathrm{~N} \\
10.1 \mathrm{~N}\end{array}$ & $\begin{array}{r}315.3 \mathrm{~W} \\
37.7 \mathrm{~W}\end{array}$ & $\begin{array}{l}55 \\
81\end{array}$ & $\begin{array}{l}E U \\
E U\end{array}$ & $\begin{array}{l}\text { NS } \\
\text { NS }\end{array}$ & $\begin{array}{l}5 \\
5\end{array}$ & $\begin{array}{l}1979 \\
1979\end{array}$ \\
\hline $\mathrm{U}$ & um & Skynd & $1.8 \mathrm{~S}$ & $331.7 \mathrm{E}$ & 72 & EU & GB & 5 & 1988 \\
\hline $\begin{array}{l}N \\
M\end{array}$ & $\begin{array}{l}\mathrm{tr} \\
\mathrm{M}\end{array}$ & $\begin{array}{l}\text { Slidr Sulci } \\
\text { Slipher }\end{array}$ & $\begin{array}{l}23.5 \mathrm{~N} \\
47.7 \mathrm{~S}\end{array}$ & $\begin{array}{c}350.0 \mathrm{E} \\
84.5 \mathrm{~W}\end{array}$ & $\begin{array}{r}0 \\
129\end{array}$ & $\begin{array}{l}\text { EU } \\
\text { NA }\end{array}$ & $\begin{array}{r}\text { NS } \\
\text { AM }\end{array}$ & $\begin{array}{l}5 \\
5\end{array}$ & $\begin{array}{r}91 \\
1973\end{array}$ \\
\hline
\end{tabular}

\section{$\mathrm{ft}$ origin}

AL Biblical; named for area next to Mare Erythraeum (Indian Ocean).

DO Classical albedo feature name.

PM Classical albedo feature name.

AA Joseph: Turkish architect (1489-1588)

VA Irish river goddess.

AA Simon; Greek benefactor (1810-1876).

AA Town in Russia.

AA Voyager who had many marvelous adventures on seven voyages.

AA Town in Sudan.

AA Town in Turkey.

SI "Seething Bay".

SI "Bay of Love".

AL Albedo feature; Antoniadi map.

SI "Bay of Roughness".

SI "Bay of Harmony".

SI "Bay of Trust".

SI "Bay of Honor".

SI "Bay of Rainbows".

SI "Lunik Bay"-landing area of Luna (Lunik) 2.

SI "Bay of the center".

AL "Middle Bay," from

Flammarion map.

SI "Bay of Dew".

AL Today's Red Sea; Saba was part of southern Arabian Peninsula.

SI "Bay of Success".

PL Pueblo (USA) hole or lake of emergence from underworld.

SU Ancient Babylonian town.

AA Elisabetta; Italian painter, etcher, printmaker (1638-1665).

FO Classical albedo feature name.

AA Sersale, Gerolamo; Italian astronomer (1584-1654).

AA Norajr M.; Soviet doctor (1907-1966)

CB Classical albedo feature name.

MO From albedo feature at $67 \mathrm{~S}, 348 \mathrm{~W}$.

AA Indian female name.

CR Norse harvest goddess.

AL Region inhabited by Sithonii; synonym of Thrace.

AA Town in Alaska, USA.

AA Edith; English poet, critic (1887-1964).

PE Nanai (Siberia) sun god.

FA Oasis oracle of Zeus-Ammon; visited by Alexander.

MO Norse mountain goddess.

AA Marie (Madame Curie); Polish physicist, chemist Nobel laureate 1867-1934

AA Marie; Polish-born French chemist (Mme P. Curie) (1867-1934)

AA Norse wolf.

AA Norse; maiden living near Yggdrasill who governed the fate of humans.

AA Troll who stole three wives of a man living in Englerup.

SU Norse river of daggers and spears.

AA Vesto M: American astronomer (1875-1969).

\footnotetext{
P: planetary system (see page xvi)

Sa: satellite (see page xvi).

lat: latitude of feature center.

long: longitude of feature center.
}

diam: diameter or long dimension of feature.

ct: continent of name origin (see page $284 \mathrm{ff}$.)

et: ethnicity of name origin (see page $284 \mathrm{ff}$.)

as: name approval status (see page xvii). ad: name approval date (year).

ref: reference source for name (see page $287 \mathrm{ff}$.).

ft: feature type (see page 290). 


\section{ALPHABETICAL LIST OF NAMES}

\section{P Sa Name}

L L Slipher

L L Slocum

H H Smetana

M M Smith

L L Smith

L L Smithson

L L Smoky Mountains

L L Smoluchowski

V V Snegurochka Planitia

L L Snellius

L L Sniadecki

H H Snorri

L L Snowman

H H Sobkou Planitia

L L Soddy

M M Sögel

M M Sokol

M M Solano

M M Solis Dorsa

M M Solis Lacus

M M Solis Planum

$\mathrm{H} \quad \mathrm{H}$ Solitudo Admetei

H H Solitudo Alarum

H H Solitudo Aphrodites

H H Solitudo Atlantis

H H Solitudo Criophori

H H Solitudo Helii

H H Solitudo Hermas

H H Solitudo Horarum

$\mathrm{H} H$ Solitudo Iovis

$\mathrm{H} \quad \mathrm{H}$ Solitudo Lycaonis

H H Solitudo Maiae

H H Solitudo Martis

H H Solitudo Neptuni

H H Solitudo Persephones

$\mathrm{H} H$ Solitudo Phoenicis

H H Solitudo Promethei

V V Somagalags Corona

L L Somerville

L L Sommerfeld

L L Sömmering

M M Soochow

V V Sophia

$55.0 \mathrm{~N}$

$15.0 \mathrm{~S}$

$25.0 \mathrm{~N}$
$35.0 \mathrm{~S}$

$35.0 \mathrm{~S}$

$30.0 \mathrm{~N}$

$0.1 \mathrm{~N}$

\section{lat long diam}

$\begin{array}{rrrrrrrr}49.5 \mathrm{~N} & 160.1 \mathrm{E} & 69 & \text { NA } & \text { AM } & 5 & 1970 & 0 \\ 3.0 \mathrm{~S} & 89.0 \mathrm{E} & 13 & \text { NA } & \text { AM } & 5 & 1976 & 0 \\ 48.5 \mathrm{~S} & 70.2 \mathrm{~W} & 190 & \text { EU } & \text { CZ } & 5 & 1985 & 59 \\ 66.1 \mathrm{~S} & 102.8 \mathrm{~W} & 71 & \text { EU } & \text { GB } & 5 & 1973 & 68 \\ 31.6 \mathrm{~S} & 150.2 \mathrm{~W} & 34 & \text { NA } & \text { AM } & 5 & 1988 & 88\end{array}$

$\begin{array}{rrrrrrrr}2.4 \mathrm{~N} & 53.6 \mathrm{E} & 5 & \text { EU } & \text { GB } & 5 & 1976 & 0 \\ 8.8 \mathrm{~S} & 15.6 \mathrm{E} & 3 & \text { NA } & \text { AM } & 5 & 1973 & 59 \\ & & & & & & & \\ 60.3 \mathrm{~N} & 96.8 \mathrm{~W} & 83 & \text { EU } & \text { PO } & 5 & 1970 & 0 \\ & & & & & & & \\ 86.6 \mathrm{~N} & 328.0 \mathrm{E} & 2773 & \text { UR } & \text { RU } & 5 & 1985 & 64 \\ & & & & & & & \\ 29.3 \mathrm{~S} & 55.7 \mathrm{E} & 82 & \text { EU } & \text { DU } & 5 & 1935 & 68 \\ & & & & & & & \\ 22.5 \mathrm{~S} & 168.9 \mathrm{~W} & 43 & \text { EU } & \text { PO } & 5 & 1970 & 0 \\ & & & & & & & \\ 9.0 \mathrm{~S} & 82.9 \mathrm{~W} & 19 & \text { EU } & \text { IC } & 5 & 1976 & 59 \\ & & & & & & & \\ 3.2 \mathrm{~S} & 23.4 \mathrm{~W} & 1 & \text { NA } & \text { AM } & 5 & 1973 & 59 \\ & & & & & & & \\ 39.9 \mathrm{~N} & 129.9 \mathrm{~W} & 0 & \text { UN } & \text { UN } & 5 & 1976 & 80 \\ 0.4 \mathrm{~N} & 121.8 \mathrm{E} & 42 & \text { EU } & \text { GB } & 5 & 1976 & 0 \\ & & & & & & & \\ 21.7 \mathrm{~N} & 55.1 \mathrm{~W} & 29 & \text { EU } & \text { GE } & 5 & 1976 & 36 \\ 42.8 \mathrm{~S} & 40.5 \mathrm{~W} & 20 & \text { UR } & \text { RU } & 5 & 1976 & 36 \\ 27.0 \mathrm{~S} & 251.0 \mathrm{~W} & 0 & \text { AS } & \text { PH } & 5 & 1989 & 74 \\ 23.4 \mathrm{~S} & 78.9 \mathrm{~W} & 771 & \text { EU } & \text { LA } & 5 & 1976 & 57 \\ 28.0 \mathrm{~S} & 90.0 \mathrm{~W} & 0 & \text { EU } & \text { LA } & 5 & 1958 & 60\end{array}$

$20.9 \mathrm{~S}$

$0.0 \mathrm{~N}$

$94.6 \mathrm{~W}$

$90.0 \mathrm{~W}$

$290.0 \mathrm{~W}$

$290.0 \mathrm{~W}$

$210.0 \mathrm{~W}$

$230.0 \mathrm{~W}$

1464

$180.0 \mathrm{~W}$

$45.0 \mathrm{~W}$

$25.0 \mathrm{~N} \quad 115.0 \mathrm{~W}$

$0.0 \mathrm{~N} \quad 0.0 \mathrm{~W}$

$0.0 \mathrm{~N} \quad 107.0 \mathrm{~W}$

$15.0 \mathrm{~S}$

$155.0 \mathrm{~W}$

$100.0 \mathrm{~W}$

$41.0 \mathrm{~S} 225.0 \mathrm{~W}$

$25.0 \mathrm{~N} \quad 225.0 \mathrm{~W}$

45.0S $142.5 \mathrm{~W}$

9.3N $\quad 348.5 \mathrm{E}$

$8.3 \mathrm{~S} \quad 64.9 \mathrm{E}$

$65.2 \mathrm{~N} \quad 162.4 \mathrm{~W}$

$7.5 \mathrm{~W}$

$16.8 \mathrm{~N}$

$28.9 \mathrm{~W}$

$28.7 \mathrm{~S} \quad 18.7 \mathrm{E}$

464
0
0
0
0

EU LA

EU RM

EU RM

EU RM

EU RM

EU RM

\section{EU RM}

EU RM

EU RM

EU RM

EU RM

EU RM

EU RM

EU RM

EU RM

$5 \quad 1973$

$5 \quad 1976$

$5 \quad 1976$

51976

51976

51976

1976

51976

51976

$5 \quad 1976$

51976

$5 \quad 1976$

$5 \quad 1976$

51976

51976

$\begin{array}{lll}0 & \text { EU } & \text { RM } \\ 0 & \text { EU } & \text { RM }\end{array}$

$5 \quad 1976$

51976

105

NA CA

$5 \quad 1994$

EU SC

51976

169

EU GE

$5 \quad 1970$

28

EU GE

51935

30
17 $\begin{array}{ll}\text { AS } & \text { CH } \\ \text { EU } & \text { GR }\end{array}$ $\begin{array}{ll}5 & 1976 \\ 5 & 1994\end{array}$
AA Earl C.; American astronomer (1883-1964); Vesto M.; American astronomer (1875-1969).

AA Frederick; American astronomer (1873-1944).

AA Bedrich; Czechoslovakian composer (1824-1884).

AA William; British geologist-engineer (1769-1839).

AA Michael John; member of the Challenger crew (1945-1986); previous designation Barringer $M$

AA James; British chemist, mineralogist (1765-1829).

LF Astronaut-named feature, Apollo 16 site.

AA Marian; Polish physicist (1872-1917).

PL Snow maiden in Russian folktales, melted in spring.

AA Snell, Willebrod van Roijen; Dutch mathematician, astronomer, optician (1591-1626).

AA Jan; Polish astronomer, mathematician (1756-1830).

AA Sturluson; Icelandic saga writer and poet (1179-1241).

LF Astronaut-named feature, Apollo 12 site.

PL Messenger god.

AA Frederick; British physicist; Nobel laureate (1877-1956).

AA Town in Germany.

AA Town in Russia.

AA Town in Phillipines.

DO Classical albedo feature name

AL "Lake of the Sun"; the so-called "Eye of Mars," connecting East with West.

PM Classical albedo feature name.

AL Albedo feature; Antoniadi map.

AL Albedo feature; Antoniadi map.

AL Albedo feature; Antoniadi map.

AL Albedo feature; Antoniadi map.

AL Albedo name for unimaged H-9 region.

AL Albedo feature; Antoniadi map.

AL Albedo name for $\mathrm{H}-11$, Discovery region.

AL Albedo feature; Antoniadi map.

AL Albedo feature; Antoniadi map.

$\mathrm{AL}$. Albedo name for $\mathrm{H}-7$, Beethoven region.

AL Albedo feature; Antoniadi map.

AL Albedo feature; Antoniadi map.

AL Albedo feature; Antoniadi map.

$\mathrm{AL}$ Albedo name for unimaged $\mathrm{H}-13$ region.

AL Albedo feature; Antoniadi map.

AL Albedo name for $\mathrm{H}-12$, Michelangelo region.

CR Bella Coola earth mother.

AA Mary Fairfax; Scottish physicist, mathematician (1780-1872).

AA Arnold J. W.; German physicist (1868-1951).

AA Samuel Thomas; German doctor (1755-1830)

AA Town in China (Kiangsu).

AA First name from Greek.

\footnotetext{
P: $\quad$ planetary system (see page xvi).

Sa: satellite (see page $\mathrm{xvi}$ ).

lat: latitude of feature center.

long: longitude of feature center.
}

diam: diameter or long dimension of feature.

ct: continent of name origin (see page $284 \mathrm{ff}$.)

et: ethnicity of name origin (see page $284 \mathrm{ff}$.)

as: name approval status (see page xvii). ad: name approval date (year).

ref: reference source for name (see page $287 \mathrm{ff}$.)

ft: feature type (see page 290). 


\section{ALPHABETICAL LIST OF NAMES}

\section{P Sa Name}

H H Sophocles

L L [Sophocles]

H H Sor Juana

L L Soraya

H H Sōseki

L L Sosigenes

H H Sōtatsu

M M Souris

L L South

M M South

L L South Cluster

L L South Massif

L L South Ray

A gs Spa

L L $\quad$ Spallanzani

M M Spallanzani

L L Spencer Jones

H H Spitteler

L L Spook

L L Spörer

L L Spot

$\mathrm{U}$ ar Sprite Vallis

M M Spry

L L Spur

M M Spur

L L Spurr

M M Srīpur

L L St. George

L L St. John

L L Stadius

V V Stanton

L L Stark

L L Statio Tranquillitatis

L L Stearns

L L Stebbins

L L Stefan

V V Stefania

M M Stege

$\mathrm{V} \quad \mathrm{V}$ Stein

L L Stein lat

long

\begin{tabular}{|c|c|c|c|c|c|c|}
\hline $\begin{array}{r}7.0 \mathrm{~S} \\
21.5 \mathrm{~S}\end{array}$ & $\begin{array}{l}145.7 \mathrm{~W} \\
119.8 \mathrm{E}\end{array}$ & $\begin{array}{r}150 \\
0\end{array}$ & $\begin{array}{l}\text { EU } \\
\text { EU }\end{array}$ & $\begin{array}{l}\text { GR } \\
\text { GR }\end{array}$ & $\begin{array}{l}5 \\
6\end{array}$ & $\begin{array}{r}1976 \\
0\end{array}$ \\
\hline $49.0 \mathrm{~N}$ & $23.9 \mathrm{~W}$ & 93 & NA & ME & 5 & 1979 \\
\hline $\begin{array}{l}12.9 \mathrm{~S} \\
38.9 \mathrm{~N}\end{array}$ & $\begin{array}{r}1.6 \mathrm{~W} \\
37.7 \mathrm{~W}\end{array}$ & $\begin{array}{r}2 \\
90\end{array}$ & $\begin{array}{l}\text { AS } \\
\text { AS }\end{array}$ & $\begin{array}{l}\text { PE } \\
\text { JA }\end{array}$ & $\begin{array}{l}5 \\
5\end{array}$ & $\begin{array}{l}1976 \\
1985\end{array}$ \\
\hline $8.7 \mathrm{~N}$ & $17.6 \mathrm{E}$ & 17 & EU & GR & 5 & 1935 \\
\hline $49.1 S$ & $18.1 \mathrm{~W}$ & 165 & AS & JA & 5 & 1976 \\
\hline $\begin{array}{l}19.7 \mathrm{~N} \\
58.0 \mathrm{~N}\end{array}$ & $\begin{array}{r}246.9 \mathrm{~W} \\
50.8 \mathrm{~W}\end{array}$ & $\begin{array}{r}0 \\
104\end{array}$ & $\begin{array}{l}\text { NA } \\
\text { EU }\end{array}$ & $\begin{array}{l}\mathrm{CA} \\
\mathrm{GB}\end{array}$ & $\begin{array}{l}5 \\
5\end{array}$ & $\begin{array}{l}1988 \\
1935\end{array}$ \\
\hline $77.0 \mathrm{~S}$ & $338.0 \mathrm{~W}$ & 111 & EU & GB & 5 & 1973 \\
\hline $26.0 \mathrm{~N}$ & $3.7 \mathrm{E}$ & 2 & NA & $\mathrm{AM}$ & 5 & 1973 \\
\hline $20.0 \mathrm{~N}$ & $30.4 \mathrm{E}$ & 16 & $\mathrm{EU}$ & FR & 5 & 1973 \\
\hline $9.2 \mathrm{~S}$ & $15.4 \mathrm{E}$ & 1 & NA & $\mathrm{AM}$ & 5 & 1973 \\
\hline $\begin{array}{l}51.5 \mathrm{~N} \\
46.3 \mathrm{~S}\end{array}$ & $\begin{array}{c}152.0 \mathrm{~W} \\
24.7 \mathrm{E}\end{array}$ & $\begin{array}{r}6 \\
32\end{array}$ & $\begin{array}{l}\mathrm{EU} \\
\mathrm{EU}\end{array}$ & $\underset{\text { IT }}{\mathrm{BE}}$ & $\begin{array}{l}5 \\
5\end{array}$ & $\begin{array}{l}1994 \\
1935\end{array}$ \\
\hline $58.4 \mathrm{~S}$ & $273.5 \mathrm{~W}$ & 72 & EU & IT & 5 & 1973 \\
\hline $13.3 \mathrm{~N}$ & $165.6 \mathrm{E}$ & 85 & EU & GB & 5 & 1970 \\
\hline $68.6 \mathrm{~S}$ & $61.8 \mathrm{~W}$ & 68 & EU & SZ & 5 & 1976 \\
\hline $9.0 \mathrm{~S}$ & $15.5 \mathrm{E}$ & 0 & NA & $\mathrm{AM}$ & 5 & 1973 \\
\hline $4.3 \mathrm{~S}$ & $1.8 \mathrm{~W}$ & 27 & EU & GE & 5 & 1935 \\
\hline $9.0 \mathrm{~S}$ & $15.5 \mathrm{E}$ & 0 & NA & $\mathrm{AM}$ & 5 & 1973 \\
\hline $\begin{array}{r}14.9 \mathrm{~S} \\
3.8 \mathrm{~S} \\
27.9 \mathrm{~N}\end{array}$ & $\begin{array}{r}340.0 \mathrm{E} \\
38.6 \mathrm{~W} \\
1.2 \mathrm{~W}\end{array}$ & $\begin{array}{r}305 \\
6 \\
13\end{array}$ & $\begin{array}{l}\text { EU } \\
\text { US } \\
\text { NA }\end{array}$ & $\begin{array}{r}\text { GB } \\
\text { UT } \\
\text { AM }\end{array}$ & $\begin{array}{l}5 \\
5 \\
5\end{array}$ & $\begin{array}{l}1988 \\
1976 \\
1973\end{array}$ \\
\hline $\begin{array}{l}22.2 \mathrm{~N} \\
27.9 \mathrm{~N}\end{array}$ & $\begin{array}{r}52.3 \mathrm{~W} \\
1.2 \mathrm{~W}\end{array}$ & $\begin{array}{r}7 \\
11\end{array}$ & $\begin{array}{l}\text { US } \\
\text { NA }\end{array}$ & $\begin{array}{r}\text { TX } \\
\text { AM }\end{array}$ & $\begin{array}{l}5 \\
5\end{array}$ & $\begin{array}{l}1976 \\
1973\end{array}$ \\
\hline $\begin{array}{l}31.1 \mathrm{~S} \\
26.0 \mathrm{~N}\end{array}$ & $\begin{array}{c}100.6 \mathrm{~W} \\
3.5 \mathrm{E}\end{array}$ & $\begin{array}{l}0 \\
2\end{array}$ & $\begin{array}{l}\text { AS } \\
\text { NA }\end{array}$ & $\begin{array}{l}\text { BA } \\
\mathrm{AM}\end{array}$ & $\begin{array}{l}5 \\
5\end{array}$ & $\begin{array}{l}1989 \\
1973\end{array}$ \\
\hline $10.2 \mathrm{~N}$ & $150.2 \mathrm{E}$ & 68 & NA & AM & 5 & 1970 \\
\hline $10.5 \mathrm{~N}$ & $13.7 \mathrm{~W}$ & 69 & EU & $\mathrm{BE}$ & 5 & 1935 \\
\hline $23.4 \mathrm{~S}$ & $199.9 \mathrm{E}$ & 110 & NA & $\mathrm{AM}$ & 5 & 1994 \\
\hline $25.5 \mathrm{~S}$ & $134.6 \mathrm{E}$ & 49 & EU & GE & 5 & 1970 \\
\hline $0.8 \mathrm{~N}$ & $23.5 \mathrm{E}$ & 0 & $\mathrm{EU}$ & LA & 5 & 1970 \\
\hline $34.8 \mathrm{~N}$ & $162.6 \mathrm{E}$ & 36 & NA & $\mathrm{AM}$ & 5 & 1979 \\
\hline $64.8 \mathrm{~N}$ & $141.8 \mathrm{~W}$ & 131 & NA & $\mathrm{AM}$ & 5 & 1970 \\
\hline $46.0 \mathrm{~N}$ & $108.3 \mathrm{~W}$ & 125 & $\mathrm{EU}$ & AS & 5 & 1970 \\
\hline $\begin{array}{r}51.3 \mathrm{~N} \\
2.6 \mathrm{~N} \\
30.1 \mathrm{~S}\end{array}$ & $\begin{array}{c}333.3 \mathrm{E} \\
58.4 \mathrm{~W} \\
345.4 \mathrm{E}\end{array}$ & $\begin{array}{l}11 \\
72 \\
15\end{array}$ & $\begin{array}{l}\text { EU } \\
\text { EU } \\
\text { NA }\end{array}$ & $\begin{array}{l}\text { RO } \\
\text { DE } \\
\mathrm{AM}\end{array}$ & $\begin{array}{l}5 \\
5 \\
5\end{array}$ & $\begin{array}{l}1985 \\
1985 \\
1991\end{array}$ \\
\hline $7.2 \mathrm{~N}$ & $179.0 \mathrm{E}$ & 33 & EU & DU & 5 & 1970 \\
\hline
\end{tabular}

AA Greek dramatist (c. 496-406 B.C.).

AA Greek philosopher (c. 495-406 B.C.)

AA Ines de la Cruz; Mexican writer (1651-1695).

AA Persian female name.

AA Natsume (Kinosaka); Japanese novelist (1867-1916).

AA Greek astronomer, chronologist (unkn-fl. 46 B.C.)

AA Tawakaya; Japanese artist (1600-1643).

AA Town in Manitoba, Canada.

AA James; British astronomer (1785-1867).

AA James; British astronomer (1785-1867).

LF Astronaut-named feature, Apollo 15 site.

LF Astronaut-named feature Apollo 17 site.

LF Astronaut-named feature, Apollo 16 site.

AA Health resort in Belgium.

AA Lazzaro; Italian natural scientist, biologist (1729-1799).

AA Lazzaro; Italian biologist (1729-1799).

AA Sir Harold; British astronomer (1890-1960).

AA Carl; Swiss epic poet (1845-1924).

LF Astronaut-named feature, Apollo 16 site.

AA Friederich Wilhelm Gustav; German astronomer (1822-1895).

LF Astronaut-named feature, Apollo 16 site.

VA Earth spirits.

AA Town in Utah, USA

LF Astronaut-named feature, Apollo 15 site.

AA Town in Texas, USA.

AA Josiah Edward; American geologist (1870-1950).

AA Town in Bangladesh.

LF Astronaut-named feature, Apollo 15 site.

AA Charles E.; American solar physicist, astronomer (1857-1935)

AA Stade, Jan; Belgian astronomer, mathematician (1527-1579).

AA Elizabeth C.; American suffragist (1815-1902).

AA Johannes; German physicist; Nobel laureate (1874-1957).

LF "Tranquility Base", Apollo 11 landing site.

AA Carl Leo; American astronomer (1892-1972).

AA Joel; American astronomer (1878-1966).

AA Josef; Austrian physicist (1835-1893).

AA Romanian first name.

AA Town in Denmark.

AA Gertrude; American writer (1874-1946)

AA J. W.; Dutch astronomer (1871-1951).

\footnotetext{
P: $\quad$ planetary system (see page $x v i)$.

Sa: satellite (see page xvi).

lat: latitude of feature center.

long: longitude of feature center.
}

diam: diameter or long dimension of feature.

ct: continent of name origin (see page $284 \mathrm{ff}$.)

et: ethnicity of name origin (see page $284 \mathrm{ff}$.)

as: name approval status (see page $x v i i)$. ad: name approval date (year)

ref: reference source for name (see page $287 \mathrm{ff}$.).

ft: feature type (see page 290). 


\section{ALPHABETICAL LIST OF NAMES}

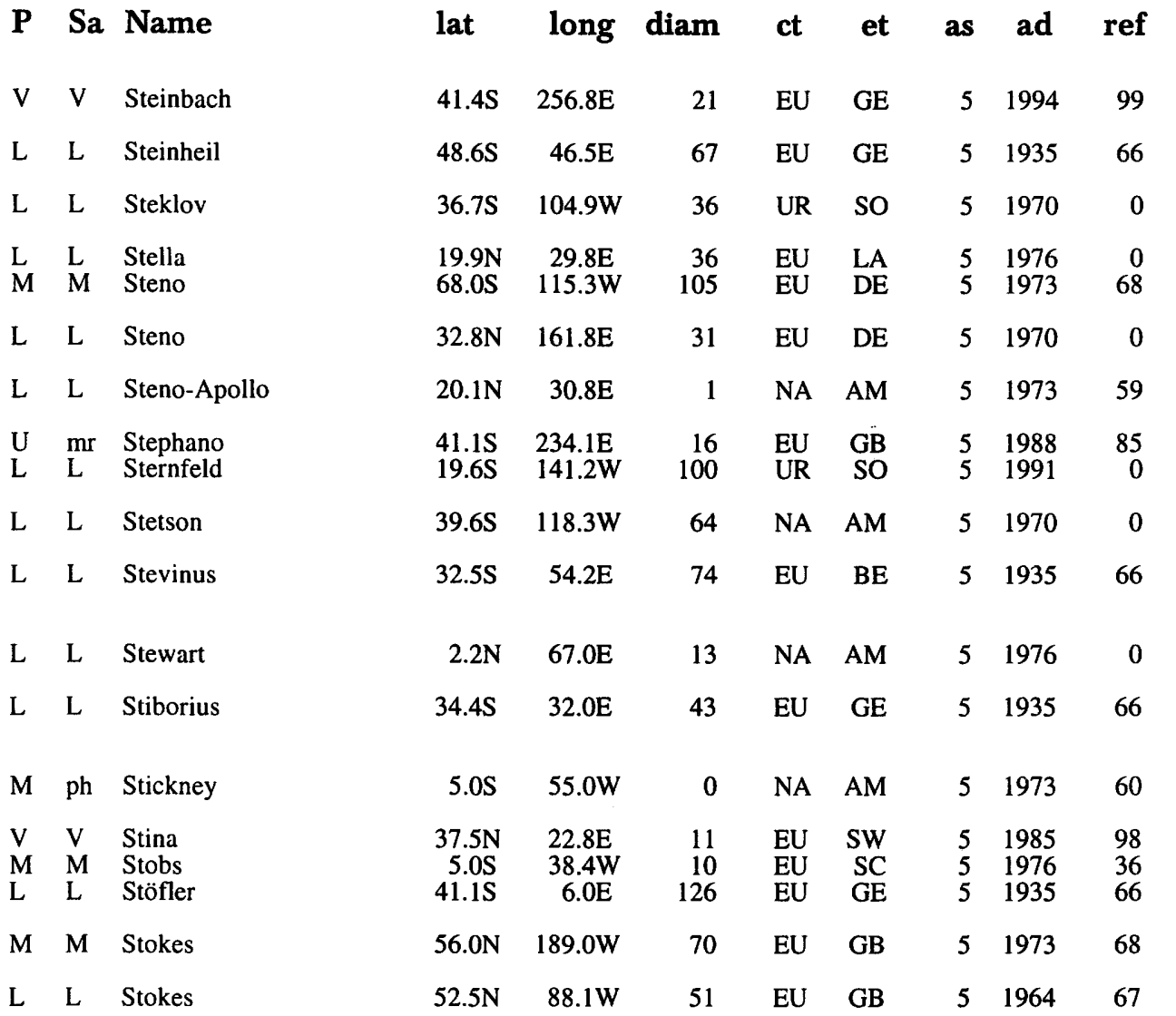

$\begin{array}{lll}\text { L } & \text { L } & \text { Stoletov } \\ \text { M } & \text { M } & \text { Ston } \\ \text { L } & \text { L } & \text { Stone Mountain } \\ \text { L } & \text { L } & \text { Stoney } \\ \text { M } & \text { M } & \text { Stoney } \\ \text { V } & \text { V } & \text { Stopes Patera } \\ \text { L } & \text { L } & \text { Störmer }\end{array}$

$\begin{array}{rrrrrrrr}45.1 \mathrm{~N} & 155.2 \mathrm{~W} & 42 & \text { UR } & \text { RU } & 5 & 1970 & 0 \\ 47.2 \mathrm{~N} & 237.4 \mathrm{~W} & 7 & \text { EU } & \text { YU } & 5 & 1979 & 36 \\ 9.1 \mathrm{~S} & 15.6 \mathrm{E} & 5 & \text { NA } & \text { AM } & 5 & 1973 & 59 \\ 55.3 \mathrm{~S} & 156.1 \mathrm{~W} & 45 & \text { EU } & \text { IR } & 5 & 1970 & 0 \\ 69.8 \mathrm{~S} & 138.4 \mathrm{~W} & 177 & \text { EU } & \text { IR } & 5 & 1973 & 68 \\ 42.6 \mathrm{~N} & 46.5 \mathrm{E} & 169 & \text { EU } & \text { GB } & 5 & 91 & 46 \\ 57.3 \mathrm{~N} & 146.3 \mathrm{E} & 69 & \text { EU } & \text { NO } & 5 & 1970 & 0\end{array}$

$\begin{array}{rrrrrrrr}9.8 \mathrm{~S} & 245.6 \mathrm{E} & 25 & \text { SA } & \text { AR } & 5 & 1994 & 99 \\ 43.2 \mathrm{~S} & 233.0 \mathrm{E} & 82 & \text { NA } & \text { AM } & 5 & 1994 & 87 \\ 61.9 \mathrm{~N} & 54.3 \mathrm{E} & 55 & \text { EU } & \text { GR } & 5 & 1935 & 66 \\ 5.8 \mathrm{~S} & 164.6 \mathrm{E} & 70 & \text { EU } & \text { GB } & 5 & 1970 & 0 \\ & & & & & & & \\ 50.5 \mathrm{~N} & 73.5 \mathrm{~W} & 190 & \text { UR } & \text { RU } & 5 & 1979 & 59 \\ & & & & & & & \\ 46.5 \mathrm{~S} & 10.5 \mathrm{~W} & 57 & \text { EU } & \text { GB } & 5 & 1935 & 66 \\ 53.7 \mathrm{~N} & 135.3 \mathrm{~W} & 190 & \text { EU } & \text { SW } & 5 & 1979 & 59\end{array}$

$21.7 \mathrm{~S} \quad 132.4 \mathrm{~W}$

$\begin{array}{llllll}61 & \mathrm{EU} & \mathrm{DE} & 5 & 1970 & 0\end{array}$

\section{ft origin}

AA Sabina; German sculptor (c. 1250).

AA Karl August von; German astronomer, physicist (1801-1870)

AA Vladimir A.; Soviet mathematician (1864-1926).

AA Latin female name.

AA Nicolaus; Danish geologist (1638-1686).

AA Nicolaus; Danish doctor (1638-1686).

LF Astronaut-named feature, Apollo 17 site.

AA A drunken butler in "The Tempest."

AA A. A.; Soviet space scientist (1905-1980).

AA Harlan T.; American astronomer, geophysicist (1885-1964).

AA Stevin, Simon; Belgian mathematician, physicist (1548-1620).

AA John Quincy; American astrophysicist (1894-1972).

AA Stoberl, Andreas; German astronomer, mathematician (1465-1515).

AA Angeline; American wife of astronomer A. Hall d.1938

AA Swedish first name.

AA Town in Scotland.

AA Johann; German astronomer, mathematician (1452-1531).

AA George G.; British physicist (1819-1903).

AA Sir George G.; British mathematician, physicist (1819-1903).

AA Aleksandr G.; Russian physicist (1839-1896).

AA Town in Yugoslavia.

LF Astronaut-named feature, Apollo 16 site.

AA George J.; Irish physicist (1826-1911).

AA George J.; Irish physicist (1826-1911).

PE Marie; English paleontologist (1880-1959).

AA F. Carl M.; Norwegian mathematician and astronomer, aurora research (1874-1957).

AA Alfonsina; Argentine poet (1892-1938).

AA Harriet B.; American novelist (1811-1896)

AA Greek geographer (54 B.C.A.D. 24).

AA Frederick J. M.; British astronomer, astrophysicist (1881-1960).

AA Igor Fyodorovich; Russian-born American composer (1882-1971).

AA Thomas; British astronomer (1621-1689).

AA August; Swedish playwright, novelist, and short-story writer (1849-1912)

AA Elis; Danish astronomer (1870-1947).

\footnotetext{
P: $\quad$ planetary system (see page $x v i)$.

Sa: $\quad$ satellite (see page xvi).

lat: latitude of feature center.

long: longitude of feature center.
}

diam: diameter or long dimension of feature. ct: continent of name origin (see page $284 \mathrm{ff}$.) et: ethnicity of name origin (see page $284 \mathrm{ff}$.) as: name approval status (see page xvii). ad: name approval date (year). ref: reference source for name (see page $287 \mathrm{ff}$.). ft: feature type (see page 290). 


\section{ALPHABETICAL LIST OF NAMES}

\section{P Sa Name}

L L Struve lat

$22.4 \mathrm{~N}$

$77.1 \mathrm{~W}$

diam

ct et

$\begin{array}{lllll}164 & \text { UR } & \text { RU } & 5 & 1964\end{array}$

$\begin{array}{rrrrrrrr}30.8 \mathrm{~S} & 20.2 \mathrm{E} & 69 & \mathrm{EU} & \mathrm{SC} & 5 & 1991 & 99 \\ 9.1 \mathrm{~S} & 15.5 \mathrm{E} & 1 & \text { NA } & \text { AM } & 5 & 1973 & 59 \\ 22.9 \mathrm{~N} & 217.6 \mathrm{~W} & 0 & \text { EU } & \text { RM } & 5 & 1985 & 56 \\ 23.6 \mathrm{~N} & 209.6 \mathrm{~W} & 99 & \text { EU } & \text { GR } & 5 & 1985 & 57 \\ 27.2 \mathrm{~N} & 210.3 \mathrm{~W} & 290 & \text { EU } & \text { GR } & 5 & 1985 & 57 \\ 30.0 \mathrm{~N} & 200.0 \mathrm{~W} & 0 & \text { EU } & \text { GR } & 5 & 1958 & 60\end{array}$

M M Styx Dorsum

M M Suata

L L Subbotin

M M Subur Vallis

M M Sucre

J ca Sudri

L L Suess

M M Suess

M M Sūf

$J$ io Sui Jen Patera

$\mathrm{H} \quad \mathrm{H}$ Suisei Planitia

M M Sulak

M M Sulci Gordii

V $\quad$ V Suliko

V V Sullivan

$\mathrm{H} \quad \mathrm{H}$ Sullivan

L L Sulpicius Gallus

$J$ ca Sumbur

M M Sumgin

L L Sumner

L L Sundman

L L Sung-Meı

V V Sunrta Corona

H H Sūr Dās

V V Surija

$\mathrm{H} \quad \mathrm{H}$ Surikov

M M Surinda Valles

M M Surius Vallis

$\mathrm{J}$ io Surt

M M Surt

L L Surveyor

L L Susan

V V Susanna

M M Suzhi

J io Svarog Patera

V V Sveta

L L Swann

L L Swasey

L L Swift

$M$ de Swift $\begin{array}{llllllll}11.6 \mathrm{~N} & 53.1 \mathrm{~W} & 30 & \mathrm{AF} & \mathrm{MA} & 5 & 1985 & 56\end{array}$

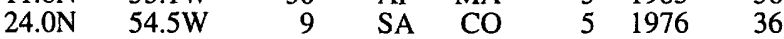

$\begin{array}{lllllll}55.4 \mathrm{~N} & 137.1 \mathrm{~W} & 69 & \mathrm{EU} & \mathrm{NS} & 5 & 1979\end{array}$

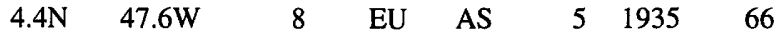

$\begin{array}{llllllll}67.1 \mathrm{~S} & 178.4 \mathrm{~W} & 72 & \mathrm{EU} & \text { AS } & 5 & 1973 & 68\end{array}$

$\begin{array}{llllllll}16.6 \mathrm{~N} & 38.2 \mathrm{~W} & 9 & \text { AS } & \text { JO } & 5 & 1976 & 36\end{array}$

$\begin{array}{llllllll}19.2 \mathrm{~S} & 4.3 \mathrm{~W} & 40 & \mathrm{AS} & \mathrm{CH} & 5 & 1985 & 30\end{array}$

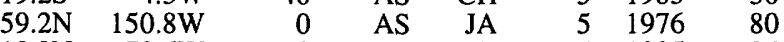

$\begin{array}{llllllll}18.3 \mathrm{~N} & 78.6 \mathrm{~W} & 0 & \text { UR } & \text { RU } & 5 & 1985 & 36\end{array}$

$\begin{array}{llllllll}17.9 \mathrm{~N} & 125.6 \mathrm{~W} & 316 & \mathrm{EU} & \mathrm{GR} & 5 & 1976 & 57\end{array}$

$\begin{array}{llrlllll}9.6 \mathrm{~N} & 214.6 \mathrm{E} & 19 & \text { UR } & \text { GE } & 5 & 1994 & 108\end{array}$

$13 \mathrm{~S} \quad 110.8 \mathrm{E} \quad 22 \quad$ NA AM

$19.6 \mathrm{~N} \quad 11.6 \mathrm{E}$

$67.1 \mathrm{~N} \quad 324.8 \mathrm{~W}$

$37.0 \mathrm{~S} \quad 48.6 \mathrm{~W}$

$37.5 \mathrm{~N} \quad 108.7 \mathrm{E}$

$10.8 \mathrm{~N} \quad 91.6 \mathrm{~W}$

$24.6 \mathrm{~N}$

$8.3 \mathrm{~N}$

47.1S

$5.3 \mathrm{~N}$

$37.1 \mathrm{~S}$

$29.3 \mathrm{~S}$

$60.3 \mathrm{~S}$

$45.5 \mathrm{~N}$

$17.0 \mathrm{~N}$

$3.2 \mathrm{~S}$

$11.0 \mathrm{~S}$

$6.0 \mathrm{~N}$

$27.6 \mathrm{~S}$

$48.3 \mathrm{~S}$

$82.6 \mathrm{~N}$

$52.0 \mathrm{~N}$

$5.5 \mathrm{~S} \quad 89.7 \mathrm{E}$

$19.3 \mathrm{~N}$

$0.0 \mathrm{~N}$
12 EU RM

UR BR

UR RU

NA AM

EU FI

AS CH

AS IN

132

15

120

UR AZ

UR RU

101 UR SO

UN UN

EU IC

AF LB

$5 \quad 1935$

$\begin{array}{ll}5 & 1979\end{array}$

$\begin{array}{ll}5 & 1979\end{array}$

51970

$5 \quad 1970$

51976

$\begin{array}{ll}5 & 1994\end{array}$

$\begin{array}{ll}5 & 1979\end{array}$

$\begin{array}{ll}5 & 1994\end{array}$

51979

51979

$5 \quad 1991$

51979

$\begin{array}{ll}5 & 1976 \\ 5 & 1973\end{array}$

EU GB

AS HE

AS $\mathrm{CH}$

UR RU

UR RU

NA AM

51976

51994

51989

51979

$5 \quad 91$

$5 \quad 1970$

51976

NA AM

$5 \quad 1976$

NA AM

$5 \quad 1973$

EU GB

$\begin{array}{llrllllr}31.4 \mathrm{~N} & 208.3 \mathrm{~W} & 0 & \text { EU } & \text { GR } & 5 & 1985 & 57 \\ 19.2 \mathrm{~S} & 253.3 \mathrm{~W} & 0 & \text { SA } & \text { VE } & 5 & 1991 & 36 \\ 29.2 \mathrm{~S} & 135.3 \mathrm{E} & 67 & \text { UR } & \text { SO } & 5 & 1970 & 0\end{array}$

16.9S $86.3 \mathrm{~W} \quad 145 \quad$ NA AM
53.4E

11.3E

$11.7 \mathrm{E}$

$178.3 \mathrm{E}$

$124.6 \mathrm{~W}$

$34.8 \mathrm{~W}$

$51.0 \mathrm{~W}$

$337.9 \mathrm{~W}$

$23.4 \mathrm{~W}$

$6.3 \mathrm{~W}$

93.3E

$273.1 \mathrm{~W}$

$267.5 \mathrm{~W}$

$112.7 \mathrm{E}$

$0.0 \mathrm{~W}$
51976

67

9

A

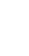

6
6
17
6
8
6
6
80
57
87

59

66

27

68

$$
0
$$

0

83

59
112

80

56

71

71
36

36
59

0
82

82

74
83

83
98
0

0$$
0
$$$$
0
$$

60

\section{ft origin}

AA Otto von; Russian astronomer (1819-1905); Otto; American astronomer (1897-1963); Friedrich G.W. von; German astronomer (1973-1864)

AA Mary; Queen of Scots (1542-1587).

LF Astronaut-named feature, Apollo 16 site.

VA Classical river east of Rome, Italy.

CA From albedo feature at $30 \mathrm{~N}, 200 \mathrm{~W}$.

FO Albedo feature name.

$\mathrm{AL}$ Great river around nether region, which souls must cross on journey from Earth.

DO Albedo feature name.

AA Town in Venezuela.

AA Mikhail F.; Soviet astronomer (1893-1966).

VA Classical river in Mauritania.

AA Town in Colombia.

AA Norse dwarf.

AA Eduard; Austrian geologist (1831-1914).

AA Eduard; Austrian t, engineer (1831-1914).

AA Town in Jordan.

PE Chinese hero who discovered fire.

PL Japanese messenger god.

AA Town in Russia.

SU Classical albedo feature name.

AA Georgian first name.

AA Anne; American teacher of Helen Keller (1866-1936).

AA Louis; American architect (1856-1924).

AA Gaius; Roman astronomer (unkn-fl. c. B.C. 166).

AA Russian (Buriat) world mountain.

AA M. I.; Russian cryopedologist.

AA Thomas H.; American geographer (1807-1876).

AA K. F.; Finnish astronomer (1873-1949).

AA Chinese female name.

CR Hindu fertility goddess.

AA Indian poet (1483-1563)

AA Azeri first name.

AA Vassily; Russian painter (1848-1916).

VA Name proposed by Soviets; found on Mars-5 Map.

VA Lowell canal name.

ER Icelandic volcano god.

AA Town in Libya.

LF Astronaut-named feature, Apollo 12 site.

AA English female name.

AA First name from Hebrew.

AA Town in China.

PE Russian smith god.

AA Russian first name.

AA William F. G.; Anglo-American physicist (1884-1962).

AA Ambrose; American inventor (1846-1937).

AA Lewis; American astronomer (1820-1913).
AA Jonathan; British writer (1667-1745).

\footnotetext{
P: $\quad$ planetary system (see page xvi).

Sa: satellite (see page $x v i$ ).

lat: latitude of feature center.

long: longitude of feature center.
}

diam: diameter or long dimension of feature.

ct: continent of name origin (see page $284 \mathrm{ff}$.)

et: ethnicity of name origin (see page $284 \mathrm{ff}$.)

as: name approval status (see page xvii). ad: name approval date (year).

ref: reference source for name (see page $287 \mathrm{ff}$.).

ft: feature type (see page 290). 
ALPHABETICAL LIST OF NAMES

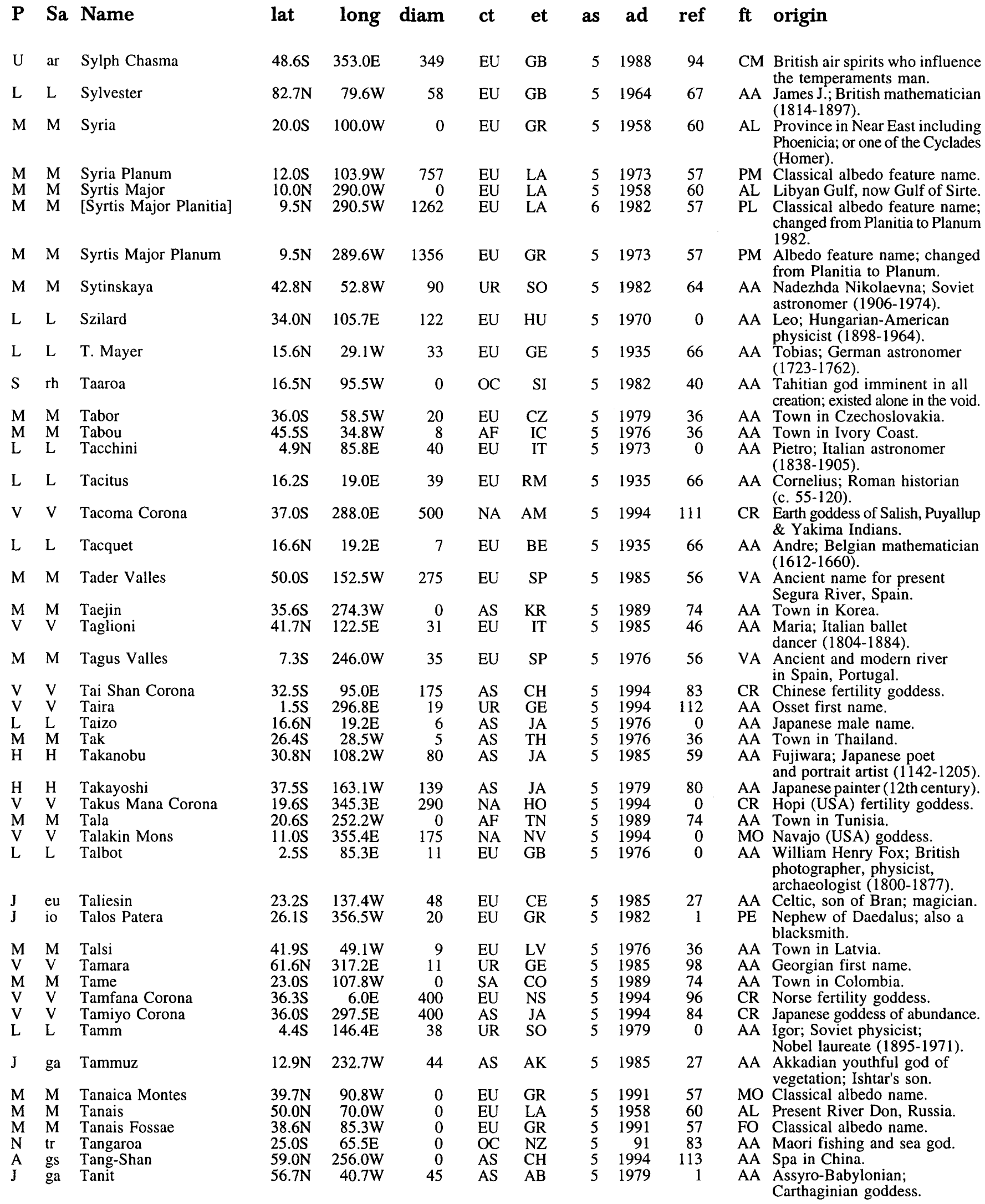

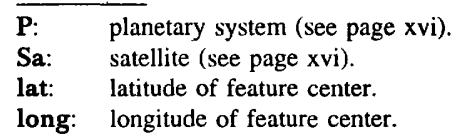

diam: diameter or long dimension of feature. ct: continent of name origin (see page $284 \mathrm{ff}$.) et: ethnicity of name origin (see page $284 \mathrm{ff}$.) as: name approval status (see page xvii) ad: name approval date (year).

ref: reference source for name (see page $287 \mathrm{ff}$.). ft: feature type (see page 290). 


\section{ALPHABETICAL LIST OF NAMES}

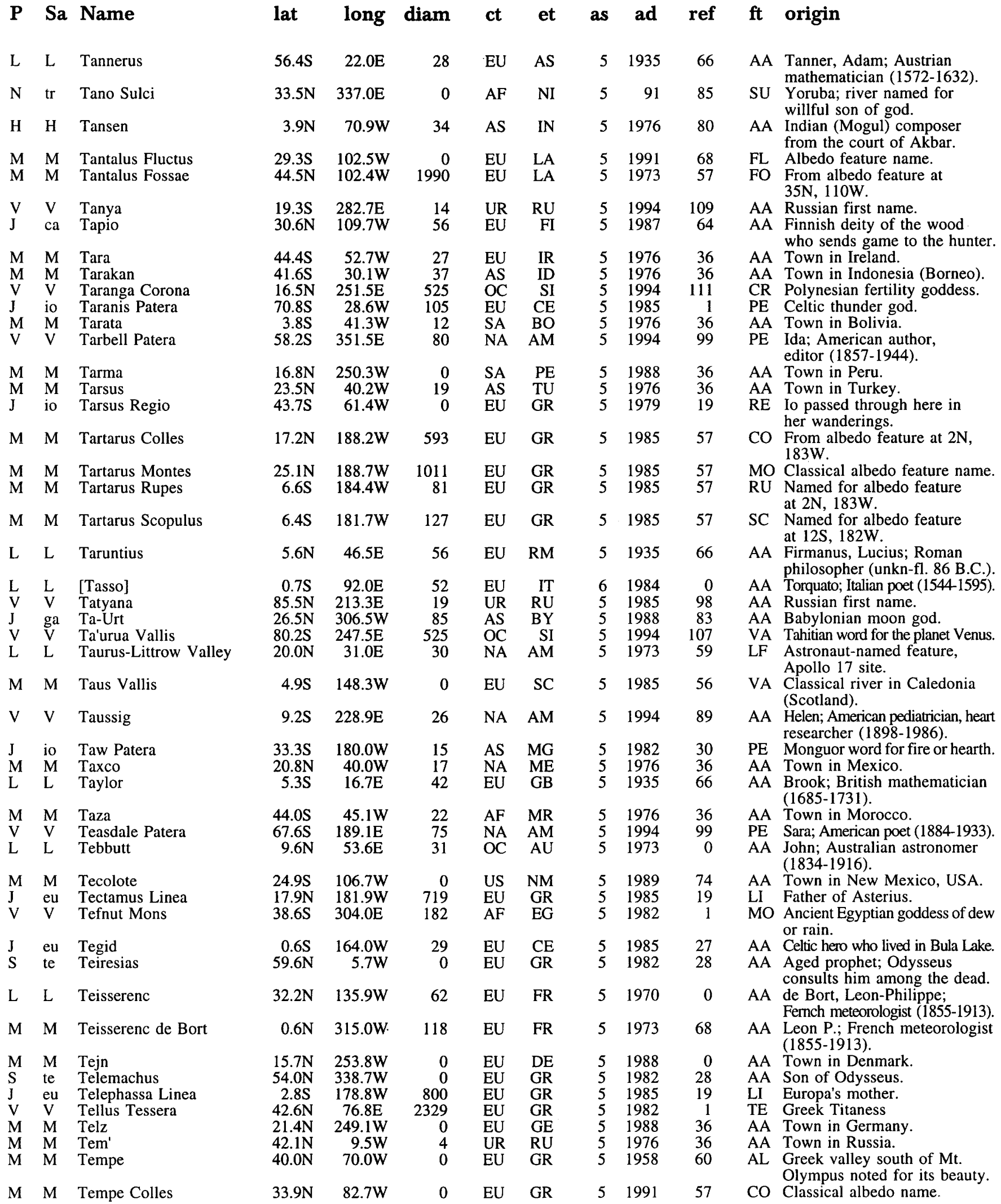

\footnotetext{
P: planetary system (see page xvi).

Sa: $\quad$ satellite (see page $x v i$ ).

lat: latitude of feature center.

long: longitude of feature center.
}

diam: diameter or long dimension of feature.

ct: continent of name origin (see page $284 \mathrm{ff}$.)

et: ethnicity of name origin (see page $284 \mathrm{ff}$.)

as: name approval status (see page $x$ vii). ad: name approval date (year).

ref: reference source for name (see page $287 \mathrm{ff}$.).

$\mathrm{ft}$ : feature type (see page 290). 


\begin{tabular}{|c|c|c|c|c|c|c|c|c|c|}
\hline $\mathbf{P}$ & $\mathbf{S a}$ & Name & lat & long & diam & ct & et & as & ad \\
\hline $\mathbf{M}$ & $\mathbf{M}$ & Tempe Fossae & $40.2 \mathrm{~N}$ & $74.5 \mathrm{~W}$ & 1553 & EU & GR & 5 & 1973 \\
\hline $\mathbf{M}$ & $\mathbf{M}$ & Tempe Mensa & $28.1 \mathrm{~N}$ & $71.5 \mathrm{~W}$ & 84 & EU & GR & 0 & 1908 \\
\hline $\mathbf{M}$ & M & Tempe Terra & $41.3 \mathrm{~N}$ & $70.5 \mathrm{~W}$ & 2055 & EU & GR & 5 & 1979 \\
\hline $\mathrm{L}$ & $\mathrm{L}$ & Tempel & $3.9 \mathrm{~N}$ & $11.9 \mathrm{E}$ & 45 & EU & GE & 5 & 1935 \\
\hline $\mathrm{L}$ & $\mathrm{L}$ & Ten Bruggencate & $9.5 \mathrm{~S}$ & $134.4 \mathrm{E}$ & 59 & EU & GE & 5 & 1970 \\
\hline $\begin{array}{l}\text { V } \\
M \\
M\end{array}$ & $\begin{array}{l}\mathrm{V} \\
\mathrm{M} \\
\mathrm{M}\end{array}$ & $\begin{array}{l}\text { Tepev Mons } \\
\text { Tepko } \\
\text { Terby }\end{array}$ & $\begin{array}{l}29.0 \mathrm{~N} \\
15.5 \mathrm{~N} \\
28.2 \mathrm{~S}\end{array}$ & $\begin{array}{c}44.3 \mathrm{E} \\
256.5 \mathrm{~W} \\
286.0 \mathrm{~W}\end{array}$ & $\begin{array}{r}301 \\
0 \\
135\end{array}$ & $\begin{array}{l}\text { SA } \\
\text { OC } \\
\text { EU }\end{array}$ & $\begin{array}{c}\text { MY } \\
\text { AU } \\
\text { BE. }\end{array}$ & $\begin{array}{l}5 \\
5 \\
5\end{array}$ & $\begin{array}{l}1985 \\
1988 \\
1973\end{array}$ \\
\hline $\begin{array}{l}\mathrm{V} \\
\mathrm{L}\end{array}$ & $\begin{array}{l}\mathrm{V} \\
\mathrm{L}\end{array}$ & $\begin{array}{l}\text { Teresa } \\
\text { Tereshkova }\end{array}$ & $\begin{array}{l}42.5 \mathrm{~S} \\
28.4 \mathrm{~N}\end{array}$ & $\begin{array}{r}9.9 \mathrm{E} \\
144.3 \mathrm{E}\end{array}$ & $\begin{array}{l}17 \\
31\end{array}$ & $\begin{array}{l}\text { EU } \\
\text { UR }\end{array}$ & $\begin{array}{l}\text { GR } \\
\text { SO }\end{array}$ & $\begin{array}{l}5 \\
5\end{array}$ & $\begin{array}{l}1994 \\
1970\end{array}$ \\
\hline M & M & Termes Vallis & $11.3 \mathrm{~S}$ & $157.1 \mathrm{~W}$ & 0 & $\mathrm{EU}$ & SP & 5 & 1988 \\
\hline $\begin{array}{l}M \\
M \\
M \\
M \\
L\end{array}$ & $\begin{array}{l}\mathrm{M} \\
\mathbf{M} \\
\mathrm{M} \\
\mathrm{M} \\
\mathrm{L}\end{array}$ & $\begin{array}{l}\text { Terra Cimmeria } \\
\text { Terra Meridiani } \\
\text { Terra Sabaea } \\
\text { Terra Sirenum } \\
\text { Terrace }\end{array}$ & $\begin{array}{r}34.0 \mathrm{~S} \\
7.2 \mathrm{~S} \\
10.4 \mathrm{~S} \\
37.0 \mathrm{~S} \\
26.1 \mathrm{~N}\end{array}$ & $\begin{array}{c}215.0 \mathrm{~W} \\
356.0 \mathrm{~W} \\
330.6 \mathrm{~W} \\
160.0 \mathrm{~W} \\
3.7 \mathrm{E}\end{array}$ & $\begin{array}{r}2285 \\
1622 \\
1367 \\
2165 \\
0\end{array}$ & $\begin{array}{l}\text { EU } \\
\text { EU } \\
\text { EU } \\
\text { EU } \\
\text { NA }\end{array}$ & $\begin{array}{l}\text { GR } \\
\text { LA } \\
\text { LA } \\
\text { GR } \\
\text { AM }\end{array}$ & $\begin{array}{l}5 \\
5 \\
5 \\
5 \\
5\end{array}$ & $\begin{array}{l}1979 \\
1979 \\
1979 \\
1979 \\
1973\end{array}$ \\
\hline $\begin{array}{l}\mathrm{J} \\
\mathrm{L}\end{array}$ & $\begin{array}{l}\text { ga } \\
\mathrm{L}\end{array}$ & $\begin{array}{l}\text { Teshub } \\
\text { Tesla }\end{array}$ & $\begin{array}{l}72.3 \mathrm{~S} \\
38.5 \mathrm{~N}\end{array}$ & $\begin{array}{l}281.0 \mathrm{~W} \\
124.7 \mathrm{E}\end{array}$ & $\begin{array}{l}60 \\
43\end{array}$ & $\begin{array}{l}\text { AS } \\
\text { EU }\end{array}$ & $\begin{array}{l}\text { AS } \\
\text { YU }\end{array}$ & $\begin{array}{l}5 \\
5\end{array}$ & $\begin{array}{l}1994 \\
1970\end{array}$ \\
\hline $\begin{array}{l}\text { V } \\
\text { V } \\
J\end{array}$ & $\begin{array}{l}\mathrm{V} \\
\mathrm{V} \\
\mathrm{ga}\end{array}$ & $\begin{array}{l}\text { Teteoinnan Corona } \\
\text { Tethus Regio } \\
\text { Tettu Facula }\end{array}$ & $\begin{array}{l}38.5 \mathrm{~S} \\
66.0 \mathrm{~N} \\
38.6 \mathrm{~N}\end{array}$ & $\begin{array}{l}149.5 \mathrm{E} \\
120.0 \mathrm{E} \\
160.9 \mathrm{~W}\end{array}$ & $\begin{array}{r}125 \\
0 \\
86\end{array}$ & $\begin{array}{l}\text { NA } \\
\text { EU } \\
\text { AF }\end{array}$ & $\begin{array}{l}\text { AZ } \\
\text { GR } \\
\text { EG }\end{array}$ & $\begin{array}{l}5 \\
5 \\
5\end{array}$ & $\begin{array}{l}1994 \\
1982 \\
1985\end{array}$ \\
\hline V & V & Tey Patera & $17.8 \mathrm{~S}$ & $349.1 \mathrm{E}$ & 20 & $\mathrm{EU}$ & SC & 5 & 1994 \\
\hline $\begin{array}{l}\mathrm{V} \\
\mathrm{H}\end{array}$ & $\begin{array}{l}\mathrm{V} \\
\mathrm{H}\end{array}$ & $\begin{array}{l}\text { Tezan Dorsa } \\
\text { Thākur }\end{array}$ & $\begin{array}{r}81.4 \mathrm{~N} \\
3.0 \mathrm{~S}\end{array}$ & $\begin{array}{l}47.1 \mathrm{E} \\
63.5 \mathrm{~W}\end{array}$ & $\begin{array}{r}1079 \\
118\end{array}$ & $\begin{array}{l}\text { EU } \\
\text { AS }\end{array}$ & IT & $\begin{array}{l}5 \\
5\end{array}$ & $\begin{array}{l}1985 \\
1976\end{array}$ \\
\hline L & $\mathrm{L}$ & Thales & $61.8 \mathrm{~N}$ & $50.3 \mathrm{E}$ & 31 & EU & GR & 5 & 1935 \\
\hline V & $\mathrm{V}$ & Thallo Mons & $72.8 \mathrm{~N}$ & $233.5 \mathrm{E}$ & 216 & EU & GR & 5 & 1985 \\
\hline $\mathbf{M}$ & $\mathbf{M}$ & Tharsis & $0.0 \mathrm{~N}$ & $0.0 \mathrm{~W}$ & 0 & EU & LA & 5 & 1973 \\
\hline $\begin{array}{l}\mathrm{M} \\
\mathrm{M} \\
\mathrm{J} \\
\mathrm{M}\end{array}$ & $\begin{array}{l}\mathrm{M} \\
\mathrm{M} \\
\mathrm{eu} \\
\mathrm{M}\end{array}$ & $\begin{array}{l}\text { Tharsis Montes } \\
\text { Tharsis Tholus } \\
\text { Thasus Linea } \\
\text { Thaumasia }\end{array}$ & $\begin{array}{l}2.8 \mathrm{~N} \\
13.4 \mathrm{~N} \\
68.7 \mathrm{~S} \\
35.0 \mathrm{~S}\end{array}$ & $\begin{array}{r}113.3 \mathrm{~W} \\
90.8 \mathrm{~W} \\
187.4 \mathrm{~W} \\
85.0 \mathrm{~W}\end{array}$ & $\begin{array}{r}2105 \\
153 \\
1027 \\
0\end{array}$ & $\begin{array}{l}\text { EU } \\
\text { EU } \\
\text { EU } \\
\text { EU }\end{array}$ & $\begin{array}{l}\text { LA } \\
\text { LA } \\
\text { GR } \\
\text { GR }\end{array}$ & $\begin{array}{l}5 \\
5 \\
5 \\
5\end{array}$ & $\begin{array}{l}1973 \\
1973 \\
1979 \\
1958\end{array}$ \\
\hline $\begin{array}{l}M \\
M \\
L\end{array}$ & $\begin{array}{l}M \\
M \\
L\end{array}$ & $\begin{array}{l}\text { Thaumasia Fossae } \\
\text { Thaumasia Planum } \\
\text { Theaetetus }\end{array}$ & $\begin{array}{l}45.9 \mathrm{~S} \\
22.0 \mathrm{~S} \\
37.0 \mathrm{~N}\end{array}$ & $\begin{array}{c}97.3 \mathrm{~W} \\
65.0 \mathrm{~W} \\
6.0 \mathrm{E}\end{array}$ & $\begin{array}{r}1118 \\
930 \\
24\end{array}$ & $\begin{array}{l}\text { EU } \\
\text { EU } \\
\text { EU }\end{array}$ & $\begin{array}{l}\text { GR } \\
\text { GR } \\
\text { GR }\end{array}$ & $\begin{array}{l}5 \\
5 \\
5\end{array}$ & $\begin{array}{l}1973 \\
1994 \\
1935\end{array}$ \\
\hline $\begin{array}{l}\mathbf{J} \\
\mathbf{L}\end{array}$ & $\begin{array}{l}\text { ga } \\
\mathrm{L}\end{array}$ & $\begin{array}{l}\text { Thebes Facula } \\
\text { Thebit }\end{array}$ & $\begin{array}{r}4.8 \mathrm{~N} \\
22.0 \mathrm{~S}\end{array}$ & $\begin{array}{r}202.4 \mathrm{~W} \\
4.0 \mathrm{~W}\end{array}$ & $\begin{array}{r}475 \\
56\end{array}$ & $\begin{array}{l}\text { AF } \\
\text { AS }\end{array}$ & $\begin{array}{l}\text { EG } \\
\text { IQ }\end{array}$ & $\begin{array}{l}5 \\
5\end{array}$ & $\begin{array}{l}1985 \\
1935\end{array}$ \\
\hline $\begin{array}{l}\text { V } \\
\mathrm{L}\end{array}$ & $\begin{array}{l}\text { V } \\
L\end{array}$ & $\begin{array}{l}\text { Theia Mons } \\
\text { Theiler }\end{array}$ & $\begin{array}{l}22.7 \mathrm{~N} \\
13.4 \mathrm{~N}\end{array}$ & $\begin{array}{r}281.0 \mathrm{E} \\
83.3 \mathrm{E}\end{array}$ & $\begin{array}{r}226 \\
7\end{array}$ & $\begin{array}{l}\mathrm{EU} \\
\mathrm{AF}\end{array}$ & $\begin{array}{l}\text { GR } \\
\text { SA }\end{array}$ & $\begin{array}{l}5 \\
5\end{array}$ & $\begin{array}{l}1979 \\
1979\end{array}$ \\
\hline $\begin{array}{l}\text { V } \\
\text { V }\end{array}$ & $\begin{array}{l}\mathrm{V} \\
\mathrm{V}\end{array}$ & $\begin{array}{l}\text { Themis Regio } \\
\text { [Theodora Patera] }\end{array}$ & $\begin{array}{l}37.4 \mathrm{~S} \\
23.0 \mathrm{~N}\end{array}$ & $\begin{array}{l}284.2 \mathrm{E} \\
280.0 \mathrm{E}\end{array}$ & $\begin{array}{r}1811 \\
0\end{array}$ & $\begin{array}{l}\mathrm{EU} \\
\mathrm{EU}\end{array}$ & $\begin{array}{l}\text { GR } \\
\text { BZ }\end{array}$ & $\begin{array}{l}5 \\
6\end{array}$ & $\begin{array}{l}1982 \\
1982\end{array}$ \\
\hline L & L & Theon Junior & $2.3 \mathrm{~S}$ & $15.8 \mathrm{E}$ & 17 & $\mathrm{EU}$ & GR & 5 & 1935 \\
\hline L & L & Theon Senior & $0.8 \mathrm{~S}$ & $15.4 \mathrm{E}$ & 18 & $\mathrm{EU}$ & GR & 5 & 1935 \\
\hline $\begin{array}{l}\mathrm{H} \\
\mathrm{L}\end{array}$ & $\begin{array}{l}\mathrm{H} \\
\mathrm{L}\end{array}$ & $\begin{array}{l}\text { Theophanes } \\
\text { Theophilus }\end{array}$ & $\begin{array}{r}4.9 \mathrm{~S} \\
11.4 \mathrm{~S}\end{array}$ & $\begin{array}{c}142.4 \mathrm{~W} \\
26.4 \mathrm{E}\end{array}$ & $\begin{array}{r}45 \\
110\end{array}$ & $\begin{array}{l}\text { EU } \\
\text { EU }\end{array}$ & $\begin{array}{l}\text { BZ } \\
\text { GR }\end{array}$ & $\begin{array}{l}5 \\
5\end{array}$ & $\begin{array}{l}1976 \\
1935\end{array}$ \\
\hline
\end{tabular}

\section{ft origin}

FO From albedo feature at $40 \mathrm{~N}, 70 \mathrm{~W}$

MN From albedo feature at $40 \mathrm{~N}, 70 \mathrm{~W}$.

TA From albedo feature at $40 \mathrm{~N}, 70 \mathrm{~W}$

AA Ernst Wilhelm Leberecht; German astronomer (1821-1889).

AA P.; German astronomer (1901-1961).

MO Quiche Mayan creator goddess.

AA Town in Australia.

AA Francois J.; Belgian astronomer (1846-1911).

AA First name from Greek.

AA Valentina Vladimirovna; Soviet cosmonaut (1937-Live).

VA Classical river in ancient Lusitania, present Tormes River, Spain.

TA Classical albedo feature name.

TA Classical albedo feature name.

TA Classical albedo feature name.

TA Classical albedo feature name.

LF Astronaut-named feature, Apollo 15 site.

AA Elamite god of the tempest.

AA Nikola; Croatian-American inventor (1856-1943)

CR Aztec fertility goddess.

RE Greek Titaness.

FA Egyptian town where Hatmenit and Osiris were worshipped.

PE Josephine; Scottish author (1897-1952)

DO Etruscan dawn goddess.

AA R.; Bengalese poet and novelist, Nobel Peace laureate (1861-1941).

AA Of Miletos; Greek mathematician, astronomer, philosopher (c. 636-546 B.C.).

MO Greek goddess of vegetation flowering (Spring Hora).

AL Connecting link between East and West; ancient Spanish town Tartessus.

MO Classical albedo feature name.

TH Classical albedo feature name.

LI Greek; brother of Europa.

AL Named for Thaumas, god of clouds; Arabia.

FO Classical albedo feature name.

PM Albedo feature at $30 \mathrm{~S}, 75 \mathrm{~W}$.

AA Greek geometrician (unkn-c. 380 B.C.)

FA Ancient capitol of upper kingdom.

AA Ben Korra; Iraqi astronomer (826-901).

MO Greek Titaness.

AA Max; South African bacteriologist; Nobel laureate (1899-1972).

RE Greek Titaness.

PE Famous Byzantine woman; not being used

AA Of Alexandria; Greek astronomer (unkn-c. 380).

AA Of Smyrna; Greek mathematician (unkn-c. 100).

AA Byzantine painter (c. 1330-1405). AA Greek astronomer d. A.D. 412.

\footnotetext{
P: $\quad$ planetary system (see page xvi)

Sa: $\quad$ satellite (see page $x v i$ ).

lat: latitude of feature center.

long: longitude of feature center.
}

diam: diameter or long dimension of feature.

ct: continent of name origin (see page $284 \mathrm{ff}$.)

et: ethnicity of name origin (see page $284 \mathrm{ff}$.)

as: name approval status (see page xvii). ad: name approval date (year)

ref: reference source for name (see page $287 \mathrm{ff}$.)

ft: feature type (see page 290). 


\section{ALPHABETICAL LIST OF NAMES}

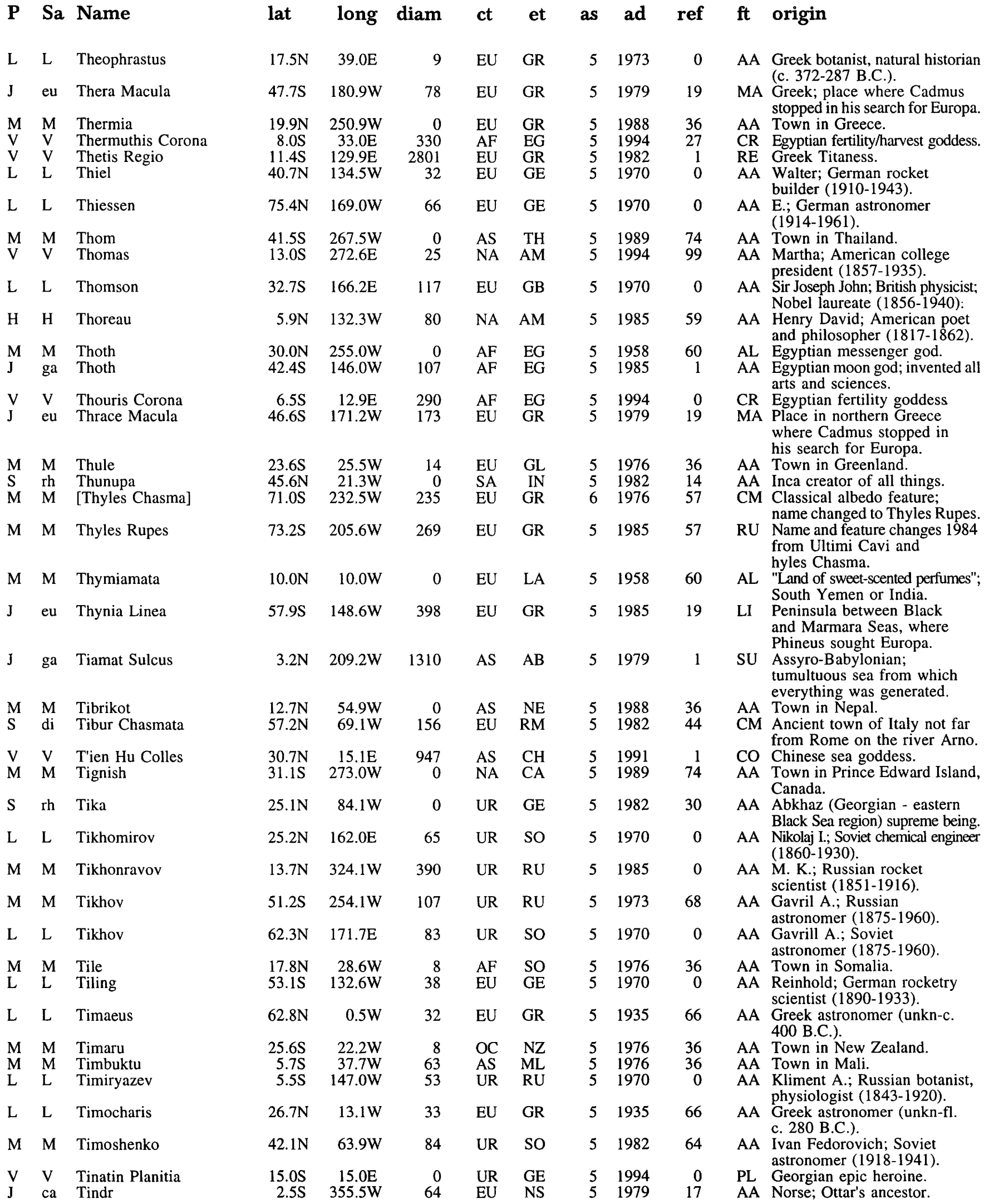

\footnotetext{
P: $\quad$ planetary system (see page xvi).

Sa: $\quad$ satellite (see page $x v i$ ).

lat: latitude of feature center.

long: longitude of feature center.
}

diam: diameter or long dimension of feature.

ct: continent of name origin (see page $284 \mathrm{ff}$.)

et: ethnicity of name origin (see page $284 \mathrm{ff}$.)

as: name approval status (see page $x$ vii). ad: name approval date (year).

ref: reference source for name (see page $287 \mathrm{ff}$.).

ft: feature type (see page 290). 


\section{ALPHABETICAL LIST OF NAMES}

\section{P Sa Name \\ M M Tinia Valles \\ M M Tinjar Valles \\ $\mathrm{S}$ mi Tintagil Chasma \\ $\mathrm{H} \quad \mathrm{H}$ Tintoretto \\ V V Tipporah Patera \\ H H Tir Planitia \\ $S$ rh Tirawa}

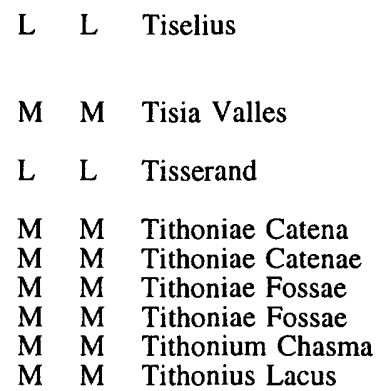

H H Titian

L L Titius

L L Titov

V V Tituba Patera

M M Tiu Vallis

M M Tiwi

M ph Todd

J io Tohil Patera

M M Tokko

V V Toklas

M M Tokma

L L Tolansky

$\mathrm{J}$ io Tol-Ava Patera

M M Tolon

H $\quad \mathrm{H} \quad$ Tolstoj

L L $\quad$ [Tolstoy]

M M Tomari

M M Tombe

V V Tomem Dorsa

M M Tōno

J ca Tontu

M M Torbay

$\mathrm{S}$ rh Tore

J ca Tornarsuk

$\mathrm{S}$ rh Torom

L L Torricelli

M M Torsö la

$\begin{array}{ccrcrrrr}4.7 \mathrm{~S} & 148.9 \mathrm{~W} & 20 & \text { EU } & \text { IT } & 5 & 1985 & 56 \\ 38.2 \mathrm{~N} & 235.7 \mathrm{~W} & 390 & \text { AS } & \text { MA } & 5 & 1985 & 36 \\ & & & & & & & \\ 49.7 \mathrm{~S} & 205.0 \mathrm{~W} & 0 & \text { EU } & \text { GB } & 5 & 1982 & 37 \\ & & & & & & & \\ 48.1 \mathrm{~S} & 22.9 \mathrm{~W} & 92 & \text { EU } & \text { IT } & 5 & 1976 & 59 \\ 38.9 \mathrm{~N} & 43.0 \mathrm{E} & 99 & \text { AS } & \text { HE } & 5 & 1985 & 80 \\ & & & & & & & \\ 0.8 \mathrm{~N} & 176.1 \mathrm{~W} & 0 & \text { EU } & \text { NS } & 5 & 1976 & 80 \\ 34.2 \mathrm{~N} & 151.7 \mathrm{~W} & 0 & \text { NA } & \text { PW } & 5 & 1987 & 83\end{array}$

$\begin{array}{rrrrrrrr}7.0 \mathrm{~N} & 176.5 \mathrm{E} & 53 & \text { EU } & \text { SW } & 5 & 1979 & 0 \\ & & & & & & & \\ 11.6 \mathrm{~S} & 313.9 \mathrm{~W} & 390 & \text { UR } & \text { UK } & 5 & 1985 & 56 \\ & & & & & & & \\ 21.4 \mathrm{~N} & 48.2 \mathrm{E} & 36 & \text { EU } & \text { FR } & 5 & 1935 & 66 \\ & & & & & & & \\ 5.5 \mathrm{~S} & 71.5 \mathrm{~W} & 380 & \text { EU } & \text { LA } & 5 & 1973 & 57 \\ 5.1 \mathrm{~S} & 78.2 \mathrm{~W} & 101 & \text { EU } & \text { LA } & 5 & 1987 & 57 \\ 1.7 \mathrm{~S} & 81.9 \mathrm{~W} & 549 & \text { EU } & \text { LA } & 5 & 1982 & 57 \\ 6.4 \mathrm{~S} & 82.5 \mathrm{~W} & 492 & \text { EU } & \text { LA } & 5 & 1988 & 57 \\ 4.6 \mathrm{~S} & 86.5 \mathrm{~W} & 904 & \text { EU } & \text { LA } & 5 & 1973 & 57 \\ 5.0 \mathrm{~S} & 85.0 \mathrm{~W} & 0 & \text { EU } & \text { LA } & 5 & 1958 & 60 \\ & & & & & & & \\ 3.6 \mathrm{~S} & 42.1 \mathrm{~W} & 121 & \text { EU } & \text { IT } & 5 & 1976 & 59\end{array}$

26.8S 100.7E

$28.6 \mathrm{~N} \quad 150.5 \mathrm{E}$

$42.4 \mathrm{~N} \quad 214.7 \mathrm{E}$

$8.6 \mathrm{~N} \quad 34.8 \mathrm{~W}$

$27.9 \mathrm{~S}$

$5.0 \mathrm{~S}$

$24.6 \mathrm{~W}$

$26.3 \mathrm{~S}$

$160.0 \mathrm{~W}$

26.35

$22.7 \mathrm{~N}$$$
21.6
$$

$\begin{array}{rrrrrrrr}1.7 \mathrm{~N} & 322.0 \mathrm{~W} & 70 & \text { UR } & \text { MO } & 5 & 1985 & 51 \\ & & & & & & & \\ 18.5 \mathrm{~N} & 255.1 \mathrm{~W} & 0 & \text { UR } & \text { RU } & 5 & 1988 & 36 \\ 16.3 \mathrm{~S} & 163.5 \mathrm{~W} & 390 & \text { UR } & \text { RU } & 5 & 1976 & 59\end{array}$

4.2

$$
20.1
$$

31.

\section{2}

$$
27.6
$$$$
18.1
$$

$28.7 \mathrm{~N} \quad 128.6 \mathrm{~W}$

68.1S $343.5 \mathrm{~W}$

4.6S $28.5 \mathrm{E}$

44.7S $51.0 \mathrm{~W}$
$5 \quad 1970$

51970

31 UR SO

163 NA AM

970 EU GB

AS OM

NA AM

SA MY

UR RU

NA AM

UR RU

EU GB

591

51973

51976

51973

51979

$\begin{array}{ll}5 & 1988\end{array}$

51994

$5 \quad 1988$

51976

6

$5 \quad 1988$

$\begin{array}{ll}5 & 1976\end{array}$

51985

12
970

UR RU

AF SU

UR SI

$5 \quad 1976$

AS JA

OC AU

$5 \quad 1988$

51982

104 NA ES

$\begin{array}{ll}5 & 1979\end{array}$

$\begin{array}{ll}5 & 1982\end{array}$

51935

$5 \quad 1976$ $\begin{array}{ll}5 & 1987\end{array}$

\section{$\mathrm{ft}$ origin}

VA Classical river in Italy.

VA Modern river in Sarawak, Malaysia

CM Home of Igraine, Arthur's mother.

AA Italian painter (1518-1594).

PE Hebrew medical scholar (1500 B.C.)

PL Norse word for Mercury.

AA Great spirit of Pawnee Tribe (USA), created first men; his messengers were the planets, stars, lightning and thunder.

AA Arne Wilhelm Kaurin; Swedish biochemist; Nobel laureate (1902-1971).

VA Ancient name for modern Tisza River, Ukraine.

AA Francois Felix; French astronomer (1845-1896).

CA Classical albedo feature name.

CA Classical albedo feature name.

FO Classical albedo name.

FO Classical albedo feature name.

CM Classical albedo feature name.

AL "Tithonian Lake"; Tithonus received from his wife Eos eternal life but not eternal youth.

AA Tiziano Vecellio; Italian Renaissance painter (c 1488/90-1576)

AA Johann D.; German astronomer (1729-1796).

AA German S.; Soviet cosmonaut (1935-Live).

PE Nurse who started Salem witch hunt (c. 1692).

VA Word for "Mars" in old English (West Germanic).

AA Town in Oman.

AA David; American astronomer 1855-1939

PE Central American god who gave fire to man.

AA Town in Russia.

AA Alice; American writer, art patron (1877-1967).

AA Town in Russia.

AA Samuel; British physicist (1907-1973).

PE Mordvinian (Russia) goddess of fire.

AA . Town in Russia.

AA Lev N.; Russian novelist (1828-1910).

AA Leo; Russian writer (1828-1910).

AA Town in Russia.

AA Town in Sudan.

DO Ketian (Siberia) Mother of the hot; lives in the sky, near the Sun.

AA Town in Japan.

AA Finnish god of housekeeping.

AA Town in Australia.

AA Pygmy lord of the world, creator of all things.

AA Greenland legendary hero.

AA Ostyak (western Siberia) sky god.

AA Evangelista; Italian physicist (1608-1647).

AA Town in Sweden.
P: $\quad$ planetary. system (see page $x v i$ ).

Sa: satellite (see page $x v i$ ).

lat: latitude of feature center.

long: longitude of feature center. diam: diameter or long dimension of feature.

ct: continent of name origin (see page $284 \mathrm{ff}$.)

et: ethnicity of name origin (see page $284 \mathrm{ff}$.)

as: name approval status (see page xvii). ad: name approval date (year).

ref: reference source for name (see page $287 \mathrm{ff}$.).

ft: feature type (see page 290). 


\section{ALPHABETICAL LIST OF NAMES}

\begin{tabular}{|c|c|c|c|c|c|c|c|c|c|c|}
\hline $\mathbf{P}$ & $\mathbf{S a}$ & Name & lat & long & diam & ct & et & as & ad & ref \\
\hline L & $\mathrm{L}$ & Tortilla Flat & $20.2 \mathrm{~N}$ & $30.7 \mathrm{E}$ & 1 & NA & AM & 5 & 1973 & 59 \\
\hline $\begin{array}{l}\mathrm{M} \\
\mathrm{L}\end{array}$ & $\begin{array}{l}\mathrm{M} \\
\mathrm{L}\end{array}$ & $\begin{array}{l}\text { Torup } \\
\text { Toscanelli }\end{array}$ & $\begin{array}{l}28.1 \mathrm{~S} \\
27.4 \mathrm{~N}\end{array}$ & $\begin{array}{r}262.2 \mathrm{~W} \\
47.5 \mathrm{~W}\end{array}$ & $\begin{array}{l}0 \\
7\end{array}$ & $\begin{array}{l}\text { EU } \\
\text { EU }\end{array}$ & $\begin{array}{c}\text { SW } \\
\text { IT }\end{array}$ & 5 & $\begin{array}{l}1989 \\
1976\end{array}$ & $\begin{array}{r}74 \\
0\end{array}$ \\
\hline $\mathrm{L}$ & $\mathrm{L}$ & Townley & $3.4 \mathrm{~N}$ & $63.3 \mathrm{E}$ & 18 & NA & AM & 5 & 1976 & 0 \\
\hline $\begin{array}{l}\mathrm{V} \\
\mathrm{M} \\
\mathrm{M} \\
\mathrm{M} \\
\mathrm{L}\end{array}$ & $\begin{array}{l}\mathrm{V} \\
\mathrm{M} \\
\mathrm{M} \\
\mathrm{M} \\
\mathrm{L}\end{array}$ & $\begin{array}{l}\text { Toyo-uke Corona } \\
\text { Tractus Albus } \\
\text { Tractus Catena } \\
\text { Tractus Fossae } \\
\text { Tralles }\end{array}$ & $\begin{array}{l}62.5 \mathrm{~S} \\
30.0 \mathrm{~N} \\
27.9 \mathrm{~N} \\
27.1 \mathrm{~N} \\
28.4 \mathrm{~N}\end{array}$ & $\begin{array}{c}41.5 \mathrm{E} \\
80.0 \mathrm{~W} \\
103.2 \mathrm{~W} \\
101.1 \mathrm{~W} \\
52.8 \mathrm{E}\end{array}$ & $\begin{array}{r}300 \\
0 \\
1234 \\
535 \\
43\end{array}$ & $\begin{array}{l}\text { AS } \\
\text { EU } \\
\text { EU } \\
\text { EU } \\
\text { EU }\end{array}$ & $\begin{array}{l}\text { JA } \\
\text { LA } \\
\text { LA } \\
\text { LA } \\
\text { GE }\end{array}$ & $\begin{array}{l}5 \\
5 \\
5 \\
5 \\
5\end{array}$ & $\begin{array}{l}1994 \\
1958 \\
1976 \\
1988 \\
1935\end{array}$ & $\begin{array}{l}83 \\
60 \\
57 \\
57 \\
66\end{array}$ \\
\hline L & L & Trap & $9.1 \mathrm{~S}$ & $15.4 \mathrm{E}$ & 1 & NA & AM & 5 & 1973 & 59 \\
\hline M & $\mathbf{M}$ & Trebia Valles & $32.4 \mathrm{~N}$ & $209.9 \mathrm{~W}$ & 0 & EU & IT & 5 & 1985 & 56 \\
\hline $\mathrm{H}$ & $\mathrm{H}$ & Tricrena & $0.0 \mathrm{~N}$ & $36.0 \mathrm{~W}$ & 0 & EU & RM & 5 & 1976 & 60 \\
\hline L & $\mathrm{L}$ & Trident & $20.2 \mathrm{~N}$ & $30.8 \mathrm{E}$ & 0 & NA & AM & 5 & 1973 & 59 \\
\hline L & L & Triesnecker & $4.2 \mathrm{~N}$ & $3.6 \mathrm{E}$ & 26 & EU & AS & 5 & 1935 & 66 \\
\hline $\begin{array}{l}\mathrm{M} \\
\mathrm{U} \\
\mathrm{M} \\
\mathrm{L}\end{array}$ & $\begin{array}{l}\mathrm{M} \\
\mathrm{mr} \\
\mathrm{M} \\
\mathrm{L}\end{array}$ & $\begin{array}{l}\text { Trinacria } \\
\text { Trinculo } \\
\text { Trinidad } \\
\text { Triplet }\end{array}$ & $\begin{array}{r}25.0 \mathrm{~S} \\
63.7 \mathrm{~S} \\
23.9 \mathrm{~S} \\
3.7 \mathrm{~S}\end{array}$ & $\begin{array}{l}268.0 \mathrm{~W} \\
163.4 \mathrm{E} \\
251.0 \mathrm{~W} \\
17.5 \mathrm{~W}\end{array}$ & $\begin{array}{r}0 \\
11 \\
0 \\
0\end{array}$ & $\begin{array}{l}\text { EU } \\
\text { EU } \\
\text { SA } \\
\text { NA }\end{array}$ & $\begin{array}{r}\text { LA } \\
\text { GB } \\
\text { PE } \\
\text { AM }\end{array}$ & $\begin{array}{l}5 \\
5 \\
5 \\
5\end{array}$ & $\begin{array}{l}1958 \\
1988 \\
1989 \\
1973\end{array}$ & $\begin{array}{l}60 \\
85 \\
74 \\
59\end{array}$ \\
\hline $\mathbf{S}$ & $\mathrm{mi}$ & Tristram & $52.2 \mathrm{~S}$ & $28.1 \mathrm{~W}$ & 0 & EU & GB & 5 & 1982 & 37 \\
\hline M & M & Trivium Charontis & $20.0 \mathrm{~N}$ & 198.0W & 0 & EU & LA & 5 & 1958 & 60 \\
\hline $\begin{array}{l}\mathrm{M} \\
\mathrm{V}\end{array}$ & $\begin{array}{l}\mathrm{M} \\
\mathrm{V}\end{array}$ & $\begin{array}{l}\text { Troika } \\
\text { Trollope }\end{array}$ & $\begin{array}{l}17.1 \mathrm{~N} \\
54.8 \mathrm{~S}\end{array}$ & $\begin{array}{l}255.0 \mathrm{~W} \\
246.4 \mathrm{E}\end{array}$ & $\begin{array}{l}13 \\
26\end{array}$ & $\begin{array}{l}\text { UR } \\
\text { EU }\end{array}$ & $\begin{array}{l}\text { RU } \\
\text { EN }\end{array}$ & $\begin{array}{l}5 \\
5\end{array}$ & $\begin{array}{l}1976 \\
1994\end{array}$ & $\begin{array}{l}36 \\
99\end{array}$ \\
\hline $\begin{array}{l}\mathrm{J} \\
\mathrm{V} \\
\mathrm{M}\end{array}$ & $\begin{array}{l}\text { ga } \\
\text { V } \\
\text { M }\end{array}$ & $\begin{array}{l}\text { Tros } \\
\text { Trotula Patera } \\
\text { Trouvelot }\end{array}$ & $\begin{array}{l}11.0 \mathrm{~N} \\
41.3 \mathrm{~N} \\
16.3 \mathrm{~N}\end{array}$ & $\begin{array}{l}31.1 \mathrm{~W} \\
18.9 \mathrm{E} \\
13.0 \mathrm{~W}\end{array}$ & $\begin{array}{l}109 \\
146 \\
168\end{array}$ & $\begin{array}{l}\text { EU } \\
\text { EU } \\
\text { NA }\end{array}$ & $\begin{array}{r}\text { GR } \\
\text { IT } \\
\mathrm{AM}\end{array}$ & $\begin{array}{l}5 \\
5 \\
5\end{array}$ & $\begin{array}{l}1979 \\
1985 \\
1973\end{array}$ & $\begin{array}{l}19 \\
80 \\
68\end{array}$ \\
\hline $\mathrm{L}$ & $\mathrm{L}$ & Trouvelot & $49.3 \mathrm{~N}$ & $5.8 \mathrm{E}$ & 9 & EU & FR & 5 & 1935 & 66 \\
\hline $\begin{array}{l}\mathrm{M} \\
\mathrm{M} \\
\mathrm{M}\end{array}$ & $\begin{array}{l}\mathrm{M} \\
\mathrm{M} \\
\mathrm{M}\end{array}$ & $\begin{array}{l}\text { Troy } \\
\text { Trud } \\
\text { Trumpler }\end{array}$ & $\begin{array}{l}23.3 \mathrm{~N} \\
17.7 \mathrm{~N} \\
61.7 \mathrm{~S}\end{array}$ & $\begin{array}{r}52.4 \mathrm{~W} \\
30.8 \mathrm{~W} \\
150.6 \mathrm{~W}\end{array}$ & $\begin{array}{r}10 \\
5 \\
77\end{array}$ & $\begin{array}{l}\text { US } \\
\text { UR } \\
\text { NA }\end{array}$ & $\begin{array}{l}\text { ID } \\
\text { RU } \\
\text { AM }\end{array}$ & $\begin{array}{l}5 \\
5 \\
5\end{array}$ & $\begin{array}{l}1976 \\
1976 \\
1973\end{array}$ & $\begin{array}{l}36 \\
36 \\
68\end{array}$ \\
\hline $\mathrm{L}$ & $\mathrm{L}$ & Trumpler & $29.3 \mathrm{~N}$ & $167.1 \mathrm{E}$ & 77 & NA & $\mathrm{AM}$ & 5 & 1970 & 0 \\
\hline V & V & Truth & $28.7 \mathrm{~N}$ & $287.7 \mathrm{E}$ & 47 & NA & AM & 5 & 1994 & 87 \\
\hline $\mathrm{H}$ & $\mathrm{H}$ & Ts'ai Wen-Chi & $22.8 \mathrm{~N}$ & $22.2 \mathrm{~W}$ & 119 & AS & $\mathrm{CH}$ & 5 & 1976 & 80 \\
\hline $\mathbf{L}$ & L & Tsander & $6.2 \mathrm{~N}$ & $149.3 \mathrm{~W}$ & 181 & UR & SO & 5 & 1970 & 0 \\
\hline $\begin{array}{l}\mathrm{H} \\
\mathrm{M} \\
\mathrm{V}\end{array}$ & $\begin{array}{l}\mathrm{H} \\
\mathrm{M} \\
\mathrm{V}\end{array}$ & $\begin{array}{l}\text { Ts'ao Chan } \\
\text { Tsau } \\
\text { Tseraskaya }\end{array}$ & $\begin{array}{l}13.4 \mathrm{~S} \\
49.8 \mathrm{~N} \\
28.6 \mathrm{~N}\end{array}$ & $\begin{array}{c}142.0 \mathrm{~W} \\
238.9 \mathrm{~W} \\
79.2 \mathrm{E}\end{array}$ & $\begin{array}{r}110 \\
6 \\
30\end{array}$ & $\begin{array}{l}\text { AS } \\
\text { AF } \\
\text { UR }\end{array}$ & $\begin{array}{l}\text { CH } \\
\text { BT } \\
\text { SO }\end{array}$ & $\begin{array}{l}5 \\
5\end{array}$ & $\begin{array}{l}1976 \\
1979 \\
1985\end{array}$ & $\begin{array}{l}59 \\
36 \\
64\end{array}$ \\
\hline $\mathrm{L}$ & $\mathrm{L}$ & Tseraskiy (Cerasky) & $49.0 \mathrm{~S}$ & $141.6 \mathrm{E}$ & 56 & UR & RU & 5 & 1970 & 0 \\
\hline $\begin{array}{l}\text { V } \\
\text { L }\end{array}$ & $\begin{array}{l}\text { V } \\
\text { L }\end{array}$ & $\begin{array}{l}\text { Tsiala } \\
\text { Tsinger }\end{array}$ & $\begin{array}{r}2.9 \mathrm{~N} \\
56.7 \mathrm{~N}\end{array}$ & $\begin{array}{l}100.0 \mathrm{E} \\
175.6 \mathrm{E}\end{array}$ & $\begin{array}{l}17 \\
44\end{array}$ & $\begin{array}{l}\text { UR } \\
\text { UR }\end{array}$ & $\begin{array}{l}\text { GE } \\
\text { RU }\end{array}$ & $\begin{array}{l}5 \\
5\end{array}$ & $\begin{array}{l}1994 \\
1970\end{array}$ & $\begin{array}{r}112 \\
0\end{array}$ \\
\hline $\mathrm{L}$ & $\mathrm{L}$ & Tsiolkovskiy & $21.2 \mathrm{~S}$ & $128.9 \mathrm{E}$ & 185 & UR & SO & 5 & 1961 & 67 \\
\hline L & $\mathrm{L}$ & Tsu Chung-Chi & $17.3 \mathrm{~N}$ & $145.1 \mathrm{E}$ & 28 & AS & $\mathrm{CH}$ & 5 & 1970 & 0 \\
\hline $\begin{array}{l}\mathrm{M} \\
\mathrm{H}\end{array}$ & $\begin{array}{l}\mathrm{M} \\
\mathbf{H}\end{array}$ & $\begin{array}{l}\text { Tsukuba } \\
\text { Tsurayuki }\end{array}$ & $\begin{array}{l}48.9 \mathrm{~N} \\
63.0 \mathrm{~S}\end{array}$ & $\begin{array}{r}225.9 \mathrm{~W} \\
21.3 \mathrm{~W}\end{array}$ & $\begin{array}{r}2 \\
87\end{array}$ & $\begin{array}{l}\text { AS } \\
\text { AS }\end{array}$ & $\begin{array}{l}\text { JA } \\
\text { JA }\end{array}$ & $\begin{array}{l}5 \\
5\end{array}$ & $\begin{array}{l}1979 \\
1976\end{array}$ & $\begin{array}{l}36 \\
80\end{array}$ \\
\hline $\begin{array}{l}\mathrm{V} \\
\mathrm{M}\end{array}$ & $\begin{array}{l}\mathrm{V} \\
\mathrm{M}\end{array}$ & $\begin{array}{l}\text { Tsvetayeva } \\
\text { Tuapi }\end{array}$ & $\begin{array}{l}64.6 \mathrm{~N} \\
17.3 \mathrm{~N}\end{array}$ & $\begin{array}{l}146.9 \mathrm{E} \\
255.8 \mathrm{~W}\end{array}$ & $\begin{array}{r}40 \\
0\end{array}$ & $\begin{array}{l}\text { UR } \\
\text { SA }\end{array}$ & $\begin{array}{l}\text { SO } \\
\text { NI }\end{array}$ & $\begin{array}{l}5 \\
5\end{array}$ & $\begin{array}{l}1985 \\
1988\end{array}$ & $\begin{array}{l}64 \\
36\end{array}$ \\
\hline
\end{tabular}

ft origin

LF Astronaut-named feature, Apollo 17 site.

AA Town in Sweden.

AA Paolo Dal Pozza; Italian doctor, cartographer (1397-1482).

AA Sidney Dean; American astronomer (1867-1946).

CR Shinto fertility goddess.

AL "White Tract" in Latin.

CA Classical albedo feature name.

FO Classical albedo feature name.

AA Johann Georg; German physicist (1763-1822).

LF Astronaut-named feature Apollo 16 site.

VA Classical name for modern Trebbia River, Italy.

AL Albedo name for $\mathrm{H}-6$, Kuiper region.

LF Astronaut-named feature, Apollo 17 site.

AA Francis A. Paula; Austrian astronomer (1745-1817).

AL Classical name for Sicily.

AA A jester in "The Tempest."

AA Town in Peru.

LF Astronaut-named feature, Apollo 14 site

AA Saved Iseult; fell in love with her.

AL "Crossroad of Charon"; meeting place of several netherworld Canals.

AA Town in Russia.

AA Frances; English novelist (1780-1863).

AA Greek; father of Ganymede.

PE Italian physician (A.D. 1097).

AA Etienne L.; Franco-American astronomer (1827-1895).

AA Etiénne Leopold; French astronomer (1827-1895).

AA Town in Idaho, USA.

AA Town in Russia.

AA Robert J.; American astronomer (1886-1956).

AA Robert J.; American astronomer (1866-1956).

AA Sojourner; American abolishionist (1797-1883).

AA Han dynasty composer (second century A.D.).

AA Friedrich A.; Soviet rocketry scientist (1887-1933).

AA Chinese writer (c. 1715-1763).

AA Town in Botswana.

AA Lidiya; Soviet astronomer (1855-1931).

AA Vitol'd K.; Polish-Russian astronomer (1849-1925).

AA Georgian first name.

AA Nikolaj Ya.; Russian astronomer (1842-1918).

AA Konstantin E.; Soviet physicist (1857-1935).

AA Chinese mathematician (430-501).

AA Japanese mission control site.

AA Ki (Kino); noted Japanese man of letters (c. 945).

AA Marina; Soviet poet (1892-1941).

AA Town in Nicaragua.

\footnotetext{
P: $\quad$ planetary system (see page $x v i)$

Sa: $\quad$ satellite (see page $x v i)$.

lat: latitude of feature center.

long: longitude of feature center.
}

diam: diameter or long dimension of feature.

ct: continent of name origin (see page $284 \mathrm{ff}$.)

et: ethnicity of name origin (see page $284 \mathrm{ff}$.)

as: name approval status (see page xvii). ad: name approval date (year)

ref: reference source for name (see page $287 \mathrm{ff}$.)

ft: feature type (see page 290). 


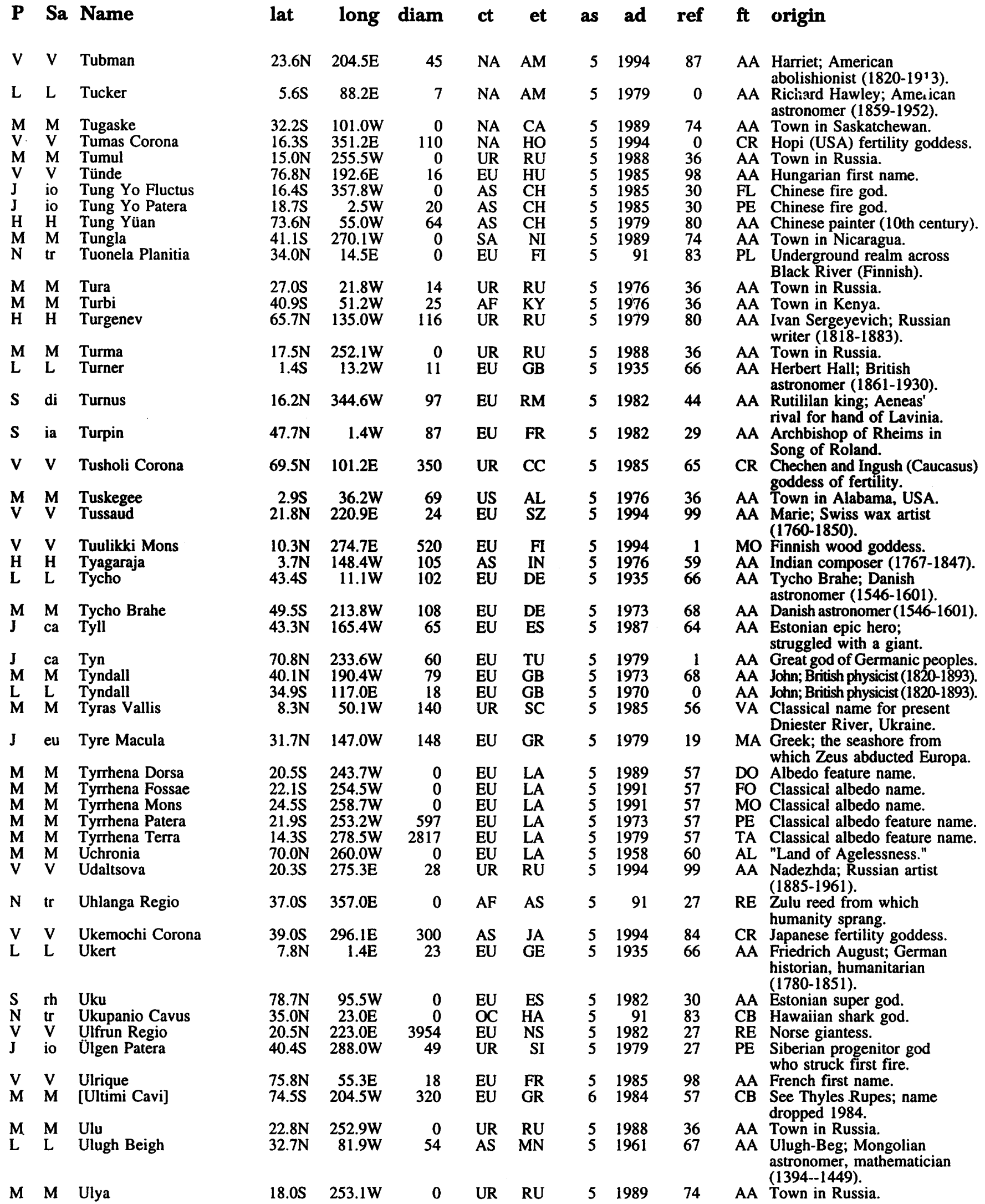

P: $\quad$ planetary system (see page xvi).

Sa: satellite (see page $\mathrm{xvi}$ ).

lat: latitude of feature center.

long: longitude of feature center. diam: diameter or long dimension of feature. ct: continent of name origin (see page $284 \mathrm{ff}$.) et: ethnicity of name origin (see page $284 \mathrm{ff}$.) as: name approval status (see page xvii). ad: name approval date (year).

ref: reference source for name (see page $287 \mathrm{ff}$.). ft: feature type (see page 290). 


\section{ALPHABETICAL LIST OF NAMES}

\section{P Sa Name}

$\begin{array}{lll}\text { M } & \text { M } & \text { Ulysses Fossae } \\ \text { M } & \text { M } & \text { Ulysses Patera } \\ \text { M } & \text { M } & \text { Ulyxis Rupes } \\ \text { M } & \text { M } & \text { Umatac } \\ \text { M } & \text { M } & \text { Umbra } \\ \text { L } & \text { L } & \text { [Undest] } \\ \text { V } & \text { V } & \text { Undset } \\ \text { V } & \text { V } & \text { Uni Dorsa } \\ \text { H } & \text { H } & \text { Unkei } \\ \text { V } & \text { V } & \text { Uorsar Rupes } \\ \text { V } & \text { V } & \text { Upunusa Tholus }\end{array}$

J ga Ur Sulcus

M M Uranius Dorsum

M M Uranius Fossae

M M Uranius Patera

M M Uranius Tholus

L L Urey

M M Urk

U ti Ursula

J ga Uruk Sulcus

V V Ushas Mons

H H Ustad Isa

V V Ut Rupes

J io Uta Fluctūs

J io Uta Patera

M M Utan

S mi Uther

M M Utopia

M M Utopia Planitia

V V Uvaysi

M M Uzboi Vallis

M M Vaals

V V Văcărecu

V V Vacuna Corona

J io Vahagn Patera

L L Väisälä

V V Vaiva Dorsum

V V Vakarine Vallis
V V

V V Valborg

V V Valentina

U ti Valeria

J ca Valfödr

M M Valga

J ca Valhalla

J ca Vali

L L Valier

V V Valkyrie Fossae lat long diam

$\begin{array}{rrrllllr}11.4 \mathrm{~N} & 123.3 \mathrm{~W} & 720 & \text { EU } & \text { GR } & 5 & 1985 & 57 \\ 2.9 \mathrm{~N} & 121.5 \mathrm{~W} & 112 & \text { EU } & \text { GR } & 5 & 1973 & 57 \\ 68.2 \mathrm{~S} & 198.8 \mathrm{~W} & 303 & \text { EU } & \text { GR } & 5 & 1976 & 57 \\ 42.7 \mathrm{~N} & 222.8 \mathrm{~W} & 12 & \text { OC } & \text { GM } & 5 & 1979 & 36 \\ 50.0 \mathrm{~N} & 290.0 \mathrm{~W} & 0 & \text { EU } & \text { LA } & 5 & 1958 & 60 \\ 26.5 \mathrm{~N} & 18.5 \mathrm{~W} & 7 & \text { EU } & \text { NO } & 6 & 1984 & 0 \\ 52.0 \mathrm{~N} & 59.5 \mathrm{E} & 28 & \text { EU } & \text { NO } & 5 & 1985 & 46 \\ 33.7 \mathrm{~N} & 114.3 \mathrm{E} & 800 & \text { EU } & \text { ET } & 5 & 1985 & 65 \\ 31.9 \mathrm{~S} & 62.7 \mathrm{~W} & 123 & \text { AS } & \text { JA } & 5 & 1976 & 59 \\ 76.8 \mathrm{~N} & 341.2 \mathrm{E} & 820 & \text { UR } & \text { CC } & 5 & 1985 & 64 \\ 66.2 \mathrm{~N} & 252.4 \mathrm{E} & 223 & \text { AS } & \text { ID } & 5 & 1985 & 64\end{array}$

$\begin{array}{lllllll}56.5 \mathrm{~N} & 177.3 \mathrm{~W} & 1774 & \text { AS } & \text { SU } & 5 & 1985\end{array}$

27

$23.2 \mathrm{~N}$

$76.3 \mathrm{~W}$

$25.8 \mathrm{~N}$

$26.7 \mathrm{~N}$

$26.5 \mathrm{~N}$

$27.9 \mathrm{~N}$

$90.1 \mathrm{~W}$

$92.6 \mathrm{~W}$

$97.8 \mathrm{~W}$

87.4E

$23.4 \mathrm{~N} \quad 248.7 \mathrm{~W}$

$12.4 \mathrm{~S} \quad 45.2 \mathrm{E}$

$8.4 \mathrm{~N} \quad 169.0 \mathrm{~W}$

352

438

276

71
38

EU GR

EU GR

EU GR

EU GR

NA AM

0
135

EU DU

EU GB

2456

AS

BY

24.3S

32.15

324.6E

413

AS

$55.3 \mathrm{~N} \quad 321.9 \mathrm{E}$

676

AS

IN

TU

UR TK

51982

$32.6 \mathrm{~S}$

$19.2 \mathrm{~W}$

$35.3 \mathrm{~S}$

$24.4 \mathrm{~N}$

$34.9 \mathrm{~S}$

$50.0 \mathrm{~N}$

$24.9 \mathrm{~W}$

246.3W

$251.1 \mathrm{~W}$

$250.0 \mathrm{~W}$

$47.6 \mathrm{~N} \quad 277.3 \mathrm{~W}$

$2.3 \mathrm{~N} \quad 198.2 \mathrm{E}$

$30.9 \mathrm{~S} \quad 35.6 \mathrm{~W}$

4.0S $33.1 \mathrm{~W}$

$63.0 \mathrm{~S}$

199.6E

$60.4 \mathrm{~N} \quad 96.0 \mathrm{E}$

23.8

$25.9 \mathrm{~N}$

53.2S $204.0 \mathrm{E}$

$5.0 \mathrm{~N} \quad 336.4 \mathrm{E}$

49.1S $167.5 \mathrm{E}$

$75.5 \mathrm{~N} \quad 272.0 \mathrm{E}$

$46.4 \mathrm{~N} \quad 144.0 \mathrm{E}$

34.5S 4.2E

$1.2 \mathrm{~S} \quad 247.8 \mathrm{~W}$

44.6S 36.3W

$15.9 \mathrm{~N} \quad 56.6 \mathrm{~W}$

0
30
0
0
0

AS SU

AS SU

UR RU

EU GB

EU

LA

3276 EU LA

UR UZ

340

8

UR

EU

DU

51985

51985

51973

$\begin{array}{ll}5 & 1973 \\ 5 & 1985\end{array}$

51985

$5 \quad 1988$

$\begin{array}{ll}5 & 1988\end{array}$

51979

$\begin{array}{ll}5 & 1982\end{array}$

51979

1985

51979

1988

1982

$\begin{array}{ll}5 & 1958\end{array}$

1973

5094

1996

$\begin{array}{ll}5 & 1976 \\ 5 & 1976\end{array}$

$\begin{array}{ll}5 & 1976 \\ 5 & 1994\end{array}$

57

57

57

57

36

36
85

1

48

80

27

22

28

36

37
60

60

57

107
36

36
36

99

51985

65

448 EU IT

51979

AS AM

51973

8
520
625
29

29

EU LI

$\begin{array}{ll}\text { EU LU } & \text { FR }\end{array}$

51994

$5 \quad 1994$

$\begin{array}{ll}5 & 1994 \\ 5 & 1994\end{array}$

$\begin{array}{lll}19 & \text { EU } & \text { DE } \\ 26 & \text { EU } & \text { LA } \\ 59 & \text { EU } & \text { GB }\end{array}$

$5 \quad 1985$

EU NS

$\begin{array}{ll}5 & 1988 \\ 5 & 1979\end{array}$

16 EU ES

$5 \quad 1976$

2748

EU NS

$5 \quad 1979$

$9.8 \mathrm{~N} \quad 325.2 \mathrm{~W}$

$6.8 \mathrm{~N} \quad 174.5 \mathrm{E}$

\section{6}

EU NS

$58.2 \mathrm{~N}^{\circ} \quad 7.0 \mathrm{E}$

$\begin{array}{rlr}46 & \text { EU } & \text { NS } \\ 67 & \text { EU } & \text { GE } \\ 357 & & \end{array}$

$\begin{array}{ll}5 & 1979\end{array}$

51970

591

\section{ft origin}

FO From albedo feature name.

PE Classical albedo feature name.

RU Classical albedo feature name.

AA Town in Guam, USA.

AL Means "Shadow" in Latin.

AA Sigrid; Norwegian novelist; Nobel laureate (1882-1949).

AA Sigrid; Norwegian author (1882-1949).

DO Etruscan goddess, same as Hera or Juno.

AA Japanese sculptor (c. 1148-1223).

RU Adygan (Caucasus) goddess of hearth.

TH Earth goddess of Leti and Babar (Southwestern islands, eastern Indonesia).

SU Ancient Sumerian seat of moon worship.

DO Named for albedo feature.

FO Classical albedo feature name.

PE Classical albedo feature name.

TH Classical albedo feature name.

AA H.; American chemist; Nobel laureate (1893-1981).

AA Town in Netherlands.

AA Attendant to hero in "Much Ado About Nothing."

SU Babylonian city ruled by Gilgamesh.

MO Indian goddess of dawn.

AA Turkish/Persian architect (17th century).

RU Siberian; Turco-Tatar goddess of the hearth fire.

FL Sumerian sun god.

PE Sumerian sun god.

AA Town in Russia.

AA Ruler of all Britain; Arthur's father.

AL Greek, meaning "nowhere"; ideal state.

PL Classical albedo feature name

AA Uzbek poet (c. 1780-c. 1850).

VA Dry riverbed in Russia.

AA Town in the Netherlands.

AA Helene; Rumanian poet, novelist (1866-1947).

CR Sabinian (Ancient Italy) goddess of harvest.

PE Armenian fire god.

AA Yrjo; Finnish astronomer (1891-1971).

DO Lithaunian rainbow goddess.

VA Lithuanian word for planet Venus.

AA Suzanne; French painter (1865-1840)

AA Danish first name.

AA Latin first name.

AA Friend to Vergilia in "Coriolanus."

AA Norse; a name for Odin, god of wisdom.

AA Town in Estonia.

LG Norse; Odin's hall, where he received the souls of slain warriors.

AA Norse; Ottar's ancestor.

AA Max; German rocketry engineer (1895-1930).

FO Norse battle maidens.
P: $\quad$ planetary system (see page xvi).

Sa: $\quad$ satellite (see page $x v i$ ).

lat: latitude of feature center.

long: longitude of feature center. diam: diameter or long dimension of feature.

ct: continent of name origin (see page $284 \mathrm{ff}$.)

et: ethnicity of name origin (see page $284 \mathrm{ff}$.)

as: name approval status (see page xvii). ad: name approval date (year)

ref: reference source for name (see page $287 \mathrm{ff}$.).

ft: feature type (see page 290). 


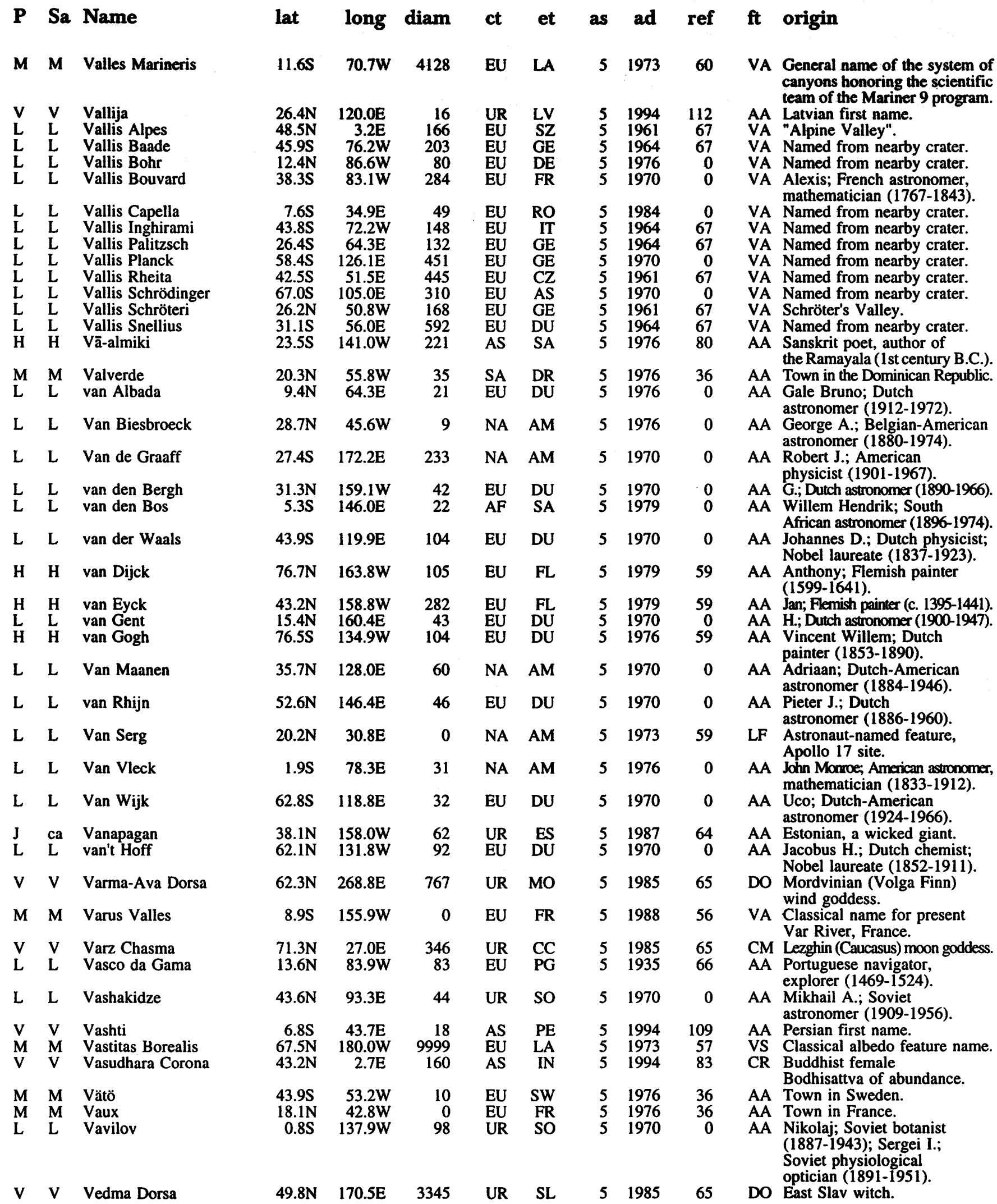

\footnotetext{
P: $\quad$ planetary system (see page $x v i)$.

Sa: $\quad$ satellite (see page $x v i)$.

lat: latitude of feature center.

long: longitude of feature center.
}

diam: diameter or long dimension of feature.

ct: continent of name origin (see page $284 \mathrm{ff}$.)

et: ethnicity of name origin (see page $284 \mathrm{ff}$.)

as: name approval status (see page xvii). ad: name approval date (year).

ref: reference source for name (see page $287 \mathrm{ff}$.).

ft: feature type (see page 290). 


\section{ALPHABETICAL LIST OF NAMES}

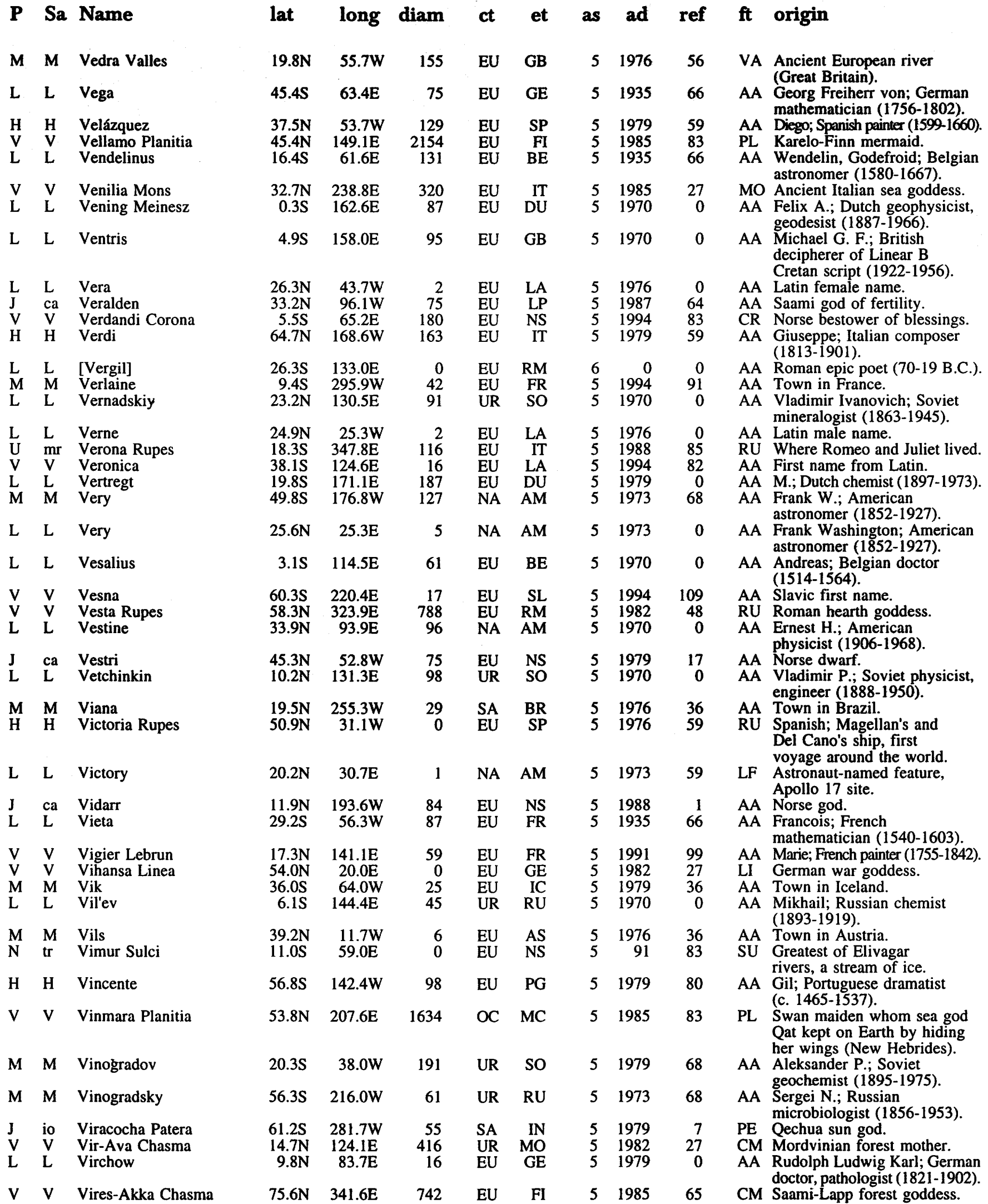

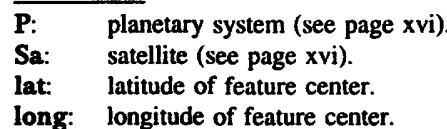

diam: diameter or long dimension of feature.

ct: continent of name origin (see page $284 \mathrm{ff}$.)

et: ethnicity of name origin (see page $284 \mathrm{ff}$.)

as: name approval status (see page xvii). ad: name approval date (year).

ref: reference source for name (see page $287 \mathrm{ff}$.)

ft: feature type (see page 290 ). 


\begin{tabular}{|c|c|c|c|c|c|c|c|c|c|c|}
\hline $\mathbf{P}$ & $\mathbf{S a}$ & Name & lat & long & diam & ct & et & a & ad & ref \\
\hline $\begin{array}{l}V \\
V\end{array}$ & $\begin{array}{l}\text { V } \\
\text { V }\end{array}$ & $\begin{array}{l}\text { Virginia } \\
\text { Virilis Tesserae }\end{array}$ & $\begin{array}{l}52.9 \mathrm{~S} \\
56.1 \mathrm{~N}\end{array}$ & $\begin{array}{l}185.9 \mathrm{E} \\
239.7 \mathrm{E}\end{array}$ & $\begin{array}{r}18 \\
782\end{array}$ & $\begin{array}{l}\text { EU } \\
\text { EU }\end{array}$ & $\begin{array}{l}\text { LA } \\
\text { RM }\end{array}$ & 5 & $\begin{array}{l}1994 \\
1985\end{array}$ & \\
\hline $\begin{array}{l}\mathbf{M} \\
\mathbf{L}\end{array}$ & $\begin{array}{l}\mathrm{M} \\
\mathrm{L}\end{array}$ & $\begin{array}{l}\text { Virrat } \\
\text { Virtanen }\end{array}$ & $\begin{array}{l}31.1 \mathrm{~S} \\
15.5 \mathrm{~N}\end{array}$ & $\begin{array}{l}102.9 \mathrm{~W} \\
176.7 \mathrm{E}\end{array}$ & $\begin{array}{r}0 \\
44\end{array}$ & $\begin{array}{l}\mathrm{EU} \\
\mathrm{EU}\end{array}$ & $\begin{array}{r}\text { SW } \\
\text { FI }\end{array}$ & & $\begin{array}{l}1989 \\
1979\end{array}$ & \\
\hline $\begin{array}{l}\text { V } \\
\mathbf{M}\end{array}$ & $\begin{array}{l}\mathrm{V} \\
\mathrm{M}\end{array}$ & $\begin{array}{l}\text { Virve } \\
\text { Vishniac }\end{array}$ & $\begin{array}{r}5.1 \mathrm{~S} \\
76.7 \mathrm{~S}\end{array}$ & $\begin{array}{l}346.8 \mathrm{E} \\
276.1 \mathrm{~W}\end{array}$ & $\begin{array}{l}19 \\
76\end{array}$ & $\begin{array}{l}\text { UR } \\
\text { NA }\end{array}$ & $\begin{array}{l}\mathrm{ES} \\
\mathrm{AM}\end{array}$ & 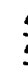 & $\begin{array}{l}1994 \\
1976\end{array}$ & $\begin{array}{r}112 \\
68\end{array}$ \\
\hline $\mathbf{M}$ & $\mathbf{M}$ & Vistula Valles & $14.2 \mathrm{~N}$ & $52.1 \mathrm{~W}$ & 190 & EU & PO & & 1985 & 5 \\
\hline L & L & Vitello & $30.4 S$ & $37.5 \mathrm{~W}$ & 42 & EU & PO & 5 & 1935 & 6 \\
\hline J & $\begin{array}{l}\text { ca } \\
\text { L }\end{array}$ & $\begin{array}{l}\text { Vitr } \\
\text { Vitruvius }\end{array}$ & $\begin{array}{l}22.4 \mathrm{~S} \\
17.6 \mathrm{~N}\end{array}$ & $\begin{array}{c}349.3 \mathrm{~W} \\
31.3 \mathrm{E}\end{array}$ & $\begin{array}{l}76 \\
29\end{array}$ & $\begin{array}{l}\text { EU } \\
\text { EU }\end{array}$ & $\begin{array}{l}\text { NS } \\
\text { RM }\end{array}$ & 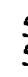 & $\begin{array}{l}1979 \\
1935\end{array}$ & $\begin{array}{l}17 \\
66\end{array}$ \\
\hline $\mathrm{H}$ & $\mathbf{H}$ & Vivaldi & $13.7 \mathrm{~N}$ & $85.0 \mathrm{~W}$ & 213 & EU & IT & 5 & 1976 & 59 \\
\hline $\begin{array}{l}\mathrm{M} \\
\mathrm{N} \\
\mathrm{L}\end{array}$ & $\begin{array}{l}M \\
\operatorname{tr} \\
L\end{array}$ & $\begin{array}{l}\text { Vivero } \\
\text { Viviane Macula } \\
\text { Viviani }\end{array}$ & $\begin{array}{c}49.4 \mathrm{~N} \\
31.0 \mathrm{~S} \\
5.2 \mathrm{~N}\end{array}$ & $\begin{array}{c}241.3 \mathrm{~W} \\
36.5 \mathrm{E} \\
117.1 \mathrm{E}\end{array}$ & $\begin{array}{r}64 \\
0 \\
26\end{array}$ & $\begin{array}{l}\text { EU } \\
\text { EU } \\
\text { EU }\end{array}$ & $\begin{array}{r}\text { SP } \\
\text { WA } \\
\text { IT }\end{array}$ & 5 & $\begin{array}{r}1979 \\
91 \\
1976\end{array}$ & $\begin{array}{l}36 \\
27\end{array}$ \\
\hline L & L & Vlacq & $53.3 \mathrm{~S}$ & $38.8 \mathrm{E}$ & 89 & EU & DU & & 1935 & 66 \\
\hline $\mathbf{H}$ & H & Vlaminck & $28.0 \mathrm{~N}$ & $12.7 \mathrm{~W}$ & 97 & $\mathrm{EU}$ & FR & 5 & 1985 & 59 \\
\hline $\begin{array}{l}\mathrm{N} \\
\mathrm{M}\end{array}$ & $\begin{array}{l}\mathrm{tr} \\
\mathrm{M}\end{array}$ & $\begin{array}{l}\text { Vodyanoy } \\
\text { Voeykov }\end{array}$ & $\begin{array}{l}17.0 \mathrm{~S} \\
32.5 \mathrm{~S}\end{array}$ & $\begin{array}{l}28.5 \mathrm{E} \\
76.1 \mathrm{~W}\end{array}$ & $\begin{array}{r}0 \\
67\end{array}$ & $\begin{array}{l}\text { EU } \\
\text { UR }\end{array}$ & $\begin{array}{l}\text { SL } \\
\text { SO }\end{array}$ & 5 & $\begin{array}{r}91 \\
1979\end{array}$ & $\begin{array}{l}83 \\
68\end{array}$ \\
\hline M & $\mathbf{M}$ & Vogel & $37.0 \mathrm{~S}$ & $13.2 \mathrm{~W}$ & 124 & $\mathrm{EU}$ & GE & & 1973 & 68 \\
\hline L & L & Vogel & $15.1 \mathrm{~S}$ & $5.9 \mathrm{E}$ & 26 & $\mathrm{EU}$ & GE & & 1935 & 66 \\
\hline $\begin{array}{l}\mathrm{M} \\
\mathrm{L}\end{array}$ & $\underset{\mathrm{L}}{\mathrm{M}}$ & $\begin{array}{l}\text { Volgograd } \\
\text { Volkov }\end{array}$ & $\begin{array}{l}48.4 \mathrm{~N} \\
13.6 \mathrm{~S}\end{array}$ & $\begin{array}{l}224.8 \mathrm{~W} \\
131.7 \mathrm{E}\end{array}$ & $\begin{array}{r}2 \\
40\end{array}$ & $\begin{array}{l}\text { UR } \\
\text { UR }\end{array}$ & $\begin{array}{l}\text { SO } \\
\text { SO }\end{array}$ & 5 & $\begin{array}{l}1979 \\
1973\end{array}$ & 36 \\
\hline $\begin{array}{l}\mathrm{V} \\
\mathrm{M} \\
\mathrm{L}\end{array}$ & $\begin{array}{l}V \\
M \\
L\end{array}$ & $\begin{array}{l}\text { Volkova } \\
\text { Vol'Sk } \\
\text { Volta }\end{array}$ & $\begin{array}{l}75.1 \mathrm{~N} \\
23.2 \mathrm{~N} \\
53.9 \mathrm{~N}\end{array}$ & $\begin{array}{c}241.9 \mathrm{E} \\
51.3 \mathrm{~W} \\
84.4 \mathrm{~W}\end{array}$ & $\begin{array}{r}48 \\
8 \\
123\end{array}$ & $\begin{array}{l}\text { UR } \\
\text { UR } \\
\text { EU }\end{array}$ & $\begin{array}{l}\text { RU } \\
\text { RU } \\
\text { IT }\end{array}$ & 5 & $\begin{array}{l}1985 \\
1976 \\
1964\end{array}$ & $\begin{array}{l}64 \\
36 \\
67\end{array}$ \\
\hline L & L & [Voltaire] & $11.9 \mathrm{~S}$ & $100.3 \mathrm{E}$ & 0 & EU & FR & 6 & 1984 & ( \\
\hline M & de & Voltaire & $0.0 \mathrm{~N}$ & $0.0 \mathrm{~W}$ & 0 & EU & FR & 5 & 1973 & 60 \\
\hline $\mathrm{L}$ & L & Volterra & $56.8 \mathrm{~N}$ & $132.2 \mathrm{E}$ & 52 & EU & IT & 5 & 1970 & 0 \\
\hline & io & Volund & $25.0 \mathrm{~N}$ & $184.3 \mathrm{~W}$ & 0 & EU & GE & 5 & 1979 & 27 \\
\hline & L & von Behring & $7.8 \mathrm{~S}$ & $71.8 \mathrm{E}$ & 38 & EU & GE & 3 & 1979 & 0 \\
\hline L & L & von Békésy & $51.9 \mathrm{~N}$ & $126.8 \mathrm{E}$ & 96 & EU & $\mathrm{HU}$ & & 1979 & 0 \\
\hline L & L & von Braun & $41.1 \mathrm{~N}$ & $78.0 \mathrm{~W}$ & 60 & EU & GE & & 1994 & 0 \\
\hline L & $\mathbf{L}$ & von der Pahlen & $24.8 S$ & $132.7 \mathrm{~W}$ & 56 & EU & GE & 5 & 1970 & 0 \\
\hline $\mathbf{M}$ & $\mathbf{M}$ & Von Karman & $64.3 \mathrm{~S}$ & $58.4 \mathrm{~W}$ & 100 & NA & $\mathbf{A M}$ & 5 & 1973 & 68 \\
\hline $\mathbf{L}$ & L & Von Kármán & $44.8 S$ & $175.9 \mathrm{E}$ & 180 & NA & AM & 5 & 1970 & 0 \\
\hline & L & Von Neumann & $40.4 \mathrm{~N}$ & $153.2 \mathrm{E}$ & 78 & NA & AM & 5 & 1970 & 0 \\
\hline V & $\begin{array}{l}\text { V } \\
\text { V }\end{array}$ & $\begin{array}{l}\text { von Paradis } \\
\text { von Schuurman }\end{array}$ & $\begin{array}{r}32.2 \mathrm{~S} \\
5.0 \mathrm{~S}\end{array}$ & $\begin{array}{l}314.8 \mathrm{E} \\
190.9 \mathrm{E}\end{array}$ & $\begin{array}{l}36 \\
29\end{array}$ & $\begin{array}{l}\text { EU } \\
\text { EU }\end{array}$ & $\begin{array}{l}\text { AS } \\
\text { DU }\end{array}$ & 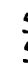 & $\begin{array}{l}1994 \\
1994\end{array}$ & $\begin{array}{l}99 \\
87\end{array}$ \\
\hline & V & von Siebold & $52.0 \mathrm{~S}$ & $36.7 \mathrm{E}$ & 36 & EU & GE & 5 & 1991 & 100 \\
\hline
\end{tabular}

ft origin

AA First name from Latin.

TE One of the names of Fortuna, Roman goddess of chance.

AA Town in Sweden.

AA Artturi Ilmari; Finnish agricultural biochemist; Nobel laureate (1895-1973).

AA Estonian first name.

AA Wolf V.; American microbiologist (1922-1974)

VA Classical name for modern Wistla River in Poland.

AA Witelo, Erazmus Ciokek; Polish physicist, mathematician (1210-1285).

AA Norse dwarf.

AA Vitruvius Pollio, Marcus; Roman engineer, architect (unkn-fl. c. 25 B.C.)

AA Antonio; Italian composer (1678-1741).

AA Town in Spain.

MA Amour of Merlin (Welsh).

AA Vincenzo; Italian physicist, mathematician (1622-1703).

AA Adriaan; Dutch mathematician (c. 1600-1667).

AA Maurice de; French painter (1876-1958)

AA Slavic water spirit.

AA A. I.; Russian climatologist and geographer (1842-1916)

AA Hermann; German astronomer (1841-1907)

AA Hermann Karl; German astronomer (1841-1907)

AA Soviet launch site.

AA Vladislav N.; Soviet engineer (1935-1971).

AA Anna; Russian chemist (1800-1876).

AA Town in Russia.

AA Count Allessandro G. A. A.; Italian physicist (1745-1827).

AA Francois; French philosopher (1694-1778).

AA Francios M.; French writer (1694-1778)

AA Vito; Italian mathematician, physicist (1860-1940).

ER Germanic supreme smith of the gods.

AA Emil Adolf; German bacteriologist; Nobel laureate (1854-1917).

AA Georg; Hungarian otological physicist; Nobel laureate (1899-1972).

AA Werner, German-American rocket pioneer (1912-1977).

AA Emanuel; German astronomer (1882-1952)

AA Theodore; American aeronautical engineer (1881-1963).

AA Theodore; American aeronautical scientist (1881-1963).

AA John; American mathematician (1903-195்7).

AA Maria; Austrian pianist (1759-1834).

AA Anna; Dutch linguist, writer, artist (1607-1678).

AA Regina; German physician, educator (1771-1849).

\footnotetext{
P: $\quad$ planetary system (see page xvi).

Sa: $\quad$ satellite (see page xvi)

lat: latitude of feature center.

long: longitude of feature center.
}

diam: diameter or long dimension of feature. ct: continent of name origin (see page $284 \mathrm{ff}$.) et: ethnicity of name origin (see page $284 \mathrm{ff}$.) as: name approval status (see page xvii) ad: name approval date (year).

ref: reference source for name (see page $287 \mathrm{ff}$.). ft: feature type (see page 290). 
ALPHABETICAL LIST OF NAMES

\begin{tabular}{|c|c|c|c|c|c|c|c|c|c|c|}
\hline $\mathbf{P}$ & $\mathbf{S a}$ & Name & lat & long & diam & ct & et & as & ad & ref \\
\hline V & V & von Suttner & $10.7 \mathrm{~S}$ & $234.9 \mathrm{E}$ & 23 & EU & AS & 5 & 1994 & \\
\hline $\mathbf{L}$ & L & von Zeipel & $42.6 \mathrm{~N}$ & $141.6 \mathrm{~W}$ & 83 & EU & SW & 5 & 1970 & \\
\hline $\begin{array}{l}\mathrm{M} \\
\mathrm{L}\end{array}$ & $\begin{array}{l}\mathbf{M} \\
\mathbf{L}\end{array}$ & $\begin{array}{l}\text { Voo } \\
\text { Voskresenskiy }\end{array}$ & $\begin{array}{l}27.3 \mathrm{~S} \\
28.0 \mathrm{~N}\end{array}$ & $\begin{array}{l}19.8 \mathrm{~W} \\
88.1 \mathrm{~W}\end{array}$ & $\begin{array}{r}2 \\
49\end{array}$ & $\begin{array}{l}\text { AF } \\
\text { UR }\end{array}$ & $\begin{array}{l}\text { KY } \\
\text { SO }\end{array}$ & $\begin{array}{l}5 \\
5\end{array}$ & $\begin{array}{l}1976 \\
1970\end{array}$ & $\begin{array}{r}36 \\
0\end{array}$ \\
\hline $\mathbf{L}$ & L & Voskresenskiy & $28.0 \mathrm{~N}$ & $88.1 \mathrm{~W}$ & 49 & UR & so & 5 & 1970 & 0 \\
\hline H & $\mathbf{H}$ & Vostok Rupes & $37.7 \mathrm{~S}$ & $19.5 \mathrm{~W}$ & 0 & UR & $\mathbf{R U}$ & 5 & 1976 & \\
\hline $\begin{array}{l}\mathbf{V} \\
\mathbf{M} \\
\mathbf{M}\end{array}$ & $\begin{array}{l}\mathbf{V} \\
\mathbf{M} \\
\mathbf{M}\end{array}$ & $\begin{array}{l}\text { Voynich } \\
\text { Voza } \\
\text { Vulcani Pelagus }\end{array}$ & $\begin{array}{l}35.4 \mathrm{~N} \\
23.5 \mathrm{~N} \\
35.0 \mathrm{~S}\end{array}$ & $\begin{array}{l}56.0 \mathrm{E} \\
53.5 \mathrm{~W} \\
15.0 \mathrm{~W}\end{array}$ & $\begin{array}{r}55 \\
0 \\
0\end{array}$ & $\begin{array}{l}\text { EU } \\
\text { OC } \\
\text { EU }\end{array}$ & $\begin{array}{r}\text { GB } \\
\text { SO } \\
\text { RM }\end{array}$ & $\begin{array}{l}5 \\
5 \\
5\end{array}$ & $\begin{array}{l}1985 \\
1988 \\
1958\end{array}$ & $\begin{array}{l}46 \\
36 \\
60\end{array}$ \\
\hline $\begin{array}{l}\mathbf{J} \\
\mathbf{J} \\
\mathrm{U} \\
\mathrm{H} \\
\mathrm{L}\end{array}$ & $\begin{array}{l}\text { ca } \\
\text { ca } \\
\text { um } \\
\mathrm{H} \\
\mathrm{L}\end{array}$ & $\begin{array}{l}\text { Vu-Murt } \\
\text { Vutash } \\
\text { Vuver } \\
\text { Vyāsa } \\
\text { W. Bond }\end{array}$ & $\begin{array}{r}22.9 \mathrm{~N} \\
31.9 \mathrm{~N} \\
4.7 \mathrm{~S} \\
48.3 \mathrm{~N} \\
65.4 \mathrm{~N}\end{array}$ & $\begin{array}{c}170.9 \mathrm{~W} \\
102.9 \mathrm{~W} \\
311.6 \mathrm{E} \\
81.1 \mathrm{~W} \\
4.5 \mathrm{~W}\end{array}$ & $\begin{array}{r}79 \\
55 \\
98 \\
290 \\
156\end{array}$ & $\begin{array}{l}\text { UR } \\
\text { UR } \\
\text { UR } \\
\text { AS } \\
\text { NA }\end{array}$ & $\begin{array}{r}\text { ES } \\
\text { ES } \\
\text { MA } \\
\text { IN } \\
\text { AM }\end{array}$ & $\begin{array}{l}5 \\
5 \\
5 \\
5 \\
5\end{array}$ & $\begin{array}{l}1987 \\
1987 \\
1988 \\
1979 \\
1935\end{array}$ & $\begin{array}{l}64 \\
64 \\
94 \\
59 \\
66\end{array}$ \\
\hline $\begin{array}{l}\mathrm{M} \\
\mathrm{M} \\
\mathrm{H}\end{array}$ & $\begin{array}{l}\mathbf{M} \\
\mathbf{M} \\
\mathbf{H}\end{array}$ & $\begin{array}{l}\text { W. Mareotis Tholus } \\
\text { Wabash } \\
\text { Wagner }\end{array}$ & $\begin{array}{l}35.8 \mathrm{~N} \\
21.5 \mathrm{~N} \\
67.4 \mathrm{~S}\end{array}$ & $\begin{array}{r}87.5 \mathrm{~W} \\
33.7 \mathrm{~W} \\
114.0 \mathrm{~W}\end{array}$ & $\begin{array}{r}0 \\
42 \\
140\end{array}$ & $\begin{array}{l}\text { EU } \\
\text { US } \\
\text { EU }\end{array}$ & $\begin{array}{l}\text { GR } \\
\text { IN } \\
\text { GE }\end{array}$ & $\begin{array}{l}5 \\
5 \\
5\end{array}$ & $\begin{array}{l}1991 \\
1976 \\
1976\end{array}$ & $\begin{array}{l}57 \\
36 \\
59\end{array}$ \\
\hline $\begin{array}{l}\mathbf{M} \\
\mathbf{M} \\
\mathbf{L}\end{array}$ & $\begin{array}{l}\mathbf{M} \\
\mathbf{M} \\
\mathbf{L}\end{array}$ & $\begin{array}{l}\text { Wahoo } \\
\text { Wajir } \\
\text { Walker }\end{array}$ & $\begin{array}{l}23.5 \mathrm{~N} \\
27.2 \mathrm{~S} \\
26.0 \mathrm{~S}\end{array}$ & $\begin{array}{r}33.6 \mathrm{~W} \\
254.5 \mathrm{~W} \\
162.2 \mathrm{~W}\end{array}$ & $\begin{array}{r}66 \\
0 \\
32\end{array}$ & $\begin{array}{l}\text { US } \\
\text { AF } \\
\text { NA }\end{array}$ & $\begin{array}{l}\mathrm{NE} \\
\mathrm{KY} \\
\mathrm{AM}\end{array}$ & $\begin{array}{l}5 \\
5 \\
5\end{array}$ & $\begin{array}{l}1976 \\
1989 \\
1970\end{array}$ & $\begin{array}{r}36 \\
74 \\
0\end{array}$ \\
\hline L & $\mathbf{L}$ & Wallace & $20.3 \mathrm{~N}$ & $8.7 \mathrm{~W}$ & 26 & EU & GB & 5 & 1935 & \\
\hline M & $\mathbf{M}$ & Wallace & $52.8 \mathrm{~S}$ & $249.2 \mathrm{~W}$ & 159 & $\mathrm{EU}$ & GB & 5 & 1973 & 8 \\
\hline $\mathbf{L}$ & $\mathrm{L}$ & Wallach & $4.9 \mathrm{~N}$ & $32.3 \mathrm{E}$ & 6 & EU & $\mathrm{GE}$ & 5 & 1979 & \\
\hline $\begin{array}{l}\mathrm{M} \\
\mathrm{L} \\
\mathrm{L}\end{array}$ & $\begin{array}{l}\text { M } \\
\text { L } \\
\text { L }\end{array}$ & $\begin{array}{l}\text { Wallops } \\
\text { Walter } \\
\text { Walter (Walther) }\end{array}$ & $\begin{array}{l}46.9 \mathrm{~N} \\
28.0 \mathrm{~N} \\
33.1 \mathrm{~S}\end{array}$ & $\begin{array}{c}227.2 \mathrm{~W} \\
33.8 \mathrm{~W} \\
1.0 \mathrm{E}\end{array}$ & $\begin{array}{r}2 \\
1 \\
128\end{array}$ & $\begin{array}{l}\text { NA } \\
\text { EU } \\
\text { EU }\end{array}$ & $\begin{array}{c}\mathrm{AM} \\
\mathrm{GE} \\
\mathrm{GE}\end{array}$ & $\begin{array}{l}5 \\
5 \\
5\end{array}$ & $\begin{array}{l}1979 \\
1979 \\
1935\end{array}$ & $\begin{array}{r}36 \\
0 \\
66\end{array}$ \\
\hline $\begin{array}{l}\text { V } \\
\mathrm{H} \\
\mathrm{V}\end{array}$ & $\begin{array}{l}\mathrm{V} \\
\mathbf{H} \\
\mathrm{V}\end{array}$ & $\begin{array}{l}\text { Wanda } \\
\text { Wang Meng } \\
\text { Wang Zhenyi }\end{array}$ & $\begin{array}{r}71.3 \mathrm{~N} \\
8.8 \mathrm{~N} \\
13.2 \mathrm{~N}\end{array}$ & $\begin{array}{l}325.0 \mathrm{E} \\
103.8 \mathrm{~W} \\
217.8 \mathrm{E}\end{array}$ & $\begin{array}{r}18 \\
165 \\
25\end{array}$ & $\begin{array}{l}\text { EU } \\
\text { AS } \\
\text { AS }\end{array}$ & $\begin{array}{l}\mathrm{PO} \\
\mathrm{CH} \\
\mathrm{CH}\end{array}$ & $\begin{array}{l}5 \\
5 \\
5\end{array}$ & $\begin{array}{l}1985 \\
1976 \\
1994\end{array}$ & $\begin{array}{r}98 \\
59 \\
105\end{array}$ \\
\hline $\begin{array}{l}\mathrm{L} \\
\mathrm{L}\end{array}$ & $\begin{array}{l}\text { L } \\
\text { L }\end{array}$ & $\begin{array}{l}\text { Wan-Hoo } \\
\text { Wargentin }\end{array}$ & $\begin{array}{r}9.8 \mathrm{~S} \\
49.6 \mathrm{~S}\end{array}$ & $\begin{array}{r}138.8 W \\
60.2 W\end{array}$ & $\begin{array}{l}52 \\
84\end{array}$ & $\begin{array}{l}\text { AS } \\
\text { EU }\end{array}$ & $\begin{array}{l}\mathrm{CH} \\
\mathrm{SW}\end{array}$ & $\begin{array}{l}5 \\
5\end{array}$ & $\begin{array}{l}1970 \\
1935\end{array}$ & $\begin{array}{l}0 \\
6\end{array}$ \\
\hline $\mathbf{L}$ & $\mathbf{L}$ & Warner & $4.0 \mathrm{~S}$ & 87.3E & 35 & NA & $\mathbf{A M}$ & 5 & 1976 & 0 \\
\hline $\begin{array}{l}\mathbf{M} \\
\mathbf{M} \\
\mathbf{V}\end{array}$ & $\begin{array}{l}\mathbf{M} \\
\mathbf{M} \\
\mathbf{V}\end{array}$ & $\begin{array}{l}\text { Warra } \\
\text { Warrego Valles } \\
\text { Warren }\end{array}$ & $\begin{array}{l}20.8 \mathrm{~N} \\
43.0 \mathrm{~S} \\
11.8 \mathrm{~S}\end{array}$ & $\begin{array}{c}37.5 W \\
93.1 W \\
176.5 E\end{array}$ & $\begin{array}{r}11 \\
164 \\
53\end{array}$ & $\begin{array}{l}\text { OC } \\
\text { OC } \\
\text { NA }\end{array}$ & $\begin{array}{l}\mathrm{AU} \\
\mathrm{AU} \\
\mathrm{AM}\end{array}$ & $\begin{array}{l}5 \\
5 \\
5\end{array}$ & $\begin{array}{l}1976 \\
1979 \\
1994\end{array}$ & $\begin{array}{l}36 \\
36 \\
87\end{array}$ \\
\hline $\begin{array}{l}M \\
M \\
L\end{array}$ & $\begin{array}{l}\mathrm{M} \\
\mathrm{M} \\
\mathrm{L}\end{array}$ & $\begin{array}{l}\text { Waspam } \\
\text { Wassamu } \\
\text { Waterman }\end{array}$ & $\begin{array}{l}20.7 \mathrm{~N} \\
25.5 \mathrm{~N} \\
25.9 \mathrm{~S}\end{array}$ & $\begin{array}{c}56.6 \mathrm{~W} \\
53.0 \mathrm{~W} \\
128.0 \mathrm{E}\end{array}$ & $\begin{array}{l}40 \\
17 \\
76\end{array}$ & $\begin{array}{l}\text { SA } \\
\text { AS } \\
\text { NA }\end{array}$ & $\begin{array}{r}\text { NI } \\
\text { JA } \\
\text { AM }\end{array}$ & $\begin{array}{l}5 \\
5 \\
5\end{array}$ & $\begin{array}{l}1976 \\
1976 \\
1970\end{array}$ & $\begin{array}{r}36 \\
36 \\
0\end{array}$ \\
\hline L & $\mathbf{L}$ & Watson & $62.6 \mathrm{~S}$ & $124.5 \mathrm{~W}$ & 62 & NA & AM & 5 & 1970 & 0 \\
\hline L & $\mathrm{L}$ & Watt & $49.5 \mathrm{~S}$ & $48.6 \mathrm{E}$ & 66 & EU & $\mathrm{SC}$ & 5 & 1935 & 66 \\
\hline $\mathbf{L}$ & $\mathbf{L}$ & Watts & $8.9 \mathrm{~N}$ & $46.3 \mathrm{E}$ & 15 & NA & $\mathrm{AM}$ & 5 & 1973 & 0 \\
\hline $\begin{array}{l}\mathrm{M} \\
\mathrm{L}\end{array}$ & $\begin{array}{l}\mathrm{M} \\
\mathrm{L}\end{array}$ & $\begin{array}{l}\text { Wau } \\
\text { Webb }\end{array}$ & $\begin{array}{r}45.2 \mathrm{~S} \\
0.9 \mathrm{~S}\end{array}$ & $\begin{array}{l}42.4 \mathrm{~W} \\
60.0 \mathrm{E}\end{array}$ & $\begin{array}{r}3 \\
21\end{array}$ & $\begin{array}{l}\text { OC } \\
\text { EU }\end{array}$ & $\begin{array}{l}\text { GU } \\
\text { GB }\end{array}$ & $\begin{array}{l}5 \\
5\end{array}$ & $\begin{array}{l}1976 \\
1935\end{array}$ & $\begin{array}{l}36 \\
66\end{array}$ \\
\hline L & L & Weber & $50.4 \mathrm{~N}$ & $123.4 \mathrm{~W}$ & 42 & EU & GE & 5 & 1970 & 0 \\
\hline $\begin{array}{l}\mathbf{M} \\
\mathbf{M}\end{array}$ & $\begin{array}{l}\mathbf{M} \\
\mathbf{M}\end{array}$ & $\begin{array}{l}\text { Weer } \\
\text { Wegener }\end{array}$ & $\begin{array}{l}19.8 \mathrm{~N} \\
64.3 \mathrm{~S}\end{array}$ & $\begin{array}{r}51.4 \mathrm{~W} \\
4.0 \mathrm{~W}\end{array}$ & $\begin{array}{r}9 \\
70\end{array}$ & $\begin{array}{l}\text { EU } \\
\text { EU }\end{array}$ & $\begin{array}{l}\mathrm{DU} \\
\mathrm{GE}\end{array}$ & $\begin{array}{l}5 \\
5\end{array}$ & $\begin{array}{l}1976 \\
1973\end{array}$ & $\begin{array}{l}36 \\
68\end{array}$ \\
\hline
\end{tabular}

ft origin

AA Bertha; Austrian journalist, pacifist (1843-1914).

AA E. H.; Swedish astronomer (1873-1959).

AA Town in Kenya.

AA Leonid A.; Soviet rocketry scientist (1913-1965).

RI Leonid A.; Soviet rocketry scientist (1913-1965).

RU Russian; Bellingshausen's ship for Antarctic exploration, 1819-21.

AA Lilian; English writer (18641960).

AA Town in Solomon Islands.

AL Named for Vulcan, Roman god of fire.

AA Estonian spirit of water.

AA Estonian spirit of water.

AA Volga Finn evil spirit.

AA Indian poet (fl. 1500 B.C.).

AA William Cranch; American astronomer (1789-1859).

TH Classical albedo name.

AA Town in Indiana, USA.

AA Richard; German composer (1813-1883)

AA Town in Nebraska, USA.

AA Town in Kenya.

AA Joseph A.; American test pilot (1921-1966).

AA Alfred Russel; British natural historian (1823-1913).

AA Alfred R.; British biologist (1823-1913).

AA Otto; German chemist; Nobel laureate (1847-1931).

AA American launch site.

AA German male name.

AA Bernard; German astronomer (1430-1504).

AA Polish first name.

AA Chinese painter (1308-1385).

AA Chinese astronomer, geophysicist (18th century).

AA Chinese inventor (unkn-c. 1500).

AA Pehr Vilhelm; Swedish astronomer (1717-1783).

AA Worcester Reed; American inventor (1846-1929).

AA Town in Australia.

VA Modern Australian River.

AA Mercy; American colonial poet, playwright, historian (1728-1814).

AA Town in Nicaragua.

AA Town in Japan.

AA Alan T.; American physicist (1892-1967)

AA James C.; American astronomer (1838-1880).

AA James; Scottish inventor (1736-1819).

AA Chester Burleigh; American astronomer (1889-1971).

AA Town in New Guinea.

AA Thomas William; British astronomer (1806-1885).

AA Wilhelm E.; German physicist (1804-1891).

AA Town in the Netherlands.

AA Alfred L.; German geophysicist (1880-1930).

\footnotetext{
P: $\quad$ planetary system (see page xvi).

Sa: satellite (see page $x v i$ ).

lat: latitude of feature center.

long: longitude of feature center.
}

diam: diameter or long dimension of feature.

ct: continent of name origin (see page $284 \mathrm{ff}$.)

et: ethnicity of name origin (see page $284 \mathrm{ff}$.)

as: name approval status (see page xvii). ad: name approval date (year)

ref: reference source for name (see page $287 \mathrm{ff}$.). ft: feature type (see page 290) 


\section{ALPHABETICAL LIST OF NAMES}

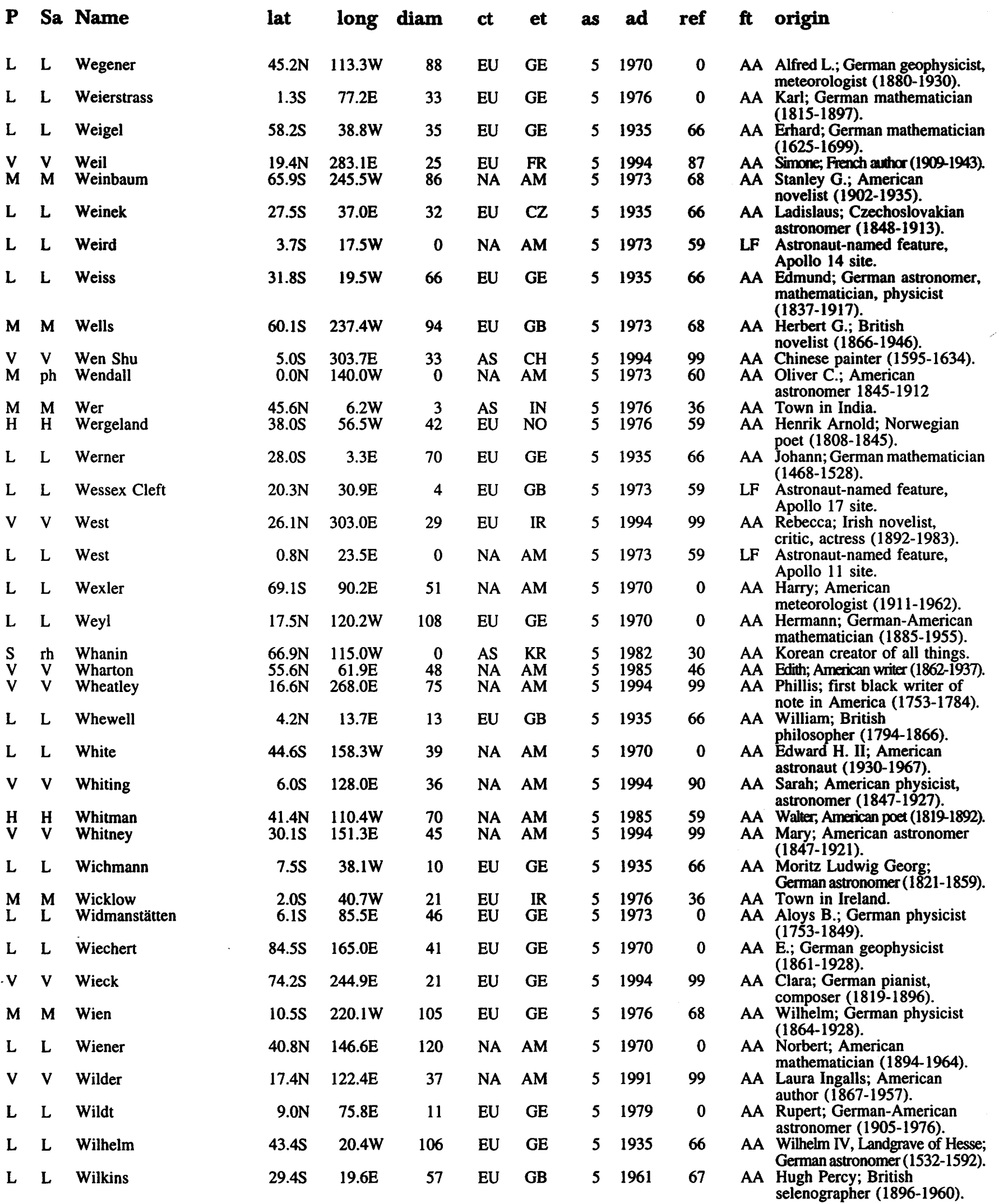

\footnotetext{
P: $\quad$ planetary system (see page $x v i)$.

Sa: $\quad$ satellite (see page $x v i$ ).

lat: latitude of feature center.

long: longitude of feature center.
}

diam: diameter or long dimension of feature.

ct: continent of name origin (see page $284 \mathrm{ff}$.)

et: ethnicity of name origin (see page $284 \mathrm{ff}$.)

as: name approval status (see page xvii). ad: name approval date (year).

ref: reference source for name (see page $287 \mathrm{ff}$.).

ft: feature type (see page 290 ). 


\section{ALPHABETICAL LIST OF NAMES}

\section{P Sa Name \\ V V Willard \\ L L Williams \\ M M Williams \\ M M Wilmington \\ $\begin{array}{lll}\text { L } & \text { L } & \text { Wilsing } \\ \text { L } & \text { L } & \text { Wilson }\end{array}$}

\begin{tabular}{|c|c|c|}
\hline $\begin{array}{l}\mathbf{M} \\
\mathbf{V}\end{array}$ & $\begin{array}{l}\mathbf{M} \\
\mathbf{V}\end{array}$ & $\begin{array}{l}\text { Windfall } \\
\text { Winema }\end{array}$ \\
\hline$\underset{\mathrm{L}}{\mathrm{M}}$ & $\begin{array}{l}\text { M } \\
\mathbf{L}\end{array}$ & $\begin{array}{l}\text { Wink } \\
\text { Winkler }\end{array}$ \\
\hline & $\mathrm{L}$ & Winlock \\
\hline V & V & Winnemucca \\
\hline & $\mathbf{L}$ & Winthrop \\
\hline M & $\mathbf{M}$ & Wirtz \\
\hline $\mathbf{M}$ & $\mathbf{M}$ & Wislicenus \\
\hline 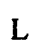 & $\mathrm{L}$ & Wöhler \\
\hline $\mathrm{U}$ & um & Wokolo \\
\hline $\mathbf{I}$ & $\mathbf{L}$ & Wolf \\
\hline & $\mathrm{L}$ & Wollaston \\
\hline $\begin{array}{l}\text { V } \\
\mathrm{L} \\
\mathrm{L}\end{array}$ & $\begin{array}{l}\text { V } \\
\text { L } \\
\text { L }\end{array}$ & $\begin{array}{l}\text { Wollstonecraft } \\
\text { Woltjer } \\
\text { Wood }\end{array}$ \\
\hline 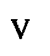 & $\mathrm{V}$ & Woodhull Patera \\
\hline $\begin{array}{l}\mathrm{V} \\
\mathrm{M} \\
\mathrm{M} \\
\mathrm{M}\end{array}$ & $\begin{array}{l}\mathrm{V} \\
\mathrm{M} \\
\mathrm{M} \\
\mathrm{M} \\
\mathrm{V}\end{array}$ & $\begin{array}{l}\text { Woolf } \\
\text { Woolgar } \\
\text { Woomera } \\
\text { Worcester } \\
\text { Workman }\end{array}$ \\
\hline & $\mathbf{L}$ & Wreck \\
\hline & $\mathbf{H}$ & Wren \\
\hline & $\mathbf{M}$ & Wright \\
\hline & $\mathbf{L}$ & Wright \\
\hline
\end{tabular}

L L Wróblewski

L L Wrottesley

V V Wu Hou

M M Wukari

M M Wum

U um Wunda

S rh Wuraka

V V Wurunsemu Tholus

$\begin{array}{lrrrrrrr}\text { lat } & \text { long } & \text { diam } & \text { ct } & \text { et } & \text { as } & \text { ad } & \text { ref } \\ 24.7 \mathrm{~S} & 296.1 \mathrm{E} & 47 & \mathrm{NA} & \mathrm{AM} & 5 & 1994 & 99 \\ 42.0 \mathrm{~N} & 37.2 \mathrm{E} & 36 & \mathrm{EU} & \text { GB } & 5 & 1935 & 66 \\ 18.8 \mathrm{~S} & 164.1 \mathrm{~W} & 125 & \mathrm{EU} & \text { GB } & 5 & 1973 & 68 \\ & & & & & & & \\ 21.9 \mathrm{~N} & 47.5 \mathrm{~W} & 1 & \text { US } & \text { DE } & 5 & 1979 & 36 \\ 21.5 \mathrm{~S} & 155.2 \mathrm{~W} & 73 & \mathrm{EU} & \text { GE } & 5 & 1970 & 0 \\ 69.2 \mathrm{~S} & 42.4 \mathrm{~W} & 69 & \text { EU } & \text { SC } & 5 & 1935 & 66\end{array}$

\begin{tabular}{|c|c|c|c|c|c|c|}
\hline $\begin{array}{l}2.1 \mathrm{~S} \\
3.1 \mathrm{~N}\end{array}$ & $\begin{array}{l}43.5 W \\
168.6 \mathrm{E}\end{array}$ & $\begin{array}{l}20 \\
22\end{array}$ & $\begin{array}{l}\text { NA } \\
\text { NA }\end{array}$ & $\begin{array}{l}\text { CA } \\
\mathbf{A M}\end{array}$ & $\begin{array}{l}5 \\
5\end{array}$ & $\begin{array}{l}1976 \\
1994\end{array}$ \\
\hline $\begin{array}{c}6.6 \mathrm{~S} \\
42.2 \mathrm{~N}\end{array}$ & $\begin{array}{r}41.5 \mathrm{~W} \\
179.0 \mathrm{~W}\end{array}$ & $\begin{array}{r}8 \\
22\end{array}$ & $\begin{array}{l}\text { US } \\
\text { EU }\end{array}$ & $\begin{array}{l}\mathrm{TX} \\
\mathrm{GE}\end{array}$ & $\begin{array}{l}5 \\
5\end{array}$ & $\begin{array}{l}1976 \\
1970\end{array}$ \\
\hline $35.6 \mathrm{~N}$ & $105.6 \mathrm{~W}$ & 64 & NA & AM & 5 & 1970 \\
\hline $15.4 \mathrm{~S}$ & $121.1 \mathrm{E}$ & 30 & NA & AM & 5 & 1994 \\
\hline $10.7 \mathrm{~S}$ & $44.4 W$ & 17 & NA & $\mathrm{AM}$ & 5 & 1976 \\
\hline $48.7 \mathrm{~S}$ & $25.8 \mathrm{~W}$ & 128 & EU & GE & 5 & 1973 \\
\hline $18.3 \mathrm{~S}$ & $348.7 \mathrm{~W}$ & 138 & $\mathrm{EU}$ & GE & 5 & 1973 \\
\hline $38.2 S$ & $31.4 \mathrm{E}$ & 27 & EU & GE & 5 & 1935 \\
\hline $30.0 \mathrm{~S}$ & $1.8 \mathrm{E}$ & 208 & $\mathrm{AF}$ & BA & 5 & 1988 \\
\hline $22.7 S$ & $16.6 \mathrm{~W}$ & 25 & EU & GE & 5 & 1935 \\
\hline $30.6 \mathrm{~N}$ & $46.9 \mathrm{~W}$ & 10 & EU & GB & 5 & 1935 \\
\hline $\begin{array}{l}39.2 \mathrm{~S} \\
45.2 \mathrm{~N} \\
43.0 \mathrm{~N}\end{array}$ & $\begin{array}{l}260.7 \mathrm{E} \\
159.6 \mathrm{~W} \\
120.8 \mathrm{~W}\end{array}$ & $\begin{array}{l}44 \\
46 \\
78\end{array}$ & $\begin{array}{l}\text { EU } \\
\text { EU } \\
\text { NA }\end{array}$ & $\begin{array}{l}\text { EN } \\
\text { DU } \\
\text { AM }\end{array}$ & $\begin{array}{l}5 \\
5 \\
5\end{array}$ & $\begin{array}{l}1994 \\
1970 \\
1970\end{array}$ \\
\hline $37.4 \mathrm{~N}$ & $305.4 \mathrm{E}$ & 83 & NA & AM & 5 & 91 \\
\hline $\begin{array}{l}37.7 \mathrm{~S} \\
34.8 \mathrm{~N} \\
48.4 \mathrm{~N} \\
26.8 \mathrm{~N} \\
12.9 \mathrm{~S}\end{array}$ & $\begin{array}{c}27.1 \mathrm{E} \\
85.5 \mathrm{~W} \\
227.3 \mathrm{~W} \\
50.3 \mathrm{~W} \\
299.9 \mathrm{E}\end{array}$ & $\begin{array}{r}25 \\
0 \\
3 \\
0 \\
19\end{array}$ & $\begin{array}{l}\text { EU } \\
\text { OC } \\
\text { OC } \\
\text { US } \\
\text { NA }\end{array}$ & $\begin{array}{l}\text { GB } \\
\text { AU } \\
\text { AU } \\
\text { NY } \\
\text { AM }\end{array}$ & $\begin{array}{l}5 \\
5 \\
5 \\
5 \\
5\end{array}$ & $\begin{array}{l}1991 \\
1991 \\
1979 \\
1988 \\
1994\end{array}$ \\
\hline $9.1 \mathrm{~S}$ & $15.5 \mathrm{E}$ & 1 & NA & $\mathrm{AM}$ & 5 & 1973 \\
\hline $24.3 \mathrm{~N}$ & $35.2 \mathrm{~W}$ & 221 & EU & EN & 5 & 1979 \\
\hline $58.6 \mathrm{~S}$ & $150.8 \mathrm{~W}$ & 106 & NA & AM & 5 & 1973 \\
\hline $31.6 \mathrm{~S}$ & $86.6 \mathrm{~W}$ & 39 & NA & AM & 5 & 1964 \\
\hline
\end{tabular}

$\begin{array}{rrrrrrr}24.0 \mathrm{~S} & 152.8 \mathrm{E} & 21 & \mathrm{EU} & \mathrm{PO} & 5 & 1976 \\ 23.9 \mathrm{~S} & 56.8 \mathrm{E} & 57 & \mathrm{EU} & \mathrm{GB} & 5 & 1935 \\ & & & & & & \\ 25.4 \mathrm{~S} & 317.4 \mathrm{E} & 30 & \mathrm{AS} & \mathrm{CH} & 5 & 1994 \\ 32.2 \mathrm{~S} & 102.8 \mathrm{~W} & 0 & \mathrm{AF} & \mathrm{NI} & 5 & 1989 \\ 16.1 \mathrm{~N} & 246.2 \mathrm{~W} & 0 & \mathrm{AF} & \mathrm{CR} & 5 & 1988 \\ 7.9 \mathrm{~S} & 273.6 \mathrm{E} & 131 & \text { OC } & \text { AU } & 5 & 1988 \\ 25.1 \mathrm{~N} & 4.0 \mathrm{~W} & 0 & \text { OC } & \text { AU } & 5 & 1982 \\ 40.6 \mathrm{~N} & 209.9 \mathrm{E} & 83 & \text { AS } & \text { TU } & 5 & 1985\end{array}$

ft origin

AA Emma; American educator (1787-1870).

AA Arthur Stanley; British astronomer (1861-1938).

AA Arthur S.; British astronomer (1861-1938).

AA American colonial town (Delaware).

AA J.; German astronomer (1856-1943).

AA Alexander; Scottish astronomer (17141786); Charles T.R.; Scottish physicist (1869-1959); Ralph E.; American astronomer (1886-1960).

AA Town in Alberta, Canada.

AA Modoc Indian heroine, peacemaker (c. 1848-1932).

AA Town in Texas, USA.

AA Johannes; German rocketry scientist (1897-1947).

AA Joseph; American astronomer (1826-1875).

AA Sarah; Piute interpreter, activist (c. 1844-1891).

AA John; American astronomer (1714-1779).

AA Carl Wilhelm; German astronomer (1876-1939).

AA Walter; German astronomer (1859-1905).

AA Friedrich; German chemist (1800-1882).

AA Baramba (West Africa) devil spirit.

AA Maxmilian Franz Joseph Comelius; German astronomer (1863-1932).

AA William Hyde; British chemist, physicist (1766-1828)

AA Mary; English author (1759-1797).

AA Jan; Dutch astronomer (1891-1946).

AA Robert W.; American physicist (1868-1955).

PE Victoria; American-English lecturer (1838-1927).

AA Virginia; British writer (1882-1941).

AA Town in Australia.

AA Australian launch site.

AA Town in New York, USA.

AA Fanny; American mountaineer, author (1859-1925).

LF Astronaut-named feature, Apollo 16 site.

AA Christopher; English architect (1632-1723)

AA William H.; American astronomer (1871-1959).

AA Frederick E; American astronomer (1878-1953); Thomas; British philosopher(1711-1786); Willian H.; American astronomer (1871-1959).

AA Sigmund von; Polish physicist (1845-1888).

AA John, Baron Wrottesley; British astronomer (1798-1867)

AA Chinese empress (c. 624-705).

AA Town in Nigeria.

AA Town in Cameroon.

AA Australian dark spirit.

AA Kakadu (Australia) ancestor of all people; a giant.

TH Hatti (proto-Hittite) sun goddess and mother of gods.

\footnotetext{
P: $\quad$ planetary system (see page xvi).

Sa: $\quad$ satellite (see page $x v i$ ).

lat: latitude of feature center.

long: longitude of feature center.
}

diam: diameter or long dimension of feature. ct: continent of name origin (see page $284 \mathrm{ff}$.) et: ethnicity of name origin (see page $284 \mathrm{ff}$.) as: name approval status (see page xvii). ad: name approval date (year).

ref: reference source for name (see page $287 \mathrm{ff}$.). ft: feature type (see page 290). 


\section{P Sa Name}

L L Wurzelbauer

L L Wyld

$S$ rh Xamba

M M Xanthe

M M Xanthe Dorsa

M M Xanthe Scopulus

M M Xanthe Terra

V V Xantippe

L L Xenophanes

L L Xenophon

V V Xiao Hong

V V Xochiquetzal Mons

$S$ rh $X u$

M M Xui

V V Yablochkina

L L Yablochkov

M M Yakima

L L Yakovkin

$\mathrm{H} \quad \mathrm{H}$ [Yakovlev]

M M Yala

M M Yalata

V V Yale

A gs Yalova

A gs Yalta

L L Yamamoto

L L Yangel'

U ar Yangoor

M M Yaonis Regio

M M Yar

V V Yaroslavna Patera

N tr Yasu Sulci

M M Yat

V V Yavine Corona

A gs Yeates Regio

M Yebra

$\begin{array}{lll}M & \text { M } & \text { Yebra } \\ \text { M } & \text { M } & \text { Yegros }\end{array}$

$\mathrm{N}$ tr Yenisey Fossa

L L Yerkes

J ca Ymir

V V Ymoja Vallis

$V$ Y Yonge

M M Yorktown

M M Yoro

L L Yoshi

V V Yoshioka

L L Young

V V Yumyn-Udyr Dorsa

H H Yun Sŏn-Do lat long

diam

ct et

33.9S

$1.4 \mathrm{~S}$

$15.9 \mathrm{~W}$

$2.1 \mathrm{~N} \quad 349.7 \mathrm{~W}$

93

EU GE

as ad

ref

51935

51970

51982

AF BU

$5 \quad 1958$

$10.0 \mathrm{~N}$

$21.3 \mathrm{~N}$

$19.6 \mathrm{~N}$

$4.2 \mathrm{~N}$

$10.8 \mathrm{~S}$

$57.5 \mathrm{~N}$

$22.8 \mathrm{~S}$

$43.6 \mathrm{~S}$

$3.5 \mathrm{~N}$

$55.0 \mathrm{~N}$

$15.3 \mathrm{~N}$

$48.2 \mathrm{~N}$

$50.0 \mathrm{~W}$

$45.5 \mathrm{~W}$

$52.1 \mathrm{~W}$

46.3W

$11.7 \mathrm{E}$

$82.0 \mathrm{~W}$

664

3074

40

122.1E

$101.7 \mathrm{E}$

$270.0 \mathrm{E}$

$71.9 \mathrm{~W}$

247.6W

$60.9 \mathrm{~N} \quad 128.3 \mathrm{E}$

$43.3 \mathrm{~N}$

54.5

$3.2 \mathrm{~W}$

$78.8 \mathrm{~W}$

EU GR

EU GR

EU LA

EU GR

EU GR

25

EU

GR

38

AS

NA

AF BU

SA BR

63 UR SO

$\begin{array}{ll}5 & 1958 \\ 5 & 1976\end{array}$

1976

51979

$\begin{array}{ll}5 & 1991\end{array}$

51935

51976

$5 \quad 1991$

$\begin{array}{ll}5 & 1994\end{array}$

$5 \quad 1982$

51988

51985

51970

$0.0 \mathrm{~N} \quad 0.0 \mathrm{~W}$

$17.5 \mathrm{~N} \quad 38.5 \mathrm{~W}$

$22.1 \mathrm{~N} 254.0 \mathrm{~W}$

13.4S 271.2E

29.0N $10.0 \mathrm{~W}$

$57.6 \mathrm{~N} \quad 261.3 \mathrm{~W}$

$58.1 \mathrm{~N} \quad 160.9 \mathrm{E}$

$17.0 \mathrm{~N}$

68.7S $\quad 279.7 \mathrm{E}$

4.7E

$40.0 \mathrm{~S} \quad 320.0 \mathrm{~W}$

99

UR

37

US

RU

$5 \quad 1976$

51985

0 UR RU

$6 \quad 1979$

$\begin{array}{ll}5 & 1976 \\ 5 & 1988\end{array}$

51994

0 OC AU

20 NA AM

51994

$22.5 \mathrm{~N}$

$39.1 \mathrm{~W}$

$38.8 \mathrm{~N}$

21.2E

$2.0 \mathrm{~N} \quad 347.0 \mathrm{E}$

$18.3 \mathrm{~N} \quad 29.1 \mathrm{~W}$

$5.5 \mathrm{~S} \quad 251.2 \mathrm{E}$

$65.0 \mathrm{~N} \quad 75.0 \mathrm{~W}$

$9.2 \mathrm{~N} \quad 34.6 \mathrm{~W}$

$21.1 \mathrm{~N} \quad 254.4 \mathrm{~W}$

$22.5 \mathrm{~S} 23.5 \mathrm{~W}$

$\begin{array}{rr}3.0 \mathrm{~N} & 56.2 \mathrm{E} \\ 14.6 \mathrm{~N} & 51.7 \mathrm{E}\end{array}$

$51.4 \mathrm{~N} \quad 101.3 \mathrm{~W}$

71.6S 204.8E

14.0S $115.1 \mathrm{E}$

$23.1 \mathrm{~N} \quad 48.7 \mathrm{~W}$

$23.0 \mathrm{~N} 28.0 \mathrm{~W}$

$24.6 \mathrm{~N} \quad 11.0 \mathrm{E}$

$32.4 \mathrm{~S} \quad 58.8 \mathrm{E}$

41.5S $\quad 50.9 \mathrm{E}$

81.4N 164.7E

$72.5 S \quad 109.4 \mathrm{~W}$
51994

51970

$\begin{array}{rrr}0 & \text { AS } & \text { TU } \\ 5 & \text { UR } & \text { UK } \\ 76 & \text { AS } & \text { JA }\end{array}$

8 UR SO

$78 \quad O C \quad A U$

51988

$\begin{array}{lllll}0 & \text { EU } & \text { LA } & 5 & 1958\end{array}$

$\begin{array}{ll}5 & 1976 \\ 5 & 1985\end{array}$

112

UR RU

$\begin{array}{lllll}0 & \text { AS } & \text { JA } & 5 & 91\end{array}$

\section{AF NG}

450 EU LI

$5 \quad 1976$

$\begin{array}{rrrrr}100 & \text { NA } & \text { AM } & 5 & 1994 \\ & \text { EU } & \text { IR } & 5 & 1976\end{array}$

$\begin{array}{lllll}0 & \text { EU } & \text { SP } & 5 & 1988\end{array}$

14 SA PA

$\begin{array}{rrr}0 & \text { UR } & \text { SI } \\ 36 & \text { NA } & \text { AM }\end{array}$

51976

$\begin{array}{rr}5 & 91 \\ 5 & 1935\end{array}$

77 EU NS

51979

390 AF NI

51994

51976

$\begin{array}{lllll}8 & \text { US } & \text { VA } & 5 & 1976 \\ 8 & \text { SA } & \text { HO } & 5 & 1976\end{array}$

1 AS JA 51976

$\begin{array}{lllll}1 & \text { AS } & \text { JA } & 5 & 1994\end{array}$

71

EU GB

51935

1086

EU FI

51985

68

AS $\mathrm{KR}$

51976
51973

$\begin{array}{lllll}26 & \mathrm{EU} & \mathrm{EN} & 5 & 1994\end{array}$

30

60

57

57

57

66

0

102

111

30

36
64

0

36

0

80

36

36
99

113

113
0

66 AA Johann Philipp von; German astronomer (1651-1725).

AA James H.; American rocketry scientist (1913-1953).

AA Bushman supreme being, creator of all things.

AL "Golden-Yellow Land."

DO Classical albedo feature name.

SC Classical albedo feature name.

TA Classical albedo feature name.

AA Wife of Socrates (5th century B.C.)

AA Of Colophon; Greek philosopher (570(?)-478(?) B.C.)

AA Greek natural philosopher, historian (c. 430-354 B.C.)

AA Chinese novelist (1911-1942).

MO Aztec goddess of flowers.

AA Bushman creator.

AA Town in Brazil.

AA Aleksandra; Soviet actress (1866-1964).

AA Pavel N.; Russian electrical engineer (1847-1894).

AA Town in Washington, USA.

AA A. A.; Soviet astronomer (1887-1974).

AA Crater name changed to Barma.

AA Town in Thailand.

AA Town in Australia.

AA Caroline; American educator of the deaf (1848-1933).

AA Health resort in Turkey.

AA Spa in Crimea, Ukraine.

AA I.; Japanese astronomer (1889-1959).

AA Mikhail Kuzmich; Soviet rocketry scientist (1911-1971).

AA Spirit that brings day.

AL Named for Chinese Emperor Yao; flood occurred during his reign.

AA Town in Russia.

PE Russian, wife of Price Igor; patiently waited for his return from captivity (12th century).

SU Japanese heavenly river; literally, "peace."

AA Town in Niger.

CR Lithuanian harvest goddess.

RE Clayne; Galileo Project manager.

AA William Butler; Irish poet and dramatist (1865-1939). 
ALPHABETICAL LIST OF NAMES

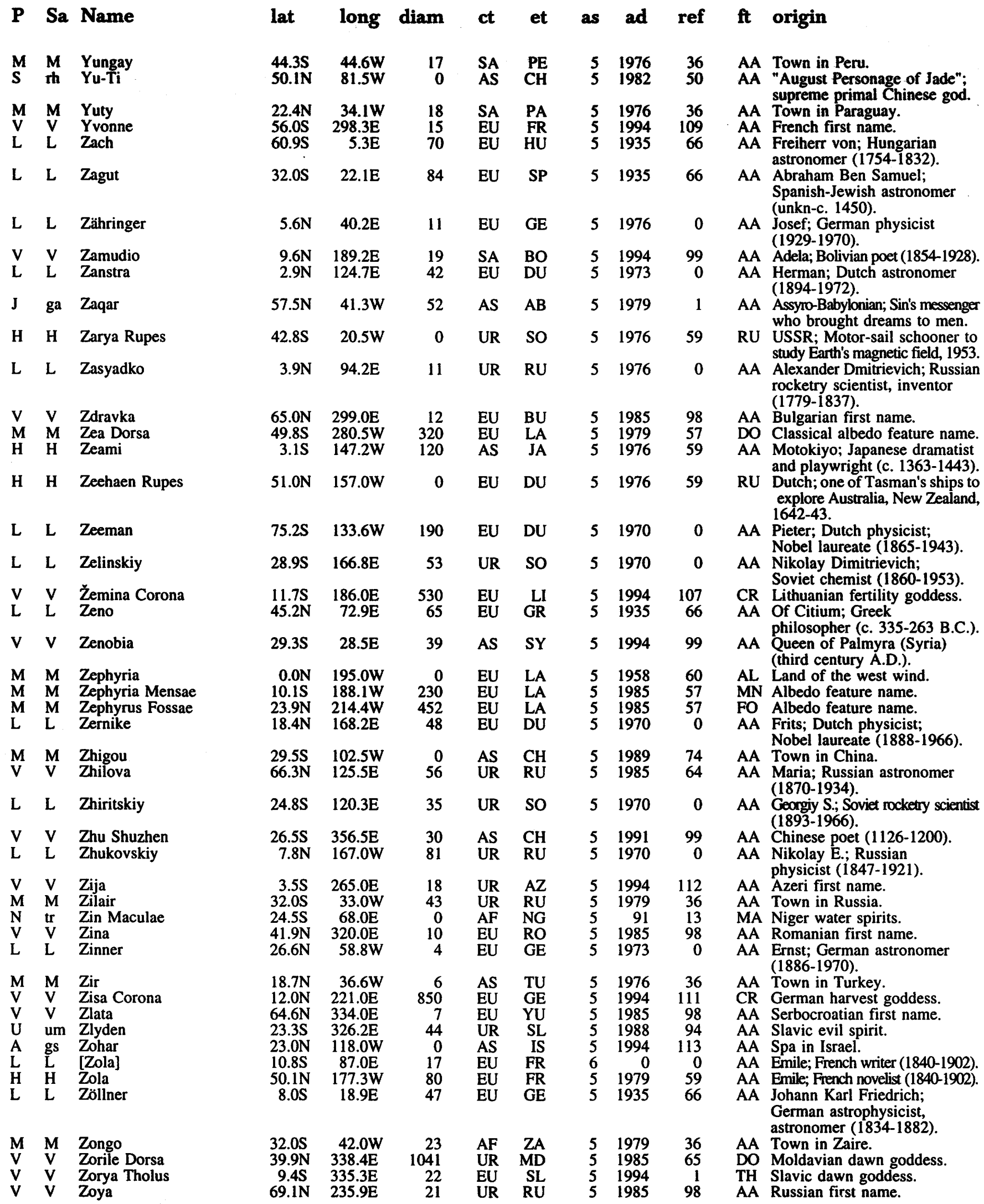

P: $\quad$ planetary system (see page xvi).

Sa: $\quad$ satellite (see page $x v i)$.

lat: latitude of feature center.

long: longitude of feature center. diam: diameter or long dimension of feature. ct: continent of name origin (see page $284 \mathrm{ff}$.)

et: ethnicity of name origin (see page $284 \mathrm{ff}$.)

as: name approval status (see page xvii). ad: name approval date (year).

ref: reference source for name (see page $287 \mathrm{ff}$.).

ft: feature type (see page 290). 


\section{ALPHABETICAL LIST OF NAMES}

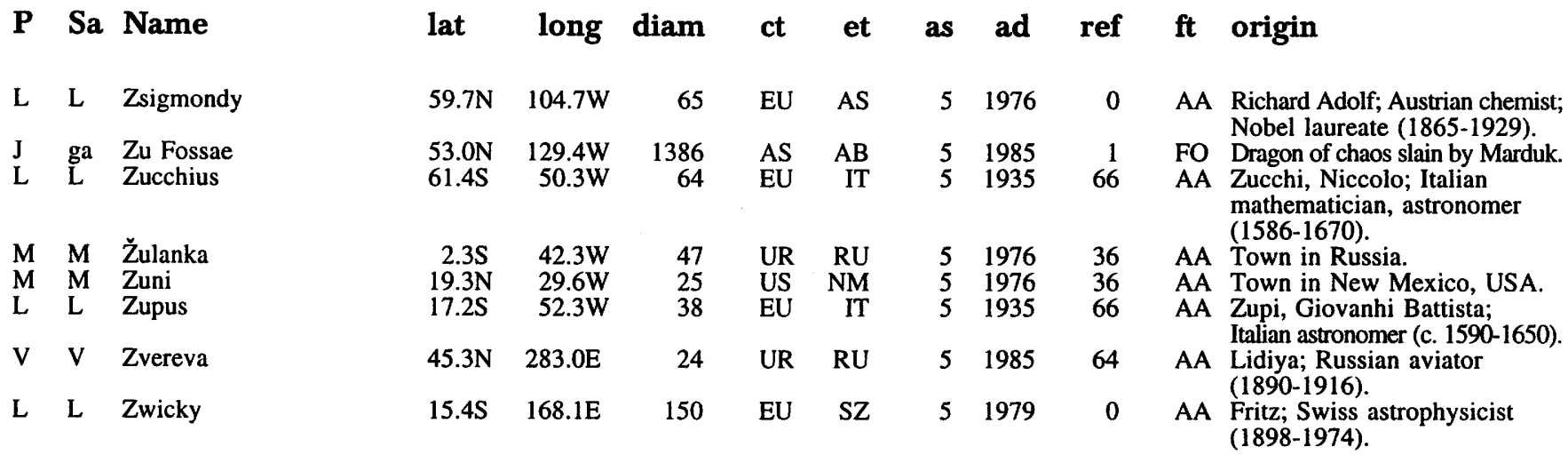

P: $\quad$ planetary system (see page $x v i)$.

Sa: satellite (see page $x v i$ ).

lat: latitude of feature center.

long: longitude of feature center. diam: diameter or long dimension of feature.

ct: continent of name origin (see page $284 \mathrm{ff}$.)

et: ethnicity of name origin (see page $284 \mathrm{ff}$.)

as: name approval status (see page $\mathrm{xvii}$ ). ad: name approval date (year)

ref: reference source for name (see page $287 \mathrm{ff}$.).

ft: feature type (see page 290). 

APPENDIXES 1-7 


\section{APPENDIX 1. CHAIRMEN OF IAU WORKING GROUP AND TASK GROUPS}

Kaare Aksnes, President, Working Group for Planetary System Nomenclature Institute for Theoretical Astrophysics

P.O. Box 1029

Blindern, N-0315

Oslo 3, Norway

V.V. Shevchenko, Chairman, Lunar Task Group

Sternberg State Astronomical Institute

Moscow University

13 Universitetsky Prospekt

119899 Moscow, Russia

David Morrison, Chairman, Mercury Task Group

Ames Research Center, MS 245-1

Moffett Field, CA 94035

G.A. Burba, Chairman, Venus Task Group

Vernadsky Institute

19 Kosygin St.

Moscow 117975, Russia

B.A. Smith, Chairman, Mars Task Group

Institute for Astronomy

P.O. Box 4729

Hilo, HI 96720

T.C. Owen, Chairman, Outer Solar System Task Group

Institute for Astronomy

2680 Woodlawn Drive

Honolulu, HI 96822

B.G. Marsden, Chairman, Asteroids and Comets Task Group

Center for Astrophysics

60 Garden St.

Cambridge, MA 02138 


\title{
APPENDIX 2. IAU TASK GROUP MEMBERS
}

\author{
Working Group for Planetary System Nomenclature
}

$\begin{array}{ll}\text { PRESIDENT: K. Aksnes } & \text { Norway } \\ \text { MEMBERS: } & \\ \text { M. Ya Marov } & \text { Russia } \\ \text { B. Marsden } & \text { U.S.A. } \\ \text { P. Moore } & \text { Great Britain } \\ \text { D. Morrison } & \text { U.S.A. } \\ \text { T.C. Owen } & \text { U.S.A. } \\ \text { V.V. Shevchenko } & \text { Russia } \\ \text { B.A. Smith } & \text { U.S.A. }\end{array}$

CONSULTANTS:

$\begin{array}{ll}\text { J. Blue } & \text { U.S.A. } \\ \text { G. Burba } & \text { Russia } \\ \text { M.E. Davies } & \text { U.S.A. } \\ \text { L. Gaddis } & \text { U.S.A. } \\ \text { P. Masson } & \text { France }\end{array}$

Task Group for Lunar Nomenclature

Task Group for Mercury Nomenclature
V. V. Shevch
F. El-Baz
H. Mizutani
P. Pinet
S. K. Runcorn
E. A. Whitaker

Russia

U.S.A.

Japan

France

Great Britain

U.S.A.
Task Group for Venus Nomenclature
G. A. Burba (Chair)
D B. Campbell
L. Gaddis
R. F. Jurgens
M. Ya. Marov
G. H. Pettengill
E. Stofan
Y. F. Tjuflin

Russia
U.S.A.

U.S.A.

U.S.A.

Russia

U.S.A.

U.S.A.

Russia
Task Group for Mars Nomenclature
B. A. Smith (Chair)
U.S.A.
M. E. Davies
U.S.A.
M. Ya. Marov
Russia
P. Masson
France
K. Saijo
Japan

Task Group for Features on Asteroids and Comets

B. G. Marsden (Chair)

J. Blue

A. Brahic

A. Carusi

M. Fulchignoni

S. Isobe

D. Lupishko

D. Morrison

P. Thomas

J. Veverka

Jia-Xiang Zhang
U.S.A.

U.S.A.

France

Italy

Italy

Japan

Ukraine

U.S.A.

U.S.A.

U.S.A.

China
Task Group for Outer Solar System Nomenclature

T. C. Owen (Chair)

U.S.A.

K. Aksnes

R. Beebe

Norway

A. Brahic

U.S.A.

G. Burba

France

M. E. Davies

Russia

B. A. Smith

U.S.A.

V. G. Tjefel
U.S.A

Kazakhstan 


\section{APPENDIX 3. ABBREVIATIONS FOR CONTINENTS AND FOR COUNTRIES OR ETHNIC GROUPS USED IN THE GAZETTEER}

Name

Abbrev.

Name

Abbrev.

AFRICA (AF)

$\begin{array}{ll}\text { Algeria } & \text { AL } \\ \text { Angola } & \text { AN } \\ \text { Bantu } & \text { BA } \\ \text { Benin } & \text { BE } \\ \text { Botswana } & \text { BT } \\ \text { Burkina Faso (Upper Volta) } & \text { BF } \\ \text { Bushman } & \text { BU } \\ \text { Bushongo } & \text { BH } \\ \text { Cameroon } & \text { CR } \\ \text { Canary Is. } & \text { CI } \\ \text { Egypt } & \text { EG } \\ \text { Ethiopia } & \text { ET } \\ \text { Gambia } & \text { GA } \\ \text { Ghana } & \text { GH } \\ \text { Gold Coast } & \text { GC } \\ \text { Ivory Coast } & \text { IC } \\ \text { Kenya } & \text { KY } \\ \text { Lesotho } & \text { LE } \\ \text { Liberia } & \text { LI } \\ \text { Libya } & \text { LB } \\ \text { Mbundu } & \text { MB } \\ \text { Mali } & \text { ML }\end{array}$

Mande

MN

Mauritania $\quad$ MU

Morocco MR

Mozambique MZ

Namibia NM

Niger NG

Nigeria NI

Pygmy PY

Semitic SE

Senegal SN

Somalia SO

South Africa SA

Sudan SU

Tanzania TA

Togo TO

Tunisia TN

Uganda UG

Yao YA

Zaire ZA

Zambia $\quad$ ZM

Zimbabwe ZI

ASIA (AS)

$\begin{array}{ll}\text { Akkadian (Accadian) } & \text { AK } \\ \text { Armenian } & \text { AM } \\ \text { Assyrian } & \text { AY } \\ \text { Assyro-Babylonian } & \text { AB } \\ \text { Babylon } & \text { BY } \\ \text { Bangladesh } & \text { BA } \\ \text { Buriat } & \text { BR } \\ \text { Burma } & \text { BU } \\ \text { Cambodia } & \text { CM } \\ \text { China } & \text { CH } \\ \text { Hebrew } & \text { HE } \\ \text { India } & \text { IN } \\ \text { Indonesia } & \text { ID } \\ \text { Iran } & \text { IR } \\ \text { Iraq } & \text { IQ } \\ \text { Israel } & \text { IS } \\ \text { Japan } & \text { JA } \\ \text { Jewish } & \text { JW } \\ \text { Jordan } & \text { JO } \\ \text { Korea } & \text { KR } \\ \text { Malaysia } & \text { MA }\end{array}$

$\begin{array}{ll}\text { Minyong } & \text { MY } \\ \text { Mongolia } & \text { MO } \\ \text { Monguor } & \text { MG } \\ \text { Nanai } & \text { NA } \\ \text { Nepal } & \text { NE } \\ \text { Oman } & \text { OM } \\ \text { Pakistan } & \text { PK } \\ \text { Persian } & \text { PE } \\ \text { Philippines } & \text { PH } \\ \text { Phoenician } & \text { PO } \\ \text { Samoyed } & \text { SM } \\ \text { Sanskrit } & \text { SA } \\ \text { Saudi Arabia } & \text { AR } \\ \text { Semitic } & \text { SE } \\ \text { Siberia } & \text { SI } \\ \text { Sumerian } & \text { SU } \\ \text { Syria } & \text { SY } \\ \text { Tibet } & \text { TB } \\ \text { Taiwan } & \text { TW } \\ \text { Thailand } & \text { TH } \\ \text { Turkey } & \text { TU } \\ \text { Vietnam } & \text { VT }\end{array}$


EUROPE (EU)

Albania
Austria
Belgium
Bulgaria
Byzantine
Celtic
Cyprus
Czechoslovakia
Denmark
England
Eskimo (Greenland)
Estonia
Finland
Flemish
France
Germany
Great Britain
Greece
Greenland
Hungary
Iceland
Ireland
Italy

AL

AS

$\mathrm{BE}$

BU

BZ

CE

CY

$\mathrm{CZ}$

DE

EN

EK

ES

FI

FL

FR

GE

GB

GR

GL

$\mathrm{HU}$

IC

IR

IT

Lapp
Latin
Latvia
Lithuania
Netherlands (Dutch)
Norse
Norway
Ostrogoth
Oscan
Poland
Portugal
Roman
Romania (Rumania)
Scandinavian
Scotland
Slavic
Spain
Sweden
Switzerland
Teutonic
Wales
Yugoslavia

LP

LA

LV

LI

DU

NS

NO

OG

OS

PO

PG

RM

RO

SD

SC

SL

SP

SW

SZ

TU

WA

YU

NORTH AMERICA (NA)

OCEANIA (OC)

Algonquin

American

Canada

Dakota

Eskimo

Hopi

Iroquois

Mandan

Mexico

Navajo

Pawnee

Pueblo

Seneca

Shoshoni
AL

AM

CA

DA

ES

HO

IR

MA

ME

NV

PW

PU

SE

SH
Australia

Caroline Is.

Guam

Hawaii

Marshall Is.

Melanesia

Micronesia

New Britain

New Guinea

New Zealand

Society Is.

Samoa

Tonga

Toamotu
AU

CI

GM

HI

MI

ME

$\mathrm{MC}$

NB

GU

NZ

SI

SA

TO

TU

SOUTH AND CENTRAL AMERICA (SA)

$\begin{array}{ll}\text { Argentina } & \text { AR } \\ \text { Auracanian } & \text { AC } \\ \text { Aztec } & \text { AZ } \\ \text { Barbados } & \text { BB } \\ \text { Bolivia } & \text { BO } \\ \text { Bororo } & \text { RR } \\ \text { Brazil } & \text { BR } \\ \text { Chile } & \text { CH } \\ \text { Colombia } & \text { CO } \\ \text { Costa Rica } & \text { CR } \\ \text { Dominican Republic } & \text { DR } \\ \text { Ecuador } & \text { EC } \\ \text { French Guiana } & \text { FG } \\ \text { Guyana } & \text { GY } \\ & \end{array}$

Haiti HA

Honduras HO

Inca IN

Jamaica JM

Mayan MY

Netherland (Dutch) Antilles DA

Nicaragua NI

Panama PM

Paraguay PA

Peru PE

Puerto Rico PR

Uruguay UR

VG Venezuela VE 
UNITED STATES (US)

$\begin{array}{llll}\text { Alabama } & \text { AL } & \text { Nebraska } & \text { NE } \\ \text { Alaska } & \text { AK } & \text { Nevada } & \text { NV } \\ \text { Arizona } & \text { AZ } & \text { New Hampshire } \\ \text { California } & \text { CA } & \text { New Jersey } & \text { NJ } \\ \text { Colorado } & \text { NO } & \text { New Mexico } & \text { NM } \\ \text { Connecticut } & \text { CT } & \text { North Dakota } & \text { NY } \\ \text { Delaware } & \text { DE } & \text { Ohio } & \text { ND } \\ \text { Florida } & \text { FL } & \text { Oklahoma } & \text { OH } \\ \text { Georgia } & \text { GA } & \text { Oregon } & \text { OK } \\ \text { Hawaii } & \text { HI } & \text { Pennsylvania } & \text { OR } \\ \text { Idaho } & \text { ID } & \text { South Carolina } & \text { PA } \\ \text { Illinois } & \text { IL } & \text { South Dakota } & \text { SC } \\ \text { Indiana } & \text { IN } & \text { Texas } & \text { SD } \\ \text { Iowa } & \text { IA } & \text { Utah } & \text { TX } \\ \text { Kansas } & \text { KS } & \text { Virginia } & \text { UT } \\ \text { Louisiana } & \text { LA } & \text { Washington } & \text { VA } \\ \text { Maryland } & \text { MD } & \text { West Virginia } & \text { WA } \\ \text { Massachusetts } & \text { MA } & \text { Wisconsin } & \text { WV } \\ \text { Mississippi } & \text { MS } & \text { Wyoming } & \text { WI } \\ \text { Montana } & \text { MT } & & \text { WY }\end{array}$

COMMONWEALTH OF INDEPENDENT STATES AND ASSOCIATED ETHNIC GROUPS (UR)

$\begin{array}{llll}\text { Altai } & \text { AL } & \text { Ostyak } & \text { OS } \\ \text { Armenia } & \text { AM } & \text { Russia } & \text { RU } \\ \text { Azerbaijan } & \text { AZ } & \text { Samoyed } & \text { SM } \\ \text { Beloruss } & \text { BE } & \text { Scythia } & \text { SC } \\ \text { Buriat } & \text { Siberia } & \text { SI } \\ \text { Caucasus } & \text { BR } & \text { Slavic (Slavonic) } & \text { SL } \\ \text { Georgia } & \text { GE } & \text { Soviet } & \text { SO } \\ \text { Karelia } & \text { KA } & \text { Tadzhikistan } & \text { TD } \\ \text { Kazakhstan } & \text { KZ } & \text { Tungu } & \text { TU } \\ \text { Mari } & \text { MJ } & \text { Turkmenistan } & \text { TK } \\ \text { Moldova } & \text { MD } & \text { Ukraine } & \text { UK } \\ \text { Mordvinian (Volga Finn) } & \text { MO } & \text { Ulci } & \text { UL } \\ \text { Nanai } & \text { NA } & \text { Uzbekistan } & \text { UZ } \\ \text { Neghidhian } & \text { NE } & \text { Yakutian } & \text { YK }\end{array}$




\section{APPENDIX 4. SOURCES OF PLANETARY NAMES}

(Identified by number in "reference" columns of Sections 1 and 2)

1. Larousse Encyclopedia of Mythology, translated by R. Aldington and D. Ames; Hamlyn Publishing Group Ltd., New York, 1976.

2. Hawaiian Folk Tales: A Collection of Native Legends, by Thomas G. Thrum; AMS Printing Inc., New York, 1907.

3. Oceanic Mythology, by Roslyn Poignant; Paul Hamlyn Ltd., London, 1967.

4. Australian Legendary Tales, collected by K. Langloh Parker; Angus \& Robertson, Brighton, 1963.

5. Aboriginal Myths and Legends, selected by Roland Robinson; Hamlyn Pub. Group Ltd., London, 1969.

6. Dreamtime: Australian Aboriginal Myths, text by Charles Mountford; Rigby Ltd., Adelaide, 1965.

7. Nomads and Empire Builders: Native Peoples and Cultures of South America, by Carleton Beals; Citadel, Secaucus, New Jersey, 1965.

8. The Indian Background of Colonial Yucatan, by R. L. Roys; Gordon Press, New York, 1976.

9. Kiowa Tales, by Elsie W. Parsons; The American Folklore Society, v. XXII, New York, 1929.

10. Myths and Tales of the Jicarilla Apache, by Morris Edward Opler; The American Folklore Society, G. E. Stechert \& Co., New York, 1938.

11. The Book of the Navajo, by Raymond Friday Locke; Mankind Pub. Co., Los Angeles, Calif., 1976.

12. Indian Mythology, by Veronica Ions; Paul Hamlyn Ltd., London, 1967.

13. African Mythology, by Geoffrey Parrinder; Paul Hamlyn Ltd., London, 1967.

14. South American Mythology, by Harold Osborne; Paul Hamlyn Ltd., London, 1968.

15. Chinese Mythology, by Anthony Christie; Paul Hamlyn Ltd., London, 1968.

16. Japanese Mythology, by Juliet Piggott; Paul Hamlyn Ltd., London, 1969.

17. Norse mythology; lists provided by Kaare Aksnes.

18. List compiled by V. G. Teifel.

19. The Greek Myths (2 volumes), by Robert Graves; Penguin Books Ltd., Harmondsworth, Middlesex, England, 1974.

21. Handbook of Greek Mythology, by H. J. Rose; E. P. Dutton \& Co., Inc., New York, 1959.

22. Gilgamesh: A Verse Narrative, by Herbert Mason; Houghton Mifflin Company, Boston, Mass., 1971.

23. The Lost Gods of England, by Brian Branston; Thames \& Hudson Ltd., London, 1957.

24. Orisha: The Gods of Yorubaland, by Judith Gleason; Atheneum, New York, 1971.

25. Ainu Creed and Cult, by Neil Gordon Munro; Columbia University Press, New York, 1963.

26. North American Indian Mythology, by Cottie Burland; Paul Hamlyn Pub. Group Ltd., London, 1968.

27. The Mythology of All Races (13 volumes), edited by John Arnott MacCulloch and George Foot Moore; Cooper Square Publishers, Inc., New York, 1964.

28. The Odyssey, by Homer, translated by W. H. D. Rouse; Thomas Nelson and Sons, Ltd., Edinburgh, 1934.

28b. The Odyssey of Homer, translated by Herbert Bates; Harper Brothers, New York, 1929.

29. Song of Roland, translated by Dorothy L. Sayers; Penguin Books Ltd., Harmondsworth, Middlesex, England, 1967.

30. Comparative Cultures; Human Relations Area File Inc., New Haven, Conn.

31. Gods, Heroes, and Men of Ancient Greece, translated by W. H. D. Rouse; The New American Library of World Literature, Inc., New York, 1957.

32. Myths of the Greeks and Romans, by Michael Grant; World Publishing Company, New York, 1962.

33. Mythology: Timeless Tales of Gods and Heros, by Edith Hamilton; Little, Brown and Company, Boston, Mass., 1942.

34. African Myths and Tales, edited by Susan Feldmann; Dell Publishing Co., Inc., New York, 1970.

35. Gods and Myths of Northern Europe, by H. R. Ellis Davidson; Penguin Books Ltd., Harmondsworth, Middlesex, England, 1974.

36. National Geographic Atlas of the World: National Geographic Society, Washington, D.C., 1970 (and other editions).

37. Malory's Le Morte d'Arthur, by Keith Baines; Clarkson N. Potter, Inc., New York, 1962.

38. Njal's Saga, by Magnus Magnusson and Hermann Paulsson; Penguin Books Ltd., Harmondsworth, Middlesex, England, 1975.

39. The Age of Fable, by Thomas Bulfinch; The Heritage Press, New York, 1942.

40. Primal Myths: Creating the World, by Barbara C. Sproul; Harper \& Row, New York, 1979. 
41. The Iliad of Homer, translated by Benjamin Smith and Walter Miller; MacMillan \& Co., New York, 1944.

42. Beowulf, translated by Burton Raffel; The New American Library of World Literature, Inc., New York, 1963.

43. Dictionary of Classical Mythology, by J. E. Zimmerman; Harper and Row, New York, 1971.

44. The Aeneid of Virgil, translated by Allen Mandelbaum; Bantam Books, Inc., New York, 1971.

45. Tales of Yoruba Gods and Heroes, by Harold Courlander; Crown Publishers, Inc., New York, 1973.

46. List of famous women provided by the National Organization for Women.

47. Giants, by David Larkin and Sarah Teale; Harry Abrams, Inc., New York, 1979.

48. Letter from G. H. Pettengill to Venus Task Group, April 27, 1977.

49. Letter from M. Ya. Marov to G. H. Pettengill, September 8, 1977.

50. List compiled by N. P. Erpylev; includes names from various Russian legends.

51. Letter from M. Ya. Marov to G. H. Pettengill, January 3, 1981.

52. Alf Laylah Wa Laylah, The Book of the Thousand Nights and a Night, by Richard F. Burton; Larsen-Harper, Colo. Press, Denver, Colo., 1900.

53. Myths and Folklore of the Temiskaming Algonquin, and Timagami Ojibwa, by F. G. Speck; Canada Department of Mines Memoir 71, Ottawa, 1915.

54. The New Century Handbook of Classical Geography, edited by Catherine B. Avery; Meredith Corp., New York, 1972.

55. Webster's New Geographical Dictionary; G. \& C. Merriam Co., Springfield, Mass., 1972.

56. Everymans Classical Atlas, by J. Oliver Thomson; J. M. Dent and Sons Ltd., London, 1963.

57. Map of albedo features of Mars, (plates 2-5), in La Planète Mars, by E.M. Antoniadi; Librairie Scientifique, Hermann et Cie., Paris, 1930.

58. Letter from M. Ya. Marov to Harold Masursky, March 19, 1979.

59. The New Encyclopaedia Britannica; Encyclopaedia Britannica Inc., Chicago, 1974.

60. Proceedings of the General Assembly in Transactions of the International Astronomical Union, v. XIVB, 1971, through XIXB, 1986, Reidel \& Co., Dordrecht, Holland, 1971, 1974, 1977, 1980, 1983, 1986.

61. List of radar scientists provided by G. H. Pettengill.

62. List of names for Mars in various languages provided by Carl Sagan.

63. List supplied by N. P. Erpylev.

64. Soviet Encyclopedia (30 volumes, in Russian).

65. Myths of the Peoples of the World (two volumes, in Russian).

66. Named Lunar Formations, by Mary A. Blagg and K. Müller; Percy Lund, Humphries and Co. Ltd., London, 1935.

67. The System of Lunar Craters, Quadrants I, II, III, IV; by D. W. G. Arthur and others; Communications of the Lunar and Planetary Laboratory, v. 2, no. 30, 1963; v. 3, no. 40, 1964; v. 4, no. 50, 1965; v. 5, no. 70, 1966.

68. World Who's Who in Science, edited by Allen G. Debus; Western Publishing Company, Hannibal, Mo., 1968; New York, 1973.

71. Commentary on Martian Nomenclature, 2d edition, by Jürgen Blunck; Exposition Press, Smithtown, New York, 1982.

72. Soviet Men of Science, by John Turkevich; Van Nostrand Company, Princeton, N.J., 1963.

73. McGraw-Hill International Atlas; McGraw-Hill Book Co., New York, 1963.

74. The Times Atlas of the World, Comprehensive Edition; The Times of London in collaboration with John Bartholomew \& Son Ltd., Edinburgh, 1971 (and other editions).

75. Webster's Biographical Dictionary; G. \& C. Merriam Company, Springfield, Mass., 1974 (and other editions).

76. Duplicate of reference 68 (World Who's Who in Science).

77. Observatories of the World, by Siegfried Marx and Werner Pfau; Van Nostrand, Reinhold Co., New York, 1982.

78. The Oxford Companion to Art; Oxford University Press, London, 1970.

79. Cassel's Encyclopaedia of World Literature; William Morrow \& Company Inc., New York, 1973.

80. Lists of names for Mercury nomenclature, provided by David Morrison.

81. Kodansha Encyclopedia of Japan; Kodansha Ltd., New York, 1983.

83. Dictionary of Mythology, Folklore, and Symbols (in 3 volumes), by Gertrude Jobes; Scarecrow Press, Inc., New York, 1962.

84. Ancient Mirrors of Womanhood, by Merlin Stone; Beacon Press, Boston, Mass., 1984.

85. The Complete Works of William Shakespeare, Illustrated; Avenel Books, a division of Crown Publishers, Inc., New York, 1975. 
86. The World Guide to Gnomes, Fairies, Elves and other Little People, by Thomas Keightley, Avenel Books, New York, 1978.

87. Webster's 3rd New International Dictionary of the English language, unabridged, Merriam Webster Editorial Staff; G. \& C. Merriam Co., Springfield, Mass., 1965.

88. Astronauts and Cosmonautics Biographical and Statistical Data, revised June 28, 1985, prepared by the Congressional Research Service, Library of Congress; U.S. Government Printing Office, Washington, D.C., 1985.

89. Dictionary of Scientific Biography, Charles Coulston Gillispie, editor in chief, v. 9; Charles Scribner's Sons, New York, 1981.

90. Encyclopedia Americana, International Edition; Grolier Inc., Danbury, Conn., 1984.

91. The Rand McNally International Atlas; Rand McNally and Co., New York, 1980.

92. Fairies, by Brian Froud and Alan Lee; Harry N. Abrams, Inc., New York, 1978.

93. Funk and Wagnalls Standard Dictionary of Folklore, Mythology, and Legend, edited by Maria Leach; Harper and Row, Inc., New York, 1984.

94. List of names supplied by T. C. Owen.

95. Poem "Rape of the Lock" by Alexander Pope.

96. Green Mansions, by W. H. Hudson; AMS Press Inc., New York, 1923.

97. The Blue Bird (printed with The Betrothal), by Maurice Maeterlinck; Philosophical Pub. Co., Quakertown, Pa., 1987.

98. Female first names supplied by Russian members of WGPSN and task groups.

99. Index to Women of the World, by N.O. Ireland; F.W. Faxon Co., Westwood, Mass., 1988.

100. List provided by Women's Study Program, Brown University, Providence, R.I.

101. An Account of the Polynesian Race, by A. Fernandez; Tuttle Press, 1969.

102. List provided by V.G. Suriquez, University of Hawaii at Manoa.

103. Macmillan Illustrated Encyclopedia of Myths and Legends, by A. Cotterell; Macmillan Publishing Co., New York, 1989.

104. Great North American Indians, by F. Dockstader; Van Nostrand Reinhold Co., New York, 1977.

105. List provided by S.V. Meschel, University of Chicago.

106. List provided by The Age (newspaper), Melbourne, Australia.

107. List provided by George Burba, Vernadsky Institute, Moscow.

108. List provided by Mikhail Ya. Marov, Academy of Science, Moscow.

109. Dictionary of First Names, by Alfred J. Kolatch; Putnam Publishing Group, New York, 1990.

110. Asimov's Biographical Encyclopedia of Science and Technology, by I. Asimov; Doubleday \& Co., Garden City, New York, 1972.

111. The Book of Goddesses and Heroines, by P. Monaghan; Llewellyn Publications, St. Paul, Minn., 1990. 


\section{APPENDIX 5. DESCRIPTOR TERMS (FEATURE TYPES)}

\section{FEATURE}

Albedo feature

Catena, catenae

Cavus, cavi

Chaos

Chasma, chasmata

Colles

Corona, coronae

Crater, craters

Dorsum, dorsa

Eruptive center

Facula, faculae

Farrum, farra

Flexus, flexūs

Fluctus, fluctūs

Fossa, fossae

Labes, labēs

Labyrinthus

Lacus ${ }^{1}$

Landing site name

Large ringed feature

Linea, lineae

Macula, maculae

Mare $^{1}$

Mensa, mensae

Mons, montes

Oceanus ${ }^{1}$

Palus ${ }^{1}$

Patera, paterae

Planitia

Planum

Plume

Promontorium ${ }^{1}$

Regio

Reticulum, reticula

Rima, rimae ${ }^{1}$

Rupes, rupēs

Scopulus

Sinus

Small satellites

Sulcus, sulci

Terra

Tessera, tesserae

Tholus, tholi

Undae

Vallis, valles

Vastitas

'Used only on the Moon.

\section{DESCRIPTION}

DESIGNATION

Hollows, irregular depressions $\quad$ CB

Distinctive area of broken terrain $\quad \mathrm{CH}$

Canyon $\quad$ CM

Small hills or knobs $\quad$ CO

Ovoid-shaped feature $\quad$ CR

Bowl-shaped depression $\quad$ AA

Ridge DO

Eruptive center $\quad$ ER

Bright spot $\quad$ FA

pancakelike structures $\quad$ FR

Cuspate, linear feature $\quad$ FE

Flow terrain $\quad$ FL

Long, narrow, shallow depression $\quad$ FO

Landslide LA

Complex of intersecting valleys $\quad$ LB

"Lake"; small plain $\quad$ LC

Feature named on Apollo map or report LF

LG

Elongate marking $\quad$ LI

Dark spot MA

"Sea"; large circular plain $\quad$ ME

Mesa, flat-topped elevation $\quad$ MN

Mountain $\quad M O$

"Ocean"; large plain $\quad$ OC

"Swamp"; small plain

Shallow crater; scalloped, complex edge PE

Low plain $\quad$ PL

Plateau or high plain $\quad$ PM

PU

"Cape"; headland $\quad$ PR

Region RE

reticular (netlike) pattern $\quad$ RT

Fissure RI

Scarp RU

Lobate or irregular scarp $\quad$ SC

"Bay"; small plain $\quad$ SI

SS

Subparallel furrows and ridges $\quad$ SU

Extensive land mass

"Tile"; terrain formed of polygonal pattern TE

Small domical mountain or hill $\quad$ TH

Dunes UN

Valley VA

Widespread lowlands $\quad$ VS

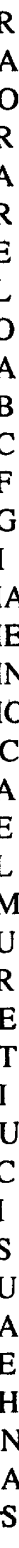




\title{
APPENDIX 6. CATEGORIES FOR NAMING FEATURES ON THE PLANETS AND SATELLITES
}

\author{
MERCURY
}

Craters

Montes

Planitiae

Rupēs

Valles
Famous deceased artists, musicians, painters, authors

Caloris, from Latin word for "hot"

Names for Mercury in various languages

Ships of discovery or scientific expeditions

Radio telescope facilities

\section{VENUS}

Chasmata

Coronae

Craters

Dorsa

Farrum

Fluctūs

Lineae

Montes

Paterae

Planitiae

Planum (one only)

Regiones

Rupēs

Tesserae

Terrae

Valles

Craters, Catenae, Dorsa, Rimae

Lacūs, Maria, Paludes, Sinūs

Montes

Rupes

Valles
Goddesses of hunt; Moon goddesses

Fertility and earth goddesses

Over $20 \mathrm{~km}$; famous women; under $20 \mathrm{~km}$, common female first names

Sky goddesses

Water goddesses

Goddess, miscellaneous

Goddesses of war

Goddesses, miscellaneous (also one radar scientist)

Famous women

Mythological heroines

Lakshmi; goddess of prosperity

Giantesses and Titanesses (also Greek alphanumeric)

Goddesses of hearth and home

Goddesses of fate or fortune

Goddesses of love

Word for planet Venus in various world languages

\section{THE MOON}

Large craters: famous deceased scientists, scholars, artists; small craters: common first names Other features named from nearby craters

Latin terms describing weather and other abstract concepts

Terrestrial mountain ranges or nearby craters

Name of nearby mountain ranges (terrestrial names)

Name of nearby features

\section{MARS AND MARTIAN SATELLITES}

\section{MARS}

Large craters

Small craters

Large valles

Small valles

Other features

DEIMOS

PHOBOS
Deceased scientists who have contributed to the study of Mars

Villages of the world (less than 100,000 population, U.N. Yearbook)

Name for Mars/star in various languages

Classical or modern names of rivers

From nearest named albedo feature on Schiaparelli or Antoniadi maps

Authors who wrote about satellites

Scientists who helped discovery 


\section{SATELLITES OF JUPITER}

\section{CALLISTO}

Large ringed features

Craters

Catenae

\section{EUROPA}

Craters

Flexūs

Lineae

Macula

\section{GANYMEDE}

Craters

Faculae

Fossae

Regiones

Sulci

IO

Active eruptive centers
Catenae
Fluctūs
Mensae
Montes
Paterae
Plana
Regiones
Tholi

\section{MIMAS}

ENCELADUS

TETHYS

DIONE

RHEA

HYPERION

IAPETUS

EPIMETHEUS

JANUS
Homes of the gods and of heroes

Heroes and heroines from northern myths

Mythological places in high latitudes

Celtic gods and heroes

Places associated with the Europa myth

People associated with the Europa myth

Places associated with the Europa myth

Gods and heroes of ancient Fertile Crescent people

Places associated with Egyptian myths

Gods (or principals) of ancient Fertile Crescent people

Astronomers who discovered Jovian satellites

Places associated with myths of ancient people

Fire, sun, thunder gods and heroes

Sun gods

Name derived from nearby named feature

People associated with Io myth

Places associated with lo myth

Fire, sun, thunder, volcano gods, heroes, goddesses, mythical blacksmiths

Places associated with lo myth

Places associated with Io myth

Places associated with Io myth

\section{SATELLITES OF SATURN}

People and places from Malory's "Le Morte d'Arthur" legends (Baines translation) People and places from Burton's "Arabian Nights"

People and places from Homer's "Odyssey"

People and places from Virgil's "Aeneid"

People and places from creation myths

Sun and Moon deities

People and places from Sayers' translation of "Chanson de Roland"

People from myth of Castor and Pollux (twins)

People from myth of Castor and Pollux (twins)

\section{SATELLITES OF URANUS}

Characters, places from Shakespeare's plays

Light spirits (individual and class)

Dark spirits (individual)

Shakespearean tragic heroes and places

Female Shakespearean characters, places

Mischievous (Pucklike) spirits (class)

Heroines from Shakespeare and Pope 


\section{SATELLITES OF NEPTUNE}

PROTEUS (1989 N1)

NEREID

TRITON

SMALL SATELLITES
Water-related spirits, gods, goddesses (excluding Greek and Roman names) Individual nereids

Aquatic names, excluding Roman and Greek. Possible categories include worldwide aquatic spirits, famous terrestrial fountains or fountain locations, terrestrial aquatic features, famous terrestrial geysers or geyser locations, terrestrial islands

Gods and goddesses associated with Neptune/Poseidon mythology or generic mythological aquatic beings 


\section{APPENDIX 7. PLANET AND SATELLITE NAMES AND DISCOVERERS}

\section{MERCURY}

VENUS

MOON

MARS

Phobos

Deimos

JUPITER

Metis

Adrastea

Amalthea

Thebe

Io

Europa

Ganymede

Callisto

Leda

Elara

Ananke

Carme

Pasiphaë

Sinope

\section{SATURN}

Pan

Atlas

Prometheus

Pandora

Janus

Epimetheus
Named Mercurius by the Romans because it appears to move so swiftly.

Roman name for the goddess of love. This planet was considered to be the brightest and most beautiful planet or star in the heavens. Other civilizations have named it for their god(ess) of love/war.

Every civilization has had a name for the satellite of Earth that is known, in English, as the Moon. The name is of Anglo-Saxon derivation.

Named by the Romans for their god of war because of its red--bloodlike--color. Other civilizations also named this planet from this attribute; for example, the Egyptians named it "Her Desher," meaning "the red one."

Inner satellite of Mars; named in 1877 by the discoverer, Asaph Hall, for one of the horses that drew Mars' chariot; also called an "attendant" or "son" of Mars, according to chapter 15, line 119 of Homer's "Iliad." This Greek word means "fear."

Outer Martian satellite, also named by Asaph Hall for one of Mars' horses/sons/companions; the word means "fear" or "terror" in Greek.

The largest and most massive of the planets was named Zeus by the Greeks and Jupiter by the Romans; he was the most important deity in both pantheons.

First wife of Zeus. He swallowed her when she became pregnant; Athena was subsequently born from the forehead of Zeus.

A nymph of Crete to whose care Rhea entrusted the infant Zeus.

Discovered by E. E. Barnard in 1892, who eventually chose a name suggested by Flammarian for the satellite. Amalthea (a goat in some accounts, a princess of Crete in others) suckled Zeus (Jupiter) as a young child.

A nymph abducted by Zeus; she is the namesake of the Greek city of Thebes.

Galileo discovered Io, Europa, Ganymede, and Callisto, in 1610. Simon Marius' claim to discovery of the Jovian satellites shortly before Galileo was not accepted. Galileo suggested that the four be known as "Medicea Sidera" to honor his patron, but the name was not accepted by other astronomers. Instead, they chose names given the four satellites by Marius in 1613; the names were of four of Jupiter's illicit loves. (Galileo refused to accept Marius' names; instead he identified the moons by Roman numerals, a secondary designation system that has been adopted for all satellite systems to the present.) Io, the daughter of Inachus, was changed by Jupiter into a cow to protect her from Hera's jealous wrath, but Hera recognized Io and sent a gadfly to torment her. Io, maddened by the fly, wandered throughout the Mediterranean region.

Beautiful daughter of Agenor, king of Tyre, she was seduced by Jupiter, who had assumed the shape of a white bull. When Europa climbed on his back he swam with her to Crete, where she bore several children, including Minos.

Beautiful young boy who was carried to Olympus by Jupiter disguised as an eagle. Ganymede then became the cupbearer of the Olympian gods.

Beautiful daughter of Lycaon, she was seduced by Jupiter, who changed her into a bear to protect her from Hera's jealousy.

Seduced by Zeus in the form of a swan, she was the mother of Pollux and Helen.

A paramour of Zeus; mother of the giant Tityus.

Moira, daughter of Zeus and Themis, as the goddess of necessity.

A nymph and attendant of Artemis; mother, by Zeus, of Britomartis.

Wife of Minos; mother of the Minotaur.

Daughter of the river god Asopus and Merope; she was abducted by Apollo.

Roman name for the Greek Cronos, father of Zeus/Jupiter. Other civilizations have given other names to Saturn, which is the farthest planet from Earth that can be observed by the naked human eye. Most of its satellites were named for Titans who, according to Greek mythology, were brothers and sisters of Saturn.

Son of Hermes and Dryope; half human, half goat god of pastoralism.

A Titan; he held the heavens on his shoulders.

Brother of Atlas and Epimetheus; he gave many gifts to humanity, including fire.

Made of clay by Hephaestus at the request of Zeus; she married Epimetheus and opened the box that loosed a host of plagues upon humanity.

Discovered by Audouin Dollfus in 1966, this small satellite was later proven to have a twin, Epimetheus, sharing the same orbit but never actually meeting. It is named for the two-faced Roman god who could look forward and backward at the same time.

Discovered by the Voyager team in 1981 and named by them for the Greek backward-looking god. 
Mimas Discovered by William Herschel in 1798 and named by his son John in the early 19th century for a Titan felled by Hephaestus (or Ares) in the war between the Titans and Olympian gods.

Enceladus Also discovered by William Herschel in 1798 and named by his son John for the Titan Enceladus. Enceladus was crushed by Athene in the battle between the Olympian gods and the Titans; earth piled on top of him became the island of Sicily.

Tethys Discovered in 1684 by Cassini, who wished to name it and the other three satellites that he discovered (Dione, Rhea, and Iapetus) for Louis XIV. However, the names used today for these satellites were applied in the early 19 th century by John Herschel, who named them for Titans and Titanesses, brothers and sisters of Saturn. Tethys was the wife of Oceanus and mother of all rivers and Oceanids.

Telesto

Calypso One of 3,000 Oceanides, water nymphs born to Oceanus and Tethys.

Dione

Rhea

A daughter of Atlas and paramour of Odysseus.

Discovered by Cassini in 1684. Dione was the sister of Cronos and mother (by Zeus) of Aphrodite.

Titan Discovered by Cassini in 1672 and named for another of Cronos' sisters, Rhea was also his wife. Her youngest son was Zeus.

Hyperion Discovered by C. and G.P. Bond and by William Lassell on the same night in 1848; named by Lassell for one of the Titans.

Iapetus Discovered by Cassini in 1671 and named by John Herschel for one of the Titans.

\section{URANUS}

Cordelia
Ophelia
Bianca
Cressida
Desdemona
Juliet
Portia
Rosalind
Belinda
Puck
Miranda
Ariel

Umbriel

Titania

Oberon

\section{NEPTUNE}

Naiad

Thalassa

Despina

Galatea

Larissa

Proteus

Triton

Nereid
Uranus was discovered by William Herschel in 1781. Several astronomers, including Flamsteed and Le Monnier, had observed it earlier but had recorded it as a fixed star. Herschel tried unsuccessfully to name his discovery "Georgian Sidus" after George III; the planet was named by Johann Bode in 1781 for the father of Saturn.

Daughter of Lear in Shakespeare's "King Lear."

Daughter of Polonius, fiancée of Hamlet in Shakespeare's "Hamlet, Prince of Denmark."

Daughter of Baptista, sister of Kate in Shakespeare's "Taming of the Shrew."

Title character in Shakespeare's "Troilus and Cressida."

Wife of Othello in Shakespeare's "Othello, the Moor of Venice."

Heroine of Shakespeare's "Romeo and Juliet."

Wife of Brutus in Shakespeare's "Julius Caesar."

Daughter of the banished duke in Shakespeare's "As You Like It."

Character in Pope's "Rape of the Lock."

Mischievous spirit in Shakespeare's "A Midsummer Night's Dream."

Discovered and named by G.P. Kuiper in 1948 for the heroine of Shakespeare's "The Tempest."

Discovered by William Lassell in 1851; named by John Herschel for the benevolent spirit in Shakespeare's "The Tempest."

Discovered by William Lassell in 1851, Umbriel was named by John Herschel for a malevolent spirit in Pope's

"Rape of the Lock."

Discovered by William Herschel in 1787; named by his son John in early 19th century for the queen of the fairies in Shakespeare's "A Midsummer Night's Dream."

Discovered by William Herschel in 1787; named by his son John in early 19th century for the king of the fairies in Shakespeare's "A Midsummer Night's Dream."

Neptune was actually "observed" as early as 1690 by John Flamsteed, who thought it was a fixed star. It was "predicted" by John Couch Adams and Urbain Le Verrier who, independently, were able to account for the irregularities in the motion of Uranus by correctly predicting the orbital elements of a trans-Uranian body. Using the predicted parameters of these two men, Johann Galle observed the planet in 1846. Galle wanted to name the planet for Le Verrier, but that was not acceptable to the international astronomical community.

The name of a group of Greek water nymphs who were guardians of lakes, fountains, springs, and rivers.

Greek sea goddess; mother of Aphrodite in some legends; others say she bore the Telchines.

Daughter of Poseidon (Neptune) and Demeter.

One: of the Nereids, attendants of Poseidon.

A lover of Poseidon.

Greek sea god, son of Oceanus and Tethys.

Discovered in 1847 by William Lassell, Triton is named for the sea-god son of Poseidon (Neptune) and Amphitrite.

The first suggestion of the name Triton has been attributed to the French astronomer Camille Flammarion.

Discovered by G. P. Kuiper at the McDonald Observatory in 1949. The Nereids were the 50 daughters of Nereus and Doris and were attendants of Neptune. 
$-$ 


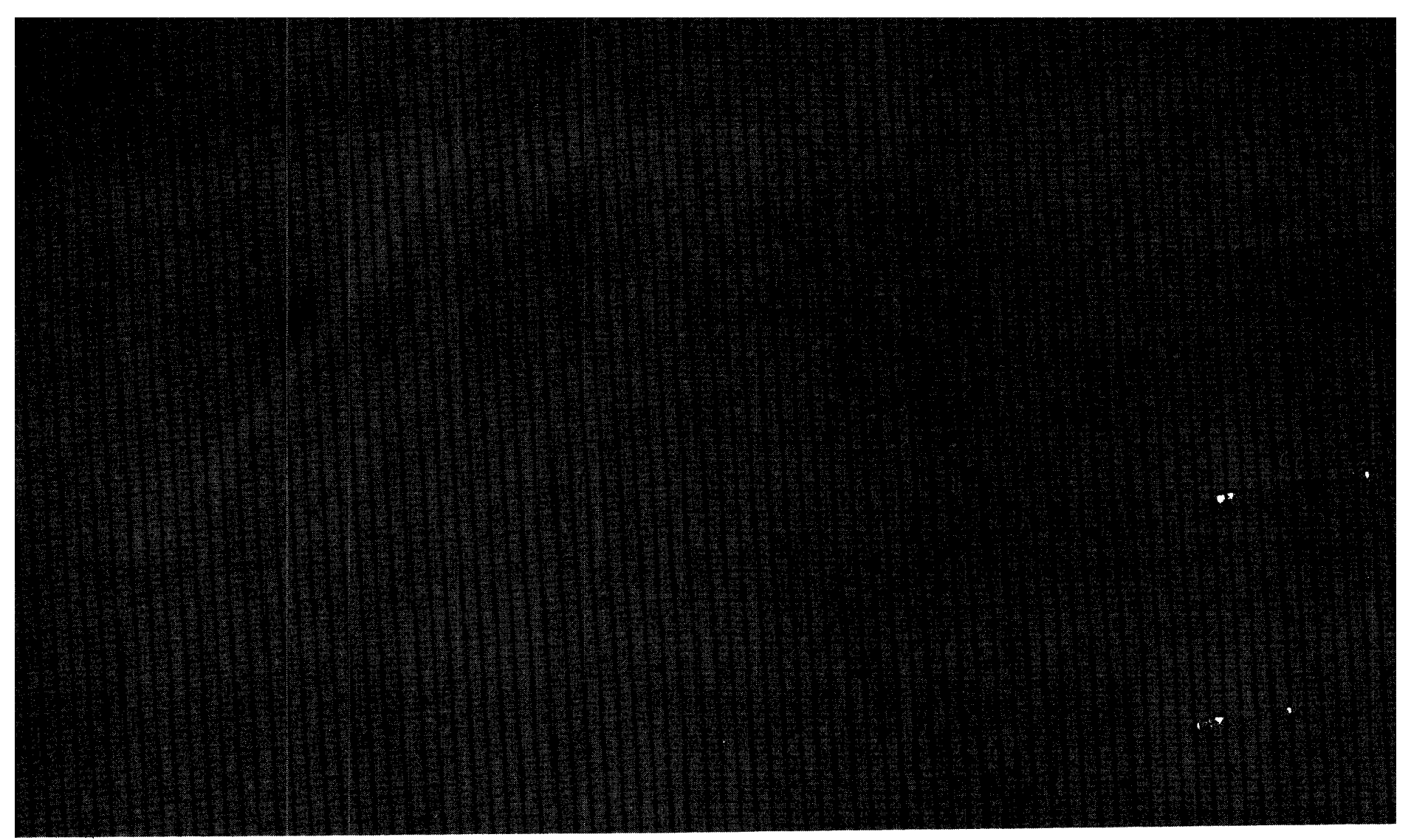

$\ldots \ldots$ 